HYDROLOGIC DATA FOR URBAN STORMWATER STUDIES IN THE DALLAS-FORT WORTH AREA, TEXAS, 1992-94

By Stanley Baldys III, T.H. Raines, B.L. Mansfield, and J.T. Sandlin

U.S. GEOLOGICAL SURVEY

Open-File Report 96-482

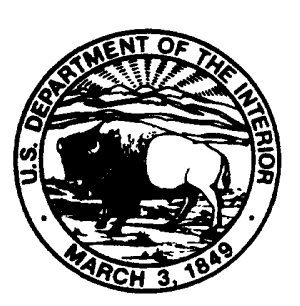

Prepared in cooperation with the NORTH CENTRAL TEXAS COUNCIL OF GOVERNMENTS 


\section{U.S. DEPARTMENT OF THE INTERIOR \\ BRUCE BABBITT, Secretary \\ U.S. GEOLOGICAL SURVEY \\ Gordon P. Eaton, Director}

Any use of trade, product, or firm names is for descriptive purposes only and does not imply endorsement by the U.S. Government.

For additional information write to:

District Chief

U.S. Geological Survey

8011 Cameron Rd.

Austin, TX 78754-3898
Copies of this report can be purchased from:

U.S. Geological Survey

Branch of Information Services

Box 25286

Denver, CO 80225-0286 


\section{CONTENTS}

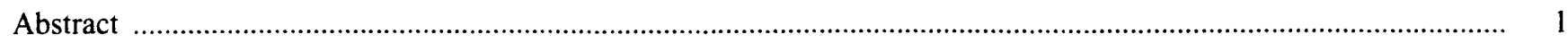

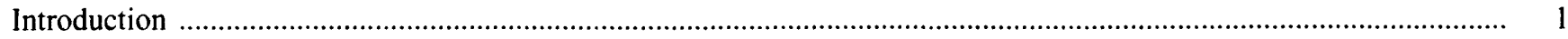

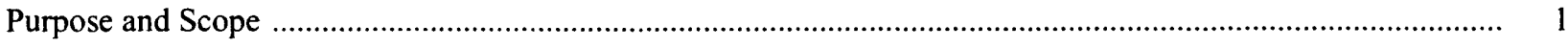

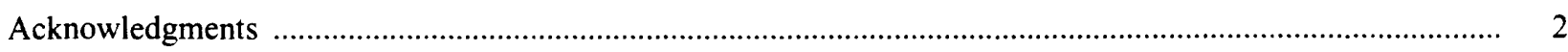

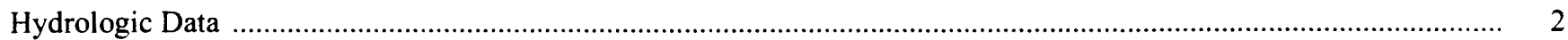

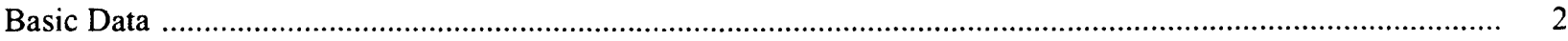

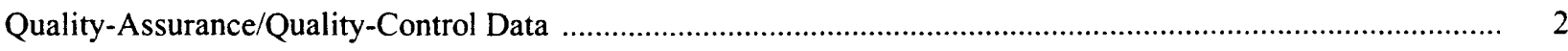

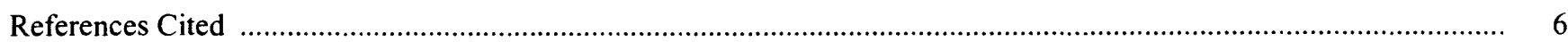

\section{FIGURES}

1. Map showing location of study area and storm-sewer outfall stations in the Dallas-Fort Worth area ................ 3

2. Graph showing accumulated precipitation, discharge, and times of composite- and grab-sample collection at 08061510 Rowlett Creek Outfall at Willow Creek Park, Plano, Texas, April 14, 1993

\section{TABLES}

1. Selected characteristics of streamflow and water-quality data-collection sites, Dallas-Fort Worth area, Texas

2-9. Water-quality data for storm-sewer outfall stations:

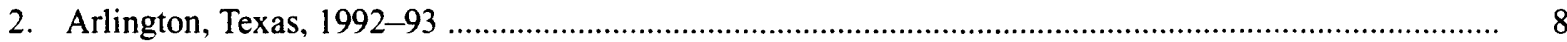

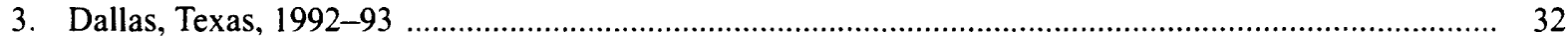

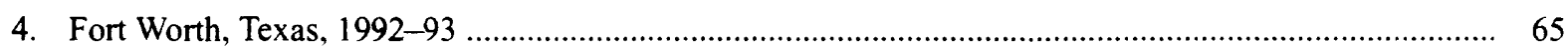

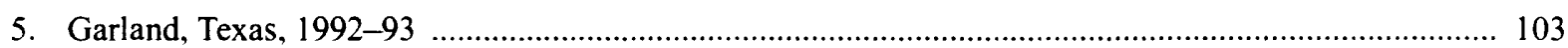

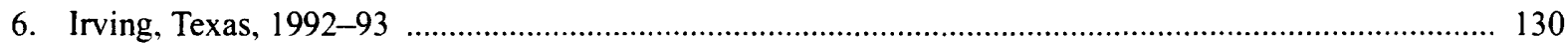

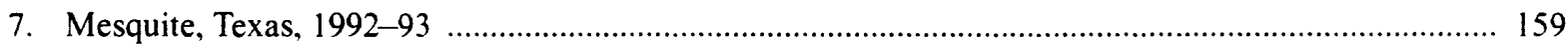

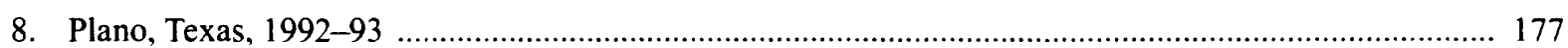

9. Texas Department of Transportation, Dallas and Fort Worth Districts, 1993-94 ................................ 195

10-17. Quality-assurance/quality-control data for storm-sewer outfall stations:

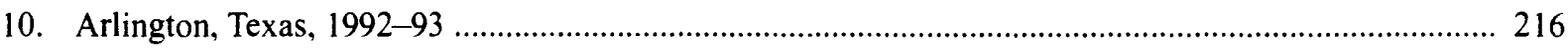

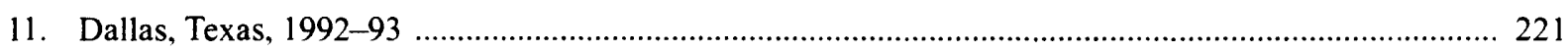

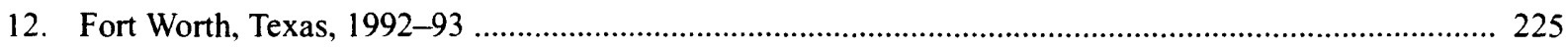

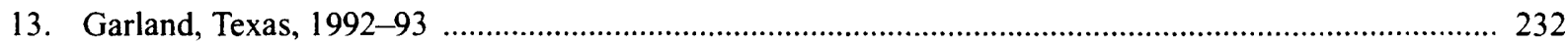

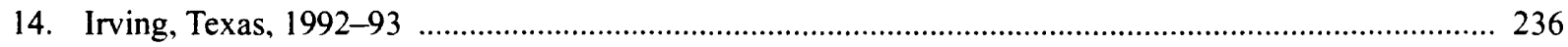

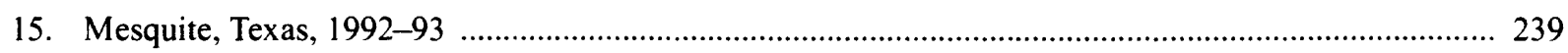

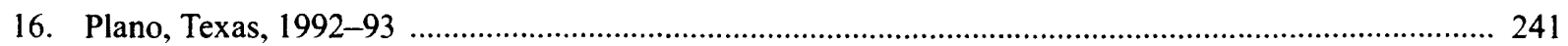

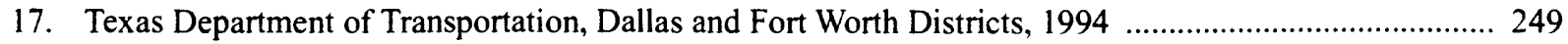




\title{
Hydrologic Data for Urban Stormwater Studies in the Dallas-Fort Worth area, Texas, 1992-94
}

\author{
By Stanley Baldys III, T.H. Raines, B.L. Mansfield, and J.T. Sandlin
}

\section{Abstract}

This report presents precipitation and waterquality data from analyses of 210 samples collected at 30 storm-sewer outfall stations in the Dallas-Fort Worth area, Texas, during February 1992-November 1994. The data were collected to fulfill requirements mandated by the U.S. Environmental Protection Agency to the cities of Arlington, Dallas, Fort Worth, Garland, Irving, Mesquite, and Plano and to the Dallas and Fort Worth Districts of the Texas Department of Transportation to obtain a National Pollution Discharge Elimination System permit. Data were collected at storm-sewer outfall stations in drainage basins classified as singular land use, either residential, commercial, industrial, or highway. Also included are qualityassurance/quality-control data for samples collected in conjunction with the stormwater samples.

\section{INTRODUCTION}

The Federal Water Pollution Control Act (FWPCA) was amended by Congress in 1972 (Public Law 92-500) and 1977 (Clean Water Act) to prohibit the discharge of any pollutant from a point source to U.S. waters unless authorized by a National Pollution Discharge Elimination System (NPDES) permit. Congress again amended the FWPCA in 1987 (Water Quality Act), directing the U.S. Environmental Protection Agency (USEPA) to establish NPDES requirements for point or nonpoint stormwater discharges, including discharges from urban areas (U.S. Environmental Protection Agency, 1992).

According to the Clean Water Act amendments, cities with populations of 100,000 or greater must obtain NPDES permits. The permits will specify waterquality criteria for stormwater discharging from city boundaries into waters of the United States. On Novem- ber 16,1990 , the USEPA published final regulations (U.S. Environmental Protection Agency, 1990) requiring these cities to complete a two-part application process to obtain an NPDES permit. Part one requires dryweather screening of all storm-sewer outfalls in each city and development of a wet-weather characterization plan to assess the quality of stormwater discharges. Part two requires each city to implement the wet-weather characterization plan.

The U.S. Geological Survey (USGS), in cooperation with the North Central Texas Council of Governments (NCTCOG), assisted seven applicant cities in the Dallas-Fort Worth area. Texas (that have populations of 100,000 or greater), and the Texas Department of Transportation (TXDOT) Dallas and Fort Worth Districts (that include population centers of 100,000 or greater) in implementing the wet-weather characterization plan. The USGS conducted sampling of stormwater from drainage basins within the boundaries of Arlington, Dallas, Fort Worth, Garland, Irving, Mesquite, and Plano; and from drainage basins in the TXDOT Dallas and Fort Worth Districts. The stormwater data are part of a USGS data base compiled to develop valuable information about the quantity and quality of stormwater from urban watersheds.

\section{Purpose and Scope}

This report presents hydrologic data for 210 samples collected from February 3, 1992, through November 9,1994 , at 30 storm-sewer outfall stations in the Dallas-Fort Worth area. Also included are qualityassurance/quality-control data for samples collected in conjunction with the stormwater samples. The data are limited to quality-assurance samples collected by the USGS office in Fort Worth and do not include internal quality-assurance/quality-control samples analyzed by the USGS National Water Quality Laboratory (NWQL), Arvada, Colo. 


\section{Acknowledgments}

The authors express their gratitude to the following people for their cooperation and assistance in collecting the stormwater samples during this study: Dr. James Caffey and Pete Cerone, City of Arlington; Larry McDaniel, City of Dallas; Gene Rattan and Brian Camp, City of Fort Worth; Philip Welsch and Mark Nelson, City of Garland; Fred Owen and Tyler Veak, City of Irving; Mike Jones, City of Mesquite; and Dale Hoelting, City of Plano.

\section{HYDROLOGIC DATA}

Thirty storm-sewer drainage basins in the study area were instrumented for streamflow and precipitation measurement and water-quality sampling. The predominant land use in most of the drainage basins is either residential, commercial, industrial, or highway. Sites for storm-sewer outfall stations were selected on the basis of hydraulic, accessibility, and safety factors. The station locations are shown in figure 1 , and selected characteristics of the drainage basin in which each station is located are given in table 1.

The USEPA-approved criteria for stormwater samples were:

1. The storm was preceded by at least 72 hours of dry weather.

2. The depth of precipitation over the basin was greater than 0.20 inch and less than 1.5 inches.

3. The amount and duration of precipitation did not vary from the average amount and duration of precipitation for the area by more than 50 percent.

The initial criterion of greater than 0.10 inch for depth of precipitation was amended based on precipitation records at long-term climatic stations in Dallas and Fort Worth and at the request of the NCTCOG. The criteria for samples were established to ensure a potential accumulation of pollutants during dry weather, an adequate flow for sampling from the storm, and a representative storm in terms of intensity, amount, and duration of precipitation.

\section{Basic Data}

Discharge at each station was computed for each storm by applying gage heights recorded during the storm to a theoretical rating based on the type of flowcontrol device at the station (weir or Palmer Bowles flume). A graph of discharge, accumulated precipitation, and time of sample collection for the storm of Apr. 14, 1993, at 08061510 Rowlett Creek Outfall at Willow Creek Park, Plano, is shown as an example of the flood hydrographs prepared for each storm (fig. 2).

Discharge is measured as the volume of stormwater flow through the storm sewer at the sampling intake during a period of time. Stormwater flow generally begins a few minutes after precipitation falls on the basin and ends after precipitation ends when either base-flow or no-flow conditions resume. The volume of flow that has occurred during this time is summed and reported in millions of gallons per day. Accumulated precipitation, reported in inches, is that precipitation falling on the basin to produce the stormwater flow. The time of sample collection represents the time that stormwater flow began at the measuring site in response to precipitation. The elapsed time of storm, reported in hours, is the time from when stormwater flow began to when it ceased.

Samples were collected for analysis of about 190 water-quality properties and constituents. One stormwater sample from each storm for analysis of about onethird of the water-quality properties and constituents was collected by grab sampling. One stormwater sample from each storm for analysis of about two-thirds of the water-quality properties and constituents was collected by automatic sampling; the automatic samplers composited into one sample a series of samples taken during each storm. Protocols established by the U.S. Environmental Protection Agency (1992) for collection, processing, packaging, and shipping of waterquality samples were followed. Biochemical oxygen demand, fecal coliform, fecal streptococci, and alkalinity were determined at the USGS laboratory in Fort Worth. Most of the constituent concentrations were determined at the NWQL. Concentrations of antimony, cyanide, silver, and thallium were determined at the Rocky Mountain Analytical Laboratory, Denver, Colo. Results of the analyses for all properties and constituents are given in tables 2-9 (at end of report).

\section{Quality-Assurance/Quality-Control Data}

The quality-assurance/quality-control data collected in conjunction with the stormwater samples are given in tables 10-17 (at end of report). USEPA and USGS quality-assurance/quality-control procedures were followed throughout the study. Chain-of-custody procedures were followed for each sample. Equipment 


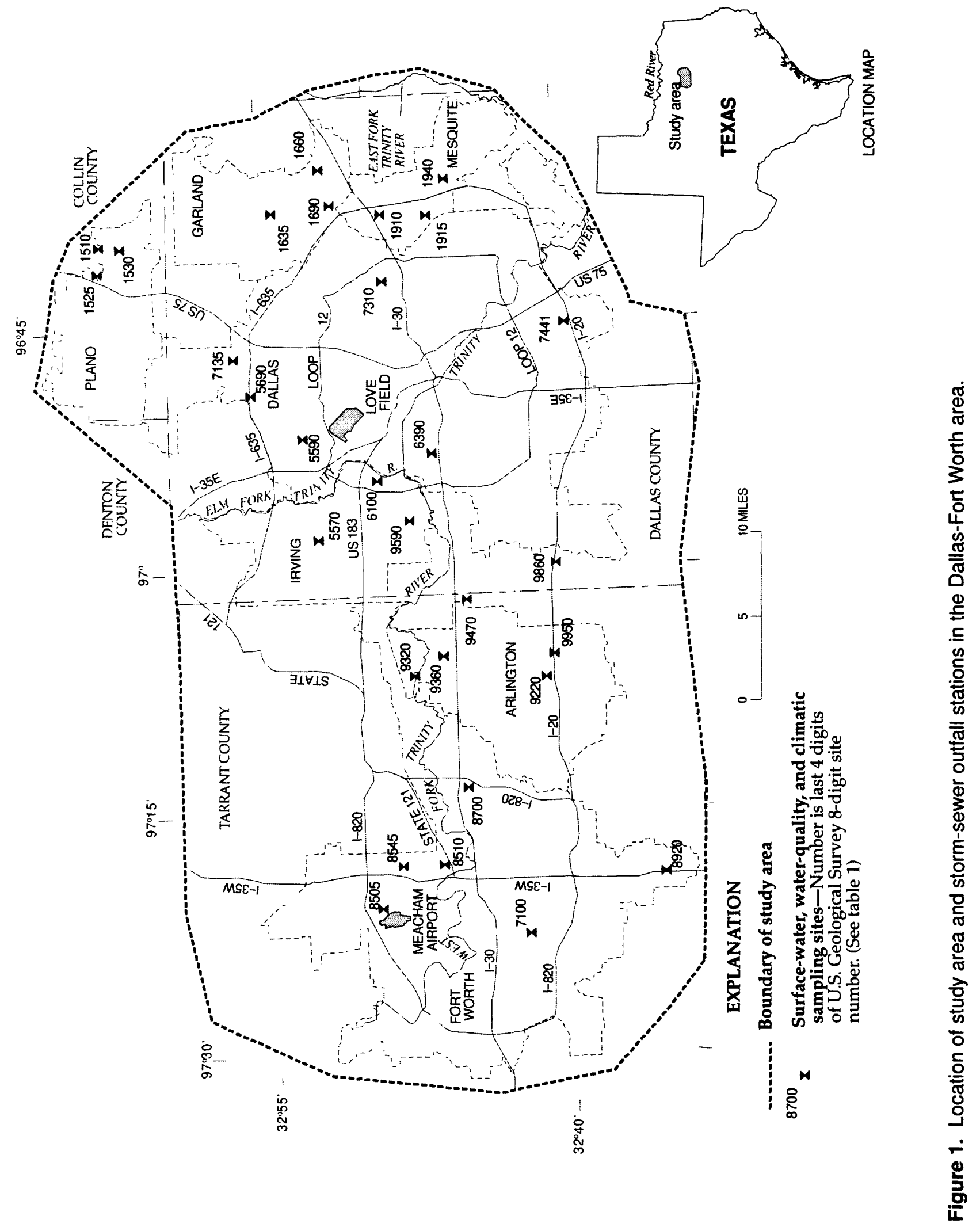

HYDROLOGIC DATA 


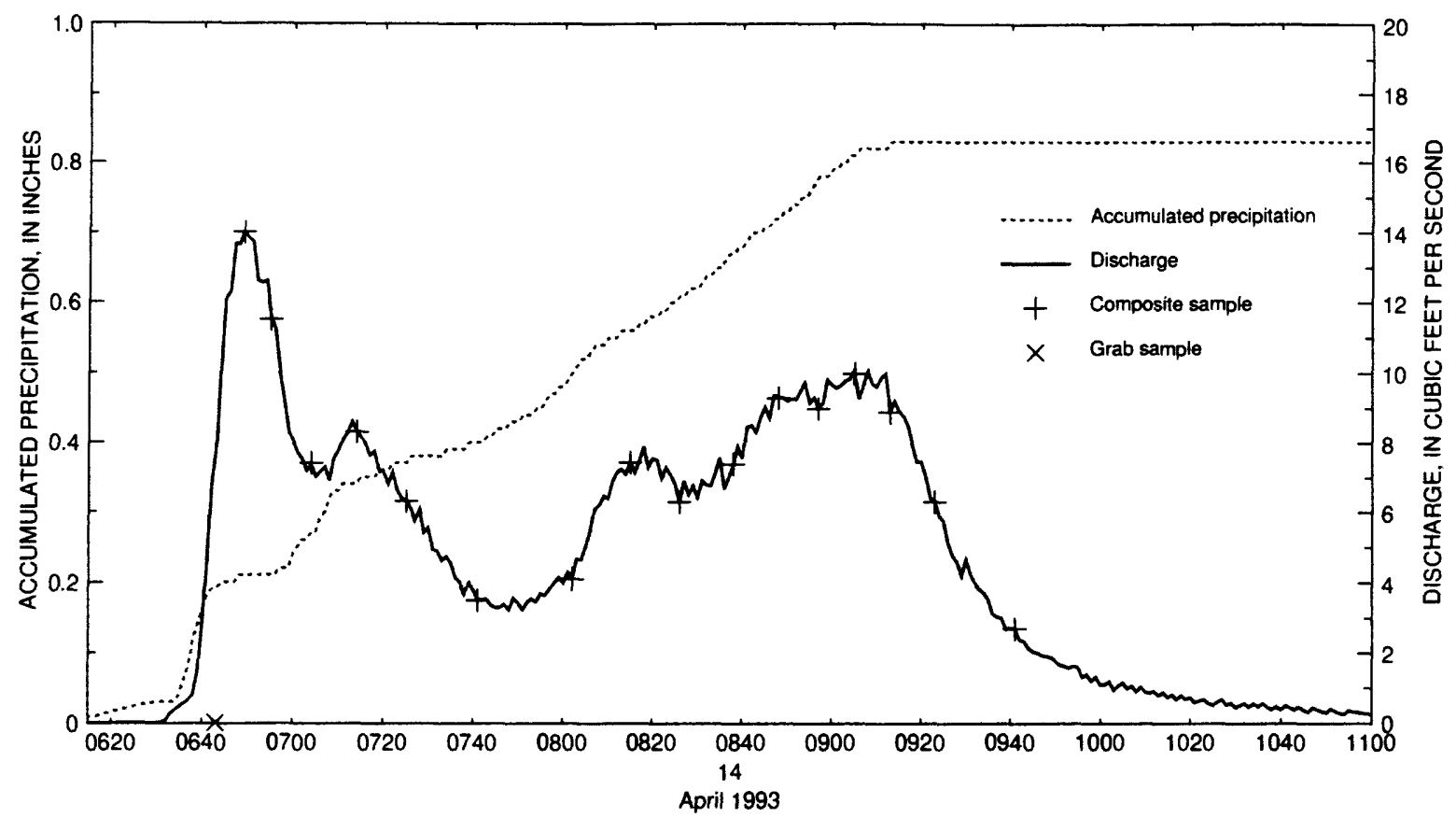

Figure 2. Accumulated precipitation, discharge, and times of composite- and grab-sample collection at 08061510 Rowlett Creek Outfall at Willow Creek Park, Plano, Texas, April 14, 1993.

Table 1. Selected characteristics of streamflow and water-quality data-collection sites, Dallas-Fort Worth area, Texas

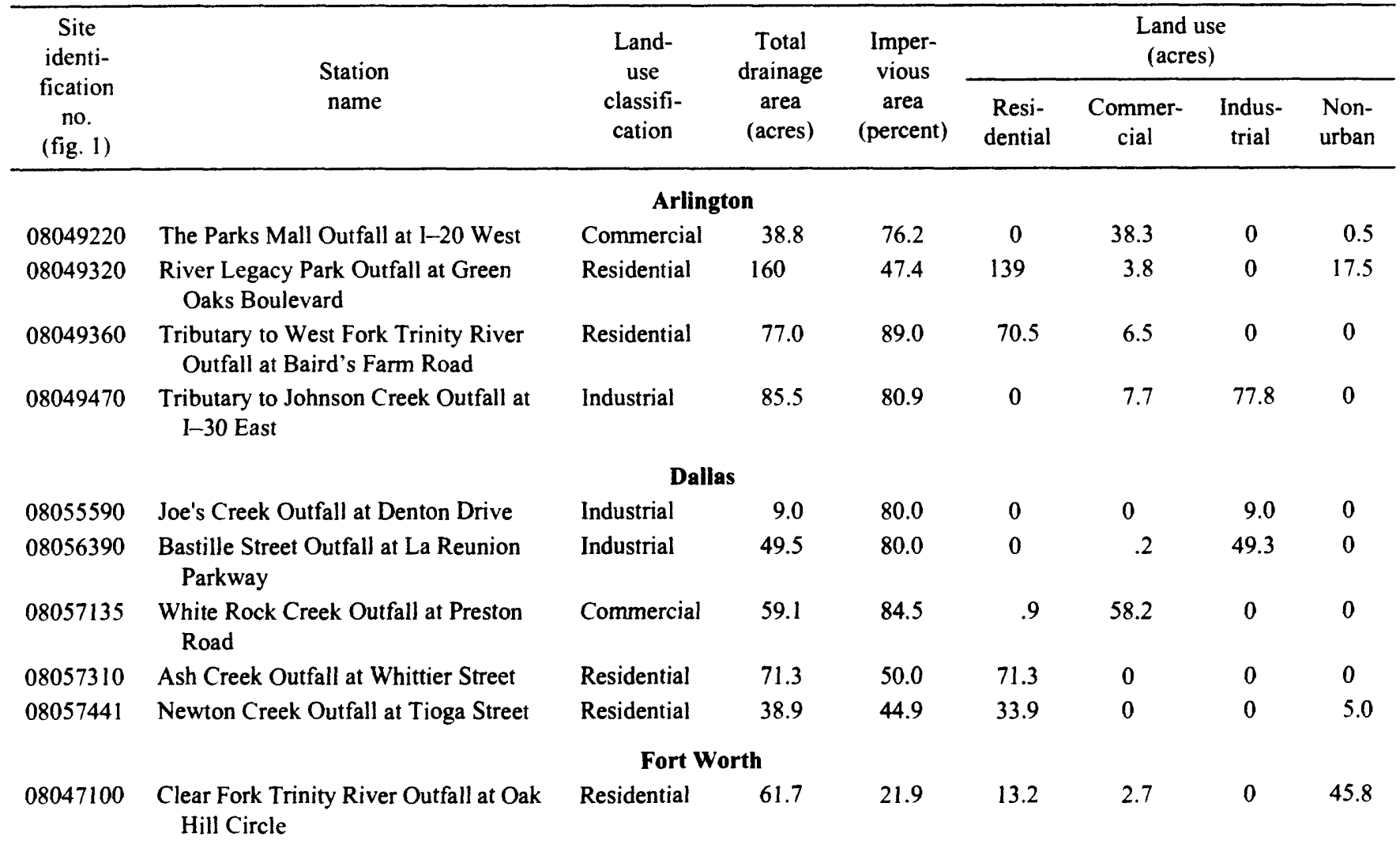


Table 1. Selected characteristics of streamflow and water-quality data-collection sites, Dallas-Fort Worth area, Texas-Continued

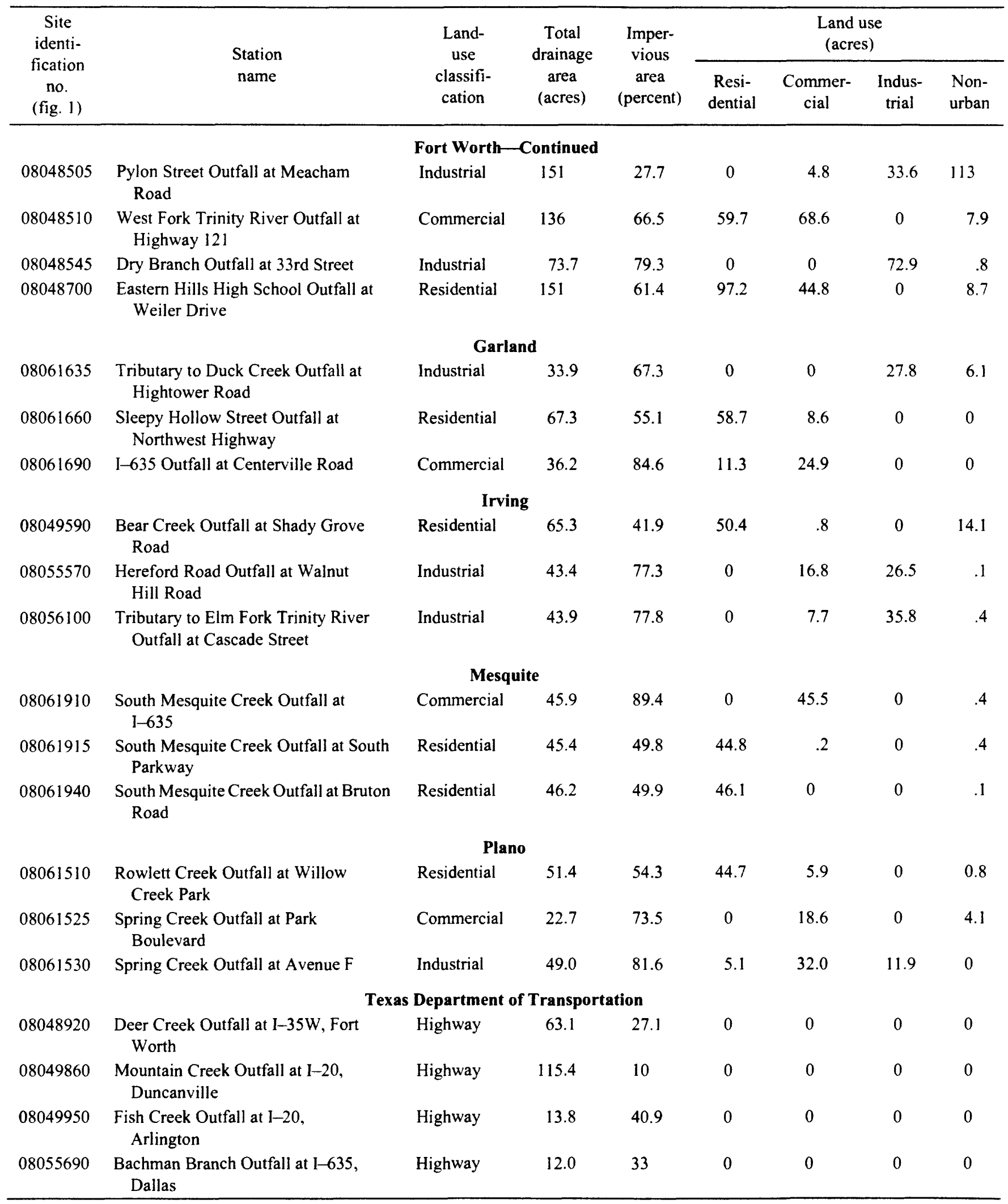


blanks for organic constituents (organic-free water) were passed through the sample-collection equipment at randomly selected stations and analyzed at the NWQL. Matrix spikes for organic constituents (predetermined concentrations of specific constituents) were injected into selected stormwater samples in the field office to determine recovery rates. Reference samples (samples with known concentrations) for nutrient and inorganic constituents were submitted to the NWQL. Trip blanks (to determine if volatile organic samples were contaminated during transit) and laboratory blanks (to determine if organic samples were contaminated in the field during compositing procedures) were submitted to the NWQL. In addition to these quality-assurance/quality-control practices, the NWQL followed similar in-house procedures-these data can be obtained from the NWQL.

\section{REFERENCES CITED}

U.S. Environmental Protection Agency, 1983, Results of the Nationwide Urban Runoff Program-Executive summary: U.S. Environmental Protection Agency Water Planning Division, National Technical Information Service accession no. PB84-185545, 24 p.

1990, National Pollutant Discharge Elimination System permit application regulations for stormwater discharges_Final rule: U.S. Federal Register, v. 55, no. 222 , p. $47,989-48,091$.

1992, NPDES stormwater sampling guidance document: $121 \mathrm{p}$. 
Tables 2-9. Water-quality data for storm-sewer outfall stations

[WY, water year; MGD, million gallons per day; US/CM, microsiemens per centimeter at $25{ }^{\circ} \mathrm{C}$; DEG C, degrees Celsius; MG/L, milligrams per liter; UM-MF, micrometer membrane filter; COLS./100 ML, colonies per 100 milliliters; $\mathrm{K}$, non-ideal colony count; -., not determined; CACO3, calcium carbonate; >, greater than; NO2+NO3, nitrite plus nitrate; EPA, analyzed by U.S. Environmental Protection Agency method; UG/L, micrograms per liter; <, less than; E, estimated] 
Table 2. Water-quality data for storm-sewer outfall stations, Arlington, Texas, 1992-93

08049220 - THE PARKS MALL OUTFALL AT I-20 WEST, ARLINGTON, TX (WY 1993)

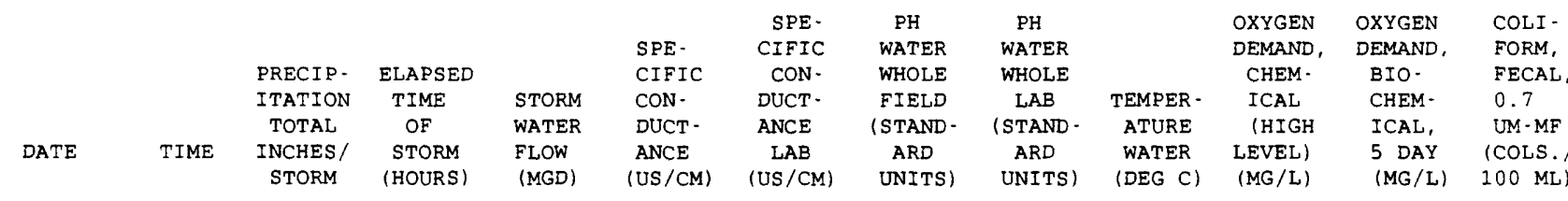

$\begin{array}{ll}\text { OCT } 1992 & \\ 28-29 & 2320 \\ \text { NOV } & \\ 19 \cdot 19 & 0015 \\ \text { DEC } & \\ 09 \cdot 09 & 0030 \\ \text { JAN } 1993 & \\ 09-09 & 0230 \\ \text { JAN } & \\ 28-28 & 1430 \\ \text { FEB } & \\ 24-24 & 1419 \\ \text { MAR } & \\ 11-12 & 1655\end{array}$
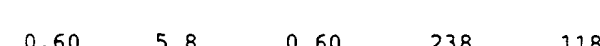

$8.0 \quad 7.5$

21.0

130

$8.3 \quad 36000$

$\begin{array}{lllll}1.5 & 11.0 & 1.4 & 722 & 110\end{array}$

7.9

$7.5 \quad 27.0$

23

4.016000

$\begin{array}{lll}0.31 & 3.5 & 0.30\end{array}$

43

80

7.6

7.9

8.5

41

$5.4 \quad 8700$

0.39

$8.0 \quad 0.32$

$74 \quad 106$

7.9

7.5

9.0

49

$3.0 \quad \mathrm{~K} 3300$

$\begin{array}{lll}0.42 & 9.5 & 0.33\end{array}$

$129 \quad 139$

$\begin{array}{ll}7.5 & 7.8\end{array}$

12.0

$0.66 \quad 5.7$

0.43

106

6.9

7.5

12.0

39

$-\cdot$

K500

$\begin{array}{llll}0.54 & 11.1 & 0.43 & 363\end{array}$

143

7.4

7.6

13.0

33

4.9

K280

41

3.8

K360

\begin{tabular}{|c|c|c|c|c|c|c|c|c|}
\hline & $\begin{array}{r}\text { STREP - } \\
\text { TOCOCCI }\end{array}$ & HARD - & $\begin{array}{l}\text { HARD- } \\
\text { NESS }\end{array}$ & $\begin{array}{c}\text { ALKA - } \\
\text { LINITY }\end{array}$ & $\begin{array}{l}\text { SOLIDS, } \\
\text { SUM OF }\end{array}$ & $\begin{array}{l}\text { RESIDUE } \\
\text { TOTAL }\end{array}$ & $\begin{array}{l}\text { SOLIDS, } \\
\text { RESIDUE }\end{array}$ & \\
\hline & FECAL & NESS & NONCARB & WAT DIS & CONSTI - & AT 105 & AT 180 & CALCIUM \\
\hline & $\begin{array}{l}\text { KF AGAR } \\
\text { ICOLS. }\end{array}$ & $\begin{array}{l}\text { TOTAL } \\
\text { (MG/L } \\
\text { AS }\end{array}$ & $\begin{array}{l}\text { DISSOLV } \\
\text { FLD. AS }\end{array}$ & $\begin{array}{l}\text { FIX END } \\
\text { FIELD } \\
\text { CACO3 }\end{array}$ & $\begin{array}{c}\text { TUENTS, } \\
\text { DI S - } \\
\text { SOLVED }\end{array}$ & $\begin{array}{l}\text { DEG. C, } \\
\text { SUS - }\end{array}$ & $\begin{array}{l}\text { DEG. C } \\
\text { DIS - }\end{array}$ & $\begin{array}{l}\text { DIS - } \\
\text { SOLVED }\end{array}$ \\
\hline DATE & $\begin{array}{c}\text { PER } \\
200 \mathrm{ML}\rangle\end{array}$ & $\begin{array}{c}\mathrm{AS} \\
\mathrm{CACO} 31\end{array}$ & $\begin{array}{c}\mathrm{CACO} 3 \\
(\mathrm{MG} / \mathrm{L})\end{array}$ & $\begin{array}{l}\mathrm{CACO} 3 \\
(\mathrm{MG} / \mathrm{L})\end{array}$ & $\begin{array}{l}\text { SOLVED } \\
\text { (MG/L }\}\end{array}$ & $\begin{array}{l}\text { PENDED } \\
(M G / L)\end{array}$ & $\begin{array}{l}\text { SOLVED } \\
\text { (MG/L) }\end{array}$ & $\begin{array}{c}(\mathrm{MG} / \mathrm{L} \\
\mathrm{AS} \mathrm{CA})\end{array}$ \\
\hline
\end{tabular}

OCT 1992

$\begin{array}{lr}\text { OCT } 1992 & \\ 28-29 & 25000 \\ \text { NOV } & \\ 19-19 & 16000 \\ \text { DEC } & \\ 09-09 & 82000 \\ \text { JAN } 1993 & \\ 09-09 & 27000 \\ \text { JAN } & \\ 28-28 & \mathrm{~K} 1700 \\ \text { FEB } & \\ 24-24 & >330000 \\ \text { MAR } & \\ 11-12 & 5400\end{array}$

$\begin{array}{rrr}45 & 20 & 25 \\ 37 & 4 & 33 \\ 25 & 2 & 23 \\ 49 & 18 & 31 \\ 49 & 5 & 44 \\ 37 & 6 & 31 \\ 55 & 12 & 43\end{array}$

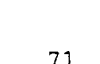

$\begin{array}{rrrr}71 & 62 & 70 & 17 \\ 51 & 3 & 62 & 14 \\ 37 & 40 & 37 & 9.7 \\ - & 72 & - & 19 \\ 71 & 15 & 74 & 19 \\ 55 & 10 & 44 & 14 \\ 77 & 16 & 87 & 21\end{array}$

MAGNE -

SIUM, SODIUM,

DIS - DIS.

SOLVED SOLVED

(MG/L (MG/L

AS MG) AS NA)

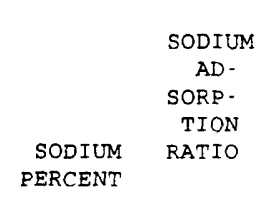

0.52

$0.43 \quad 2.5$

0.26

1.3

0.421 .3

0.47

2.9

0.39

3.1

$0.55 \quad 3.9$

$\begin{array}{ll}12 & 0.2 \\ 13 & 0.2 \\ 10 & 0.1 \\ 5 & 0.1 \\ 11 & 0.2 \\ 15 & 0.2 \\ 13 & 0.2\end{array}$

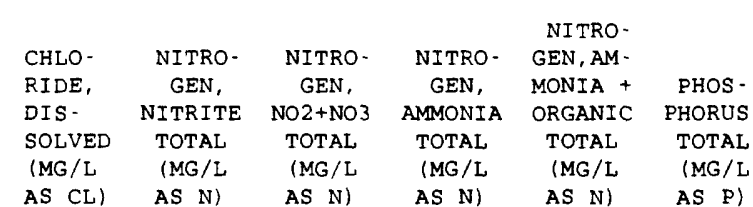

P
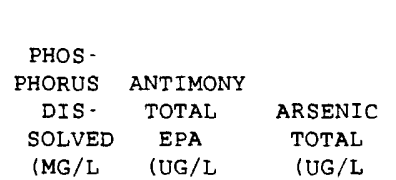

BERYL -

LIUM,

TOTAL

RECOV -

$\begin{array}{llllcccr}\text { DIS - } & \text { DIS - } & \text { DIS - } & \text { NITRITE } & \text { NO2+NO3 } & \text { AMMONIA } & \text { ORGANIC } & \text { PHORUS } \\ \text { SOLVED } & \text { SOLVED } & \text { SOLVED } & \text { TOTAL } & \text { TOTAL } & \text { TOTAL } & \text { TOTAL } & \text { TOTAL } \\ \text { (MG/L } & \text { (MG/L } & \text { (MG/L } & \text { (MG/L } & \text { (MG/L } & \text { (MG/L } & \text { (MG/L } & \text { (MG/L }\end{array}$

AS K) AS SO4) AS CL) AS N) AS N) AS N) AS N

AS P)

(UG/L

(UG/L

ERABLE

(UG/L

AS BE)

$\begin{array}{lll}\text { OCT } 1992 & & \\ 28.29 & 1.1 & 13 \\ \text { NOV } & & \\ 19-19 & 0.80 & 7.3 \\ \text { DEC } & & \\ 09.09 & 0.70 & 4.9 \\ \text { JAN } 1993 & & \\ 09-09 & 0.80 & \ldots \\ \text { JAN } & & \\ 28.28 & 0.90 & 11 \\ \text { FEB } & & \\ 24.24 & 0.70 & 7.8 \\ \text { MAR } & & 16 \\ 11-12 & 0.90 & 16\end{array}$

$\begin{array}{llllllllll}3.2 & 0.030 & 0.950 & 0.430 & 1.8 & 0.200 & 0.080 & <10.0 & 2 & <10 \\ 2.4 & 0.050 & 0.390 & 0.080 & 0.40 & 0.060 & 0.010 & <20.0 & <1 & <10 \\ 1.1 & 0.050 & 0.380 & 0.190 & 0.60 & 0.090 & 0.040 & <10.0 & 1 & <10 \\ \ldots & \ldots & 0.610 & \ldots & 0.50 & 0.060 & <0.010 & <10.0 & <1 & \cdots \\ 2.1 & \ldots & 0.340 & \ldots & 0.60 & 0.070 & 0.030 & <20.0 & 2 & <10 \\ 4.2 & \ldots & 0.900 & \ldots & 0.80 & 0.050 & 0.040 & <10.0 & <1 & <10 \\ 2.5 & \ldots & 0.540 & \ldots & 0.80 & 0.050 & 0.040<10.0 & 1 & <10\end{array}$


Table 2. Water-quality data for storm-sewer outfall stations, Arlington, Texas, 1992-93-Continued

08049220 - THE PARKS MALL OUTFALL AT I-20 WEST, ARLINGTON, TX (WY 1993)-Continued

\begin{tabular}{|c|c|c|c|c|c|c|c|c|c|c|c|c|}
\hline DATE & $\begin{array}{l}\text { CADMIUM } \\
\text { TOTAL } \\
\text { RECOV- } \\
\text { ERABLE } \\
\text { (UG/L } \\
\text { AS CD) }\end{array}$ & $\begin{array}{l}\text { CHRO- } \\
\text { MIUM, } \\
\text { TOTAL } \\
\text { RECOV- } \\
\text { ERABLE } \\
\text { (UG/L } \\
\text { AS CR) }\end{array}$ & $\begin{array}{l}\text { COPPER, } \\
\text { TOTAL } \\
\text { RECOV- } \\
\text { ERABLE } \\
\text { (UG/L } \\
\text { AS CU) }\end{array}$ & $\begin{array}{c}\text { CYANIDE } \\
\text { TOTAL } \\
\text { EPA } \\
\text { (MG/L } \\
\text { AS CN) }\end{array}$ & $\begin{array}{c}\text { CYANIDE } \\
\text { TOTAL } \\
\text { (MG/L } \\
\text { AS CN) }\end{array}$ & $\begin{array}{l}\text { LEAD, } \\
\text { TOTAL } \\
\text { RECOV - } \\
\text { ERABLE } \\
\text { (UG/L } \\
\text { AS PB) }\end{array}$ & $\begin{array}{l}\text { MERCURY } \\
\text { TOTAL } \\
\text { RECOV- } \\
\text { ERABLE } \\
\text { (UG/L } \\
\text { AS HG) }\end{array}$ & $\begin{array}{l}\text { NICKEL, } \\
\text { TOTAL } \\
\text { RECOV- } \\
\text { ERABLE } \\
\text { (UG/L } \\
\text { AS NI) }\end{array}$ & $\begin{array}{l}\text { SELE - } \\
\text { NIUM, } \\
\text { TOTAL } \\
\text { (UG/L } \\
\text { AS SE) }\end{array}$ & $\begin{array}{l}\text { SILVER, } \\
\text { TOTAL } \\
\text { RECOV- } \\
\text { ERABLE } \\
\text { (UG/L } \\
\text { AS AG) }\end{array}$ & $\begin{array}{l}\text { SILVER, } \\
\text { TOTAL } \\
\text { RECOV- } \\
\text { ERABLE } \\
\text { EPA } \\
\text { (UG/L } \\
\text { AS AG) }\end{array}$ & $\begin{array}{l}\text { THAL- } \\
\text { LIUM, } \\
\text { TOTAL } \\
\text { (UG/L } \\
\text { AS TL) }\end{array}$ \\
\hline СT 1992 & & & & & & & & & & & & \\
\hline $\begin{array}{l}28-29 \\
\text { Nov }\end{array}$ & $<1$ & 13 & 9 & 0.013 & 0.010 & 65 & $<0.10$ & 4 & $<2$ & $<1$ & $<0.500$ & $<5$ \\
\hline${ }_{\mathrm{DEC}}^{19-19}$ & $<1$ & $<1$ & 2 & $<0.010$ & $<0.010$ & 16 & $<0.10$ & $<1$ & $<2$ & $<1$ & $<0.500$ & $<5$ \\
\hline $\begin{array}{c}09-09 \\
\text { JAN } 1993\end{array}$ & $<1$ & $<1$ & 4 & $<0.010$ & $<0.010$ & 30 & $<0.10$ & 2 & $<2$ & $<1$ & $<0.500$ & $<10$ \\
\hline $\begin{array}{l}09-09 \\
\text { JAN }\end{array}$ & - & $\cdots$ & $\cdots$ & $<0.010$ & $<0.010$ & $\cdots$ & - & . & $<2$ & - & $<0.500$ & $<5$ \\
\hline${ }_{F E B}^{28-28}$ & $<1$ & 8 & 4 & $<0.010$ & $<0.010$ & 34 & $<0.10$ & 2 & $<2$ & $<1$ & $<0.500$ & $<10$ \\
\hline $24-24$ & $<1$ & 7 & 4 & $<0.010$ & $<0.010$ & 19 & $<0.10$ & 2 & $<2$ & $<1$ & $<0.500$ & $<50$ \\
\hline IAR & & & & & & & & & & & & \\
\hline $11 \cdot 12$ & $<1$ & 4 & 3 & $<0.010$ & $<0.010$ & 12 & $<0.10$ & 2 & $<2$ & $<1$ & $<0.500$ & $<5$ \\
\hline
\end{tabular}

\begin{tabular}{|c|c|c|c|c|c|c|c|c|c|c|c|c|}
\hline & & & OIL AND & & & & & & METHANE & & & BENZENE \\
\hline & ZINC, & & GREASE, & & & & & BROMO- & BROMO & & BENZENE & SEC \\
\hline & TOTAL & CARBON, & TOTAL & & & & & BENZENE & CHLORO- & & N-BUTYL & BUTYL - \\
\hline & RECOV - & ORGANIC & RECOV. & & ACRO - & ACRYLO. & & WATER, & WAT & BROMO - & WATER & WATER \\
\hline & ERABLE & TOTAL & GRAVI - & PHENOLS & LEIN & NITRILE & BENZENE & WHOLE, & UNFLTRD & FORM & UNFLTRD & UNFLTRD \\
\hline & (UG/L & (MG/L & METRIC & TOTAL & TOTAL & TOTAL & TOTAL & TOTAL & REC & TOTAL & REC & REC \\
\hline & AS ZN) & AS C) & (MG /L) & (UG/L) & (UG/L) & (UG/L) & (UG/L) & (UG/L) & (UG/L) & (UG/L) & $(\mathrm{UG} / \mathrm{L})$ & (UG/L) \\
\hline
\end{tabular}

OCT 1992

28. 29

NOV

$19 \cdot 19$

$110 \quad 24$

09.09

JAN 1993

$09 \cdot 09$

JAN

$28-28$

FEB

$24-24$

$\begin{array}{lll}110 & 24 & <1 \\ 40 & 6.5 & <1 \\ 50 & 9.2 & 6\end{array}$

(UG/L)

UG / L)

(UG/L)

UG/L)

(UG/L)

(UG/L)

(UG/L)

$\begin{array}{cccccc}<0.2 & <0.2 & \ldots & <0.2 & <0.20 & <0.20 \\ \cdots & \cdots & \ldots & \ldots & \ldots & \cdots \\ 0.8 & <0.2 & <0.20 & <0.2 & <0.20 & <0.20 \\ <0.2 & <0.2 & <0.20 & <0.2 & <0.20 & <0.20 \\ <0.2 & <0.2 & <0.20 & <0.2 & <0.20 & <0.20 \\ <0.2 & <0.2 & <0.20 & <0.2 & <0.20 & <0.20 \\ <0.2 & <0.2 & <0.20 & <0.2 & <0.20 & <0.20\end{array}$

11- 12

$\begin{array}{ll} & 8.5\end{array}$

$\begin{array}{lll}50 & 9.9 & 2\end{array}$

$\begin{array}{lll}40 & 9.7<1\end{array}$

$\begin{array}{rrr}9 & <20 & <20 \\ <1 & \cdots & - \\ 6 & <20 & <20 \\ 2 & <20 & <20 \\ 7 & <20 & <20 \\ 12 & <20 & <20 \\ 13 & <20 & <20\end{array}$

$<0.2$

$<0$.

$\begin{array}{cclcc}\text { BENZENE } & & & \\ \text { TERT - } & \text { CARBON - } & & \text { CHLORO- } \\ \text { BUTYL - } & \text { TETRA- } & & \text { DI- } \\ \text { WATER } & \text { CHLO- } & \text { CHLORO- } & \text { BROMO- } & \text { CHLORO- } \\ \text { UNFLTRD } & \text { RIDE } & \text { BENZENE } & \text { METHANE } & \text { ETHANE } \\ \text { REC } & \text { TOTAL } & \text { TOTAL } & \text { TOTAL } & \text { TOTAL } \\ \text { (UG } / L) & \text { (UG/L) } & \text { (UG/L) } & \text { (UG/L) } & \text { (UG/L) }\end{array}$

2 -

CHLORO -

ETHYL -

VINYL -

ETHER

TOTAL

(UG/L)

\begin{tabular}{|c|c|c|c|c|c|}
\hline \multirow[b]{4}{*}{ CHLORO - } & & $0-$ & & DIBROMO & DI - \\
\hline & & CHLORO - & TOLUENE & CHLORO- & BROMO - \\
\hline & METHYL - & TOLUENE & P-CHLOR & PROPANE & METHANE \\
\hline & CHLO- & WATER & WATER & WATER & WATER \\
\hline FORM & RIDE & WHOLE & UNFLTRD & WHOLE & WHOLE \\
\hline $\begin{array}{l}\text { TOTAL } \\
\text { (UG/L) }\end{array}$ & $\begin{array}{l}\text { TOTAL } \\
\text { (UG/L) }\end{array}$ & $\begin{array}{l}\text { TOTAL } \\
\text { (UG/L) }\end{array}$ & $\begin{array}{c}\text { REC } \\
(U G / L)\end{array}$ & $\begin{array}{l}\text { TOT . REC } \\
\text { (UG } / \mathrm{L})\end{array}$ & $\begin{array}{l}\text { RECOVER } \\
\text { (UG/L) }\end{array}$ \\
\hline
\end{tabular}

\begin{tabular}{|c|c|c|c|c|c|c|c|c|c|c|c|c|}
\hline \multicolumn{13}{|l|}{ OCT 1992} \\
\hline $28-29$ & $<0.20$ & $<0.2$ & $<0.20$ & $<0.2$ & $<0.2$ & $<1.0$ & $<0.2$ & $<0.2$ & $<0.2$ & $<0.20$ & $<1.0$ & $<0.2$ \\
\hline \multicolumn{13}{|l|}{ Nov } \\
\hline $19-19$ & $\cdots$ & $\cdots$ & $\cdots$ & $\cdots$ & $\cdots$ & $\cdots$ & $\cdots$ & $\cdots$ & $\cdots$ & $\cdots$ & $\cdots$ & $\cdots$ \\
\hline \multicolumn{13}{|l|}{$\mathrm{DEC}$} \\
\hline 09.09 & $<0.20$ & $<0.2$ & $<0.20$ & $<0.2$ & $<0.2$ & $<1.0$ & $<0.2$ & $<0.2$ & $<0.2$ & $<0.20$ & $<1.0$ & $<0.2$ \\
\hline \multicolumn{13}{|l|}{ JAN 1993} \\
\hline $09-09$ & $<0.20$ & $<0.2$ & $<0.20$ & $<0.2$ & $<0.2$ & $<1.0$ & $<0.2$ & $<0.2$ & $<0.2$ & $<0.20$ & $<1.0$ & $<0.2$ \\
\hline \multicolumn{13}{|l|}{ JAN } \\
\hline $28-28$ & $<0.20$ & $<0.2$ & $<0.20$ & $<0.2$ & $<0.2$ & $<1.0$ & $<0.2$ & $<0.2$ & $<0.2$ & $<0.20$ & $<1.0$ & $<0.2$ \\
\hline \multicolumn{13}{|l|}{ FEB } \\
\hline $24-24$ & $<0.20$ & $<0.2$ & $<0.20$ & $<0.2$ & $<0.2$ & $<1.0$ & $<0.2$ & $<0.2$ & $<0.2$ & $<0.20$ & $<1.0$ & $<0.2$ \\
\hline \multicolumn{13}{|l|}{ MAR } \\
\hline $11-12$ & $<0.20$ & $<0.2$ & $<0.20$ & $<0.2$ & $<0.2$ & $<1.0$ & $<0.2$ & $<0.2$ & $<0.2$ & $<0.20$ & $<1.0$ & $<0.2$ \\
\hline
\end{tabular}


Table 2. Water-quality data for storm-sewer outfall stations, Arlington, Texas, 1992-93-Continued

08049220 - THE PARKS MALL OUTFALL AT I-20 WEST, ARLINGTON, TX (WY 1993)-Continued

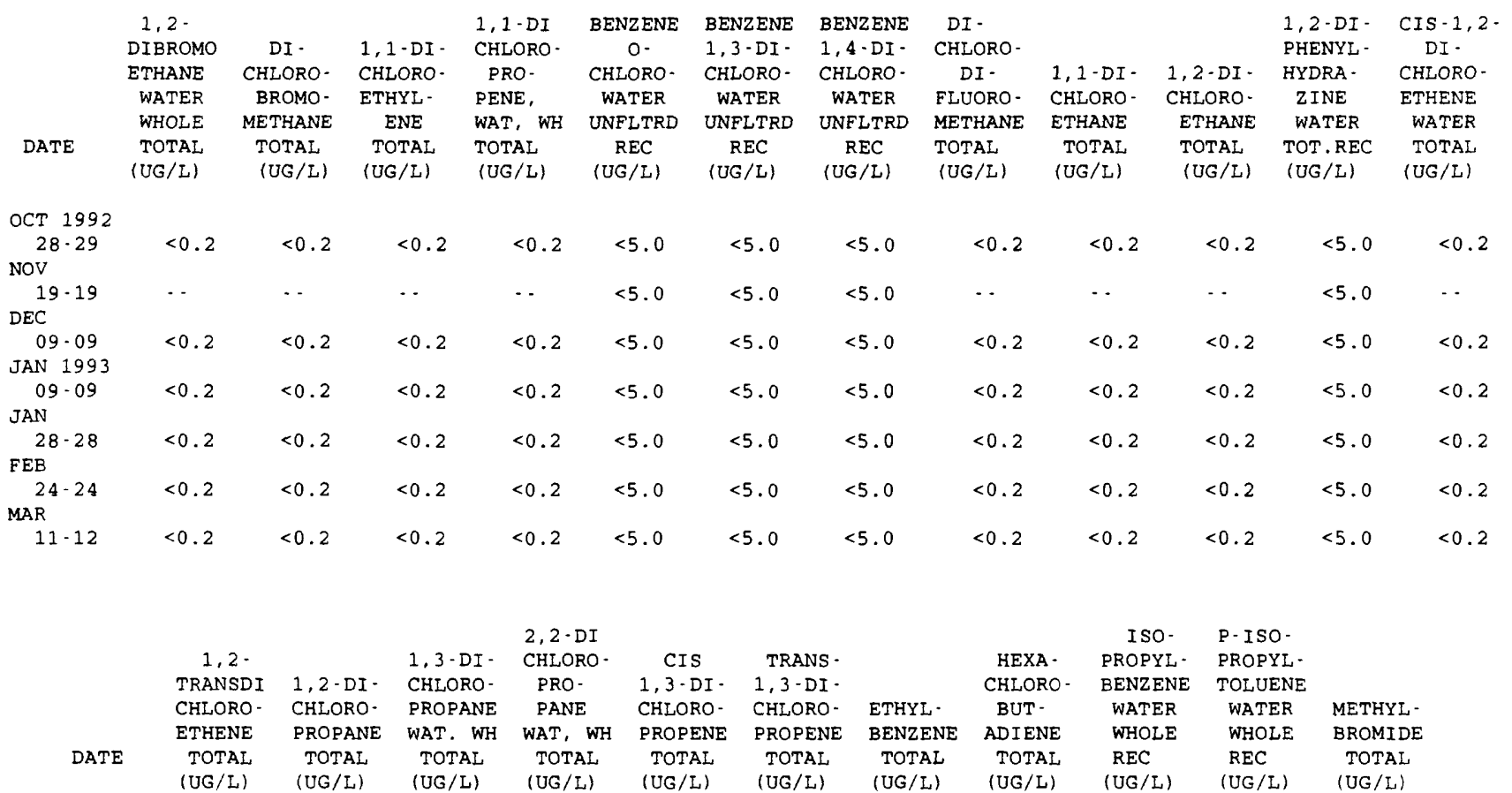

\begin{tabular}{|c|c|c|c|c|c|c|c|c|c|c|c|}
\hline \multicolumn{12}{|l|}{ OCT 1992} \\
\hline $28-29$ & $<0.2$ & $<0.2$ & $<0.2$ & $<0.2$ & $<0.2$ & $<0.2$ & $<0.2$ & $<5.0$ & $<0.20$ & $<0.20$ & $<0.2$ \\
\hline \multicolumn{12}{|l|}{ Nov } \\
\hline $19-19$ & $\cdots$ & $\cdots$ & $\cdots$ & $\cdots$ & -- & $\cdots$ & $-\cdot$ & $<5.0$ & $\cdots$ & $\cdots$ & $\cdots$ \\
\hline \multicolumn{12}{|l|}{$\mathrm{DEC}$} \\
\hline 09.09 & $<0.2$ & $<0.2$ & $<0.2$ & $<0.2$ & $<0.2$ & $<0.2$ & $<0.2$ & $<5.0$ & $<0.20$ & $<0.20$ & $<0.2$ \\
\hline \multicolumn{12}{|l|}{ JAN 1993} \\
\hline $09-09$ & $<0.2$ & $<0.2$ & $<0.2$ & $<0.2$ & $<0.2$ & $<0.2$ & $<0.2$ & $<5.0$ & $<0.20$ & $<0.20$ & $<0.2$ \\
\hline \multicolumn{12}{|l|}{ JAN } \\
\hline $28-28$ & $<0.2$ & $<0.2$ & $<0.2$ & $<0.2$ & $<0.2$ & $<0.2$ & $<0.2$ & $<5.0$ & $<0.20$ & $<0.20$ & $<0.2$ \\
\hline \multicolumn{12}{|l|}{ FEB } \\
\hline $24-24$ & $<0.2$ & $<0.2$ & $<0.2$ & $<0.2$ & $<0.2$ & $<0.2$ & $<0.2$ & $<5.0$ & $<0.20$ & 0.90 & $<0.2$ \\
\hline \multicolumn{12}{|l|}{ MAR } \\
\hline $11 \cdot 12$ & $<0.2$ & $<0.2$ & $<0.2$ & $<0.2$ & $<0.2$ & $<0.2$ & $<0.2$ & $<5.0$ & $<0.20$ & $<0.20$ & $<0.2$ \\
\hline
\end{tabular}


Table 2. Water-quality data for storm-sewer outfall stations, Arlington, Texas, 1992-93-Continued

08049220 - THE PARKS MALL OUTFALL AT I-20 WEST, ARLINGTON, TX (WY 1993)-Continued

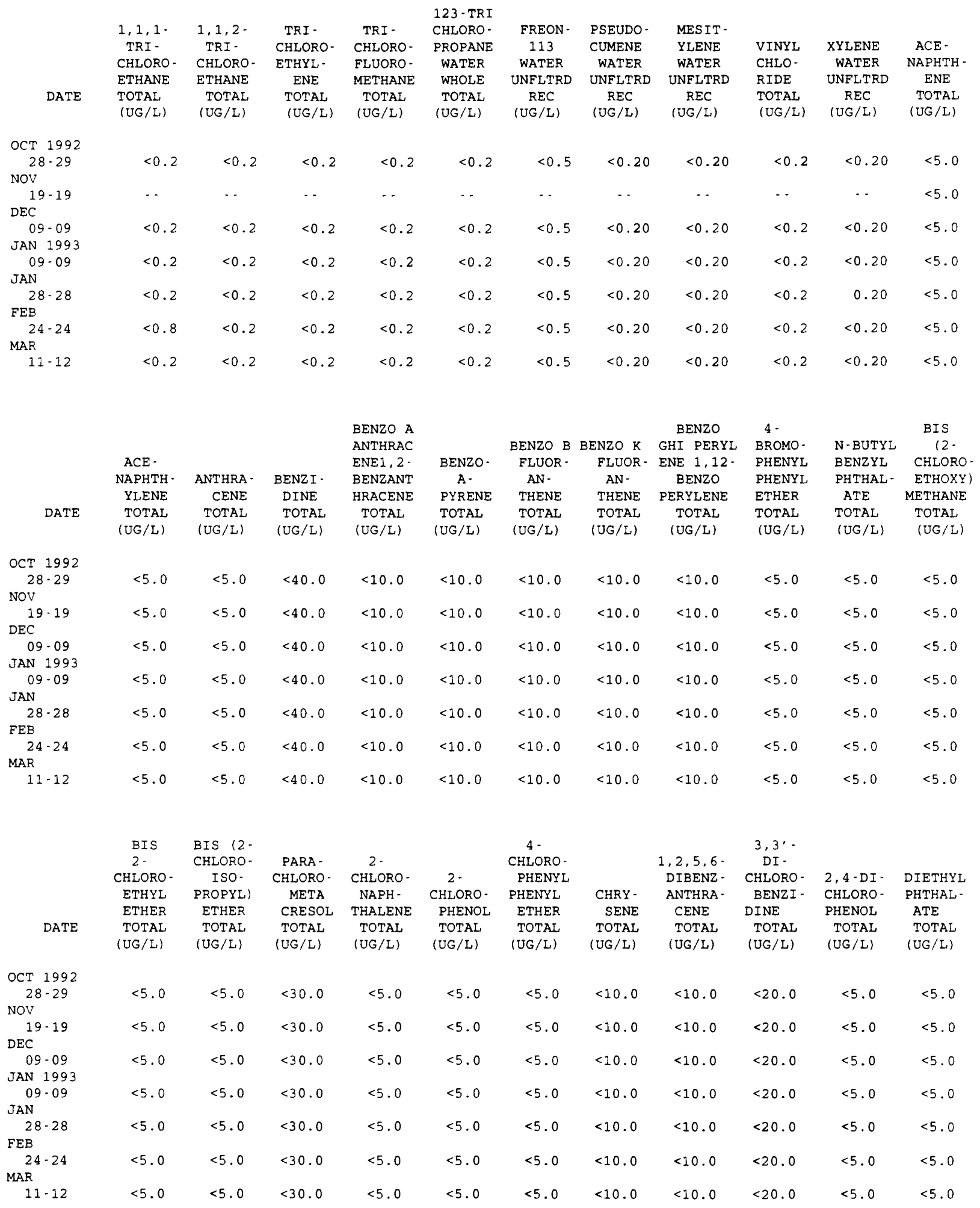


Table 2. Water-quality data for storm-sewer outfall stations, Arlington, Texas, 1992-93-Continued

08049220 - THE PARKS MALL OUTFALL AT I-20 WEST, ARLINGTON, TX (WY 1993)-Continued

\begin{tabular}{|c|c|c|c|c|c|c|c|c|c|c|c|}
\hline DATE & $\begin{array}{l}\text { DI - } \\
\text { METHYL } \\
\text { PHTHAL- } \\
\text { ATE } \\
\text { TOTAL } \\
\text { (UG/L) }\end{array}$ & $\begin{array}{l}2,4 \text { - DI - } \\
\text { METHYL - } \\
\text { PHENOL } \\
\text { TOTAL } \\
(U G / L)\end{array}$ & $\begin{array}{c}\text { DI - N- } \\
\text { BUTYL } \\
\text { PHTHAL- } \\
\text { ATE } \\
\text { TOTAL } \\
(\text { UG/L) }\end{array}$ & $\begin{array}{c}4,6- \\
\text { DINITRO- } \\
\text { ORTHO- } \\
\text { CRESOL } \\
\text { TOTAL } \\
\text { (UG/L) }\end{array}$ & $\begin{array}{c}2,4,- \\
\text { DI - } \\
\text { NITRO- } \\
\text { PHENOL } \\
\text { TOTAL } \\
\text { (UG/L) }\end{array}$ & $\begin{array}{l}2,4-\mathrm{DI}- \\
\text { NITRO- } \\
\text { TOLUENE } \\
\text { TOTAL } \\
(\mathrm{UG} / \mathrm{L})\end{array}$ & $\begin{array}{l}2,6-\mathrm{DI}- \\
\text { NITRO- } \\
\text { TOLUENE } \\
\text { TOTAL } \\
\text { (UG/L) }\end{array}$ & $\begin{array}{l}\text { DI - N- } \\
\text { OCTYL } \\
\text { PHTHAL- } \\
\text { ATE } \\
\text { TOTAL } \\
\text { (UG/L) }\end{array}$ & $\begin{array}{l}\text { BIS }(2- \\
\text { ETHYL } \\
\text { HEXYL) } \\
\text { PHTHAL - } \\
\text { ATE } \\
\text { TOTAL } \\
\text { (UG/L) }\end{array}$ & $\begin{array}{l}\text { FLUOR - } \\
\text { ANTHENE } \\
\text { TOTAL } \\
\text { (UG / L) }\end{array}$ & $\begin{array}{c}\text { FLUOR- } \\
\text { ENE } \\
\text { TOTAL } \\
\text { (UG/L) }\end{array}$ \\
\hline OCT 1992 & & & & & & & & & & & \\
\hline $\begin{array}{l}28-29 \\
\text { NoV }\end{array}$ & $<5.0$ & $<5.0$ & $<5.0$ & $<30.0$ & $<20.0$ & $<5.0$ & $<5.0$ & $<10.0$ & 9.0 & $<5.0$ & $<5.0$ \\
\hline $\begin{array}{l}19 \cdot 19 \\
\text { DEC }\end{array}$ & $<5.0$ & $<5.0$ & $<5.0$ & $<30.0$ & $<20.0$ & $<5.0$ & $<5.0$ & $<10.0$ & 5.0 & $<5.0$ & $<5.0$ \\
\hline $\begin{array}{c}09-09 \\
\text { JAN } 1993\end{array}$ & $<5.0$ & $<5.0$ & $<5.0$ & $<30.0$ & $<20.0$ & $<5.0$ & $<5.0$ & $<10.0$ & 7.0 & $<5.0$ & $<5.0$ \\
\hline $\begin{array}{l}09-09 \\
\text { JAN }\end{array}$ & $<5.0$ & $<5.0$ & $<5.0$ & $<30.0$ & $<20.0$ & $<5.0$ & $<5.0$ & $<10.0$ & $<5.0$ & $<5.0$ & $<5.0$ \\
\hline $\begin{array}{l}28-28 \\
\text { FEB }\end{array}$ & $<5.0$ & $<5.0$ & $<5.0$ & $<30.0$ & $<20.0$ & $<5.0$ & $<5.0$ & $<10.0$ & 7.0 & $<5.0$ & $<5.0$ \\
\hline $\begin{array}{l}24-24 \\
\text { MAR }\end{array}$ & $<5.0$ & $<5.0$ & $<5.0$ & $<30.0$ & $<20.0$ & $<5.0$ & $<5.0$ & $<10.0$ & 6.0 & $<5.0$ & $<5.0$ \\
\hline $11-12$ & $\begin{array}{l}\text { HEXA - } \\
\text { CHLORO - } \\
\text { BENZENE } \\
\text { TOTAL } \\
\text { (UG/L) }\end{array}$ & $\begin{array}{l}\text { HEXA- } \\
\text { CHLORO - } \\
\text { CYCLO - } \\
\text { PENT - } \\
\text { ADIENE } \\
\text { TOTAL } \\
\text { (UG/L) }\end{array}$ & $\begin{array}{l}\text { HEXA- } \\
\text { CHLORO- } \\
\text { ETHANE } \\
\text { TOTAL } \\
\text { (UG/L) }\end{array}$ & $\begin{array}{c}\text { INDENO } \\
(1,2,3- \\
\text { CD) } \\
\text { PYRENE } \\
\text { TOTAL } \\
\text { (UG/L) }\end{array}$ & $\begin{array}{l}\text { ISO- } \\
\text { PHORONE } \\
\text { TOTAL } \\
\text { (UG/L) }\end{array}$ & $\begin{array}{l}\text { NITRO- } \\
\text { BENZENE } \\
\text { TOTAL } \\
\text { (UG/L) }\end{array}$ & $\begin{array}{c}\text { N- NITRO- } \\
\text { SODI - } \\
\text { METHY - } \\
\text { LAMINE } \\
\text { TOTAL } \\
\text { (UG/L) }\end{array}$ & $\begin{array}{l}2 \text { - } \\
\text { NITRO- } \\
\text { PHENOL } \\
\text { TOTAL } \\
\text { (UG/L) }\end{array}$ & $\begin{array}{l}4- \\
\text { NITRO- } \\
\text { PHENOL } \\
\text { TOTAL } \\
\text { (UG/L) }\end{array}$ & $\begin{array}{l}\text { N- } \\
\text { NITRO- } \\
\text { SODI - N- } \\
\text { PROPYL- } \\
\text { AMINE } \\
\text { TOTAL } \\
\text { (UG/L) }\end{array}$ & $\begin{array}{c}\text { N-NITRO - } \\
\text { SODI - } \\
\text { PHENY - } \\
\text { LAMINE } \\
\text { TOTAL } \\
\text { (UG/L) }\end{array}$ \\
\hline $\begin{array}{c}\text { OCT } 1992 \\
28.29\end{array}$ & $<5.0$ & $<5.0$ & $<5.0$ & $<10.0$ & $<5,0$ & $<5,0$ & $<50$ & $<50$ & $<300$ & $<50$ & \\
\hline Nov & & & -3.0 & & & -3.0 & -3.0 & $<3.0$ & $<30.0$ & $<3.0$ & $<5.0$ \\
\hline $\begin{array}{l}19 \cdot 19 \\
\mathrm{DEC}\end{array}$ & $<5.0$ & $<5.0$ & $<5.0$ & $<10.0$ & $<5.0$ & $<5.0$ & $<5.0$ & $<5.0$ & $<30.0$ & $<5.0$ & $<5.0$ \\
\hline $\begin{array}{c}09.09 \\
\text { JAN } 1993\end{array}$ & $<5.0$ & $<5.0$ & $<5.0$ & $<10.0$ & $<5.0$ & $<5.0$ & $<5.0$ & $<5.0$ & $<30.0$ & $<5.0$ & $<5.0$ \\
\hline $\begin{array}{l}09.09 \\
\text { JAN }\end{array}$ & $<5.0$ & $<5.0$ & $<5.0$ & $<10.0$ & $<5.0$ & $<5.0$ & $<5.0$ & $<5.0$ & $<30.0$ & $<5.0$ & $<5.0$ \\
\hline $\begin{array}{l}28 \cdot 28 \\
F E B\end{array}$ & $<5.0$ & $<5.0$ & $<5.0$ & $<10.0$ & $<5.0$ & $<5.0$ & $<5.0$ & $<5.0$ & $<30.0$ & $<5.0$ & $<5.0$ \\
\hline $\begin{array}{l}24-24 \\
\text { MAR }\end{array}$ & $<5.0$ & $<5.0$ & $<5.0$ & $<10.0$ & $<5.0$ & $<5.0$ & $<5.0$ & $<5.0$ & $<30.0$ & $<5.0$ & $<5.0$ \\
\hline $11-12$ & $\begin{array}{l}\text { PENTA - } \\
\text { CHLORO - } \\
\text { PHENOL } \\
\text { TOTAL } \\
(U G / L)\end{array}$ & $\begin{array}{l}\text { PHENAN- } \\
\text { THRENE } \\
\text { TOTAL } \\
\text { (UG/L) }\end{array}$ & $\begin{array}{l}\text { PHENOL } \\
\text { (C6H - } \\
5 O H) \\
\text { TOTAL } \\
(U G / L)\end{array}$ & $\begin{array}{c}\text { PYRENE } \\
\text { TOTAL } \\
\text { (UG/L) }\end{array}$ & $\begin{array}{c}2,4,6- \\
\text { TRI - } \\
\text { CHLORO- } \\
\text { PHENOL } \\
\text { TOTAL } \\
\text { (UG/L) }\end{array}$ & $\begin{array}{l}\text { ALDRIN, } \\
\text { TOTAL } \\
\text { (UG/L) }\end{array}$ & $\begin{array}{c}\text { P, } P^{\prime} \\
\text { DDT, } \\
\text { TOTAL } \\
\text { (UG/L) }\end{array}$ & $\begin{array}{l}\text { ALPHA } \\
\text { BHC } \\
\text { TOTAL } \\
\text { (UG/L) }\end{array}$ & $\begin{array}{c}\text { BETA } \\
\text { BENZENE } \\
\text { HEXA- } \\
\text { CHLOR - } \\
\text { IDE } \\
\text { TOTAL } \\
\text { (UG/L) }\end{array}$ & $\begin{array}{l}\text { DELTA } \\
\text { BENZENE } \\
\text { HEXA - } \\
\text { CHLOR - } \\
\text { IDE } \\
\text { TOTAL } \\
\text { (UG/L) }\end{array}$ & $\begin{array}{c}\text { LINDANE } \\
\text { TOTAL } \\
\text { (UG/L) }\end{array}$ \\
\hline OCT 1992 & & & & & & & & & & & \\
\hline $\begin{array}{l}28-29 \\
\text { Nov }\end{array}$ & $<30.0$ & $<5.0$ & $<5.0$ & $<5.0$ & $<20.0$ & $<0.040$ & $<0.10$ & $<0.03$ & $<0.03$ & $<0.09$ & $<0.030$ \\
\hline DEC $19 \cdot 19$ & $<30.0$ & $<5.0$ & $<5.0$ & $<5.0$ & $<20.0$ & $<0.040$ & $<0.10$ & $<0.03$ & $<0.03$ & $<0.09$ & $<0.030$ \\
\hline $\begin{array}{c}09-09 \\
\text { JAN } 1993\end{array}$ & $<30.0$ & $<5.0$ & $<5.0$ & $<5.0$ & $<20.0$ & $<0.040$ & $<0.10$ & $<0.03$ & $<0.03$ & $<0.09$ & $<0.030$ \\
\hline $\begin{array}{l}09-09 \\
\text { JAN }\end{array}$ & $<30.0$ & $<5.0$ & $<5.0$ & $<5.0$ & $<20.0$ & $<0.040$ & $<0.10$ & $<0.03$ & $<0.03$ & $<0.09$ & $<0.030$ \\
\hline $28-28$ & $<30.0$ & $<5.0$ & $<5.0$ & $<5.0$ & $<20.0$ & $<0.040$ & $<0.10$ & $<0.03$ & $<0.03$ & $<0.09$ & $<0.030$ \\
\hline $\begin{array}{l}\text { FEB } \\
24-24\end{array}$ & $<30.0$ & $<5.0$ & $<5.0$ & $<5.0$ & $<20.0$ & $<0.040$ & $<0.10$ & $<0.03$ & $<0.03$ & $<0.09$ & $<0.030$ \\
\hline $\begin{array}{l}\text { MAR } \\
\quad 11-12\end{array}$ & $<30.0$ & $<5.0$ & $<5.0$ & $<5.0$ & $<20.0$ & $<0.040$ & $<0.10$ & $<0.03$ & $<0.03$ & $<0.09$ & $<0.030$ \\
\hline
\end{tabular}


Table 2. Water-quality data for storm-sewer outfall stations, Arlington, Texas, 1992-93-Continued

\begin{tabular}{|c|c|c|c|c|c|c|c|c|c|c|c|}
\hline & 08049220 & \multicolumn{2}{|c|}{ - THE PARKS MALL } & OUTFALL $A^{\prime}$ & $\mathrm{T}$ I-20 WE & & \multicolumn{2}{|c|}{ (wY 1993)-Continued } & & \\
\hline DATE & $\begin{array}{c}\text { CHLOR - } \\
\text { DANE } \\
\text { CIS } \\
\text { WATER } \\
\text { WHOLE } \\
\text { TOTAL } \\
\text { (UG/L) }\end{array}$ & $\begin{array}{c}\text { CHLOR - } \\
\text { DANE } \\
\text { TRANS } \\
\text { WATER } \\
\text { WHOLE } \\
\text { TOTAL } \\
\text { (UG/L) }\end{array}$ & $\begin{array}{l}\text { CHLOR- } \\
\text { DANE, } \\
\text { TOTAL } \\
\text { (UG/L) }\end{array}$ & $\begin{array}{c}\text { P, } P^{\prime} \\
\text { DDD, } \\
\text { TOTAL } \\
(U G / L)\end{array}$ & $\begin{array}{l}P, P^{\prime} \\
\text { DDE, } \\
\text { TOTAL } \\
\text { (UG/L) }\end{array}$ & $\begin{array}{l}\text { DI - } \\
\text { ELDRIN } \\
\text { TOTAL } \\
(U G / L)\end{array}$ & $\begin{array}{c}\text { ENDO - } \\
\text { SULFAN - } \\
\text { I } \\
\text { WATER } \\
\text { WHOLE } \\
\text { REC } \\
\text { (UG/L) }\end{array}$ & $\begin{array}{l}\text { ENDO- } \\
\text { SULFAN } \\
\text { BETA } \\
\text { TOTAL } \\
(U G / L)\end{array}$ & $\begin{array}{l}\text { ENDO- } \\
\text { SULFAN } \\
\text { SULFATE } \\
\text { TOTAL } \\
\text { (UG/L) }\end{array}$ & $\begin{array}{c}\text { ENDR IN } \\
\text { WATER } \\
\text { UNFLTRD } \\
\text { REC } \\
(\mathrm{UG} / \mathrm{L})\end{array}$ & $\begin{array}{c}\text { ENDRIN } \\
\text { ALDE - } \\
\text { HYDE } \\
\text { TOTAL } \\
\text { (UG/L) }\end{array}$ \\
\hline OCT 1992 & & & & & & & & & & & \\
\hline $\begin{array}{l}28-29 \\
\text { Nov }\end{array}$ & $<0.10$ & $<0.10$ & $<0.1$ & $<0.10$ & $<0.04$ & $<0.020$ & $<0.10$ & $<0.04$ & $<0.60$ & $<0.060$ & $<0.20$ \\
\hline $\mathrm{DEC}^{19-19}$ & $<0.10$ & $<0.10$ & $<0.1$ & $<0.10$ & $<0.04$ & $<0.020$ & $<0.10$ & $<0.04$ & $<0.60$ & $<0.060$ & $<0.20$ \\
\hline $\begin{array}{c}09-09 \\
\text { JAN } 1993\end{array}$ & $<0.10$ & $<0.10$ & $<0.1$ & $<0.10$ & $<0.04$ & $<0.020$ & $<0.10$ & $<0.04$ & $<0.60$ & $<0.060$ & $<0.20$ \\
\hline $\begin{array}{l}09 \cdot 09 \\
\text { JAN }\end{array}$ & $<0.10$ & $<0.10$ & $<0.1$ & $<0.10$ & $<0.04$ & $<0.020$ & $<0.10$ & $<0.04$ & $<0.60$ & $<0.060$ & $<0.20$ \\
\hline $\begin{array}{l}28 \cdot 28 \\
F E B\end{array}$ & $<0.10$ & $<0.10$ & $<0.1$ & $<0.10$ & $<0.04$ & $<0.020$ & $<0.10$ & $<0.04$ & $<0.60$ & $<0.060$ & $<0.20$ \\
\hline $\begin{array}{l}24 \cdot 24 \\
\text { MAR }\end{array}$ & $<0.10$ & $<0.10$ & $<0.1$ & $<0.10$ & $<0.04$ & $<0.020$ & $<0.10$ & $<0.04$ & $<0.60$ & $<0.060$ & $<0.20$ \\
\hline $11 \cdot 12$ & $<0.10$ & $<0.10$ & $<0.1$ & $<0.10$ & $<0.04$ & $<0.020$ & $<0.10$ & $<0.04$ & $<0.60$ & $<0.060$ & $<0.20$ \\
\hline DATE & $\begin{array}{l}\text { HEPTA- } \\
\text { CHLOR, } \\
\text { TOTAL } \\
\text { (UG/L) }\end{array}$ & $\begin{array}{l}\text { HEPTA- } \\
\text { CHLOR } \\
\text { EPOXIDE } \\
\text { TOTAL } \\
\text { (UG/L) }\end{array}$ & $\begin{array}{c}\text { TOX - } \\
\text { APHENE, } \\
\text { TOTAL } \\
\text { (UG/L) }\end{array}$ & $\begin{array}{c}\text { AROCLOR } \\
1016 \\
\text { PCB } \\
\text { TOTAL } \\
\text { (UG/L) }\end{array}$ & $\begin{array}{c}\text { AROCLOR } \\
1221 \\
\text { PCB } \\
\text { TOTAL } \\
\text { (UG/L) }\end{array}$ & $\begin{array}{c}\text { AROCLOR } \\
1232 \\
\text { PCB } \\
\text { TOTAL } \\
(U G / L)\end{array}$ & $\begin{array}{c}\text { AROCLOR } \\
1242 \\
\text { PCB } \\
\text { TOTAL } \\
\text { (UG/L) }\end{array}$ & $\begin{array}{c}\text { AROCLOR } \\
1248 \\
\text { PCB } \\
\text { TOTAL } \\
(U G / L)\end{array}$ & $\begin{array}{c}\text { AROCLOR } \\
1254 \\
\text { PCB } \\
\text { TOTAL } \\
\text { (UG/L) }\end{array}$ & $\begin{array}{c}\text { AROCLOR } \\
1260 \\
\text { PCB } \\
\text { TOTAL } \\
(\mathrm{UG} / \mathrm{L})\end{array}$ & $\begin{array}{c}\text { DI - } \\
\text { AZINON, } \\
\text { TOTAL } \\
\text { (UG/L) }\end{array}$ \\
\hline OCT 1992 & & & & & & & & & & & \\
\hline $\begin{array}{l}28-29 \\
\text { Nov }\end{array}$ & $<0.030$ & $<0.80$ & $<2$ & $<0.1$ & $<1.0$ & $<0.1$ & $<0.1$ & $<0.1$ & $<0.1$ & $<0.1$ & 0.20 \\
\hline $\mathrm{DEC}^{19-19}$ & $<0.030$ & $<0.80$ & $<2$ & $<0.1$ & $<1.0$ & $<0.1$ & $<0.1$ & $<0.1$ & $<0.1$ & $<0.1$ & $<0.10$ \\
\hline $\begin{array}{c}09-09 \\
\text { JAN } 1993\end{array}$ & $<0.030$ & $<0.80$ & $<2$ & $<0.1$ & $<1.0$ & $<0.1$ & $<0.1$ & $<0.1$ & $<0.1$ & $<0.1$ & $<0.10$ \\
\hline $\begin{array}{l}09-09 \\
\text { JAN }\end{array}$ & $<0.030$ & $<0.80$ & $<2$ & $<0.1$ & $<1.0$ & $<0.1$ & $<0.1$ & $<0.1$ & $<0.1$ & $<0.1$ & 0.10 \\
\hline $\begin{array}{l}28-28 \\
\text { FEB }\end{array}$ & $<0.030$ & $<0.80$ & $<2$ & $<0.1$ & $<1.0$ & $<0.1$ & $<0.1$ & $<0.1$ & $<0.1$ & $<0.1$ & 0.10 \\
\hline $\begin{array}{l}24-24 \\
M A R\end{array}$ & $<0.030$ & $<0.80$ & $<2$ & $<0.1$ & $<1.0$ & $<0.1$ & $<0.1$ & $<0.1$ & $<0.1$ & $<0.1$ & $<0.05$ \\
\hline $11-12$ & $<0.030$ & $<0.80$ & $<2$ & $<0.1$ & $<1.0$ & $<0.1$ & $<0.1$ & $<0.1$ & $<0.1$ & $<0.1$ & $<0.05$ \\
\hline
\end{tabular}


Table 2. Water-quality data for storm-sewer outfall stations, Arlington, Texas, 1992-93-Continued

08049320 - RIVER LEGACY PARK OUTFALL AT GREEN OAKS BOULEVARD, ARLINGTON, TX (WY 1993)

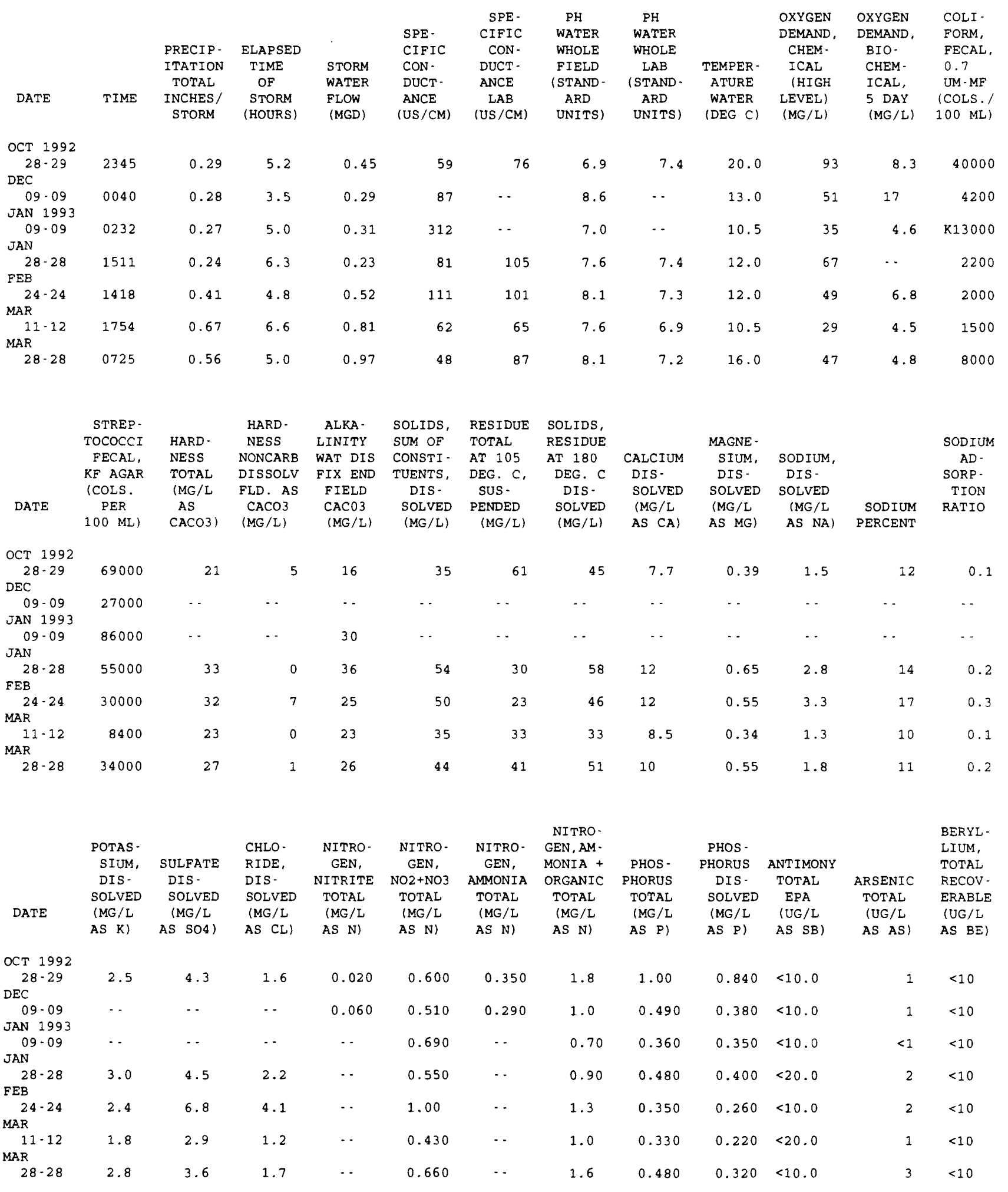


Table 2. Water-quality data for storm-sewer outfall stations, Arlington, Texas, 1992-93-Continued

08049320 - RIVER LEGACY PARK OUTFALL AT GREEN OAKS BOULEVARD, ARLINGTON, TX (WY 1993)-COntinUEd

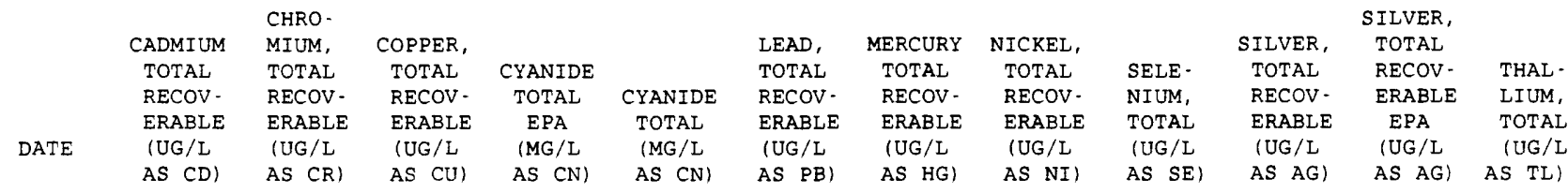

OCT 1992

$28-29$

DEC

09.09

JAN 1993

09-09

JAN

$28-28$

FEB

$24-24$

MAR
$11-12$

MAR

$28-28$

AS CD) AS CU) AS CN)

AS $\mathrm{CN}$ )

AS NI)

AS SE)

AS $A G)$

S AG) AS TL)

\begin{tabular}{|c|c|c|c|c|c|c|c|c|c|c|c|c|}
\hline & & & OIL AND & & & & & & METHANE & & & BENZENE \\
\hline & ZINC, & & GREASE, & & & & & BROMO - & BROMO & & BENZENE & SEC \\
\hline & TOTAL & CARBON, & TOTAL & & & & & BENZENE & CHLORO- & & N-BUTYL & BUTYL - \\
\hline & RECOV - & ORGANIC & RECOV. & & ACRO - & ACRYLO - & & WATER, & WAT & BROMO - & WATER & WATER \\
\hline & ERABLE & TOTAL & GRAVI - & PHENOLS & LEIN & NITRILE & BENZENE & WHOLE, & UNFLTRD & FORM & UNFLTRD & UNFLTRD \\
\hline DATE & (UG/L & (MG/L & METRIC & TOTAL & TOTAL & TOTAL & TOTAL & TOTAL & REC & TOTAL & REC & REC \\
\hline & $A S \quad Z N)$ & AS C) & $(\mathrm{MG} / \mathrm{L})$ & $(\mathrm{UG} / \mathrm{L})$ & (UG/L) & (UG/L) & $(U G / L)$ & $(U G / L)$ & (UG/L) & $(\mathrm{UG} / \mathrm{L})$ & (UG/L) & (UG/L) \\
\hline OCT 1992 & & & & & & & & & & & & \\
\hline $28-29$ & 70 & 19 & 1 & 4 & $<20$ & $<20$ & $<0.2$ & $<0.2$ & $<0.20$ & $<0.2$ & $<0.20$ & $<0.20$ \\
\hline $\mathrm{DEC}$ & & & & & & & & & & & & \\
\hline $09-09$ & 40 & 18 & $<1$ & 6 & $<20$ & $<20$ & $<0.2$ & $<0.2$ & $<0.20$ & $<0.2$ & $<0.20$ & $<0.20$ \\
\hline JAN 1993 & & & & & & & & & & & & \\
\hline $09-09$ & 40 & 11 & 2 & 3 & $<20$ & $<20$ & $<0.2$ & $<0.2$ & $<0.20$ & $<0.2$ & $<0.20$ & $<0.20$ \\
\hline JAN & & & & & & & & & & & & \\
\hline $28-28$ & 40 & 8.9 & 2 & 4 & $<20$ & $<20$ & $<0.2$ & $<0.2$ & $<0.20$ & $<0.2$ & $<0.20$ & $<0.20$ \\
\hline FEB & & & & & & & & & & & & \\
\hline $24-24$ & 50 & 10 & $<1$ & 6 & $<20$ & $<20$ & 0.3 & $<0.2$ & $<0.20$ & $<0.2$ & $<0.20$ & $<0.20$ \\
\hline MAR & & & & & & & & & & & & \\
\hline $11 \cdot 12$ & 30 & 9.1 & $<1$ & 1 & $<20$ & $<20$ & $<0.2$ & $<0.2$ & $<0.20$ & $<0.2$ & $<0.20$ & $<0.20$ \\
\hline MAR & & & & & & & & & & & & \\
\hline $28-28$ & 50 & 12 & $<1$ & $<1$ & $<20$ & $<20$ & $<0.2$ & $<0.2$ & $<0.20$ & $<0.2$ & $<0.20$ & $<0.20$ \\
\hline & BENZENE & & & & & 2 & & & 0 & & DIBROMO & DI - \\
\hline & TERT - & CARBON - & & CHLORO - & & CHLORO- & & & CHLORO - & TOLUENE & CHLORO - & BROMO - \\
\hline & BUTYL - & TETRA - & & DI - & & ETHYL - & & METHYL - & TOLUENE & P-CHLOR & PROPANE & METHANE \\
\hline & WATER & CHLO - & CHLORO - & BROMO - & CHLORO - & VINYL - & CHLORO- & CHLO - & WATER & WATER & WATER & WATER \\
\hline & UNFLTRD & RIDE & BENZENE & METHANE & ETHANE & ETHER & FORM & RIDE & WHOLE & UNFLTRD & WHOLE & WHOLE \\
\hline DATE & $\begin{array}{c}\text { REC } \\
(U G / L)\end{array}$ & $\begin{array}{l}\text { TOTAL } \\
\text { (UG/L) }\end{array}$ & $\begin{array}{l}\text { TOTAL } \\
\text { (UG/L) }\end{array}$ & $\begin{array}{l}\text { TOTAL } \\
\text { (UG/L) }\end{array}$ & $\begin{array}{l}\text { TOTAL } \\
\text { (UG/L) }\end{array}$ & $\begin{array}{l}\text { TOTAL } \\
\text { (UG/L) }\end{array}$ & $\begin{array}{l}\text { TOTAL } \\
\text { (UG/L) }\end{array}$ & $\begin{array}{l}\text { TOTAL } \\
\text { (UG/L) }\end{array}$ & $\begin{array}{l}\text { TOTAL } \\
\text { (UG/L) }\end{array}$ & $\begin{array}{c}\text { REC } \\
(U G / L)\end{array}$ & $\begin{array}{l}\text { TOT.REC } \\
\text { (UG/L) }\end{array}$ & $\begin{array}{l}\text { RECOVER } \\
\text { (UG/L) }\end{array}$ \\
\hline
\end{tabular}

\begin{tabular}{|c|c|c|c|c|c|c|c|c|c|c|c|c|}
\hline \multicolumn{13}{|l|}{ OCT 1992} \\
\hline $28-29$ & $<0.20$ & $<0.2$ & $<0.20$ & $<0.2$ & $<0.2$ & $<1.0$ & $<0.2$ & $<0.2$ & $<0.2$ & $<0.20$ & $<1.0$ & $<0.2$ \\
\hline \multicolumn{13}{|l|}{$\mathrm{DEC}$} \\
\hline $09-09$ & $<0.20$ & $<0.2$ & $<0.20$ & $<0.2$ & $<0.2$ & $<1.0$ & $<0.2$ & $<0.2$ & $<0.2$ & $<0.20$ & $<1.0$ & $<0.2$ \\
\hline \multicolumn{13}{|l|}{ JAN 1993} \\
\hline $09-09$ & $<0.20$ & $<0.2$ & $<0.20$ & $<0.2$ & $<0.2$ & $<1.0$ & $<0.2$ & $<0.2$ & $<0.2$ & $<0.20$ & $<1.0$ & $<0.2$ \\
\hline \multicolumn{13}{|l|}{ JAN } \\
\hline $28-28$ & $<0.20$ & $<0.2$ & $<0.20$ & $<0.2$ & $<0.2$ & $<1.0$ & $<0.2$ & $<0.2$ & $<0.2$ & $<0.20$ & $<1.0$ & $<0.2$ \\
\hline \multicolumn{13}{|l|}{$F E B$} \\
\hline $24-24$ & $<0.20$ & $<0.2$ & $<0.20$ & $<0.2$ & $<0.2$ & $<1.0$ & $<0.2$ & $<0.2$ & $<0.2$ & $<0.20$ & $<1.0$ & $<0.2$ \\
\hline \multicolumn{13}{|l|}{ MAR } \\
\hline $11-12$ & $<0.20$ & $<0.2$ & $<0.20$ & $<0.2$ & $<0.2$ & $<1.0$ & $<0.2$ & $<0.2$ & $<0.2$ & $<0.20$ & $<1.0$ & $<0.2$ \\
\hline \multicolumn{13}{|l|}{ MAR } \\
\hline $28-28$ & $<0.20$ & $<0.2$ & $<0.20$ & $<0.2$ & $<0.2$ & $<1.0$ & $<0.2$ & 0.4 & $<0.2$ & $<0.20$ & $<1.0$ & $<0.2$ \\
\hline
\end{tabular}


Table 2. Water-quality data for storm-sewer outfall stations, Arlington, Texas, 1992-93-Continued

08049320 - RIVER LEGACY PARK OUTFALL AT GREEN OAKS BOULEVARD, ARLINGTON, TX (WY 1993)-COntinued

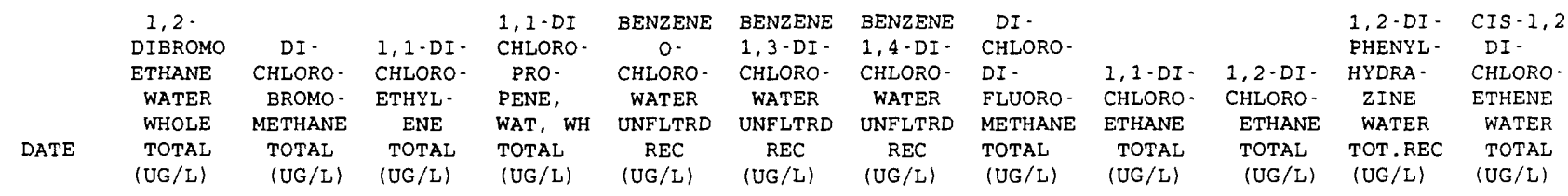

\begin{tabular}{|c|c|c|c|c|c|c|c|c|c|c|c|c|}
\hline OCT 1992 & & & & & & & & & & & & \\
\hline $28-29$ & $<0.2$ & $<0.2$ & $<0.2$ & $<0.2$ & $<5.0$ & $<5.0$ & $<5.0$ & $<0.2$ & $<0.2$ & $<0.2$ & $<5.0$ & $<0.2$ \\
\hline $\mathrm{DEC}$ & & & & & & & & & & & & \\
\hline 09.09 & $<0.2$ & $<0.2$ & $<0.2$ & $<0.2$ & $<5.0$ & $<5.0$ & $<5.0$ & $<0.2$ & $<0.2$ & $<0.2$ & $<5.0$ & $<0.2$ \\
\hline JAN 1993 & & & & & & & & & & & & \\
\hline 09.09 & $<0.2$ & $<0.2$ & $<0.2$ & $<0.2$ & $<5.0$ & $<5.0$ & $<5.0$ & $<0.2$ & $<0.2$ & $<0.2$ & $<5.0$ & $<0.2$ \\
\hline JAN & & & & & & & & & & & & \\
\hline $28 \cdot 28$ & $<0.2$ & $<0.2$ & $<0.2$ & $<0.2$ & $<5.0$ & $<5.0$ & $<5.0$ & $<0.2$ & $<0.2$ & $<0.2$ & $<5.0$ & $<0.2$ \\
\hline $\mathrm{FEB}$ & & & & & & & & & & & & \\
\hline $24-24$ & $<0.2$ & $<0.2$ & $<0.2$ & $<0.2$ & $<5.0$ & $<5.0$ & $<5.0$ & $<0.2$ & $<0.2$ & $<0.2$ & $<5.0$ & $<0.2$ \\
\hline MAR & & & & & & & & & & & & \\
\hline $11 \cdot 12$ & $<0.2$ & $<0.2$ & $<0.2$ & $<0.2$ & $<5.0$ & $<5.0$ & $<5.0$ & $<0.2$ & $<0.2$ & $<0.2$ & $<5.0$ & $<0.2$ \\
\hline MAR & & & & & & & & & & & & \\
\hline $28-28$ & $<0.2$ & $<0.2$ & $<0.2$ & $<0.2$ & $<5.0$ & $<5.0$ & $<5.0$ & $<0.2$ & $<0.2$ & $<0.2$ & $<5.0$ & $<0.2$ \\
\hline
\end{tabular}

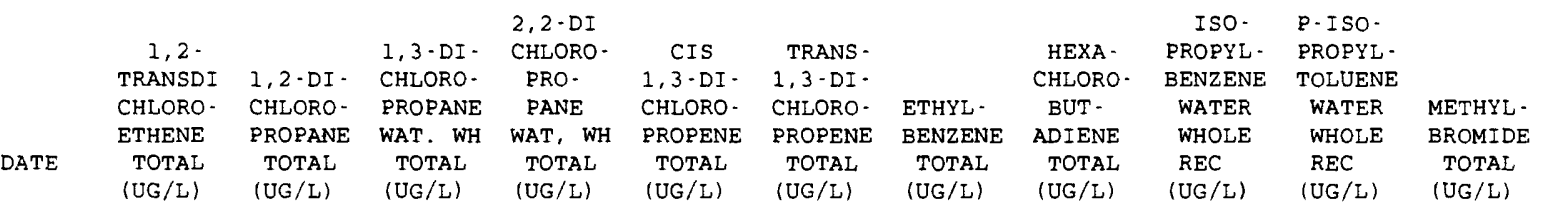

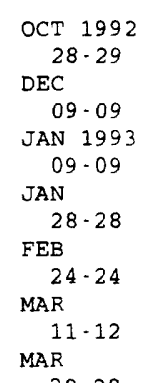

$28-28$

\begin{tabular}{|c|c|c|c|}
\hline$<0.2$ & $<0.2$ & $<0.2$ & $<0.2$ \\
\hline$<0.2$ & $<0.2$ & $<0.2$ & $<0.2$ \\
\hline$<0.2$ & $<0.2$ & $<0.2$ & $<0.2$ \\
\hline$<0.2$ & $<0.2$ & $<0.2$ & $<0.2$ \\
\hline$<0.2$ & $<0.2$ & $<0.2$ & $<0.2$ \\
\hline$<0.2$ & $<0.2$ & $<0.2$ & $<0.2$ \\
\hline$<0.2$ & $<0.2$ & $<0.2$ & $<0.2$ \\
\hline \multicolumn{4}{|c|}{ METHYL } \\
\hline THYL - & ETHER & & BENZENE \\
\hline ENE & TERT - & & $\mathrm{N} \cdot \mathrm{PROPY}$ \\
\hline HLO - & BUTYL & NAPHTH - & WATER \\
\hline IDE & WAT UNF & ALENE & UNFLTRD \\
\hline TAL & REC & TOTAL & REC \\
\hline$J G / L)$ & $\{U G / L\rangle$ & (UG/L) & (UG/L) \\
\hline
\end{tabular}

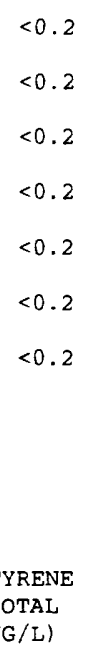

\begin{abstract}
$<0.2$
\end{abstract}
$<0.2$

$<0.2$

$<0.2$

$<0.2$

$<0.2$

$<0.2$

ETHANE, ETHANE,

1112- 1,1,2,2 TETRA-

TETRA - TETRA - CHLORO -

CHLORO- CHLORO- ETHYL-

WAT UNF WAT UNF ENE

REC

(UG/L)
REC (UG/L)

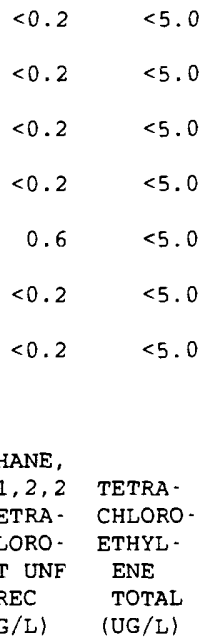

$<0.20$

$<0.20$

$<0.2$

$<0.20$

$<0.20$

$<0.2$

$<0.20$

$<0.20$

$<0.2$

$<0.20$

$<0.20$

$<0.2$

$<0.20$

$<0.20$

$<0.2$

$<0.20$

$<0.20$

$<0.2$

$<0.20$

$<0.20$

$<0.2$
OCT 1992

$28-29$

DEC

09.09

JAN 1993

09.09

JAN

$28-28$

FEB

24-24

MAR

11. 12

MAR
$28 \cdot 28$

$\begin{array}{llllllllllll}<0.2 & <1.0 & <5.0 & <0.20 & <0.2 & <0.2 & <0.2 & <0.2 & <0.2 & <0.20 & <5.0 \\ <0.2 & <1.0 & <5.0 & <0.20 & <0.2 & <0.2 & <0.2 & <0.2 & <0.2 & <0.20 & <5.0 \\ <0.2 & <1.0 & <5.0 & <0.20 & <0.2 & <0.2 & <0.2 & <0.2 & <0.2 & <0.20 & <5.0 \\ <0.2 & <1.0 & <5.0 & <0.20 & <0.2 & <0.2 & <0.2 & <0.2 & <0.2 & <0.20 & <5.0 \\ <0.3 & <1.0 & <5.0 & <0.20 & <0.2 & <0.2 & <0.2 & <0.2 & 1.8 & <0.20 & <5.0 \\ <0.2 & <1.0 & <5.0 & <0.20 & <0.2 & <0.2 & <0.2 & <0.2 & 0.2 & <0.20 & <5.0 \\ <0.2 & <1.0 & <5.0 & <0.20 & <0.2 & <0.2 & <0.2 & <0.2 & <0.2 & <0.20\end{array}$


Table 2. Water-quality data for storm-sewer outfall stations, Arlington, Texas, 1992-93-Continued

08049320 - RIVER LEGACY PARK OUTFALL AT GREEN OAKS BOULEVARD, ARLINGTON, TX (WY 1993)-Continued

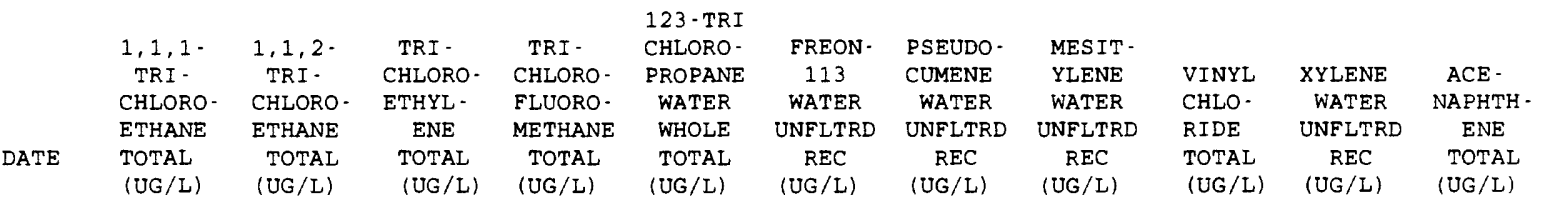

OCT 1992
$28-29$
DEC
$09-09$
JAN 1993
$09-09$
JAN
$28-28$
FEB
$24-24$
MAR
$11-12$
MAR
$28-28$

$\begin{array}{rrrrrrrrrrrr}<0.2 & <0.2 & <0.2 & <0.2 & <0.2 & <0.5 & <0.20 & <0.20 & <0.2 & <0.20 & <5.0 \\ <0.2 & <0.2 & <0.2 & <0.2 & <0.2 & <0.5 & <0.20 & <0.20 & <0.2 & <0.20 & <5.0 \\ <0.2 & <0.2 & <0.2 & <0.2 & <0.2 & <0.5 & <0.20 & <0.20 & <0.2 & <0.20 & <5.0 \\ <0.2 & <0.2 & <0.2 & <0.2 & <0.2 & <0.5 & <0.20 & <0.20 & <0.2 & <0.20 & <5.0 \\ <0.2 & <0.2 & <0.2 & <0.2 & <0.2 & <0.5 & 2.0 & 0.20 & <0.2 & 3.7 & <5.0 \\ <0.2 & <0.2 & <0.2 & <0.2 & <0.2 & <0.5 & 0.30 & <0.20 & <0.2 & 0.40 & <5.0 \\ <0.2 & <0.2 & <0.2 & <0.2 & <0.2 & <0.5 & <0.20 & <0.20 & <0.2 & <0.20 & <5.0\end{array}$

\begin{tabular}{|c|c|c|c|c|c|c|c|c|c|c|c|}
\hline DATE & $\begin{array}{l}\text { ACE - } \\
\text { NAPHTH - } \\
\text { YLENE } \\
\text { TOTAL } \\
\text { (UG/L) }\end{array}$ & $\begin{array}{l}\text { ANTHRA - } \\
\text { CENE } \\
\text { TOTAL } \\
\text { (UG/L) }\end{array}$ & $\begin{array}{l}\text { BENZI - } \\
\text { DINE } \\
\text { TOTAL } \\
(U G / L)\end{array}$ & $\begin{array}{l}\text { BENZO A } \\
\text { ANTHRAC } \\
\text { ENE 1,2- } \\
\text { BENZANT } \\
\text { HRACENE } \\
\text { TOTAL } \\
\text { (UG/L) }\end{array}$ & $\begin{array}{l}\text { BENZO- } \\
\text { A- } \\
\text { PYRENE } \\
\text { TOTAL } \\
\text { (UG/L) }\end{array}$ & $\begin{array}{l}\text { BENZO B } \\
\text { FLUOR- } \\
\text { AN- } \\
\text { THENE } \\
\text { TOTAL } \\
\text { (UG/L) }\end{array}$ & $\begin{array}{l}\text { BENZO K } \\
\text { FLUOR - } \\
\text { AN- } \\
\text { THENE } \\
\text { TOTAL } \\
\text { (UG/L) }\end{array}$ & $\begin{array}{c}\text { BENZO } \\
\text { GHI PERYL } \\
\text { ENE1, } 12- \\
\text { BENZO } \\
\text { PERYLENE } \\
\text { TOTAL } \\
\text { (UG/L) }\end{array}$ & $\begin{array}{c}4- \\
\text { L BROMO- } \\
\text { PHENYL } \\
\text { PHENYL } \\
\text { ETHER } \\
\text { TOTAL } \\
\text { (UG/L) }\end{array}$ & $\begin{array}{l}\text { N-BUTYL } \\
\text { BENZYL } \\
\text { PHTHAL- } \\
\text { ATE } \\
\text { TOTAL } \\
(U G / L)\end{array}$ & $\begin{array}{l}\text { BIS } \\
\text { (2- } \\
\text { CHLORO- } \\
\text { ETHOXY) } \\
\text { METHANE } \\
\text { TOTAL } \\
\text { (UG/L) }\end{array}$ \\
\hline OCT 1992 & & & & & & & & & & & \\
\hline${ }^{28-29}$ & $<5.0$ & $<5.0$ & $<40.0$ & $<10.0$ & $<10.0$ & $<10.0$ & $<10.0$ & $<10.0$ & $<5.0$ & $<5.0$ & $<5.0$ \\
\hline $\begin{array}{c}09-09 \\
\text { JAN } 1993\end{array}$ & $<5.0$ & $<5.0$ & $<40.0$ & $<10.0$ & $<10.0$ & $<10.0$ & $<10.0$ & $<10.0$ & $<5.0$ & $<5.0$ & $<5.0$ \\
\hline $\begin{array}{l}09-09 \\
\text { JAN }\end{array}$ & $<5.0$ & $<5.0$ & $<40.0$ & $<10.0$ & $<10.0$ & $<10.0$ & $<10.0$ & $<10.0$ & $<5.0$ & $<5.0$ & $<5.0$ \\
\hline $\begin{array}{l}28-28 \\
\text { FEB }\end{array}$ & $<5.0$ & $<5.0$ & $<40.0$ & $<10.0$ & $<10.0$ & $<10.0$ & $<10.0$ & $<10.0$ & $<5.0$ & $<5.0$ & $<5.0$ \\
\hline $\begin{array}{l}24 \cdot 24 \\
\text { MAR }\end{array}$ & $<5.0$ & $<5.0$ & $<40.0$ & $<10.0$ & $<10.0$ & $<10.0$ & $<10.0$ & $<10.0$ & $<5.0$ & $<5.0$ & $<5.0$ \\
\hline MAR $11-12$ & $<5.0$ & $<5.0$ & $<40.0$ & $<10.0$ & $<10.0$ & $<10.0$ & $<10.0$ & $<10.0$ & $<5.0$ & $<5.0$ & $<5.0$ \\
\hline $28-28$ & $<5.0$ & $<5.0$ & $<40.0$ & $<10.0$ & $<10.0$ & $<10.0$ & $<10.0$ & $<10.0$ & $<5.0$ & $<5.0$ & $<5.0$ \\
\hline DATE & $\begin{array}{l}\text { BIS } \\
2- \\
\text { CHLORO- } \\
\text { ETHYL } \\
\text { ETHER } \\
\text { TOTAL } \\
\text { (UG/L) }\end{array}$ & $\begin{array}{l}\text { BIS } 12- \\
\text { CHLORO- } \\
\text { ISO- } \\
\text { PROPYL) } \\
\text { ETHER } \\
\text { TOTAL } \\
\text { (UG/L) }\end{array}$ & $\begin{array}{l}\text { PARA- } \\
\text { CHLORO- } \\
\text { META } \\
\text { CRESOL } \\
\text { TOTAL } \\
\text { (UG/L) }\end{array}$ & $\begin{array}{c}2- \\
\text { CHLORO- } \\
\text { NAPH- } \\
\text { THALENE } \\
\text { TOTAL } \\
\text { (UG/L) }\end{array}$ & $\begin{array}{l}2- \\
\text { CHLORO- } \\
\text { PHENOL } \\
\text { TOTAL } \\
\text { (UG/L) }\end{array}$ & $\begin{array}{c}4- \\
\text { CHLORO - } \\
\text { PHENYL } \\
\text { PHENYL } \\
\text { ETHER } \\
\text { TOTAL } \\
(U G / L)\end{array}$ & $\begin{array}{l}\text { CHRY - } \\
\text { SENE } \\
\text { TOTAL } \\
(U G / L)\end{array}$ & $\begin{array}{l}1,2,5,6- \\
\text { DIBENZ - } \\
\text { ANTHRA - } \\
\text { CENE } \\
\text { TOTAL } \\
\text { (UG/L) }\end{array}$ & $\begin{array}{l}3,3^{\prime}- \\
\text { DI- } \\
\text { CHLORO- } \\
\text { BENZI- } \\
\text { DINE } \\
\text { TOTAL } \\
\text { (UG/L) }\end{array}$ & $\begin{array}{c}2,4 \text { - DI - } \\
\text { CHLORO- } \\
\text { PHENOL } \\
\text { TOTAL } \\
\text { (UG/L) }\end{array}$ & $\begin{array}{l}\text { DIETHYL } \\
\text { PHTHAL- } \\
\text { ATE } \\
\text { TOTAL } \\
\text { (UG/L) }\end{array}$ \\
\hline $\begin{array}{c}\text { OCT } 1992 \\
28-29\end{array}$ & $<5.0$ & $<5.0$ & $<30.0$ & $<5.0$ & $<5.0$ & $<5.0$ & $<10.0$ & $<10.0$ & $<20.0$ & $<5.0$ & $<5.0$ \\
\hline DEC & & & & & & & & & & & \\
\hline $\begin{array}{c}09-09 \\
\text { JAN } 1993\end{array}$ & $<5.0$ & $<5.0$ & $<30.0$ & $<5.0$ & $<5.0$ & $<5.0$ & $<10.0$ & $<10.0$ & $<20.0$ & $<5.0$ & $<5.0$ \\
\hline $\begin{array}{l}09-09 \\
\text { JAN }\end{array}$ & $<5.0$ & $<5.0$ & $<30.0$ & $<5.0$ & $<5.0$ & $<5.0$ & $<10.0$ & $<10.0$ & $<20.0$ & $<5.0$ & $<5.0$ \\
\hline $\begin{array}{l}28-28 \\
\text { FEB }\end{array}$ & $<5.0$ & $<5.0$ & $<30.0$ & $<5.0$ & $<5.0$ & $<5.0$ & $<10.0$ & $<10.0$ & $<20.0$ & $<5.0$ & $<5.0$ \\
\hline $\begin{array}{l}24-24 \\
\text { MAR }\end{array}$ & $<5.0$ & $<5.0$ & $<30.0$ & $<5.0$ & $<5.0$ & $<5.0$ & $<10.0$ & $<10.0$ & $<20.0$ & $<5.0$ & $<5.0$ \\
\hline $11 \cdot 12$ & $<5.0$ & $<5.0$ & $<30.0$ & $<5.0$ & $<5.0$ & $<5.0$ & $<10.0$ & $<10.0$ & $<20.0$ & $<5.0$ & $<5.0$ \\
\hline $\begin{array}{l}\text { MAR } \\
\quad 28-28\end{array}$ & $<5.0$ & $<5.0$ & $<30.0$ & $<5.0$ & $<5.0$ & $<5.0$ & $<10.0$ & $<10.0$ & $<20.0$ & $<5.0$ & $<5.0$ \\
\hline
\end{tabular}


Table 2. Water-quality data for storm-sewer outfall stations, Arlington, Texas, 1992-93-Continued

08049320 - RIVER LEGACY PARK OUTFALL AT GREEN OAKS BOULEVARD, ARLINGTON, TX (WY 1993)-COntinUEd

\begin{tabular}{|c|c|c|c|c|c|c|c|c|c|c|c|}
\hline DATE & $\begin{array}{c}\text { DI- } \\
\text { METHYL } \\
\text { PHTHAL- } \\
\text { ATE } \\
\text { TOTAL } \\
\text { (UG/L) }\end{array}$ & $\begin{array}{c}2,4-D I- \\
\text { METHYL- } \\
\text { PHENOL } \\
\text { TOTAL } \\
\text { (UG/L) }\end{array}$ & $\begin{array}{c}\text { DI-N- } \\
\text { BUTYL } \\
\text { PHTHAL- } \\
\text { ATE } \\
\text { TOTAL } \\
\text { (UG/L) }\end{array}$ & $\begin{array}{c}4,6- \\
\text { DINITRO- } \\
\text { ORTHO- } \\
\text { CRESOL } \\
\text { TOTAL } \\
\text { (UG/L) }\end{array}$ & $\begin{array}{c}2,4,- \\
\text { DI- } \\
\text { NITRO- } \\
\text { PHENOL } \\
\text { TOTAL } \\
(\text { UG } / L)\end{array}$ & $\begin{array}{l}2,4-D I- \\
\text { NITRO- } \\
\text { TOLUENE } \\
\text { TOTAL } \\
\text { (UG/L) }\end{array}$ & $\begin{array}{l}2,6 \cdot D I- \\
\text { NITRO- } \\
\text { TOLUENE } \\
\text { TOTAL } \\
(U G / L)\end{array}$ & $\begin{array}{c}\text { DI - N- } \\
\text { OCTYL } \\
\text { PHTHAL- } \\
\text { ATE } \\
\text { TOTAL } \\
\text { (UG/L) }\end{array}$ & $\begin{array}{c}\text { BIS }\{2- \\
\text { ETHYL } \\
\text { HEXYL) } \\
\text { PHTHAL- } \\
\text { ATE } \\
\text { TOTAL } \\
\text { (UG/L) }\end{array}$ & $\begin{array}{l}\text { FLUOR - } \\
\text { ANTHENE } \\
\text { TOTAL } \\
\text { (UG/L) }\end{array}$ & $\begin{array}{c}\text { FLUOR- } \\
\text { ENE } \\
\text { TOTAL } \\
\text { (UG/L) }\end{array}$ \\
\hline $\begin{array}{c}\text { OCT } 1992 \\
28-29\end{array}$ & & & & & & & & & & & \\
\hline DEC & $<5.0$ & $<5.0$ & $<5.0$ & $<30.0$ & $<20.0$ & $<5.0$ & $<5.0$ & $<10.0$ & $<5.0$ & $<5.0$ & $<5.0$ \\
\hline $\begin{array}{c}09-09 \\
\text { JAN } 1993\end{array}$ & $<5.0$ & $<5.0$ & $<5.0$ & $<30.0$ & $<20.0$ & $<5.0$ & $<5.0$ & $<10.0$ & $<5.0$ & $<5.0$ & $<5.0$ \\
\hline $\begin{array}{l}09 \cdot 09 \\
\text { JAN }\end{array}$ & $<5.0$ & $<5.0$ & $<5.0$ & $<30.0$ & $<20.0$ & $<5.0$ & $<5.0$ & $<10.0$ & $<5.0$ & $<5.0$ & $<5.0$ \\
\hline $\begin{array}{l}28-28 \\
\text { FEB }\end{array}$ & $<5.0$ & $<5.0$ & $<5.0$ & $<30.0$ & $<20.0$ & $<5.0$ & $<5.0$ & $<10.0$ & $<5.0$ & $<5.0$ & $<5.0$ \\
\hline $\begin{array}{l}24-24 \\
\text { MAR }\end{array}$ & $<5.0$ & $<5.0$ & $<5.0$ & $<30.0$ & $<20.0$ & $<5.0$ & $<5.0$ & $<10.0$ & 5.0 & $<5.0$ & $<5.0$ \\
\hline $\begin{array}{l}11 \cdot 12 \\
\operatorname{MAR}\end{array}$ & $<5.0$ & $<5.0$ & $<5.0$ & $<30.0$ & $<20.0$ & $<5.0$ & $<5.0$ & $<10.0$ & $<5.0$ & $<5.0$ & $<5.0$ \\
\hline $28-28$ & $<5.0$ & $<5.0$ & $<5.0$ & $<30.0$ & $<20.0$ & $<5.0$ & $<5.0$ & $<10.0$ & $<5.0$ & $<5.0$ & $<5.0$ \\
\hline DATE & $\begin{array}{l}\text { HEXA- } \\
\text { CHLORO- } \\
\text { BENZENE } \\
\text { TOTAL } \\
\text { (UG/L) }\end{array}$ & $\begin{array}{c}\text { HEXA- } \\
\text { CHLORO- } \\
\text { CYCLO- } \\
\text { PENT- } \\
\text { ADIENE } \\
\text { TOTAL } \\
\text { (UG/L) }\end{array}$ & $\begin{array}{l}\text { HEXA- } \\
\text { CHLORO- } \\
\text { ETHANE } \\
\text { TOTAL } \\
\text { (UG/L) }\end{array}$ & $\begin{array}{c}\text { INDENO } \\
(1,2,3- \\
\text { CD) } \\
\text { PYRENE } \\
\text { TOTAL } \\
\text { (UG/L) }\end{array}$ & $\begin{array}{l}\text { ISO- } \\
\text { PHORONE } \\
\text { TOTAL } \\
\text { (UG/L) }\end{array}$ & $\begin{array}{l}\text { NITRO- } \\
\text { BENZENE } \\
\text { TOTAL } \\
\text { (UG/L) }\end{array}$ & $\begin{array}{c}\text { N-NITRO- } \\
\text { SODI - } \\
\text { METHY - } \\
\text { LAMINE } \\
\text { TOTAL } \\
\text { (UG/L) }\end{array}$ & $\begin{array}{l}\text { 2- } \\
\text { NITRO- } \\
\text { PHENOL } \\
\text { TOTAL } \\
\text { (UG/L) }\end{array}$ & $\begin{array}{l}4- \\
\text { NITRO- } \\
\text { PHENOL } \\
\text { TOTAL } \\
\text { (UG/L) }\end{array}$ & $\begin{array}{c}\text { N- } \\
\text { NITRO- } \\
\text { SODI-N- } \\
\text { PROPYL- } \\
\text { AMINE } \\
\text { TOTAL } \\
\text { (UG/L) }\end{array}$ & $\begin{array}{c}\text { N-NITRO- } \\
\text { SODI- } \\
\text { PHENY - } \\
\text { LAMINE } \\
\text { TOTAL } \\
\text { (UG/L) }\end{array}$ \\
\hline OCT 1992 & & & & & & & & & & & \\
\hline $\begin{array}{l}28-29 \\
\text { DEC }\end{array}$ & $<5.0$ & $<5.0$ & $<5.0$ & $<10.0$ & $<5.0$ & $<5.0$ & $<5.0$ & $<5.0$ & $<30.0$ & $<5.0$ & $<5.0$ \\
\hline $\begin{array}{c}09 \cdot 09 \\
\text { JAN } 1993\end{array}$ & $<5.0$ & $<5.0$ & $<5.0$ & $<10.0$ & $<5.0$ & $<5.0$ & $<5.0$ & $<5.0$ & $<30.0$ & $<5.0$ & $<5.0$ \\
\hline $\begin{array}{l}09-09 \\
\text { JAN }\end{array}$ & $<5.0$ & $<5.0$ & $<5.0$ & $<10.0$ & $<5.0$ & $<5.0$ & $<5.0$ & $<5.0$ & $<30.0$ & $<5.0$ & $<5.0$ \\
\hline $\begin{array}{l}28-28 \\
\text { FEB }\end{array}$ & $<5.0$ & $<5.0$ & $<5.0$ & $<10.0$ & $<5.0$ & $<5.0$ & $<5.0$ & $<5.0$ & $<30.0$ & $<5.0$ & $<5.0$ \\
\hline $\begin{array}{l}24-24 \\
\text { MAR }\end{array}$ & $<5.0$ & $<5.0$ & $<5.0$ & $<10.0$ & $<5.0$ & $<5.0$ & $<5.0$ & $<5.0$ & $<30.0$ & $<5.0$ & $<5.0$ \\
\hline $\begin{array}{l}11 \cdot 12 \\
\text { MAR }\end{array}$ & $<5.0$ & $<5.0$ & $<5.0$ & $<10.0$ & $<5.0$ & $<5.0$ & $<5.0$ & $<5.0$ & $<30.0$ & $<5.0$ & $<5.0$ \\
\hline $28-28$ & $<5.0$ & $<5.0$ & $<5.0$ & $<10.0$ & $<5.0$ & $<5.0$ & $<5.0$ & $<5.0$ & $<30.0$ & $<5.0$ & $<5.0$ \\
\hline DATE & $\begin{array}{c}\text { PENTA- } \\
\text { CHLORO- } \\
\text { PHENOL } \\
\text { TOTAL } \\
\text { (UG/L) }\end{array}$ & $\begin{array}{l}\text { PHENAN- } \\
\text { THRENE } \\
\text { TOTAL } \\
\text { (UG/L) }\end{array}$ & $\begin{array}{c}\text { PHENOL } \\
\text { (C6H- } \\
5 O H) \\
\text { TOTAL } \\
\text { (UG/L) }\end{array}$ & $\begin{array}{c}\text { PYRENE } \\
\text { TOTAL } \\
\text { (UG/L) }\end{array}$ & $\begin{array}{c}2,4,6- \\
\text { TRI- } \\
\text { CHLORO- } \\
\text { PHENOL } \\
\text { TOTAL } \\
\text { (UG/L) }\end{array}$ & $\begin{array}{l}\text { ALDRIN, } \\
\text { TOTAL } \\
\text { (UG/L) }\end{array}$ & $\begin{array}{c}\text { P, } P^{\prime} \\
\text { DDT, } \\
\text { TOTAL } \\
\text { (UG/L) }\end{array}$ & $\begin{array}{l}\text { ALPHA } \\
\text { BHC } \\
\text { TOTAL } \\
\text { (UG/L) }\end{array}$ & $\begin{array}{l}\text { BETA } \\
\text { BENZENE } \\
\text { HEXA- } \\
\text { CHLOR- } \\
\text { IDE } \\
\text { TOTAL } \\
\text { (UG/L) }\end{array}$ & $\begin{array}{c}\text { DELTA } \\
\text { BENZENE } \\
\text { HEXA- } \\
\text { CHLOR- } \\
\text { IDE } \\
\text { TOTAL } \\
\text { (UG/L) }\end{array}$ & $\begin{array}{l}\text { LINDANE } \\
\text { TOTAL } \\
\text { (UG/L) }\end{array}$ \\
\hline $\begin{array}{l}\text { OCT } 1992 \\
28-29 \\
\text { DEC }\end{array}$ & $<30.0$ & $<5.0$ & $<5.0$ & $<5.0$ & $<20.0$ & $<0.040$ & $<0.10$ & $<0.03$ & $<0.03$ & $<0.09$ & $<0.030$ \\
\hline $\begin{array}{c}09-09 \\
\text { JAN } 1993\end{array}$ & $<30.0$ & $<5.0$ & $<5.0$ & $<5.0$ & $<20.0$ & $<0.040$ & $<0.10$ & $<0.03$ & $<0.03$ & $<0.09$ & $<0.030$ \\
\hline $\begin{array}{l}09 \cdot 09 \\
\text { JAN }\end{array}$ & $<30.0$ & $<5.0$ & $<5.0$ & $<5.0$ & $<20.0$ & $<0.080$ & $<0.20$ & $<0.06$ & $<0.06$ & $<0.20$ & $<0.060$ \\
\hline $\begin{array}{l}28-28 \\
\text { FEB }\end{array}$ & $<30.0$ & $<5.0$ & $<5.0$ & $<5.0$ & $<20.0$ & $<0.040$ & $<0.10$ & $<0.03$ & $<0.03$ & $<0.09$ & $<0.030$ \\
\hline $24 \cdot 24$ & $<30.0$ & $<5.0$ & $<5.0$ & $<5.0$ & $<20.0$ & $<0.040$ & $<0.10$ & $<0.03$ & $<0.03$ & $<0.09$ & $<0.030$ \\
\hline $11 \cdot 12$ & $<30.0$ & $<5.0$ & $<5.0$ & $<5.0$ & $<20.0$ & $<0.040$ & $<0.10$ & $<0.03$ & $<0.03$ & $<0.09$ & $<0.030$ \\
\hline $28 \cdot 28$ & $<30.0$ & $<5.0$ & $<5.0$ & $<5.0$ & $<20.0$ & $<0.040$ & $<0.10$ & $<0.03$ & $<0.03$ & $<0.09$ & $<0.030$ \\
\hline
\end{tabular}


Table 2. Water-quality data for storm-sewer outfall stations, Arlington, Texas, 1992-93-Continued

08049320 - RIVER LEGACY PARK OUTFALL AT GREEN OAKS BOULEVARD, ARLINGTON, TX (WY 1993)-CONtinUed

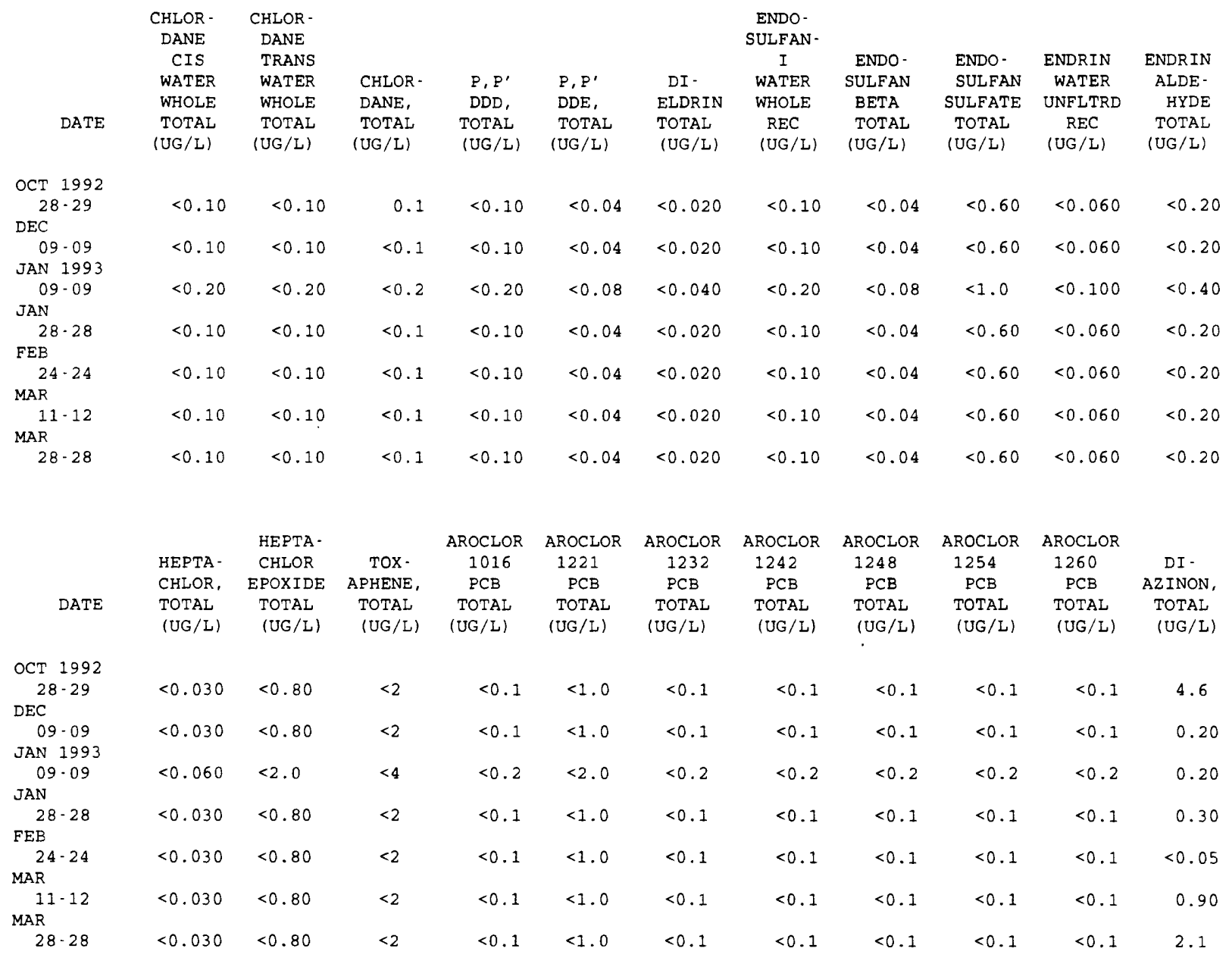


Table 2. Water-quality data for storm-sewer outfall stations, Arlington, Texas, 1992-93-Continued

08049360 - TRIBUTARY TO WEST FORK TRINITY RIVER OUTFALL, AT BAIRD'S FARM ROAD, ARLINGTON, TX (WY 1993)

\begin{tabular}{|c|c|c|c|c|c|c|c|c|c|c|c|c|}
\hline DATE & TIME & $\begin{array}{l}\text { PRECIP- } \\
\text { ITATION } \\
\text { TOTAL } \\
\text { INCHES/ } \\
\text { STORM }\end{array}$ & $\begin{array}{l}\text { ELAPSED } \\
\text { TIME } \\
\text { OF } \\
\text { STORM } \\
\text { (HOURS) }\end{array}$ & $\begin{array}{l}\text { STORM } \\
\text { WATER } \\
\text { FLOW } \\
\text { (MGD) }\end{array}$ & $\begin{array}{l}\text { SPE- } \\
\text { CIFIC } \\
\text { CON- } \\
\text { DUCT - } \\
\text { ANCE } \\
\text { (US/CM) }\end{array}$ & $\begin{array}{c}\text { SPE- } \\
\text { CIFIC } \\
\text { CON- } \\
\text { DUCT- } \\
\text { ANCE } \\
\text { LAB } \\
\text { (US/CM) }\end{array}$ & $\begin{array}{c}\text { PH } \\
\text { WATER } \\
\text { WHOLE } \\
\text { FIELD } \\
\text { (STAND - } \\
\text { ARD } \\
\text { UNITS) }\end{array}$ & $\begin{array}{c}\text { PH } \\
\text { WATER } \\
\text { WHOLE } \\
\text { LAB } \\
\text { (STAND- } \\
\text { ARD } \\
\text { UNITS) }\end{array}$ & $\begin{array}{c}\text { TEMPER - } \\
\text { ATURE } \\
\text { WATER } \\
\text { (DEG C) }\end{array}$ & $\begin{array}{l}\text { OXYGEN } \\
\text { DEMAND, } \\
\text { CHEM- } \\
\text { ICAL } \\
\text { (HIGH } \\
\text { LEVEL) } \\
\text { (MG/L) }\end{array}$ & $\begin{array}{l}\text { OXYGEN } \\
\text { DEMAND, } \\
\text { BIO- } \\
\text { CHEM- } \\
\text { ICAL, } \\
5 \text { DAY } \\
\text { (MG/L) }\end{array}$ & $\begin{array}{l}\text { COLI - } \\
\text { FORM, } \\
\text { FECAL, } \\
0.7 \\
\text { UM-MF } \\
\text { (COLS./ } \\
100 \mathrm{ML)}\end{array}$ \\
\hline OCT 1992 & & & & & & & & & & & & \\
\hline $\begin{array}{l}29 \cdot 29 \\
\text { Nov }\end{array}$ & 0005 & 0.49 & 4.3 & 0.42 & 152 & 104 & 7.8 & 7.7 & 21.0 & 47 & 6.5 & 170000 \\
\hline $\begin{array}{c}19 \cdot 19 \\
\text { FEB } 1993\end{array}$ & 0100 & 1.2 & 10.0 & 1.3 & 94 & 108 & 7.4 & 7.3 & 17.0 & 35 & 3.9 & 28000 \\
\hline $\begin{array}{l}03-03 \\
\text { FEB }\end{array}$ & 0552 & 0.44 & 8.7 & 0.35 & 108 & 153 & 6.2 & 7.6 & 12.0 & 67 & 5.2 & 19000 \\
\hline $\begin{array}{l}10 \cdot 10 \\
\text { MAR }\end{array}$ & 0346 & 0.19 & 4.2 & 0.12 & 842 & 134 & 7.4 & 7.3 & 13.0 & 51 & 4.5 & K9 700 \\
\hline $\operatorname{MAR}^{11-12}$ & 1806 & 0.53 & 7.4 & 0.22 & 102 & 91 & 8.0 & 7.1 & 10.5 & 31 & 4.1 & 3700 \\
\hline $\begin{array}{l}28 \cdot 28 \\
A P R\end{array}$ & 0739 & 0.50 & 3.8 & 0.39 & 84 & 100 & 7.6 & 7.2 & 15.0 & 48 & 4.9 & 25000 \\
\hline $14-14$ & 0624 & 0.31 & 0.80 & 0.23 & 585 & 82 & 8.3 & 6.8 & 19.0 & 53 & 7.8 & 16000 \\
\hline DATE & $\begin{array}{l}\text { STREP - } \\
\text { TOCOCCI } \\
\text { FECAL, } \\
\text { RF AGAR } \\
\text { (COLS. } \\
\text { PER } \\
100 \mathrm{ML})\end{array}$ & $\begin{array}{l}\text { HARD- } \\
\text { NESS } \\
\text { TOTAL } \\
\text { (MG/L } \\
\text { AS } \\
\text { CACO3) }\end{array}$ & $\begin{array}{l}\text { HARD- } \\
\text { NESS } \\
\text { NONCARB } \\
\text { DISSOLV } \\
\text { FLD. AS } \\
\text { CACO3 } \\
\text { (MG/L) }\end{array}$ & $\begin{array}{l}\text { ALKA- } \\
\text { LINITY } \\
\text { WAT DIS } \\
\text { FIX END } \\
\text { FIELD } \\
\text { CAC03 } \\
\text { (MG/L) }\end{array}$ & $\begin{array}{l}\text { SOLIDS, } \\
\text { SUM OF } \\
\text { CONSTI - } \\
\text { TUENTS, } \\
\text { DIS- } \\
\text { SOLVED } \\
\text { (MG/L) }\end{array}$ & $\begin{array}{l}\text { RESIDUE } \\
\text { TOTAL } \\
\text { AT } 105 \\
\text { DEG. C. } \\
\text { SUS - } \\
\text { PENDED } \\
\text { (MG/L) }\end{array}$ & $\begin{array}{l}\text { SOLIDS, } \\
\text { RESIDUE } \\
\text { AT } 180 \\
\text { DEG. C } \\
\text { DIS - } \\
\text { SOLVED } \\
\text { (MG/L) }\end{array}$ & $\begin{array}{l}\text { CALCIUM } \\
\text { DIS- } \\
\text { SOLVED } \\
\text { (MG/L } \\
\text { AS CA) }\end{array}$ & $\begin{array}{l}\text { MAGNE - } \\
\text { SIUM, } \\
\text { DIS - } \\
\text { SOLVED } \\
\text { (MG/L } \\
\text { AS MG) }\end{array}$ & $\begin{array}{l}\text { SODIUM, } \\
\text { DIS- } \\
\text { SOLVED } \\
\text { (MG/L } \\
\text { AS NA) }\end{array}$ & $\begin{array}{l}\text { SODIUM } \\
\text { PERCENT }\end{array}$ & $\begin{array}{c}\text { SODIUM } \\
\text { AD- } \\
\text { SORP- } \\
\text { TION } \\
\text { RATIO }\end{array}$ \\
\hline OCT 1992 & & & & & & & & & & & & \\
\hline $\begin{array}{l}29-29 \\
\text { NoV }\end{array}$ & 320000 & 24 & 4 & 20 & 57 & 114 & 56 & 8.7 & 0.62 & 3.0 & 19 & 0.3 \\
\hline $\begin{array}{c}19.19 \\
F E B \quad 1993\end{array}$ & 51000 & 31 & 0 & 31 & 52 & 13 & 61 & 11 & 0.94 & 4.8 & 23 & 0.4 \\
\hline $\begin{array}{l}03-03 \\
F E B\end{array}$ & 72000 & 36 & 0 & 41 & 68 & 78 & 49 & 13 & 0.92 & 4.9 & 21 & 0.4 \\
\hline $\begin{array}{l}10 \cdot 10 \\
\text { MAR }\end{array}$ & K7200 & 36 & 0 & 36 & 68 & 30 & 73 & 13 & 0.96 & 5.9 & 24 & 0.4 \\
\hline $\operatorname{MAR}^{11 \cdot 12}$ & 19000 & 30 & 2 & 28 & 48 & 21 & 56 & 11 & 0.73 & 3.4 & 18 & 0.3 \\
\hline $\begin{array}{l}28-28 \\
\text { APR }\end{array}$ & 670000 & 33 & 7 & 26 & 51 & 27 & 55 & 12 & 0.79 & 2.9 & 15 & 0.2 \\
\hline $14 \cdot 14$ & 24000 & 21 & 5 & 16 & 43 & 78 & 48 & 7.4 & 0.52 & 2.8 & 21 & 0.3 \\
\hline DATE & $\begin{array}{l}\text { POTAS - } \\
\text { SIUM, } \\
\text { DIS - } \\
\text { SOLVED } \\
\text { (MG/L } \\
\text { AS K) }\end{array}$ & $\begin{array}{l}\text { SULFATE } \\
\text { DIS- } \\
\text { SOLVED } \\
\text { (MG/L } \\
\text { AS SO4) }\end{array}$ & $\begin{array}{l}\text { CHLO- } \\
\text { RIDE, } \\
\text { DIS - } \\
\text { SOLVED } \\
\text { (MG/L } \\
\text { AS CL) }\end{array}$ & $\begin{array}{l}\text { NITRO- } \\
\text { GEN, } \\
\text { NITRITE } \\
\text { TOTAL } \\
\text { (MG/L } \\
\text { AS N) }\end{array}$ & $\begin{array}{l}\text { NITRO- } \\
\text { GEN, } \\
\text { NO2+NO3 } \\
\text { TOTAL } \\
\text { (MG/L } \\
\text { AS N) }\end{array}$ & $\begin{array}{l}\text { NITRO- } \\
\text { GEN, } \\
\text { AMMONIA } \\
\text { TOTAL } \\
\text { (MG/L } \\
\text { AS N) }\end{array}$ & $\begin{array}{l}\text { NITRO- } \\
\text { GEN, AM- } \\
\text { MONIA + } \\
\text { ORGANIC } \\
\text { TOTAL } \\
\text { (MG/L } \\
\text { AS N) }\end{array}$ & $\begin{array}{l}\text { PHOS - } \\
\text { PHORUS } \\
\text { TOTAL } \\
\text { (MG/L } \\
\text { AS P) }\end{array}$ & $\begin{array}{l}\text { PHOS - } \\
\text { PHORUS } \\
\text { DIS - } \\
\text { SOLVED } \\
\text { (MG/L } \\
\text { AS P) }\end{array}$ & $\begin{array}{c}\text { ANTIMONY } \\
\text { TOTAL } \\
\text { EPA } \\
\text { (UG/L } \\
\text { AS SB) }\end{array}$ & $\begin{array}{c}\text { ARSENIC } \\
\text { TOTAL } \\
\text { (UG/L } \\
\text { AS AS) }\end{array}$ & $\begin{array}{l}\text { BERYL - } \\
\text { LIUM, } \\
\text { TOTAL } \\
\text { RECOV- } \\
\text { ERABLE } \\
\text { (UG/L } \\
\text { AS BE) }\end{array}$ \\
\hline $\begin{array}{c}\text { OCT } 1992 \\
29.29\end{array}$ & 2.4 & 75 & 4,1 & 0.030 & 0.490 & 0.130 & 12 & $0 \begin{array}{lll}0 & 390\end{array}$ & 0210 & $<100$ & 3 & $<10$ \\
\hline Nov & 2.7 & 1.0 & 7.2 & ט & 0.490 & 0.120 & 1.2 & 0.290 & 0.210 & -10.0 & & 20 \\
\hline $\begin{array}{cc}19.19 \\
\text { FEB } 1993\end{array}$ & 3.0 & 7.2 & 4.5 & 0.120 & 0.590 & 0.130 & 0.80 & 0.330 & 0.290 & $<20.0$ & 2 & $<10$ \\
\hline $\begin{array}{l}03.03 \\
\text { FEB }\end{array}$ & 2.5 & 13 & 4.4 & $\cdots$ & 0.610 & $\cdots$ & 0.70 & 0.200 & 0.150 & $<10.0$ & 3 & $<10$ \\
\hline $\begin{array}{l}10 \cdot 10 \\
\text { MAR }\end{array}$ & 2.7 & 14 & 5.1 & $\cdots$ & 0.670 & $\cdots$ & 0.70 & 0.200 & 0.140 & $<10.0$ & 3 & $<10$ \\
\hline $11-12$ & 2.1 & 6.9 & 3.1 & $\cdots$ & 0.460 & $\cdots$ & 0.80 & 0.210 & 0.150 & $<20.0$ & 2 & $<10$ \\
\hline MAR & & & & & & & & & & & & \\
\hline $\begin{array}{l}28-28 \\
\text { APR }\end{array}$ & 2.8 & 8.1 & 2.7 & $\cdots$ & 0.570 & $\cdots$ & 1.2 & 0.380 & 0.210 & $<10.0$ & 2 & $<10$ \\
\hline $14-14$ & 2.2 & 6.5 & 3.3 & $\cdots$ & 0.600 & $\cdots$ & 0.80 & 0.270 & 0.170 & $<10.0$ & 2 & $<10$ \\
\hline
\end{tabular}


Table 2. Water-quality data for storm-sewer outfall stations, Arlington, Texas, 1992-93-Continued

08049360 - TRIBUTARY TO WEST FORK TRINITY RIVER OUTFALL, AT BAIRD'S FARM ROAD, ARLINGTON, TX (WY 1993)-CONEINUEd

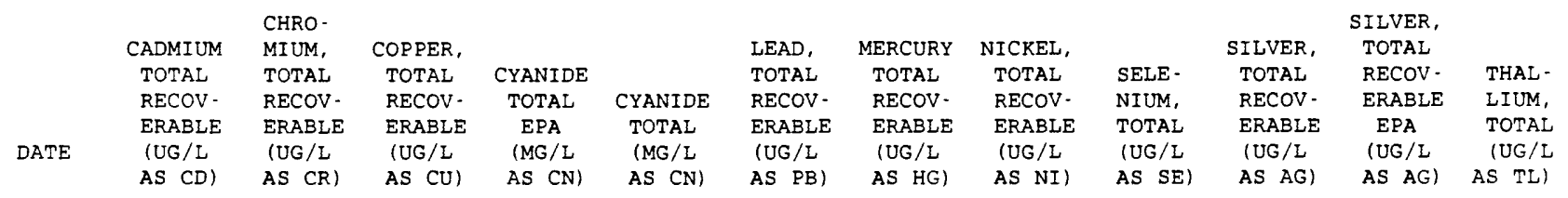

\begin{tabular}{|c|c|c|c|c|c|c|c|c|c|c|c|c|}
\hline \multicolumn{13}{|l|}{ OCT 1992} \\
\hline $29 \cdot 29$ & $<1$ & 8 & 8 & $<0.010$ & 0.020 & 24 & $<0.10$ & 4 & $<2$ & $<1$ & $<0.500$ & $<5$ \\
\hline \multicolumn{13}{|l|}{ Nov } \\
\hline $19-19$ & $<1$ & 9 & 4 & $<0.010$ & $<0.010$ & 7 & $<0.10$ & 3 & $<2$ & $<1$ & $<0.500$ & $<10$ \\
\hline \multicolumn{13}{|l|}{ FEB 1993} \\
\hline 03.03 & $<1$ & 7 & 8 & $<0.010$ & $<0.010$ & 22 & $<0.10$ & 3 & $<2$ & $<1$ & $<0.500$ & $<5$ \\
\hline \multicolumn{13}{|l|}{ FEB } \\
\hline $10-10$ & $<1$ & 6 & 5 & $<0.010$ & $<0.010$ & 10 & $<0.10$ & 4 & $<2$ & $<1$ & $<0.500$ & $<10$ \\
\hline \multicolumn{13}{|l|}{ MAR } \\
\hline $11-12$ & $<1$ & 2 & 4 & $<0.010$ & $<0.010$ & 5 & $<0.10$ & 2 & $<2$ & $<1$ & $<0.500$ & $<5$ \\
\hline \multicolumn{13}{|l|}{ MAR } \\
\hline $28 \cdot 28$ & $<1$ & 1 & 4 & $<0.010$ & 0.080 & 9 & $<0.10$ & 3 & $<2$ & $<1$ & $<1.00$ & $<5$ \\
\hline \multicolumn{13}{|l|}{ APR } \\
\hline $14 \cdot 14$ & $<1$ & $<10$ & 9 & $<0.010$ & $<0.010$ & 20 & $<0.10$ & 3 & $<2$ & $<1$ & $<0.500$ & $<5$ \\
\hline
\end{tabular}

\begin{tabular}{|c|c|c|c|c|c|c|c|c|c|c|c|c|}
\hline & & & OIL AND & & & & \multicolumn{5}{|c|}{ METHANE } & \multirow{2}{*}{$\begin{array}{c}\text { BENZENE } \\
\text { SEC }\end{array}$} \\
\hline & ZINC, & & GREASE, & & & & & BROMO - & BROMO & & BENZENE & \\
\hline & TOTAL & CARBON, & TOTAL & & & & & BENZENE & CHLORO - & & N-BUTYL & BUTYL - \\
\hline & RECOV - & ORGANIC & RECOV. & & ACRO - & ACRYLO- & & WATER, & WAT & BROMO - & WATER & WATER \\
\hline & ERABLE & TOTAL & GRAVI - & PHENOLS & LEIN & NITRILE & BENZENE & WHOLE, & UNFLTRD & FORM & UNFLTRD & UNFLTRD \\
\hline DATE & (UG / L & (MG/L & METRIC & TOTAL & TOTAL & TOTAL & TOTAL & TOTAL & REC & TOTAL & REC & REC \\
\hline & AS $\mathrm{ZN}$ ) & AS C) & $(\mathrm{MG} / \mathrm{L})$ & (UG/L) & (UG/L) & (UG/L) & (UG/L) & (UG/L) & (UG/L) & & $(U G / L)$ & $\langle U G / L\rangle$ \\
\hline
\end{tabular}

\begin{tabular}{|c|c|c|c|c|c|c|c|c|c|c|c|c|}
\hline $29-29$ & 60 & 14 & $<1$ & 6 & $<20$ & $<20$ & $<0.2$ & $<0.2$ & $<0.20$ & $<0.2$ & $<0.20$ & $<0.20$ \\
\hline \multicolumn{13}{|l|}{ Nov } \\
\hline $19-19$ & 50 & 8.9 & $<1$ & 2 & $<20$ & $<20$ & $<0.2$ & $<0.2$ & $<0.20$ & $<0.2$ & $<0.20$ & $<0.20$ \\
\hline \multicolumn{13}{|l|}{ FEB 1993} \\
\hline 03.03 & 90 & 14 & 3 & 4 & $<20$ & $<20$ & $<0.2$ & $<0.2$ & $<0.20$ & $<0.2$ & $<0.20$ & $<0.20$ \\
\hline \multicolumn{13}{|l|}{ FEB } \\
\hline $10-10$ & 40 & 11 & 5 & 2 & $<20$ & $<20$ & $<0.2$ & $<0.2$ & $<0.20$ & $<0.2$ & $<0.20$ & $<0.20$ \\
\hline \multicolumn{13}{|l|}{ MAR } \\
\hline $11-12$ & 20 & 8.2 & $<1$ & 4 & $<20$ & $<20$ & $<0.2$ & $<0.2$ & $<0.20$ & $<0.2$ & $<0.20$ & $<0.20$ \\
\hline \multicolumn{13}{|l|}{ MAR } \\
\hline $28-28$ & 40 & 12 & $<1$ & 1 & $<20$ & $<20$ & $<0.2$ & $<0.2$ & $<0.20$ & $<0.2$ & $<0.20$ & $<0.20$ \\
\hline \multicolumn{13}{|l|}{ APR } \\
\hline $14-14$ & 70 & 14 & 4 & 5 & $<20$ & $<20$ & $<0.2$ & $<0.2$ & $<0.20$ & $<0.2$ & $<0.20$ & $<0.20$ \\
\hline
\end{tabular}

\begin{tabular}{|c|c|c|c|c|c|c|c|c|c|c|c|c|}
\hline & BENZENE & & & & & 2 & & & 0 & & DIBROMO & DI - \\
\hline & $\begin{array}{l}\text { TERT - } \\
\text { BUTYL - }\end{array}$ & $\begin{array}{l}\text { CARBON - } \\
\text { TETRA - }\end{array}$ & & $\begin{array}{c}\text { CHLORO - } \\
\text { DI - }\end{array}$ & & $\begin{array}{l}\text { CHLORO - } \\
\text { ETHYL - }\end{array}$ & & METHYL - & $\begin{array}{l}\text { CHLORO- } \\
\text { TOLUENE }\end{array}$ & $\begin{array}{l}\text { TOLUENE } \\
\text { P-CHLOR }\end{array}$ & $\begin{array}{l}\text { CHLORO- } \\
\text { PROPANE }\end{array}$ & $\begin{array}{l}\text { BROMO- } \\
\text { METHANE }\end{array}$ \\
\hline & $\begin{array}{l}\text { WATER } \\
\text { UNFLTRD }\end{array}$ & $\begin{array}{l}\text { CHLO- } \\
\text { RIDE }\end{array}$ & $\begin{array}{l}\text { CHLORO- } \\
\text { BENZENE }\end{array}$ & $\begin{array}{l}\text { BROMO- } \\
\text { METHANE }\end{array}$ & $\begin{array}{l}\text { CHLORO- } \\
\text { ETHANE }\end{array}$ & $\begin{array}{c}\text { VINYL- } \\
\text { ETHER }\end{array}$ & $\begin{array}{l}\text { CHLORO- } \\
\text { FORM }\end{array}$ & $\begin{array}{l}\text { CHLO- } \\
\text { RIDE }\end{array}$ & $\begin{array}{l}\text { WATER } \\
\text { WHOLE }\end{array}$ & $\begin{array}{l}\text { WATER } \\
\text { UNFLTRD }\end{array}$ & $\begin{array}{l}\text { WATER } \\
\text { WHOLE }\end{array}$ & $\begin{array}{l}\text { WATER } \\
\text { WHOLE }\end{array}$ \\
\hline DATE & $\begin{array}{c}\text { REC } \\
\text { (UG/L) }\end{array}$ & $\begin{array}{l}\text { TOTAL } \\
\text { (UG/L) }\end{array}$ & $\begin{array}{l}\text { TOTAL } \\
\text { (UG/L) }\end{array}$ & $\begin{array}{l}\text { TOTAL } \\
\text { (UG/L) }\end{array}$ & $\begin{array}{l}\text { TOTAL } \\
\text { (UG/L) }\end{array}$ & $\begin{array}{l}\text { TOTAL } \\
\text { (UG/L) }\end{array}$ & $\begin{array}{l}\text { TOTAL } \\
\text { (UG/L) }\end{array}$ & $\begin{array}{l}\text { TOTAL } \\
\text { (UG/L) }\end{array}$ & $\begin{array}{l}\text { TOTAL } \\
\text { (UG/L) }\end{array}$ & $\begin{array}{c}\text { REC } \\
(U G / L)\end{array}$ & $\begin{array}{l}\text { TOT . REC } \\
\text { (UG/L) }\end{array}$ & $\begin{array}{l}\text { RECOVER } \\
(\mathrm{UG} / \mathrm{L})\end{array}$ \\
\hline
\end{tabular}

\begin{tabular}{|c|c|c|c|c|c|c|c|c|c|c|c|c|}
\hline \multicolumn{13}{|l|}{ OCT 1992} \\
\hline $29-29$ & $<0.20$ & $<0.2$ & $<0.20$ & $<0.2$ & $<0.2$ & $<1.0$ & $<0.2$ & $<0.2$ & $<0.2$ & $<0.20$ & $<1.0$ & $<0.2$ \\
\hline \multicolumn{13}{|l|}{ NOV } \\
\hline $19-19$ & $<0.20$ & $<0.2$ & $<0.20$ & $<0.2$ & $<0.2$ & $<1.0$ & $<0.2$ & $<0.2$ & $<0.2$ & $<0.20$ & $<1.0$ & $<0.2$ \\
\hline \multicolumn{13}{|l|}{ FEB 1993} \\
\hline $03-03$ & $<0.20$ & $<0.2$ & $<0.20$ & $<0.2$ & $<0.2$ & $<1.0$ & $<0.2$ & $<0.2$ & $<0.2$ & $<0.20$ & $<1.0$ & $<0.2$ \\
\hline \multicolumn{13}{|l|}{ FEB } \\
\hline $10 \cdot 10$ & $<0.20$ & $<0.2$ & $<0.20$ & $<0.2$ & $<0.2$ & $<1.0$ & $<0.2$ & $<0.2$ & $<0.2$ & $<0.20$ & $<1.0$ & $<0.2$ \\
\hline \multicolumn{13}{|l|}{ MAR } \\
\hline $11-12$ & $<0.20$ & $<0.2$ & $<0.20$ & $<0.2$ & $<0.2$ & $<1.0$ & $<0.2$ & $<0.2$ & $<0.2$ & $<0.20$ & $<1.0$ & $<0.2$ \\
\hline \multicolumn{13}{|l|}{ MAR } \\
\hline $28-28$ & $<0.20$ & $<0.2$ & $<0.20$ & $<0.2$ & $<0.2$ & $<1.0$ & $<0.2$ & $<0.2$ & $<0.2$ & $<0.20$ & $<1.0$ & $<0.2$ \\
\hline \multicolumn{13}{|l|}{ APR } \\
\hline $14-14$ & $<0.20$ & $<0.2$ & $<0.20$ & $<0.2$ & $<0.2$ & $<1.0$ & $<0.2$ & $<0.2$ & $<0.2$ & $<0.20$ & $<1.0$ & $<0.2$ \\
\hline
\end{tabular}


Table 2. Water-quality data for storm-sewer outfall stations, Arlington, Texas, 1992-93-Continued

08049360 - TRIBUTARY TO WEST FORK TRINITY RIVER OUTFALL, AT BAIRD'S FARM ROAD, ARLINGTON, TX (WY 1993)-CONEINUEd

\begin{tabular}{|c|c|c|c|c|c|c|c|c|c|c|c|c|}
\hline & $\begin{array}{c}3,2- \\
\text { DIBROMO }\end{array}$ & - ד & & $\begin{array}{l}1,1-D I \\
\text { CHLORO. }\end{array}$ & $\begin{array}{c}\text { BENZENE } \\
0 .\end{array}$ & & $\begin{array}{l}\text { BENZENE } \\
1,4-D I-\end{array}$ & $\begin{array}{l}\text { DI - } \\
\text { CHLORO. }\end{array}$ & & & $\begin{array}{l}1,2-D I \text { - } \\
\text { PHENYL. }\end{array}$ & $\begin{array}{c}\text { CIS }-1,2 \\
\text { DI }\end{array}$ \\
\hline & $\begin{array}{r}\text { ETHANE } \\
\text { WATER }\end{array}$ & $\begin{array}{l}\text { CHLORO- } \\
\text { BROMO- }\end{array}$ & $\begin{array}{l}\text { CHLORO- } \\
\text { ETHYL- }\end{array}$ & $\begin{array}{l}\text { PRO- } \\
\text { PENE. }\end{array}$ & $\begin{array}{c}\text { CHLORO- } \\
\text { WATER }\end{array}$ & $\begin{array}{l}\text { CHLORO- } \\
\text { WATER }\end{array}$ & $\begin{array}{l}\text { CHLORO- } \\
\text { WATER }\end{array}$ & $\begin{array}{l}\text { DI - } \\
\text { FLUORO- }\end{array}$ & $\begin{array}{l}1,1-D I- \\
\text { CHLORO- }\end{array}$ & $\begin{array}{l}1,2 \text {-DI - } \\
\text { CHLORO- }\end{array}$ & $\begin{array}{l}\text { HYDRA - } \\
\text { ZINE }\end{array}$ & $\begin{array}{l}\text { CHLORO } \\
\text { ETHENE }\end{array}$ \\
\hline & $\begin{array}{l}\text { WATER } \\
\text { WHOLE }\end{array}$ & $\begin{array}{l}\text { BROMO- } \\
\text { METHANE }\end{array}$ & $\begin{array}{c}\text { ETHYL- } \\
\text { ENE }\end{array}$ & WAT, WH & $\begin{array}{l}\text { WATER } \\
\text { UNFLTRD }\end{array}$ & $\begin{array}{l}\text { WATER } \\
\text { UNFLTRD }\end{array}$ & UNFLTRD & METHANE & ETHANE & ETHANE & WATER & $\begin{array}{l}\text { ETHEIVE } \\
\text { WATER }\end{array}$ \\
\hline & $\begin{array}{l}\text { TOTAL } \\
\text { (UG/L) }\end{array}$ & $\begin{array}{l}\text { TOTAL } \\
\text { (UG/L) }\end{array}$ & $\begin{array}{l}\text { TOTAL } \\
(U G / L)\end{array}$ & $\begin{array}{l}\text { TOTAL } \\
\text { (UG/L) }\end{array}$ & $\begin{array}{c}\text { REC } \\
(U G / L)\end{array}$ & $\begin{array}{c}\text { REC } \\
(U G / L)\end{array}$ & $\begin{array}{c}\text { REC } \\
(\mathrm{UG} / \mathrm{L})\end{array}$ & $\begin{array}{l}\text { TOTAL } \\
\text { (UG/L) }\end{array}$ & $\begin{array}{l}\text { TOTAL } \\
\text { (UG } / L)\end{array}$ & $\begin{array}{l}\text { TOTAL } \\
\text { (UG/L) }\end{array}$ & $\begin{array}{l}\text { TOT.REC } \\
\text { (UG/L) }\end{array}$ & $\begin{array}{l}\text { TOTAL } \\
\text { (UG/L) }\end{array}$ \\
\hline
\end{tabular}

\begin{tabular}{|c|c|c|c|c|c|c|c|c|c|c|c|c|}
\hline OCT 1992 & & & & & & & & & & & & \\
\hline $\begin{array}{l}29-29 \\
\text { NOV }\end{array}$ & $<0.2$ & $<0.2$ & $<0.2$ & $<0.2$ & $<5.0$ & $<5.0$ & $<5.0$ & $<0.2$ & $<0.2$ & $<0.2$ & $<5.0$ & $<0.2$ \\
\hline $\begin{array}{c}19-19 \\
\text { FEB } 1993\end{array}$ & $<0.2$ & $<0.2$ & $<0.2$ & $<0.2$ & $<5.0$ & $<5.0$ & $<5.0$ & $<0.2$ & $<0.2$ & $<0.2$ & $<5.0$ & $<0.2$ \\
\hline $\begin{array}{l}03.03 \\
\text { FEB }\end{array}$ & $<0.2$ & $<0.2$ & $<0.2$ & $<0.2$ & $<5.0$ & $<5.0$ & $<5.0$ & $<0.2$ & $<0.2$ & $<0.2$ & $<5.0$ & $<0.2$ \\
\hline${ }_{\text {MAR }}^{10-10}$ & $<0.2$ & $<0.2$ & $<0.2$ & $<0.2$ & $<5.0$ & $<5.0$ & $<5.0$ & $<0.2$ & $<0.2$ & $<0.2$ & $<5.0$ & $<0.2$ \\
\hline${ }_{\text {MAR }}^{11-12}$ & $<0.2$ & $<0.2$ & $<0.2$ & $<0.2$ & $<5.0$ & $<5.0$ & $<5.0$ & $<0.2$ & $<0.2$ & $<0.2$ & $<5.0$ & \\
\hline $\begin{array}{l}28 \cdot 28 \\
\text { APR }\end{array}$ & $<0.2$ & $<0.2$ & $<0.2$ & $<0.2$ & $<5.0$ & $<5.0$ & $<5.0$ & $<0.2$ & $<0.2$ & $<0.2$ & $<5.0$ & $<0.2$ \\
\hline $14-14$ & $<0.2$ & $<0,2$ & $<0,2$ & $<0.2$ & $<5.0$ & $<5,0$ & $<5,0$ & $<0.2$ & $<0.2$ & $<0,2$ & $<5,0$ & \\
\hline
\end{tabular}

\begin{tabular}{|c|c|c|c|c|c|}
\hline \multirow[b]{2}{*}{1,2} & \multicolumn{5}{|c|}{$2,2 \cdot D I$} \\
\hline & & $1,3-\mathrm{DI}$ - & CHLORO- & CIS & TRANS - \\
\hline TRANSDI & $1,2 \cdot \mathrm{DI}-$ & CHLORO- & PRO- & $1,3-\mathrm{DI}-$ & $1,3-D I-$ \\
\hline CHLORO - & CHLORO - & PROPANE & PANE & CHLORO - & CHLORO - \\
\hline ETHENE & PROPANE & WAT. WH & WAT, WH & PROPENE & PROPENE \\
\hline $\begin{array}{l}\text { TOTAL } \\
\text { (UG/L) }\end{array}$ & $\begin{array}{c}\text { TOTAL } \\
\text { (UG/L) }\end{array}$ & $\begin{array}{l}\text { TOTAL } \\
\text { (UG/L) }\end{array}$ & $\begin{array}{l}\text { TOTAL } \\
\text { (UG/L) }\end{array}$ & $\begin{array}{l}\text { TOTAL } \\
\text { (UG/L) }\end{array}$ & $\begin{array}{l}\text { TOTAL } \\
\text { (UG/L) }\end{array}$ \\
\hline
\end{tabular}

$\begin{array}{cccc} & \text { ISO - } & \text { P-ISO- } & \\ \text { HEXA- } & \text { PROPYL- } & \text { PROPYL - } & \\ \text { CHLORO- } & \text { BENZENE } & \text { TOLUENE } & \\ \text { BUT - } & \text { WATER } & \text { WATER } & \text { METHYL - } \\ \text { ADIENE } & \text { WHOLE } & \text { WHOLE } & \text { BROMIDE } \\ \text { TOTAL } & \text { REC } & \text { REC } & \text { TOTAL } \\ \text { (UG/L) } & (\text { UG } / \text { L) } & \text { (UG/L) } & \text { (UG/L) }\end{array}$

$\begin{array}{rlrllllllll}<0.2 & <0.2 & <0.2 & <0.2 & <0.2 & <0.2 & <0.2 & <5.0 & <0.20 & <0.20 & <0.2 \\ <0.2 & <0.2 & <0.2 & <0.2 & <0.2 & <0.2 & <0.2 & <5.0 & <0.20 & <0.20 & <0.2 \\ <0.2 & <0.2 & <0.2 & <0.2 & <0.2 & <0.2 & <0.2 & <5.0 & <0.20 & <0.20 & <0.2 \\ <0.2 & <0.2 & <0.2 & <0.2 & <0.2 & <0.2 & <0.2 & <5.0 & <0.20 & <0.20 & <0.2 \\ <0.2 & <0.2 & <0.2 & <0.2 & <0.2 & <0.2 & <0.2 & <5.0 & <0.20 & <0.20 & <0.2 \\ <0.2 & <0.2 & <0.2 & <0.2 & <0.2 & <0.2 & <0.2 & <5.0 & <0.20 & <0.20 & <0.2 \\ <0.2 & <0.2 & <0.2 & <0.2 & <0.2 & <0.2 & <0.2 & <5.0 & <0.20 & <0.20 & <0.2\end{array}$

$\begin{array}{cccc} & \text { METHYL } & & \\ \text { METHYL- } & \text { ETHER } & & \text { BENZENE } \\ \text { ENE } & \text { TERT - } & & \text { N-PROPY } \\ \text { CHLO- } & \text { BUTYL } & \text { NAPHTH - } & \text { WATER } \\ \text { RIDE } & \text { WAT UNF } & \text { ALENE } & \text { UNFLTRD } \\ \text { TOTAL } & \text { REC } & \text { TOTAL } & \text { REC }\end{array}$

$\begin{array}{ccc}\text { ETHANE, } & \text { ETHANE, } & \\ 1112- & 1,1,2,2 & \text { TETRA- } \\ \text { TETRA- } & \text { TETRA- } & \text { CHLORO- } \\ \text { CHLORO- } & \text { CHLORO- } & \text { ETHYL- } \\ \text { WAT UNF } & \text { WAT UNF } & \text { ENE } \\ \text { REC } & \text { REC } & \text { TOTAL } \\ \text { (UG } / L) & \text { (UG } / L) & \text { (UG } / L)\end{array}$

$\begin{array}{ccc} & 1,2,3- & \text { BENZENE } \\ & \text { TRI - } & 1,2,4- \\ & \text { CHLORO } & \text { TRI - } \\ & \text { BENZENE } & \text { CHLORO- } \\ \text { TOLUENE } & \text { WAT, WH } & \text { WAT UNF } \\ \text { TOTAL } & \text { REC } & \text { REC } \\ \text { (UG } / \text { L) } & \text { (UG/L) } & \text { (UG/L) }\end{array}$

OCT 1992

$29 \cdot 29$

NOV

$19-19$

FEB 1993

$03-03$

FEB

$10-10$

MAR

11- 12

MAR

$28 \cdot 28$

APR

14-14

$\begin{array}{llllllllllll}<0.2 & <1.0 & <5.0 & <0.20 & <0.2 & <0.2 & <0.2 & <0.2 & <0.2 & <0.20 & <5.0 \\ <0.2 & <1.0 & <5.0 & <0.20 & <0.2 & <0.2 & <0.2 & <0.2 & <0.2 & <0.20 & <5.0 \\ <0.2 & <1.0 & <5.0 & <0.20 & <0.2 & <0.2 & <0.2 & <0.2 & <0.2 & <0.20 & <5.0 \\ <0.2 & <1.0 & <5.0 & <0.20 & <0.2 & <0.2 & <0.2 & <0.2 & <0.2 & <0.20 & <5.0 \\ <0.2 & <1.0 & <5.0 & <0.20 & <0.2 & <0.2 & <0.2 & <0.2 & <0.2 & <0.20 & <5.0 \\ <0.2 & <1.0 & <5.0 & <0.20 & <0.2 & <0.2 & <0.2 & <0.2 & <0.2 & <0.20 & <5.0 \\ <0.2 & <1.0 & <5.0 & <0.20 & <0.2 & <0.2 & <0.2 & <0.2 & <0.2 & <0.20 & <5.0\end{array}$


Table 2. Water-quality data for storm-sewer outfall stations, Arlington, Texas, 1992-93-Continued

08049360 - TRIBUTARY TO WEST FORK TRINITY RIVER OUTFALL, AT BAIRD'S FARM ROAD, ARLINGTON, TX (WY 1993)-CONtInUEd

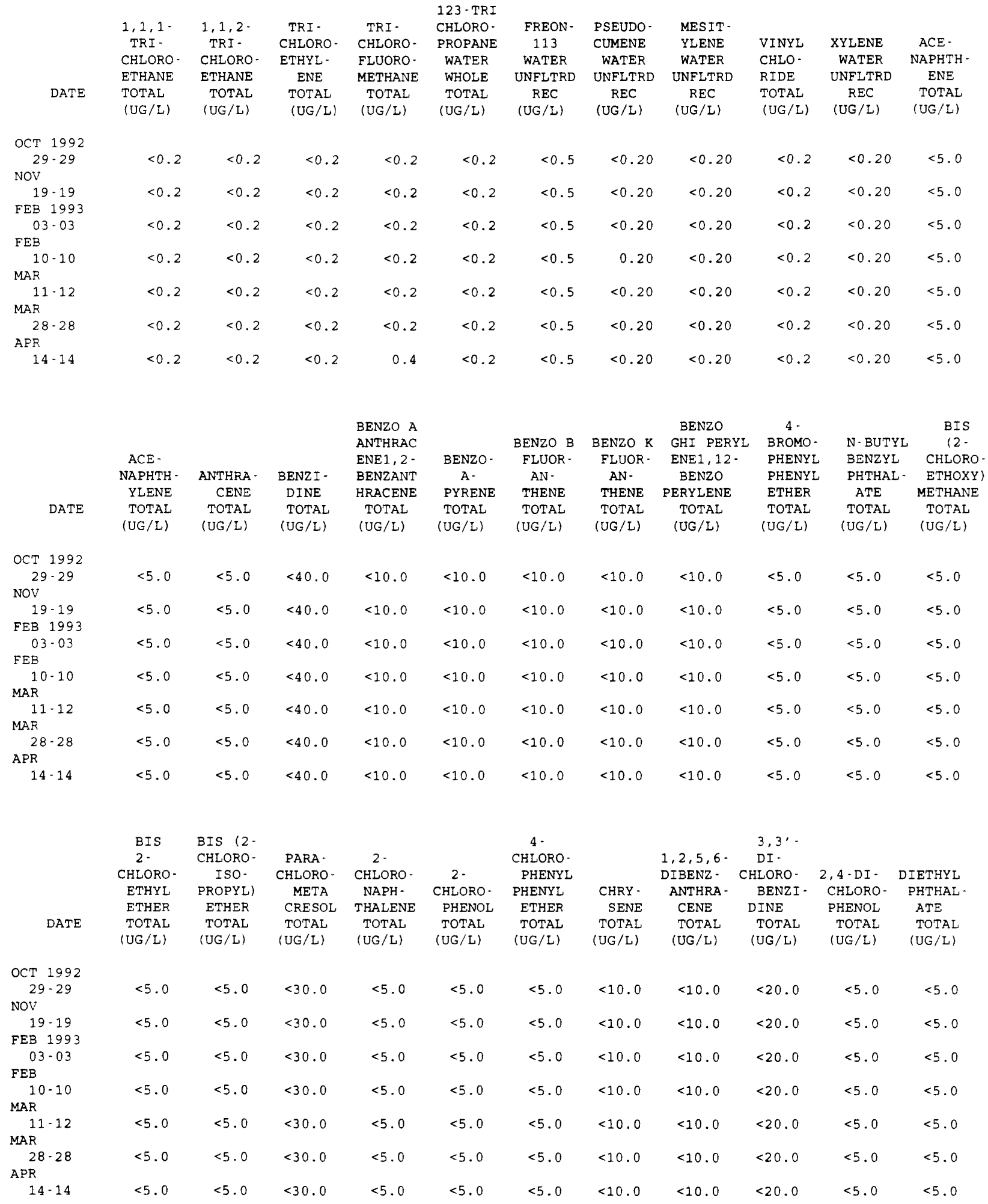


Table 2. Water-quality data for storm-sewer outfall stations, Arlington, Texas, 1992-93-Continued

08049360 - TRIBUTARY TO WEST FORK TRINITY RIVER OUTFALL, AT BAIRD'S FARM ROAD, ARLINGTON, TX (WY 1993)-CONTINUEd

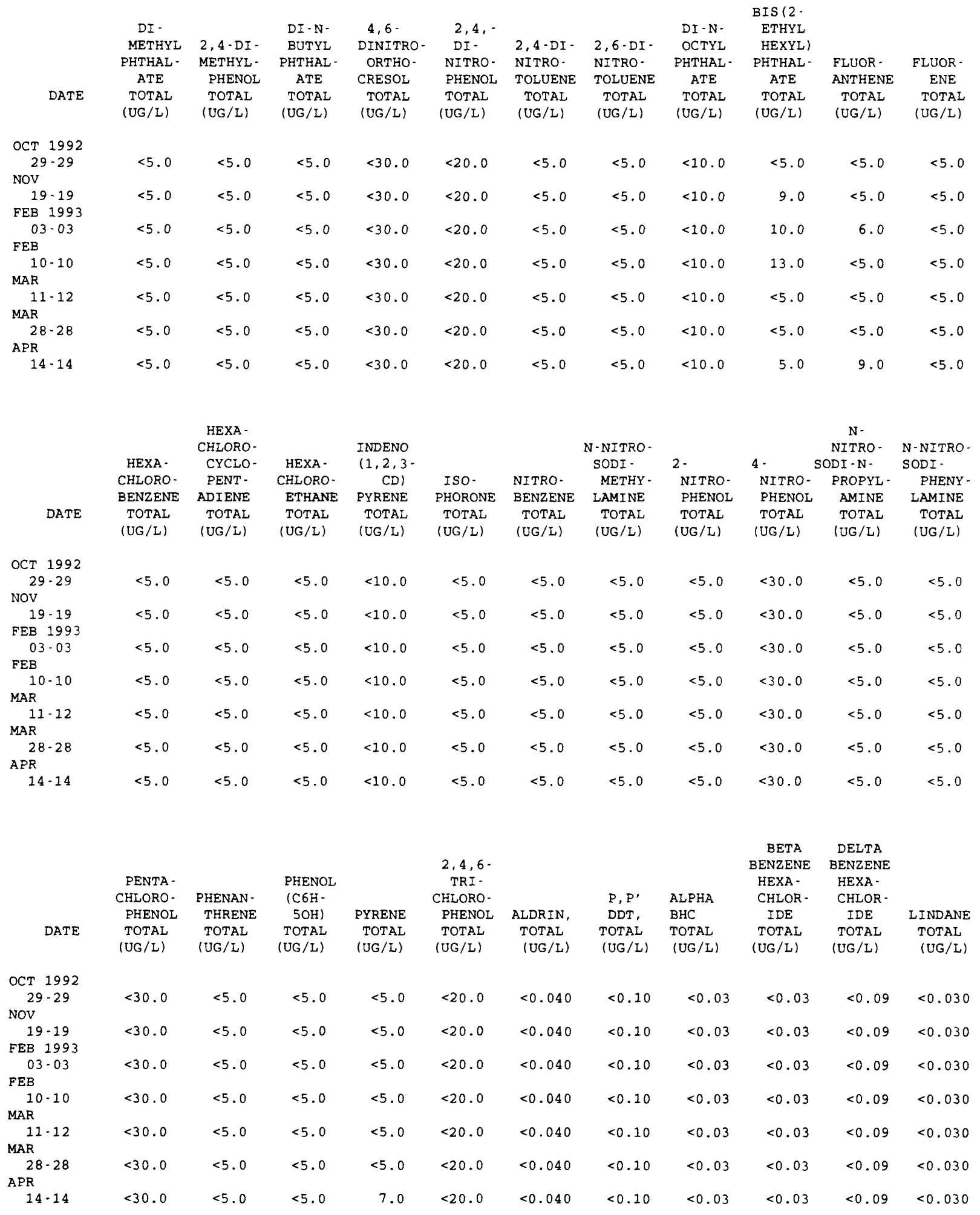


Table 2. Water-quality data for storm-sewer outfall stations, Arlington, Texas, 1992-93-Continued

08049360 - TRIBUTARY TO WEST FORK TRINITY RIVER OUTFALL, AT BAIRD'S FARM ROAD, ARLINGTON, TX (WY 1993)-CONTINUEd

\begin{tabular}{|c|c|c|c|c|c|c|c|c|c|c|c|}
\hline DATE & $\begin{array}{c}\text { CHLOR - } \\
\text { DANE } \\
\text { CIS } \\
\text { WATER } \\
\text { WHOLE } \\
\text { TOTAL } \\
\text { (UG/L) }\end{array}$ & $\begin{array}{l}\text { CHLOR - } \\
\text { DANE } \\
\text { TRANS } \\
\text { WATER } \\
\text { WHOLE } \\
\text { TOTAL } \\
\text { (UG/L) }\end{array}$ & $\begin{array}{l}\text { CHLOR - } \\
\text { DANE, } \\
\text { TOTAL } \\
\text { (UG/L) }\end{array}$ & $\begin{array}{c}\text { P, } P^{\prime} \\
\text { DDD, } \\
\text { TOTAL } \\
\text { (UG /L) }\end{array}$ & $\begin{array}{l}\mathrm{P}, \mathrm{P}^{\prime} \\
\text { DDE, } \\
\text { TOTAL } \\
\text { (UG/L) }\end{array}$ & $\begin{array}{l}\text { DI - } \\
\text { ELDRIN } \\
\text { TOTAL } \\
\text { (UG/L) }\end{array}$ & $\begin{array}{c}\text { ENDO- } \\
\text { SULFAN - } \\
\text { I } \\
\text { WATER } \\
\text { WHOLE } \\
\text { REC } \\
\text { (UG/L) }\end{array}$ & $\begin{array}{l}\text { ENDO- } \\
\text { SULFAN } \\
\text { BETA } \\
\text { TOTAL } \\
\text { (UG/L) }\end{array}$ & $\begin{array}{l}\text { ENDO- } \\
\text { SULFAN } \\
\text { SULFATE } \\
\text { TOTAL } \\
\text { (UG/L) }\end{array}$ & $\begin{array}{c}\text { ENDRIN } \\
\text { WATER } \\
\text { UNFLTRD } \\
\text { REC } \\
(U G / L)\end{array}$ & $\begin{array}{c}\text { ENDRIN } \\
\text { ALDE - } \\
\text { HYDE } \\
\text { TOTAL } \\
(U G / L)\end{array}$ \\
\hline OCT 1992 & & & & & & & & & & & \\
\hline $\begin{array}{l}29-29 \\
\text { Nov }\end{array}$ & $<0.10$ & $<0.10$ & $<0.1$ & $<0.10$ & $<0.04$ & $<0.020$ & $<0.10$ & $<0.04$ & $<0.60$ & $<0.060$ & $<0.20$ \\
\hline $\begin{array}{c}19-19 \\
\text { FEB } 1993\end{array}$ & $<0.10$ & $<0.10$ & $<0.1$ & $<0.10$ & $<0.04$ & $<0.020$ & $<0.10$ & $<0.04$ & $<0.60$ & $<0.060$ & $<0.20$ \\
\hline $\begin{array}{l}03.03 \\
\mathrm{FEB}\end{array}$ & $<0.10$ & $<0.10$ & $<0.1$ & $<0.10$ & $<0.04$ & $<0.020$ & $<0.10$ & $<0.04$ & $<0.60$ & $<0.060$ & $<0.20$ \\
\hline $\begin{array}{l}10-10 \\
\text { MAR }\end{array}$ & $<0.10$ & $<0.10$ & $<0.1$ & $<0.10$ & $<0.04$ & 0.020 & $<0.10$ & $<0.04$ & $<0.60$ & $<0.060$ & $<0.20$ \\
\hline $\begin{array}{l}11-12 \\
\text { MAR }\end{array}$ & $<0.10$ & $<0.10$ & $<0.1$ & $<0.10$ & $<0.04$ & $<0.020$ & $<0.10$ & $<0.04$ & $<0.60$ & $<0.060$ & $<0.20$ \\
\hline $\begin{array}{l}28-28 \\
A P R\end{array}$ & $<0.10$ & $<0.10$ & $<0.1$ & $<0.10$ & $<0.04$ & $<0.020$ & $<0.10$ & $<0.04$ & $<0.60$ & $<0.060$ & $<0.20$ \\
\hline $14-14$ & $<0.10$ & $<0.10$ & $<0.1$ & $<0.10$ & $<0.04$ & $<0.020$ & $<0.10$ & $<0.04$ & $<0.60$ & $<0.060$ & $<0.20$ \\
\hline DATE & $\begin{array}{l}\text { HEPTA- } \\
\text { CHLOR, } \\
\text { TOTAL } \\
\text { (UG/L) }\end{array}$ & $\begin{array}{l}\text { HEPTA - } \\
\text { CHLOR } \\
\text { EPOXIDE } \\
\text { TOTAL } \\
\text { (UG/L) }\end{array}$ & $\begin{array}{c}\text { TOX- } \\
\text { APHENE, } \\
\text { TOTAL } \\
\text { (UG/L) }\end{array}$ & $\begin{array}{c}\text { AROCLOR } \\
1016 \\
\text { PCB } \\
\text { TOTAL } \\
\text { (UG/L) }\end{array}$ & $\begin{array}{c}\text { AROCLOR } \\
1221 \\
\text { PCB } \\
\text { TOTAL } \\
\text { (UG/L) }\end{array}$ & $\begin{array}{c}\text { AROCLOR } \\
1232 \\
\text { PCB } \\
\text { TOTAL } \\
\text { (UG/L) }\end{array}$ & $\begin{array}{c}\text { AROCLOR } \\
1242 \\
\text { PCB } \\
\text { TOTAL } \\
\text { (UG/L) }\end{array}$ & $\begin{array}{c}\text { AROCLOR } \\
1248 \\
\text { PCB } \\
\text { TOTAL } \\
\text { (UG/L) }\end{array}$ & $\begin{array}{c}\text { AROCLOR } \\
1254 \\
\text { PCB } \\
\text { TOTAL } \\
\text { (UG/L) }\end{array}$ & $\begin{array}{c}\text { AROCLOR } \\
1260 \\
\text { PCB } \\
\text { TOTAL } \\
\text { (UG/L) }\end{array}$ & $\begin{array}{c}\text { DI - } \\
\text { AZINON, } \\
\text { TOTAL } \\
\text { (UG/L) }\end{array}$ \\
\hline OCT 1992 & & & & & & & & & & & \\
\hline $\begin{array}{l}29-29 \\
\text { NoV }\end{array}$ & $<0.030$ & $<0.80$ & $<2$ & $<0.1$ & $<1.0$ & $<0.1$ & $<0.1$ & $<0.1$ & $<0.1$ & $<0.1$ & 2.1 \\
\hline $\begin{array}{c}19-19 \\
\text { FEB } 1993\end{array}$ & $<0.030$ & $<0.80$ & $<2$ & $<0.1$ & $<1.0$ & $<0.1$ & $<0.1$ & $<0.1$ & $<0.1$ & $<0.1$ & 0.20 \\
\hline $\begin{array}{l}03-03 \\
\text { FEB }\end{array}$ & $<0.030$ & $<0.80$ & $<2$ & $<0.1$ & $<1.0$ & $<0.1$ & $<0.1$ & $<0.1$ & $<0.1$ & $<0.1$ & 0.20 \\
\hline $\begin{array}{l}10-10 \\
\text { MAR }\end{array}$ & $<0.030$ & $<0.80$ & $<2$ & $<0.1$ & $<1.0$ & $<0.1$ & $<0.1$ & $<0.1$ & $<0.1$ & $<0.1$ & $<0.05$ \\
\hline $11-12$ & $<0.030$ & $<0.80$ & $<2$ & $<0.1$ & $<1.0$ & $<0.1$ & $<0.1$ & $<0.1$ & $<0.1$ & $<0.1$ & $<0.05$ \\
\hline $\begin{array}{l}\text { MAR } \\
28 \cdot 28\end{array}$ & $<0.030$ & $<0.80$ & $<2$ & $<0.1$ & $<1.0$ & $<0.1$ & $<0.1$ & $<0.1$ & $<0.1$ & $<0.1$ & 0.60 \\
\hline $\begin{array}{l}\text { APR } \\
\qquad 14-14\end{array}$ & $<0.030$ & $<0.80$ & $<2$ & $<0.1$ & $<1.0$ & $<0.1$ & $<0.1$ & $<0.1$ & $<0.1$ & $<0.1$ & 0.40 \\
\hline
\end{tabular}


Table 2. Water-quality data for storm-sewer outfall stations, Arlington, Texas, 1992-93-Continued

08049470 - TRIBUTARY TO JOHNSON CREEK OUTFALI AT I-30 EAST, ARLINGTON, TX (WY 1993)

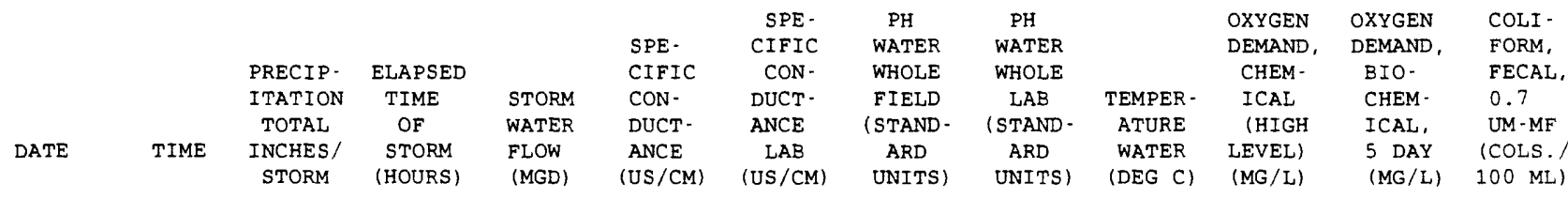

DEC 1992

$09.09 \quad 0045$

$\begin{array}{cc}\text { JAN } 1993 \\ 09.09 & 0250\end{array}$

JAN

$19.19 \quad 1540$

FEB
$10.10 \quad 0357$

FEB

$24 \cdot 24 \quad 1433$

MAR

$11.12 \quad 1750$

MAR

$28-28 \quad 0530$

0.30

3.2

0.46

0.24

5.5

0.25

7.9

0.20

4.4

0.43

6.0

0.42

10.2

0.39

7.0

0.38

71

161

$8 \cdot 5$

8.5
7.3

10.0

85

7.5

10.0

120

$0.41 \quad 154$

0.30

6.5

7.7

8.5

120

7.8

7.2

14.0

12.0

0.81

116

162

8.0

7.1

11.0

7.5

0.63

96

153

6.8

7.5

10.5

MAGNE -

HARD- ALKA- SOLIDS, RESIDUE SOLIDS,

NESS LINITY SUM OF TOTAL RESIDUE

NONCARB WAT DIS CONSTI-

DISSOLV FIX END TUENTS,

FID AS

I - 105
DEG. C,

AT 180 CALCIUM

DEG. C

DIS -

ICOIS.

PER

(MG / L

$\mathrm{CACO} 3$

FIELD DIS - SUS -

CACO3 SOLVED PENDED

(MG/L) (MG/L)

(MG/L)

SOLVED

(MG/L)

DIS -

SOLVED

(MG/I
AS CA)

SIUM, SODIUM,
DIS - DIS-

SOLVED SOLVED

(MG / L (MG/L

AS MG) AS NA)

$\begin{array}{rrrr}67 & 38 & 57 & 11 \\ 115 & 165 & 80 & 21 \\ 98 & 282 & 72 & 18 \\ 102 & 110 & 85 & 22 \\ 86 & 53 & 57 & 16 \\ 85 & 84 & 94 & 16 \\ 76 & 84 & 59 & 16\end{array}$

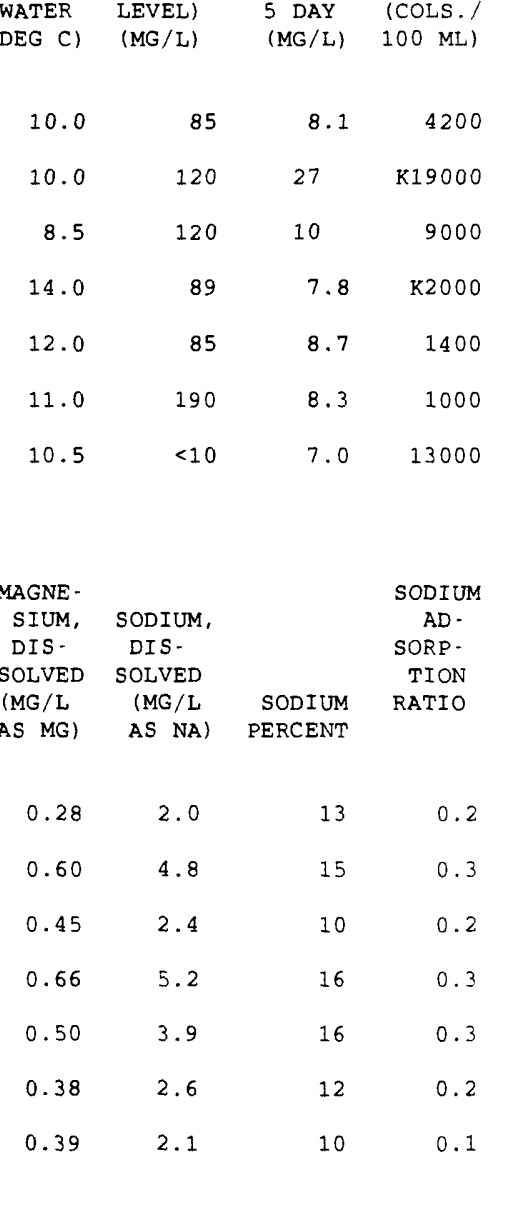

NITRO -

POTAS -

SIUM, SULFATE

DIS - DIS.

SOLVED SOLVED

DATE

(MG/L (MG/L

AS K) AS SO4)

RIDE,

RIS.

SOLVED

(MG/L

AS CL)

NITRO -

GEN,

NTRITE NO2+NO3

TOTAL

(MG/L

AS N) $\begin{array}{lll}0.080 & 0.630 & 0.140\end{array}$

$0.60 \quad 0.230$

$1.0 \quad 0.270$

1.0

0.240

0.80

0.620

$0.90 \quad 0.160$

0.90

0.210

0.80

0.130

0.620

-

\begin{abstract}
$$
0.3
$$
\end{abstract}


Table 2. Water-quality data for storm-sewer outfall stations, Arlington, Texas, 1992-93-Continued

08049470 - TRIBUTARY TO JOHNSON CREEK OUTFALL AT I-30 EAST, ARLINGTON, TX (WY 1993)-COntinUEd

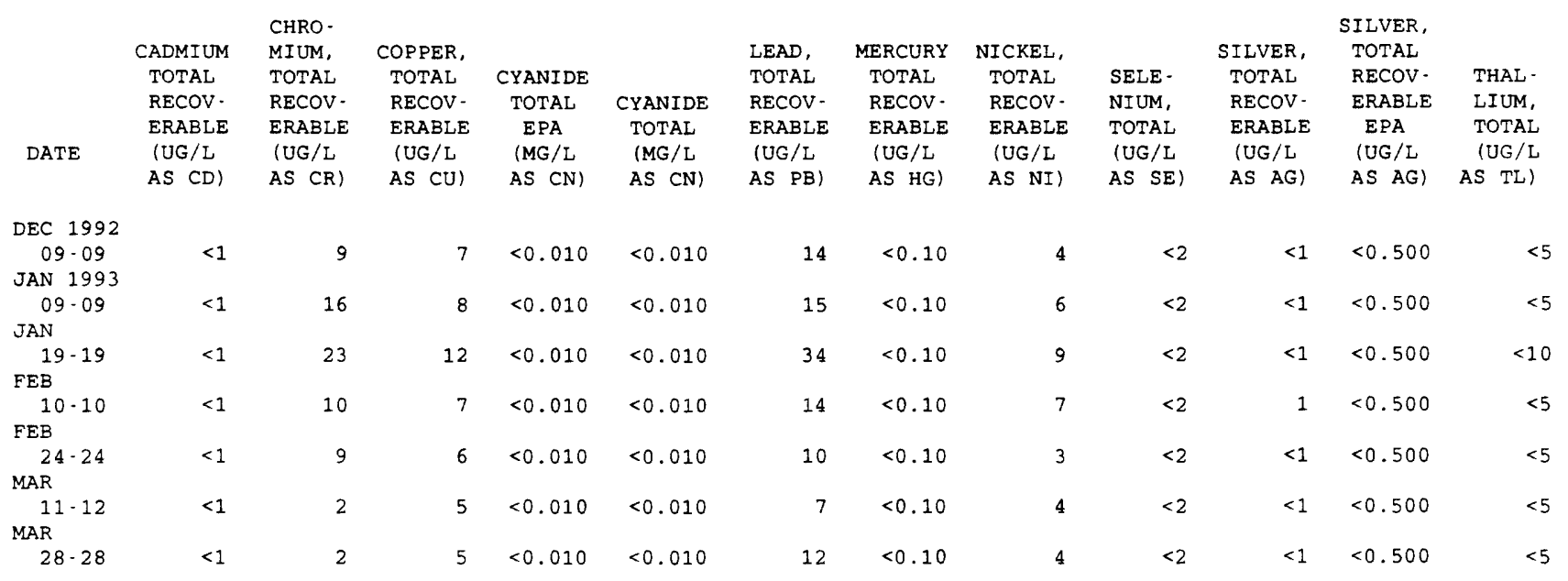

\begin{tabular}{|c|c|c|c|c|c|c|c|c|c|c|c|c|}
\hline & & & OIL AND & & & & & & METHANE & & & BENZENE \\
\hline & ZINC, & & GREASE, & & & & & BROMO - & BROMO & & BENZENE & SEC \\
\hline & TOTAL & CARBON, & TOTAL & & & & & BENZENE & CHLORO- & & $\mathrm{N}$-BUTYL & BUTYL - \\
\hline & RECOV - & ORGANIC & RECOV. & & ACRO - & ACRYLO- & & WATER, & WAT & BROMO - & WATER & WATER \\
\hline & ERABLE & TOTAL & GRAVI - & PHENOLS & LEIN & NI TRILE & BENZENE & WHOLE, & UNFLTRD & FORM & UNFLTRD & UNFLTRD \\
\hline DATE & $\begin{array}{c}\text { (UG/L } \\
\text { AS ZN) }\end{array}$ & $\begin{array}{l}(\mathrm{MG} / \mathrm{L} \\
\mathrm{AS} \mathrm{C})\end{array}$ & $\begin{array}{c}\text { METRIC } \\
(\mathrm{MG} / \mathrm{L})\end{array}$ & $\begin{array}{l}\text { TOTAL } \\
\text { (UG/L) }\end{array}$ & $\begin{array}{l}\text { TOTAL } \\
\text { (UG/L) }\end{array}$ & $\begin{array}{c}\text { TOTAL } \\
\text { (UG/L) }\end{array}$ & $\begin{array}{c}\text { TOTAL } \\
\text { (UG/L) }\end{array}$ & $\begin{array}{l}\text { TOTAL } \\
\text { (UG/L) }\end{array}$ & $\begin{array}{c}\text { REC } \\
(U G / L)\end{array}$ & $\begin{array}{l}\text { TOTAL } \\
\text { (UG/L) }\end{array}$ & $\begin{array}{c}\text { REC } \\
\text { (UG/L) }\end{array}$ & $\begin{array}{c}\text { REC } \\
(U G / L)\end{array}$ \\
\hline
\end{tabular}

\begin{tabular}{|c|c|c|c|c|c|c|c|c|c|c|c|c|}
\hline 09.09 & 100 & 26 & $<1$ & 5 & $<20$ & $<20$ & $<0.2$ & $<0.2$ & $<0.20$ & $<0.2$ & $<0.20$ & $<0.20$ \\
\hline \multicolumn{13}{|l|}{ JAN 1993} \\
\hline 09.09 & 170 & 37 & 2 & 2 & $<20$ & $<20$ & $<0.2$ & $<0.2$ & $<0.20$ & $<0.2$ & $<0.20$ & $<0.20$ \\
\hline \multicolumn{13}{|l|}{ JAN } \\
\hline $19 \cdot 19$ & 200 & 27 & $<1$ & 8 & $<20$ & $<20$ & $<0.2$ & $<0.2$ & $<0.20$ & $<0.2$ & $<0.20$ & $<0.20$ \\
\hline \multicolumn{13}{|l|}{ FEB } \\
\hline $10-10$ & 140 & 26 & 1 & $<1$ & $<20$ & $<20$ & $<0.2$ & $<0.2$ & $<0.20$ & $<0.2$ & $<0.20$ & $<0.20$ \\
\hline \multicolumn{13}{|l|}{ FEB } \\
\hline $24-24$ & 120 & 25 & 2 & 8 & $<20$ & $<20$ & $<0.2$ & $<0.2$ & $<0.20$ & $<0.2$ & $<0.20$ & $<0.20$ \\
\hline \multicolumn{13}{|l|}{ MAR } \\
\hline $11-12$ & 100 & 58 & $<1$ & 3 & $<20$ & $<20$ & $<0.2$ & $<0.2$ & $<0.20$ & $<0.2$ & $<0.20$ & $<0.20$ \\
\hline \multicolumn{13}{|l|}{ MAR } \\
\hline $28-28$ & 100 & 24 & 1 & 2 & $<20$ & $<20$ & $<0.2$ & $<0.2$ & $<0.20$ & $<0.2$ & $<0.20$ & $<0.20$ \\
\hline
\end{tabular}

\begin{tabular}{|c|c|c|c|c|c|c|c|c|c|c|c|c|}
\hline & BENZENE & & & & & 2- & & & 0. & & DI BROMO & DI - \\
\hline & TERT - & CARBON - & & CHLORO - & & CHLORO - & & & CHLORO - & TOLUENE & CHLORO - & BROMO - \\
\hline & BUTYL - & TETRA - & & DI - & & ETHYL - & & METHYL - & TOLUENE & P.CHLOR & PROPANE & METHANE \\
\hline & WATER & CHLO - & CHLORO - & BROMO - & CHLORO- & VINYL - & CHLORO - & CHLO - & WATER & WATER & WATER & WATER \\
\hline & UNFLTRD & RIDE & BENZENE & METHANE & ETHANE & ETHER & FORM & RIDE & WHOLE & UNFLTRD & WHOLE & WHOLE \\
\hline DATE & $\begin{array}{c}\text { REC } \\
(U G / L)\end{array}$ & $\begin{array}{l}\text { TOTAL } \\
\text { (UG/L) }\end{array}$ & $\begin{array}{l}\text { TOTAL } \\
\text { (UG/L) }\end{array}$ & $\begin{array}{l}\text { TOTAL } \\
\text { (UG/L) }\end{array}$ & $\begin{array}{l}\text { TOTAL } \\
\text { (UG/L) }\end{array}$ & $\begin{array}{l}\text { TOTAL } \\
\text { (UG/L) }\end{array}$ & $\begin{array}{l}\text { TOTAL } \\
\text { (UG/L) }\end{array}$ & $\begin{array}{l}\text { TOTAL } \\
\text { (UG/L) }\end{array}$ & $\begin{array}{l}\text { TOTAL } \\
\text { (UG/L) }\end{array}$ & $\begin{array}{c}\text { REC } \\
(U G / L)\end{array}$ & $\begin{array}{l}\text { TOT.REC } \\
\text { (UG/L) }\end{array}$ & $\begin{array}{l}\text { RECOVER } \\
\text { (UG/L) }\end{array}$ \\
\hline
\end{tabular}

\begin{tabular}{|c|c|c|c|c|c|c|c|c|c|c|c|c|}
\hline $09-09$ & $<0.20$ & $<0.2$ & $<0.20$ & $<0.2$ & $<0.2$ & $<1.0$ & $<0.2$ & $<0.2$ & $<0.2$ & $<0.20$ & $<1.0$ & $<0.2$ \\
\hline JAN 1993 & & & & & & & & & & & & \\
\hline $09-09$ & $<0.20$ & $<0.2$ & $<0.20$ & $<0.2$ & $<0.2$ & $<1.0$ & $<0.2$ & $<0.2$ & $<0.2$ & $<0.20$ & $<1.0$ & $<0.2$ \\
\hline JAN & & & & & & & & & & & & \\
\hline $19 \cdot 19$ & $<0.20$ & $<0.2$ & $<0.20$ & $<0.2$ & $<0.2$ & $<1.0$ & $<0.2$ & $<0.2$ & $<0.2$ & $<0.20$ & $<1.0$ & $<0.2$ \\
\hline FEB & & & & & & & & & & & & \\
\hline $10-10$ & $<0.20$ & $<0.2$ & $<0.20$ & $<0.2$ & $<0.2$ & $<1.0$ & $<0.2$ & $<0.2$ & $<0.2$ & $<0.20$ & $<1.0$ & $<0.2$ \\
\hline FEB & & & & & & & & & & & & \\
\hline $24-24$ & $<0.20$ & $<0.2$ & $<0.20$ & $<0.2$ & $<0.2$ & $<1.0$ & $<0.2$ & $<0.2$ & $<0.2$ & $<0.20$ & $<1.0$ & $<0.2$ \\
\hline MAR & & & & & & & & & & & & \\
\hline $11-12$ & $<0.20$ & $<0.2$ & $<0.20$ & $<0.2$ & $<0.2$ & $<1.0$ & $<0.2$ & $<0.2$ & $<0.2$ & $<0.20$ & $<1.0$ & $<0.2$ \\
\hline MAR & & & & & & & & & & & & \\
\hline $28-28$ & $<0.20$ & $<0.2$ & $<0.20$ & $<0.2$ & $<0.2$ & $<1.0$ & $<0.2$ & $<0.2$ & $<0.2$ & $<0.20$ & $<1.0$ & $<0.2$ \\
\hline
\end{tabular}


Table 2. Water-quality data for storm-sewer outfall stations, Arlington, Texas, 1992-93-Continued

08049470 - TRIBUTARY TO JOHNSON CREEK OUTFALL AT I-30 EAST, ARLINGTON, TX (WY 1993)-COntinUed

\begin{tabular}{|c|c|c|c|c|c|c|c|c|c|c|c|c|}
\hline & $\begin{array}{c}1,2- \\
\text { DI BROMO }\end{array}$ & $D I$ & $1,1 \cdot D I$ & $\begin{array}{l}1,1-D I \\
\text { CHLORO- }\end{array}$ & $\begin{array}{c}\text { BENZENE } \\
0-\end{array}$ & $\begin{array}{l}\text { BENZENE } \\
1,3 \text {-DI - }\end{array}$ & $\begin{array}{l}\text { BENZENE } \\
1,4 \text {-DI- }\end{array}$ & $\begin{array}{l}\text { DI- } \\
\text { CHLORO- }\end{array}$ & & & $\begin{array}{l}1,2-D I- \\
\text { PHENYL - }\end{array}$ & $\begin{array}{c}\text { CIS - 1,2- } \\
\text { DI. }\end{array}$ \\
\hline & ETHANE & CHLORO- & CHLORO- & PRO- & CHLORO- & CHLORO- & CHLORO- & & $1,1 \cdot D I$ & $1,2-D I$ & HYDRA. & CHLORO- \\
\hline & WATER & BROMO - & ETHYL - & PENE, & WATER & WATER & WATER & FLUORO- & CHLORO- & CHLORO & ZINE & ETHENE \\
\hline & $\begin{array}{l}\text { TOTAL } \\
\text { (UG/L) }\end{array}$ & $\begin{array}{l}\text { TOTAL } \\
\text { (UG/L) }\end{array}$ & $\begin{array}{l}\text { ENE } \\
\text { TOTAL } \\
\text { (UG/L) }\end{array}$ & $\begin{array}{l}\text { WAT, WH } \\
\text { TOTAL } \\
\text { (UG/L) }\end{array}$ & $\begin{array}{c}\text { UNFLTR } \\
\text { REC } \\
\text { (UG/L) }\end{array}$ & $\begin{array}{l}\text { REC } \\
\text { (UG/L) }\end{array}$ & $\begin{array}{l}\text { REC } \\
\text { (UG/L) }\end{array}$ & $\begin{array}{l}\text { METHAN } \\
\text { TOTAL } \\
\text { (UG/L) }\end{array}$ & $\begin{array}{l}\text { ETHANE } \\
\text { TOTAL } \\
\text { (UG/L) }\end{array}$ & $\begin{array}{l}\text { ETHANE } \\
\text { TOTAL } \\
\text { (UG/L) }\end{array}$ & $\begin{array}{l}\text { TOT . REC } \\
\text { (UG/L) }\end{array}$ & $\begin{array}{l}\text { WATER } \\
\text { TOTAL } \\
\text { (UG/L) }\end{array}$ \\
\hline
\end{tabular}

\begin{tabular}{|c|c|c|c|c|c|c|c|c|c|c|c|c|}
\hline \multicolumn{13}{|l|}{ DEC 1992} \\
\hline 09.09 & $<0.2$ & $<0.2$ & $<0.2$ & $<0.2$ & $<5.0$ & $<5.0$ & $<5.0$ & $<0.2$ & $<0.2$ & $<0.2$ & $<5.0$ & $<0.2$ \\
\hline \multicolumn{13}{|l|}{ JAN 1993} \\
\hline $09-09$ & $<0.2$ & $<0.2$ & $<0.2$ & $<0.2$ & $<5.0$ & $<5.0$ & $<5.0$ & $<0.2$ & $<0.2$ & $<0.2$ & $<5.0$ & $<0.2$ \\
\hline \multicolumn{13}{|l|}{ JAN } \\
\hline $19-19$ & $<0.2$ & $<0.2$ & $<0.2$ & $<0.2$ & $<5.0$ & $<5.0$ & $<5.0$ & $<0.2$ & $<0.2$ & $<0.2$ & $<5.0$ & $<0.2$ \\
\hline \multicolumn{13}{|l|}{ FEB } \\
\hline $10 \cdot 10$ & $<0.2$ & $<0.2$ & $<0.2$ & $<0.2$ & $<5.0$ & $<5.0$ & $<5.0$ & $<0.2$ & $<0.2$ & $<0.2$ & $<5.0$ & $<0.2$ \\
\hline \multicolumn{13}{|l|}{ FEB } \\
\hline $24-24$ & $<0.2$ & $<0.2$ & $<0.2$ & $<0.2$ & $<5.0$ & $<5.0$ & $<5.0$ & $<0.2$ & $<0.2$ & $<0.2$ & $<5.0$ & $<0.2$ \\
\hline \multicolumn{13}{|l|}{ MAR } \\
\hline $11 \cdot 12$ & $<0.2$ & $<0.2$ & $<0.2$ & $<0.2$ & $<5.0$ & $<5.0$ & $<5.0$ & $<0.2$ & $<0.2$ & $<0.2$ & $<5.0$ & $<0.2$ \\
\hline \multicolumn{13}{|l|}{ MAR } \\
\hline $28-28$ & $<0.2$ & $<0.2$ & $<0.2$ & $<0.2$ & $<5.0$ & $<5.0$ & $<5.0$ & $<0.2$ & $<0.2$ & $<0.2$ & $<5.0$ & $<0.2$ \\
\hline
\end{tabular}

\begin{tabular}{|c|c|c|c|c|c|c|c|c|c|c|}
\hline & & & $2,2 \cdot \mathrm{DI}$ & & & & & ISO- & P.ISO- & \\
\hline $1,2-$ & & $1,3-D I-$ & CHLORO - & CIS & TRANS - & & HEXA - & PROPYL - & PROPYL - & \\
\hline TRANSDI & $1,2 \cdot D I=$ & CHLORO - & PRO - & $1,3 \cdot \mathrm{DI}$ - & $1,3-D I$ - & & CHLORO - & BENZENE & TOLUENE & \\
\hline CHLORO- & CHLORO- & PROPANE & PANE & CHLORO- & CHLORO- & ETHYL - & BUT - & WATER & WATER & METHYL - \\
\hline $\begin{array}{c}\text { ETHENE } \\
\text { TOTAL } \\
\text { (UG/L) }\end{array}$ & $\begin{array}{l}\text { PROPANE } \\
\text { TOTAL } \\
\text { (UG/L) }\end{array}$ & $\begin{array}{l}\text { WAT. WH } \\
\text { TOTAL } \\
\text { (UG/L) }\end{array}$ & $\begin{array}{l}\text { WAT, WH } \\
\text { TOTAL } \\
\text { (UG/L) }\end{array}$ & $\begin{array}{l}\text { PROPENE } \\
\text { TOTAL } \\
\text { (UG/L) }\end{array}$ & $\begin{array}{l}\text { PROPENE } \\
\text { TOTAL } \\
\text { (UG/L) }\end{array}$ & $\begin{array}{l}\text { BENZENE } \\
\text { TOTAL } \\
\text { (UG/L) }\end{array}$ & $\begin{array}{l}\text { ADIENE } \\
\text { TOTAL } \\
\text { (UG/L) }\end{array}$ & $\begin{array}{l}\text { WHOLE } \\
\text { REC } \\
\text { (UG/L) }\end{array}$ & $\begin{array}{l}\text { WHOLE } \\
\text { REC } \\
\text { (UG/L) }\end{array}$ & $\begin{array}{c}\text { BROMIDE } \\
\text { TOTAL } \\
\text { (UG/L) }\end{array}$ \\
\hline
\end{tabular}

DEC 1992
$09-09$
JAN 1993
$09 \cdot 09$
JAN
$19-19$
FEB
$10-10$
FEB
$24-24$
MAR
$11-12$
MAR
$28-28$

$\begin{array}{rrrrrrrrrrr}<0.2 & <0.2 & <0.2 & <0.2 & <0.2 & <0.2 & <0.2 & <5.0 & <0.20 & <0.20 & <0.2 \\ <0.2 & <0.2 & <0.2 & <0.2 & <0.2 & <0.2 & <0.2 & <5.0 & <0.20 & <0.20 & <0.2 \\ <0.2 & <0.2 & <0.2 & <0.2 & <0.2 & <0.2 & <0.2 & <5.0 & <0.20 & <0.20 & <0.2 \\ <0.2 & <0.2 & <0.2 & <0.2 & <0.2 & <0.2 & <0.2 & <5.0 & <0.20 & <0.20 & <0.2 \\ <0.2 & <0.2 & <0.2 & <0.2 & <0.2 & <0.2 & <0.2 & <5.0 & <0.20 & <0.20 & <0.2 \\ <0.2 & <0.2 & <0.2 & <0.2 & <0.2 & <0.2 & <0.2 & <5.0 & <0.20 & <0.20 & <0.2 \\ <0.2 & <0.2 & <0.2 & <0.2 & <0.2 & <0.2 & <0.2 & <5.0 & <0.20 & <0.20\end{array}<0.2<$

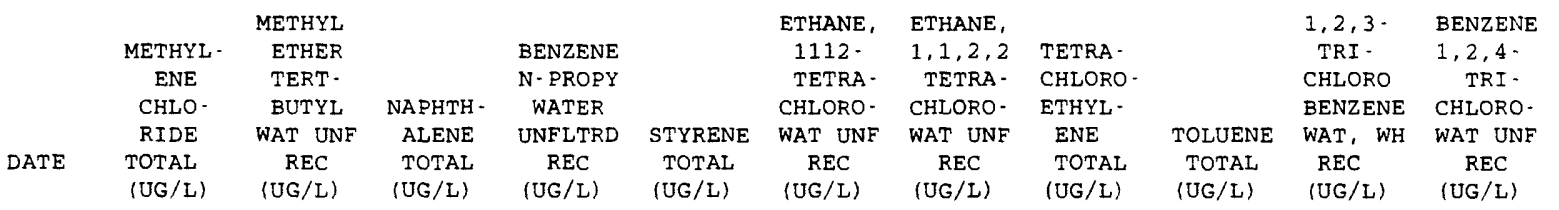

\begin{tabular}{|c|c|c|c|c|c|c|c|c|c|c|c|}
\hline \multicolumn{12}{|l|}{ DEC 1992} \\
\hline $09-09$ & $<0.2$ & $<1.0$ & $<5.0$ & $<0.20$ & $<0.2$ & $<0.2$ & $<0.2$ & $<0.2$ & 0.2 & $<0.20$ & $<5.0$ \\
\hline \multicolumn{12}{|l|}{ JAN 1993} \\
\hline 09.09 & $<0.3$ & $<1.0$ & $<5.0$ & $<0.20$ & $<0.2$ & $<0.2$ & $<0.2$ & $<0.2$ & $<0.2$ & $<0.20$ & $<5.0$ \\
\hline \multicolumn{12}{|l|}{ JAN } \\
\hline $19-19$ & $<0.2$ & $<1.0$ & $<5.0$ & $<0.20$ & $<0.2$ & $<0.2$ & $<0.2$ & $<0.2$ & 0.2 & $<0.20$ & $<5.0$ \\
\hline \multicolumn{12}{|l|}{ FEB } \\
\hline $10-10$ & $<0.2$ & $<1.0$ & $<5.0$ & $<0.20$ & $<0.2$ & $<0.2$ & $<0.2$ & $<0.2$ & $<0.2$ & $<0.20$ & $<5.0$ \\
\hline \multicolumn{12}{|l|}{ FEB } \\
\hline $24-24$ & $<0.2$ & $<1.0$ & $<5.0$ & $<0.20$ & $<0.2$ & $<0.2$ & $<0.2$ & $<0.2$ & $<0.2$ & $<0.20$ & $<5.0$ \\
\hline \multicolumn{12}{|l|}{ MAR } \\
\hline $11-12$ & $<0.2$ & $<1.0$ & $<5.0$ & $<0.20$ & $<0.2$ & $<0.2$ & $<0.2$ & $<0.2$ & 0.2 & $<0.20$ & $<5.0$ \\
\hline \multicolumn{12}{|l|}{ MAR } \\
\hline $28-28$ & $<0.2$ & $<1.0$ & $<5.0$ & $<0.20$ & $<0.2$ & $<0.2$ & $<0.2$ & $<0.2$ & $<0.2$ & $<0.20$ & $<5.0$ \\
\hline
\end{tabular}


Table 2. Water-quality data for storm-sewer outfall stations, Arlington, Texas, 1992-93-Continued

08049470 - TRIBUTARY TO JOHNSON CREEK OUTFALL AT I-30 EAST, ARLINGTON,TX (WY 1993)-COntinUed

\begin{tabular}{|c|c|c|c|c|c|c|c|c|c|c|}
\hline $\begin{array}{c}1,1,1- \\
\text { TRI. }\end{array}$ & $\begin{array}{c}1,1,2- \\
\text { TRI. }\end{array}$ & $\begin{array}{l}\text { TRI - } \\
\text { CHLORO- }\end{array}$ & $\begin{array}{c}\text { TRI - } \\
\text { CHLORO- }\end{array}$ & $\begin{array}{l}123-T R I \\
\text { CHLORO- } \\
\text { PROPANE }\end{array}$ & $\begin{array}{c}\text { FREON - } \\
113\end{array}$ & $\begin{array}{l}\text { PSEUDO- } \\
\text { CUMENE }\end{array}$ & $\begin{array}{l}\text { MESIT- } \\
\text { YLENE }\end{array}$ & VINYL & XYLENE & ACE - \\
\hline $\begin{array}{l}\text { CHLORO- } \\
\text { ETHANE }\end{array}$ & $\begin{array}{l}\text { CHLORO- } \\
\text { ETHANE }\end{array}$ & $\begin{array}{l}\text { ETHYL- } \\
\text { ENE }\end{array}$ & $\begin{array}{l}\text { FLUORO- } \\
\text { METHANE }\end{array}$ & $\begin{array}{l}\text { WATER } \\
\text { WHOLE }\end{array}$ & $\begin{array}{l}\text { WATER } \\
\text { UNFLTRD }\end{array}$ & $\begin{array}{l}\text { WATER } \\
\text { UNFLTRD }\end{array}$ & $\begin{array}{l}\text { WATER } \\
\text { UNFLTRD }\end{array}$ & $\begin{array}{l}\text { CHLO- } \\
\text { RIDE }\end{array}$ & $\begin{array}{l}\text { WATER } \\
\text { UNFLTRD }\end{array}$ & $\begin{array}{l}\text { NAPHTH - } \\
\text { ENE }\end{array}$ \\
\hline $\begin{array}{l}\text { TOTAL } \\
\text { (UG/L) }\end{array}$ & $\begin{array}{l}\text { TOTAL } \\
\text { (UG/L) }\end{array}$ & $\begin{array}{l}\text { TOTAL } \\
\text { (UG/L) }\end{array}$ & $\begin{array}{l}\text { TOTAL } \\
\text { (UG/L) }\end{array}$ & $\begin{array}{l}\text { TOTAL } \\
\text { (UG/L) }\end{array}$ & $\begin{array}{c}\text { REC } \\
(U G / L)\end{array}$ & $\begin{array}{c}\text { REC } \\
(\mathrm{UG} / \mathrm{L})\end{array}$ & $\begin{array}{c}\text { REC } \\
(U G / L)\end{array}$ & $\begin{array}{l}\text { TOTAL } \\
\text { (UG/L) }\end{array}$ & $\begin{array}{c}R E C \\
(U G / L)\end{array}$ & $\begin{array}{l}\text { TOTAL } \\
\text { (UG/L) }\end{array}$ \\
\hline
\end{tabular}

\begin{tabular}{|c|c|c|c|c|c|c|c|c|c|c|c|}
\hline \multicolumn{12}{|l|}{ DEC 1992} \\
\hline $09-09$ & $<0.2$ & $<0.2$ & $<0.2$ & $<0.2$ & $<0.2$ & $<0.5$ & $<0.20$ & $<0.20$ & $<0.2$ & $<0.20$ & $<5.0$ \\
\hline \multicolumn{12}{|l|}{ JAN 1993} \\
\hline $09-09$ & $<0.2$ & $<0.2$ & $<0.2$ & $<0.2$ & $<0.2$ & $<0.5$ & $<0.20$ & $<0.20$ & $<0.2$ & $<0.20$ & $<5.0$ \\
\hline \multicolumn{12}{|l|}{ JAN } \\
\hline $19-19$ & $<0.2$ & $<0.2$ & $<0.2$ & $<0.2$ & $<0.2$ & $<0.5$ & $<0.20$ & $<0.20$ & $<0.2$ & 0.40 & $<5.0$ \\
\hline \multicolumn{12}{|l|}{ FEB } \\
\hline $10-10$ & $<0.2$ & $<0.2$ & $<0.2$ & $<0.2$ & $<0.2$ & $<0.5$ & $<0.20$ & $<0.20$ & $<0.2$ & $<0.20$ & $<5.0$ \\
\hline \multicolumn{12}{|l|}{ FEB } \\
\hline $24 \cdot 24$ & $<0.2$ & $<0.2$ & $<0.2$ & $<0.2$ & $<0.2$ & $<0.5$ & $<0.20$ & $<0.20$ & $<0.2$ & $<0.20$ & $<5.0$ \\
\hline \multicolumn{12}{|l|}{ MAR } \\
\hline $11-12$ & $<0.2$ & $<0.2$ & $<0.2$ & $<0.2$ & $<0.2$ & $<0.5$ & $<0.20$ & $<0.20$ & $<0.2$ & 0.90 & $<5.0$ \\
\hline \multicolumn{12}{|l|}{ MAR } \\
\hline $28-28$ & $<0.2$ & $<0.2$ & $<0.2$ & $<0.2$ & $<0.2$ & $<0.5$ & $<0.20$ & $<0.20$ & $<0.2$ & $<0.20$ & $<5.0$ \\
\hline
\end{tabular}

\begin{tabular}{|c|c|c|c|c|c|c|c|c|c|c|c|}
\hline DATE & $\begin{array}{l}\text { ACE - } \\
\text { NAPHTH - } \\
\text { YLENE } \\
\text { TOTAL } \\
\text { (UG/L) }\end{array}$ & $\begin{array}{c}\text { ANTHRA - } \\
\text { CENE } \\
\text { TOTAL } \\
(\mathrm{UG} / \mathrm{L})\end{array}$ & $\begin{array}{l}\text { BENZI - } \\
\text { DINE } \\
\text { TOTAL } \\
\text { (UG/L) }\end{array}$ & $\begin{array}{l}\text { BENZO A } \\
\text { ANTHRAC } \\
\text { ENE1, 2- } \\
\text { BENZANT } \\
\text { HRACENE } \\
\text { TOTAL } \\
\text { (UG/L) }\end{array}$ & $\begin{array}{l}\text { BENZO- } \\
\text { A- } \\
\text { PYRENE } \\
\text { TOTAL } \\
\text { (UG/L) }\end{array}$ & $\begin{array}{l}\text { BENZO B } \\
\text { FLUOR- } \\
\text { AN- } \\
\text { THENE } \\
\text { TOTAL } \\
\text { (UG/L) }\end{array}$ & $\begin{array}{l}\text { BENZO K } \\
\text { FLUOR - } \\
\text { AN- } \\
\text { THENE } \\
\text { TOTAL } \\
\text { (UG/L) }\end{array}$ & $\begin{array}{l}\text { BENZO } \\
\text { GHI PERYL } \\
\text { ENE1, 12- } \\
\text { BENZO } \\
\text { PERYLENE } \\
\text { TOTAL } \\
\text { (UG/L) }\end{array}$ & $\begin{array}{c}4- \\
\text { L } \\
\text { BROMO- } \\
\text { PHENYL } \\
\text { PHENYL } \\
\text { ETHER } \\
\text { TOTAL } \\
\text { (UG/L) }\end{array}$ & $\begin{array}{l}\text { N-BUTYL } \\
\text { BENZYL } \\
\text { PHTHAL- } \\
\text { ATE } \\
\text { TOTAL } \\
\text { (UG/L) }\end{array}$ & $\begin{array}{c}\text { BIS } \\
\text { (2- } \\
\text { CHLORO - } \\
\text { ETHOXY) } \\
\text { METHANE } \\
\text { TOTAL } \\
\text { (UG/L) }\end{array}$ \\
\hline EC 1992 & & & & & & & & & & & \\
\hline $\begin{array}{c}09-09 \\
\text { JAN } 1993\end{array}$ & $<5.0$ & $<5.0$ & $<40.0$ & $<10.0$ & $<10.0$ & $<10.0$ & $<10.0$ & $<10.0$ & $<5.0$ & $<5.0$ & $<5.0$ \\
\hline $\begin{array}{l}09-09 \\
\text { IAN }\end{array}$ & $<5.0$ & $<5.0$ & $<40.0$ & $<10.0$ & $<10.0$ & $<10.0$ & $<10.0$ & $<10.0$ & $<5.0$ & $<5.0$ & $<5.0$ \\
\hline $\begin{array}{l}19-19 \\
E B\end{array}$ & $<5.0$ & $<5.0$ & $<40.0$ & $<10.0$ & $<10.0$ & $<10.0$ & $<10.0$ & $<10.0$ & $<5.0$ & $<5.0$ & $<5.0$ \\
\hline $\begin{array}{l}10-10 \\
\text { EB }\end{array}$ & $<5.0$ & $<5.0$ & $<40.0$ & $<10.0$ & $<10.0$ & $<10.0$ & $<10.0$ & $<10.0$ & $<5.0$ & $<5.0$ & $<5.0$ \\
\hline $\begin{array}{l}24 \cdot 24 \\
A R\end{array}$ & $<5.0$ & $<5.0$ & $<40.0$ & $<10.0$ & $<10.0$ & $<10.0$ & $<10.0$ & $<10.0$ & $<5.0$ & $<5.0$ & $<5.0$ \\
\hline $\begin{array}{l}11-12 \\
A R\end{array}$ & $<5.0$ & $<5.0$ & $<40.0$ & $<10.0$ & $<10.0$ & $<10.0$ & $<10.0$ & $<10.0$ & $<5.0$ & $<5.0$ & $<5.0$ \\
\hline $28-28$ & $<5.0$ & 7.0 & $<40.0$ & 24.0 & 20.0 & 18.0 & 12.0 & $<10.0$ & $<5.0$ & $<5.0$ & $<5.0$ \\
\hline DATE & $\begin{array}{l}\text { BIS } \\
2 \cdot \\
\text { CHLORO- } \\
\text { ETHYL } \\
\text { ETHER } \\
\text { TOTAL } \\
(\text { UG/L) }\end{array}$ & $\begin{array}{l}\text { BIS }(2- \\
\text { CHLORO- } \\
\text { ISO- } \\
\text { PROPYL) } \\
\text { ETHER } \\
\text { TOTAL } \\
\text { (UG/L) }\end{array}$ & $\begin{array}{l}\text { PARA- } \\
\text { CHLORO- } \\
\text { META } \\
\text { CRESOL } \\
\text { TOTAL } \\
\text { (UG/L) }\end{array}$ & $\begin{array}{l}2 \text { - } \\
\text { CHLORO- } \\
\text { NAPH - } \\
\text { THALENE } \\
\text { TOTAL } \\
\text { (UG/L) }\end{array}$ & $\begin{array}{c}2 \text { - } \\
\text { CHLORO- } \\
\text { PHENOL } \\
\text { TOTAL } \\
(\text { UG/L) }\end{array}$ & $\begin{array}{l}4 \text { - } \\
\text { CHLORO - } \\
\text { PHENYL } \\
\text { PHENYL } \\
\text { ETHER } \\
\text { TOTAL } \\
\text { (UG/L) }\end{array}$ & $\begin{array}{l}\text { CHRY - } \\
\text { SENE } \\
\text { TOTAL } \\
\text { (UG/L) }\end{array}$ & $\begin{array}{l}1,2,5,6- \\
\text { DIBENZ- C } \\
\text { ANTHRA- } \\
\text { CENE } \\
\text { TOTAL } \\
\text { (UG/L) }\end{array}$ & $\begin{array}{c}3,3 \cdot \\
\text { DI - } \\
\text { CHLORO- } \\
\text { BENZI- } \\
\text { DINE } \\
\text { TOTAL } \\
\text { (UG/L) }\end{array}$ & $\begin{array}{r}2,4 \text { - DI - } \\
\text { CHLORO- } \\
\text { PHENOL } \\
\text { TOTAL } \\
(U G / L)\end{array}$ & $\begin{array}{l}\text { IETHYL } \\
\text { PHTHAL- } \\
\text { ATE } \\
\text { TOTAL } \\
\text { (UG/L) }\end{array}$ \\
\hline $\begin{array}{cc}\text { OEC } 1992 \\
09-09 \\
\text { JAN } 1993\end{array}$ & $<5.0$ & $<5.0$ & $<30.0$ & $<5.0$ & $<5.0$ & $<5.0$ & $<10.0$ & $<10.0$ & $<20.0$ & $<5.0$ & $<5.0$ \\
\hline $\begin{array}{l}09 \cdot 09 \\
\text { AN }\end{array}$ & $<5.0$ & $<5.0$ & $<30.0$ & $<5.0$ & $<5.0$ & $<5.0$ & $<10.0$ & $<10.0$ & $<20.0$ & $<5.0$ & $<5.0$ \\
\hline $\mathrm{EB}^{19 \cdot 19}$ & $<5.0$ & $<5.0$ & $<30.0$ & $<5.0$ & $<5.0$ & $<5.0$ & $<10.0$ & $<10.0$ & $<20.0$ & $<5.0$ & $<5.0$ \\
\hline $\begin{array}{l}10-10 \\
\mathrm{~EB}\end{array}$ & $<5.0$ & $<5.0$ & $<30.0$ & $<5,0$ & $<5.0$ & $<5.0$ & $<10.0$ & $<10.0$ & $<20.0$ & $<5.0$ & $<5.0$ \\
\hline $\begin{array}{l}24-24 \\
\mathrm{AR}\end{array}$ & $<5.0$ & $<5.0$ & $<30.0$ & $<5.0$ & $<5.0$ & $<5.0$ & $<10.0$ & $<10.0$ & $<20.0$ & $<5.0$ & $<5.0$ \\
\hline $\begin{array}{l}11 \cdot 12 \\
A R\end{array}$ & $<5.0$ & $<5.0$ & $<30.0$ & $<5.0$ & $<5.0$ & $<5.0$ & $<10.0$ & $<10.0$ & $<20.0$ & $<5.0$ & $<5.0$ \\
\hline $28 \cdot 28$ & $<5.0$ & $<5.0$ & $<30.0$ & $<5.0$ & $<5.0$ & $<5.0$ & 27.0 & $<10.0$ & $<20.0$ & $<5.0$ & $<5.0$ \\
\hline
\end{tabular}


Table 2. Water-quality data for storm-sewer outfall stations, Arlington, Texas, 1992-93-Continued

08049470 - TRIBUTARY TO JOHNSON CREEK OUTFALL AT I-30 EAST, ARLINGTON, TX (WY 1993)-CONTINUEd

\begin{tabular}{|c|c|c|c|c|c|c|c|c|c|c|c|}
\hline DATE & $\begin{array}{c}\text { DI - } \\
\text { METHYL } \\
\text { PHTHAL- } \\
\text { ATE } \\
\text { TOTAL } \\
\text { (UG/L) }\end{array}$ & $\begin{array}{c}2,4-\mathrm{DI} \text { - } \\
\text { METHYL- } \\
\text { PHENOL } \\
\text { TOTAL } \\
\text { (UG/L) }\end{array}$ & $\begin{array}{c}\text { DI-N- } \\
\text { BUTYL } \\
\text { PHTHAL- } \\
\text { ATE } \\
\text { TOTAL } \\
\text { (UG/L) }\end{array}$ & $\begin{array}{c}4,6 \cdot \\
\text { DINITRO- } \\
\text { ORTHO- } \\
\text { CRESOL } \\
\text { TOTAL } \\
\text { (UG/L) }\end{array}$ & $\begin{array}{c}2,4,- \\
\text { DI- } \\
\text { NITRO- } \\
\text { PHENOL } \\
\text { TOTAL } \\
\text { (UG/L) }\end{array}$ & $\begin{array}{l}2,4-\mathrm{DI} \text { - } \\
\text { NITRO- } \\
\text { TOLUENE } \\
\text { TOTAL } \\
(\mathrm{UG} / \mathrm{L})\end{array}$ & $\begin{array}{l}2,6 \text {-DI - } \\
\text { NITRO- } \\
\text { TOLUENE } \\
\text { TOTAL } \\
\text { (UG/L) }\end{array}$ & $\begin{array}{c}\text { DI-N- } \\
\text { OCTYL } \\
\text { PHTHAL- } \\
\text { ATE } \\
\text { TOTAL } \\
(\text { UG/L) }\end{array}$ & $\begin{array}{c}\text { BIS }(2- \\
\text { ETHYL } \\
\text { HEXYL) } \\
\text { PHTHAL- } \\
\text { ATE } \\
\text { TOTAL } \\
\text { (UG/L) }\end{array}$ & $\begin{array}{l}\text { FLUOR - } \\
\text { ANTHENE } \\
\text { TOTAL } \\
\text { (UG/L) }\end{array}$ & $\begin{array}{c}\text { FLUOR- } \\
\text { ENE } \\
\text { TOTAL } \\
\text { (UG/L) }\end{array}$ \\
\hline EC 1992 & & & & & & & & & & & \\
\hline $\begin{array}{c}09-09 \\
\text { JAN } 1993\end{array}$ & $<5.0$ & $<5.0$ & $<5.0$ & $<30.0$ & $<20.0$ & $<5.0$ & $<5.0$ & $<10.0$ & $<5.0$ & $<5.0$ & $<5.0$ \\
\hline $\begin{array}{l}09 \cdot 09 \\
\text { JAN }\end{array}$ & $<5.0$ & $<5.0$ & $<5.0$ & $<30.0$ & $<20.0$ & $<5.0$ & $<5.0$ & $<10.0$ & $<5.0$ & $<5.0$ & $<5.0$ \\
\hline $\begin{array}{l}19-19 \\
\text { FEB }\end{array}$ & $<5.0$ & $<5.0$ & $<5.0$ & $<30.0$ & $<20.0$ & $<5.0$ & $<5.0$ & $<10.0$ & 7.0 & $<5.0$ & $<5.0$ \\
\hline $\begin{array}{l}10-10 \\
\text { FEB }\end{array}$ & $<5.0$ & $<5.0$ & $<5.0$ & $<30.0$ & $<20.0$ & $<5.0$ & $<5.0$ & $<10.0$ & $<5.0$ & 5.0 & $<5.0$ \\
\hline $\begin{array}{l}24-24 \\
\text { MAR }\end{array}$ & $<5.0$ & $<5.0$ & $<5.0$ & $<30.0$ & $<20.0$ & $<5.0$ & $<5.0$ & $<10.0$ & $<5.0$ & $<5.0$ & $<5.0$ \\
\hline${ }_{\text {MAR }}^{11 \cdot 12}$ & $<5.0$ & $<5.0$ & $<5.0$ & $<30.0$ & $<20.0$ & $<5.0$ & $<5.0$ & $<10.0$ & $<5.0$ & 7.0 & $<5.0$ \\
\hline $28-28$ & $<5.0$ & $<5.0$ & $<5.0$ & $<30.0$ & $<20.0$ & $<5.0$ & $<5.0$ & $<10.0$ & $<5.0$ & 52.0 & $<5.0$ \\
\hline DATE & $\begin{array}{l}\text { HEXA- } \\
\text { CHLORO- } \\
\text { BENZENE } \\
\text { TOTAL } \\
\text { (UG/L) }\end{array}$ & $\begin{array}{l}\text { HEXA- } \\
\text { CHLORO- } \\
\text { CYCLO- } \\
\text { PENT- } \\
\text { ADIENE } \\
\text { TOTAL } \\
\text { (UG/L) }\end{array}$ & $\begin{array}{l}\text { HEXA- } \\
\text { CHLORO- } \\
\text { ETHANE } \\
\text { TOTAL } \\
\text { (UG/L) }\end{array}$ & $\begin{array}{c}\text { INDENO } \\
(1,2,3- \\
\text { CD) } \\
\text { PYRENE } \\
\text { TOTAL } \\
\text { (UG/L) }\end{array}$ & $\begin{array}{l}\text { ISO- } \\
\text { PHORONE } \\
\text { TOTAL } \\
\text { (UG/L) }\end{array}$ & $\begin{array}{l}\text { NITRO- } \\
\text { BENZENE } \\
\text { TOTAL } \\
\text { (UG/L) }\end{array}$ & $\begin{array}{c}\text { N-NITRO- } \\
\text { SODI- } \\
\text { METHY- } \\
\text { LAMINE } \\
\text { TOTAL } \\
\text { (UG/L) }\end{array}$ & $\begin{array}{l}2- \\
\text { NITRO- } \\
\text { PHENOL } \\
\text { TOTAL } \\
(\text { UG } / L)\end{array}$ & $\begin{array}{c}4- \\
\text { NITRO- } \\
\text { PHENOL } \\
\text { TOTAL } \\
\text { (UG/L) }\end{array}$ & $\begin{array}{c}\text { N- } \\
\text { NITRO- } \\
\text { SODI-N- } \\
\text { PROPYL- } \\
\text { AMINE } \\
\text { TOTAL } \\
\text { (UG/L) }\end{array}$ & $\begin{array}{l}\mathrm{N}-\mathrm{NITRO} \\
\text { SODI - } \\
\text { PHENY - } \\
\text { LAMINE } \\
\text { TOTAL } \\
\text { (UG/L) }\end{array}$ \\
\hline $\begin{array}{c}\text { DEC } 1992 \\
09.09\end{array}$ & & & & & & & & & & & \\
\hline $\begin{array}{r}09-09 \\
\text { JAN } 1993\end{array}$ & $<5.0$ & $<5.0$ & $<5.0$ & $<10.0$ & $<5.0$ & $<5.0$ & $<5.0$ & $<5.0$ & $<30.0$ & $<5.0$ & $<5.0$ \\
\hline $\begin{array}{l}09-09 \\
\text { JAN }\end{array}$ & $<5.0$ & $<5.0$ & $<5.0$ & $<10.0$ & $<5.0$ & $<5.0$ & $<5.0$ & $<5.0$ & $<30.0$ & $<5.0$ & $<5.0$ \\
\hline $\begin{array}{l}19-19 \\
\text { FEB }\end{array}$ & $<5.0$ & $<5.0$ & $<5.0$ & $<10.0$ & $<5.0$ & $<5.0$ & $<5.0$ & $<5.0$ & $<30.0$ & $<5.0$ & $<5.0$ \\
\hline${ }^{10-10}$ & $<5.0$ & $<5.0$ & $<5.0$ & $<10.0$ & $<5.0$ & $<5.0$ & $<5.0$ & $<5.0$ & $<30.0$ & $<5.0$ & $<5.0$ \\
\hline $\begin{array}{l}24-24 \\
\text { MAR }\end{array}$ & $<5.0$ & $<5.0$ & $<5.0$ & $<10.0$ & $<5.0$ & $<5.0$ & $<5.0$ & $<5.0$ & $<30.0$ & $<5.0$ & $<5.0$ \\
\hline $\begin{array}{l}11-12 \\
\text { MAR }\end{array}$ & $<5.0$ & $<5.0$ & $<5.0$ & $<10.0$ & $<5.0$ & $<5.0$ & $<5.0$ & $<5.0$ & $<30.0$ & $<5.0$ & $<5.0$ \\
\hline $28 \cdot 28$ & $<5.0$ & $<5.0$ & $<5.0$ & $<10.0$ & $<5.0$ & $<5.0$ & $<5.0$ & $<5.0$ & $<30.0$ & $<5.0$ & $<5.0$ \\
\hline DATE & $\begin{array}{l}\text { PENTA- } \\
\text { CHLORO- } \\
\text { PHENOL } \\
\text { TOTAL } \\
(U G / L)\end{array}$ & $\begin{array}{c}\text { PHENAN - } \\
\text { THRENE } \\
\text { TOTAL } \\
\text { (UG/L) }\end{array}$ & $\begin{array}{l}\text { PHENOL } \\
\text { (C6H - } \\
5 O H \text { ) } \\
\text { TOTAL } \\
\text { (UG/L) }\end{array}$ & $\begin{array}{c}\text { PYRENE } \\
\text { TOTAL } \\
\text { (UG/L) }\end{array}$ & $\begin{array}{c}2,4,6- \\
\text { TRI- } \\
\text { CHLORO- } \\
\text { PHENOL } \\
\text { TOTAL } \\
\text { (UG/L) }\end{array}$ & $\begin{array}{l}\text { ALDRIN, } \\
\text { TOTAL } \\
\text { (UG/L) }\end{array}$ & $\begin{array}{l}\text { P, P' } \\
\text { DDT, } \\
\text { TOTAL } \\
\text { (UG/L) }\end{array}$ & $\begin{array}{l}\text { ALPHA } \\
\text { BHC } \\
\text { TOTAL } \\
\text { (UG/L) }\end{array}$ & $\begin{array}{c}\text { BETA } \\
\text { BENZENE } \\
\text { HEXA- } \\
\text { CHLOR- } \\
\text { IDE } \\
\text { TOTAL } \\
\text { (UG/L) }\end{array}$ & $\begin{array}{l}\text { DELTA } \\
\text { BENZENE } \\
\text { HEXA- } \\
\text { CHLOR- } \\
\text { IDE } \\
\text { TOTAL } \\
\text { (UG/L) }\end{array}$ & $\begin{array}{l}\text { LINDANE } \\
\text { TOTAL } \\
\text { (UG/L) }\end{array}$ \\
\hline DEC 1992 & & & & & & & & & & & \\
\hline $\begin{array}{c}09-09 \\
\text { JAN } 1993\end{array}$ & $<30.0$ & $<5.0$ & $<5.0$ & $<5.0$ & $<20.0$ & $<0.040$ & $<0.10$ & $<0.03$ & $<0.03$ & $<0.09$ & $<0.030$ \\
\hline $\begin{array}{l}09-09 \\
\text { JAN }\end{array}$ & $<30.0$ & $<5.0$ & $<5.0$ & $<5.0$ & $<20.0$ & $<0.040$ & $<0.10$ & $<0.03$ & $<0.03$ & $<0.09$ & $<0.030$ \\
\hline FEB $19-19$ & $<30.0$ & $<5.0$ & $<5.0$ & $<5.0$ & $<20.0$ & $<0.040$ & $<0.10$ & $<0.03$ & $<0.03$ & $<0.09$ & $<0.030$ \\
\hline $\begin{array}{l}10-10 \\
F E B\end{array}$ & $<30.0$ & $<5.0$ & $<5.0$ & $<5.0$ & $<20.0$ & $<0.040$ & $<0.10$ & $<0.03$ & $<0.03$ & $<0.09$ & $<0.030$ \\
\hline $\begin{array}{l}24-24 \\
\text { MAR }\end{array}$ & $<30.0$ & $<5.0$ & $<5.0$ & $<5.0$ & $<20.0$ & $<0.040$ & $<0.10$ & $<0.03$ & $<0.03$ & $<0.09$ & $<0.030$ \\
\hline $11-12$ & $<30.0$ & $<5.0$ & $<5.0$ & 8.0 & $<20.0$ & $<0.040$ & $<0.10$ & $<0.03$ & $<0.03$ & $<0.09$ & $<0.030$ \\
\hline $28-28$ & $<30.0$ & 27.0 & $<5.0$ & 43.0 & $<20.0$ & $<0.040$ & $<0.10$ & $<0.03$ & $<0.03$ & $<0.09$ & $<0.030$ \\
\hline
\end{tabular}


Table 2. Water-quality data for storm-sewer outfall stations, Arlington, Texas, 1992-93-Continued 08049470 - TRIBUTARY TO JOHNSON CREEK OUTFALL AT I-30 EAST, ARLINGTON, TX (WY 1993)-CONtInUEd

\begin{tabular}{|c|c|c|c|c|c|c|c|c|c|c|c|}
\hline & $\begin{array}{c}\text { CHLOR - } \\
\text { DANE }\end{array}$ & $\begin{array}{l}\text { CHLOR - } \\
\text { DANE }\end{array}$ & & & & & $\begin{array}{l}\text { ENDO- } \\
\text { SULFAN - }\end{array}$ & & & & \\
\hline & CIS & TRANS & & & & & I & ENDO - & ENDO - & ENDRIN & ENDRIN \\
\hline & WATER & WATER & CHLOR - & $P, P^{\prime}$ & $P, P^{\prime}$ & DI - & WATER & SULFAN & SULFAN & WATER & ALDE - \\
\hline & WHOLE & WHOLE & DANE, & DDD & DDE , & ELDRIN & WHOLE & BETA & SULFATE & UNFLTRD & HYDE \\
\hline DATE & $\begin{array}{l}\text { TOTAL } \\
\text { (UG/L) }\end{array}$ & $\begin{array}{l}\text { TOTAL } \\
\text { (UG/L) }\end{array}$ & $\begin{array}{l}\text { TOTAL } \\
\text { (UG/L) }\end{array}$ & $\begin{array}{l}\text { TOTAL } \\
\text { (UG/L) }\end{array}$ & $\begin{array}{l}\text { TOTAL } \\
\text { (UG/L) }\end{array}$ & $\begin{array}{l}\text { TOTAL } \\
\text { (UG/L) }\end{array}$ & $\begin{array}{c}\text { REC } \\
(U G / L)\end{array}$ & $\begin{array}{l}\text { TOTAL } \\
\text { (UG/L) }\end{array}$ & $\begin{array}{l}\text { TOTAL } \\
(U G / L)\end{array}$ & $\begin{array}{c}\text { REC } \\
(U G / L)\end{array}$ & $\begin{array}{c}\text { TOTAL } \\
(\mathrm{UG} / \mathrm{L})\end{array}$ \\
\hline \multicolumn{12}{|l|}{ DEC 1992} \\
\hline 09.09 & $<0.10$ & $<0.10$ & $<0.1$ & $<0.10$ & $<0.04$ & $<0.020$ & $<0.10$ & $<0.04$ & $<0.60$ & $<0.060$ & $<0.20$ \\
\hline \multicolumn{12}{|l|}{ JAN 1993} \\
\hline $09-09$ & $<0.10$ & $<0.10$ & $<0.1$ & $<0.10$ & $<0.04$ & $<0.020$ & $<0.10$ & $<0.04$ & $<0.60$ & $<0.060$ & $<0.20$ \\
\hline \multicolumn{12}{|l|}{ JAN } \\
\hline $19-19$ & $<0.10$ & $<0.10$ & 0.1 & $<0.10$ & $<0.04$ & $<0.020$ & $<0.10$ & $<0.04$ & $<0.60$ & $<0.060$ & $<0.20$ \\
\hline \multicolumn{12}{|l|}{ FEB } \\
\hline $10 \cdot 10$ & $<0.10$ & $<0.10$ & $<0.1$ & $<0.10$ & $<0.04$ & $<0.020$ & $<0.10$ & $<0.04$ & $<0.60$ & $<0.060$ & $<0.20$ \\
\hline \multicolumn{12}{|l|}{ FEB } \\
\hline $24-24$ & $<0.10$ & $<0.10$ & $<0.1$ & $<0.10$ & $<0.04$ & $<0.020$ & $<0.10$ & $<0.04$ & $<0.60$ & $<0.060$ & $<0.20$ \\
\hline \multicolumn{12}{|l|}{ MAR } \\
\hline $11 \cdot 12$ & $<0.10$ & $<0.10$ & $<0.1$ & $<0.10$ & $<0.04$ & $<0.020$ & $<0.10$ & $<0.04$ & $<0.60$ & $<0.060$ & $<0.20$ \\
\hline \multicolumn{12}{|l|}{ MAR } \\
\hline \multirow[t]{4}{*}{$28-28$} & $<0.10$ & $<0.10$ & $<0.1$ & $<0.10$ & $<0.04$ & $<0.020$ & $<0.10$ & $<0.04$ & $<0.60$ & $<0.060$ & $<0.20$ \\
\hline & & HEPTA- & & AROCLOR & AROCLOR & AROCLOR & AROCLOR & AROCLOR & AROCLOR & AROCLOR & \\
\hline & HEPTA - & CHLOR & TOX - & 1016 & 1221 & 1232 & 1242 & 1248 & 1254 & 1260 & DI - \\
\hline & CHLOR, & EPOXIDE & APHENE, & PCB & $\mathrm{PCB}$ & PCB & PCB & PCB & PCB & PCB & AZINON, \\
\hline DATE & $\begin{array}{l}\text { TOTAL } \\
\text { (UG/L) }\end{array}$ & $\begin{array}{l}\text { TOTAL } \\
\text { (UG/L) }\end{array}$ & $\begin{array}{l}\text { TOTAL } \\
\text { (UG/L) }\end{array}$ & $\begin{array}{l}\text { TOTAL } \\
\text { (UG/L) }\end{array}$ & $\begin{array}{l}\text { TOTAL } \\
\text { (UG/L) }\end{array}$ & $\begin{array}{l}\text { TOTAL } \\
\text { (UG/L) }\end{array}$ & $\begin{array}{l}\text { TOTAL } \\
\text { (UG/L) }\end{array}$ & $\begin{array}{l}\text { TOTAL } \\
\text { (UG/L) }\end{array}$ & $\begin{array}{l}\text { TOTAL } \\
\text { (UG/L) }\end{array}$ & $\begin{array}{l}\text { TOTAL } \\
\text { (UG/L) }\end{array}$ & $\begin{array}{l}\text { TOTAL } \\
\text { (UG/L) }\end{array}$ \\
\hline \multicolumn{12}{|l|}{ DEC 1992} \\
\hline $09-09$ & $<0.030$ & $<0.80$ & $<2$ & $<0.1$ & $<1.0$ & $<0.1$ & $<0.1$ & $<0.1$ & $<0.1$ & $<0.1$ & 0.70 \\
\hline \multicolumn{12}{|l|}{ JAN 1993} \\
\hline $09-09$ & $<0.030$ & $<0.80$ & $<2$ & $<0.1$ & $<1.0$ & $<0.1$ & $<0.1$ & $<0.1$ & $<0.1$ & $<0.1$ & $<0.05$ \\
\hline \multicolumn{12}{|l|}{ JAN } \\
\hline $19-19$ & $<0.030$ & $<0.80$ & $<2$ & $<0.1$ & $<1.0$ & $<0.1$ & $<0.1$ & $<0.1$ & $<0.1$ & $<0.1$ & 0.20 \\
\hline \multicolumn{12}{|l|}{ FEB } \\
\hline $10-10$ & $<0.030$ & $<0.80$ & $<2$ & $<0.1$ & $<1.0$ & $<0.1$ & $<0.1$ & $<0.1$ & $<0.1$ & $<0.1$ & $<0.05$ \\
\hline \multicolumn{12}{|l|}{ FEB } \\
\hline $24-24$ & $<0.030$ & $<0.80$ & $<2$ & $<0.1$ & $<1.0$ & $<0.1$ & $<0.1$ & $<0.1$ & $<0.1$ & $<0.1$ & $<0.20$ \\
\hline \multicolumn{12}{|l|}{ MAR } \\
\hline $11 \cdot 12$ & $<0.030$ & $<0.80$ & $<2$ & $<0.1$ & $<1.0$ & $<0.1$ & $<0.1$ & $<0.1$ & $<0.1$ & $<0.1$ & $<0.10$ \\
\hline \multicolumn{12}{|l|}{ MAR } \\
\hline $28-28$ & $<0.030$ & $<0.80$ & $<2$ & $<0.1$ & $<1.0$ & $<0.1$ & $<0.1$ & $<0.1$ & $<0.1$ & $<0.1$ & $<0.05$ \\
\hline
\end{tabular}


Table 3. Water-quality data for storm-sewer outfall stations, Dallas, Texas, 1992-93

08055590 - JOE'S CREEK OUTFALL AT DENTON DRIVE, DALLAS, TX (WY 1992)

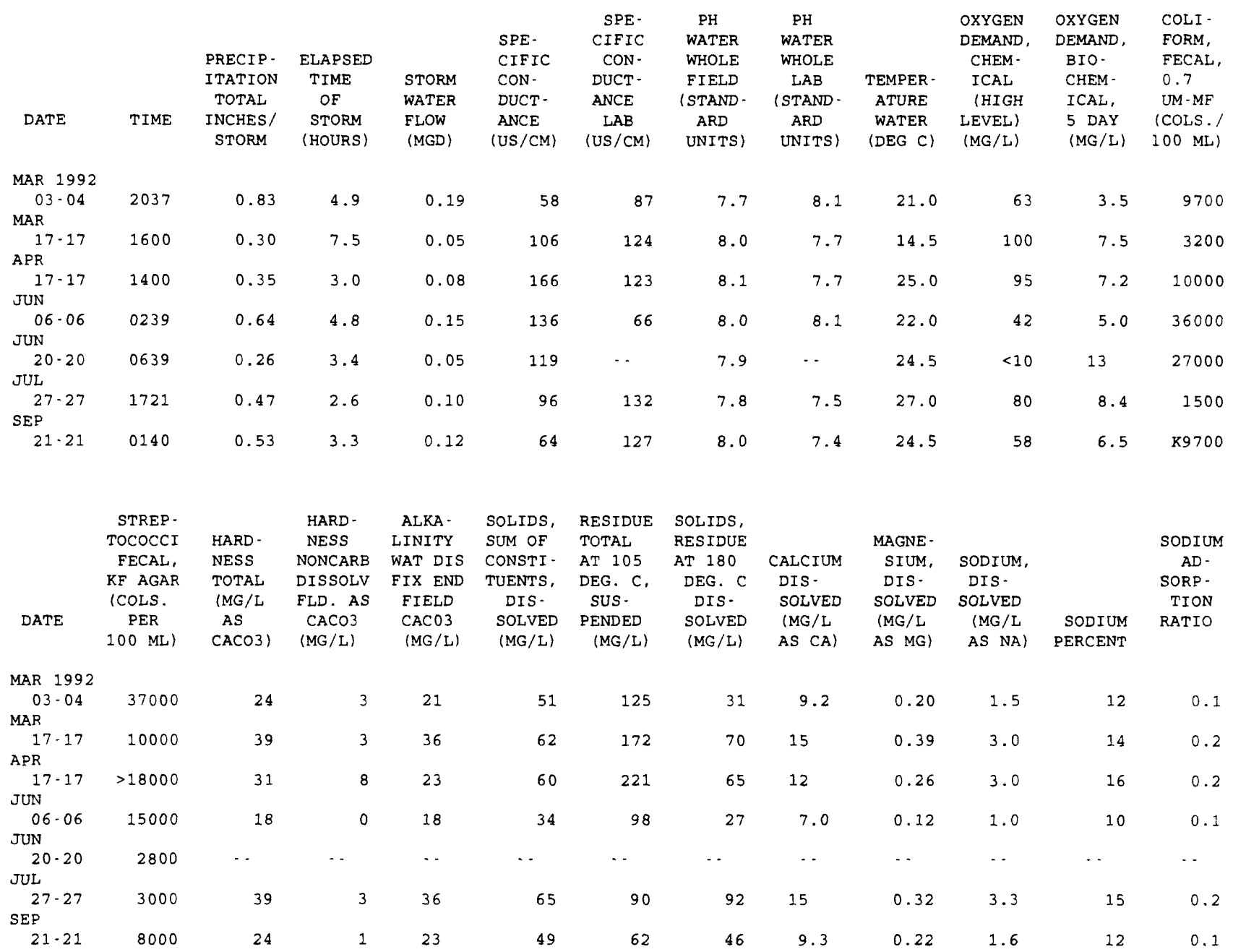

\begin{tabular}{|c|c|c|c|c|c|c|c|c|c|c|c|c|}
\hline ATE & $\begin{array}{l}\text { POTAS - } \\
\text { SIUM, } \\
\text { DIS - } \\
\text { SOLVED } \\
\text { (MG/L }\end{array}$ & $\begin{array}{l}\text { SULFATE } \\
\text { DIS - } \\
\text { SOLVED } \\
\text { (MG/L }\end{array}$ & $\begin{array}{l}\text { CHLO- } \\
\text { RIDE, } \\
\text { DIS - } \\
\text { SOLVED } \\
(\mathrm{MG} / \mathrm{L}\end{array}$ & $\begin{array}{l}\text { NITRO- } \\
\text { GEN, } \\
\text { NITRITE } \\
\text { TOTAL } \\
\text { (MG /L }\end{array}$ & $\begin{array}{c}\text { NITRO- } \\
\text { GEN, } \\
\text { NO2+NO3 } \\
\text { TOTAL } \\
\text { (MG/L }\end{array}$ & $\begin{array}{l}\text { NITRO- } \\
\text { GEN, } \\
\text { AMMONIA } \\
\text { TOTAL } \\
\text { (MG/L }\end{array}$ & $\begin{array}{l}\text { NITRO- } \\
\text { GEN, AM- } \\
\text { MONIA + } \\
\text { ORGANIC } \\
\text { TOTAL } \\
\text { (MG/L }\end{array}$ & $\begin{array}{l}\text { PHOS - } \\
\text { PHORUS } \\
\text { TOTAL } \\
\text { (MG/L }\end{array}$ & $\begin{array}{c}\text { PHOS - } \\
\text { PHORUS } \\
\text { DIS - } \\
\text { SOLVED } \\
\text { (MG/L }\end{array}$ & $\begin{array}{l}\text { ANTIMONY } \\
\text { TOTAL } \\
\text { EPA } \\
\text { (UG/L }\end{array}$ & $\begin{array}{c}\text { ARSENIC } \\
\text { TOTAL } \\
\text { (UG/L }\end{array}$ & $\begin{array}{l}\text { BERYL- } \\
\text { LIUM, } \\
\text { TOTAL } \\
\text { RECOV- } \\
\text { ERABLE } \\
\text { (UG /L }\end{array}$ \\
\hline & AS $\mathrm{K}$ ) & AS SO4) & AS CL) & AS N) & AS $N$ ) & AS N> & AS $N\}$ & AS P) & AS P\} & AS SB) & AS AS) & AS BE) \\
\hline
\end{tabular}

\begin{tabular}{|c|c|c|c|c|c|c|c|c|c|c|c|c|}
\hline \multicolumn{13}{|l|}{ MAR 1992} \\
\hline 03.04 & 0.90 & 3.2 & 1.0 & 0.050 & 0.320 & 0.080 & 0.70 & 0.170 & 0.060 & $<10.0$ & 1 & $<10$ \\
\hline \multicolumn{13}{|l|}{ MAR } \\
\hline $17-17$ & 1.4 & 6.8 & 2.0 & 0.110 & 0.600 & 0.310 & 0.90 & 0.330 & 0.200 & $<10.0$ & 2 & $<10$ \\
\hline \multicolumn{13}{|l|}{$A P R$} \\
\hline $17-17$ & 1.6 & 7.3 & 2.4 & 0.070 & 0.820 & 0.190 & 1.0 & 0.490 & 0.390 & $<20.0$ & 2 & $<10$ \\
\hline \multicolumn{13}{|l|}{ JUN } \\
\hline 06.06 & 0.70 & 2.2 & 0.80 & 0.020 & 0.300 & 0.120 & 0.30 & 0.080 & 0.070 & $<20.0$ & $<1$ & $<10$ \\
\hline \multicolumn{13}{|l|}{ JUN } \\
\hline $20-20$ & $\cdots$ & $\cdots$ & $\cdots$ & 0.070 & 0.790 & 0.310 & 1.4 & 0.310 & 0.190 & $<20.0$ & 1 & $<10$ \\
\hline \multicolumn{13}{|l|}{ JUL } \\
\hline $27 \cdot 27$ & 1.8 & 7.4 & 3.7 & 0.070 & 0.840 & 0.290 & 1.3 & 0.450 & 0.360 & $<10.0$ & 2 & $<10$ \\
\hline \multicolumn{13}{|l|}{ SEP } \\
\hline $21 \cdot 21$ & 1.7 & 4.0 & 1.5 & 0.030 & 0.350 & 0.110 & 1.0 & 0.300 & 0.230 & $<10.0$ & $<1$ & $<10$ \\
\hline
\end{tabular}


Table 3. Water-quality data for storm-sewer outfall stations, Dallas, Texas, 1992-93-Continued

08055590 - JOE'S CREEK OUTFALL AT DENTON DRIVE, DALLAS, TX (WY 1992)-Continued

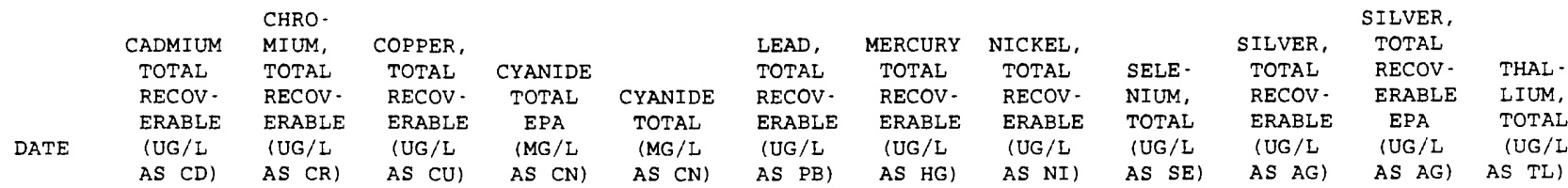

MAR 1992
03.04

MAR 17.17

$17 \cdot 17$
APR
$17 \cdot 17$

JUN

06-06

JUN

$20-20$

JUL

$27-27$

SEP

21-21

AS CD

AS CR)

AS CU)

AS PB)

AS HG)

AS NI)

AS SE)

AS AG)

AS AG) AS TL)

$<1$
$<1$
1
8
$<1$
2
$<1$

$\begin{array}{lll}11 & <0.010 & <0.010 \\ 10 & <0.010 & <0.010 \\ 14 & <0.010 & <0.010 \\ 8 & <10.0 & <0.010 \\ 12 & <10.0 & <0.010 \\ 16 & <10.0 & <0.010 \\ 4 & <0.010 & <0.010\end{array}$

$\begin{array}{ll}39 & <0.10 \\ 33 & <0.10 \\ 44 & <0.10 \\ 20 & <0.10 \\ 15 & <0.10 \\ 25 & 0.40 \\ <1 & <0.10\end{array}$

$<2$
$<2$
$<2$
$<2$
$<2$
$<1$
$<2$

$\begin{array}{lll}<1 & <1.00 & <10 \\ <1 & <1.00 & <5 \\ <1 & <1.00 & <5 \\ <1 & <1.00 & <5 \\ <1 & <1.00 & <10 \\ <1 & <0.500 & <5 \\ <1 & <1.00 & <10\end{array}$

$\begin{array}{lll} & & \\ & \text { ZINC, } & \\ & \text { TOTAL } & \text { CARBON, } \\ & \text { RECOV - } & \text { ORGANIC } \\ & \text { ERABLE } & \text { TOTAL } \\ \text { DATE } & \text { (UG } / L & \text { (MG } / L \\ & \text { AS ZN) } & \text { AS C) }\end{array}$

OIL AND

GREASE,

TOTAL

RECOV.

ACRO-

METRIC PHENOLS LEIN

(MG/L)

(UG/L) (UG/L)

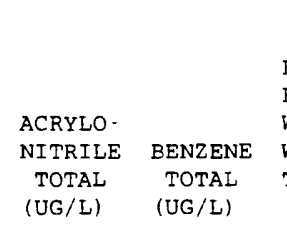

$\begin{array}{ll}\text { BROMO- } & \\ \text { BENZENE } & \\ \text { WATER, } & \text { BROMO- } \\ \text { WHOLE, } & \text { FORM } \\ \text { TOTAL } & \text { TOTAL } \\ \text { (UG } / \text { L) } & \text { (UG/L) }\end{array}$

$\begin{array}{ccc} & \text { BENZENE } & \text { BENZENE } \\ \text { BENZENE } & \text { SEC } & \text { TERT- } \\ \text { N-BUTYL } & \text { BUTYL- } & \text { BUTYL- } \\ \text { WATER } & \text { WATER } & \text { WATER } \\ \text { UNFLTRD } & \text { UNFLTRD } & \text { UNFLTRD } \\ \text { REC } & \text { REC } & \text { REC } \\ \text { (UG/L) } & \text { (UG/L) } & \text { (UG/L) }\end{array}$

MAR 1992

$03-04$
MAR
$17-17$
APR

$100 \quad 14$

$\begin{array}{llll}2 & 2 & <20 & <20 \\ 1 & 2 & <20 & <20 \\ <1 & 4 & <20 & <20 \\ <1 & 4 & <20 & <20 \\ <1 & 4 & <20 & <20 \\ <1 & 9 & <20 & <20 \\ <1 & 1 & <20 & <20\end{array}$

$<0.2$
$<0.2$
$<0.2$
$<0.2$
$<0.2$
$<0.2$
$<0.2$

$<0.2$

$<0.2$

$<0.20$

$<0.20<0.20$

$17-17$

$110 \quad 27$

$170 \quad 24$

$70 \quad 10$

06-06

JUN

$20-20$

$27-27$

SEP

$21-21$

$70 \quad 29$

$140 \quad 27$

$110 \quad 16$

$<1$

$<20$

$<0.2$

$<0.2$

$<0.20$

$<0.20$

$<0.20$

$<0.2$

$<0.2<0.20$

$<0.20$

$<0.20$

$<0.2$

$<0.2<0.20$

$<0.20$

$<0.20$

$<0.2$

$<0.2$

$<0.20$

$<0.20$

$<0.20$

$<0.2$

$<0.2<0.20$

$<0.20$

$<0.20$

$<0.2$

$<0.2$

$<0.20$

$<0.20<0.20$

\begin{tabular}{clcc} 
CARBON- & \multicolumn{3}{c}{ CHLORO- } \\
DETRA - \\
CHLO - & CHLORO- & BROMO- & CHLORO- \\
RIDE & BENZENE & METHANE & ETHANE \\
TOTAL & TOTAL & TOTAL & TOTAL \\
$(U G / L)$ & $(U G / L)$ & (UG $/ L)$ & $($ UG $/ L)$
\end{tabular}

2 -

CHLORO-

ETHYL-

VINYL -

ETHER

TOTAL
$(U G / L)$

MAR 1992

03-04

MAR

$17-17$

$<0.2<0.20$

$<0.2<0.2$

$<1.0 \quad<0.2$

$<0.2$

O-

CHLORO- TOLUENE

CHLORO -

$\begin{array}{ccccc}\text { METHYL- } & \text { TOLUENE } & \text { P-CHLOR PROPANE } & \text { METHANE ETHANE } \\ \text { CHLO- } & \text { WATER } & \text { WATER } & \text { WATER } & \text { WATER } \\ \end{array}$

DIBROMO

DI. 1,2-

FORM RIDE WHOLE UNFLTRD WHOLE WHOLE WHOLE

TOTAL TOTAL TOTAL REC TOT.REC RECOVER TOTAL

$(U G / L) \quad(U G / L) \quad(U G / L)$

$(U G / L)$

(UG/L)

(UG/L) (UG/L)

$<0.2<0.20$

$<0.2$

$<0.2$

$<1.0$

$<0.2$

$<0.2$

$<0.2$

$<0.20$

$<1.0$

$<0.2<0.2$

$<0.2$

$<0.20$

$<1.0$

$<0.2$

$<0.2$

APR
$17-17$

$<0.2<0.20$

$<0.2$

$<0.2$

$<1.0$

$<0.2$

$<0.2$

$06-06$

$<0.2<0.20$

$<0.2$

$<0.2$

$<1.0$

$<0$.

$<0.2$

$<0.2<0.20$

$<1.0$

$<0.2$

$<0.2$

JUN $20 \cdot 20$

$<0.2<0.20$

$<0.2$

$<0$.

$<1.0$

$<0.2$

$<0.2$

$<0.2<0.20$

$<1.0$

$<0.2<0.2$

JUL

$27-27$

$<0.2<0.20$

$<0.2$

$<0.2$

$<1.0$

0.5

$<0.2$

$<0$.

$<0.20$

$<1.0$

$<0.2$

$<0.2$

$21 \cdot 21$

$<0.2<0.20$

$<0.2$

$<0.2$

$<1.0$

$<0.2$

$<0.2$

$<0.2<0.20$

$<1.0$

$<0.2<0.2$

$<0.2<0.20$

$<1$. 
Table 3. Water-quality data for storm-sewer outfall stations, Dallas, Texas, 1992-93-Continued

08055590 - JOE'S CREEK OUTFALL AT DENTON DRIVE, DALLAS, TX (WY 1992)-Continued

\begin{tabular}{|c|c|c|c|c|c|c|c|c|c|c|c|c|}
\hline DATE & $\begin{array}{c}\text { DI - } \\
\text { CHLORO- } \\
\text { BROMO- } \\
\text { METHANE } \\
\text { TOTAL } \\
\text { (UG/L) }\end{array}$ & $\begin{array}{c}1,1 \text { - DI - } \\
\text { CHLORO- } \\
\text { ETHYL - } \\
\text { ENE } \\
\text { TOTAL } \\
\text { (UG/L) }\end{array}$ & $\begin{array}{l}1,1 \text { - DI } \\
\text { CHLORO- } \\
\text { PRO- } \\
\text { PENE, } \\
\text { WAT, WH } \\
\text { TOTAL } \\
\text { (UG/L) }\end{array}$ & $\begin{array}{c}\text { BENZENE } \\
0^{-} \\
\text {CHLORO- } \\
\text { WATER } \\
\text { UNFLTRD } \\
\text { REC } \\
\text { (UG/L) }\end{array}$ & $\begin{array}{c}\text { BENZENE } \\
1,3-D I- \\
\text { CHLORO- } \\
\text { WATER } \\
\text { UNFLTRD } \\
\text { REC } \\
\text { (UG/L) }\end{array}$ & $\begin{array}{l}\text { BENZENE } \\
1,4-D I- \\
\text { CHLORO- } \\
\text { WATER } \\
\text { UNFLTRD } \\
\text { REC } \\
\text { (UG/L) }\end{array}$ & $\begin{array}{l}\text { DI- } \\
\text { CHLORO- } \\
\text { DI- } \\
\text { FLUORO- } \\
\text { METHANE } \\
\text { TOTAL } \\
\text { (UG/L) }\end{array}$ & $\begin{array}{c}1,1-D I- \\
\text { CHLORO- } \\
\text { ETHANE } \\
\text { TOTAL } \\
\text { (UG/L) }\end{array}$ & $\begin{array}{c}1,2-D I- \\
\text { CHLORO- } \\
\text { ETHANE } \\
\text { TOTAL } \\
\text { (UG/L) }\end{array}$ & $\begin{array}{l}1,2-D I- \\
\text { PHENYL- } \\
\text { HYDRA- } \\
\text { ZINE } \\
\text { WATER } \\
\text { TOT.REC } \\
(\text { UG } / L)\end{array}$ & $\begin{array}{c}\text { CIS - } 1,2- \\
\text { DI- } \\
\text { CHLORO- } \\
\text { ETHENE } \\
\text { WATER } \\
\text { TOTAL } \\
\text { (UG/L) }\end{array}$ & $\begin{array}{l}1,2- \\
\text { TRANSDI } \\
\text { CHLORO- } \\
\text { ETHENE } \\
\text { TOTAL } \\
\text { (UG/L) }\end{array}$ \\
\hline $\begin{array}{c}\text { MAR } 1992 \\
03-04\end{array}$ & $<0.2$ & $<0.2$ & $<0,2$ & $<5,0$ & $<50$ & $<50$ & $<02$ & $<02$ & $<0$ & $<50$ & $<0 ?$ & $<0.2$ \\
\hline MAR & & & & & & & & & & & & \\
\hline $\begin{array}{l}17 \cdot 17 \\
\text { APR }\end{array}$ & $<0.2$ & $<0.2$ & $<0.2$ & $<5.0$ & $<5.0$ & $<5.0$ & $<0.2$ & $<0.2$ & $<0.2$ & $<5.0$ & $<0.2$ & $<0.2$ \\
\hline${ }_{\text {JUN }}^{17-17}$ & $<0.2$ & $<0.2$ & $<0.2$ & $<5.0$ & $<5.0$ & $<5.0$ & $<0.2$ & $<0.2$ & $<0.2$ & $<5.0$ & $<0.2$ & $<0.2$ \\
\hline $\begin{array}{l}06-06 \\
\text { JUN }\end{array}$ & $<0.2$ & $<0.2$ & $<0.2$ & $<5.0$ & $<5.0$ & $<5.0$ & $<0.2$ & $<0.2$ & $<0.2$ & $<5.0$ & $<0.2$ & $<0.2$ \\
\hline $\begin{array}{l}20 \cdot 20 \\
\text { JUL }\end{array}$ & $<0.2$ & $<0.2$ & $<0.2$ & $<5.0$ & $<5.0$ & $<5.0$ & $<0.2$ & $<0.2$ & $<0.2$ & $<5.0$ & $<0.2$ & $<0.2$ \\
\hline $\begin{array}{l}27-27 \\
\text { EP }\end{array}$ & $<0.2$ & $<0.2$ & $<0.2$ & $<5.0$ & $<5.0$ & $<5.0$ & $<0.2$ & $<0.2$ & $<0.2$ & $<5.0$ & $<0.2$ & $<0.2$ \\
\hline $21 \cdot 21$ & $<0.2$ & $<0.2$ & $<0.2$ & $<5.0$ & $<5.0$ & $<5.0$ & $<0.2$ & $<0.2$ & $<0.2$ & $<5.0$ & $<0.2$ & $<0.2$ \\
\hline
\end{tabular}

\begin{tabular}{|c|c|c|c|c|c|c|c|c|c|c|c|c|}
\hline & 2-DI- & $\begin{array}{l}\text { 1, } 3 \text {-DI - } \\
\text { CHLORO- }\end{array}$ & $\begin{array}{l}2,2 \text {-DI } \\
\text { CHLORO- } \\
\text { PRO- }\end{array}$ & $\begin{array}{c}\text { CIS } \\
1,3 \text {-DI - }\end{array}$ & $\begin{array}{r}\text { TRANS- } \\
1,3 \text {-DI - }\end{array}$ & & $\begin{array}{l}\text { HEXA- } \\
\text { CHLORO- }\end{array}$ & $\begin{array}{c}\text { ISO- } \\
\text { PROPYL - } \\
\text { BENZENE }\end{array}$ & $\begin{array}{l}\text { P-ISO- } \\
\text { PROPYL- } \\
\text { TOLUENE }\end{array}$ & & $\begin{array}{l}\text { METHYL - } \\
\text { ENE }\end{array}$ & \\
\hline & HLORO- & PROPANE & PANE & CHLORO- & CHLORO- & ETHYL- & BUT - & WATER & WATER & METHYL- & CHLO- & NAPHTH - \\
\hline $\mathrm{TE}$ & $\begin{array}{l}\text { PROPANE } \\
\text { TOTAL } \\
\text { (UG/L) }\end{array}$ & $\begin{array}{l}\text { WAT. WH } \\
\text { TOTAL } \\
\text { (UG/L) }\end{array}$ & $\begin{array}{l}\text { WAT, WH } \\
\text { TOTAL } \\
\text { (UG/L) }\end{array}$ & $\begin{array}{l}\text { PROPENE } \\
\text { TOTAL } \\
\text { (UG/L) }\end{array}$ & $\begin{array}{l}\text { PROPENE } \\
\text { TOTAL } \\
\text { (UG/L) }\end{array}$ & $\begin{array}{l}\text { BENZENE } \\
\text { TOTAL } \\
\text { (UG/L) }\end{array}$ & $\begin{array}{l}\text { ADIENE } \\
\text { TOTAL } \\
\text { (UG/L) }\end{array}$ & $\begin{array}{l}\text { WHOLE } \\
\text { REC } \\
\text { (UG/L) }\end{array}$ & $\begin{array}{l}\text { WHOLE } \\
\text { REC } \\
\text { (UG/L) }\end{array}$ & $\begin{array}{l}\text { BROMIDE } \\
\text { TOTAL } \\
\text { (UG/L) }\end{array}$ & $\begin{array}{l}\text { RIDE } \\
\text { TOTAL } \\
\text { (UG/L) }\end{array}$ & $\begin{array}{l}\text { ALENE } \\
\text { TOTAL } \\
\text { (UG/L) }\end{array}$ \\
\hline
\end{tabular}

\begin{tabular}{|c|c|c|c|c|c|c|c|c|c|c|c|c|}
\hline MAR 1992 & & & & & & & & & & & & \\
\hline $03-04$ & $<0.2$ & $<0.2$ & $<0.2$ & $<0.2$ & $<0.2$ & $<0.2$ & $<5.0$ & $<0.20$ & $<0.20$ & $<0.2$ & $<0.2$ & $<5.0$ \\
\hline MAR & & & & & & & & & & & & \\
\hline $17-17$ & $<0.2$ & $<0.2$ & $<0.2$ & $<0.2$ & $<0.2$ & $<0.2$ & $<5.0$ & $<0.20$ & $<0.20$ & $<0.2$ & $<0.2$ & $<5.0$ \\
\hline APR & & & & & & & & & & & & \\
\hline $17-17$ & $<0.2$ & $<0.2$ & $<0.2$ & $<0.2$ & $<0.2$ & $<0.2$ & $<5.0$ & $<0.20$ & $<0.20$ & $<0.2$ & $<0.2$ & $<5.0$ \\
\hline JUN & & & & & & & & & & & & \\
\hline 06.06 & $<0.2$ & $<0.2$ & $<0.2$ & $<0.2$ & $<0.2$ & $<0.2$ & $<5.0$ & $<0.20$ & $<0.20$ & $<0.2$ & $<0.3$ & $<5.0$ \\
\hline JUN & & & & & & & & & & & & \\
\hline $20-20$ & $<0.2$ & $<0.2$ & $<0.2$ & $<0.2$ & $<0.2$ & $<0.2$ & $<5.0$ & $<0.20$ & $<0.20$ & $<0.2$ & $<0.2$ & $<5.0$ \\
\hline JUL & & & & & & & & & & & & \\
\hline $27-27$ & $<0.2$ & $<0.2$ & $<0.2$ & $<0.2$ & $<0.2$ & $<0.2$ & $<5.0$ & $<0.20$ & $<0.20$ & $<0.2$ & $<0.2$ & $<5.0$ \\
\hline $21-21$ & $<0.2$ & $<0.2$ & $<0.2$ & $<0.2$ & $<0.2$ & $<0.2$ & $<5.0$ & $<0.20$ & $<0.20$ & $<0.2$ & $<0.2$ & $<5.0$ \\
\hline
\end{tabular}

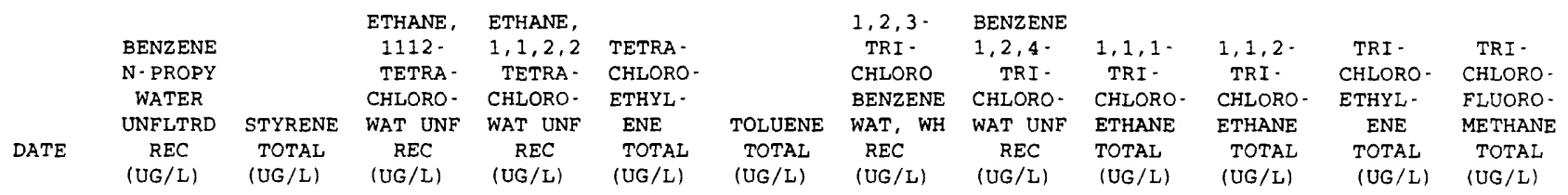

\begin{tabular}{|c|c|c|c|c|c|c|c|c|c|c|c|c|}
\hline MAR 1992 & & & & & & & & & & & & \\
\hline $03-04$ & $<0.20$ & $<0.2$ & $<0.2$ & $<0.2$ & $<0.2$ & $<0.2$ & $<0.20$ & $<5.0$ & $<0.2$ & $<0.2$ & $<0.2$ & $<0.2$ \\
\hline MAR & & & & & & & & & & & & \\
\hline $17-17$ & $<0.20$ & $<0.2$ & $<0.2$ & $<0.2$ & 1.9 & $<0.2$ & $<0.20$ & $<5.0$ & $<0.2$ & $<0.2$ & $<0.2$ & $<0.2$ \\
\hline APR & & & & & & & & & & & & \\
\hline $17 \cdot 17$ & $<0.20$ & $<0.2$ & $<0.2$ & $<0.2$ & $<0.2$ & $<0.2$ & $<0.20$ & $<5.0$ & $<0.2$ & $<0.2$ & $<0.2$ & $<0.2$ \\
\hline JUN & & & & & & & & & & & & \\
\hline $06-06$ & $<0.20$ & $<0.2$ & $<0.2$ & $<0.2$ & $<0.2$ & $<0.2$ & $<0.20$ & $<5.0$ & $<0.2$ & $<0.2$ & $<0.2$ & $<0.2$ \\
\hline$\pi N$ & & & & & & & & & & & & \\
\hline $20-20$ & $<0.20$ & $<0.2$ & $<0.2$ & $<0.2$ & $<0.2$ & $<0.2$ & $<0.20$ & $<5.0$ & $<0.2$ & $<0.2$ & $<0.2$ & $<0.2$ \\
\hline$\pi$ & & & & & & & & & & & & \\
\hline $27-27$ & $<0.20$ & $<0.2$ & $<0.2$ & $<0.2$ & $<0.2$ & $<0.2$ & $<0.20$ & $<5.0$ & $<0.2$ & $<0.2$ & $<0.2$ & $<0.2$ \\
\hline SEP & & & & & & & & & & & & \\
\hline $21-21$ & $<0.20$ & $<0.2$ & $<0.2$ & $<0.2$ & $<0.2$ & $<0.2$ & $<0.20$ & $<5,0$ & $<0.2$ & $<0.2$ & $<0.2$ & $<0.2$ \\
\hline
\end{tabular}


Table 3. Water-quality data for storm-sewer outfall stations, Dallas, Texas, 1992-93-Continued

08055590 - JOE'S CREEK OUTFALL AT DENTON DRIVE, DALLAS, TX (WY 1992)-Continued

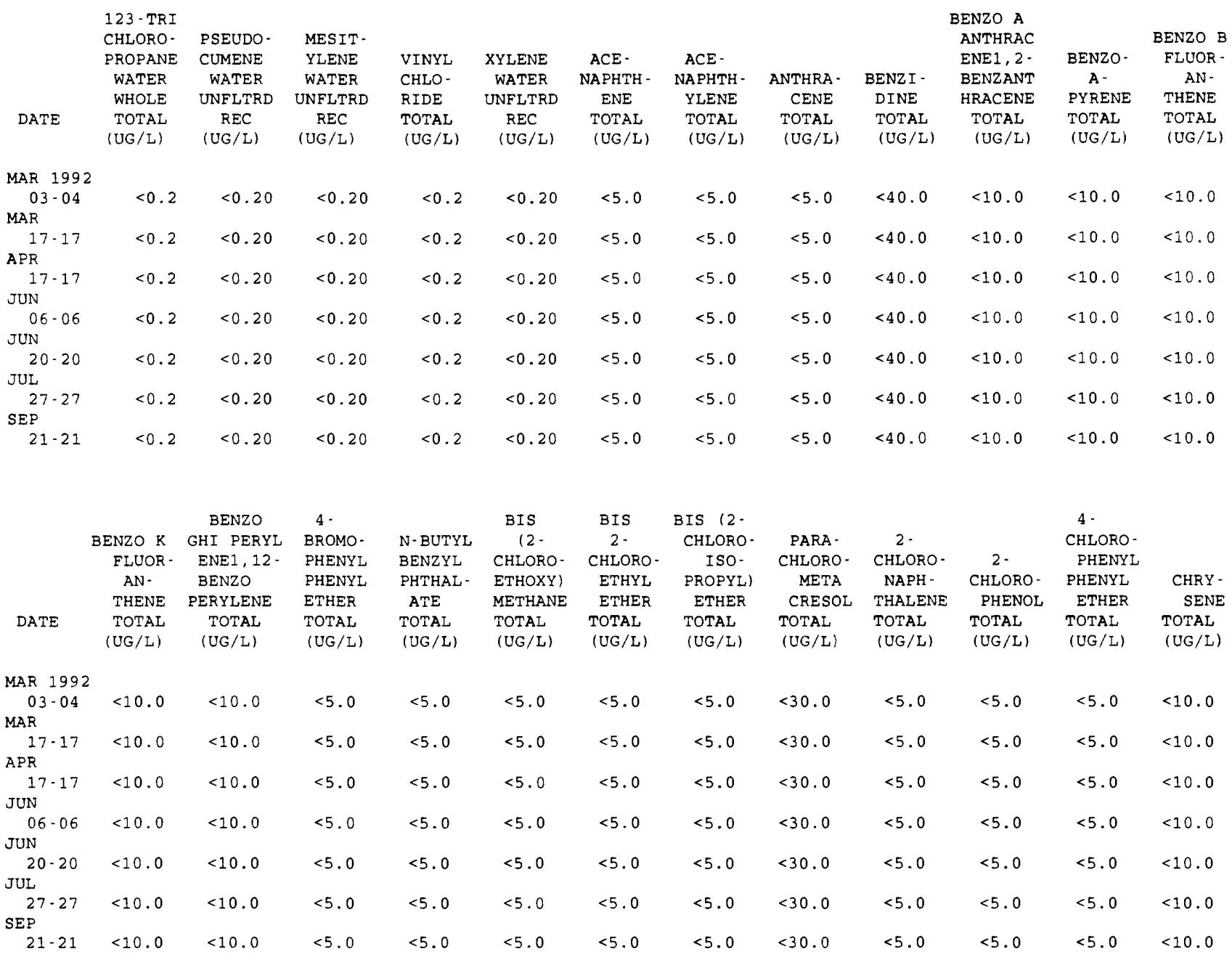

\begin{tabular}{|c|c|c|c|c|c|c|c|c|c|c|c|c|}
\hline DATE & $\begin{array}{c}1,2,5,6- \\
\text { DIBENZ- } \\
\text { ANTHRA- } \\
\text { CENE } \\
\text { TOTAL } \\
\text { (UG/L) }\end{array}$ & $\begin{array}{c}3,3^{\prime}- \\
\text { DI - } \\
\text { CHLORO- } \\
\text { BENZI - } \\
\text { DINE } \\
\text { TOTAL } \\
(\text { UG } / L)\end{array}$ & $\begin{array}{c}2,4-D I- \\
\text { CHLORO- } \\
\text { PHENOL } \\
\text { TOTAL } \\
\text { (UG/L) }\end{array}$ & $\begin{array}{l}\text { DIETHYL } \\
\text { PHTHAL - } \\
\text { ATE } \\
\text { TOTAL } \\
\text { (UG/L) }\end{array}$ & $\begin{array}{c}\text { DI - } \\
\text { METHYL } \\
\text { PHTHAL- } \\
\text { ATE } \\
\text { TOTAL } \\
\text { (UG/L) }\end{array}$ & $\begin{array}{c}2,4 \cdot \mathrm{DI}- \\
\text { METHYL- } \\
\text { PHENOL } \\
\text { TOTAL } \\
\text { (UG/L) }\end{array}$ & $\begin{array}{l}\text { DI - N- } \\
\text { BUTYL } \\
\text { PHTHAL- } \\
\text { ATE } \\
\text { TOTAL } \\
(\text { UG } / L)\end{array}$ & $\begin{array}{c}4,6- \\
\text { DINITRO- } \\
\text { ORTHO- } \\
\text { CRESOL } \\
\text { TOTAL } \\
\text { (UG/L) }\end{array}$ & $\begin{array}{c}2,4,- \\
\text { DI- } \\
\text { NITRO- } \\
\text { PHENOL } \\
\text { TOTAL } \\
\text { (UG/L) }\end{array}$ & $\begin{array}{l}2,4-\mathrm{DI}- \\
\text { NITRO- } \\
\text { TOLUENE } \\
\text { TOTAL } \\
(\mathrm{UG} / \mathrm{L})\end{array}$ & $\begin{array}{l}2,6 \text {-DI - } \\
\text { NITRO- } \\
\text { TOLUENE } \\
\text { TOTAL } \\
\text { (UG/L) }\end{array}$ & $\begin{array}{c}\text { DI-N- } \\
\text { OCTYL } \\
\text { PHTHAL- } \\
\text { ATE } \\
\text { TOTAL } \\
(U G / L)\end{array}$ \\
\hline MAR 1992 & & & & & & & & & & & & \\
\hline $\begin{array}{l}03-04 \\
\text { MAR }\end{array}$ & $<10.0$ & $<20.0$ & $<5.0$ & $<5.0$ & $<5.0$ & $<5.0$ & $<5.0$ & $<30.0$ & $<20.0$ & $<5.0$ & $<5.0$ & $<10.0$ \\
\hline $\begin{array}{l}17 \cdot 17 \\
A P R\end{array}$ & $<10.0$ & $<20.0$ & $<5.0$ & $<5.0$ & $<5.0$ & $<5.0$ & $<5.0$ & $<30.0$ & $<20.0$ & $<5.0$ & $<5.0$ & $<10.0$ \\
\hline${ }_{\text {JUN }}^{17-17}$ & $<10.0$ & $<20.0$ & $<5.0$ & $<5.0$ & $<5.0$ & $<5.0$ & $<5.0$ & $<30.0$ & $<20.0$ & $<5.0$ & $<5.0$ & $<10.0$ \\
\hline $\begin{array}{l}06-06 \\
\text { JUN }\end{array}$ & $<10.0$ & $<20.0$ & $<5.0$ & $<5.0$ & $<5.0$ & $<5.0$ & $<5.0$ & $<30.0$ & $<20.0$ & $<5.0$ & $<5.0$ & $<10.0$ \\
\hline${ }_{\text {JUL }}^{20-20}$ & $<10.0$ & $<20.0$ & $<5.0$ & $<5.0$ & $<5.0$ & $<5.0$ & $<5.0$ & $<30.0$ & $<20.0$ & $<5.0$ & $<5.0$ & $<10.0$ \\
\hline $\begin{array}{l}27-27 \\
\text { SEP }\end{array}$ & $<10.0$ & $<20.0$ & $<5.0$ & $<5.0$ & $<5.0$ & $<5.0$ & $<5.0$ & $<30.0$ & $<20.0$ & $<5.0$ & $<5.0$ & $<10.0$ \\
\hline $21 \cdot 21$ & $<10.0$ & $<20.0$ & $<5.0$ & $<5.0$ & $<5.0$ & $<5.0$ & $<5.0$ & $<30.0$ & $<20.0$ & $<5.0$ & $<5.0$ & $<10.0$ \\
\hline
\end{tabular}


Table 3. Water-quality data for storm-sewer outfall stations, Dallas, Texas, 1992-93-Continued 08055590 - JOE'S CREEK OUTFALL AT DENTON DRIVE, DALLAS, TX (WY 1992)-Continued

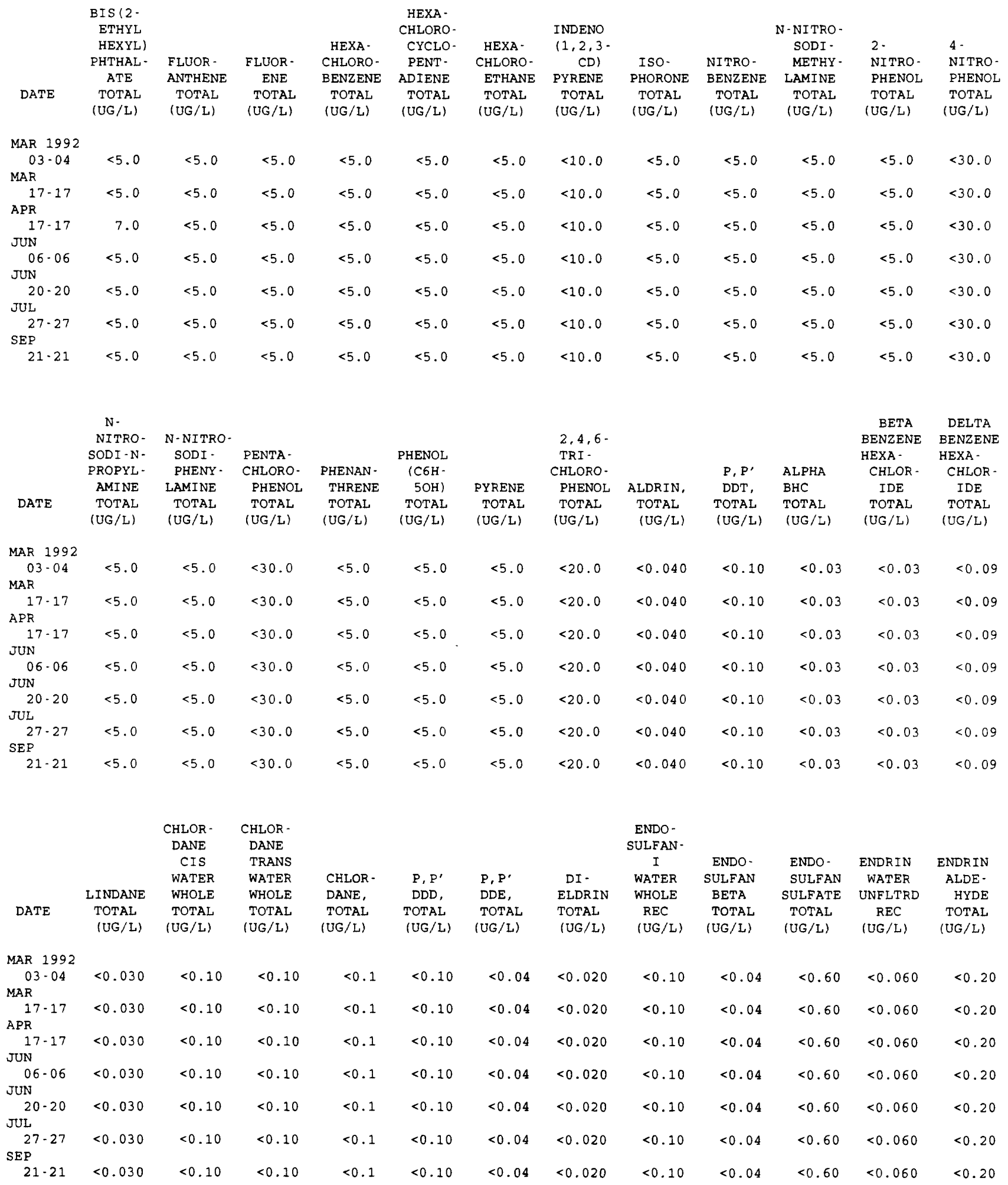


Table 3. Water-quality data for storm-sewer outfall stations, Dallas, Texas, 1992-93-Continued

08055590 - JOE'S CREEK OUTFALL AT DENTON DRIVE, DALLAS, TX (WY 1992)-Continued

\begin{tabular}{|c|c|c|c|c|c|c|c|c|c|c|c|}
\hline & & HEPTA - & & AROCLOR & AROCLOR & AROCLOR & AROCLOR & AROCLOR & AROCLOR & AROCLOR & \\
\hline & HEPTA - & CHLOR & TOX - & 1016 & 1221 & 1232 & 1242 & 1248 & 1254 & 1260 & DI - \\
\hline DATE & $\begin{array}{l}\text { CHLOR, } \\
\text { TOTAL } \\
\text { (UG/L) }\end{array}$ & $\begin{array}{c}\text { EPOXIDE } \\
\text { TOTAL } \\
\text { (UG/L) }\end{array}$ & $\begin{array}{c}\text { APHENE, } \\
\text { TOTAL } \\
\text { (UG/L) }\end{array}$ & $\begin{array}{c}\text { PCB } \\
\text { TOTAL } \\
\text { (UG/L) }\end{array}$ & $\begin{array}{c}\text { PCB } \\
\text { TOTAL } \\
(\mathrm{UG} / \mathrm{L})\end{array}$ & $\begin{array}{c}\text { PCB } \\
\text { TOTAL } \\
\text { (UG/L) }\end{array}$ & $\begin{array}{c}\text { PCB } \\
\text { TOTAL } \\
\text { (UG/L) }\end{array}$ & $\begin{array}{c}\text { PCB } \\
\text { TOTAL } \\
\text { (UG/L) }\end{array}$ & $\begin{array}{c}\text { PCB } \\
\text { TOTAL } \\
\text { (UG/L) }\end{array}$ & $\begin{array}{c}\text { PCB } \\
\text { TOTAL } \\
\text { (UG/L) }\end{array}$ & $\begin{array}{c}\text { AZINON, } \\
\text { TOTAL } \\
\text { (UG/L) }\end{array}$ \\
\hline
\end{tabular}

\begin{tabular}{|c|c|c|c|c|c|c|c|c|c|c|c|}
\hline \multicolumn{12}{|l|}{ MAR 1992} \\
\hline $03-04$ & $<0.030$ & $<0.80$ & $<2$ & $<0.1$ & $<1.0$ & $<0.1$ & $<0.1$ & $<0.1$ & $<0.1$ & $<0.1$ & 0.03 \\
\hline \multicolumn{12}{|l|}{ MAR } \\
\hline $17-17$ & $<0.030$ & $<0.80$ & $<2$ & $<0.1$ & $<1.0$ & $<0.1$ & $<0.1$ & $<0.1$ & $<0.1$ & $<0.1$ & 0.20 \\
\hline \multicolumn{12}{|l|}{ APR } \\
\hline $17-17$ & $<0.030$ & $<0.80$ & $<2$ & $<0.1$ & $<1.0$ & $<0.1$ & $<0.1$ & $<0.1$ & $<0.1$ & $<0.1$ & $<0.10$ \\
\hline \multicolumn{12}{|l|}{ JUN } \\
\hline $06-06$ & $<0.030$ & $<0.80$ & $<2$ & $<0.1$ & $<1.0$ & $<0.1$ & $<0.1$ & $<0.1$ & $<0.1$ & $<0.1$ & $<0.10$ \\
\hline \multicolumn{12}{|l|}{ JUN } \\
\hline $20-20$ & $<0.030$ & $<0.80$ & $<2$ & $<0.1$ & $<1.0$ & $<0.1$ & $<0.1$ & $<0.1$ & $<0.1$ & $<0.1$ & $<0.10$ \\
\hline \multicolumn{12}{|l|}{ JUL } \\
\hline $27-27$ & $<0.030$ & $<0.80$ & $<2$ & $<0.1$ & $<1.0$ & $<0.1$ & $<0.1$ & $<0.1$ & $<0.1$ & $<0.1$ & $<0.10$ \\
\hline \multicolumn{12}{|l|}{ SEP } \\
\hline $21-21$ & $<0.030$ & $<0.80$ & $<2$ & $<0.1$ & $<1.0$ & $<0.1$ & $<0.1$ & $<0.1$ & $<0.1$ & $<0.1$ & $<0.10$ \\
\hline
\end{tabular}


Table 3. Water-quality data for storm-sewer outfall stations, Dallas, Texas, 1992-93-Continued

08056390 - BASTILLE STREET OUTFALL AT LA REUNION PARKWAY, DALLAS, TX (WY 1992)

\begin{tabular}{|c|c|c|c|c|c|c|c|c|c|c|c|c|}
\hline DATE & TIME & $\begin{array}{l}\text { PRECIP - } \\
\text { ITATION } \\
\text { TOTAL } \\
\text { INCHES/ } \\
\text { STORM }\end{array}$ & $\begin{array}{l}\text { ELAPSED } \\
\text { TIME } \\
\text { OF } \\
\text { STORM } \\
\text { (HOURS) }\end{array}$ & $\begin{array}{l}\text { STORM } \\
\text { WATER } \\
\text { FLOW } \\
\text { (MGD) }\end{array}$ & $\begin{array}{l}\text { SPE- } \\
\text { CIFIC } \\
\text { CON- } \\
\text { DUCT- } \\
\text { ANCE } \\
\text { (US/CM) }\end{array}$ & $\begin{array}{c}\text { SPE - } \\
\text { CIFIC } \\
\text { CON- } \\
\text { DUCT - } \\
\text { ANCE } \\
\text { LAB } \\
\text { (US/CM) }\end{array}$ & $\begin{array}{c}\text { PH } \\
\text { WATER } \\
\text { WHOLE } \\
\text { FIELD } \\
\text { (STAND. } \\
\text { ARD } \\
\text { UNITS) }\end{array}$ & $\begin{array}{c}\text { PH } \\
\text { WATER } \\
\text { WHOLE } \\
\text { LAB } \\
\text { (STAND- } \\
\text { ARD } \\
\text { UNITS) }\end{array}$ & $\begin{array}{c}\text { TEMPER - } \\
\text { ATURE } \\
\text { WATER } \\
\text { (DEG C) }\end{array}$ & $\begin{array}{c}\text { OXYGEN } \\
\text { DEMAND. } \\
\text { CHEM- } \\
\text { ICAL } \\
\text { (HIGH } \\
\text { LEVEL) } \\
\text { (MG/L) }\end{array}$ & $\begin{array}{l}\text { OXYGEN } \\
\text { DEMAND, } \\
\text { BIO- } \\
\text { CHEM- } \\
\text { ICAL, } \\
5 \text { DAY } \\
(M G / L)\end{array}$ & $\begin{array}{l}\text { COLI. } \\
\text { FORM, } \\
\text { FECAL, } \\
0.7 \\
\text { UM-MF } \\
\text { (COLS.) } \\
100 \mathrm{ML)}\end{array}$ \\
\hline MAR 1992 & & & & & & & & & & & & \\
\hline $\begin{array}{l}03-04 \\
\text { MAR }\end{array}$ & 2031 & 0.58 & 5.0 & 0.70 & 131 & 221 & 8.6 & 7.9 & 15.5 & 65 & 8.6 & 1200 \\
\hline $\begin{array}{l}09.09 \\
\text { APR }\end{array}$ & 0145 & 0.56 & 1.9 & 0.64 & 234 & 136 & 9.0 & 8.2 & 17.0 & 30 & 3.9 & 2500 \\
\hline $\begin{array}{l}19-19 \\
\text { MAY }\end{array}$ & 1212 & 0.25 & 7.3 & 0.27 & 124 & $\cdots$ & 8.3 & $\cdots$ & 18.5 & $\cdots$ & 8.4 & 2400 \\
\hline $\begin{array}{l}14-14 \\
\text { JUN }\end{array}$ & 1235 & 0.49 & 1.9 & 0.56 & 402 & 146 & 8.0 & 8.2 & 23.5 & 56 & 6.3 & 19000 \\
\hline $\begin{array}{l}01-01 \\
\text { JUN }\end{array}$ & 2000 & 0.90 & 4.0 & 1.1 & 232 & 120 & 8.0 & 7.9 & 22.5 & 43 & 3.6 & 22000 \\
\hline $\begin{array}{l}06.06 \\
\text { JUL }\end{array}$ & 0250 & 0.49 & 4.7 & 0.63 & 270 & 119 & 8.4 & 7.6 & 30.0 & 25 & 4.8 & 13000 \\
\hline $27 \cdot 27$ & 1437 & 1.2 & 1.4 & 1.2 & 91 & 144 & 8.3 & 7.6 & 25.0 & 33 & 6.8 & 8000 \\
\hline
\end{tabular}

\begin{tabular}{|c|c|c|c|c|c|c|c|c|c|c|c|c|}
\hline & $\begin{array}{r}\text { STREP - } \\
\text { TOCOCCI }\end{array}$ & HARD - & $\begin{array}{l}\text { HARD - } \\
\text { NESS }\end{array}$ & $\begin{array}{l}\text { ALKA - } \\
\text { LINITY }\end{array}$ & $\begin{array}{l}\text { SOLIDS, } \\
\text { SUM OF }\end{array}$ & $\begin{array}{l}\text { RESIDUE } \\
\text { TOTAL }\end{array}$ & $\begin{array}{l}\text { SOLIDS, } \\
\text { RESIDUE }\end{array}$ & & MAGNE - & & & SODIUM \\
\hline & FECAL, & NESS & NONCARB & WAT DIS & CONSTI - & AT 105 & $\mathrm{AT} 180$ & CALCIUM & SIUM, & SODIUM, & & $A D-$ \\
\hline & $\begin{array}{l}\text { KF AGAR } \\
\text { (COLS. }\end{array}$ & $\begin{array}{l}\text { TOTAL } \\
\text { (MG/L }\end{array}$ & $\begin{array}{l}\text { DISSOLV } \\
\text { FLD, AS }\end{array}$ & $\begin{array}{l}\text { FIX END } \\
\text { FIELD }\end{array}$ & $\begin{array}{c}\text { TUENTS, } \\
\text { DIS - }\end{array}$ & $\begin{array}{l}\text { DEG. C, } \\
\text { SUS - }\end{array}$ & $\begin{array}{l}\text { DEG. C } \\
\text { DIS - }\end{array}$ & $\begin{array}{l}\text { DIS - } \\
\text { SOLVED }\end{array}$ & $\begin{array}{l}\text { DIS - } \\
\text { SOLVED }\end{array}$ & $\begin{array}{l}\text { DIS - } \\
\text { SOLVED }\end{array}$ & & $\begin{array}{l}\text { SORP - } \\
\text { TION }\end{array}$ \\
\hline $\mathrm{TE}$ & $\begin{array}{c}\text { PER } \\
100 \mathrm{ML})\end{array}$ & $\begin{array}{c}\mathrm{AS} \\
\mathrm{CACO} 31\end{array}$ & $\begin{array}{c}\mathrm{CACO} 3 \\
(\mathrm{MG} / \mathrm{L})\end{array}$ & $\begin{array}{l}\mathrm{CACO} 3 \\
(\mathrm{MG} / \mathrm{L})\end{array}$ & $\begin{array}{l}\text { SOLVED } \\
\text { (MG/L) }\end{array}$ & $\begin{array}{l}\text { PENDED } \\
(M G / L)\end{array}$ & $\begin{array}{l}\text { SOLVED } \\
(M G / L)\end{array}$ & $\begin{array}{l}\text { (MG/L } \\
\text { AS CA) }\end{array}$ & $\begin{array}{l}\text { (MG/L } \\
\text { AS MG) }\end{array}$ & $\begin{array}{l}\text { (MG/L } \\
\text { AS NA) }\end{array}$ & $\begin{array}{r}\text { SODIUM } \\
\text { PERCENT }\end{array}$ & RATIO \\
\hline
\end{tabular}

\begin{tabular}{|c|c|c|c|c|c|c|c|c|c|c|c|c|}
\hline \multicolumn{13}{|l|}{ MAR 1992} \\
\hline $03-04$ & 7800 & 48 & 13 & 35 & 100 & 191 & 84 & 18 & 0.68 & 7.1 & 23 & 0.4 \\
\hline \multicolumn{13}{|l|}{ MAR } \\
\hline 09.09 & 17000 & 37 & 11 & 26 & 64 & 48 & 63 & 14 & 0.44 & 4.2 & 19 & 0.3 \\
\hline \multicolumn{13}{|l|}{ APR } \\
\hline $19-19$ & $\cdots$ & $\cdots$ & $\cdots$ & 34 & -- & - & $\cdots$ & $\cdots$ & -- & - & - & $\cdots$ \\
\hline \multicolumn{13}{|l|}{ MAY } \\
\hline $14-14$ & 20000 & 34 & $\cdots$ & E28 & 61 & 68 & 94 & 13 & 0.35 & 2.4 & 13 & 0.2 \\
\hline \multicolumn{13}{|l|}{ JUN } \\
\hline $01-01$ & 32000 & 39 & 10 & 29 & 68 & 189 & 69 & 15 & 0.49 & 3.2 & 14 & 0.2 \\
\hline \multicolumn{13}{|l|}{ JUN } \\
\hline 06.06 & 8900 & 37 & 8 & 29 & 59 & 36 & 58 & 14 & 0.42 & 3.7 & 17 & 0.3 \\
\hline \multicolumn{13}{|l|}{ JUL } \\
\hline $27-27$ & 1600 & 38 & 15 & 23 & 74 & 177 & 72 & 14 & 0.73 & 4.0 & 18 & 0.3 \\
\hline
\end{tabular}

\begin{tabular}{|c|c|c|c|c|c|c|c|c|c|c|c|c|}
\hline $\mathrm{TE}$ & $\begin{array}{l}\text { POTAS - } \\
\text { SIUM, } \\
\text { DIS - } \\
\text { SOLVED } \\
\text { (MG/L }\end{array}$ & $\begin{array}{l}\text { SULFATE } \\
\text { DIS - } \\
\text { SOLVED } \\
\text { (MG/L }\end{array}$ & $\begin{array}{l}\text { CHLO - } \\
\text { RIDE, } \\
\text { DIS - } \\
\text { SOLVED } \\
\text { (MG/L }\end{array}$ & $\begin{array}{l}\text { NITRO- } \\
\text { GEN, } \\
\text { NITRITE } \\
\text { TOTAL } \\
\text { (MG/L }\end{array}$ & $\begin{array}{l}\text { NITRO- } \\
\text { GEN, } \\
\text { NO2+NO3 } \\
\text { TOTAL } \\
\text { (MG/L }\end{array}$ & $\begin{array}{l}\text { NITRO- } \\
\text { GEN, } \\
\text { AMMONIA } \\
\text { TOTAL } \\
\text { (MG/L }\end{array}$ & $\begin{array}{l}\text { GEN, AM- } \\
\text { MONIA + } \\
\text { ORGANIC } \\
\text { TOTAL } \\
\text { (MG/L }\end{array}$ & $\begin{array}{r}\text { PHOS - } \\
\text { PHORUS } \\
\text { TOTAL } \\
\text { (MG / L }\end{array}$ & $\begin{array}{l}\text { PHOS - } \\
\text { PHORUS } \\
\text { DIS - } \\
\text { SOLVED } \\
\text { (MG/L }\end{array}$ & $\begin{array}{l}\text { ANT IMONY } \\
\text { TOTAL } \\
\text { EPA } \\
\text { (UG/L }\end{array}$ & $\begin{array}{c}\text { ARSENIC } \\
\text { TOTAL } \\
\text { (UG/L }\end{array}$ & $\begin{array}{l}\text { LIUM, } \\
\text { TOTAL } \\
\text { RECOV - } \\
\text { ERABLE } \\
\text { (UG/L }\end{array}$ \\
\hline & AS $K$ ) & AS SO4) & AS CL) & AS N) & AS N) & AS N) & AS N) & AS P) & AS P) & AS SB) & AS AS) & AS $\mathrm{BE}$ ) \\
\hline
\end{tabular}

\begin{tabular}{|c|c|c|c|c|c|c|c|c|c|c|c|c|}
\hline \multicolumn{13}{|l|}{ MAR 1992} \\
\hline $03-04$ & 3.2 & 23 & 3.5 & 0.120 & 0.730 & 0.120 & 0.50 & 0.110 & 0.040 & $<10.0$ & 2 & $<10$ \\
\hline \multicolumn{13}{|l|}{ MAR } \\
\hline $09-09$ & 1.6 & 16 & 2.2 & 0.060 & 0.480 & 0.110 & 0.70 & 0.110 & 0.030 & $<20.0$ & 1 & $<10$ \\
\hline \multicolumn{13}{|l|}{ APR } \\
\hline $19 \cdot 19$ & $\cdots$ & $\cdots$ & $\cdots$ & 0.110 & 1.40 & 0.140 & 1.1 & 0.130 & 0.040 & $<20.0$ & $\cdots$ & $<10$ \\
\hline \multicolumn{13}{|l|}{ MAY } \\
\hline $14 \cdot 14$ & 1.7 & 9.8 & 2.2 & 0.050 & 0.700 & 0.210 & 0.40 & 0.060 & 0.060 & $<10.0$ & 2 & $<10$ \\
\hline \multicolumn{13}{|l|}{ JUN } \\
\hline 01.01 & 1.7 & 15 & 2.2 & 0.070 & 0.660 & 0.150 & 0.60 & 0.130 & 0.040 & $<10.0$ & 1 & $<10$ \\
\hline \multicolumn{13}{|l|}{ JUN } \\
\hline $06-06$ & 1.7 & 13 & 2.6 & 0.060 & 0.680 & 0.140 & 0.50 & 0.060 & 0.050 & $<20.0$ & 1 & $<10$ \\
\hline \multicolumn{13}{|l|}{ JUL } \\
\hline $27-27$ & 1.3 & 20 & 4.0 & 0.030 & 0.490 & 0.180 & 0.50 & 0.050 & 0.050 & $<10.0$ & 1 & $<10$ \\
\hline
\end{tabular}


Table 3. Water-quality data for storm-sewer outfall stations, Dallas, Texas, 1992-93-Continued

08056390 - BASTILLE STREET OUTFALL AT LA REUNION PARKWAY, DALLAS, TX (WY 1992)-Continued

\begin{tabular}{|c|c|c|c|c|c|c|c|c|c|c|c|c|}
\hline DATE & $\begin{array}{l}\text { CADMIUM } \\
\text { TOTAL } \\
\text { RECOV- } \\
\text { ERABLE } \\
\text { (UG/L }\end{array}$ & $\begin{array}{l}\text { CHRO- } \\
\text { MIUM, } \\
\text { TOTAL } \\
\text { RECOV- } \\
\text { ERABLE } \\
\text { (UG/L }\end{array}$ & $\begin{array}{l}\text { COPPER, } \\
\text { TOTAL } \\
\text { RECOV- } \\
\text { ERABLE } \\
\text { /UG/L }\end{array}$ & $\begin{array}{c}\text { CYANIDE } \\
\text { TOTAL } \\
\text { EPA } \\
\text { (MG/L }\end{array}$ & $\begin{array}{l}\text { CYANIDE } \\
\text { TOTAL } \\
\text { (MG/L }\end{array}$ & $\begin{array}{l}\text { LEAD, } \\
\text { TOTAL } \\
\text { RECOV - } \\
\text { ERABLE } \\
\text { /UG/L }\end{array}$ & $\begin{array}{l}\text { MERCURY } \\
\text { TOTAL } \\
\text { RECOV- } \\
\text { ERABLE } \\
\text { (UG/L }\end{array}$ & $\begin{array}{c}\text { NICKEL, } \\
\text { TOTAL } \\
\text { RECOV- } \\
\text { ERABLE } \\
\text { /UG/L }\end{array}$ & $\begin{array}{l}\text { SELE - } \\
\text { NIUM, } \\
\text { TOTAL } \\
\text { (UG/L }\end{array}$ & $\begin{array}{l}\text { SILVER, } \\
\text { TOTAL } \\
\text { RECOV- } \\
\text { ERABLE } \\
\text { (UG/L }\end{array}$ & $\begin{array}{l}\text { SILVER, } \\
\text { TOTAL } \\
\text { RECOV- } \\
\text { ERABLE } \\
\text { EPA } \\
\text { (UG/L }\end{array}$ & $\begin{array}{l}\text { THAL - } \\
\text { LIUM, } \\
\text { TOTAL } \\
\text { (UG/L }\end{array}$ \\
\hline & AS CD) & AS CR) & AS CU) & AS $C N$ ) & AS CN) & AS $P B)$ & AS HG) & AS NI) & AS SE) & AS AG) & AS AG) & AS TL\} \\
\hline
\end{tabular}

\begin{tabular}{|c|c|c|c|c|c|c|c|c|c|c|c|c|}
\hline $\begin{array}{c}\text { MAR } 1992 \\
03-04\end{array}$ & $<1$ & 9 & 16 & $<0.010$ & $<0.010$ & 28 & $<0.10$ & 8 & $<2$ & $<1$ & $<1.00$ & $<50$ \\
\hline \multicolumn{13}{|l|}{ MAR } \\
\hline $09-09$ & $<1$ & 4 & 10 & $<0.010$ & $<0.010$ & 14 & $<0.10$ & 4 & $<2$ & $<1$ & $<1.00$ & $<50$ \\
\hline \multicolumn{13}{|l|}{ APR } \\
\hline $19-19$ & $<1$ & $\cdots$ & $\cdots$ & 0.540 & $<0.010$ & $\cdots$ & $<0.10$ & $\cdots$ & $<2$ & $<1$ & $<1.00$ & $<5$ \\
\hline \multicolumn{13}{|l|}{ MAY } \\
\hline $14-14$ & $<1$ & 3 & 23 & $<10.0$ & $<0.010$ & 23 & $<0.10$ & 5 & $<2$ & $<1$ & $<1.00$ & $<5$ \\
\hline \multicolumn{13}{|l|}{ JUN } \\
\hline 01.01 & $<1$ & 20 & 21 & $<10.0$ & $\cdots$ & 44 & $<0.10$ & 35 & $<2$ & $<1$ & $<1.00$ & $<5$ \\
\hline \multicolumn{13}{|l|}{ JUN } \\
\hline 06.06 & $<1$ & 2 & 13 & $<10.0$ & $<0.010$ & 12 & 0.20 & 2 & $<2$ & $<1$ & $<1.00$ & $<5$ \\
\hline \multicolumn{13}{|l|}{ JUL } \\
\hline $27-27$ & $<1$ & 8 & 30 & $<10.0$ & $<0.010$ & 47 & $<0.10$ & 5 & $<1$ & $<1$ & $<0.500$ & $<5$ \\
\hline
\end{tabular}

\begin{tabular}{|c|c|c|c|c|c|c|c|c|c|c|c|c|}
\hline & & & OIL AND & & & & & & & & BENZENE & BENZENE \\
\hline & ZINC, & & GREASE, & & & & & BROMO - & & BENZENE & SEC & TERT - \\
\hline & TOTAL & CARBON, & TOTAL & & & & & BENZENE & & N-BUTYL & BUTYL - & BUTYL - \\
\hline & RECOV - & ORGANIC & RECOV. & & ACRO - & ACRYLO - & & WATER, & BROMO - & WATER & WATER & WATER \\
\hline & ERABLE & TOTAL & GRAVI - & PHENOLS & LEIN & NITRILE & BENZENE & WHOLE, & FORM & UNFLTRD & UNFLTRD & UNFLTRD \\
\hline DATE & $\begin{array}{l}\text { (UG/L } \\
\text { AS } \quad \mathrm{ZN} \text { ) }\end{array}$ & $\begin{array}{l}(\mathrm{MG} / \mathrm{L} \\
\mathrm{AS} C)\end{array}$ & $\begin{array}{c}\text { METRIC } \\
(\mathrm{MG} / \mathrm{L})\end{array}$ & $\begin{array}{c}\text { TOTAL } \\
\text { (UG/L) }\end{array}$ & $\begin{array}{l}\text { TOTAL } \\
\text { (UG/L) }\end{array}$ & $\begin{array}{l}\text { TOTAL } \\
\text { (UG/L) }\end{array}$ & $\begin{array}{l}\text { TOTAL } \\
\text { (UG/L) }\end{array}$ & $\begin{array}{l}\text { TOTAL } \\
\text { (UG/L) }\end{array}$ & $\begin{array}{l}\text { TOTAL } \\
\text { (UG/L) }\end{array}$ & $\begin{array}{c}\text { REC } \\
\text { (UG/L) }\end{array}$ & $\begin{array}{c}\text { REC } \\
(U G / L)\end{array}$ & $\begin{array}{c}\text { REC } \\
(U G / L)\end{array}$ \\
\hline
\end{tabular}

\begin{tabular}{|c|c|c|c|c|c|c|c|c|c|c|c|c|}
\hline MAR 1992 & & & & & & & & & & & & \\
\hline $03-04$ & 110 & 15 & $<1$ & 2 & $<20$ & $<20$ & $<0.2$ & $<0.2$ & $<0.2$ & $<0.20$ & $<0.20$ & $<0.20$ \\
\hline MAR & & & & & & & & & & & & \\
\hline $09-09$ & 50 & 7.6 & $<1$ & 3 & $<20$ & $<20$ & $<0.2$ & $<0.2$ & $<0.2$ & $<0.20$ & $<0.20$ & $<0.20$ \\
\hline$A P R$ & & & & & & & & & & & & \\
\hline $19-19$ & $\cdots$ & 24 & $<1$ & 3 & $<20$ & $<20$ & $<0.2$ & $<0.2$ & $<0.2$ & $<0.20$ & $<0.20$ & $<0.20$ \\
\hline MAY & & & & & & & & & & & & \\
\hline $14-14$ & 120 & 13 & $<1$ & 7 & $<20$ & $<20$ & $<0.2$ & $<0.2$ & $<0.2$ & $<0.20$ & $<0.20$ & $<0.20$ \\
\hline JUN & & & & & & & & & & & & \\
\hline $01-01$ & 90 & 9.2 & $<1$ & 4 & $<20$ & $<20$ & $<0.2$ & $<0.2$ & $<0.2$ & $<0.20$ & $<0.20$ & $<0.20$ \\
\hline JUN & & & & & & & & & & & & \\
\hline 06.06 & 50 & 8.0 & $<1$ & 4 & $<20$ & $<20$ & $<0.2$ & $<0.2$ & $<0.2$ & $<0.20$ & $<0.20$ & $<0.20$ \\
\hline JUL & & & & & & & & & & & & \\
\hline $27-27$ & 130 & 11 & $<1$ & 6 & $<20$ & $<20$ & $<0.2$ & $<0.2$ & $<0.2$ & $<0.20$ & $<0.20$ & $<0.20$ \\
\hline
\end{tabular}

\begin{tabular}{|c|c|c|c|c|c|c|c|c|c|c|c|c|}
\hline & & & & & 2 . & & & 0 . & & DIBROMO & DI - & $1,2-$ \\
\hline & CARBON- & & CHLORO - & & CHLORO- & & & CHLORO - & TOLUENE & CHLORO- & BROMO - & DIBROMO \\
\hline & TETRA - & & DI - & & ETHYL - & & METHYL - & TOLUENE & P-CHLOR & PROPANE & METHANE & ETHANE \\
\hline & CHLO - & CHLORO- & BROMO - & CHLORO - & VINYL - & CHLORO - & CHLO - & WATER & WATER & WATER & WATER & WATER \\
\hline & RIDE & BENZENE & METHANE & ETHANE & ETHER & FORM & RIDE & WHOLE & UNFLTRD & WHOLE & WHOLE & WHOLE \\
\hline DATE & $\begin{array}{l}\text { TOTAL } \\
\text { (UG/L) }\end{array}$ & $\begin{array}{l}\text { TOTAL } \\
\text { (UG/L) }\end{array}$ & $\begin{array}{l}\text { TOTAL } \\
\text { (UG/L) }\end{array}$ & $\begin{array}{l}\text { TOTAL } \\
\text { (UG/L) }\end{array}$ & $\begin{array}{l}\text { TOTAL } \\
\text { (UG/L) }\end{array}$ & $\begin{array}{l}\text { TOTAL } \\
\text { (UG/L) }\end{array}$ & $\begin{array}{l}\text { TOTAL } \\
\text { (UG/L) }\end{array}$ & $\begin{array}{l}\text { TOTAL } \\
\text { (UG/L) }\end{array}$ & $\begin{array}{c}\text { REC } \\
(U G / L)\end{array}$ & $\begin{array}{l}\text { TOT.REC } \\
\text { (UG/L) }\end{array}$ & $\begin{array}{l}\text { RECOVER } \\
\text { (UG/L) }\end{array}$ & $\begin{array}{l}\text { TOTAL } \\
\text { (UG/L) }\end{array}$ \\
\hline
\end{tabular}

\begin{tabular}{|c|c|c|c|c|c|c|c|c|c|c|c|c|}
\hline \multicolumn{13}{|l|}{ MAR 1992} \\
\hline $03-04$ & $<0.2$ & $<0.20$ & $<0.2$ & $<0.2$ & $<1.0$ & $<0.2$ & $<0.2$ & $<0.2$ & $<0.20$ & $<1.0$ & $<0.2$ & $<0.2$ \\
\hline \multicolumn{13}{|l|}{ MAR } \\
\hline 09.09 & $<0.2$ & $<0.20$ & $<0.2$ & $<0.2$ & $<1.0$ & $<0.2$ & $<0.2$ & $<0.2$ & $<0.20$ & $<1.0$ & $<0.2$ & $<0.2$ \\
\hline \multicolumn{13}{|l|}{ APR } \\
\hline $19 \cdot 19$ & $<0.2$ & $<0.20$ & $<0.2$ & $<0.2$ & $<1.0$ & $<0.2$ & $<0.2$ & $<0.2$ & $<0.20$ & $<1.0$ & $<0.2$ & $<0.2$ \\
\hline \multicolumn{13}{|l|}{ MAY } \\
\hline $14-14$ & $<0.2$ & $<0.20$ & $<0.2$ & $<0.2$ & $<1.0$ & $<0.2$ & $<0.2$ & $<0.2$ & $<0.20$ & $<1.0$ & $<0.2$ & $<0.2$ \\
\hline \multicolumn{13}{|l|}{ JUN } \\
\hline $01-01$ & $<0.2$ & $<0.20$ & $<0.2$ & $<0.2$ & $<1.0$ & $<0.2$ & $<0.2$ & $<0.2$ & $<0.20$ & $<1.0$ & $<0.2$ & $<0.2$ \\
\hline \multicolumn{13}{|l|}{ JUN } \\
\hline $06-06$ & $<0.2$ & $<0.20$ & $<0.2$ & $<0.2$ & $<1.0$ & $<0.2$ & $<0.2$ & $<0.2$ & $<0.20$ & $<1.0$ & $<0.2$ & $<0.2$ \\
\hline \multicolumn{13}{|l|}{ JUL } \\
\hline $27 \cdot 27$ & $<0.2$ & $<0.20$ & $<0.2$ & $<0.2$ & $<1.0$ & $<0.2$ & $<0.2$ & $<0.2$ & $<0.20$ & $<1.0$ & $<0.2$ & $<0.2$ \\
\hline
\end{tabular}


Table 3. Water-quality data for storm-sewer outfall stations, Dallas, Texas, 1992-93-Continued

08056390 - BASTILLE STREET OUTFALL AT LA REUNION PARKWAY, DALLAS, TX (WY 1992)-ContinUed

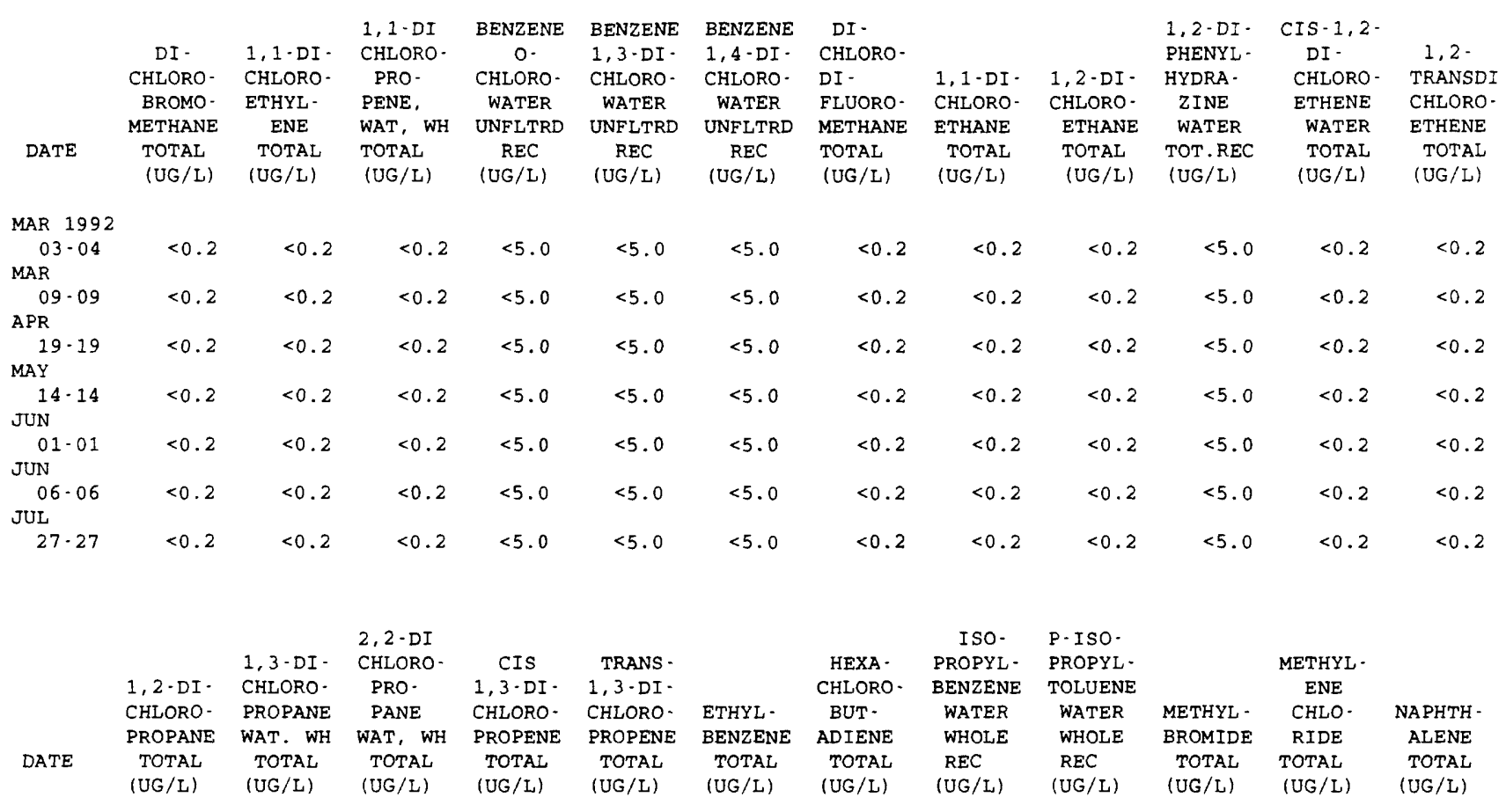

MAR 1992

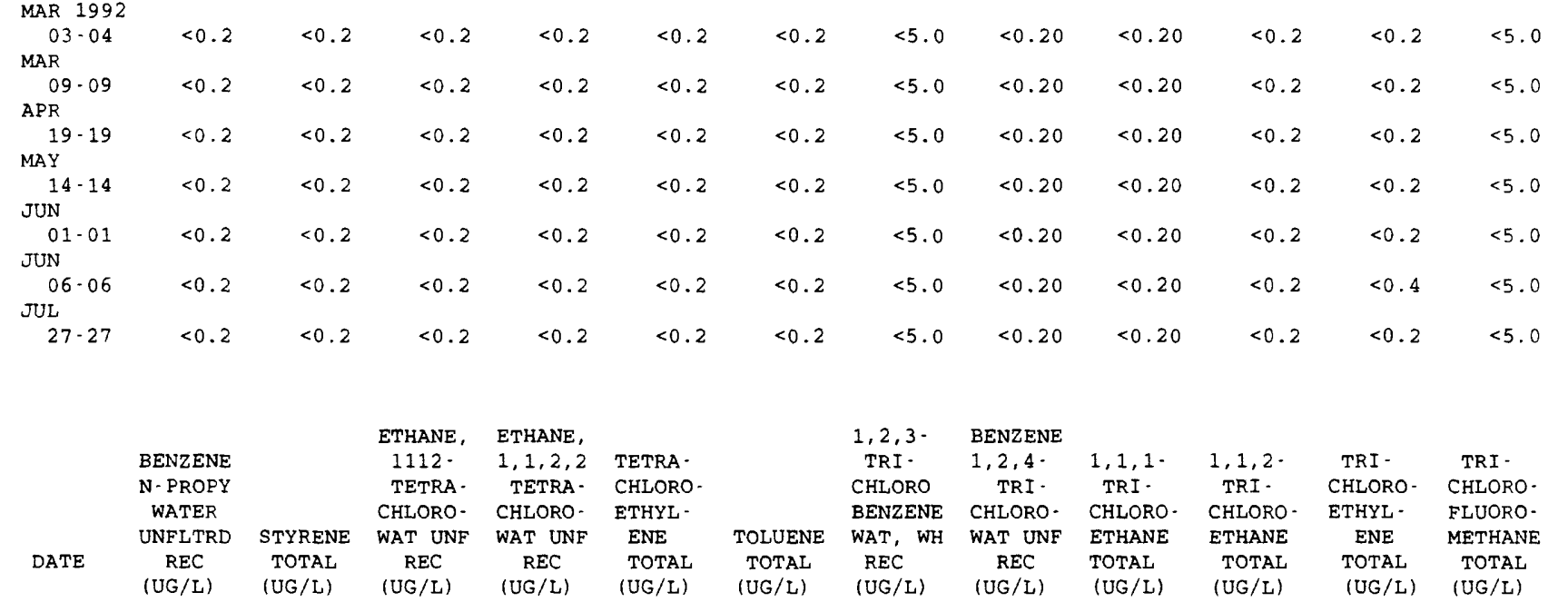

\begin{tabular}{|c|c|c|c|c|c|c|c|c|c|c|c|c|}
\hline \multicolumn{13}{|l|}{ MAR 1992} \\
\hline $03-04$ & $<0.20$ & $<0.2$ & $<0.2$ & $<0.2$ & $<0.2$ & $<0.2$ & $<0.20$ & $<5.0$ & $<0.2$ & $<0.2$ & $<0.2$ & $<0.2$ \\
\hline \multicolumn{13}{|l|}{ MAR } \\
\hline $09-09$ & $<0.20$ & $<0.2$ & $<0.2$ & $<0.2$ & $<0.2$ & $<0.2$ & $<0.20$ & $<5.0$ & $<0.2$ & $<0.2$ & $<0.2$ & $<0.2$ \\
\hline \multicolumn{13}{|l|}{ APR } \\
\hline $19-19$ & $<0.20$ & $<0.2$ & $<0.2$ & $<0.2$ & $<0.2$ & $<0.2$ & $<0.20$ & $<5.0$ & $<0.2$ & $<0.2$ & $<0.2$ & $<0.2$ \\
\hline \multicolumn{13}{|l|}{ MAY } \\
\hline $14 \cdot 14$ & $<0.20$ & $<0.2$ & $<0.2$ & $<0.2$ & $<0.2$ & $<0.2$ & $<0.20$ & $<5.0$ & $<0.2$ & $<0.2$ & $<0.2$ & $<0.2$ \\
\hline \multicolumn{13}{|l|}{ JUN } \\
\hline $01-01$ & $<0.20$ & $<0.2$ & $<0.2$ & $<0.2$ & $<0.2$ & $<0.2$ & $<0.20$ & $<5.0$ & $<0.2$ & $<0.2$ & $<0.2$ & $<0.2$ \\
\hline \multicolumn{13}{|l|}{ JUN } \\
\hline 06.06 & $<0.20$ & $<0.2$ & $<0.2$ & $<0.2$ & $<0.2$ & $<0.2$ & $<0.20$ & $<5.0$ & $<0.2$ & $<0.2$ & $<0.2$ & $<0.2$ \\
\hline \multicolumn{13}{|l|}{ JUL } \\
\hline $27-27$ & $<0.20$ & $<0.2$ & $<0.2$ & $<0.2$ & $<0.2$ & $<0.2$ & $<0.20$ & $<5.0$ & $<0.2$ & $<0.2$ & $<0.2$ & $<0.2$ \\
\hline
\end{tabular}


Table 3. Water-quality data for storm-sewer outfall stations, Dallas, Texas, 1992-93-Continued

08056390 - BASTILLE STREET OUTFALI AT LA REUNION PARKWAY, DALLAS, TX (WY 1992)-Continued

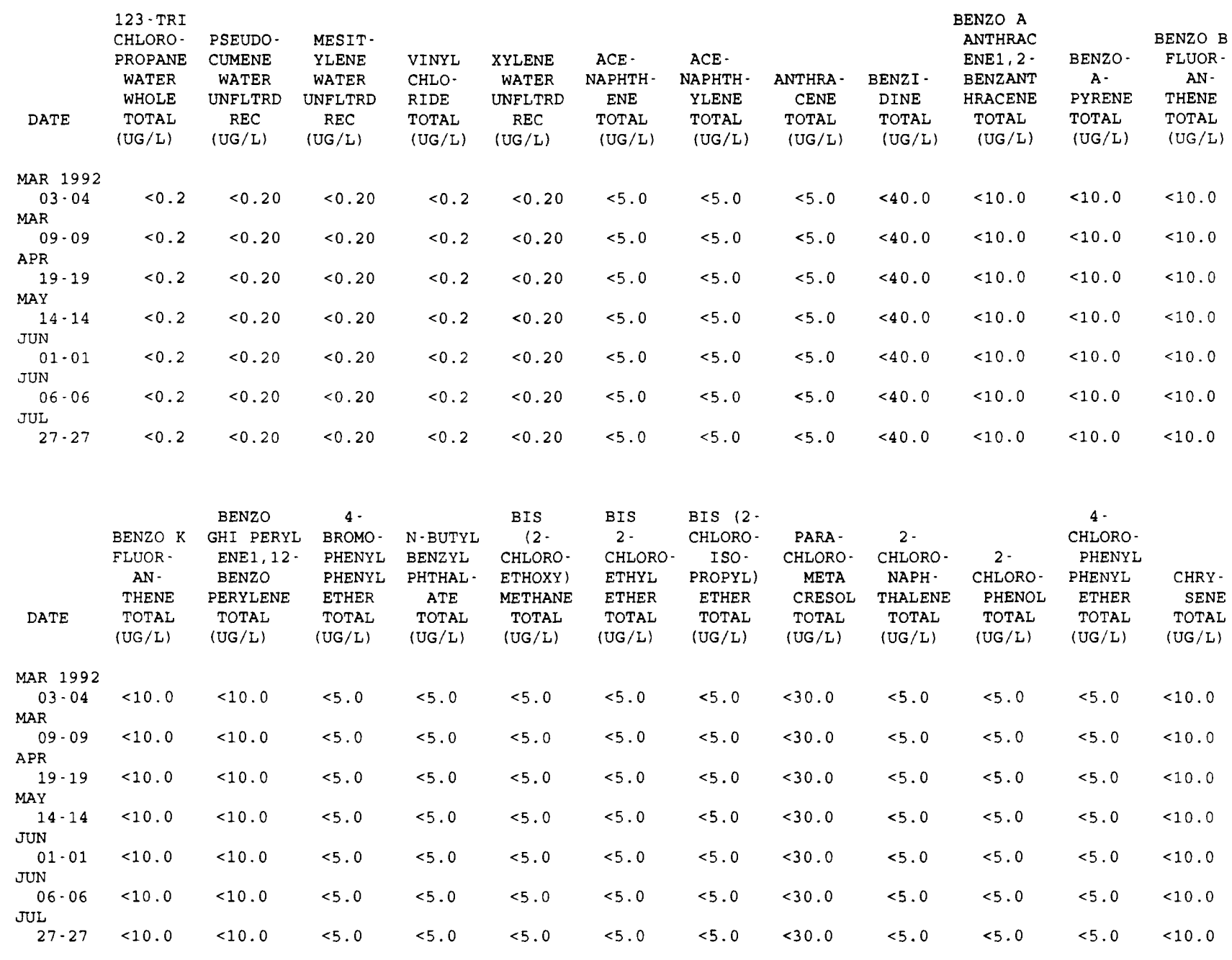

\begin{tabular}{|c|c|c|c|c|c|c|c|c|c|c|c|c|}
\hline DATE & $\begin{array}{c}1,2,5,6- \\
\text { DIBENZ - } \\
\text { ANTHRA - } \\
\text { CENE } \\
\text { TOTAL } \\
\text { (UG/L) }\end{array}$ & $\begin{array}{c}3,3^{\prime}- \\
\text { DI- } \\
\text { CHLORO- } \\
\text { BENZI- } \\
\text { DINE } \\
\text { TOTAL } \\
\text { (UG/L) }\end{array}$ & $\begin{array}{c}2,4 \text {-DI - } \\
\text { CHLORO- } \\
\text { PHENOL } \\
\text { TOTAL } \\
\text { (UG/L) }\end{array}$ & $\begin{array}{c}\text { DIETHYL } \\
\text { PHTHAL - } \\
\text { ATE } \\
\text { TOTAL } \\
\text { (UG/L) }\end{array}$ & $\begin{array}{c}\text { DI - } \\
\text { METHYL } \\
\text { PHTHAL- } \\
\text { ATE } \\
\text { TOTAL } \\
\text { (UG } / L)\end{array}$ & $\begin{array}{c}2,4 \text { - DI - } \\
\text { METHYL - } \\
\text { PHENOL } \\
\text { TOTAL } \\
\text { (UG/L) }\end{array}$ & $\begin{array}{c}\text { DI - N - } \\
\text { BUTYL } \\
\text { PHTHAL- } \\
\text { ATE } \\
\text { TOTAL } \\
(\mathrm{UG} / \mathrm{L})\end{array}$ & $\begin{array}{c}4,6- \\
\text { DINITRO- } \\
\text { ORTHO- } \\
\text { CRESOL } \\
\text { TOTAL } \\
\text { (UG/L) }\end{array}$ & $\begin{array}{c}2,4,- \\
\text { DI- } \\
\text { NITRO- } \\
\text { PHENOL } \\
\text { TOTAL } \\
\text { (UG/L) }\end{array}$ & $\begin{array}{l}2,4 \text {-DI- } \\
\text { NITRO- } \\
\text { TOLUENE } \\
\text { TOTAL } \\
\text { (UG/L) }\end{array}$ & $\begin{array}{l}2,6-D I- \\
\text { NITRO- } \\
\text { TOLUENE } \\
\text { TOTAL } \\
\text { (UG/L) }\end{array}$ & $\begin{array}{c}\text { DI - N - } \\
\text { OCTYL } \\
\text { PHTHAL - } \\
\text { ATE } \\
\text { TOTAL } \\
\text { (UG/L) }\end{array}$ \\
\hline MAR 1992 & & & & & & & & & & & & \\
\hline $\begin{array}{l}03-04 \\
\text { MAR }\end{array}$ & $<10.0$ & $<20.0$ & $<5.0$ & $<5.0$ & $<5.0$ & $<5.0$ & $<5.0$ & $<30.0$ & $<20.0$ & $<5.0$ & $<5.0$ & $<10.0$ \\
\hline $\begin{array}{l}09-09 \\
\text { APR }\end{array}$ & $<10.0$ & $<20.0$ & $<5.0$ & $<5.0$ & $<5.0$ & $<5.0$ & $<5.0$ & $<30.0$ & $<20.0$ & $<5.0$ & $<5.0$ & $<10.0$ \\
\hline${ }_{\text {MAY }}^{19 \cdot 19}$ & $<10.0$ & $<20.0$ & $<5.0$ & $<5.0$ & $<5.0$ & $<5.0$ & $<5.0$ & $<30.0$ & $<20.0$ & $<5.0$ & $<5.0$ & $<10.0$ \\
\hline $\begin{array}{l}14-14 \\
\text { JUN }\end{array}$ & $<10.0$ & $<20.0$ & $<5.0$ & $<5.0$ & $<5.0$ & $<5.0$ & $<5.0$ & $<30.0$ & $<20.0$ & $<5.0$ & $<5.0$ & $<10.0$ \\
\hline $\begin{array}{l}01-01 \\
\text { JUN }\end{array}$ & $<10.0$ & $<20.0$ & $<5.0$ & $<5.0$ & $<5.0$ & $<5.0$ & $<5.0$ & $<30.0$ & $<20.0$ & $<5.0$ & $<5.0$ & $<10.0$ \\
\hline $\begin{array}{l}06.06 \\
\text { JUL }\end{array}$ & $<10.0$ & $<20.0$ & $<5.0$ & $<5.0$ & $<5.0$ & $<5.0$ & $<5.0$ & $<30.0$ & $<20.0$ & $<5.0$ & $<5.0$ & $<10.0$ \\
\hline $27-27$ & $<10.0$ & $<20.0$ & $<5.0$ & $<5.0$ & $<5.0$ & $<5.0$ & $<5.0$ & $<30.0$ & $<20.0$ & $<5.0$ & $<5.0$ & $<10.0$ \\
\hline
\end{tabular}


Table 3. Water-quality data for storm-sewer outfall stations, Dallas, Texas, 1992-93-Continued 08056390 - BASTILLE STREET OUTFALL AT LA REUNION PARKWAY, DALLAS, TX (WY 1992)-Continued

\begin{tabular}{|c|c|c|c|c|c|c|c|c|c|c|c|c|}
\hline & $\begin{array}{c}\text { BIS (2- } \\
\text { ETHYL }\end{array}$ & & & & $\begin{array}{c}\text { HEXA- } \\
\text { CHLORO - }\end{array}$ & & INDENO & & & N-NITRO- & & \\
\hline & HEXYL) & & & HEXA - & CYCLO- & HEXA - & $(1,2,3-$ & & & SODI - & $2-$ & 4- \\
\hline & $\begin{array}{c}\text { PHTHAL - } \\
\text { ATE }\end{array}$ & $\begin{array}{l}\text { FLUOR - } \\
\text { ANTHENE }\end{array}$ & $\begin{array}{c}\text { FLUOR - } \\
\text { ENE }\end{array}$ & $\begin{array}{l}\text { CHLORO - } \\
\text { BENZENE }\end{array}$ & $\begin{array}{c}\text { PENT - } \\
\text { ADIENE }\end{array}$ & $\begin{array}{l}\text { CHLORO- } \\
\text { ETHANE }\end{array}$ & $\begin{array}{r}\text { CD) } \\
\text { PYRENE }\end{array}$ & $\begin{array}{l}\text { I SO- } \\
\text { PHORONE }\end{array}$ & $\begin{array}{l}\text { NITRO- } \\
\text { BENZENE }\end{array}$ & $\begin{array}{l}\text { METHY - } \\
\text { LAMINE }\end{array}$ & $\begin{array}{l}\text { NITRO- } \\
\text { PHENOL }\end{array}$ & $\begin{array}{l}\text { NITRO } \\
\text { PHENO }\end{array}$ \\
\hline & $\begin{array}{l}\text { TOTAL } \\
(U G / L)\end{array}$ & $\begin{array}{l}\text { TOTAL } \\
\text { (UG/L) }\end{array}$ & $\begin{array}{l}\text { TOTAL } \\
\text { (UG/L) }\end{array}$ & $\begin{array}{l}\text { TOTAL } \\
(\mathrm{UG} / \mathrm{L})\end{array}$ & $\begin{array}{l}\text { TOTAL } \\
\text { (UG/L) }\end{array}$ & $\begin{array}{l}\text { TOTAL } \\
(\mathrm{UG} / \mathrm{L})\end{array}$ & $\begin{array}{l}\text { TOTAL } \\
\text { (UG/L) }\end{array}$ & $\begin{array}{l}\text { TOTAL } \\
\text { (UG/L) }\end{array}$ & $\begin{array}{l}\text { TOTAL } \\
\text { (UG/L) }\end{array}$ & $\begin{array}{l}\text { TOTAL } \\
\text { (UG/L) }\end{array}$ & $\begin{array}{l}\text { TOTAL } \\
\text { (UG/L) }\end{array}$ & $\begin{array}{l}\text { TOTAL } \\
(U G / L)\end{array}$ \\
\hline
\end{tabular}

\begin{tabular}{|c|c|c|c|c|c|c|c|c|c|c|c|c|}
\hline \multicolumn{13}{|l|}{ MAR 1992} \\
\hline $03-04$ & $<5.0$ & $<5.0$ & $<5.0$ & $<5.0$ & $<5.0$ & $<5.0$ & $<10.0$ & $<5.0$ & $<5.0$ & $<5.0$ & $<5.0$ & $<30.0$ \\
\hline \multicolumn{13}{|l|}{ MAR } \\
\hline 09.09 & $<5.0$ & $<5.0$ & $<5.0$ & $<5.0$ & $<5.0$ & $<5.0$ & $<10.0$ & $<5.0$ & $<5.0$ & $<5.0$ & $<5.0$ & $<30.0$ \\
\hline \multicolumn{13}{|l|}{ APR } \\
\hline $19 \cdot 19$ & $<5.0$ & $<5.0$ & $<5.0$ & $<5.0$ & $<5.0$ & $<5.0$ & $<10.0$ & $<5.0$ & $<5.0$ & $<5.0$ & $<5.0$ & $<30.0$ \\
\hline \multicolumn{13}{|l|}{ MAY } \\
\hline $14 \cdot 14$ & $<5.0$ & $<5.0$ & $<5.0$ & $<5.0$ & $<5.0$ & $<5.0$ & $<10.0$ & $<5.0$ & $<5.0$ & $<5.0$ & $<5.0$ & $<30.0$ \\
\hline \multicolumn{13}{|l|}{ JUN } \\
\hline 01.01 & $<5.0$ & 14.0 & $<5.0$ & $<5.0$ & $<5.0$ & $<5.0$ & $<10.0$ & $<5.0$ & $<5.0$ & $<5.0$ & $<5.0$ & $<30.0$ \\
\hline \multicolumn{13}{|l|}{ JUN } \\
\hline $06-06$ & $<5.0$ & $<5.0$ & $<5.0$ & $<5.0$ & $<5.0$ & $<5.0$ & $<10.0$ & $<5.0$ & $<5.0$ & $<5.0$ & $<5.0$ & $<30.0$ \\
\hline \multicolumn{13}{|l|}{ JUL } \\
\hline $27-27$ & $<5.0$ & 18.0 & $<5.0$ & $<5.0$ & $<5.0$ & $<5.0$ & $<10.0$ & $<5.0$ & $<5.0$ & $<5.0$ & $<5.0$ & $<30.0$ \\
\hline
\end{tabular}

\begin{tabular}{|c|c|c|c|c|c|c|c|c|c|c|c|c|}
\hline & $\mathrm{N}-$ & & & & & & & & & & BETA & DELTA \\
\hline & NITRO- & N-NITRO- & & & & & $2,4,6=$ & & & & BENZENE & BENZENE \\
\hline & SODI - N- & SODI - & PENTA - & & PHENOL & & TRI - & & & & HEXA - & HEXA- \\
\hline & $\begin{array}{l}\text { PROPYL - } \\
\text { AMINE }\end{array}$ & $\begin{array}{l}\text { PHENY - } \\
\text { LAMINE }\end{array}$ & $\begin{array}{l}\text { CHLORO- } \\
\text { PHENOL }\end{array}$ & $\begin{array}{l}\text { PHENAN - } \\
\text { THRENE }\end{array}$ & $\begin{array}{r}(\mathrm{C} 6 \mathrm{H}- \\
5 \mathrm{OH})\end{array}$ & PYRENE & $\begin{array}{l}\text { CHLORO- } \\
\text { PHENOL }\end{array}$ & ALDRIN, & $\begin{array}{l}P, P^{\prime} \\
\text { DDT, }\end{array}$ & $\begin{array}{l}\text { ALPHA } \\
\text { BHC }\end{array}$ & $\begin{array}{l}\text { CHLOR - } \\
\text { IDE }\end{array}$ & $\begin{array}{c}\text { CHLOR } \\
\text { IDE }\end{array}$ \\
\hline ATE & $\begin{array}{l}\text { TOTAL } \\
(\mathrm{UG} / \mathrm{L})\end{array}$ & $\begin{array}{l}\text { TOTAL } \\
\text { (UG/L) }\end{array}$ & $\begin{array}{l}\text { TOTAL } \\
\text { (UG/L) }\end{array}$ & $\begin{array}{l}\text { TOTAL } \\
(U G / L)\end{array}$ & $\begin{array}{l}\text { TOTAL } \\
\text { (UG/L) }\end{array}$ & $\begin{array}{l}\text { TOTAL } \\
\text { (UG/L) }\end{array}$ & $\begin{array}{l}\text { TOTAL } \\
(\mathrm{UG} / \mathrm{L})\end{array}$ & $\begin{array}{l}\text { TOTAL } \\
\text { (UG/L) }\end{array}$ & $\begin{array}{l}\text { TOTAL } \\
\text { (UG/L) }\end{array}$ & $\begin{array}{l}\text { TOTAL } \\
\text { (UG/L) }\end{array}$ & $\begin{array}{l}\text { TOTAL } \\
\text { (UG/L) }\end{array}$ & $\begin{array}{r}\text { TOTAL } \\
\text { (UG/L) }\end{array}$ \\
\hline
\end{tabular}

\begin{tabular}{|c|c|c|c|c|c|c|c|c|c|c|c|c|}
\hline \multicolumn{13}{|l|}{ MAR 1992} \\
\hline 03.04 & $<5.0$ & $<5.0$ & $<30.0$ & $<5.0$ & $<5.0$ & $<5.0$ & $<20.0$ & $<0.040$ & $<0.10$ & $<0.03$ & $<0.03$ & $<0.09$ \\
\hline \multicolumn{13}{|l|}{ MAR } \\
\hline $09-09$ & $<5.0$ & $<5.0$ & $<30.0$ & $<5.0$ & $<5.0$ & $<5.0$ & $<20.0$ & $<0.040$ & $<0.10$ & $<0.03$ & $<0.03$ & $<0.09$ \\
\hline \multicolumn{13}{|l|}{ APR } \\
\hline $19-19$ & $<5.0$ & $<5.0$ & $<30.0$ & $<5.0$ & $<5.0$ & $<5.0$ & $<20.0$ & $<0.040$ & $<0.10$ & $<0.03$ & $<0.03$ & $<0.09$ \\
\hline \multicolumn{13}{|l|}{ MAY } \\
\hline $14-14$ & $<5.0$ & $<5.0$ & $<30.0$ & $<5.0$ & $<5.0$ & $<5.0$ & $<20.0$ & $<0.040$ & $<0.10$ & $<0.03$ & $<0.03$ & $<0.09$ \\
\hline \multicolumn{13}{|l|}{ JUN } \\
\hline $01-01$ & $<5.0$ & $<5.0$ & $<30.0$ & 6.0 & $<5.0$ & 12.0 & $<20.0$ & $<0.040$ & $<0.10$ & $<0.03$ & $<0.03$ & $<0.09$ \\
\hline \multicolumn{13}{|l|}{ JUN } \\
\hline $06-06$ & $<5.0$ & $<5.0$ & $<30.0$ & $<5.0$ & $<5.0$ & $<5.0$ & $<20.0$ & $<0.040$ & $<0.10$ & $<0.03$ & $<0.03$ & $<0.09$ \\
\hline \multicolumn{13}{|l|}{ JUL } \\
\hline $27-27$ & $<5.0$ & $<5.0$ & $<30.0$ & 11.0 & $<5.0$ & 14.0 & $<20.0$ & $<0.040$ & $<0.10$ & $<0.03$ & $<0.03$ & $<0.09$ \\
\hline
\end{tabular}

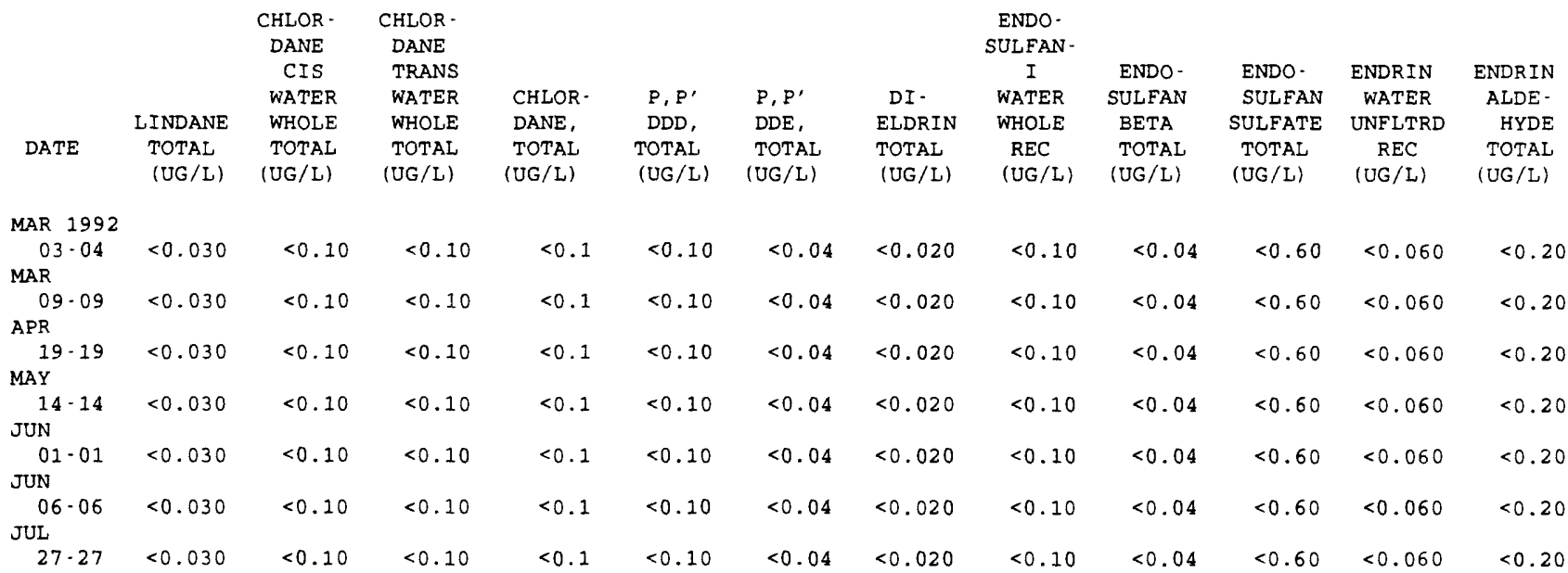


Table 3. Water-quality data for storm-sewer outfall stations, Dallas, Texas, 1992-93-Continued

08056390 - BASTILLE STREET OUTFALL AT LA REUNION PARKWAY, DALLAS, TX (WY 1992)-Continued

\begin{tabular}{|c|c|c|c|c|c|c|c|c|c|c|c|}
\hline & HEPTA - & HEPTA - & & AROCLOR & AROCLOR & AROCLOR & AROCLOR & AROCLOR & AROCLOR & AROCLOR & DI - \\
\hline & CHLOR, & EPOXIDE & TPHENE, & PCB & PCB & PCB & PCB & PCB & PCB & $\begin{array}{r}1260 \\
\text { PCB }\end{array}$ & AZINON, \\
\hline DATE & $\begin{array}{l}\text { TOTAL } \\
\text { (UG/L) }\end{array}$ & $\begin{array}{l}\text { TOTAL } \\
\text { (UG/L) }\end{array}$ & $\begin{array}{l}\text { TOTAL } \\
\text { (UG/L) }\end{array}$ & $\begin{array}{l}\text { TOTAL } \\
\text { (UG/L) }\end{array}$ & $\begin{array}{l}\text { TOTAL } \\
\text { (UG/L) }\end{array}$ & $\begin{array}{l}\text { TOTAL } \\
\text { (UG/L) }\end{array}$ & $\begin{array}{l}\text { TOTAL } \\
\text { (UG/L) }\end{array}$ & $\begin{array}{l}\text { TOTAL } \\
\text { (UG/L) }\end{array}$ & $\begin{array}{l}\text { TOTAL } \\
\text { (UG/L) }\end{array}$ & $\begin{array}{l}\text { TOTAL } \\
\text { (UG/L) }\end{array}$ & $\begin{array}{l}\text { TOTAL } \\
\text { (UG/L) }\end{array}$ \\
\hline MAR 1992 & & & & & & & & & & & \\
\hline $\begin{array}{l}03-04 \\
\text { MAR }\end{array}$ & $<0.030$ & $<0.80$ & $<2$ & $<0.1$ & $<1.0$ & $<0.1$ & $<0.1$ & $<0.1$ & $<0.1$ & $<0.1$ & 0.05 \\
\hline $\begin{array}{l}09.09 \\
\text { APR }\end{array}$ & $<0.030$ & $<0.80$ & $<2$ & $<0.1$ & $<1.0$ & $<0.1$ & $<0.1$ & $<0.1$ & $<0.1$ & $<0.1$ & $<0.10$ \\
\hline $\begin{array}{l}19 \cdot 19 \\
\text { MAY }\end{array}$ & $<0.030$ & $<0.80$ & $<2$ & $<0.1$ & $<1.0$ & $<0.1$ & $<0.1$ & $<0.1$ & $<0.1$ & $<0.1$ & 0.10 \\
\hline $\mathrm{JUN}^{14 \cdot 14}$ & $<0.030$ & $<0.80$ & $<2$ & $<0.1$ & $<1.0$ & $<0.1$ & $<0.1$ & $<0.1$ & $<0.1$ & $<0.1$ & $<0.10$ \\
\hline $\begin{array}{l}01-01 \\
\text { JUN }\end{array}$ & $<0.030$ & $<0.80$ & $<2$ & $<0.1$ & $<1.0$ & $<0.1$ & $<0.1$ & $<0.1$ & $<0.1$ & $<0.1$ & $<0.10$ \\
\hline JUL $^{06-06}$ & $<0.030$ & $<0.80$ & $<2$ & $<0.1$ & $<1.0$ & $<0.1$ & $<0.1$ & $<0.1$ & $<0.1$ & $<0.1$ & 0.10 \\
\hline $27 \cdot 27$ & $<0.030$ & $<0.80$ & $<2$ & $<0.1$ & $<1.0$ & $<0.1$ & $<0.1$ & $<0.1$ & $<0.1$ & $<0.1$ & $<0.10$ \\
\hline
\end{tabular}


Table 3. Water-quality data for storm-sewer outfall stations, Dallas, Texas, 1992-93-Continued 08057135 - WHITE ROCK CREEK OUTFALL AT PRESTON ROAD, DALLAS, TX (WY 1992)

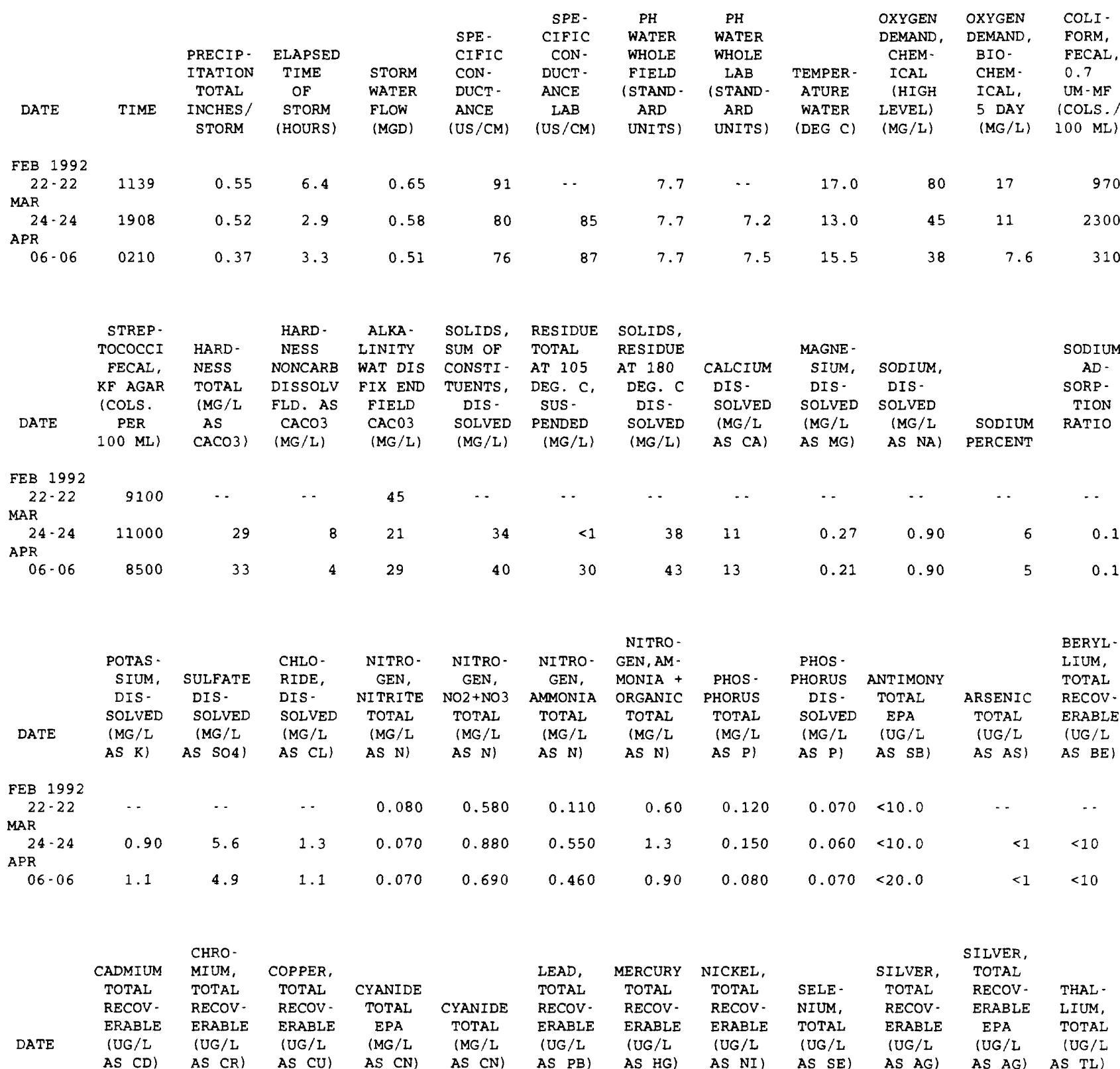

FEB 1992

\begin{tabular}{|c|c|c|c|c|c|c|c|c|c|c|c|c|}
\hline $22-22$ & $\cdots$ & & $\cdots$ & $<0.010$ & $<0.010$ & $\cdots$ & $\cdots$ & - & - & - & $<1.00$ & $<5$ \\
\hline MAR & & & & & & & & & & & & \\
\hline $\begin{array}{l}24-24 \\
P R\end{array}$ & $<1$ & $<1$ & $\cdots$ & $<0.010$ & $<0.010$ & 7 & $<0.10$ & 2 & $<2$ & $<1$ & $<1.00$ & $<5$ \\
\hline $06-06$ & $<1$ & 2 & 13 & $<0.010$ & $<0.010$ & 7 & $<0.10$ & $<1$ & $<2$ & $<1$ & $<1.00$ & $<10$ \\
\hline
\end{tabular}


Table 3. Water-quality data for storm-sewer outfall stations, Dallas, Texas, 1992-93-Continued

08057135 - WHITE ROCK CREEK OUTFALL AT PRESTON ROAD, DALLAS, TX (WY 1992)-Continued

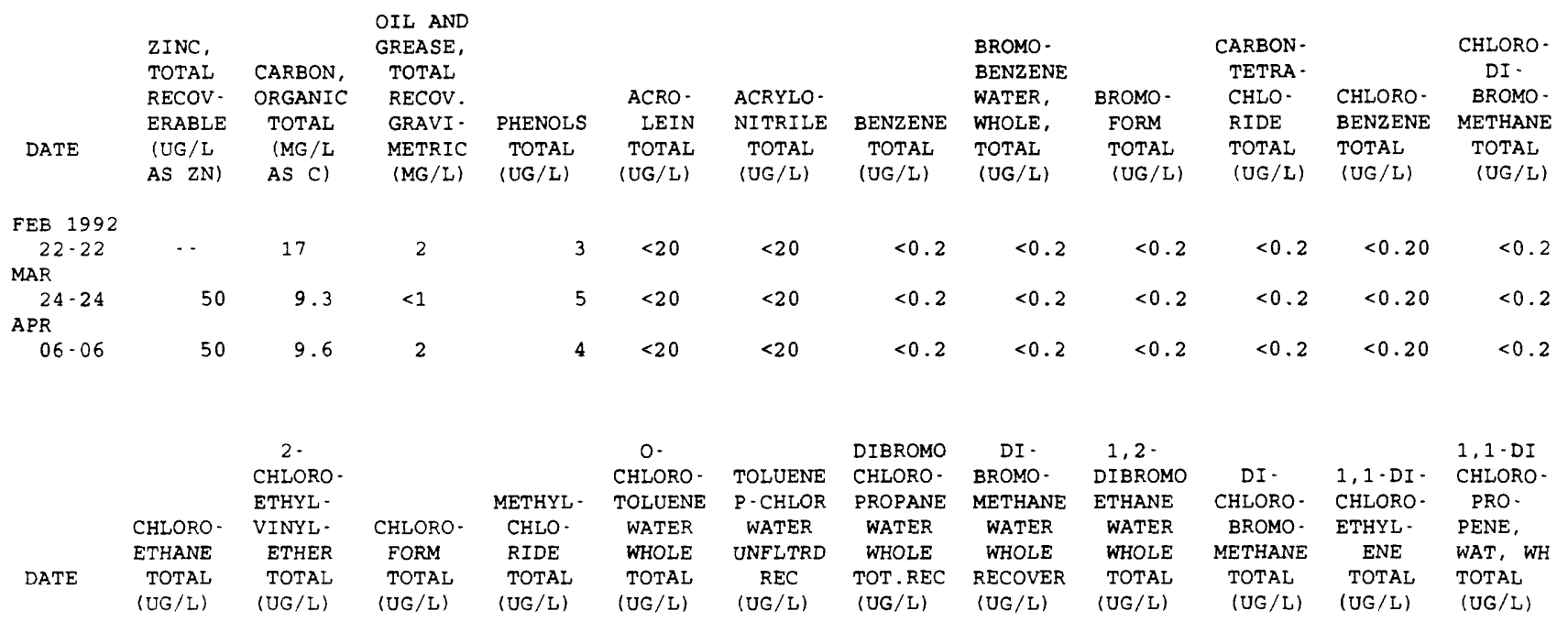

\begin{tabular}{|c|c|c|c|c|c|c|c|c|c|c|c|c|}
\hline \multicolumn{13}{|l|}{ FEB 1992} \\
\hline $22 \cdot 22$ & $<0.2$ & $<1.0$ & $<0.2$ & $<0.2$ & $<0.2$ & $<0.20$ & $<1.0$ & $<0.2$ & $<0.2$ & $<0.2$ & $<0.2$ & $<0.2$ \\
\hline \multicolumn{13}{|l|}{ MAR } \\
\hline $24-24$ & $<0.2$ & $<1.0$ & $<0.2$ & $<0.2$ & $<0.2$ & $<0.20$ & $<1.0$ & $<0.2$ & $<0.2$ & $<0.2$ & $<0.2$ & $<0.2$ \\
\hline \multicolumn{13}{|l|}{ APR } \\
\hline 06.06 & $<0.2$ & $<1.0$ & $<0.2$ & $<0.2$ & $<0.2$ & $<0.20$ & $<1.0$ & $<0.2$ & $<0.2$ & $<0.2$ & $<0.2$ & $<0.2$ \\
\hline
\end{tabular}

BENZENE BENZENE BENZENE DI -

$\begin{array}{clll}0- & 1,3-\mathrm{DI}- & 1,4-\mathrm{DI} \text { - CHLORO- }\end{array}$

CHLORO- CHLORO- CHLORO- DI -

WATER WATER WATER FLUORO-

UNFLTRD UNFLTRD UNFLTRD METHANE

REC REC REC TOTAL

$(U G / L) \quad(U G / L) \quad(U G / L) \quad(U G / L)$

$1,1-D I-$
CHLORO-
ETHANE
TOTAL
(UG/L)$$
\text { 1,2-DI - CIS-1,2 - }
$$$$
\text { PHENYL - DI- }
$$

TRANSDI

, 2-DI - HYDRA - CHLORO-

ZINE ETHENE

WATER WATER

TOT. REC

TOTAL

(UG/L) (UG/L) (UG/L)

CHLORO- CHLORO- PROPANE

ETHENE PROPANE WAT. WH

TOTAL TOTAL TOTAL

$(U G / L) \quad(U G / L) \quad(U G / L)$

\begin{tabular}{|c|c|c|c|c|c|c|c|c|c|c|c|}
\hline \multicolumn{12}{|l|}{ FEB 1992} \\
\hline $22 \cdot 22$ & $<5.0$ & $<5.0$ & $<5.0$ & $<0.2$ & $<0.2$ & $<0.2$ & $<5.0$ & $<0.2$ & $<0.2$ & $<0.2$ & $<0.2$ \\
\hline \multicolumn{12}{|l|}{ MAR } \\
\hline $24-24$ & $<5.0$ & $<5.0$ & $<5.0$ & $<0.2$ & $<0.2$ & $<0.2$ & $<5.0$ & $<0.2$ & $<0.2$ & $<0.2$ & $<0.2$ \\
\hline \multicolumn{12}{|l|}{$A P R$} \\
\hline \multirow[t]{6}{*}{06.06} & $<5.0$ & $<5.0$ & $<5.0$ & $<0.2$ & $<0.2$ & $<0.2$ & $<5.0$ & $<0.2$ & $<0.2$ & $<0.2$ & $<0.2$ \\
\hline & $2,2-\mathrm{DI}$ & & & & & & & & & ETHANE, & ETHANE, \\
\hline & CHLORO - & CIS & TRANS - & & HEXA- & & METHYL - & & & $11120^{\circ}$ & $1,1,2,2$ \\
\hline & PRO. & $1,3 \cdot \mathrm{DI}-$ & $1,3-D I$. & & CHLORO - & & ENE & & & TETRA - & TETRA. \\
\hline & PANE & CHLORO. & CHLORO. & ETHYL - & BUT - & METHYL - & CHLO - & NAPHTH - & & CHLORO- & CHLORO - \\
\hline & WAT, WH & PROPENE & PROPENE & BENZENE & ADIENE & BROMIDE & RIDE & ALENE & STYRENE & WAT UNF & WAT UNF \\
\hline DATE & $\begin{array}{l}\text { TOTAL } \\
\text { (UG/L) }\end{array}$ & $\begin{array}{l}\text { TOTAL } \\
\text { (UG/L) }\end{array}$ & $\begin{array}{l}\text { TOTAL } \\
\text { (UG/L) }\end{array}$ & $\begin{array}{l}\text { TOTAL } \\
\text { (UG/L) }\end{array}$ & $\begin{array}{c}\text { TOTAL } \\
\text { (UG/L) }\end{array}$ & $\begin{array}{l}\text { TOTAL } \\
\text { (UG/L) }\end{array}$ & $\begin{array}{l}\text { TOTAL } \\
\text { (UG/L) }\end{array}$ & $\begin{array}{l}\text { TOTAL } \\
\text { (UG/L) }\end{array}$ & $\begin{array}{l}\text { TOTAL } \\
\text { (UG/L) }\end{array}$ & $\begin{array}{c}\text { REC } \\
(\mathrm{UG} / \mathrm{L})\end{array}$ & $\begin{array}{c}\text { REC } \\
\text { (UG/L) }\end{array}$ \\
\hline \multicolumn{12}{|l|}{ FEB 1992} \\
\hline $22 \cdot 22$ & $<0.2$ & $<0.2$ & $<0.2$ & $<0.2$ & $<5.0$ & $<0.2$ & $<0.2$ & $<5.0$ & $<0.2$ & $<0.2$ & $<0.2$ \\
\hline \multicolumn{12}{|l|}{ MAR } \\
\hline $24-24$ & $<0.2$ & $<0.2$ & $<0.2$ & $<0.2$ & $<5.0$ & $<0.2$ & $<0.2$ & $<5.0$ & $<0.2$ & $<0.2$ & $<0.2$ \\
\hline \multicolumn{12}{|l|}{ APR } \\
\hline $06-06$ & $<0.2$ & $<0.2$ & $<0.2$ & $<0.2$ & $<5.0$ & $<0.2$ & $<0.2$ & $<5.0$ & $<0.2$ & $<0.2$ & $<0.2$ \\
\hline
\end{tabular}


Table 3. Water-quality data for storm-sewer outfall stations, Dallas, Texas, 1992-93-Continued 08057135 - WHITE ROCK CREEK OUTFALL AT PRESTON ROAD, DALLAS, TX (WY 1992)-Continued

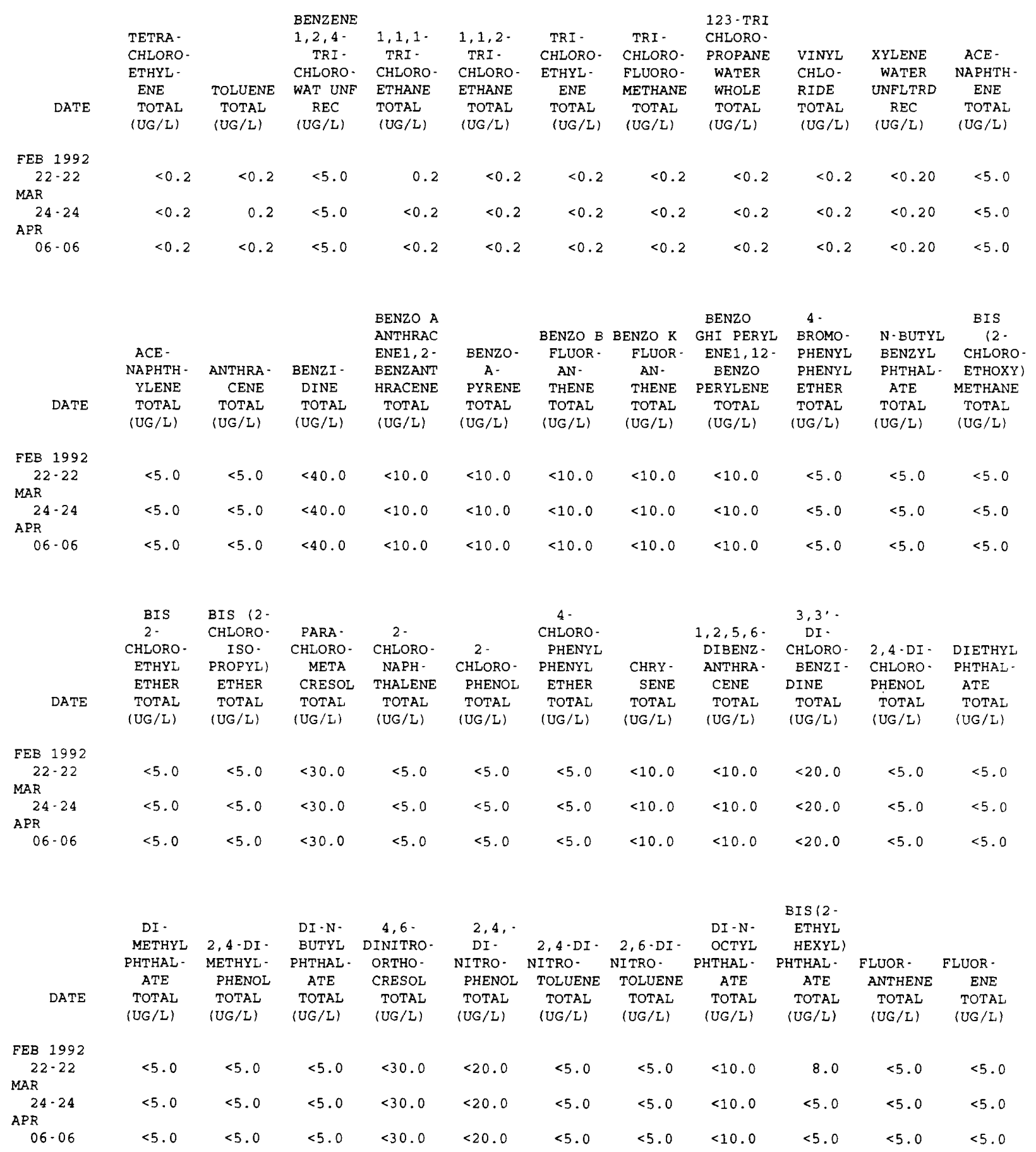


Table 3. Water-quality data for storm-sewer outfall stations, Dallas, Texas, 1992-93-Continued

08057135 - WHITE ROCK CREEK OUTFALL AT PRESTON ROAD, DALLAS, TX (WY 1992)-Continued

\begin{tabular}{|c|c|c|c|c|c|c|c|c|c|c|c|}
\hline DATE & $\begin{array}{l}\text { HEXA- } \\
\text { CHLORO- } \\
\text { BENZENE } \\
\text { TOTAL } \\
\text { (UG/L) }\end{array}$ & $\begin{array}{c}\text { HEXA- } \\
\text { CHLORO- } \\
\text { CYCLO- } \\
\text { PENT- } \\
\text { ADIENE } \\
\text { TOTAL } \\
\text { (UG/L) }\end{array}$ & $\begin{array}{c}\text { HEXA- } \\
\text { CHLORO- } \\
\text { ETHANE } \\
\text { TOTAL } \\
\text { (UG/L) }\end{array}$ & $\begin{array}{r}\text { INDENO } \\
(1,2,3- \\
\text { CD) } \\
\text { PYRENE } \\
\text { TOTAL } \\
\text { (UG/L) }\end{array}$ & $\begin{array}{l}\text { ISO- } \\
\text { PHORONE } \\
\text { TOTAL } \\
\text { (UG/L) }\end{array}$ & $\begin{array}{l}\text { NITRO- } \\
\text { BENZENE } \\
\text { TOTAL } \\
\text { (UG/L) }\end{array}$ & $\begin{array}{c}\text { N-NITRO- } \\
\text { SODI - } \\
\text { METHY - } \\
\text { LAMINE } \\
\text { TOTAL } \\
\text { (UG/L) }\end{array}$ & $\begin{array}{l}2- \\
\text { NITRO- } \\
\text { PHENOL } \\
\text { TOTAL } \\
\text { (UG/L) }\end{array}$ & $\begin{array}{l}4- \\
\text { NITRO- } \\
\text { PHENOL } \\
\text { TOTAL } \\
\text { (UG/L) }\end{array}$ & $\begin{array}{l}\text { N- } \\
\text { NITRO- } \\
\text { SODI-N- } \\
\text { PROPYL- } \\
\text { AMINE } \\
\text { TOTAL } \\
\text { (UG/L) }\end{array}$ & $\begin{array}{c}\text { N-NITRO- } \\
\text { SODI - } \\
\text { PHENY - } \\
\text { LAMINE } \\
\text { TOTAL } \\
\text { (UG/L) }\end{array}$ \\
\hline $\begin{array}{c}\text { EB } 1992 \\
22.22\end{array}$ & & & & & & & & & & & \\
\hline $\begin{array}{l}22-22 \\
\text { MAR }\end{array}$ & $<5.0$ & $<5.0$ & $<5.0$ & $<10.0$ & $<5.0$ & $<5.0$ & $<5.0$ & $<5.0$ & $<30.0$ & $<5.0$ & $<5.0$ \\
\hline $\begin{array}{l}24-24 \\
\text { APR }\end{array}$ & $<5.0$ & $<5.0$ & $<5.0$ & $<10.0$ & $<5.0$ & $<5.0$ & $<5.0$ & $<5.0$ & $<30.0$ & $<5.0$ & $<5.0$ \\
\hline 06.06 & $\begin{array}{l}\text { PENTA- } \\
\text { CHLORO- } \\
\text { PHENOL } \\
\text { TOTAL } \\
(\mathrm{UG} / \mathrm{L})\end{array}$ & $\begin{array}{c}\text { PHENAN- } \\
\text { THRENE } \\
\text { TOTAL } \\
\text { (UG/L) }\end{array}$ & $\begin{array}{c}\text { PHENOL } \\
\text { (C6H- } \\
50 \mathrm{H}) \\
\text { TOTAL } \\
\text { (UG/L) }\end{array}$ & $\begin{array}{l}\text { PYRENE } \\
\text { TOTAL } \\
\text { (UG/L) }\end{array}$ & $\begin{array}{c}2,4,6- \\
\text { TRI - } \\
\text { CHLORO- } \\
\text { PHENOL } \\
\text { TOTAL } \\
\text { (UG/L) }\end{array}$ & $\begin{array}{l}\text { ALDRIN, } \\
\text { TOTAL } \\
\text { (UG/L) }\end{array}$ & $\begin{array}{l}\text { P, P' } \\
\text { DDT, } \\
\text { TOTAL } \\
\text { (UG/L) }\end{array}$ & $\begin{array}{l}\text { ALPHA } \\
\text { BHC } \\
\text { TOTAL } \\
\text { (UG/L) }\end{array}$ & $\begin{array}{c}\text { BETA } \\
\text { BENZENE } \\
\text { HEXA- } \\
\text { CHLOR- } \\
\text { IDE } \\
\text { TOTAL } \\
\text { (UG/L) }\end{array}$ & $\begin{array}{l}\text { DELTA } \\
\text { BENZENE } \\
\text { HEXA- } \\
\text { CHLOR- } \\
\text { IDE } \\
\text { TOTAL } \\
\text { (UG/L) }\end{array}$ & $\begin{array}{c}\text { LINDANE } \\
\text { TOTAL } \\
\text { (UG/L) }\end{array}$ \\
\hline $\begin{array}{c}\mathrm{FEB} \quad 1992 \\
22-22\end{array}$ & $<30.0$ & $<5.0$ & $<5.0$ & $<5.0$ & $<20.0$ & $<0.040$ & $<0.10$ & $<0.03$ & $<0.03$ & $<0.09$ & $<0.030$ \\
\hline MAR & & & & & & & & & & & 0.050 \\
\hline $\begin{array}{l}24-24 \\
\text { APR }\end{array}$ & $<30.0$ & $<5.0$ & $<5.0$ & $<5.0$ & $<20.0$ & $<0.040$ & $<0.10$ & $<0.03$ & $<0.03$ & $<0.09$ & $<0.030$ \\
\hline $06-06$ & $<30.0$ & $<5.0$ & $<5.0$ & $<5.0$ & $<20.0$ & $<0.040$ & $<0.10$ & $<0.03$ & $<0.03$ & $<0.09$ & $<0.030$ \\
\hline & $\begin{array}{l}\text { CHLOR- } \\
\text { DANE }\end{array}$ & $\begin{array}{l}\text { CHLOR- } \\
\text { DANE }\end{array}$ & & & & & $\begin{array}{c}\text { ENDO- } \\
\text { SULFAN- }\end{array}$ & & & & \\
\hline & CIS & TRANS & & & & & I & ENDO- & ENDO- & ENDRIN & ENDRIN \\
\hline & $\begin{array}{l}\text { WATER } \\
\text { WHOLE }\end{array}$ & $\begin{array}{l}\text { WATER } \\
\text { WHOLE }\end{array}$ & $\begin{array}{l}\text { CHLOR- } \\
\text { DANE, }\end{array}$ & $\begin{array}{l}P, P^{\prime} \\
D D D\end{array}$ & $\begin{array}{l}P, P^{\prime} \\
D D E\end{array}$ & $\begin{array}{c}\text { DI - } \\
\text { ELDRIN }\end{array}$ & $\begin{array}{l}\text { WATER } \\
\text { WHOLE }\end{array}$ & $\begin{array}{l}\text { SULFAN } \\
\text { BETA }\end{array}$ & $\begin{array}{r}\text { SULFAN } \\
\text { SULFATE }\end{array}$ & $\begin{array}{l}\text { WATER } \\
\text { UNFLTRD }\end{array}$ & $\begin{array}{c}\text { ALDE - } \\
\text { HYDE }\end{array}$ \\
\hline DATE & $\begin{array}{l}\text { TOTAL } \\
\text { (UG/L) }\end{array}$ & $\begin{array}{l}\text { TOTAL } \\
\text { (UG/L) }\end{array}$ & $\begin{array}{l}\text { TOTAL } \\
\text { (UG/L) }\end{array}$ & $\begin{array}{l}\text { TOTAL } \\
\text { (UG/L) }\end{array}$ & $\begin{array}{l}\text { TOTAL } \\
\text { (UG/L) }\end{array}$ & $\begin{array}{l}\text { TOTAL } \\
\text { (UG/L) }\end{array}$ & $\begin{array}{l}\mathrm{REC} \\
(\mathrm{UG} / \mathrm{L}\rangle\end{array}$ & $\begin{array}{l}\text { TOTAL } \\
\text { (UG/L) }\end{array}$ & $\begin{array}{l}\text { TOTAL } \\
\text { (UG/L) }\end{array}$ & $\begin{array}{c}\text { REC } \\
\text { (UG/L) }\end{array}$ & $\begin{array}{l}\text { TOTAL } \\
(\mathrm{UG} / \mathrm{L})\end{array}$ \\
\hline FEB 1992 & & & & & & & & & & & \\
\hline $\begin{array}{l}22-22 \\
\text { MAR }\end{array}$ & $<0.10$ & $<0.10$ & $<0.1$ & $<0.10$ & $<0.04$ & $<0.020$ & $<0.10$ & $<0.04$ & $<0.60$ & $<0.060$ & $<0.20$ \\
\hline $\begin{array}{l}24-24 \\
\mathrm{APR}\end{array}$ & $<0.10$ & $<0.10$ & $<0.1$ & $<0.10$ & $<0.04$ & $<0.020$ & $<0.10$ & $<0.04$ & $<0.60$ & $<0.060$ & $<0.20$ \\
\hline 06.06 & $<0.10$ & $<0.10$ & $<0.1$ & $<0.10$ & $<0.04$ & $<0.020$ & $<0.10$ & $<0.04$ & $<0.60$ & $<0.060$ & $<0.20$ \\
\hline & & HEPTA- & & AROCLOR & AROCLOR & AROCLOR & AROCLOR & AROCLOR & AROCLOR & AROCLOR & \\
\hline & $\begin{array}{l}\text { HEPTA- } \\
\text { CHLOR, }\end{array}$ & $\begin{array}{l}\text { CHLOR } \\
\text { EPOXIDE }\end{array}$ & $\begin{array}{c}\text { TOX - } \\
\text { APHENE, }\end{array}$ & $\begin{array}{l}1016 \\
\text { PCB }\end{array}$ & $\begin{array}{r}1221 \\
\text { PCB }\end{array}$ & $\begin{array}{l}1232 \\
\text { PCB }\end{array}$ & $\begin{array}{r}1242 \\
\text { PCB }\end{array}$ & $\begin{array}{r}1248 \\
\text { PCB }\end{array}$ & $\begin{array}{r}1254 \\
\text { PCB }\end{array}$ & $\begin{array}{r}1260 \\
\text { PCB }\end{array}$ & $\begin{array}{c}\text { DI- } \\
\text { AZINON, }\end{array}$ \\
\hline DATE & $\begin{array}{l}\text { CHLOR, } \\
\text { TOTAL } \\
\text { (UG/L) }\end{array}$ & $\begin{array}{l}\text { EPOXIDE } \\
\text { TOTAL } \\
\text { (UG/L) }\end{array}$ & $\begin{array}{c}\text { APHENE, } \\
\text { TOTAL } \\
\text { (UG/L) }\end{array}$ & $\begin{array}{c}\text { PCB } \\
\text { TOTAL } \\
(U G / L)\end{array}$ & $\begin{array}{l}\text { TOTAL } \\
\text { (UG/L) }\end{array}$ & $\begin{array}{l}\text { TOTAL } \\
\text { (UG/L) }\end{array}$ & $\begin{array}{l}\text { TOTAL } \\
\text { (UG/L) }\end{array}$ & $\begin{array}{l}\text { TOTAL } \\
\text { (UG/L) }\end{array}$ & $\begin{array}{l}\text { TOTAL } \\
\text { (UG/L) }\end{array}$ & $\begin{array}{l}\text { TOTAL } \\
\text { (UG/L) }\end{array}$ & $\begin{array}{l}\text { TOTAL } \\
\text { (UG/L) }\end{array}$ \\
\hline FEB 1992 & & & & & & & & & & & \\
\hline $\begin{array}{l}22 \cdot 22 \\
A R\end{array}$ & $<0.030$ & $<0.80$ & $<2$ & $<0.1$ & $<1.0$ & $<0.1$ & $<0.1$ & $<0.1$ & $<0.1$ & $<0.1$ & 0.13 \\
\hline $\begin{array}{l}24-24 \\
P R\end{array}$ & $<0.030$ & $<0.80$ & $<2$ & $<0.1$ & $<1.0$ & $<0.1$ & $<0.1$ & $<0.1$ & $<0.1$ & $<0.1$ & 0.20 \\
\hline $06-06$ & $<0.030$ & $<0.80$ & $<2$ & $<0.1$ & $<1.0$ & $<0.1$ & $<0.1$ & $<0.1$ & $<0.1$ & $<0.1$ & 0.30 \\
\hline
\end{tabular}


Table 3. Water-quality data for storm-sewer outfall stations, Dallas, Texas, 1992-93-Continued 08057135 - WHITE ROCK CREEK OUTFALL AT PRESTON ROAD, DALLAS, TX (WY 1993)

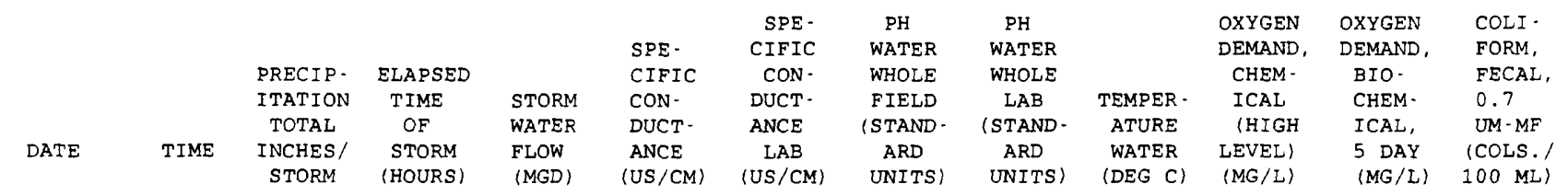

$\begin{array}{ll}\text { NOV } 1992 & \\ 19-19 & 0200 \\ \text { JAN } 1993 & \\ 09-09 & 0245 \\ \text { JAN } & \\ \quad 19-20 & 1541 \\ \text { FEB } & \\ 10-10 & 0427\end{array}$

$\begin{array}{rrrrrrrrrrr}0.76 & 8.8 & 1.1 & 72 & 80 & 7.2 & 6.8 & 18.5 & 23 & 4.1 & 11000 \\ 0.41 & 5.2 & 0.58 & 54 & 85 & 8.4 & 7.8 & 9.5 & 47 & 3.7 & \mathrm{k} 4700 \\ 0.56 & 7.0 & 0.69 & 115 & 80 & 8.1 & 7.5 & 6.5 & 91 & 2.7 & 1700 \\ 0.22 & 2.1 & 0.29 & 114 & 111 & 7.8 & 7.3 & 13.0 & 110 & 5.1 & \mathrm{k} 2100\end{array}$

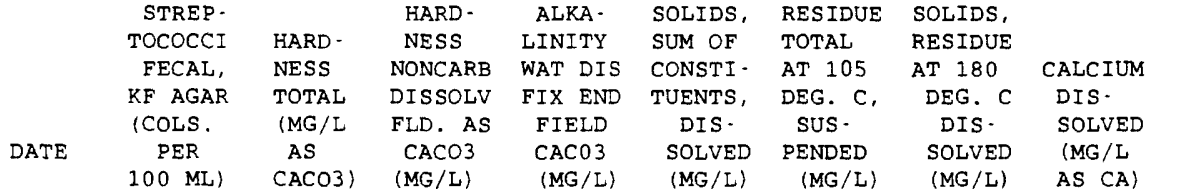

MAGNE -

SIUM, SODIUM,

DIS - DIS -

SOLVED SOLVED

(MG/L (MG / L

$\begin{array}{lcr}\text { (MG / L } & \text { (MG/L } & \text { SODIUM } \\ \text { AS MG) AS NA) } & \text { PERCENT }\end{array}$

SODIUM

NOV 1992

$$
19 \cdot 19
$$

78000

\begin{abstract}
26
\end{abstract}
JAN 1993

$09.09 \quad 67000$

JAN $19 \cdot 20 \quad 36000$

FEB

10-10 K150000

$\begin{array}{lll}26 & 1 & 25 \\ 28 & 2 & 26 \\ 23 & 2 & 21 \\ 34 & 1 & 33\end{array}$

$\begin{array}{ll}35 & <1 \\ 42 & 39 \\ 40 & 92 \\ 54 & 24\end{array}$

$\begin{array}{cc}41 & 10 \\ 35 & 11 \\ 29 & 9.0 \\ 63 & 13\end{array}$

$\begin{array}{ll}0.30 & 1.4 \\ 0.24 & 1.3 \\ 0.17 & 0.70 \\ 0.34 & 1.9\end{array}$

10

0.1

0.1

0.1

0.1

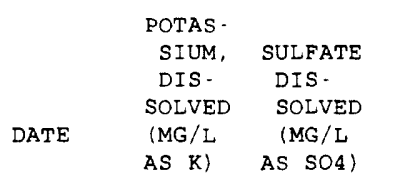

$\begin{array}{lcc}\text { CHLO } & \text { NITRO- } & \text { NITRO- } \\ \text { RIDE, } & \text { GEN, } & \text { GEN, } \\ \text { DIS- } & \text { NITRITE } & \text { NO2+NO3 } \\ \text { SOLVED } & \text { TOTAL } & \text { TOTAL } \\ \text { (MG } / \text { L } & \text { (MG/L } & \text { (MG/L } \\ \text { AS CL) } & \text { AS N) } & \text { AS N) }\end{array}$

$\begin{array}{ccc} & \text { NITRO- } & \\ \text { NITRO- } & \text { GEN, AM- } & \\ \text { GEN, } & \text { MONIA + } & \text { PHOS- } \\ \text { AMMONIA } & \text { ORGANIC } & \text { PHORUS } \\ \text { TOTAL } & \text { TOTAL } & \text { TOTAL } \\ \text { (MG } / L & \text { (MG } / L & \text { (MG } / L \\ \text { AS N) } & \text { AS N) } & \text { AS P) }\end{array}$

PHOS -

PHORUS ANTIMONY

DIS - TOTAL

SOLVED EPA

(MG/L (UG/L

AS P) AS SB)

NOV 1992

$19-19$
JAN 1993

$0.90 \quad 4.4$

1.4

0.080

0.450

0.110

0.40

0.080

$0.060<20.0$

$<1$

$09-09$

$\begin{array}{ll}1.8 & 2.8\end{array}$

1.6

0.520

-

$0.50 \quad 0.070$

$0.010<10.0$

$1.0 \quad 0.480$

$0.020<10.0$

0.250

$0.030<10.0$

$<10$

$19-20$

$\begin{array}{ll}0.70 & 3.1 \\ 1.0 & 7.7\end{array}$

1.6

0.530

-

1. 3

CHRO-

MIUM, COPPER,

CADMIUM MIUM

TOTAL TOTAL TOTAL, CYANTDE

RECOV - RECOV - RECOV - TOTAL

ERABLE CYANIDE

(UG/L (UG/L (UG/L T

LEAD, MERCURY NICKEL,

TOTAL TOTAL TOTAL SELE-

RECOV - RECOV - RECOV - NIUM,

ERABLE ERABLE ERABLE TOTAL

(UG/L (UG/L (UG/L (UG /L

AS CU) AS CN) AS CN

AS PB) AS HG)

AS NI)

AS SE)

$\begin{array}{ccc} & \text { SILVER, } \\ \text { SILVER, } & \text { TOTAL } \\ \text { TOTAL } & \text { RECOV- } & \text { THAL- } \\ \text { RECOV. } & \text { ERABLE } & \text { LIUM, } \\ \text { ERABLE } & \text { EPA } & \text { TOTAL } \\ \text { (UG/L } & \text { (UG/L } & \text { (UG/L } \\ \text { AS AG) } & \text { AS AG) } & \text { AS TL) }\end{array}$

NOV 1992

$19-19$
JAN 1993

$09 \cdot 09$

JAN

$19 \cdot 20$

FEB

$10-10$

$\begin{array}{rr}<1 & <1 \\ <1 & 8 \\ <1 & 100 \\ <1 & 11\end{array}$

$\begin{array}{rlr}4 & <0.010 & <0.010 \\ 6 & <0.010 & <0.010 \\ 82 & <0.010 & <0.010 \\ 32 & <0.010 & <0.010\end{array}$

$\begin{aligned} 5 & <0.10 \\ 16 & <0.10 \\ 61 & <0.10 \\ 15 & <0.10\end{aligned}$

$\begin{array}{ll}2 & <2 \\ 2 & <2 \\ 4 & <2 \\ 5 & <2\end{array}$

$\begin{array}{ll}<1 & <0.500 \\ <1 & <0.500 \\ <1 & <0.500 \\ <1 & <0.500\end{array}$

$<10$
$<5$
$<5$
$<10$ 
Table 3. Water-quality data for storm-sewer outfall stations, Dallas, Texas, 1992-93-Continued

08057135 - WHITE ROCK CREEK OUTFALL AT PRESTON ROAD, DALLAS, TX (WY 1993)-Continued

\begin{tabular}{|c|c|c|c|c|c|c|c|c|c|c|c|c|}
\hline DATE & $\begin{array}{l}\text { 2INC, } \\
\text { TOTAL } \\
\text { RECOV- } \\
\text { ERABLE } \\
\text { (UG/L } \\
\text { AS ZN) }\end{array}$ & $\begin{array}{l}\text { CARBON, } \\
\text { ORGANIC } \\
\text { TOTAL } \\
\text { (MG/L } \\
\text { AS C) }\end{array}$ & $\begin{array}{l}\text { OIL AND } \\
\text { GREASE, } \\
\text { TOTAL } \\
\text { RECOV. } \\
\text { GRAVI- } \\
\text { METRIC } \\
\text { (MG/L) }\end{array}$ & $\begin{array}{l}\text { PHENOLS } \\
\text { TOTAL } \\
\text { (UG/L) }\end{array}$ & $\begin{array}{l}\text { ACRO- } \\
\text { LEIN } \\
\text { TOTAL } \\
(U G / L)\end{array}$ & $\begin{array}{c}\text { ACRYLO- } \\
\text { NITRILE } \\
\text { TOTAL } \\
\text { (UG/L) }\end{array}$ & $\begin{array}{l}\text { BENZENE } \\
\text { TOTAL } \\
\text { (UG/L) }\end{array}$ & $\begin{array}{l}\text { BROMO- } \\
\text { BENZENE } \\
\text { WATER, } \\
\text { WHOLE, } \\
\text { TOTAL } \\
\text { (UG/L) }\end{array}$ & $\begin{array}{l}\text { METHANE } \\
\text { BROMO } \\
\text { CHLORO- } \\
\text { WAT } \\
\text { UNFLTRD } \\
\text { REC } \\
\text { (UG/L) }\end{array}$ & $\begin{array}{l}\text { BROMO- } \\
\text { FORM } \\
\text { TOTAL } \\
\text { (UG/L) }\end{array}$ & $\begin{array}{c}\text { BENZENE } \\
\text { N-BUTYL } \\
\text { WATER } \\
\text { UNFLTRD } \\
\text { REC } \\
(U G / L)\end{array}$ & $\begin{array}{l}\text { BENZENE } \\
\text { SEC } \\
\text { BUTYL- } \\
\text { WATER } \\
\text { UNFLTRD } \\
\text { REC } \\
\text { (UG/L) }\end{array}$ \\
\hline $\begin{array}{c}\text { NOV } 1992 \\
19-19 \\
\text { JAN } 1993\end{array}$ & 40 & 6.9 & $<1$ & 2 & $<20$ & $<20$ & $<0.2$ & $<0.2$ & $<0.20$ & $<0.2$ & $<0.20$ & $<0.20$ \\
\hline $\begin{array}{l}09-09 \\
\text { JAN } \\
19-20 \\
\text { FEB } \\
10-10\end{array}$ & 220 & 8.2 & 2 & 7 & $<20$ & $<20$ & $<0.2$ & $<0.2$ & $<0.20$ & $<0.2$ & $<0.20$ & $<0.20$ \\
\hline DATE & $\begin{array}{l}\text { BENZENE } \\
\text { TERT- } \\
\text { BUTYL- } \\
\text { WATER } \\
\text { UNFLTRD } \\
\text { REC } \\
\text { (UG/L) }\end{array}$ & $\begin{array}{l}\text { CARBON- } \\
\text { TETRA- } \\
\text { CHLO- } \\
\text { RIDE } \\
\text { TOTAL } \\
(\text { UG } / L)\end{array}$ & $\begin{array}{l}\text { CHLORO- } \\
\text { BENZENE } \\
\text { TOTAL } \\
\text { (UG/L) }\end{array}$ & $\begin{array}{c}\text { CHLORO- } \\
\text { DI - } \\
\text { BROMO- } \\
\text { METHANE } \\
\text { TOTAL } \\
\text { (UG/L) }\end{array}$ & $\begin{array}{l}\text { CHLORO- } \\
\text { ETHANE } \\
\text { TOTAL } \\
\text { (UG/L) }\end{array}$ & $\begin{array}{c}2- \\
\text { CHLORO- } \\
\text { ETHYL- } \\
\text { VINYL- } \\
\text { ETHER } \\
\text { TOTAL } \\
\text { (UG/L) }\end{array}$ & $\begin{array}{c}\text { CHLORO- } \\
\text { FORM } \\
\text { TOTAL } \\
\text { (UG/L) }\end{array}$ & $\begin{array}{l}\text { METHYL- } \\
\text { CHLO- } \\
\text { RIDE } \\
\text { TOTAL } \\
\text { (UG /L) }\end{array}$ & $\begin{array}{c}\text { O- } \\
\text { CHLORO- } \\
\text { TOLUENE } \\
\text { WATER } \\
\text { WHOLE } \\
\text { TOTAL } \\
\text { (UG/L) }\end{array}$ & $\begin{array}{l}\text { TOLUENE } \\
\text { P. CHLOR } \\
\text { WATER } \\
\text { UNFLTRD } \\
\text { REC } \\
\text { (UG/L) }\end{array}$ & $\begin{array}{l}\text { DIBROMO } \\
\text { CHLORO- } \\
\text { PROPANE } \\
\text { WATER } \\
\text { WHOLE } \\
\text { TOT.REC } \\
\text { (UG/L) }\end{array}$ & $\begin{array}{c}\text { DI - } \\
\text { BROMO- } \\
\text { METHANE } \\
\text { WATER } \\
\text { WHOLE } \\
\text { RECOVER } \\
\text { (UG/L) }\end{array}$ \\
\hline $\begin{array}{c}\text { NOV } 1992 \\
19 \cdot 19 \\
\text { JAN } 1993\end{array}$ & $<0.20$ & $<0.2$ & $<0.20$ & $<0.2$ & $<0.2$ & $<1.0$ & $<0.2$ & $<0.2$ & $<0.2$ & $<0.20$ & $<1.0$ & $<0.2$ \\
\hline $\begin{array}{l}09-09 \\
\text { JAN } \\
19 \cdot 20\end{array}$ & $<0.20$ & $<0.2$ & $<0.20$ & $<0.2$ & $<0.2$ & $<1.0$ & $<0.2$ & $<0.2$ & $<0.2$ & $<0.20$ & $<1.0$ & $<0.2$ \\
\hline $\begin{array}{l}E B \\
10-10\end{array}$ & $<0.20$ & $<0.2$ & $<0.20$ & $<0.2$ & $<0.2$ & $<1.0$ & 1.1 & $<0.2$ & $<0.2$ & $<0.20$ & $<1.0$ & $<0.2$ \\
\hline
\end{tabular}

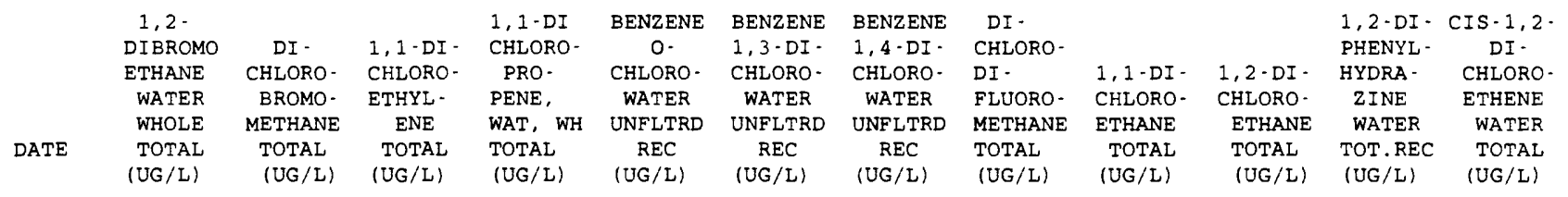

Nov 1992

\begin{tabular}{|c|c|c|c|c|c|c|c|c|c|c|c|c|}
\hline 19.19 & & & & & & & & & & & & \\
\hline \multicolumn{13}{|l|}{ JAN 1993} \\
\hline 09.09 & $<0.2$ & $<0.2$ & $<0.2$ & $<0.2$ & $<5.0$ & $<5.0$ & $<5.0$ & $<0.2$ & $<0.2$ & $<0.2$ & $<5.0$ & $<0.2$ \\
\hline \multicolumn{13}{|l|}{ JAN } \\
\hline $19-20$ & $<0.2$ & 0.3 & $<0.2$ & $<0.2$ & $<5.0$ & $<5.0$ & $<5.0$ & $<0.2$ & $<0.2$ & $<0.2$ & $<5.0$ & $<0.2$ \\
\hline \multicolumn{13}{|l|}{ FEB } \\
\hline $10-10$ & $<0.2$ & 0.4 & $<0.2$ & $<0.2$ & $<5.0$ & $<5.0$ & $<5.0$ & $<0.2$ & $<0.2$ & $<0.2$ & $<5.0$ & $<0.2$ \\
\hline
\end{tabular}

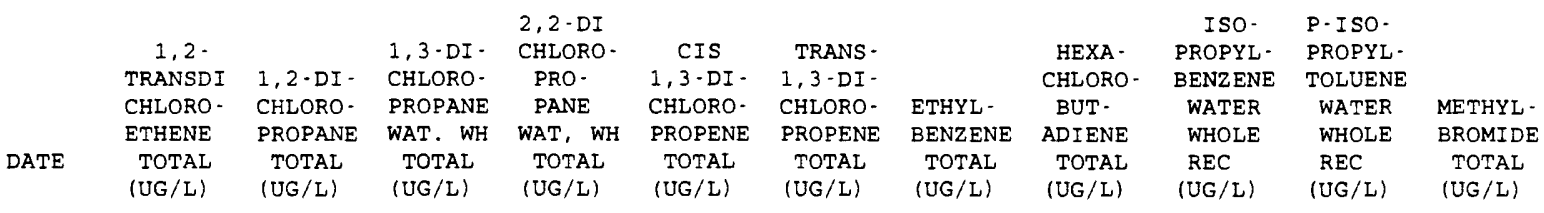

\begin{tabular}{|c|c|c|c|c|c|c|c|c|c|c|c|}
\hline \multicolumn{12}{|l|}{ Nov 1992} \\
\hline $19-19$ & $<0.2$ & $<0.2$ & $<0.2$ & $<0.2$ & $<0.2$ & $<0.2$ & $<0.2$ & $<5.0$ & $<0.20$ & $<0.20$ & $<0.2$ \\
\hline \multicolumn{12}{|l|}{ JAN 1993} \\
\hline $09-09$ & $<0.2$ & $<0.2$ & $<0.2$ & $<0.2$ & $<0.2$ & $<0.2$ & $<0.2$ & $<5.0$ & $<0.20$ & $<0.20$ & $<0.2$ \\
\hline \multicolumn{12}{|l|}{ JAN } \\
\hline $19-20$ & $<0.2$ & $<0.2$ & $<0.2$ & $<0.2$ & $<0.2$ & $<0.2$ & $<0.2$ & $<5.0$ & $<0.20$ & $<0.20$ & $<0.2$ \\
\hline \multicolumn{12}{|l|}{ FEB } \\
\hline $10 \cdot 10$ & $<0.2$ & $<0.2$ & $<0.2$ & $<0.2$ & $<0.2$ & $<0.2$ & $<0.2$ & $<5.0$ & $<0.20$ & $<0.20$ & $<0.2$ \\
\hline
\end{tabular}


Table 3. Water-quality data for storm-sewer outfall stations, Dallas, Texas, 1992-93-Continued 08057135 - WHITE ROCK CREEK OUTFALL AT PRESTON ROAD, DALLAS, TX (WY 1993)-Continued

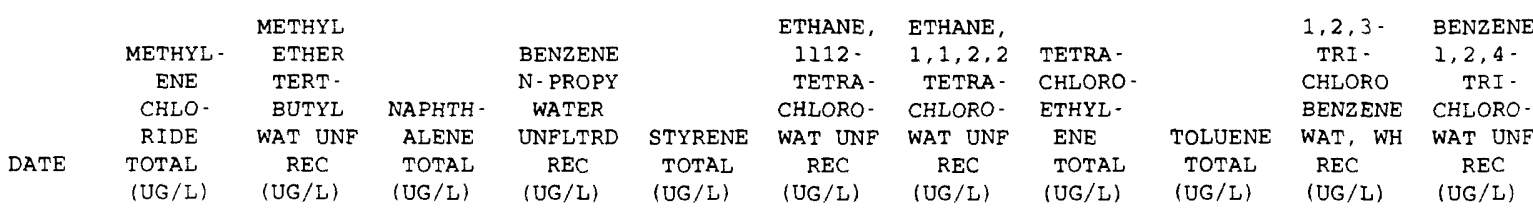

$$
\begin{gathered}
\text { NOV } 1992 \\
19-19 \\
\text { JAN } 1993 \\
09-09 \\
\text { JAN } \\
19-20 \\
\text { FEB } \\
10-10
\end{gathered}
$$

$$
\begin{array}{llll}
<0.2 & <1.0 & <5.0 & <0.20 \\
<0.2 & <1.0 & <5.0 & <0.20 \\
<0.2 & <1.0 & <5.0 & <0.20 \\
<0.2 & <1.0 & <5.0 & <0.20
\end{array}
$$$$
<0.2
$$$$
<0.2
$$$$
<0.2
$$$$
<0.2
$$$$
<0.2
$$$$
<0.20
$$$$
<5.0
$$$$
<0.2
$$$$
<0.2
$$$$
<0.2
$$$$
<0.2
$$$$
<0.2<0.20
$$$$
<5.0
$$

$<0.2$

$<0.2$

$<0.2$

$<0.2$

$0.2<0.20$

$<5.0$

$<0.2$

$<0.2$

$<0.2$

$<0.2$

$<0.2<0.20$

$<5.0$

DATE
1992
199
-09
-20
-10

$$
\begin{array}{lc}
1,1,1- & 1,1,2- \\
\text { TRI- } & \text { TRI- } \\
\text { CHLORO- } & \text { CHLORO- } \\
\text { ETHANE } & \text { ETHANE } \\
\text { TOTAL } & \text { TOTAL } \\
\text { (UG/L) } & (\text { UG } / L)
\end{array}
$$$$
\begin{aligned}
& \text { TRI - } \\
& \text { CHLORO- } \\
& \text { ETHYL- } \\
& \text { ENE } \\
& \text { TOTAL } \\
& \text { (UG/L) }
\end{aligned}
$$

TRI -
CHLORO-
FLUORO-
METHANE
TOTAL
(UG/L)

123 - TRI
CHLORO -

FREON - PSEUDO - MESIT -

WATER

WHOLE

\begin{tabular}{|c|c|c|c|}
\hline & & & BENZO A \\
\hline $\mathrm{ACE}-$ & & & ENE1, 2 - \\
\hline NAPHTH - & ANTHRA - & BENZI - & BENZANT \\
\hline YLENE & CENE & DINE & HRACENE \\
\hline $\begin{array}{l}\text { TOTAL } \\
\text { (UG/L) }\end{array}$ & $\begin{array}{l}\text { TOTAL } \\
\text { (UG/L) }\end{array}$ & $\begin{array}{l}\text { TOTAL } \\
\text { (UG/L) }\end{array}$ & $\begin{array}{l}\text { TOTAL } \\
(\mathrm{UG} / \mathrm{L})\end{array}$ \\
\hline
\end{tabular}

TOTAL

(UG/L)

$\begin{array}{ccc}113 & \text { CUMENE } & \text { YLENE } \\ \text { WATER } & \text { WATER } & \text { WATER } \\ \text { UNFLTRD } & \text { UNFLTRD } & \text { UNFLTRD } \\ \text { REC } & \text { REC } & \text { REC } \\ \text { (UG/L) } & \text { (UG/L) } & \text { (UG/L) }\end{array}$

NOV 1992
$19-19$
JAN 1993
$09-09$
JAN
$19-20$
FEB
$10-10$

$\begin{array}{lll}<0.2 & <0.2 & <0.2 \\ <0.2 & <0.2 & <0.2 \\ <0.2 & <0.2 & <0.2 \\ <0.2 & <0.2 & <0.2\end{array}$

$<0.2$
$<0.2$
$<0.2$
$<0.2$

$<0.2$
$<0.2$
$<0.2$
$<0.2$

$<0.5$
$<0.5$
$<0.5$
$<0.5$

$\begin{array}{ll}<0.20 & <0.20 \\ <0.20 & <0.20 \\ 0.20 & <0.20 \\ <0.20 & <0.20\end{array}$

$<0$.

$<0.2<0.20$

$<0.2$

0.30

$<0.2$

$<0.20$

$<5.0$
$(2$

DATE

NOV 1992 $19-19$ JAN 1993

09-09

JAN

$19 \cdot 20$ FEB

$10-10$

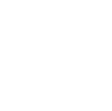

$$
\begin{array}{llll}
<5.0 & <5.0 & <40.0 & <10.0 \\
<5.0 & <5.0 & <40.0 & <10.0 \\
<5.0 & <5.0 & <40.0 & <10.0 \\
<5.0 & <5.0 & <40.0 & <10.0
\end{array}
$$

$\begin{array}{lc} & \\ \text { BENZO- } & \text { FLUOR - } \\ \text { A- } & \text { AN - } \\ \text { PYRENE } & \text { THENE } \\ \text { TOTAL } & \text { TOTAL } \\ \text { (UG } / \text { L }) & \text { (UG/L) }\end{array}$
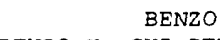

BENZO

4 -

BENZO $\mathrm{K}$ GHI PERYL BROMO FLUOR - ENE1,12 - PHENYL

AN- BENZO PHENYL

THENE PERYLENE ETHER

TOTAL

TOTAL

(UG/L)

TOTAL

(UG/L)

$<10.0<10.0$

$<10.0<10.0$

$<5.0$

$<10.0<10.0<10.0<10.0$

$<10.0$

$<10.0$

$<10.0$

$<10.0$

$<5.0$

$<5.0$

$<5.0$

$<10.0<10.0<10.0<10.0$

4 -

CHLORO -

BIS BIS 12 -

CHLORO-

ETHYL PROPYL

TOTAL TOTAL

(UG/L)

(UG/L)

PARA - 2 -

CHLORO- CHLORO-

META NAPH -

CHLORO- PHENYL

CRESOL THALENE PHENOL ETHER

TOTAL TOTAL

(UG/L)

(UG/L)

TOTAL TOTAL

(UG/L) (UG/L)

NOV 1992

$19-19$

JAN 1993

$09-09$

JAN

$19-20$

FEB

$10-10$

$\begin{array}{lllllllllll}<5.0 & <5.0 & <30.0 & <5.0 & <5.0 & <5.0 & <10.0 & <10.0 & <20.0 & <5.0 & 6.0 \\ <5.0 & <5.0 & <30.0 & <5.0 & <5.0 & <5.0 & <10.0 & <10.0 & <20.0 & <5.0 & <5.0 \\ <5.0 & <5.0 & <30.0 & <5.0 & <5.0 & <5.0 & <10.0 & <10.0 & <20.0 & <5.0 & <5.0 \\ <5.0 & <5.0 & <30.0 & <5.0 & <5.0 & <5.0 & <10.0 & <10.0 & <20.0 & <5.0 & <5.0\end{array}$


Table 3. Water-quality data for storm-sewer outfall stations, Dallas, Texas, 1992-93-Continued

08057135 - WHITE ROCK CREEK OUTFALL AT PRESTON ROAD, DALLAS, TX (WY 1993)-Continued

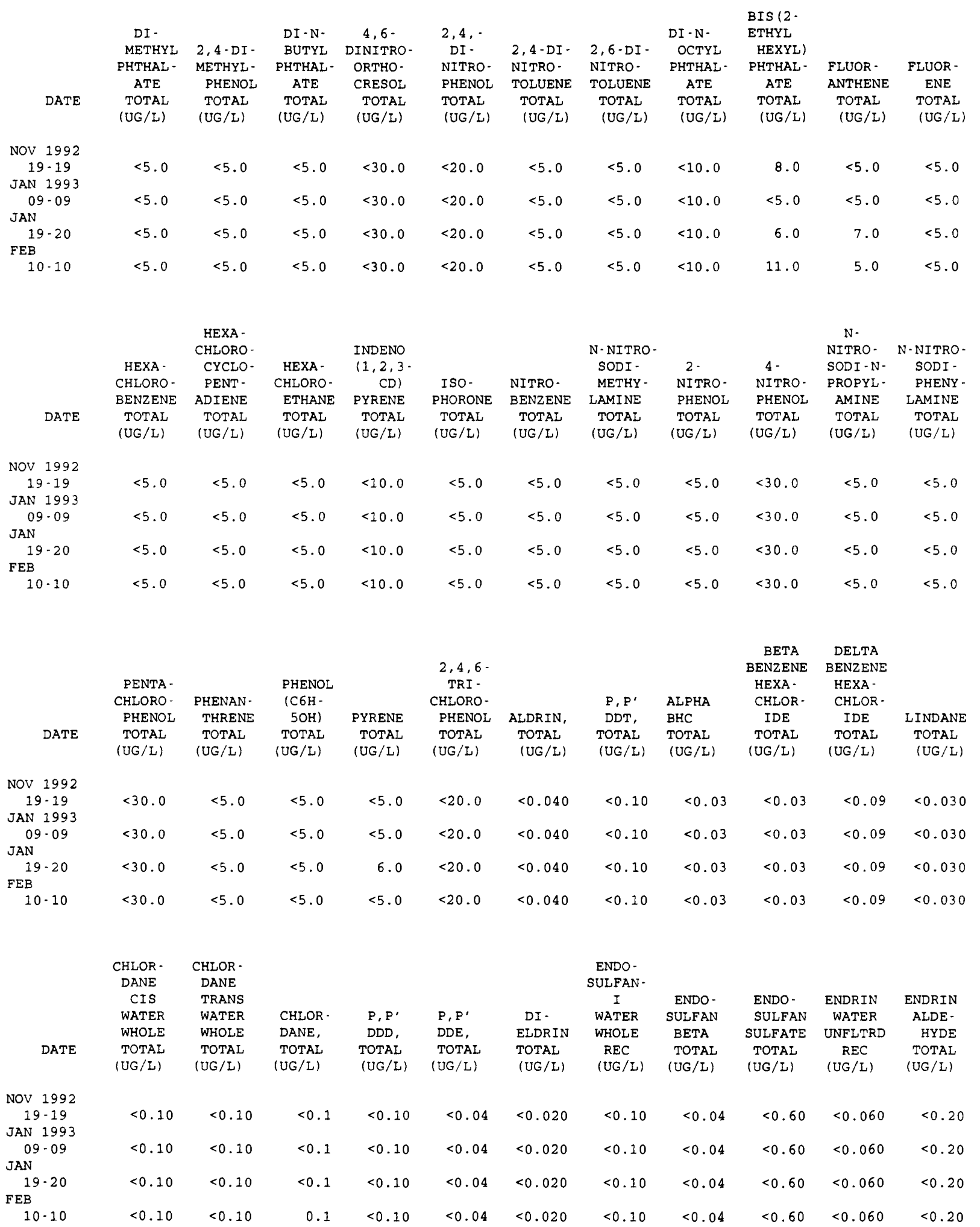


Table 3. Water-quality data for storm-sewer outfall stations, Dallas, Texas, 1992-93-Continued 08057135 - WHITE ROCK CREEK OUTFALL AT PRESTON ROAD, DALLAS, TX (WY 1993)-Continued

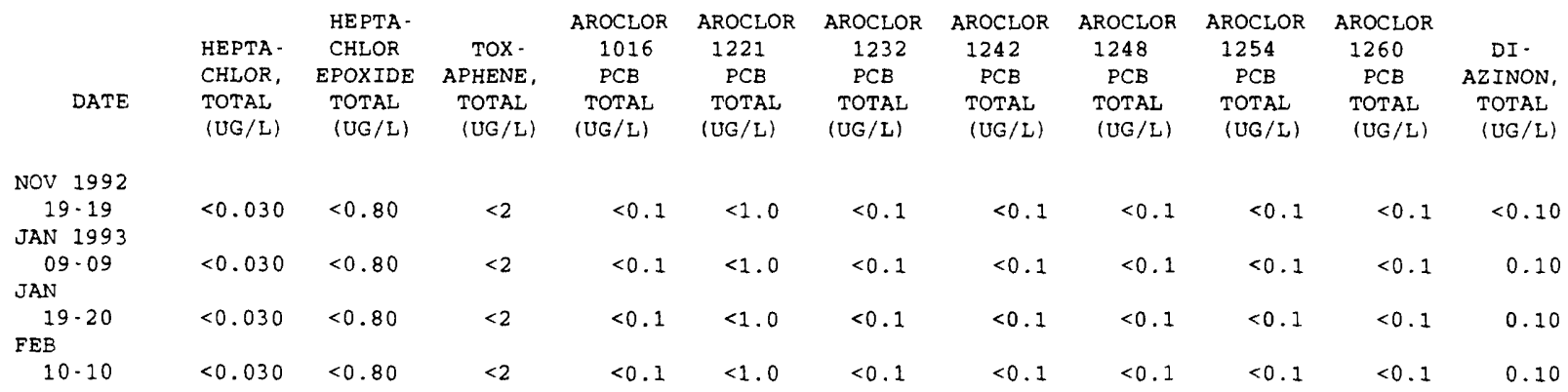


Table 3. Water-quality data for storm-sewer outfall stations, Dallas, Texas, 1992-93-Continued

08057310 - ASH CREEK OUTFALl AT WHITTIER STREET, DALLAS, TX (WY 1992)

\begin{tabular}{|c|c|c|c|c|c|c|c|c|c|c|c|c|}
\hline DATE & TIME & $\begin{array}{l}\text { PRECIP - } \\
\text { ITATION } \\
\text { TOTAL } \\
\text { INCHES/ } \\
\text { STORM }\end{array}$ & $\begin{array}{l}\text { ELAPSED } \\
\text { TIME } \\
\text { OF } \\
\text { STORM } \\
\text { (HOURS) }\end{array}$ & $\begin{array}{l}\text { STORM } \\
\text { WATER } \\
\text { FLOW } \\
\text { (MGD) }\end{array}$ & $\begin{array}{l}\text { SPE- } \\
\text { CIFIC } \\
\text { CON- } \\
\text { DUCT- } \\
\text { ANCE } \\
\text { (US/CM) }\end{array}$ & $\begin{array}{c}\text { SPE - } \\
\text { CIFIC } \\
\text { CON- } \\
\text { DUCT- } \\
\text { ANCE } \\
\text { LAB } \\
\text { (US/CM) }\end{array}$ & $\begin{array}{c}\text { PH } \\
\text { WATER } \\
\text { WHOLE } \\
\text { FIELD } \\
\text { (STAND- } \\
\text { ARD } \\
\text { UNITS) }\end{array}$ & $\begin{array}{c}\text { PH } \\
\text { WATER } \\
\text { WHOLE } \\
\text { LAB } \\
\text { (STAND - } \\
\text { ARD } \\
\text { UNITS) }\end{array}$ & $\begin{array}{c}\text { TEMPER - } \\
\text { ATURE } \\
\text { WATER } \\
\text { (DEG C) }\end{array}$ & $\begin{array}{l}\text { OXYGEN } \\
\text { DEMAND, } \\
\text { CHEM- } \\
\text { ICAL } \\
\text { (HIGH } \\
\text { LEVEL) } \\
\text { (MG/L) }\end{array}$ & $\begin{array}{l}\text { OXYGEN } \\
\text { DEMAND, } \\
\text { BIO- } \\
\text { CHEM- } \\
\text { ICAL, } \\
5 \text { DAY } \\
\text { (MG/L) }\end{array}$ & $\begin{array}{l}\text { COLI - } \\
\text { FORM, } \\
\text { FECAL, } \\
0.7 \\
\text { UM-MF } \\
\text { (COLS./ } \\
100 \mathrm{ML})\end{array}$ \\
\hline \multicolumn{13}{|l|}{ FEB 1992} \\
\hline $12 \cdot 12$ & 1610 & 0.40 & 2.8 & 0.13 & 208 & $\cdots$ & 7.8 & $\cdots$ & 16.5 & 260 & 7.9 & 30000 \\
\hline \multicolumn{13}{|l|}{ FEB } \\
\hline $22 \cdot 22$ & 1129 & 0.77 & 9.5 & 0.27 & 256 & 215 & 7.9 & 7.8 & 16.0 & 84 & 7.0 & 2300 \\
\hline \multicolumn{13}{|l|}{ MAR } \\
\hline $17-18$ & 1700 & 0.56 & 9.5 & 0.27 & 416 & 204 & 7.7 & 7.6 & 18.5 & 160 & 19 & $\mathrm{~K} 62000$ \\
\hline \multicolumn{13}{|l|}{ APR } \\
\hline 06.06 & 0120 & 0.61 & 5.2 & 0.12 & 120 & 160 & 9.9 & 7.7 & 15.0 & 90 & 8.1 & 13000 \\
\hline \multicolumn{13}{|l|}{ MAY } \\
\hline $14 \cdot 14$ & 1200 & 0.48 & 2.5 & 0.13 & 196 & 227 & 6.4 & 7.7 & 22.0 & 190 & 7.6 & 140000 \\
\hline \multicolumn{13}{|l|}{ JUN } \\
\hline $06-06$ & 0252 & 0.72 & 4.0 & 0.38 & 305 & 126 & 6.7 & 8.2 & 21.5 & 64 & 7.5 & $\mathrm{~K} 170000$ \\
\hline \multicolumn{13}{|l|}{ SEP } \\
\hline $21-21$ & 0142 & 0.98 & 2.5 & 0.20 & 130 & 157 & 7.5 & 7.5 & 23.5 & 47 & 6.8 & K14000 \\
\hline
\end{tabular}

\begin{tabular}{|c|c|c|c|c|c|c|c|c|c|c|c|c|}
\hline & $\begin{array}{l}\text { STREP } \\
\text { TOCOCCI }\end{array}$ & HARD - & $\begin{array}{l}\text { HARD } \\
\text { NESS }\end{array}$ & $\begin{array}{l}\text { ALKA- } \\
\text { LINITY }\end{array}$ & $\begin{array}{l}\text { SOLIDS, } \\
\text { SUM OF }\end{array}$ & $\begin{array}{l}\text { RESIDUE } \\
\text { TOTAL }\end{array}$ & $\begin{array}{l}\text { SOLIDS, } \\
\text { RESIDUE }\end{array}$ & & MAGNE - & & & SODIUM \\
\hline & FECAL, & NESS & NONCARB & WAT DIS & CONSTI - & AT 105 & AT 180 & CALCIUM & SIUM, & SODIUM, & & $A D$ \\
\hline & $\begin{array}{l}\text { KF AGAR } \\
\text { (COLS. }\end{array}$ & $\begin{array}{l}\text { TOTAL } \\
\text { (MG/L }\end{array}$ & $\begin{array}{l}\text { DISSOLV } \\
\text { FLD. AS }\end{array}$ & $\begin{array}{c}\text { FIX END } \\
\text { FIELD }\end{array}$ & $\begin{array}{c}\text { TUENTS, } \\
\text { DIS - }\end{array}$ & $\begin{array}{l}\text { DEG. C, } \\
\text { SUS - }\end{array}$ & $\begin{array}{l}\text { DEG. C } \\
\text { DIS. }\end{array}$ & $\begin{array}{l}\text { DIS - } \\
\text { SOLVED }\end{array}$ & $\begin{array}{c}\text { DIS - } \\
\text { SOLVED }\end{array}$ & $\begin{array}{c}\text { DIS - } \\
\text { SOLVED }\end{array}$ & & $\begin{array}{l}\text { SORP - } \\
\text { TION }\end{array}$ \\
\hline TE & $\begin{array}{c}\text { PER } \\
100 \mathrm{ML})\end{array}$ & $\begin{array}{c}\text { AS } \\
\text { (ACO3) }\end{array}$ & $\begin{array}{c}\mathrm{CACO} 3 \\
(\mathrm{MG} / \mathrm{L})\end{array}$ & $\begin{array}{l}\mathrm{CAC0} 3 \\
(\mathrm{MG} / \mathrm{L})\end{array}$ & $\begin{array}{l}\text { SOLVED } \\
(M G / L)\end{array}$ & $\begin{array}{l}\text { PENDED } \\
(M G / L)\end{array}$ & $\begin{array}{l}\text { SOLVED } \\
(M G / L)\end{array}$ & $\begin{array}{c}(M G / L \\
A S \quad C A)\end{array}$ & $\begin{array}{l}\text { (MG / L } \\
\text { AS MG) }\end{array}$ & $\begin{array}{c}(M G / L \\
\text { AS NA) }\end{array}$ & $\begin{array}{l}\text { SODIUM } \\
\text { PERCENT }\end{array}$ & RATIO \\
\hline
\end{tabular}
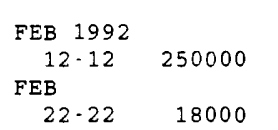

$\begin{array}{cr}22 \cdot 22 & 18000 \\ \text { MAR } & \\ 17 \cdot 18 & \times 350000\end{array}$

$\begin{array}{cr}17-18 & K 350000 \\ \text { APR } & \\ 06-06 & 45000\end{array}$

MAY $14-14 \quad 200000$

JUN $06-06 \quad K 510000$

SEP
$21-21 \quad K 35000$

$\begin{array}{lrl}\cdots & \cdots & \cdots \\ 50 & 18 & 32 \\ 60 & 7 & 49 \\ 45 & 6 & 54 \\ 27 & 11 & 34 \\ 29 & 1 & 26 \\ & 3 & 26\end{array}$

$\begin{array}{cccc}\cdots & \cdots & \cdots & \cdots \\ 81 & 320 & 89 & 19 \\ 97 & 103 & 100 & 21 \\ 82 & 65 & 100 & 23 \\ 104 & 284 & 103 & 17 \\ 60 & 352 & 54 & 10 \\ 61 & 55 & 51 & 11\end{array}$

$\begin{array}{ll}\cdots & \cdots \\ 0.69 & 1.9 \\ 0.79 & 2.0 \\ 0.65 & 1.8 \\ 0.71 & 2.1 \\ 0.38 & 1.1 \\ 0.46 & 1.3\end{array}$

$\begin{array}{ll}\ldots & \cdots \\ 7 & 0.1 \\ 6 & 0.1 \\ 5 & 0.1 \\ 7 & 0.1 \\ 7 & 0.1 \\ 7 & 0.1\end{array}$

\begin{tabular}{|c|c|c|c|c|c|c|c|c|c|c|c|c|}
\hline DATE & $\begin{array}{l}\text { POTAS - } \\
\text { SIUM, } \\
\text { DIS - } \\
\text { SOLVED } \\
\text { (MG/L }\end{array}$ & $\begin{array}{l}\text { SULFATE } \\
\text { DIS - } \\
\text { SOLVED } \\
\text { (MG / L }\end{array}$ & $\begin{array}{l}\text { CHLO- } \\
\text { RIDE, } \\
\text { DIS - } \\
\text { SOLVED } \\
(M G / L\end{array}$ & $\begin{array}{l}\text { NITRO- } \\
\text { GEN, } \\
\text { NITRITE } \\
\text { TOTAL } \\
\text { (MG/L }\end{array}$ & $\begin{array}{l}\text { NITRO- } \\
\text { GEN, } \\
\text { NO2+NO3 } \\
\text { TOTAL } \\
\text { (MG/L }\end{array}$ & $\begin{array}{l}\text { NITRO- } \\
\text { GEN, } \\
\text { AMMONIA } \\
\text { TOTAL } \\
\text { (MG / L }\end{array}$ & $\begin{array}{l}\text { NITRO- } \\
\text { GEN, AM- } \\
\text { MONIA + } \\
\text { ORGANIC } \\
\text { TOTAL } \\
\text { (MG/L }\end{array}$ & $\begin{array}{l}\text { PHOS - } \\
\text { PHORUS } \\
\text { TOTAL } \\
\text { (MG / L }\end{array}$ & $\begin{array}{l}\text { PHOS - } \\
\text { PHORUS } \\
\text { DIS - } \\
\text { SOLVED } \\
\text { (MG/L }\end{array}$ & $\begin{array}{l}\text { ANT IMONY } \\
\text { TOTAL } \\
\text { EPA } \\
\text { IUG/L }\end{array}$ & $\begin{array}{c}\text { ARSENIC } \\
\text { TOTAL } \\
\text { IUG/L }\end{array}$ & $\begin{array}{l}\text { BERYL- } \\
\text { LIUM, } \\
\text { TOTAL } \\
\text { RECOV- } \\
\text { ERABLE } \\
\text { IUG /L }\end{array}$ \\
\hline & AS $\mathrm{K}$ ) & AS SO4) & AS CL) & AS N) & AS N) & AS $\mathrm{N}$ ) & AS N) & AS P) & AS P) & AS $S B$ & AS AS) & AS BE) \\
\hline
\end{tabular}

\begin{tabular}{|c|c|c|c|c|c|c|c|c|c|c|c|c|}
\hline \multicolumn{13}{|l|}{ FEB 1992} \\
\hline $12 \cdot 12$ & $\cdots$ & $\cdots$ & $\cdots$ & 0.050 & 0.830 & 0.270 & 1.2 & 0.460 & 0.460 & $<10.0$ & $\cdots$ & $<10$ \\
\hline \multicolumn{13}{|l|}{ FEB } \\
\hline $22-22$ & 2.0 & 8.6 & 2.6 & 0.110 & 0.500 & 0.130 & 0.90 & 0.270 & 0.190 & $<10.0$ & 3 & $<10$ \\
\hline \multicolumn{13}{|l|}{ MAR } \\
\hline $17-18$ & 5.5 & 7.8 & 3.5 & 0.120 & 0.630 & 0.390 & 1.8 & 0.470 & 0.290 & $<20.0$ & 4 & $<10$ \\
\hline \multicolumn{13}{|l|}{ APR } \\
\hline 06.06 & 6.5 & 8.2 & 2.5 & 0.090 & 0.760 & 0.470 & 1.9 & 0.390 & 0.350 & $<20.0$ & 4 & $<10$ \\
\hline \multicolumn{13}{|l|}{ MAY } \\
\hline $14 \cdot 14$ & 8.6 & 11 & 2.9 & 0.080 & 1.00 & 0.540 & 2.5 & 0.480 & 0.360 & $<10.0$ & 4 & $<10$ \\
\hline \multicolumn{13}{|l|}{ JUN } \\
\hline $06-06$ & 4.5 & 4.4 & 1.7 & 0.070 & 0.580 & 0.180 & 0.80 & 0.250 & 0.240 & $<20.0$ & 4 & $<10$ \\
\hline \multicolumn{13}{|l|}{ SEP } \\
\hline $21 \cdot 21$ & 4.9 & 5.5 & 2.9 & 0.030 & 0.370 & 0.090 & 1.4 & 0.450 & 0.270 & $<10.0$ & 2 & $<10$ \\
\hline
\end{tabular}


Table 3. Water-quality data for storm-sewer outfall stations, Dallas, Texas, 1992-93-Continued

08057310 - ASH CREEK OUTFALI AT WHITTIER STREET, DALLAS, TX (WY 1992)-Continued

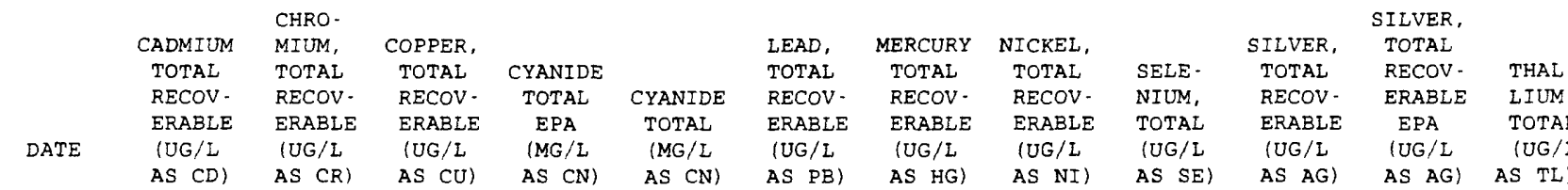

FEB 1992
$12-12$

$\mathrm{FEB}$

$22 \cdot 22$

MAR

17-18

APR

$06-06$

MAY

$14-14$

JUN

$06-06$ SEP

$21-21$
OIL AND

GREASE,

TOTAL CARBON, TOTAL

RECOV - ORGANIC RECOV.

ERABLE TOTAL GRAVI.

DATE

METRIC

(MG / L)

$\begin{array}{cr} & \text { ACRO- } \\ \text { PHENOLS } & \text { LEIN } \\ \text { TOTAL } & \text { TOTAL } \\ (U G / L) & (U G / L)\end{array}$

$<0.010$

$<0.010$

$10<0.010<0.010$

$8<0.010<0.010$

$16<10$.

$<0.010$

$12<10.0$

$<0.010$

$0.014<0.010$

$\begin{array}{ll}\cdots & <0.10 \\ 33 & <0.10 \\ 51 & <0.10 \\ 19 & <0.10 \\ 89 & <0.10 \\ 66 & <0.10 \\ 21 & <0.10\end{array}$

AS AG)

AS AG) AS TL)

FEB 1992

$12 \cdot 12$

FEB

$22-22$

MAR

$17 \cdot 18$

APR

06.06

MAY

$14 \cdot 14$

JUN

06.06

SEP

21- 21

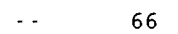

$\begin{array}{ll}70 & 27\end{array}$

$110 \quad 43$

$50 \quad 22$

$140 \quad 51$

$100 \quad 24$

40

$<1$
$<1$
$<1$
1
$<1$
$<1$
$<1$

$\begin{array}{lll}3 & <20 & <20 \\ 5 & <20 & <20 \\ 2 & <20 & <20 \\ 3 & <20 & <20 \\ 6 & <20 & <20 \\ 6 & <20 & <20 \\ 1 & <20 & <20\end{array}$

ACRYZO -

NITRILE BENZENE

TOTAL

(UG/L)

\section{TOTAL}

(UG/L)

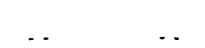

$<2$
$<2$
$<2$
$<2$
$<2$
$<2$

$\begin{array}{lll}<1 & <1.00 & <10 \\ <1 & <1.00 & <5 \\ <1 & <1.00 & <10 \\ <1 & <1.00 & <10 \\ <1 & <1.00 & <10 \\ <1 & <1.00 & <10 \\ <1 & <1.00 & <10\end{array}$

$\begin{array}{ll}<0.2 & <0.2 \\ <0.2 & <0.2 \\ 0.2 & <0.2 \\ <0.2 & <0.2 \\ <0.2 & <0.2 \\ <0.2 & <0.2 \\ <0.2 & <0.2\end{array}$

$\begin{array}{llll}<0.2 & <0.20 & <0.20 & <0.20 \\ <0.2 & <0.20 & <0.20 & <0.20 \\ <0.2 & <0.20 & <0.20 & <0.20 \\ <0.2 & <0.20 & <0.20 & <0.20 \\ <0.2 & <0.20 & <0.20 & <0.20 \\ <0.2 & <0.20 & <0.20 & <0.20 \\ <0.2 & <0.20 & <0.20 & <0.20\end{array}$

\begin{tabular}{|c|c|c|c|c|}
\hline & & & & 2 . \\
\hline $\begin{array}{l}\text { CARBON - } \\
\text { TETRA - }\end{array}$ & & $\begin{array}{c}\text { CHLORO- } \\
\text { DI - }\end{array}$ & & $\begin{array}{l}\text { CHLORO- } \\
\text { ETHYL - }\end{array}$ \\
\hline CHLO - & CHLORO - & BROMO - & CHLORO - & VINYL - \\
\hline RIDE & BENZENE & METHANE & ETHANE & ETHER \\
\hline $\begin{array}{l}\text { TOTAL } \\
\text { (UG/L) }\end{array}$ & $\begin{array}{l}\text { TOTAL } \\
\text { (UG/L) }\end{array}$ & $\begin{array}{l}\text { TOTAL } \\
(U G / L)\end{array}$ & $\begin{array}{c}\text { TOTAL } \\
(\mathrm{UG} / \mathrm{L})\end{array}$ & $\begin{array}{l}\text { TOTAL } \\
\text { (UG/L) }\end{array}$ \\
\hline
\end{tabular}

$\begin{array}{ll} & \\ \text { CHLORO- } & \text { CHLO- } \\ \text { FORM } & \text { RIDE } \\ \text { TOTAL } & \text { TOTAL } \\ \text { (UG/L) } & \text { (UG/L) }\end{array}$

$$
0-
$$

CHLORO - TOLUENE

CHLORO - TOLUENE WATER WATER WHOLE UNFLTRD

TOTAL

(UG/L)
UNFLTRD
REC

(UG/L)

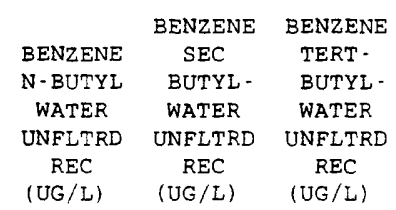

BENZENE BENZENE BUTYL - BUTYLUNFLTRD UNFLTRD L

\begin{tabular}{|c|c|c|c|c|c|c|c|c|c|c|c|c|}
\hline \multicolumn{13}{|l|}{ FEB 1992} \\
\hline $12-12$ & $<0.2$ & $<0.20$ & $<0.2$ & $<0.2$ & $<1.0$ & $<0.2$ & $<0.2$ & $<0.2$ & $<0.20$ & $<1.0$ & $<0.2$ & $<0.2$ \\
\hline \multicolumn{13}{|l|}{ FEB } \\
\hline $22-22$ & $<0.2$ & $<0.20$ & $<0.2$ & $<0.2$ & $<1.0$ & $<0.2$ & $<0.2$ & $<0.2$ & $<0.20$ & $<1.0$ & $<0.2$ & $<0.2$ \\
\hline \multicolumn{13}{|l|}{ MAR } \\
\hline $17 \cdot 18$ & $<0.2$ & $<0.20$ & $<0.2$ & $<0.2$ & $<1.0$ & 0.7 & $<0.2$ & $<0.2$ & $<0.20$ & $<1.0$ & $<0.2$ & $<0.2$ \\
\hline \multicolumn{13}{|l|}{ APR } \\
\hline 06.06 & $<0.2$ & $<0.20$ & $<0.2$ & $<0.2$ & $<1.0$ & $<0.2$ & $<0.2$ & $<0.2$ & $<0.20$ & $<1.0$ & $<0.2$ & $<0.2$ \\
\hline \multicolumn{13}{|l|}{ MAY } \\
\hline $14 \cdot 14$ & $<0.2$ & $<0.20$ & $<0.2$ & $<0.2$ & $<1.0$ & $<0.2$ & $<0.2$ & $<0.2$ & $<0.20$ & $<1.0$ & $<0.2$ & $<0.2$ \\
\hline \multicolumn{13}{|l|}{ JUN } \\
\hline 06.06 & $<0.2$ & $<0.20$ & $<0.2$ & $<0.2$ & $<1.0$ & 0.2 & $<0.2$ & $<0.2$ & $<0.20$ & $<1.0$ & $<0.2$ & $<0.2$ \\
\hline \multicolumn{13}{|l|}{ SEP } \\
\hline $21-21$ & $<0.2$ & $<0.20$ & $<0.2$ & $<0.2$ & $<1.0$ & $<0.2$ & $<0.2$ & $<0.2$ & $<0.20$ & $<1.0$ & $<0.2$ & $<0.2$ \\
\hline
\end{tabular}


Table 3. Water-quality data for storm-sewer outfall stations, Dallas, Texas, 1992-93-Continued

08057310 - ASH CREEK OUTFALL AT WHITTIER STREET, DALLAS, TX (WY 1992)-Continued

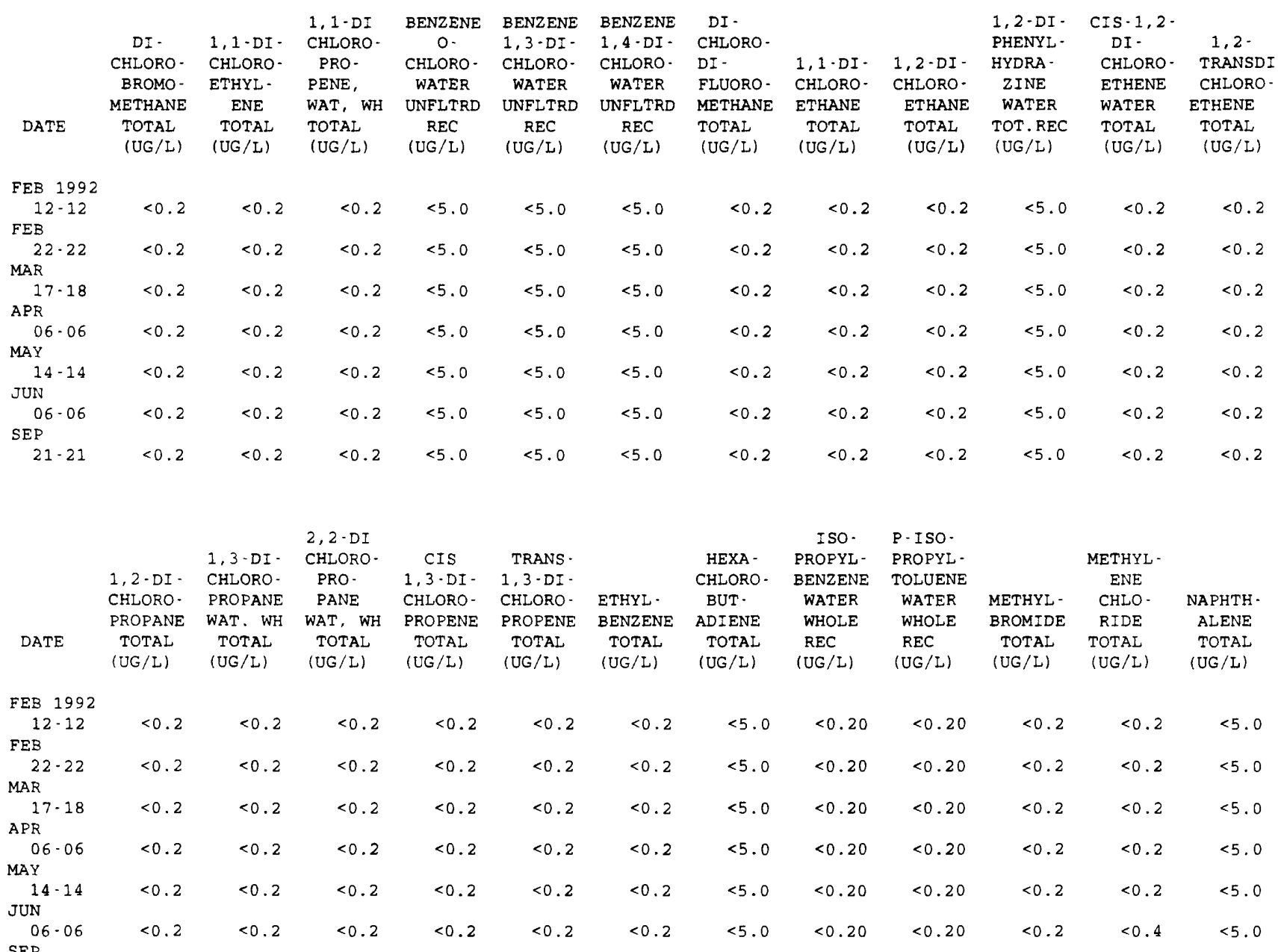

\begin{tabular}{|c|c|c|c|c|c|c|c|c|c|c|c|c|}
\hline & BENZENE & & $\begin{array}{c}\text { ETHANE, } \\
1112-\end{array}$ & $\begin{array}{l}\text { ETHANE, } \\
1,1,2,2\end{array}$ & TETRA - & & $\begin{array}{c}1,2,3 . \\
\text { TRI. }\end{array}$ & $\begin{array}{l}\text { BENZENE } \\
1,2,4 .\end{array}$ & $1,1,1$. & $1,1,2$. & TRI. & TRI \\
\hline & N-PROPY & & TETRA - & TETRA- & CHLORO- & & CHLORO & TRI. & TRI. & TRI . & CHLORO - & CHLORO- \\
\hline & WATER & & CHLORO- & CHLORO- & ETHYL- & & BENZENE & CHLORO- & CHLORO- & CHLORO- & ETHYL - & FLUORO- \\
\hline & UNFLTRD & STYRENE & WAT UNF & WAT UNF & ENE & TOLUENE & WAT, WH & WAT UNF & ETHANE & ETH & ENE & METHANE \\
\hline & $\begin{array}{c}\text { REC } \\
(U G / L)\end{array}$ & $\begin{array}{l}\text { TOTAL } \\
(\mathrm{UG} / \mathrm{L})\end{array}$ & $\begin{array}{c}\text { REC } \\
(\mathrm{UG} / \mathrm{L})\end{array}$ & $\begin{array}{c}\text { REC } \\
(U G / L)\end{array}$ & $\begin{array}{c}\text { TOTAL } \\
(U G / L)\end{array}$ & $\begin{array}{l}\text { TOTAL } \\
\text { (UG/L) }\end{array}$ & $\begin{array}{c}R E C \\
(U G / L)\end{array}$ & $\begin{array}{c}\text { REC } \\
(U G / L)\end{array}$ & $\begin{array}{l}\text { TOTAL } \\
\text { (UG/L) }\end{array}$ & $\begin{array}{c}\text { TOTAL } \\
\text { (UG/L) }\end{array}$ & $\begin{array}{l}\text { TOTAL } \\
\text { (UG/L) }\end{array}$ & $\begin{array}{c}\text { TOTAL } \\
(U G / L)\end{array}$ \\
\hline
\end{tabular}

\begin{tabular}{|c|c|c|c|c|c|c|c|c|c|c|c|c|}
\hline \multicolumn{13}{|l|}{ FEB 1992} \\
\hline $12-12$ & $<0.20$ & $<0.2$ & $<0.2$ & $<0.2$ & $<0.2$ & $<0.2$ & $<0.20$ & $<5.0$ & $<0.2$ & $<0.2$ & $<0.2$ & $<0.2$ \\
\hline \multicolumn{13}{|l|}{$F E B$} \\
\hline $22-22$ & $<0.20$ & $<0.2$ & $<0.2$ & $<0.2$ & $<0.2$ & $<0.2$ & $<0.20$ & $<5.0$ & $<0.2$ & $<0.2$ & $<0.2$ & $<0.2$ \\
\hline \multicolumn{13}{|l|}{ MAR } \\
\hline $17 \cdot 18$ & $<0.20$ & $<0.2$ & $<0.2$ & $<0.2$ & $<0.2$ & $<0.2$ & $<0.20$ & $<5.0$ & $<0.2$ & $<0.2$ & $<0.2$ & $<0.2$ \\
\hline \multicolumn{13}{|l|}{ APR } \\
\hline $06-06$ & $<0.20$ & $<0.2$ & $<0.2$ & $<0.2$ & $<0.2$ & $<0.2$ & $<0.20$ & $<5.0$ & 0.3 & $<0.2$ & $<0.2$ & $<0.2$ \\
\hline \multicolumn{13}{|l|}{ MAY } \\
\hline $14-14$ & $<0.20$ & $<0.2$ & $<0.2$ & $<0.2$ & $<0.2$ & $<0.2$ & $<0.20$ & $<5.0$ & $<0.2$ & $<0.2$ & $<0.2$ & $<0.2$ \\
\hline \multicolumn{13}{|l|}{ JUN } \\
\hline $06-06$ & $<0.20$ & $<0.2$ & $<0.2$ & $<0.2$ & $<0.2$ & $<0.2$ & $<0.20$ & $<5.0$ & $<0.2$ & $<0.2$ & $<0.2$ & $<0.2$ \\
\hline \multicolumn{13}{|l|}{ SEP } \\
\hline $21-21$ & $<0.20$ & $<0.2$ & $<0.2$ & $<0.2$ & $<0.2$ & $<0.2$ & $<0.20$ & $<5.0$ & $<0.2$ & $<0.2$ & $<0.2$ & $<0.2$ \\
\hline
\end{tabular}


Table 3. Water-quality data for storm-sewer outfall stations, Dallas, Texas, 1992-93-Continued

08057310 - ASH CREEK OUTFALL AT WHITTIER STREET, DALLAS, TX (WY 1992)-Continued

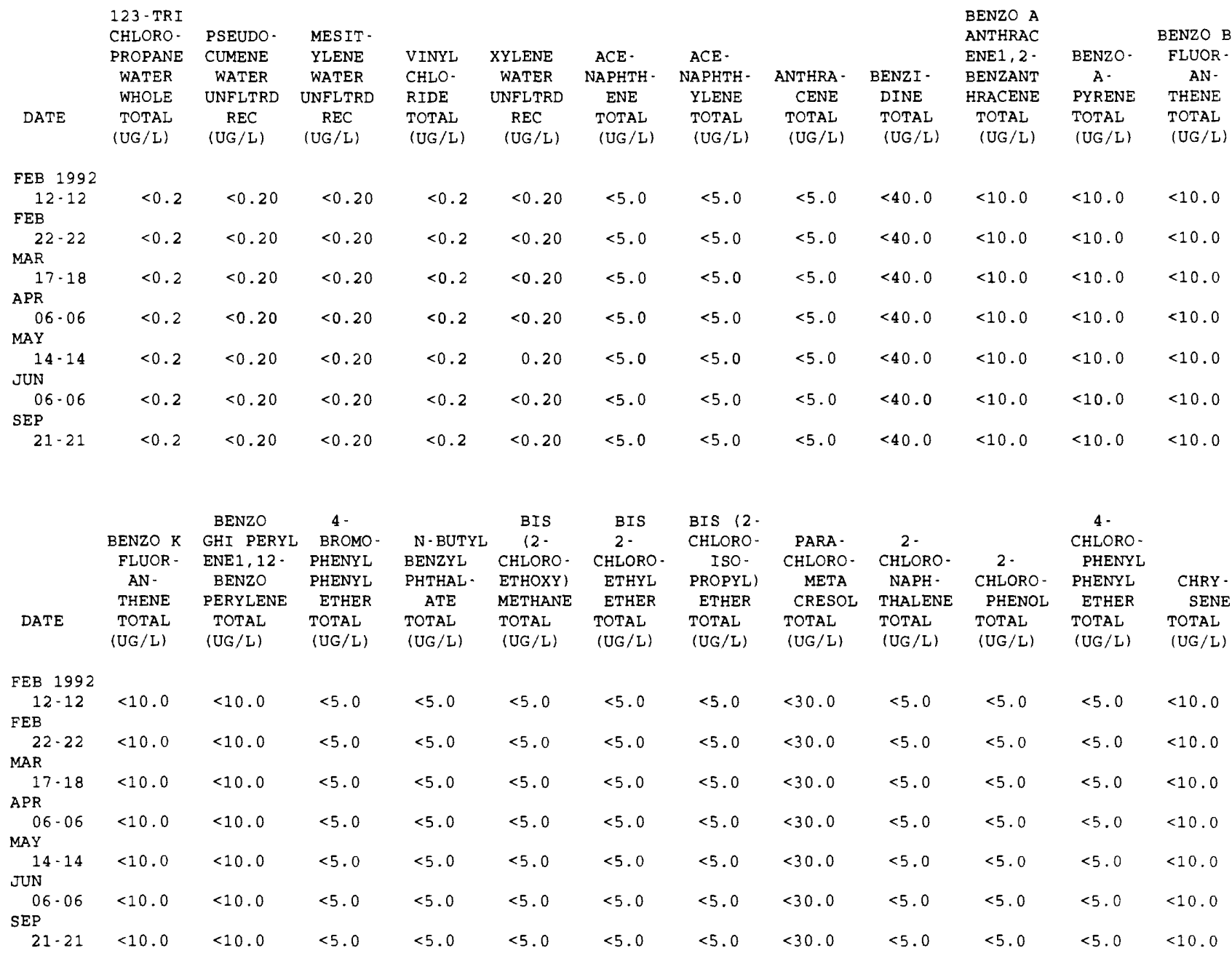

\begin{tabular}{|c|c|c|c|c|c|c|c|c|c|c|c|c|}
\hline DATE & $\begin{array}{l}1,2,5,6- \\
\text { DIBENZ- } \\
\text { ANTHRA- } \\
\text { CENE } \\
\text { TOTAL } \\
\text { (UG/L) }\end{array}$ & $\begin{array}{c}3,3^{\prime}- \\
\text { DI- } \\
\text { CHLORO- } \\
\text { BENZI- } \\
\text { DINE } \\
\text { TOTAL } \\
\text { (UG/L) }\end{array}$ & $\begin{array}{c}2,4 \cdot D I- \\
\text { CHLORO- } \\
\text { PHENOL } \\
\text { TOTAL } \\
\text { (UG/L) }\end{array}$ & $\begin{array}{l}\text { DIETHYL } \\
\text { PHTHAL- } \\
\text { ATE } \\
\text { TOTAL } \\
\text { (UG/L) }\end{array}$ & $\begin{array}{c}\text { DI- } \\
\text { METHYL } \\
\text { PHTHAL- } \\
\text { ATE } \\
\text { TOTAL } \\
\text { (UG/L) }\end{array}$ & $\begin{array}{c}2,4-D I- \\
\text { METHYL- } \\
\text { PHENOL } \\
\text { TOTAL } \\
\text { (UG/L) }\end{array}$ & $\begin{array}{l}\text { DI-N- } \\
\text { BUTYL } \\
\text { PHTHAL- } \\
\text { ATE } \\
\text { TOTAL } \\
\text { (UG/L) }\end{array}$ & $\begin{array}{c}4,6- \\
\text { DINITRO- } \\
\text { ORTHO- } \\
\text { CRESOL } \\
\text { TOTAL } \\
(\mathrm{UG} / \mathrm{L})\end{array}$ & $\begin{array}{c}2,4 \mathbf{1}^{-} \\
\text {DI- } \\
\text { NITRO- } \\
\text { PHENOL } \\
\text { TOTAL } \\
\text { (UG/L) }\end{array}$ & $\begin{array}{l}2,4 \cdot D I- \\
\text { NITRO- } \\
\text { TOLUENE } \\
\text { TOTAL } \\
(U G / L)\end{array}$ & $\begin{array}{l}2,6 \cdot \mathrm{DI}- \\
\text { NITRO- } \\
\text { TOLUENE } \\
\text { TOTAL } \\
\text { (UG/L) }\end{array}$ & $\begin{array}{c}\text { DI-N- } \\
\text { OCTYL } \\
\text { PHTHAL } \\
\text { ATE } \\
\text { TOTAL } \\
\text { (UG/L) }\end{array}$ \\
\hline FEB 1992 & & & & & & & & & & & & \\
\hline $\mathrm{FEB}^{12 \cdot 12}$ & $<10.0$ & $<20.0$ & $<5.0$ & $<5.0$ & $<5.0$ & $<5.0$ & $<5.0$ & $<30.0$ & $<20.0$ & $<5.0$ & $<5.0$ & $<10.0$ \\
\hline $\begin{array}{l}22 \cdot 22 \\
\text { MAR }\end{array}$ & $<10.0$ & $<20.0$ & $<5.0$ & $<5.0$ & $<5.0$ & $<5.0$ & $<5.0$ & $<30.0$ & $<20.0$ & $<5.0$ & $<5.0$ & $<10.0$ \\
\hline${ }_{\text {APR }}^{17-18}$ & $<10.0$ & $<20.0$ & $<5.0$ & $<5.0$ & $<5.0$ & $<5.0$ & $<5.0$ & $<30.0$ & $<20.0$ & $<5.0$ & $<5.0$ & $<10.0$ \\
\hline $\begin{array}{l}06-06 \\
\text { MAY }\end{array}$ & $<10.0$ & $<20.0$ & $<5.0$ & $<5.0$ & $<5.0$ & $<5.0$ & $<5.0$ & $<30.0$ & $<20.0$ & $<5.0$ & $<5.0$ & $<10.0$ \\
\hline${ }_{\text {JUN }}^{14-14}$ & $<10.0$ & $<20.0$ & $<5.0$ & $<5.0$ & $<5.0$ & $<5.0$ & $<5.0$ & $<30.0$ & $<20.0$ & $<5.0$ & $<5.0$ & $<10.0$ \\
\hline $\begin{array}{l}06.06 \\
\text { SEP }\end{array}$ & $<10.0$ & $<20.0$ & $<5.0$ & $<5.0$ & $<5.0$ & $<5.0$ & $<5.0$ & $<30.0$ & $<20.0$ & $<5.0$ & $<5.0$ & $<10.0$ \\
\hline $21-21$ & $<10.0$ & $<20.0$ & $<5.0$ & $<5.0$ & $<5.0$ & $<5.0$ & $<5.0$ & $<30.0$ & $<20.0$ & $<5.0$ & $<5.0$ & $<10.0$ \\
\hline
\end{tabular}


Table 3. Water-quality data for storm-sewer outfall stations, Dallas, Texas, 1992-93-Continued

08057310 - ASH CREEK OUTFALL AT WHITTIER STREET, DALLAS, TX (WY 1992)-Continued

\begin{tabular}{|c|c|c|c|c|c|c|c|c|c|c|c|c|}
\hline DATE & $\begin{array}{c}\text { BIS }(2- \\
\text { ETHYL } \\
\text { HEXYL) } \\
\text { PHTHAL- } \\
\text { ATE } \\
\text { TOTAL } \\
\text { (UG/L) }\end{array}$ & $\begin{array}{l}\text { FLUOR- } \\
\text { ANTHENE } \\
\text { TOTAL } \\
\text { (UG/L) }\end{array}$ & $\begin{array}{c}\text { FLUOR - } \\
\text { ENE } \\
\text { TOTAL } \\
\text { (UG/L) }\end{array}$ & $\begin{array}{l}\text { HEXA- } \\
\text { CHLORO- } \\
\text { BENZENE } \\
\text { TOTAL } \\
\text { (UG/L) }\end{array}$ & $\begin{array}{l}\text { HEXA- } \\
\text { CHLORO- } \\
\text { CYCLO- } \\
\text { PENT- } \\
\text { ADIENE } \\
\text { TOTAL } \\
\text { (UG/L) }\end{array}$ & $\begin{array}{l}\text { HEXA- } \\
\text { CHLORO- } \\
\text { ETHANE } \\
\text { TOTAL } \\
\text { (UG/L) }\end{array}$ & $\begin{array}{c}\text { INDENO } \\
(1,2,3- \\
\text { CD) } \\
\text { PYRENE } \\
\text { TOTAL } \\
(\mathrm{UG} / \mathrm{L})\end{array}$ & $\begin{array}{l}\text { ISO- } \\
\text { PHORONE } \\
\text { TOTAL } \\
\text { (UG/L) }\end{array}$ & $\begin{array}{l}\text { NITRO- } \\
\text { BENZENE } \\
\text { TOTAL } \\
\text { (UG/L) }\end{array}$ & $\begin{array}{l}\text { N-NITRO- } \\
\text { SODI- } \\
\text { METHY - } \\
\text { LAMINE } \\
\text { TOTAL } \\
\text { (UG/L) }\end{array}$ & $\begin{array}{l}2- \\
\text { NITRO- } \\
\text { PHENOL } \\
\text { TOTAL } \\
(\text { UG/L) }\end{array}$ & $\begin{array}{c}4- \\
\text { NITRO- } \\
\text { PHENOL } \\
\text { TOTAL } \\
\text { (UG/L) }\end{array}$ \\
\hline $\begin{array}{r}\text { FEB } 1992 \\
12-12\end{array}$ & 7.0 & $<5.0$ & $<5.0$ & $<5.0$ & $<5.0$ & $<5.0$ & $<10.0$ & $<5.0$ & $<5.0$ & $<5.0$ & $<5.0$ & $<30.0$ \\
\hline $\begin{array}{l}\text { FEB } \\
22 \cdot 22\end{array}$ & $<5.0$ & $<5.0$ & $<5.0$ & $<5.0$ & $<5.0$ & $<5.0$ & $<10.0$ & $<5.0$ & $<5.0$ & $<5.0$ & $<5.0$ & $<30.0$ \\
\hline $\begin{array}{l}\text { MAR } \\
17-18 \\
\text { APR }\end{array}$ & $<5.0$ & $<5.0$ & $<5.0$ & $<5.0$ & $<5.0$ & $<5.0$ & $<10.0$ & $<5.0$ & $<5.0$ & $<5.0$ & $<5.0$ & $<30.0$ \\
\hline $\begin{array}{l}06-06 \\
\text { MAY }\end{array}$ & $<5.0$ & $<5.0$ & $<5.0$ & $<5.0$ & $<5.0$ & $<5.0$ & $<10.0$ & $<5.0$ & $<5.0$ & $<5.0$ & $<5.0$ & $<30.0$ \\
\hline${ }_{\text {JUN }}^{14-14}$ & $<5.0$ & $<5.0$ & $<5.0$ & $<5.0$ & $<5.0$ & $<5.0$ & $<10.0$ & $<5.0$ & $<5.0$ & $<5.0$ & $<5.0$ & $<30.0$ \\
\hline $\begin{array}{l}06-06 \\
\text { SEP }\end{array}$ & $<5.0$ & $<5.0$ & $<5.0$ & $<5.0$ & $<5.0$ & $<5.0$ & $<10.0$ & $<5.0$ & $<5.0$ & $<5.0$ & $<5.0$ & $<30.0$ \\
\hline $21-21$ & $<5.0$ & $<5.0$ & $<5.0$ & $<5.0$ & $<5.0$ & $<5.0$ & $<10.0$ & $<5.0$ & $<5.0$ & $<5.0$ & $<5.0$ & $<30.0$ \\
\hline & $\mathrm{N}$ - & & & & & & & & & & BETA & DELTA \\
\hline & $\begin{array}{l}\text { NITRO- } \\
\text { SODI - N- }\end{array}$ & $\begin{array}{c}\mathrm{N} \cdot \mathrm{NITRO}- \\
\text { SODI - }\end{array}$ & PENTA - & & PHENOL & & $\begin{array}{l}2,4,6- \\
\text { TRI. }\end{array}$ & & & & $\begin{array}{l}\text { BENZENE } \\
\text { HEXA- }\end{array}$ & $\begin{array}{l}\text { BENZENE } \\
\text { HEXA - }\end{array}$ \\
\hline & $\begin{array}{l}\text { PROPYL - } \\
\text { AMINE }\end{array}$ & $\begin{array}{l}\text { PHENY - } \\
\text { LAMINE }\end{array}$ & $\begin{array}{l}\text { CHLORO- } \\
\text { PHENOL }\end{array}$ & $\begin{array}{l}\text { PHENAN- } \\
\text { THRENE }\end{array}$ & $\begin{array}{c}\text { (C6H - } \\
5 \mathrm{OH})\end{array}$ & PYRENE & $\begin{array}{l}\text { CHLORO- } \\
\text { PHENOL }\end{array}$ & ALDRIN, & $\begin{array}{l}\mathrm{P}, \mathrm{P}^{\prime} \\
\mathrm{DDT}\end{array}$ & $\begin{array}{l}\text { ALPHA } \\
\text { BHC }\end{array}$ & $\begin{array}{l}\text { CHLOR - } \\
\text { IDE }\end{array}$ & $\begin{array}{l}\text { CHLOR - } \\
\text { IDE }\end{array}$ \\
\hline DATE & $\begin{array}{c}\text { TOTAL } \\
\text { (UG/L) }\end{array}$ & $\begin{array}{l}\text { TOTAL } \\
\text { (UG/L) }\end{array}$ & $\begin{array}{l}\text { TOTAL } \\
\text { (UG/L) }\end{array}$ & $\begin{array}{l}\text { TOTAL } \\
\text { (UG/L) }\end{array}$ & $\begin{array}{l}\text { TOTAL } \\
\text { (UG/L) }\end{array}$ & $\begin{array}{l}\text { TOTAL } \\
\text { (UG/L) }\end{array}$ & $\begin{array}{l}\text { TOTAL } \\
\text { (UG/L) }\end{array}$ & $\begin{array}{l}\text { TOTAL } \\
\text { (UG/L) }\end{array}$ & $\begin{array}{l}\text { TOTAL } \\
\text { (UG/L) }\end{array}$ & $\begin{array}{l}\text { TOTAL } \\
\text { (UG/L) }\end{array}$ & $\begin{array}{l}\text { TOTAL } \\
\text { (UG/L) }\end{array}$ & $\begin{array}{l}\text { TOTAL } \\
\text { (UG/L) }\end{array}$ \\
\hline FEB 1992 & & & & & & & & & & & & \\
\hline $\begin{array}{l}12-12 \\
F E B\end{array}$ & $<5.0$ & $<5.0$ & $<30.0$ & $<5.0$ & $<5.0$ & $<5.0$ & $<20.0$ & $<0.040$ & $<0.10$ & $<0.03$ & $<0.03$ & $<0.09$ \\
\hline $\begin{array}{l}22-22 \\
\text { MAR }\end{array}$ & $<5.0$ & $<5.0$ & $<30.0$ & $<5.0$ & $<5.0$ & $<5.0$ & $<20.0$ & $<0.040$ & $<0.10$ & $<0.03$ & $<0.03$ & $<0.09$ \\
\hline $\begin{array}{l}17 \cdot 18 \\
A P R\end{array}$ & $<5.0$ & $<5.0$ & $<30.0$ & $<5.0$ & $<5.0$ & $<5.0$ & $<20.0$ & $<0.040$ & $<0.10$ & $<0.03$ & $<0.03$ & $<0.09$ \\
\hline $\begin{array}{l}06-06 \\
\text { MAY }\end{array}$ & $<5.0$ & $<5.0$ & $<30.0$ & $<5.0$ & $<5.0$ & $<5.0$ & $<20.0$ & $<0.040$ & $<0.10$ & $<0.03$ & $<0.03$ & $<0.09$ \\
\hline $\int_{\text {JUN }}^{14-14}$ & $<5.0$ & $<5.0$ & $<30.0$ & $<5.0$ & $<5.0$ & $<5.0$ & $<20.0$ & $<0.040$ & $<0.10$ & $<0.03$ & $<0.03$ & $<0.09$ \\
\hline $\begin{array}{l}06-06 \\
\text { SEP }\end{array}$ & $<5.0$ & $<5.0$ & $<30.0$ & $<5.0$ & $<5.0$ & $<5.0$ & $<20.0$ & $<0.040$ & $<0.10$ & $<0.03$ & $<0.03$ & $<0.09$ \\
\hline $21-21$ & $<5.0$ & $<5.0$ & $<30.0$ & $<5.0$ & $<5.0$ & $<5.0$ & $<20.0$ & $<0.040$ & $<0.10$ & $<0.03$ & $<0.03$ & $<0.09$ \\
\hline
\end{tabular}

\begin{tabular}{|c|c|c|c|c|c|c|c|c|c|c|c|c|}
\hline & & $\begin{array}{l}\text { CHLOR - } \\
\text { DANE }\end{array}$ & $\begin{array}{l}\text { CHLOR- } \\
\text { DANE }\end{array}$ & & & & & $\begin{array}{l}\text { ENDO- } \\
\text { SULFAN- }\end{array}$ & & & & \\
\hline & & CIS & TRANS & & & & & I & ENDO & ENDO- & ENDRIN & ENDRIN \\
\hline & & WATER & WATER & CHLOR - & $P, P^{\prime}$ & $P, P^{\prime}$ & DI - & WATER & SULFAN & SULFAN & WATER & ALDE - \\
\hline & LINDANE & WHOLE & WHOLE & DANE, & DDD & $D D E$ & ELDRIN & WHOLE & BETA & SULFATE & UNFLTRD & HYDE \\
\hline DATE & $\begin{array}{l}\text { TOTAL } \\
\text { (UG/L) }\end{array}$ & $\begin{array}{l}\text { TOTAL } \\
\text { (UG/L) }\end{array}$ & $\begin{array}{l}\text { TOTAL } \\
\text { (UG/L) }\end{array}$ & $\begin{array}{l}\text { TOTAL } \\
\text { (UG/L) }\end{array}$ & $\begin{array}{l}\text { TOTAL } \\
\text { (UG/L) }\end{array}$ & $\begin{array}{l}\text { TOTAL } \\
\text { (UG/L) }\end{array}$ & $\begin{array}{l}\text { TOTAL } \\
\text { (UG/L) }\end{array}$ & $\begin{array}{l}\mathrm{REC} \\
(\mathrm{UG} / \mathrm{L})\end{array}$ & $\begin{array}{l}\text { TOTAL } \\
\text { (UG/L) }\end{array}$ & $\begin{array}{c}\text { TOTAL } \\
\text { (UG/L) }\end{array}$ & $\begin{array}{c}\text { REC } \\
(\mathrm{UG} / \mathrm{L})\end{array}$ & $\begin{array}{l}\text { TOTAL } \\
\text { (UG/L) }\end{array}$ \\
\hline FEB 199 & & & & & & & & & & & & \\
\hline $12-12$ & $<0.030$ & $<0.10$ & $<0.10$ & $<0.1$ & $<0.10$ & $<0.04$ & 0.030 & $<0.10$ & $<0.04$ & $<0.60$ & $<0.060$ & $<0.20$ \\
\hline FEB & & & & & & & & & & & & \\
\hline $22 \cdot 22$ & $<0.030$ & $<0.10$ & $<0.10$ & $<0.1$ & $<0.10$ & $<0.04$ & $<0.020$ & $<0.10$ & $<0.04$ & $<0.60$ & $<0.060$ & $<0.20$ \\
\hline MAR & & & & & & & & & & & & \\
\hline $17-18$ & $<0.030$ & $<0.10$ & $<0.10$ & $<0.1$ & $<0.10$ & $<0.04$ & 0.020 & $<0.10$ & $<0.04$ & $<0.60$ & $<0.060$ & $<0.20$ \\
\hline APR & & & & & & & & & & & & \\
\hline 06.06 & $<0.030$ & $<0.10$ & $<0.10$ & $<0.1$ & $<0.10$ & $<0.04$ & $<0.020$ & $<0.10$ & $<0.04$ & $<0.60$ & $<0.060$ & $<0.20$ \\
\hline MAY & & & & & & & & & & & & \\
\hline $14-14$ & $<0.030$ & $<0.10$ & $<0.10$ & $<0.1$ & $<0.10$ & $<0.04$ & 0.020 & $<0.10$ & $<0.04$ & $<0.60$ & $<0.060$ & $<0.20$ \\
\hline JUN & & & & & & & & & & & & \\
\hline 06.06 & 0.080 & $<0.10$ & $<0.10$ & $<0.1$ & $<0.10$ & $<0.04$ & 0.050 & $<0.10$ & $<0.04$ & $<0.60$ & $<0.060$ & $<0.20$ \\
\hline SEP & & & & & & & & & & & & \\
\hline $21-21$ & $<0.030$ & $<0.10$ & $<0.10$ & $<0.1$ & $<0.10$ & $<0.04$ & 0.020 & $<0.10$ & $<0.04$ & $<0.60$ & $<0.060$ & $<0.20$ \\
\hline
\end{tabular}


Table 3. Water-quality data for storm-sewer outfall stations, Dallas, Texas, 1992-93-Continued 08057310 - ASH CREEK OUTFALL AT WHITTIER STREET, DALLAS, TX (WY 1992)-Continued

\begin{tabular}{|c|c|c|c|c|c|c|c|c|c|c|c|}
\hline & HEPTA - & $\begin{array}{l}\text { HEPTA - } \\
\text { CHLOR }\end{array}$ & TOX - & AROCLOR & AROCLOR & AROCLOR & AROCLOR & $\begin{array}{c}\text { AROCLOR } \\
1248\end{array}$ & $\begin{array}{l}\text { AROCLOR } \\
1254\end{array}$ & $\begin{array}{l}\text { AROCLOR } \\
1260\end{array}$ & $D I$ \\
\hline & CHLOR, & EPOXIDE & APHENE, & PCB & PCB & PCB & PCB & PCB & PCB & PCB & AZINON, \\
\hline DATE & $\begin{array}{l}\text { TOTAL } \\
\text { (UG/L) }\end{array}$ & $\begin{array}{l}\text { TOTAL } \\
\text { (UG/I) }\end{array}$ & $\begin{array}{l}\text { TOTAL } \\
\text { (UG/L) }\end{array}$ & $\begin{array}{l}\text { TOTAL } \\
\text { (UG/L) }\end{array}$ & $\begin{array}{l}\text { TOTAL } \\
\text { (UG/L) }\end{array}$ & $\begin{array}{l}\text { TOTAL } \\
\text { (UG/L) }\end{array}$ & $\begin{array}{l}\text { TOTAL } \\
\text { (UG/L) }\end{array}$ & $\begin{array}{l}\text { TOTAL } \\
\text { (UG/L) }\end{array}$ & $\begin{array}{l}\text { TOTAL } \\
\text { (UG/L) }\end{array}$ & $\begin{array}{l}\text { TOTAL } \\
\text { (UG/L) }\end{array}$ & $\begin{array}{l}\text { TOTAL } \\
\text { (UG/L) }\end{array}$ \\
\hline
\end{tabular}

\begin{tabular}{|c|c|c|c|c|c|c|c|c|c|c|c|}
\hline \multicolumn{12}{|l|}{ FEB 1992} \\
\hline $12-12$ & $<0.030$ & $<0.80$ & $<2$ & $<0.1$ & $<1.0$ & $<0.1$ & $<0.1$ & $<0.1$ & $<0.1$ & $<0.1$ & $\cdots$ \\
\hline \multicolumn{12}{|l|}{ FEB } \\
\hline $22-22$ & $<0.030$ & $<0.80$ & $<2$ & $<0.1$ & $<1.0$ & $<0.1$ & $<0.1$ & $<0.1$ & $<0.1$ & $<0.1$ & 0.66 \\
\hline \multicolumn{12}{|l|}{ MAR } \\
\hline $17-18$ & $<0.030$ & $<0.80$ & $<2$ & $<0.1$ & $<1.0$ & $<0.1$ & $<0.1$ & $<0.1$ & $<0.1$ & $<0.1$ & 1.2 \\
\hline \multicolumn{12}{|l|}{$A P R$} \\
\hline 06.06 & $<0.030$ & $<0.80$ & $<2$ & $<0.1$ & $<1.0$ & $<0.1$ & $<0.1$ & $<0.1$ & $<0.1$ & $<0.1$ & 0.70 \\
\hline \multicolumn{12}{|l|}{ MAY } \\
\hline $14-14$ & $<0.030$ & $<0.80$ & $<2$ & $<0.1$ & $<1.0$ & $<0.1$ & $<0.1$ & $<0.1$ & $<0.1$ & $<0.1$ & 0.60 \\
\hline \multicolumn{12}{|l|}{ JUN } \\
\hline 06.06 & $<0.030$ & $<0.80$ & $<2$ & $<0.1$ & $<1.0$ & $<0.1$ & $<0.1$ & $<0.1$ & $<0.1$ & $<0.1$ & 3.3 \\
\hline \multicolumn{12}{|l|}{ SEP } \\
\hline $21-21$ & $<0.030$ & $<0.80$ & $<2$ & $<0.1$ & $<1.0$ & $<0.1$ & $<0.1$ & $<0.1$ & $<0.1$ & $<0.1$ & 2.0 \\
\hline
\end{tabular}


Table 3. Water-quality data for storm-sewer outfall stations, Dallas, Texas, 1992-93-Continued

08057441 - NEWTON CREEK OUTFALL AT TIOGA STREET, DALLAS, TX (WY 1992)

\begin{tabular}{|c|c|c|c|c|c|c|c|c|c|c|c|c|}
\hline DATE & TIME & $\begin{array}{l}\text { PRECIP- } \\
\text { ITATION } \\
\text { TOTAL } \\
\text { INCHES/ } \\
\text { STORM }\end{array}$ & $\begin{array}{l}\text { ELAPSED } \\
\text { TIME } \\
\text { OF } \\
\text { STORM } \\
\text { (HOURS) }\end{array}$ & $\begin{array}{l}\text { STORM } \\
\text { WATER } \\
\text { FLOW } \\
\text { (MGD) }\end{array}$ & $\begin{array}{l}\text { SPE - } \\
\text { CIFIC } \\
\text { CON- } \\
\text { DUCT - } \\
\text { ANCE } \\
\text { (US/CM) }\end{array}$ & $\begin{array}{c}\text { SPE- } \\
\text { CIFIC } \\
\text { CON- } \\
\text { DUCT- } \\
\text { ANCE } \\
\text { LAB } \\
\text { (US/CM) }\end{array}$ & $\begin{array}{c}\text { PH } \\
\text { WATER } \\
\text { WHOLE } \\
\text { FIELD } \\
\text { (STAND- } \\
\text { ARD } \\
\text { UNITS) }\end{array}$ & $\begin{array}{c}\text { PH } \\
\text { WATER } \\
\text { WHOLE } \\
\text { LAB } \\
\text { (STAND- } \\
\text { ARD } \\
\text { UNITS) }\end{array}$ & $\begin{array}{c}\text { TEMPER - } \\
\text { ATURE } \\
\text { WATER } \\
\text { (DEG C) }\end{array}$ & $\begin{array}{c}\text { OXYGEN } \\
\text { DEMAND, } \\
\text { CHEM- } \\
\text { ICAL } \\
\text { (HIGH } \\
\text { LEVEL) } \\
\text { (MG/L) }\end{array}$ & $\begin{array}{l}\text { OXYGEN } \\
\text { DEMAND, } \\
\text { BIO- } \\
\text { CHEM- } \\
\text { ICAL, } \\
5 \text { DAY } \\
\text { (MG } / \mathrm{L})\end{array}$ & $\begin{array}{l}\text { COLI- } \\
\text { FORM, } \\
\text { FECAL, } \\
0.7 \\
\text { UM-MF } \\
\text { (COLS. / } \\
100 \mathrm{ML} \text { ) }\end{array}$ \\
\hline FEB 1992 & & & & & & & & & & & & \\
\hline $\begin{array}{l}22-22 \\
\text { MAR }\end{array}$ & 0118 & 0.70 & 2.5 & 0.18 & 480 & 196 & 7.4 & 8.0 & 16.0 & 92 & 6.5 & 2200 \\
\hline $\begin{array}{l}09 \cdot 09 \\
\text { APR }\end{array}$ & 0255 & 0.47 & 0.80 & 0.14 & 48 & 215 & 8.5 & 7.9 & 18.0 & 110 & 8.1 & 220000 \\
\hline $\begin{array}{l}06-06 \\
\text { APR }\end{array}$ & 0051 & 0.41 & 5.4 & 0.09 & 77 & 109 & 7.4 & 7.3 & 16.5 & 1300 & 8.0 & 7000 \\
\hline $\mathrm{JUL}^{28-29}$ & 2355 & 0.30 & 0.90 & 0.09 & 72 & 152 & 8.3 & 7.9 & 19.5 & 140 & 3.9 & $>200000$ \\
\hline $\operatorname{SEP}^{18 \cdot 18}$ & 1344 & 0.56 & 2.0 & 0.12 & 78 & 102 & 8.2 & 7.6 & 27.0 & 59 & $\cdots$ & 46000 \\
\hline $\begin{array}{l}01-01 \\
\operatorname{SEP}\end{array}$ & 1324 & 0.34 & 0.60 & 0.08 & 70 & 235 & 8.1 & 7.5 & 27.0 & 110 & 12 & 490000 \\
\hline $21 \cdot 21$ & 0155 & 0.91 & 2.1 & 0.24 & 110 & 58 & 7.4 & 6.7 & 24.5 & 100 & 6.8 & $\mathrm{~K} 260000$ \\
\hline
\end{tabular}

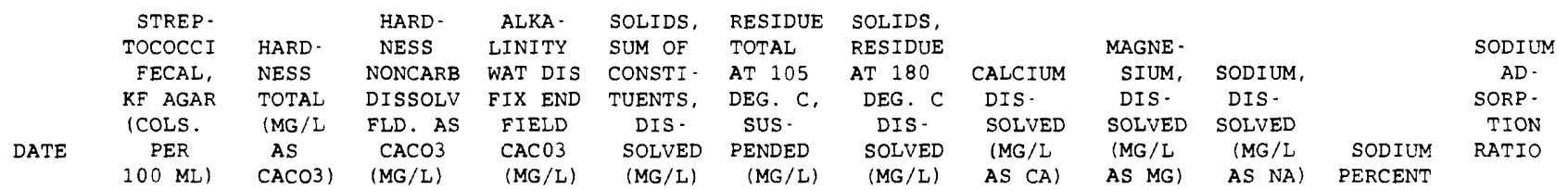

\begin{tabular}{|c|c|c|c|c|c|c|c|c|c|c|c|c|}
\hline FEB $\quad 1992$ & & & & & & & & & & & & \\
\hline $22-22$ & 25000 & 28 & 1 & 27 & 73 & 209 & 53 & 11 & 0.23 & 0.90 & 6 & 0.1 \\
\hline MAR & & & & & & & & & & & & \\
\hline 09.09 & 220000 & 28 & 4 & 24 & 62 & 230 & 40 & 11 & 0.24 & 1.6 & 10 & 0.1 \\
\hline APR & & & & & & & & & & & & \\
\hline 06.06 & 29000 & 33 & 0 & 33 & 54 & 59 & 61 & 13 & 0.21 & 1.4 & 8 & 0.1 \\
\hline APR & & & & & & & & & & & & \\
\hline $28-29$ & $>330000$ & 26 & 6 & 20 & 66 & 228 & 66 & 10 & 0.24 & 1.0 & 7 & 0.1 \\
\hline JUL & & & & & & & & & & & & \\
\hline $18-18$ & 16000 & 29 & 6 & 23 & 55 & 100 & 60 & 11 & 0.26 & 1.2 & 8 & 0.1 \\
\hline SEP & & & & & & & & & & & & \\
\hline 01.01 & 260000 & 22 & 6 & 16 & 82 & 365 & 35 & 8.3 & 0.22 & 0.90 & 7 & 0.1 \\
\hline SEP & & & & & & & & & & & & \\
\hline $21-21$ & K300000 & 21 & 1 & 20 & 32 & 5 & 959 & 8.1 & 0.23 & 0.90 & 7 & 0.1 \\
\hline
\end{tabular}

\begin{tabular}{|c|c|c|c|c|c|c|c|c|c|c|c|c|}
\hline DATE & $\begin{array}{l}\text { POTAS- } \\
\text { SIUM, } \\
\text { DIS- } \\
\text { SOLVED } \\
\text { (MG/L } \\
\text { AS K) }\end{array}$ & $\begin{array}{l}\text { SULFATE } \\
\text { DIS- } \\
\text { SOLVED } \\
\text { (MG/L } \\
\text { AS SO4) }\end{array}$ & $\begin{array}{l}\text { CHLO- } \\
\text { RIDE, } \\
\text { DIS- } \\
\text { SOLVED } \\
\text { (MG/L } \\
\text { AS CL) }\end{array}$ & $\begin{array}{l}\text { NITRO- } \\
\text { GEN, } \\
\text { NITRITE } \\
\text { TOTAL } \\
\text { (MG /L } \\
\text { AS N) }\end{array}$ & $\begin{array}{l}\text { NITRO- } \\
\text { GEN, } \\
\text { NO2 +NO3 } \\
\text { TOTAL } \\
\text { (MG/L } \\
\text { AS N) }\end{array}$ & $\begin{array}{l}\text { NITRO- } \\
\text { GEN, } \\
\text { AMMONIA } \\
\text { TOTAL } \\
\text { (MG/L } \\
\text { AS N) }\end{array}$ & $\begin{array}{l}\text { NITRO- } \\
\text { GEN, AM- } \\
\text { MONIA + } \\
\text { ORGANIC } \\
\text { TOTAL } \\
\text { (MG/L } \\
\text { AS N) }\end{array}$ & $\begin{array}{l}\text { PHOS- } \\
\text { PHORUS } \\
\text { TOTAL } \\
\text { (MG/L } \\
\text { AS P) }\end{array}$ & $\begin{array}{l}\text { PHOS - } \\
\text { PHORUS } \\
\text { DIS - } \\
\text { SOLVED } \\
\text { (MG/L } \\
\text { AS P) }\end{array}$ & $\begin{array}{l}\text { ANT IMONY } \\
\text { TOTAL } \\
\text { EPA } \\
\text { (UG/L } \\
\text { AS SB) }\end{array}$ & $\begin{array}{c}\text { ARSENIC } \\
\text { TOTAL } \\
\text { (UG/L } \\
\text { AS AS) }\end{array}$ & $\begin{array}{l}\text { BERYL- } \\
\text { LIUM, } \\
\text { TOTAL } \\
\text { RECOV- } \\
\text { ERABLE } \\
\text { (UG /L } \\
\text { AS BE) }\end{array}$ \\
\hline & AS K) & AS SO4) & AS CL) & AS N) & AS N) & AS N) & AS N) & AS P) & AS P) & & AS AS) & \\
\hline
\end{tabular}

\begin{tabular}{|c|c|c|c|c|c|c|c|c|c|c|c|c|}
\hline $\begin{array}{c}\text { FEB } 1992 \\
22-22\end{array}$ & 19 & 40 & 14 & 0040 & 0330 & 0120 & 10 & & & & & \\
\hline MAR & & & & & & & & 0.300 & 0.200 & 80.0 & & \\
\hline $09-09$ & 1.9 & 5.2 & 1.6 & 0.040 & 0.390 & 0.140 & 1.7 & 0.350 & 0.120 & $<20.0$ & 2 & $<10$ \\
\hline APR & & & & & & & & & & & & \\
\hline 06.06 & 2.6 & 3.7 & 0.90 & 0.040 & 0.380 & 0.300 & 1.1 & 0.280 & 0.190 & $<10.0$ & 1 & $<10$ \\
\hline $\begin{array}{l}\text { APR } \\
28 \cdot 29 \\
\text { JUL }\end{array}$ & 2.8 & 4.6 & 0.70 & 0.030 & 0.470 & 0.290 & 1.5 & 0.300 & 0.200 & $<10.0$ & $<1$ & $<10$ \\
\hline $\begin{array}{l}18-18 \\
\text { SEP }\end{array}$ & 2.8 & 2.8 & 1.2 & 0.030 & 0.390 & 0.120 & 1.7 & 0.370 & 0.200 & $<10.0$ & 3 & $<10$ \\
\hline $\begin{array}{l}\text { 01-01 } \\
\text { SEP }\end{array}$ & 2.7 & 2.4 & 0.60 & 0.020 & 0.270 & 0.110 & 1.1 & 0.290 & 0.170 & $<20.0$ & 2 & $<10$ \\
\hline 21-21 & 3.2 & 3.5 & 2.6 & 0.020 & 0.340 & 0.130 & 2.0 & 0.530 & 0.230 & $<10.0$ & 2 & $<10$ \\
\hline
\end{tabular}


Table 3. Water-quality data for storm-sewer outfall stations, Dallas, Texas, 1992-93-Continued 08057441 - NEWTON CREEK OUTFALL AT TIOGA STREET, DALLAS, TX (WY 1992)-Continued

\begin{tabular}{|c|c|c|c|c|c|c|c|c|c|c|c|c|}
\hline DATE & $\begin{array}{l}\text { CADMIUM } \\
\text { TOTAL } \\
\text { RECOV- } \\
\text { ERABLE } \\
\text { (UG/L }\end{array}$ & $\begin{array}{l}\text { CHRO- } \\
\text { MIUM, } \\
\text { TOTAL } \\
\text { RECOV- } \\
\text { ERABLE } \\
\text { (UG / L }\end{array}$ & $\begin{array}{c}\text { COPPER, } \\
\text { TOTAL } \\
\text { RECOV- } \\
\text { ERABLE } \\
\text { (UG / L }\end{array}$ & $\begin{array}{c}\text { CYANIDE } \\
\text { TOTAL } \\
\text { EPA } \\
\text { /MG/L }\end{array}$ & $\begin{array}{c}\text { CYANIDE } \\
\text { TOTAL } \\
\text { (MG/L }\end{array}$ & $\begin{array}{l}\text { LEAD, } \\
\text { TOTAL } \\
\text { RECOV - } \\
\text { ERABLE } \\
\text { (UG / L }\end{array}$ & $\begin{array}{l}\text { MERCURY } \\
\text { TOTAL } \\
\text { RECOV - } \\
\text { ERABLE } \\
\text { (UG /L }\end{array}$ & $\begin{array}{c}\text { NICKEL, } \\
\text { TOTAL } \\
\text { RECOV - } \\
\text { ERABLE } \\
\text { /UG /L }\end{array}$ & $\begin{array}{l}\text { SELE- } \\
\text { NIUM, } \\
\text { TOTAL } \\
\text { (UG/L }\end{array}$ & $\begin{array}{c}\text { SILVER, } \\
\text { TOTAL } \\
\text { RECOV- } \\
\text { ERABLE } \\
\text { (UG/L }\end{array}$ & $\begin{array}{l}\text { SILVER, } \\
\text { TOTAL } \\
\text { RECOV- } \\
\text { ERABLE } \\
\text { EPA } \\
\text { (UG/L }\end{array}$ & $\begin{array}{l}\text { THAL - } \\
\text { LIUM, } \\
\text { TOTAL } \\
\text { (UG/L }\end{array}$ \\
\hline & AS CD) & AS (R) & AS CU) & AS $\mathrm{CN}$ ) & AS $(\mathrm{N})$ & $A S \quad P B)$ & AS HG) & AS NI) & AS SE) & AS AG) & $A S \quad A G)$ & AS TL) \\
\hline \multicolumn{13}{|l|}{ FEB 1992} \\
\hline $22 \cdot 22$ & $<1$ & 3 & 12 & $<0.010$ & $<0.010$ & 48 & $<0.10$ & 8 & $<2$ & $<1$ & $<1.00$ & $<5$ \\
\hline \multicolumn{13}{|l|}{ MAR } \\
\hline 09.09 & $<1$ & 7 & 14 & $<0.010$ & $<0.010$ & 46 & $<0.10$ & 9 & $<2$ & $<1$ & $<1.00$ & $<10$ \\
\hline \multicolumn{13}{|l|}{ APR } \\
\hline 06.06 & $<1$ & 3 & 11 & $<0.010$ & $<0.010$ & 17 & $<0.10$ & 2 & $<2$ & $<1$ & $<1.00$ & $<10$ \\
\hline \multicolumn{13}{|l|}{ APR } \\
\hline $28-29$ & $<1$ & 5 & 13 & $<0.010$ & $<0.010$ & 48 & $<0.10$ & 12 & $<2$ & $<1$ & $<1.00$ & $<5$ \\
\hline \multicolumn{13}{|l|}{ JUL } \\
\hline $18-18$ & $<1$ & 1 & 7 & $<10.0$ & $<0.010$ & 41 & $<0.10$ & 5 & $<2$ & $<1$ & $<0.500$ & $<5$ \\
\hline \multicolumn{13}{|l|}{ SEP } \\
\hline $01-01$ & $<1$ & 7 & 13 & 0.140 & $<0.010$ & 39 & $<0.10$ & 13 & $<2$ & $<1$ & $<0.500$ & $<5$ \\
\hline \multicolumn{13}{|l|}{ SEP } \\
\hline $21 \cdot 21$ & $<1$ & 10 & 14 & $<0.010$ & $<0.010$ & 57 & $<0.10$ & 18 & $<2$ & $<1$ & $<0.500$ & $<10$ \\
\hline
\end{tabular}

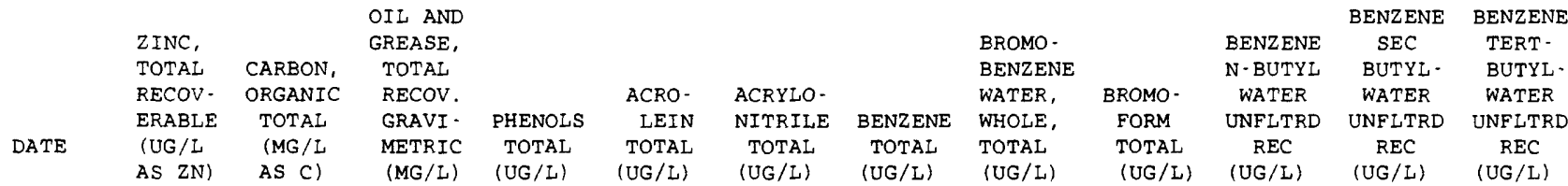

\begin{tabular}{|c|c|c|c|c|c|c|c|c|c|c|c|c|}
\hline \multicolumn{13}{|l|}{ FEB 1992} \\
\hline $22-22$ & 100 & 20 & 1 & 1 & $<20$ & $<20$ & $<0.2$ & $<0.2$ & $<0.2$ & $<0.20$ & $<0.20$ & $<0.20$ \\
\hline \multicolumn{13}{|l|}{ MAR } \\
\hline 09.09 & 180 & 27 & $<1$ & 3 & $<20$ & $<20$ & $<0.2$ & $<0.2$ & $<0.2$ & $<0.20$ & $<0.20$ & $<0.20$ \\
\hline \multicolumn{13}{|l|}{ APR } \\
\hline 06.06 & 70 & 370 & 4 & 6 & $<20$ & $<20$ & $<0.2$ & $<0.2$ & $<0.2$ & $<0.20$ & $<0.20$ & $<0.20$ \\
\hline \multicolumn{13}{|l|}{ APR } \\
\hline $28-29$ & 130 & 28 & 8 & 3 & $<20$ & $<20$ & $<0.2$ & $<0.2$ & $<0.2$ & $<0.20$ & $<0.20$ & $<0.20$ \\
\hline \multicolumn{13}{|l|}{ JUL } \\
\hline $18-18$ & 60 & 26 & 6 & 4 & $<20$ & $<20$ & $<0.2$ & $<0.2$ & $<0.2$ & $<0.20$ & $<0.20$ & $<0.20$ \\
\hline \multicolumn{13}{|l|}{ SEP } \\
\hline 01.01 & 140 & 40 & 10 & 4 & $<20$ & $<20$ & $<0.2$ & $<0.2$ & $<0.2$ & $<0.20$ & $<0.20$ & $<0.20$ \\
\hline \multicolumn{13}{|l|}{ SEP } \\
\hline $21-21$ & 170 & 37 & 5 & 8 & $<20$ & $<20$ & $<0.2$ & $<0.2$ & $<0.2$ & $<0.20$ & $<0.20$ & $<0.20$ \\
\hline
\end{tabular}

\begin{tabular}{|c|c|c|c|c|c|c|c|c|c|c|c|c|}
\hline & & & & & $2-$ & & & 0 & & DIBROMO & DI - & $1,2-$ \\
\hline & CARBON - & & CHLORO - & & CHLORO - & & & CHLORO- & TOLUENE & CHLORO - & BROMO - & DIBROMO \\
\hline & TETRA - & & DI. & & ETHYL - & & METHYL - & TOLUENE & P-CHLOR & PROPANE & METHANE & ETHANE \\
\hline & CHLO - & CHLORO - & BROMO - & CHLORO - & VINYL - & CHLORO - & CHLO. & WATER & WATER & WATER & WATER & WATER \\
\hline & RIDE & BENZENE & METHANE & ETHANE & ETHER & FORM & RIDE & WHOLE & UNFLTRD & WHOLE & WHOLE & WHOLE \\
\hline DATE & $\begin{array}{l}\text { TOTAL } \\
\text { (UG/I) }\end{array}$ & $\begin{array}{l}\text { TOTAL } \\
\text { (UG /L) }\end{array}$ & $\begin{array}{l}\text { TOTAL } \\
\text { (UG/L) }\end{array}$ & $\begin{array}{l}\text { TOTAL } \\
\text { (UG/L) }\end{array}$ & $\begin{array}{l}\text { TOTAL } \\
\text { (UG/L) }\end{array}$ & $\begin{array}{l}\text { TOTAL } \\
\text { (UG/L) }\end{array}$ & $\begin{array}{l}\text { TOTAL } \\
\text { (UG/L) }\end{array}$ & $\begin{array}{r}\text { TOTAL } \\
(U G / L)\end{array}$ & $\begin{array}{c}\text { REC } \\
\text { (UG/I) }\end{array}$ & $\begin{array}{l}\text { TOT . REC } \\
\text { (UG/I) }\end{array}$ & $\begin{array}{l}\text { RECOVER } \\
\text { (UG/L) }\end{array}$ & $\begin{array}{l}\text { TOTAL } \\
\text { (UG/L) }\end{array}$ \\
\hline
\end{tabular}

\begin{tabular}{|c|c|c|c|c|c|c|c|c|c|c|c|c|}
\hline \multicolumn{13}{|l|}{ FEB 1992} \\
\hline $22-22$ & $<0.2$ & $<0.20$ & $<0.2$ & $<0.2$ & $<1.0$ & $<0.2$ & $<0.2$ & $<0.2$ & $<0.20$ & $<1.0$ & $<0.2$ & $<0.2$ \\
\hline \multicolumn{13}{|l|}{ MAR } \\
\hline 09.09 & $<0.2$ & $<0.20$ & $<0.2$ & $<0.2$ & $<1.0$ & $<0.2$ & $<0.2$ & $<0.2$ & $<0.20$ & $<1.0$ & $<0.2$ & $<0.2$ \\
\hline \multicolumn{13}{|l|}{ APR } \\
\hline 06.06 & $<0.2$ & $<0.20$ & $<0.2$ & $<0.2$ & $<1.0$ & $<0.2$ & $<0.2$ & $<0.2$ & $<0.20$ & $<1.0$ & $<0.2$ & $<0.2$ \\
\hline \multicolumn{13}{|l|}{ APR } \\
\hline $28 \cdot 29$ & $<0.2$ & $<0.20$ & $<0.2$ & $<0.2$ & $<1.0$ & $<0.2$ & $<0.2$ & $<0.2$ & $<0.20$ & $<1.0$ & $<0.2$ & $<0.2$ \\
\hline \multicolumn{13}{|l|}{ JUL } \\
\hline $18-18$ & $<0.2$ & $<0.20$ & $<0.2$ & $<0.2$ & $<1.0$ & $<0.2$ & $<0.2$ & $<0.2$ & $<0.20$ & $<1.0$ & $<0.2$ & $<0.2$ \\
\hline \multicolumn{13}{|l|}{ SEP } \\
\hline $01 \cdot 01$ & $<0.2$ & $<0.20$ & $<0.2$ & $<0.2$ & $<1.0$ & $<0.2$ & $<0.2$ & $<0.2$ & $<0.20$ & $<1.0$ & $<0.2$ & $<0.2$ \\
\hline \multicolumn{13}{|l|}{ SEP } \\
\hline $21-21$ & $<0.2$ & $<0.20$ & $<0.2$ & $<0.2$ & $<1.0$ & $<0.2$ & $<0.2$ & $<0.2$ & $<0.20$ & $<1.0$ & $<0.2$ & $<0.2$ \\
\hline
\end{tabular}


Table 3. Water-quality data for storm-sewer outfall stations, Dallas, Texas, 1992-93-Continued

08057441 - NEWTON CREEK OUTFALL AT TIOGA STREET, DALLAS, TX (WY 1992)-Continued

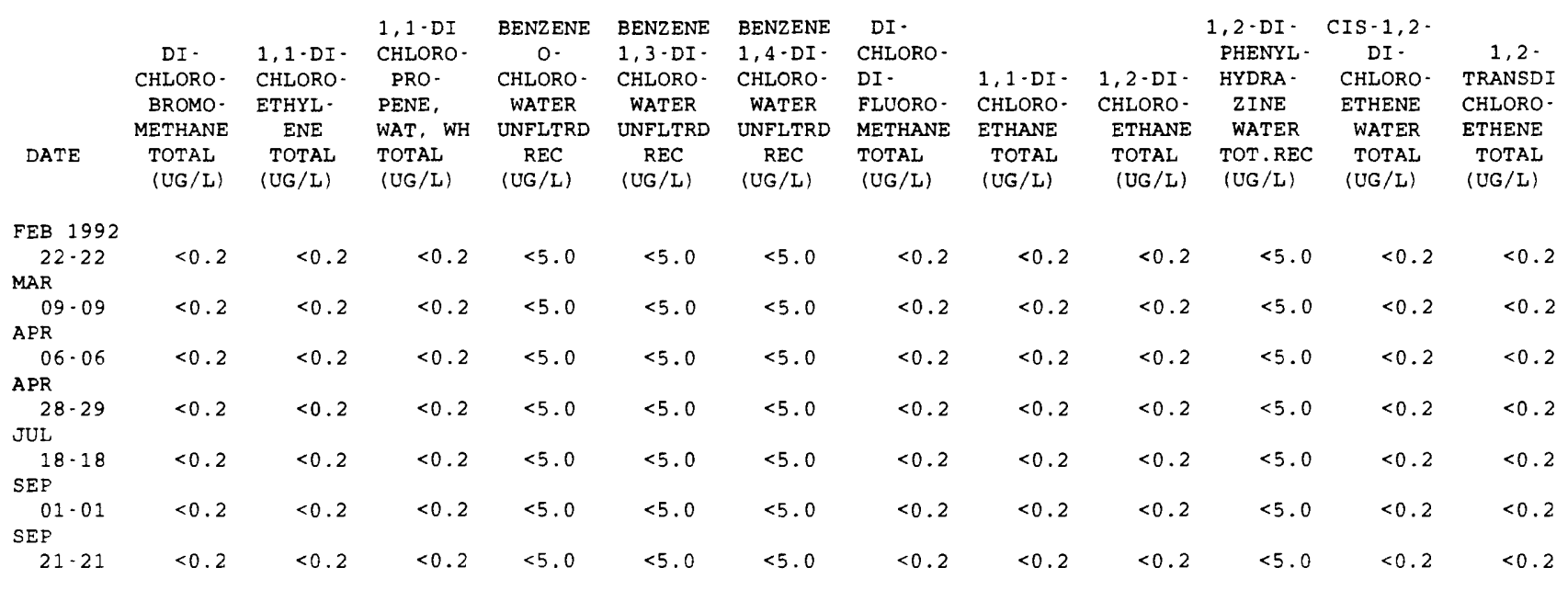

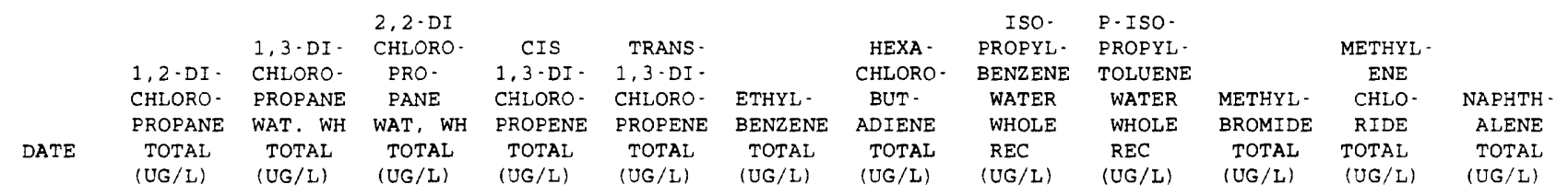

\begin{tabular}{|c|c|c|c|c|c|c|c|c|c|c|c|c|}
\hline \multicolumn{13}{|c|}{ FEB $\quad 1992$} \\
\hline $22 \cdot 22$ & $<0.2$ & $<0.2$ & $<0.2$ & $<0.2$ & $<0.2$ & $<0.2$ & $<5.0$ & $<0.20$ & $<0.20$ & $<0.2$ & $<0.2$ & $<5.0$ \\
\hline \multicolumn{13}{|l|}{ MAR } \\
\hline 09.09 & $<0.2$ & $<0.2$ & $<0.2$ & $<0.2$ & $<0.2$ & $<0.2$ & $<5.0$ & $<0.20$ & $<0.20$ & $<0.2$ & $<0.2$ & $<5.0$ \\
\hline \multicolumn{13}{|l|}{ APR } \\
\hline 06.06 & $<0.2$ & $<0.2$ & $<0.2$ & $<0.2$ & $<0.2$ & $<0.2$ & $<5.0$ & $<0.20$ & $<0.20$ & $<0.2$ & $<0.2$ & $<5.0$ \\
\hline \multicolumn{13}{|l|}{ APR } \\
\hline $28-29$ & $<0.2$ & $<0.2$ & $<0.2$ & $<0.2$ & $<0.2$ & $<0.2$ & $<5.0$ & $<0.20$ & $<0.20$ & $<0.2$ & $<0.2$ & $<5.0$ \\
\hline \multicolumn{13}{|l|}{ JUL } \\
\hline $18-18$ & $<0.2$ & $<0.2$ & $<0.2$ & $<0.2$ & $<0.2$ & $<0.2$ & $<5.0$ & $<0.20$ & $<0.20$ & $<0.2$ & $<0.2$ & $<5.0$ \\
\hline \multicolumn{13}{|l|}{ SEP } \\
\hline 01.01 & $<0.2$ & $<0.2$ & $<0.2$ & $<0.2$ & $<0.2$ & $<0.2$ & $<5.0$ & $<0.20$ & $<0.20$ & $<0.2$ & $<0.2$ & $<5.0$ \\
\hline \multicolumn{13}{|l|}{ SEP } \\
\hline $21-21$ & $<0.2$ & $<0.2$ & $<0.2$ & $<0.2$ & $<0.2$ & $<0.2$ & $<5.0$ & $<0.20$ & $<0.20$ & $<0.2$ & $<0.2$ & $<5.0$ \\
\hline
\end{tabular}

\begin{tabular}{|c|c|c|c|c|c|c|c|c|c|c|c|c|}
\hline DATE & $\begin{array}{l}\text { BENZENE } \\
\text { N-PROPY } \\
\text { WATER } \\
\text { UNFLTRD } \\
\text { REC } \\
\text { (UG/L) }\end{array}$ & $\begin{array}{c}\text { STYRENE } \\
\text { TOTAL } \\
\text { (UG/L) }\end{array}$ & $\begin{array}{l}\text { ETHANE, } \\
1112- \\
\text { TETRA- } \\
\text { CHLORO- } \\
\text { WAT UNF } \\
\text { REC } \\
\text { (UG/L) }\end{array}$ & $\begin{array}{l}\text { ETHANE, } \\
1,1,2,2 \\
\text { TETRA- } \\
\text { CHLORO- } \\
\text { WAT UNF } \\
\text { REC } \\
(\text { UG/L) }\end{array}$ & $\begin{array}{l}\text { TETRA- } \\
\text { CHLORO- } \\
\text { ETHYL- } \\
\text { ENE } \\
\text { TOTAL } \\
\text { (UG/L) }\end{array}$ & $\begin{array}{c}\text { TOLUENE } \\
\text { TOTAL } \\
\text { (UG/L) }\end{array}$ & $\begin{array}{l}1,2,3- \\
\text { TRI- } \\
\text { CHLORO } \\
\text { BENZENE } \\
\text { WAT, WH } \\
\text { REC } \\
\text { (UG/L) }\end{array}$ & $\begin{array}{c}\text { BENZENE } \\
1,2,4- \\
\text { TRI - } \\
\text { CHLORO- } \\
\text { WAT UNF } \\
\text { REC } \\
\text { (UG/L) }\end{array}$ & $\begin{array}{l}1,1,1- \\
\text { TRI- } \\
\text { CHLORO- } \\
\text { ETHANE } \\
\text { TOTAL } \\
\text { (UG/L) }\end{array}$ & $\begin{array}{c}1,1,2- \\
\text { TRI- } \\
\text { CHLORO- } \\
\text { ETHANE } \\
\text { TOTAL } \\
\text { (UG/L) }\end{array}$ & $\begin{array}{l}\text { TRI - } \\
\text { CHLORO- } \\
\text { ETHYL- } \\
\text { ENE } \\
\text { TOTAL } \\
\text { (UG/L) }\end{array}$ & $\begin{array}{l}\text { TRI- } \\
\text { CHLORO- } \\
\text { FLUORO- } \\
\text { METHANE } \\
\text { TOTAL } \\
\text { (UG/L) }\end{array}$ \\
\hline $\begin{array}{c}\text { FEB } 1992 \\
22-22\end{array}$ & $<0,20$ & 20 & $<0 ?$ & $<0 ?$ & $<0 ?$ & & & & & & & \\
\hline MAR & & & & & & & & & & & & \\
\hline $\begin{array}{l}09-09 \\
\text { APR }\end{array}$ & $<0.20$ & $<0.2$ & $<0.2$ & $<0.2$ & $<0.2$ & $<0.2$ & $<0.20$ & $<5.0$ & $<0.2$ & $<0.2$ & $<0.2$ & $<0.2$ \\
\hline $\begin{array}{l}06.06 \\
\text { APR }\end{array}$ & $<0.20$ & $<0.2$ & $<0.2$ & $<0.2$ & $<0.2$ & $<0.2$ & $<0.20$ & $<5.0$ & $<0.2$ & $<0.2$ & $<0.2$ & $<0.2$ \\
\hline $\begin{array}{l}28-29 \\
\text { JUL }\end{array}$ & $<0.20$ & $<0.2$ & $<0.2$ & $<0.2$ & $<0.2$ & $<0.2$ & $<0.20$ & $<5.0$ & $<0.2$ & $<0.2$ & $<0.2$ & $<0.2$ \\
\hline $\begin{array}{l}18 \cdot 18 \\
\text { SEP }\end{array}$ & $<0.20$ & $<0.2$ & $<0.2$ & $<0.2$ & $<0.2$ & $<0.2$ & $<0.20$ & $<5.0$ & $<0.2$ & $<0.2$ & $<0.2$ & $<0.2$ \\
\hline $\begin{array}{l}01-01 \\
\text { SEP }\end{array}$ & $<0.20$ & $<0.2$ & $<0.2$ & $<0.2$ & $<0.2$ & 0.2 & $<0.20$ & $<5.0$ & $<0.2$ & $<0.2$ & $<0.2$ & $<0.2$ \\
\hline $21-21$ & $<0.20$ & $<0.2$ & $<0.2$ & $<0.2$ & $<0.2$ & $<0.2$ & $<0.20$ & $<5.0$ & $<0.2$ & $<0.2$ & $<0.2$ & $<0.2$ \\
\hline
\end{tabular}


Table 3. Water-quality data for storm-sewer outfall stations, Dallas, Texas, 1992-93-Continued

08057441 - NEWTON CREEK OUTFALI AT TIOGA STREET, DALLAS, TX (WY 1992)-Continued

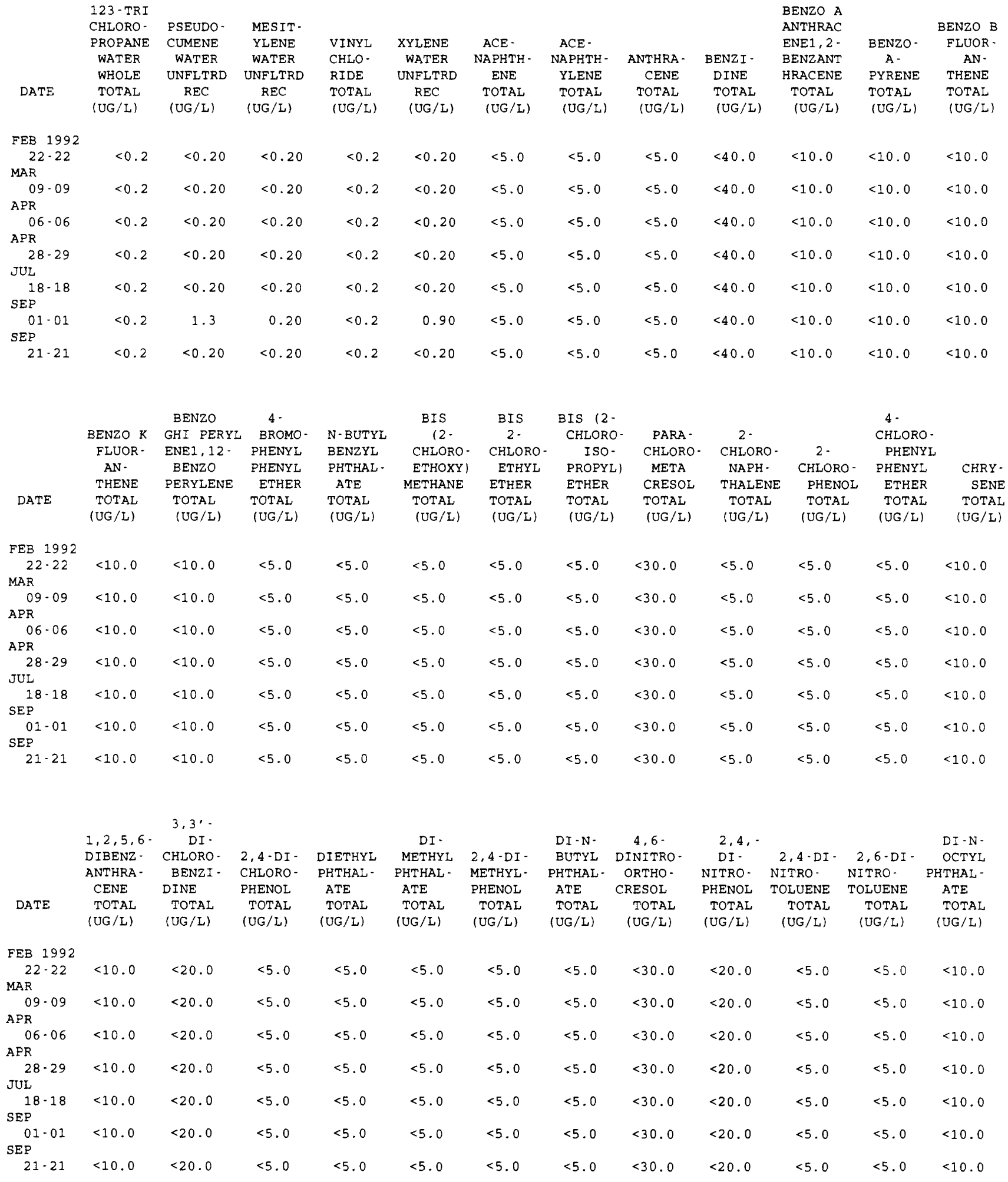


Table 3. Water-quality data for storm-sewer outfall stations, Dallas, Texas, 1992-93-Continued

08057441 - NEWTON CREEK OUTFALL AT TIOGA STREET, DALLAS, TX (WY 1992)-Continued

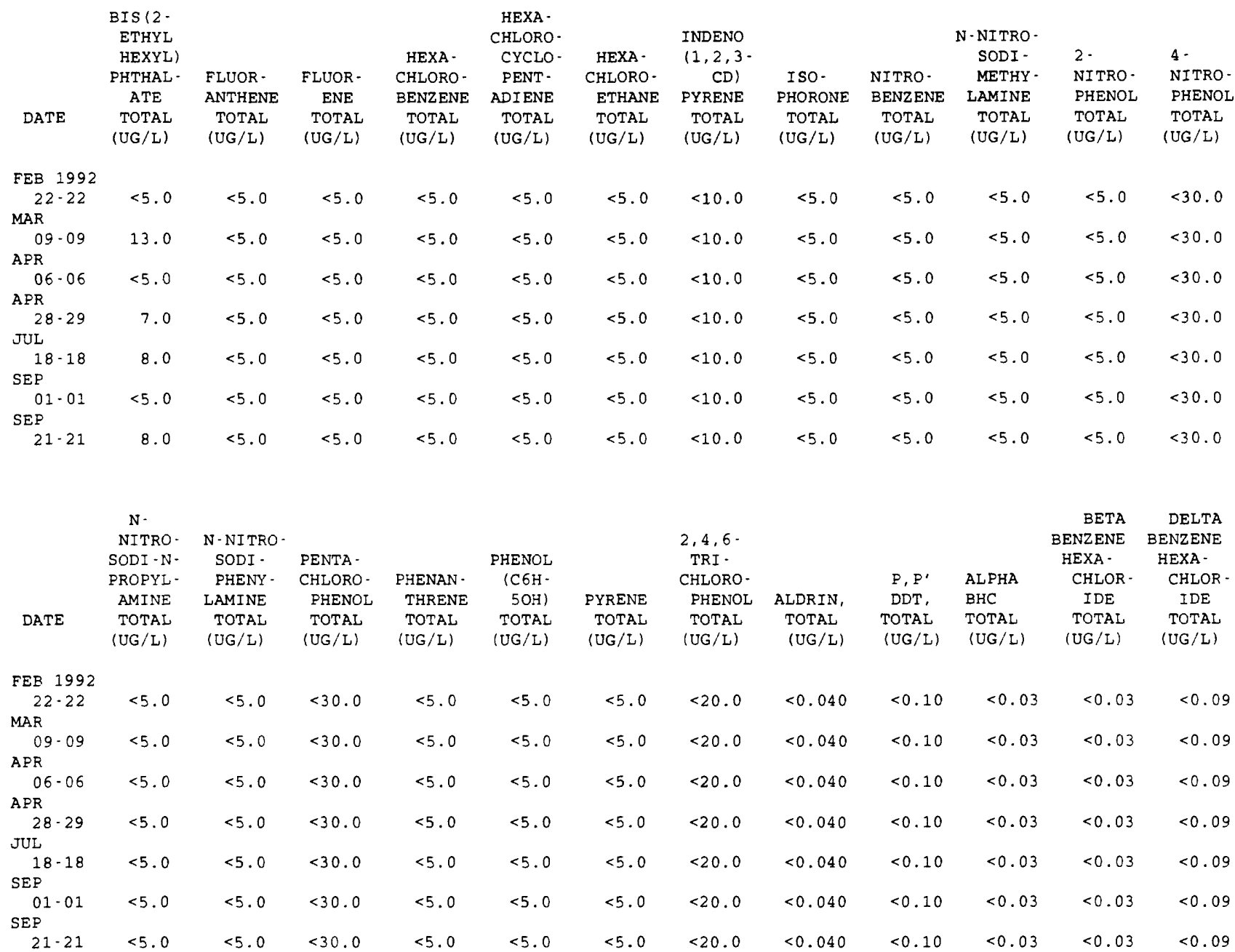

\begin{tabular}{|c|c|c|c|c|c|c|c|c|c|c|c|c|}
\hline & & $\begin{array}{l}\text { CHLOR- } \\
\text { DANE }\end{array}$ & $\begin{array}{l}\text { CHLOR - } \\
\text { DANE }\end{array}$ & & & & & $\begin{array}{l}\text { ENDO- } \\
\text { SULFAN- }\end{array}$ & & & & \\
\hline & & $\begin{array}{c}\text { CIS } \\
\text { WATER }\end{array}$ & $\begin{array}{l}\text { TRANS } \\
\text { WATER }\end{array}$ & CHLOR - & $p, p^{\prime}$ & $p, p^{\prime}$ & DI - & $\stackrel{I}{I}$ & $\begin{array}{l}\text { ENDO- } \\
\text { SULFAN }\end{array}$ & $\begin{array}{l}\text { ENDO- } \\
\text { SULFAN }\end{array}$ & $\begin{array}{r}\text { ENDRIN } \\
\text { NATER }\end{array}$ & ENDRIN \\
\hline & LINDANE & $\begin{array}{l}\text { WATEK } \\
\text { WHOLE }\end{array}$ & WHOLE & DANE, & DDD, & DDE, & ELDRIN & WHOLE & BETA & SULFATE & UNFLTRD & HYDE \\
\hline DATE & $\begin{array}{l}\text { TOTAL } \\
\text { (UG/L) }\end{array}$ & $\begin{array}{l}\text { TOTAL } \\
\text { (UG/L) }\end{array}$ & $\begin{array}{l}\text { TOTAL } \\
\text { (UG/L) }\end{array}$ & $\begin{array}{l}\text { TOTAL } \\
\text { (UG/L) }\end{array}$ & $\begin{array}{l}\text { TOTAL } \\
\text { (UG/L) }\end{array}$ & $\begin{array}{l}\text { TOTAL } \\
\text { (UG/L) }\end{array}$ & $\begin{array}{l}\text { TOTAL } \\
\text { (UG/L) }\end{array}$ & $\begin{array}{c}\text { REC } \\
(\mathrm{UG} / \mathrm{L})\end{array}$ & $\begin{array}{c}\text { TOTAL } \\
\text { (UG/L) }\end{array}$ & $\begin{array}{l}\text { TOTAL } \\
\text { (UG/L) }\end{array}$ & $\begin{array}{c}R E C \\
(U G / L)\end{array}$ & $\begin{array}{l}\text { TOTAL } \\
\text { (UG/L) }\end{array}$ \\
\hline FEB 1992 & & & & & & & & & & & & \\
\hline $\begin{array}{l}22 \cdot 22 \\
\text { MAR }\end{array}$ & $<0.030$ & $<0.10$ & $<0.10$ & $<0.1$ & $<0.10$ & $<0.04$ & $<0.020$ & $<0.10$ & $<0.04$ & $<0.60$ & $<0.060$ & $<0.20$ \\
\hline $\begin{array}{l}09-09 \\
A P R\end{array}$ & $<0.030$ & $<0.10$ & $<0.10$ & $<0.1$ & $<0.10$ & $<0.04$ & $<0.020$ & $<0.10$ & $<0.04$ & $<0.60$ & $<0.060$ & $<0.20$ \\
\hline $\begin{array}{l}06-06 \\
\text { APR }\end{array}$ & $<0.030$ & $<0.10$ & $<0.10$ & $<0.1$ & $<0.10$ & $<0.04$ & $<0.020$ & $<0.10$ & $<0.04$ & $<0.60$ & $<0.060$ & $<0.20$ \\
\hline JUL $^{28-29}$ & 0.030 & $<0.10$ & $<0.10$ & $<0.1$ & $<0.10$ & $<0.04$ & $<0.020$ & $<0.10$ & $<0.04$ & $<0.60$ & $<0.060$ & $<0.20$ \\
\hline $\operatorname{SEP}^{18-18}$ & $<0.030$ & $<0.10$ & $<0.10$ & $<0.1$ & $<0.10$ & $<0.04$ & $<0.020$ & $<0.10$ & $<0.04$ & $<0.60$ & $<0.060$ & $<0.20$ \\
\hline $\begin{array}{l}01-01 \\
\text { SEP }\end{array}$ & $<0.030$ & $<0.10$ & $<0.10$ & 0.3 & $<0.10$ & $<0.04$ & $<0.020$ & $<0.10$ & $<0.04$ & $<0.60$ & $<0.060$ & $<0.20$ \\
\hline $21 \cdot 21$ & $<0.030$ & $<0.10$ & $<0.10$ & 0.5 & $<0.10$ & $<0.04$ & $<0.020$ & $<0.10$ & $<0.04$ & $<0.60$ & $<0.060$ & $<0.20$ \\
\hline
\end{tabular}


Table 3. Water-quality data for storm-sewer outfall stations, Dallas, Texas, 1992-93-Continued 08057441 - NEWTON CREEK OUTFALL AT TIOGA STREET, DALLAS, TX (WY 1992)-Continued

\begin{tabular}{|c|c|c|c|c|c|c|c|c|c|c|c|}
\hline & HEPTA - & $\begin{array}{l}\text { HEPTA - } \\
\text { CHLOR }\end{array}$ & TOX & $\begin{array}{c}\text { AROCLOR } \\
1016\end{array}$ & $\begin{array}{c}\text { AROCLOR } \\
1221\end{array}$ & $\begin{array}{c}\text { AROCLOR } \\
1232\end{array}$ & $\begin{array}{c}\text { AROCLOR } \\
1242\end{array}$ & $\begin{array}{c}\text { AROCLOR } \\
1248\end{array}$ & $\begin{array}{c}\text { AROCLOR } \\
1254\end{array}$ & $\begin{array}{c}\text { AROCLOR } \\
1260\end{array}$ & DI - \\
\hline DATE & $\begin{array}{l}\text { CHLOR, } \\
\text { TOTAL } \\
\text { (UG /L) }\end{array}$ & $\begin{array}{c}\text { EPOXIDE } \\
\text { TOTAL } \\
\text { (UG/L) }\end{array}$ & $\begin{array}{l}\text { APHENE, } \\
\text { TOTAL } \\
\text { (UG/L) }\end{array}$ & $\begin{array}{c}\text { PCB } \\
\text { TOTAL } \\
(\mathrm{UG} / L)\end{array}$ & $\begin{array}{c}\text { PCB } \\
\text { TOTAL } \\
\text { (UG/L) }\end{array}$ & $\begin{array}{c}\text { PCB } \\
\text { TOTAL } \\
\text { (UG/L) }\end{array}$ & $\begin{array}{c}\text { PCB } \\
\text { TOTAL } \\
(\mathrm{UG} / \mathrm{L})\end{array}$ & $\begin{array}{c}\text { PCB } \\
\text { TOTAL } \\
\text { (UG/L) }\end{array}$ & $\begin{array}{c}\text { PCB } \\
\text { TOTAL } \\
\text { (UG/L) }\end{array}$ & $\begin{array}{c}\text { PCB } \\
\text { TOTAL } \\
\text { (UG/L) }\end{array}$ & $\begin{array}{c}\text { AZINON, } \\
\text { TOTAL } \\
\text { (UG/L) }\end{array}$ \\
\hline
\end{tabular}

\begin{tabular}{|c|c|c|c|c|c|c|c|c|c|c|c|}
\hline \multicolumn{12}{|l|}{ FEB $\quad 1992$} \\
\hline $22-22$ & $<0.030$ & $<0.80$ & $<2$ & $<0.1$ & $<1.0$ & $<0.1$ & $<0.1$ & $<0.1$ & $<0.1$ & $<0.1$ & 0.13 \\
\hline \multicolumn{12}{|l|}{ MAR } \\
\hline 09.09 & $<0.030$ & $<0.80$ & $<2$ & $<0.1$ & $<1.0$ & $<0.1$ & $<0.1$ & $<0.1$ & $<0.1$ & $<0.1$ & 2.4 \\
\hline \multicolumn{12}{|l|}{ APR } \\
\hline 06.06 & $<0.030$ & $<0.80$ & $<2$ & $<0.1$ & $<1.0$ & $<0.1$ & $<0.1$ & $<0.1$ & $<0.1$ & $<0.1$ & 0.80 \\
\hline \multicolumn{12}{|l|}{ APR } \\
\hline $28-29$ & $<0.030$ & $<0.80$ & $<2$ & $<0.1$ & $<1.0$ & $<0.1$ & $<0.1$ & $<0.1$ & $<0.1$ & $<0.1$ & 3.3 \\
\hline \multicolumn{12}{|l|}{ JUL } \\
\hline $18-18$ & $<0.030$ & $<0.80$ & $<2$ & $<0.1$ & $<1.0$ & $<0.1$ & $<0.1$ & $<0.1$ & $<0.1$ & $<0.1$ & 7.4 \\
\hline \multicolumn{12}{|l|}{ SEP } \\
\hline $01-01$ & $<0.030$ & $<0.80$ & $<2$ & $<0.1$ & $<1.0$ & $<0.1$ & $<0.1$ & $<0.1$ & $<0.1$ & $<0.1$ & 0.40 \\
\hline \multicolumn{12}{|l|}{$S E P$} \\
\hline $21 \cdot 21$ & $<0.030$ & $<0.80$ & $<2$ & $<0.1$ & $<1.0$ & $<0.1$ & $<0.1$ & $<0.1$ & $<0.1$ & $<0.1$ & 0.50 \\
\hline
\end{tabular}


Table 4. Water-quality data for storm-sewer outfall stations, Fort worth, Texas, 1992-93

08047100 - CLEAR FORK TRINITY RIVER OUTFALL AT OAK HILL CIRCLE, FORT WORTH, TX (WY 1992)

\begin{tabular}{|c|c|c|c|c|c|c|c|c|c|c|c|c|}
\hline & & $\begin{array}{l}\text { PRECIP- } \\
\text { ITATION } \\
\text { TOTAL }\end{array}$ & $\begin{array}{c}\text { ELAPSED } \\
\text { TIME } \\
\text { OF }\end{array}$ & $\begin{array}{l}\text { STORM } \\
\text { WATER }\end{array}$ & $\begin{array}{l}\text { SPE- } \\
\text { CIFIC } \\
\text { CON- } \\
\text { DUCT- }\end{array}$ & $\begin{array}{l}\text { SPE- } \\
\text { CIFIC } \\
\text { CON- } \\
\text { DUCT- } \\
\text { ANCE }\end{array}$ & $\begin{array}{c}\text { PH } \\
\text { WATER } \\
\text { WHOLE } \\
\text { FIELD } \\
\text { (STAND. }\end{array}$ & $\begin{array}{c}\text { PH } \\
\text { WATER } \\
\text { WHOIE } \\
\text { LAB } \\
\text { (STAND. }\end{array}$ & $\begin{array}{l}\text { TEMPER - } \\
\text { ATURE }\end{array}$ & $\begin{array}{l}\text { OXYGEN } \\
\text { DEMAND, } \\
\text { CHEM- } \\
\text { ICAL } \\
\text { (HIGH }\end{array}$ & $\begin{array}{l}\text { OXYGEN } \\
\text { DEMAND, } \\
\text { BIO- } \\
\text { CHEM- } \\
\text { ICAL, }\end{array}$ & $\begin{array}{l}\text { COLI. } \\
\text { FORM, } \\
\text { FECAL, } \\
0.7 \\
\text { UM-MF }\end{array}$ \\
\hline $\mathrm{TE}$ & IME & $\begin{array}{l}\text { INCHES / } \\
\text { STORM }\end{array}$ & $\begin{array}{c}\text { STORM } \\
\text { (HOURS) }\end{array}$ & $\begin{array}{l}\text { FLOW } \\
\text { (MGD) }\end{array}$ & $\begin{array}{l}\text { ANCE } \\
\text { (US/CM) }\end{array}$ & $\begin{array}{c}\text { LAB } \\
\text { (US/CM) }\end{array}$ & $\begin{array}{l}\text { ARD } \\
\text { UNITS) }\end{array}$ & $\begin{array}{c}\text { ARD } \\
\text { UNITS) }\end{array}$ & $\begin{array}{l}\text { WATER } \\
\text { (DEG C) }\end{array}$ & $\begin{array}{c}\text { LEVEL) } \\
(M G / L)\end{array}$ & $\begin{array}{l}5 \text { DAY } \\
\text { (MG/L) }\end{array}$ & $\begin{array}{l}\text { (COLS. } \\
100 \mathrm{ML} \text { ) }\end{array}$ \\
\hline
\end{tabular}

\begin{tabular}{|c|c|c|c|c|c|c|c|c|c|c|c|c|}
\hline FEB 1992 & & & & & & & & & & & & \\
\hline $22-22$ & 1213 & 0.55 & 4.6 & 0.18 & 68 & 108 & 8.4 & 7.8 & 16.0 & 34 & 5.3 & 1000 \\
\hline \multicolumn{13}{|l|}{ MAR } \\
\hline 09.09 & 0040 & 0.73 & 3.2 & 0.29 & 75 & 106 & 8.7 & 8.2 & 17.5 & 40 & 7.4 & K150 \\
\hline \multicolumn{13}{|l|}{ APR } \\
\hline $05-06$ & 2340 & 0.30 & 5.3 & 0.14 & 113 & 138 & 7.5 & 7.8 & 15.0 & 54 & 8.2 & 2500 \\
\hline \multicolumn{13}{|l|}{ APR } \\
\hline $\begin{array}{l}28-28 \\
\text { JUN }\end{array}$ & 2130 & 0.43 & 1.5 & 0.15 & 119 & 101 & 8.6 & 8.7 & 22.0 & 72 & 8.1 & $>200000$ \\
\hline $06-06$ & 0232 & 0.41 & 2.7 & 0.17 & 173 & 104 & 9.6 & 8.1 & 25.0 & 36 & 4.1 & 80000 \\
\hline \multicolumn{13}{|l|}{ JUN } \\
\hline $21-21$ & 0800 & 0.52 & 4.5 & 0.19 & 145 & 110 & 8.2 & 7.3 & 24.0 & 43 & 8.9 & 11000 \\
\hline \multicolumn{13}{|l|}{ AUG } \\
\hline $12-12$ & 1532 & 0.22 & 2.5 & 0.09 & 290 & 148 & 7.8 & 7.2 & 29.0 & 45 & 6.7 & 63000 \\
\hline
\end{tabular}

\begin{tabular}{|c|c|c|c|c|c|c|c|c|c|c|c|c|}
\hline & $\begin{array}{l}\text { STREP - } \\
\text { TOCOCCI }\end{array}$ & HARD & $\begin{array}{l}\text { HARD - } \\
\text { NESS }\end{array}$ & $\begin{array}{l}\text { ALKA - } \\
\text { LINITY }\end{array}$ & $\begin{array}{l}\text { SOLIDS, } \\
\text { SUM OF }\end{array}$ & $\begin{array}{l}\text { RESIDUE } \\
\text { TOTAL }\end{array}$ & $\begin{array}{l}\text { SOLIDS, } \\
\text { RESIDUE }\end{array}$ & & MAGNE - & & & SODIUM \\
\hline & FECAL & NESS & NONCARB & WAT DIS & CONSTI - & AT 105 & AT 180 & CALCIUM & SIUM, & SODIUM, & & $A D$ \\
\hline & $\begin{array}{l}\text { KF AGAR } \\
\text { (COLS. }\end{array}$ & $\begin{array}{l}\text { TOTAL } \\
\text { (MG/L }\end{array}$ & $\begin{array}{l}\text { DISSOLV } \\
\text { FLD. AS }\end{array}$ & $\begin{array}{l}\text { FIX END } \\
\text { FIELD }\end{array}$ & $\begin{array}{c}\text { TUENTS, } \\
\text { DIS - }\end{array}$ & $\begin{array}{l}\text { DEG. C, } \\
\text { SUS - }\end{array}$ & $\begin{array}{l}\text { DEG. C } \\
\text { DIS - }\end{array}$ & $\begin{array}{l}\text { DIS- } \\
\text { SOLVED }\end{array}$ & $\begin{array}{l}\text { DIS - } \\
\text { SOLVED }\end{array}$ & $\begin{array}{c}\text { DIS - } \\
\text { SOLVED }\end{array}$ & & $\begin{array}{l}\text { SORP- } \\
\text { TION }\end{array}$ \\
\hline & PER & AS & $\mathrm{CACO} 3$ & $\mathrm{CACO} 3$ & SOLVED & PENDED & SOLVED & (MG / L & (MG / L & (MG/L & SODIUM & RATIO \\
\hline & $100 \mathrm{ML}\rangle$ & $\mathrm{CACO} 31$ & (MG/L) & $(M G / L)$ & $(M G / L)$ & $(M G / L)$ & $(M G / L)$ & AS CA) & AS MG) & AS NA) & PERCENT & \\
\hline
\end{tabular}

\begin{tabular}{|c|c|c|c|c|c|c|c|c|c|c|c|c|}
\hline $\begin{array}{c}\text { FEB } \quad 1992 \\
22 \cdot 22\end{array}$ & 18000 & 25 & 0 & 30 & $5 ?$ & 74 & 58 & 06 & 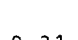 & 2 & 27 & \\
\hline \multicolumn{13}{|l|}{ MAR } \\
\hline 09.09 & K46000 & 26 & 3 & 23 & 47 & 74 & 36 & 9.7 & 0.43 & 1.7 & 11 & 0.1 \\
\hline \multicolumn{13}{|l|}{ APR } \\
\hline 05.06 & 17000 & 34 & 4 & 30 & 63 & 51 & 60 & 13 & 0.48 & 2.1 & 10 & 0.2 \\
\hline \multicolumn{13}{|l|}{ APR } \\
\hline \multirow{2}{*}{\multicolumn{13}{|c|}{ JUN }} \\
\hline & & & & & & & & & & & & \\
\hline \multicolumn{13}{|l|}{ JUN } \\
\hline $21-21$ & 20000 & 32 & 6 & 26 & 54 & 44 & 73 & 12 & 0.53 & 2.3 & 12 & 0.2 \\
\hline AUG & & & & & & & & & & & & \\
\hline $12-12$ & 93000 & 42 & 9 & 33 & 71 & 32 & 74 & 15 & 1.0 & 4.4 & 17 & 0.3 \\
\hline
\end{tabular}

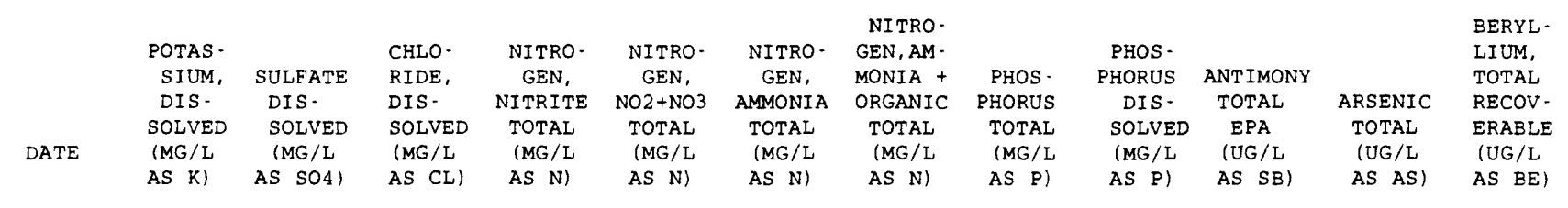

\begin{tabular}{|c|c|c|c|c|c|c|c|c|c|c|c|c|}
\hline FEB 1992 & & & & & & & & & & & & \\
\hline $22-22$ & 4.1 & 5.3 & 1.9 & 0.050 & 0.280 & 0.110 & 0.70 & 0.200 & 0.170 & $<10.0$ & 2 & $<10$ \\
\hline $\begin{array}{l}\text { MAR } \\
09.09\end{array}$ & 35 & 40 & 23 & & & & & & & & & \\
\hline APR & & & & 0.040 & . & 0.480 & 1.7 & 0.690 & 0.540 & $<20.0$ & 3 & $<10$ \\
\hline $\begin{array}{r}05-06 \\
A P R\end{array}$ & 6.0 & 8.2 & 1.7 & 0.050 & 0.880 & 0.490 & 1.6 & 0.290 & 0.230 & $<10.0$ & 2 & $<10$ \\
\hline $\begin{array}{l}28 \cdot 28 \\
\text { JUN }\end{array}$ & 3.9 & 5.4 & 3.8 & 0.060 & 0.510 & 0.170 & 0.70 & 0.170 & 0.130 & $<20.0$ & 1 & $<10$ \\
\hline $\begin{array}{l}06-06 \\
\text { JUN }\end{array}$ & 4.9 & 5.5 & 2.3 & 0.050 & 0.710 & 0.320 & 0.80 & 0.180 & 0.180 & $<20.0$ & 5 & $<10$ \\
\hline 21-21 & 4.8 & 4.4 & 3.4 & 0.050 & 0.630 & 0.200 & 1.0 & 0.230 & 0.170 & $<20.0$ & 1 & $<10$ \\
\hline $\begin{array}{l}\text { AUG } \\
12-12\end{array}$ & 5.0 & 11 & 5.4 & 0.070 & 0.810 & 0.200 & 0.80 & 0.290 & 0.220 & $<10.0$ & 11 & $<10$ \\
\hline
\end{tabular}


Table 4. Water-quality data for storm-sewer outfall stations, Fort worth, Texas, 1992-93-Continued

08047100 - CLEAR FORK TRINITY RIVER OUTFALL AT OAK HILL CIRCLE, FORT WORTH, TX (WY 1992)-CONEINUEd

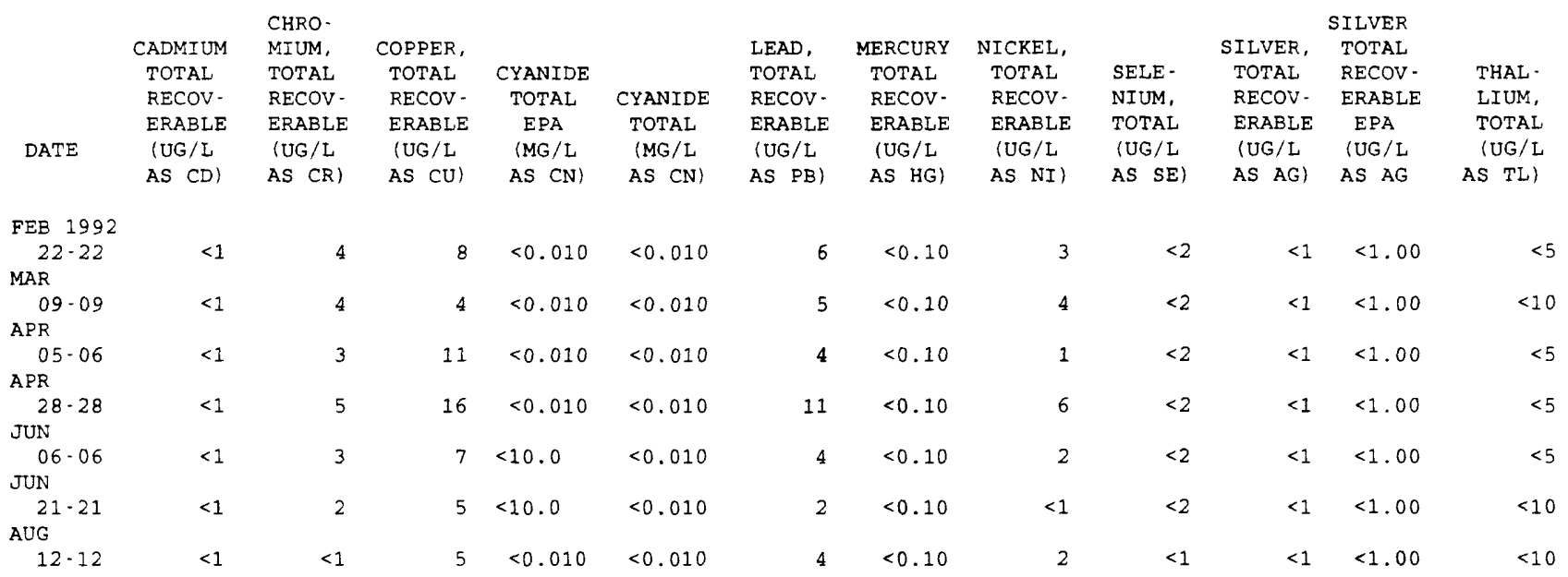

\begin{tabular}{|c|c|c|c|c|c|c|c|c|c|c|c|c|}
\hline & & & OIL AND & & & & & & & & BENZENE & BENZENE \\
\hline & ZINC, & & GREASE, & & & & & BROMO - & & BENZENE & SEC & TERT . \\
\hline & TOTAL & CARBON, & TOTAL & & & & & BENZENE & & $\mathrm{N}$-BUTYL & BUTYL - & BUTYL - \\
\hline & RECOV & ORGANIC & RECOV. & & ACRO- & ACRYLO- & & WATER, & BROMO - & WATER & WATER & WATER \\
\hline & ERABLE & TOTAL & GRAVI - & PHENOLS & LEIN & NITRILE & BENZENE & WHOLE, & FORM & UNFLTRD & UNFLTRD & UNFLTRD \\
\hline & $\begin{array}{l}(\mathrm{UG} / \mathrm{L} \\
\mathrm{AS} \quad \mathrm{zN})\end{array}$ & $\begin{array}{l}(M G / L \\
\text { AS C) }\end{array}$ & $\begin{array}{c}\text { METRIC } \\
(\mathrm{MG} / \mathrm{L})\end{array}$ & $\begin{array}{l}\text { TOTAL } \\
\text { (UG/L) }\end{array}$ & $\begin{array}{l}\text { TOTAL } \\
\text { (UG/L) }\end{array}$ & $\begin{array}{l}\text { TOTAL } \\
\text { (UG/L) }\end{array}$ & $\begin{array}{l}\text { TOTAL } \\
\text { (UG/L) }\end{array}$ & $\begin{array}{l}\text { TOTAL } \\
\text { (UG/L) }\end{array}$ & $\begin{array}{l}\text { TOTAL } \\
\text { (UG/L) }\end{array}$ & $\begin{array}{c}\text { REC } \\
(U G / L)\end{array}$ & $\begin{array}{c}\text { REC } \\
\text { (UG/L) }\end{array}$ & $\begin{array}{c}R E C \\
(U G / L)\end{array}$ \\
\hline
\end{tabular}

\begin{tabular}{|c|c|c|c|c|c|c|c|c|c|c|c|c|}
\hline FEB 1992 & & & & & & & & & & & & \\
\hline $22-22$ & 40 & 10 & $<1$ & 2 & $<20$ & $<20$ & $<0.2$ & $<0.2$ & $<0.2$ & $<0.20$ & $<0.20$ & $<0.20$ \\
\hline MAR & & & & & & & & & & & & \\
\hline 09.09 & 30 & 12 & 2 & 5 & $<20$ & $<20$ & $<0.2$ & $<0.2$ & $<0.2$ & $<0.20$ & $<0.20$ & $<0.20$ \\
\hline APR & & & & & & & & & & & & \\
\hline $05-06$ & 50 & 11 & $<1$ & 4 & $<20$ & $<20$ & $<0.2$ & $<0.2$ & $<0.2$ & $<0.20$ & $<0.20$ & $<0.20$ \\
\hline APR & & & & & & & & & & & & \\
\hline $28-28$ & 70 & 16 & $<1$ & 2 & $<20$ & $<20$ & $<0.2$ & $<0.2$ & $<0.2$ & $<0.20$ & $<0.20$ & $<0.20$ \\
\hline JUN & & & & & & & & & & & & \\
\hline $06-06$ & 40 & 12 & $<1$ & 3 & $<20$ & $<20$ & $<0.2$ & $<0.2$ & $<0.2$ & $<0.20$ & $<0.20$ & $<0.20$ \\
\hline JUN & & & & & & & & & & & & \\
\hline $21 \cdot 21$ & 30 & 13 & $<1$ & 3 & $<20$ & $<20$ & $<0.2$ & $<0.2$ & $<0.2$ & $<0.20$ & $<0.20$ & $<0.20$ \\
\hline AUG & & & & & & & & & & & & \\
\hline $12 \cdot 12$ & 50 & 12 & $<1$ & 5 & $<20$ & $<20$ & $<0.2$ & $<0.2$ & $<0.2$ & $<0.20$ & $<0.20$ & $<0.20$ \\
\hline
\end{tabular}

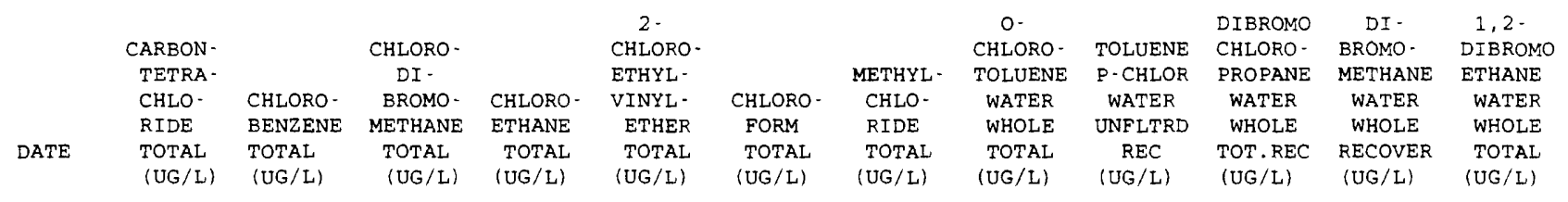

\begin{tabular}{|c|c|c|c|c|c|c|c|c|c|c|c|c|}
\hline FEB 1992 & & & & & & & & & & & & \\
\hline $\begin{array}{l}22-22 \\
\text { MAR }\end{array}$ & $<0.2$ & $<0.20$ & $<0.2$ & $<0.2$ & $<1.0$ & $<0.2$ & $<0.2$ & $<0.2$ & $<0.20$ & $<1.0$ & $<0.2$ & $<0.2$ \\
\hline $09-09$ & $<0.2$ & $<0.20$ & $<0.2$ & $<0.2$ & $<1.0$ & $<0.2$ & $<0.2$ & $<0.2$ & $<0.20$ & $<1.0$ & $<0.2$ & $<0.2$ \\
\hline APR & & & & & & & & & & & & \\
\hline $\begin{array}{l}05 \cdot 06 \\
\text { APR }\end{array}$ & $<0.2$ & $<0.20$ & $<0.2$ & $<0.2$ & $<1.0$ & $<0.2$ & $<0.2$ & $<0.2$ & $<0.20$ & $<1.0$ & $<0.2$ & $<0.2$ \\
\hline${ }_{\text {JUN }}^{28 \cdot 28}$ & $<0.2$ & $<0.20$ & $<0.2$ & $<0.2$ & $<1.0$ & $<0.2$ & $<0.2$ & $<0.2$ & $<0.20$ & $<1.0$ & $<0.2$ & $<0.2$ \\
\hline $\begin{array}{l}06-06 \\
\text { JUN }\end{array}$ & $<0.2$ & $<0.20$ & $<0.2$ & $<0.2$ & $<1.0$ & $<0.2$ & $<0.2$ & $<0.2$ & $<0.20$ & $<1.0$ & $<0.2$ & $<0.2$ \\
\hline $\begin{array}{l}21 \cdot 21 \\
\text { AUG }\end{array}$ & $<0.2$ & $<0.20$ & $<0.2$ & $<0.2$ & $<1.0$ & $<0.2$ & $<0.2$ & $<0.2$ & $<0.20$ & $<1.0$ & $<0.2$ & $<0.2$ \\
\hline $12 \cdot 12$ & $<0.2$ & $<0.20$ & 0.2 & $<0.2$ & $<1.0$ & 0.2 & $<0.2$ & $<0.2$ & $<0.20$ & $<1.0$ & $<0.2$ & $<0.2$ \\
\hline
\end{tabular}


Table 4. Water-quality data for storm-sewer outfall stations, Fort worth, Texas, 1992-93-Continued

08047100 - CLEAR FORK TRINITY RIVER OUTFALL AT OAK HILL CIRCLE, FORT WORTH, TX (WY 1992)-CONEINUEd

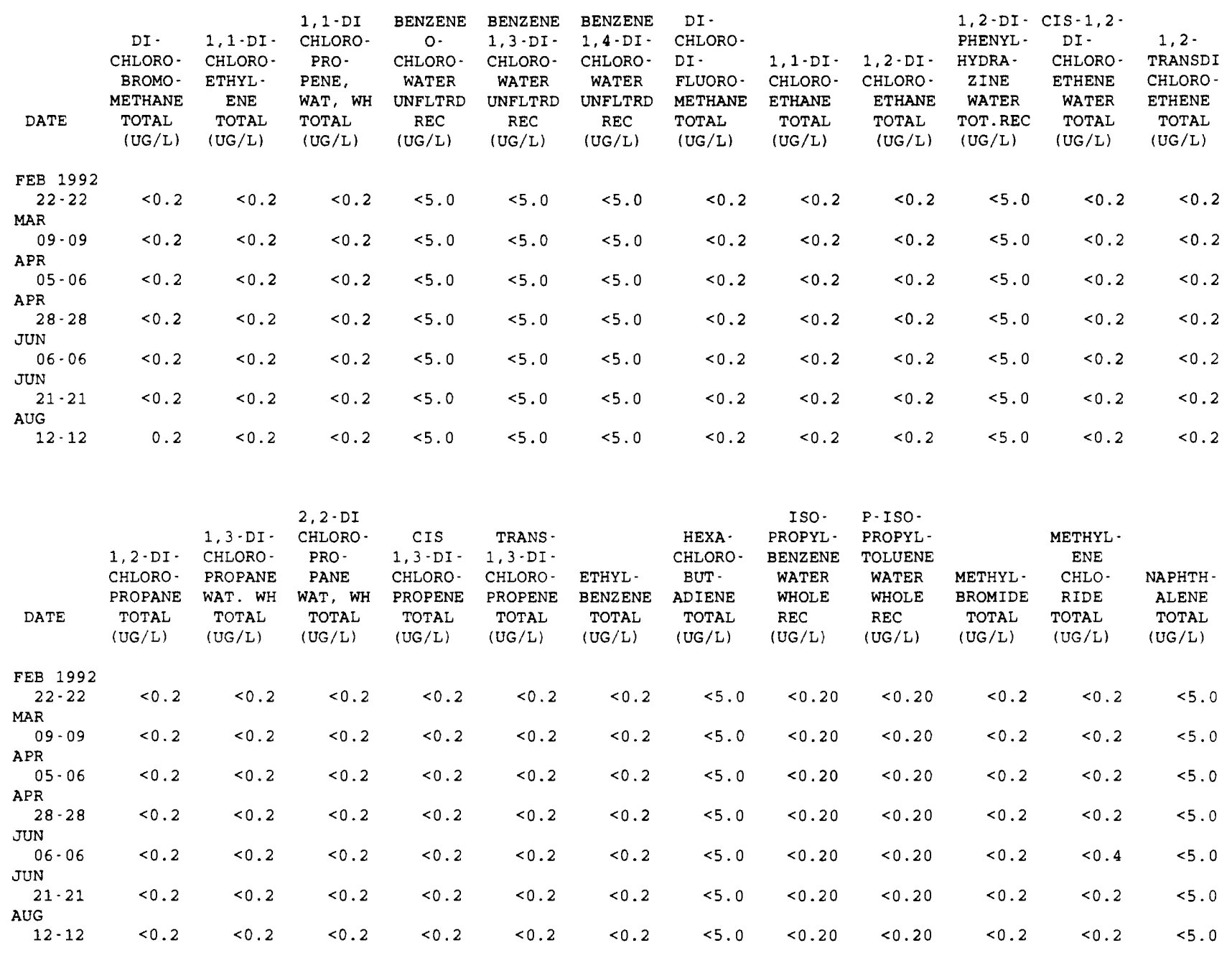

\begin{tabular}{|c|c|c|c|c|c|c|c|c|c|c|c|c|}
\hline & BENZENE & & $\begin{array}{c}\text { ETHANE, } \\
1112-\end{array}$ & $\begin{array}{l}\text { ETHANE, } \\
1,1,2,2\end{array}$ & TETRA- & & $\begin{array}{l}1,2,3- \\
\text { TRI- }\end{array}$ & $\begin{array}{l}\text { BENZENE } \\
1,2,4-\end{array}$ & $1,1,1-$ & $1,1,2$. & & \\
\hline & N-PROPY & & TETRA - & TETRA - & CHLORO- & & CHLORO & TRI - & TRI- & TRI - & CHLORO- & CHLORO- \\
\hline & WATER & & CHLORO - & CHLORO - & ETHYL - & & BENZENE & CHLORO - & CHLORO- & CHLORO- & ETHYL - & FLUORO- \\
\hline$T E$ & $\begin{array}{l}\text { RECTRD } \\
\text { (UG/L) }\end{array}$ & $\begin{array}{l}\text { STYRENEE } \\
\text { TOTAL } \\
\text { (UG/L) }\end{array}$ & $\begin{array}{c}\text { REC } \\
\text { (UG/L) }\end{array}$ & $\begin{array}{l}\text { REC } \\
\text { (UG/L) }\end{array}$ & $\begin{array}{l}\text { ENE } \\
\text { TOTAL } \\
\text { (UG/L) }\end{array}$ & $\begin{array}{l}\text { TOLUENE } \\
\text { TOTAL } \\
\text { (UG/L) }\end{array}$ & $\begin{array}{l}\text { WAT, WH } \\
\text { REC } \\
(U G / L)\end{array}$ & $\begin{array}{l}\text { RAT UNF } \\
\text { REC } \\
(U G / L)\end{array}$ & $\begin{array}{l}\text { ETHANE } \\
\text { TOTAL } \\
\text { (UG/L) }\end{array}$ & $\begin{array}{l}\text { ETHANA } \\
\text { TOTAL } \\
\text { (UG/L) }\end{array}$ & $\begin{array}{l}\text { ENE } \\
\text { TOTAL } \\
\text { (UG/L) }\end{array}$ & $\begin{array}{l}\text { METHANE } \\
\text { TOTAL } \\
\text { (UG/L) }\end{array}$ \\
\hline
\end{tabular}

\begin{tabular}{|c|c|c|c|c|c|c|c|c|c|c|c|c|}
\hline \multicolumn{13}{|l|}{ FEB 1992} \\
\hline $22-22$ & $<0.20$ & $<0.2$ & $<0.2$ & $<0.2$ & $<0.2$ & $<0.2$ & $<0.20$ & $<5.0$ & $<0.2$ & $<0.2$ & $<0.2$ & $<0.2$ \\
\hline \multicolumn{13}{|l|}{ MAR } \\
\hline 09.09 & $<0.20$ & $<0.2$ & $<0.2$ & $<0.2$ & $<0.2$ & $<0.2$ & $<0.20$ & $<5.0$ & $<0.2$ & $<0.2$ & $<0.2$ & $<0.2$ \\
\hline \multicolumn{13}{|l|}{ APR } \\
\hline $05-06$ & $<0.20$ & $<0.2$ & $<0.2$ & $<0.2$ & $<0.2$ & $<0.2$ & $<0.20$ & $<5.0$ & $<0.2$ & $<0.2$ & $<0.2$ & $<0.2$ \\
\hline \multicolumn{13}{|l|}{ APR } \\
\hline $28-28$ & $<0.20$ & $<0.2$ & $<0.2$ & $<0.2$ & $<0.2$ & 0.2 & $<0.20$ & $<5.0$ & $<0.2$ & $<0.2$ & $<0.2$ & $<0.2$ \\
\hline \multicolumn{13}{|l|}{ JUN } \\
\hline $06-06$ & $<0.20$ & $<0.2$ & $<0.2$ & $<0.2$ & $<0.2$ & $<0.2$ & $<0.20$ & $<5.0$ & $<0.2$ & $<0.2$ & $<0.2$ & $<0.2$ \\
\hline \multicolumn{13}{|l|}{ JUN } \\
\hline $21-21$ & $<0.20$ & $<0.2$ & $<0.2$ & $<0.2$ & $<0.2$ & $<0.2$ & $<0.20$ & $<5.0$ & $<0.2$ & $<0.2$ & $<0.2$ & $<0.2$ \\
\hline \multicolumn{13}{|l|}{ AUG } \\
\hline $12-12$ & $<0.20$ & $<0.2$ & $<0.2$ & $<0.2$ & $<0.2$ & $<0.2$ & $<0.20$ & $<5.0$ & $<0.2$ & $<0.2$ & $<0.2$ & $<0.2$ \\
\hline
\end{tabular}


Table 4. Water-quality data for storm-sewer outfal1 stations, Fort Worth, Texas, 1992-93-Continued

08047100 - CLEAR FORK TRINITY RIVER OUTFALL AT OAK HILL CIRCLE, FORT WORTH, TX (WY 1992)-CONEINUEd

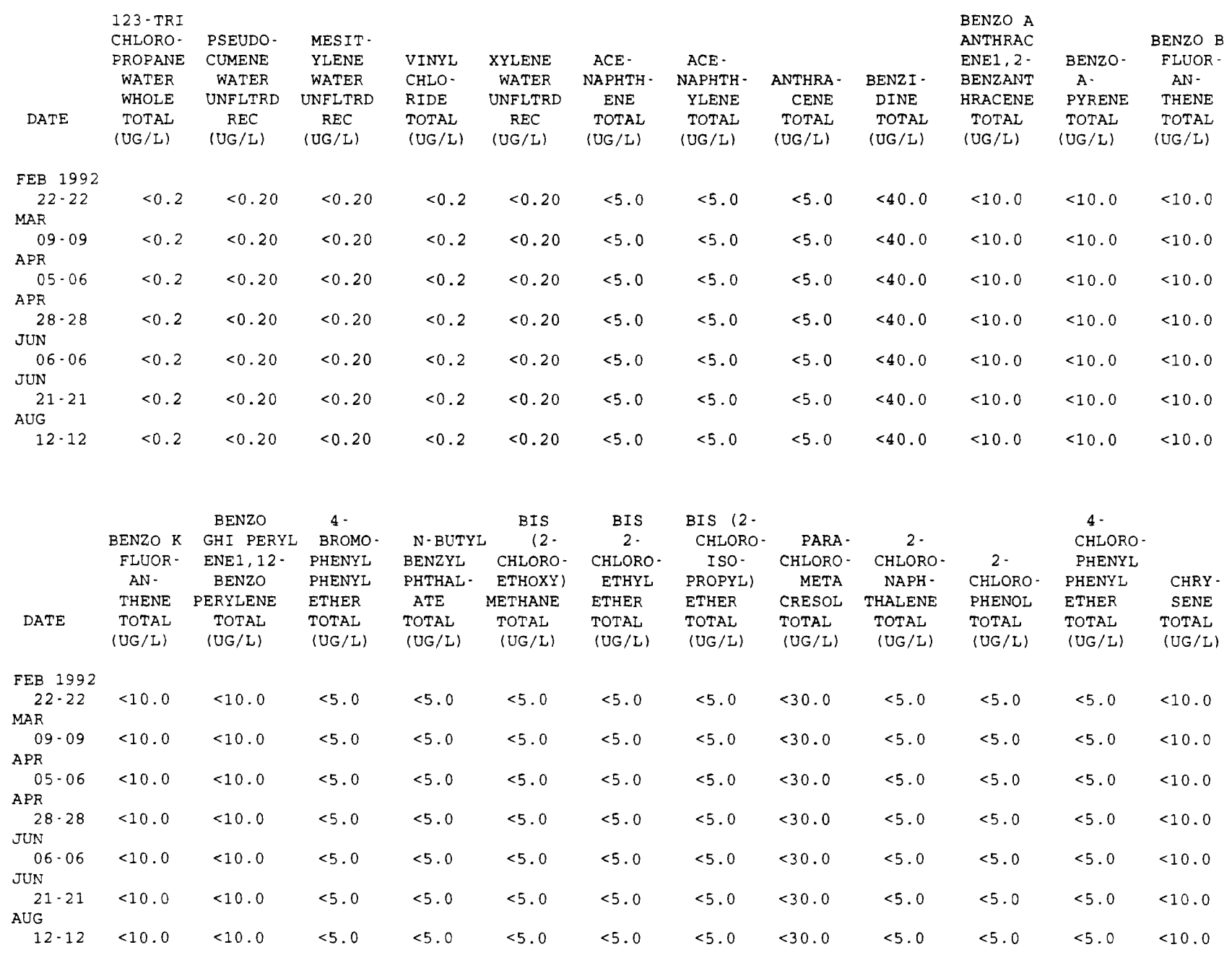

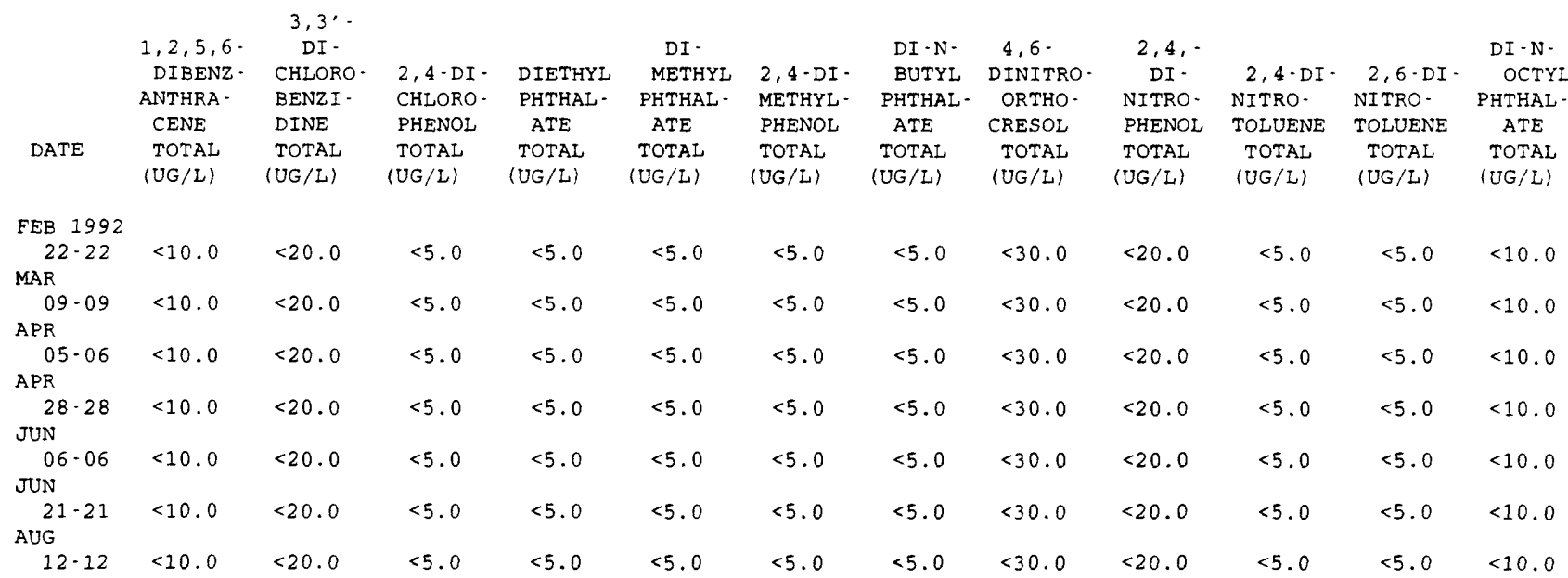


Table 4. Water-quality data for storm-sewer outfall stations, Fort worth, Texas, 1992-93-Continued

08047100 - CLEAR FORK TRINITY RIVER OUTFALL AT OAK HILL CIRCLE, FORT WORTH, TX (WY 1992)-CONEINUEd

\begin{tabular}{|c|c|c|c|c|c|c|c|c|c|c|c|c|}
\hline DATE & $\begin{array}{c}\text { BIS }(2- \\
\text { ETHYL } \\
\text { HEXYL) } \\
\text { PHTHAL- } \\
\text { ATE } \\
\text { TOTAL } \\
\text { (UG/L) }\end{array}$ & $\begin{array}{l}\text { FLUOR- } \\
\text { ANTHENE } \\
\text { TOTAL } \\
\text { (UG/L) }\end{array}$ & $\begin{array}{c}\text { FLUOR- } \\
\text { ENE } \\
\text { TOTAL } \\
\text { (UG/L) }\end{array}$ & $\begin{array}{l}\text { HEXA- } \\
\text { CHLORO- } \\
\text { BENZENE } \\
\text { TOTAL } \\
\text { (UG/L) }\end{array}$ & $\begin{array}{l}\text { HEXA- } \\
\text { CHLORO- } \\
\text { CYCLO- } \\
\text { PENT- } \\
\text { ADIENE } \\
\text { TOTAL } \\
\text { (UG/L) }\end{array}$ & $\begin{array}{l}\text { HEXA- } \\
\text { CHLORO- } \\
\text { ETHANE } \\
\text { TOTAL } \\
\text { (UG/L) }\end{array}$ & $\begin{array}{r}\text { INDENO } \\
(1,2,3- \\
\text { CD) } \\
\text { PYRENE } \\
\text { TOTAL } \\
\text { (UG/L) }\end{array}$ & $\begin{array}{l}\text { ISO- } \\
\text { PHORONE } \\
\text { TOTAL } \\
\text { (UG/L) }\end{array}$ & $\begin{array}{l}\text { NITRO- } \\
\text { BENZENE } \\
\text { TOTAL } \\
\text { (UG/L) }\end{array}$ & $\begin{array}{c}\text { N-NITRO- } \\
\text { SODI- } \\
\text { METHY- } \\
\text { LAMINE } \\
\text { TOTAL } \\
\text { (UG/L) }\end{array}$ & $\begin{array}{l}2 \cdot \\
\text { NITRO- } \\
\text { PHENOL } \\
\text { TOTAL } \\
\text { (UG/L) }\end{array}$ & $\begin{array}{c}4- \\
\text { NITRO- } \\
\text { PHENOL } \\
\text { TOTAL } \\
\text { (UG/L) }\end{array}$ \\
\hline FEB 1992 & & & & & & & & & & & & \\
\hline$\underset{\text { MAR }}{22 \cdot 22}$ & $<5.0$ & $<5.0$ & $<5.0$ & $<5.0$ & $<5.0$ & $<5.0$ & $<10.0$ & $<5.0$ & $<5.0$ & $<5.0$ & $<5.0$ & $<30.0$ \\
\hline $\begin{array}{l}09-09 \\
A P R\end{array}$ & $<5.0$ & $<5.0$ & $<5.0$ & $<5.0$ & $<5.0$ & $<5.0$ & $<10.0$ & $<5.0$ & $<5.0$ & $<5.0$ & $<5.0$ & $<30.0$ \\
\hline $\begin{array}{l}\text { APK } \\
\text { OPR } \\
\text { APR }\end{array}$ & $<5.0$ & $<5.0$ & $<5.0$ & $<5.0$ & $<5.0$ & $<5.0$ & $<10.0$ & $<5.0$ & $<5.0$ & $<5.0$ & $<5.0$ & $<30.0$ \\
\hline $\begin{array}{l}28-28 \\
\text { JUN }\end{array}$ & $<5.0$ & $<5.0$ & $<5.0$ & $<5.0$ & $<5.0$ & $<5.0$ & $<10.0$ & $<5.0$ & $<5.0$ & $<5.0$ & $<5.0$ & $<30.0$ \\
\hline $\begin{array}{l}06 \cdot 06 \\
\text { JUN }\end{array}$ & $<5.0$ & $<5.0$ & $<5.0$ & $<5.0$ & $<5.0$ & $<5.0$ & $<10.0$ & $<5.0$ & $<5.0$ & $<5.0$ & $<5.0$ & $<30.0$ \\
\hline AUG $21-21$ & $<5.0$ & $<5.0$ & $<5.0$ & $<5.0$ & $<5.0$ & $<5.0$ & $<10.0$ & $<5.0$ & $<5.0$ & $<5.0$ & $<5.0$ & $<30.0$ \\
\hline $12 \cdot 12$ & $<5.0$ & $<5.0$ & $<5.0$ & $<5.0$ & $<5.0$ & $<5.0$ & $<10.0$ & $<5.0$ & $<5.0$ & $<5.0$ & $<5.0$ & $<30.0$ \\
\hline
\end{tabular}

\begin{tabular}{|c|c|c|c|c|c|c|c|c|c|c|c|c|}
\hline DATE & $\begin{array}{l}\text { N- } \\
\text { NITRO- } \\
\text { SODI-N- } \\
\text { PROPYL- } \\
\text { AMINE } \\
\text { TOTAL } \\
\text { (UG/L) }\end{array}$ & $\begin{array}{l}\text { N-NITRO- } \\
\text { SODI - } \\
\text { PHENY - } \\
\text { LAMINE } \\
\text { TOTAL } \\
\text { (UG/L) }\end{array}$ & $\begin{array}{l}\text { PENTA- } \\
\text { CHLORO- } \\
\text { PHENOL } \\
\text { TOTAL } \\
\text { (UG/L) }\end{array}$ & $\begin{array}{l}\text { PHENAN- } \\
\text { THRENE } \\
\text { TOTAL } \\
\text { (UG/L) }\end{array}$ & $\begin{array}{l}\text { PHENOL } \\
\text { (C6H- } \\
5 \mathrm{OH}) \\
\text { TOTAL } \\
\text { (UG/L) }\end{array}$ & $\begin{array}{l}\text { PYRENE } \\
\text { TOTAL } \\
\text { (UG/L) }\end{array}$ & $\begin{array}{c}2,4,6 \\
\text { TRI- } \\
\text { CHLORO- } \\
\text { PHENOL } \\
\text { TOTAL } \\
\text { (UG/L) }\end{array}$ & $\begin{array}{c}\text { ALDRIN, } \\
\text { TOTAL } \\
\text { (UG/L) }\end{array}$ & $\begin{array}{l}\text { P, } P^{\prime} \\
\text { DDT, } \\
\text { TOTAL } \\
\text { (UG/L) }\end{array}$ & $\begin{array}{l}\text { ALPHA } \\
\text { BHC } \\
\text { TOTAL } \\
\text { (UG/L) }\end{array}$ & $\begin{array}{c}\text { BETA } \\
\text { BENZENE } \\
\text { HEXA- } \\
\text { CHLOR- } \\
\text { IDE } \\
\text { TOTAL } \\
\text { (UG/L) }\end{array}$ & $\begin{array}{l}\text { DELTA } \\
\text { BENZENE } \\
\text { HEXA- } \\
\text { CHLOR- } \\
\text { IDE } \\
\text { TOTAL } \\
\text { (UG/L) }\end{array}$ \\
\hline EB 1992 & & & & & & & & & & & & \\
\hline $\begin{array}{l}22-22 \\
\text { MAR }\end{array}$ & $<5.0$ & $<5.0$ & $<30.0$ & $<5.0$ & $<5.0$ & $<5.0$ & $<20.0$ & $<0.040$ & $<0.10$ & $<0.03$ & $<0.03$ & $<0.09$ \\
\hline $\begin{array}{l}09-09 \\
\text { APR }\end{array}$ & $<5.0$ & $<5.0$ & $<30.0$ & $<5.0$ & $<5.0$ & $<5.0$ & $<20.0$ & $<0.040$ & $<0.10$ & $<0.03$ & $<0.03$ & $<0.09$ \\
\hline $\begin{array}{l}05-06 \\
A P R\end{array}$ & $<5.0$ & $<5.0$ & $<30.0$ & $<5.0$ & $<5.0$ & $<5.0$ & $<20.0$ & $<0.040$ & $<0.10$ & $<0.03$ & $<0.03$ & $<0.09$ \\
\hline $\begin{array}{l}28-28 \\
\text { JUN }\end{array}$ & $<5.0$ & $<5.0$ & $<30.0$ & $<5.0$ & $<5.0$ & $<5.0$ & $<20.0$ & $<0.040$ & $<0.10$ & $<0.03$ & $<0.03$ & $<0.09$ \\
\hline $\begin{array}{l}06-06 \\
\text { JUN }\end{array}$ & $<5.0$ & $<5.0$ & $<30.0$ & $<5.0$ & $<5.0$ & $<5.0$ & $<20.0$ & $<0.040$ & $<0.10$ & $<0.03$ & $<0.03$ & $<0.09$ \\
\hline $\begin{array}{l}21-21 \\
\text { UG }\end{array}$ & $<5.0$ & $<5.0$ & $<30.0$ & $<5.0$ & $<5.0$ & $<5.0$ & $<20.0$ & $<0.040$ & $<0.10$ & $<0.03$ & $<0.03$ & $<0.09$ \\
\hline $12 \cdot 12$ & $<5.0$ & $<5.0$ & $<30.0$ & $<5.0$ & $<5.0$ & $<5.0$ & $<20.0$ & $<0.040$ & $<0.10$ & $<0.03$ & $<0.03$ & $<0.09$ \\
\hline
\end{tabular}

\begin{tabular}{|c|c|c|c|c|c|c|c|c|c|c|c|c|}
\hline & & $\begin{array}{l}\text { CHLOR- } \\
\text { DANE }\end{array}$ & $\begin{array}{l}\text { CHLOR. } \\
\text { DANE }\end{array}$ & & & & & $\begin{array}{l}\text { ENDO- } \\
\text { SULFAN- }\end{array}$ & & & & \\
\hline & & CIS & TRANS & & & & & I & ENDO - & ENDO- & ENDRIN & ENDRIN \\
\hline & & WATER & WATER & CHLOR - & $P, P^{\prime}$ & $P, P^{\prime}$ & DI - & WATER & SULFAN & SULFAN & WATER & ALDE- \\
\hline & LINDANE & WHOLE & WHOLE & DANE, & DDD & DDE & ELDRIN & WHOLE & BETA & SULFATE & UNFLTRD & HYDE \\
\hline DATE & $\begin{array}{l}\text { TOTAL } \\
(U G / L)\end{array}$ & $\begin{array}{l}\text { TOTAL } \\
\text { (UG/L) }\end{array}$ & $\begin{array}{l}\text { TOTAL } \\
\text { (UG/L) }\end{array}$ & $\begin{array}{l}\text { TOTAL } \\
\text { (UG/L) }\end{array}$ & $\begin{array}{l}\text { TOTAL } \\
\text { (UG/L) }\end{array}$ & $\begin{array}{l}\text { TOTAL } \\
(\text { UG } / L)\end{array}$ & $\begin{array}{l}\text { TOTAL } \\
\text { (UG/L) }\end{array}$ & $\begin{array}{l}\text { REC } \\
\text { (UG/L) }\end{array}$ & $\begin{array}{l}\text { TOTAL } \\
\text { (UG/L) }\end{array}$ & $\begin{array}{l}\text { TOTAL } \\
\text { (UG/L) }\end{array}$ & $\begin{array}{c}\text { REC } \\
(U G / L)\end{array}$ & $\begin{array}{l}\text { TOTAL } \\
\text { (UG/L) }\end{array}$ \\
\hline FEB 1992 & & & & & & & & & & & & \\
\hline $22-22$ & $<0.030$ & $<0.10$ & $<0.10$ & $<0.1$ & $<0.10$ & $<0.04$ & $<0.020$ & $<0.10$ & $<0.04$ & $<0.60$ & $<0.060$ & $<0.20$ \\
\hline MAR & & & & & & & & & & & & \\
\hline $09-09$ & $<0.030$ & $<0.10$ & $<0.10$ & $<0.1$ & $<0.10$ & $<0.04$ & $<0.020$ & $<0.10$ & $<0.04$ & $<0.60$ & $<0.060$ & $<0.20$ \\
\hline APR & & & & & & & & & & & & \\
\hline $\begin{array}{l}05.06 \\
A P R\end{array}$ & $<0.030$ & $<0.10$ & $<0.10$ & $<0.1$ & $<0.10$ & $<0.04$ & $<0.020$ & $<0.10$ & $<0.04$ & $<0.60$ & $<0.060$ & $<0.20$ \\
\hline $28 \cdot 28$ & $<0.030$ & $<0.10$ & $<0.10$ & $<0.1$ & $<0.10$ & $<0.04$ & $<0.020$ & $<0.10$ & $<0.04$ & $<0.60$ & $<0.060$ & $<0.20$ \\
\hline JUN & & & & & & & & & & & & \\
\hline 06.06 & $<0.030$ & $<0.10$ & $<0.10$ & $<0.1$ & $<0.10$ & $<0.04$ & $<0.020$ & $<0.10$ & $<0.04$ & $<0.60$ & $<0.060$ & $<0.20$ \\
\hline JUN & & & & & & & & & & & & \\
\hline $21-21$ & $<0.030$ & $<0.10$ & $<0.10$ & $<0.1$ & $<0.10$ & $<0.04$ & $<0.020$ & $<0.10$ & $<0.04$ & $<0.60$ & $<0.060$ & $<0.20$ \\
\hline AUG & & & & & & & & & & & & \\
\hline $12 \cdot 12$ & $<0.030$ & $<0.10$ & $<0.10$ & $<0.1$ & $<0.10$ & $<0.04$ & $<0.020$ & $<0.10$ & $<0.04$ & $<0.60$ & $<0.060$ & $<0.20$ \\
\hline
\end{tabular}


Table 4. Water-quality data for storm-sewer outfall stations, Fort worth, Texas, 1992-93-Continued 08047100 - CLEAR FORK TRINITY RIVER OUTFALL AT OAK HILL CIRCLE, FORT WORTH, TX (WY 1992)-CONTINUEd

\begin{tabular}{|c|c|c|c|c|c|c|c|c|c|c|c|}
\hline & & HEPTA - & & $\begin{array}{c}\text { AROCLOR } \\
1016\end{array}$ & $\begin{array}{l}\text { AROCLOR } \\
1221\end{array}$ & $\begin{array}{c}\text { AROCLOR } \\
1232\end{array}$ & $\begin{array}{l}\text { AROCLOR } \\
1242\end{array}$ & $\begin{array}{l}\text { AROCLOR } \\
1248\end{array}$ & $\begin{array}{c}\text { AROCLOR } \\
1254\end{array}$ & AROCLOR & \\
\hline DATE & $\begin{array}{l}\text { HEPTA- } \\
\text { CHLOR, } \\
\text { TOTAL } \\
\text { (UG/L) }\end{array}$ & $\begin{array}{l}\text { CHLOR } \\
\text { EPOXIDE } \\
\text { TOTAL } \\
\text { (UG/L) }\end{array}$ & $\begin{array}{c}\text { TOX - } \\
\text { APHENE, } \\
\text { TOTAL } \\
\text { (UG/L) }\end{array}$ & $\begin{array}{l}1016 \\
\text { PCB } \\
\text { TOTAL } \\
\text { (UG/L) }\end{array}$ & $\begin{array}{c}1221 \\
\text { PCB } \\
\text { TOTAL } \\
\text { (UG/L) }\end{array}$ & $\begin{array}{c}1232 \\
\text { PCB } \\
\text { TOTAL } \\
\text { (UG/L) }\end{array}$ & $\begin{array}{c}1242 \\
\text { PCB } \\
\text { TOTAL } \\
\text { (UG/L) }\end{array}$ & $\begin{array}{c}1248 \\
\text { PCB } \\
\text { TOTAL } \\
\text { (UG/L) }\end{array}$ & $\begin{array}{c}1254 \\
\text { PCB } \\
\text { TOTAL } \\
\text { (UG/L) }\end{array}$ & $\begin{array}{c}1260 \\
\text { PCB } \\
\text { TOTAL } \\
(U G / L)\end{array}$ & $\begin{array}{c}\text { DI - } \\
\text { AZINON, } \\
\text { TOTAL } \\
\text { (UG/L) }\end{array}$ \\
\hline \multicolumn{12}{|l|}{ FEB 1992} \\
\hline $22 \cdot 22$ & $<0.030$ & $<0.80$ & $<2$ & $<0.1$ & $<1.0$ & $<0.1$ & $<0.1$ & $<0.1$ & $<0.1$ & $<0.1$ & 0.90 \\
\hline \multicolumn{12}{|l|}{ MAR } \\
\hline 09.09 & $<0.030$ & $<0.80$ & $<2$ & $<0.1$ & $<1.0$ & $<0.1$ & $<0.1$ & $<0.1$ & $<0.1$ & $<0.1$ & 2.3 \\
\hline \multicolumn{12}{|l|}{ APR } \\
\hline $05-06$ & $<0.030$ & $<0.80$ & $<2$ & $<0.1$ & $<1.0$ & $<0.1$ & $<0.1$ & $<0.1$ & $<0.1$ & $<0.1$ & 1.0 \\
\hline \multicolumn{12}{|l|}{ APR } \\
\hline $28-28$ & $<0.030$ & $<0.80$ & $<2$ & $<0.1$ & $<1.0$ & $<0.1$ & $<0.1$ & $<0.1$ & $<0.1$ & $<0.1$ & 1.3 \\
\hline \multicolumn{12}{|l|}{ JUN } \\
\hline 06.06 & $<0.030$ & $<0.80$ & $<2$ & $<0.1$ & $<1.0$ & $<0.1$ & $<0.1$ & $<0.1$ & $<0.1$ & $<0.1$ & 0.50 \\
\hline \multicolumn{12}{|l|}{ JUN } \\
\hline $21 \cdot 21$ & $<0.030$ & $<0.80$ & $<2$ & $<0.1$ & $<1.0$ & $<0.1$ & $<0.1$ & $<0.1$ & $<0.1$ & $<0.1$ & 0.10 \\
\hline \multicolumn{12}{|l|}{$A U G$} \\
\hline $12-12$ & $<0.030$ & $<0.80$ & $<2$ & $<0.1$ & $<1,0$ & $<0.1$ & $<0.1$ & $<0.1$ & $<0.1$ & $<0.1$ & 0.10 \\
\hline
\end{tabular}


Table 4. Water-quality data for storm-sewer outfall stations, Fort Worth, Texas, 1992-93-Continued

08048505 - PYLON STREET OUTFALL AT MEACHAM ROAD, FORT WORTH, TX (WY 1992)

\begin{tabular}{|c|c|c|c|c|c|c|c|c|c|c|c|c|}
\hline DATE & TIME & $\begin{array}{l}\text { PRECIP. } \\
\text { ITATION } \\
\text { TOTAL } \\
\text { INCHES/ } \\
\text { STORM }\end{array}$ & $\begin{array}{l}\text { ELAPSED } \\
\text { TIME } \\
\text { OF } \\
\text { STORM } \\
\text { (HOURS) }\end{array}$ & $\begin{array}{l}\text { STORM } \\
\text { WATER } \\
\text { FLOW } \\
\text { (MGD) }\end{array}$ & $\begin{array}{l}\text { SPE- } \\
\text { CIFIC } \\
\text { CON- } \\
\text { DUCT- } \\
\text { ANCE } \\
\text { (US/CM) }\end{array}$ & $\begin{array}{c}\text { SPE- } \\
\text { CIFIC } \\
\text { CON- } \\
\text { DUCT- } \\
\text { ANCE } \\
\text { LAB } \\
\text { (US/CM) }\end{array}$ & $\begin{array}{c}\text { PH } \\
\text { WATER } \\
\text { WHOLE } \\
\text { FIELD } \\
\text { (STAND- } \\
\text { ARD } \\
\text { UNITS) }\end{array}$ & $\begin{array}{c}\text { PH } \\
\text { WATER } \\
\text { WHOLE } \\
\text { LAB } \\
\text { (STAND- } \\
\text { ARD } \\
\text { UNITS) }\end{array}$ & $\begin{array}{c}\text { TEMPER- } \\
\text { ATURE } \\
\text { WATER } \\
\text { (DEG C) }\end{array}$ & $\begin{array}{c}\text { OXYGEN } \\
\text { DEMAND, } \\
\text { CHEM- } \\
\text { ICAL } \\
\text { (HIGH } \\
\text { LEVEL) } \\
\text { (MG/L) }\end{array}$ & $\begin{array}{l}\text { OXYGEN } \\
\text { DEMAND, } \\
\text { BIO- } \\
\text { CHEM- } \\
\text { ICAL, } \\
5 \text { DAY } \\
\text { (MG } / \mathrm{L})\end{array}$ & $\begin{array}{l}\text { COLI- } \\
\text { FORM, } \\
\text { FECAL, } \\
0.7 \\
\text { UM-MF } \\
\text { (COLS. / } \\
100 \mathrm{ML} \text { ) }\end{array}$ \\
\hline FEB 1992 & & & & & & & & & & & & \\
\hline $\begin{array}{l}03-04 \\
F E B\end{array}$ & 1150 & 0.25 & 20.5 & 0.14 & 637 & 539 & 8.6 & 7.5 & 10.5 & 36 & 10 & 320 \\
\hline $22 \cdot 22$ & 1215 & 0.39 & 5.4 & 0.20 & 319 & 178 & 7.6 & 7.6 & 16.0 & 86 & 8.8 & 1600 \\
\hline $\begin{array}{c}03-04 \\
\text { APR }\end{array}$ & 2245 & 0.41 & 5.0 & 0.22 & 240 & 264 & 7.7 & 7.2 & 16.0 & 73 & 10 & 4100 \\
\hline $\begin{array}{l}17 \cdot 17 \\
\text { MAY }\end{array}$ & 0530 & 0.29 & 3.5 & 0.13 & 85 & 258 & 8.0 & 7.3 & 18.0 & 55 & 8.7 & $>18000$ \\
\hline${ }_{\text {JUN }}^{14 \cdot 14}$ & 1132 & 0.29 & 2.5 & 0.16 & 132 & 213 & 8.2 & 7.2 & 21.0 & 97 & 7.6 & 5400 \\
\hline $\begin{array}{l}06-06 \\
\text { SEP }\end{array}$ & 0230 & 0.57 & 2.8 & 0.37 & 195 & 174 & 7.9 & 7.5 & 21.5 & 34 & 9.1 & 290000 \\
\hline $21 \cdot 21$ & 0055 & 0.88 & 4.1 & 0.58 & 110 & 166 & 8.2 & 7.9 & 24.0 & 43 & 7.0 & K17000 \\
\hline
\end{tabular}

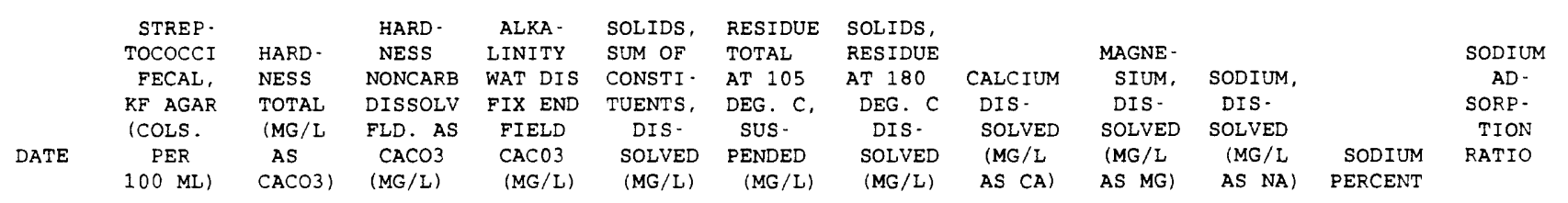

\begin{tabular}{|c|c|c|c|c|c|c|c|c|c|c|c|c|}
\hline $\begin{array}{l}03-04 \\
\text { FEB }\end{array}$ & 2600 & 160 & 22 & 140 & 296 & 23 & 318 & 59 & 2.6 & 41 & 35 & 1 \\
\hline $22-22$ & 8400 & 42 & 6 & 36 & 89 & 98 & 89 & 16 & 050 & 80 & 28 & 0.5 \\
\hline MAR & & & & & & & & & & & & \\
\hline $\begin{array}{l}03-04 \\
A P R\end{array}$ & 17000 & 63 & 11 & 52 & 145 & 25 & 135 & 23 & 1.3 & 23 & 43 & 1 \\
\hline${ }_{\text {MAY }}^{17-17}$ & $>33000$ & 63 & 12 & 51 & 126 & 74 & 137 & 23 & 1.3 & 19 & 38 & 1 \\
\hline $\begin{array}{l}14 \cdot 14 \\
\text { JUN }\end{array}$ & 65000 & 51 & 15 & 36 & 100 & 27 & 116 & 19 & 0.90 & 11 & 30 & 0.7 \\
\hline $\begin{array}{l}06-06 \\
\text { SEP }\end{array}$ & 150000 & 43 & 2 & 41 & 85 & 29 & 92 & 16 & 0.76 & 11 & 34 & 0.7 \\
\hline $21 \cdot 21$ & K10000 & 23 & 5 & 18 & 71 & 171 & 48 & 8.6 & 0.37 & 4.9 & 30 & 0.4 \\
\hline
\end{tabular}

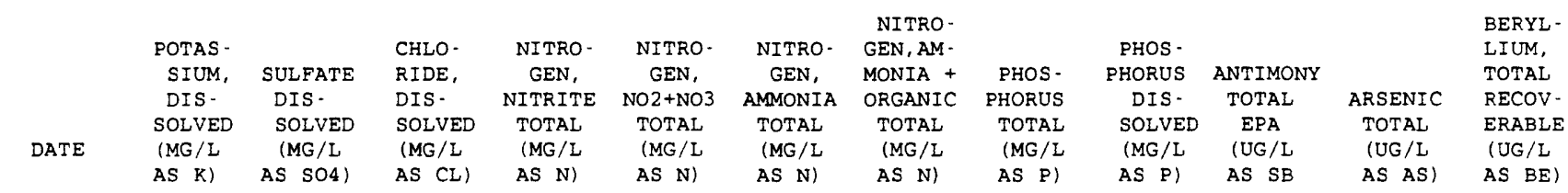

\begin{tabular}{|c|c|c|c|c|c|c|c|c|c|c|c|c|}
\hline \multicolumn{13}{|l|}{ FEB 1992} \\
\hline 03-04 & 3.5 & 57 & 50 & 0.050 & 1.10 & 0.020 & 0.50 & 0.100 & 0.060 & $<20.0$ & 3 & $<10$ \\
\hline \multicolumn{13}{|l|}{$F E B$} \\
\hline $22 \cdot 22$ & 2.1 & 16 & 8.2 & 0.070 & 0.610 & 0.070 & 0.80 & 0.320 & 0.200 & $<10.0$ & 1 & $<10$ \\
\hline \multicolumn{13}{|l|}{ MAR } \\
\hline 03.04 & 2.0 & 21 & 27 & 0.050 & 0.710 & 0.090 & 0.80 & 0.660 & 0.060 & $<10.0$ & 1 & $<10$ \\
\hline \multicolumn{13}{|l|}{ APR } \\
\hline $17-17$ & 2.8 & 17 & 22 & 0.050 & 0.650 & 0.190 & 1.1 & 0.410 & 0.320 & $<10.0$ & 2 & $<10$ \\
\hline \multicolumn{13}{|l|}{ MAY } \\
\hline $14 \cdot 14$ & 3.9 & 15 & 16 & 0.100 & 1.10 & 0.340 & 1.2 & 0.440 & 0.420 & $<10.0$ & 2 & $<10$ \\
\hline \multicolumn{13}{|l|}{ JUN } \\
\hline $06-06$ & 1.9 & 12 & 13 & 0.030 & 0.480 & 0.120 & 0.50 & 0.110 & 0.080 & $<20.0$ & $<1$ & $<10$ \\
\hline \multicolumn{13}{|l|}{ SEP } \\
\hline $21-21$ & 1.4 & 4.8 & 6.9 & 0.030 & 0.310 & 0.110 & 0.70 & 0.200 & 0.140 & $<10.0$ & 1 & $<10$ \\
\hline
\end{tabular}


Table 4. Water-quality data for storm-sewer outfall stations, Fort worth, Texas, 1992-93-Continued

08048505 - PYLON STREET OUTFALL AT MEACHAM ROAD, FORT WORTH, TX (WY 1992)-Continued

\begin{tabular}{|c|c|c|c|c|c|c|c|c|c|c|c|c|}
\hline & $\begin{array}{l}\text { CADMIUM } \\
\text { TOTAL }\end{array}$ & $\begin{array}{l}\text { CHRO- } \\
\text { MIUM, } \\
\text { TOTAL }\end{array}$ & $\begin{array}{c}\text { COPPER, } \\
\text { TOTAL }\end{array}$ & CYANIDE & & $\begin{array}{l}\text { LEAD, } \\
\text { TOTAL }\end{array}$ & $\begin{array}{c}\text { MERCURY } \\
\text { TOTAL }\end{array}$ & $\begin{array}{c}\text { NICKEL, } \\
\text { TOTAL }\end{array}$ & SELE- & $\begin{array}{c}\text { SILVER, } \\
\text { TOTAL }\end{array}$ & $\begin{array}{l}\text { SILVER, } \\
\text { TOTAL } \\
\text { RECOV- }\end{array}$ & THAL - \\
\hline DATE & $\begin{array}{l}\text { RECOV- } \\
\text { ERABLE } \\
\text { (UG/L }\end{array}$ & $\begin{array}{l}\text { RECOV - } \\
\text { ERABLE } \\
\text { (UG/L }\end{array}$ & $\begin{array}{l}\text { RECOV } \\
\text { ERABLE } \\
\text { (UG/L }\end{array}$ & $\begin{array}{l}\text { TOTAL } \\
\text { EPA } \\
\text { (MG/L }\end{array}$ & $\begin{array}{c}\text { CYANIDE } \\
\text { TOTAL } \\
\text { (MG/L }\end{array}$ & $\begin{array}{l}\text { RECOV - } \\
\text { ERABLE } \\
\text { (UG/L }\end{array}$ & $\begin{array}{l}\text { RECOV - } \\
\text { ERABLE } \\
\text { (UG/L }\end{array}$ & $\begin{array}{l}\text { RECOV - } \\
\text { ERABLE } \\
\text { (UG/L }\end{array}$ & $\begin{array}{l}\text { NIUM, } \\
\text { TOTAL } \\
\text { (UG/L }\end{array}$ & $\begin{array}{l}\text { RECOV - } \\
\text { ERABLE } \\
(U G / L\end{array}$ & $\begin{array}{c}\text { ERABLE } \\
\text { EPA } \\
(\mathrm{UG} / \mathrm{L}\end{array}$ & $\begin{array}{l}\text { LIUM, } \\
\text { TOTAL } \\
\text { (UG/L }\end{array}$ \\
\hline & AS CD) & AS CR) & AS CU) & AS CN) & AS CN) & AS $P B)$ & AS HG) & AS NI) & AS SE) & AS AG) & AS AG) & AS TL) \\
\hline
\end{tabular}

$\begin{array}{ll}\text { FEB } 1992 & \\ 03-04 & <1 \\ \text { FEB } & \\ 22-22 & <1 \\ \text { MAR } & \\ 03-04 & <1 \\ \text { APR } & \\ 17-17 & <1 \\ \text { MAY } & \\ 14-14 & <1 \\ \text { JUN } & \\ 06-06 & <1 \\ \text { SEP } & \\ 21-21 & <1\end{array}$

$\begin{array}{ccc}5 & <0.010 & <0.010 \\ 18 & <0.010 & <0.010 \\ 8 & <0.010 & <0.010 \\ 8 & <0.010 & <0.010 \\ 9 & <10.0 & <0.010 \\ 4 & <10.0 & <0.010 \\ 7 & <0.010 & <0.010\end{array}$

$\begin{aligned} 3 & <0.10 \\ 32 & <0.10 \\ 14 & <0.10 \\ 10 & <0.10 \\ 12 & <0.10 \\ 6 & <0.10 \\ 26 & <0.10\end{aligned}$

As

SE)

2
$<2$
$<2$
$<2$
$<2$
$<2$
$<2$

$\begin{array}{lrr}\text { AG) } & \text { AS AG) } & \text { AS TL) } \\ <1 & <1.00 & <10 \\ <1 & <1.00 & <5 \\ <1 & <1.00 & <10 \\ <1 & <1.00 & <5 \\ <1 & <1.00 & <10 \\ <1 & <1.00 & <5 \\ <1 & <0.500 & <10\end{array}$

\begin{tabular}{|c|c|c|c|c|c|c|c|c|c|c|c|c|}
\hline DATE & $\begin{array}{l}\text { ZINC, } \\
\text { TOTAL } \\
\text { RECOV- } \\
\text { ERABLE } \\
\text { (UG/L } \\
\text { AS ZN) }\end{array}$ & $\begin{array}{c}\text { CARBON, } \\
\text { ORGANIC } \\
\text { TOTAL } \\
\text { (MG/L } \\
\text { AS C) }\end{array}$ & $\begin{array}{l}\text { OIL AND } \\
\text { GREASE, } \\
\text { TOTAL } \\
\text { RECOV. } \\
\text { GRAVI - } \\
\text { METRIC } \\
\text { (MG/L) }\end{array}$ & $\begin{array}{c}\text { PHENOLS } \\
\text { TOTAL } \\
\text { (UG/L) }\end{array}$ & $\begin{array}{l}\text { ACRO- } \\
\text { LEIN } \\
\text { TOTAL } \\
(U G / L)\end{array}$ & $\begin{array}{l}\text { ACRYLO- } \\
\text { NITRILE } \\
\text { TOTAL } \\
\text { (UG/L) }\end{array}$ & $\begin{array}{c}\text { BENZENE } \\
\text { TOTAL } \\
\text { (UG/L) }\end{array}$ & $\begin{array}{l}\text { BROMO- } \\
\text { BENZENE } \\
\text { WATER, } \\
\text { WHOLE, } \\
\text { TOTAL } \\
\text { (UG/L) }\end{array}$ & $\begin{array}{l}\text { BROMO- } \\
\text { FORM } \\
\text { TOTAL } \\
\text { (UG/L) }\end{array}$ & $\begin{array}{c}\text { BENZENE } \\
\text { N-BUTYL } \\
\text { WATER } \\
\text { UNFLTRD } \\
\text { REC } \\
\text { (UG/L) }\end{array}$ & $\begin{array}{l}\text { BENZENE } \\
\text { SEC } \\
\text { BUTYL- } \\
\text { WATER } \\
\text { UNFLTRD } \\
\text { REC } \\
\text { (UG/L) }\end{array}$ & $\begin{array}{c}\text { BENZENE } \\
\text { TERT - } \\
\text { BUTYL- } \\
\text { WATER } \\
\text { UNFLTRD } \\
\text { REC } \\
\text { (UG/L) }\end{array}$ \\
\hline $\begin{array}{c}\text { FEB } 1992 \\
03-04\end{array}$ & 20 & 8.8 & $<1$ & 3 & $<20$ & $<20$ & $<0.2$ & $<0.2$ & $<0.2$ & $<0.20$ & $<0.20$ & $<0,20$ \\
\hline$F E B$ & & & & & & & & & & & & \\
\hline $\begin{array}{l}22-22 \\
\text { MAR }\end{array}$ & 110 & 19 & 1 & 5 & $<20$ & $<20$ & $<0.2$ & $<0.2$ & $<0.2$ & $<0.20$ & $<0.20$ & $<0.20$ \\
\hline $\begin{array}{l}03-04 \\
\text { APR }\end{array}$ & 70 & 14 & $<1$ & 3 & $<20$ & $<20$ & $<0.2$ & $<0.2$ & $<0.2$ & $<0.20$ & $<0.20$ & $<0.20$ \\
\hline $\operatorname{MAY}^{17 \cdot 17}$ & 120 & 18 & $<1$ & 2 & $<20$ & $<20$ & $<0.2$ & $<0.2$ & $<0.2$ & $<0.20$ & $<0.20$ & $<0.20$ \\
\hline $\begin{array}{l}14-14 \\
\text { JUN }\end{array}$ & 110 & 24 & $<1$ & 11 & $<20$ & $<20$ & $<0.2$ & $<0.2$ & $<0.2$ & $<0.20$ & $<0.20$ & $<0.20$ \\
\hline $\begin{array}{l}06.06 \\
\text { SEP }\end{array}$ & 40 & 8.0 & $<1$ & 2 & $<20$ & $<20$ & $<0.2$ & $<0.2$ & $<0.2$ & $<0.20$ & $<0.20$ & $<0.20$ \\
\hline $21-21$ & 80 & 17 & $<1$ & 3 & $2-$ & $<20$ & $<0.2$ & $<0.2$ & $<0.2$ & DIBROMO & $<0.20$ & 1,2 \\
\hline & $\begin{array}{l}\text { CARBON - } \\
\text { TETRA- } \\
\text { CHLO- } \\
\text { RIDE }\end{array}$ & $\begin{array}{l}\text { CHLORO - } \\
\text { BENZENE }\end{array}$ & $\begin{array}{c}\text { CHLORO- } \\
\text { DI - } \\
\text { BROMO- } \\
\text { METHANE }\end{array}$ & $\begin{array}{l}\text { CHLORO- } \\
\text { ETHANE }\end{array}$ & $\begin{array}{l}\text { CHLORO- } \\
\text { ETHYL - } \\
\text { VINYL- } \\
\text { ETHER }\end{array}$ & $\begin{array}{l}\text { CHLORO- } \\
\text { FORM }\end{array}$ & $\begin{array}{l}\text { METHYL - } \\
\text { CHLO- } \\
\text { RIDE }\end{array}$ & $\begin{array}{c}\text { CHLORO- } \\
\text { TOLUENE } \\
\text { WATER } \\
\text { WHOLE }\end{array}$ & $\begin{array}{c}\text { TOLUENE } \\
\text { P-CHLOR } \\
\text { WATER } \\
\text { UNFLTRD }\end{array}$ & $\begin{array}{l}\text { CHLORO- } \\
\text { PROPANE } \\
\text { WATER } \\
\text { WHOLE }\end{array}$ & $\begin{array}{l}\text { BROMO- } \\
\text { METHANE } \\
\text { WATER } \\
\text { WHOLE }\end{array}$ & $\begin{array}{l}\text { DIBROMO } \\
\text { ETHANE } \\
\text { WATER } \\
\text { WHOLE }\end{array}$ \\
\hline DATE & $\begin{array}{l}\text { TOTAL } \\
\text { (UG/L) }\end{array}$ & $\begin{array}{l}\text { TOTAL } \\
(\mathrm{UG} / \mathrm{L})\end{array}$ & $\begin{array}{l}\text { TOTAL } \\
\text { (UG/L) }\end{array}$ & $\begin{array}{l}\text { TOTAL } \\
\text { (UG/L) }\end{array}$ & $\begin{array}{l}\text { TOTAL } \\
\text { (UG/L) }\end{array}$ & $\begin{array}{c}\text { TOTAL } \\
(U G / L)\end{array}$ & $\begin{array}{l}\text { TOTAL } \\
\text { (UG/L) }\end{array}$ & $\begin{array}{c}\text { TOTAL } \\
\text { (UG/L) }\end{array}$ & $\begin{array}{c}R E C \\
(U G / L)\end{array}$ & $\begin{array}{l}\text { TOT.REC } \\
(U G / L)\end{array}$ & $\begin{array}{l}\text { RECOVER } \\
\text { (UG/L) }\end{array}$ & $\begin{array}{l}\text { TOTAL } \\
\text { (UG/L) }\end{array}$ \\
\hline FEB 1992 & & & & & & & & & & & & \\
\hline $\begin{array}{l}03-04 \\
F E B\end{array}$ & $<0.2$ & $<0.20$ & $<0.2$ & $<0.2$ & $<1.0$ & $<0.2$ & $<0.2$ & $<0.2$ & $<0.20$ & $<1.0$ & $<0.2$ & $<0.2$ \\
\hline $\begin{array}{l}22-22 \\
\text { MAR }\end{array}$ & $<0.2$ & $<0.20$ & $<0.2$ & $<0.2$ & $<1.0$ & $<0.2$ & $<0.2$ & $<0.2$ & $<0.20$ & $<1.0$ & $<0.2$ & $<0.2$ \\
\hline $\begin{array}{l}03-04 \\
\text { APR }\end{array}$ & $<0.2$ & $<0.20$ & $<0.2$ & $<0.2$ & $<1.0$ & $<0.2$ & $<0.2$ & $<0.2$ & $<0.20$ & $<1.0$ & $<0.2$ & $<0.2$ \\
\hline $\begin{array}{l}17-17 \\
\text { MAY }\end{array}$ & $<0.2$ & $<0.20$ & $<0.2$ & $<0.2$ & $<1.0$ & $<0.2$ & $<0.2$ & $<0.2$ & $<0.20$ & $<1.0$ & $<0.2$ & $<0.2$ \\
\hline JUN $^{14-14}$ & $<0.2$ & $<0.20$ & $<0.2$ & $<0.2$ & $<1.0$ & $<0.2$ & $<0.2$ & $<0.2$ & $<0.20$ & $<1.0$ & $<0.2$ & $<0.2$ \\
\hline 06.06 & $<0.2$ & $<0.20$ & $<0.2$ & $<0.2$ & $<1.0$ & $<0.2$ & $<0.2$ & $<0.2$ & $<0.20$ & $<1.0$ & $<0.2$ & $<0.2$ \\
\hline SEP & & & & & & & & & & 10 & & \\
\hline $21 \cdot 21$ & $<0.2$ & $<0.20$ & $<0.2$ & $<0.2$ & $<1.0$ & $<0.2$ & $<0.2$ & $<0.2$ & $<0.20$ & $<1.0$ & $<0.2$ & $<0.2$ \\
\hline
\end{tabular}


Table 4. Water-quality data for storm-sewer outfall stations, Fort Worth, Texas, 1992-93-Continued

08048505 - PYLON STREET OUTFALL AT MEACHAM ROAD, FORT WORTH, TX (WY 1992)-Continued

\begin{tabular}{|c|c|c|c|c|c|c|c|c|c|c|c|c|}
\hline DATE & $\begin{array}{c}\text { DI- } \\
\text { CHLORO- } \\
\text { BROMO- } \\
\text { METHANE } \\
\text { TOTAL } \\
\text { (UG/L) }\end{array}$ & $\begin{array}{c}1,1-D I- \\
\text { CHLORO- } \\
\text { ETHYL- } \\
\text { ENE } \\
\text { TOTAL } \\
\text { (UG/L) }\end{array}$ & $\begin{array}{l}\text { 1, I-DI } \\
\text { CHLORO- } \\
\text { PRO- } \\
\text { PENE, } \\
\text { WAT, WH } \\
\text { TOTAL } \\
\text { (UG/L) }\end{array}$ & $\begin{array}{c}\text { BENZENE } \\
\text { O- } \\
\text { CHLORO- } \\
\text { WATER } \\
\text { UNFLTRD } \\
\text { REC } \\
\text { (UG/L) }\end{array}$ & $\begin{array}{c}\text { BENZENE } \\
1,3-D I- \\
\text { CHLORO- } \\
\text { WATER } \\
\text { UNFLTRD } \\
\text { REC } \\
(U G / L)\end{array}$ & $\begin{array}{c}\text { BENZENE } \\
1,4-D I- \\
\text { CHLORO- } \\
\text { WATER } \\
\text { UNFLTRD } \\
\text { REC } \\
\text { (UG/L) }\end{array}$ & $\begin{array}{l}\text { DI- } \\
\text { CHLORO- } \\
\text { DI- } \\
\text { FLUORO- } \\
\text { METHANE } \\
\text { TOTAL } \\
\text { (UG/L) }\end{array}$ & $\begin{array}{l}1,1 \text { - DI - } \\
\text { CHLORO- } \\
\text { ETHANE } \\
\text { TOTAL } \\
\text { (UG/L) }\end{array}$ & $\begin{array}{c}1,2-D I- \\
\text { CHLORO- } \\
\text { ETHANE } \\
\text { TOTAL } \\
\text { (UG/L) }\end{array}$ & $\begin{array}{l}1,2-D I- \\
\text { PHENYL- } \\
\text { HYDRA- } \\
\text { ZINE } \\
\text { WATER } \\
\text { TOT.REC } \\
\text { (UG/L) }\end{array}$ & $\begin{array}{c}\text { CIS-1,2- } \\
\text { DI- } \\
\text { CHLORO- } \\
\text { ETHENE } \\
\text { WATER } \\
\text { TOTAL } \\
\text { (UG/L) }\end{array}$ & $\begin{array}{l}1,2- \\
\text { TRANSDI } \\
\text { CHLORO- } \\
\text { ETHENE } \\
\text { TOTAL } \\
\text { (UG/L) }\end{array}$ \\
\hline EB 199 & & & & & & & & & & & & \\
\hline $\begin{array}{l}03-04 \\
F E B\end{array}$ & $<0.2$ & $<0.2$ & $<0.2$ & $<5.0$ & $<5.0$ & $<5.0$ & $<0.2$ & $<0.2$ & $<0.2$ & $<5.0$ & $<0.2$ & $<0.2$ \\
\hline $22-22$ & $<0.2$ & $<0.2$ & $<0.2$ & $<5.0$ & $<5.0$ & $<5.0$ & $<0.2$ & $<0.2$ & $<0.2$ & $<5.0$ & $<0.2$ & $<0.2$ \\
\hline $\begin{array}{l}03-04 \\
A P R\end{array}$ & $<0.2$ & $<0.2$ & $<0.2$ & $<5.0$ & $<5.0$ & $<5.0$ & $<0.2$ & $<0.2$ & $<0.2$ & $<5.0$ & $<0.2$ & $<0.2$ \\
\hline $\operatorname{MAY}^{17-17}$ & $<0.2$ & $<0.2$ & $<0.2$ & $<5.0$ & $<5.0$ & $<5.0$ & $<0.2$ & $<0.2$ & $<0.2$ & $<5.0$ & $<0.2$ & $<0.2$ \\
\hline $\begin{array}{l}14-14 \\
\text { JUN }\end{array}$ & $<0.2$ & $<0.2$ & $<0.2$ & $<5.0$ & $<5.0$ & $<5.0$ & $<0.2$ & $<0.2$ & $<0.2$ & $<5.0$ & $<0.2$ & $<0.2$ \\
\hline $\begin{array}{l}06.06 \\
\text { SEP }\end{array}$ & $<0.2$ & $<0.2$ & $<0.2$ & $<5.0$ & $<5.0$ & $<5.0$ & $<0.2$ & $<0.2$ & $<0.2$ & $<5.0$ & $<0.2$ & $<0.2$ \\
\hline $21 \cdot 21$ & $<0.2$ & $<0.2$ & $<0.2$ & $<5.0$ & $<5.0$ & $<5.0$ & $<0.2$ & $<0.2$ & $<0.2$ & $<5.0$ & $<0.2$ & $<0.2$ \\
\hline
\end{tabular}

\begin{tabular}{|c|c|c|c|c|c|c|c|c|c|c|c|c|}
\hline & $1,2 \cdot \mathrm{DI}$ - & $\begin{array}{l}\text { 1, } 3 \text {-DI - } \\
\text { CHLORO- }\end{array}$ & $\begin{array}{l}2,2 \text { - DI } \\
\text { CHLORO- } \\
\text { PRO- }\end{array}$ & $\begin{array}{c}\text { CIS } \\
1,3 \cdot \mathrm{DI} \text {. }\end{array}$ & $\begin{array}{r}\text { TRANS - } \\
1,3 \cdot \mathrm{DI} \text { - }\end{array}$ & & $\begin{array}{c}\text { HEXA- } \\
\text { CHLORO- }\end{array}$ & $\begin{array}{l}\text { ISO- } \\
\text { PROPYL- } \\
\text { BENZENE }\end{array}$ & $\begin{array}{l}\text { P-ISO- } \\
\text { PROPYL- } \\
\text { TOLUENE }\end{array}$ & & $\begin{array}{l}\text { METHYL - } \\
\text { ENE }\end{array}$ & \\
\hline & CHLORO- & PROPANE & PANE & CHLORO- & CHLORO- & ETHYL - & BUT- & WATER & WATER & METHYL - & CHLO- & NAPHTH \\
\hline & PROPANE & WAT. WH & WAT, WH & PROPENE & PROPENE & BENZENE & ADIENE & WHOLE & WHOLE & BROMIDE & RIDE & ALENE \\
\hline & $\begin{array}{l}\text { TOTAL } \\
(U G / L)\end{array}$ & $\begin{array}{l}\text { TOTAL } \\
(U G / L)\end{array}$ & $\begin{array}{l}\text { TOTAL } \\
\text { (UG/L) }\end{array}$ & $\begin{array}{l}\text { TOTAL } \\
\text { (UG/L) }\end{array}$ & $\begin{array}{l}\text { TOTAL } \\
\text { (UG/L) }\end{array}$ & $\begin{array}{l}\text { TOTAL } \\
\text { (UG/L) }\end{array}$ & $\begin{array}{l}\text { TOTAL } \\
\text { (UG/L) }\end{array}$ & $\begin{array}{l}\text { REC } \\
(\mathrm{UG} / \mathrm{L})\end{array}$ & $\begin{array}{c}\text { REC } \\
(U G / L)\end{array}$ & $\begin{array}{l}\text { TOTAL } \\
(U G / L)\end{array}$ & $\begin{array}{l}\text { TOTAL } \\
\text { (UG/L) }\end{array}$ & $\begin{array}{l}\text { TOTAL } \\
(U G / L)\end{array}$ \\
\hline
\end{tabular}

\begin{tabular}{|c|c|c|c|c|c|c|c|c|c|c|c|c|}
\hline $\begin{array}{c}\text { FEB } 1992 \\
03-04\end{array}$ & $<0.2$ & $<0.2$ & $<0.2$ & $<0.2$ & $<0.2$ & $<0.2$ & $<5.0$ & $<0.20$ & $<0.20$ & $<0.2$ & $<0.2$ & $<5.0$ \\
\hline FEB & & & & & & & & & & & & \\
\hline $22-22$ & $<0.2$ & $<0.2$ & $<0.2$ & $<0.2$ & $<0.2$ & $<0.2$ & $<5.0$ & $<0.20$ & $<0.20$ & $<0.2$ & $<0.2$ & $<5.0$ \\
\hline MAR & & & & & & & & & & & & \\
\hline $03-04$ & $<0.2$ & $<0.2$ & $<0.2$ & $<0.2$ & $<0.2$ & $<0.2$ & $<5.0$ & $<0.20$ & $<0.20$ & $<0.2$ & $<0.2$ & $<5.0$ \\
\hline APR & & & & & & & & & & & & \\
\hline $17 \cdot 17$ & $<0.2$ & $<0.2$ & $<0.2$ & $<0.2$ & $<0.2$ & $<0.2$ & $<5.0$ & $<0.20$ & $<0.20$ & $<0.2$ & 0.8 & $<5.0$ \\
\hline MAY & & & & & & & & & & & & \\
\hline $\mathrm{JUN}^{14-14}$ & $<0.2$ & $<0.2$ & $<0.2$ & $<0.2$ & $<0.2$ & $<0.2$ & $<5.0$ & $<0.20$ & $<0.20$ & $<0.2$ & 0.3 & $<5.0$ \\
\hline $\begin{array}{l}06-06 \\
\text { SEP }\end{array}$ & $<0.2$ & $<0.2$ & $<0.2$ & $<0.2$ & $<0.2$ & $<0.2$ & $<5.0$ & $<0.20$ & $<0.20$ & $<0.2$ & $<0.4$ & $<5.0$ \\
\hline $21-21$ & $<0.2$ & $<0.2$ & $<0.2$ & $<0.2$ & $<0.2$ & $<0.2$ & $<5.0$ & $<0.20$ & $<0.20$ & $<0.2$ & $<0.2$ & $<5.0$ \\
\hline
\end{tabular}

\begin{tabular}{|c|c|c|c|c|c|c|c|c|c|c|c|c|}
\hline & BENZENE & & $\begin{array}{c}\text { ETHANE, } \\
1112 .\end{array}$ & $\begin{array}{l}\text { ETHANE, } \\
1,1,2,2\end{array}$ & TETRA - & & $\begin{array}{l}1,2,3- \\
\text { TRI. }\end{array}$ & $\begin{array}{l}\text { BENZENE } \\
1,2,4 .\end{array}$ & $1,1,1$. & $1,1,2$ & TRI & TRI - \\
\hline & N-PROPY & & TETRA - & TETRA - & CHLORO- & & CHLORO & TRI - & TRI. & TRI - & CHLORO- & CHLORO- \\
\hline & WATER & & CHLORO - & CHLORO- & ETHYL - & & BENZENE & CHLORO- & CHLORO- & CHLO & ETHYL - & FLUORO - \\
\hline & UNFLTRD & STYRENE & WAT UNF & WAT UNF & ENE & TOLUENE & WAT, WH & WAT UNF & ETHANE & ETH & ENE & METHANE \\
\hline & $\begin{array}{c}\text { REC } \\
(U G / L)\end{array}$ & $\begin{array}{l}\text { TOTAL } \\
(U G / L)\end{array}$ & $\begin{array}{c}\text { REC } \\
(U G / L)\end{array}$ & $\begin{array}{c}\text { REC } \\
(U G / L)\end{array}$ & $\begin{array}{l}\text { TOTAL } \\
\text { (UG/L) }\end{array}$ & $\begin{array}{l}\text { TOTAL } \\
(\mathrm{UG} / \mathrm{L})\end{array}$ & $\begin{array}{c}\text { REC } \\
(U G / L)\end{array}$ & $\begin{array}{c}\text { REC } \\
(U G / L)\end{array}$ & $\begin{array}{l}\text { TOTAL } \\
\text { (UG/L) }\end{array}$ & $\begin{array}{l}\text { TOTAL } \\
\text { (UG/L) }\end{array}$ & $\begin{array}{l}\text { TOTAL } \\
\text { (UG/L) }\end{array}$ & $\begin{array}{l}\text { TOTAL } \\
\text { (UG/L) }\end{array}$ \\
\hline
\end{tabular}

\begin{tabular}{|c|c|c|c|c|c|c|c|c|c|c|c|c|}
\hline \multicolumn{13}{|l|}{ FEB 1992} \\
\hline 03.04 & $<0.20$ & $<0.2$ & $<0.2$ & $<0.2$ & $<0.2$ & 0.2 & $<0.20$ & $<5.0$ & $<0.2$ & $<0.2$ & $<0.2$ & $<0.2$ \\
\hline $\begin{array}{l}\text { FEB } \\
22 \cdot 22\end{array}$ & $<0.20$ & $<0.2$ & $<0.2$ & $<0.2$ & $<0.2$ & $<0,2$ & $<0,20$ & $<5,0$ & $<0,2$ & $<0,2$ & $<0.2$ & $<0.2$ \\
\hline MAR & & & & & & & & & .0 .2 & .0 .2 & & \\
\hline $03-04$ & $<0.20$ & $<0.2$ & $<0.2$ & $<0.2$ & $<0.2$ & $<0.2$ & $<0.20$ & $<5.0$ & $<0.2$ & $<0.2$ & $<0.2$ & $<0.2$ \\
\hline APR & & & & & & & & & & & & \\
\hline $17-17$ & $<0.20$ & $<0.2$ & $<0.2$ & $<0.2$ & $<0.2$ & $<0.2$ & $<0.20$ & $<5.0$ & $<0.2$ & $<0.2$ & $<0.2$ & $<0.2$ \\
\hline MAY & & & & & & & & & & & & \\
\hline $14-14$ & $<0.20$ & $<0.2$ & $<0.2$ & $<0.2$ & $<0.2$ & $<0.2$ & $<0.20$ & $<5.0$ & $<0.2$ & $<0.2$ & $<0.2$ & $<0.2$ \\
\hline JUN & & & & & & & & & & & & \\
\hline $06-06$ & $<0.20$ & $<0.2$ & $<0.2$ & $<0.2$ & $<0.2$ & 0.2 & $<0.20$ & $<5.0$ & $<0.2$ & $<0.2$ & $<0.2$ & $<0.2$ \\
\hline SEP & & & & & & & & & & & & \\
\hline $21-21$ & $<0.20$ & $<0.2$ & $<0.2$ & $<0.2$ & $<0.2$ & $<0.2$ & $<0.20$ & $<5.0$ & $<0.2$ & $<0.2$ & $<0.2$ & $<0.2$ \\
\hline
\end{tabular}


Table 4. Water-quality data for storm-sewer outfall stations, Fort Worth, Texas, 1992-93-Continued

08048505 - PYLON STREET OUTFALL AT MEACHAM ROAD, FORT WORTH, TX (WY 1992)-Continued

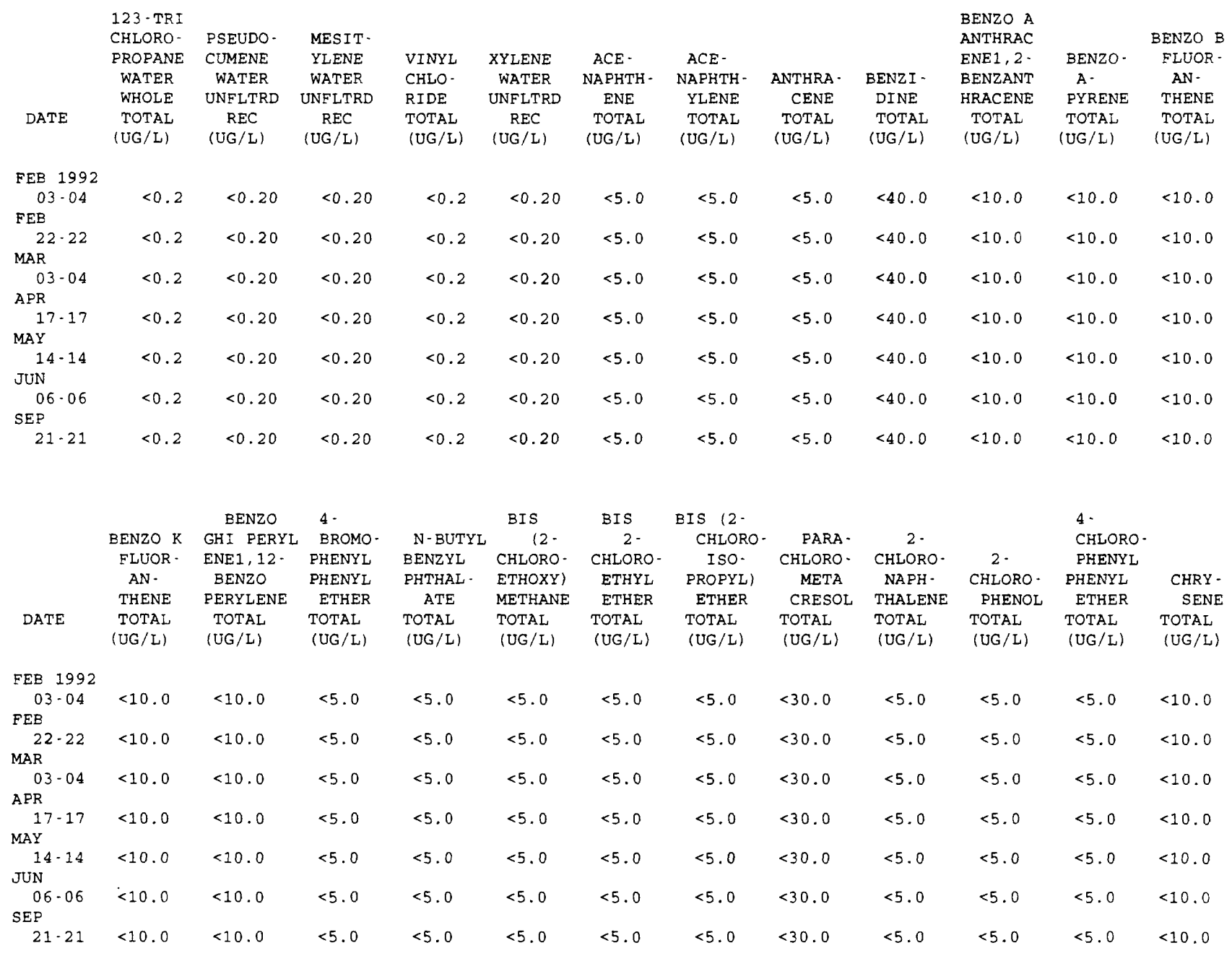

\begin{tabular}{|c|c|c|c|c|c|c|c|c|c|c|c|c|}
\hline DATE & $\begin{array}{l}1,2,5,6- \\
\text { DIBENZ- } \\
\text { ANTHRA- } \\
\text { CENE } \\
\text { TOTAL } \\
\{\text { UG } / L\rangle\end{array}$ & $\begin{array}{c}3,3 '- \\
\text { DI - } \\
\text { CHLORO- } \\
\text { BENZI- } \\
\text { DINE } \\
\text { TOTAL } \\
\text { (UG/L) }\end{array}$ & $\begin{array}{l}2,4-D I- \\
\text { CHLORO- } \\
\text { PHENOL } \\
\text { TOTAL } \\
(\text { UG/L) }\end{array}$ & $\begin{array}{l}\text { DIETHYL } \\
\text { PHTHAL- } \\
\text { ATE } \\
\text { TOTAL } \\
\text { (UG/L) }\end{array}$ & $\begin{array}{c}\text { DI - } \\
\text { METHYL } \\
\text { PHTHAL- } \\
\text { ATE } \\
\text { TOTAL } \\
\text { (UG/L) }\end{array}$ & $\begin{array}{c}2,4 \text {-DI - } \\
\text { METHYL - } \\
\text { PHENOL } \\
\text { TOTAL } \\
(U G / L)\end{array}$ & $\begin{array}{l}\text { DI - N- } \\
\text { BUTYL } \\
\text { PHTHAL - } \\
\text { ATE } \\
\text { TOTAL } \\
\text { (UG/L) }\end{array}$ & $\begin{array}{l}4,6- \\
\text { DINITRO- } \\
\text { ORTHO- } \\
\text { CRESOL } \\
\text { TOTAL } \\
\text { (UG/L) }\end{array}$ & $\begin{array}{c}2,4,- \\
\text { DI- } \\
\text { NITRO- } \\
\text { PHENOL } \\
\text { TOTAL } \\
\text { (UG/L) }\end{array}$ & $\begin{array}{l}2,4-D I- \\
\text { NITRO- } \\
\text { TOLUENE } \\
\text { TOTAL } \\
\text { (UG/L) }\end{array}$ & $\begin{array}{l}2,6 \cdot D I- \\
\text { NITRO- } \\
\text { TOLUENE } \\
\text { TOTAL } \\
\{U G / L\rangle\end{array}$ & $\begin{array}{l}\text { DI - N - } \\
\text { OCTYL } \\
\text { PHTHAL- } \\
\text { ATE } \\
\text { TOTAL } \\
(U G / L)\end{array}$ \\
\hline \multicolumn{13}{|l|}{ FEB 1992} \\
\hline $03-04$ & $<10.0$ & $<20.0$ & $<5.0$ & $<5.0$ & $<5.0$ & $<5.0$ & $<5.0$ & $<30.0$ & $<20.0$ & $<5.0$ & $<5.0$ & $<10.0$ \\
\hline \multicolumn{13}{|l|}{ FEB } \\
\hline $22-22$ & $<10.0$ & $<20.0$ & $<5.0$ & $<5.0$ & $<5.0$ & $<5.0$ & $<5.0$ & $<30.0$ & $<20.0$ & $<5.0$ & $<5.0$ & $<10.0$ \\
\hline \multicolumn{13}{|l|}{ MAR } \\
\hline $03 \cdot 04$ & $<10.0$ & $<20.0$ & $<5.0$ & $<5.0$ & $<5.0$ & $<5.0$ & $<5.0$ & $<30.0$ & $<20.0$ & $<5.0$ & $<5.0$ & $<10.0$ \\
\hline \multicolumn{13}{|l|}{ APR } \\
\hline $17-17$ & $<10.0$ & $<20.0$ & $<5.0$ & $<5.0$ & $<5.0$ & $<5.0$ & $<5.0$ & $<30.0$ & $<20.0$ & $<5.0$ & $<5.0$ & $<10.0$ \\
\hline \multicolumn{13}{|l|}{ MAY } \\
\hline $14-14$ & $<10.0$ & $<20.0$ & $<5.0$ & $<5.0$ & $<5.0$ & $<5.0$ & $<5.0$ & $<30.0$ & $<20.0$ & $<5.0$ & $<5.0$ & $<10.0$ \\
\hline \multicolumn{13}{|l|}{ JUN } \\
\hline $06-06$ & $<10.0$ & $<20.0$ & $<5.0$ & $<5.0$ & $<5.0$ & $<5.0$ & $<5.0$ & $<30.0$ & $<20.0$ & $<5.0$ & $<5.0$ & $<10.0$ \\
\hline \multicolumn{13}{|l|}{ SEP } \\
\hline $21-21$ & $<10.0$ & $<20.0$ & $<5.0$ & $<5.0$ & $<5.0$ & $<5.0$ & $<5.0$ & $<30.0$ & $<20.0$ & $<5.0$ & $<5.0$ & $<10.0$ \\
\hline
\end{tabular}


Table 4. Water-quality data for storm-sewer outfall stations, Fort worth, Texas, 1992-93-Continued

08048505 - PYLON STREET OUTFALL AT MEACHAM ROAD, FORT WORTH, TX (WY 1992)-Continued

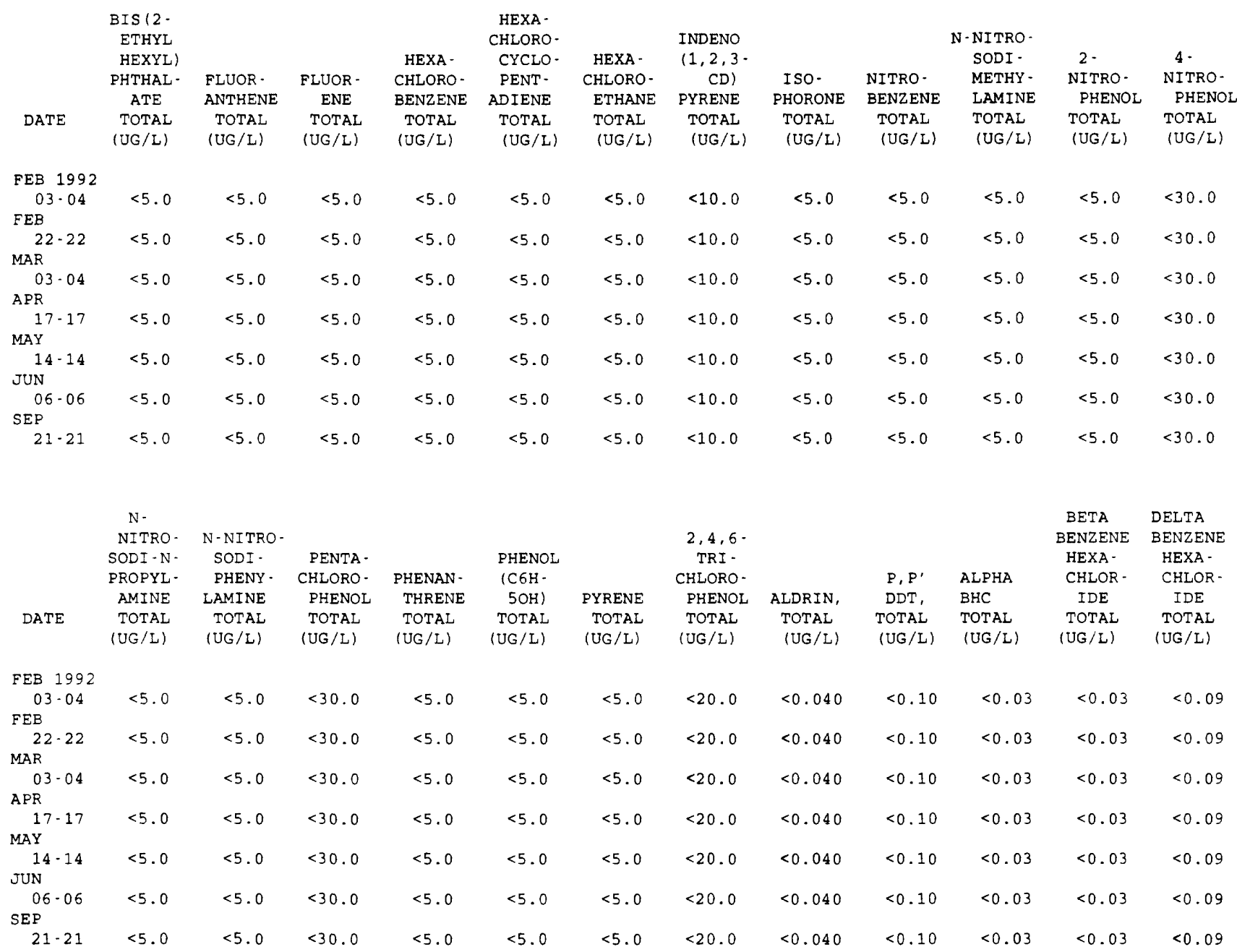

\begin{tabular}{|c|c|c|c|c|c|c|c|c|c|c|c|c|}
\hline DATE & $\begin{array}{c}\text { LINDANE } \\
\text { TOTAL } \\
\text { (UG/L) }\end{array}$ & $\begin{array}{c}\text { CHLOR- } \\
\text { DANE } \\
\text { CIS } \\
\text { WATER } \\
\text { WHOLE } \\
\text { TOTAL } \\
\text { (UG/L) }\end{array}$ & $\begin{array}{l}\text { CHLOR- } \\
\text { DANE } \\
\text { TRANS } \\
\text { WATER } \\
\text { WHOLE } \\
\text { TOTAL } \\
\text { (UG/L) }\end{array}$ & $\begin{array}{l}\text { CHLOR- } \\
\text { DANE, } \\
\text { TOTAL } \\
\text { (UG/L) }\end{array}$ & $\begin{array}{l}\text { P, P' } \\
\text { DDD, } \\
\text { TOTAL } \\
\text { (UG/L) }\end{array}$ & $\begin{array}{l}\text { P, P' } \\
\text { DDE, } \\
\text { TOTAL } \\
\text { (UG/L) }\end{array}$ & $\begin{array}{l}\text { DI - } \\
\text { ELDRIN } \\
\text { TOTAL } \\
\text { (UG/L) }\end{array}$ & $\begin{array}{c}\text { ENDO- } \\
\text { SULFAN- } \\
\text { I } \\
\text { WATER } \\
\text { WHOLE } \\
\text { REC } \\
\text { (UG/L) }\end{array}$ & $\begin{array}{l}\text { ENDO- } \\
\text { SULFAN } \\
\text { BETA } \\
\text { TOTAL } \\
(U G / L)\end{array}$ & $\begin{array}{l}\text { ENDO- } \\
\text { SULFAN } \\
\text { SULFATE } \\
\text { TOTAL } \\
\text { (UG/L) }\end{array}$ & $\begin{array}{l}\text { ENDRIN } \\
\text { WATER } \\
\text { UNFLTRD } \\
\text { REC } \\
\text { (UG/L) }\end{array}$ & $\begin{array}{c}\text { ENDRIN } \\
\text { ALDE- } \\
\text { HYDE } \\
\text { TOTAL } \\
\text { (UG/L) }\end{array}$ \\
\hline FEB 1992 & & & & & & & & & & & & \\
\hline $\begin{array}{l}03 \cdot 04 \\
\text { FEB }\end{array}$ & $<0.030$ & $<0.10$ & $<0.10$ & $<0.1$ & $<0.10$ & $<0.04$ & $<0.020$ & $<0.10$ & $<0.04$ & $<0.60$ & $<0.060$ & $<0.20$ \\
\hline $\begin{array}{l}22-22 \\
\text { MAR }\end{array}$ & $<0.030$ & $<0.10$ & $<0.10$ & $<0.1$ & $<0.10$ & $<0.04$ & $<0.020$ & $<0.10$ & $<0.04$ & $<0.60$ & $<0.060$ & $<0.20$ \\
\hline $\begin{array}{l}03-04 \\
\text { APR }\end{array}$ & $<0.030$ & $<0.10$ & $<0.10$ & $<0.1$ & $<0.10$ & $<0.04$ & $<0.020$ & $<0.10$ & $<0.04$ & $<0.60$ & $<0.060$ & $<0.20$ \\
\hline $\mathrm{MAY}^{17-17}$ & $<0.030$ & $<0.10$ & $<0.10$ & $<0.1$ & $<0.10$ & $<0.04$ & $<0.020$ & $<0.10$ & $<0.04$ & $<0.60$ & $<0.060$ & $<0.20$ \\
\hline${ }_{\text {JUN }}^{14-14}$ & $<0.030$ & $<0.10$ & $<0.10$ & $<0.1$ & $<0.10$ & $<0.04$ & $<0.020$ & $<0.10$ & $<0.04$ & $<0.60$ & $<0.060$ & $<0.20$ \\
\hline $\begin{array}{l}06-06 \\
\text { SEP }\end{array}$ & $<0.030$ & $<0.10$ & $<0.10$ & $<0.1$ & $<0.10$ & $<0.04$ & $<0.020$ & $<0.10$ & $<0.04$ & $<0.60$ & $<0.060$ & $<0.20$ \\
\hline $21-21$ & $<0.030$ & $<0.10$ & $<0.10$ & $<0.1$ & $<0.10$ & $<0.04$ & $<0.020$ & $<0.10$ & $<0.04$ & $<0.60$ & $<0.060$ & $<0.20$ \\
\hline
\end{tabular}


Table 4. Water-quality data for storm-sewer outfall stations, Fort worth, Texas, 1992-93-Continued 08048505 - PYLON STREET OUTFALI AT MEACHAM ROAD, FORT WORTH, TX (WY 1992)-Continued

\begin{tabular}{|c|c|c|c|c|c|c|c|c|c|c|c|}
\hline & & HEPTA - & & AROCLOR & AROCLOR & AROCLOR & AROCLOR & AROCLOR & AROCLOR & AROCLOR & \\
\hline & HEPTA - & CHLOR & TOX - & 1016 & 1221 & 1232 & 1242 & 1248 & 1254 & 1260 & DI. \\
\hline & CHLOR, & EPOXIDE & APHENE, & PCB & PCB & PCB & PCB & PCB & $\mathrm{PCB}$ & PCB & AZINON, \\
\hline DATE & $\begin{array}{l}\text { TOTAL } \\
\text { (UG/L) }\end{array}$ & $\begin{array}{l}\text { TOTAL } \\
\text { (UG/L) }\end{array}$ & $\begin{array}{l}\text { TOTAL } \\
\text { (UG/L) }\end{array}$ & $\begin{array}{l}\text { TOTAL } \\
\text { (UG/L) }\end{array}$ & $\begin{array}{l}\text { TOTAL } \\
\text { (UG/L) }\end{array}$ & $\begin{array}{l}\text { TOTAL } \\
\text { (UG/L) }\end{array}$ & $\begin{array}{l}\text { TOTAL } \\
\text { (UG/L) }\end{array}$ & $\begin{array}{l}\text { TOTAL } \\
\text { (UG/I) }\end{array}$ & $\begin{array}{l}\text { TOTAL } \\
\text { (UG/L) }\end{array}$ & $\begin{array}{l}\text { TOTAL } \\
\text { (UG/L) }\end{array}$ & $\begin{array}{l}\text { TOTAL } \\
\text { (UG/L) }\end{array}$ \\
\hline
\end{tabular}

\begin{tabular}{|c|c|c|c|c|c|c|c|c|c|c|c|}
\hline \multicolumn{12}{|l|}{ FEB 1992} \\
\hline $03-04$ & $<0.030$ & $<0.80$ & $<2$ & $<0.1$ & $<1.0$ & $<0.1$ & $<0.1$ & $<0.1$ & $<0.1$ & $<0.1$ & $<0.01$ \\
\hline \multicolumn{12}{|l|}{ FEB } \\
\hline $22-22$ & $<0.030$ & $<0.80$ & $<2$ & $<0.1$ & $<1.0$ & $<0.1$ & $<0.1$ & $<0.1$ & $<0.1$ & $<0.1$ & 0.04 \\
\hline \multicolumn{12}{|l|}{ MAR } \\
\hline 03.04 & $<0.030$ & $<0.80$ & $<2$ & $<0.1$ & $<1.0$ & $<0.1$ & $<0.1$ & $<0.1$ & $<0.1$ & $<0.1$ & 0.04 \\
\hline \multicolumn{12}{|l|}{ APR } \\
\hline $17 \cdot 17$ & $<0.030$ & $<0.80$ & $<2$ & $<0.1$ & $<1.0$ & $<0.1$ & $<0.1$ & $<0.1$ & $<0.1$ & $<0.1$ & $<0.10$ \\
\hline \multicolumn{12}{|l|}{ MAY } \\
\hline $14 \cdot 14$ & $<0.030$ & $<0.80$ & $<2$ & $<0.1$ & $<1.0$ & $<0.1$ & $<0.1$ & $<0.1$ & $<0.1$ & $<0.1$ & $<0.10$ \\
\hline \multicolumn{12}{|l|}{ JUN } \\
\hline $06-06$ & $<0.030$ & $<0.80$ & $<2$ & $<0.1$ & $<1.0$ & $<0.1$ & $<0.1$ & $<0.1$ & $<0.1$ & $<0.1$ & $<0.10$ \\
\hline \multicolumn{12}{|l|}{ SEP } \\
\hline $21-21$ & $<0.030$ & $<0.80$ & $<2$ & $<0.1$ & $<1.0$ & $<0.1$ & $<0.1$ & $<0.1$ & $<0.1$ & $<0.1$ & $<0.10$ \\
\hline
\end{tabular}


Table 4. Water-quality data for storm-sewer outfall stations, Fort worth, Texas, 1992-93-Continued

08048510 - WEST FORK TRINITY RIVER OUTFALI AT HIGHWAY 121, FORT WORTH, TX (WY 1992)

\begin{tabular}{|c|c|c|c|c|c|c|c|c|c|c|c|c|}
\hline DATE & TIME & $\begin{array}{l}\text { PRECIP. } \\
\text { ITATION } \\
\text { TOTAL } \\
\text { INCHES/ } \\
\text { STORM }\end{array}$ & $\begin{array}{l}\text { ELAPSED } \\
\text { TIME } \\
\text { OF } \\
\text { STORM } \\
\text { (HOURS) }\end{array}$ & $\begin{array}{l}\text { STORM } \\
\text { WATER } \\
\text { FLOW } \\
\text { (MGD) }\end{array}$ & $\begin{array}{l}\text { SPE- } \\
\text { CIFIC } \\
\text { CON- } \\
\text { DUCT- } \\
\text { ANCE } \\
\text { (US/CM) }\end{array}$ & $\begin{array}{c}\text { SPE- } \\
\text { CIFIC } \\
\text { CON- } \\
\text { DUCT - } \\
\text { ANCE } \\
\text { LAB } \\
\text { (US/CM) }\end{array}$ & $\begin{array}{c}\text { PH } \\
\text { WATER } \\
\text { WHOLE } \\
\text { FIELD } \\
\text { (STAND- } \\
\text { ARD } \\
\text { UNITS) }\end{array}$ & $\begin{array}{c}\text { PH } \\
\text { WATER } \\
\text { WHOLE } \\
\text { LAB } \\
\text { (STAND- } \\
\text { ARD } \\
\text { UNITS) }\end{array}$ & $\begin{array}{c}\text { TEMPER - } \\
\text { ATURE } \\
\text { WATER } \\
\text { (DEG C) }\end{array}$ & $\begin{array}{c}\text { OXYGEN } \\
\text { DEMAND, } \\
\text { CHEM- } \\
\text { ICAL } \\
\text { (HIGH } \\
\text { LEVEL) } \\
\text { (MG/L) }\end{array}$ & $\begin{array}{l}\text { OXYGEN } \\
\text { DEMAND, } \\
\text { BIO- } \\
\text { CHEM- } \\
\text { ICAL, } \\
5 \text { DAY } \\
\text { (MG } / \mathrm{L} \text { ) }\end{array}$ & $\begin{array}{l}\text { COLI- } \\
\text { FORM, } \\
\text { FECAL, } \\
0.7 \\
\text { UM-MF } \\
\text { (COLS./ } \\
100 \mathrm{ML} \text { ) }\end{array}$ \\
\hline $\begin{array}{c}\text { AR } 1992 \\
09-09\end{array}$ & 0040 & 0.61 & 4.0 & 1.3 & 44 & 126 & 8.4 & 8.1 & 15.5 & 70 & 7.7 & 19000 \\
\hline MAR & & & & & & & & & & & & \\
\hline${ }_{\text {MAY }}^{24-24}$ & 1904 & 0.20 & 4.4 & 0.31 & 155 & 193 & 7.5 & 7.2 & 15.0 & 180 & 15 & 9700 \\
\hline${ }_{\text {JUN }}^{14-14}$ & 1130 & 0.53 & 3.5 & 0.89 & 978 & 258 & 8.1 & 7.2 & 23.0 & 160 & 7.6 & K810000 \\
\hline $\begin{array}{l}06-06 \\
\text { JUN }\end{array}$ & 0230 & 0.51 & 3.5 & 0.73 & 155 & 105 & 8.3 & 7.6 & 20.5 & 64 & 10 & 68000 \\
\hline $\begin{array}{l}21 \cdot 21 \\
\text { AUG }\end{array}$ & 0930 & 0.40 & 3.0 & 0.42 & 112 & 148 & 7.7 & 7.1 & 23.0 & 110 & 16 & 9000 \\
\hline $18-19$ & 1450 & 0.32 & 10.2 & 0.26 & 192 & 255 & 7.3 & 6.9 & 24.0 & 140 & 7.2 & 440000 \\
\hline & $\begin{array}{l}\text { STREP. } \\
\text { TOCOCCI }\end{array}$ & HARD - & $\begin{array}{l}\text { HARD - } \\
\text { NESS }\end{array}$ & $\begin{array}{l}\text { ALKA- } \\
\text { LINITY }\end{array}$ & $\begin{array}{l}\text { SOLIDS, } \\
\text { SUM OF }\end{array}$ & $\begin{array}{l}\text { RESIDUE } \\
\text { TOTAL }\end{array}$ & $\begin{array}{l}\text { SOLIDS, } \\
\text { RESIDUE }\end{array}$ & & MAGNE - & & & SODIUM \\
\hline & FECAL, & NESS & NONCARB & WAT DIS & CONSTI - & AT 105 & AT 180 & CALCIUM & SIUM, & SODIUM, & & AD - \\
\hline & $\begin{array}{l}\text { KF AGAR } \\
\text { (COLS. }\end{array}$ & $\begin{array}{l}\text { TOTAL } \\
\text { (MG/L }\end{array}$ & $\begin{array}{l}\text { DISSOLV } \\
\text { FLD. AS }\end{array}$ & $\begin{array}{l}\text { FIX END } \\
\text { FIELD }\end{array}$ & $\begin{array}{c}\text { TUENTS, } \\
\text { DIS - }\end{array}$ & $\begin{array}{l}\text { DEG. C, } \\
\text { SUS- }\end{array}$ & $\begin{array}{l}\text { DEG. C } \\
\text { DIS. }\end{array}$ & $\begin{array}{l}\text { DIS - } \\
\text { SOLVED }\end{array}$ & $\begin{array}{l}\text { DIS- } \\
\text { SOLVED }\end{array}$ & $\begin{array}{c}\text { DIS- } \\
\text { SOLVED }\end{array}$ & & $\begin{array}{l}\text { SORP- } \\
\text { TION }\end{array}$ \\
\hline DATE & $\begin{array}{c}\text { PER } \\
100 \mathrm{ML})\end{array}$ & $\begin{array}{c}\text { AS } \\
\text { CACO3) }\end{array}$ & $\begin{array}{c}\mathrm{CACO} 3 \\
(\mathrm{MG} / \mathrm{L})\end{array}$ & $\begin{array}{l}\mathrm{CAC} 03 \\
(\mathrm{MG} / \mathrm{L})\end{array}$ & $\begin{array}{l}\text { SOLVED } \\
\text { (MG/L) }\end{array}$ & $\begin{array}{l}\text { PENDED } \\
\text { (MG/L) }\end{array}$ & $\begin{array}{l}\text { SOLVED } \\
\text { (MG/L) }\end{array}$ & $\begin{array}{l}\text { (MG/L } \\
\text { AS CA) }\end{array}$ & $\begin{array}{l}\text { (MG/L } \\
\text { AS MG) }\end{array}$ & $\begin{array}{c}(\mathrm{MG} / \mathrm{L} \\
\mathrm{AS} \mathrm{NA})\end{array}$ & $\begin{array}{l}\text { SODIUM } \\
\text { PERCENT }\end{array}$ & RATIO \\
\hline
\end{tabular}

\section{MAR 1992}

MAR 1992
MAR
MA

$24-24 \quad 100000$

MAY

$14-14 \quad 270000$

JUN

$06.06 \quad 49000$

JUN

$21 \cdot 21 \quad 23000$

AUG

$18-19 \quad 350000$

$\begin{array}{rrrrrrr}32 & 7 & 25 & 47 & 119 & 55 & 12 \\ 40 & 10 & 30 & 87 & <1 & 128 & 15 \\ 70 & 36 & 34 & 119 & 146 & 132 & 25 \\ 26 & 1 & 25 & 51 & 85 & 48 & 10 \\ 45 & 18 & 27 & 51 & 68 & 105 & 17 \\ 87 & 26 & 61 & 126 & 74 & 151 & 32\end{array}$

$\begin{array}{ll}0.41 & 2.3 \\ 0.72 & 2.8 \\ 1.8 & 8.6 \\ 0.37 & 1.6 \\ 0.72 & 3.0 \\ 1.8 & 8.0\end{array}$

\section{POTAS -}

SIUM, SULFATE

DIS - DIS - DIS -

SOLVED SOLVED

DATE $\begin{array}{lcc}\text { RIDE, } & \text { GEN, } & \text { GEN, } \\ \text { DIS- } & \text { NITRITE } & \text { NO2+NO3 } \\ \text { SOLVED } & \text { TOTAL } & \text { TOTAL }\end{array}$

(MG/L (MG/L (MG/L

AS N) AS N)
NITRO-

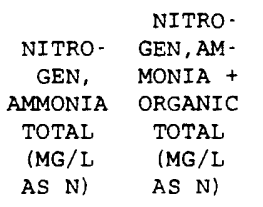

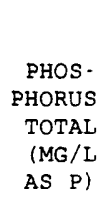

PHOS -

PHORUS ANTIMONY

DIS - TOTAL

SOLVED EPA

(MG/L (UG/L

AS P) AS SB)

$\begin{array}{ll}13 & 0.2 \\ 12 & 0.2 \\ 20 & 0.4 \\ 11 & 0.1 \\ 12 & 0.2 \\ 16 & 0.4\end{array}$

BERYL LIUM,

TOTAL

ARSENIC RECOV -

TOTAL ERABLE

(UG/L (UG/L

AS AS) AS BE)

\begin{tabular}{|c|c|c|c|c|c|c|c|c|c|c|c|c|}
\hline \multicolumn{13}{|l|}{ MAR 1992} \\
\hline $09-09$ & 1.6 & 4.9 & 2.2 & 0.040 & 0.320 & 0.160 & 0.90 & 0.210 & 0.130 & $<10.0$ & 1 & $<10$ \\
\hline \multicolumn{13}{|l|}{ MAR } \\
\hline $24-24$ & 3.9 & 9.1 & 4.7 & 0.070 & 1.20 & 0.730 & 4.2 & 0.740 & 0.310 & $<10.0$ & 1 & $<10$ \\
\hline \multicolumn{13}{|l|}{ MAY } \\
\hline $14-14$ & 4.8 & 15 & 9.5 & 0.060 & 1.20 & 0.660 & 1.8 & 0.540 & 0.470 & $<10.0$ & 1 & $<10$ \\
\hline \multicolumn{13}{|l|}{ JUN } \\
\hline $06-06$ & 2.0 & 4.4 & 1.8 & 0.030 & 0.820 & 0.280 & 0.90 & 0.240 & 0.200 & $<10.0$ & $<1$ & $<10$ \\
\hline \multicolumn{13}{|l|}{ JUN } \\
\hline $21 \cdot 21$ & 3.0 & 7.4 & 3.4 & 0.060 & 0.740 & 0.350 & 1.5 & 0.290 & 0.250 & $<20.0$ & $<1$ & $<10$ \\
\hline \multicolumn{13}{|l|}{ AUG } \\
\hline $18 \cdot 19$ & 4.6 & 17 & 7.7 & 0.100 & 1.30 & 0.170 & 1.4 & 0.380 & 0.240 & $<10.0$ & 3 & $<10$ \\
\hline
\end{tabular}


Table 4. Water-quality data for storm-sewer outfall stations, Fort worth, Texas, 1992-93-Continued 08048510 - WEST FORK TRINITY RIVER OUTFALL AT HIGHWA 121, FORT WORTH, TX (WY 1992)-CONTInUEd

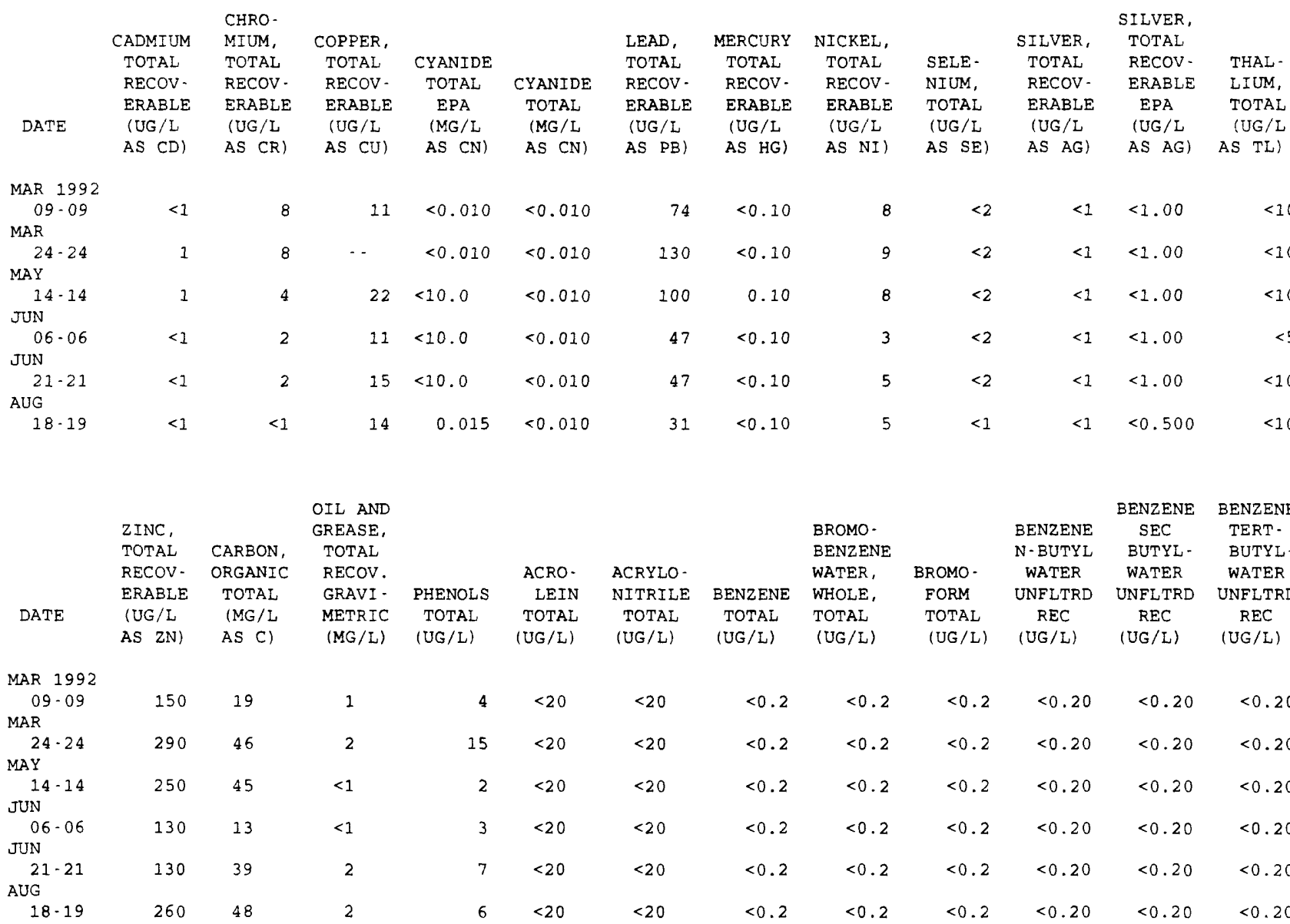

\begin{tabular}{|c|c|c|c|c|c|c|c|c|c|c|c|c|}
\hline & & & & & $2-$ & & & 0. & & DIBROMO & DI - & 1,2 \\
\hline & CARBON - & & CHLORO - & & CHLORO - & & & CHLORO - & TOLUENE & CHLORO - & BROMO - & DIBROMO \\
\hline & TETRA - & & DI- & & ETHYL- & & METHYL- & TOLUENE & P.CHLOR & PROPANE & METHANE & ETHANE \\
\hline & CHLO & CHLORO - & BROMO - & CHLORO - & VINYL - & CHLORO - & CHLO- & WATER & WATER & WATER & WATER & WATER \\
\hline & RIDE & BENZENE & METHANE & ETHANE & ETHER & FORM & RIDE & WHOLE & UNFLTRD & WHOLE & WHOLE & WHOLE \\
\hline DATE & $\begin{array}{l}\text { TOTAL } \\
\text { (UG/L) }\end{array}$ & $\begin{array}{l}\text { TOTAL } \\
\text { (UG/L) }\end{array}$ & $\begin{array}{l}\text { TOTAL } \\
\text { (UG/L) }\end{array}$ & $\begin{array}{l}\text { TOTAL } \\
\text { (UG/L) }\end{array}$ & $\begin{array}{l}\text { TOTAL } \\
\text { (UG/L) }\end{array}$ & $\begin{array}{l}\text { TOTAL } \\
\text { (UG/L) }\end{array}$ & $\begin{array}{l}\text { TOTAL } \\
\text { (UG/L) }\end{array}$ & $\begin{array}{c}\text { TOTAL } \\
\text { (UG/L) }\end{array}$ & $\begin{array}{c}\text { REC } \\
(U G / L)\end{array}$ & $\begin{array}{l}\text { TOT.REC } \\
\text { (UG/L) }\end{array}$ & $\begin{array}{l}\text { RECOVER } \\
(\mathrm{UG} / \mathrm{L})\end{array}$ & $\begin{array}{l}\text { TOTAL } \\
\text { (UG/L) }\end{array}$ \\
\hline
\end{tabular}

\begin{tabular}{|c|c|c|c|c|c|c|c|c|c|c|c|c|}
\hline \multicolumn{13}{|l|}{ MAR 1992} \\
\hline $09-09$ & $<0.2$ & $<0.20$ & $<0.2$ & $<0.2$ & $<1.0$ & $<0.2$ & $<0.2$ & $<0.2$ & $<0.20$ & $<1.0$ & $<0.2$ & $<0.2$ \\
\hline \multicolumn{13}{|l|}{ MAR } \\
\hline $24-24$ & $<0.2$ & $<0.20$ & $<0.2$ & $<0.2$ & $<1.0$ & $<0.2$ & $<0.2$ & $<0.2$ & $<0.20$ & $<1.0$ & $<0.2$ & $<0.2$ \\
\hline \multicolumn{13}{|l|}{ MAY } \\
\hline $14 \cdot 14$ & $<0.2$ & $<0.20$ & $<0.2$ & $<0.2$ & $<1.0$ & $<0.2$ & $<0.2$ & $<0.2$ & $<0.20$ & $<1.0$ & $<0.2$ & $<0.2$ \\
\hline \multicolumn{13}{|l|}{ JUN } \\
\hline 06.06 & $<0.2$ & $<0.20$ & $<0.2$ & $<0.2$ & $<1.0$ & $<0.2$ & $<0.2$ & $<0.2$ & $<0.20$ & $<1.0$ & $<0.2$ & $<0.2$ \\
\hline \multicolumn{13}{|l|}{ JUN } \\
\hline $21-21$ & $<0.2$ & $<0.20$ & $<0.2$ & $<0.2$ & $<1.0$ & $<0.2$ & $<0.2$ & $<0.2$ & $<0.20$ & $<1.0$ & $<0.2$ & $<0.2$ \\
\hline \multicolumn{13}{|l|}{ AUG } \\
\hline $18 \cdot 19$ & $<0.2$ & $<0.20$ & $<0.2$ & $<0.2$ & $<1.0$ & $<0.2$ & $<0.2$ & $<0.2$ & $<0.20$ & $<1.0$ & $<0.2$ & $<0.2$ \\
\hline
\end{tabular}


Table 4. Water-quality data for storm-sewer outfall stations, Fort Worth, Texas, 1992-93-Continued

08048510 - WEST FORK TRINITY RIVER OUTFALL AT HIGHWAY 121, FORT WORTH, TX (WY 1992)-COntinued

\begin{tabular}{|c|c|c|c|c|c|c|c|c|c|c|c|c|}
\hline & & & & SENZENE & BEI & & & & & 1,2-DI- & CIs - 1,2 & \\
\hline & DI - & 1, 1-DI - & CHLORC & 0 - & 1,3-DI - & 1,4 -DI - & CHLORO- & & & PHENYL - & DI - & $\begin{array}{l}1,2- \\
\text { TRANSDI }\end{array}$ \\
\hline & BROMO- & ETHYL - & PENE, & $\begin{array}{l}\text { CHLURU- } \\
\text { WATER }\end{array}$ & WATER & WATER & FLUORO- & CHLORO- & CHLORO- & $\begin{array}{l}\text { ZINE } \\
\text { ZINA- }\end{array}$ & ETHENE & CHLORO- \\
\hline & METHANE & ENE & WAT, WH & UNFLTRD & UNFLTRD & UNFLTRD & METHANE & ETHANE & ETHANE & WATER & WATER & ETHENE \\
\hline & $\begin{array}{l}\text { TOTAL } \\
\text { (UG/L) }\end{array}$ & $\begin{array}{l}\text { TOTAL } \\
\text { (UG/L) }\end{array}$ & $\begin{array}{l}\text { TOTAL } \\
\text { (UG/L) }\end{array}$ & $\begin{array}{c}\text { REC } \\
(U G / L)\end{array}$ & $\begin{array}{c}\text { REC } \\
(\mathrm{UG} / \mathrm{L})\end{array}$ & $\begin{array}{c}\text { REC } \\
(\mathrm{UG} / \mathrm{L})\end{array}$ & $\begin{array}{l}\text { TOTAL } \\
\text { (UG/L) }\end{array}$ & $\begin{array}{l}\text { TOTAL } \\
\text { (UG/L) }\end{array}$ & $\begin{array}{l}\text { TOTAL } \\
\text { (UG/L) }\end{array}$ & $\begin{array}{l}\text { TOT.REC } \\
\text { (UG/L) }\end{array}$ & $\begin{array}{l}\text { TOTAL } \\
\text { (UG/L) }\end{array}$ & $\begin{array}{l}\text { TOTAL } \\
\text { (UG/L) }\end{array}$ \\
\hline
\end{tabular}

\begin{tabular}{|c|c|c|c|c|c|c|c|c|c|c|c|c|}
\hline $\begin{array}{c}\text { MAR } 1992 \\
09.09\end{array}$ & $<0.2$ & $<0.2$ & $<0.2$ & $<5.0$ & $<5.0$ & $<5.0$ & $<0.2$ & $<0.2$ & $<0.2$ & $<5.0$ & $<0.2$ & $<0.2$ \\
\hline MAR & & & & & & & & & & & & \\
\hline $24-24$ & $<0.2$ & $<0.2$ & $<0.2$ & $<5.0$ & $<5.0$ & $<5.0$ & $<0.2$ & $<0.2$ & $<0.2$ & $<5.0$ & $<0.2$ & $<0.2$ \\
\hline MAY & & & & & & & & & & & & \\
\hline $\begin{array}{l}14-14 \\
\text { JUN }\end{array}$ & $<0.2$ & $<0.2$ & $<0.2$ & $<0.20$ & $<0.20$ & $<0.20$ & $<0.2$ & $<0.2$ & $<0.2$ & $<5.0$ & $<0.2$ & $<0.2$ \\
\hline $\begin{array}{l}06-06 \\
\text { JUN }\end{array}$ & $<0.2$ & $<0.2$ & $<0.2$ & $<5.0$ & $<5.0$ & $<5.0$ & $<0.2$ & $<0.2$ & $<0.2$ & $<5.0$ & $<0.2$ & $<0.2$ \\
\hline $\begin{array}{l}21-21 \\
A \cup G\end{array}$ & $<0.2$ & $<0.2$ & $<0.2$ & $<5.0$ & $<5.0$ & $<5.0$ & $<0.2$ & $<0.2$ & $<0.2$ & $<5.0$ & $<0.2$ & $<0.2$ \\
\hline $18-19$ & $<0.2$ & $<0.2$ & $<0.2$ & $<5.0$ & $<5.0$ & $<5.0$ & $<0.2$ & $<0.2$ & $<0.2$ & $<5.0$ & $<0.2$ & $<0.2$ \\
\hline
\end{tabular}

\begin{tabular}{|c|c|c|c|c|c|c|c|c|c|c|c|c|}
\hline & & $\begin{array}{l}1,3 \text {-DI - } \\
\text { CHLORO- }\end{array}$ & $\begin{array}{c}2,2-D I \\
\text { CHLORO- } \\
\text { PRO- }\end{array}$ & $\begin{array}{c}\text { CIS } \\
1,3-D I-\end{array}$ & $\begin{array}{l}\text { TRANS - } \\
1,3-\mathrm{DI} \text { - }\end{array}$ & & $\begin{array}{c}\text { HEXA- } \\
\text { CHLORO- }\end{array}$ & $\begin{array}{c}\text { ISO - } \\
\text { PROPYL - } \\
\text { BENZENE }\end{array}$ & $\begin{array}{l}\text { P-ISO- } \\
\text { PROPYL- } \\
\text { TOLUENE }\end{array}$ & & $\begin{array}{c}\text { METHYL - } \\
\text { ENE }\end{array}$ & \\
\hline & HLORO- & PROPANE & PANE & CHLORO- & CHLORO- & ETHYL - & BUT - & WATER & WATER & METHYL - & CHLO - & NAPHTH - \\
\hline & & WAT. WH & WAT, WH & PROPENE & & BENZENE & ADIENE & WHOLE & WHOLE & BROMIDE & RIDE & ALENE \\
\hline & $\begin{array}{l}\text { TOTAL } \\
\text { (UG/L) }\end{array}$ & $\begin{array}{l}\text { TOTAL } \\
(\text { UG/L) }\end{array}$ & $\begin{array}{c}\text { TOTAL } \\
(\text { UG } / L)\end{array}$ & $\begin{array}{l}\text { TOTAL } \\
\text { (UG/L) }\end{array}$ & $\begin{array}{l}\text { TOTAL } \\
(\mathrm{UG} / \mathrm{L})\end{array}$ & $\begin{array}{l}\text { TOTAL } \\
\text { (UG/L) }\end{array}$ & $\begin{array}{l}\text { TOTAL } \\
\text { (UG/L) }\end{array}$ & $\begin{array}{l}\text { REC } \\
(U G / L)\end{array}$ & $\begin{array}{l}\text { REC } \\
(\mathrm{UG} / \mathrm{L})\end{array}$ & $\begin{array}{l}\text { TOTAL } \\
\text { (UG/L) }\end{array}$ & $\begin{array}{l}\text { TOTAL } \\
\text { (UG/L) }\end{array}$ & $\begin{array}{c}\text { TOTAL } \\
\text { (UG/L) }\end{array}$ \\
\hline
\end{tabular}

\begin{tabular}{|c|c|c|c|c|c|c|c|c|c|c|c|c|}
\hline $\begin{array}{c}\text { MAR } 1992 \\
09.09\end{array}$ & $<0,2$ & & & & & & & & & & & \\
\hline MAR & 0.0 & 0.8 & 8.0 & 0.2 & $<0.2$ & 20.2 & $<3.0$ & $<0.20$ & $<0.20$ & $<0.2$ & $<0.2$ & \\
\hline $24-24$ & $<0.2$ & $<0.2$ & $<0.2$ & $<0.2$ & $<0.2$ & $<0.2$ & $<5.0$ & $<0.20$ & $<0.20$ & $<0.2$ & 0.3 & $<5.0$ \\
\hline MAY & & & & & & & & & & & & \\
\hline $\mathrm{JUN}^{14-14}$ & $<0.2$ & $<0.2$ & $<0.2$ & $<0.2$ & $<0.2$ & $<0.2$ & $<0.2$ & $<0.20$ & $<0.20$ & $<0.2$ & $<0.2$ & $<0.2$ \\
\hline $\begin{array}{l}06-06 \\
\text { JUN }\end{array}$ & $<0.2$ & $<0.2$ & $<0.2$ & $<0.2$ & $<0.2$ & $<0.2$ & $<5.0$ & $<0.20$ & $<0.20$ & $<0.2$ & $<0.4$ & $<5.0$ \\
\hline $21 \cdot 21$ & $<0.2$ & $<0.2$ & $<0.2$ & $<0.2$ & $<0.2$ & $<0.2$ & $<5.0$ & $<0.20$ & $<0.20$ & $<0.2$ & $<0.2$ & $<5.0$ \\
\hline AUG & & & & & & & & & & & & \\
\hline $18-19$ & $<0.2$ & $<0.2$ & $<0.2$ & $<0.2$ & $<0.2$ & $<0.2$ & $<5.0$ & $<0.20$ & $<0.20$ & $<0.2$ & $<0.2$ & $<5.0$ \\
\hline
\end{tabular}

\begin{tabular}{|c|c|c|c|c|c|c|c|c|c|c|c|c|}
\hline & BENZENE & & $\begin{array}{c}\text { ETHANE, } \\
1112-\end{array}$ & $\begin{array}{l}\text { ETHANE, } \\
1,1,2,2\end{array}$ & TETRA - & & $\begin{array}{c}1,2,3 . \\
\text { TRI- }\end{array}$ & $\begin{array}{l}\text { BENZENE } \\
1,2,4-\end{array}$ & $1,1,1$ & $1212-$ & TRI & \\
\hline & $N-P R O P Y$ & & TETRA & TETRA & CHLORO- & & CHLORO & TRI - & TRI & TRI - & CHLORO- & CHLORO- \\
\hline & WATER & & CHLORO- & CHLORO - & ETHYL - & & BENZENE & CHLORO- & CHLORO - & CHLORO- & ETHYL - & FLUORO- \\
\hline & UNFLTRD & STYRENE & WAT UNF & WAT UNF & ENE & TOLUENE & WAT, WH & WAT UNF & ETHANE & ETH & ENE & METHANE \\
\hline & $\begin{array}{c}\text { REC } \\
(U G / L)\end{array}$ & $\begin{array}{l}\text { TOTAL } \\
\text { (UG/L) }\end{array}$ & $\begin{array}{c}\text { REC } \\
(U G / L)\end{array}$ & $\begin{array}{c}R E C \\
(U G / L)\end{array}$ & $\begin{array}{l}\text { TOTAL } \\
\text { (UG/L) }\end{array}$ & $\begin{array}{c}\text { TOTAL } \\
\text { (UG/L) }\end{array}$ & $\begin{array}{c}\text { REC } \\
(U G / L)\end{array}$ & $\begin{array}{c}\text { REC } \\
(U G / L)\end{array}$ & $\begin{array}{l}\text { TOTAL } \\
\text { (UG/L) }\end{array}$ & $\begin{array}{l}\text { TOTAL } \\
\text { (UG/L) }\end{array}$ & $\begin{array}{l}\text { TOTAL } \\
\text { (UG/L) }\end{array}$ & $\begin{array}{c}\text { TOTAL } \\
(\mathrm{UG} / \mathrm{L})\end{array}$ \\
\hline
\end{tabular}

\begin{tabular}{|c|c|c|c|c|c|c|c|c|c|c|c|c|}
\hline MAR 1992 & & & & & & & & & & & & \\
\hline $09-09$ & $<0.20$ & $<0.2$ & $<0.2$ & $<0.2$ & $<0.2$ & $<0.2$ & $<0.20$ & $<5.0$ & $<0.2$ & $<0.2$ & $<0.2$ & $<0.2$ \\
\hline $\begin{array}{l}\text { MAR } \\
24-24\end{array}$ & $<0.20$ & $<0.2$ & $<0.2$ & $<0.2$ & $<0.2$ & $<0.2$ & $<0.20$ & $<5,0$ & $<0,2$ & $<0,2$ & $<0.2$ & $<0.2$ \\
\hline MAY & & & & & & & & & & & & \\
\hline $\operatorname{JUN}^{14 \cdot 14}$ & $<0.20$ & $<0.2$ & $<0.2$ & $<0.2$ & $<0.2$ & $<0.2$ & $<0.20$ & $<0.20$ & $<0.2$ & $<0.2$ & $<0.2$ & $<0.2$ \\
\hline $\begin{array}{l}06-06 \\
\text { JUN }\end{array}$ & $<0.20$ & $<0.2$ & $<0.2$ & $<0.2$ & $<0.2$ & $<0.2$ & $<0.20$ & $<5.0$ & $<0.2$ & $<0.2$ & $<0.2$ & $<0.2$ \\
\hline${ }_{A U G}^{21-21}$ & $<0.20$ & $<0.2$ & $<0.2$ & $<0.2$ & $<0.2$ & $<0.2$ & $<0.20$ & $<5.0$ & $<0.2$ & $<0.2$ & $<0.2$ & $<0.2$ \\
\hline $18-19$ & $<0.20$ & $<0.2$ & $<0.2$ & $<0.2$ & $<0.2$ & $<0.2$ & $<0.20$ & $<5.0$ & $=0 ?$ & $=0 ?$ & $<0,2$ & $<0.2$ \\
\hline
\end{tabular}


Table 4. Water-quality data for storm-sewer outfall stations, Fort worth, Texas, 1992-93-Continued

08048510 - WEST FORK TRINITY RIVER OUTFALI AT HIGHWAY 121, FORT WORTH, TX (WY 1992)-COntinued

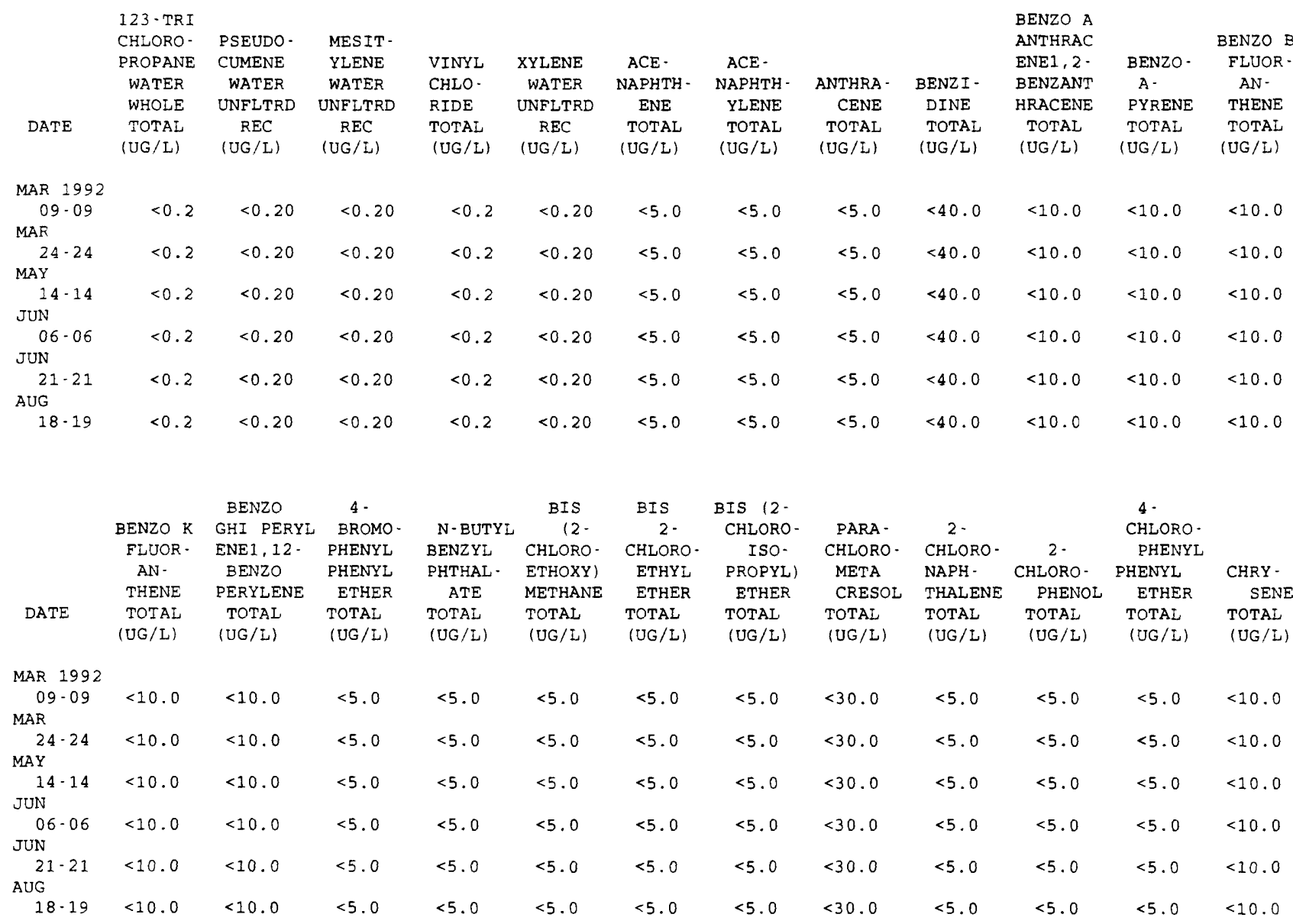

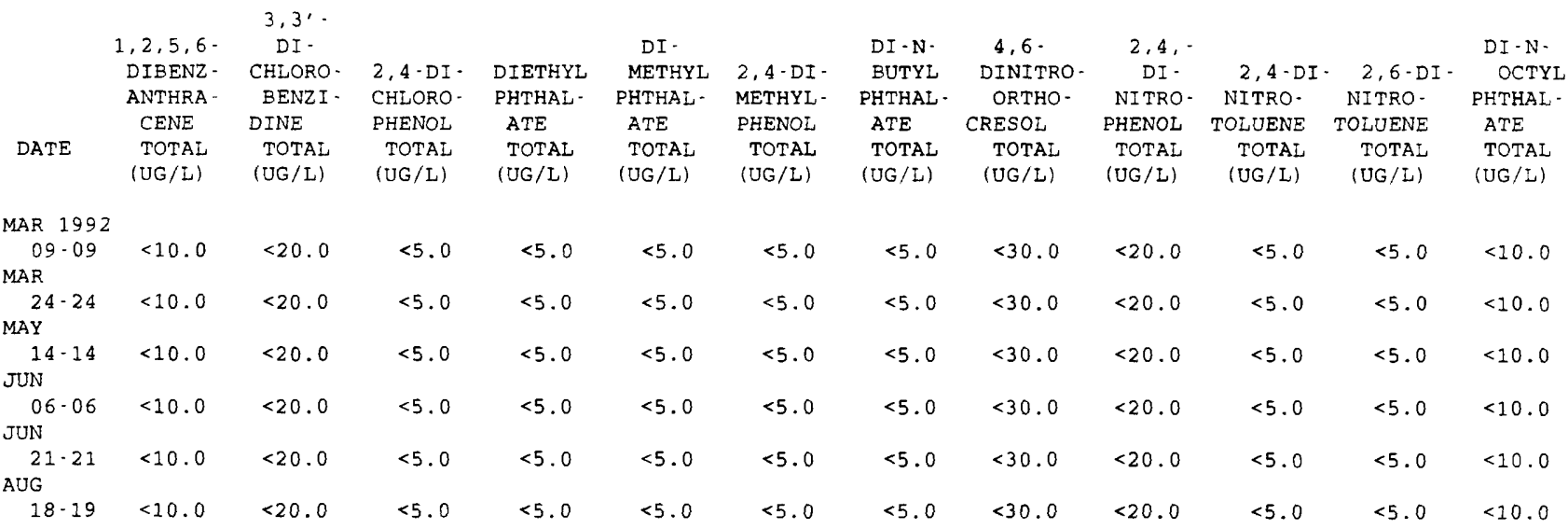


Table 4. Water-quality data for storm-sewer outfall stations, Fort worth, Texas, 1992-93-Continued

08048510 - WEST FORK TRINITY RIVER OUTFALL AT HIGHWAY 121, FORT WORTH, TX (WY 1992)-CONEINUEd

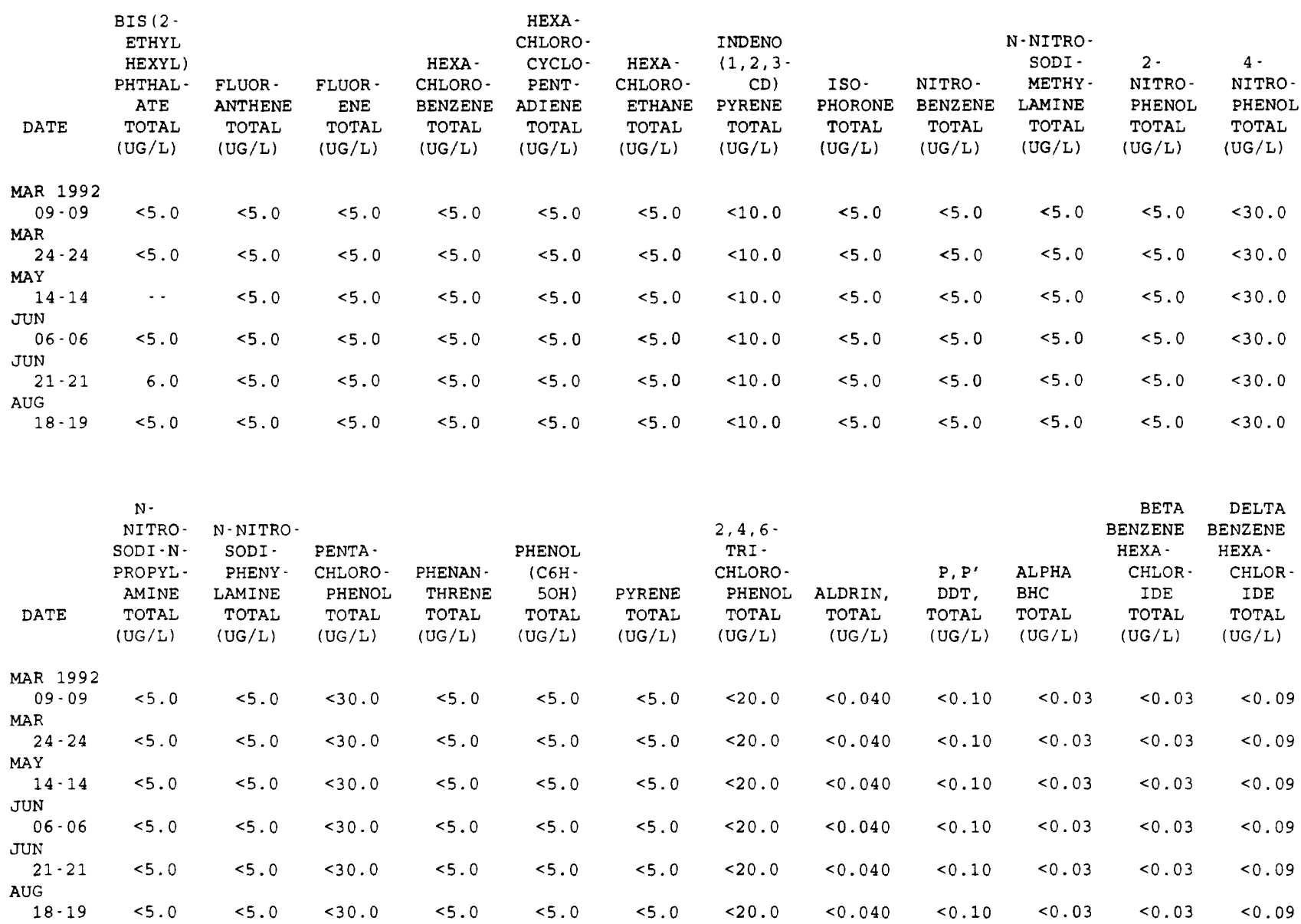

\begin{tabular}{|c|c|c|c|c|c|c|c|c|c|c|c|c|}
\hline & & $\begin{array}{l}\text { CHLOR - } \\
\text { DANE }\end{array}$ & $\begin{array}{l}\text { CHLOR. } \\
\text { DANE }\end{array}$ & & & & & $\begin{array}{l}\text { ENDO- } \\
\text { SULFAN - }\end{array}$ & & & & \\
\hline & & CIS & TRANS & & & & & I & ENDO- & ENDO- & ENDR IN & ENDRIN \\
\hline & & $\begin{array}{l}\text { WATER } \\
\text { WHOLE }\end{array}$ & $\begin{array}{l}\text { WATER } \\
\text { WHOLE }\end{array}$ & $\begin{array}{l}\text { CHLOR - } \\
\text { DANE }\end{array}$ & $\begin{array}{l}P, P^{\prime} \\
D D D\end{array}$ & $\begin{array}{l}P, P^{\prime} \\
D D E\end{array}$ & $\begin{array}{l}\text { DI. } \\
\text { ELDRIN }\end{array}$ & $\begin{array}{l}\text { WATER } \\
\text { WHOLE }\end{array}$ & $\begin{array}{l}\text { SULFAN } \\
\text { BETA }\end{array}$ & $\begin{array}{r}\text { SULFAN } \\
\text { SULFATE }\end{array}$ & $\begin{array}{l}\text { WATER } \\
\text { UNFLTRD }\end{array}$ & $\begin{array}{l}\text { ALDE - } \\
\text { HYDE }\end{array}$ \\
\hline 8 & $\begin{array}{l}\text { LINDANE } \\
\text { TOTAL } \\
\text { (UG/L) }\end{array}$ & $\begin{array}{l}\text { WHOLE } \\
\text { TOTAL } \\
\text { (UG/L) }\end{array}$ & $\begin{array}{l}\text { WHULE } \\
\text { TOTAL } \\
\text { (UG/L) }\end{array}$ & $\begin{array}{l}\text { TOTAL } \\
\text { (UG/L) }\end{array}$ & $\begin{array}{l}\text { TOTAL } \\
\text { (UG/L) }\end{array}$ & $\begin{array}{l}\text { TOTAL } \\
\text { (UG/L) }\end{array}$ & $\begin{array}{l}\text { TOTAL } \\
\text { (UG/L) }\end{array}$ & $\begin{array}{l}\text { REC } \\
(U G / L)\end{array}$ & $\begin{array}{l}\text { TOTAL } \\
\text { (UG/L) }\end{array}$ & $\begin{array}{l}\text { TOTAL } \\
\text { (UG/L) }\end{array}$ & $\begin{array}{c}\text { REC } \\
\text { (UG/L) }\end{array}$ & $\begin{array}{l}\text { TOTAL } \\
\text { (UG/L) }\end{array}$ \\
\hline
\end{tabular}

\begin{tabular}{|c|c|c|c|c|c|c|c|c|c|c|c|c|}
\hline \multicolumn{13}{|l|}{ MAR 1992} \\
\hline $09-09$ & $<0.030$ & $<0.10$ & $<0.10$ & $<0.1$ & $<0.10$ & $<0.04$ & $<0.020$ & $<0.10$ & $<0.04$ & $<0.60$ & $<0.060$ & $<0.20$ \\
\hline \multicolumn{13}{|l|}{ MAR } \\
\hline $24-24$ & $<0.030$ & $<0.10$ & $<0.10$ & $<0.1$ & $<0.10$ & $<0.04$ & $<0.020$ & $<0.10$ & $<0.04$ & $<0.60$ & $<0.060$ & $<0.20$ \\
\hline \multicolumn{13}{|l|}{ MAY } \\
\hline $14-14$ & $<0.030$ & $<0.10$ & $<0.10$ & $<0.1$ & $<0.10$ & $<0.04$ & $<0.020$ & $<0.10$ & $<0.04$ & $<0.60$ & $<0.060$ & $<0.20$ \\
\hline \multicolumn{13}{|l|}{ JUN } \\
\hline 06.06 & $<0.030$ & $<0.10$ & $<0.10$ & $<0.1$ & $<0.10$ & $<0.04$ & $<0.020$ & $<0.10$ & $<0.04$ & $<0.60$ & $<0.060$ & $<0.20$ \\
\hline \multicolumn{13}{|l|}{ JUN } \\
\hline $21-21$ & $<0.030$ & $<0.10$ & $<0.10$ & 0.1 & $<0.10$ & $<0.04$ & $<0.020$ & $<0.10$ & $<0.04$ & $<0.60$ & $<0.060$ & $<0.20$ \\
\hline \multicolumn{13}{|l|}{ AUG } \\
\hline $18-19$ & $<0.030$ & $<0.10$ & $<0.10$ & $<0.1$ & $<0.10$ & $<0.04$ & $<0.020$ & $<0.10$ & $<0.04$ & $<0.60$ & $<0.060$ & $<0.20$ \\
\hline
\end{tabular}


Table 4. Water-quality data for storm-sewer outfall stations, Fort worth, Texas, 1992-93-Continued 08048510 - WEST FORK TRINITY RIVER OUTFALL AT HIGHWAY 121, FORT WORTH, TX (WY 1992)-COntIIUUEd

\begin{tabular}{|c|c|c|c|c|c|c|c|c|c|c|c|}
\hline & & HEPTA - & & AROCLOR & AROCLOR & AROCLOR & AROCLOR & AROCLOR & AROCLOR & AROCLOR & \\
\hline & HEPTA - & CHLOR & TOX - & 1016 & 1221 & 1232 & 1242 & 1248 & 1254 & 1260 & DI - \\
\hline & CHLOR, & EPOXIDE & APHENE, & PCB & $\mathrm{PCB}$ & PCB & $\mathrm{PCB}$ & $\mathrm{PCB}$ & $\mathrm{PCB}$ & PCB & AZINON, \\
\hline DATE & $\begin{array}{l}\text { TOTAL } \\
\text { (UG/L) }\end{array}$ & $\begin{array}{l}\text { TOTAL } \\
\text { (UG/L) }\end{array}$ & $\begin{array}{l}\text { TOTAL } \\
\text { (UG/L) }\end{array}$ & $\begin{array}{l}\text { TOTAL } \\
\text { (UG/L) }\end{array}$ & $\begin{array}{l}\text { TOTAL } \\
\text { (UG/L) }\end{array}$ & $\begin{array}{l}\text { TOTAL } \\
\text { (UG/L) }\end{array}$ & $\begin{array}{l}\text { TOTAL } \\
\text { (UG/L) }\end{array}$ & $\begin{array}{l}\text { TOTAL } \\
\text { (UG/L) }\end{array}$ & $\begin{array}{l}\text { TOTAL } \\
\text { (UG/L) }\end{array}$ & $\begin{array}{l}\text { TOTAL } \\
\text { (UG/L) }\end{array}$ & $\begin{array}{l}\text { TOTAL } \\
\text { (UG/L) }\end{array}$ \\
\hline
\end{tabular}

\begin{tabular}{|c|c|c|c|c|c|c|c|c|c|c|c|}
\hline \multicolumn{12}{|l|}{ MAR 1992} \\
\hline 09.09 & $<0.030$ & $<0.80$ & $<2$ & $<0.1$ & $<1.0$ & $<0.1$ & $<0.1$ & $<0.1$ & $<0.1$ & $<0.1$ & 0.30 \\
\hline \multicolumn{12}{|l|}{ MAR } \\
\hline $24-24$ & $<0.030$ & $<0.80$ & $<2$ & $<0.1$ & $<1.0$ & $<0.1$ & $<0.1$ & $<0.1$ & $<0.1$ & $<0.1$ & 0.30 \\
\hline \multicolumn{12}{|l|}{ MAY } \\
\hline $14-14$ & $<0.030$ & $<0.80$ & $<2$ & $<0.1$ & $<1.0$ & $<0.1$ & $<0.1$ & $<0.1$ & $<0.1$ & $<0.1$ & 0.20 \\
\hline \multicolumn{12}{|l|}{ JUN } \\
\hline 06.06 & $<0.030$ & $<0.80$ & $<2$ & $<0.1$ & $<1.0$ & $<0.1$ & $<0.1$ & $<0.1$ & $<0.1$ & $<0.1$ & 0.30 \\
\hline \multicolumn{12}{|l|}{ JUN } \\
\hline $21-21$ & $<0.030$ & $<0.80$ & $<2$ & $<0.1$ & $<1.0$ & $<0.1$ & $<0.1$ & $<0.1$ & $<0.1$ & $<0.1$ & 0.20 \\
\hline \multicolumn{12}{|l|}{ AUG } \\
\hline $18-19$ & $<0.030$ & $<0.80$ & $<2$ & $<0.1$ & $<1.0$ & $<0.1$ & $<0.1$ & $<0.1$ & $<0.1$ & $<0.1$ & 0.40 \\
\hline
\end{tabular}


Table 4. Water-quality data for storm-sewer outfall stations, Fort worth, Texas, 1992-93-Continued

08048510 - WEST FORK TRINITY RIVER OUTFALI AT HIGHWAY 121, FORT WORTH, TX (WY 1993)

\begin{tabular}{|c|c|c|c|c|c|c|c|c|c|c|c|c|}
\hline DATE & TIME & $\begin{array}{l}\text { PRECIP- } \\
\text { ITATION } \\
\text { TOTAL } \\
\text { INCHES/ } \\
\text { STORM }\end{array}$ & $\begin{array}{l}\text { ELAPSED } \\
\text { TIME } \\
\text { OF } \\
\text { STORM } \\
\text { (HOURS) }\end{array}$ & $\begin{array}{l}\text { STORM } \\
\text { WATER } \\
\text { FLOW } \\
\text { (MGD) }\end{array}$ & $\begin{array}{l}\text { SPE- } \\
\text { CIFIC } \\
\text { CON- } \\
\text { DUCT - } \\
\text { ANCE } \\
\text { (US/CM) }\end{array}$ & $\begin{array}{c}\text { SPE- } \\
\text { CIFIC } \\
\text { CON- } \\
\text { DUCT- } \\
\text { ANCE } \\
\text { LAB } \\
\text { (US/CM) }\end{array}$ & $\begin{array}{c}\text { PH } \\
\text { WATER } \\
\text { WHOLE } \\
\text { FIEID } \\
\text { (STAND- } \\
\text { ARD } \\
\text { UNITS) }\end{array}$ & $\begin{array}{c}\text { PH } \\
\text { WATER } \\
\text { WHOLE } \\
\text { LAB } \\
\text { (STAND- } \\
\text { ARD } \\
\text { UNITS) }\end{array}$ & $\begin{array}{c}\text { TEMPER - } \\
\text { ATURE } \\
\text { WATER } \\
\text { (DEG C) }\end{array}$ & $\begin{array}{l}\text { OXYGEN } \\
\text { DEMAND, } \\
\text { CHEM. } \\
\text { ICAL } \\
\text { (HIGH } \\
\text { LEVEL) } \\
\text { (MG/L) }\end{array}$ & $\begin{array}{l}\text { OXYGEN } \\
\text { DEMAND, } \\
\text { BIO- } \\
\text { CHEM- } \\
\text { ICAL, } \\
5 \text { DAY } \\
\text { (MG/L) }\end{array}$ & $\begin{array}{l}\text { COLI- } \\
\text { FORM, } \\
\text { FECAL, } \\
0.7 \\
\text { UM-MF } \\
\text { (COLS. } \\
100 \mathrm{ML} \text { ) }\end{array}$ \\
\hline $\begin{array}{c}\text { CT } 1992 \\
28-29\end{array}$ & 2320 & 0.39 & 7.7 & 0.56 & 87 & 128 & 8.7 & 7.4 & 19.5 & 93 & 7.7 & 200000 \\
\hline DATE & $\begin{array}{l}\text { STREP- } \\
\text { TOCOCCI } \\
\text { FECAL, } \\
\text { KF AGAR } \\
\text { (COLS. } \\
\text { PER } \\
100 \mathrm{ML} \text { ) }\end{array}$ & $\begin{array}{l}\text { HARD- } \\
\text { NESS } \\
\text { TOTAL } \\
\text { (MG/L } \\
\text { AS } \\
\text { CACO3) }\end{array}$ & $\begin{array}{l}\text { HARD- } \\
\text { NESS } \\
\text { NONCARB } \\
\text { DISSOLV } \\
\text { FLD. AS } \\
\text { CACO3 } \\
(\mathrm{MG} / \mathrm{L})\end{array}$ & $\begin{array}{l}\text { ALKA- } \\
\text { LINITY } \\
\text { WAT DIS } \\
\text { FIX END } \\
\text { FIELD } \\
\text { CAC03 } \\
\text { (MG/L) }\end{array}$ & $\begin{array}{l}\text { SOLIDS, } \\
\text { SUM OF } \\
\text { CONSTI - } \\
\text { TUENTS, } \\
\text { DIS - } \\
\text { SOLVED } \\
\text { (MG/L) }\end{array}$ & $\begin{array}{l}\text { RESIDUE } \\
\text { TOTAL } \\
\text { AT } 105 \\
\text { DEG. C, } \\
\text { SUS - } \\
\text { PENDED } \\
\text { (MG/L) }\end{array}$ & $\begin{array}{l}\text { SOLIDS, } \\
\text { RESIDUE } \\
\text { AT } 180 \\
\text { DEG. C } \\
\text { DIS. } \\
\text { SOLVED } \\
\text { (MG/L) }\end{array}$ & $\begin{array}{l}\text { CALCIUM } \\
\text { DIS- } \\
\text { SOLVED } \\
\text { (MG/L } \\
\text { AS CA) }\end{array}$ & $\begin{array}{l}\text { MAGNE - } \\
\text { SIUM, } \\
\text { DIS - } \\
\text { SOLVED } \\
\text { (MG/L } \\
\text { AS MG) }\end{array}$ & $\begin{array}{l}\text { SODIUM, } \\
\text { DIS. } \\
\text { SOLVED } \\
\text { (MG/L } \\
\text { AS NA) }\end{array}$ & $\begin{array}{l}\text { SODIUM } \\
\text { PERCENT }\end{array}$ & $\begin{array}{l}\text { SODIUM } \\
\text { AD- } \\
\text { SORP. } \\
\text { TION } \\
\text { RATIO }\end{array}$ \\
\hline $\begin{array}{c}\text { OCT } 1992 \\
28.29\end{array}$ & 160000 & 35 & 4 & 31 & 71 & 89 & 71 & 13 & 0.67 & 2.2 & 11 & 0.2 \\
\hline DATE & $\begin{array}{l}\text { POTAS- } \\
\text { SIUM, } \\
\text { DIS- } \\
\text { SOLVED } \\
\text { (MG/L } \\
\text { AS K) }\end{array}$ & $\begin{array}{l}\text { SULFATE } \\
\text { DIS- } \\
\text { SOLVED } \\
\text { (MG/L } \\
\text { AS SO4) }\end{array}$ & $\begin{array}{l}\text { CHLO- } \\
\text { RIDE. } \\
\text { DIS- } \\
\text { SOLVED } \\
\text { (MG/L } \\
\text { AS CL) }\end{array}$ & $\begin{array}{l}\text { NITRO- } \\
\text { GEN, } \\
\text { NITRITE } \\
\text { TOTAL } \\
\text { (MG/L } \\
\text { AS N) }\end{array}$ & $\begin{array}{l}\text { NITRO- } \\
\text { GEN, } \\
\text { NO2+NO3 } \\
\text { TOTAL } \\
\text { (MG/L } \\
\text { AS N) }\end{array}$ & $\begin{array}{l}\text { NITRO- } \\
\text { GEN, } \\
\text { AMMONIA } \\
\text { TOTAL } \\
\text { (MG/L } \\
\text { AS N) }\end{array}$ & $\begin{array}{l}\text { NITRO- } \\
\text { GEN, AM- } \\
\text { MONIA + } \\
\text { ORGANIC } \\
\text { TOTAL } \\
\text { (MG/L } \\
\text { AS N) }\end{array}$ & $\begin{array}{l}\text { PHOS- } \\
\text { PHORUS } \\
\text { TOTAL } \\
\text { (MG/L } \\
\text { AS P) }\end{array}$ & $\begin{array}{c}\text { PHOS - } \\
\text { PHORUS } \\
\text { DIS - } \\
\text { SOLVED } \\
\text { (MG /L } \\
\text { AS P) }\end{array}$ & $\begin{array}{c}\text { ANTIMONY } \\
\text { TOTAL } \\
\text { EPA } \\
\text { (UG/L } \\
\text { AS SB) }\end{array}$ & $\begin{array}{c}\text { ARSENIC } \\
\text { TOTAL } \\
\text { (UG/L } \\
\text { AS AS) }\end{array}$ & $\begin{array}{l}\text { BERYL- } \\
\text { LIUM, } \\
\text { TOTAL } \\
\text { RECOV- } \\
\text { ERABLE } \\
\text { (UG/L } \\
\text { AS BE) }\end{array}$ \\
\hline $\begin{array}{c}\text { OCT } 1992 \\
28.29\end{array}$ & 2.3 & 6.1 & 2.6 & 0.030 & 0.580 & 0.180 & 1.2 & 0.280 & 0.130 & $<10.0$ & 2 & $<10$ \\
\hline DATE & $\begin{array}{l}\text { CADMIUM } \\
\text { TOTAL } \\
\text { RECOV- } \\
\text { ERABLE } \\
\text { (UG/L } \\
\text { AS CD) }\end{array}$ & $\begin{array}{l}\text { CHRO- } \\
\text { MIUM, } \\
\text { TOTAL } \\
\text { RECOV- } \\
\text { ERABLE } \\
\text { (UG/L } \\
\text { AS CR) }\end{array}$ & $\begin{array}{l}\text { COPPER, } \\
\text { TOTAL } \\
\text { RECOV- } \\
\text { ERABLE } \\
\text { (UG/L } \\
\text { AS CU) }\end{array}$ & $\begin{array}{c}\text { CYANIDE } \\
\text { TOTAL } \\
\text { EPA } \\
\text { (MG/L } \\
\text { AS CN) }\end{array}$ & $\begin{array}{l}\text { CYANIDE } \\
\text { TOTAL } \\
\text { (MG/L } \\
\text { AS CN) }\end{array}$ & $\begin{array}{l}\text { LEAD, } \\
\text { TOTAL } \\
\text { RECOV- } \\
\text { ERABLE } \\
\text { (UG/L } \\
\text { AS PB) }\end{array}$ & $\begin{array}{l}\text { MERCURY } \\
\text { TOTAL } \\
\text { RECOV- } \\
\text { ERABLE } \\
\text { (UG/L } \\
\text { AS HG) }\end{array}$ & $\begin{array}{l}\text { NICKEL, } \\
\text { TOTAL } \\
\text { RECOV- } \\
\text { ERABLE } \\
\text { (UG/L } \\
\text { AS NI) }\end{array}$ & $\begin{array}{l}\text { SELE- } \\
\text { NIUM, } \\
\text { TOTAL } \\
\text { (UG/L } \\
\text { AS SE) }\end{array}$ & $\begin{array}{l}\text { SILVER, } \\
\text { TOTAL } \\
\text { RECOV. } \\
\text { ERABLE } \\
\text { (UG/L } \\
\text { AS AG) }\end{array}$ & $\begin{array}{l}\text { SILVER, } \\
\text { TOTAL } \\
\text { RECOV- } \\
\text { ERABLE } \\
\text { EPA } \\
\text { (UG/L } \\
\text { AS AG) }\end{array}$ & $\begin{array}{l}\text { THAL- } \\
\text { LIUM, } \\
\text { TOTAL } \\
\text { (UG/L } \\
\text { AS TL) }\end{array}$ \\
\hline $\begin{array}{c}\text { OCT } 1992 \\
28-29\end{array}$ & $<1$ & 3 & 11 & $<0.010$ & 0.020 & 56 & $<0.10$ & 4 & $<2$ & $<1$ & $<0.500$ & $<5$ \\
\hline DATE & $\begin{array}{l}\text { ZINC, } \\
\text { TOTAL } \\
\text { RECOV. } \\
\text { ERABLE } \\
\text { (UG/L } \\
\text { AS ZN) }\end{array}$ & $\begin{array}{l}\text { CARBON, } \\
\text { ORGANIC } \\
\text { TOTAL } \\
\text { (MG/L } \\
\text { AS C) }\end{array}$ & $\begin{array}{c}\text { OIL AND } \\
\text { GREASE, } \\
\text { TOTAL } \\
\text { RECOV. } \\
\text { GRAVI- } \\
\text { METRIC } \\
\text { (MG/L) }\end{array}$ & $\begin{array}{l}\text { PHENOLS } \\
\text { TOTAL } \\
\text { (UG/L) }\end{array}$ & $\begin{array}{l}\text { ACRO- } \\
\text { LEIN } \\
\text { TOTAL } \\
\text { (UG/L) }\end{array}$ & $\begin{array}{l}\text { ACRYLO- } \\
\text { NITRILE } \\
\text { TOTAL } \\
\text { (UG/L) }\end{array}$ & $\begin{array}{l}\text { BENZENE } \\
\text { TOTAL } \\
\text { (UG/L) }\end{array}$ & $\begin{array}{l}\text { BROMO- } \\
\text { BENZENE } \\
\text { WATER, } \\
\text { WHOLE, } \\
\text { TOTAL } \\
\text { (UG/L) }\end{array}$ & $\begin{array}{l}\text { BROMO- } \\
\text { FORM } \\
\text { TOTAL } \\
\text { (UG/L) }\end{array}$ & $\begin{array}{c}\text { BENZENE } \\
\text { N-BUTYL } \\
\text { WATER } \\
\text { UNFLTRD } \\
\text { REC } \\
\text { (UG/L) }\end{array}$ & $\begin{array}{l}\text { BENZENE } \\
\text { SEC } \\
\text { BUTYL- } \\
\text { WATER } \\
\text { UNFLTRD } \\
\text { REC } \\
(\text { UG } / \mathrm{L})\end{array}$ & $\begin{array}{l}\text { BENZENE } \\
\text { TERT - } \\
\text { BUTYL - } \\
\text { WATER } \\
\text { UNFLTRD } \\
\text { REC } \\
\text { (UG/L) }\end{array}$ \\
\hline $\begin{array}{c}\text { CT } 1992 \\
28-29\end{array}$ & 110 & 23 & 1 & 7 & $<20$ & $<20$ & $<0.2$ & $<0.2$ & $<0.2$ & $<0.20$ & $<0.20$ & $<0.20$ \\
\hline DATE & $\begin{array}{l}\text { CARBON- } \\
\text { TETRA- } \\
\text { CHLO- } \\
\text { RIDE } \\
\text { TOTAL } \\
\text { (UG/L) }\end{array}$ & $\begin{array}{l}\text { CHLORO- } \\
\text { BENZENE } \\
\text { TOTAL } \\
\text { (UG/L) }\end{array}$ & $\begin{array}{l}\text { CHLORO- } \\
\text { DI - } \\
\text { BROMO- } \\
\text { METHANE } \\
\text { TOTAL } \\
\text { (UG/L) }\end{array}$ & $\begin{array}{l}\text { CHLORO- } \\
\text { ETHANE } \\
\text { TOTAL } \\
(U G / L)\end{array}$ & $\begin{array}{c}2- \\
\text { CHLORO- } \\
\text { ETHYL- } \\
\text { VINYL- } \\
\text { ETHER } \\
\text { TOTAL } \\
\text { (UG/L) }\end{array}$ & $\begin{array}{l}\text { CHLORO - } \\
\text { FORM } \\
\text { TOTAL } \\
\text { (UG/L) }\end{array}$ & $\begin{array}{l}\text { METHYL- } \\
\text { CHLO- } \\
\text { RIDE } \\
\text { TOTAL } \\
\text { (UC/L) }\end{array}$ & $\begin{array}{c}\text { O- } \\
\text { CHLORO- } \\
\text { TOLUENE } \\
\text { WATER } \\
\text { WHOLE } \\
\text { TOTAL } \\
\text { (UG/L) }\end{array}$ & $\begin{array}{c}\text { TOLUENE } \\
\text { P-CHLOR } \\
\text { WATER } \\
\text { UNFLTRD } \\
\text { REC } \\
(U G / L)\end{array}$ & $\begin{array}{l}\text { DIBROMO } \\
\text { CHLORO- } \\
\text { PROPANE } \\
\text { WATER } \\
\text { WHOLE } \\
\text { TOT.REC } \\
\text { (UG/L) }\end{array}$ & $\begin{array}{l}\text { DI- } \\
\text { BROMO- } \\
\text { METHANE } \\
\text { WATER } \\
\text { WHOLE } \\
\text { RECOVER } \\
(\mathrm{UG} / \mathrm{L})\end{array}$ & $\begin{array}{c}1,2- \\
\text { DIBROMO } \\
\text { ETHANE } \\
\text { WATER } \\
\text { WHOLE } \\
\text { TOTAL } \\
\text { (UG/L) }\end{array}$ \\
\hline
\end{tabular}

OCT 1992

$<0.2<0.20<0.2<0.2<1.0$

$<0.2<0.2<0.2<0.20$

$<1.0$

$<0.2<0.2$ 
Table 4. Water-quality data for storm-sewer outfall stations, Fort worth, Texas, 1992-93-Continued

08048510 - WEST FORK TRINITY RIVER OUTFALL AT HIGHWAY 121, FORT WORTH, TX (WY 1993)-CONtINUEd

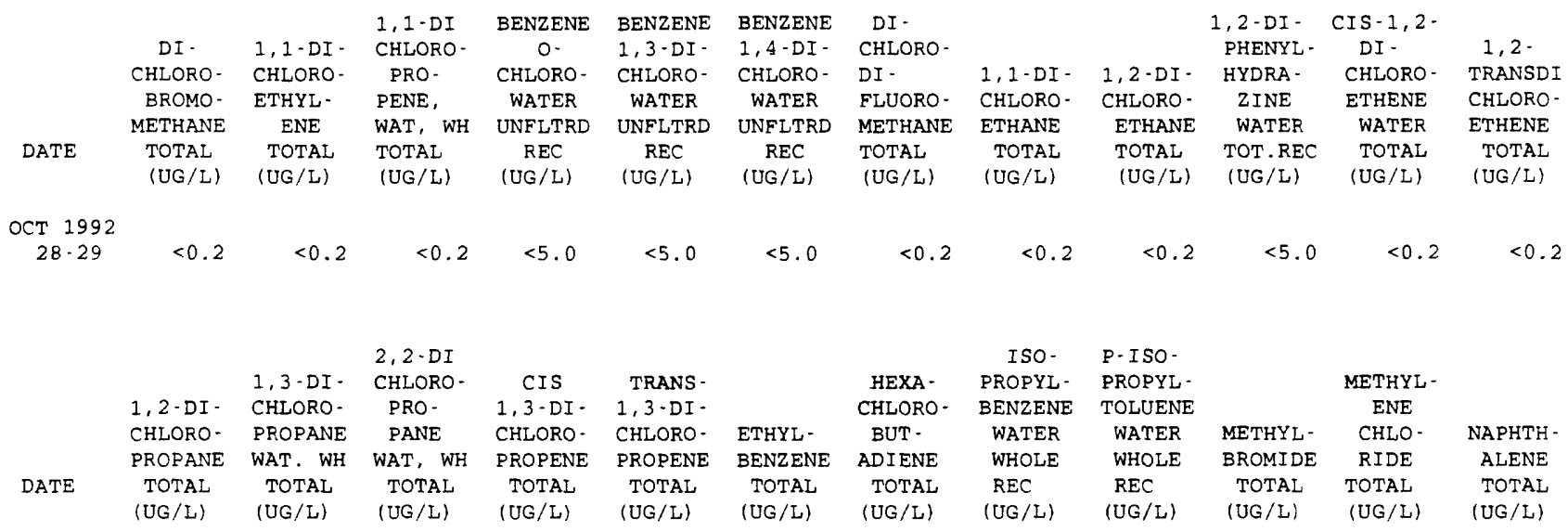

OCT 1992

$28-29$

$<0.2$

$<0.2$

$<0.2$

$<0.2$

$<0.2$

$<0.2$

$<5.0$

$<0.20$

$<0.20$

$<0.2$

$<0.2$

$<5.0$

DATE

$\begin{array}{ccc} & & \text { ETHANE, } \\ \text { BENZENE } & & 1112- \\ \text { N-PROPY } & & \text { TETRA- } \\ \text { WATER } & & \text { CHLORO- } \\ \text { UNFLTRD } & \text { STYRENE } & \text { WAT UNF } \\ \text { REC } & \text { TOTAL } & \text { REC } \\ (U G / L) & (U G / L) & \text { (UG } / L)\end{array}$

ETHANE,

$1,1,2,2$ TETRA.

TETRA - CHLORO -

CHLORO- ETHYL-

WAT UNF ENE

REC TOTAL

$\begin{array}{cc} & 1,2,3- \\ & \text { TRI- } \\ & \text { CHLORO } \\ & \text { BENZENE } \\ \text { TOLUENE } & \text { WAT, WH } \\ \text { TOTAL } & \text { REC } \\ \text { (UG/L) } & (\text { UG } / L)\end{array}$

BENZENE

$1,2,4$ -

TRI -

CHLORO-

1,1,1

(1)

$1,1,2-$

TRI -

TRI -

CHLORO - CHLORO -

CHLORO- CHLORO- ETHYL- FLUORO-

(UG/L) (UG/L)

REC

METHANE

(UG/L)

(UG/L)$$
\text { (UG/L) }
$$

123. TRI

CHLORO- PSEUDO- MESIT-

CHLORO-

$\begin{array}{lll}\text { PROPANE } & \text { CUMENE } & \text { YLENE } \\ \text { WATER } & \text { WATER } & \text { WATER }\end{array}$

DATE

WHOLE UNFLTRD UNFLTRD

TOTAL
(UG/L)

(UG/L)

(UG/L)

VINYL
CHLO-

XYLENE ACE -

RIDE UNFLTRD

ENE

TOTAL

YAPHTH

(UG/L)

REC

(UG/L)

(UOTAL)

(UG/L)

(UG/L)

TOTAL

TOTAL

(UG/L)

(UG/L)

OCT 1992

ANTHRA - BENZI -

CENE DINE

TOTAL

TOTAL

(UG/L)

(UG/L)

BENZO A

ANTHRAC

ENE1, 2-

BENZANT

HRACENE

TOTAL

(UG/L)

BENZO B

A. $\quad A N$ -

PYRENE THENE

TOTAL TOTAL

(UG/L) (UG/L)

$28-29$

$<0.2$

$<0.20$

$<0.20$

$<0.2$

$<0.20$

$<5.0$

$<5.0$

BENZO 4 -

BENZO $K$ GHI PERYL BROMO.

FLUOR - ENE1,12 - PHENYL

AN - BENZO PHENYL

THENE PERYLENE ETHER

DATE

TOTAL TOTAL TOTAL

(UG/L)

(UG/L)

(UG/L)

N-BUTYL BIS

BENZYL CHLORO-
PHTHAL - ETHOXY)

ATE METHANE

TOTAL TOTAL

(UG/L)

(UG/L)

BIS
$2-$
CHLORO-
ETHYL
ETHER
TOTAL
(UG/L)

BIS 12

$<5.0$

$<40.0$

$<10.0$

$<10.0$

$<10.0$

OCT 1992

28-29

$<10.0$

$<10.0$

$<5.0$

$<5.0$

$<5.0$

$<5.0$

$<5$.

CHLORO.

ISO.

PROPYL)

ETHER

TOTAL

(UG/L)

PARA-
CHLORO-
META
CRESOL
TOTAL
(UG/L)

2 -

CHLORO

NAPH -

THALENE

TOTAL

(UG/L)

$<30.0$

$<5.0$

$<5.0$

2 -

CHLORO-

PHENYL CHRY -

TOTAL TOTAL TOTAL

(UG/L) (UG/L) (UG/L)

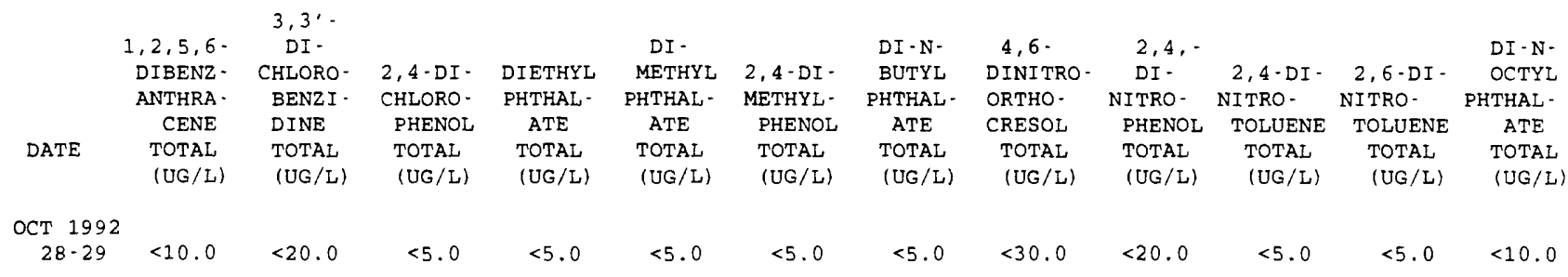


Table 4. Water-quality data for storm-sewer outfall stations, Fort Worth, Texas, 1992-93-Continued

08048510 WEST FORK TRINITY RIVER OUTFALL AT HIGHWAY 121, FORT WORTH, TX (WY 1993)-COntInUEd

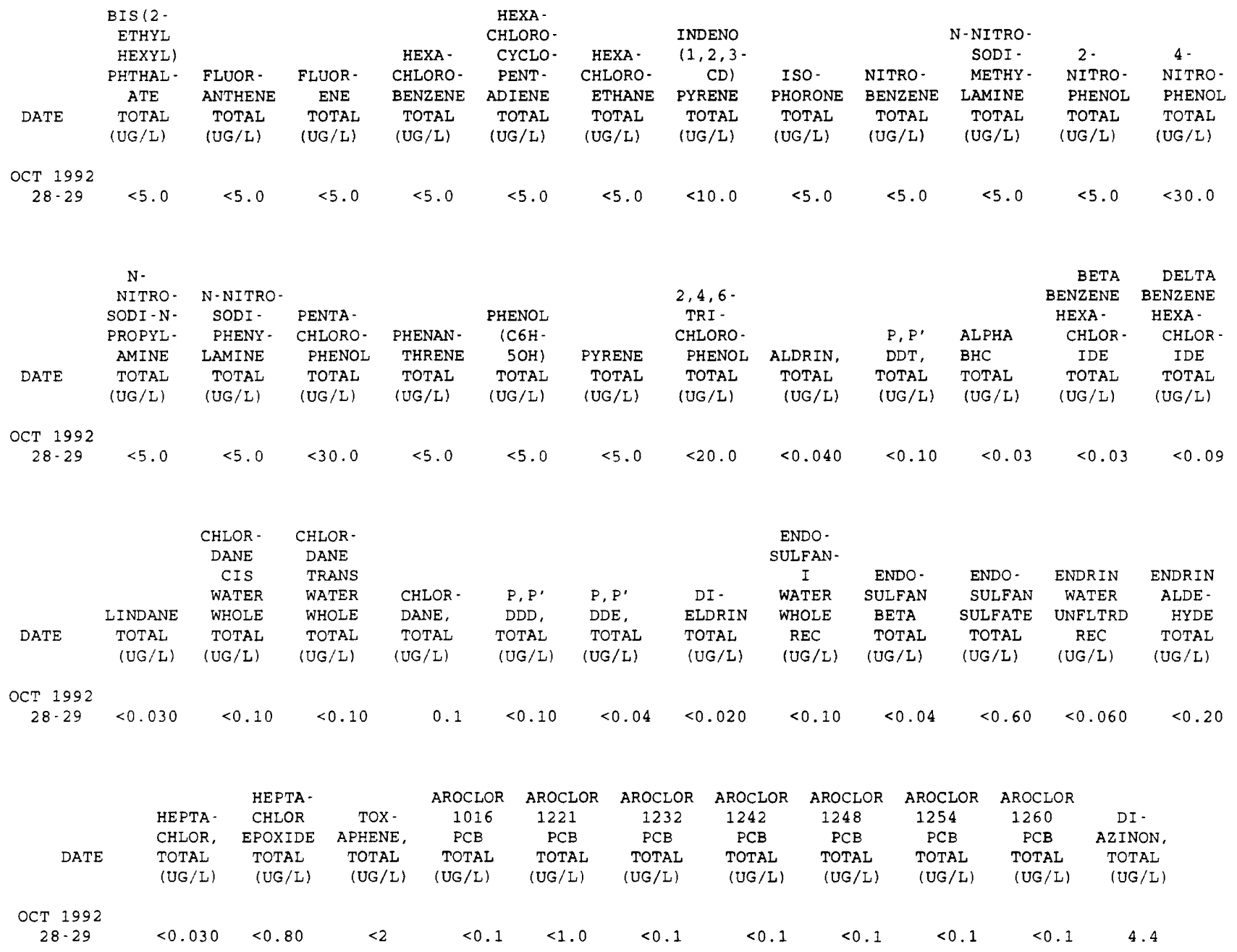


Table 4. Water-quality data for storm-sewer outfall stations, Fort Worth, Texas, 1992-93-Continued 08048545 - DRY BRANCH OUTFALL AT 33RD STREET, FORT WORTH, TX (WY 1992)

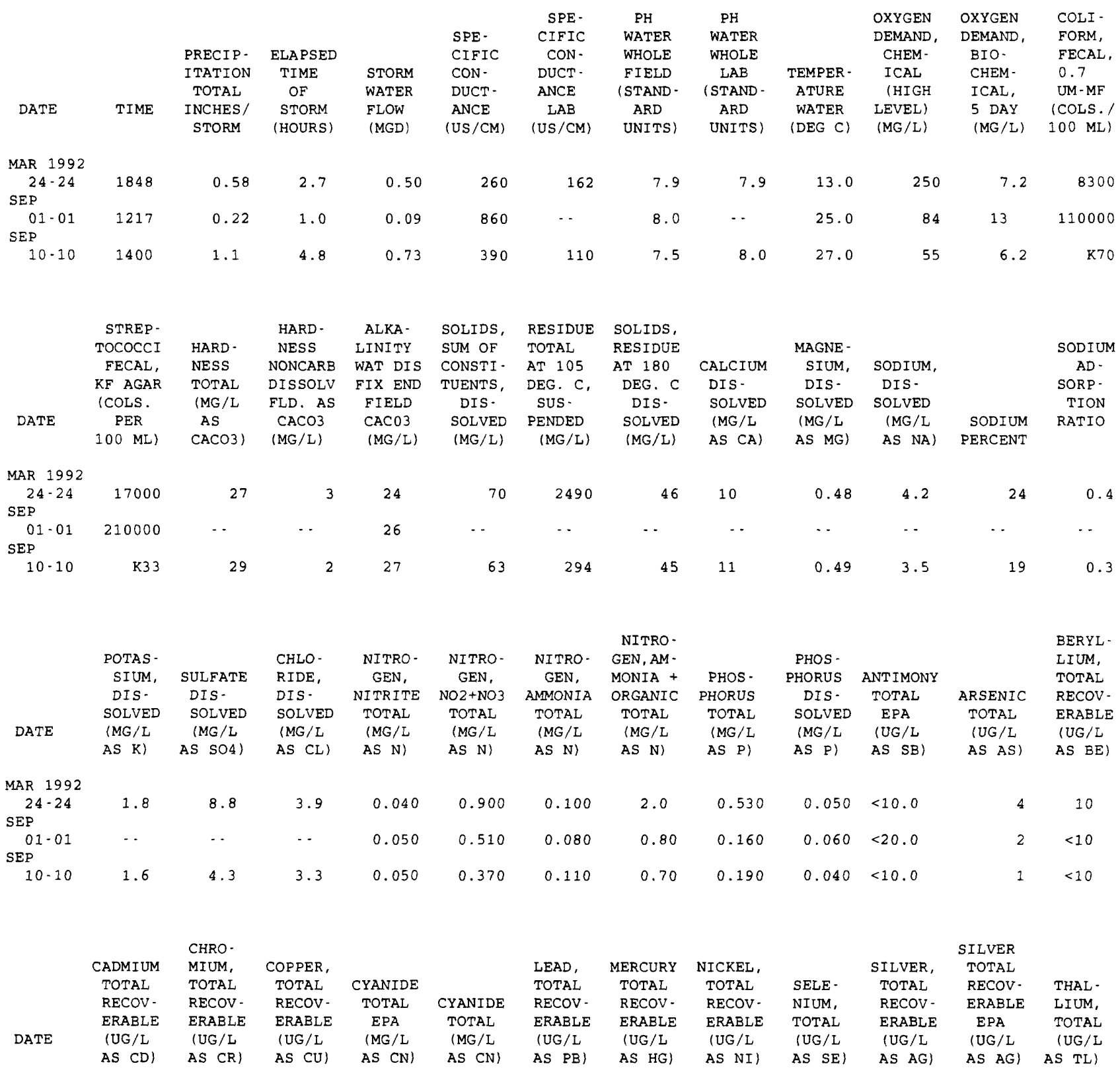

\begin{tabular}{|c|c|c|c|c|c|c|c|c|c|c|c|c|}
\hline \multicolumn{13}{|l|}{ MAR 1992} \\
\hline $24-24$ & 4 & 100 & $\cdots$ & $<0.010$ & $<0.010$ & 270 & $<0.10$ & 59 & $<2$ & $<1$ & $<1.00$ & $<10$ \\
\hline \multicolumn{13}{|l|}{ SEP } \\
\hline $01-01$ & 1 & 15 & 40 & $\cdots$ & $<0.010$ & 45 & $<0.10$ & 11 & $<2$ & $<1$ & $<0.500$ & $<5$ \\
\hline \multicolumn{13}{|l|}{ SEP } \\
\hline $10-10$ & $<1$ & 18 & 27 & 0.041 & 0.040 & 250 & $<0.10$ & 7 & $<1$ & $<1$ & $<0.500$ & $<5$ \\
\hline
\end{tabular}


Table 4. Water-quality data for storm-sewer outfall stations, Fort Worth, Texas, 1992-93-Continued

08048545 - DRY BRANCH OUTFALL AT 33RD STREET, FORT WORTH, TX (WY 1992)-Continued

\begin{tabular}{|c|c|c|c|c|c|c|c|c|c|c|c|c|}
\hline DATE & $\begin{array}{l}\text { ZINC, } \\
\text { TOTAL } \\
\text { RECOV. } \\
\text { ERABLE } \\
\text { (UG/L } \\
\text { AS ZN) }\end{array}$ & $\begin{array}{c}\text { CARBON, } \\
\text { ORGANIC } \\
\text { TOTAL } \\
\text { (MG/L } \\
\text { AS C) }\end{array}$ & $\begin{array}{l}\text { OIL AND } \\
\text { GREASE, } \\
\text { TOTAL } \\
\text { RECOV. } \\
\text { GRAVI- } \\
\text { METRIC } \\
\text { (MG/L) }\end{array}$ & $\begin{array}{l}\text { PHENOLS } \\
\text { TOTAL } \\
\text { (UG/L) }\end{array}$ & $\begin{array}{l}\text { ACRO- } \\
\text { LEIN } \\
\text { TOTAL } \\
\text { (UG/L) }\end{array}$ & $\begin{array}{l}\text { ACRYLO- } \\
\text { NITRILE } \\
\text { TOTAL } \\
\text { (UG/L) }\end{array}$ & $\begin{array}{l}\text { BENZENE } \\
\text { TOTAL } \\
\text { (UG/L) }\end{array}$ & $\begin{array}{l}\text { BROMO- } \\
\text { BENZENE } \\
\text { WATER, } \\
\text { WHOLE, } \\
\text { TOTAL } \\
\text { (UG/L) }\end{array}$ & $\begin{array}{l}\text { BROMO- } \\
\text { FORM } \\
\text { TOTAL } \\
\text { (UG/L) }\end{array}$ & $\begin{array}{c}\text { BENZENE } \\
\text { N-BUTYL } \\
\text { WATER } \\
\text { UNFLTRD } \\
\text { REC } \\
\text { (UG/L) }\end{array}$ & $\begin{array}{l}\text { BENZENE } \\
\text { SEC } \\
\text { BUTYL- } \\
\text { WATER } \\
\text { UNFLTRD } \\
\text { REC } \\
\text { (UG/L) }\end{array}$ & $\begin{array}{l}\text { BENZENE } \\
\text { TERT- } \\
\text { BUTYL- } \\
\text { WATER } \\
\text { UNFLTRD } \\
\text { REC } \\
\text { (UG/L) }\end{array}$ \\
\hline $\begin{array}{l}\text { MAR } 1992 \\
24-24 \\
\text { SEP }\end{array}$ & 1400 & 46 & $<1$ & 5 & $<20$ & $<20$ & $<0.2$ & $<0.2$ & $<0.2$ & $<0.20$ & $<0.20$ & $<0.20$ \\
\hline $\begin{array}{l}01-01 \\
\text { SEP } \\
10-10\end{array}$ & 710 & 27 & 3 & 3 & $\begin{array}{l}<20 \\
<20\end{array}$ & $\begin{array}{l}<20 \\
<20\end{array}$ & $<0.2$ & $<0.2$ & $<0.2$ & $<0.20$ & $<0.20$ & $<0.20$ \\
\hline DATE & $\begin{array}{l}\text { CARBON - } \\
\text { TETRA - } \\
\text { CHLO- } \\
\text { RIDE } \\
\text { TOTAL } \\
\text { (UG/L) }\end{array}$ & $\begin{array}{l}\text { CHLORO- } \\
\text { BENZENE } \\
\text { TOTAL } \\
\text { (UG/L) }\end{array}$ & $\begin{array}{l}\text { CHLORO- } \\
\text { DI- } \\
\text { BROMO- } \\
\text { METHANE } \\
\text { TOTAL } \\
\text { (UG/L) }\end{array}$ & $\begin{array}{l}\text { CHLORO- } \\
\text { ETHANE } \\
\text { TOTAL } \\
\text { (UG/L) }\end{array}$ & $\begin{array}{c}2- \\
\text { CHLORO- } \\
\text { ETHYL- } \\
\text { VINYL- } \\
\text { ETHER } \\
\text { TOTAL } \\
\text { (UG/L) }\end{array}$ & $\begin{array}{l}\text { CHLORO- } \\
\text { FORM } \\
\text { TOTAL } \\
\text { (UG/L) }\end{array}$ & $\begin{array}{l}\text { METHYL- } \\
\text { CHLO- } \\
\text { RIDE } \\
\text { TOTAL } \\
\text { (UG/L) }\end{array}$ & $\begin{array}{c}\text { O- } \\
\text { CHLORO- } \\
\text { TOLUENE } \\
\text { WATER } \\
\text { WHOLE } \\
\text { TOTAL } \\
\text { (UG/L) }\end{array}$ & $\begin{array}{c}\text { TOLUENE } \\
\text { P-CHLOR } \\
\text { WATER } \\
\text { UNFLTRD } \\
\text { REC } \\
\text { (UG/L) }\end{array}$ & $\begin{array}{l}\text { DIBROMO } \\
\text { CHLORO- } \\
\text { PROPANE } \\
\text { WATER } \\
\text { WHOLE } \\
\text { TOT.REC } \\
\text { (UG/L) }\end{array}$ & $\begin{array}{c}\text { DI- } \\
\text { BROMO- } \\
\text { METHANE } \\
\text { WATER } \\
\text { WHOLE } \\
\text { RECOVER } \\
\text { (UG/L) }\end{array}$ & $\begin{array}{l}1,2- \\
\text { DIBROMO } \\
\text { ETHANE } \\
\text { WATER } \\
\text { WHOLE } \\
\text { TOTAL } \\
\text { (UG } / \mathrm{L})\end{array}$ \\
\hline $\begin{array}{l}\text { MAR } 1992 \\
24-24 \\
\text { SEP }\end{array}$ & $<0.2$ & $<0.20$ & $<0.2$ & $<0.2$ & $<1.0$ & $<0.2$ & $<0.2$ & $<0.2$ & $<0.20$ & $<1.0$ & $<0.2$ & $<0.2$ \\
\hline $\begin{array}{l}01.01 \\
\text { SEP } \\
10-10\end{array}$ & $<0.2$ & $<0.20$ & $<0.2$ & $<0.2$ & $<1.0$ & $\begin{array}{r}<0.2 \\
0.5\end{array}$ & $<0.2$ & $\begin{array}{l}<0.2 \\
<0.2\end{array}$ & $\begin{array}{l}<0.20 \\
<0.20\end{array}$ & $\begin{array}{l}<1.0 \\
<1.0\end{array}$ & $\begin{array}{l}<0.2 \\
<0.2\end{array}$ & $\begin{array}{l}<0.2 \\
<0.2\end{array}$ \\
\hline DATE & $\begin{array}{c}\text { DI - } \\
\text { CHLORO- } \\
\text { BROMO- } \\
\text { METHANE } \\
\text { TOTAL } \\
\text { (UG/L) }\end{array}$ & $\begin{array}{c}1,1-D I- \\
\text { CHLORO- } \\
\text { ETHYL- } \\
\text { ENE } \\
\text { TOTAL } \\
\text { (UG/L) }\end{array}$ & $\begin{array}{l}\text { 1, I-DI } \\
\text { CHLORO- } \\
\text { PRO- } \\
\text { PENE, } \\
\text { WAT, WH } \\
\text { TOTAL } \\
\text { (UG } / L)\end{array}$ & $\begin{array}{c}\text { BENZENE } \\
0- \\
\text { CHLORO- } \\
\text { WATER } \\
\text { UNFLTRD } \\
\text { REC } \\
\text { (UG/L) }\end{array}$ & $\begin{array}{l}\text { BENZENE } \\
1,3 \cdot D I- \\
\text { CHLORO- } \\
\text { WATER } \\
\text { UNFLTRD } \\
\text { REC } \\
\text { (UG/L) }\end{array}$ & $\begin{array}{c}\text { BENZENE } \\
1,4 \text {-DI - } \\
\text { CHLORO- } \\
\text { WATER } \\
\text { UNFLTRD } \\
\text { REC } \\
(U G / L)\end{array}$ & $\begin{array}{l}\text { DI- } \\
\text { CHLORO- } \\
\text { DI- } \\
\text { FLUORO- } \\
\text { METHANE } \\
\text { TOTAL } \\
\text { (UG/L) }\end{array}$ & $\begin{array}{c}1,1-D I- \\
\text { CHLORO- } \\
\text { ETHANE } \\
\text { TOTAL } \\
\text { (UG/L) }\end{array}$ & $\begin{array}{c}1,2-D I- \\
\text { CHLORO- } \\
\text { ETHANE } \\
\text { TOTAL } \\
\text { (UG/L) }\end{array}$ & $\begin{array}{l}1,2-D I- \\
\text { PHENYL- } \\
\text { HYDRA- } \\
\text { ZINE } \\
\text { WATER } \\
\text { TOT.REC } \\
\text { (UG/L) }\end{array}$ & $\begin{array}{c}\text { CIS-1, } 2- \\
\text { DI - } \\
\text { CHLORO- } \\
\text { ETHENE } \\
\text { WATER } \\
\text { TOTAL } \\
(U G / L)\end{array}$ & $\begin{array}{l}1,2- \\
\text { TRANSDI } \\
\text { CHLORO- } \\
\text { ETHENE } \\
\text { TOTAL } \\
\text { (UG/L) }\end{array}$ \\
\hline $\begin{array}{l}\text { MAR } 1992 \\
24-24 \\
\text { SEP }\end{array}$ & $<0.2$ & 1.9 & $<0.2$ & $<5.0$ & $<5.0$ & $<5.0$ & $<0.2$ & 0.6 & $<0.2$ & $<5.0$ & 10 & 0.3 \\
\hline $\begin{array}{l}01-01 \\
\operatorname{SEP} \\
10-10\end{array}$ & $<0.2$ & $<0.2$ & $<0.2$ & $\begin{array}{l}<5.0 \\
<5.0\end{array}$ & $\begin{array}{l}<5.0 \\
<5.0\end{array}$ & $\begin{array}{l}<5.0 \\
<5.0\end{array}$ & $<0.2$ & $\begin{array}{r}<0.2 \\
0.3\end{array}$ & $<0.2$ & $\begin{array}{l}<5.0 \\
<5.0\end{array}$ & 1.4 & $\begin{array}{l}<0.2 \\
<0.2\end{array}$ \\
\hline DATE & $\begin{array}{l}1,2-D I- \\
\text { CHLORO- } \\
\text { PROPANE } \\
\text { TOTAL } \\
\text { (UG/L) }\end{array}$ & $\begin{array}{l}1,3-D I- \\
\text { CHLORO- } \\
\text { PROPANE } \\
\text { WAT. WH } \\
\text { TOTAL } \\
\text { (UG/L) }\end{array}$ & $\begin{array}{l}2,2 \text { - DI } \\
\text { CHLORO- } \\
\text { PRO- } \\
\text { PANE } \\
\text { WAT, WH } \\
\text { TOTAL } \\
\text { (UG } / \mathrm{L} \text { ) }\end{array}$ & $\begin{array}{c}\text { CIS } \\
1,3 \cdot \mathrm{DI}- \\
\text { CHLORO- } \\
\text { PROPENE } \\
\text { TOTAL } \\
\text { (UG/L) }\end{array}$ & $\begin{array}{l}\text { TRANS - } \\
1,3-\mathrm{DI}- \\
\text { CHLORO- } \\
\text { PROPENE } \\
\text { TOTAL } \\
\text { (UG/L) }\end{array}$ & $\begin{array}{l}\text { ETHYL - } \\
\text { BENZENE } \\
\text { TOTAL } \\
\text { (UG/L) }\end{array}$ & $\begin{array}{l}\text { HEXA- } \\
\text { CHLORO- } \\
\text { BUT- } \\
\text { ADIENE } \\
\text { TOTAL } \\
\text { (UG/L) }\end{array}$ & $\begin{array}{l}\text { ISO- } \\
\text { PROPYL- } \\
\text { BENZENE } \\
\text { WATER } \\
\text { WHOLE } \\
\text { REC } \\
\text { (UG/L) }\end{array}$ & $\begin{array}{l}\text { P-ISO- } \\
\text { PROPYL- } \\
\text { TOLUENE } \\
\text { WATER } \\
\text { WHOLE } \\
\text { REC } \\
\text { (UG/L) }\end{array}$ & $\begin{array}{c}\text { METHYL- } \\
\text { BROMIDE } \\
\text { TOTAL } \\
\text { (UG/L) }\end{array}$ & $\begin{array}{l}\text { METHYL - } \\
\text { ENE } \\
\text { CHLO- } \\
\text { RIDE } \\
\text { TOTAL } \\
\text { (UG /L) }\end{array}$ & $\begin{array}{c}\text { NAPHTH - } \\
\text { ALENE } \\
\text { TOTAL } \\
\text { (UG/L) }\end{array}$ \\
\hline $\begin{array}{l}\text { IAR } 1992 \\
24-24 \\
\text { SEP }\end{array}$ & $<0.2$ & $<0.2$ & $<0.2$ & $<0.2$ & $<0.2$ & $<0.2$ & $<5.0$ & $<0.20$ & $<0.20$ & $<0.2$ & 3.3 & $<5.0$ \\
\hline$E^{01-01}$ & $<0.2$ & 0.2 & $<0.2$ & $<0$ & $<0.2$ & $<0.2$ & $<5.0$ & $<0.20$ & $<0.20$ & $<0.2$ & $<0.2$ & $<5.0$ \\
\hline $10 \cdot 10$ & 0.2 & 0.2 & $<0.2$ & 0.2 & $<0.2$ & 1.5 & $<5.0$ & 0.20 & $<0.20$ & $<0.2$ & 0.4 & $<5.0$ \\
\hline
\end{tabular}


Table 4. Water-quality data for storm-sewer outfall stations, Fort worth, Texas, 1992-93-Continued 08048545 - DRY BRANCH OUTFALL AT 33RD STREET, FORT WORTH, TX (WY 1992)-Continued

\begin{tabular}{|c|c|c|c|c|c|c|c|c|c|c|c|c|}
\hline & $\begin{array}{c}\text { BENZENE } \\
\text { N-PROPY } \\
\text { WATER }\end{array}$ & & $\begin{array}{c}\text { ETHANE, } \\
1112- \\
\text { TETRA - } \\
\text { CHLORO - }\end{array}$ & $\begin{array}{r}\text { ETHANE, } \\
1,1,2,2 \\
\text { TETRA- } \\
\text { CHLORO- }\end{array}$ & $\begin{array}{l}\text { TETRA - } \\
\text { CHLORO- } \\
\text { ETHYL- }\end{array}$ & & $\begin{array}{c}1,2,3- \\
\text { TRI- } \\
\text { CHLORO } \\
\text { BENZENE }\end{array}$ & $\begin{array}{c}\text { BENZENE } \\
1,2,4- \\
\text { TRI - } \\
\text { CHLORO- }\end{array}$ & $\begin{array}{c}1,1,1- \\
\text { TRI. } \\
\text { CHLORO. }\end{array}$ & $\begin{array}{c}1,1,2- \\
\text { TRI. } \\
\text { CHLORO- }\end{array}$ & $\begin{array}{l}\text { TRI - } \\
\text { CHLORO- } \\
\text { ETHYL - }\end{array}$ & $\begin{array}{c}\text { TRI - } \\
\text { CHLORO- } \\
\text { FLUORO- }\end{array}$ \\
\hline & UNFLTRD & STYRENE & WAT UNF & WAT UNF & ENE & TOLUENE & WAT, WH & WAT UNF & ETHANE & ETHANE & ENE & METHANE \\
\hline 5 & $\begin{array}{c}\text { REC } \\
(U G / L)\end{array}$ & $\begin{array}{l}\text { TOTAL } \\
\text { (UG/L) }\end{array}$ & $\begin{array}{c}\text { REC } \\
(U G / L)\end{array}$ & $\begin{array}{c}\text { REC } \\
(U G / L)\end{array}$ & $\begin{array}{l}\text { TOTAL } \\
\text { (UG/L) }\end{array}$ & $\begin{array}{l}\text { TOTAL } \\
\text { (UG/L) }\end{array}$ & $\begin{array}{l}\text { REC } \\
(U G / L)\end{array}$ & $\begin{array}{c}\text { REC } \\
(U G / L)\end{array}$ & $\begin{array}{l}\text { TOTAL } \\
\text { (UG/L) }\end{array}$ & $\begin{array}{l}\text { TOTAL } \\
\text { (UG/L) }\end{array}$ & $\begin{array}{l}\text { TOTAL } \\
\text { (UG/L) }\end{array}$ & $\begin{array}{l}\text { TOTAL } \\
\text { (UG/L) }\end{array}$ \\
\hline
\end{tabular}

\begin{tabular}{|c|c|c|c|c|c|c|c|c|c|c|c|c|}
\hline \multicolumn{13}{|l|}{ MAR 1992} \\
\hline $24-24$ & $<0.20$ & $<0.2$ & $<0.2$ & $<0.2$ & 13 & 0.3 & $<0.20$ & $<5.0$ & 5.4 & $<0.2$ & 7.6 & $<0.2$ \\
\hline \multicolumn{13}{|l|}{ SEP } \\
\hline $01-01$ & $<0.20$ & $<0.2$ & $<0.2$ & $<0.2$ & 0.9 & $<0.2$ & $<0.20$ & $<5.0$ & 0.8 & $<0.2$ & 0.5 & $<0.2$ \\
\hline \multicolumn{13}{|l|}{ SEP } \\
\hline $10 \cdot 10$ & 0.30 & $<0.2$ & $<0.2$ & $<0.2$ & 2.3 & 0.7 & $<0.20$ & $<5.0$ & 0.5 & 0.2 & 1.3 & $<0.2$ \\
\hline
\end{tabular}

\begin{tabular}{|c|c|c|c|c|c|c|c|c|c|c|c|c|}
\hline & 123 -TRI & & & & & & & & & BENZO A & & \\
\hline & CHLORO- & PSEUDO - & MESIT - & & & & & & & ANTHRAC & & BENZO B \\
\hline & PROPANE & CUMENE & YLENE & VINYL & XYLENE & $A C E-$ & ACE - & & & ENE 1, 2- & BENZO - & FLUOR - \\
\hline & WATER & WATER & WATER & CHLO - & WATER & NAPHTH - & NAPHTH - & ANTHRA - & BENZI - & BENZANT & A. & $\mathrm{AN}$ - \\
\hline & WHOLE & UNFLTRD & UNFLTRD & RIDE & UNFLTRD & ENE & YLENE & CENE & DINE & HRACENE & PYRENE & THENE \\
\hline DATE & $\begin{array}{l}\text { TOTAL } \\
\text { (UG/L) }\end{array}$ & $\begin{array}{c}\text { REC } \\
\text { (UG/L) }\end{array}$ & $\begin{array}{c}\text { REC } \\
\text { (UG } / L)\end{array}$ & $\begin{array}{l}\text { TOTAL } \\
\text { (UG/L) }\end{array}$ & $\begin{array}{c}\text { REC } \\
(U G / L)\end{array}$ & $\begin{array}{l}\text { TOTAL } \\
\text { (UG/L) }\end{array}$ & $\begin{array}{l}\text { TOTAL } \\
\text { (UG/L) }\end{array}$ & $\begin{array}{l}\text { TOTAL } \\
\text { (UG/L) }\end{array}$ & $\begin{array}{l}\text { TOTAL } \\
\text { (UG/L) }\end{array}$ & $\begin{array}{l}\text { TOTAL } \\
(U G / L)\end{array}$ & $\begin{array}{l}\text { TOTAL } \\
\text { (UG/L) }\end{array}$ & $\begin{array}{l}\text { TOTAL } \\
\text { (UG/L) }\end{array}$ \\
\hline \multicolumn{13}{|l|}{ MAR 1992} \\
\hline $24-24$ & $<0.2$ & $<0.20$ & $<0.20$ & 0.4 & 1.5 & $<5.0$ & $<5.0$ & $<5.0$ & $<40.0$ & $<10.0$ & $<10.0$ & $<10.0$ \\
\hline \multicolumn{13}{|l|}{ SEP } \\
\hline 01.01 & $<0.2$ & $<0.20$ & $<0.20$ & $<0.2$ & $<0.20$ & $<5.0$ & $<5.0$ & $<5.0$ & $<40.0$ & $<10.0$ & $<10.0$ & $<10.0$ \\
\hline \multicolumn{13}{|l|}{ SEP } \\
\hline \multirow[t]{6}{*}{$10-10$} & $<0.2$ & 1.3 & 0.30 & 0.7 & 10 & $<5.0$ & $<5.0$ & $<5.0$ & $<40.0$ & $<20.0$ & $<10.0$ & $<10.0$ \\
\hline & & BENZO & 4 & & BIS & BIS & BIS 12. & & & & $4-$ & \\
\hline & BENZO K & GHI PERYL & BROMO - & $N \cdot B U T Y L$ & $12-$ & 2 & CHLORO- & PARA - & 2 & & CHLORO - & \\
\hline & FLUOR - & ENE1, 12 - & PHENYL & BENZYL & CHLORO - & CHLORO - & ISO- & CHLORO - & CHLORO - & $2-$ & PHENYL & \\
\hline & $\mathrm{AN}-$ & BENZO & PHENYL & PHTHAL - & ЕTHOXY) & ETHYL & PROPYL) & META & NAPH - & CHLORO - & PHENYL & CHRY - \\
\hline & THENE & PERYLENE & ETHER & ATE & METHANE & ETHER & ETHER & CRESOL & THALENE & PHENOL & ETHER & SENE \\
\hline DATE & $\begin{array}{l}\text { TOTAL } \\
(\text { UG/L) }\end{array}$ & $\begin{array}{l}\text { TOTAL } \\
\text { (UG/L) }\end{array}$ & $\begin{array}{l}\text { TOTAL } \\
(U G / L)\end{array}$ & $\begin{array}{l}\text { TOTAL } \\
\text { (UG/L) }\end{array}$ & $\begin{array}{l}\text { TOTAL } \\
(U G / L)\end{array}$ & $\begin{array}{l}\text { TOTAL } \\
\text { (UG/L) }\end{array}$ & $\begin{array}{l}\text { TOTAL } \\
\text { (UG/L) }\end{array}$ & $\begin{array}{l}\text { TOTAL } \\
\text { (UG/L) }\end{array}$ & $\begin{array}{l}\text { TOTAL } \\
\text { (UG/L) }\end{array}$ & $\begin{array}{l}\text { TOTAL } \\
\text { (UG/L) }\end{array}$ & $\begin{array}{l}\text { TOTAL } \\
\text { (UG/L) }\end{array}$ & $\begin{array}{l}\text { TOTAL } \\
\text { (UG/L) }\end{array}$ \\
\hline \multicolumn{13}{|l|}{ MAR 1992} \\
\hline $24-24$ & $<10.0$ & 25.0 & $<5.0$ & $<5.0$ & $<5.0$ & $<5.0$ & $<5.0$ & $<30.0$ & $<5.0$ & $<5.0$ & $<5.0$ & $<10.0$ \\
\hline \multicolumn{13}{|l|}{ SEP } \\
\hline $01-01$ & $<10.0$ & $<10.0$ & $<5.0$ & $<5.0$ & $<5.0$ & $<5.0$ & $<5.0$ & $<30.0$ & $<5.0$ & $<5.0$ & $<5.0$ & $<10.0$ \\
\hline \multicolumn{13}{|l|}{ SEP } \\
\hline $10 \cdot 10$ & $<10.0$ & $<10.0$ & $<5.0$ & $<5.0$ & $<5.0$ & $<5.0$ & $<5.0$ & $<30.0$ & $<5.0$ & $<5.0$ & $<5.0$ & $<10.0$ \\
\hline
\end{tabular}

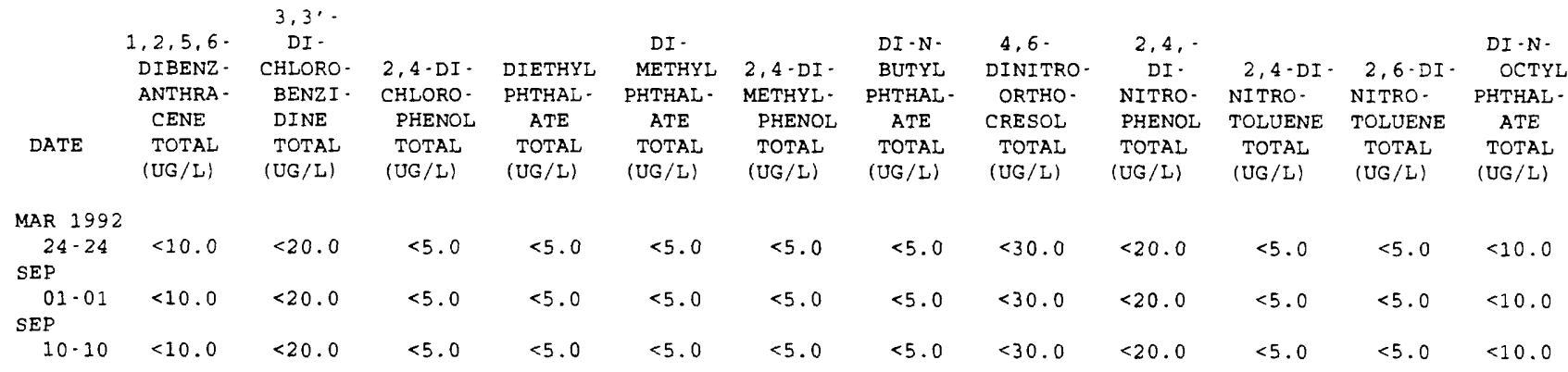


Table 4. Water-quality data for storm-sewer outfall stations, Fort worth, Texas, 1992-93-Continued

08048545 - DRY BRANCH OUTFALL AT 33RD STREET, FORT WORTH, TX (WY 1992)-Continued

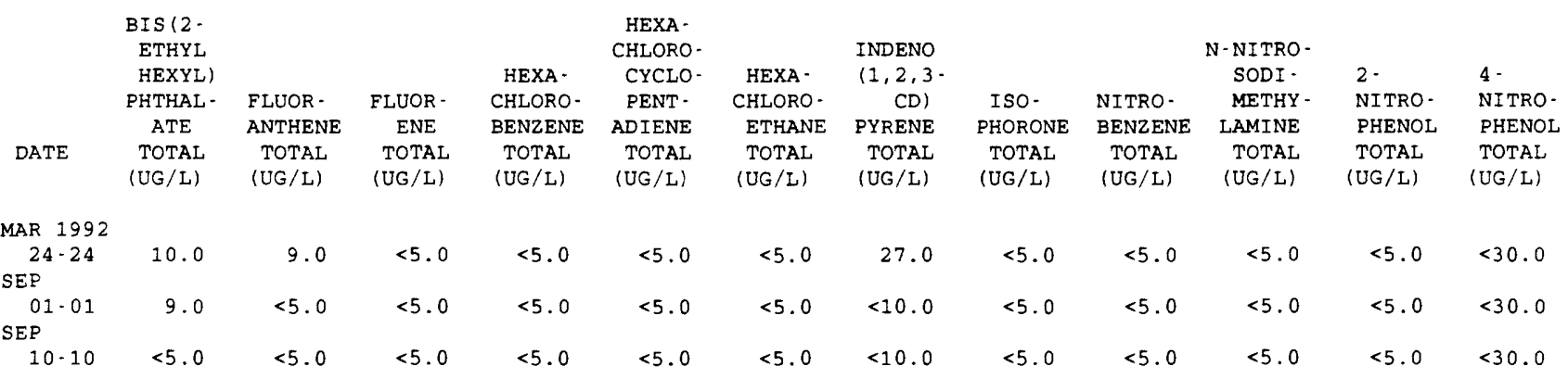

\begin{tabular}{|c|c|c|c|c|c|c|c|c|c|c|c|c|}
\hline DATE & $\begin{array}{l}\text { N- } \\
\text { NITRO- } \\
\text { SODI-N- } \\
\text { PROPYL- } \\
\text { AMINE } \\
\text { TOTAL } \\
(U G / L)\end{array}$ & $\begin{array}{l}\text { N-NITRO- } \\
\text { SODI - } \\
\text { PHENY - } \\
\text { LAMINE } \\
\text { TOTAL } \\
\text { (UG/L) }\end{array}$ & $\begin{array}{l}\text { PENTA- } \\
\text { CHLORO- } \\
\text { PHENOL } \\
\text { TOTAL } \\
\text { (UG/L) }\end{array}$ & $\begin{array}{c}\text { PHENAN - } \\
\text { THRENE } \\
\text { TOTAL } \\
\text { (UG/L) }\end{array}$ & $\begin{array}{l}\text { PHENOL } \\
\text { (C6H- } \\
5 O H) \\
\text { TOTAL } \\
\text { (UG/L) }\end{array}$ & $\begin{array}{l}\text { PYRENE } \\
\text { TOTAL } \\
\text { (UG/L) }\end{array}$ & $\begin{array}{c}2,4,6- \\
\text { TRI - } \\
\text { CHLORO- } \\
\text { PHENOL } \\
\text { TOTAL } \\
\text { (UG/L) }\end{array}$ & $\begin{array}{l}\text { ALDRIN, } \\
\text { TOTAL } \\
\text { (UG/L) }\end{array}$ & $\begin{array}{l}\text { P, } P^{\prime} \\
\text { DDT, } \\
\text { TOTAL } \\
\text { (UG/L) }\end{array}$ & $\begin{array}{l}\text { ALPHA } \\
\text { BHC } \\
\text { TOTAL } \\
\text { (UG/L) }\end{array}$ & $\begin{array}{c}\text { BETA } \\
\text { BENZENE } \\
\text { HEXA- } \\
\text { CHLOR- } \\
\text { IDE } \\
\text { TOTAL } \\
\text { (UG/L) }\end{array}$ & $\begin{array}{c}\text { DELTA } \\
\text { BENZENE } \\
\text { HEXA- } \\
\text { CHLOR- } \\
\text { IDE } \\
\text { TOTAL } \\
\text { (UG/L) }\end{array}$ \\
\hline MAR 1992 & & & & & & & & & & & & \\
\hline $\begin{array}{l}24-24 \\
\text { SEP }\end{array}$ & $<5.0$ & $<5.0$ & $<30.0$ & 5.0 & $<5.0$ & 6.0 & $<20.0$ & $<0.040$ & $<0.10$ & $<0.03$ & $<0.03$ & $<0.09$ \\
\hline $\begin{array}{l}01.01 \\
\text { SEP }\end{array}$ & $<5.0$ & $<5.0$ & $<30.0$ & $<5.0$ & $<5.0$ & $<5.0$ & $<20.0$ & $<0.040$ & $<0.10$ & $<0.03$ & $<0.03$ & $<0.09$ \\
\hline $10 \cdot 10$ & $<5.0$ & $<5.0$ & $<30.0$ & $<5.0$ & $<5.0$ & $<5.0$ & $<20.0$ & $<0.040$ & $<0.10$ & $<0.03$ & $<0.03$ & $<0.09$ \\
\hline
\end{tabular}

\begin{tabular}{|c|c|c|c|c|c|c|c|c|c|c|c|c|}
\hline & & $\begin{array}{l}\text { CHLOR- } \\
\text { DANE }\end{array}$ & $\begin{array}{l}\text { CHLOR - } \\
\text { DANE }\end{array}$ & & & & & $\begin{array}{l}\text { ENDO- } \\
\text { SULFAN- }\end{array}$ & & & & \\
\hline & & CIS & TRANS & & - & & & $I$ & ENDO- & ENDO - & ENDRIN & ENDRIN \\
\hline & & WATER & WATER & CHLOR - & $P, P^{\prime}$ & $P, P^{\prime}$ & DI - & WATER & SULFAN & SULFAN & WATER & ALDE - \\
\hline & LINDANE & WHOLE & WHOLE & DANE, & DDD, & DDE, & ELDRIN & WHOLE & BETA & SULFATE & UNFLTRD & HYDE \\
\hline ATE & $\begin{array}{l}\text { TOTAL } \\
\text { (UG/L) }\end{array}$ & $\begin{array}{l}\text { TOTAL } \\
(U G / L)\end{array}$ & $\begin{array}{l}\text { TOTAL } \\
(\mathrm{UG} / \mathrm{L})\end{array}$ & $\begin{array}{l}\text { TOTAL } \\
\text { (UG/L) }\end{array}$ & $\begin{array}{l}\text { TOTAL } \\
\text { (UG/L) }\end{array}$ & $\begin{array}{l}\text { TOTAI } \\
(U G / L)\end{array}$ & $\begin{array}{l}\text { TOTAL } \\
\text { (UG/L) }\end{array}$ & $\begin{array}{l}\text { REC } \\
(U G / L)\end{array}$ & $\begin{array}{l}\text { TOTAL } \\
\text { (UG/L) }\end{array}$ & $\begin{array}{l}\text { TOTAL } \\
\text { (UG/L) }\end{array}$ & $\begin{array}{c}\text { REC } \\
(U G / L)\end{array}$ & $\begin{array}{l}\text { TOTAL } \\
\text { (UG/L) }\end{array}$ \\
\hline
\end{tabular}

\begin{tabular}{|c|c|c|c|c|c|c|c|c|c|c|c|c|}
\hline MAR 1992 & & & & & & & & & & & & \\
\hline $24-24$ & $<0.030$ & $<0.10$ & $<0.10$ & $<0.1$ & $<0.10$ & $<0.04$ & $<0.020$ & $<0.10$ & $<0.04$ & $<0.60$ & $<0.060$ & $<0.20$ \\
\hline \multicolumn{13}{|l|}{ SEP } \\
\hline $01-01$ & $<0.030$ & $<0.10$ & $<0.10$ & $<0.1$ & $<0.10$ & $<0.04$ & $<0.020$ & $<0.10$ & $<0.04$ & $<0.60$ & $<0.060$ & $<0.20$ \\
\hline \multicolumn{13}{|l|}{ SEP } \\
\hline $10-10$ & $<0.030$ & $<0.10$ & $<0.10$ & $<0.1$ & $<0.10$ & $<0.04$ & $<0.020$ & $<0.10$ & $<0.04$ & $<0.60$ & $<0.060$ & $<0.20$ \\
\hline
\end{tabular}

\begin{tabular}{|c|c|c|c|c|c|c|c|c|c|c|c|}
\hline & & HEPTA - & & AROCLOR & AROCLOR & AROCLOR & AROCLOR & AROCLOR & AROCLOR & AROCLOR & \\
\hline & HEPTA - & CHLOR & Tox- & 1016 & 1221 & 1232 & 1242 & 1248 & 1254 & 1260 & DI - \\
\hline DATE & $\begin{array}{l}\text { CHLOR, } \\
\text { TOTAL } \\
\text { (UG/L) }\end{array}$ & $\begin{array}{l}\text { EPOXIDE } \\
\text { TOTAL } \\
\text { (UG/L) }\end{array}$ & $\begin{array}{c}\text { APHENE, } \\
\text { TOTAL } \\
\text { (UG/L) }\end{array}$ & $\begin{array}{c}\text { PCB } \\
\text { TOTAL } \\
(U G / L)\end{array}$ & $\begin{array}{c}\text { PCB } \\
\text { TOTAL } \\
\text { (UG/L) }\end{array}$ & $\begin{array}{c}\text { PCB } \\
\text { TOTAL } \\
(U G / L)\end{array}$ & $\begin{array}{l}\text { PCB } \\
\text { TOTAL } \\
\text { (UG/L) }\end{array}$ & $\begin{array}{l}\text { PCB } \\
\text { TOTAL } \\
(U G / L)\end{array}$ & $\begin{array}{l}\text { PCB } \\
\text { TOTAL } \\
\text { (UG/L) }\end{array}$ & $\begin{array}{c}\text { PCB } \\
\text { TOTAL } \\
\text { (UG/L) }\end{array}$ & $\begin{array}{c}\text { AZINON, } \\
\text { TOTAL } \\
\text { (UG/L) }\end{array}$ \\
\hline
\end{tabular}

\begin{tabular}{|c|c|c|c|c|c|c|c|c|c|c|c|}
\hline MAR 1992 & & & & & & & & & & & \\
\hline $24 \cdot 24$ & $<0.030$ & $<0.80$ & $<2$ & $<0.1$ & $<1.0$ & $<0.1$ & $<0.1$ & $<0.1$ & 0.8 & $<0.1$ & 0.50 \\
\hline \multicolumn{12}{|l|}{ SEP } \\
\hline $01-01$ & $<0.030$ & $<0.80$ & $<2$ & $<0.1$ & $<1.0$ & $<0.1$ & $<0.1$ & $<0.1$ & 0.1 & $<0.1$ & $<0.10$ \\
\hline \multicolumn{12}{|l|}{ SEP } \\
\hline $10-10$ & $<0.030$ & $<0.80$ & $<2$ & $<0.1$ & $<1.0$ & $<0.1$ & $<0.1$ & $<0.1$ & $<0.1$ & $<0.1$ & $<0.10$ \\
\hline
\end{tabular}


Table 4. Water-quality data for storm-sewer outfall stations, Fort Worth, Texas, 1992-93-Continued 08048545 - DRY BRANCH OUTFALL AT 33RD STREET, FORT WORTH, TX (WY 1993)

\begin{tabular}{|c|c|c|c|c|c|c|c|c|c|c|c|c|}
\hline & & $\begin{array}{l}\text { PRECIP - } \\
\text { ITATION } \\
\text { TOTAL }\end{array}$ & $\begin{array}{c}\text { ELAPSED } \\
\text { TIME } \\
\text { OF }\end{array}$ & $\begin{array}{l}\text { STORM } \\
\text { WATER }\end{array}$ & $\begin{array}{l}\text { SPE- } \\
\text { CIFIC } \\
\text { CON- } \\
\text { DUCT - }\end{array}$ & $\begin{array}{r}\text { SPE - } \\
\text { CIFIC } \\
\text { CON - } \\
\text { DUCT - } \\
\text { ANCE }\end{array}$ & $\begin{array}{c}\text { PH } \\
\text { WATER } \\
\text { WHOLE } \\
\text { FIELD } \\
\text { (STAND- }\end{array}$ & $\begin{array}{c}\text { PH } \\
\text { WATER } \\
\text { WHOLE } \\
\text { LAB } \\
\text { (STAND - }\end{array}$ & $\begin{array}{c}\text { TEMPER - } \\
\text { ATURE }\end{array}$ & $\begin{array}{c}\text { OXYGEN } \\
\text { DEMAND, } \\
\text { CHEM- } \\
\text { ICAL } \\
\text { (HIGH }\end{array}$ & $\begin{array}{l}\text { OXYGEN } \\
\text { DEMAND, } \\
\text { BIO- } \\
\text { CHEM- } \\
\text { ICAL, }\end{array}$ & $\begin{array}{l}\text { COLI - } \\
\text { FORM, } \\
\text { FECAL, } \\
0.7 \\
\text { UM-MF }\end{array}$ \\
\hline DATE & TIME & $\begin{array}{l}\text { INCHES / } \\
\text { STORM }\end{array}$ & $\begin{array}{c}\text { STORM } \\
\text { (HOURS) }\end{array}$ & $\begin{array}{l}\text { FLOW } \\
\text { (MGD) }\end{array}$ & $\begin{array}{l}\text { ANCE } \\
\text { (US/CM) }\end{array}$ & $\begin{array}{c}\text { LAB } \\
\text { (US/CM) }\end{array}$ & $\begin{array}{c}\text { ARD } \\
\text { UNITS) }\end{array}$ & $\begin{array}{l}\text { ARD } \\
\text { UNITS) }\end{array}$ & $\begin{array}{c}\text { WATER } \\
\text { (DEG C) }\end{array}$ & $\begin{array}{c}\text { LEVEL) } \\
(M G / L)\end{array}$ & $\begin{array}{l}5 \mathrm{DAY} \\
(\mathrm{MG} / \mathrm{L})\end{array}$ & $\begin{array}{l}\text { (COLS. } \\
100 \mathrm{ML})\end{array}$ \\
\hline
\end{tabular}

$\begin{array}{ll}\text { OCT } 1992 & \\ 15-15 & 1847 \\ \text { OCT } & \\ 28-29 & 2340 \\ \text { NOV } & \\ 19-19 & 0025 \\ \text { DEC } & \\ 09-09 & 0013\end{array}$

$\begin{array}{rrrrrrrrrrr}0.48 & 1.1 & 0.19 & 410 & 95 & 7.6 & 7.6 & 25.0 & 81 & 8.1 & 7700 \\ 0.54 & 4.2 & 0.35 & 94 & 121 & 8.6 & 7.8 & 19.0 & 89 & 5.8 & 50000 \\ 0.97 & 8.8 & 1.1 & 111 & 180 & 8.8 & 7.5 & 16.5 & 59 & 6.9 & 29000 \\ 0.31 & 1.7 & 0.26 & 110 & 181 & 6.6 & 7.9 & 9.5 & 61 & 8.5 & 1000\end{array}$

\begin{tabular}{|c|c|c|c|c|c|c|c|c|c|c|c|c|}
\hline & $\begin{array}{l}\text { STREP - } \\
\text { TOCOCCI }\end{array}$ & HARD - & $\begin{array}{l}\text { HARD - } \\
\text { NESS }\end{array}$ & $\begin{array}{l}\text { ALKA - } \\
\text { LINITY }\end{array}$ & $\begin{array}{l}\text { SOLIDS, } \\
\text { SUM OF }\end{array}$ & $\begin{array}{l}\text { RESIDUE } \\
\text { TOTAL }\end{array}$ & $\begin{array}{l}\text { SOLIDS, } \\
\text { RESIDUE }\end{array}$ & & MAGNE - & & & SODIUM \\
\hline & FECAL, & NESS & NONCARB & WAT DIS & CONSTI - & AT 105 & AT 180 & CALCIUM & SIUM, & SODIUM, & & $A D$ \\
\hline & $\begin{array}{l}\text { KF AGAR } \\
\text { ICOLS. }\end{array}$ & $\begin{array}{l}\text { TOTAL } \\
\text { (MG/L }\end{array}$ & $\begin{array}{l}\text { DISSOLV } \\
\text { FLD. AS }\end{array}$ & $\begin{array}{l}\text { FIX END } \\
\text { FIELD }\end{array}$ & $\begin{array}{c}\text { TUENTS, } \\
\text { DIS - }\end{array}$ & $\begin{array}{l}\text { DEG. C } \\
\text { SUS - }\end{array}$ & $\begin{array}{l}\text { DEG. C } \\
\text { DIS. }\end{array}$ & $\begin{array}{l}\text { DIS - } \\
\text { SOLVED }\end{array}$ & $\begin{array}{c}\text { DIS - } \\
\text { SOLVED }\end{array}$ & $\begin{array}{l}\text { DIS - } \\
\text { SOLVED }\end{array}$ & & $\begin{array}{l}\text { SORP - } \\
\text { TION }\end{array}$ \\
\hline & $\begin{array}{c}\text { PER } \\
100 \mathrm{ML})\end{array}$ & $\begin{array}{c}\mathrm{AS} \\
\mathrm{CACO} 3)\end{array}$ & $\begin{array}{c}\mathrm{CACO} 3 \\
(\mathrm{MG} / \mathrm{L})\end{array}$ & $\begin{array}{l}\mathrm{CACO} 3 \\
(\mathrm{MG} / \mathrm{L})\end{array}$ & $\begin{array}{c}\text { SOLVED } \\
\text { (MG/L) }\end{array}$ & $\begin{array}{c}\text { PENDED } \\
(M G / L)\end{array}$ & $\begin{array}{l}\text { SOLVED } \\
(M G / L)\end{array}$ & $\begin{array}{l}(M G / L \\
A S C A)\end{array}$ & $\begin{array}{l}\text { (MG/L } \\
\text { AS MG) }\end{array}$ & $\begin{array}{c}\text { (MG/L } \\
\text { AS NA) }\end{array}$ & $\begin{array}{l}\text { SODIUM } \\
\text { PERCENT }\end{array}$ & RATIO \\
\hline
\end{tabular}

$\begin{array}{ll}\text { OCT } 1992 & \\ 15 \cdot 15 & 16000 \\ \text { OCT } & \\ 28-29 & 50000 \\ \text { NOV } & \\ 19-19 & 28000 \\ \text { DEC } & \\ 09-09 & 6600\end{array}$

$\begin{array}{lllllll}29 & 9 & 20 & 68 & 343 & 74 & 11 \\ 30 & 4 & 26 & 71 & 576 & 67 & 11 \\ 44 & 5 & 39 & 84 & 209 & 69 & 16 \\ 38 & 10 & 28 & 87 & 207 & 79 & 14\end{array}$

$\begin{array}{ll}0.42 & 4.3 \\ 0.52 & 4.5 \\ 0.92 & 6.0 \\ 0.66 & 7.8\end{array}$

$\begin{array}{ll}23 & 0.3 \\ 24 & 0.4 \\ 22 & 0.4 \\ 30 & 0.6\end{array}$

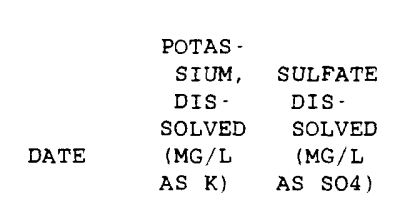

$\begin{array}{lcc}\text { CHLO- } & \text { NITRO- } & \text { NITRO- } \\ \text { RIDE, } & \text { GEN, } & \text { GEN, } \\ \text { DIS- } & \text { NITRITE } & \text { NO2+NO3 } \\ \text { SOLVED } & \text { TOTAL } & \text { TOTAL } \\ \text { (MG/L } & \text { (MG/L } & \text { (MG/L } \\ \text { AS CL) } & \text { AS N) } & \text { AS N) }\end{array}$

$\begin{array}{ccc} & \text { NITRO- } \\ \text { NITRO- } & \text { GEN, AM- } \\ \text { GEN, } & \text { MONIA + } & \text { PHOS- } \\ \text { AMMONIA } & \text { ORGANIC } & \text { PHORUS } \\ \text { TOTAL } & \text { TOTAL } & \text { TOTAL } \\ \text { (MG/L } & \text { (MG/L } & \text { (MG/L } \\ \text { AS N) } & \text { AS N) } & \text { AS P) }\end{array}$

$\begin{array}{lll}\text { OCT } 1992 & & \\ 15-15 & 2.2 & 6.3 \\ \text { OCT } & & \\ 28-29 & 1.5 & 6.6 \\ \text { NOV } & & \\ 19-19 & 2.5 & 11 \\ \text { DEC } & & \\ \text { O9-09 } & 1.8 & 12\end{array}$

$\begin{array}{llll}4.7 & 0.060 & 0.640 & 0.190 \\ 3.9 & 0.030 & 0.590 & 0.100 \\ 6.2 & 0.120 & 0.810 & 0.090 \\ 9.0 & 0.100 & 0.630 & 0.180\end{array}$

$\begin{array}{llll}0.80 & 0.180 & 0.090 & <10.0 \\ 0.90 & 0.260 & 0.070 & <10.0 \\ 0.70 & 0.160 & 0.100 & <20.0 \\ 0.60 & 0.180 & 0.080 & <10.0\end{array}$

\begin{tabular}{|c|c|}
\hline PHOS - & \\
\hline PHORUS & ANTIMONY \\
\hline DIS - & TOTAL \\
\hline SOLVED & $\mathrm{EPA}$ \\
\hline (MG / L & (UG/L \\
\hline AS P) & $A S \quad S B$ \\
\hline
\end{tabular}

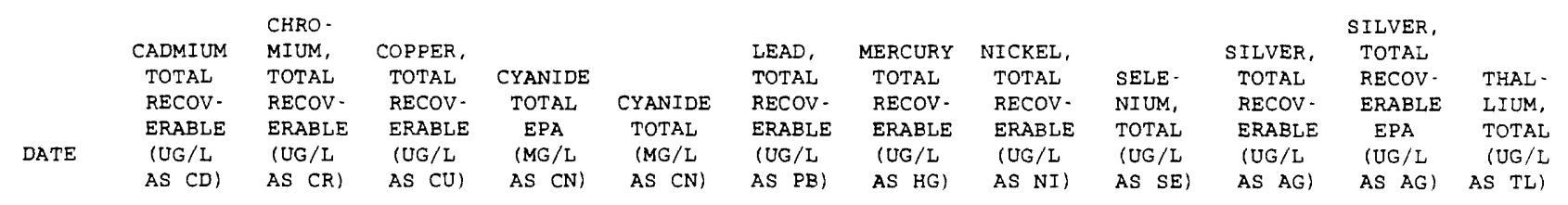

\begin{tabular}{|c|c|c|c|c|c|}
\hline \multicolumn{6}{|l|}{ OCT 1992} \\
\hline $15-15$ & 1 & 12 & 19 & $<0.010$ & 0.010 \\
\hline \multicolumn{6}{|l|}{ OCT } \\
\hline $28-29$ & 2 & 46 & 37 & $<0.010$ & 0.010 \\
\hline \multicolumn{6}{|l|}{ NOV } \\
\hline $19-19$ & 1 & 18 & 13 & $<0.010$ & $<0.010$ \\
\hline \multicolumn{6}{|l|}{$\mathrm{DEC}$} \\
\hline 09.09 & $<1$ & 16 & 24 & $<0.010$ & 0.010 \\
\hline
\end{tabular}

$\begin{array}{rrrrrrr}36 & <0.10 & 10 & <2 & <1 & <0.500 & <10 \\ 62 & <0.10 & 14 & <2 & <1 & <0.500 & <10 \\ 24 & 0.30 & 5 & <2 & <1 & <0.500 & <10 \\ 28 & 9.2 & 7 & <2 & <1 & <0.500 & <10\end{array}$


Table 4. Water-quality data for storm-sewer outfall stations, Fort worth, Texas, 1992-93-Continued

08048545 - DRY BRANCH OUTFALL AT 33RD STREET, FORT WORTH, TX (WY 1993)-Continued

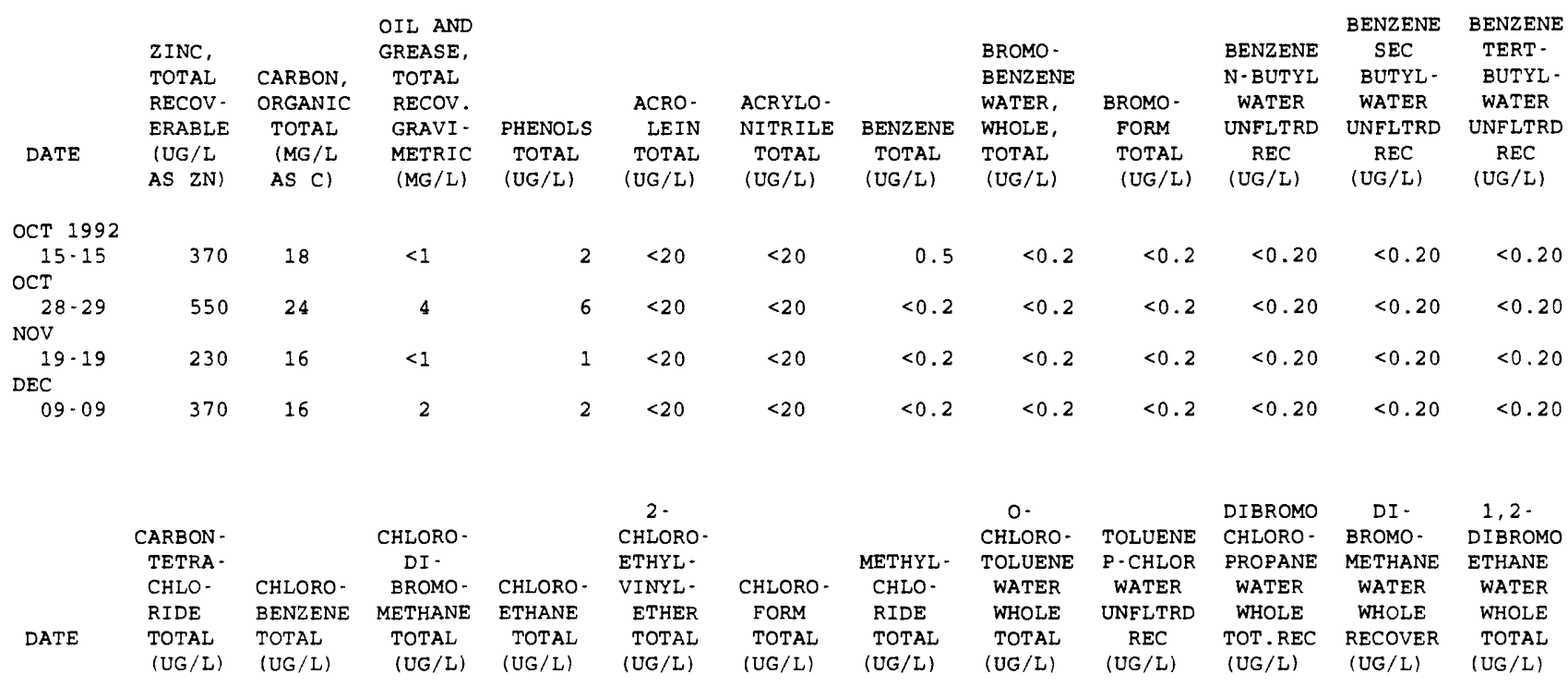

\begin{tabular}{|c|c|c|c|c|c|c|c|c|c|c|c|c|}
\hline $\begin{array}{c}\text { OCT } 1992 \\
15.15\end{array}$ & $<0.2$ & $<0.20$ & $<0.2$ & $<0.2$ & $<1.0$ & $<0.2$ & $<0.2$ & $<0.2$ & $<0.20$ & $<1.0$ & $<0.2$ & $<0.2$ \\
\hline \multicolumn{13}{|l|}{ OCT } \\
\hline $28-29$ & $<0.2$ & $<0.20$ & $<0.2$ & $<0.2$ & $<1.0$ & $<0.2$ & $<0.2$ & $<0.2$ & $<0.20$ & $<1.0$ & $<0.2$ & $<0.2$ \\
\hline \multicolumn{13}{|l|}{ Nov } \\
\hline DEC $19 \cdot 19$ & \multicolumn{11}{|c|}{$\mathrm{DEC}$} & $<0.2$ \\
\hline 09.09 & $<0.2$ & $<0.20$ & $<0.2$ & $<0.2$ & $<1.0$ & 0.3 & $<0.2$ & $<0.2$ & $<0.20$ & $<1.0$ & $<0.2$ & $<0.2$ \\
\hline
\end{tabular}

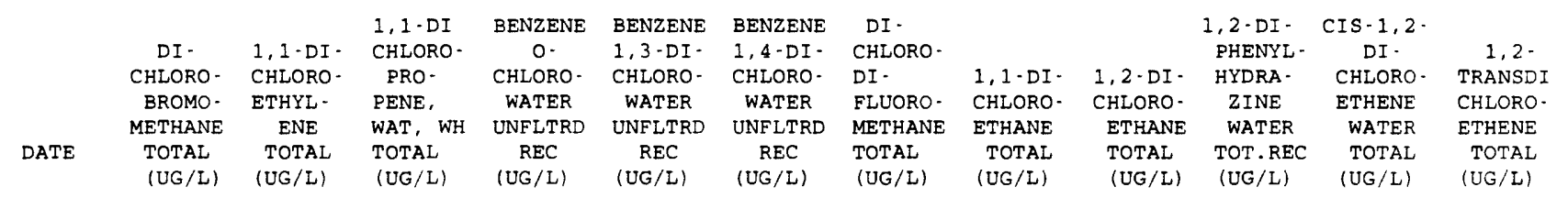

\begin{tabular}{|c|c|c|c|c|c|c|c|c|c|c|c|c|}
\hline \multicolumn{13}{|l|}{ OCT 1992} \\
\hline $15-15$ & $<0.2$ & 13 & $<0.2$ & $<5.0$ & $<5.0$ & $<5.0$ & $<0.2$ & 8.3 & $<0.2$ & $<5.0$ & 170 & 3.8 \\
\hline \multicolumn{13}{|l|}{ OCT } \\
\hline $28-29$ & $<0.2$ & 0.5 & $<0.2$ & $<5.0$ & $<5.0$ & $<5.0$ & $<0.2$ & $<0.2$ & $<0.2$ & $<5.0$ & 3.8 & $<0.2$ \\
\hline \multicolumn{13}{|l|}{ Nov } \\
\hline $19-19$ & $<0.2$ & $<0.2$ & $<0.2$ & $<5.0$ & $<5.0$ & $<5.0$ & $<0.2$ & $<0.2$ & $<0.2$ & $<5.0$ & 1.0 & $<0.2$ \\
\hline \multicolumn{13}{|l|}{ DEC } \\
\hline 09.09 & $<0.2$ & 1.3 & $<0.2$ & $<5.0$ & $<5.0$ & $<5.0$ & $<0.2$ & 0.5 & $<0.2$ & $<5.0$ & 12 & 0.9 \\
\hline
\end{tabular}

\begin{tabular}{|c|c|c|c|c|c|c|c|c|c|c|c|c|}
\hline & $1,2-D I$ & $\begin{array}{l}1,3-D I- \\
\text { CHLORO - }\end{array}$ & $\begin{array}{l}2,2 \text {-DI } \\
\text { CHLORO- } \\
\text { PRO- }\end{array}$ & $\begin{array}{c}\text { CIS } \\
1,3-\mathrm{DI} \text { - }\end{array}$ & $\begin{array}{l}\text { TRANS - } \\
1,3-\mathrm{DI} \text { - }\end{array}$ & & $\begin{array}{l}\text { HEXA - } \\
\text { CHLORO- }\end{array}$ & $\begin{array}{c}\text { ISO- } \\
\text { PROPYL- } \\
\text { BENZENE }\end{array}$ & $\begin{array}{l}\text { P-ISO- } \\
\text { PROPYL- } \\
\text { TOLUENE }\end{array}$ & & $\begin{array}{c}\text { METHYL - } \\
\text { ENE }\end{array}$ & \\
\hline DATE & $\begin{array}{l}\text { CHLORO- } \\
\text { PROPANE } \\
\text { TOTAL } \\
\text { (UG/L) }\end{array}$ & $\begin{array}{c}\text { PROPANE } \\
\text { WAT. WH } \\
\text { TOTAL } \\
\text { (UG/L) }\end{array}$ & $\begin{array}{l}\text { PANE } \\
\text { WAT, WH } \\
\text { TOTAL } \\
\text { (UG/L) }\end{array}$ & $\begin{array}{l}\text { CHLORO- } \\
\text { PROPENE } \\
\text { TOTAL } \\
\text { (UG } / L \text { ) }\end{array}$ & $\begin{array}{c}\text { CHLORO- } \\
\text { PROPENE } \\
\text { TOTAL } \\
\text { (UG/L) }\end{array}$ & $\begin{array}{l}\text { ETHYL - } \\
\text { BENZENE } \\
\text { TOTAL } \\
\text { (UG/L) }\end{array}$ & $\begin{array}{c}\text { BUT - } \\
\text { ADIENE } \\
\text { TOTAL } \\
\text { (UG/L) }\end{array}$ & $\begin{array}{l}\text { WATER } \\
\text { WHOLE } \\
\text { REC } \\
\text { (UG/L) }\end{array}$ & $\begin{array}{l}\text { WATER } \\
\text { WHOLE } \\
\text { REC } \\
\text { (UG/L) }\end{array}$ & $\begin{array}{c}\text { METHYL - } \\
\text { BROMIDE } \\
\text { TOTAL } \\
\text { (UG/L) }\end{array}$ & $\begin{array}{l}\text { CHLO- } \\
\text { RIDE } \\
\text { TOTAL } \\
\text { (UG/L) }\end{array}$ & $\begin{array}{c}\text { NAPHTH - } \\
\text { ALENE } \\
\text { TOTAL } \\
\text { (UG/L) }\end{array}$ \\
\hline
\end{tabular}

\begin{tabular}{|c|c|c|c|c|c|c|c|c|c|c|c|c|}
\hline \multicolumn{13}{|l|}{ OCT 1992} \\
\hline $15 \cdot 15$ & $<0.2$ & $<0.2$ & $<0.2$ & $<0.2$ & $<0.2$ & $<0.2$ & $<5.0$ & $<0.20$ & $<0.20$ & $<0.2$ & $<0.2$ & $<5.0$ \\
\hline \multicolumn{13}{|l|}{ OCT } \\
\hline $28 \cdot 29$ & $<0.2$ & $<0.2$ & $<0.2$ & $<0.2$ & $<0.2$ & $<0.2$ & $<5.0$ & $<0.20$ & $<0.20$ & $<0.2$ & $<0.3$ & $<5.0$ \\
\hline \multicolumn{13}{|l|}{ NoV } \\
\hline $19 \cdot 19$ & $<0.2$ & $<0.2$ & $<0.2$ & $<0.2$ & $<0.2$ & $<0.2$ & $<5.0$ & $<0.20$ & $<0.20$ & $<0.2$ & 0.2 & $<5.0$ \\
\hline \multicolumn{13}{|l|}{ DEC } \\
\hline 09.09 & $<0.2$ & $<0.2$ & $<0.2$ & $<0.2$ & $<0.2$ & $<0.2$ & $<5.0$ & $<0.20$ & $<0.20$ & $<0.2$ & $<0.2$ & $<5.0$ \\
\hline
\end{tabular}


Table 4. Water-quality data for storm-sewer outfall stations, Fort Worth, Texas, 1992-93-Continued

08048545 - DRY BRANCH OUTFALL AT 33RD STREET, FORT WORTH, TX (WY 1993)-Continued

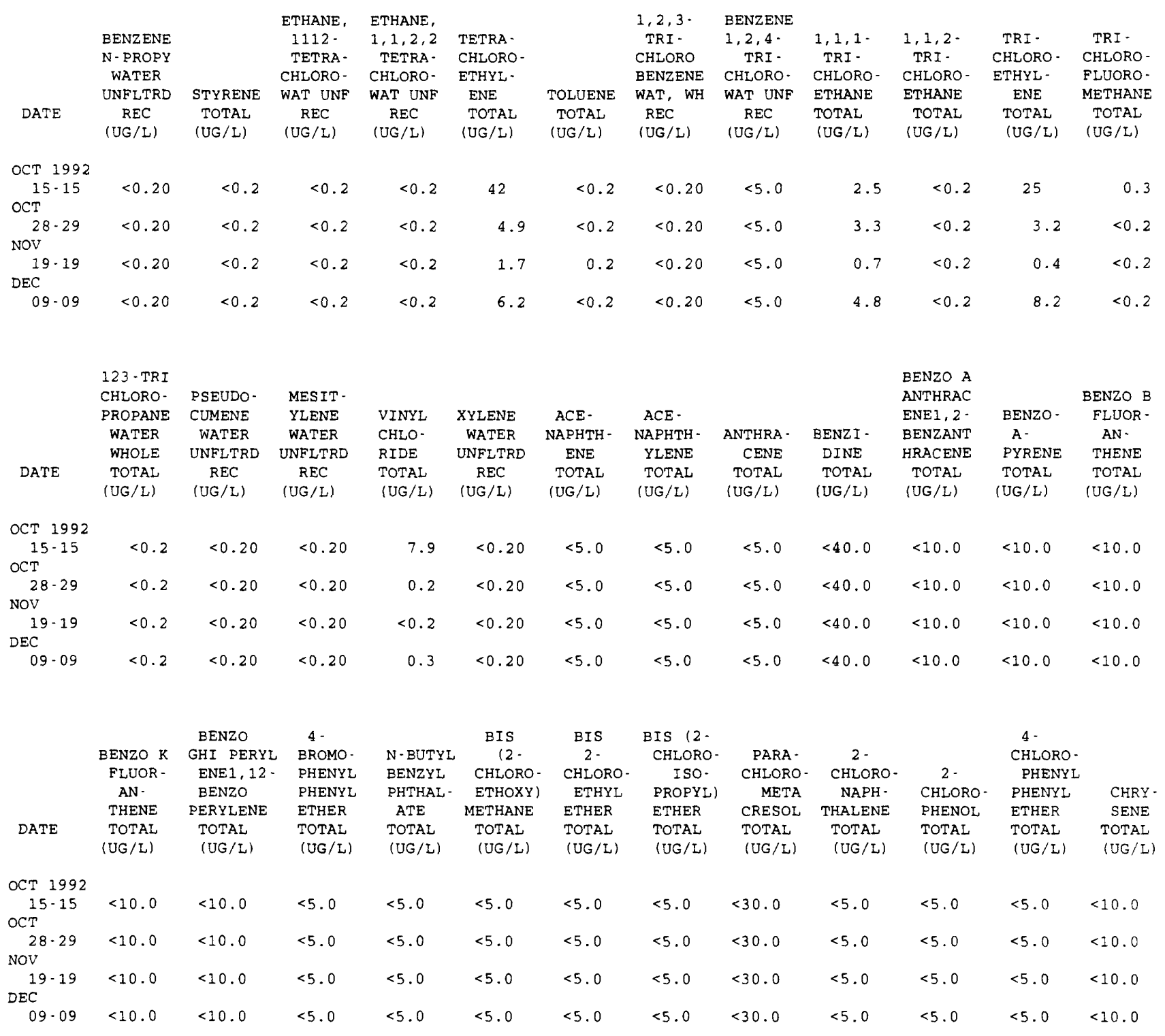

\begin{tabular}{|c|c|c|c|c|c|c|c|c|c|c|c|c|}
\hline DATE & $\begin{array}{l}1,2,5,6- \\
\text { DIBENZ- } \\
\text { ANTHRA - } \\
\text { CENE } \\
\text { TOTAL } \\
\text { (UG/L) }\end{array}$ & $\begin{array}{c}3,3^{\prime}- \\
\text { DI. } \\
\text { CHLORO- } \\
\text { BENZI- } \\
\text { DINE } \\
\text { TOTAL } \\
\text { (UG/L) }\end{array}$ & $\begin{array}{l}2,4 \text {-DI- } \\
\text { CHLORO- } \\
\text { PHENOL } \\
\text { TOTAL } \\
\text { (UG/L) }\end{array}$ & $\begin{array}{l}\text { DIETHYL } \\
\text { PHTHAL- } \\
\text { ATE } \\
\text { TOTAL } \\
\text { (UG/L) }\end{array}$ & $\begin{array}{l}\text { DI- } \\
\text { METHYL } \\
\text { PHTHAL- } \\
\text { ATE } \\
\text { TOTAL } \\
\text { (UG/L) }\end{array}$ & $\begin{array}{c}2,4-D I- \\
\text { METHYL- } \\
\text { PHENOL } \\
\text { TOTAL } \\
\text { (UG/L) }\end{array}$ & $\begin{array}{l}\text { DI - N - } \\
\text { BUTYL } \\
\text { PHTHAL- } \\
\text { ATE } \\
\text { TOTAL } \\
(U G / L)\end{array}$ & $\begin{array}{c}4,6- \\
\text { DINITRO- } \\
\text { ORTHO- } \\
\text { CRESOL } \\
\text { TOTAL } \\
\text { (UG/L) }\end{array}$ & $\begin{array}{c}2,4,- \\
\text { DI - } \\
\text { NITRO- } \\
\text { PHENOL } \\
\text { TOTAL } \\
\text { (UG/L) }\end{array}$ & $\begin{array}{c}2,4-D I- \\
\text { NITRO- } \\
\text { TOLUENE } \\
\text { TOTAL } \\
\text { (UG/L) }\end{array}$ & $\begin{array}{c}2,6-\mathrm{DI}- \\
\text { NITRO- } \\
\text { TOLUENE } \\
\text { TOTAL } \\
\text { (UG/L) }\end{array}$ & $\begin{array}{c}\text { DI-N- } \\
\text { OCTYL } \\
\text { PHTHAL- } \\
\text { ATE } \\
\text { TOTAL } \\
(\text { UG/L) }\end{array}$ \\
\hline CT 19 & & & & & & & & & & & & \\
\hline${ }_{C T}^{15-15}$ & $<10.0$ & $<20.0$ & $<5.0$ & $<5.0$ & $<5.0$ & $<5.0$ & $<5.0$ & $<30.0$ & $<20.0$ & $<5.0$ & $<5.0$ & $<10.0$ \\
\hline $\begin{array}{l}28-29 \\
\text { NoV }\end{array}$ & $<10.0$ & $<20.0$ & $<5.0$ & $<5.0$ & $<5.0$ & $<5.0$ & $<5.0$ & $<30.0$ & $<20.0$ & $<5.0$ & $<5.0$ & $<10.0$ \\
\hline${ }_{\text {DEC }}^{19-19}$ & $<10.0$ & $<20.0$ & $<5.0$ & $<5.0$ & $<5.0$ & $<5.0$ & $<5.0$ & $<30.0$ & $<20.0$ & $<5.0$ & $<5.0$ & $<10.0$ \\
\hline $09-09$ & $<10.0$ & $<20.0$ & $<5.0$ & $<5.0$ & $<5.0$ & $<5.0$ & $<5.0$ & $<30.0$ & $<20.0$ & $<5.0$ & $<5.0$ & $<10.0$ \\
\hline
\end{tabular}


Table 4. Water-quality data for storm-sewer outfall stations, Fort worth, Texas, 1992-93-Continued

08048545 - DRY BRANCH OUTFALL AT 33RD STREET, FORT WORTH, TX (WY 1993)-Continued

\begin{tabular}{|c|c|c|c|c|c|c|c|c|c|c|c|c|}
\hline DATE & $\begin{array}{c}\text { BIS }(2- \\
\text { ETHYL } \\
\text { HEXYL) } \\
\text { PHTHAL- } \\
\text { ATE } \\
\text { TOTAL } \\
\text { (UG/L) }\end{array}$ & $\begin{array}{l}\text { FLUOR - } \\
\text { ANTHENE } \\
\text { TOTAL } \\
\text { (UG/L) }\end{array}$ & $\begin{array}{l}\text { FLUOR- } \\
\text { ENE } \\
\text { TOTAL } \\
\text { (UG/L) }\end{array}$ & $\begin{array}{l}\text { HEXA- } \\
\text { CHLORO- } \\
\text { BENZENE } \\
\text { TOTAL } \\
\text { (UG/L) }\end{array}$ & $\begin{array}{l}\text { HEXA- } \\
\text { CHLORO- } \\
\text { CYCLO- } \\
\text { PENT- } \\
\text { ADIENE } \\
\text { TOTAL } \\
\text { (UG/L) }\end{array}$ & $\begin{array}{l}\text { HEXA- } \\
\text { CHLORO- } \\
\text { ETHANE } \\
\text { TOTAL } \\
\text { (UG/L) }\end{array}$ & $\begin{array}{c}\text { INDENO } \\
(1,2,3- \\
\text { CD) } \\
\text { PYRENE } \\
\text { TOTAL } \\
\text { (UG/L) }\end{array}$ & $\begin{array}{l}\text { ISO- } \\
\text { PHORONE } \\
\text { TOTAL } \\
\text { (UG/L) }\end{array}$ & $\begin{array}{l}\text { NITRO- } \\
\text { BENZENE } \\
\text { TOTAL } \\
\text { (UG/L) }\end{array}$ & $\begin{array}{c}\text { N-NITRO- } \\
\text { SODI- } \\
\text { METHY - } \\
\text { LAMINE } \\
\text { TOTAL } \\
\text { (UG/L) }\end{array}$ & $\begin{array}{l}2- \\
\text { NITRO- } \\
\text { PHENOL } \\
\text { TOTAI } \\
\text { (UG/L) }\end{array}$ & $\begin{array}{c}4- \\
\text { NITRO- } \\
\text { PHENOL } \\
\text { TOTAL } \\
\text { (UG/L) }\end{array}$ \\
\hline $\begin{array}{l}\text { OCT } 1992 \\
15 \cdot 15 \\
\text { OCT }\end{array}$ & 9.0 & $<5.0$ & $<5.0$ & $<5.0$ & $<5.0$ & $<5.0$ & $<10.0$ & $<5.0$ & $<5.0$ & $<5.0$ & $<5.0$ & $<30.0$ \\
\hline $\begin{array}{l}28-29 \\
\text { NoV }\end{array}$ & 10.0 & $<5.0$ & $<5.0$ & $<5.0$ & $<5.0$ & $<5.0$ & $<10.0$ & $<5.0$ & $<5.0$ & $<5.0$ & $<5.0$ & $<30.0$ \\
\hline $\begin{array}{l}19.19 \\
\text { DEC } \\
09.09\end{array}$ & 8.0 & $\begin{array}{l}<5.0 \\
<5.0\end{array}$ & $\begin{array}{l}<5.0 \\
<5.0\end{array}$ & $\begin{array}{l}<5.0 \\
<5.0\end{array}$ & $<5.0$ & $\begin{array}{l}<5.0 \\
<5.0\end{array}$ & $\begin{array}{l}<10.0 \\
<10.0\end{array}$ & $\begin{array}{l}<5.0 \\
<5.0\end{array}$ & $\begin{array}{l}<5.0 \\
<5.0\end{array}$ & $\begin{array}{l}<5.0 \\
<5.0\end{array}$ & $<5.0$ & $\begin{array}{l}<30.0 \\
<30.0\end{array}$ \\
\hline DATE & $\begin{array}{l}\text { N- } \\
\text { NITRO- } \\
\text { SODI - N- } \\
\text { PROPYL- } \\
\text { AMINE } \\
\text { TOTAL } \\
\text { (UG/L) }\end{array}$ & $\begin{array}{c}\text { N-NITRO- } \\
\text { SODI - } \\
\text { PHENY- } \\
\text { LAMINE } \\
\text { TOTAL } \\
\text { (UG/L) }\end{array}$ & $\begin{array}{l}\text { PENTA- } \\
\text { CHLORO- } \\
\text { PHENOL } \\
\text { TOTAL } \\
\text { (UG/L) }\end{array}$ & $\begin{array}{l}\text { PHENAN- } \\
\text { THRENE } \\
\text { TOTAL } \\
\text { (UG/L) }\end{array}$ & $\begin{array}{l}\text { PHENOL } \\
\text { (C6H - } \\
5 O H) \\
\text { TOTAL } \\
\text { (UG/L) }\end{array}$ & $\begin{array}{l}\text { PYRENE } \\
\text { TOTAI } \\
\text { (UG/L) }\end{array}$ & $\begin{array}{c}2,4,6- \\
\text { TRI - } \\
\text { CHLORO- } \\
\text { PHENOL } \\
\text { TOTAL } \\
\text { (UG/L) }\end{array}$ & $\begin{array}{l}\text { ALDRIN, } \\
\text { TOTAL } \\
\text { (UG/L) }\end{array}$ & $\begin{array}{l}\text { P, P' } \\
\text { DDT, } \\
\text { TOTAL } \\
\text { (UG/L) }\end{array}$ & $\begin{array}{l}\text { ALPHA } \\
\text { BHC } \\
\text { TOTAL } \\
\text { (UG/L) }\end{array}$ & $\begin{array}{c}\text { BETA } \\
\text { BENZENE } \\
\text { HEXA- } \\
\text { CHLOR- } \\
\text { IDE } \\
\text { TOTAL } \\
\text { (UG/L) }\end{array}$ & $\begin{array}{c}\text { DELTA } \\
\text { BENZENE } \\
\text { HEXA- } \\
\text { CHLOR- } \\
\text { IDE } \\
\text { TOTAL } \\
\text { (UG/L) }\end{array}$ \\
\hline $\begin{array}{c}\text { OCT } 1992 \\
15-15\end{array}$ & $<5.0$ & $<5.0$ & $<30.0$ & $<5.0$ & $<5.0$ & $<5.0$ & $<20.0$ & $<0.040$ & $<0.10$ & $<0.03$ & $<0.03$ & $<0.09$ \\
\hline $\begin{array}{l}\text { OCT } \\
28 \cdot 29 \\
\text { NOV }\end{array}$ & $<5.0$ & $<5.0$ & $<30.0$ & $<5.0$ & $<5.0$ & $<5.0$ & $<20.0$ & $<0.040$ & $<0.10$ & $<0.03$ & $<0.03$ & $<0.09$ \\
\hline $\mathrm{DEC}^{19 \cdot 19}$ & $<5.0$ & $<5.0$ & $<30.0$ & $<5.0$ & $<5.0$ & $<5.0$ & $<20.0$ & $<0.040$ & $<0.10$ & $<0.03$ & $<0.03$ & $<0.09$ \\
\hline 09.09 & $<5.0$ & $<5.0$ & $<30.0$ & $<5.0$ & $<5.0$ & $<5.0$ & $<20.0$ & $<0.040$ & $<0.10$ & $<0.03$ & $<0.03$ & $<0.09$ \\
\hline & & $\begin{array}{l}\text { CHLOR- } \\
\text { DANE }\end{array}$ & $\begin{array}{l}\text { CHLOR- } \\
\text { DANE }\end{array}$ & & & & & $\begin{array}{l}\text { ENDO- } \\
\text { SULFAN. }\end{array}$ & & & & \\
\hline & LINDANE & $\begin{array}{l}\text { CIS } \\
\text { WATER } \\
\text { WHOLE }\end{array}$ & $\begin{array}{l}\text { TRANS } \\
\text { WATER } \\
\text { WHOLE }\end{array}$ & $\begin{array}{l}\text { CHLOR- } \\
\text { DANE }\end{array}$ & $\begin{array}{l}\text { P, P' } \\
\text { DDD }\end{array}$ & $\begin{array}{l}P, P^{\prime} \\
D D E\end{array}$ & $\begin{array}{c}\text { DI- } \\
\text { ELDRIN }\end{array}$ & $\begin{array}{c}\text { I } \\
\text { WATER } \\
\text { WHOLE }\end{array}$ & $\begin{array}{l}\text { ENDO- } \\
\text { SULFAN } \\
\text { BETA }\end{array}$ & $\begin{array}{l}\text { ENDO- } \\
\text { SULFAN } \\
\text { SULFATE }\end{array}$ & $\begin{array}{l}\text { ENDRIN } \\
\text { WATER } \\
\text { UNFLTRD }\end{array}$ & $\begin{array}{c}\text { ENDRIN } \\
\text { ALDE- } \\
\text { HYDE }\end{array}$ \\
\hline DATE & $\begin{array}{l}\text { TOTAL } \\
\text { (UG/L) }\end{array}$ & $\begin{array}{l}\text { TOTAL } \\
\text { (UG/L) }\end{array}$ & $\begin{array}{l}\text { TOTAL } \\
\text { (UG/L) }\end{array}$ & $\begin{array}{l}\text { TOTAL } \\
\text { (UG/L) }\end{array}$ & $\begin{array}{l}\text { TOTAL } \\
\text { (UG/L) }\end{array}$ & $\begin{array}{l}\text { TOTAL } \\
\text { (UG/L) }\end{array}$ & $\begin{array}{l}\text { TOTAL } \\
\text { (UG/L) }\end{array}$ & $\begin{array}{l}\text { REC } \\
(U G / L)\end{array}$ & $\begin{array}{l}\text { TOTAL } \\
\text { (UG/L) }\end{array}$ & $\begin{array}{l}\text { TOTAL } \\
\text { (UG/L) }\end{array}$ & $\begin{array}{c}\text { REC } \\
(U G / L)\end{array}$ & $\begin{array}{l}\text { TOTAL } \\
\text { (UG/L) }\end{array}$ \\
\hline $\begin{array}{c}\text { OCT } 1992 \\
15.15\end{array}$ & $<0.030$ & $<0.10$ & $<0.10$ & $<0.1$ & $<0.10$ & $<0.04$ & $<0.020$ & $<0.10$ & $<0.04$ & $<0.60$ & $<0.060$ & $<0.20$ \\
\hline $\begin{array}{l}\text { OCT } \\
\quad 28-29 \\
\text { NoV }\end{array}$ & $<0.030$ & $<0.10$ & $<0.10$ & $<0.1$ & $<0.10$ & $<0.04$ & $<0.020$ & $<0.10$ & $<0.04$ & $<0.60$ & $<0.060$ & $<0.20$ \\
\hline DEC & $<0.030$ & $<0.10$ & $<0.10$ & $<0.1$ & $<0.10$ & $<0.04$ & $<0.020$ & $<0.10$ & $<0.04$ & $<0.60$ & $<0.060$ & $<0.20$ \\
\hline 09.09 & $<0.030$ & $<0.10$ & $<0.10$ & $<0.1$ & $<0.10$ & $<0.04$ & $<0.020$ & $<0.10$ & $<0.04$ & $<0.60$ & $<0.060$ & $<0.20$ \\
\hline
\end{tabular}

\begin{tabular}{|c|c|c|c|c|c|c|c|c|c|c|c|}
\hline & & HEPTA - & & AROCLOR & AROCLOR & AROCLOR & AROCLOR & AROCLOR & AROCLOR & AROCLOR & \\
\hline & HEPTA - & CHLOR & TOX - & 1016 & 1221 & 1232 & 1242 & 1248 & 1254 & 1260 & DI - \\
\hline DATE & $\begin{array}{l}\text { CHLOR, } \\
\text { TOTAL } \\
\text { (UG/L) }\end{array}$ & $\begin{array}{c}\text { EPOXIDE } \\
\text { TOTAL } \\
\text { (UG/L) }\end{array}$ & $\begin{array}{c}\text { APHENE, } \\
\text { TOTAL } \\
\text { (UG/L) }\end{array}$ & $\begin{array}{c}\text { PCB } \\
\text { TOTAL } \\
\text { (UG/L) }\end{array}$ & $\begin{array}{c}\text { PCB } \\
\text { TOTAL } \\
\text { (UG/L) }\end{array}$ & $\begin{array}{c}\text { PCB } \\
\text { TOTAL } \\
\text { (UG/L) }\end{array}$ & $\begin{array}{c}\text { PCB } \\
\text { TOTAL } \\
\text { (UG/L) }\end{array}$ & $\begin{array}{l}\text { PCB } \\
\text { TOTAL } \\
\text { (UG/L) }\end{array}$ & $\begin{array}{l}\text { PCB } \\
\text { TOTAL } \\
\text { (UG/L) }\end{array}$ & $\begin{array}{l}\text { PCB } \\
\text { TOTAL } \\
\text { (UG/L) }\end{array}$ & $\begin{array}{c}\text { AZINON, } \\
\text { TOTAL } \\
\text { (UG/L) }\end{array}$ \\
\hline 1992 & & & & & & & & & & & \\
\hline $5 \cdot 15$ & $<0.030$ & $<0.80$ & $<2$ & $<0.1$ & $<1.0$ & $<0.1$ & $<0.1$ & $<0.1$ & 0.1 & $<0.1$ & $<0.10$ \\
\hline $28-29$ & $<0.030$ & $<0.80$ & $<2$ & $<0.1$ & $<1.0$ & $<0.1$ & $<0.1$ & $<0.1$ & 0.1 & $<0.1$ & $<0.10$ \\
\hline $19 \cdot 19$ & $<0.030$ & $<0.80$ & $<2$ & $<0.1$ & $<1.0$ & $<0.1$ & $<0.1$ & $<0.1$ & $<0.1$ & $<0.1$ & $<0.10$ \\
\hline $09-09$ & $<0.030$ & $<0.80$ & $<2$ & $<0.1$ & $<1.0$ & $<0.1$ & $<0.1$ & $<0.1$ & $<0.1$ & $<0.1$ & $<0.10$ \\
\hline
\end{tabular}


Table 4. Water-quality data for storm-sewer outfall stations, Fort worth, Texas, 1992-93-Continued 08048700 - EASTERN HILLS HIGH SCHOOL OUTFALL AT WEILER DRIVE, FORT WORTH, TX (WY 1992)

\begin{tabular}{|c|c|c|c|c|c|c|c|c|c|c|c|c|}
\hline & & $\begin{array}{l}\text { PRECIP- } \\
\text { ITATION } \\
\text { TOTAL }\end{array}$ & $\begin{array}{c}\text { ELAPSED } \\
\text { TIME } \\
\text { OF }\end{array}$ & $\begin{array}{l}\text { STORM } \\
\text { WATER }\end{array}$ & $\begin{array}{l}\text { SPE- } \\
\text { CIFIC } \\
\text { CON- } \\
\text { DUCT- }\end{array}$ & $\begin{array}{l}\text { SPE- } \\
\text { CIFIC } \\
\text { CON- } \\
\text { DUCT- } \\
\text { ANCE }\end{array}$ & $\begin{array}{c}\text { PH } \\
\text { WATER } \\
\text { WHOLE } \\
\text { FIELD } \\
\text { (STAND- }\end{array}$ & $\begin{array}{l}\text { PH } \\
\text { WATER } \\
\text { WHOLE } \\
\text { LAB } \\
\text { (STAND- }\end{array}$ & $\begin{array}{l}\text { TEMPER - } \\
\text { ATURE }\end{array}$ & $\begin{array}{l}\text { OXYGEN } \\
\text { DEMAND, } \\
\text { CHEM- } \\
\text { ICAL } \\
\text { (HIGH }\end{array}$ & $\begin{array}{l}\text { OXYGEN } \\
\text { DEMAND, } \\
\text { BIO- } \\
\text { CHEM- } \\
\text { ICAL }\end{array}$ & $\begin{array}{l}\text { COLI- } \\
\text { FORM, } \\
\text { FECAL, } \\
0.7 \\
\text { UM-MF }\end{array}$ \\
\hline DATE & TIME & $\begin{array}{l}\text { INCHES / } \\
\text { STORM }\end{array}$ & $\begin{array}{c}\text { STORM } \\
\text { (HOURS) }\end{array}$ & $\begin{array}{l}\text { FLOW } \\
\text { (MGD) }\end{array}$ & $\begin{array}{c}\text { ANCE } \\
\text { (US /CM) }\end{array}$ & $\begin{array}{c}\text { LAB } \\
\text { (US /CM) }\end{array}$ & $\begin{array}{l}\text { ARD } \\
\text { UNITS) }\end{array}$ & $\begin{array}{c}\text { ARD } \\
\text { UNITS) }\end{array}$ & $\begin{array}{l}\text { WATER } \\
\text { (DEG C) }\end{array}$ & $\begin{array}{l}\text { LEVEL) } \\
(M G / L)\end{array}$ & $\begin{array}{l}5 \text { DAY } \\
(M G / L)\end{array}$ & $\begin{array}{l}\text { (COLS.) } \\
100 \mathrm{ML} \text { ) }\end{array}$ \\
\hline
\end{tabular}

\begin{tabular}{|c|c|c|c|c|c|c|c|c|c|c|c|c|}
\hline \multicolumn{13}{|l|}{ APR 1992} \\
\hline $17-17$ & 0526 & 0.39 & 3.6 & 0.34 & 262 & 236 & 7.6 & 7.8 & 17.5 & 72 & 8.1 & $>100000$ \\
\hline \multicolumn{13}{|l|}{ MAY } \\
\hline $14 \cdot 14$ & 1039 & 0.70 & 2.8 & 0.31 & 202 & 187 & 7.8 & 7.7 & 24.5 & 93 & 8.0 & $\mathrm{~K} 500000$ \\
\hline \multicolumn{13}{|l|}{ SEP } \\
\hline \multirow[t]{6}{*}{$21 \cdot 21$} & 0106 & 0.83 & 2.9 & 0.48 & 158 & 206 & 8.1 & 7.6 & 23.0 & 72 & 7.1 & $\mathrm{~K} 20000$ \\
\hline & STREP - & & HARD - & ALKA - & SOLIDS, & RESIDUE & SOLIDS, & & & & & \\
\hline & TOCOCCI & HARD - & NESS & LINITY & SUM OF & TOTAL & RESIDUE & & MAGNE - & & & SODIUM \\
\hline & FECAL, & NESS & NONCARB & WAT DIS & CONSTI - & AT 105 & AT 180 & CALCIUM & SIUM, & SODIUM, & & $A D$ \\
\hline & KF AGAR & TOTAL & DISSOLV & FIX END & TUEN'T, & DEG. $C$, & DEG. C & DIS - & DIS - & DIS - & & SORP - \\
\hline & 1COLS. & (MG / L & FLD. AS & FIELD & DIS - & SUS- & DIS - & SOLVED & SOLVED & SOLVED & & TION \\
\hline \multirow[t]{2}{*}{ DATE } & PER & AS & $\mathrm{CACO} 3$ & $\mathrm{CAC} 03$ & SOLVED & PENDED & SOLVED & (MG / L & $(M G / L$ & (MG / L & SODIUM & RATIO \\
\hline & $100 \mathrm{ML})$ & $(\mathrm{CACO} 3)$ & $(M G / L)$ & $(M G / L)$ & $(M G / L)$ & $(M G / L)$ & $(M G / L)$ & AS (A) & AS MG) & AS NA) & PERCENT & \\
\hline
\end{tabular}

\begin{tabular}{|c|c|c|c|c|c|c|c|c|c|c|c|c|}
\hline \multicolumn{13}{|l|}{ APR 1992} \\
\hline $17 \cdot 17$ & $>97000$ & 69 & 25 & 44 & 115 & 190 & 111 & 22 & 3.4 & 8.1 & 20 & 0.4 \\
\hline \multicolumn{13}{|l|}{ MAY } \\
\hline $14-14$ & $\mathrm{~K} 420000$ & 54 & 20 & 34 & 87 & 203 & 72 & 19 & 1.5 & 3.4 & 11 & 0.2 \\
\hline \multicolumn{13}{|l|}{ SEP } \\
\hline $21-21$ & K20000 & 40 & 14 & 26 & 90 & 485 & 68 & 13 & 1.8 & 4.9 & 20 & 0.3 \\
\hline
\end{tabular}

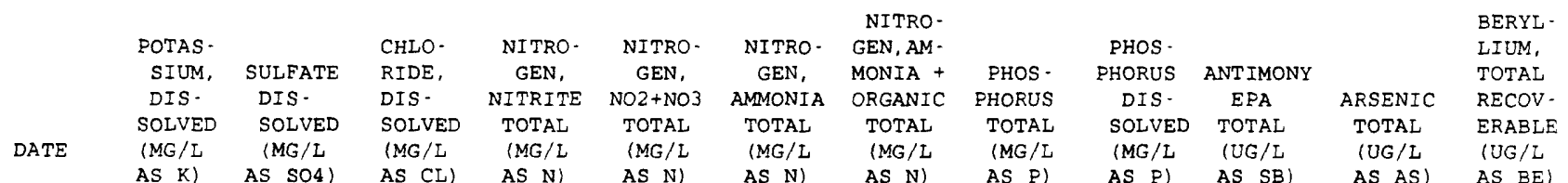

\begin{tabular}{|c|c|c|c|c|c|c|c|c|c|c|c|c|}
\hline \multicolumn{13}{|l|}{ APR 1992} \\
\hline $17-17$ & 2.7 & 27 & 9.6 & 0.040 & 0.620 & 0.270 & 1.2 & 0.230 & 0.110 & $<10.0$ & 1 & $<10$ \\
\hline \multicolumn{13}{|l|}{ MAY } \\
\hline $14-14$ & 3.1 & 13 & 4.7 & 0.070 & 0.990 & 0.390 & 1.3 & 0.170 & 0.130 & $<10.0$ & 2 & $<10$ \\
\hline \multicolumn{13}{|l|}{ SEP } \\
\hline $21-21$ & 2.3 & 15 & 6.7 & 0.030 & 0.420 & 0.190 & 0.70 & 0.190 & 0.180 & $<10.0$ & 2 & $<10$ \\
\hline
\end{tabular}

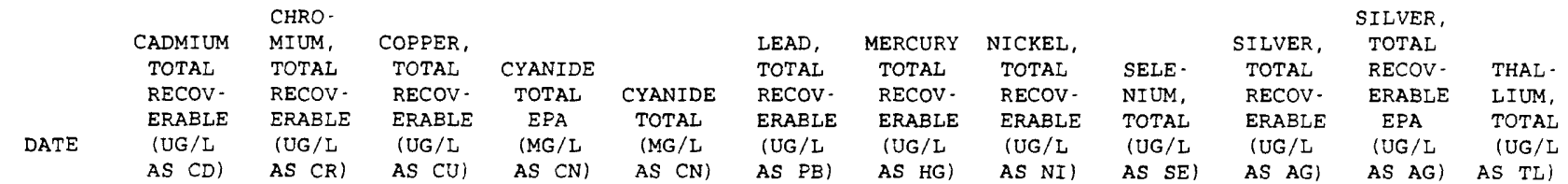

\begin{tabular}{|c|c|c|c|c|c|c|c|c|c|c|c|c|}
\hline \multicolumn{13}{|l|}{ APR 1992} \\
\hline $17 \cdot 17$ & $<1$ & 7 & 6 & $<0.010$ & $<0.010$ & 31 & $<0.10$ & 5 & $<2$ & $<1$ & $<1.00$ & $<1000$ \\
\hline \multicolumn{13}{|l|}{ MAY } \\
\hline $14 \cdot 14$ & $<1$ & 5 & 13 & $<10.0$ & $<0.010$ & 40 & $<0.10$ & 6 & $<2$ & $<1$ & $<1.00$ & $<10$ \\
\hline \multicolumn{13}{|l|}{ SEP } \\
\hline $21-21$ & $<1$ & 11 & 7 & $<0.010$ & $<0.010$ & 12 & $<0.10$ & 5 & $<2$ & $<1$ & $<1.00$ & $<10$ \\
\hline
\end{tabular}


Table 4. Water-quality data for storm-sewer outfall stations, Fort Worth, Texas, 1992-93-Continued 08048700 - EASTERN HILLS HIGH SCHOOL OUTFALL AT WEILER DRIVE, FORT WORTH, TX (WY 1992)-ContinUed

\begin{tabular}{|c|c|c|c|c|c|c|c|c|c|c|c|c|}
\hline DATE & $\begin{array}{l}\text { ZINC, } \\
\text { TOTAL } \\
\text { RECOV- } \\
\text { ERABLE } \\
\text { (UG/L } \\
\text { AS ZN) }\end{array}$ & $\begin{array}{c}\text { CARBON, } \\
\text { ORGANIC } \\
\text { TOTAL } \\
\text { (MG/L } \\
\text { AS C) }\end{array}$ & $\begin{array}{l}\text { OIL AND } \\
\text { GREASE, } \\
\text { TOTAL } \\
\text { RECOV. } \\
\text { GRAVI - } \\
\text { METRIC } \\
\text { (MG/L) }\end{array}$ & $\begin{array}{l}\text { PHENOLS } \\
\text { TOTAL } \\
\text { (UG/L) }\end{array}$ & $\begin{array}{l}\text { ACRO- } \\
\text { LEIN } \\
\text { TOTAL } \\
\text { (UG/L) }\end{array}$ & $\begin{array}{c}\text { ACRYLO- } \\
\text { NITRILE } \\
\text { TOTAL } \\
\text { (UG/L) }\end{array}$ & $\begin{array}{c}\text { BENZENE } \\
\text { TOTAL } \\
\text { (UG/L) }\end{array}$ & $\begin{array}{l}\text { BROMO- } \\
\text { BENZENE } \\
\text { WATER, } \\
\text { WHOLE, } \\
\text { TOTAL } \\
\text { (UG/L) }\end{array}$ & $\begin{array}{c}\text { BROMO- } \\
\text { FORM } \\
\text { TOTAL } \\
\text { (UG/L) }\end{array}$ & $\begin{array}{c}\text { BENZENE } \\
\text { N-BUTYL } \\
\text { WATER } \\
\text { UNFLTRD } \\
\text { REC } \\
(U G / L)\end{array}$ & $\begin{array}{c}\text { BENZENE } \\
\text { SEC } \\
\text { BUTYL. } \\
\text { WATER } \\
\text { UNFLTRD } \\
\text { REC } \\
\text { (UG/L) }\end{array}$ & $\begin{array}{c}\text { BENZENE } \\
\text { TERT- } \\
\text { BUTYL- } \\
\text { WATER } \\
\text { UNFLTRD } \\
\text { REC } \\
\text { (UG/L) }\end{array}$ \\
\hline APR 1992 & & & & & & & & & & & & \\
\hline $\begin{array}{l}17-17 \\
\text { MAY }\end{array}$ & 100 & 17 & $<1$ & 3 & $<20$ & $<20$ & $<0.2$ & $<0.2$ & $<0.2$ & $<0.20$ & $<0.20$ & $<0.20$ \\
\hline $14-14$ & 100 & 24 & $<1$ & 6 & $<20$ & $<20$ & $<0.2$ & $<0.2$ & $<0.2$ & $<0.20$ & $<0.20$ & $<0.20$ \\
\hline $\begin{array}{l}\text { SEP } \\
21 \cdot 21\end{array}$ & 90 & 23 & $<1$ & 5 & $<20$ & $<20$ & $<0.2$ & $<0.2$ & $<0.2$ & $<0.20$ & $<0.20$ & $<0.20$ \\
\hline DATE & $\begin{array}{l}\text { CARBON - } \\
\text { TETRA - } \\
\text { CHLO- } \\
\text { RIDE } \\
\text { TOTAL } \\
\text { (UG/L) }\end{array}$ & $\begin{array}{l}\text { CHLORO- } \\
\text { BENZENE } \\
\text { TOTAL } \\
\text { (UG/L) }\end{array}$ & $\begin{array}{c}\text { CHLORO - } \\
\text { DI - } \\
\text { BROMO - } \\
\text { METHANE } \\
\text { TOTAL } \\
\text { (UG /L) }\end{array}$ & $\begin{array}{l}\text { CHLORO- } \\
\text { ETHANE } \\
\text { TOTAL } \\
\text { (UG/L) }\end{array}$ & $\begin{array}{c}2- \\
\text { CHLORO- } \\
\text { ETHYL- } \\
\text { VINYL - } \\
\text { ETHER } \\
\text { TOTAL } \\
\text { (UG/L) }\end{array}$ & $\begin{array}{l}\text { CHLORO- } \\
\text { FORM } \\
\text { TOTAL } \\
\text { (UG/L) }\end{array}$ & $\begin{array}{c}\text { METHYL- } \\
\text { CHLO- } \\
\text { RIDE } \\
\text { TOTAL } \\
(U G / L)\end{array}$ & $\begin{array}{c}\text { O- } \\
\text { CHLORO- } \\
\text { TOLUENE } \\
\text { WATER } \\
\text { WHOLE } \\
\text { TOTAL } \\
\text { (UG/L) }\end{array}$ & $\begin{array}{c}\text { TOLUENE } \\
\text { P-CHLOR } \\
\text { WATER } \\
\text { UNFLTRD } \\
\text { REC } \\
(\text { UG } / L)\end{array}$ & $\begin{array}{c}\text { DIBROMO } \\
\text { CHLORO- } \\
\text { PROPANE } \\
\text { WATER } \\
\text { WHOLE } \\
\text { TOT.REC } \\
\text { (UG/L) }\end{array}$ & $\begin{array}{c}\text { DI - } \\
\text { BROMO- } \\
\text { METHANE } \\
\text { WATER } \\
\text { WHOLE } \\
\text { RECOVER } \\
\text { (UG/L) }\end{array}$ & $\begin{array}{c}1,2- \\
\text { DIBROMO } \\
\text { ETHANE } \\
\text { WATER } \\
\text { WHOLE } \\
\text { TOTAL } \\
\text { (UG/L) }\end{array}$ \\
\hline $\begin{array}{c}\text { APR } 1992 \\
17-17\end{array}$ & $<0.2$ & $<0,20$ & $<02$ & 0.2 & $<10$ & $<02$ & $<02$ & $<0 ?$ & $<0>20$ & $<1 \quad 0$ & $<02$ & $<02$ \\
\hline $\begin{array}{l}\text { MAY } \\
\qquad 14-14\end{array}$ & $<0.2$ & $<0.20$ & $<0.2$ & $<0.2$ & $<1.0$ & $<0.2$ & $<0.2$ & $<0.2$ & $<0.20$ & $<1.0$ & $<0.2$ & $<0.2$ \\
\hline $\begin{array}{l}\text { SEP } \\
\quad 21-21\end{array}$ & $<0.2$ & $<0.20$ & $<0.2$ & $<0.2$ & $<1.0$ & 0.2 & $<0.2$ & $<0.2$ & $<0.20$ & $<1.0$ & $<0.2$ & $<0.2$ \\
\hline DATE & $\begin{array}{c}\text { DI- } \\
\text { CHLORO- } \\
\text { BROMO- } \\
\text { METHANE } \\
\text { TOTAL } \\
\text { (UG/L) }\end{array}$ & $\begin{array}{c}1,1-D I- \\
\text { CHLORO- } \\
\text { ETHYL- } \\
\text { ENE } \\
\text { TOTAL } \\
\text { (UG/L) }\end{array}$ & $\begin{array}{l}1,1 \text { - DI } \\
\text { CHLORO- } \\
\text { PRO- } \\
\text { PENE, } \\
\text { WAT, WH } \\
\text { TOTAL } \\
\text { (UG/L) }\end{array}$ & $\begin{array}{c}\text { BENZENE } \\
\text { O- } \\
\text { CHLORO- } \\
\text { WATER } \\
\text { UNFLTRD } \\
\text { REC } \\
\text { (UG/L) }\end{array}$ & $\begin{array}{l}\text { BENZENE } \\
1,3 \text { - DI - } \\
\text { CHLORO- } \\
\text { WATER } \\
\text { UNFLTRD } \\
\text { REC } \\
\text { (UG/L) }\end{array}$ & $\begin{array}{c}\text { BENZENE } \\
1,4 \text {-DI- } \\
\text { CHLORO- } \\
\text { WATER } \\
\text { UNFLTRD } \\
\text { REC } \\
\text { (UG/L) }\end{array}$ & \begin{tabular}{l}
\multicolumn{1}{c}{ DI - } \\
CHLORO- \\
DI - \\
FLUORO- \\
METHANE \\
TOTAL \\
(UG/L)
\end{tabular} & $\begin{array}{c}1,1 \text { - DI - } \\
\text { CHLORO- } \\
\text { ETHANE } \\
\text { TOTAL } \\
(\text { UG/L) }\end{array}$ & $\begin{array}{c}1,2 \text {-DI - } \\
\text { CHLORO- } \\
\text { ETHANE } \\
\text { TOTAL } \\
\text { (UG/L) }\end{array}$ & $\begin{array}{c}1,2-D I- \\
\text { PHENYL - } \\
\text { HYDRA - } \\
\text { ZINE } \\
\text { WATER } \\
\text { TOT.REC } \\
(U G / L)\end{array}$ & $\begin{array}{c}\text { IS - } 1,2- \\
\text { DI- } \\
\text { CHLORO- } \\
\text { ETHENE } \\
\text { WATER } \\
\text { TOTAL } \\
\text { (UG/L) }\end{array}$ & $\begin{array}{c}1,2- \\
\text { TRANSDI } \\
\text { CHLORO- } \\
\text { ETHENE } \\
\text { TOTAL } \\
\text { (UG/L) }\end{array}$ \\
\hline $\begin{array}{c}\text { APR } 1992 \\
17-17\end{array}$ & $<0.2$ & 5.9 & $<0.2$ & $<5.0$ & $<5.0$ & $<5.0$ & $<0.2$ & $<0.2$ & $<0.2$ & $<5.0$ & $<0.2$ & $<0.2$ \\
\hline $\begin{array}{l}\text { MAY } \\
\qquad 14-14\end{array}$ & $<0.2$ & $<0.2$ & $<0.2$ & $<5.0$ & $<5.0$ & $<5.0$ & $<0.2$ & $<0.2$ & $<0.2$ & $<5.0$ & $<0.2$ & $<0.2$ \\
\hline $\begin{array}{l}\text { SEP } \\
\quad 21 \cdot 21\end{array}$ & $<0.2$ & $<0.2$ & $<0.2$ & $<5.0$ & $<5.0$ & $<5.0$ & $<0.2$ & $<0.2$ & $<0.2$ & $<5.0$ & $<0.2$ & $<0.2$ \\
\hline DATE & $\begin{array}{l}1,2-D I- \\
\text { CHLORO- } \\
\text { PROPANE } \\
\text { TOTAL } \\
(\text { UG /L) }\end{array}$ & $\begin{array}{c}1,3-D I- \\
\text { CHLORO- } \\
\text { PROPANE } \\
\text { WAT . WH } \\
\text { TOTAL } \\
\text { (UG/L) }\end{array}$ & $\begin{array}{l}2,2-D I \\
\text { CHLORO- } \\
\text { PRO- } \\
\text { PANE } \\
\text { WAT, WH } \\
\text { TOTAL } \\
\text { (UG/L) }\end{array}$ & $\begin{array}{c}\text { CIS } \\
1,3 \text {-DI - } \\
\text { CHLORO- } \\
\text { PROPENE } \\
\text { TOTAL } \\
\text { (UG/L) }\end{array}$ & $\begin{array}{l}\text { TRANS - } \\
1,3-\mathrm{DI}- \\
\text { CHLORO- } \\
\text { PROPENE } \\
\text { TOTAL } \\
\text { (UG/L) }\end{array}$ & $\begin{array}{l}\text { ETHYL - } \\
\text { BENZENE } \\
\text { TOTAL } \\
\text { (UG/L) }\end{array}$ & $\begin{array}{c}\text { HEXA- } \\
\text { CHLORO- } \\
\text { BUT - } \\
\text { ADIENE } \\
\text { TOTAL } \\
\text { (UG/L) }\end{array}$ & $\begin{array}{l}\text { ISO- } \\
\text { PROPYL- } \\
\text { BENZENE } \\
\text { WATER } \\
\text { WHOLE } \\
\text { REC } \\
\text { (UG/L) }\end{array}$ & $\begin{array}{l}\text { P-ISO- } \\
\text { PROPYL- } \\
\text { TOLUENE } \\
\text { WATER } \\
\text { WHOLE } \\
\text { REC } \\
(U G / L)\end{array}$ & $\begin{array}{c}\text { METHYL - } \\
\text { BROMIDE } \\
\text { TOTAL } \\
\text { (UG/L) }\end{array}$ & $\begin{array}{l}\text { METHYL- } \\
\text { ENE } \\
\text { CHLO- } \\
\text { RIDE } \\
\text { TOTAL } \\
\text { (UG/L) }\end{array}$ & $\begin{array}{c}\text { NAPHTH - } \\
\text { ALENE } \\
\text { TOTAL } \\
\text { (UG/L) }\end{array}$ \\
\hline APR 1992 & & & & & & & & & & & & \\
\hline $\begin{array}{l}17-17 \\
\text { MAY }\end{array}$ & $<0.2$ & $<0.2$ & $<0.2$ & $<0.2$ & $<0.2$ & $<0.2$ & $<5.0$ & $<0.20$ & $<0.20$ & $<0.2$ & $<0.2$ & $<5.0$ \\
\hline $\begin{array}{l}14-14 \\
\text { SEP }\end{array}$ & $<0.2$ & $<0.2$ & $<0.2$ & $<0.2$ & $<0.2$ & $<0.2$ & $<5.0$ & $<0.20$ & $<0.20$ & $<0.2$ & 0.8 & $<5.0$ \\
\hline $21-21$ & $<0.2$ & $<0.2$ & $<0.2$ & $<0.2$ & $<0.2$ & $<0.2$ & $<5.0$ & $<0.20$ & $<0.20$ & $<0.2$ & $<0.2$ & $<5.0$ \\
\hline
\end{tabular}


Table 4. Water-quality data for storm-sewer outfall stations, Fort Worth, Texas, 1992-93-Continued

08048700 - EASTERN HILLS HIGH SCHOOL OUTFALI AT WEILER DRIVE, FORT WORTH, TX (WY 1992)-CONTInUEd

\begin{tabular}{|c|c|c|c|c|c|c|c|c|c|c|c|c|}
\hline & $\begin{array}{c}\text { BENZENE } \\
\text { N-PROPY } \\
\text { WATER }\end{array}$ & & $\begin{array}{l}\text { ETHANE, } \\
1112- \\
\text { TETRA - } \\
\text { CHLORO- }\end{array}$ & $\begin{array}{r}\text { ETHANE, } \\
1,1,2,2 \\
\text { TETRA- } \\
\text { CHLORO- }\end{array}$ & $\begin{array}{l}\text { TETRA - } \\
\text { CHLORO- } \\
\text { ETHYL - }\end{array}$ & & $\begin{array}{c}1,2,3- \\
\text { TRI- } \\
\text { CHLORO } \\
\text { BENZENE }\end{array}$ & $\begin{array}{c}\text { BENZENE } \\
1,2,4- \\
\text { TRI - } \\
\text { CHLORO - }\end{array}$ & $\begin{array}{l}1,1,1 \text { - } \\
\text { TRI - } \\
\text { CHLORO- }\end{array}$ & $\begin{array}{c}1,1,2 \text { - } \\
\text { TRI - } \\
\text { CHLORO- }\end{array}$ & $\begin{array}{l}\text { TRI - } \\
\text { CHLORO- } \\
\text { ETHYL - }\end{array}$ & $\begin{array}{l}\text { TRI - } \\
\text { CHLORO - } \\
\text { FLUORO - }\end{array}$ \\
\hline & $\begin{array}{c}\text { UNFLTRD } \\
\text { REC } \\
\text { (UG/L) }\end{array}$ & $\begin{array}{l}\text { STYRENE } \\
\text { TOTAL } \\
\text { (UG/L) }\end{array}$ & $\begin{array}{c}\text { WAT UNF } \\
\text { REC } \\
\text { (UG/L) }\end{array}$ & $\begin{array}{l}\text { WAT UNF } \\
\text { REC } \\
\text { (UG/L) }\end{array}$ & $\begin{array}{l}\text { ENE } \\
\text { TOTAL } \\
\text { (UG/L) }\end{array}$ & $\begin{array}{c}\text { TOLUENE } \\
\text { TOTAL } \\
\text { (UG/L) }\end{array}$ & $\begin{array}{l}\text { WAT, WH } \\
\text { REC } \\
\text { (UG/L) }\end{array}$ & $\begin{array}{l}\text { WAT UNF } \\
\text { REC } \\
\text { (UG/L) }\end{array}$ & $\begin{array}{l}\text { ETHANE } \\
\text { TOTAL } \\
\text { (UG /L) }\end{array}$ & $\begin{array}{c}\text { ETHANE } \\
\text { TOTAL } \\
\text { (UG/L) }\end{array}$ & $\begin{array}{c}\text { ENE } \\
\text { TOTAL } \\
\text { (UG/L) }\end{array}$ & $\begin{array}{l}\text { METHANE } \\
\text { TOTAL } \\
\text { (UG/L) }\end{array}$ \\
\hline
\end{tabular}

\begin{tabular}{|c|c|c|c|c|c|c|c|c|c|c|c|c|}
\hline $17 \cdot 17$ & $<0.20$ & $<0.2$ & $<0.2$ & $<0.2$ & $<0.2$ & 0.2 & $<0.20$ & $<5.0$ & $<0.2$ & $<0.2$ & $<0.2$ & $<0.2$ \\
\hline MAY & & & & & & & & & & & & \\
\hline $14-14$ & $<0.20$ & $<0.2$ & $<0.2$ & $<0.2$ & $<0.2$ & 0.2 & $<0.20$ & $<5.0$ & $<0.2$ & $<0.2$ & $<0.2$ & $<0.2$ \\
\hline $\begin{array}{l}E P \\
21-21\end{array}$ & $<0.20$ & $<0.2$ & $<0.2$ & $<0.2$ & $<0.2$ & $<0.2$ & $<0.20$ & $<5.0$ & $<0.2$ & $<0.2$ & $<0.2$ & $<0.2$ \\
\hline
\end{tabular}

\begin{tabular}{|c|c|c|c|c|c|c|c|c|c|c|c|c|}
\hline & $123-\mathrm{TRI}$ & & & & & & & & & BENZO A & & \\
\hline & CHLORO- & FSEUDO - & MESIT - & & & & & & & ANTHRAC & & BENZO B \\
\hline & PROPANE & CUMENE & YLENE & VINYI & XYLENE & $\mathrm{ACE}-$ & $\mathrm{ACE}-$ & & & ENE 1, 2- & BENZO - & FLUOR - \\
\hline & WATER & WATER & WATER & CHLO- & WATER & NAPHTH - & NAPHTH - & ANTHRA - & BENZI - & BENZANT & A - & $A N-$ \\
\hline & WHOLE & UNFLTRD & UNFLTRD & RIDE & UNFLTRD & ENE & YLENE & CENE & DINE & HRACENE & PYRENE & THENE \\
\hline DATE & $\begin{array}{l}\text { TOTAL } \\
\text { (UG/L) }\end{array}$ & $\begin{array}{c}R E C \\
(U G / L)\end{array}$ & $\begin{array}{c}R E C \\
(U G / L)\end{array}$ & $\begin{array}{l}\text { TOTAL, } \\
\text { (UG/L) }\end{array}$ & $\begin{array}{c}\text { REC } \\
(U G / L)\end{array}$ & $\begin{array}{l}\text { TOTAL } \\
\text { (UG/L) }\end{array}$ & $\begin{array}{l}\text { TOTAL } \\
\text { (UG/L) }\end{array}$ & $\begin{array}{l}\text { TOTAL } \\
\text { (UG/L) }\end{array}$ & $\begin{array}{l}\text { TOTAL } \\
\text { (UG/L) }\end{array}$ & $\begin{array}{l}\text { TOTAL } \\
\text { (UG/L) }\end{array}$ & $\begin{array}{l}\text { TOTAL } \\
\text { (UG/L) }\end{array}$ & $\begin{array}{l}\text { TOTAL } \\
(U G / L)\end{array}$ \\
\hline \multicolumn{13}{|l|}{ APR 1992} \\
\hline $17-17$ & $<0.2$ & $<0.20$ & $<0.20$ & $<0.2$ & $<0.20$ & $<5.0$ & $<5.0$ & $<5.0$ & $<40.0$ & $<10.0$ & $<10.0$ & $<10.0$ \\
\hline \multicolumn{13}{|l|}{ MAY } \\
\hline $14 \cdot 14$ & $<0.2$ & $<0.20$ & $<0.20$ & $<0.2$ & $<0.20$ & $<5.0$ & $<5.0$ & $<5.0$ & $<40.0$ & $<10.0$ & $<10.0$ & $<10.0$ \\
\hline \multicolumn{13}{|l|}{ SEP } \\
\hline \multirow[t]{6}{*}{$21-21$} & $<0.2$ & $<0.20$ & $<0.20$ & $<0.2$ & $<0.20$ & $<5.0$ & $<5.0$ & $<5.0$ & $<40.0$ & $<10.0$ & $<10.0$ & $<10.0$ \\
\hline & & BENZO & 4 & & BIS & BIS & BIS 12 - & & & & 4 & \\
\hline & BENZO $\mathrm{K}$ & GHI PERYL & BROMO- & N-BUTYL & $12-$ & $2-$ & CHLORO- & PARA - & 2- & & CHLORO- & \\
\hline & FLUOR - & ENE1, 12- & PHENYL & BENZYL & CHLORO- & CHLORO - & ISO- & CHLORO - & CHLORO- & 2- & PHENYL & \\
\hline & $\mathrm{AN}-$ & BENZO & PHENYL & PHTHAL - & ETHOXY) & ETHYL & PROPYL) & META & NAPH - & CHLORO- & PHENYL & CHRY - \\
\hline & THENE & PERYLENE & ETHER & ATE & METHANE & ETHER & ETHER & CRESOL & THALENE & PHENOL & ETHER & SENE \\
\hline DATE & $\begin{array}{l}\text { TOTAL } \\
(U G / L)\end{array}$ & $\begin{array}{l}\text { TOTAL } \\
\text { (UG/L) }\end{array}$ & $\begin{array}{l}\text { TOTAL } \\
(U G / L)\end{array}$ & $\begin{array}{l}\text { TOTAL } \\
\text { (UG/L) }\end{array}$ & $\begin{array}{l}\text { TOTAL } \\
\text { (UG/L) }\end{array}$ & $\begin{array}{l}\text { TOTAL } \\
\text { (UG/L) }\end{array}$ & $\begin{array}{l}\text { TOTAL } \\
\text { (UG/L) }\end{array}$ & $\begin{array}{l}\text { TOTAL } \\
(U G / L)\end{array}$ & $\begin{array}{l}\text { TOTAL } \\
(\mathrm{UG} / \mathrm{L})\end{array}$ & $\begin{array}{l}\text { TOTAL } \\
\text { (UG/L) }\end{array}$ & $\begin{array}{l}\text { TOTAL } \\
\text { (UG/L) }\end{array}$ & $\begin{array}{l}\text { TOTAL } \\
\text { (UG/L) }\end{array}$ \\
\hline \multicolumn{13}{|l|}{ APR 1992} \\
\hline $17-17$ & $<10.0$ & $<10.0$ & $<5.0$ & $<5.0$ & $<5.0$ & $<5.0$ & $<5.0$ & $<30.0$ & $<5.0$ & $<5.0$ & $<5.0$ & $<10.0$ \\
\hline \multicolumn{13}{|l|}{ MAY } \\
\hline $14-14$ & $<10.0$ & $<10.0$ & $<5.0$ & $<5.0$ & $<5.0$ & $<5.0$ & $<5.0$ & $<30.0$ & $<5.0$ & $<5.0$ & $<5.0$ & $<10.0$ \\
\hline \multicolumn{13}{|l|}{ SEP } \\
\hline $21-21$ & $<10.0$ & $<10.0$ & $<5.0$ & $<5.0$ & $<5.0$ & $<5.0$ & $<5.0$ & $<30.0$ & $<5.0$ & $<5.0$ & $<5.0$ & $<10.0$ \\
\hline
\end{tabular}

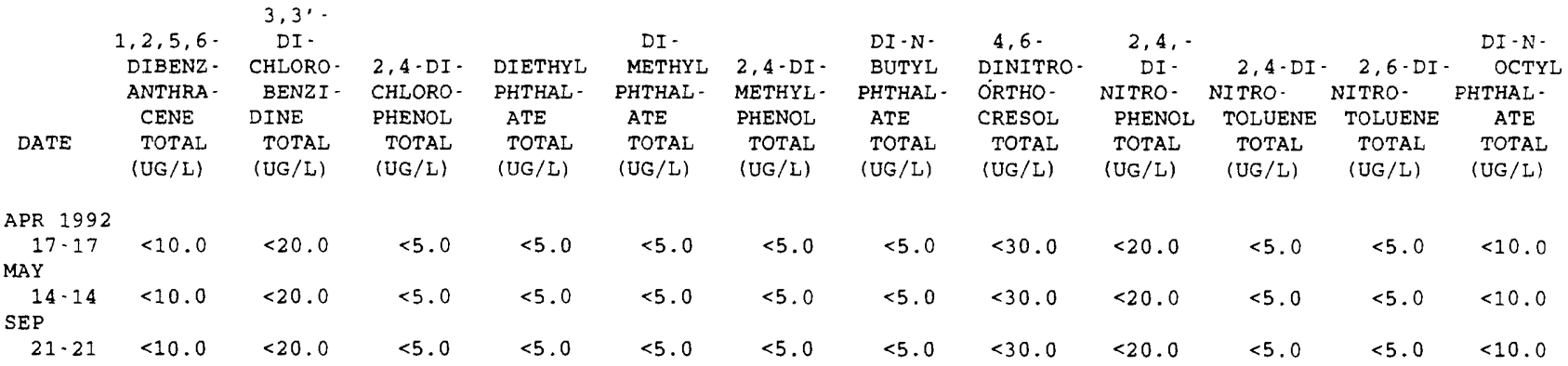


Table 4. Water-quality data for storm-sewer outfall stations, Fort worth, Texas, 1992-93-Continued

08048700 - EASTERN HILLS HIGH SCHOOL OUTFALL AT WEILER DRIVE, FORT WORTH, TX (WY 1992)-CONtINUEd

\begin{tabular}{|c|c|c|c|c|c|c|c|c|c|c|c|c|}
\hline & $\begin{array}{l}\text { BIS (2- } \\
\text { ETHYL }\end{array}$ & & & & $\begin{array}{c}\text { HEXA- } \\
\text { CHLORO- }\end{array}$ & & INDENO & & & N-NITRO- & & \\
\hline & HEXYL) & & & HEXA - & CYCLO- & HEXA- & $(1,2,3$. & & & SODI - & & 4. \\
\hline & $\begin{array}{c}\text { PHTHAL- } \\
\text { ATE }\end{array}$ & $\begin{array}{l}\text { FLUOR - } \\
\text { ANTHENE }\end{array}$ & $\begin{array}{c}\text { FLUOR - } \\
\text { ENE }\end{array}$ & $\begin{array}{l}\text { CHLORO- } \\
\text { BENZENE }\end{array}$ & $\begin{array}{c}\text { PENT - } \\
\text { ADIENE }\end{array}$ & $\begin{array}{l}\text { CHLORO- } \\
\text { ETHANE }\end{array}$ & $\begin{array}{r}\text { CD) } \\
\text { PYRENE }\end{array}$ & $\begin{array}{l}\text { ISO- } \\
\text { PHORONE }\end{array}$ & $\begin{array}{l}\text { NITRO- } \\
\text { BENZENE }\end{array}$ & $\begin{array}{l}\text { METHY - } \\
\text { LAMINE }\end{array}$ & $\begin{array}{l}\text { NITRO- } \\
\text { PHENOL }\end{array}$ & $\begin{array}{l}\text { NITRO } \\
\text { PHENOI }\end{array}$ \\
\hline & $\begin{array}{l}\text { TOTAL } \\
\text { (UG/L) }\end{array}$ & $\begin{array}{l}\text { TOTAL } \\
\text { (UG/L) }\end{array}$ & $\begin{array}{l}\text { TOTAL } \\
\text { (UG/L) }\end{array}$ & $\begin{array}{l}\text { TOTAL } \\
\text { (UG/L) }\end{array}$ & $\begin{array}{l}\text { TOTAL } \\
\text { (UG/L) }\end{array}$ & $\begin{array}{l}\text { TOTAL } \\
\text { (UG/L) }\end{array}$ & $\begin{array}{l}\text { TOTAL } \\
\text { (UG/L) }\end{array}$ & $\begin{array}{l}\text { TOTAL } \\
\text { (UG/L) }\end{array}$ & $\begin{array}{l}\text { TOTAL } \\
\text { (UG/L) }\end{array}$ & $\begin{array}{l}\text { TOTAL } \\
\text { (UG/L) }\end{array}$ & $\begin{array}{l}\text { TOTAL } \\
\text { (UG/L) }\end{array}$ & $\begin{array}{l}\text { TOTAL } \\
\text { (UG/L) }\end{array}$ \\
\hline
\end{tabular}

\begin{tabular}{|c|c|c|c|c|c|c|c|c|c|c|c|c|}
\hline APR 1992 & & & & & & & & & & & & \\
\hline MAY & $<5.0$ & $<5.0$ & $<5.0$ & $<5.0$ & $<5.0$ & $<5.0$ & $<10.0$ & $<5.0$ & $<5.0$ & $<5.0$ & $<5.0$ & $<30.0$ \\
\hline $\operatorname{SEP}^{14 \cdot 14}$ & $<5.0$ & $<5.0$ & $<5.0$ & $<5.0$ & $<5.0$ & $<5.0$ & $<10.0$ & $<5.0$ & $<5.0$ & $<5.0$ & $<5.0$ & $<30.0$ \\
\hline $21 \cdot 21$ & $<5.0$ & 13.0 & $<5.0$ & $<5.0$ & $<5.0$ & $<5.0$ & $<10.0$ & $<5.0$ & $<5.0$ & $<5.0$ & $<5.0$ & $<30.0$ \\
\hline
\end{tabular}

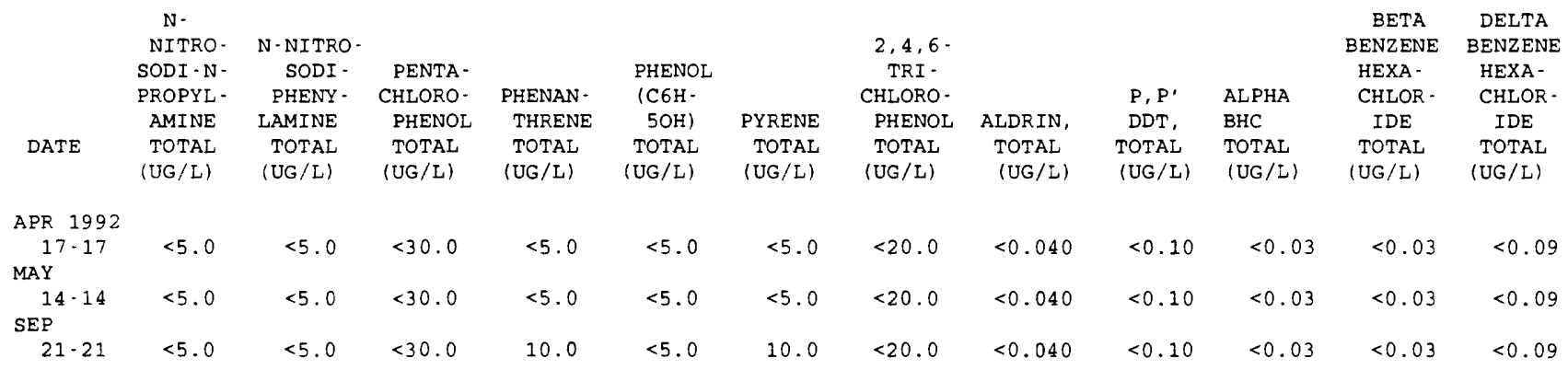

\begin{tabular}{|c|c|c|c|c|c|c|c|c|c|c|c|c|}
\hline & & $\begin{array}{l}\text { CHLOR - } \\
\text { DANE }\end{array}$ & $\begin{array}{l}\text { CHLOR - } \\
\text { DANE }\end{array}$ & & & & & $\begin{array}{l}\text { ENDO- } \\
\text { SULFAN- }\end{array}$ & & & & \\
\hline & & CIS & TRANS & & & & & I & ENDO - & ENDO - & ENDRIN & ENDRIN \\
\hline & & WATER & WATER & CHLOR - & $P, P^{\prime}$ & $P, P^{\prime}$ & DI - & WATER & SULFAN & SULFAN & WATER & ALDE - \\
\hline & LINDANE & WHOLE & WHOLE & DANE, & DDD, & DDE, & ELDRIN & WHOLE & BETA & SULFATE & UNFLTRD & HYDE \\
\hline DATE & $\begin{array}{l}\text { TOTAL } \\
\text { (UG/L) }\end{array}$ & $\begin{array}{l}\text { TOTAL } \\
\text { (UG/L) }\end{array}$ & $\begin{array}{l}\text { TOTAL } \\
\text { (UG/L) }\end{array}$ & $\begin{array}{l}\text { TOTAL } \\
(\text { UG/L) }\end{array}$ & $\begin{array}{l}\text { TOTAL } \\
\text { (UG/L) }\end{array}$ & $\begin{array}{l}\text { TOTAL } \\
\text { (UG/L) }\end{array}$ & $\begin{array}{l}\text { TOTAL } \\
\text { (UG/L) }\end{array}$ & $\begin{array}{l}\text { REC } \\
\text { (UG/L) }\end{array}$ & $\begin{array}{l}\text { TOTAL } \\
\text { (UG } / \mathrm{L})\end{array}$ & $\begin{array}{l}\text { TOTAL } \\
\text { (UG/L) }\end{array}$ & $\begin{array}{c}\text { REC } \\
(U G / L)\end{array}$ & $\begin{array}{l}\text { TOTAL } \\
\text { (UG/L) }\end{array}$ \\
\hline
\end{tabular}

\begin{tabular}{|c|c|c|c|c|c|c|c|c|c|c|c|c|}
\hline \multicolumn{13}{|l|}{ APR 1992} \\
\hline $17 \cdot 17$ & $<0.030$ & $<0.10$ & $<0.10$ & $<0.1$ & $<0.10$ & $<0.04$ & 0.040 & $<0.10$ & $<0.04$ & $<0.60$ & $<0.060$ & $<0.20$ \\
\hline MAY & & & & & & & & & & & & \\
\hline $14-14$ & $<0.030$ & 0.10 & 0.10 & 1.2 & $<0.10$ & $<0.04$ & 0.060 & $<0.10$ & $<0.04$ & $<0.60$ & $<0.060$ & $<0.20$ \\
\hline SEP & & & & & & & & & & & & \\
\hline $21 \cdot 21$ & $<0.030$ & $<0.10$ & $<0.10$ & 0.2 & $<0.10$ & $<0.04$ & $<0.020$ & $<0.10$ & $<0.04$ & $<0.60$ & $<0.060$ & $<0.20$ \\
\hline
\end{tabular}

\begin{tabular}{|c|c|c|c|c|c|c|c|c|c|c|c|}
\hline & & HEPTA- & & AROCLOR & AROCLOR & AROCLOR & AROCLOR & AROCLOR & AROCLOR & AROCLOR & \\
\hline & HEPTA - & CHLOR & TOX - & 1016 & 1221 & 1232 & 1242 & 1248 & 1254 & 1260 & DI. \\
\hline & CHLOR, & EPOXIDE & APHENE, & $\mathrm{PCB}$ & PCB & $\mathrm{PCB}$ & $\mathrm{PCB}$ & PCB & $\mathrm{PCB}$ & PCB & AZINON, \\
\hline$A$ & $\begin{array}{l}\text { TOTAL } \\
\text { (UG/L) }\end{array}$ & $\begin{array}{l}\text { TOTAL } \\
\text { (UG/L) }\end{array}$ & $\begin{array}{l}\text { TOTAL } \\
\text { \{UG/L\} }\end{array}$ & $\begin{array}{l}\text { TOTAL } \\
\text { (UG/L) }\end{array}$ & $\begin{array}{l}\text { TOTAL } \\
\text { (UG/L) }\end{array}$ & $\begin{array}{l}\text { TOTAL } \\
\text { (UG/L) }\end{array}$ & $\begin{array}{l}\text { TOTAL } \\
\text { (UG/L) }\end{array}$ & $\begin{array}{l}\text { TOTAL } \\
\text { (UG/L) }\end{array}$ & $\begin{array}{l}\text { TOTAL } \\
\text { (UG/L) }\end{array}$ & $\begin{array}{l}\text { TOTAL } \\
\text { (UG/L) }\end{array}$ & $\begin{array}{l}\text { TOTAL } \\
\text { (UG/L) }\end{array}$ \\
\hline
\end{tabular}

\begin{tabular}{|c|c|c|c|c|c|c|c|c|c|c|c|}
\hline \multicolumn{12}{|l|}{ APR 1992} \\
\hline $17-17$ & $<0.030$ & $<0.80$ & $<2$ & $<0.1$ & $<1.0$ & $<0.1$ & $<0.1$ & $<0.1$ & $<0.1$ & $<0.1$ & 0.40 \\
\hline MAY & & & & & & & & & & & \\
\hline $14-14$ & 0.20 & $<0.80$ & $<2$ & $<0.1$ & $<1.0$ & $<0.1$ & $<0.1$ & $<0.1$ & $<0.1$ & $<0.1$ & 0.10 \\
\hline SEP & & & & & & & & & & & \\
\hline $21 \cdot 21$ & $<0.030$ & $<0.80$ & $<2$ & $<0.1$ & $<1.0$ & $<0.1$ & $<0.1$ & $<0.1$ & $<0.1$ & $<0.1$ & 1.5 \\
\hline
\end{tabular}


Table 4. Water-quality data for storm-sewer outfall stations, Fort Worth, Texas, 1992-93-Continued 08048700 - EASTERN HILLS HIGH SCHOOL OUTFALL AT WEILER DRIVE, FORT WORTH, TX (WY 1993)

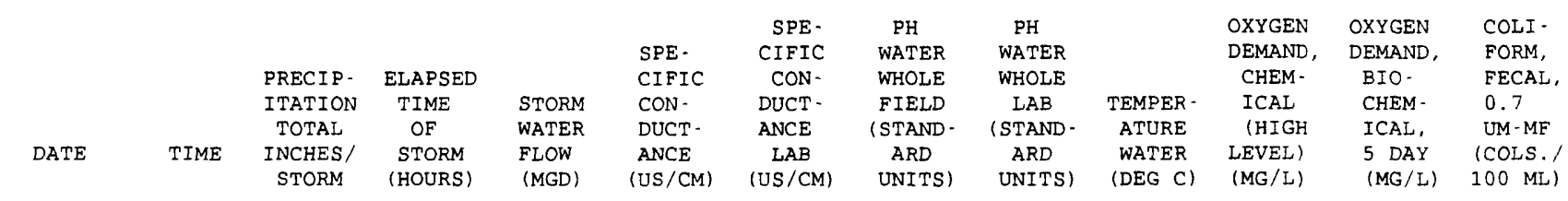

\begin{tabular}{|c|c|c|c|c|c|c|c|c|c|c|c|c|}
\hline $\begin{array}{c}\text { OCT } 1992 \\
28.29\end{array}$ & 2330 & 0.38 & 4.5 & 0.20 & 81 & 127 & 9.0 & 7.9 & 20.0 & 99 & 6.5 & 50000 \\
\hline NoV & & & & & & & & & & & & \\
\hline $\begin{array}{l}19-19 \\
D E C\end{array}$ & 0020 & 0.86 & 10.2 & 0.99 & 178 & 190 & 8.7 & 7.6 & 17.5 & 55 & 6.3 & 17000 \\
\hline $\begin{array}{c}09-09 \\
\text { JAN } 1993\end{array}$ & 0030 & 0.24 & 3.3 & 0.21 & 144 & 216 & 7.1 & 7.9 & 9.5 & 65 & 9.4 & 15000 \\
\hline 09.09 & 0215 & 0.25 & 6.0 & 0.21 & 158 & 297 & 9.2 & 8.8 & 9.5 & 93 & 4.8 & K2100 \\
\hline
\end{tabular}

\begin{tabular}{|c|c|c|c|c|c|c|c|c|c|c|c|c|}
\hline & $\begin{array}{l}\text { STREP- } \\
\text { TOCOCCI }\end{array}$ & HARD & $\begin{array}{l}\text { HARD - } \\
\text { NESS }\end{array}$ & $\begin{array}{l}\text { ALKA- } \\
\text { LINITY }\end{array}$ & $\begin{array}{l}\text { SOLIDS, } \\
\text { SUM OF }\end{array}$ & $\begin{array}{l}\text { RESIDUE } \\
\text { TOTAL }\end{array}$ & $\begin{array}{l}\text { SOLIDS, } \\
\text { RESIDUE }\end{array}$ & & MAGNE - & & & SODIUM \\
\hline & FECAL, & NESS & NONCARB & WAT DIS & CONSTI - & AT 105 & AT 180 & CALCIUM & SIUM, & SODIUM, & & AD - \\
\hline & $\begin{array}{l}\text { KF AGAR } \\
\text { (COLS. }\end{array}$ & $\begin{array}{l}\text { TOTAL } \\
\text { (MG/L }\end{array}$ & $\begin{array}{l}\text { DISSOLV } \\
\text { FLD. AS }\end{array}$ & $\begin{array}{l}\text { FIX END } \\
\text { FIELD }\end{array}$ & $\begin{array}{l}\text { TUENTS, } \\
\text { DIS - }\end{array}$ & $\begin{array}{l}\text { DEG. C, } \\
\text { SUS. }\end{array}$ & $\begin{array}{l}\text { DEG. C } \\
\text { DIS. }\end{array}$ & $\begin{array}{l}\text { DIS- } \\
\text { SOLVED }\end{array}$ & $\begin{array}{c}\text { DIS- } \\
\text { SOLVED }\end{array}$ & $\begin{array}{l}\text { DIS - } \\
\text { SOLVED }\end{array}$ & & $\begin{array}{l}\text { SORP. } \\
\text { TION }\end{array}$ \\
\hline & PER & AS & $\mathrm{CACO}$ & CACO3 & SOLVED & PENDED & SOLVED & (MG/L & (MG /L & (MG/L & SODIUM & RATIO \\
\hline & $100 \mathrm{ML})$ & (ACO3) & (MG/L) & $(M G / L)$ & $(M G / L)$ & $(M G / L)$ & (MG/L) & AS $(A)$ & AS MG) & AS NA) & PERCENT & \\
\hline
\end{tabular}

OCT 1992

$\begin{array}{ll}28-29 & 42000 \\ \text { NOV } & \\ \text { 19- } 19 & 55000 \\ \text { DEC } & \\ 09 \cdot 09 & 20000 \\ \text { JAN } 1993 & \\ 09-09 & 36000\end{array}$

$\begin{array}{rrr}39 & 11 & 28 \\ 54 & 6 & 48 \\ 50 & 14 & 36 \\ 100 & 39 & 64\end{array}$

$\begin{array}{rrrr}83 & 686 & 81 & 14 \\ 94 & 98 & 104 & 18 \\ 96 & 192 & 78 & 17 \\ 173 & 644 & 163 & 33\end{array}$

$\begin{array}{ll} & \\ \text { POTAS- } & \\ \text { SIUM, } & \text { SULFATE } \\ \text { DIS- } & \text { DIS- } \\ \text { SOLVED } & \text { SOLVED } \\ \text { (MG/L } & \text { (MG/L } \\ \text { AS K) } & \text { AS SO4) }\end{array}$

$\begin{array}{lcc}\text { CHLO- } & \text { NITRO- } & \text { NITRO- } \\ \text { RIDE, } & \text { GEN, } & \text { GEN, } \\ \text { DIS- } & \text { NITRITE } & \text { NO2+NO3 } \\ \text { SOLVED } & \text { TOTAL } & \text { TOTAL } \\ \text { (MG/L } & \text { (MG/L } & \text { (MG/L } \\ \text { AS CL) } & \text { AS N) } & \text { AS N) }\end{array}$

$\begin{array}{ccr} & \text { NITRO. } & \\ \text { NITRO- } & \text { GEN, AM- } & \\ \text { GEN, } & \text { MONIA + } & \text { PHOS } \\ \text { AMMONIA } & \text { ORGANIC } & \text { PHORUS } \\ \text { TOTAL } & \text { TOTAL } & \text { TOTAL } \\ \text { (MG/L } & \text { (MG } / L & \text { (MG/L } \\ \text { AS N) } & \text { AS N) } & \text { AS P) }\end{array}$

$$
\begin{array}{ll}
1.0 & 2.7 \\
2.1 & 6.2 \\
1.9 & 5.7 \\
5.1 & 17
\end{array}
$$

$\begin{array}{cc}\text { PHOS- } & \\ \text { PHORUS } & \text { ANTIMONY } \\ \text { DIS- } & \text { TOTAL } \\ \text { SOLVED } & \text { EPA } \\ \text { (MG/L } & \text { (UG/L } \\ \text { AS P) } & \text { AS SB) }\end{array}$

$\begin{array}{cl} & \text { BERYL- } \\ & \text { LIUM, } \\ & \text { TOTAL } \\ \text { ARSENIC } & \text { RECOV- } \\ \text { TOTAL } & \text { ERABLE } \\ \text { (UG/L } & \text { (UG/L } \\ \text { AS AS) } & \text { AS BE) }\end{array}$

\begin{tabular}{|c|c|c|c|c|c|c|c|c|c|c|c|c|}
\hline & $\begin{array}{l}\text { CADMIUM } \\
\text { TOTAL }\end{array}$ & $\begin{array}{l}\text { CHRO- } \\
\text { MIUM, } \\
\text { TOTAL }\end{array}$ & $\begin{array}{c}\text { COPPER, } \\
\text { TOTAL }\end{array}$ & CYANIDE & & $\begin{array}{l}\text { LEAD, } \\
\text { TOTAL }\end{array}$ & $\begin{array}{l}\text { MERCURY } \\
\text { TOTAL }\end{array}$ & $\begin{array}{l}\text { NICKEL, } \\
\text { TOTAL }\end{array}$ & SELE - & $\begin{array}{l}\text { SILVER, } \\
\text { TOTAL }\end{array}$ & $\begin{array}{l}\text { SILVER, } \\
\text { TOTAL } \\
\text { RECOV. }\end{array}$ & THAL - \\
\hline & RECOV- & RECOV - & RECOV - & TOTAL & CYANIDE & RECOV - & RECOV - & RECOV - & NIUM, & RECOV - & ERABLE & LIUM, \\
\hline DATE & $\begin{array}{l}\text { ERABLE } \\
\text { (UG/L }\end{array}$ & $\begin{array}{l}\text { ERABLE } \\
\text { (UG/L }\end{array}$ & $\begin{array}{l}\text { ERABLE } \\
\text { (UG/L }\end{array}$ & $\begin{array}{c}\text { EPA } \\
(\mathrm{MG} / \mathrm{L}\end{array}$ & $\begin{array}{l}\text { TOTAL } \\
\text { (MG/L }\end{array}$ & $\begin{array}{l}\text { ERABLE } \\
\text { (UG/L }\end{array}$ & & $\begin{array}{l}\text { ERABLE } \\
\text { (UG/L }\end{array}$ & $\begin{array}{l}\text { TOTAL } \\
\text { (UG/L }\end{array}$ & $\begin{array}{l}\text { ERABLE } \\
\text { (UG/L }\end{array}$ & $\begin{array}{l}\text { EPA } \\
\text { (UG/L }\end{array}$ & $\begin{array}{l}\text { TOTAL } \\
\text { (UG/L }\end{array}$ \\
\hline & AS $C D$ ) & AS (R) & AS CUl & AS CN) & AS $C N$ ) & AS PB) & AS $\mathrm{HG}$ ) & AS NI) & AS SE) & AS AG) & AS AG) & AS TL) \\
\hline
\end{tabular}

3.4
6.0
5.6
15

$\begin{array}{ccc}0.040 & 0.600 & 0.120 \\ 0.100 & 0.470 & 0.060 \\ 0.150 & 0.540 & 0.170 \\ \ldots & 0.850 & \cdots\end{array}$

$\begin{array}{llll}0.80 & 0.200 & 0.130 & <10.0 \\ 0.60 & 0.250 & 0.200 & <20.0 \\ 0.60 & 0.170 & 0.040 & <10.0 \\ 0.60 & 0.160 & 0.060 & <10.0\end{array}$

AS AS )

$\begin{array}{ll}12 & 0.2 \\ 18 & 0.4 \\ 19 & 0.3 \\ 26 & 0.7\end{array}$

0.7

\section{OCT 1992}

$28-29$
NOV
$19-19$
DEC
$09-09$
JAN 1993
$09-09$

$\begin{array}{rrrrrrr}<1 & 15 & 86 & <0.010 & 0.010 & 89 & 0.10 \\ <1 & 7 & 5 & <0.010 & <0.010 & 9 & <0.10 \\ <1 & <1 & 9 & <0.010 & <0.010 & 19 & <0.10 \\ <1 & 26 & 10 & <0.010 & <0.010 & 51 & <0.10\end{array}$

$\begin{array}{rlrrr}10 & <2 & <1 & <0.500 & <5 \\ 3 & <2 & <1 & <0.500 & <5 \\ 5 & <2 & <1 & <0.500 & <10 \\ 9 & <2 & <1 & <0.500 & <10\end{array}$


Table 4. Water-quality data for storm-sewer outfall stations, Fort worth, Texas, 1992-93-Continued

08048700 - EASTERN HILLS HIGH SCHOOL OUTFALL AT WEILER DRIVE, FORT WORTH, TX (WY 1993)-COntinued

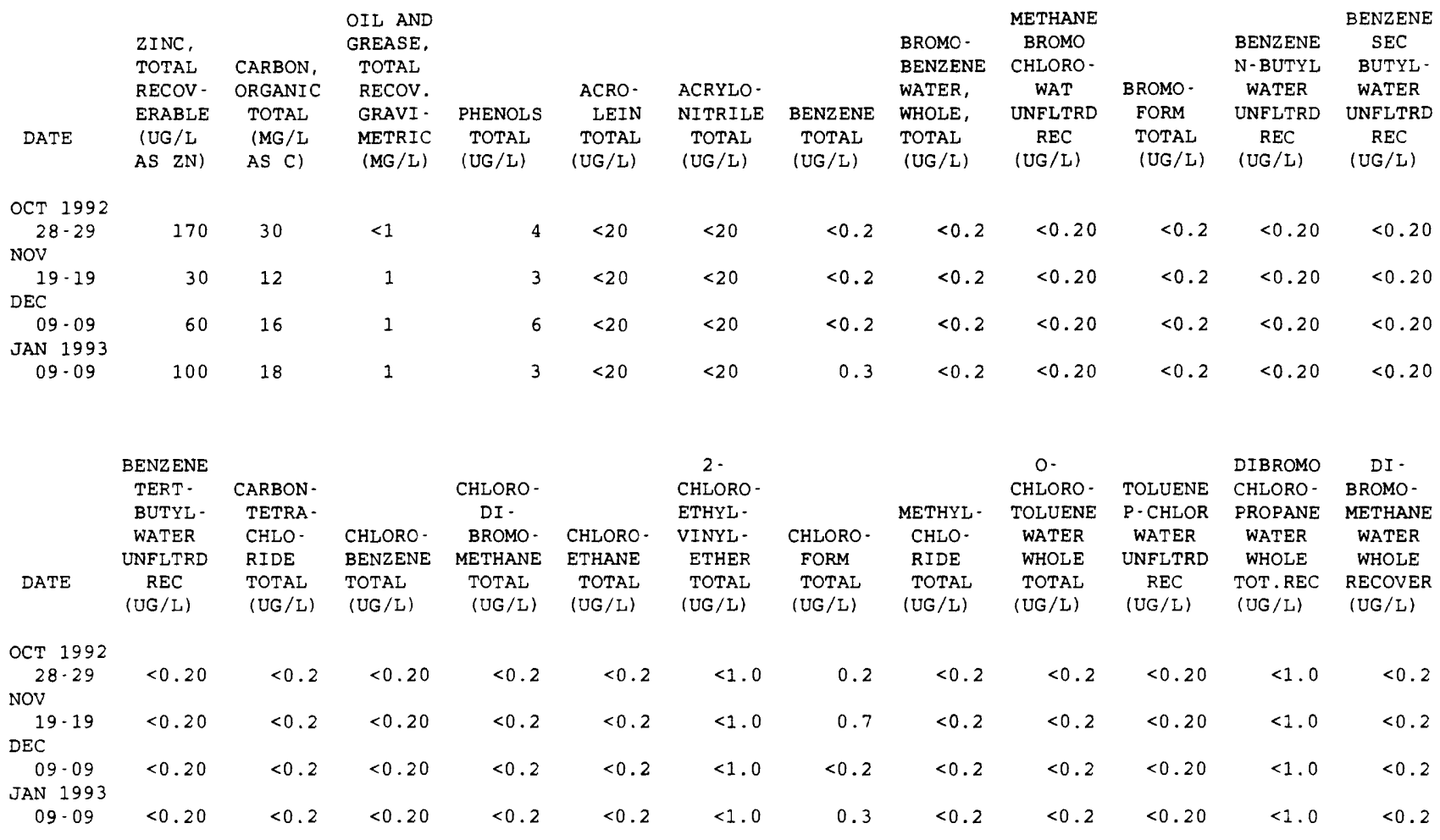

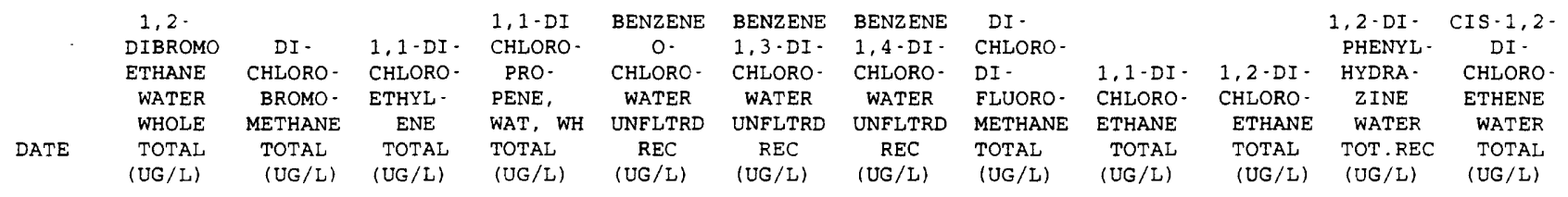

\begin{tabular}{|c|c|c|c|c|c|c|c|c|c|c|c|c|}
\hline \multicolumn{13}{|l|}{ OCT 1992} \\
\hline $28-29$ & $<0.2$ & $<0.2$ & $<0.2$ & $<0.2$ & $<5.0$ & $<5.0$ & $<5.0$ & $<0.2$ & $<0.2$ & $<0.2$ & $<5.0$ & $<0.2$ \\
\hline \multicolumn{13}{|l|}{ NOV } \\
\hline $19-19$ & $<0.2$ & $<0.2$ & $<0.2$ & $<0.2$ & $<5.0$ & $<5.0$ & $<5.0$ & $<0.2$ & $<0.2$ & $<0.2$ & $<5.0$ & $<0.2$ \\
\hline \multicolumn{13}{|l|}{ DEC } \\
\hline $\begin{array}{c}09 \cdot 09 \\
\text { JAN } 1993\end{array}$ & $<0.2$ & $<0.2$ & $<0.2$ & $<0.2$ & $<5.0$ & $<5.0$ & $<5.0$ & $<0.2$ & $<0.2$ & $<0.2$ & $<5.0$ & $<0.2$ \\
\hline 09.09 & $<0.2$ & $<0.2$ & $<0.2$ & $<0.2$ & $<0.20$ & $<0.20$ & $<0.20$ & $<0.2$ & $<0.2$ & $<0.2$ & $<5.0$ & $<0.2$ \\
\hline
\end{tabular}

\begin{tabular}{|c|c|c|c|c|c|c|c|c|c|c|c|}
\hline & $\begin{array}{c}1,2- \\
\text { RANSDI }\end{array}$ & 2- - & $\begin{array}{l}\text { 1,3-DI - } \\
\text { CHLORO- }\end{array}$ & $\begin{array}{l}2,2 \text { - DI } \\
\text { CHLORO - } \\
\text { PRO- }\end{array}$ & $\begin{array}{c}\text { CIS } \\
1,3-D I-\end{array}$ & $\begin{array}{l}\text { TRANS - } \\
1,3-\mathrm{DI} \text { - }\end{array}$ & & $\begin{array}{c}\text { HEXA- } \\
\text { CHLORO- }\end{array}$ & $\begin{array}{c}\text { ISO- } \\
\text { PROPYL- } \\
\text { BENZENE }\end{array}$ & $\begin{array}{l}\text { P-ISO- } \\
\text { PROPYL- } \\
\text { TOLUENE }\end{array}$ & \\
\hline & CHLORO- & CHLORO- & PROPANE & PANE & CHLORO- & CHLORO- & ETHY & BUT. & WATER & WATER & METHYL- \\
\hline & ETHENE & PROPANE & WAT. WH & WAT, WH & PROPENE & PROPENE & BENZENE & ADIENE & WHOLE & WHOLE & BROMIDE \\
\hline & $\begin{array}{l}\text { TOTAL } \\
\text { (UG/L) }\end{array}$ & $\begin{array}{l}\text { TOTAL } \\
\text { (UG/L) }\end{array}$ & $\begin{array}{l}\text { TOTAL } \\
(\mathrm{UG} / \mathrm{L})\end{array}$ & $\begin{array}{l}\text { TOTAL } \\
(\mathrm{UG} / \mathrm{L})\end{array}$ & $\begin{array}{l}\text { TOTAL } \\
(\mathrm{UG} / \mathrm{L})\end{array}$ & $\begin{array}{l}\text { TOTAL } \\
\text { (UG/L) }\end{array}$ & $\begin{array}{l}\text { TOTAL } \\
\text { (UG/L) }\end{array}$ & $\begin{array}{l}\text { TOTAL } \\
\text { (UG/L) }\end{array}$ & $\begin{array}{l}\text { REC } \\
\text { (UG/L) }\end{array}$ & $\begin{array}{l}\text { REC } \\
(\mathrm{UG} / \mathrm{L})\end{array}$ & $\begin{array}{l}\text { TOTAL } \\
\text { (UG/L) }\end{array}$ \\
\hline
\end{tabular}

\begin{tabular}{|c|c|c|c|c|c|c|c|c|c|c|c|}
\hline $\begin{array}{c}\text { OCT } 1992 \\
28-29\end{array}$ & $<0.2$ & $<0.2$ & $<0.2$ & $<0.2$ & $<0.2$ & $<0.2$ & $<0.2$ & $<5.0$ & $<0.20$ & $<0.20$ & $<0.2$ \\
\hline Nov & & & & & & & & & & & \\
\hline $19-19$ & $<0.2$ & $<0.2$ & $<0.2$ & $<0.2$ & $<0.2$ & $<0.2$ & $<0.2$ & $<5.0$ & $<0.20$ & $<0.20$ & $<0.2$ \\
\hline $\mathrm{DEC}$ & & & & & & & & & & & \\
\hline $\begin{array}{c}09-09 \\
\text { JAN } 1993\end{array}$ & $<0.2$ & $<0.2$ & $<0.2$ & $<0.2$ & $<0.2$ & $<0.2$ & $<0.2$ & $<5.0$ & $<0.20$ & $<0.20$ & $<0.2$ \\
\hline 09.09 & $<0.2$ & $<0.2$ & $<0.2$ & $<0.2$ & $<0.2$ & $<0.2$ & 0.2 & $<0.2$ & $<0.20$ & $<0.20$ & $<0.2$ \\
\hline
\end{tabular}


Table 4. Water-quality data for storm-sewer outfall stations, Fort worth, Texas, 1992-93-Continued 08048700 - EASTERN HILLS HIGH SCHOOL OUTFALL AT WEILER DRIVE, FORT WORTH, TX (WY 1993)-CONtInUEd

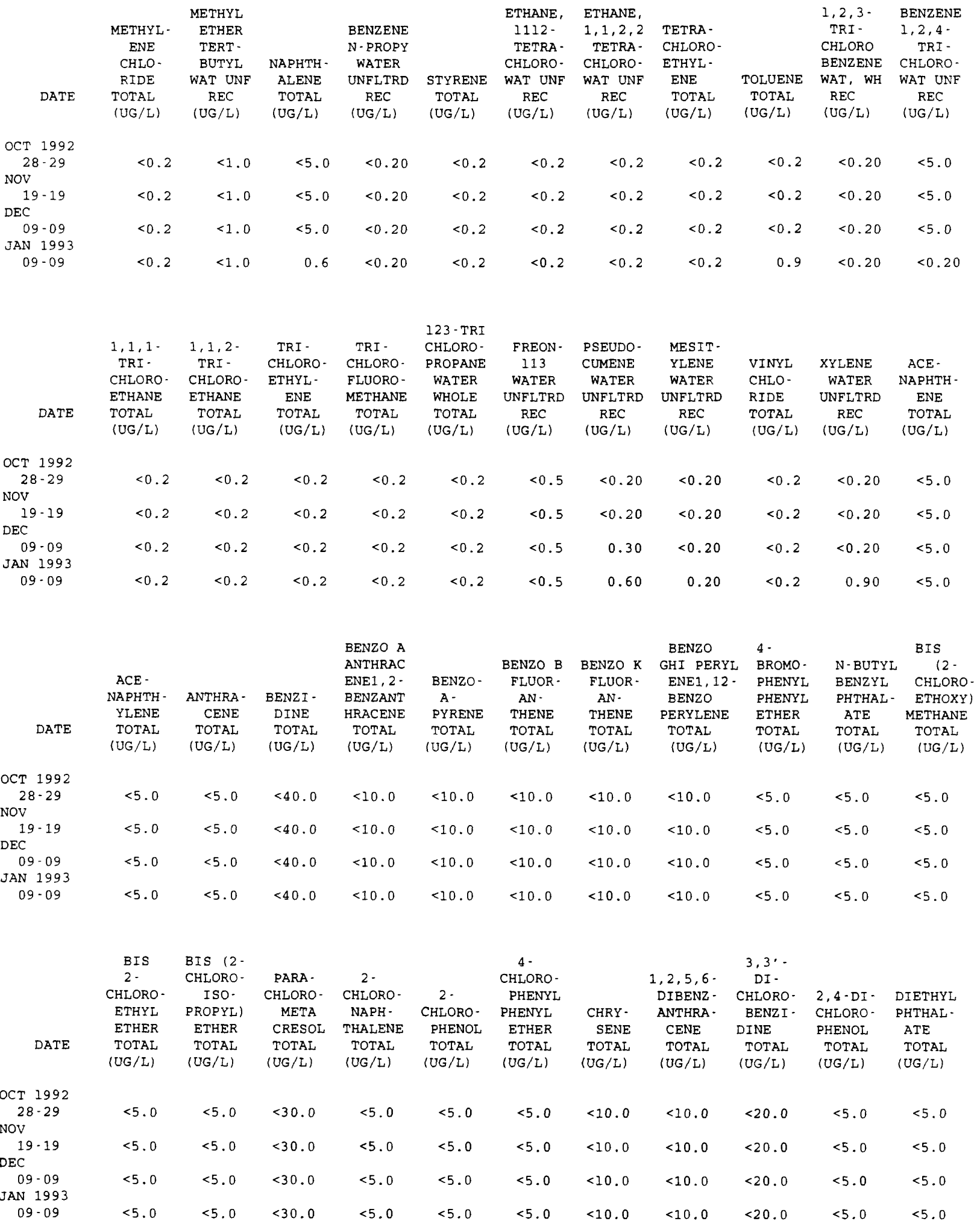


Table 4. Water-quality data for storm-sewer outfall stations, Fort worth, Texas, 1992-93-Continued

08048700 - EASTERN HILLS HIGH SCHOOL OUTFALL AT WEILER DRIVE, FORT WORTH, TX (WY 1993)-CONTINUEd

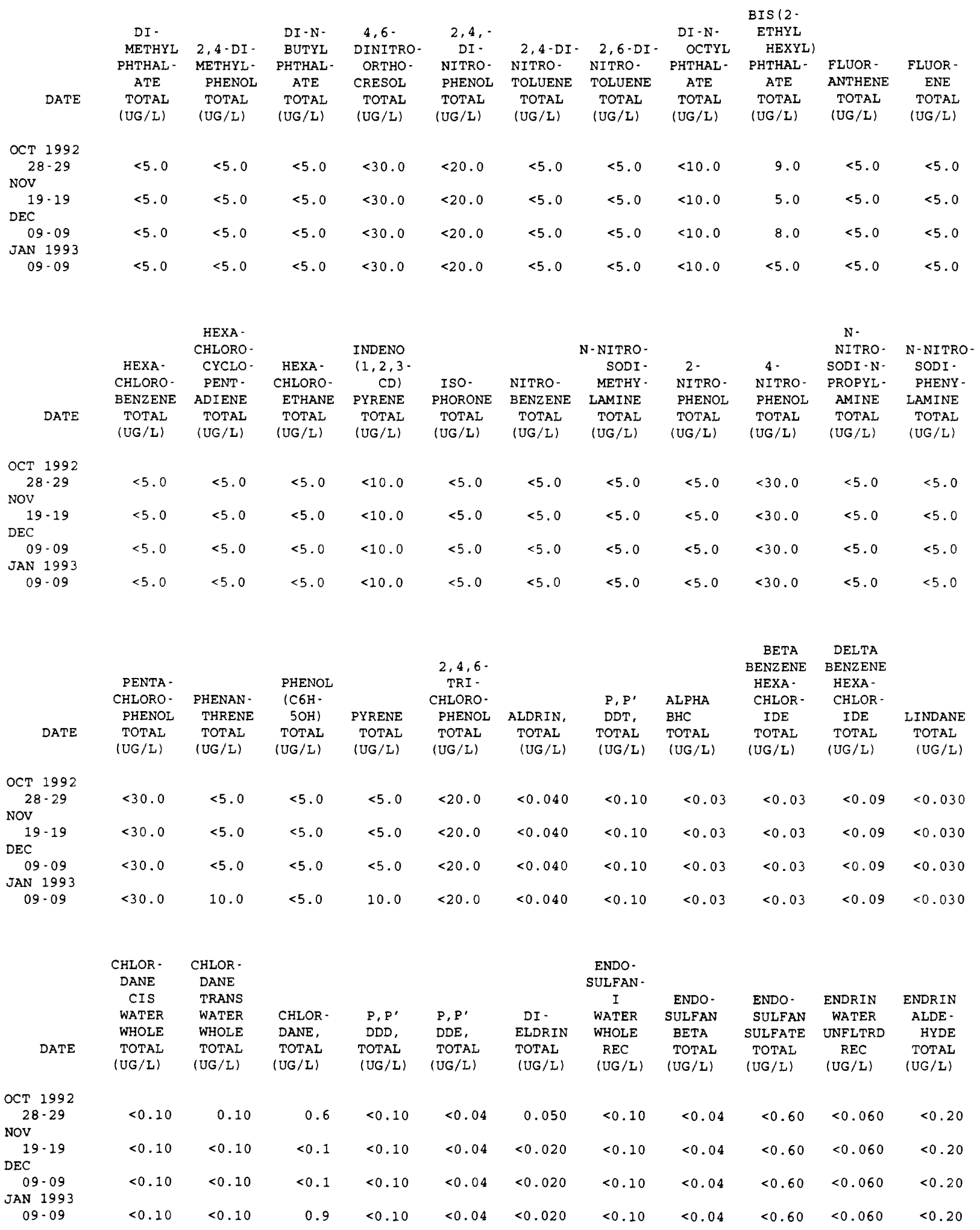


Table 4. Water-quality data for storm-sewer outfall stations, Fort worth, Texas, 1992-93-Continued 08048700 - EASTERN HILLS HIGH SCHOOL OUTFALL AT WEILER DRIVE, FORT WORTH, TX (WY 1993)-CONTINUEd

\begin{tabular}{|c|c|c|c|c|c|c|c|c|c|c|}
\hline & HEPTA - & & AROCLOR & AROCLOR & AROCLOR & AROCLOR & AROCLOR & AROCLOR & AROCLOR & \\
\hline HEPTA - & CHLOR & TOX - & 1016 & 1221 & 1232 & 1242 & 1248 & 1254 & 1260 & $\mathrm{DI}$ \\
\hline CHLOR, & EPOXIDE & APHENE, & PCB & PCB & PCB & PCB & PCB & PCB & PCB & AZINON, \\
\hline $\begin{array}{l}\text { TOTAL } \\
\text { (UG/L) }\end{array}$ & $\begin{array}{l}\text { TOTAL } \\
(U G / L)\end{array}$ & $\begin{array}{l}\text { TOTAL } \\
\text { (UG/L) }\end{array}$ & $\begin{array}{l}\text { TOTAL } \\
\text { (UG/L) }\end{array}$ & $\begin{array}{l}\text { TOTAL } \\
\text { (UG/L) }\end{array}$ & $\begin{array}{l}\text { TOTAL } \\
\text { (UG/L) }\end{array}$ & $\begin{array}{l}\text { TOTAL } \\
\text { (UG/L) }\end{array}$ & $\begin{array}{l}\text { TOTAL } \\
\text { (UG/L) }\end{array}$ & $\begin{array}{l}\text { TOTAL } \\
\text { (UG/L) }\end{array}$ & $\begin{array}{l}\text { TOTAL } \\
\text { (UG/L) }\end{array}$ & $\begin{array}{l}\text { TOTAL } \\
\text { (UG/L) }\end{array}$ \\
\hline
\end{tabular}

\begin{tabular}{|c|c|c|c|c|c|c|c|c|c|c|c|}
\hline \multicolumn{12}{|l|}{ OCT 1992} \\
\hline $28-29$ & $<0.030$ & $<0.80$ & $<2$ & $<0.1$ & $<1.0$ & $<0.1$ & $<0.1$ & $<0.1$ & $<0.1$ & $<0.1$ & 0.20 \\
\hline \multicolumn{12}{|l|}{ NOV } \\
\hline $19-19$ & $<0.030$ & $<0.80$ & $<2$ & $<0.1$ & $<1.0$ & $<0.1$ & $<0.1$ & $<0.1$ & $<0.1$ & $<0.1$ & 0.10 \\
\hline \multicolumn{12}{|l|}{$\mathrm{DEC}$} \\
\hline 09.09 & $<0.030$ & $<0.80$ & $<2$ & $<0.1$ & $<1.0$ & $<0.1$ & $<0.1$ & $<0.1$ & $<0.1$ & $<0.1$ & $<0.10$ \\
\hline \multicolumn{12}{|l|}{ JAN 1993} \\
\hline 09.09 & $<0.030$ & $<0.80$ & $<2$ & $<0.1$ & $<1.0$ & $<0.1$ & $<0.1$ & $<0.1$ & $<0.1$ & $<0.1$ & 0.10 \\
\hline
\end{tabular}


Table 5. Water-quality data for storm-sewer outfall stations, Garland, Texas, 1992-93

08061635 - TRIBUTARY TO DUCK CREEK OUTFALL AT HIGHTOWER ROAD, GARLAND, TX (WY 1992)

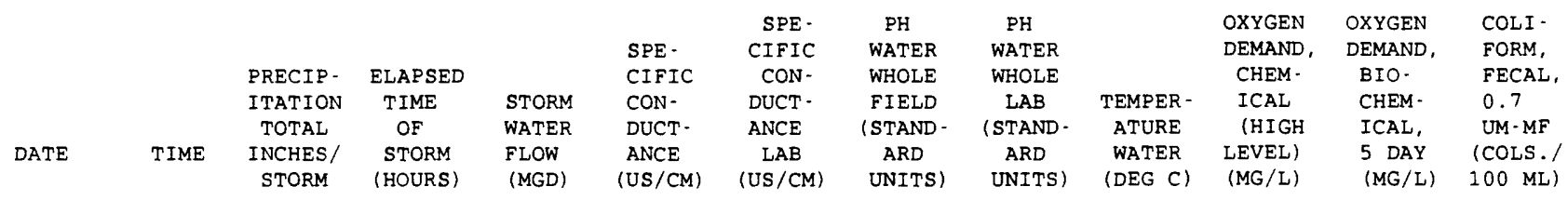

\begin{tabular}{|c|c|c|c|c|c|c|c|c|c|c|c|c|}
\hline $\begin{array}{c}\text { JUN } 1992 \\
20-20\end{array}$ & 0640 & 0.39 & 3.3 & 0.25 & 235 & 125 & 7.2 & 7.3 & 23.5 & 67 & 8.9 & 13000 \\
\hline AUG & & & & & & & & & & & & \\
\hline $\operatorname{SEP}^{12-12}$ & 1645 & 0.34 & 1.5 & 0.24 & 85 & 96 & 7.9 & 8.0 & 26.5 & 52 & 5.0 & 14000 \\
\hline $\begin{array}{l}01-01 \\
\text { SEP }\end{array}$ & 1328 & 0.31 & 2.0 & 0.23 & 61 & 175 & 7.1 & 7.6 & 25.5 & 76 & 7.5 & 73000 \\
\hline $21-21$ & 0150 & 0.80 & 3.2 & 0.66 & 195 & 139 & 7.0 & 8.1 & 24.0 & 62 & 5.2 & K15000 \\
\hline
\end{tabular}

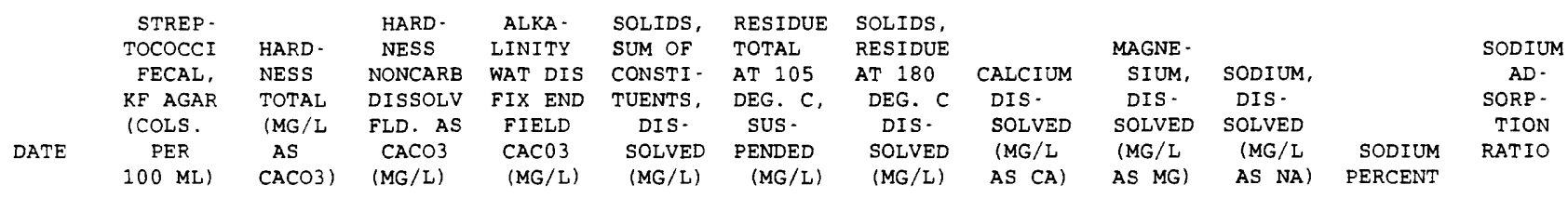

JUN 1992

\begin{tabular}{|c|c|c|c|c|}
\hline \multirow{2}{*}{\multicolumn{5}{|c|}{$\begin{array}{l}20-20 \\
\text { AUG }\end{array}$}} \\
\hline & & & & \\
\hline $12 \cdot 12$ & 11000 & 22 & 4 & 18 \\
\hline SEP & & & & \\
\hline 01.01 & 6500 & 34 & 7 & 27 \\
\hline SEP & & & & \\
\hline $21-21$ & $\mathrm{~K} 6200$ & 16 & 1 & 15 \\
\hline
\end{tabular}

$\begin{array}{rr}67 & 91 \\ 47 & 94 \\ 66 & 144 \\ 42 & 99\end{array}$

$\begin{array}{ll}64 & 15 \\ 34 & 8.5 \\ 61 & 13 \\ 21 & 6.1\end{array}$

0.28
0.16
0.31
0.14

2.2

1.3

2.3

1.0

NITRO -

$\begin{array}{ll}\text { POTAS- } & \\ \text { SIUM, } & \text { SULFATE } \\ \text { DIS. } & \text { DIS- } \\ \text { SOLVED } & \text { SOLVED } \\ \text { (MG/L } & \text { (MG/L } \\ \text { AS K) } & \text { AS SO4) }\end{array}$

$\begin{array}{lcc}\text { CHLO- } & \text { NITRO- } & \text { NITRO- } \\ \text { RIDE, } & \text { GEN, } & \text { GEN, } \\ \text { DIS- } & \text { NITRITE } & \text { NO2+NO3 } \\ \text { SOLVED } & \text { TOTAL } & \text { TOTAL } \\ \text { (MG/L } & \text { (MG/L } & \text { (MG/L } \\ \text { AS CL) } & \text { AS N) } & \text { AS N) }\end{array}$

$\begin{array}{ccr} & \text { NITRO- } \\ \text { NITRO- } & \text { GEN, AM- } & \\ \text { GEN, } & \text { MONIA + } & \text { PHOS- } \\ \text { AMMONIA } & \text { ORGANIC } & \text { PHORUS } \\ \text { TOTAL } & \text { TOTAL } & \text { TOTAL } \\ \text { (MG/L } & \text { (MG/L } & \text { (MG/L } \\ \text { AS N) } & \text { AS N) } & \text { AS P) }\end{array}$

PHOS -

PHORUS ANTIMONY

DIS - TOTAL

SOLVED EPA

(MG/L (UG/L

AS P) AS $S B$

$\begin{array}{ll}1.5 & 0.200 \\ 0.50 & 0.090 \\ 0.90 & 0.190 \\ 0.70 & 0.160\end{array}$

$0.120<20.0$

$0.060<10.0$

$0.120<10.0$

$0.090<10.0$

0.120

0.70

0.160

$0.090<10.0$

LEAD, MERCURY NICKEL, TOTAL TOTAL TOTAL RECOV - RECOV - RECOV.

ERABLE ERABLE ERABLE

(UG/L (UG/L (UG/L

$\begin{array}{ll}\text { (UG/L } & \text { (UG/L } \\ \text { AS NI) } & \text { AS SE) }\end{array}$

AS PB) AS HG)

$\begin{array}{lccc} & & & \\ & \text { SILVER, } & \\ \text { SELE. } & \text { TOTAL, } & \text { TOTAL } & \\ \text { NIUM, } & \text { RECOV. } & \text { ERABLE } & \text { THAL. } \\ \text { TOTAL } & \text { ERABLE } & \text { EPA } & \text { TOTAL } \\ \text { (UG/L } & \text { (UG/L } & \text { (UG/L } & \text { (UG/L } \\ \text { AS SE) } & \text { AS AG) } & \text { AS AG) } & \text { AS TL) }\end{array}$
JUN 1992
$20-20$
AUG
$12 \cdot 12$
SEP
01.01 SEP

\begin{abstract}
$12<10.0<0.010$
$14<0.010<0.010$

$17 \quad 0.690<0.010$

$13<0.010<0.010$
\end{abstract}

48

$<0.10$

52

$<0.10$

$80<0.10$

3
4
6
11
$<2$
$<1$
$<2$
$<2$

$<1<1.00$

$<10$

$<1<0.500$

$<5$

52

0.10

$<1<0.500$

$<10$

$<1<0.500$

$<5$ 
Table 5. Water-quality data for storm-sewer outfall stations, Garland, Texas, 1992-93-Continued

08061635 - TRIBUTARY TO DUCK CREEK OUTFALL AT HIGHTOWER ROAD, GARLAND, TX (WY 1992)-ContinUEd

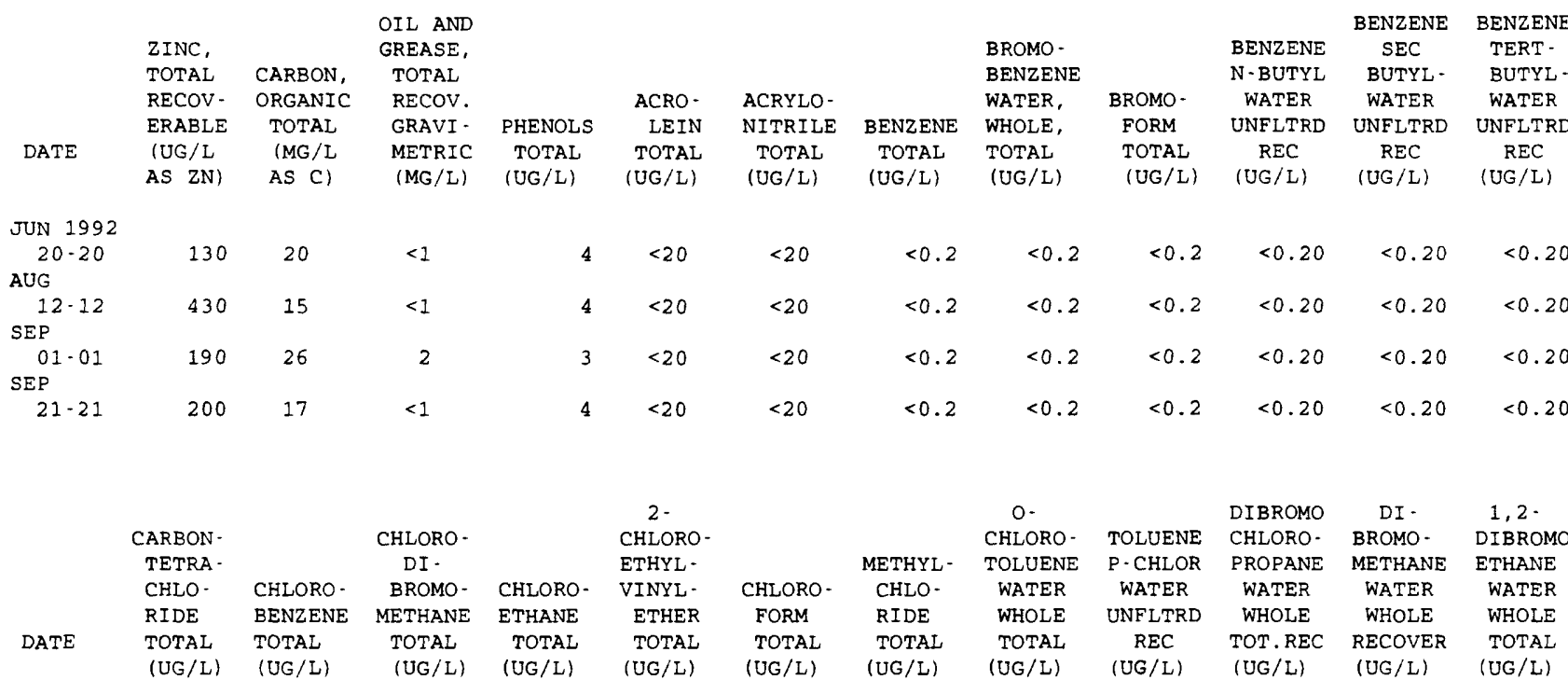

JUN 1992

$20-20$

AUG

$12-12$

SEP

$01 \cdot 01$

SEP

$21 \cdot 21$

$$
<0.2<0.20
$$

$<0.2<0.20$

$<0.2<0.20$

$<0.2$

$<0.20$

$<0.2$

$<0.2$

$<0.2$

$<0.2$

$<0.2$

$<0.2$

$<1.0$

$<0.2$

$<0.2$

$<0.2<0.20$

$<0.2$

$<0.2$

$<0.2$

$<0.20$

$<0.2$

$<0.2$

0.2

0.20

$<0.2$

$<0.2$

$<1.0$

0.7

$<0.2$

$<0.2$

$<0.20$

1, 1-DI

DI

$1,1-D I-$
CHLORO-
ETHYL-
ENE
TOTAL
(UG/L)

CHLORO-

CHLORO-

BROMO- ETHYL - PENE,

$\begin{array}{cc}\text { METHANE } & \text { ENE } \\ \text { TOTAL } & \text { TOTAL } \\ (U G / L) & (U G / L)\end{array}$

PENE, WH

BENZENE

BENZENE

BENZENE

DI -

O- 1,3-DI - 1,4-DI - CHLORO-

WATER WATER WATER

NNFLTRD

TOTAL

(UG/L)

REC

(UG/L)

UNFLTRD

REC

(UG/L)
DI -

METHANE

TOTAL

(UG/L)
1, I-DI - 1,2-DI -

CHLORO- CHLORO.

ETHANE

TOTAL

(UG/L)
ETHANE

TOTAL

(UG/L)
1,2-DI - CIS-1,2-

PHENYL - DI -

HYDRA- CHLORO- TRANSDI

ZINE ETHENE CHLORO

WATER WATER ETHENE

TOT.REC TOTAL TOTAL

$\begin{array}{lll}\text { TOT.REC } & \text { TOTAL } & \text { (UG/L) } \\ (U G / L)\end{array}$

\begin{tabular}{|c|c|c|c|c|c|c|c|c|c|c|c|c|}
\hline $20-20$ & $<0.2$ & $<0.2$ & $<0.2$ & $<5.0$ & $<5.0$ & $<5.0$ & $<0.2$ & $<0.2$ & $<0.2$ & $<5.0$ & $<0.2$ & $<0.2$ \\
\hline AUG & & & & & & & & & & & & \\
\hline $12-12$ & $<0.2$ & $<0.2$ & $<0.2$ & $<5.0$ & $<5.0$ & $<5.0$ & $<0.2$ & $<0.2$ & $<0.2$ & $<5.0$ & $<0.2$ & $<0.2$ \\
\hline SEP & & & & & & & & & & & & \\
\hline $01-01$ & $<0.2$ & $<0.2$ & $<0.2$ & $<5.0$ & $<5.0$ & $<5.0$ & $<0.2$ & $<0.2$ & $<0.2$ & $<5.0$ & $<0.2$ & $<0.2$ \\
\hline SEP & & & & & & & & & & & & \\
\hline $21-21$ & $<0.2$ & $<0.2$ & $<0.2$ & $<5.0$ & $<5.0$ & $<5.0$ & $<0.2$ & $<0.2$ & $<0.2$ & $<5.0$ & $<0.2$ & $<0.2$ \\
\hline
\end{tabular}

\begin{tabular}{|c|c|c|c|c|c|c|c|c|c|c|c|c|}
\hline & $1,2 \cdot \mathrm{DI}-$ & $\begin{array}{l}1,3-\mathrm{DI} \text { - } \\
\text { CHLORO- }\end{array}$ & $\begin{array}{l}2,2 \text {-DI } \\
\text { CHLORO- } \\
\text { PRO- }\end{array}$ & $\begin{array}{c}\text { CIS } \\
1,3-D I-\end{array}$ & $\begin{array}{l}\text { TRANS - } \\
1,3-\mathrm{DI} \text { - }\end{array}$ & & $\begin{array}{l}\text { HEXA- } \\
\text { CHLORO- }\end{array}$ & $\begin{array}{c}\text { ISO- } \\
\text { PROPYL- } \\
\text { BENZENE }\end{array}$ & $\begin{array}{l}\text { P-ISO- } \\
\text { PROPYL - } \\
\text { TOLUENE }\end{array}$ & & $\begin{array}{c}\text { METHYL - } \\
\text { ENE }\end{array}$ & \\
\hline & CHLORO- & PROPANE & PANE & CHLORO- & CHLORO- & ETHYL - & BUT - & WATER & WATER & METHYL- & CHLO- & NAPHTH- \\
\hline DATE & $\begin{array}{c}\text { PROPANE } \\
\text { TOTAL } \\
\text { (UG/L) }\end{array}$ & $\begin{array}{l}\text { WAT. WH } \\
\text { TOTAL } \\
\text { (UG/L) }\end{array}$ & $\begin{array}{l}\text { WAT, WH } \\
\text { TOTAL } \\
\text { (UG/L) }\end{array}$ & $\begin{array}{l}\text { PROPENE } \\
\text { TOTAL } \\
\text { (UG/L) }\end{array}$ & $\begin{array}{l}\text { PROPENE } \\
\text { TOTAL } \\
\text { (UG/L) }\end{array}$ & $\begin{array}{l}\text { BENZENE } \\
\text { TOTAL } \\
\text { (UG/L) }\end{array}$ & $\begin{array}{c}\text { ADIENE } \\
\text { TOTAL } \\
\text { (UG/L) }\end{array}$ & $\begin{array}{l}\text { WHOLE } \\
\text { REC } \\
(U G / L)\end{array}$ & $\begin{array}{l}\text { WHOLE } \\
\text { REC } \\
\text { (UG/L) }\end{array}$ & $\begin{array}{l}\text { BROMIDE } \\
\text { TOTAL } \\
\text { (UG/L) }\end{array}$ & $\begin{array}{l}\text { RIDE } \\
\text { TOTAL } \\
\text { (UG/L) }\end{array}$ & $\begin{array}{c}\text { ALENE } \\
\text { TOTAL } \\
(U G / L)\end{array}$ \\
\hline
\end{tabular}

JUN 1992

\begin{tabular}{|c|c|c|c|c|c|c|c|c|c|c|c|c|}
\hline $20-20$ & $<0.2$ & $<0.2$ & $<0.2$ & $<0.2$ & $<0.2$ & $<0.2$ & $<5.0$ & $<0.20$ & $<0.20$ & $<0.2$ & $<0.2$ & $<5.0$ \\
\hline AUG & & & & & & & & & & & & \\
\hline $12 \cdot 12$ & $<0.2$ & $<0.2$ & $<0.2$ & $<0.2$ & $<0.2$ & $<0.2$ & $<5.0$ & $<0.20$ & $<0.20$ & $<0.2$ & $<0.2$ & $<5.0$ \\
\hline SEP & & & & & & & & & & & & \\
\hline 01.01 & $<0.2$ & $<0.2$ & $<0.2$ & $<0.2$ & $<0.2$ & $<0.2$ & $<5.0$ & $<0.20$ & $<0.20$ & $<0.2$ & $<0.2$ & $<5.0$ \\
\hline SEP & & & & & & & & & & & & \\
\hline $21-21$ & $<0.2$ & $<0.2$ & $<0.2$ & $<0.2$ & $<0.2$ & $<0.2$ & $<5.0$ & $<0.20$ & $<0.20$ & $<0.2$ & $<0.2$ & $<5.0$ \\
\hline
\end{tabular}


Table 5. Water-quality data for storm-sewer outfall stations, Garland, Texas, 1992-93-Continued

08061635 - TRIBUTARY TO DUCK CREEK OUTFALL AT HIGHTOWER ROAD, GARLAND, TX (WY 1992)-CONTINUEd

\begin{tabular}{|c|c|c|c|c|c|c|c|c|c|c|c|c|}
\hline & BENZENE & & $\begin{array}{c}\text { ETHANE, } \\
1112-\end{array}$ & $\begin{array}{l}\text { ETHANE, } \\
1,1,2,2\end{array}$ & TETRA - & & $\begin{array}{c}1,2,3- \\
\text { TRI- }\end{array}$ & $\begin{array}{l}\text { BENZENE } \\
1,2,4 .\end{array}$ & $1,1,1$. & $1,1,2-$ & $\mathrm{TR}$ & \\
\hline & N-PROPY & & TETRA - & TETRA - & CHLORO- & & CHLORO & TRI - & TRI - & TRI - & CHLORO- & CHLORO- \\
\hline & WATER & & CHLORO- & CHLORO- & ETHYL - & & BENZENE & CHLORO- & CHLORO- & CHLORO - & & \\
\hline & UNFLTRD & STY & WAT UNF & WAT UNF & ENE & TOLUENE & WAT, WH & WAT UNF & ETHANE & ETHANE & ENE & MET \\
\hline TE & $\begin{array}{c}\text { REC } \\
(U G / L)\end{array}$ & $\begin{array}{l}\text { TOTAL } \\
\text { (UG/L) }\end{array}$ & $\begin{array}{c}\text { REC } \\
\text { (UG/L) }\end{array}$ & $\begin{array}{c}\text { REC } \\
(U G / L)\end{array}$ & $\begin{array}{l}\text { TOTAL } \\
(U G / L)\end{array}$ & $\begin{array}{l}\text { TOTAL } \\
\text { (UG/L) }\end{array}$ & $\begin{array}{l}\text { REC } \\
(U G / L)\end{array}$ & $\begin{array}{c}\text { REC } \\
\text { (UG / L ) }\end{array}$ & $\begin{array}{l}\text { TOTAL } \\
\text { (UG/L) }\end{array}$ & $\begin{array}{l}\text { TOTAL } \\
\text { (UG/L) }\end{array}$ & $\begin{array}{l}\text { TOTAL } \\
\text { (UG/L) }\end{array}$ & $\begin{array}{l}\text { TOTAL } \\
(\text { UG/L) }\end{array}$ \\
\hline
\end{tabular}

\begin{tabular}{|c|c|c|c|c|c|c|c|c|c|c|c|c|}
\hline $\begin{array}{c}\text { JUN } 1992 \\
20-20\end{array}$ & $<0.20$ & $<0,2$ & $<0,2$ & $<0.2$ & $<0.2$ & $<0.2$ & $<0.20$ & $<5,0$ & $<0.2$ & $<0.2$ & $<0.2$ & $<0.2$ \\
\hline \multicolumn{13}{|l|}{ AUG } \\
\hline $12-12$ & $<0.20$ & $<0.2$ & $<0.2$ & $<0.2$ & $<0.2$ & $<0.2$ & $<0.20$ & $<5.0$ & $<0.2$ & $<0.2$ & $<0.2$ & $<0.2$ \\
\hline \multicolumn{13}{|l|}{ SEP } \\
\hline $01-01$ & $<0.20$ & $<0.2$ & $<0.2$ & $<0.2$ & $<0.2$ & $<0.2$ & $<0.20$ & $<5.0$ & $<0.2$ & $<0.2$ & $<0.2$ & $<0.2$ \\
\hline \multicolumn{13}{|l|}{ SEP } \\
\hline $21-21$ & $<0.20$ & $<0.2$ & $<0.2$ & $<0.2$ & $<0.2$ & $<0.2$ & $<0.20$ & $<5.0$ & $<0.2$ & $<0.2$ & $<0.2$ & $<0.2$ \\
\hline
\end{tabular}

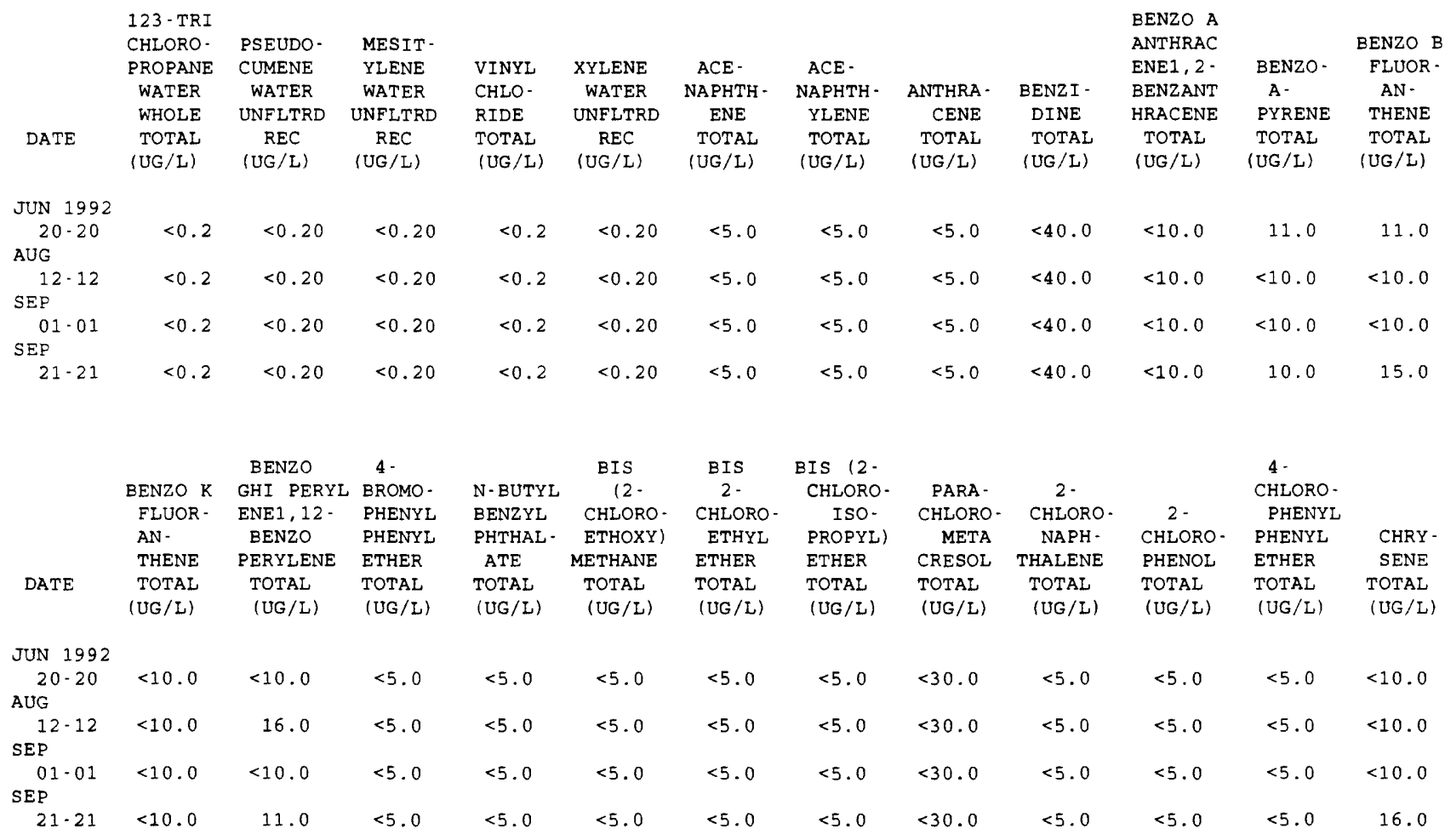

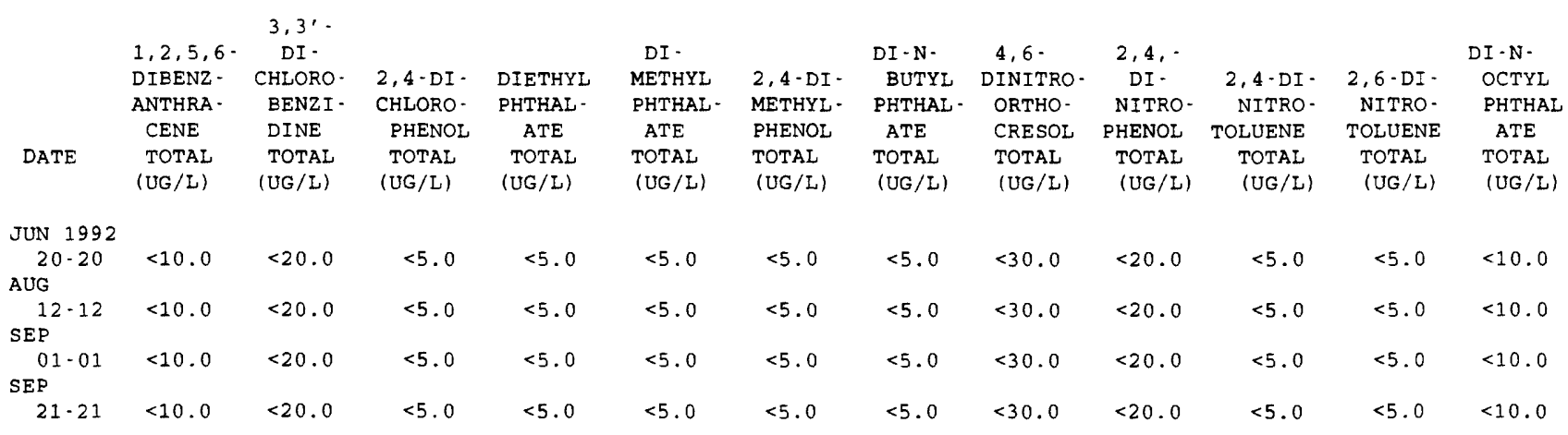


Table 5. Water-quality data for storm-sewer outfall stations, Garland, Texas, 1992-93-Continued

08061635 - TRIBUTARY TO DUCK CREEK OUTFALL AT HIGHTOWER ROAD, GARLAND, TX (WY 1992)-COntInUEd

\begin{tabular}{|c|c|c|c|c|c|c|c|c|c|c|c|c|}
\hline DATE & $\begin{array}{l}\text { BIS ( } 2 \text { - } \\
\text { ETHYL } \\
\text { HEXYL) } \\
\text { PHTHAL- } \\
\text { ATE } \\
\text { TOTAL } \\
\text { (UG/L) }\end{array}$ & $\begin{array}{l}\text { FLUOR - } \\
\text { ANTHENE } \\
\text { TOTAL } \\
\text { (UG/L) }\end{array}$ & $\begin{array}{c}\text { FLUOR - } \\
\text { ENE } \\
\text { TOTAL } \\
\text { (UG/L) }\end{array}$ & $\begin{array}{l}\text { HEXA- } \\
\text { CHLORO- } \\
\text { BENZENE } \\
\text { TOTAL } \\
\text { (UG/L) }\end{array}$ & $\begin{array}{l}\text { HEXA- } \\
\text { CHLORO- } \\
\text { CYCLO- } \\
\text { PENT- } \\
\text { ADIENE } \\
\text { TOTAL } \\
\text { (UG/L) }\end{array}$ & $\begin{array}{l}\text { HEXA- } \\
\text { CHLORO- } \\
\text { ETHANE } \\
\text { TOTAL } \\
\text { (UG/L) }\end{array}$ & $\begin{array}{c}\text { INDENO } \\
(1,2,3- \\
\text { CD) } \\
\text { PYRENE } \\
\text { TOTAL } \\
(U G / L)\end{array}$ & $\begin{array}{l}\text { ISO- } \\
\text { PHORONE } \\
\text { TOTAL } \\
\text { (UG/L) }\end{array}$ & $\begin{array}{l}\text { NITRO- } \\
\text { BENZENE } \\
\text { TOTAL } \\
\text { (UG/L) }\end{array}$ & $\begin{array}{c}\text { N-NITRO- } \\
\text { SODI- } \\
\text { METHY - } \\
\text { LAMINE } \\
\text { TOTAL } \\
(\text { UG/L) }\end{array}$ & $\begin{array}{l}2 \text { - } \\
\text { NITRO- } \\
\text { PHENOL } \\
\text { TOTAL } \\
\text { (UG/L) }\end{array}$ & $\begin{array}{l}\quad 4- \\
\text { NITRO- } \\
\text { PHENOL } \\
\text { TOTAL } \\
\text { (UG/L) }\end{array}$ \\
\hline JUN 1992 & & & & & & & & & & & & \\
\hline $\begin{array}{l}20-20 \\
\text { AUG }\end{array}$ & 6.0 & 23.0 & $<5.0$ & $<5.0$ & $<5.0$ & $<5.0$ & $<10.0$ & $<5.0$ & $<5.0$ & $<5.0$ & $<5.0$ & $<30.0$ \\
\hline $\begin{array}{l}12-12 \\
\text { SEP }\end{array}$ & 5.0 & 20.0 & $<5.0$ & $<5.0$ & $<5.0$ & $<5.0$ & 18.0 & $<5.0$ & $<5.0$ & $<5.0$ & $<5.0$ & $<30.0$ \\
\hline $\begin{array}{l}01-01 \\
\operatorname{SEP}\end{array}$ & $<5.0$ & 19.0 & $<5.0$ & $<5.0$ & $<5.0$ & $<5.0$ & $<10.0$ & $<5.0$ & $<5.0$ & $<5.0$ & $<5.0$ & $<30.0$ \\
\hline $21-21$ & $<5.0$ & 35.0 & $<5.0$ & $<5.0$ & $<5.0$ & $<5.0$ & 11.0 & $<5.0$ & $<5.0$ & $<5.0$ & $<5.0$ & $<30.0$ \\
\hline DATE & $\begin{array}{c}\text { N- } \\
\text { NITRO- } \\
\text { SODI - N- } \\
\text { PROPYL- } \\
\text { AMINE } \\
\text { TOTAL } \\
\text { (UG/L) }\end{array}$ & $\begin{array}{l}\text { N-NITRO- } \\
\text { SODI - } \\
\text { PHENY - } \\
\text { LAMINE } \\
\text { TOTAL } \\
(U G / L)\end{array}$ & $\begin{array}{l}\text { PENTA- } \\
\text { CHLORO- } \\
\text { PHENOL } \\
\text { TOTAL } \\
\text { (UG/L) }\end{array}$ & $\begin{array}{l}\text { PHENAN - } \\
\text { THRENE } \\
\text { TOTAL } \\
\text { (UG/L) }\end{array}$ & $\begin{array}{l}\text { PHENOL } \\
\text { (C6H - } \\
5 O H) \\
\text { TOTAL } \\
(U G / L)\end{array}$ & $\begin{array}{c}\text { PYRENE } \\
\text { TOTAL } \\
\text { (UG/L) }\end{array}$ & $\begin{array}{l}2,4,6- \\
\text { TRI- } \\
\text { CHLORO- } \\
\text { PHENOL } \\
\text { TOTAL } \\
\text { (UG/L) }\end{array}$ & $\begin{array}{c}\text { ALDRIN, } \\
\text { TOTAL } \\
\text { (UG/L) }\end{array}$ & $\begin{array}{c}\text { P, } P^{\prime} \\
\text { DDT, } \\
\text { TOTAL } \\
\text { (UG/L) }\end{array}$ & $\begin{array}{l}\text { AL PHA } \\
\text { BHC } \\
\text { TOTAL } \\
\text { (UG/L) }\end{array}$ & $\begin{array}{c}\text { BETA } \\
\text { BENZENE } \\
\text { HEXA- } \\
\text { CHLOR- } \\
\text { IDE } \\
\text { TOTAL } \\
\text { (UG/L) }\end{array}$ & $\begin{array}{c}\text { DELTA } \\
\text { BENZENE } \\
\text { HEXA- } \\
\text { CHLOR- } \\
\text { IDE } \\
\text { TOTAL } \\
(U G / L)\end{array}$ \\
\hline JUN 1992 & & & & & & & & & & & & \\
\hline $\begin{array}{l}20-20 \\
\text { AUG }\end{array}$ & $<5.0$ & $<5.0$ & $<30.0$ & 11.0 & $<5.0$ & 16.0 & $<20.0$ & $<0.040$ & $<0.10$ & $<0.03$ & $<0.03$ & $<0.09$ \\
\hline $\begin{array}{l}12-12 \\
\operatorname{SEP}\end{array}$ & $<5.0$ & $<5.0$ & $<30.0$ & 9.0 & $<5.0$ & 14.0 & $<20.0$ & $<0.040$ & $<0.10$ & $<0.03$ & $<0.03$ & $<0.09$ \\
\hline $\begin{array}{l}01-01 \\
\text { SEP }\end{array}$ & $<5.0$ & $<5.0$ & $<30.0$ & 7.0 & $<5.0$ & 14.0 & $<20.0$ & $<0.040$ & $<0.10$ & $<0.03$ & $<0.03$ & $<0.09$ \\
\hline $21 \cdot 21$ & $<5.0$ & $<5.0$ & $<30.0$ & 17.0 & $<5.0$ & 26.0 & $<20.0$ & $<0.040$ & $<0.10$ & $<0.03$ & $<0.03$ & $<0.09$ \\
\hline DATE & $\begin{array}{l}\text { LINDANE } \\
\text { TOTAL } \\
\text { (UG/L) }\end{array}$ & $\begin{array}{l}\text { CHLOR - } \\
\text { DANE } \\
\text { CIS } \\
\text { WATER } \\
\text { WHOLE } \\
\text { TOTAL } \\
\text { (UG/L) }\end{array}$ & $\begin{array}{l}\text { CHLOR - } \\
\text { DANE } \\
\text { TRANS } \\
\text { WATER } \\
\text { WHOLE } \\
\text { TOTAL } \\
\text { (UG/L) }\end{array}$ & $\begin{array}{l}\text { CHLOR- } \\
\text { DANE, } \\
\text { TOTAL } \\
\text { (UG/L) }\end{array}$ & $\begin{array}{l}\text { P, } P^{\prime} \\
\text { DDD, } \\
\text { TOTAL } \\
\text { (UG/L) }\end{array}$ & $\begin{array}{l}\text { P, } P^{\prime} \\
\text { DDE, } \\
\text { TOTAL } \\
(U G / L)\end{array}$ & $\begin{array}{l}\text { DI- } \\
\text { ELDRIN } \\
\text { TOTAL } \\
(U G / L)\end{array}$ & $\begin{array}{c}\text { ENDO- } \\
\text { SULFAN- } \\
\text { I } \\
\text { WATER } \\
\text { WHOLE } \\
\text { REC } \\
\text { (UG/L) }\end{array}$ & $\begin{array}{l}\text { ENDO- } \\
\text { SULFAN } \\
\text { BETA } \\
\text { TOTAL } \\
\text { (UG/L) }\end{array}$ & $\begin{array}{l}\text { ENDO- } \\
\text { SULFAN } \\
\text { SULFATE } \\
\text { TOTAL } \\
\text { (UG/L) }\end{array}$ & $\begin{array}{c}\text { ENDRIN } \\
\text { WATER } \\
\text { UNFLTRD } \\
\text { REC } \\
(U G / L)\end{array}$ & $\begin{array}{c}\text { ENDRIN } \\
\text { ALDE - } \\
\text { HYDE } \\
\text { TOTAL } \\
\text { (UG/L) }\end{array}$ \\
\hline $\begin{array}{c}\text { JUN } 1992 \\
20-20\end{array}$ & $<0.030$ & $<0.10$ & $<0.10$ & $<0.1$ & $<0.10$ & $<0.04$ & $<0.020$ & $<0.10$ & $<0.04$ & $<0.60$ & $<0.060$ & $<0.20$ \\
\hline AUG & & & & & & & & & & & & \\
\hline $\begin{array}{l}12-12 \\
S E P\end{array}$ & $<0.030$ & $<0.10$ & $<0.10$ & $<0.1$ & $<0.10$ & $<0.04$ & $<0.020$ & $<0.10$ & $<0.04$ & $<0.60$ & $<0.060$ & $<0.20$ \\
\hline $\begin{array}{l}01-01 \\
\text { EP }\end{array}$ & $<0.030$ & $<0.10$ & $<0.10$ & $<0.1$ & $<0.10$ & $<0.04$ & $<0.020$ & $<0.10$ & $<0.04$ & $<0.60$ & $<0.060$ & $<0.20$ \\
\hline $21-21$ & $<0.030$ & $<0.10$ & $<0.10$ & $<0.1$ & $<0.10$ & $<0.04$ & $<0.020$ & $<0.10$ & $<0.04$ & $<0.60$ & $<0.060$ & $<0.20$ \\
\hline
\end{tabular}

\begin{tabular}{|c|c|c|c|c|c|c|c|c|c|c|c|}
\hline & & HEPTA - & & AROCLOR & AROCLOR & AROCLOR & AROCLOR & AROCLOR & AROCLOR & AROCLOR & \\
\hline & HEPTA - & CHLOR & TOX - & 1016 & 1221 & 1232 & 1242 & 1248 & 1254 & 1260 & DI - \\
\hline DATE & $\begin{array}{l}\text { CHLOR, } \\
\text { TOTAL } \\
\text { (UG/L) }\end{array}$ & $\begin{array}{c}\text { EPOXIDE } \\
\text { TOTAL } \\
\text { (UG/L) }\end{array}$ & $\begin{array}{c}\text { APHENE, } \\
\text { TOTAL } \\
\text { (UG/L) }\end{array}$ & $\begin{array}{c}\text { PCB } \\
\text { TOTAL } \\
\text { (UG/L) }\end{array}$ & $\begin{array}{c}\text { PCB } \\
\text { TOTAL } \\
\text { (UG/L) }\end{array}$ & $\begin{array}{c}\text { PCB } \\
\text { TOTAL } \\
\text { (UG/L) }\end{array}$ & $\begin{array}{l}\text { PCB } \\
\text { TOTAL } \\
\text { (UG/L) }\end{array}$ & $\begin{array}{l}\text { PCB } \\
\text { TOTAL } \\
\text { (UG/L) }\end{array}$ & $\begin{array}{l}\text { PCB } \\
\text { TOTAL } \\
\text { (UG/L) }\end{array}$ & $\begin{array}{l}\text { PCB } \\
\text { TOTAL } \\
\text { (UG/L) }\end{array}$ & $\begin{array}{c}\text { AZINON, } \\
\text { TOTAL } \\
\text { (UG/L) }\end{array}$ \\
\hline \multicolumn{12}{|l|}{ JUN 1992} \\
\hline $20-20$ & $<0.030$ & $<0.80$ & $<2$ & $<0.1$ & $<1.0$ & $<0.1$ & $<0.1$ & $<0.1$ & $<0.1$ & $<0.1$ & 0.20 \\
\hline \multicolumn{12}{|l|}{ AUG } \\
\hline $12-12$ & $<0.030$ & $<0.80$ & $<2$ & $<0.1$ & $<1.0$ & $<0.1$ & $<0.1$ & $<0.1$ & $<0.1$ & $<0.1$ & 0.30 \\
\hline \multicolumn{12}{|l|}{ SEP } \\
\hline $01-01$ & $<0.030$ & $<0.80$ & $<2$ & $<0.1$ & $<1.0$ & $<0.1$ & $<0.1$ & $<0.1$ & $<0.1$ & $<0.1$ & $<0.10$ \\
\hline \multicolumn{12}{|l|}{ SEP } \\
\hline $21-21$ & $<0.030$ & $<0.80$ & $<2$ & $<0.1$ & $<1.0$ & $<0.1$ & $<0.1$ & $<0.1$ & $<0.1$ & $<0.1$ & 0.10 \\
\hline
\end{tabular}


Table 5. Water-quality data for storm-sewer outfall stations, Garland, Texas, 1992-93-Continued

08061635 - TRIBUTARY TO DUCK CREEK OUTFALI AT HIGHTOWER ROAD, GARLAND, TX (WY 1993)

\begin{tabular}{|c|c|c|c|c|c|c|c|c|c|c|c|c|}
\hline & & $\begin{array}{l}\text { PRECIP- } \\
\text { ITATION } \\
\text { TOTAL }\end{array}$ & $\begin{array}{l}\text { ELAPSED } \\
\text { TIME } \\
\text { OF }\end{array}$ & $\begin{array}{l}\text { STORM } \\
\text { WATER }\end{array}$ & $\begin{array}{l}\text { SPE- } \\
\text { CIFIC } \\
\text { CON- } \\
\text { DUCT. }\end{array}$ & $\begin{array}{l}\text { SPE- } \\
\text { CIFIC } \\
\text { CON- } \\
\text { DUCT- } \\
\text { ANCE }\end{array}$ & $\begin{array}{c}\text { PH } \\
\text { WATER } \\
\text { WHOLE } \\
\text { FIELD } \\
\text { (STAND- }\end{array}$ & $\begin{array}{c}\text { PH } \\
\text { WATER } \\
\text { WHOLE } \\
\text { LAB } \\
\text { (STAND- }\end{array}$ & $\begin{array}{l}\text { TEMPER - } \\
\text { ATURE }\end{array}$ & $\begin{array}{c}\text { OXYGEN } \\
\text { DEMAND, } \\
\text { CHEM- } \\
\text { ICAL } \\
\text { (HIGH }\end{array}$ & $\begin{array}{l}\text { OXYGEN } \\
\text { DEMAND, } \\
\text { BIO- } \\
\text { CHEM- } \\
\text { ICAL, }\end{array}$ & $\begin{array}{l}\text { COLI. } \\
\text { FORM, } \\
\text { FECAL, } \\
0.7 \\
\text { UM-MF }\end{array}$ \\
\hline TE & TIME & $\begin{array}{l}\text { INCHES / } \\
\text { STORM }\end{array}$ & $\begin{array}{c}\text { STORM } \\
\text { (HOURS) }\end{array}$ & $\begin{array}{l}\text { FLOW } \\
\text { (MGD) }\end{array}$ & $\begin{array}{l}\text { ANCE } \\
\text { (US /CM) }\end{array}$ & $\begin{array}{c}\text { LAB } \\
\text { (US/CM) }\end{array}$ & $\begin{array}{l}\text { ARD } \\
\text { UNITS) }\end{array}$ & $\begin{array}{l}\text { ARD } \\
\text { UNITS) }\end{array}$ & $\begin{array}{c}\text { WATER } \\
\text { (DEG C) }\end{array}$ & $\begin{array}{l}\text { LEVEL) } \\
\text { (MG/L) }\end{array}$ & $\begin{array}{l}5 \text { DAY } \\
(\mathrm{MG} / \mathrm{L})\end{array}$ & $\begin{array}{l}\text { (COLS./ } \\
100 \mathrm{ML} \text { ) }\end{array}$ \\
\hline
\end{tabular}

$\begin{array}{ll}\text { OCT } 1992 & \\ 15-15 & 2045 \\ \text { OCT } & \\ 29-29 & 0100 \\ \text { NOV } & \end{array}$

NOV $19-19 \quad 0235$

$\begin{array}{lll}0.93 & 1.1 & 0.78 \\ 0.53 & 4.8 & 0.39\end{array}$

0.549 .9

0.48

50
66
115

137
83
120

$\begin{array}{ll}9.2 & 7.8 \\ 7.0 & 7.8 \\ 8.5 & 7.9\end{array}$
21.0
19.0
17.5

63

$5.1 \quad 14000$

$6.8 \quad 11000$

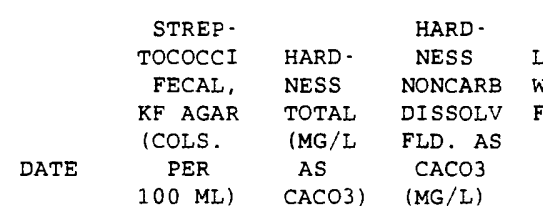

$\begin{array}{lll}\text { ALKA- } & \text { SOLIDS, } & \text { RESIDUE } \\ \text { LINITY } & \text { SUM OF } & \text { TOTAL } \\ \text { WAT DIS } & \text { CONSTI- } & \text { AT } 105 \\ \text { FIX END } & \text { TUENTS, } & \text { DEG. C. } \\ \text { FIELD } & \text { DIS- } & \text { SUS - } \\ \text { CACO3 } & \text { SOLVED } & \text { PENDED } \\ (M G / L) & \text { (MG/L) } & \text { (MG/L) }\end{array}$

SOLIDS,

SOLIDS,

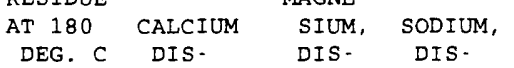

DIS -

$\begin{array}{ll}\text { SOLVED } & \text { (MG/L } \\ (\mathrm{MG} / L) & \text { AS CA) }\end{array}$

AS CA)

SOLVED

(MG/L

SOLVED

(MG/I

AS MG)

AS NA)

PERCENT

\begin{tabular}{|c|c|c|c|c|c|c|c|c|c|c|c|c|}
\hline $\begin{array}{l}\text { OCT } 1992 \\
15-15 \\
\text { OCT }\end{array}$ & $\mathrm{K} 1800$ & 13 & 2 & 11 & 40 & 115 & 30 & 5.1 & 0.11 & 0.70 & 10 & 0.1 \\
\hline $\begin{array}{l}29-29 \\
\text { NOV }\end{array}$ & 2500 & 21 & 3 & 18 & 54 & 158 & 54 & 8.2 & 0.17 & 1.0 & 9 & 0.1 \\
\hline $19 \cdot 19$ & 10000 & 29 & 1 & 28 & 53 & 44 & 43 & 11 & 0.28 & 2.3 & 14 & 0.2 \\
\hline
\end{tabular}

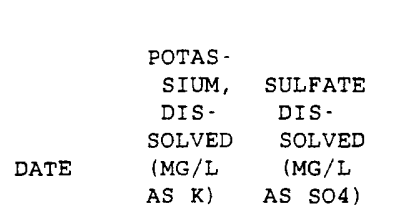

$\begin{array}{lcccc}\text { CHLO } & \text { NITRO } & \text { NITRO } & \text { NITRO- } & \text { GEN, AM- } \\ \text { RIDE, } & \text { GEN, } & \text { GEN, } & \text { GEN, } & \text { MONIA + } \\ \text { DIS- } & \text { NITRITE } & \text { NO2+NO3 } & \text { AMMONIA } & \text { ORGANIC } \\ \text { SOLVED } & \text { TOTAL } & \text { TOTAL } & \text { TOTAL } & \text { TOTAL } \\ \text { (MG } / L & \text { (MG } / \text { L } & \text { (MG } / \text { L } & \text { (MG } / L & \text { (MG } / L \\ \text { AS CL) } & \text { AS N) } & \text { AS N) } & \text { AS N) } & \text { AS N) }\end{array}$
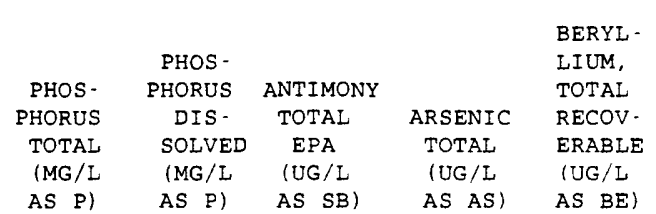

OCT 1992

$15-15$
OCT
$29-29$
NOV

$0.90 \quad 2.8$

$0.80 \quad 0.030$

0.270

0.140

0.70

0.160

$0.050<10.0$

0.90

0.180

$0.060<10.0$

0.60

0.140

$0.080<20.0$

$0.560 \quad 0.120$

0.110

LEAD

RECOV. TOTAL TOTAL

ERABLE ERABIE ERABLE

(UG/L) (UG/L (UG/L

(UG/L
AS NI)

CADMIUM
TOTAL
RECOV.
ERABLE
(UG $/$ L
AS CD)

CHRO

TOTAL

(UG/L (UG/L

AS $(R)$
$\begin{array}{ccc}(\mathrm{UG} / \mathrm{L} & (\mathrm{MG} / \mathrm{L} & (\mathrm{MG} / \mathrm{L} \\ \mathrm{AS} \mathrm{CU}) & \mathrm{AS} \mathrm{CN}) & \mathrm{AS} \mathrm{CN})\end{array}$
AS PB)

AS HG)

SELE-
NIUM,
TOTAL
(UG/L
AS SE)

$\begin{array}{ccc} & \text { SILVER, } \\ \text { SILVER, } & \text { TOTAL } & \\ \text { TOTAL } & \text { RECOV- } & \text { THAL- } \\ \text { RECOV- } & \text { ERABLE } & \text { LIUM, } \\ \text { ERABLE } & \text { EPA } & \text { TOTAL } \\ \text { (UG/L } & \text { (UG/L } & \text { (UG/L } \\ \text { AS AG) } & \text { AS AG) } & \text { AS TL) }\end{array}$

OCT 1992

$15-15$
OCT
$29-29$
NOV

$19-19$

$\begin{array}{rrr}24 & <0.010 & <0.010 \\ 11 & <0.010 & 0.020 \\ 7 & <0.010 & <0.010\end{array}$

$71<0.10$

$57 \quad 0.10$

$\begin{array}{rr}1 & 10 \\ <1 & 3\end{array}$

$7<0.010<0.010$

17

$<0.10$

7
5
3

$\begin{array}{llll}<2 & <1 & <0.500 & <5 \\ <2 & <1 & <0.500 & <5 \\ <2 & <1 & <0.500 & <5\end{array}$


Table 5. Water-quality data for storm-sewer outfall stations, Garland, Texas, 1992-93-Continued

08061635 - TRIBUTARY TO DUCK CREEK OUTFALL AT HIGHTOWER ROAD, GARLAND, TX (WY 1993)-CONTIIUEd

\begin{tabular}{|c|c|c|c|c|c|c|c|c|c|c|c|c|}
\hline DATE & $\begin{array}{l}\text { ZINC, } \\
\text { TOTAL } \\
\text { RECOV. } \\
\text { ERABLE } \\
\text { (UG/L } \\
\text { AS ZN) }\end{array}$ & $\begin{array}{c}\text { CARBON, } \\
\text { ORGANIC } \\
\text { TOTAL } \\
\text { (MG/L } \\
\text { AS C) }\end{array}$ & $\begin{array}{l}\text { OIL AND } \\
\text { GREASE, } \\
\text { TOTAL } \\
\text { RECOV. } \\
\text { GRAVI - } \\
\text { METRIC } \\
\text { (MG/L) }\end{array}$ & $\begin{array}{l}\text { PHENOLS } \\
\text { TOTAL } \\
\text { (UG/L) }\end{array}$ & $\begin{array}{l}\text { ACRO- } \\
\text { LEIN } \\
\text { TOTAL } \\
\text { (UG/L) }\end{array}$ & $\begin{array}{c}\text { ACRYLO- } \\
\text { NITRILE } \\
\text { TOTAL } \\
\text { (UG/L) }\end{array}$ & $\begin{array}{c}\text { BENZENE } \\
\text { TOTAL } \\
\text { (UG/L) }\end{array}$ & $\begin{array}{l}\text { BROMO- } \\
\text { BENZENE } \\
\text { WATER, } \\
\text { WHOLE, } \\
\text { TOTAL } \\
\text { (UG/L) }\end{array}$ & $\begin{array}{l}\text { BROMO - } \\
\text { FORM } \\
\text { TOTAL } \\
\text { (UG/L) }\end{array}$ & $\begin{array}{c}\text { BENZENE } \\
\text { N-BUTYL } \\
\text { WATER } \\
\text { UNFLTRD } \\
\text { REC } \\
\text { (UG/L) }\end{array}$ & $\begin{array}{l}\text { BENZENE } \\
\text { SEC } \\
\text { BUTYL- } \\
\text { WATER } \\
\text { UNFLTRD } \\
\text { REC } \\
\text { (UG/L) }\end{array}$ & $\begin{array}{l}\text { BENZENE } \\
\text { TERT- } \\
\text { BUTYL- } \\
\text { WATER } \\
\text { UNFLTRD } \\
\text { REC } \\
\text { (UG/L) }\end{array}$ \\
\hline $\begin{array}{l}\text { OCT } 1992 \\
15.15 \\
\text { OCT }\end{array}$ & 190 & 13 & $<1$ & 5 & $<20$ & $<20$ & $<0.2$ & $<0.2$ & $<0.2$ & $<0.20$ & $<0.20$ & $<0.20$ \\
\hline $\begin{array}{l}29-29 \\
\text { NoV }\end{array}$ & 170 & 14 & 1 & 5 & $<20$ & $<20$ & $<0.2$ & $<0.2$ & $<0.2$ & $<0.20$ & $<0.20$ & $<0.20$ \\
\hline $19-19$ & 90 & 14 & 4 & 3 & $<20$ & $<20$ & $<0.2$ & $<0.2$ & $<0.2$ & DIBROMO & $<0.20$ & $<0.20$ \\
\hline & $\begin{array}{l}\text { CARBON- } \\
\text { TETRA - } \\
\text { CHLO- } \\
\text { RIDE }\end{array}$ & $\begin{array}{l}\text { CHLORO- } \\
\text { BENZENE }\end{array}$ & $\begin{array}{c}\text { CHLORO- } \\
\text { DI - } \\
\text { BROMO - } \\
\text { METHANE }\end{array}$ & $\begin{array}{l}\text { CHLORO- } \\
\text { ETHANE }\end{array}$ & $\begin{array}{l}\text { CHLORO- } \\
\text { ETHYL- } \\
\text { VINYL- } \\
\text { ETHER }\end{array}$ & $\begin{array}{l}\text { CHLORO- } \\
\text { FORM }\end{array}$ & $\begin{array}{l}\text { METHYL- } \\
\text { CHLO- } \\
\text { RIDE }\end{array}$ & $\begin{array}{c}\text { CHLORO- } \\
\text { TOLUENE } \\
\text { WATER } \\
\text { WHOLE }\end{array}$ & $\begin{array}{c}\text { TOLUENE } \\
\text { P-CHLOR } \\
\text { WATER } \\
\text { UNFLTRD }\end{array}$ & $\begin{array}{l}\text { CHLORO - } \\
\text { PROPANE } \\
\text { WATER } \\
\text { WHOLE }\end{array}$ & $\begin{array}{l}\text { BROMO- } \\
\text { METHANE } \\
\text { WATER } \\
\text { WHOLE }\end{array}$ & $\begin{array}{l}\text { DIBROMO } \\
\text { ETHANE } \\
\text { WATER } \\
\text { WHOLE }\end{array}$ \\
\hline DATE & $\begin{array}{l}\text { TOTAL } \\
(U G / L)\end{array}$ & $\begin{array}{l}\text { TOTAL } \\
(U G / L)\end{array}$ & $\begin{array}{l}\text { TOTAL } \\
\text { (UG/L) }\end{array}$ & $\begin{array}{l}\text { TOTAL } \\
\text { (UG/L) }\end{array}$ & $\begin{array}{l}\text { TOTAL } \\
\text { (UG/L) }\end{array}$ & $\begin{array}{l}\text { TOTAL } \\
\text { (UG/L) }\end{array}$ & $\begin{array}{l}\text { TOTAL } \\
\text { (UG/L) }\end{array}$ & $\begin{array}{l}\text { TOTAL } \\
\text { (UG/L) }\end{array}$ & $\begin{array}{c}\text { REC } \\
\text { (UG/L) }\end{array}$ & $\begin{array}{l}\text { TOT.REC } \\
\text { (UG/L) }\end{array}$ & $\begin{array}{l}\text { RECOVER } \\
(U G / L)\end{array}$ & $\begin{array}{l}\text { TOTAL } \\
\text { (UG/L) }\end{array}$ \\
\hline OCT 1992 & & & & & & & & & & & & \\
\hline $\begin{array}{l}15-15 \\
\text { OCT }\end{array}$ & $<0.2$ & $<0.20$ & $<0.2$ & $<0.2$ & $<1.0$ & $<0.2$ & $<0.2$ & $<0.2$ & $<0.20$ & $<1.0$ & $<0.2$ & $<0.2$ \\
\hline $\begin{array}{l}29-29 \\
\text { NOV }\end{array}$ & $<0.2$ & $<0.20$ & $<0.2$ & $<0.2$ & $<1.0$ & $<0.2$ & $<0.2$ & $<0.2$ & $<0.20$ & $<1.0$ & $<0.2$ & $<0.2$ \\
\hline $19-19$ & $<0.2$ & $<0.20$ & $<0.2$ & $<0.2$ & $<1.0$ & $<0.2$ & $<0.2$ & $<0.2$ & $<0.20$ & $<1.0$ & $<0.2$ & $<0.2$ \\
\hline DATE & $\begin{array}{c}\text { DI - } \\
\text { CHLORO- } \\
\text { BROMO- } \\
\text { METHANE } \\
\text { TOTAL } \\
\text { (UG/L) }\end{array}$ & $\begin{array}{l}1,1 \text {-DI - } \\
\text { CHLORO- } \\
\text { ETHYL- } \\
\text { ENE } \\
\text { TOTAL } \\
\text { (UG/L) }\end{array}$ & $\begin{array}{l}1,1-D I \\
\text { CHLORO- } \\
\text { PRO- } \\
\text { PENE, } \\
\text { WAT, WH } \\
\text { TOTAL } \\
\text { (UG/L) }\end{array}$ & $\begin{array}{c}\text { BENZENE } \\
\text { O- } \\
\text { CHLORO- } \\
\text { WATER } \\
\text { UNFLTRD } \\
\text { REC } \\
\text { (UG /L) }\end{array}$ & $\begin{array}{c}\text { BENZENE } \\
1,3 \text { - DI - } \\
\text { CHLORO- } \\
\text { WATER } \\
\text { UNFLTRD } \\
\text { REC } \\
\text { (UG/L) }\end{array}$ & $\begin{array}{l}\text { BENZENE } \\
1,4-D I- \\
\text { CHLORO- } \\
\text { WATER } \\
\text { UNFLTRD } \\
\text { REC } \\
\text { (UG/L) }\end{array}$ & $\begin{array}{l}\text { DI - } \\
\text { CHLORO- } \\
\text { DI - } \\
\text { FLUORO- } \\
\text { METHANE } \\
\text { TOTAL } \\
\text { (UG/L) }\end{array}$ & $\begin{array}{l}1,1-D I- \\
\text { CHLORO- } \\
\text { ETHANE } \\
\text { TOTAL } \\
\text { (UG/L) }\end{array}$ & $\begin{array}{l}1,2 \text {-DI - } \\
\text { CHLORO- } \\
\text { ETHANE } \\
\text { TOTAL } \\
(U G / L)\end{array}$ & $\begin{array}{l}1,2 \text {-DI - } \\
\text { PHENYL- } \\
\text { HYDRA- } \\
\text { ZINE } \\
\text { WATER } \\
\text { TOT.REC } \\
\text { (UG/L) }\end{array}$ & $\begin{array}{c}\text { CIS - 1,2- } \\
\text { DI - } \\
\text { CHLORO- } \\
\text { ETHENE } \\
\text { WATER } \\
\text { TOTAL } \\
\text { (UG/L) }\end{array}$ & $\begin{array}{c}1,2- \\
\text { TRANSDI } \\
\text { CHLORO- } \\
\text { ETHENE } \\
\text { TOTAL } \\
\text { (UG/L) }\end{array}$ \\
\hline
\end{tabular}

\begin{tabular}{|c|c|c|c|c|c|c|c|c|c|c|c|c|}
\hline \multicolumn{13}{|l|}{ OCT 1992} \\
\hline $15-15$ & $<0.2$ & $<0.2$ & $<0.2$ & $<5.0$ & $<5.0$ & $<5.0$ & $<0.2$ & $<0.2$ & $<0.2$ & $<5.0$ & $<0.2$ & $<0.2$ \\
\hline \multicolumn{13}{|l|}{ OCT } \\
\hline $29-29$ & $<0.2$ & $<0.2$ & $<0.2$ & $<5.0$ & $<5.0$ & $<5.0$ & $<0.2$ & $<0.2$ & $<0.2$ & $<5.0$ & $<0.2$ & $<0.2$ \\
\hline \multicolumn{13}{|l|}{ NOV } \\
\hline $19 \cdot 19$ & $<0.2$ & $<0.2$ & $<0.2$ & $<5.0$ & $<5.0$ & $<5.0$ & $<0.2$ & $<0.2$ & $<0.2$ & $<5.0$ & $<0.2$ & $<0.2$ \\
\hline
\end{tabular}

\begin{tabular}{|c|c|c|c|c|c|c|c|c|c|c|c|c|}
\hline & $1,2-\mathrm{DI}-$ & $\begin{array}{l}1,3-D I- \\
\text { CHLORO- }\end{array}$ & $\begin{array}{c}2,2-D I \\
\text { CHLORO- } \\
\text { PRO- }\end{array}$ & $\begin{array}{l}\text { CIS } \\
1,3-\mathrm{DI} .\end{array}$ & $\begin{array}{l}\text { TRANS - } \\
1,3-\mathrm{DI} \text { - }\end{array}$ & & $\begin{array}{l}\text { HEXA- } \\
\text { CHLORO- }\end{array}$ & $\begin{array}{c}\text { ISO- } \\
\text { PROPYL - } \\
\text { BENZENE }\end{array}$ & $\begin{array}{l}\text { P.ISO- } \\
\text { PROPYL- } \\
\text { TOLUENE }\end{array}$ & & $\begin{array}{c}\text { METHYL - } \\
\text { ENE }\end{array}$ & \\
\hline & CHLORO - & PROPANE & PANE & CHLORO- & CHLORO - & ETHYL - & BUT - & WATER & WATER & METHYL - & CHLO - & NAPHTH - \\
\hline DATE & $\begin{array}{l}\text { PROPANE } \\
\text { TOTAL } \\
\text { (UG/L) }\end{array}$ & $\begin{array}{l}\text { WAT. WH } \\
\text { TOTAL } \\
\text { (UG/L) }\end{array}$ & $\begin{array}{l}\text { WAT, WH } \\
\text { TOTAL } \\
\text { (UG/L) }\end{array}$ & $\begin{array}{l}\text { PROPENE } \\
\text { TOTAL } \\
\text { (UG /L) }\end{array}$ & $\begin{array}{c}\text { PROPENE } \\
\text { TOTAL } \\
\text { (UG/L) }\end{array}$ & $\begin{array}{l}\text { BENZENE } \\
\text { TOTAL } \\
\text { (UG/L) }\end{array}$ & $\begin{array}{c}\text { ADIENE } \\
\text { TOTAL } \\
\text { (UG/L) }\end{array}$ & $\begin{array}{l}\text { WHOLE } \\
\text { REC } \\
\text { (UG/L) }\end{array}$ & $\begin{array}{l}\text { WHOLE } \\
\text { REC } \\
\text { (UG/L) }\end{array}$ & $\begin{array}{l}\text { BROMIDE } \\
\text { TOTAL } \\
\text { (UG/L) }\end{array}$ & $\begin{array}{l}\text { RIDE } \\
\text { TOTAL } \\
\text { (UG/L) }\end{array}$ & $\begin{array}{c}\text { ALENE } \\
\text { TOTAL } \\
\text { (UG/L) }\end{array}$ \\
\hline
\end{tabular}

\begin{tabular}{|c|c|c|c|c|c|c|c|c|c|c|c|c|}
\hline \multicolumn{13}{|l|}{ OCT 1992} \\
\hline $15-15$ & $<0.2$ & $<0.2$ & $<0.2$ & $<0.2$ & $<0.2$ & $<0.2$ & $<5.0$ & $<0.20$ & $<0.20$ & $<0.2$ & $<0.2$ & $<5.0$ \\
\hline \multicolumn{13}{|l|}{ OCT } \\
\hline $29 \cdot 29$ & $<0.2$ & $<0.2$ & $<0.2$ & $<0.2$ & $<0.2$ & $<0.2$ & $<5.0$ & $<0.20$ & $<0.20$ & $<0.2$ & $<0.2$ & $<5.0$ \\
\hline \multicolumn{13}{|l|}{ NoV } \\
\hline $19 \cdot 19$ & $<0.2$ & $<0.2$ & $<0.2$ & $<0.2$ & $<0.2$ & $<0.2$ & $<5.0$ & $<0.20$ & $<0.20$ & $<0.2$ & $<0.2$ & $<5.0$ \\
\hline
\end{tabular}


Table 5. Water-quality data for storm-sewer outfall stations, Garland, Texas, 1992-93-Continued

08061635 - TRIBUTARY TO DUCK CREEK OUTFALL AT HIGHTOWER ROAD, GARLAND, TX (WY 1993)-ContinUed

\begin{tabular}{|c|c|c|c|c|c|c|c|c|c|c|c|c|}
\hline & $\begin{array}{c}\text { BENZENE } \\
\text { N-PROPY } \\
\text { WATER }\end{array}$ & & $\begin{array}{c}\text { ETHANE, } \\
1112- \\
\text { TETRA- } \\
\text { CHLORO - }\end{array}$ & $\begin{array}{r}\text { ETHANE, } \\
1,1,2,2 \\
\text { TETRA - } \\
\text { CHLORO - }\end{array}$ & $\begin{array}{l}\text { TETRA- } \\
\text { CHLORO- } \\
\text { ETHYL- }\end{array}$ & & $\begin{array}{c}1,2,3- \\
\text { TRI- } \\
\text { CHLORO } \\
\text { BENZENE }\end{array}$ & $\begin{array}{c}\text { BENZENE } \\
1,2,4 \text { - } \\
\text { TRI - } \\
\text { CHLORO- }\end{array}$ & $\begin{array}{c}1,1,1- \\
\text { TRI - } \\
\text { CHLORO- }\end{array}$ & $\begin{array}{c}1,1,2- \\
\text { TRI - } \\
\text { CHLORO. }\end{array}$ & $\begin{array}{l}\text { TRI - } \\
\text { CHLORO - } \\
\text { ETHYL - }\end{array}$ & $\begin{array}{l}\text { TRI - } \\
\text { CHLORO - } \\
\text { FLUORO - }\end{array}$ \\
\hline & $\begin{array}{l}\text { UNFLTRD } \\
\text { REC } \\
\text { (UG/L) }\end{array}$ & $\begin{array}{l}\text { STYRENE } \\
\text { TOTAL } \\
\text { (UG/L) }\end{array}$ & $\begin{array}{l}\text { WAT UNF } \\
\text { REC } \\
\text { (UG/L) }\end{array}$ & $\begin{array}{l}\text { WAT UNF } \\
\text { REC } \\
\text { (UG/L) }\end{array}$ & $\begin{array}{c}\text { ENE } \\
\text { TOTAL } \\
\text { (UG/L) }\end{array}$ & $\begin{array}{l}\text { TOLUENE } \\
\text { TOTAL } \\
\text { (UG/L) }\end{array}$ & $\begin{array}{l}\text { WAT, WH } \\
\text { REC } \\
\text { (UG/L) }\end{array}$ & $\begin{array}{c}\text { WAT UNF } \\
\text { REC } \\
\text { (UG/L) }\end{array}$ & $\begin{array}{l}\text { ETHANE } \\
\text { TOTAL } \\
\text { (UG/L) }\end{array}$ & $\begin{array}{c}\text { ETHANE } \\
\text { TOTAL } \\
\text { (UG/L) }\end{array}$ & $\begin{array}{c}\text { ENE } \\
\text { TOTAL } \\
\text { (UG/L) }\end{array}$ & $\begin{array}{c}\text { METHANE } \\
\text { TOTAL } \\
\text { (UG/L) }\end{array}$ \\
\hline
\end{tabular}

\begin{tabular}{|c|c|c|c|c|c|c|c|c|c|c|c|c|}
\hline \multicolumn{13}{|c|}{ OCT 1992} \\
\hline $15-15$ & $<0.20$ & $<0.2$ & $<0.2$ & $<0.2$ & $<0.2$ & $<0.2$ & $<0.20$ & $<5.0$ & $<0.2$ & $<0.2$ & $<0.2$ & $<0.2$ \\
\hline \multicolumn{13}{|l|}{ OCT } \\
\hline $29-29$ & $<0.20$ & $<0.2$ & $<0.2$ & $<0.2$ & $<0.2$ & $<0.2$ & $<0.20$ & $<5.0$ & $<0.2$ & $<0.2$ & $<0.2$ & $<0.2$ \\
\hline \multicolumn{13}{|l|}{ NOV } \\
\hline $19-19$ & $<0.20$ & $<0.2$ & $<0.2$ & $<0.2$ & $<0.2$ & $<0.2$ & $<0.20$ & $<5.0$ & $<0.2$ & $<0.2$ & $<0.2$ & $<0.2$ \\
\hline
\end{tabular}

\begin{tabular}{|c|c|c|c|c|c|c|c|c|c|c|c|c|}
\hline & $123-\mathrm{TRI}$ & & & & & & & & & BENZO A & & \\
\hline & CHLORO- & PSEUDO- & MESIT- & & & & & & & ANTHRAC & & BENZO B \\
\hline & PROPANE & CUMENE & YLENE & VINYL & XYLENE & $\mathrm{ACE}-$ & $\mathrm{ACE}$ - & & & ENE1, 2 - & BENZO - & FLUOR - \\
\hline & WATER & WATER & WATER & CHLO- & WATER & NAPHTH - & NAPHTH - & ANTHRA - & BENZ I - & BENZANT & A - & $\mathrm{AN}$ - \\
\hline & WHOLE & UNFLTRD & UNFLTRD & RIDE & UNFLTRD & ENE & YLENE & CENE & DINE & HRACENE & PYRENE & THENE \\
\hline DATE & $\begin{array}{l}\text { TOTAL } \\
\text { (UG/L) }\end{array}$ & $\begin{array}{c}\text { REC } \\
(U G / L)\end{array}$ & $\begin{array}{c}\text { REC } \\
(U G / L)\end{array}$ & $\begin{array}{l}\text { TOTAL } \\
\text { (UG/L) }\end{array}$ & $\begin{array}{c}R E C \\
(U G / L)\end{array}$ & $\begin{array}{c}\text { TOTAL } \\
\text { (UG/L) }\end{array}$ & $\begin{array}{l}\text { TOTAL } \\
\text { (UG/L) }\end{array}$ & $\begin{array}{l}\text { TOTAL } \\
\text { (UG/L) }\end{array}$ & $\begin{array}{r}\text { TOTAL } \\
\text { (UG/L) }\end{array}$ & $\begin{array}{l}\text { TOTAL } \\
\text { (UG/L) }\end{array}$ & $\begin{array}{l}\text { TOTAL } \\
\text { (UG/L) }\end{array}$ & $\begin{array}{l}\text { TOTAL } \\
\text { (UG/L) }\end{array}$ \\
\hline \multicolumn{13}{|c|}{ OCT 1992} \\
\hline $15 \cdot 15$ & $<0.2$ & $<0.20$ & $<0.20$ & $<0.2$ & $<0.20$ & $<5.0$ & $<5.0$ & $<5.0$ & $<40.0$ & 26.0 & 19.0 & 23.0 \\
\hline \multicolumn{13}{|l|}{ OCT } \\
\hline $29-29$ & $<0.2$ & $<0.20$ & $<0.20$ & $<0.2$ & $<0.20$ & $<5.0$ & $<5.0$ & $<5.0$ & $<40.0$ & $<10.0$ & $<10.0$ & $<10.0$ \\
\hline \multicolumn{13}{|l|}{ NOV } \\
\hline $19-19$ & $<0.2$ & $<0.20$ & $<0.20$ & $<0.2$ & 0.40 & $<5.0$ & $<5.0$ & $<5.0$ & $<40.0$ & $<10.0$ & $<10.0$ & $<10.0$ \\
\hline
\end{tabular}

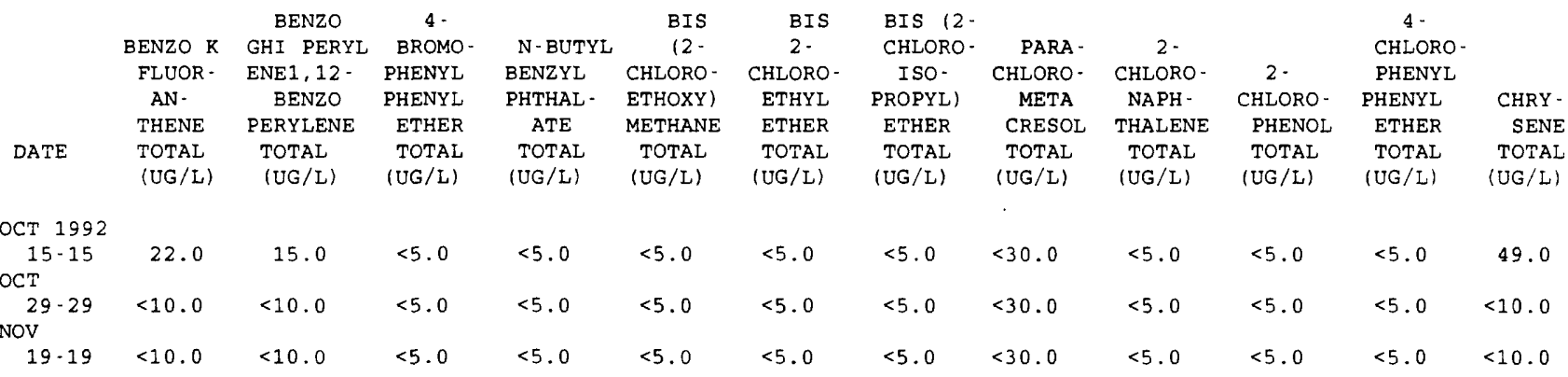

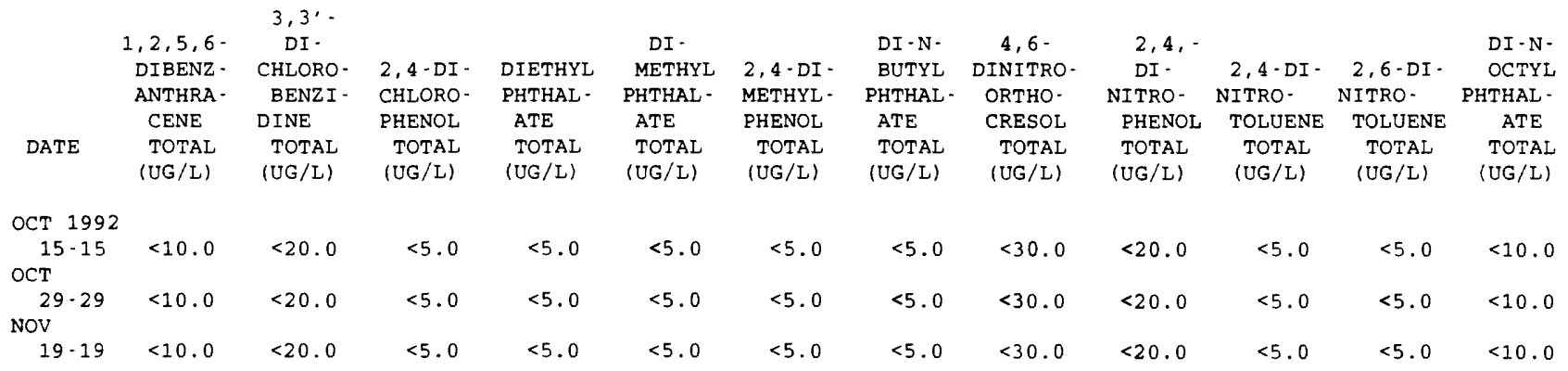


Table 5. Water-quality data for storm-sewer outfall stations, Garland, Texas, 1992-93-Continued

08061635 - TRIBUTARY TO DUCK CREEK OUTFALL AT HIGHTOWER ROAD, GARLAND, TX (WY 1993)-Continued

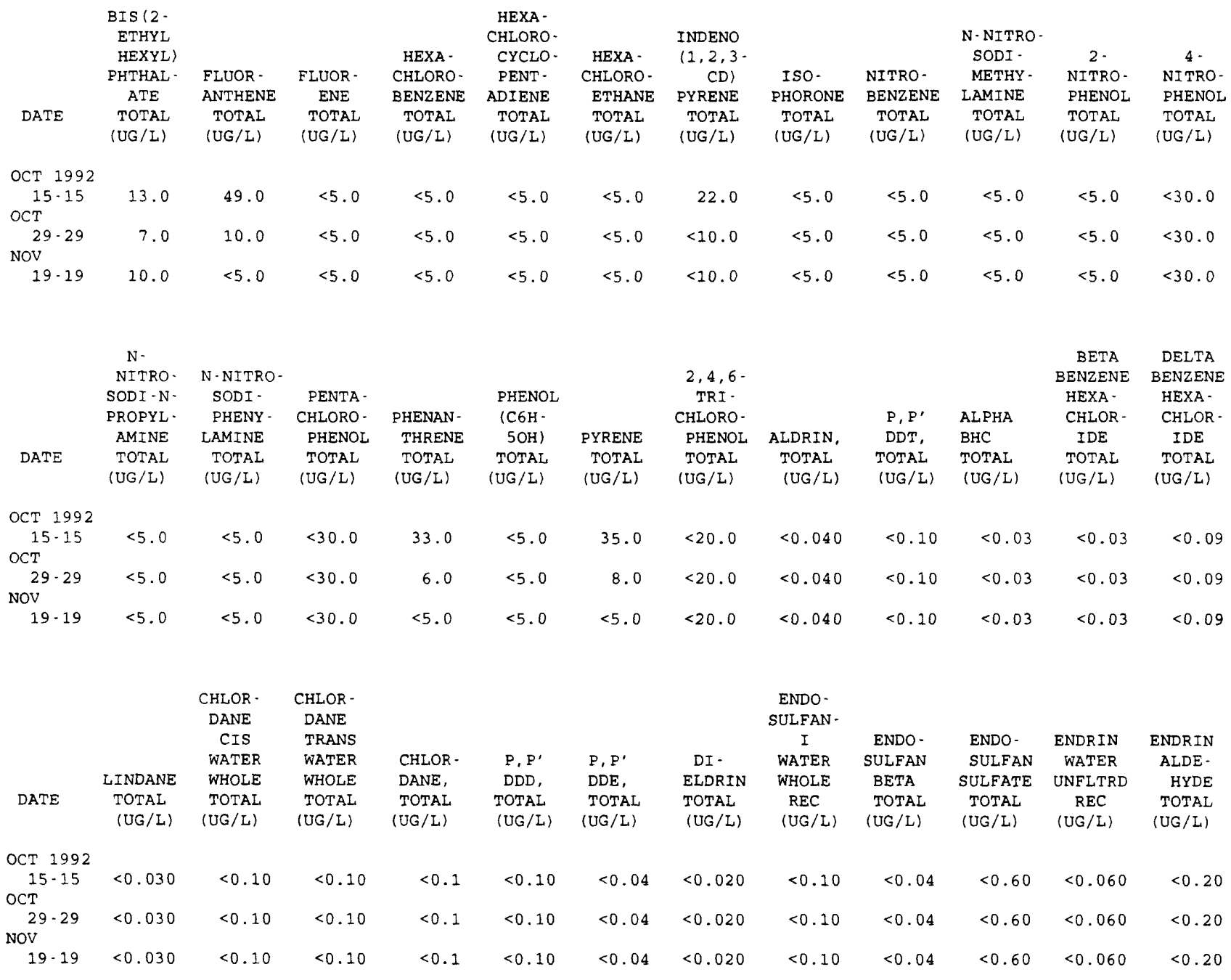

\begin{tabular}{|c|c|c|c|c|c|c|c|c|c|c|}
\hline & HEPTA - & & AROCLOR & AROCLOR & AROCLOR & AROCLOR & AROCLOR & AROCLOR & AROCLOR & \\
\hline HEPTA - & CHLOR & TOX - & 1016 & 1221 & 1232 & 1242 & 1248 & 1254 & 1260 & $\mathrm{DI}$ \\
\hline CHLOR, & EPOXIDE & APHENE, & PCB & PCB & PCB & $\mathrm{PCB}$ & PCB & PCB & $\mathrm{PCB}$ & AZINON, \\
\hline $\begin{array}{l}\text { TOTAL } \\
\text { (UG/L) }\end{array}$ & $\begin{array}{l}\text { TOTAL } \\
\text { (UG/L) }\end{array}$ & $\begin{array}{l}\text { TOTAL } \\
\text { (UG/L) }\end{array}$ & $\begin{array}{l}\text { TOTAL } \\
\text { (UG/L) }\end{array}$ & $\begin{array}{l}\text { TOTAL } \\
(\mathrm{UG} / \mathrm{L})\end{array}$ & $\begin{array}{l}\text { TOTAL } \\
\text { (UG/L) }\end{array}$ & $\begin{array}{l}\text { TOTAL } \\
\text { (UG/L) }\end{array}$ & $\begin{array}{l}\text { TOTAL } \\
\text { (UG/L) }\end{array}$ & $\begin{array}{l}\text { TOTAL } \\
\text { (UG/L) }\end{array}$ & $\begin{array}{l}\text { TOTAL } \\
\text { (UG/L) }\end{array}$ & $\begin{array}{l}\text { TOTAL } \\
\text { (UG/L) }\end{array}$ \\
\hline
\end{tabular}

\begin{tabular}{|c|c|c|c|c|c|c|c|c|c|c|c|}
\hline \multicolumn{12}{|l|}{ OCT 1992} \\
\hline $15-15$ & $<0.030$ & $<0.80$ & $<2$ & $<0.1$ & $<1.0$ & $<0.1$ & $<0.1$ & $<0.1$ & 0.2 & $<0.1$ & 0.10 \\
\hline \multicolumn{12}{|l|}{$\mathrm{OCT}$} \\
\hline $29-29$ & $<0.030$ & $<0.80$ & $<2$ & $<0.1$ & $<1.0$ & $<0.1$ & $<0.1$ & $<0.1$ & $<0.1$ & $<0.1$ & $<0.10$ \\
\hline \multicolumn{12}{|l|}{ NOV } \\
\hline $19 \cdot 19$ & $<0.030$ & $<0.80$ & $<2$ & $<0.1$ & $<1.0$ & $<0.1$ & $<0.1$ & $<0.1$ & $<0.1$ & $<0.1$ & $<0.10$ \\
\hline
\end{tabular}


Table 5. Water-quality data for storm-sewer outfall stations, Garland, Texas, 1992-93-Continued

08061660 - SLEEPY HOLLOW STREET OUTFALL AT NORTHWEST HIGHWAY, GARLAND, TX (WY 1992)

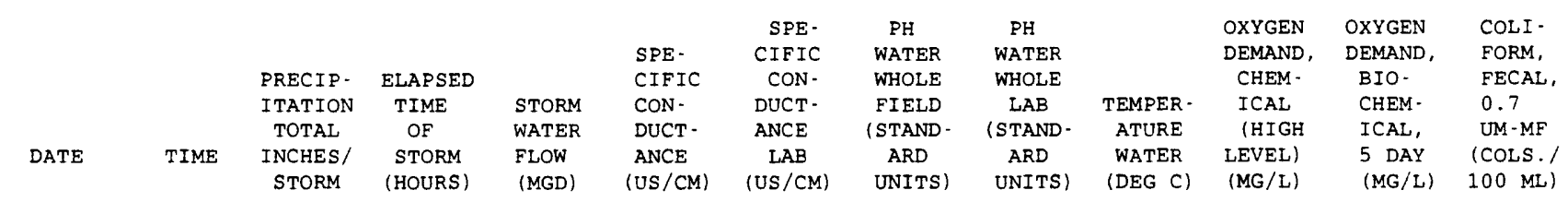

$\begin{array}{cccccccccccccc}\text { SEP 1992 } \\ \begin{array}{l}01.01 \\ \text { SEP }\end{array} & 1340 & 0.39 & 1.3 & 0.14 & 80 & 138 & 8.2 & 6.8 & 26.5 & 63 & 13 & 100000 \\ 21.21 & 0155 & 0.89 & 2.2 & 0.34 & 115 & 72 & 8.0 & 7.4 & 24.5 & 50 & 6.5 & 8000\end{array}$

\begin{tabular}{|c|c|c|c|c|c|c|c|c|c|c|c|c|}
\hline & $\begin{array}{l}\text { STREP- } \\
\text { TOCOCCI }\end{array}$ & HARD - & $\begin{array}{l}\text { HARD - } \\
\text { NESS }\end{array}$ & $\begin{array}{l}\text { ALKA - } \\
\text { LINITY }\end{array}$ & $\begin{array}{l}\text { SOLIDS, } \\
\text { SUM OF }\end{array}$ & $\begin{array}{l}\text { RESIDUE } \\
\text { TOTAL }\end{array}$ & $\begin{array}{l}\text { SOLIDS, } \\
\text { RESIDUE }\end{array}$ & & MAGNE - & & & SODIUM \\
\hline & FECAL, & NESS & NONCARB & WAT DIS & CONSTI - & AT 105 & AT 180 & CALCIUM & SIUM, & SODIUM, & & $A D$ \\
\hline & $\begin{array}{l}\text { KF AGAR } \\
\text { ICOLS. }\end{array}$ & $\begin{array}{l}\text { TOTAL } \\
\text { (MG/L }\end{array}$ & $\begin{array}{l}\text { DISSOLV } \\
\text { FLD. AS }\end{array}$ & $\begin{array}{l}\text { FIX END } \\
\text { FIELD }\end{array}$ & $\begin{array}{l}\text { TUENTS, } \\
\text { DIS. }\end{array}$ & $\begin{array}{l}\text { DEG. C, } \\
\text { SUS - }\end{array}$ & $\begin{array}{l}\text { DEG. } C \\
\text { DIS - }\end{array}$ & $\begin{array}{c}\text { DIS- } \\
\text { SOLVED }\end{array}$ & $\begin{array}{c}\text { DIS - } \\
\text { SOLVED }\end{array}$ & $\begin{array}{l}\text { DIS - } \\
\text { SOLVED }\end{array}$ & & $\begin{array}{l}\text { SORP - } \\
\text { TION }\end{array}$ \\
\hline DATE & $\begin{array}{c}\text { PER } \\
100 \mathrm{ML})\end{array}$ & $\begin{array}{c}\text { AS } \\
\text { (ACO3) }\end{array}$ & $\begin{array}{r}\mathrm{CACO} 3 \\
(\mathrm{MG} / \mathrm{L})\end{array}$ & $\begin{array}{l}\mathrm{CACO} 3 \\
(\mathrm{MG} / \mathrm{L})\end{array}$ & $\begin{array}{l}\text { SOLVED } \\
\text { (MG/L) }\end{array}$ & $\begin{array}{l}\text { PENDED } \\
(\mathrm{MG} / \mathrm{L})\end{array}$ & $\begin{array}{l}\text { SOLVED } \\
(M G / L)\end{array}$ & $\begin{array}{c}(\mathrm{MG} / \mathrm{L} \\
\mathrm{AS} \mathrm{CA})\end{array}$ & $\begin{array}{l}\text { (MG/L } \\
\text { AS } M G)\end{array}$ & $\begin{array}{c}\text { (MG/L } \\
\text { AS NA) }\end{array}$ & $\begin{array}{l}\text { SODIUM } \\
\text { PERCENT }\end{array}$ & RATIO \\
\hline
\end{tabular}

SEP 1992

\begin{tabular}{|c|c|c|c|c|c|c|c|c|c|c|c|c|}
\hline $01-01$ & 69000 & 21 & 4 & 17 & 28 & $<1$ & 29 & 8.0 & 0.25 & 1.4 & 11 & 0.1 \\
\hline$S E P$ & & & & & & & & & & & & \\
\hline $21-21$ & K4000 & 17 & 1 & 16 & 32 & 84 & 55 & 6.3 & 0.20 & 0.90 & 9 & 0.1 \\
\hline
\end{tabular}

\begin{tabular}{|c|c|c|c|c|c|c|c|c|c|c|c|c|}
\hline DATE & $\begin{array}{l}\text { POTAS - } \\
\text { SIUM, } \\
\text { DIS- } \\
\text { SOLVED } \\
\text { (MG /L }\end{array}$ & $\begin{array}{l}\text { SULFATE } \\
\text { DIS - } \\
\text { SOLVED } \\
\text { (MG/L }\end{array}$ & $\begin{array}{l}\text { CHLO - } \\
\text { RIDE, } \\
\text { DIS - } \\
\text { SOLVED } \\
\text { (MG /L }\end{array}$ & $\begin{array}{l}\text { NITRO- } \\
\text { GEN, } \\
\text { NITRITE } \\
\text { TOTAL } \\
\text { (MG/L }\end{array}$ & $\begin{array}{c}\text { NITRO- } \\
\text { GEN, } \\
\text { NO2+NO3 } \\
\text { TOTAL } \\
\text { (MG / L }\end{array}$ & $\begin{array}{l}\text { NITRO- } \\
\text { GEN, } \\
\text { AMMONIA } \\
\text { TOTAL } \\
\text { (MG/L }\end{array}$ & $\begin{array}{l}\text { NITRO- } \\
\text { GEN, AM- } \\
\text { MONIA + } \\
\text { ORGANIC } \\
\text { TOTAL } \\
\text { (MG/L }\end{array}$ & $\begin{array}{l}\text { PHOS - } \\
\text { PHORUS } \\
\text { TOTAL } \\
\text { (MG/L }\end{array}$ & $\begin{array}{l}\text { PHOS - } \\
\text { PHORUS } \\
\text { DIS - } \\
\text { SOLVED } \\
\text { (MG / L }\end{array}$ & $\begin{array}{c}\text { ANT IMONY } \\
\text { TOTAL } \\
\text { EPA } \\
\text { (UG/L }\end{array}$ & $\begin{array}{c}\text { ARSENIC } \\
\text { TOTAL } \\
\text { (UG/L }\end{array}$ & $\begin{array}{l}\text { BERYL- } \\
\text { LIUM, } \\
\text { TOTAL } \\
\text { RECOV- } \\
\text { ERABLE } \\
\text { (UG /L }\end{array}$ \\
\hline & AS K) & AS SO4) & AS (L) & AS N) & AS N) & AS N) & AS N) & AS P) & AS P) & AS $S B$ ) & AS AS) & AS BE) \\
\hline
\end{tabular}

\begin{tabular}{|c|c|c|c|c|c|c|c|c|c|c|c|c|}
\hline SEP 1992 & & & & & & & & & & & & \\
\hline $01-01$ & 2.5 & 3.9 & 0.60 & 0.030 & 0.430 & 0.160 & 1.0 & 0.320 & 0.210 & $<10.0$ & 6 & $<10$ \\
\hline \multicolumn{13}{|l|}{ SEP } \\
\hline $21-21$ & 2.3 & 3.3 & 1.5 & 0.020 & 0.350 & 0.170 & 1.4 & 0.330 & 0.180 & $<10.0$ & 4 & $<10$ \\
\hline
\end{tabular}

\begin{tabular}{|c|c|c|c|c|c|c|c|c|c|c|c|c|}
\hline DATE & $\begin{array}{l}\text { CADMIUM } \\
\text { TOTAL } \\
\text { RECOV- } \\
\text { ERABLE } \\
\text { (UG/L }\end{array}$ & $\begin{array}{l}\text { CHRO- } \\
\text { MIUM, } \\
\text { TOTAL } \\
\text { RECOV- } \\
\text { ERABLE } \\
\text { (UG/L }\end{array}$ & $\begin{array}{c}\text { COPPER, } \\
\text { TOTAL } \\
\text { RECOV- } \\
\text { ERABLE } \\
\text { (UG / L }\end{array}$ & $\begin{array}{c}\text { CYANIDE } \\
\text { TOTAL } \\
\text { EPA } \\
\text { (MG/L }\end{array}$ & $\begin{array}{l}\text { CYANIDE } \\
\text { TOTAL } \\
\text { (MG/L }\end{array}$ & $\begin{array}{l}\text { LEAD, } \\
\text { TOTAL } \\
\text { RECOV - } \\
\text { ERABLE } \\
\text { (UG/L }\end{array}$ & $\begin{array}{l}\text { MERCURY } \\
\text { TOTAL } \\
\text { RECOV- } \\
\text { ERABLE } \\
\text { (UG/L }\end{array}$ & $\begin{array}{l}\text { NICKEL, } \\
\text { TOTAL } \\
\text { RECOV - } \\
\text { ERABLE } \\
\text { (UG/L }\end{array}$ & $\begin{array}{l}\text { SELE- } \\
\text { NIUM, } \\
\text { TOTAL } \\
\text { (UG/L }\end{array}$ & $\begin{array}{l}\text { SILVER, } \\
\text { TOTAL } \\
\text { RECOV- } \\
\text { ERABLE } \\
\text { (UG/L }\end{array}$ & $\begin{array}{l}\text { SILVER, } \\
\text { TOTAL } \\
\text { RECOV- } \\
\text { ERABLE } \\
\text { EPA } \\
\text { (UG/L }\end{array}$ & $\begin{array}{l}\text { THAL - } \\
\text { LIUM, } \\
\text { TOTAL } \\
\text { (UG/L }\end{array}$ \\
\hline & AS CD) & AS (R) & AS CU) & AS CN) & AS CN) & AS PB) & AS HG) & AS NI) & AS SE) & AS AG) & AS AG) & AS TL) \\
\hline
\end{tabular}

SEP 1992

01-01

SEP

$21-21$

CR) AS

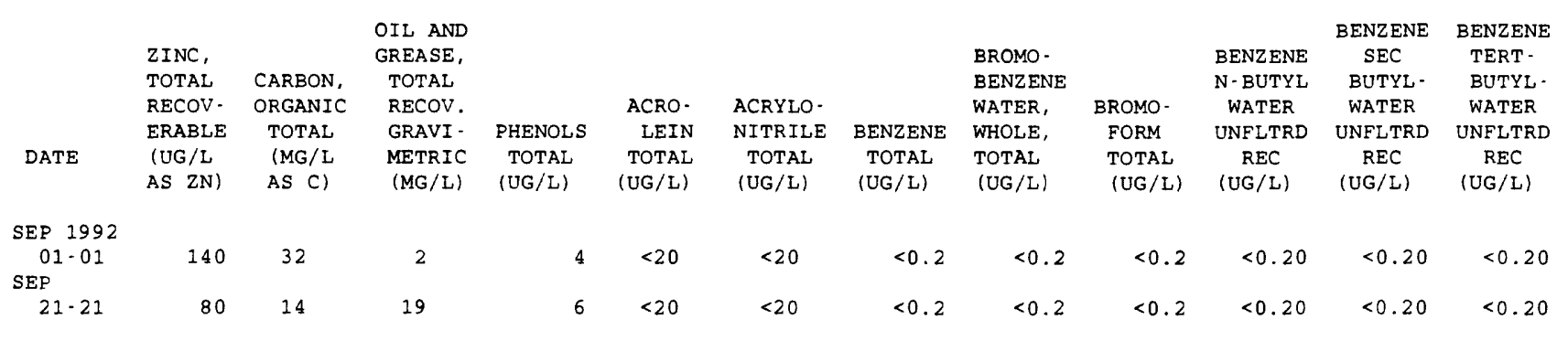


Table 5. Water-quality data for storm-sewer outfa11 stations, Garland, Texas, 1992-93-Continued

08061660 - SLEEPY HOLLOW STREET OUTFALL AT NORTHWEST HIGHWAY, GARLAND, TX (WY 1992)-COntinUed

\begin{tabular}{|c|c|c|c|c|c|c|c|c|c|c|c|c|}
\hline & \multirow{5}{*}{$\begin{array}{l}\text { CARBON- } \\
\text { TETRA- } \\
\text { CHLO- } \\
\text { RIDE }\end{array}$} & \multirow{3}{*}{\multicolumn{2}{|c|}{$\begin{array}{l}\text { CHLORO- } \\
\text { DI - }\end{array}$}} & & & & & \\
\hline & & & & & $\begin{array}{l}2- \\
\text { CHLORO- }\end{array}$ & & & CHLORO- & TOLUENE & $\begin{array}{l}\text { DIBROMO } \\
\text { CHLORO- }\end{array}$ & $\begin{array}{c}\text { DI- } \\
\text { BROMO- }\end{array}$ & $\begin{array}{c}1,2- \\
\text { DIBROMO }\end{array}$ \\
\hline & & & & & ETHYL- & & METHYL - & TOLUENE & P-CHLOR & PROPANE & METHANE & ETHANE \\
\hline & & CHLORO - & BROMO- & CHLORO- & VINYL. & CHLORO - & CHLO- & WATER & WATER & WATER & WATER & WATER \\
\hline & & BENZENE & METHANE & ETHANE & ETHER & FORM & RIDE & WHOLE & UNFLTRD & WHOLE & WHOLE & WHOLE \\
\hline DATE & $\begin{array}{l}\text { TOTAL } \\
\text { (UG/L) }\end{array}$ & $\begin{array}{l}\text { TOTAL } \\
\text { (UG/L) }\end{array}$ & $\begin{array}{l}\text { TOTAL } \\
\text { (UG/L) }\end{array}$ & $\begin{array}{l}\text { TOTAL } \\
\text { (UG/L) }\end{array}$ & $\begin{array}{l}\text { TOTAL } \\
\text { (UG/L) }\end{array}$ & $\begin{array}{l}\text { TOTAL } \\
\text { (UG/L) }\end{array}$ & $\begin{array}{l}\text { TOTAL } \\
\text { (UG/L) }\end{array}$ & $\begin{array}{l}\text { TOTAL } \\
\text { (UG/L) }\end{array}$ & $\begin{array}{c}\text { REC } \\
(\mathrm{UG} / \mathrm{L})\end{array}$ & $\begin{array}{l}\text { TOT.REC } \\
\text { (UG/L) }\end{array}$ & $\begin{array}{l}\text { RECOVER } \\
(\mathrm{UG} / \mathrm{L})\end{array}$ & $\begin{array}{l}\text { TOTAL } \\
\text { (UG/L) }\end{array}$ \\
\hline \multicolumn{13}{|l|}{ SEP 1992} \\
\hline $01-01$ & $<0.2$ & $<0.20$ & $<0.2$ & $<0.2$ & $<1.0$ & $<0.2$ & $<0.2$ & $<0.2$ & $<0.20$ & $<1.0$ & $<0.2$ & $<0.2$ \\
\hline \multicolumn{13}{|l|}{ SEP } \\
\hline \multirow[t]{6}{*}{$21-21$} & \multirow[t]{2}{*}{$<0.2$} & \multirow[t]{2}{*}{$<0.20$} & $<0.2$ & $<0.2$ & $<1.0$ & $<0.2$ & \multicolumn{2}{|r|}{$<0.2$} & $<0.20$ & $<1.0$ & $<0.2$ & $<0.2$ \\
\hline & & & $1,1-D I$ & BENZENE & BENZENE & BENZENE & DI - & & & $1,2-D I-$ & CIS-1,2- & \\
\hline & DI - & $1,1-\mathrm{DI}-$ & CHLORO- & 0 & $1,3-\mathrm{DI}$. & $1,4-\mathrm{DI}$ - & CHLORO - & & & PHENYL - & DI - & $1,2-$ \\
\hline & CHLORO- & CHLORO- & PRO- & CHLORO- & CHLORO- & CHLORO- & DI - & 1,1 -DI - & $1,2-D I-$ & HYDRA - & CHLORO- & TRANSDI \\
\hline & BROMO - & ETHYL - & PENE, & WATER & WATER & WATER & FLUORO- & CHLORO - & CHLORO- & ZINE & ETHENE & CHLORO- \\
\hline & METHANE & ENE & WAT, WH & UNFLTRD & UNFLTRD & UNFLTRD & METHANE & ETHANE & ETHANE & WATER & WATER & ETHENE \\
\hline DATE & TOTAL & TOTAL & TOTAL & REC & REC & REC & TOTAL & TOTAL & TOTAL & TOT.REC & TOTAL & TOTAL \\
\hline & (UG/L) & (UG/L) & (UG/L) & $(U G / L)$ & (UG/L) & (UG/L) & (UG/L) & (UG/L) & (UG/L) & $(U G / L)$ & $(U G / L)$ & $(U G / L)$ \\
\hline \multirow{2}{*}{$\begin{array}{c}\text { SEP } 1992 \\
01-01\end{array}$} & & & & & & & & & & & & \\
\hline & $<0.2$ & $<0.2$ & $<0.2$ & $<5.0$ & $<5.0$ & $<5.0$ & $<0.2$ & $<0.2$ & $<0.2$ & $<5.0$ & $<0.2$ & $<0.2$ \\
\hline \multicolumn{13}{|l|}{ SEP } \\
\hline \multirow[t]{5}{*}{$21-21$} & $<0.2$ & $<0.2$ & $<0.2$ & $<5.0$ & $<5.0$ & $<5.0$ & $<0.2$ & $<0.2$ & $<0.2$ & $<5.0$ & $<0.2$ & $<0.2$ \\
\hline & & & $2,2 \cdot D I$ & & & & & ISO- & P-ISO- & & & \\
\hline & $1,2 \cdot \mathrm{DI}-$ & $\begin{array}{l}1,3-D I- \\
\text { CHLORO. }\end{array}$ & $\begin{array}{l}\text { CHLORO- } \\
\text { PRO. }\end{array}$ & $\begin{array}{c}\text { CIS } \\
1,3-D I-\end{array}$ & $\begin{array}{r}\text { TRANS - } \\
1,3 \text {-DI - }\end{array}$ & & $\begin{array}{l}\text { HEXA- } \\
\text { CHLORO- }\end{array}$ & $\begin{array}{l}\text { PROPYL- } \\
\text { BENZENE }\end{array}$ & $\begin{array}{l}\text { PROPYL - } \\
\text { TOLUENE }\end{array}$ & & $\begin{array}{l}\text { METHYL - } \\
\text { ENE }\end{array}$ & \\
\hline & CHLORO- & PROPANE & PANE & CHLORO- & CHLORO- & ETHYL - & BUT - & WATER & WATER & METHYL - & CHLO- & NAPHTH - \\
\hline & PROPANE & WAT. WH & WAT, WH & PROPENE & PROPENE & BENZENE & ADIENE & WHOLE & WHOLE & BROMIDE & RIDE & ALENE \\
\hline DATE & $\begin{array}{c}\text { TOTAL } \\
\text { (UG/L) }\end{array}$ & $\begin{array}{l}\text { TOTAL } \\
\text { (UG/L) }\end{array}$ & $\begin{array}{c}\text { TOTAL } \\
\text { (UG/L) }\end{array}$ & $\begin{array}{l}\text { TOTAL } \\
\text { (UG/L) }\end{array}$ & $\begin{array}{l}\text { TOTAL } \\
\text { (UG/L) }\end{array}$ & $\begin{array}{c}\text { TOTAL } \\
\text { (UG/L) }\end{array}$ & $\begin{array}{l}\text { TOTAL } \\
\text { (UG/L) }\end{array}$ & $\begin{array}{l}\text { REC } \\
(U G / L)\end{array}$ & $\begin{array}{l}\text { REC } \\
\text { (UG/L) }\end{array}$ & $\begin{array}{c}\text { TOTAL } \\
\text { (UG/L) }\end{array}$ & $\begin{array}{l}\text { TOTAL } \\
\text { (UG/L) }\end{array}$ & $\begin{array}{l}\text { TOTAL } \\
\text { (UG/L) }\end{array}$ \\
\hline
\end{tabular}

\begin{tabular}{|c|c|c|c|c|c|c|c|c|c|c|c|c|}
\hline SEP 1992 & & & & & & & & & & & & \\
\hline $01-01$ & $<0.2$ & $<0.2$ & $<0.2$ & $<0.2$ & $<0.2$ & $<0.2$ & $<5.0$ & $<0.20$ & $<0.20$ & $<0.2$ & $<0.2$ & $<5.0$ \\
\hline SEP & & & & & & & & & & & & \\
\hline $21-21$ & $<0.2$ & $<0.2$ & $<0.2$ & $<0.2$ & $<0.2$ & $<0.2$ & $<5.0$ & $<0,20$ & $<0,20$ & $<0,2$ & $<0,2$ & \\
\hline
\end{tabular}

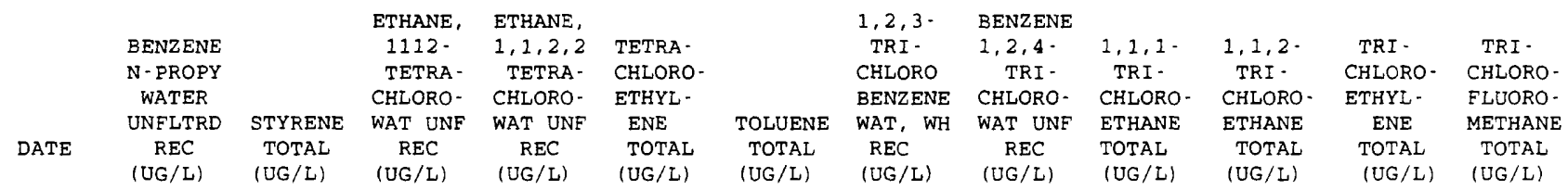

\begin{tabular}{|c|c|c|c|c|c|c|c|c|c|c|c|c|}
\hline SEP 1992 & & & & & & & & & & & & \\
\hline 01-01 & $<0.20$ & $<0.2$ & $<0.2$ & $<0.2$ & $<0.2$ & $<0.2$ & $<0.20$ & $<5.0$ & $<0.2$ & $<0.2$ & $<0.2$ & $<0.2$ \\
\hline $21-21$ & $<0.20$ & $<0.2$ & $<0.2$ & $<0.2$ & $<0.2$ & $<0.2$ & $<0.20$ & $<5.0$ & $<0.2$ & $<0.2$ & $<0,2$ & $<0.2$ \\
\hline
\end{tabular}

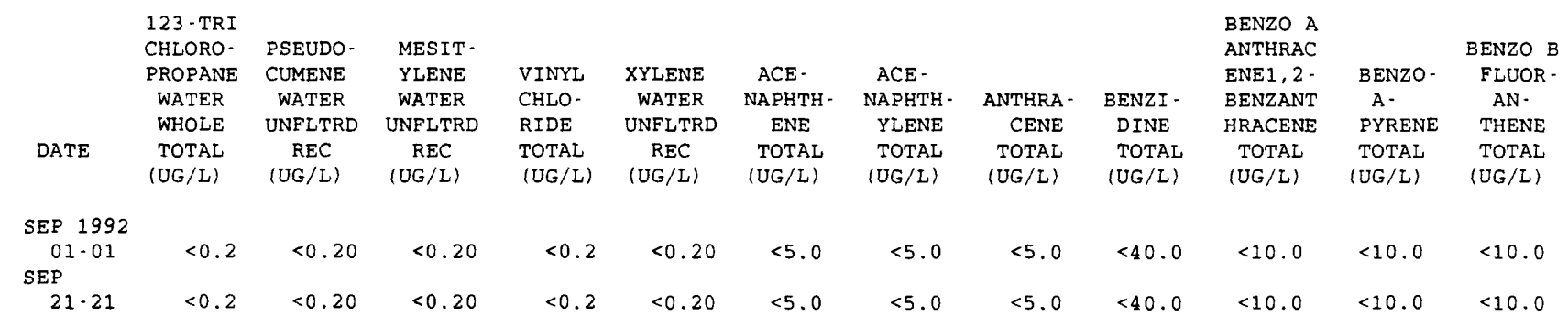


Table 5. Water-quality data for storm-sewer outfall stations, Garland, Texas, 1992-93-Continued

08061660 - SLEEPY HOLLOW STREET OUTFALL AT NORTHWEST HIGHWAY, GARLAND, TX (WY 1992)-ContINUEd

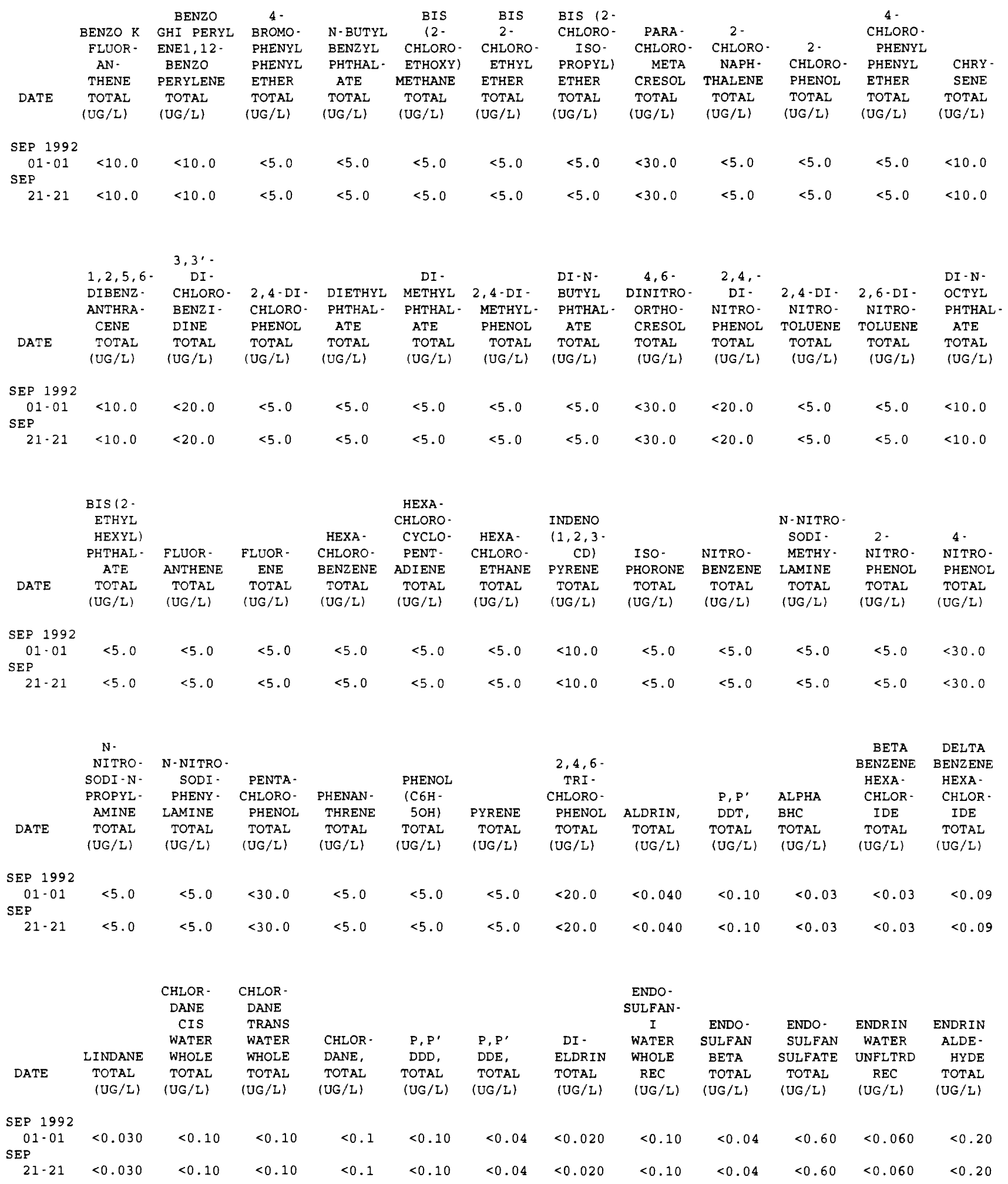


Table 5. Water-quality data for storm-sewer outfall stations, Garland, Texas, 1992-93-Continued

08061660 - SLEEPY HOLLOW STREET OUTFALL AT NORTHWEST HIGHWAY, GARLAND, TX (WY 1992)-ContinUed

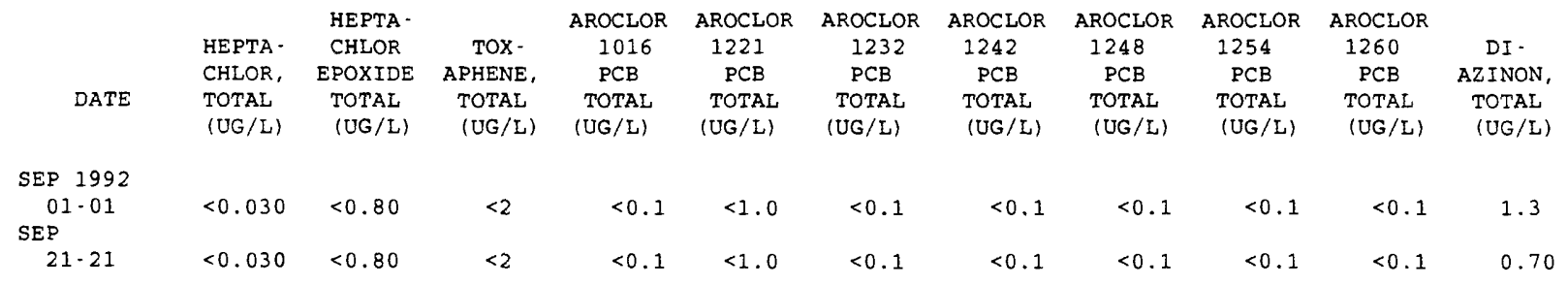


Table 5. Water-quality data for storm-sewer outfall stations, Garland, Texas, 1992-93-Continued

08061660 - SLEEPY HOLLOW STREET OUTFALL AT NORTHWEST HIGHWAY, GARLAND, TX (WY 1993)

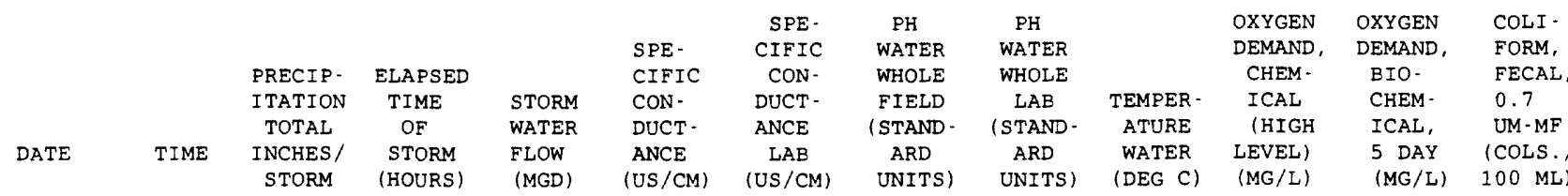

\begin{tabular}{|c|c|c|c|c|c|c|c|c|c|c|c|c|}
\hline \multicolumn{13}{|l|}{ OCT 1992} \\
\hline 07.07 & 1752 & 0.34 & 2.5 & 0.11 & 911 & 126 & 7.6 & 6.9 & 21.0 & 120 & 7.5 & 17000 \\
\hline \multicolumn{13}{|l|}{ NOV } \\
\hline $10-10$ & 1330 & 0.56 & 5.5 & 0.16 & 142 & 107 & 7.5 & 7.3 & 20.0 & 190 & 7.8 & 49000 \\
\hline \multicolumn{13}{|l|}{ NOV } \\
\hline $19-19$ & 0330 & 0.56 & 8.8 & 0.16 & 135 & 101 & 8.1 & 7.1 & 17.0 & 79 & 7.7 & 6500 \\
\hline \multicolumn{13}{|l|}{ JAN 1993} \\
\hline $09-09$ & 0335 & 0.34 & 7.4 & 0.11 & 89 & 177 & 7.0 & 7.9 & 11.0 & 85 & 5.9 & K19000 \\
\hline \multicolumn{13}{|l|}{ JAN } \\
\hline $23-23$ & 1801 & 0.34 & 2.4 & 0.23 & 169 & 96 & 7.3 & 7.6 & 14.0 & 120 & 9.7 & 52000 \\
\hline
\end{tabular}

\begin{tabular}{|c|c|c|c|c|c|c|c|c|c|c|c|c|}
\hline & $\begin{array}{r}\text { STREP - } \\
\text { TOCOCCI }\end{array}$ & HARD - & $\begin{array}{l}\text { HARD- } \\
\text { NESS }\end{array}$ & $\begin{array}{c}\text { ALKA - } \\
\text { LINITY }\end{array}$ & $\begin{array}{l}\text { SOLIDS, } \\
\text { SUM OF }\end{array}$ & $\begin{array}{l}\text { RESIDUE } \\
\text { TOTAL }\end{array}$ & $\begin{array}{l}\text { SOLIDS, } \\
\text { RESIDUE }\end{array}$ & & MAGNE - & & & SODIUM \\
\hline & FECAL , & NESS & NONCARB & WAT DIS & CONSTI - & AT 105 & AT 180 & CALCIUM & SIUM, & SODIUM, & & $\mathrm{AD}$ - \\
\hline & $\begin{array}{l}\text { KF AGAR } \\
\text { (COLS. }\end{array}$ & $\begin{array}{l}\text { TOTAL } \\
(\mathrm{MG} / \mathrm{L}\end{array}$ & $\begin{array}{l}\text { DISSOLV } \\
\text { FLD. AS }\end{array}$ & $\begin{array}{l}\text { FIX END } \\
\text { FIELD }\end{array}$ & $\begin{array}{c}\text { TUENTS, } \\
\text { DIS - }\end{array}$ & $\begin{array}{l}\text { DEG. C, } \\
\text { SUS - }\end{array}$ & $\begin{array}{l}\text { DEG. C } \\
\text { DIS - }\end{array}$ & $\begin{array}{l}\text { DIS - } \\
\text { SOLVED }\end{array}$ & $\begin{array}{l}\text { DIS - } \\
\text { SOLVED }\end{array}$ & $\begin{array}{l}\text { DIS - } \\
\text { SOLVED }\end{array}$ & & $\begin{array}{l}\text { SORP - } \\
\text { TION }\end{array}$ \\
\hline DATE & $\begin{array}{c}\text { PER } \\
100 \mathrm{ML})\end{array}$ & $\begin{array}{c}\text { AS } \\
\text { CACO3) }\end{array}$ & $\begin{array}{c}\mathrm{CACO} 3 \\
(\mathrm{MG} / \mathrm{L})\end{array}$ & $\begin{array}{l}\mathrm{CAC03} \\
(\mathrm{MG} / \mathrm{L})\end{array}$ & $\begin{array}{l}\text { SOLVED } \\
\text { (MG/L) }\end{array}$ & $\begin{array}{l}\text { PENDED } \\
(\mathrm{MG} / \mathrm{L})\end{array}$ & $\begin{array}{l}\text { SOLVED } \\
\text { (MG/L) }\end{array}$ & $\begin{array}{c}\text { (MG/L } \\
\text { AS CA) }\end{array}$ & $\begin{array}{l}\text { (MG/L } \\
\text { AS MG) }\end{array}$ & $\begin{array}{l}\text { (MG/L } \\
\text { AS NA) }\end{array}$ & $\begin{array}{l}\text { SODIUM } \\
\text { PERCENT }\end{array}$ & RATIO \\
\hline
\end{tabular}

\begin{tabular}{|c|c|c|c|c|c|c|c|c|c|c|c|c|}
\hline OCT 1992 & & & & & & & & & & & & \\
\hline 07.07 & 13000 & 32 & 17 & 15 & 58 & 106 & 68 & 12 & 0.44 & 1.7 & 9 & 0.1 \\
\hline NOV & & & & & & & & & & & & \\
\hline $10 \cdot 10$ & 280000 & 26 & 3 & 23 & 48 & 120 & 45 & 9.9 & 0.35 & 1.5 & 10 & 0.1 \\
\hline NOV & & & & & & & & & & & & \\
\hline $19 \cdot 19$ & 91000 & 51 & 13 & 38 & 61 & 54 & 59 & 19 & 0.74 & 2.5 & 9 & 0.2 \\
\hline JAN 1993 & & & & & & & & & & & & \\
\hline $09 \cdot 09$ & 200000 & 43 & 2 & 41 & 76 & 252 & 71 & 16 & 0.72 & 3.2 & 13 & 0.2 \\
\hline JAN & & & & & & & & & & & & \\
\hline $23 \cdot 23$ & 250000 & 32 & 4 & 28 & 65 & 322 & 59 & 12 & 0.50 & 2.5 & 13 & 0.2 \\
\hline
\end{tabular}

\begin{tabular}{|c|c|c|c|c|c|c|c|c|c|c|c|c|}
\hline DATE & $\begin{array}{l}\text { POTAS - } \\
\text { SIUM, } \\
\text { DIS - } \\
\text { SOLVED } \\
\text { (MG/L }\end{array}$ & $\begin{array}{l}\text { SULFATE } \\
\text { DIS - } \\
\text { SOLVED } \\
\text { (MG/L }\end{array}$ & $\begin{array}{l}\text { CHLO - } \\
\text { RIDE, } \\
\text { DIS - } \\
\text { SOLVED } \\
\text { (MG / L }\end{array}$ & $\begin{array}{l}\text { NITRO- } \\
\text { GEN, } \\
\text { NITRITE } \\
\text { TOTAL } \\
\text { (MG/L }\end{array}$ & $\begin{array}{c}\text { NITRO- } \\
\text { GEN, } \\
\text { NO2+NO3 } \\
\text { TOTAL } \\
\text { (MG/L }\end{array}$ & $\begin{array}{l}\text { NITRO- } \\
\text { GEN, } \\
\text { AMMONIA } \\
\text { TOTAL } \\
\text { (MG/L }\end{array}$ & $\begin{array}{l}\text { NITRO- } \\
\text { GEN, AM- } \\
\text { MONIA + } \\
\text { ORGANIC } \\
\text { TOTAL } \\
\text { (MG/L }\end{array}$ & $\begin{array}{l}\text { PHOS - } \\
\text { PHORUS } \\
\text { TOTAL } \\
\text { (MG /L }\end{array}$ & $\begin{array}{c}\text { PHOS - } \\
\text { PHORUS } \\
\text { DIS - } \\
\text { SOLVED } \\
\text { (MG / L }\end{array}$ & $\begin{array}{l}\text { ANTIMONY } \\
\text { TOTAL } \\
\text { EPA } \\
\text { (UG/L }\end{array}$ & $\begin{array}{c}\text { ARSENIC } \\
\text { TOTAL } \\
\text { (UG/L }\end{array}$ & $\begin{array}{l}\text { BERYL - } \\
\text { LIUM, } \\
\text { TOTAL } \\
\text { RECOV- } \\
\text { ERABLE } \\
\text { (UG/L }\end{array}$ \\
\hline & AS K) & AS SO4) & AS CL) & AS N) & AS N) & AS N) & AS N) & AS P) & AS P) & AS SB) & AS AS) & AS BE) \\
\hline
\end{tabular}

\begin{tabular}{|c|c|c|c|c|c|c|c|c|c|c|c|c|}
\hline \multicolumn{13}{|l|}{ OCT 1992} \\
\hline $07-07$ & 3.8 & 8.6 & 2.4 & 0.050 & 0.960 & 0.530 & 2.6 & 0.520 & 0.290 & $<10.0$ & 8 & $<10$ \\
\hline \multicolumn{13}{|l|}{ NOV } \\
\hline $10-10$ & 2.8 & 4.4 & 2.1 & 0.060 & 0.270 & 0.140 & 1.1 & 0.350 & 0.200 & $<10.0$ & 3 & $<10$ \\
\hline \multicolumn{13}{|l|}{ NOV } \\
\hline $19 \cdot 19$ & 4.0 & 4.9 & 2.6 & 0.080 & 0.400 & 0.090 & 0.80 & 0.310 & 0.190 & $<20.0$ & 4 & $<10$ \\
\hline \multicolumn{13}{|l|}{ JAN 1993} \\
\hline 09.09 & 4.1 & 4.7 & 2.9 & $\cdots$ & 0.580 & $\cdots$ & 0.80 & 0.240 & 0.160 & $<10.0$ & 5 & $<10$ \\
\hline \multicolumn{13}{|l|}{ JAN } \\
\hline $23-23$ & 3.3 & 6.1 & 2.5 & $\cdots$ & 0.650 & $\cdots$ & 2.7 & 0.870 & 0.410 & $<10.0$ & 5 & $<10$ \\
\hline
\end{tabular}


Table 5. Water-quality data for storm-sewer outfall stations, Garland, Texas, 1992-93-Continued

08061660 - SLEEPY HOLLOW STREET OUTFALL AT NORTHWEST HIGHWAY, GARLAND, TX (WY 1993)-COntinUEd

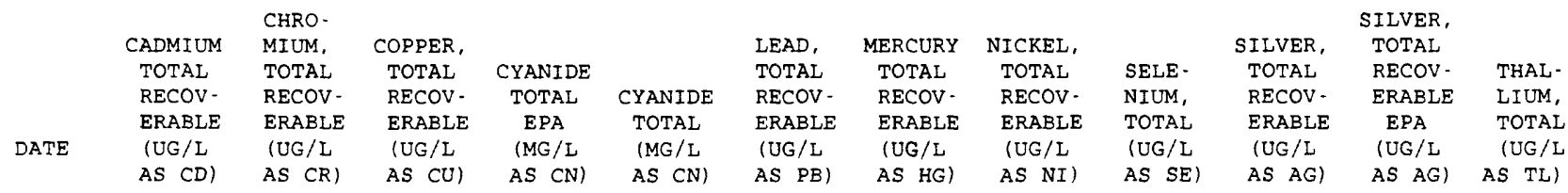

OCT 1992
$07-07$

$07-07$
NOV
$10-10$

Nov $10-10$

$19-19$
JAN 1993

JAN 1993
$09-09$

JAN

$23 \cdot 23$

$\begin{array}{lll} & & \\ & \text { ZINC, } & \\ & \text { TOTAL } & \text { CARBON, } \\ & \text { RECOV - } & \text { ORGANIC } \\ & \text { ERABLE } & \text { TOTAL } \\ \text { DATE } & \text { (UG } / L & \text { (MG } / \text { L } \\ & \text { AS ZN) } & \text { AS C) }\end{array}$

OIL AND

GREASE,

TOTAL

RECOV.

TOTAL TOTAL

NITRILE

TOTAL

(UG/L)

$\begin{array}{ll}40 & <0.10 \\ 25 & <0.10 \\ 11 & 0.10 \\ 24 & <0.10 \\ 52 & <0.10\end{array}$

\section{6}

5

2

$<1<0.500$

$<1<0.500$

$<1<0.500$

$<1<0.500$

$<1<0.500$

$<5$

OCT 1992

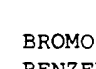

METHANE

BROMO

BENZENE CHLORO-

BENZENE

WATER,

CHLORO-

WHOLE,

(UG/L)

UNFLTRD

(UG/L)

$\begin{array}{lll}07.07 & 150 & 41 \\ \text { NOV } & & \\ 10.10 & 90 & 22 \\ \text { NOV } & & \\ 19-19 & 70 & 22 \\ \text { JAN } 1993 & & \\ 09-09 & 90 & 21 \\ \text { JAN } & & \\ 23-23 & 170 & 30\end{array}$

2
2
$<1$
1
$<1$

$\begin{array}{rll}10 & <20 & <20 \\ 5 & <20 & <20 \\ 5 & <20 & <20 \\ 3 & <20 & <20 \\ 2 & <20 & <20\end{array}$$$
<0.2
$$

$<0.2$

$<0.2<0.20$

$<0.2<0.20$

$<0.2$

$<0.2$

0.20

$<0.2$

$<0.2$

$<0.20$

$<0.2$

$<0$.

$<0.20$

O.

CHLORO

CHLORO-

ETHYL -

TERT - CARBON -

BUTYL- TETRA

$\begin{array}{cl}\text { WATER } & \text { CHLO } \\ \text { UNFLTRD } & \text { RIDE }\end{array}$

DATE

REC

RIDE BENZENE METHANE ETHANE

TOTAL TOTAL TOTAL TOTAL TOTAL

(UG/L)

(UG/L)

TOTAL

(UG/L)

$\begin{array}{lllll}<0.2 & <0.20 & <0.2 & <0.2 & <1.0 \\ <0.2 & <0.20 & <0.2 & <0.2 & <1.0 \\ <0.2 & <0.20 & <0.2 & <0.2 & <1.0 \\ <0.2 & <0.20 & <0.2 & <0.2 & <1.0 \\ <0.2 & <0.20 & <0.2 & <0.2 & <1.0\end{array}$

$<0$.

$<0.2$

$<0.2$

$<0$

$<0.2$

$<0.2$

$<0$.

$<0.2$

$<0.2$

$<0$.

$<0.20$

$<0$.

$<0.20$

$<0.20$

$<0$.

$<0.20$

$<0.20$

$<0.2$

$<0.20$

$<0.20$

NOV

$19-19$
JAN 1993

$<0.20$

$<0.20$

$<0.2<0.20$

$<0.2$

$<0.2$

$<0.2$

$<0.2$

$<0.20$

DIBROMO

DI -

CHLORO - BROMO-

JAN

$23-23<0.20$

$<0.2$

$<0.2$

$<0.2$

$<0.20$

$<0.2<0.20$

$\begin{array}{ll}<1.0 & <0.2 \\ <1.0 & <0.2 \\ <1.0 & <0.2 \\ <1.0 & <0.2 \\ <1.0 & <0.2\end{array}$


Table 5. Water-quality data for storm-sewer outfall stations, Garland, Texas, 1992-93-Continued

08061660 - SLEEPY HOLLOW STREET OUTFALL AT NORTHWEST HIGHWAY, GARLAND, TX (WY 1993)-ContinUEd

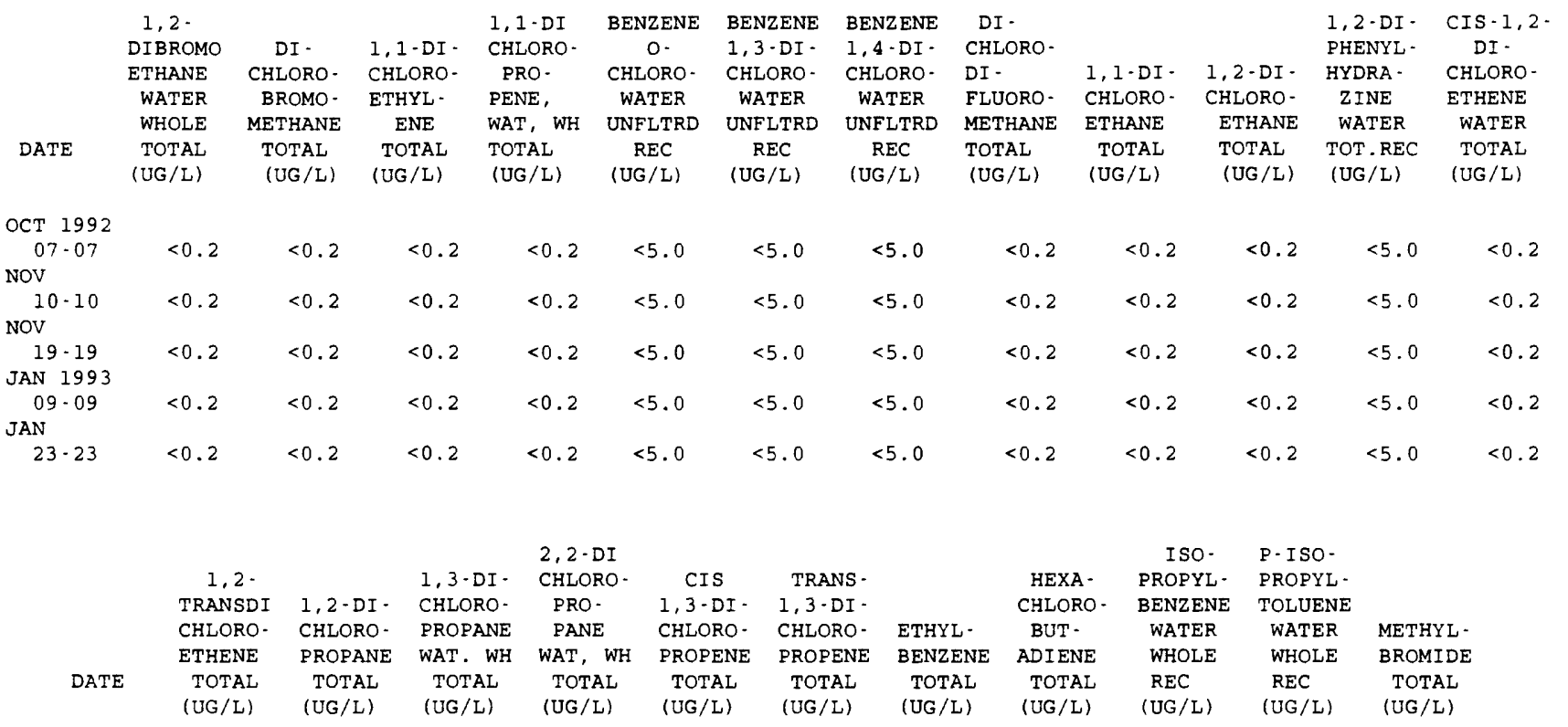

\begin{tabular}{|c|c|c|c|c|c|c|c|c|c|c|c|}
\hline \multicolumn{12}{|l|}{ OCT 1992} \\
\hline $07-07$ & $<0.2$ & $<0.2$ & $<0.2$ & $<0.2$ & $<0.2$ & $<0.2$ & $<0.2$ & $<5.0$ & $<0.20$ & $<0.20$ & $<0.2$ \\
\hline \multicolumn{12}{|l|}{ NOV } \\
\hline $10-10$ & $<0.2$ & $<0.2$ & $<0.2$ & $<0.2$ & $<0.2$ & $<0.2$ & $<0.2$ & $<5.0$ & $<0.20$ & $<0.20$ & $<0.2$ \\
\hline \multicolumn{12}{|l|}{ NOV } \\
\hline $\begin{array}{c}19-19 \\
\text { JAN } 1993\end{array}$ & $<0.2$ & $<0.2$ & $<0.2$ & $<0.2$ & $<0.2$ & $<0.2$ & $<0.2$ & $<5.0$ & $<0.20$ & $<0.20$ & $<0.2$ \\
\hline $09-09$ & $<0.2$ & $<0.2$ & $<0.2$ & $<0.2$ & $<0.2$ & $<0.2$ & $<0.2$ & $<5.0$ & $<0.20$ & $<0.20$ & $<0.2$ \\
\hline \multicolumn{12}{|l|}{ JAN } \\
\hline \multirow[t]{6}{*}{$23 \cdot 23$} & $<0.2$ & $<0.2$ & $<0.2$ & $<0.2$ & $<0.2$ & $<0.2$ &.$<0.2$ & $<5.0$ & $<0.20$ & $<0.20$ & $<0.2$ \\
\hline & & METHYL & & & & ETHANE, & ETHANE, & & & $1,2,3-$ & BENZENE \\
\hline & METHYL - & ETHER & & BENZENE & & $1112-$ & $1,1,2,2$ & TETRA - & & TRI - & $1,2,4-$ \\
\hline & ENE & TERT - & & $\mathrm{N}-\mathrm{PROPY}$ & & TETRA - & TETRA - & CHLORO - & & CHLORO & TRI - \\
\hline & CHLO - & BUTYL & NAPHTH - & WATER & & CHLORO- & CHLORO- & ETHYL - & & BENZENE & CHLORO - \\
\hline & RIDE & WAT UNF & ALENE & UNFLTRD & STYRENE & WAT UNF & WAT UNF & ENE & TOLUENE & WAT, WH & WAT UNF \\
\hline DATE & $\begin{array}{l}\text { TOTAL } \\
\text { (UG/L) }\end{array}$ & $\begin{array}{c}\text { REC } \\
(U G / L)\end{array}$ & $\begin{array}{l}\text { TOTAL } \\
\text { (UG/L) }\end{array}$ & $\begin{array}{c}\text { REC } \\
\text { (UG/L) }\end{array}$ & $\begin{array}{l}\text { TOTAL } \\
\text { (UG/L) }\end{array}$ & $\begin{array}{c}\text { REC } \\
(U G / L)\end{array}$ & $\begin{array}{c}\text { REC } \\
(\mathrm{UG} / \mathrm{L})\end{array}$ & $\begin{array}{l}\text { TOTAL } \\
\text { (UG/L) }\end{array}$ & $\begin{array}{l}\text { TOTAL } \\
\text { (UG/L) }\end{array}$ & $\begin{array}{c}\text { REC } \\
(U G / L)\end{array}$ & $\begin{array}{c}\text { REC } \\
(U G / L)\end{array}$ \\
\hline
\end{tabular}

\begin{tabular}{|c|c|c|c|c|c|c|c|c|c|c|c|}
\hline \multicolumn{12}{|l|}{ OCT 1992} \\
\hline $07-07$ & $<0.2$ & $<1.0$ & $<5.0$ & $<0.20$ & $<0.2$ & $<0.2$ & $<0.2$ & $<0.2$ & $<0.2$ & $<0.20$ & $<5.0$ \\
\hline \multicolumn{12}{|l|}{ Nov } \\
\hline $10-10$ & $<0.2$ & $<1.0$ & $<5.0$ & $<0.20$ & $<0.2$ & $<0.2$ & $<0.2$ & $<0.2$ & $<0.2$ & $<0.20$ & $<5.0$ \\
\hline \multicolumn{12}{|l|}{ NOV } \\
\hline $19-19$ & $<0.2$ & $<1.0$ & $<5.0$ & $<0.20$ & $<0.2$ & $<0.2$ & $<0.2$ & $<0.2$ & $<0.2$ & $<0.20$ & $<5.0$ \\
\hline \multicolumn{12}{|l|}{ JAN 1993} \\
\hline 09.09 & $<0.2$ & $<1.0$ & $<5.0$ & $<0.20$ & $<0.2$ & $<0.2$ & $<0.2$ & $<0.2$ & $<0.2$ & $<0.20$ & $<5.0$ \\
\hline \multicolumn{12}{|l|}{ JAN } \\
\hline $23-23$ & $<0.2$ & $<1.0$ & $<5.0$ & $<0.20$ & $<0.2$ & $<0.2$ & $<0.2$ & $<0.2$ & $<0.2$ & $<0.20$ & $<5.0$ \\
\hline
\end{tabular}


Table 5. Water-quality data for storm-sewer outfall stations, Garland, Texas, 1992-93-Continued

08061660 - SLEEPY HOLLOW STREET OUTFALL AT NORTHWEST HIGHWAY, GARLAND, TX (WY 1993)-Continued

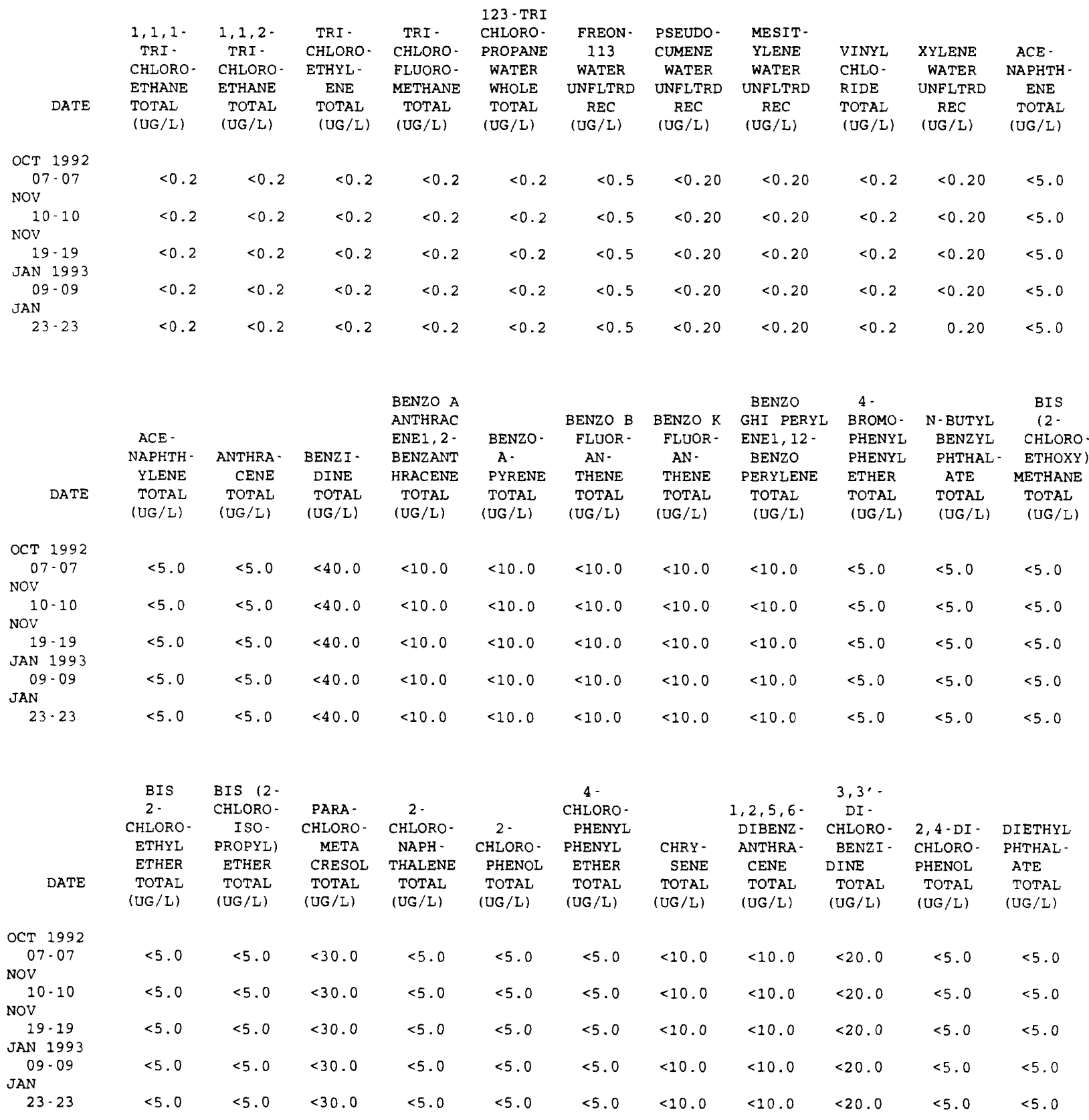


Table 5. Water-quality data for storm-sewer outfall stations, Garland, Texas, 1992-93-Continued 08061660 - SLEEPY HOLLOW STREET OUTFALL AT NORTHWEST HIGHWAY, GARLAND, TX (WY 1993)-CONEINUEd

\begin{tabular}{|c|c|c|c|c|c|c|c|c|c|c|c|}
\hline DATE & $\begin{array}{c}\text { DI - } \\
\text { METHYL } \\
\text { PHTHAL- } \\
\text { ATE } \\
\text { TOTAL } \\
\text { (UG/L) }\end{array}$ & $\begin{array}{c}2,4 \cdot D I- \\
\text { METHYL - } \\
\text { PHENOL } \\
\text { TOTAL } \\
(U G / L)\end{array}$ & $\begin{array}{c}\text { DI - N - } \\
\text { BUTYL } \\
\text { PHTHAL- } \\
\text { ATE } \\
\text { TOTAL } \\
\text { (UG/L) }\end{array}$ & $\begin{array}{c}4,6- \\
\text { DINITRO- } \\
\text { ORTHO- } \\
\text { CRESOL } \\
\text { TOTAL } \\
\text { (UG/L) }\end{array}$ & $\begin{array}{c}2,4,- \\
\text { DI- } \\
\text { NITRO- } \\
\text { PHENOL } \\
\text { TOTAL } \\
\text { (UG/L) }\end{array}$ & $\begin{array}{l}2,4-D I- \\
\text { NITRO- } \\
\text { TOLUENE } \\
\text { TOTAL } \\
\text { (UG/L) }\end{array}$ & $\begin{array}{l}2,6-D I- \\
\text { NITRO- } \\
\text { TOLUENE } \\
\text { TOTAL } \\
\text { (UG/L) }\end{array}$ & $\begin{array}{c}\text { DI - N- } \\
\text { OCTYL } \\
\text { PHTHAL- } \\
\text { ATE } \\
\text { TOTAL } \\
(\text { UG /L) }\end{array}$ & $\begin{array}{c}\text { BIS }(2 \cdot \\
\text { ETHYL } \\
\text { HEXYL) } \\
\text { PHTHAL- } \\
\text { ATE } \\
\text { TOTAL } \\
\text { (UG/L) }\end{array}$ & $\begin{array}{l}\text { FLUOR - } \\
\text { ANTHENE } \\
\text { TOTAL } \\
\text { (UG/L) }\end{array}$ & $\begin{array}{c}\text { FLUOR- } \\
\text { ENE } \\
\text { TOTAL } \\
\text { (UG/L) }\end{array}$ \\
\hline OCT 1992 & & & & & & & & & & & \\
\hline $\begin{array}{l}07-07 \\
\text { NOV }\end{array}$ & $<5.0$ & $<5.0$ & $<5.0$ & $<30.0$ & $<20.0$ & $<5.0$ & $<5.0$ & $<10.0$ & 11.0 & $<5.0$ & $<5.0$ \\
\hline $\begin{array}{l}10-10 \\
\text { Nov }\end{array}$ & $<5.0$ & $<5.0$ & $<5.0$ & $<30.0$ & $<20.0$ & $<5.0$ & $<5.0$ & $<10.0$ & 8.0 & $<5.0$ & $<5.0$ \\
\hline $\begin{array}{c}19-19 \\
\text { JAN } 1993\end{array}$ & $<5.0$ & $<5.0$ & $<5.0$ & $<30.0$ & $<20.0$ & $<5.0$ & $<5.0$ & $<10.0$ & 12.0 & $<5.0$ & $<5.0$ \\
\hline $\begin{array}{l}09-09 \\
\text { JAN }\end{array}$ & $<5.0$ & $<5.0$ & $<5.0$ & $<30.0$ & $<20.0$ & $<5.0$ & $<5.0$ & $<10.0$ & $<5.0$ & $<5.0$ & $<5.0$ \\
\hline $23-23$ & $<5.0$ & $<5.0$ & $<5.0$ & $<30.0$ & $<20.0$ & $<5.0$ & $<5.0$ & $<10.0$ & 8.0 & $<5.0$ & $<5.0$ \\
\hline DATE & $\begin{array}{l}\text { HEXA- } \\
\text { CHLORO- } \\
\text { BENZENE } \\
\text { TOTAL } \\
\text { (UG/L) }\end{array}$ & $\begin{array}{c}\text { HEXA- } \\
\text { CHLORO- } \\
\text { CYCLO- } \\
\text { PENT- } \\
\text { ADIENE } \\
\text { TOTAL } \\
\text { (UG/L) }\end{array}$ & $\begin{array}{l}\text { HEXA- } \\
\text { CHLORO- } \\
\text { ETHANE } \\
\text { TOTAL } \\
\text { (UG /L) }\end{array}$ & $\begin{array}{c}\text { INDENO } \\
(1,2,3- \\
\text { CD) } \\
\text { PYRENE } \\
\text { TOTAL } \\
\text { (UG/L) }\end{array}$ & $\begin{array}{l}\text { ISO- } \\
\text { PHORONE } \\
\text { TOTAL } \\
\text { (UG/L) }\end{array}$ & $\begin{array}{l}\text { NITRO - } \\
\text { BENZENE } \\
\text { TOTAL } \\
(U G / L)\end{array}$ & $\begin{array}{c}\text { N- NITRO- } \\
\text { SODI - } \\
\text { METHY - } \\
\text { LAMINE } \\
\text { TOTAL } \\
\text { (UG/L) }\end{array}$ & $\begin{array}{l}2- \\
\text { NITRO- } \\
\text { PHENOL } \\
\text { TOTAL } \\
\text { (UG/L) }\end{array}$ & $\begin{array}{c}4- \\
\text { NITRO- } \\
\text { PHENOL } \\
\text { TOTAL } \\
\text { (UG/L) }\end{array}$ & $\begin{array}{c}\text { N- } \\
\text { NITRO- } \\
\text { SODI-N- } \\
\text { PROPYL- } \\
\text { AMINE } \\
\text { TOTAL } \\
\text { (UG/L) }\end{array}$ & $\begin{array}{l}\text { N-NITRO- } \\
\text { SODI - } \\
\text { PHENY - } \\
\text { LAMINE } \\
\text { TOTAL } \\
\text { (UG/L) }\end{array}$ \\
\hline $\begin{array}{c}\text { OCT } 1992 \\
07.07\end{array}$ & $<5.0$ & $<5.0$ & $<5.0$ & $<10.0$ & $<5.0$ & $<5.0$ & $<5.0$ & $<5.0$ & $<30.0$ & $<5.0$ & $<5.0$ \\
\hline $\begin{array}{l}\text { Nov } \\
10-10 \\
\text { NOV }\end{array}$ & $<5.0$ & $<5.0$ & $<5.0$ & $<10.0$ & $<5.0$ & $<5.0$ & $<5.0$ & $<5.0$ & $<30.0$ & $<5.0$ & $<5.0$ \\
\hline $\begin{array}{c}19-19 \\
\text { JAN } 1993\end{array}$ & $<5.0$ & $<5.0$ & $<5.0$ & $<10.0$ & $<5.0$ & $<5.0$ & $<5.0$ & $<5.0$ & $<30.0$ & $<5.0$ & $<5.0$ \\
\hline $\begin{array}{l}09-09 \\
\text { JAN }\end{array}$ & $<5.0$ & $<5.0$ & $<5.0$ & $<10.0$ & $<5.0$ & $<5.0$ & $<5.0$ & $<5.0$ & $<30.0$ & $<5.0$ & $<5.0$ \\
\hline $23-23$ & $<5.0$ & $<5.0$ & $<5.0$ & $<10.0$ & $<5.0$ & $<5.0$ & $<5.0$ & $<5.0$ & $<30.0$ & $<5.0$ & $<5.0$ \\
\hline & $\begin{array}{r}\text { PENTA - } \\
\text { CHLORO - } \\
\text { PHENOL }\end{array}$ & $\begin{array}{l}\text { PHENAN - } \\
\text { THRENE }\end{array}$ & $\begin{array}{l}\text { PHENOL } \\
\text { (C6H - } \\
5 \mathrm{OH})\end{array}$ & PYRENE & $\begin{array}{c}2,4,6- \\
\text { TRI - } \\
\text { CHLORO- } \\
\text { PHENOL }\end{array}$ & ALDRIN, & $\begin{array}{l}\text { P, P' } \\
\text { DDT, }\end{array}$ & $\begin{array}{l}\text { ALPHA } \\
\text { BHC }\end{array}$ & $\begin{array}{c}\text { BETA } \\
\text { BENZENE } \\
\text { HEXA- } \\
\text { CHLOR- } \\
\text { IDE }\end{array}$ & $\begin{array}{l}\text { DELTA } \\
\text { BENZENE } \\
\text { HEXA- } \\
\text { CHLOR - } \\
\text { IDE }\end{array}$ & LINDANE \\
\hline DATE & $\begin{array}{l}\text { TOTAL } \\
\text { (UG/L) }\end{array}$ & $\begin{array}{l}\text { TOTAL } \\
\text { (UG/L) }\end{array}$ & $\begin{array}{l}\text { TOTAL } \\
(U G / L)\end{array}$ & $\begin{array}{l}\text { TOTAL } \\
\text { (UG/L) }\end{array}$ & $\begin{array}{l}\text { TOTAL } \\
\text { (UG/L) }\end{array}$ & $\begin{array}{l}\text { TOTAL } \\
\text { (UG/L) }\end{array}$ & $\begin{array}{l}\text { TOTAL } \\
\text { (UG/L) }\end{array}$ & $\begin{array}{l}\text { TOTAL } \\
\text { (UG/L) }\end{array}$ & $\begin{array}{l}\text { TOTAL } \\
\text { (UG/L) }\end{array}$ & $\begin{array}{c}\text { TOTAL } \\
\text { (UG/L) }\end{array}$ & $\begin{array}{l}\text { TOTAL } \\
\text { (UG/L) }\end{array}$ \\
\hline OCT 1992 & & & & & & & & & & & \\
\hline $\begin{array}{l}07-07 \\
\text { Jov }\end{array}$ & $<30.0$ & $<5.0$ & $<5.0$ & $<5.0$ & $<20.0$ & $<0.040$ & $<0.10$ & $<0.03$ & $<0.03$ & $<0.09$ & 0.21 \\
\hline $\begin{array}{l}10-10 \\
\text { NOV }\end{array}$ & $<30.0$ & $<5.0$ & $<5.0$ & $<5.0$ & $<20.0$ & $<0.040$ & $<0.10$ & $<0.03$ & $<0.03$ & $<0.09$ & $<0.030$ \\
\hline $\begin{array}{c}19-19 \\
\text { JAN } 1993\end{array}$ & $<30.0$ & $<5.0$ & $<5.0$ & $<5.0$ & $<20.0$ & $<0.040$ & $<0.10$ & $<0.03$ & $<0.03$ & $<0.09$ & $<0.030$ \\
\hline $\begin{array}{l}09-09 \\
\text { JAN }\end{array}$ & $<30.0$ & $<5.0$ & $<5.0$ & $<5.0$ & $<20.0$ & $<0.040$ & $<0.10$ & $<0.03$ & $<0.03$ & $<0.09$ & $<0.030$ \\
\hline $23-23$ & $<30.0$ & $<5.0$ & $<5.0$ & $<5.0$ & $<20.0$ & $<0.040$ & $<0.10$ & 60.0 & $<0.03$ & $=0.09$ & $<0.030$ \\
\hline
\end{tabular}


Table 5. Water-quality data for storm-sewer outfall stations, Garland, Texas, 1992-93-Continued

08061660 - SLEEPY HOLLOW STREET OUTFALL AT NORTHWEST HIGHWAY, GARLAND, TX (WY 1993)-CONTINUEd

\begin{tabular}{|c|c|c|c|c|c|c|c|c|c|c|c|}
\hline DATE & $\begin{array}{c}\text { CHLOR - } \\
\text { DANE } \\
\text { CIS } \\
\text { WATER } \\
\text { WHOLE } \\
\text { TOTAL } \\
\text { (UG/L) }\end{array}$ & $\begin{array}{c}\text { CHLOR - } \\
\text { DANE } \\
\text { TRANS } \\
\text { WATER } \\
\text { WHOLE } \\
\text { TOTAL } \\
\text { (UG/L) }\end{array}$ & $\begin{array}{l}\text { CHLOR- } \\
\text { DANE, } \\
\text { TOTAL } \\
\text { (UG/L) }\end{array}$ & $\begin{array}{c}\text { P, } P^{\prime} \\
\text { DDD, } \\
\text { TOTAL } \\
\text { (UG/L) }\end{array}$ & $\begin{array}{c}\text { P, } P^{\prime} \\
\text { DDE, } \\
\text { TOTAL } \\
\text { (UG/L) }\end{array}$ & $\begin{array}{l}\text { DI - } \\
\text { ELDRIN } \\
\text { TOTAL } \\
\text { (UG/L) }\end{array}$ & $\begin{array}{c}\text { ENDO- } \\
\text { SULFAN- } \\
\text { I } \\
\text { WATER } \\
\text { WHOLE } \\
\text { REC } \\
\text { (UG/L) }\end{array}$ & $\begin{array}{c}\text { ENDO- } \\
\text { SULFAN } \\
\text { BETA } \\
\text { TOTAL } \\
\text { (UG/L) }\end{array}$ & $\begin{array}{l}\text { ENDO- } \\
\text { SULFAN } \\
\text { SULFATE } \\
\text { TOTAL } \\
\text { (UG/L) }\end{array}$ & $\begin{array}{c}\text { ENDRIN } \\
\text { WATER } \\
\text { UNFLTRD } \\
\text { REC } \\
\text { (UG/L) }\end{array}$ & $\begin{array}{l}\text { ENDRIN } \\
\text { ALDE - } \\
\text { HYDE } \\
\text { TOTAL } \\
\text { (UG/L) }\end{array}$ \\
\hline OCT 1992 & & & & & & & & & & & \\
\hline $\begin{array}{l}07-07 \\
\text { Nov }\end{array}$ & $<0.10$ & $<0.10$ & $<0.1$ & $<0.10$ & $<0.04$ & $<0.020$ & $<0.10$ & $<0.04$ & $<0.60$ & $<0.060$ & $<0.20$ \\
\hline $\begin{array}{l}10-10 \\
\text { NOV }\end{array}$ & $<0.10$ & $<0.10$ & 0.1 & $<0.10$ & $<0.04$ & $<0.020$ & $<0.10$ & $<0.04$ & $<0.60$ & $<0.060$ & $<0.20$ \\
\hline $\begin{array}{c}19-19 \\
\text { JAN } 1993\end{array}$ & $<0.10$ & $<0.10$ & $<0.1$ & $<0.10$ & $<0.04$ & $<0.020$ & $<0.10$ & $<0.04$ & $<0.60$ & $<0.060$ & $<0.20$ \\
\hline $\begin{array}{l}09-09 \\
\text { JAN }\end{array}$ & $<0.10$ & $<0.10$ & $<0.1$ & $<0.10$ & $<0.04$ & $<0.020$ & $<0.10$ & $<0.04$ & $<0.60$ & $<0.060$ & $<0.20$ \\
\hline $23-23$ & $<0.10$ & $<0.10$ & 0.2 & $<0.10$ & $<0.04$ & $<0.020$ & $<0.10$ & $<0.04$ & $<0.60$ & $<0.060$ & $<0.20$ \\
\hline DATE & $\begin{array}{l}\text { HEPTA- } \\
\text { CHLOR, } \\
\text { TOTAL } \\
\text { (UG/L) }\end{array}$ & $\begin{array}{l}\text { HEPTA - } \\
\text { CHLOR } \\
\text { EPOXIDE } \\
\text { TOTAL } \\
\text { (UG/L) }\end{array}$ & $\begin{array}{c}\text { TOX - } \\
\text { APHENE, } \\
\text { TOTAL } \\
\text { (UG/L) }\end{array}$ & $\begin{array}{c}\text { AROCLOR } \\
1016 \\
\text { PCB } \\
\text { TOTAL } \\
\text { (UG/L) }\end{array}$ & $\begin{array}{c}\text { AROCLOR } \\
1221 \\
\text { PCB } \\
\text { TOTAL } \\
\text { (UG/L) }\end{array}$ & $\begin{array}{c}\text { AROCLOR } \\
1232 \\
\text { PCB } \\
\text { TOTAL } \\
\text { (UG/L) }\end{array}$ & $\begin{array}{c}\text { AROCLOR } \\
1242 \\
\text { PCB } \\
\text { TOTAL } \\
\text { (UG/L) }\end{array}$ & $\begin{array}{c}\text { AROCLOR } \\
1248 \\
\text { PCB } \\
\text { TOTAL } \\
\text { (UG/L) }\end{array}$ & $\begin{array}{c}\text { AROCLOR } \\
1254 \\
\text { PCB } \\
\text { TOTAL } \\
\text { (UG/L) }\end{array}$ & $\begin{array}{c}\text { AROCLOR } \\
1260 \\
\text { PCB } \\
\text { TOTAL } \\
\text { (UG/L) }\end{array}$ & $\begin{array}{c}\text { DI - } \\
\text { AZINON, } \\
\text { TOTAL } \\
\text { (UG/L) }\end{array}$ \\
\hline OCT 1992 & & & & & & & & & & & \\
\hline $\begin{array}{l}07-07 \\
\text { Nov }\end{array}$ & $<0.030$ & $<0.80$ & $<2$ & $<0.1$ & $<1.0$ & $<0.1$ & $<0.1$ & $<0.1$ & $<0.1$ & $<0.1$ & 4.7 \\
\hline $\begin{array}{l}10-10 \\
\text { Nov }\end{array}$ & $<0.030$ & $<0.80$ & $<2$ & $<0.1$ & $<1.0$ & $<0.1$ & $<0.1$ & $<0.1$ & $<0.1$ & $<0.1$ & 0.40 \\
\hline $\begin{array}{c}19-19 \\
\text { JAN } 1993\end{array}$ & $<0.030$ & $<0.80$ & $<2$ & $<0.1$ & $<1.0$ & $<0.1$ & $<0.1$ & $<0.1$ & $<0.1$ & $<0.1$ & 0.50 \\
\hline $\begin{array}{l}09.09 \\
\text { JAN }\end{array}$ & $<0.030$ & $<0.80$ & $<2$ & $<0.1$ & $<1.0$ & $<0.1$ & $<0.1$ & $<0.1$ & $<0.1$ & $<0.1$ & 0.10 \\
\hline $23-23$ & $<0.030$ & $<0.80$ & $<2$ & $<0.1$ & $<1.0$ & $<0.1$ & $<0.1$ & $<0.1$ & $<0.1$ & $<0.1$ & $<0.20$ \\
\hline
\end{tabular}


Table 5. Water-quality data for storm-sewer outfall stations, Garland, Texas, 1992-93-Continued

08061690 - I-635 OUTFALL AT CENTERVILLE ROAD, GARLAND, TX (WY 1992)

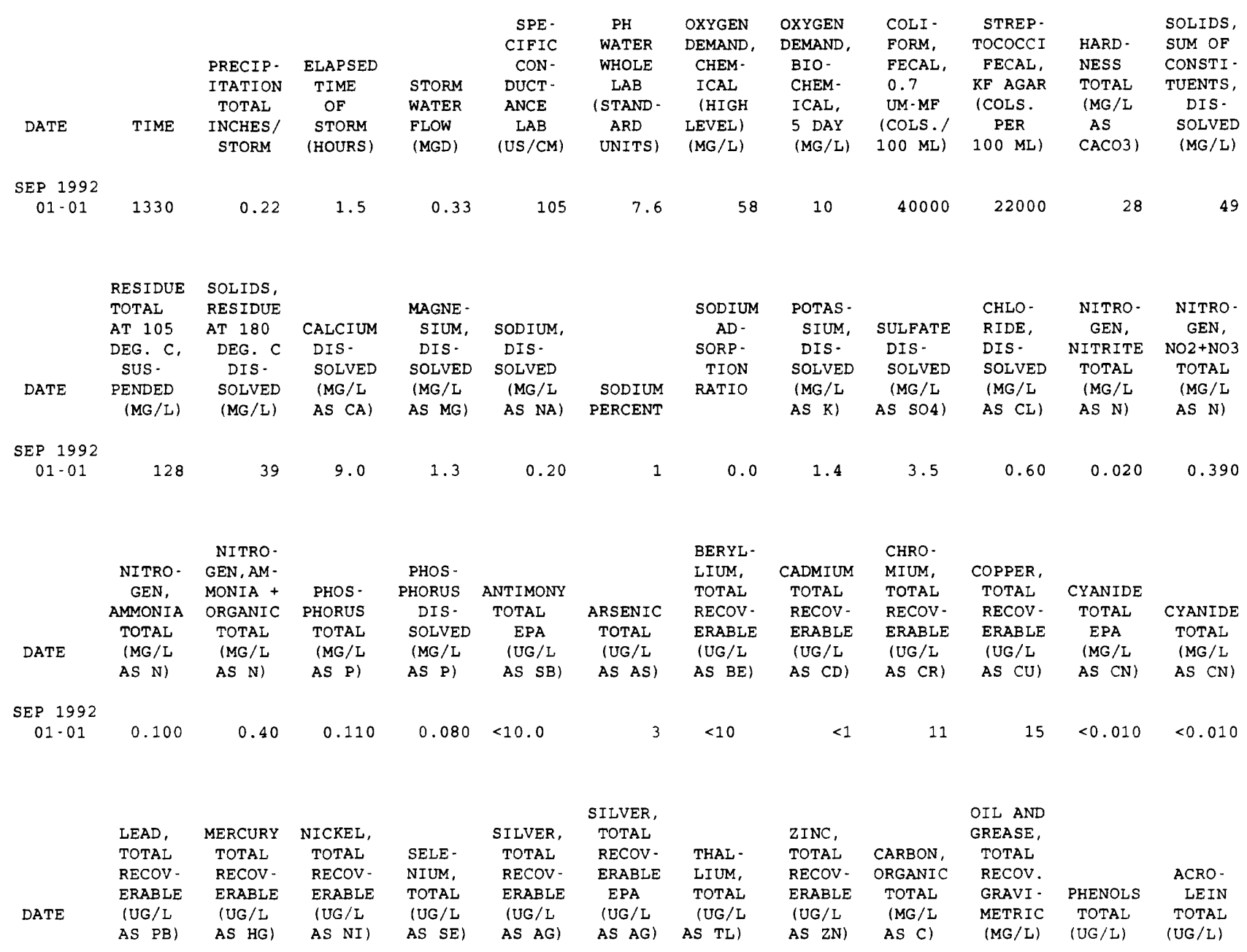

SEP 1992

$01-01$

$$
67
$$

$(0,10$

MAGNE -

SIUM, SODIUM,

SOLVED SOLVED

(MG/L (MG/L

SODIUM

SODIUM POTAS -

$\begin{array}{rcl}\text { AD - } & \text { SIUM, } & \text { SULFATE } \\ \text { SORP- } & \text { DIS - } & \text { DIS - } \\ \text { TION } & \text { SOLVED } & \text { SOLVED }\end{array}$

RIDE, GEN, GEN, DIS - NITRITE NO2+NO3 $\begin{array}{lll}\text { SOLVED } & \text { TOTAL } & \text { TOTAL } \\ \text { (MG } / \mathrm{L} & \text { (MG/L } & \text { (MG/L }\end{array}$ AS K) AS SO4)

$\mathrm{N})$

9$$
\text { 1 } 01
$$

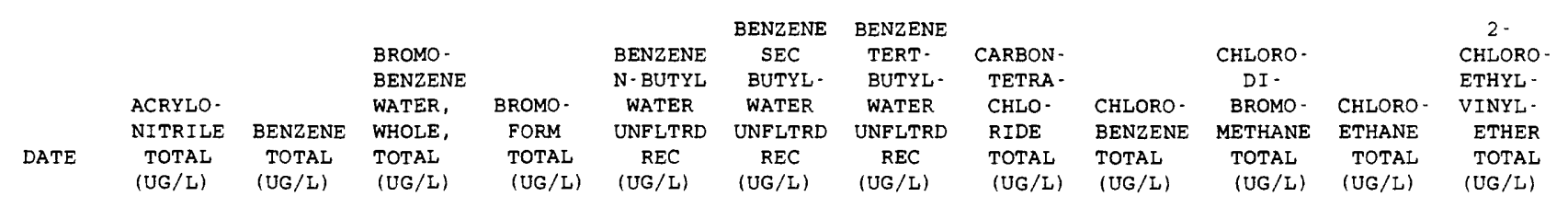

$$
\text { SEP } 1992
$$

$$
<0.2
$$

$$
<0.2
$$$$
<0.2
$$$$
<0.20
$$

$$
<0.20<0.20
$$$$
<0.2
$$$$
<0.20
$$$$
<0.2
$$$$
<0.2
$$$$
<1.0
$$

0 -

CHLOROMO DI- 1,2.

CHLORO- TOLUENE CHLORO- BROMO- DIBROMO

TOLUENE P-CHLOR PROPANE METHANE ETHANE

WATER WATER WATER WATER WATER

WHOLE UNFLTRD WHOLE WHOLE WHOLE

TOTAL

(UG/L)
REC TOT.REC RECOVER TOTAL

$\begin{array}{llll}(U G / L) & \text { (UG/L) } & \text { (UG/L) } & \text { (UG/L) }\end{array}$

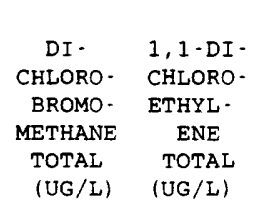

$1,1-D I$
CHLORO-

PRO - CHLORO- CHLORO.

PENE, WATER WATER

WAT, WH UNFLTRD UNFLTRD

TOTAL REC REC

(UG/L) (UG/L) (UG/L)

SEP 1992

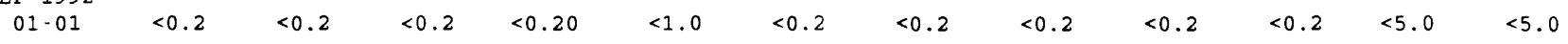


Table 5. Water-quality data for storm-sewer outfall stations, Garland, Texas, 1992-93-Continued

08061690 - I-635 OUTFALL AT CENTERVILLE ROAD, GARLAND, TX (WY 1992)-Continued

\begin{tabular}{|c|c|c|c|c|c|c|c|c|c|c|c|c|}
\hline DATE & $\begin{array}{c}\text { BENZENE } \\
1.4-D I- \\
\text { CHLORO- } \\
\text { WATER } \\
\text { UNFLTRD } \\
\text { REC } \\
\text { (UG/L) }\end{array}$ & $\begin{array}{c}\text { DI - } \\
\text { CHLORO- } \\
\text { DI - } \\
\text { FLUORO - } \\
\text { METHANE } \\
\text { TOTAL } \\
\text { (UG/L) }\end{array}$ & $\begin{array}{c}1,1 \cdot \mathrm{DI}- \\
\text { CHLORO- } \\
\text { ETHANE } \\
\text { TOTAL } \\
(\mathrm{UG} / \mathrm{L})\end{array}$ & $\begin{array}{c}1,2 \text {-DI - } \\
\text { CHLORO- } \\
\text { ETHANE } \\
\text { TOTAL } \\
\text { (UG/L) }\end{array}$ & $\begin{array}{l}1,2-D I- \\
\text { PHENYL- } \\
\text { HYDRA- } \\
\text { ZINE } \\
\text { WATER } \\
\text { TOT.REC } \\
\text { (UG/L) }\end{array}$ & $\begin{array}{c}\text { CIS - 1,2- } \\
\text { DI - } \\
\text { CHLORO- } \\
\text { ETHENE } \\
\text { WATER } \\
\text { TOTAL } \\
\text { (UG/L) }\end{array}$ & $\begin{array}{c}1,2- \\
\text { TRANSDI } \\
\text { CHLORO- } \\
\text { ETHENE } \\
\text { TOTAL } \\
\text { (UG/L) }\end{array}$ & $\begin{array}{l}1,2-D I- \\
\text { CHLORO- } \\
\text { PROPANE } \\
\text { TOTAL } \\
(U G / L)\end{array}$ & $\begin{array}{c}1,3 \text {-DI - } \\
\text { CHLORO- } \\
\text { PROPANE } \\
\text { WAT. WH } \\
\text { TOTAL } \\
\text { (UG/L) }\end{array}$ & $\begin{array}{l}2,2-D I \\
\text { CHLORO- } \\
\text { PRO- } \\
\text { PANE } \\
\text { WAT, WH } \\
\text { TOTAL } \\
\text { (UG/L) }\end{array}$ & $\begin{array}{c}\text { CIS } \\
1,3-D I- \\
\text { CHLORO- } \\
\text { PROPENE } \\
\text { TOTAL } \\
\text { (UG/L) }\end{array}$ & $\begin{array}{l}\text { TRANS - } \\
1,3 \text {-DI - } \\
\text { CHLORO- } \\
\text { PROPENE } \\
\text { TOTAL } \\
\text { (UG/L) }\end{array}$ \\
\hline $\begin{array}{c}\text { EP } 1992 \\
01.01\end{array}$ & $<5.0$ & $<0.2$ & $<0.2$ & $<0.2$ & $<5.0$ & $<0.2$ & $<0.2$ & $<0.2$ & $<0.2$ & $<0.2$ & $<0.2$ & $<0.2$ \\
\hline DATE & $\begin{array}{l}\text { ETHYL - } \\
\text { BENZENE } \\
\text { TOTAL } \\
\text { (UG/L) }\end{array}$ & $\begin{array}{l}\text { HEXA- } \\
\text { CHLORO- } \\
\text { BUT- } \\
\text { ADIENE } \\
\text { TOTAL } \\
\text { (UG/L) }\end{array}$ & $\begin{array}{l}\text { ISO- } \\
\text { PROPYL- } \\
\text { BENZENE } \\
\text { WATER } \\
\text { WHOLE } \\
\text { REC } \\
\text { (UG/L) }\end{array}$ & $\begin{array}{l}\text { P-ISO- } \\
\text { PROPYL- } \\
\text { TOLUENE } \\
\text { WATER } \\
\text { WHOLE } \\
\text { REC } \\
\text { (UG/L) }\end{array}$ & $\begin{array}{l}\text { METHYL - } \\
\text { BROMIDE } \\
\text { TOTAL } \\
\text { (UG/L) }\end{array}$ & $\begin{array}{l}\text { METHYL- } \\
\text { ENE } \\
\text { CHLO- } \\
\text { RIDE } \\
\text { TOTAL } \\
\text { (UG/L) }\end{array}$ & $\begin{array}{l}\text { NAPHTH - } \\
\text { ALENE } \\
\text { TOTAL } \\
\text { (UG/L) }\end{array}$ & $\begin{array}{c}\text { BENZENE } \\
\text { N-PROPY } \\
\text { WATER } \\
\text { UNFLTRD } \\
\text { REC } \\
(U G / L)\end{array}$ & $\begin{array}{l}\text { STYRENE } \\
\text { TOTAL } \\
\text { (UG/L) }\end{array}$ & $\begin{array}{c}\text { ETHANE, } \\
1112- \\
\text { TETRA- } \\
\text { CHLORO- } \\
\text { WAT UNF } \\
\text { REC } \\
\text { (UG/L) }\end{array}$ & $\begin{array}{l}\text { ETHANE, } \\
1,1,2,2 \\
\text { TETRA- } \\
\text { CHLORO- } \\
\text { WAT UNF } \\
\text { REC } \\
\text { (UG/L) }\end{array}$ & $\begin{array}{l}\text { TETRA- } \\
\text { CHLORO- } \\
\text { ETHYL- } \\
\text { ENE } \\
\text { TOTAL } \\
\text { (UG/L) }\end{array}$ \\
\hline
\end{tabular}

SEP 1992

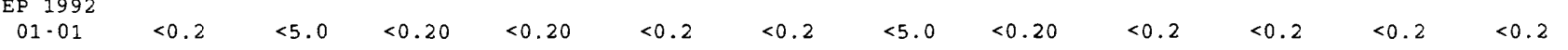

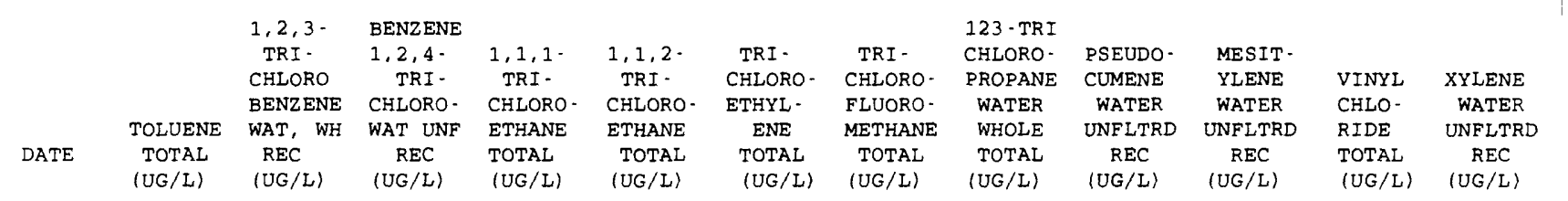

SEP 1992

$\begin{array}{lllllllllllll}01.01 & 0.3 & <0.20 & <5.0 & <0.2 & <0.2 & <0.2 & <0.2 & <0.2 & 0.20 & <0.20 & <0.2<0.40\end{array}$

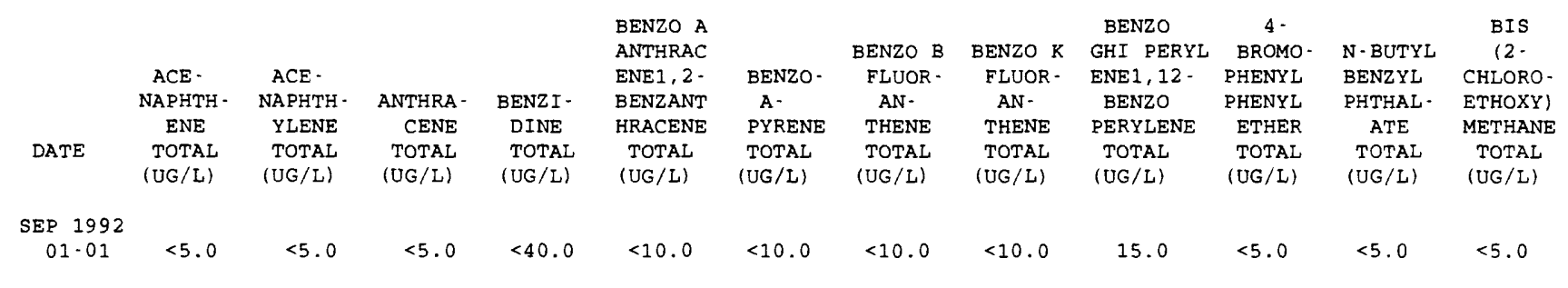

\begin{tabular}{|c|c|c|c|c|c|c|c|c|c|c|c|}
\hline DATE & $\begin{array}{c}\text { BIS } \\
2- \\
\text { CHLORO- } \\
\text { ETHYL } \\
\text { ETHER } \\
\text { TOTAL } \\
\text { (UG/L) }\end{array}$ & $\begin{array}{l}\text { BIS }(2- \\
\text { CHLORO- } \\
\text { ISO- } \\
\text { PROPYL) } \\
\text { ETHER } \\
\text { TOTAL } \\
\text { (UG/L) }\end{array}$ & $\begin{array}{l}\text { PARA - } \\
\text { CHLORO- } \\
\text { META } \\
\text { CRESOL } \\
\text { TOTAL } \\
\text { (UG/L) }\end{array}$ & $\begin{array}{c}2- \\
\text { CHLORO- } \\
\text { NAPH- } \\
\text { THALENE } \\
\text { TOTAL } \\
\text { (UG/L) }\end{array}$ & $\begin{array}{l}2 \text { - } \\
\text { CHLORO- } \\
\text { PHENOL } \\
\text { TOTAL } \\
\text { (UG/L) }\end{array}$ & $\begin{array}{c}4- \\
\text { CHLORO- } \\
\text { PHENYL } \\
\text { PHENYL } \\
\text { ETHER } \\
\text { TOTAL } \\
\text { (UG/L) }\end{array}$ & $\begin{array}{r}\text { CHRY - } \\
\text { SENE } \\
\text { TOTAL } \\
\text { (UG/L) }\end{array}$ & $\begin{array}{l}1,2,5,6- \\
\text { DIBENZ- } \\
\text { ANTHRA- } \\
\text { CENE } \\
\text { TOTAL } \\
\text { (UG/L) }\end{array}$ & $\begin{array}{c}3,3^{\prime}- \\
\text { DI - } \\
\text { CHLORO- } \\
\text { BENZI- } \\
\text { DINE } \\
\text { TOTAL } \\
\text { (UG/L) }\end{array}$ & $\begin{array}{l}2,4 \text {-DI - } \\
\text { CHLORO- } \\
\text { PHENOL } \\
\text { TOTAL } \\
(\text { UG } / L)\end{array}$ & $\begin{array}{l}\text { DIETHYL } \\
\text { PHTHAL- } \\
\text { ATE } \\
\text { TOTAL } \\
\text { (UG/L) }\end{array}$ \\
\hline $\begin{array}{c}\text { SEP } 1992 \\
01.01\end{array}$ & $<5.0$ & $<5.0$ & $<30.0$ & $<5.0$ & $<5.0$ & $<5.0$ & $<10.0$ & $<10.0$ & $<20.0$ & $<5.0$ & $<5.0$ \\
\hline DATE & $\begin{array}{c}\text { DI - } \\
\text { METHYL } \\
\text { PHTHAL- } \\
\text { ATE } \\
\text { TOTAL } \\
\text { (UG/L) }\end{array}$ & $\begin{array}{c}2,4-D I- \\
\text { METHYL - } \\
\text { PHENOL } \\
\text { TOTAL } \\
(U G / L)\end{array}$ & $\begin{array}{l}\text { DI - N- } \\
\text { BUTYL } \\
\text { PHTHAL- } \\
\text { ATE } \\
\text { TOTAL } \\
(U G / L)\end{array}$ & $\begin{array}{c}4,6- \\
\text { DINITRO- } \\
\text { ORTHO- } \\
\text { CRESOL } \\
\text { TOTAL } \\
\text { (UG/L) }\end{array}$ & $\begin{array}{c}2,4,- \\
\text { DI- } \\
\text { NITRO- } \\
\text { PHENOL } \\
\text { TOTAL } \\
\text { (UG/L) }\end{array}$ & $\begin{array}{l}2,4-D I- \\
\text { NITRO- } \\
\text { TOLUENE } \\
\text { TOTAL } \\
(U G / L)\end{array}$ & $\begin{array}{l}2,6-D I- \\
\text { NITRO- } \\
\text { TOLUENE } \\
\text { TOTAL } \\
\text { (UG/L) }\end{array}$ & $\begin{array}{c}\text { DI - N- } \\
\text { OCTYL } \\
\text { PHTHAL- } \\
\text { ATE } \\
\text { TOTAL } \\
\text { (UG/L) }\end{array}$ & $\begin{array}{c}\text { BIS }(2- \\
\text { ETHYL } \\
\text { HEXYL) } \\
\text { PHTHAL } \\
\text { ATE } \\
\text { TOTAL } \\
\text { (UG/L) }\end{array}$ & $\begin{array}{l}\text { FLUOR - } \\
\text { ANTHENE } \\
\text { TOTAL } \\
\text { (UG/L) }\end{array}$ & $\begin{array}{c}\text { FLUOR - } \\
\text { ENE } \\
\text { TOTAL } \\
\text { (UG/L) }\end{array}$ \\
\hline $\begin{array}{c}\text { SEP } 1992 \\
01-01\end{array}$ & $<5.0$ & $<5.0$ & $<5.0$ & $<30.0$ & $<20.0$ & $<5.0$ & $<5.0$ & $<10.0$ & $<5.0$ & 9.0 & $<5.0$ \\
\hline
\end{tabular}


Table 5. Water-quality data for storm-sewer outfall stations, Garland, Texas, 1992-93-Continued 08061690 - I-635 OUTFALL AT CENTERVILLE ROAD, GARLAND, TX (WY 1992)-Continued

\begin{tabular}{|c|c|c|c|c|c|c|c|c|c|c|c|}
\hline DATE & $\begin{array}{c}\text { HEXA- } \\
\text { CHLORO- } \\
\text { BENZENE } \\
\text { TOTAL } \\
(\mathrm{UG} / \mathrm{L})\end{array}$ & $\begin{array}{l}\text { HEXA- } \\
\text { CHLORO- } \\
\text { CYCLO- } \\
\text { PENT - } \\
\text { ADIENE } \\
\text { TOTAL } \\
\text { (UG/L) }\end{array}$ & $\begin{array}{c}\text { HEXA - } \\
\text { CHLORO- } \\
\text { ETHANE } \\
\text { TOTAL } \\
\text { (UG/L) }\end{array}$ & $\begin{array}{c}\text { INDENO } \\
(1,2,3- \\
\text { CD) } \\
\text { PYRENE } \\
\text { TOTAL } \\
(\text { UG /L) }\end{array}$ & $\begin{array}{l}\text { ISO- } \\
\text { PHORONE } \\
\text { TOTAL } \\
\text { (UG/L) }\end{array}$ & $\begin{array}{l}\text { NITRO- } \\
\text { BENZENE } \\
\text { TOTAL } \\
\text { (UG/L) }\end{array}$ & $\begin{array}{l}\text { N-NITRO- } \\
\text { SODI - } \\
\text { METHY - } \\
\text { LAMINE } \\
\text { TOTAL } \\
\text { (UG/L) }\end{array}$ & $\begin{array}{c}2 \text { - } \\
\text { NITRO- } \\
\text { PHENOL } \\
\text { TOTAL } \\
\text { (UG/L) }\end{array}$ & $\begin{array}{l}4- \\
\text { NITRO- } \\
\text { PHENOL } \\
\text { TOTAL } \\
(U G / L)\end{array}$ & $\begin{array}{c}\text { N- } \\
\text { NITRO- } \\
\text { SODI - N - } \\
\text { PROPYL - } \\
\text { AMINE } \\
\text { TOTAL } \\
\text { (UG/L) }\end{array}$ & $\begin{array}{l}\text { N- NITRO - } \\
\text { SODI - } \\
\text { PHENY - } \\
\text { LAMINE } \\
\text { TOTAL } \\
\text { (UG/L) }\end{array}$ \\
\hline $\begin{array}{c}\text { SEP } 1992 \\
01-01\end{array}$ & $<5.0$ & $<5.0$ & $<5.0$ & 15.0 & $<5.0$ & $<5.0$ & $<5.0$ & $<5.0$ & $<30.0$ & $<5.0$ & $<5.0$ \\
\hline DATE & $\begin{array}{l}\text { PENTA - } \\
\text { CHLORO - } \\
\text { PHENOL } \\
\text { TOTAL } \\
(\text { UG/L) }\end{array}$ & $\begin{array}{c}\text { PHENAN- } \\
\text { THRENE } \\
\text { TOTAL } \\
\text { (UG/L) }\end{array}$ & $\begin{array}{c}\text { PHENOL } \\
\text { (C6H - } \\
5 O H) \\
\text { TOTAL } \\
(U G / L)\end{array}$ & $\begin{array}{c}\text { PYRENE } \\
\text { TOTAL } \\
\text { (UG/L) }\end{array}$ & $\begin{array}{c}2,4,6- \\
\text { TRI- } \\
\text { CHLORO- } \\
\text { PHENOL } \\
\text { TOTAL } \\
\text { (UG/L) }\end{array}$ & $\begin{array}{c}\text { ALDRIN, } \\
\text { TOTAL } \\
\text { (UG/L) }\end{array}$ & $\begin{array}{c}\text { P, } P^{\prime} \\
\text { DDT, } \\
\text { TOTAL } \\
\text { (UG/L) }\end{array}$ & $\begin{array}{l}\text { ALPHA } \\
\text { BHC } \\
\text { TOTAL } \\
\text { (UG/L) }\end{array}$ & $\begin{array}{c}\text { BETA } \\
\text { BENZENE } \\
\text { HEXA- } \\
\text { CHLOR - } \\
\text { IDE } \\
\text { TOTAL } \\
\text { (UG/L) }\end{array}$ & $\begin{array}{c}\text { DELTA } \\
\text { BENZENE } \\
\text { HEXA- } \\
\text { CHLOR - } \\
\text { IDE } \\
\text { TOTAL } \\
\text { (UG/L) }\end{array}$ & $\begin{array}{c}\text { LINDANE } \\
\text { TOTAL } \\
\text { (UG/L) }\end{array}$ \\
\hline $\begin{array}{c}\text { SEP } 1992 \\
01-01\end{array}$ & $<30.0$ & $<5.0$ & $<5.0$ & 6.0 & $<20.0$ & $<0.040$ & $<0.10$ & $<0.03$ & $<0.03$ & $<0.09$ & $<0.030$ \\
\hline DATE & $\begin{array}{c}\text { CHLOR - } \\
\text { DANE } \\
\text { CIS } \\
\text { WATER } \\
\text { WHOLE } \\
\text { TOTAL } \\
\text { (UG/L) }\end{array}$ & $\begin{array}{c}\text { CHLOR - } \\
\text { DANE } \\
\text { TRANS } \\
\text { WATER } \\
\text { WHOLE } \\
\text { TOTAL } \\
\text { (UG/L) }\end{array}$ & $\begin{array}{l}\text { CHLOR- } \\
\text { DANE, } \\
\text { TOTAL } \\
\text { (UG/L) }\end{array}$ & $\begin{array}{c}\text { P, } P^{\prime} \\
\text { DDD, } \\
\text { TOTAL } \\
\text { (UG/L) }\end{array}$ & $\begin{array}{l}\mathrm{P}, \mathrm{P}^{\prime} \\
\mathrm{DDE} \\
\text { TOTAL } \\
\text { (UG/L) }\end{array}$ & $\begin{array}{l}\text { DI - } \\
\text { ELDRIN } \\
\text { TOTAL } \\
\text { (UG/L) }\end{array}$ & $\begin{array}{c}\text { ENDO- } \\
\text { SULFAN- } \\
\text { I } \\
\text { WATER } \\
\text { WHOLE } \\
\text { REC } \\
\text { (UG/L) }\end{array}$ & $\begin{array}{l}\text { ENDO- } \\
\text { SULFAN } \\
\text { BETA } \\
\text { TOTAL } \\
\text { (UG/L) }\end{array}$ & $\begin{array}{l}\text { ENDO- } \\
\text { SULFAN } \\
\text { SULFATE } \\
\text { TOTAL } \\
\text { (UG/L) }\end{array}$ & $\begin{array}{c}\text { ENDRIN } \\
\text { WATER } \\
\text { UNFLTRD } \\
\text { REC } \\
(\mathrm{UG} / \mathrm{L})\end{array}$ & $\begin{array}{c}\text { ENDRIN } \\
\text { ALDE - } \\
\text { HYDE } \\
\text { TOTAL } \\
\text { (UG/L) }\end{array}$ \\
\hline $\begin{array}{c}\text { SEP } 1992 \\
01.01\end{array}$ & $<0.10$ & $<0.10$ & 0.1 & $<0.10$ & $<0.04$ & $<0.020$ & $<0.10$ & $<0.04$ & $<0.60$ & $<0.060$ & $<0.20$ \\
\hline $\begin{array}{c}\text { SEP } 1992 \\
01-01\end{array}$ & $<0.030$ & $<0.80$ & $<2$ & $<0.1$ & $<1.0$ & $<0.1$ & $<0.1$ & $<0.1$ & $<0.1$ & $<0.1$ & 0.30 \\
\hline
\end{tabular}


Table 5. Water-quality data for storm-sewer outfall stations, Garland, Texas, 1992-93-Continued

$$
08061690 \text { - I-635 OUTFALL AT CENTERVILLE ROAD, GARLAND, TX (WY 1993) }
$$

\begin{tabular}{|c|c|c|c|c|c|c|c|c|c|c|c|c|}
\hline & & $\begin{array}{l}\text { PRECIP- } \\
\text { ITATION } \\
\text { TOTAL }\end{array}$ & $\begin{array}{l}\text { ELAPSED } \\
\text { TIME } \\
\text { OF }\end{array}$ & $\begin{array}{l}\text { STORM } \\
\text { WATER }\end{array}$ & $\begin{array}{l}\text { SPE- } \\
\text { CIFIC } \\
\text { CON- } \\
\text { DUCT- }\end{array}$ & $\begin{array}{c}\text { SPE- } \\
\text { CIFIC } \\
\text { CON- } \\
\text { DUCT - } \\
\text { ANCE }\end{array}$ & $\begin{array}{c}\text { PH } \\
\text { WATER } \\
\text { WHOLE } \\
\text { FIELD } \\
\text { (STAND- }\end{array}$ & $\begin{array}{c}\text { PH } \\
\text { WATER } \\
\text { WHOLE } \\
\text { LAB } \\
\text { (STAND - }\end{array}$ & $\begin{array}{l}\text { TEMPER - } \\
\text { ATURE }\end{array}$ & $\begin{array}{c}\text { OXYGEN } \\
\text { DEMAND, } \\
\text { CHEM- } \\
\text { ICAL } \\
\text { (HIGH }\end{array}$ & $\begin{array}{l}\text { OXYGEN } \\
\text { DEMAND, } \\
\text { BIO- } \\
\text { CHEM- } \\
\text { ICAL, }\end{array}$ & $\begin{array}{l}\text { COLI - } \\
\text { FORM, } \\
\text { FECAL, } \\
0.7 \\
\text { UM-MF }\end{array}$ \\
\hline DATE & TIME & $\begin{array}{l}\text { INCHES/ } \\
\text { STORM }\end{array}$ & $\begin{array}{c}\text { STORM } \\
\text { (HOURS) }\end{array}$ & $\begin{array}{l}\text { FLOW } \\
\text { (MGD) }\end{array}$ & $\begin{array}{c}\text { ANCE } \\
\text { (US/CM) }\end{array}$ & $\begin{array}{c}\text { LAB } \\
\text { (US/CM) }\end{array}$ & $\begin{array}{c}\text { ARD } \\
\text { UNITS) }\end{array}$ & $\begin{array}{c}\text { ARD } \\
\text { UNITS) }\end{array}$ & $\begin{array}{l}\text { WATER } \\
(\text { DEG C) }\end{array}$ & $\begin{array}{c}\text { LEVEL) } \\
\text { (MG/L) }\end{array}$ & $\begin{array}{l}5 \mathrm{DAY} \\
(\mathrm{MG} / \mathrm{L})\end{array}$ & $\begin{array}{l}\text { (COLS./ } \\
100 \mathrm{ML})\end{array}$ \\
\hline
\end{tabular}

$\begin{array}{cc}\text { OCT } 1992 & \\ 07-07 & 1750 \\ \text { OCT } & \\ 15-15 & 2049 \\ \text { NOV } & \\ 10-10 & 1420 \\ \text { NOV } & \\ 19-19 & 0230 \\ \text { JAN } 1993 & \\ 09-09 & 0300 \\ \text { JAN } & \\ 23-23 & 1806\end{array}$

\begin{tabular}{|c|c|c|c|c|c|c|c|c|c|c|}
\hline 0.26 & 2.2 & 0.24 & 111 & 120 & 7.6 & 7.0 & 20.5 & 56 & 8.6 & 36000 \\
\hline 0.81 & 1.1 & 0.55 & 82 & 85 & 8.1 & 7.3 & 21.0 & 63 & 7.6 & 26000 \\
\hline 0.28 & 4.4 & 0.23 & 123 & 120 & 8.2 & 7.4 & 20.0 & 97 & 6.1 & 9000 \\
\hline 0.55 & 9.8 & 0.50 & 125 & 120 & 8.1 & 7.3 & 16.5 & 51 & 7.6 & 48000 \\
\hline 0.30 & 5.0 & 0.28 & 69 & 104 & 7.8 & 8.0 & 10.5 & 81 & 5.1 & $\mathrm{~K} 6700$ \\
\hline 0.36 & 2.5 & 0.34 & 77 & 82 & 7.8 & 7.6 & 14.5 & 120 & 4.7 & 54000 \\
\hline
\end{tabular}

STREP -
TOCOCCI
FECAL,
KF AGAR
(COLS.
PETE $\quad$ PER
100 ML)

$\begin{array}{lllllll} & \text { HARD - } & \text { ALKA- } & \text { SOLIDS, } & \text { RESIDUE } & \text { SOLIDS, } & \\ \text { HARD- } & \text { NESS } & \text { LINITY } & \text { SUM OF } & \text { TOTAL } & \text { RESIDUE } & \\ \text { NESS } & \text { NONCARB } & \text { WAT DIS } & \text { CONSTI- } & \text { AT 105 } & \text { AT 180 } & \text { CALCIUM } \\ \text { TOTAL } & \text { DISSOLV } & \text { FIX END } & \text { TUENTS, } & \text { DEG. C, } & \text { DEG. C } & \text { DIS- } \\ \text { (MG } / L & \text { FLD. AS } & \text { FIELD } & \text { DIS- } & \text { SUS- } & \text { DIS- } & \text { SOLVED } \\ \text { AS } & \text { CACO3 } & \text { CAC03 } & \text { SOLVED } & \text { PENDED } & \text { SOLVED } & \text { (MG/L } \\ \text { CACO3) } & (M G / L) & (M G / L) & \text { (MG/L) } & \text { (MG/L) } & \text { (MG/L) } & \text { AS CA) }\end{array}$

MAGNE -

SIUM, SODIUM, DIS - DIS. SOLVED SOLVED (MG/L AS MG)

(MG/L AS NA)

$\begin{array}{cc} & \text { SODIUM } \\ & \text { AD - } \\ & \text { SORP. } \\ & \text { TION } \\ \text { SODIUM } & \text { RATIO } \\ \text { PERCENT } & \end{array}$

\begin{tabular}{cc} 
OCT 1992 & \\
$07-07$ & 7500 \\
OCT & \\
$15-15$ & 20000 \\
NOV & \\
$10-10$ & 10000 \\
NOV & \\
$19-19$ & 46000 \\
JAN 1993 & \\
09-09 & 24000 \\
JAN & \\
\hline
\end{tabular}

$\begin{array}{lll}29 & 11 & 18 \\ 14 & 3 & 11 \\ 29 & 4 & 25 \\ 34 & 3 & 31 \\ 36 & 6 & 30 \\ 24 & 1 & 23\end{array}$

$\begin{array}{rr}54 & 125 \\ 32 & 80 \\ 56 & 78 \\ 52 & 34 \\ - & 89 \\ 43 & 156\end{array}$

$\begin{array}{ll}55 & 11 \\ 30 & 5.5 \\ 32 & 11 \\ 36 & 13 \\ 39 & 14 \\ 47 & 9.2\end{array}$

$\begin{array}{ll}0.31 & 1.7 \\ 0.15 & 0.80 \\ 0.31 & 2.1 \\ 0.33 & 2.3 \\ 0.31 & 2.1 \\ 0.21 & 1.2\end{array}$

$\begin{array}{ll}10 & 0.1 \\ 10 & 0.1 \\ 13 & 0.2 \\ 12 & 0.2 \\ 10 & 0.2 \\ 9 & 0.1\end{array}$

\begin{tabular}{|c|c|c|}
\hline \multirow{5}{*}{ DATE } & \multirow{3}{*}{$\begin{array}{c}\text { POTAS - } \\
\text { SIUM, } \\
\text { DIS - } \\
\text { SOLVED }\end{array}$} & \multirow{4}{*}{$\begin{array}{l}\text { SULFATE } \\
\text { DIS - } \\
\text { SOLVED } \\
\text { (MG /L }\end{array}$} \\
\hline & & \\
\hline & & \\
\hline & (MG /L & \\
\hline & AS K) & AS SO4) \\
\hline
\end{tabular}

$\begin{array}{lccccc} & & & & \text { NITRO- } \\ \text { CHLO- } & \text { NITRO } & \text { NITRO- } & \text { NITRO- } & \text { GEN, AM- } \\ \text { RIDE, } & \text { GEN, } & \text { GEN, } & \text { GEN, } & \text { MONIA + } & \text { PHOS - } \\ \text { DIS - } & \text { NITRITE } & \text { NO2+NO3 } & \text { AMMONIA } & \text { ORGANIC } & \text { PHORUS } \\ \text { SOLVED } & \text { TOTAL } & \text { TOTAL } & \text { TOTAL } & \text { TOTAL } & \text { TOTAL } \\ \text { (MG/L } & \text { (MG/L } & \text { (MG/L } & \text { (MG/L } & \text { (MG/L } & \text { (MG/L } \\ \text { AS CL) } & \text { AS N) } & \text { AS N) } & \text { AS N) } & \text { AS N) } & \text { AS P) }\end{array}$

\begin{tabular}{|c|c|}
\hline $\begin{array}{r}\text { PHOS - } \\
\text { PHORUS }\end{array}$ & ANT IMONY \\
\hline DIS - & TOTAL \\
\hline SOLVED & EPA \\
\hline (MG/L & (UG/L \\
\hline AS P) & $A S \quad S B)$ \\
\hline
\end{tabular}

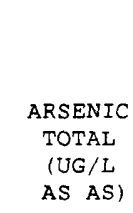

BERYL -

LIUM,

TOTAL

ERABLE

(UG/L

OCT 1992

\begin{tabular}{|c|c|c|c|c|c|c|c|c|c|c|c|c|}
\hline 07.07 & 2.2 & 7.4 & 1.9 & 0.050 & 0.790 & 0.430 & 2.2 & 0.400 & 0.150 & $<10.0$ & 2 & $<10$ \\
\hline \multicolumn{13}{|l|}{ OCT } \\
\hline $15-15$ & 1.2 & 2.8 & 0.90 & 0.030 & 0.230 & 0.180 & 0.80 & 0.180 & 0.080 & $<10.0$ & 2 & $<10$ \\
\hline \multicolumn{13}{|l|}{ Nov } \\
\hline $10-10$ & 2.5 & 6.0 & 2.5 & 0.080 & 0.400 & 0.140 & 0.80 & 0.190 & 0.090 & $<10.0$ & 2 & $<10$ \\
\hline \multicolumn{13}{|l|}{ Nov } \\
\hline $\begin{array}{cc}19-19 \\
\text { JAN } 1993\end{array}$ & 2.5 & 5.9 & 1.8 & 0.120 & 0.640 & 0.120 & 0.60 & 0.160 & 0.120 & $<10.0$ & 1 & $<10$ \\
\hline 09.09 & 2.4 & M3. 5 & 0.80 & $\cdots$ & 0.730 & $\cdots$ & 0.70 & 0.140 & 0.040 & $<10.0$ & 1 & $<10$ \\
\hline \multicolumn{13}{|l|}{ JAN } \\
\hline $23-23$ & 1.6 & 3.8 & 1.2 & $\cdots$ & 0.390 & $\cdots$ & 0.90 & 0.180 & 0.060 & $<10.0$ & 2 & $<10$ \\
\hline
\end{tabular}


Table 5. Water-quality data for storm-sewer outfall stations, Garland, Texas, 1992-93-Continued

08061690 - I-635 OUTFALL AT CENTERVILLE ROAD, GARLAND, TX (WY 1993)-Continued

\begin{tabular}{|c|c|c|c|c|c|c|c|c|c|c|c|c|}
\hline DATE & $\begin{array}{l}\text { CADMIUM } \\
\text { TOTAL } \\
\text { RECOV- } \\
\text { ERABLE } \\
\text { (UG/L } \\
\text { AS CD) }\end{array}$ & $\begin{array}{l}\text { CHRO- } \\
\text { MIUM, } \\
\text { TOTAL } \\
\text { RECOV- } \\
\text { ERABLE } \\
\text { (UG/L } \\
\text { AS CR) }\end{array}$ & $\begin{array}{l}\text { COPPER, } \\
\text { TOTAL } \\
\text { RECOV - } \\
\text { ERABLE } \\
\text { (UG/L } \\
\text { AS CU) }\end{array}$ & $\begin{array}{c}\text { CYANIDE } \\
\text { TOTAL } \\
\text { EPA } \\
\text { (MG/L } \\
\text { AS CN) }\end{array}$ & $\begin{array}{c}\text { CYANIDE } \\
\text { TOTAL } \\
\text { (MG/L } \\
\text { AS CN) }\end{array}$ & $\begin{array}{l}\text { LEAD, } \\
\text { TOTAL } \\
\text { RECOV- } \\
\text { ERABLE } \\
\text { (UG/L } \\
\text { AS PB) }\end{array}$ & $\begin{array}{l}\text { MERCURY } \\
\text { TOTAL } \\
\text { RECOV- } \\
\text { ERABLE } \\
\text { (UG/L } \\
\text { AS HG) }\end{array}$ & $\begin{array}{l}\text { NICKEL, } \\
\text { TOTAL } \\
\text { RECOV - } \\
\text { ERABLE } \\
\text { (UG/L } \\
\text { AS NI) }\end{array}$ & $\begin{array}{l}\text { SELE- } \\
\text { NIUM, } \\
\text { TOTAL } \\
\text { (UG/L } \\
\text { AS SE) }\end{array}$ & $\begin{array}{l}\text { SILVER, } \\
\text { TOTAL } \\
\text { RECOV- } \\
\text { ERABLE } \\
\text { (UG / L } \\
\text { AS AG) }\end{array}$ & $\begin{array}{l}\text { SILVER, } \\
\text { TOTAL } \\
\text { RECOV. } \\
\text { ERABLE } \\
\text { EPA } \\
\text { (UG / L } \\
\text { AS AG) }\end{array}$ & $\begin{array}{l}\text { THAL- } \\
\text { LIUM, } \\
\text { TOTAL } \\
\text { (UG/L } \\
\text { AS TL) }\end{array}$ \\
\hline OCT 1992 & & & & & & & & & & & & \\
\hline $\begin{array}{l}07.07 \\
\text { OCT }\end{array}$ & $<1$ & 5 & 25 & $<0.010$ & 0.080 & 46 & $<0.10$ & 6 & $<2$ & $<1$ & $<0.500$ & $<10$ \\
\hline $\begin{array}{l}15-15 \\
\text { Nov }\end{array}$ & $<1$ & 6 & 8 & $<0.010$ & $<0.010$ & 48 & $<0.10$ & 3 & $<2$ & $<1$ & $<0.500$ & $<5$ \\
\hline $\begin{array}{l}10 \cdot 10 \\
\text { Nov }\end{array}$ & $<1$ & 5 & 11 & $<0.010$ & $<0.010$ & 31 & $<0.10$ & 5 & $<2$ & $<1$ & $<1.00$ & $<5$ \\
\hline $\begin{array}{c}19-19 \\
\text { JAN } 1993\end{array}$ & $<1$ & 2 & 6 & $<0.010$ & $<0.010$ & 13 & 0.10 & 2 & $<2$ & $<1$ & $<0.500$ & $<10$ \\
\hline $\begin{array}{l}09-09 \\
\text { JAN }\end{array}$ & $<1$ & 7 & 10 & $<0.010$ & $<0.010$ & 30 & $<0.10$ & 4 & $<2$ & $<1$ & $<0.500$ & $<5$ \\
\hline $23-23$ & $<1$ & 11 & 11 & $<0.010$ & $<0.010$ & 39 & $<0.10$ & 4 & $<2$ & $<1$ & $<0.500$ & $<5$ \\
\hline
\end{tabular}

\begin{tabular}{|c|c|c|c|c|c|c|c|c|c|c|c|c|}
\hline & & & OIL AND & & & & & & METHANE & & & BENZENE \\
\hline & ZINC, & & GREASE, & & & & & BROMO - & BROMO & & BENZENE & SEC \\
\hline & TOTAL & CARBON, & TOTAL & & & & & BENZENE & CHLORO- & & N-BUTYL & BUTYL- \\
\hline & RECOV - & ORGANIC & RECOV. & & ACRO - & ACRYLO & & WATER, & WAT & BROMO - & WATER & WATER \\
\hline & ERABLE & TOTAL & GRAVI - & PHENOLS & LEIN & NITRILE & BENZENE & WHOLE, & UNFLTRD & FORM & UNFLTRD & UNFLTRD \\
\hline DATE & $\begin{array}{l}\text { (UG /L } \\
\text { AS ZN) }\end{array}$ & $\begin{array}{l}\text { (MG /L } \\
\text { AS C) }\end{array}$ & $\begin{array}{c}\text { METRIC } \\
\text { (MG/L) }\end{array}$ & $\begin{array}{l}\text { TOTAL } \\
\text { (UG/L) }\end{array}$ & $\begin{array}{l}\text { TOTAL } \\
\text { (UG/L) }\end{array}$ & $\begin{array}{l}\text { TOTAL } \\
(U G / L)\end{array}$ & $\begin{array}{c}\text { TOTAL } \\
(U G / L)\end{array}$ & $\begin{array}{l}\text { TOTAL } \\
\text { (UG/L) }\end{array}$ & $\begin{array}{c}\text { REC } \\
(U G / L)\end{array}$ & $\begin{array}{l}\text { TOTAL } \\
\text { (UG/L) }\end{array}$ & $\begin{array}{c}\text { REC } \\
(U G / L)\end{array}$ & $\begin{array}{c}\text { REC } \\
(\mathrm{UG} / \mathrm{L})\end{array}$ \\
\hline OCT 1992 & & & & & & & & & & & & \\
\hline $\begin{array}{l}07.07 \\
\text { OCT }\end{array}$ & 190 & 33 & 4 & 4 & $<20$ & $<20$ & 0.6 & $<0.2$ & $<0.20$ & $<0.2$ & $<0.20$ & $<0.20$ \\
\hline $15 \cdot 15$ & 80 & 13 & $<1$ & 3 & $<20$ & $<20$ & 0.3 & $<0.2$ & $<0.20$ & $<0.2$ & $<0.20$ & $<0.20$ \\
\hline NOV & & & & & & & & & & & & \\
\hline $10-10$ & 130 & 20 & 2 & 5 & $<20$ & $<20$ & 0.2 & $<0.2$ & $<0.20$ & $<0.2$ & $<0.20$ & $<0.20$ \\
\hline NOV & & & & & & & & & & & & \\
\hline $\begin{array}{c}19.19 \\
\text { JAN } 1993\end{array}$ & 70 & 14 & 2 & 3 & $<20$ & $<20$ & 0.4 & $<0.2$ & $<0.20$ & $<0.2$ & $<0.20$ & $<0.20$ \\
\hline 09.09 & 120 & 16 & 5 & 2 & $<20$ & $<20$ & 0.2 & $<0.2$ & $<0.20$ & $<0.2$ & $<0.20$ & $<0.20$ \\
\hline AN & & & & & & & & & & & & \\
\hline $23-23$ & 110 & 8.3 & 1 & 3 & $<20$ & $<20$ & 0.6 & $<0.2$ & $<0.20$ & $<0.2$ & $<0.20$ & $<0.20$ \\
\hline
\end{tabular}

\begin{tabular}{|c|c|c|c|c|c|c|c|c|c|c|c|}
\hline \multirow{2}{*}{$\begin{array}{c}\text { BENZENE } \\
\text { TERT }\end{array}$} & \multirow[b]{2}{*}{ CARBON - } & \multirow{2}{*}{\multicolumn{2}{|c|}{ CHLORO- }} & \multicolumn{3}{|c|}{2 . } & \multicolumn{3}{|c|}{$0-$} & DIBROMO & DI - \\
\hline & & & & & CHLORO - & & & CHLORO- & TOLUENE & CHLORO- & BROMO - \\
\hline BUTYL - & TETRA. & & DI - & & ETHYL - & & METHYL - & TOLUENE & P-CHLOR & PROPANE & METHANE \\
\hline WATER & CHLO- & CHLORO - & BROMO - & CHLORO - & VINYL - & CHLORO- & CHLO. & WATER & WATER & WATER & WATER \\
\hline UNFLTRD & RIDE & BENZENE & METHANE & ETHANE & ETHER & FORM & RIDE & WHOLE & UNFLTRD & WHOLE & WHOLE \\
\hline $\begin{array}{c}\text { REC } \\
(U G / L)\end{array}$ & $\begin{array}{l}\text { TOTAL } \\
\text { (UG/L) }\end{array}$ & $\begin{array}{l}\text { TOTAL } \\
\text { (UG/L) }\end{array}$ & $\begin{array}{l}\text { TOTAL } \\
\text { (UG/L) }\end{array}$ & $\begin{array}{l}\text { TOTAL } \\
\text { (UG/L) }\end{array}$ & $\begin{array}{l}\text { TOTAL } \\
\text { (UG/L) }\end{array}$ & $\begin{array}{l}\text { TOTAL } \\
\text { (UG/L) }\end{array}$ & $\begin{array}{l}\text { TOTAL } \\
\text { (UG/L) }\end{array}$ & $\begin{array}{l}\text { TOTAL } \\
\text { (UG/L) }\end{array}$ & $\begin{array}{c}\text { REC } \\
(U G / L)\end{array}$ & $\begin{array}{l}\text { TOT.REC } \\
\text { (UG/L) }\end{array}$ & $\begin{array}{l}\text { RECOVER } \\
\text { (UG/L) }\end{array}$ \\
\hline
\end{tabular}

\begin{tabular}{|c|c|c|c|c|c|c|c|c|c|c|c|c|}
\hline 07.07 & $<0.20$ & $<0.2$ & $<0.20$ & $<0.2$ & $<0.2$ & $<1.0$ & $<0.2$ & $<0.2$ & $<0.2$ & $<0.20$ & $<1.0$ & $<0.2$ \\
\hline \multicolumn{13}{|l|}{ OCT } \\
\hline $15-15$ & $<0.20$ & $<0.2$ & $<0.20$ & $<0.2$ & $<0.2$ & $<1.0$ & $<0.2$ & $<0.2$ & $<0.2$ & $<0.20$ & $<1.0$ & $<0.2$ \\
\hline \multicolumn{13}{|l|}{ NOV } \\
\hline $10-10$ & $<0.20$ & $<0.2$ & $<0.20$ & $<0.2$ & $<0.2$ & $<1.0$ & $<0.2$ & $<0.2$ & $<0.2$ & $<0.20$ & $<1.0$ & $<0.2$ \\
\hline \multicolumn{13}{|l|}{ NOV } \\
\hline $19-19$ & $<0.20$ & $<0.2$ & $<0.20$ & $<0.2$ & $<0.2$ & $<1.0$ & $<0.2$ & $<0.2$ & $<0.2$ & $<0.20$ & $<1.0$ & $<0.2$ \\
\hline \multicolumn{13}{|l|}{ JAN 1993} \\
\hline 09.09 & $<0.20$ & $<0.2$ & $<0.20$ & $<0.2$ & $<0.2$ & $<1.0$ & $<0.2$ & $<0.2$ & $<0.2$ & $<0.20$ & $<1.0$ & $<0.2$ \\
\hline \multicolumn{13}{|l|}{ JAN } \\
\hline $23 \cdot 23$ & $<0.20$ & $<0.2$ & $<0.20$ & $<0.2$ & $<0.2$ & $<1.0$ & $<0.2$ & $<0.2$ & $<0.2$ & $<0.20$ & $<1.0$ & $<0.2$ \\
\hline
\end{tabular}


rable 5. Water-quality data for storm-sewer outfall stations, Garland, Texas, 1992-93-Continued

08061690 - I-635 OUTFALL AT CENTERVILLE ROAD, GARLAND, TX (WY 1993)-Continued

\begin{tabular}{|c|c|c|c|c|c|c|c|c|c|c|c|c|}
\hline & 1,2 & & & $1,1-D I$ & BENZENE & BENZENE & BENZENE & DI - & & & $1,2-D I-$ & CIS- 1,2 \\
\hline & DIBROMO & DI - & $1,1-\mathrm{DI}$ - & CHLORO- & 0 & $1,3 \cdot \mathrm{DI}$ - & $1,4 \cdot \mathrm{DI}-$ & CHLORO - & & & PHENYI - & DI - \\
\hline & $\begin{array}{r}\text { ETHANE } \\
\text { WATER }\end{array}$ & $\begin{array}{l}\text { CHLORO - } \\
\text { BROMO - }\end{array}$ & $\begin{array}{l}\text { CHLORO- } \\
\text { ETHYL - }\end{array}$ & $\begin{array}{l}\text { PRO- } \\
\text { PENE, }\end{array}$ & $\begin{array}{c}\text { CHLORO- } \\
\text { WATER }\end{array}$ & $\begin{array}{c}\text { CHLORO- } \\
\text { WATER }\end{array}$ & $\begin{array}{c}\text { CHLORO- } \\
\text { WATER }\end{array}$ & $\begin{array}{l}\text { DI - } \\
\text { FLUORO- }\end{array}$ & $\begin{array}{l}1,1 \text {-DI - } \\
\text { CHLORO- }\end{array}$ & $\begin{array}{l}\text { 1,2-DI - } \\
\text { CHLORO- }\end{array}$ & $\begin{array}{c}\text { HYDRA - } \\
\text { ZINE }\end{array}$ & $\begin{array}{l}\text { CHLORO- } \\
\text { ETHENE }\end{array}$ \\
\hline & WHOLE & METHANE & ENE & WAT, WH & UNFLTRD & UNFLTRD & UNFLTRD & METHANE & ETHANE & ETHANE & WATER & WATER \\
\hline & $\begin{array}{l}\text { TOTAL } \\
\text { (UG/L) }\end{array}$ & $\begin{array}{l}\text { TOTAL } \\
\text { (UG/L) }\end{array}$ & $\begin{array}{l}\text { TOTAL } \\
\text { (UG/L) }\end{array}$ & $\begin{array}{l}\text { TOTAL } \\
(U G / L)\end{array}$ & $\begin{array}{c}\text { REC } \\
(U G / L)\end{array}$ & $\begin{array}{c}\text { REC } \\
(U G / L)\end{array}$ & $\begin{array}{c}\text { REC } \\
(U G / L)\end{array}$ & $\begin{array}{l}\text { TOTAL } \\
\text { (UG/L) }\end{array}$ & $\begin{array}{l}\text { TOTAL } \\
\text { (UG/L) }\end{array}$ & $\begin{array}{l}\text { TOTAL } \\
\text { (UG/L) }\end{array}$ & $\begin{array}{l}\text { TOT . REC } \\
\text { (UG/L) }\end{array}$ & $\begin{array}{l}\text { TOTAL } \\
\text { (UG/L) }\end{array}$ \\
\hline
\end{tabular}

\begin{tabular}{|c|c|c|c|c|c|c|c|c|c|c|c|c|}
\hline \multicolumn{13}{|l|}{ OCT 1992} \\
\hline $07-07$ & $<0.2$ & $<0.2$ & $<0.2$ & $<0.2$ & $<5.0$ & $<5.0$ & $<5.0$ & $<0.2$ & $<0.2$ & $<0.2$ & $<5.0$ & $<0.2$ \\
\hline \multicolumn{13}{|l|}{ OCT } \\
\hline $15-15$ & $<0.2$ & $<0.2$ & $<0.2$ & $<0.2$ & $<5.0$ & $<5.0$ & $<5.0$ & $<0.2$ & $<0.2$ & $<0.2$ & $<5.0$ & $<0.2$ \\
\hline \multicolumn{13}{|l|}{ Nov } \\
\hline $10-10$ & $<0.2$ & $<0.2$ & $<0.2$ & $<0.2$ & $<5.0$ & $<5.0$ & $<5.0$ & $<0.2$ & $<0.2$ & $<0.2$ & $<5.0$ & $<0.2$ \\
\hline \multicolumn{13}{|l|}{ NOV } \\
\hline $19 \cdot 19$ & $<0.2$ & $<0.2$ & $<0.2$ & $<0.2$ & $<5.0$ & $<5.0$ & $<5.0$ & $<0.2$ & $<0.2$ & $<0.2$ & $<5.0$ & $<0.2$ \\
\hline \multicolumn{13}{|l|}{ JAN 1993} \\
\hline 09.09 & $<0.2$ & $<0.2$ & $<0.2$ & $<0.2$ & $<5.0$ & $<5.0$ & $<5.0$ & $<0.2$ & $<0.2$ & $<0.2$ & $<5.0$ & $<0.2$ \\
\hline \multicolumn{13}{|l|}{ JAN } \\
\hline $23-23$ & $<0.2$ & $<0.2$ & $<0.2$ & $<0.2$ & $<5.0$ & $<5.0$ & $<5.0$ & $<0.2$ & $<0.2$ & $<0.2$ & $<5.0$ & $<0.2$ \\
\hline
\end{tabular}

DATE

\begin{tabular}{|c|c|c|c|c|c|c|c|c|c|c|}
\hline $\begin{array}{c}1,2- \\
\text { RANSDI }\end{array}$ & $1,2-\mathrm{DI}-$ & $\begin{array}{l}1,3-\mathrm{DI} \text { - } \\
\text { CHLORO- }\end{array}$ & $\begin{array}{c}2,2 \text { - DI } \\
\text { CHLORO- } \\
\text { PRO- }\end{array}$ & $\begin{array}{l}\text { CIS } \\
1,3-D I-\end{array}$ & $\begin{array}{l}\text { TRANS - } \\
1,3 \text {-DI - }\end{array}$ & & $\begin{array}{l}\text { HEXA- } \\
\text { CHLORO- }\end{array}$ & $\begin{array}{c}\text { ISO- } \\
\text { PROPYL- } \\
\text { BENZENE }\end{array}$ & $\begin{array}{l}\text { P-ISO- } \\
\text { PROPYL- } \\
\text { TOLUENE }\end{array}$ & \\
\hline HLORO- & CHLORO - & PROPANE & PANE & CHLORO- & CHLORO - & ETHYL - & BUT - & WATER & WATER & METHYI - \\
\hline
\end{tabular}

OCT 1992

$07-07$
OCT
$15-15$
NOV
$10-10$
NOV
$19-19$
JAN 1993
O9-09
JAN

$\begin{array}{lllllllllll}<0.2 & <0.2 & <0.2 & <0.2 & <0.2 & <0.2 & 0.6 & <5.0 & <0.20 & <0.20 & <0.2 \\ <0.2 & <0.2 & <0.2 & <0.2 & <0.2 & <0.2 & 0.3 & <5.0 & <0.20 & <0.20 & <0.2 \\ <0.2 & <0.2 & <0.2 & <0.2 & <0.2 & <0.2 & 0.2 & <5.0 & <0.20 & <0.20 & <0.2 \\ <0.2 & <0.2 & <0.2 & <0.2 & <0.2 & <0.2 & 0.3 & <5.0 & <0.20 & <0.20 & <0.2 \\ <0.2 & <0.2 & <0.2 & <0.2 & <0.2 & <0.2 & 0.2 & <5.0 & <0.20 & <0.20 & <0.2 \\ <0.2 & <0.2 & <0.2 & <0.2 & <0.2 & <0.2 & 0.6 & <5.0 & <0.20 & <0.20 & <0.2\end{array}$

$23-23$

$<0.2$

$<0.2$

$<0.2$

$<0.2$

$<0.2$

0.6

$<5.0$

ETHANE, ETHANE,

$\begin{array}{cccccc} & \text { METHYL } & & & \text { ETHANE, } \\ \text { METHYL- } & \text { ETHER } & & \text { BENZENE } & & 1112- \\ \text { ENE } & \text { TERT - } & & \text { N-PROPY } & & \text { TETRA- } \\ \text { CHLO- } & \text { BUTYL } & \text { NAPHTH - } & \text { WATER } & & \text { CHLORO- } \\ \text { RIDE } & \text { WAT UNF } & \text { ALENE } & \text { UNFLTRD } & \text { STYRENE } & \text { WAT UNF } \\ \text { TOTAL } & \text { REC } & \text { TOTAL } & \text { REC } & \text { TOTAL } & \text { REC } \\ \text { (UG/L) } & \text { (UG/L) } & \text { (UG/L) } & \text { (UG/L) } & \text { (UG/L) } & \text { (UG/L) }\end{array}$

ETHANE,
$1,1,2,2$

$1,1,2,2$ TETRA -

TETRA- CHLORO-

WAT UNF ENE

REC TOTAL

$\begin{array}{cc}\text { REC } & \text { TOTAL } \\ \text { (UG/L) } & \text { (UG/L) }\end{array}$

$\begin{array}{ccc} & 1,2,3- & \text { BENZENE } \\ & \text { TRI- } & 1,2,4 \\ & \text { CHLORO } & \text { TRI- } \\ & \text { BENZENE } & \text { CHLORO- } \\ \text { TOLUENE } & \text { WAT, WH } & \text { WAT UNF } \\ \text { TOTAL } & \text { REC } & \text { REC } \\ (U G / L) & \text { (UG/L) } & \text { (UG/L) }\end{array}$

OCT 1992

07-07

OCT

$15-15$

NOV

$10-10$

NOV

$19-19$

JAN 1993

$09-09$

JAN

$23-23$

$\begin{array}{lllllllllll}<0.2 & 8.3 & <5.0 & <0.20 & <0.2 & <0.2 & <0.2 & <0.2 & 2.0 & <0.20 & <5.0 \\ <0.2 & 3.0 & <5.0 & <0.20 & <0.2 & <0.2 & <0.2 & <0.2 & 1.0 & <0.20 & <5.0 \\ <0.2 & 1.5 & <5.0 & <0.20 & <0.2 & <0.2 & <0.2 & <0.2 & 0.6 & <0.20 & <5.0 \\ <0.2 & 4.1 & <5.0 & <0.20 & <0.2 & <0.2 & <0.2 & <0.2 & 1.2 & <0.20 & <5.0 \\ <0.2 & 2.1 & <5.0 & <0.20 & <0.2 & <0.2 & <0.2 & <0.2 & 0.6 & <0.20 & <5.0 \\ <0.2 & 8.7 & <5.0 & <0.20 & <0.2 & <0.2 & <0.2 & <0.2 & 2.4 & <0.20 & <5.0\end{array}$


Table 5. Water-quality data for storm-sewer outfall stations, Garland, Texas, 1992-93-Continued

$$
08061690 \text { - I-635 OUTFALL AT CENTERVILLE ROAD, GARLAND, TX (WY 1993)-Continued }
$$

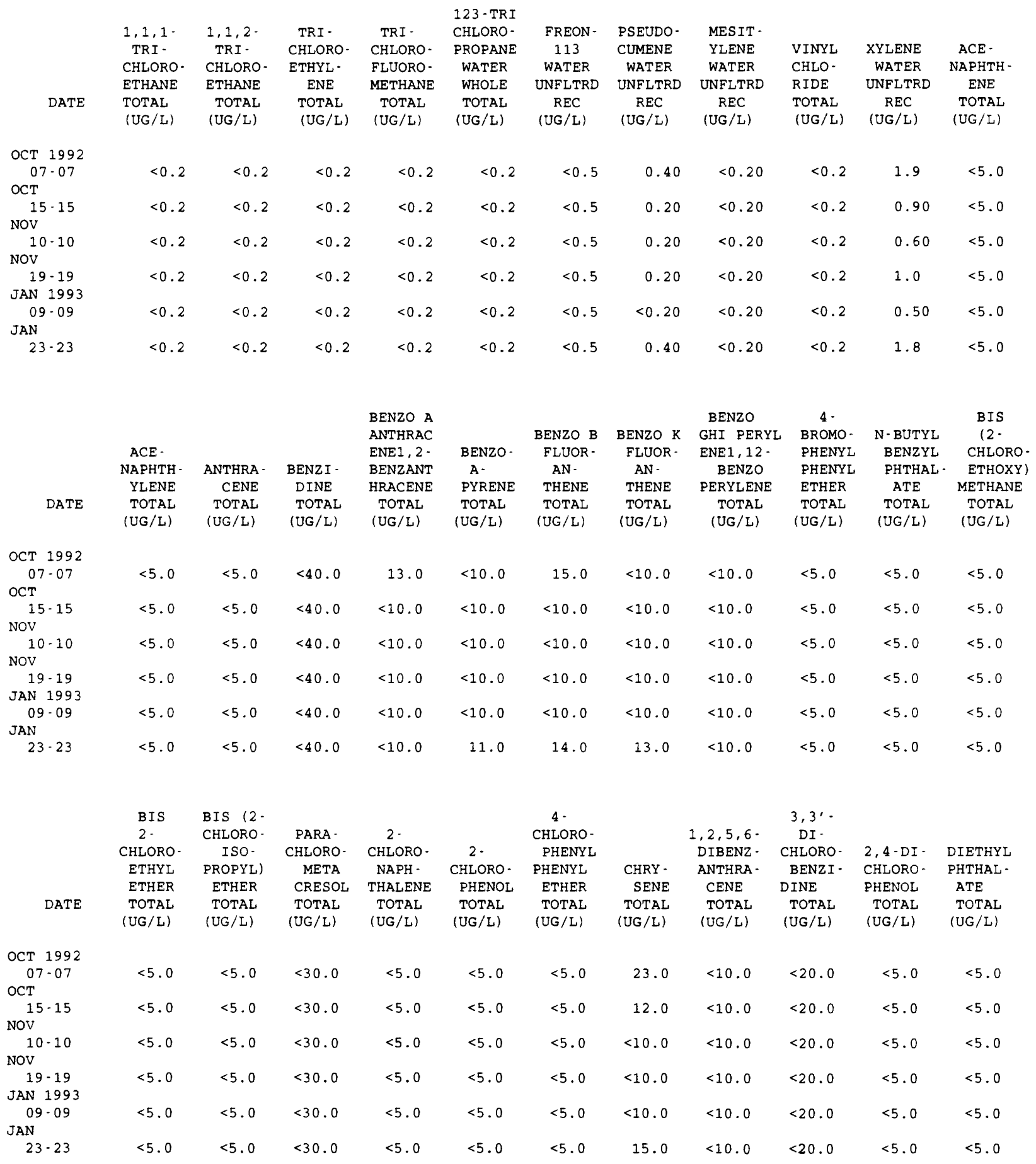


Table 5. Water-quality data for storm-sewer outfall stations, Garland, Texas, 1992-93-Continued

08061690 - I-635 OUTFALL AT CENTERVILLE ROAD, GARLAND, TX (WY 1993)-Continued

\begin{tabular}{|c|c|c|c|c|c|c|c|c|c|c|c|}
\hline DATE & $\begin{array}{c}\text { DI - } \\
\text { METHYL } \\
\text { PHTHAL- } \\
\text { ATE } \\
\text { TOTAL } \\
\text { (UG/L) }\end{array}$ & $\begin{array}{c}2,4-\mathrm{DI} \text { - } \\
\text { METHYL- } \\
\text { PHENOL } \\
\text { TOTAL } \\
\text { (UG/L) }\end{array}$ & $\begin{array}{c}\text { DI - N- } \\
\text { BUTYL } \\
\text { PHTHAL- } \\
\text { ATE } \\
\text { TOTAL } \\
\text { (UG/L) }\end{array}$ & $\begin{array}{c}4,6- \\
\text { DINITRO- } \\
\text { ORTHO- } \\
\text { CRESOL } \\
\text { TOTAL } \\
\text { (UG/L) }\end{array}$ & $\begin{array}{c}2,4,- \\
\text { DI- } \\
\text { NITRO- } \\
\text { PHENOL } \\
\text { TOTAL } \\
\text { (UG/L) }\end{array}$ & $\begin{array}{l}2,4-D I- \\
\text { NITRO- } \\
\text { TOLUENE } \\
\text { TOTAL } \\
\text { (UG/L) }\end{array}$ & $\begin{array}{l}2,6-\mathrm{DI} \text { - } \\
\text { NITRO- } \\
\text { TOLUENE } \\
\text { TOTAL } \\
\text { (UG/L) }\end{array}$ & $\begin{array}{c}\text { DI - N- } \\
\text { OCTYL } \\
\text { PHTHAL- } \\
\text { ATE } \\
\text { TOTAL } \\
\text { (UG/L) }\end{array}$ & $\begin{array}{c}\text { BIS }(2- \\
\text { ETHYL } \\
\text { HEXYL) } \\
\text { PHTHAL- } \\
\text { ATE } \\
\text { TOTAL } \\
\text { (UG/L) }\end{array}$ & $\begin{array}{l}\text { FLUOR - } \\
\text { ANTHENE } \\
\text { TOTAL } \\
(U G / L)\end{array}$ & $\begin{array}{c}\text { FLUOR - } \\
\text { ENE } \\
\text { TOTAL } \\
\text { (UG/L) }\end{array}$ \\
\hline $\begin{array}{c}\text { OCT } 1992 \\
07-07\end{array}$ & $<5.0$ & $<5.0$ & $<5.0$ & $<30.0$ & $<20.0$ & $<5.0$ & $<5.0$ & $<10.0$ & 13.0 & 21.0 & $<5.0$ \\
\hline OCT & & & & - & 20. & (5.0 & or & - 0 & 20.0 & -1.0 & \\
\hline $\begin{array}{l}15 \cdot 15 \\
\text { NOV }\end{array}$ & $<5.0$ & $<5.0$ & $<5.0$ & $<30.0$ & $<20.0$ & $<5.0$ & $<5.0$ & $<10.0$ & 8.0 & 9.0 & $<5.0$ \\
\hline $\begin{array}{l}10-10 \\
\mathrm{NOV}\end{array}$ & $<5.0$ & $<5.0$ & $<5.0$ & $<30.0$ & $<20.0$ & $<5.0$ & $<5.0$ & $<10.0$ & 13.0 & 5.0 & $<5.0$ \\
\hline $\begin{array}{c}19 \cdot 19 \\
\text { JAN } 1993\end{array}$ & $<5.0$ & $<5.0$ & $<5.0$ & $<30.0$ & $<20.0$ & $<5.0$ & $<5.0$ & $<10.0$ & 11.0 & $<5.0$ & $<5.0$ \\
\hline $\begin{array}{l}09-09 \\
\text { JAN }\end{array}$ & $<5.0$ & $<5.0$ & $<5.0$ & $<30.0$ & $<20.0$ & $<5.0$ & $<5.0$ & $<10.0$ & 6.0 & 6.0 & $<5.0$ \\
\hline $23-23$ & $<5.0$ & $<5.0$ & $<5.0$ & $<30.0$ & $<20.0$ & $<5.0$ & $<5.0$ & $<10.0$ & 6.0 & 23.0 & $<5.0$ \\
\hline DATE & $\begin{array}{l}\text { HEXA- } \\
\text { CHLORO- } \\
\text { BENZENE } \\
\text { TOTAL } \\
\text { (UG/L) }\end{array}$ & $\begin{array}{c}\text { HEXA- } \\
\text { CHLORO- } \\
\text { CYCLO- } \\
\text { PENT- } \\
\text { ADIENE } \\
\text { TOTAL } \\
\text { (UG/L) }\end{array}$ & $\begin{array}{l}\text { HEXA- } \\
\text { CHLORO- } \\
\text { ETHANE } \\
\text { TOTAL } \\
\text { (UG/L) }\end{array}$ & $\begin{array}{c}\text { INDENO } \\
(1,2,3- \\
\text { CD }) \\
\text { PYRENE } \\
\text { TOTAL } \\
(\mathrm{UG} / \mathrm{L})\end{array}$ & $\begin{array}{l}\text { ISO- } \\
\text { PHORONE } \\
\text { TOTAL } \\
\text { (UG/L) }\end{array}$ & $\begin{array}{l}\text { NITRO- } \\
\text { BENZENE } \\
\text { TOTAL } \\
\text { (UG/L) }\end{array}$ & $\begin{array}{l}\text { N-NITRO- } \\
\text { SODI- } \\
\text { METHY - } \\
\text { LAMINE } \\
\text { TOTAL } \\
\text { (UG/L) }\end{array}$ & $\begin{array}{l}2- \\
\text { NITRO- } \\
\text { PHENOL } \\
\text { TOTAL } \\
\text { (UG/L) }\end{array}$ & $\begin{array}{c}4- \\
\text { NITRO- } \\
\text { PHENOL } \\
\text { TOTAL } \\
\text { (UG/L) }\end{array}$ & $\begin{array}{c}\text { N- } \\
\text { NITRO- } \\
\text { SODI-N- } \\
\text { PROPYL- } \\
\text { AMINE } \\
\text { TOTAL } \\
\text { (UG/L) }\end{array}$ & $\begin{array}{l}\text { N-NITRO- } \\
\text { SODI - } \\
\text { PHENY - } \\
\text { LAMINE } \\
\text { TOTAL } \\
(U G / L)\end{array}$ \\
\hline OCT 1992 & & & & & & & & & & & \\
\hline $\begin{array}{l}07-07 \\
\text { OCT }\end{array}$ & $<5.0$ & $<5.0$ & $<5.0$ & $<10.0$ & $<5.0$ & $<5.0$ & $<5.0$ & $<5.0$ & $<30.0$ & $<5.0$ & $<5.0$ \\
\hline $\begin{array}{l}15-15 \\
\text { Nov }\end{array}$ & $<5.0$ & $<5.0$ & $<5.0$ & $<10.0$ & $<5.0$ & $<5.0$ & $<5.0$ & $<5.0$ & $<30.0$ & $<5.0$ & $<5.0$ \\
\hline $\begin{array}{l}10-10 \\
\text { Nov }\end{array}$ & $<5.0$ & $<5.0$ & $<5.0$ & $<10.0$ & $<5.0$ & $<5.0$ & $<5.0$ & $<5.0$ & $<30.0$ & $<5.0$ & $<5.0$ \\
\hline $\begin{array}{c}19-19 \\
\text { JAN } 1993\end{array}$ & $<5.0$ & $<5.0$ & $<5.0$ & $<10.0$ & $<5.0$ & $<5.0$ & $<5.0$ & $<5.0$ & $<30.0$ & $<5.0$ & $<5.0$ \\
\hline $\begin{array}{l}09-09 \\
\text { JAN }\end{array}$ & $<5.0$ & $<5.0$ & $<5.0$ & $<10.0$ & $<5.0$ & $<5.0$ & $<5.0$ & $<5.0$ & $<30.0$ & $<5.0$ & $<5.0$ \\
\hline $23-23$ & $<5.0$ & $<5.0$ & $<5.0$ & $<10.0$ & $<5.0$ & $<5.0$ & $<5.0$ & $<5.0$ & $<30.0$ & $<5.0$ & $<5.0$ \\
\hline DATE & $\begin{array}{l}\text { PENTA- } \\
\text { CHLORO- } \\
\text { PHENOL } \\
\text { TOTAL } \\
(U G / L)\end{array}$ & $\begin{array}{l}\text { PHENAN- } \\
\text { THRENE } \\
\text { TOTAL } \\
\text { (UG/L) }\end{array}$ & $\begin{array}{l}\text { PHENOL } \\
\text { (C6H- } \\
5 O H) \\
\text { TOTAL } \\
\text { (UG/L) }\end{array}$ & $\begin{array}{l}\text { PYRENE } \\
\text { TOTAL } \\
\text { (UG/L) }\end{array}$ & $\begin{array}{c}2,4,6- \\
\text { TRI- } \\
\text { CHLORO- } \\
\text { PHENOL } \\
\text { TOTAL } \\
\text { (UG/L) }\end{array}$ & $\begin{array}{c}\text { ALDRIN, } \\
\text { TOTAL } \\
\text { (UG/L) }\end{array}$ & $\begin{array}{c}\text { P, } P^{\prime} \\
\text { DDT, } \\
\text { TOTAL } \\
\text { (UG } / L \text { ) }\end{array}$ & $\begin{array}{l}\text { ALPHA } \\
\text { BHC } \\
\text { TOTAL } \\
\text { (UG/L) }\end{array}$ & $\begin{array}{c}\text { BETA } \\
\text { BENZENE } \\
\text { HEXA- } \\
\text { CHLOR- } \\
\text { IDE } \\
\text { TOTAL } \\
\text { (UG/L) }\end{array}$ & $\begin{array}{c}\text { DELTA } \\
\text { BENZENE } \\
\text { HEXA- } \\
\text { CHLOR- } \\
\text { IDE } \\
\text { TOTAL } \\
\text { (UG/L) }\end{array}$ & $\begin{array}{c}\text { LINDANE } \\
\text { TOTAL } \\
\text { (UG/L) }\end{array}$ \\
\hline $\begin{array}{l}\text { OCT } 1992 \\
07-07 \\
\text { OCT }\end{array}$ & $<30.0$ & 6.0 & $<5.0$ & 17.0 & $<20.0$ & $<0.040$ & $<0.10$ & $<0.03$ & $<0.03$ & $<0.09$ & $<0.030$ \\
\hline Nov $15-15$ & $<30.0$ & $<5.0$ & $<5.0$ & 7.0 & $<20.0$ & $<0.040$ & $<0.10$ & $<0.03$ & $<0.03$ & $<0.09$ & $<0.030$ \\
\hline $\begin{array}{l}10-10 \\
\text { vov }\end{array}$ & $<30.0$ & $<5.0$ & $<5.0$ & $<5.0$ & $<20.0$ & $<0.040$ & $<0.10$ & $<0.03$ & $<0.03$ & $<0.09$ & $<0.030$ \\
\hline $\begin{array}{c}19-19 \\
\text { JAN } 1993\end{array}$ & $<30.0$ & $<5.0$ & $<5.0$ & $<5.0$ & $<20.0$ & $<0.040$ & $<0.10$ & $<0.03$ & $<0.03$ & $<0.09$ & $<0.030$ \\
\hline $\begin{array}{l}09-09 \\
\text { AN }\end{array}$ & $<30.0$ & $<5.0$ & $<5.0$ & 5.0 & $<20.0$ & $<0.040$ & $<0.10$ & $<0.03$ & $<0.03$ & $<0.09$ & $<0.030$ \\
\hline $23 \cdot 23$ & $<30.0$ & 7.0 & $<5.0$ & 18.0 & $<20.0$ & $<0.040$ & $<0.10$ & $<0.03$ & $<0.03$ & $<0.09$ & $<0.030$ \\
\hline
\end{tabular}


Table 5. Water-quality data for storm-sewer outfall stations, Garland, Texas, 1992-93-Continued

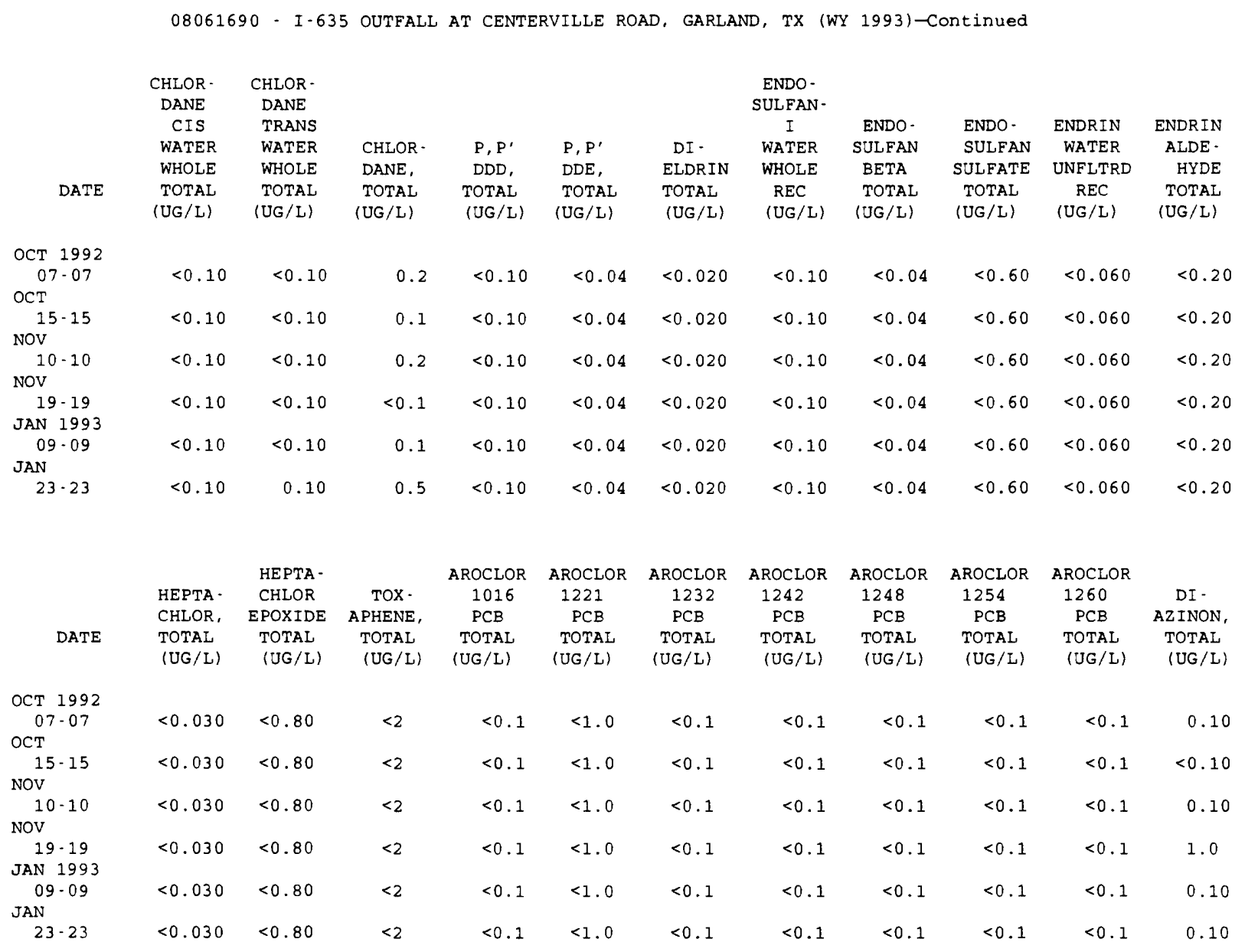


Table 6. Water-quality data for storm-sewer outfall stations, Irving, Texas, 1992-93

08049590 - BEAR CREEK OUTFALI AT SHADY GROVE ROAD, IRVING, TX (WY 1992)

\begin{tabular}{|c|c|c|c|c|c|c|c|c|c|c|c|c|}
\hline DATE & TIME & $\begin{array}{l}\text { PRECIP- } \\
\text { ITATION } \\
\text { TOTAL } \\
\text { INCHES/ } \\
\text { STORM }\end{array}$ & $\begin{array}{l}\text { ELAPSED } \\
\text { TIME } \\
\text { OF } \\
\text { STORM } \\
\text { (HOURS) }\end{array}$ & $\begin{array}{l}\text { STORM } \\
\text { WATER } \\
\text { FLOW } \\
\text { (MGD) }\end{array}$ & $\begin{array}{l}\text { SPE- } \\
\text { CIFIC } \\
\text { CON- } \\
\text { DUCT- } \\
\text { ANCE } \\
\text { (US/CM) }\end{array}$ & $\begin{array}{l}\text { SPE- } \\
\text { CIFIC } \\
\text { CON- } \\
\text { DUCT- } \\
\text { ANCE } \\
\text { LAB } \\
\text { (US/CM) }\end{array}$ & $\begin{array}{c}\text { PH } \\
\text { WATER } \\
\text { WHOLE } \\
\text { FIELD } \\
\text { (STAND- } \\
\text { ARD } \\
\text { UNITS) }\end{array}$ & $\begin{array}{c}\text { PH } \\
\text { WATER } \\
\text { WHOLE } \\
\text { LAB } \\
\text { (STAND- } \\
\text { ARD } \\
\text { UNITS) }\end{array}$ & $\begin{array}{c}\text { TEMPER - } \\
\text { ATURE } \\
\text { WATER } \\
\text { (DEG C) }\end{array}$ & $\begin{array}{c}\text { OXYGEN } \\
\text { DEMAND, } \\
\text { CHEM- } \\
\text { ICAL } \\
\text { (HIGH } \\
\text { LEVEL) } \\
\text { (MG/L) }\end{array}$ & $\begin{array}{l}\text { OXYGEN } \\
\text { DEMAND, } \\
\text { BIO- } \\
\text { CHEM- } \\
\text { ICAL, } \\
5 \text { DAY } \\
\text { (MG/L) }\end{array}$ & $\begin{array}{l}\text { COLI- } \\
\text { FORM, } \\
\text { FECAL, } \\
0.7 \\
\text { UM-MF } \\
\text { (COLS./ } \\
100 \mathrm{ML} \text { ) }\end{array}$ \\
\hline $\begin{array}{l}\text { SEP } 1992 \\
03.03 \\
\text { SEP } \\
10-10\end{array}$ & 0122 & 0.37 & 2.0 & 0.14 & 78 & 98 & 7.8 & 7.0 & 25.0 & 56 & 8.5 & 210000 \\
\hline DATE & $\begin{array}{l}\text { STREP- } \\
\text { TOCOCCI } \\
\text { FECAL, } \\
\text { KF AGAR } \\
\text { (COLS. } \\
\text { PER } \\
100 \mathrm{ML} \text { ) }\end{array}$ & $\begin{array}{l}\text { HARD- } \\
\text { NESS } \\
\text { TOTAL } \\
\text { (MG/L } \\
\text { AS } \\
\text { CACO3) }\end{array}$ & $\begin{array}{l}\text { HARD- } \\
\text { NESS } \\
\text { NONCARB } \\
\text { DISSOLV } \\
\text { FLD. AS } \\
\text { CACO3 } \\
(M G / L)\end{array}$ & $\begin{array}{l}\text { ALKA- } \\
\text { LINITY } \\
\text { WAT DIS } \\
\text { FIX END } \\
\text { FIELD } \\
\text { CACO3 } \\
(M G / L)\end{array}$ & $\begin{array}{l}\text { SOLIDS, } \\
\text { SUM OF } \\
\text { CONSTI- } \\
\text { TUENTS, } \\
\text { DIS - } \\
\text { SOLVED } \\
\text { (MG/L) }\end{array}$ & $\begin{array}{l}\text { RESIDUE } \\
\text { TOTAL } \\
\text { AT } 105 \\
\text { DEG. C, } \\
\text { SUS. } \\
\text { PENDED } \\
\text { (MG/L) }\end{array}$ & $\begin{array}{l}\text { SOLIDS, } \\
\text { RESIDUE } \\
\text { AT } 180 \\
\text { DEG. C } \\
\text { DIS- } \\
\text { SOLVED } \\
\text { (MG/L) }\end{array}$ & $\begin{array}{l}\text { CALCIUM } \\
\text { DIS - } \\
\text { SOLVED } \\
\text { (MG/L } \\
\text { AS CA) }\end{array}$ & $\begin{array}{l}\text { MAGNE- } \\
\text { SIUM, } \\
\text { DIS - } \\
\text { SOLVED } \\
\text { (MG/L } \\
\text { AS MG) }\end{array}$ & $\begin{array}{l}\text { SODIUM, } \\
\text { DIS- } \\
\text { SOLVED } \\
\text { (MG/L } \\
\text { AS NA) }\end{array}$ & $\begin{array}{r}\text { SODIUM } \\
\text { PERCENT }\end{array}$ & $\begin{array}{l}\text { SODIUM } \\
\text { AD- } \\
\text { SORP- } \\
\text { TION } \\
\text { RATIO }\end{array}$ \\
\hline $\begin{array}{l}\text { SEP } 1992 \\
03-03 \\
\text { SEP } \\
10-10\end{array}$ & 280000 & 22 & 2 & 29 & 49 & 24 & 62 & 11 & 0.65 & 2.9 & 20 & 0.3 \\
\hline DATE & $\begin{array}{l}\text { POTAS - } \\
\text { SIUM, } \\
\text { DIS - } \\
\text { SOLVED } \\
\text { (MG/L } \\
\text { AS K) }\end{array}$ & $\begin{array}{l}\text { SULFATE } \\
\text { DIS - } \\
\text { SOLVED } \\
\text { (MG/L } \\
\text { AS SO4) }\end{array}$ & $\begin{array}{l}\text { CHLO- } \\
\text { RIDE, } \\
\text { DIS- } \\
\text { SOLVED } \\
\text { (MG/L } \\
\text { AS CL) }\end{array}$ & $\begin{array}{l}\text { NITRO- } \\
\text { GEN, } \\
\text { NITRITE } \\
\text { TOTAL } \\
\text { (MG/L } \\
\text { AS N) }\end{array}$ & $\begin{array}{c}\text { NITRO- } \\
\text { GEN, } \\
\text { NO2+NO3 } \\
\text { TOTAL } \\
\text { (MG/L } \\
\text { AS N) }\end{array}$ & $\begin{array}{c}\text { NITRO- } \\
\text { GEN, } \\
\text { AMMONIA } \\
\text { TOTAL } \\
\text { (MG/L } \\
\text { AS N) }\end{array}$ & $\begin{array}{l}\text { NITRO- } \\
\text { GEN, AM- } \\
\text { MONIA + } \\
\text { ORGANIC } \\
\text { TOTAL } \\
\text { (MG/L } \\
\text { AS N) }\end{array}$ & $\begin{array}{l}\text { PHOS - } \\
\text { PHORUS } \\
\text { TOTAL } \\
\text { (MG/L } \\
\text { AS P) }\end{array}$ & $\begin{array}{c}\text { PHOS - } \\
\text { PHORUS } \\
\text { DIS - } \\
\text { SOLVED } \\
\text { (MG/L } \\
\text { AS P) }\end{array}$ & $\begin{array}{c}\text { ANTIMONY } \\
\text { TOTAL } \\
\text { EPA } \\
\text { (UG/I } \\
\text { AS SB) }\end{array}$ & $\begin{array}{c}\text { ARSENIC } \\
\text { TOTAL } \\
\text { (UG/L } \\
\text { AS AS) }\end{array}$ & $\begin{array}{l}\text { BERYL- } \\
\text { LIUM, } \\
\text { TOTAL } \\
\text { RECOV- } \\
\text { ERABLE } \\
\text { (UG/L } \\
\text { AS BE) }\end{array}$ \\
\hline $\begin{array}{l}\text { SEP } 1992 \\
03-03 \\
\text { SEP } \\
\quad 10-10\end{array}$ & 3.9 & 5.6 & 3.5 & 0.030 & 0.580 & 0.240 & 0.90 & 0.430 & 0.390 & $<10.0$ & 3 & $\begin{array}{l}<10 \\
<10\end{array}$ \\
\hline DATE & $\begin{array}{l}\text { CADMIUM } \\
\text { TOTAL } \\
\text { RECOV- } \\
\text { ERABLE } \\
\text { (UG/L } \\
\text { AS CD) }\end{array}$ & $\begin{array}{l}\text { CHRO- } \\
\text { MIUM, } \\
\text { TOTAL } \\
\text { RECOV- } \\
\text { ERABLE } \\
\text { (UG/L } \\
\text { AS CR) }\end{array}$ & $\begin{array}{l}\text { COPPER, } \\
\text { TOTAL } \\
\text { RECOV- } \\
\text { ERABLE } \\
\text { (UG/L } \\
\text { AS CU) }\end{array}$ & $\begin{array}{l}\text { CYANIDE } \\
\text { TOTAL } \\
\text { EPA } \\
\text { (MG/L } \\
\text { AS CN) }\end{array}$ & $\begin{array}{c}\text { CYANIDE } \\
\text { TOTAL } \\
\text { (MG/L } \\
\text { AS CN) }\end{array}$ & $\begin{array}{l}\text { LEAD, } \\
\text { TOTAL } \\
\text { RECOV- } \\
\text { ERABLE } \\
\text { (UG/I } \\
\text { AS PB) }\end{array}$ & $\begin{array}{l}\text { MERCURY } \\
\text { TOTAL } \\
\text { RECOV- } \\
\text { ERABLE } \\
\text { (UG/L } \\
\text { AS HG) }\end{array}$ & $\begin{array}{l}\text { NICKEL, } \\
\text { TOTAL } \\
\text { RECOV- } \\
\text { ERABLE } \\
\text { (UG/L } \\
\text { AS NI) }\end{array}$ & $\begin{array}{l}\text { SELE- } \\
\text { NIUM, } \\
\text { TOTAL } \\
\text { (UG/L } \\
\text { AS SE) }\end{array}$ & $\begin{array}{l}\text { SILVER, } \\
\text { TOTAL } \\
\text { RECOV } \\
\text { ERABLE } \\
\text { (UG/L } \\
\text { AS AG) }\end{array}$ & $\begin{array}{c}\text { SILVER, } \\
\text { TOTAL } \\
\text { RECOV- } \\
\text { ERABLE } \\
\text { EPA } \\
\text { (UG/L } \\
\text { AS AG) }\end{array}$ & $\begin{array}{l}\text { THAL- } \\
\text { LIUM, } \\
\text { TOTAL } \\
\text { (UG/L } \\
\text { AS TL) }\end{array}$ \\
\hline $\begin{array}{c}\text { SEP } 1992 \\
03-03 \\
\text { SEP } \\
10-10\end{array}$ & $<1$ & 3 & 5 & $<0.010$ & $<0.010$ & 13 & $<0.10$ & 3 & $<2$ & $<1$ & $\begin{array}{l}<0.500 \\
<0.500\end{array}$ & $<5$ \\
\hline DATE & $\begin{array}{l}\text { ZINC, } \\
\text { TOTAL } \\
\text { RECOV- } \\
\text { ERABLE } \\
\text { (UG/I } \\
\text { AS ZN) }\end{array}$ & $\begin{array}{c}\text { CARBON, } \\
\text { ORGANIC } \\
\text { TOTAL } \\
\text { (MG / L } \\
\text { AS C) }\end{array}$ & $\begin{array}{l}\text { OIL AND } \\
\text { GREASE, } \\
\text { TOTAL } \\
\text { RECOV. } \\
\text { GRAVI- } \\
\text { METRIC } \\
\text { (MG/L) }\end{array}$ & $\begin{array}{l}\text { PHENOLS } \\
\text { TOTAL } \\
\text { (UG/L) }\end{array}$ & $\begin{array}{l}\text { ACRO- } \\
\text { LEIN } \\
\text { TOTAL } \\
\text { (UG/L) }\end{array}$ & $\begin{array}{l}\text { ACRYLO- } \\
\text { NITRILE } \\
\text { TOTAL } \\
\text { (UG/L) }\end{array}$ & $\begin{array}{l}\text { BENZENE } \\
\text { TOTAL } \\
\text { (UG/L) }\end{array}$ & $\begin{array}{l}\text { BROMO- } \\
\text { BENZENE } \\
\text { WATER, } \\
\text { WHOLE, } \\
\text { TOTAL } \\
\text { (UG/L) }\end{array}$ & $\begin{array}{l}\text { BROMO- } \\
\text { FORM } \\
\text { TOTAL } \\
\text { (UG/L) }\end{array}$ & $\begin{array}{c}\text { BENZENE } \\
\text { N-BUTYL } \\
\text { WATER } \\
\text { UNFLTRD } \\
\text { REC } \\
(U G / L)\end{array}$ & $\begin{array}{l}\text { BENZENE } \\
\text { SEC } \\
\text { BUTYL- } \\
\text { WATER } \\
\text { UNFLTRD } \\
\text { REC } \\
\text { (UG/L) }\end{array}$ & $\begin{array}{l}\text { BENZENE } \\
\text { TERT - } \\
\text { BUTYL- } \\
\text { WATER } \\
\text { UNFLTRD } \\
\text { REC } \\
\text { (UG/L) }\end{array}$ \\
\hline $\begin{array}{l}\text { EP } 1992 \\
03-03 \\
\text { EP }\end{array}$ & 60 & 27 & $<1$ & 2 & $<20$ & $<20$ & $<0.2$ & $<0.2$ & $<0.2$ & $<0.20$ & $<0.20$ & $<0.20$ \\
\hline $10-10$ & 60 & 15 & 3 & 21 & $<20$ & $<20$ & $<1.0$ & $<0.2$ & $<0.2$ & $<0.20$ & $<0.20$ & $<0.20$ \\
\hline
\end{tabular}


Table 6. Water-quality data for storm-sewer outfall stations, Irving, Texas, 1992-93-Continued

08049590 - BEAR CREEK OUTFALL AT SHADY GROVE ROAD, IRVING, TX (WY 1992)-Continued

\begin{tabular}{|c|c|c|c|c|c|c|c|c|c|c|c|c|}
\hline & & & & & $2 \cdot$ & & & $0-$ & & DI BROMO & DI - & $1,2-$ \\
\hline & CARBON - & & CHLORO - & & CHLORO - & & & CHLORO - & TOLUENE & CHLORO - & BROMO - & $\begin{array}{l}\text { DIBROMO } \\
\text { ETHANE }\end{array}$ \\
\hline & TETRA - & & DI - & & ETHYL - & & METHYL - & TOLUENE & P.CHLOR & PROPANE & METHANE & ETHANE \\
\hline & $\begin{array}{l}\text { CHLO- } \\
\text { RIDE }\end{array}$ & $\begin{array}{l}\text { CHLORO - } \\
\text { BENZENE }\end{array}$ & $\begin{array}{l}\text { BROMO - } \\
\text { METHANE }\end{array}$ & $\begin{array}{l}\text { CHLORO- } \\
\text { ETHANE }\end{array}$ & $\begin{array}{l}\text { VINYL - } \\
\text { ETHER }\end{array}$ & $\begin{array}{l}\text { CHLORO- } \\
\text { FORM }\end{array}$ & $\begin{array}{l}\text { CHLO - } \\
\text { RIDE }\end{array}$ & $\begin{array}{l}\text { WATER } \\
\text { WHOLE }\end{array}$ & $\begin{array}{l}\text { WATER } \\
\text { UNFLTRD }\end{array}$ & $\begin{array}{l}\text { WATER } \\
\text { WHOLE }\end{array}$ & $\begin{array}{l}\text { WATER } \\
\text { WHOLE }\end{array}$ & $\begin{array}{l}\text { WATER } \\
\text { WHOLE }\end{array}$ \\
\hline DATE & $\begin{array}{l}\text { TOTAL } \\
\text { (UG/L) }\end{array}$ & $\begin{array}{l}\text { TOTAL } \\
\text { (UG/L) }\end{array}$ & $\begin{array}{l}\text { TOTAL } \\
\text { (UG/L) }\end{array}$ & $\begin{array}{l}\text { TOTAL } \\
\text { (UG/L) }\end{array}$ & $\begin{array}{r}\text { TOTAL } \\
(U G / L)\end{array}$ & $\begin{array}{l}\text { TOTAL } \\
\text { (UG/L) }\end{array}$ & $\begin{array}{l}\text { TOTAL } \\
\text { (UG/L) }\end{array}$ & $\begin{array}{l}\text { TOTAL } \\
\text { (UG/L) }\end{array}$ & $\begin{array}{c}\text { REC } \\
(\mathrm{UG} / \mathrm{L})\end{array}$ & $\begin{array}{l}\text { TOT.REC } \\
\text { (UG/L) }\end{array}$ & $\begin{array}{l}\text { RECOVER } \\
\text { (UG/L) }\end{array}$ & $\begin{array}{l}\text { TOTAL } \\
\text { (UG/L) }\end{array}$ \\
\hline
\end{tabular}

\begin{tabular}{|c|c|c|c|c|c|c|c|c|c|c|c|c|}
\hline $\begin{array}{r}\text { SEP } 199 \\
03-03\end{array}$ & $<0.2$ & $<0.20$ & $<0.2$ & $<0.2$ & $<1.0$ & $<0.2$ & $<0.2$ & $<0.2$ & $<0.20$ & $<1.0$ & $<0.2$ & $<0.2$ \\
\hline \multicolumn{13}{|l|}{ SEP } \\
\hline \multirow[t]{5}{*}{$10-10$} & $<0.2$ & $<1.0$ & $<0.2$ & $<0.2$ & $<1.0$ & $<0.2$ & $<0.2$ & $<0.2$ & $<0.20$ & $<1.0$ & $<0.2$ & $<0.2$ \\
\hline & & & $1,1-\mathrm{DI}$ & BENZENE & BENZENE & BENZENE & DI - & & & $1,2-D I$ - & CIS 1,2 - & \\
\hline & DI - & $1,1-D I-$ & CHLORO - & O- & $1,3-D I$ & 1,4-DI - & CHLORO - & & & PHENYL - & DI - & 1,2- \\
\hline & CHLORO - & CHLORO - & PRO - & CHLORO - & CHLORO- & CHLORO- & DI - & 1,1 -DI - & $1,2-D I$ - & HYDRA- & CHLORO- & TRANSDI \\
\hline & $\begin{array}{l}\text { BROMO- } \\
\text { METHANE }\end{array}$ & $\begin{array}{c}\text { ETHYL- } \\
\text { ENE }\end{array}$ & $\begin{array}{l}\text { PENE, } \\
\text { WAT, WH }\end{array}$ & $\begin{array}{l}\text { WATER } \\
\text { UNFLTRD }\end{array}$ & $\begin{array}{l}\text { WATER } \\
\text { UNFLTRD }\end{array}$ & $\begin{array}{l}\text { WATER } \\
\text { UNFLTRD }\end{array}$ & $\begin{array}{l}\text { FLUORO- } \\
\text { METHANE }\end{array}$ & $\begin{array}{l}\text { CHLORO- } \\
\text { ETHANE }\end{array}$ & $\begin{array}{l}\text { CHLORO - } \\
\text { ETHANE }\end{array}$ & $\begin{array}{l}\text { ZINE } \\
\text { WATER }\end{array}$ & $\begin{array}{r}\text { ETHENE } \\
\text { WATER }\end{array}$ & $\begin{array}{l}\text { CHLORO- } \\
\text { ETHENE }\end{array}$ \\
\hline DATE & $\begin{array}{l}\text { TOTAL } \\
\text { (UG/L) }\end{array}$ & $\begin{array}{l}\text { TOTAL } \\
\text { (UG/L) }\end{array}$ & $\begin{array}{l}\text { TOTAL } \\
\text { (UG/L) }\end{array}$ & $\begin{array}{c}\text { REC } \\
\text { (UG/L) }\end{array}$ & $\begin{array}{c}\text { REC } \\
(U G / L)\end{array}$ & $\begin{array}{c}\text { REC } \\
\text { (UG/L) }\end{array}$ & $\begin{array}{l}\text { TOTAL } \\
\text { (UG/L) }\end{array}$ & $\begin{array}{l}\text { TOTAL } \\
(\mathrm{UG} / \mathrm{L})\end{array}$ & $\begin{array}{l}\text { TOTAL } \\
\text { (UG/L) }\end{array}$ & $\begin{array}{l}\text { TOT.REC } \\
\text { (UG/L) }\end{array}$ & $\begin{array}{l}\text { TOTAL } \\
(U G / L)\end{array}$ & $\begin{array}{l}\text { TOTAL } \\
\{\mathrm{UG} / \mathrm{L}\}\end{array}$ \\
\hline
\end{tabular}

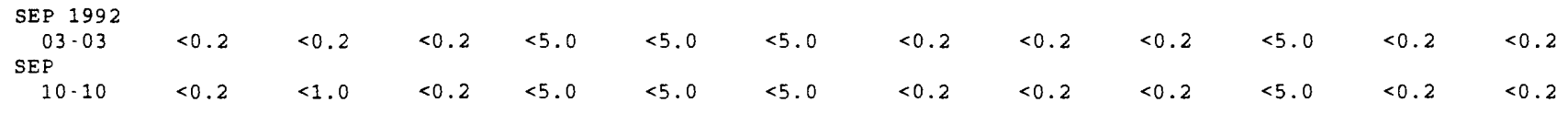

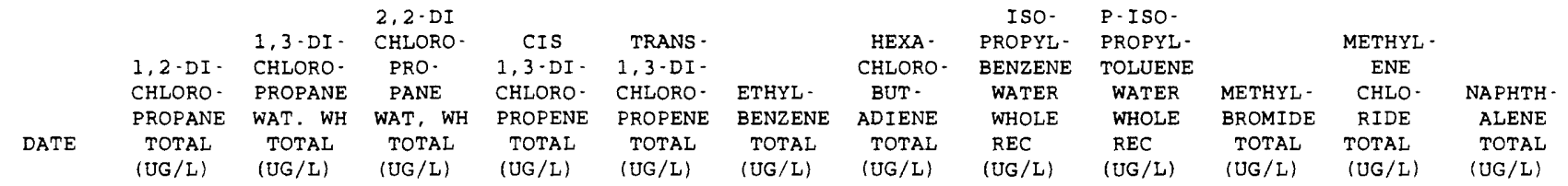

SEP 1992

\begin{tabular}{|c|c|c|c|c|c|c|c|c|c|c|c|c|}
\hline $03-03$ & $<0.2$ & $<0.2$ & $<0.2$ & $<0.2$ & $<0.2$ & $<0.2$ & $<5.0$ & $<0.20$ & $<0.20$ & $<0.2$ & $<0.2$ & $<5.0$ \\
\hline \multicolumn{13}{|l|}{ SEP } \\
\hline $10-10$ & $<0.2$ & $<0.2$ & $<0.2$ & $<0.2$ & $<0.2$ & $<0.2$ & $<5.0$ & $<0.20$ & $<0.20$ & $<0.2$ & $<0.2$ & $<5.0$ \\
\hline
\end{tabular}

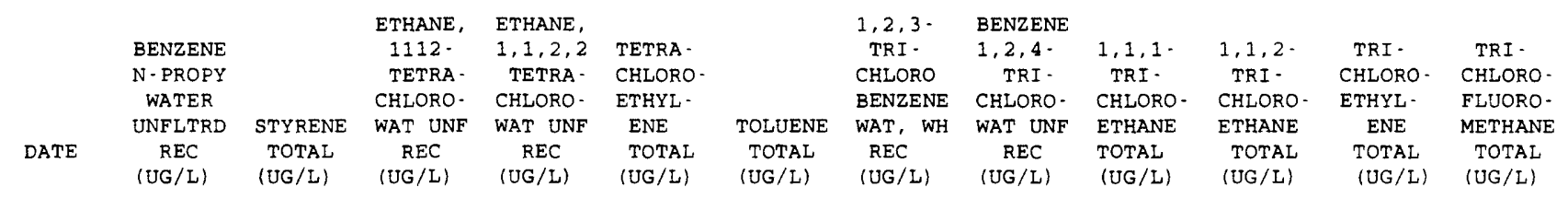

\begin{tabular}{|c|c|c|c|c|c|c|c|c|c|c|c|c|}
\hline \multicolumn{13}{|c|}{ SEP 1992} \\
\hline 03.03 & $<0.20$ & $<0.2$ & $<0.2$ & $<0.2$ & $<0.2$ & $<0.2$ & $<0.20$ & $<5.0$ & $<0.2$ & $<0.2$ & $<0.2$ & $<0.2$ \\
\hline \multicolumn{13}{|l|}{ SEP } \\
\hline $10-10$ & $<0.20$ & $<0.2$ & $<0.2$ & $<0.2$ & $<0.2$ & $<1.0$ & $<0.20$ & $<5.0$ & $<0.2$ & $<0.2$ & $<1.0$ & $<0.2$ \\
\hline
\end{tabular}

\begin{tabular}{|c|c|c|c|c|c|c|c|c|c|c|c|c|}
\hline \multicolumn{13}{|c|}{$123-$ TRI } \\
\hline & CHLORO - & PSEUDO- & MESIT - & & & & & & & ANTHRAC & & BENZO B \\
\hline \multirow[b]{4}{*}{ DATE } & PROPANE & CUMENE & YLENE & VINYL & XYLENE & ACE - & $A C E-$ & & & ENE1, 2- & BENZO - & FLUOR - \\
\hline & WATER & WATER & WATER & CHLO - & WATER & NAPHTH - & NAPHTH - & ANTHRA - & BENZI - & BENZANT & A - & AN- \\
\hline & WHOLE & UNFLTRD & UNFLTRD & RIDE & UNFLTRD & ENE & YLENE & CENE & DINE & HRACENE & PYRENE & THENE \\
\hline & $\begin{array}{l}\text { TOTAL } \\
\text { (UG/L) }\end{array}$ & $\begin{array}{c}\text { REC } \\
(U G / L)\end{array}$ & $\begin{array}{c}\text { REC } \\
(U G / L)\end{array}$ & $\begin{array}{l}\text { TOTAL } \\
\text { (UG/L) }\end{array}$ & $\begin{array}{c}\text { REC } \\
\text { (UG/L) }\end{array}$ & $\begin{array}{l}\text { TOTAL } \\
\text { (UG/L) }\end{array}$ & $\begin{array}{l}\text { TOTAL } \\
\text { (UG/L) }\end{array}$ & $\begin{array}{l}\text { TOTAL } \\
\text { (UG/L) }\end{array}$ & $\begin{array}{l}\text { TOTAL } \\
\text { (UG/L) }\end{array}$ & $\begin{array}{l}\text { TOTAL } \\
(\mathrm{UG} / \mathrm{L})\end{array}$ & $\begin{array}{l}\text { TOTAL } \\
(\mathrm{UG} / \mathrm{L})\end{array}$ & $\begin{array}{l}\text { TOTAL } \\
(\mathrm{UG} / \mathrm{L})\end{array}$ \\
\hline \multicolumn{13}{|c|}{ SEP 1992} \\
\hline $03-03$ & $<0.2$ & $<0.20$ & $<0.20$ & $<0.2$ & $<0.20$ & $<5.0$ & $<5.0$ & $<5.0$ & $<40.0$ & $<10.0$ & $<10.0$ & $<10.0$ \\
\hline \multicolumn{13}{|l|}{ SEP } \\
\hline $10-10$ & $<0.2$ & $<0.20$ & $<0.20$ & $<0.2$ & $<0.20$ & $<5.0$ & $<5.0$ & $<5.0$ & $<40.0$ & $<10.0$ & $<10.0$ & $<10.0$ \\
\hline
\end{tabular}


Table 6. Water-quality data for storm-sewer outfall stations, Irving, Texas, 1992-93-Continued

08049590 - BEAR CREEK OUTFALL AT SHADY GROVE ROAD, IRVING, TX (WY 1992)-Continued

\begin{tabular}{|c|c|c|c|c|c|c|c|c|c|c|c|c|}
\hline & & BENZO & $4-$ & & BIS & BIS & BIS $12-$ & & & & $4-$ & \\
\hline & $\begin{array}{l}\text { BENZO K } \\
\text { FLUOR - } \\
\text { AN- } \\
\text { THENE }\end{array}$ & $\begin{array}{l}\text { GHI PERYL } \\
\text { ENE1, } 12- \\
\text { BENZO } \\
\text { PERYLENE }\end{array}$ & $\begin{array}{l}\text { BROMO- } \\
\text { PHENYL } \\
\text { PHENYL } \\
\text { ETHER }\end{array}$ & $\begin{array}{l}\text { N-BUTYL } \\
\text { BENZYL } \\
\text { PHTHAL- } \\
\text { ATE }\end{array}$ & $\begin{array}{c}\text { (2- } \\
\text { CHLORO- } \\
\text { ETHOXY) } \\
\text { METHANE }\end{array}$ & $\begin{array}{c}2- \\
\text { CHLORO- } \\
\text { ETHYL } \\
\text { ETHER }\end{array}$ & $\begin{array}{l}\text { CHLORO- } \\
\quad \text { ISO- } \\
\text { PROPYL) } \\
\text { ETHER }\end{array}$ & $\begin{array}{l}\text { PARA - } \\
\text { CHLORO- } \\
\text { META } \\
\text { CRESOL }\end{array}$ & $\begin{array}{c}2- \\
\text { CHLORO- } \\
\text { NAPH- } \\
\text { THALENE }\end{array}$ & $\begin{array}{c}2- \\
\text { CHLORO- } \\
\text { PHENOL }\end{array}$ & $\begin{array}{l}\text { CHLORO- } \\
\text { PHENYL } \\
\text { PHENYL } \\
\text { ETHER }\end{array}$ & $\begin{array}{l}\text { CHRY - } \\
\text { SENE }\end{array}$ \\
\hline DATE & $\begin{array}{l}\text { TOTAL } \\
\text { (UG/L) }\end{array}$ & $\begin{array}{l}\text { TOTAL } \\
\text { (UG/L) }\end{array}$ & $\begin{array}{l}\text { TOTAL } \\
\text { (UG/L) }\end{array}$ & $\begin{array}{l}\text { TOTAL } \\
\text { (UG/L) }\end{array}$ & $\begin{array}{l}\text { TOTAL } \\
(\mathrm{UG} / \mathrm{L})\end{array}$ & $\begin{array}{l}\text { TOTAL } \\
(\mathrm{UG} / \mathrm{L})\end{array}$ & $\begin{array}{l}\text { TOTAL } \\
\text { (UG/L) }\end{array}$ & $\begin{array}{l}\text { TOTAL } \\
\text { (UG/L) }\end{array}$ & $\begin{array}{l}\text { TOTAL } \\
\text { (UG/L) }\end{array}$ & $\begin{array}{l}\text { TOTAL } \\
\text { (UG/L) }\end{array}$ & $\begin{array}{l}\text { TOTAL } \\
\text { (UG/L) }\end{array}$ & $\begin{array}{l}\text { TOTAL } \\
\text { (UG/L) }\end{array}$ \\
\hline \multicolumn{13}{|l|}{ SEP 1992} \\
\hline $\begin{array}{l}03-03 \\
\text { SEP }\end{array}$ & $<10.0$ & $<10.0$ & $<5.0$ & $<5.0$ & $<5.0$ & $<5.0$ & $<5.0$ & $<30.0$ & $<5.0$ & $<5.0$ & $<5.0$ & $<10.0$ \\
\hline $10-10$ & $<10.0$ & $<10.0$ & $<5.0$ & $<5.0$ & $<5.0$ & $<5.0$ & $<5.0$ & $<30.0$ & $<5.0$ & $<5.0$ & $<5.0$ & $<10.0$ \\
\hline
\end{tabular}

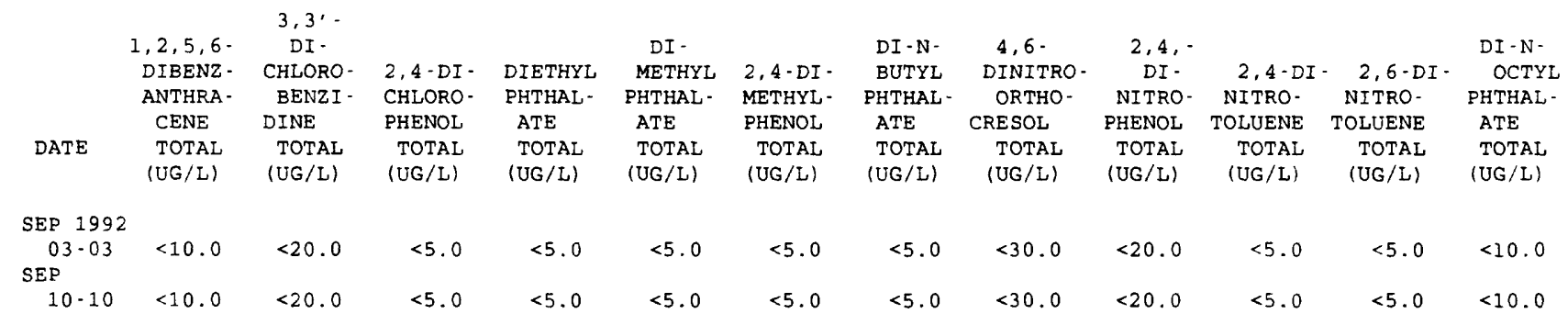

\begin{tabular}{|c|c|c|c|c|c|c|c|c|c|c|c|c|}
\hline DATE & $\begin{array}{l}\text { BIS }(2- \\
\text { ETHYL } \\
\text { HEXYL) } \\
\text { PHTHAL- } \\
\text { ATE } \\
\text { TOTAL } \\
\text { (UG/L) }\end{array}$ & $\begin{array}{l}\text { FLUOR - } \\
\text { ANTHENE } \\
\text { TOTAL } \\
\text { (UG/L) }\end{array}$ & $\begin{array}{c}\text { FLUOR- } \\
\text { ENE } \\
\text { TOTAL } \\
\text { (UG/L) }\end{array}$ & $\begin{array}{c}\text { HEXA- } \\
\text { CHLORO- } \\
\text { BENZENE } \\
\text { TOTAL } \\
\text { (UG/L) }\end{array}$ & $\begin{array}{l}\text { HEXA- } \\
\text { CHLORO- } \\
\text { CYCLO- } \\
\text { PENT - } \\
\text { ADIENE } \\
\text { TOTAL } \\
\text { (UG/L) }\end{array}$ & $\begin{array}{l}\text { HEXA- } \\
\text { CHLORO- } \\
\text { ETHANE } \\
\text { TOTAL } \\
\text { (UG/L) }\end{array}$ & $\begin{array}{c}\text { INDENO } \\
(1,2,3- \\
\text { CD) } \\
\text { PYRENE } \\
\text { TOTAL } \\
\text { (UG/L) }\end{array}$ & $\begin{array}{l}\text { ISO- } \\
\text { PHORONE } \\
\text { TOTAL } \\
\text { (UG/L) }\end{array}$ & $\begin{array}{l}\text { NITRO- } \\
\text { BENZENE } \\
\text { TOTAL } \\
\text { (UG/L) }\end{array}$ & $\begin{array}{l}\text { N-NITRO- } \\
\text { SODI- } \\
\text { METHY - } \\
\text { LAMINE } \\
\text { TOTAL } \\
\text { (UG/L) }\end{array}$ & $\begin{array}{c}2- \\
\text { NITRO- } \\
\text { PHENOL } \\
\text { TOTAL } \\
\text { (UG/L) }\end{array}$ & $\begin{array}{c}4- \\
\text { NITRO- } \\
\text { PHENOL } \\
\text { TOTAL } \\
\text { (UG/L) }\end{array}$ \\
\hline SEP 1992 & & & & & & & & & & & & \\
\hline $\begin{array}{l}03-03 \\
\text { SEP }\end{array}$ & $<5.0$ & $<5.0$ & $<5.0$ & $<5.0$ & $<5.0$ & $<5.0$ & $<10.0$ & $<5.0$ & $<5.0$ & $<5.0$ & $<5.0$ & $<30.0$ \\
\hline $10-10$ & $<5.0$ & $<5.0$ & $<5.0$ & $<5.0$ & $<5.0$ & $<5.0$ & $<10.0$ & $<5.0$ & $<5.0$ & $<5.0$ & $<5.0$ & $<30.0$ \\
\hline
\end{tabular}

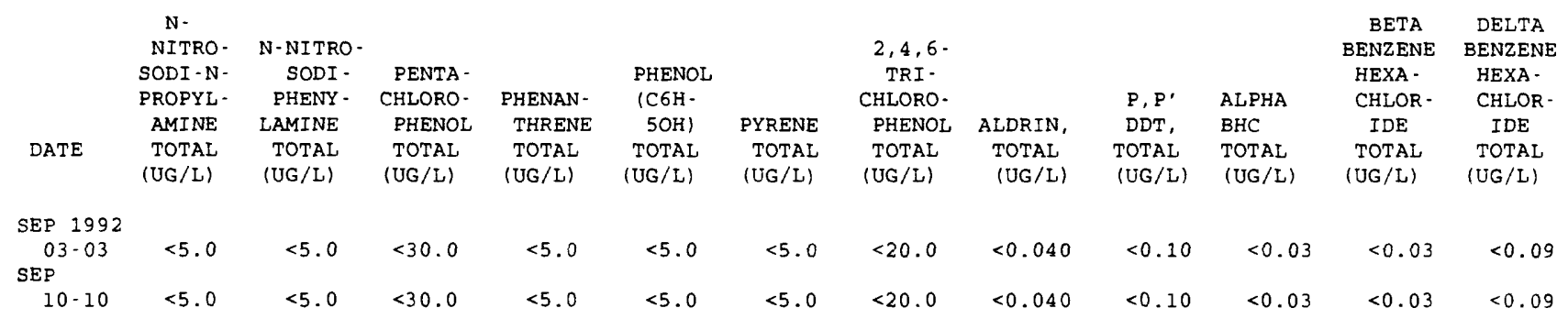

\begin{tabular}{|c|c|c|c|c|c|c|c|c|c|c|c|c|}
\hline & & $\begin{array}{c}\text { CHLOR - } \\
\text { DANE }\end{array}$ & $\begin{array}{c}\text { CHLOR - } \\
\text { DANE }\end{array}$ & & & & & $\begin{array}{l}\text { ENDO- } \\
\text { SULFAN- }\end{array}$ & & & & \\
\hline & & CIS & TRANS & & & & & I & ENDO - & ENDO - & ENDRIN & ENDR IN \\
\hline & & WATER & WATER & CHLOR - & $P, P^{\prime}$ & $P, P^{\prime}$ & DI - & WATER & SULFAN & SULFAN & WATER & ALDE - \\
\hline & LINDANE & WHOLE & WHOLE & DANE, & DDD, & DDE, & ELDRIN & WHOLE & BETA & SULFATE & UNFLTRD & HYDE \\
\hline DATE & $\begin{array}{l}\text { TOTAL } \\
\text { (UG/L) }\end{array}$ & $\begin{array}{r}\text { TOTAL } \\
\text { \{UG/L\} }\end{array}$ & $\begin{array}{l}\text { TOTAL } \\
\text { (UG/L) }\end{array}$ & $\begin{array}{l}\text { TOTAL } \\
\text { (UG/L) }\end{array}$ & $\begin{array}{l}\text { TOTAL } \\
\text { (UG/L) }\end{array}$ & $\begin{array}{l}\text { TOTAL } \\
\text { (UG/L) }\end{array}$ & $\begin{array}{l}\text { TOTAL } \\
\text { (UG/L) }\end{array}$ & $\begin{array}{c}\text { REC } \\
\{U G / L\}\end{array}$ & $\begin{array}{l}\text { TOTAL } \\
\text { (UG/L) }\end{array}$ & $\begin{array}{l}\text { TOTAL } \\
\text { (UG/L) }\end{array}$ & $\begin{array}{c}\text { REC } \\
(U G / L)\end{array}$ & $\begin{array}{l}\text { TOTAL } \\
\text { (UG/L) }\end{array}$ \\
\hline
\end{tabular}

\begin{tabular}{|c|c|c|c|c|c|c|c|c|c|c|c|c|}
\hline $\begin{array}{c}\text { SEP } 1992 \\
03-03\end{array}$ & $<0.030$ & $<0.10$ & $<0.10$ & 0.1 & $<0.10$ & $<0.04$ & $<0.020$ & $<0.10$ & $<0.04$ & $<0.60$ & $<0.060$ & $<0.20$ \\
\hline \multicolumn{13}{|l|}{ SEP } \\
\hline $10-10$ & $<0.030$ & $<0.10$ & $<0.10$ & $<0.1$ & $<0.10$ & $<0.04$ & $<0.020$ & $<0.10$ & $<0.04$ & $<0.60$ & $<0.060$ & $<0.20$ \\
\hline
\end{tabular}


Table 6. Water-quality data for storm-sewer outfall stations, Irving, Texas, 1992-93-Continued 08049590 - BEAR CREEK OUTFALL AT SHADY GROVE ROAD, IRVING, TX (WY 1992)-Continued

\begin{tabular}{|c|c|c|c|c|c|c|c|c|c|c|c|}
\hline & & HEPTA - & & AROCLOR & AROCLOR & AROCLOR & AROCLOR & AROCLOR & AROCLOR & AROCLOR & \\
\hline & HEPTA - & CHLOR & TOX - & 1016 & 1221 & 1232 & 1242 & 1248 & 1254 & 1260 & DI - \\
\hline DATE & $\begin{array}{l}\text { CHLOR, } \\
\text { TOTAL } \\
\text { (UG/L) }\end{array}$ & $\begin{array}{c}\text { EPOXIDE } \\
\text { TOTAL } \\
\text { (UG/L) }\end{array}$ & $\begin{array}{c}\text { APHENE, } \\
\text { TOTAL } \\
\text { (UG/L) }\end{array}$ & $\begin{array}{c}\text { PCB } \\
\text { TOTAL } \\
(\mathrm{UG} / \mathrm{L})\end{array}$ & $\begin{array}{c}\text { PCB } \\
\text { TOTAL } \\
(\mathrm{UG} / \mathrm{L})\end{array}$ & $\begin{array}{c}\mathrm{PCB} \\
\text { TOTAL } \\
(\mathrm{UG} / \mathrm{L})\end{array}$ & $\begin{array}{c}\text { PCB } \\
\text { TOTAL } \\
(U G / L)\end{array}$ & $\begin{array}{c}\text { PCB } \\
\text { TOTAL } \\
\text { (UG /L) }\end{array}$ & $\begin{array}{c}\text { PCB } \\
\text { TOTAL } \\
\text { (UG/L) }\end{array}$ & $\begin{array}{c}\text { PCB } \\
\text { TOTAL } \\
(U G / L)\end{array}$ & $\begin{array}{c}\text { AZINON, } \\
\text { TOTAL } \\
\text { (UG/L) }\end{array}$ \\
\hline \multicolumn{12}{|l|}{ SEP 1992} \\
\hline 03.03 & $<0.030$ & $<0.80$ & $<2$ & $<0.1$ & $<1.0$ & $<0.1$ & $<0.1$ & $<0.1$ & $<0.1$ & $<0.1$ & 0.80 \\
\hline \multicolumn{12}{|l|}{ SEP } \\
\hline $10-10$ & $<0.030$ & $<0.80$ & $<2$ & $<0.1$ & $<1.0$ & $<0.1$ & $<0.1$ & $<0.1$ & $<0.1$ & $<0.1$ & 0.50 \\
\hline
\end{tabular}


Table 6. Water-quality data for storm-sewer outfall stations, Irving, Texas, 1992-93-Continued

08049590 - BEAR CREEK OUTFALL AT SHADY GROVE ROAD, IRVING, TX (WY 1993)

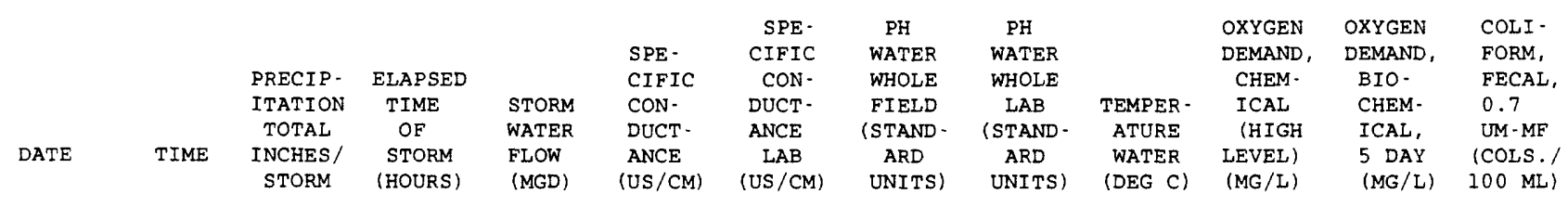

\begin{tabular}{cc} 
OCT 1992 & \\
$07-07$ & 1730 \\
OCT & \\
$15-15$ & 2020 \\
NOV & \\
$19-19$ & 0122 \\
FEB 1993 & \\
$03-03$ & 0557 \\
FEB & \\
\hline
\end{tabular}

\begin{tabular}{|c|c|c|c|c|c|c|c|c|c|c|}
\hline 0.20 & 1.4 & 0.07 & 78 & $\cdots$ & 7.9 & $\cdots$ & 23.5 & 140 & 8.7 & 12000 \\
\hline 0.45 & 1.1 & 0.11 & 160 & 110 & 7.8 & 7.1 & 24.0 & 220 & 8.3 & 48000 \\
\hline 0.94 & 10.0 & 0.24 & 66 & 90 & 7.7 & 7.0 & 17.0 & 45 & 7.4 & 22000 \\
\hline 0.92 & 11.3 & 0.32 & 93 & 103 & 8.2 & 7.7 & 12.5 & 55 & 7.4 & 6000 \\
\hline 0.42 & 5.1 & 0.10 & 113 & 111 & 8.1 & 7.2 & 12.0 & 43 & 6.3 & 70000 \\
\hline
\end{tabular}

\begin{tabular}{|c|c|c|c|c|c|c|c|c|c|c|c|c|}
\hline & $\begin{array}{r}\text { STREP - } \\
\text { TOCOCCI }\end{array}$ & HARD- & $\begin{array}{l}\text { HARD - } \\
\text { NESS }\end{array}$ & $\begin{array}{l}\text { ALKA - } \\
\text { LINITY }\end{array}$ & $\begin{array}{l}\text { SOLIDS, } \\
\text { SUM OF }\end{array}$ & $\begin{array}{l}\text { RESIDUE } \\
\text { TOTAL }\end{array}$ & $\begin{array}{l}\text { SOLIDS, } \\
\text { RESIDUE }\end{array}$ & & MAGNE - & & & SODIUM \\
\hline & FECAL, & NESS & NONCARB & WAT DIS & CONSTI- & AT 105 & AT 180 & CALCIUM & SIUM, & SODIUM, & & $A D$ \\
\hline & $\begin{array}{l}\text { KF AGAR } \\
\text { (COLS. }\end{array}$ & $\begin{array}{l}\text { TOTAL } \\
(\mathrm{MG} / \mathrm{L}\end{array}$ & $\begin{array}{l}\text { DISSOLV } \\
\text { FLD. AS }\end{array}$ & $\begin{array}{l}\text { FIX END } \\
\text { FIELD }\end{array}$ & $\begin{array}{c}\text { TUENTS, } \\
\text { DIS - }\end{array}$ & $\begin{array}{l}\text { DEG. C, } \\
\text { SUS - }\end{array}$ & $\begin{array}{l}\text { DEG. C } \\
\text { DIS - }\end{array}$ & $\begin{array}{l}\text { DIS - } \\
\text { SOLVED }\end{array}$ & $\begin{array}{l}\text { DIS - } \\
\text { SOLVED }\end{array}$ & $\begin{array}{l}\text { DIS - } \\
\text { SOLVED }\end{array}$ & & $\begin{array}{l}\text { SORP - } \\
\text { TION }\end{array}$ \\
\hline DATE & $\begin{array}{c}\text { PER } \\
100 \mathrm{ML})\end{array}$ & $\begin{array}{c}\text { AS } \\
\text { (ACO3) }\end{array}$ & $\begin{array}{r}\mathrm{CACO} 3 \\
(\mathrm{MG} / \mathrm{L})\end{array}$ & $\begin{array}{l}\text { CAC03 } \\
(\mathrm{MG} / \mathrm{L})\end{array}$ & $\begin{array}{c}\text { SOLVED } \\
(M G / L)\end{array}$ & $\begin{array}{l}\text { PENDED } \\
(M G / L)\end{array}$ & $\begin{array}{l}\text { SOLVED } \\
(M G / L)\end{array}$ & $\begin{array}{c}(\mathrm{MG} / \mathrm{L} \\
\mathrm{AS} \quad \mathrm{CA})\end{array}$ & $\begin{array}{l}\text { (MG /L } \\
\text { AS MG) }\end{array}$ & $\begin{array}{l}\text { (MG/L } \\
\text { AS NA) }\end{array}$ & $\begin{array}{l}\text { SODIUM } \\
\text { PERCENT }\end{array}$ & RATIO \\
\hline
\end{tabular}

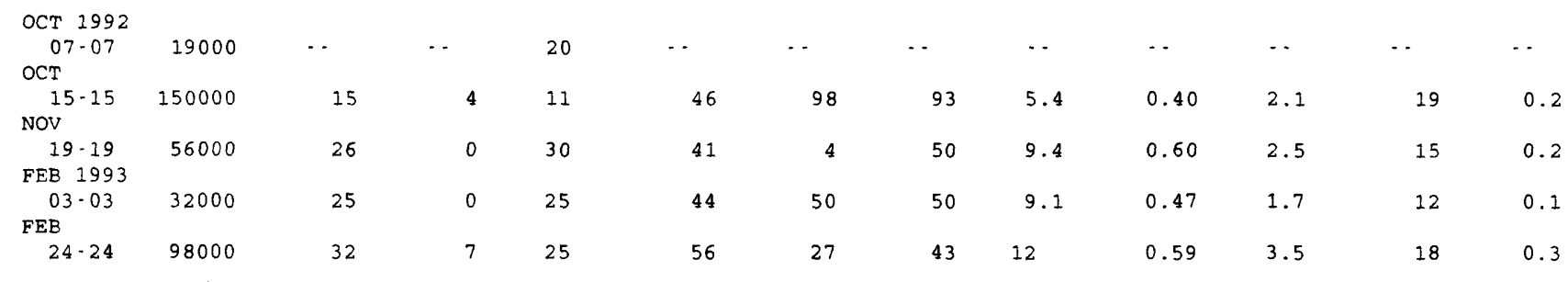

\begin{tabular}{|c|c|c|c|c|c|c|c|c|c|c|c|c|}
\hline DATE & $\begin{array}{l}\text { POTAS - } \\
\text { SIUM, } \\
\text { DIS - } \\
\text { SOIVED } \\
\text { (MG/L }\end{array}$ & $\begin{array}{l}\text { SULFATE } \\
\text { DIS- } \\
\text { SOLVED } \\
\text { (MG/L }\end{array}$ & $\begin{array}{l}\text { CHLO - } \\
\text { RIDE, } \\
\text { DIS - } \\
\text { SOLVED } \\
\text { (MG / I }\end{array}$ & $\begin{array}{l}\text { NITRO- } \\
\text { GEN, } \\
\text { NITRITE } \\
\text { TOTAL } \\
\text { (MG/L }\end{array}$ & $\begin{array}{c}\text { NITRO- } \\
\text { GEN, } \\
\text { NO2+NO3 } \\
\text { TOTAL } \\
\text { (MG/I. }\end{array}$ & $\begin{array}{l}\text { NITRO- } \\
\text { GEN, } \\
\text { AMMONIA } \\
\text { TOTAL } \\
\text { (MG/L }\end{array}$ & $\begin{array}{l}\text { NITRO- } \\
\text { GEN, AM- } \\
\text { MONIA + } \\
\text { ORGANIC } \\
\text { TOTAL } \\
\text { (MG/L }\end{array}$ & $\begin{array}{r}\text { PHOS - } \\
\text { PHORUS } \\
\text { TOTAL } \\
\text { (MG / L }\end{array}$ & $\begin{array}{c}\text { PHOS - } \\
\text { PHORUS } \\
\text { DIS - } \\
\text { SOIVED } \\
\text { (MG / L }\end{array}$ & $\begin{array}{l}\text { ANTIMONY } \\
\text { TOTAL } \\
\text { EPA } \\
\text { (UG/L }\end{array}$ & $\begin{array}{l}\text { ARSENIC } \\
\text { TOTAL } \\
\text { IUG/L }\end{array}$ & $\begin{array}{l}\text { BERYL- } \\
\text { LIUM, } \\
\text { TOTAL } \\
\text { RECOV- } \\
\text { ERABLE } \\
\text { (UG /L }\end{array}$ \\
\hline & AS K) & AS SO4) & AS CL & AS $\mathrm{N}$ ) & AS N) & AS N) & AS N) & AS P) & AS P) & AS SB) & AS AS) & AS BE? \\
\hline
\end{tabular}

\begin{tabular}{|c|c|c|c|c|c|c|c|c|c|c|c|c|}
\hline \multicolumn{13}{|l|}{ OCT 1992} \\
\hline 07.07 & $\cdots$ & $\cdots$ & $\cdots$ & 0.060 & 1.10 & 0.650 & 4.3 & 0.850 & 0.380 & $<10.0$ & 6 & $<10$ \\
\hline \multicolumn{13}{|l|}{ OCT } \\
\hline $15-15$ & 3.5 & 13 & 4.5 & 0.030 & 0.430 & 0.330 & 2.2 & 0.620 & 0.350 & $<10.0$ & 3 & $<10$ \\
\hline \multicolumn{13}{|l|}{ NOV } \\
\hline $19-19$ & 3.9 & 4.5 & 2.9 & 0.110 & 0.610 & 0.150 & 0.90 & 0.400 & 0.330 & $<20.0$ & 2 & $<10$ \\
\hline FEB 1993 & & & & & & & & & & & & \\
\hline 03.03 & 2.6 & 3.8 & 1.9 & $\cdots$ & 0.450 & $\cdots$ & 1.1 & 0.370 & 0.240 & $<10.0$ & 3 & $<10$ \\
\hline \multicolumn{13}{|l|}{ FEB } \\
\hline $24-24$ & 2.6 & 7.5 & 5.2 & $\cdots$ & 1.20 & $\cdots$ & 1.1 & 0.210 & 0.150 & $<10.0$ & 1 & $<10$ \\
\hline
\end{tabular}


Table 6. Water-quality data for storm-sewer outfall stations, Irving, Texas, 1992-93-Continued

08049590 - BEAR CREEK OUTFALL AT SHADY GROVE ROAD, IRVING, TX (WY 1993)-Continued

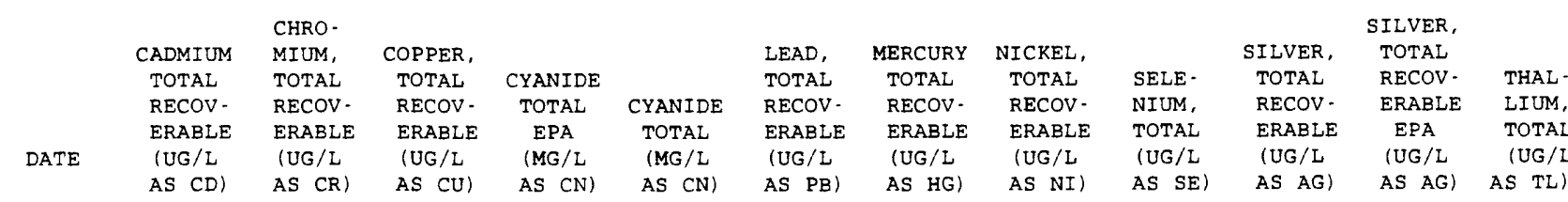

\begin{tabular}{|c|c|c|c|c|c|c|c|c|c|c|c|c|}
\hline $\begin{array}{c}\text { OCT } 1992 \\
07.07\end{array}$ & $<1$ & 3 & 15 & $<0.010$ & $<0.010$ & 31 & $<0.10$ & 6 & $<2$ & $<1$ & $<0.500$ & $<5$ \\
\hline OCT & & & & & & & & & & & & \\
\hline $\begin{array}{l}15 \cdot 15 \\
\text { Nov }\end{array}$ & $<1$ & 4 & 6 & $<0.010$ & $<0.010$ & 21 & $<0.10$ & 3 & $<2$ & $<1$ & $<0.500$ & $<5$ \\
\hline $\begin{array}{c}19-19 \\
\text { FEB } \quad 1993\end{array}$ & $<1$ & 3 & 5 & $<0.010$ & $<0.010$ & 8 & $<0.10$ & $<1$ & $<2$ & $<1$ & $<0.500$ & $<5$ \\
\hline $03-03$ & $<1$ & 3 & 6 & $<0.010$ & $\cdots$ & 18 & $<0.10$ & 3 & $<2$ & $<1$ & $<0.500$ & $<10$ \\
\hline $24-24$ & $<1$ & 6 & 7 & $<0.010$ & $<0.010$ & 16 & $<0.10$ & 3 & $<2$ & $<1$ & $<0.500$ & $<5$ \\
\hline
\end{tabular}

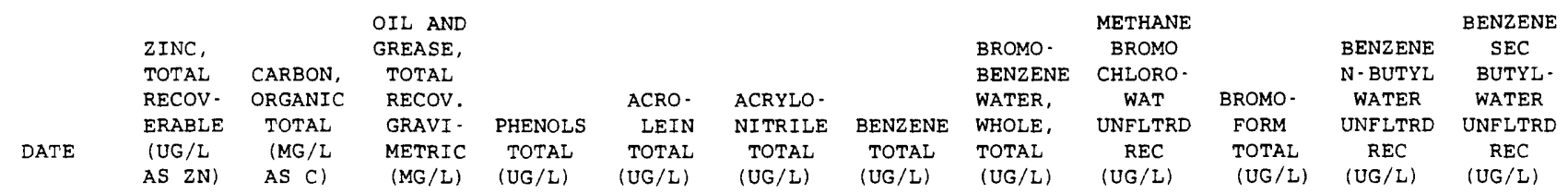

\begin{tabular}{|c|c|c|c|c|c|c|c|c|c|c|c|c|}
\hline $\begin{array}{c}\text { OCT } 1992 \\
07.07\end{array}$ & 150 & 49 & 2 & 8 & $<20$ & $<20$ & $<0.2$ & $<0.2$ & $<0.20$ & $<0.2$ & $<0.20$ & $<0.20$ \\
\hline OCT & & & & & & & & & & & & \\
\hline $\begin{array}{l}15-15 \\
\text { Nov }\end{array}$ & 60 & 60 & 6 & 11 & $<20$ & $<20$ & $<0.2$ & $<0.2$ & $<0.20$ & $<0.2$ & $<0.20$ & $<0.20$ \\
\hline $\begin{array}{c}19-19 \\
\text { FEB } 1993\end{array}$ & 50 & 16 & $<1$ & 3 & $<20$ & $<20$ & $<0.2$ & $<0.2$ & $<0.20$ & $<0.2$ & $<0.20$ & $<0.20$ \\
\hline $\begin{array}{l}03.03 \\
\text { FEB }\end{array}$ & 70 & 14 & 7 & 6 & $<20$ & $<20$ & $<0.2$ & $<0.2$ & $<0.20$ & $<0.2$ & $<0.20$ & $<0.20$ \\
\hline $24-24$ & 60 & 13 & 2 & 11 & $<20$ & $<20$ & $<0.2$ & $<0.2$ & $<0.20$ & $<0.2$ & $<0.20$ & $<0.20$ \\
\hline
\end{tabular}

\begin{tabular}{|c|c|c|c|c|c|c|c|c|c|c|c|c|}
\hline & $\begin{array}{l}\text { BENZENE } \\
\text { TERT - }\end{array}$ & CARBON - & & CHLORO- & & $\begin{array}{c}2- \\
\text { CHLORO- }\end{array}$ & & & $\begin{array}{c}\text { O- } \\
\text { CHLORO- }\end{array}$ & TOLUENE & $\begin{array}{l}\text { DIBROMO } \\
\text { CHLORO- }\end{array}$ & $\begin{array}{c}\text { DI - } \\
\text { BROMO - }\end{array}$ \\
\hline & & TETRA. & & DI - & & FTHYT. & & METHYL - & TOLUENE & P-CHLOR & PROPANE & METHAN \\
\hline & WATER & CHLO- & CHLORO- & BROMO - & CHLORO- & VINYL - & CHLORO- & CHLO- & WATER & WATER & WATER & WATER \\
\hline & UNFLTRD & RIDE & BENZENE & METHANE & ETHANE & ETHER & FORM & RIDE & & UNFLTRD & WHOLE & WHOLE \\
\hline & $\begin{array}{c}\text { REC } \\
(U G / L)\end{array}$ & $\begin{array}{l}\text { TOTAL } \\
\text { (UG/L) }\end{array}$ & $\begin{array}{l}\text { TOTAL } \\
\text { (UG/L) }\end{array}$ & $\begin{array}{l}\text { TOTAL } \\
\text { (UG/L) }\end{array}$ & $\begin{array}{l}\text { TOTAL } \\
\text { (UG/L) }\end{array}$ & $\begin{array}{l}\text { TOTAL } \\
\text { (UG/L) }\end{array}$ & $\begin{array}{l}\text { TOTAL } \\
\text { (UG/L) }\end{array}$ & $\begin{array}{l}\text { TOTAL } \\
\text { (UG/L) }\end{array}$ & $\begin{array}{l}\text { TOTAL } \\
\text { (UG/L) }\end{array}$ & $\begin{array}{c}\text { REC } \\
(\mathrm{UG} / \mathrm{L})\end{array}$ & $\begin{array}{l}\text { TOT.REC } \\
\text { (UG } / L)\end{array}$ & $\begin{array}{l}\text { RECOVER } \\
\text { (UG/L) }\end{array}$ \\
\hline
\end{tabular}

\begin{tabular}{|c|c|c|c|c|c|c|c|c|c|c|c|c|}
\hline OCT 1992 & & & & & & & & & & & & \\
\hline $07-07$ & $<0.20$ & $<0.2$ & $<0.20$ & $<0.2$ & $<0.2$ & $<1.0$ & $<0.2$ & $<0.2$ & $<0.2$ & $<0.20$ & $<1.0$ & $<0.2$ \\
\hline OCT & & & & & & & & & & & & \\
\hline $\begin{array}{l}15 \cdot 15 \\
\text { Nov }\end{array}$ & $<0.20$ & $<0.2$ & $<0.20$ & $<0.2$ & $<0.2$ & $<1.0$ & $<0.2$ & $<0.2$ & $<0.2$ & $<0.20$ & $<1.0$ & $<0.2$ \\
\hline $\begin{array}{c}19-19 \\
\text { FEB } \quad 1993\end{array}$ & $<0.20$ & $<0.2$ & $<0.20$ & $<0.2$ & $<0.2$ & $<1.0$ & $<0.2$ & $<0.2$ & $<0.2$ & $<0.20$ & $<1.0$ & $<0.2$ \\
\hline 03.03 & $<0.20$ & $<0.2$ & $<0.20$ & $<0.2$ & $<0.2$ & $<1.0$ & $<0.2$ & $<0.2$ & $<0.2$ & $<0.20$ & $<1.0$ & $<0.2$ \\
\hline FEB & & & & & & & & & & & & \\
\hline $24-24$ & $<0.20$ & $<0.2$ & $<0.20$ & $<0.2$ & $<0.2$ & $<1.0$ & $<0.2$ & $<0.2$ & $<0.2$ & $<0.20$ & $<1,0$ & $<0.2$ \\
\hline
\end{tabular}


Table 6. Water-quality data for storm-sewer outfall stations, Irving, Texas, 1992-93-Continued

08049590 - BEAR CREEK OUTFALL AT SHADY GROVE ROAD, IRVING, TX (WY 1993)-Continued

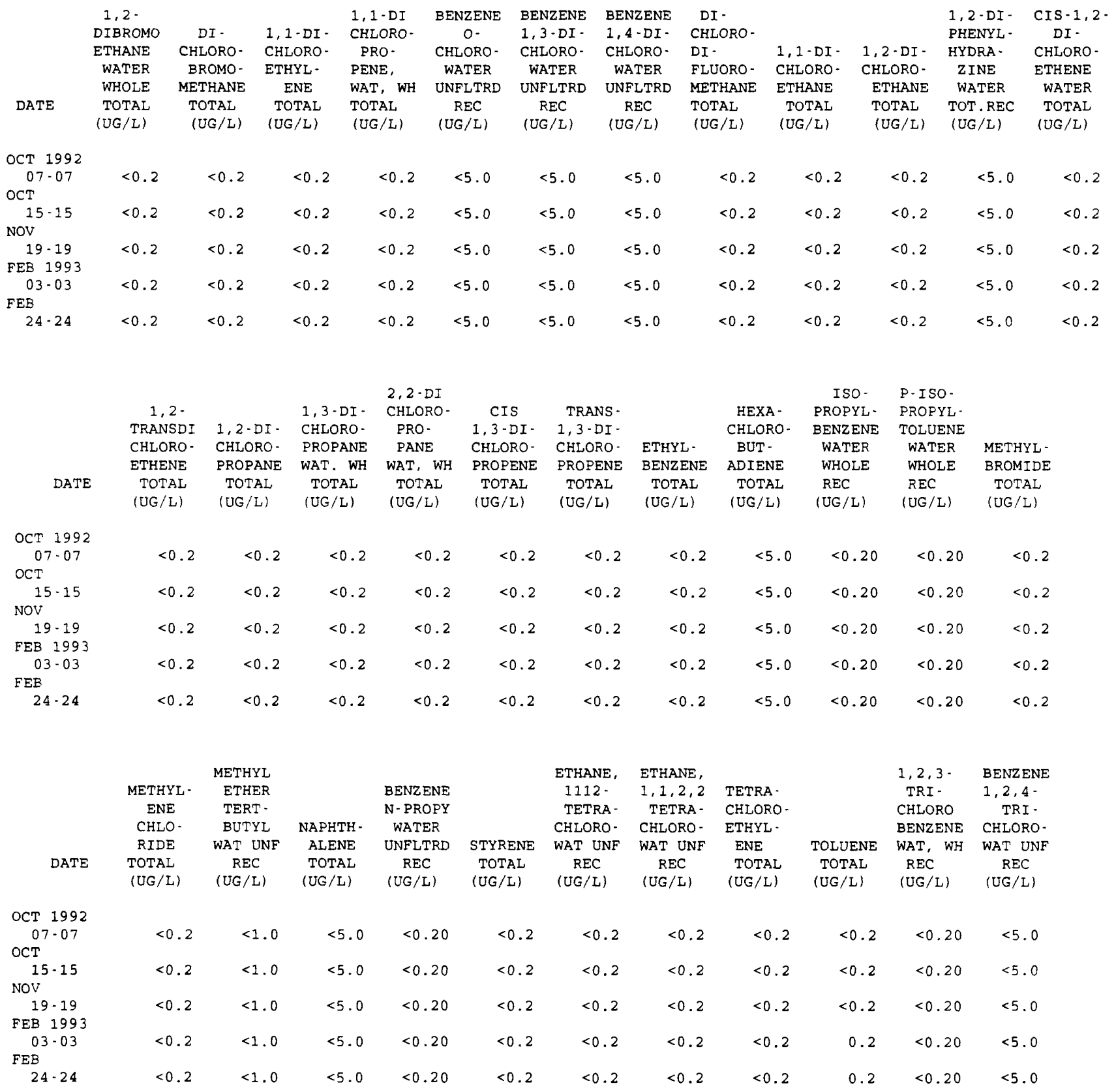


Table 6. Water-quality data for storm-sewer outfall stations, Irving, Texas, 1992-93-Continued

08049590 - BEAR CREEK OUTFALL AT SHADY GROVE ROAD, IRVING, TX (WY 1993)-Continued

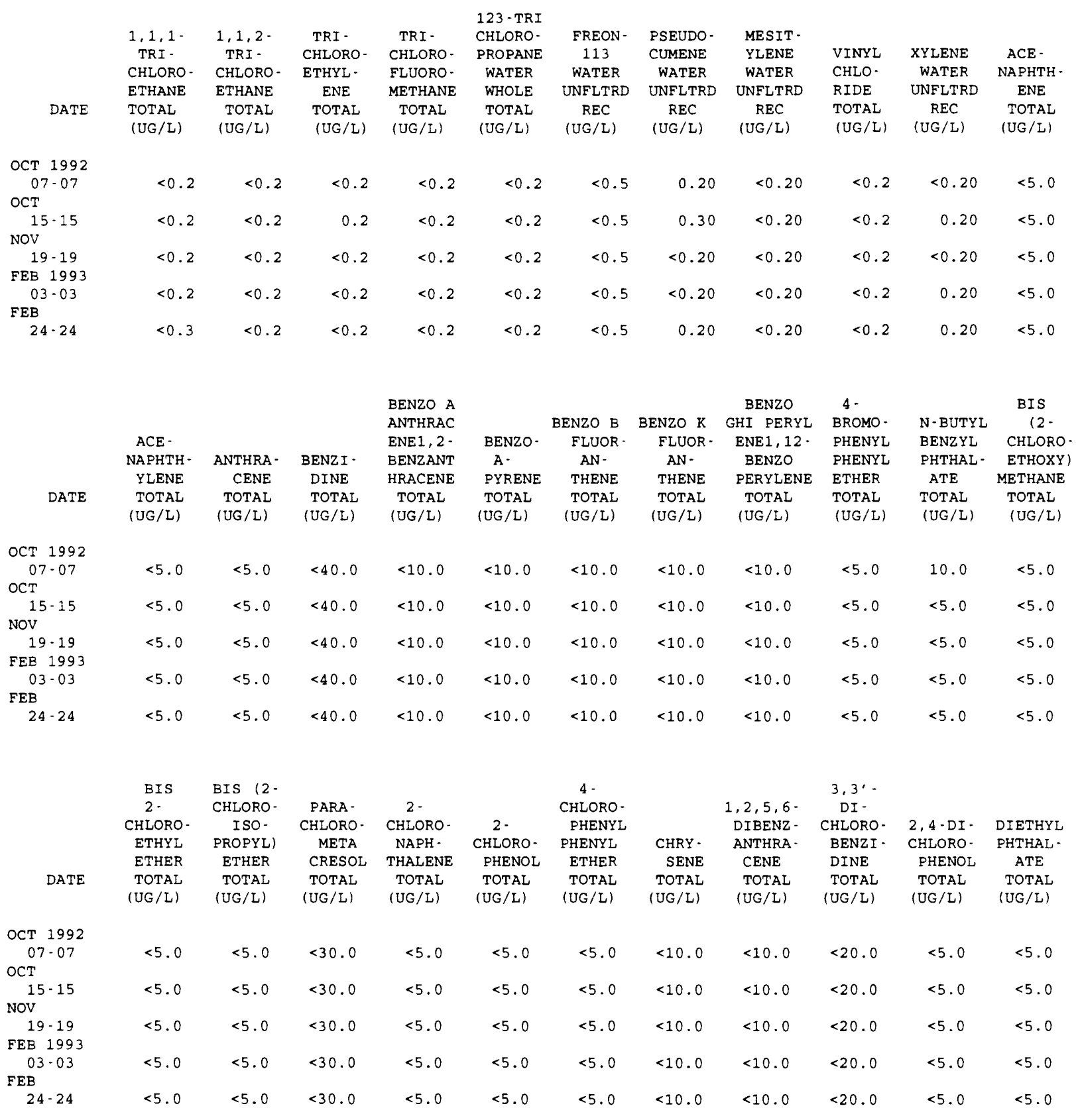


Table 6. Water-quality data for storm-sewer outfall stations, Irving, Texas, 1992-93-Continued

08049590 - BEAR CREEK OUTFALL AT SHADY GROVE ROAD, IRVING, TX (WY 1993)-Continued

\begin{tabular}{|c|c|c|c|c|c|c|c|c|c|c|c|}
\hline DATE & $\begin{array}{c}\text { DI - } \\
\text { METHYL } \\
\text { PHTHAL- } \\
\text { ATE } \\
\text { TOTAL } \\
\text { (UG/L) }\end{array}$ & $\begin{array}{c}2,4-\mathrm{DI}- \\
\text { METHYL - } \\
\text { PHENOL } \\
\text { TOTAL } \\
\text { (UG/L) }\end{array}$ & $\begin{array}{c}\text { DI - N- } \\
\text { BUTYL } \\
\text { PHTHAL- } \\
\text { ATE } \\
\text { TOTAL } \\
\text { (UG/L) }\end{array}$ & $\begin{array}{c}4,6- \\
\text { DINITRO- } \\
\text { ORTHO- } \\
\text { CRESOL } \\
\text { TOTAL } \\
\text { (UG/L) }\end{array}$ & $\begin{array}{c}2,4,- \\
\text { DI- } \\
\text { NITRO- } \\
\text { PHENOL } \\
\text { TOTAL } \\
\text { (UG/L) }\end{array}$ & $\begin{array}{l}2,4-\mathrm{DI}- \\
\text { NITRO- } \\
\text { TOLUENE } \\
\text { TOTAL } \\
\text { (UG/L) }\end{array}$ & $\begin{array}{l}2,6 \cdot \mathrm{DI}- \\
\text { NITRO- } \\
\text { TOLUENE } \\
\text { TOTAL } \\
\text { (UG/L) }\end{array}$ & $\begin{array}{l}\text { DI-N- } \\
\text { OCTYL } \\
\text { PHTHAL- } \\
\text { ATE } \\
\text { TOTAL } \\
\text { (UG/L) }\end{array}$ & $\begin{array}{l}\text { BIS (2- } \\
\text { ETHYL } \\
\text { HEXYL) } \\
\text { PHTHAL- } \\
\text { ATE } \\
\text { TOTAL } \\
\text { (UG/L) }\end{array}$ & $\begin{array}{l}\text { FLUOR - } \\
\text { ANTHENE } \\
\text { TOTAL } \\
\text { (UG/L) }\end{array}$ & $\begin{array}{c}\text { FLUOR - } \\
\text { ENE } \\
\text { TOTAL } \\
\text { (UG/L) }\end{array}$ \\
\hline OCT 1992 & & & & & & & & & & & \\
\hline $\begin{array}{l}07-07 \\
\text { OCT }\end{array}$ & $<5.0$ & $<5.0$ & $<5.0$ & $<30.0$ & $<20.0$ & $<5.0$ & $<5.0$ & $<10.0$ & 24.0 & $<5.0$ & $<5.0$ \\
\hline $\begin{array}{l}15-15 \\
\text { NoV }\end{array}$ & $<5.0$ & $<5.0$ & $<5.0$ & $<30.0$ & $<20.0$ & $<5.0$ & $<5.0$ & $<10.0$ & 17.0 & $<5.0$ & $<5.0$ \\
\hline $\begin{array}{cc}19-19 \\
\text { FEB } 1993\end{array}$ & $<5.0$ & $<5.0$ & $<5.0$ & $<30.0$ & $<20.0$ & $<5.0$ & $<5.0$ & $<10.0$ & 8.0 & $<5.0$ & $<5.0$ \\
\hline $\begin{array}{l}03.03 \\
\text { FEB }\end{array}$ & $<5.0$ & $<5.0$ & $<5.0$ & $<30.0$ & $<20.0$ & $<5.0$ & $<5.0$ & $<10.0$ & 7.0 & $<5.0$ & $<5.0$ \\
\hline DATE & $\begin{array}{l}\text { HEXA- } \\
\text { CHLORO- } \\
\text { BENZENE } \\
\text { TOTAL } \\
\text { (UG/L) }\end{array}$ & $\begin{array}{l}\text { HEXA- } \\
\text { CHLORO- } \\
\text { CYCLO- } \\
\text { PENT- } \\
\text { ADIENE } \\
\text { TOTAL } \\
\text { (UG/L) }\end{array}$ & $\begin{array}{l}\text { HEXA- } \\
\text { CHLORO- } \\
\text { ETHANE } \\
\text { TOTAL } \\
\text { (UG/L) }\end{array}$ & $\begin{array}{c}\text { INDENO } \\
(1,2,3- \\
\text { CD) } \\
\text { PYRENE } \\
\text { TOTAL } \\
\text { (UG/L) }\end{array}$ & $\begin{array}{l}\text { ISO- } \\
\text { PHORONE } \\
\text { TOTAL } \\
(U G / L)\end{array}$ & $\begin{array}{l}\text { NITRO- } \\
\text { BENZENE } \\
\text { TOTAL } \\
\text { (UG/L) }\end{array}$ & $\begin{array}{l}\text { N - NITRO- } \\
\text { SODI - } \\
\text { METHY - } \\
\text { LAMINE } \\
\text { TOTAL } \\
\text { (UG/L) }\end{array}$ & $\begin{array}{l}2 \text { - } \\
\text { NITRO- } \\
\text { PHENOL } \\
\text { TOTAL } \\
(\text { UG/L) }\end{array}$ & $\begin{array}{c}4- \\
\text { NITRO- } \\
\text { PHENOL } \\
\text { TOTAL } \\
\text { (UG/L) }\end{array}$ & $\begin{array}{l}\text { N- } \\
\text { NITRO- } \\
\text { SODI - N- } \\
\text { PROPYL- } \\
\text { AMINE } \\
\text { TOTAL } \\
\text { (UG/L) }\end{array}$ & $\begin{array}{l}\text { N-NITRO- } \\
\text { SODI- } \\
\text { PHENY- } \\
\text { LAMINE } \\
\text { TOTAL } \\
\text { (UG/L) }\end{array}$ \\
\hline OCT 1992 & & & & & & & & & & & \\
\hline $\begin{array}{l}07-07 \\
\text { OCT }\end{array}$ & $<5.0$ & $<5.0$ & $<5.0$ & $<10.0$ & $<5.0$ & $<5.0$ & $<5.0$ & $<5.0$ & $<30.0$ & $<5.0$ & $<5.0$ \\
\hline $\begin{array}{l}15 \cdot 15 \\
\text { NOV }\end{array}$ & $<5.0$ & $<5.0$ & $<5.0$ & $<10.0$ & $<5.0$ & $<5.0$ & $<5.0$ & $<5.0$ & $<30.0$ & $<5.0$ & $<5.0$ \\
\hline $\begin{array}{c}19-19 \\
\text { FEB } 1993\end{array}$ & $<5.0$ & $<5.0$ & $<5.0$ & $<10.0$ & $<5.0$ & $<5.0$ & $<5.0$ & $<5.0$ & $<30.0$ & $<5.0$ & $<5.0$ \\
\hline $\begin{array}{l}03.03 \\
\text { FEB }\end{array}$ & $<5.0$ & $<5.0$ & $<5.0$ & $<10.0$ & $<5.0$ & $<5.0$ & $<5.0$ & $<5.0$ & $<30.0$ & $<5.0$ & $<5.0$ \\
\hline $24-24$ & $<5.0$ & $<5.0$ & $<5.0$ & $<10.0$ & $<5.0$ & $<5.0$ & $<5.0$ & $<5.0$ & $<30.0$ & $<5.0$ & $<5.0$ \\
\hline DATE & $\begin{array}{l}\text { PENTA- } \\
\text { CHLORO- } \\
\text { PHENOL } \\
\text { TOTAL } \\
\text { (UG/L) }\end{array}$ & $\begin{array}{l}\text { PHENAN - } \\
\text { THRENE } \\
\text { TOTAL } \\
\text { (UG/L) }\end{array}$ & $\begin{array}{c}\text { PHENOL } \\
\text { (C6H- } \\
5 O H) \\
\text { TOTAL } \\
(U G / L)\end{array}$ & $\begin{array}{r}\text { PYRENE } \\
\text { TOTAL } \\
\text { (UG/L) }\end{array}$ & $\begin{array}{c}2,4,6- \\
\text { TRI- } \\
\text { CHLORO- } \\
\text { PHENOL } \\
\text { TOTAL } \\
\text { (UG/L) }\end{array}$ & $\begin{array}{c}\text { ALDRIN, } \\
\text { TOTAL } \\
\text { (UG/L) }\end{array}$ & $\begin{array}{l}\text { P, } P^{\prime} \\
\text { DDT, } \\
\text { TOTAL } \\
\text { (UG/L) }\end{array}$ & $\begin{array}{l}\text { ALPHA } \\
\text { BHC } \\
\text { TOTAL } \\
\text { (UG/L) }\end{array}$ & $\begin{array}{l}\text { BETA } \\
\text { BENZENE } \\
\text { HEXA- } \\
\text { CHLOR- } \\
\text { IDE } \\
\text { TOTAL } \\
\text { (UG/L) }\end{array}$ & $\begin{array}{l}\text { DELTA } \\
\text { BENZENE } \\
\text { HEXA- } \\
\text { CHLOR- } \\
\text { IDE } \\
\text { TOTAL } \\
\text { (UG/L) }\end{array}$ & $\begin{array}{c}\text { LINDANE } \\
\text { TOTAL } \\
\text { (UG/L) }\end{array}$ \\
\hline OCT 1992 & & & & & & & & & & & \\
\hline $\begin{array}{l}07-07 \\
\text { OCT }\end{array}$ & $<30.0$ & $<5.0$ & $<5.0$ & $<5.0$ & $<20.0$ & $<0.040$ & $<0.10$ & $<0.03$ & $<0.03$ & $<0.09$ & $<0.030$ \\
\hline $\operatorname{NOV}^{15-15}$ & $<30.0$ & $<5.0$ & $<5.0$ & $<5.0$ & $<20.0$ & $<0.040$ & $<0.10$ & $<0.03$ & $<0.03$ & $<0.09$ & $<0.030$ \\
\hline $\begin{array}{c}19-19 \\
\text { FEB } 1993\end{array}$ & $<30.0$ & $<5.0$ & $<5.0$ & $<5.0$ & $<20.0$ & $<0.040$ & $<0.10$ & $<0.03$ & $<0.03$ & $<0.09$ & $<0.030$ \\
\hline $\begin{array}{l}03-03 \\
\text { FEB }\end{array}$ & $<30.0$ & $<5.0$ & $<5.0$ & $<5.0$ & $<20.0$ & $<0.040$ & $<0.10$ & $<0.03$ & $<0.03$ & $<0.09$ & $<0.030$ \\
\hline $24-24$ & $<30.0$ & $<5.0$ & $<5.0$ & $<5.0$ & $<20.0$ & $<0.040$ & $<0.10$ & $<0.03$ & $<0.03$ & $<0.09$ & $<0.030$ \\
\hline
\end{tabular}


Table 6. Water-quality data for storm-sewer outfall stations, Irving, Texas, 1992-93-Continued

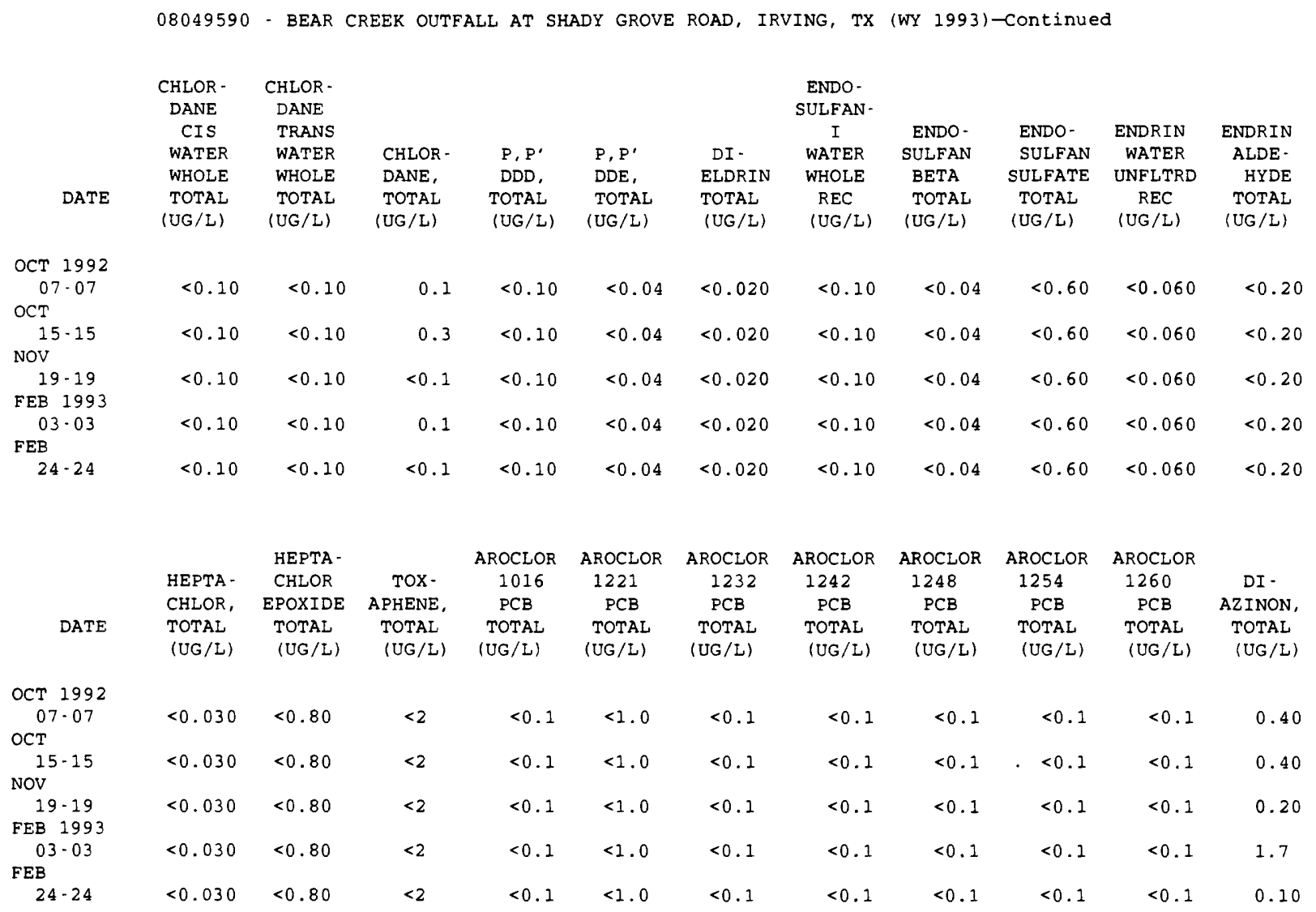


Table 6. Water-quality data for storm-sewer outfall stations, Irving, Texas, 1992-93-Continued 08055570 - HEREFORD ROAD OUTFALL AT WALNUT HILL ROAD, IRVING, TX (WY 1992)

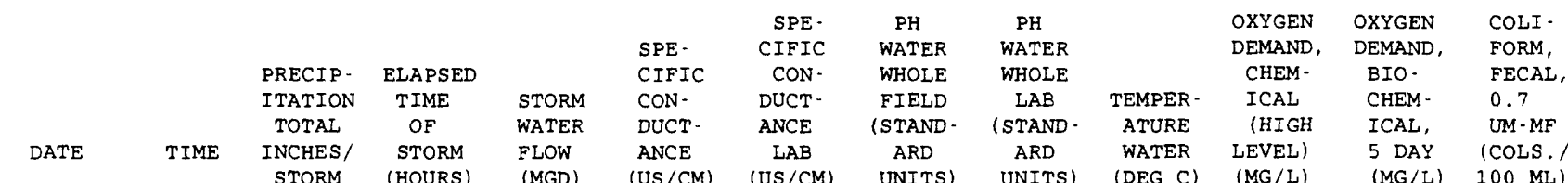

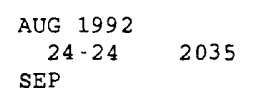

0.26

2.3

0.20

186

107

125

218

7.5

$\begin{array}{ll}7.2 & 27.0 \\ 7.7 & 25.0\end{array}$

25.0

40

78

8. $3 \quad 5100$

8.7130000

$\begin{array}{ccll} & \text { STREP } & & \text { HARD - } \\ & \text { TOCOCCI } & \text { HARD } & \text { NESS } \\ & \text { FECAL, } & \text { NESS } & \text { NONCARB } \\ & \text { KF AGAR } & \text { TOTAL } & \text { DISSOLV } \\ & \text { (COLS. } & \text { (MG } / L & \text { FLD. AS } \\ \text { DATE } & \text { PER } & \text { AS } & \text { CACO3 } \\ & 100 \mathrm{ML}) & \text { CACO3) } & (\mathrm{MG} / \mathrm{L})\end{array}$

AUG 1992

$\begin{array}{ll}24-24 & 9300 \\ \text { SEP } & \end{array}$

$03.03 \quad 180000$

$\begin{array}{rrr}33 & 11 & 22 \\ 26 & 6 & 20\end{array}$

ALKA -

LINITY

WAT DIS

FIX END

FIELD

$\mathrm{CAC} 03$

(MG/L)
SOLIDS, RESIDUE SOLIDS,

SUM OF TOTAL RESIDUE

CONSTI. AT 105 AT 180

TUENTS, DEG. C, DEG. C

DIS - SUS -

SOIVED PENDED

(MG/L) (MG/L) 
Table 6. Water-quality data for storm-sewer outfall stations, Irving, Texas, 1992-93-Continued

08055570 - HEREFORD ROAD OUTFALL AT WALNUT HILL ROAD, IRVING, TX (WY 1992)-Continued

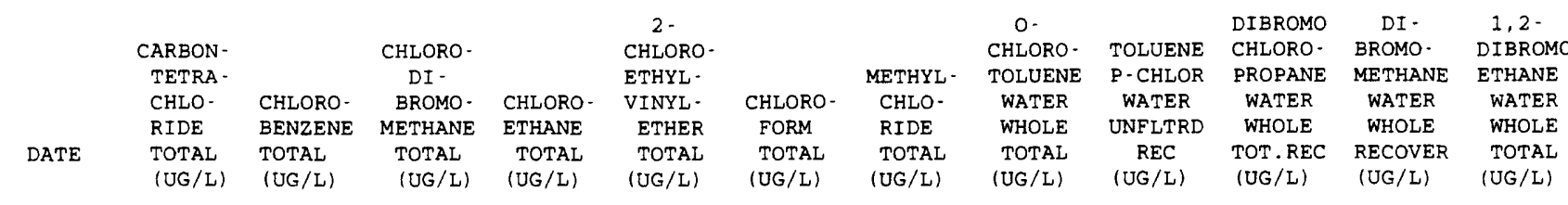

AUG 1992

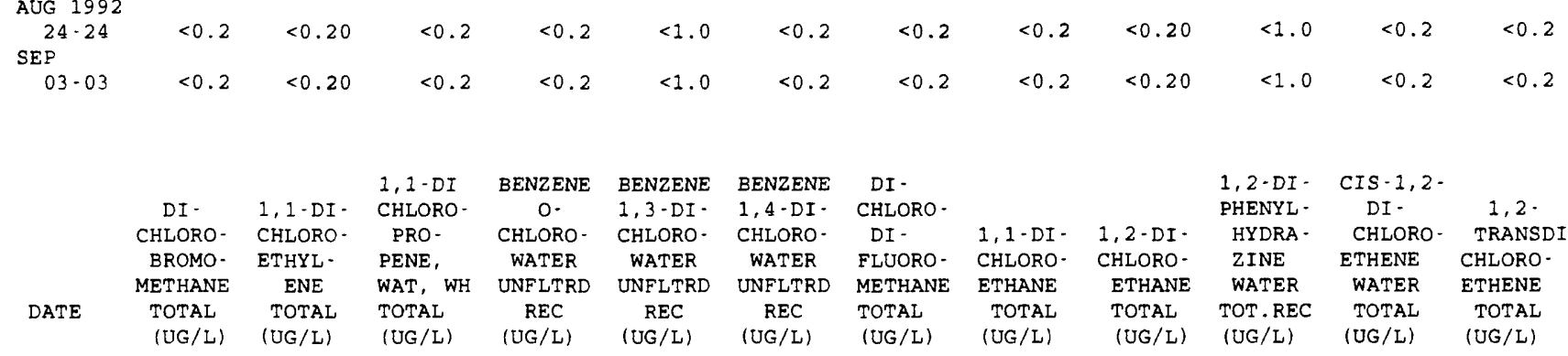

AUG 1992
$24 \cdot 24$ SEP

$03-03$

$$
<0.2<0.2
$$$$
<0.2
$$$$
<5.0
$$$$
<5.0
$$$$
<5.0
$$$$
<0.2
$$$$
<0.2
$$$$
<0.2
$$$$
<5 \text {. }
$$$$
<0.2
$$

$<0.2$

$<0.2$

$<0.2$

$<5.0$

$<0.2<0.2$
$<0.2<0.2$

$$
\text { 2, } 2 \text {-DI }
$$

$$
1,2 \text {-DI - } \begin{aligned}
& 1,3-\mathrm{DI}- \\
& \text { CHLORO- CHLORO- } \\
& \text { PRO- }
\end{aligned}
$$

PROPANE WAT. WH WAT, WH PROPENE PROPENE

DATE

TOTAL (UG/L)

TOTAL

TOTAL (UG/L) (UG/L) (UG/L)

$$
\text { ISO- P-ISO- }
$$

PROPYL - PROPYL .

BENZENE TOLUENE

WATER WATER

\begin{tabular}{|c|c|c|c|c|c|c|c|c|c|c|c|c|}
\hline $\begin{array}{l}\text { AUG } 1992 \\
24 \cdot 24 \\
\text { SEP }\end{array}$ & $<0.2$ & $<0.2$ & $<0.2$ & $<0.2$ & $<0.2$ & $<0.2$ & $<5.0$ & $<0.20$ & $<0.20$ & $<0.2$ & $<0.2$ & $<5.0$ \\
\hline \multirow[t]{5}{*}{03.03} & $<0.2$ & $<0.2$ & $<0.2$ & $<0.2$ & $<0.2$ & $<0.2$ & $<5.0$ & $<0.20$ & $<0.20$ & $<0.2$ & $<0.2$ & $<5.0$ \\
\hline & & & ETHANE, & ETHANE, & & & $1,2,3-$ & BENZENE & & & & \\
\hline & $\begin{array}{l}\text { BENZENE } \\
\mathrm{N} \text { - PROPY }\end{array}$ & & $\begin{array}{l}1112- \\
\text { TETRA - }\end{array}$ & $\begin{array}{r}1,1,2,2 \\
\text { TETRA. }\end{array}$ & $\begin{array}{l}\text { TETRA - } \\
\text { CHLORO- }\end{array}$ & & $\begin{array}{l}\text { TRI - } \\
\text { CHLORO }\end{array}$ & $\begin{array}{r}1,2,4- \\
\text { TRI. }\end{array}$ & $\begin{array}{c}1,1,1- \\
\text { TRI. }\end{array}$ & $\begin{array}{c}1,1,2- \\
\text { TRI. }\end{array}$ & $\begin{array}{l}\text { TRI- } \\
\text { CHLORO- }\end{array}$ & $\begin{array}{c}\text { TRI - } \\
\text { CHLORO- }\end{array}$ \\
\hline & WATER & & CHLORO- & CHLORO- & ETHYL - & & BENZENE & CHLORO- & CHLORO- & CHLORO- & ETHYL - & FLUORO- \\
\hline & UNFLTRD & STYRENE & WAT UNF & WAT UNF & ENE & TOLUENE & WAT, WH & WAT UNF & ETHANE & ETHANE & ENE & METHANE \\
\hline DATE & $\begin{array}{c}\text { REC } \\
(U G / L)\end{array}$ & $\begin{array}{l}\text { TOTAL } \\
\text { (UG/L) }\end{array}$ & $\begin{array}{c}\text { REC } \\
(\mathrm{UG} / \mathrm{L})\end{array}$ & $\begin{array}{c}\text { REC } \\
(U G / L)\end{array}$ & $\begin{array}{l}\text { TOTAL } \\
\text { (UG/L) }\end{array}$ & $\begin{array}{l}\text { TOTAL } \\
\text { (UG/L) }\end{array}$ & $\begin{array}{l}\text { REC } \\
\text { (UG/L) }\end{array}$ & $\begin{array}{c}\text { REC } \\
(U G / L)\end{array}$ & $\begin{array}{l}\text { TOTAL } \\
\text { (UG/L) }\end{array}$ & $\begin{array}{l}\text { TOTAL } \\
\text { (UG/L) }\end{array}$ & $\begin{array}{l}\text { TOTAL } \\
\text { (UG/L) }\end{array}$ & $\begin{array}{l}\text { TOTAL } \\
\text { (UG/L) }\end{array}$ \\
\hline
\end{tabular}

WHOLE WHOLE

\begin{tabular}{|c|c|c|c|c|c|c|c|c|c|c|c|c|}
\hline $\begin{array}{c}\text { AUG } 1992 \\
24 \cdot 24\end{array}$ & & & & & & & & & & & & \\
\hline SEP & -0.20 & 20.2 & $<0.2$ & $<0.2$ & $<0.2$ & $<0.2$ & $<0.20$ & $<5.0$ & $<0.2$ & $<0.2$ & $<0.2$ & $<0.2$ \\
\hline $03-03$ & $<0.20$ & $<0.2$ & $<0.2$ & $<0.2$ & $<0.2$ & $<0.2$ & $<0.20$ & $<5.0$ & $<0.2$ & $<0.2$ & $<0.2$ & $<0.2$ \\
\hline
\end{tabular}

REC REC

(UG/L)

\begin{tabular}{ccc}
\multicolumn{3}{c}{ METHYL- } \\
ENE \\
METHYL - & CHLO- & NAPHTH - \\
BROMIDE & RIDE & ALENE \\
TOTAL & TOTAL & TOTAL \\
(UG/L) & (UG/L) & (UG/L)
\end{tabular}

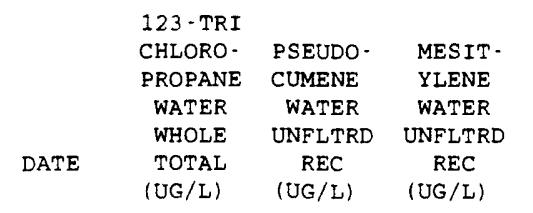

VINYL XYLENE ACE - ACE-

CHLO- WATER NAPHTH - NAPHTH -

RIDE UNFLTRD ENE YLENE

TOTAL REC TOTAL TOTAL $\begin{array}{llll}(U G / L) & (U G / L) & (U G / L) & \text { (UG/L) }\end{array}$
ANTHRA - BENZI -

CENE DINE

TOTAL TOTAL

(UG/L)
(UG/L)
BENZO A

ANTHRAC

ENE1, 2 -

BENZANT

HRACENE

TOTAL

(UG/L)
BENZO B

- FLUOR -

\begin{tabular}{|c|c|c|c|c|c|c|c|c|c|c|c|c|}
\hline $\begin{array}{c}\text { AUG } 1992 \\
24 \cdot 24\end{array}$ & $<0.2$ & $<0.20$ & $<0,20$ & $<0,2$ & $<0,20$ & $<5,0$ & $<5,0$ & $<5,0$ & $<400$ & $<10$ & $<100$ & $<100$ \\
\hline EP & & & & & & & & & & & & \\
\hline 03.03 & $<0.2$ & $<0.20$ & $<0.20$ & $<0.2$ & $<0.20$ & $<5.0$ & $<5.0$ & $<5.0$ & $<40.0$ & $<10.0$ & $<10,0$ & $<10.0$ \\
\hline
\end{tabular}

A. $A N$ -

PYRENE THENE

TOTAL TOTAL (UG/L) (UG/L) 
Table 6. Water-quality data for storm-sewer outfall stations, Irving, Texas, 1992-93-Continued

08055570 - HEREFORD ROAD OUTFALL AT WALNUT HILL ROAD, IRVING, TX (WY 1992)-Continued

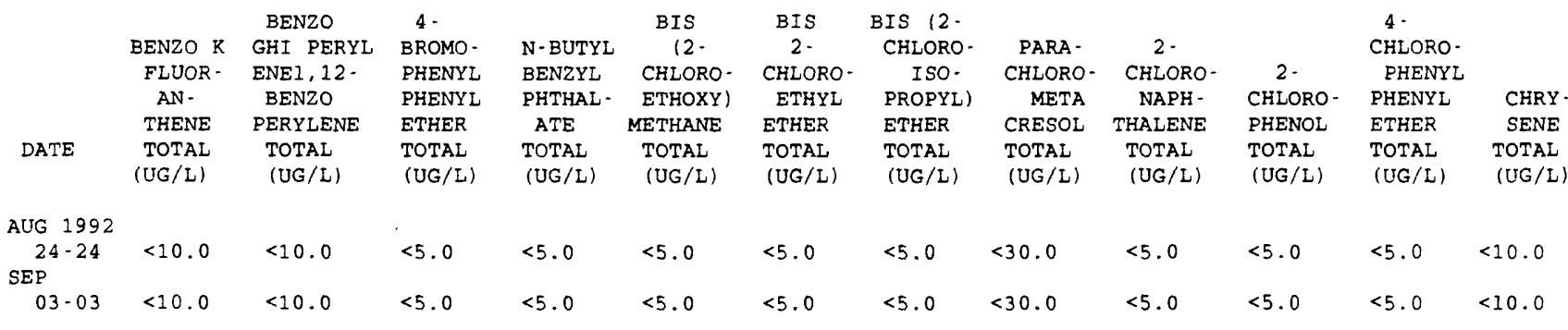

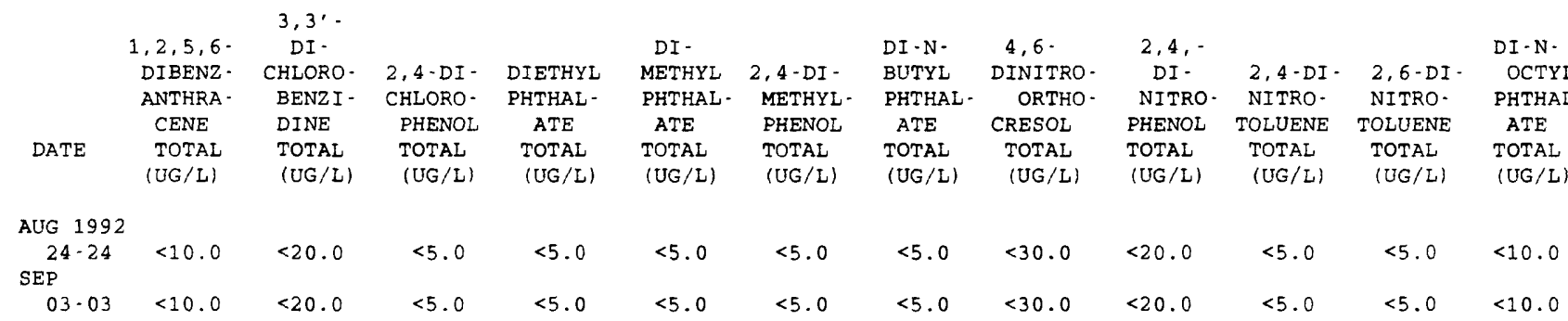

\begin{tabular}{|c|c|c|c|c|c|c|c|c|c|c|c|c|}
\hline & $\begin{array}{l}\text { BIS (2* } \\
\text { ETHYL }\end{array}$ & & & & $\begin{array}{l}\text { HEXA- } \\
\text { CHLORO- }\end{array}$ & & INDENO & & & N-NITRO- & & \\
\hline & HEXYL) & & & HEXA. & CYCLO- & HEXA - & $(1,2,3$. & & & SODI - & $2 \cdot$ & $4-$ \\
\hline & $\begin{array}{c}\text { PHTHAL- } \\
\text { ATE }\end{array}$ & $\begin{array}{l}\text { FLUOR - } \\
\text { ANTHENE }\end{array}$ & $\begin{array}{l}\text { FLUOR - } \\
\text { ENE }\end{array}$ & $\begin{array}{l}\text { CHLORO- } \\
\text { BENZENE }\end{array}$ & $\begin{array}{l}\text { PENT - } \\
\text { ADIENE }\end{array}$ & $\begin{array}{l}\text { CHLORO- } \\
\text { ETHANE }\end{array}$ & $\begin{array}{r}\text { CD) } \\
\text { PYRENE }\end{array}$ & $\begin{array}{l}\text { ISO- } \\
\text { PHORONE }\end{array}$ & $\begin{array}{l}\text { NITRO- } \\
\text { BENZENE }\end{array}$ & $\begin{array}{l}\text { METHY - } \\
\text { LAMINE }\end{array}$ & $\begin{array}{l}\text { NITRO- } \\
\text { PHENOL }\end{array}$ & $\begin{array}{l}\text { NITRO- } \\
\text { PHENOL }\end{array}$ \\
\hline AT & $\begin{array}{l}\text { TOTAL } \\
(U G / L)\end{array}$ & $\begin{array}{l}\text { TOTAL } \\
\text { (UG/L) }\end{array}$ & $\begin{array}{l}\text { TOTAL } \\
\text { (UG/L) }\end{array}$ & $\begin{array}{l}\text { TOTAL } \\
\text { (UG/L) }\end{array}$ & $\begin{array}{l}\text { TOTAL } \\
\text { (UG/L) }\end{array}$ & $\begin{array}{l}\text { TOTAL } \\
\text { (UG/L) }\end{array}$ & $\begin{array}{l}\text { TOTAL } \\
\text { (UG/L) }\end{array}$ & $\begin{array}{l}\text { TOTAL } \\
\text { (UG/L) }\end{array}$ & $\begin{array}{l}\text { TOTAL } \\
\text { (UG/L) }\end{array}$ & $\begin{array}{l}\text { TOTAL } \\
\text { (UG/L) }\end{array}$ & $\begin{array}{l}\text { TOTAL } \\
\text { (UG/L) }\end{array}$ & $\begin{array}{c}\text { TOTAL } \\
\text { (UG/L) }\end{array}$ \\
\hline
\end{tabular}

\begin{tabular}{|c|c|c|c|c|c|c|c|c|c|c|c|c|}
\hline \multicolumn{13}{|l|}{ AUG 1992} \\
\hline $24-24$ & $<5.0$ & $<5.0$ & $<5.0$ & $<5.0$ & $<5.0$ & $<5.0$ & $<10.0$ & $<5.0$ & $<5.0$ & $<5.0$ & $<5.0$ & $<30.0$ \\
\hline \multicolumn{13}{|l|}{ SEP } \\
\hline $03-03$ & $<5.0$ & 17.0 & $<5.0$ & $<5.0$ & $<5.0$ & $<5.0$ & $<10.0$ & $<5.0$ & $<5.0$ & $<5.0$ & $<5.0$ & $<30.0$ \\
\hline
\end{tabular}

\begin{tabular}{|c|c|c|c|c|c|c|c|c|c|c|c|c|}
\hline & $\mathrm{N}-$ & & & & & & & & & & BETA & DELTA \\
\hline & NITRO - & N-NITRO & & & & & $2,4,6=$ & & & & BENZENE & BENZENE \\
\hline & SODI - N- & SODI - & PENTA - & & PHENOL & & TRI - & & & & HEXA - & HEXA- \\
\hline & $\begin{array}{c}\text { PROPYL- } \\
\text { AMINE }\end{array}$ & $\begin{array}{l}\text { PHENY - } \\
\text { LAMINE }\end{array}$ & $\begin{array}{l}\text { CHLORO- } \\
\text { PHENOL }\end{array}$ & $\begin{array}{l}\text { PHENAN - } \\
\text { THRENE }\end{array}$ & $\begin{array}{r}(\mathrm{C} 6 \mathrm{H}- \\
5 \mathrm{OH})\end{array}$ & PYRENE & $\begin{array}{l}\text { CHLORO- } \\
\text { PHENOL }\end{array}$ & ALDRIN, & $\begin{array}{l}\text { P, } P^{\prime} \\
\text { DDT, }\end{array}$ & $\begin{array}{l}\text { ALPHA } \\
\text { BHC }\end{array}$ & $\begin{array}{l}\text { CHLOR - } \\
\text { IDE }\end{array}$ & $\begin{array}{l}\text { CHLOR - } \\
\text { IDE }\end{array}$ \\
\hline DATE & $\begin{array}{l}\text { TOTAL } \\
(U G / L)\end{array}$ & $\begin{array}{l}\text { TOTAL } \\
(U G / L)\end{array}$ & $\begin{array}{l}\text { TOTAL } \\
(U G / L)\end{array}$ & $\begin{array}{l}\text { TOTAL } \\
(U G / L)\end{array}$ & $\begin{array}{l}\text { TOTAL } \\
(U G / L)\end{array}$ & $\begin{array}{r}\text { TOTAL } \\
(U G / L)\end{array}$ & $\begin{array}{l}\text { TOTAL } \\
(U G / L)\end{array}$ & $\begin{array}{l}\text { TOTAL } \\
\text { (UG/L) }\end{array}$ & $\begin{array}{l}\text { TOTAL } \\
\text { (UG/L) }\end{array}$ & $\begin{array}{l}\text { TOTAL } \\
\text { (UG/L) }\end{array}$ & $\begin{array}{l}\text { TOTAL } \\
\text { (UG/L) }\end{array}$ & $\begin{array}{l}\text { TOTAL } \\
(U G / L)\end{array}$ \\
\hline AUG 1992 & & & & & & & & & & & & \\
\hline $24-24$ & $<5.0$ & $<5.0$ & $<30.0$ & $<5.0$ & $<5.0$ & $<5.0$ & $<20.0$ & $<0.040$ & $<0.10$ & $<0.03$ & $<0.03$ & $<0.09$ \\
\hline SEP & & & & & & & & & & & & \\
\hline $03-03$ & $<5.0$ & $<5.0$ & $<30.0$ & 8.0 & $<5.0$ & 15.0 & $<20.0$ & $<0.040$ & $<0.10$ & $<0.03$ & $<0.03$ & $<0.09$ \\
\hline
\end{tabular}

\begin{tabular}{|c|c|c|c|c|c|c|c|c|c|c|c|c|}
\hline & & $\begin{array}{l}\text { CHLOR - } \\
\text { DANE }\end{array}$ & $\begin{array}{l}\text { CHLOR - } \\
\text { DANE }\end{array}$ & & & & & $\begin{array}{l}\text { ENDO- } \\
\text { SULFAN- }\end{array}$ & & & & \\
\hline & & CIS & TRANS & & & & & I & ENDO- & ENDO - & ENDRIN & ENDRIN \\
\hline & & WATER & WATER & CHLOR - & $P, P^{\prime}$ & $P, P^{\prime}$ & DI - & WATER & SULFAN & SULFAN & WATER & ALDE - \\
\hline & LINDANE & WHOLE & WHOLE & DANE, & $\mathrm{DDD}$ & $\mathrm{DDE}$ & ELDRIN & WHOLE & BETA & SULFATE & UNFLTRD & HYDE \\
\hline ATE & $\begin{array}{l}\text { TOTAL } \\
\text { (UG/I) }\end{array}$ & $\begin{array}{l}\text { TOTAL } \\
\text { (UG/L) }\end{array}$ & $\begin{array}{l}\text { TOTAL } \\
\text { (UG/L) }\end{array}$ & $\begin{array}{l}\text { TOTAL } \\
\text { (UG/L) }\end{array}$ & $\begin{array}{l}\text { TOTAL } \\
\text { (UG/L) }\end{array}$ & $\begin{array}{l}\text { TOTAL } \\
\text { (UG/L) }\end{array}$ & $\begin{array}{l}\text { TOTAL } \\
\text { (UG/L) }\end{array}$ & $\begin{array}{c}\text { REC } \\
(U G / L)\end{array}$ & $\begin{array}{l}\text { TOTAL } \\
\text { (UG/L) }\end{array}$ & $\begin{array}{l}\text { TOTAL } \\
\text { (UG/L) }\end{array}$ & $\begin{array}{c}\text { REC } \\
(U G / L)\end{array}$ & $\begin{array}{l}\text { TOTAL } \\
\text { (UG/L) }\end{array}$ \\
\hline
\end{tabular}

\begin{tabular}{|c|c|c|c|c|c|c|c|c|c|c|c|c|}
\hline $24-24$ & $<0.030$ & $<0.10$ & $<0.10$ & $<0.1$ & $<0.10$ & $<0.04$ & $<0.020$ & $<0.10$ & $<0.04$ & $<0.60$ & $<0.060$ & $<0.20$ \\
\hline \multicolumn{13}{|l|}{ SEP } \\
\hline 03.03 & $<0.030$ & $<0.10$ & $<0.10$ & $<0.1$ & $<0.10$ & $<0.04$ & $<0.020$ & $<0.10$ & $<0.04$ & $<0.60$ & $<0.060$ & $<0.20$ \\
\hline
\end{tabular}


Table 6. Water-quality data for storm-sewer outfall stations, Irving, Texas, 1992-93-Continued

08055570 - HEREFORD ROAD OUTFALL AT WALNUT HILL ROAD, IRVING, TX (WY 1992)-Continued

\begin{tabular}{|c|c|c|c|c|c|c|c|c|c|c|c|}
\hline & & HEPTA- & & AROCLOR & AROCLOR & AROCLOR & AROCLOR & AROCLOR & AROCLOR & AROCLOR & \\
\hline & HEPTA - & CHLOR & TOX - & 1016 & 1221 & 1232 & 1242 & 1248 & 1254 & 1260 & DI - \\
\hline & CHLOR, & EPOXIDE & APHENE, & PCB & PCB & $\mathrm{PCB}$ & $\mathrm{PCB}$ & PCB & PCB & PCB & AZINON, \\
\hline DATE & $\begin{array}{l}\text { TOTAL } \\
\text { (UG/L) }\end{array}$ & $\begin{array}{l}\text { TOTAL } \\
\text { (UG/L) }\end{array}$ & $\begin{array}{l}\text { TOTAL } \\
\text { (UG/L) }\end{array}$ & $\begin{array}{l}\text { TOTAL } \\
\text { (UG/L) }\end{array}$ & $\begin{array}{l}\text { TOTAL } \\
\text { (UG/L) }\end{array}$ & $\begin{array}{c}\text { TOTAL } \\
\text { (UG/L) }\end{array}$ & $\begin{array}{l}\text { TOTAL } \\
\text { (UG/L) }\end{array}$ & $\begin{array}{l}\text { TOTAL } \\
\text { (UG/L) }\end{array}$ & $\begin{array}{l}\text { TOTAL } \\
\text { (UG/L) }\end{array}$ & $\begin{array}{l}\text { TOTAL } \\
\text { (UG/L) }\end{array}$ & $\begin{array}{l}\text { TOTAL } \\
\text { (UG/L) }\end{array}$ \\
\hline
\end{tabular}

\begin{tabular}{|c|c|c|c|c|c|c|c|c|c|c|c|}
\hline & & & & & & & & & & & \\
\hline $24-24$ & $<0.030$ & $<0.80$ & $<2$ & $<0.1$ & $<1.0$ & $<0.1$ & $<0.1$ & $<0.1$ & $<0.1$ & $<0.1$ & $<0.10$ \\
\hline \multicolumn{12}{|l|}{ SEP } \\
\hline 03.03 & $<0.030$ & $<0.80$ & $<2$ & $<0.1$ & $<1.0$ & $<0.1$ & $<0.1$ & $<0.1$ & $<0.1$ & $<0.1$ & $<0.10$ \\
\hline
\end{tabular}


Table 6. Water-quality data for storm-sewer outfall stations, Irving, Texas, 1992-93-Continued 08055570 - HEREFORD ROAD OUTFALI AT WALNUT HILL ROAD, IRVING, TX (WY 1993)

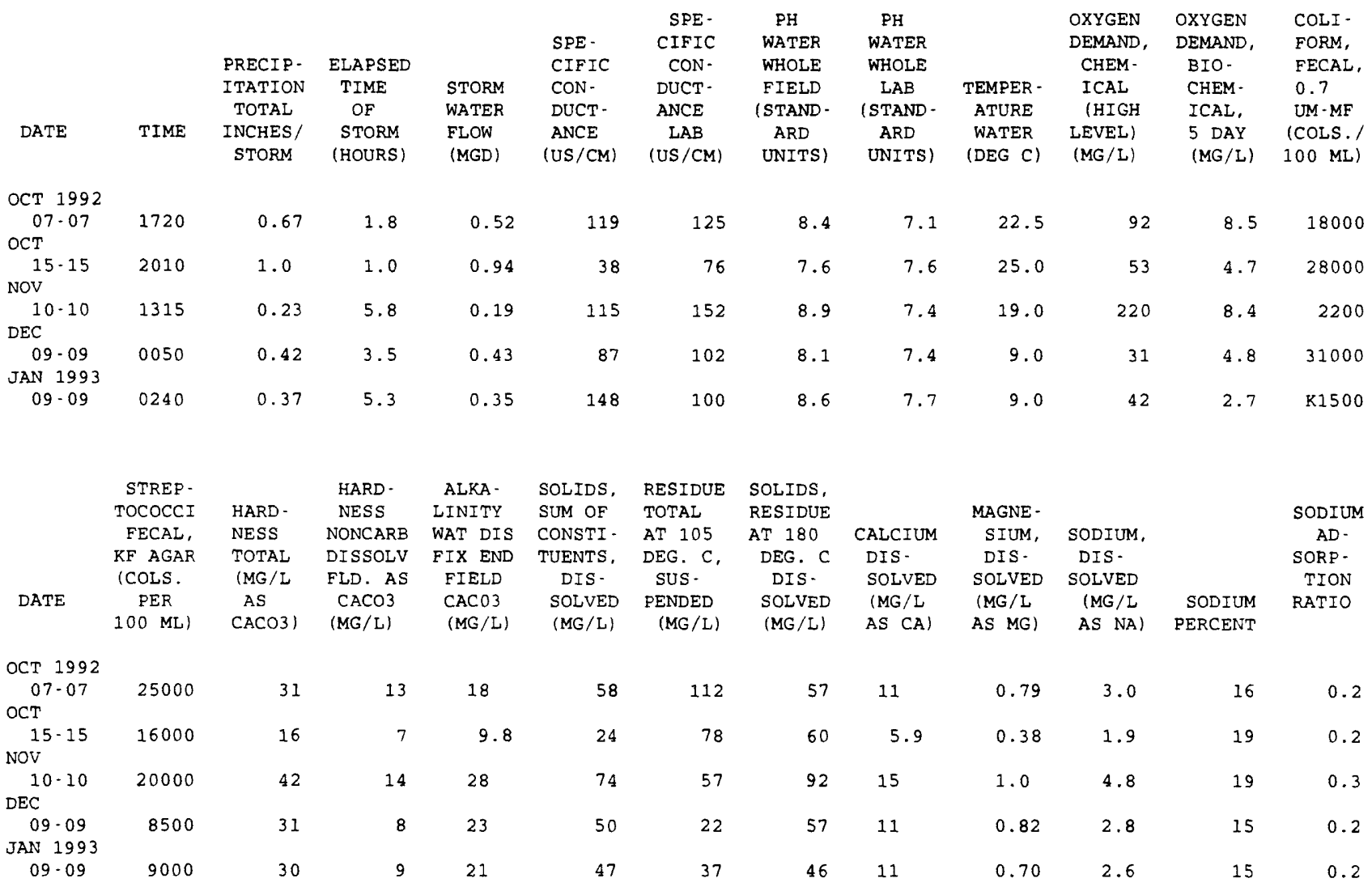

\begin{tabular}{|c|c|c|c|c|c|c|c|c|c|c|c|c|}
\hline DATE & $\begin{array}{l}\text { POTAS - } \\
\text { SIUM, } \\
\text { DIS - } \\
\text { SOLVED } \\
\text { (MG/L }\end{array}$ & $\begin{array}{l}\text { SULFATE } \\
\text { DIS - } \\
\text { SOLVED } \\
\text { (MG / L }\end{array}$ & $\begin{array}{l}\text { CHLO - } \\
\text { RIDE. } \\
\text { DIS - } \\
\text { SOLVED } \\
\text { (MG / L }\end{array}$ & $\begin{array}{l}\text { NITRO- } \\
\text { GEN, } \\
\text { NITRITE } \\
\text { TOTAL } \\
\text { (MG /L }\end{array}$ & $\begin{array}{l}\text { NITRO- } \\
\text { GEN, } \\
\text { NO2+NO3 } \\
\text { TOTAL } \\
\text { (MG/L }\end{array}$ & $\begin{array}{l}\text { NITRO- } \\
\text { GEN, } \\
\text { AMMONIA } \\
\text { TOTAL } \\
\text { (MG /L }\end{array}$ & $\begin{array}{l}\text { NITRO- } \\
\text { GEN, AM- } \\
\text { MONIA + } \\
\text { ORGANIC } \\
\text { TOTAL } \\
\text { (MG/L }\end{array}$ & $\begin{array}{l}\text { PHOS - } \\
\text { PHORUS } \\
\text { TOTAL } \\
\text { (MG/L }\end{array}$ & $\begin{array}{c}\text { PHOS - } \\
\text { PHORUS } \\
\text { DIS - } \\
\text { SOLVED } \\
\text { (MG/L }\end{array}$ & $\begin{array}{l}\text { ANTIMONY } \\
\text { TOTAL } \\
\text { EPA } \\
\text { (UG/L }\end{array}$ & $\begin{array}{c}\text { ARSENIC } \\
\text { TOTAL } \\
\text { (UG/L }\end{array}$ & $\begin{array}{l}\text { BERYL- } \\
\text { LIUM, } \\
\text { TOTAL } \\
\text { RECOV- } \\
\text { ERABLE } \\
\text { (UG /L }\end{array}$ \\
\hline & AS K) & AS SO4) & AS CL) & AS N) & AS N) & AS N) & AS N) & AS P) & AS P) & AS SB) & AS AS) & $A S \quad B E)$ \\
\hline
\end{tabular}

\begin{tabular}{|c|c|c|c|c|c|c|c|c|c|c|c|c|}
\hline \multicolumn{13}{|l|}{ OCT 1992} \\
\hline 07.07 & 2.3 & 12 & 3.0 & 0.040 & 0.650 & 0.280 & 1.9 & 0.360 & 0.120 & $<10.0$ & 3 & $<10$ \\
\hline \multicolumn{13}{|l|}{ OCT } \\
\hline $15 \cdot 15$ & 1.4 & 5.7 & 1.7 & 0.020 & 0.260 & 0.250 & 1.0 & 0.190 & 0.090 & $<10.0$ & 2 & $<10$ \\
\hline \multicolumn{13}{|l|}{ NOV } \\
\hline $10-10$ & 2.6 & 15 & 4.3 & 0.070 & 0.770 & 0.190 & 1.0 & 0.180 & 0.090 & $<10.0$ & 2 & $<10$ \\
\hline \multicolumn{13}{|l|}{$\mathrm{DEC}$} \\
\hline $09-09$ & 1.9 & 11 & 2.4 & 0.050 & 2.70 & 0.180 & 1.1 & 0.160 & 0.080 & $<10.0$ & 2 & $<10$ \\
\hline \multicolumn{13}{|l|}{ JAN 1993} \\
\hline $09-09$ & 2.1 & 7.5 & 1.5 & $\cdots$ & 0.830 & $\cdots$ & 0.60 & 0.070 & 0.030 & $<10.0$ & 1 & $<10$ \\
\hline
\end{tabular}


Table 6. Water-quality data for storm-sewer outfall stations, Irving, Texas, 1992-93-Continued

08055570 - HEREFORD ROAD OUTFALL AT WALNUT HILL ROAD, IRVING, TX (WY 1993)-Continued

\begin{tabular}{|c|c|c|c|c|c|c|c|c|c|c|c|c|}
\hline DATE & $\begin{array}{l}\text { CADMIUM } \\
\text { TOTAL } \\
\text { RECOV - } \\
\text { ERABLE } \\
\text { (UG /L }\end{array}$ & $\begin{array}{l}\text { CHRO- } \\
\text { MIUM, } \\
\text { TOTAL } \\
\text { RECOV- } \\
\text { ERABLE } \\
\text { (UG / L }\end{array}$ & $\begin{array}{c}\text { COPPER, } \\
\text { TOTAL } \\
\text { RECOV- } \\
\text { ERABLE } \\
\text { (UG/L }\end{array}$ & $\begin{array}{c}\text { CYANIDE } \\
\text { TOTAL } \\
\text { EPA } \\
\text { (MG/L }\end{array}$ & $\begin{array}{c}\text { CYANIDE } \\
\text { TOTAL } \\
\text { (MG/L }\end{array}$ & $\begin{array}{l}\text { LEAD, } \\
\text { TOTAL } \\
\text { RECOV- } \\
\text { ERABLE } \\
\text { (UG/L }\end{array}$ & $\begin{array}{c}\text { MERCURY } \\
\text { TOTAL } \\
\text { RECOV- } \\
\text { ERABLE } \\
\text { (UG/L }\end{array}$ & $\begin{array}{c}\text { NICKEL, } \\
\text { TOTAL } \\
\text { RECOV- } \\
\text { ERABLE } \\
\text { (UG/L }\end{array}$ & $\begin{array}{l}\text { SELE- } \\
\text { NIUM, } \\
\text { TOTAL } \\
\text { (UG/L }\end{array}$ & $\begin{array}{c}\text { SILVER, } \\
\text { TOTAL } \\
\text { RECOV - } \\
\text { ERABLE } \\
\text { (UG/L }\end{array}$ & $\begin{array}{l}\text { SILVER, } \\
\text { TOTAL } \\
\text { RECOV- } \\
\text { ERABLE } \\
\text { EPA } \\
\text { IUG/L }\end{array}$ & $\begin{array}{l}\text { THAL - } \\
\text { LIUM, } \\
\text { TOTAL } \\
\text { (UG /L }\end{array}$ \\
\hline & AS CD) & AS CR) & AS CU) & AS CN) & AS CN) & AS PB) & AS HG) & AS NI) & AS SE) & AS AG) & $A S \quad A G)$ & AS TL) \\
\hline \multicolumn{13}{|l|}{ OCT 1992} \\
\hline 07.07 & $<1$ & 66 & 20 & $<0.010$ & $<0.010$ & 320 & $<0.10$ & 12 & $<2$ & $<1$ & $<0.500$ & $<5$ \\
\hline \multicolumn{13}{|l|}{ OCT } \\
\hline $15-15$ & $<1$ & 16 & 10 & $<0.010$ & $<0.010$ & 60 & $<0.10$ & 7 & $<2$ & $<1$ & $<0.500$ & $<5$ \\
\hline \multicolumn{13}{|l|}{ NOV } \\
\hline $10-10$ & 2 & 4 & 16 & $<0.010$ & $<0.010$ & 35 & $<0.10$ & 6 & $<2$ & $<1$ & $<0.500$ & $<5$ \\
\hline \multicolumn{13}{|l|}{$D E C$} \\
\hline 09.09 & $<1$ & 4 & 7 & $<0.010$ & $<0.010$ & 17 & $<0.10$ & 4 & $<2$ & $<1$ & $<0.500$ & $<25$ \\
\hline \multicolumn{13}{|l|}{ JAN 1993} \\
\hline $09-09$ & $<1$ & 11 & 4 & $<0.010$ & $<0.010$ & 9 & $<0.10$ & 2 & $<2$ & $<1$ & $<1.00$ & $<5$ \\
\hline
\end{tabular}

\begin{tabular}{|c|c|c|c|c|c|c|c|c|c|c|c|c|}
\hline & & & OIL AND & & & & & & METHANE & & & BENZENE \\
\hline & ZINC, & & GREASE, & & & & & BROMO - & BROMO & & BENZENE & SEC \\
\hline & TOTAL & CARBON, & TOTAL & & & & & BENZENE & CHLORO- & & N-BUTYL & BUTYL - \\
\hline & RECOV - & ORGANIC & RECOV. & & ACRO- & ACRYLO- & & WATER, & WAT & BROMO - & WATER & WATER \\
\hline & ERABLE & TOTAL & GRAVI - & PHENOLS & LEIN & NITRILE & BENZENE & WHOLE, & UNFLTRD & FORM & UNFLTRD & UNFLTRD \\
\hline DATE & $\begin{array}{l}\text { (UG/L } \\
\text { AS } \quad \text { ZN) }\end{array}$ & $\begin{array}{l}\text { (MG / L } \\
\text { AS C) }\end{array}$ & $\begin{array}{c}\text { METRIC } \\
(\mathrm{MG} / \mathrm{L})\end{array}$ & $\begin{array}{l}\text { TOTAL } \\
(U G / L)\end{array}$ & $\begin{array}{l}\text { TOTAL } \\
(U G / L)\end{array}$ & $\begin{array}{l}\text { TOTAL } \\
\text { (UG/L) }\end{array}$ & $\begin{array}{l}\text { TOTAL } \\
(U G / L)\end{array}$ & $\begin{array}{l}\text { TOTAL } \\
\text { (UG/L) }\end{array}$ & $\begin{array}{c}\text { REC } \\
(U G / L)\end{array}$ & $\begin{array}{l}\text { TOTAL } \\
(\mathrm{UG} / \mathrm{L})\end{array}$ & $\begin{array}{c}\text { REC } \\
\text { (UG/L) }\end{array}$ & $\begin{array}{c}\text { REC } \\
(U G / L)\end{array}$ \\
\hline
\end{tabular}

\begin{tabular}{|c|c|c|c|c|c|c|c|c|c|c|c|c|}
\hline \multicolumn{13}{|l|}{ OCT 1992} \\
\hline $07-07$ & 270 & 25 & 6 & 4 & $<20$ & $<20$ & $<0.2$ & $<0.2$ & $<0.20$ & $<0.2$ & $<0.20$ & $<0.20$ \\
\hline \multicolumn{13}{|l|}{ OCT } \\
\hline $15 \cdot 15$ & 110 & 11 & $<1$ & 7 & $<20$ & $<20$ & $<0.2$ & $<0.2$ & $<0.20$ & $<0.2$ & $<0.20$ & $<0.20$ \\
\hline \multicolumn{13}{|l|}{ Nov } \\
\hline $10-10$ & 160 & 19 & $<1$ & 5 & $<20$ & $<20$ & $<0.2$ & $<0.2$ & $<0.20$ & $<0.2$ & $<0.20$ & $<0.20$ \\
\hline \multicolumn{13}{|l|}{ DEC } \\
\hline $09-09$ & 80 & 8.1 & $<1$ & 2 & $<20$ & $<20$ & $<0.2$ & $<0.2$ & $<0.20$ & $<0.2$ & $<0.20$ & $<0.20$ \\
\hline \multicolumn{13}{|l|}{ JAN 1993} \\
\hline $09-09$ & 70 & 11 & 1 & 2 & $<20$ & $<20$ & $<0.2$ & $<0.2$ & $<0.20$ & $<0.2$ & $<0.20$ & $<0.20$ \\
\hline
\end{tabular}

\begin{tabular}{|c|c|c|c|c|c|c|c|c|c|c|c|c|}
\hline & BENZENE & & & & & $2-$ & & & 0 & & DIBROMO & DI - \\
\hline & TERT - & CARBON - & & CHLORO - & & CHLORO - & & & CHLORO- & TOLUENE & CHLORO - & BROMO - \\
\hline & BUTYL - & TETRA - & & DI - & & ETHYL - & & METHYL - & TOLUENE & P.CHLOR & PROPANE & METHANE \\
\hline & WATER & CHLO- & CHLORO - & BROMO - & CHLORO- & VINYL - & CHLORO - & CHLO - & WATER & WATER & WATER & WATER \\
\hline & UNFLTRD & RIDE & BENZENE & METHANE & ETHANE & ETHER & FORM & RIDE & WHOLE & UNFLTRD & WHOLE & WHOLE \\
\hline ATE & $\begin{array}{c}\text { REC } \\
\langle U G / L\rangle\end{array}$ & $\begin{array}{l}\text { TOTAL } \\
\text { (UG/L) }\end{array}$ & $\begin{array}{l}\text { TOTAL } \\
\text { (UG/L) }\end{array}$ & $\begin{array}{l}\text { TOTAL } \\
\text { (UG/L) }\end{array}$ & $\begin{array}{l}\text { TOTAL } \\
\text { (UG/L) }\end{array}$ & $\begin{array}{l}\text { TOTAL } \\
\text { (UG/L) }\end{array}$ & $\begin{array}{l}\text { TOTAL } \\
\text { (UG } / L)\end{array}$ & $\begin{array}{l}\text { TOTAL } \\
\text { (UG/L) }\end{array}$ & $\begin{array}{l}\text { TOTAL } \\
\text { (UG/L) }\end{array}$ & $\begin{array}{c}\text { REC } \\
(U G / L)\end{array}$ & $\begin{array}{l}\text { TOT . REC } \\
\text { (UG/L) }\end{array}$ & $\begin{array}{l}\text { RECOVER } \\
\text { (UG/L) }\end{array}$ \\
\hline
\end{tabular}

\begin{tabular}{|c|c|c|c|c|c|c|c|c|c|c|c|c|}
\hline 07.07 & $<0.20$ & $<0.2$ & $<0.20$ & $<0.2$ & $<0.2$ & $<1.0$ & $<0.2$ & $<0.2$ & $<0.2$ & $<0.20$ & $<1.0$ & $<0.2$ \\
\hline OCT & & & & & & & & & & & & \\
\hline $15 \cdot 15$ & $<0.20$ & $<0.2$ & $<0.20$ & $<0.2$ & $<0.2$ & $<1.0$ & $<0.2$ & $<0.2$ & $<0.2$ & $<0.20$ & $<1.0$ & $<0.2$ \\
\hline NOV & & & & & & & & & & & & \\
\hline $10-10$ & $<0.20$ & $<0.2$ & $<0.20$ & $<0.2$ & $<0.2$ & $<1.0$ & $<0.2$ & $<0.2$ & $<0.2$ & $<0.20$ & $<1.0$ & $<0.2$ \\
\hline DEC & & & & & & & & & & & & \\
\hline $\begin{array}{c}09 \cdot 09 \\
\text { JAN } 1993\end{array}$ & $<0.20$ & $<0.2$ & $<0.20$ & $<0.2$ & $<0.2$ & $<1.0$ & $<0.2$ & $<0.2$ & $<0.2$ & $<0.20$ & $<1.0$ & $<0.2$ \\
\hline 09.09 & $<0.20$ & $<0.2$ & $<0.20$ & $<0.2$ & $<0.2$ & $<1.0$ & $<0.2$ & $<0.2$ & $<0.2$ & $<0.20$ & $<1.0$ & $<0.2$ \\
\hline
\end{tabular}


Table 6. Water-quality data for storm-sewer outfall stations, Irving, Texas, 1992-93-Continued

08055570 - HEREFORD ROAD OUTFALL AT WALNUT HILL ROAD, IRVING, TX (WY 1993)-Continued

\begin{tabular}{|c|c|c|c|c|c|c|c|c|c|c|c|c|}
\hline & $\begin{array}{c}1,2- \\
\text { DIBROMO }\end{array}$ & DI - & $1,1 \cdot \mathrm{DI}$ & $\begin{array}{l}1,1-D I \\
\text { CHLORO. }\end{array}$ & $\begin{array}{c}\text { BENZENE } \\
0-\end{array}$ & $\begin{array}{l}\text { BENZENE } \\
1,3-\mathrm{DI} \text { - }\end{array}$ & $\begin{array}{l}\text { BENZENE } \\
I, 4 \text {-DI - }\end{array}$ & $\begin{array}{l}\text { DI - } \\
\text { CHLORO- }\end{array}$ & & & $\begin{array}{l}1,2-\mathrm{DI}- \\
\text { PHENYL }\end{array}$ & $\begin{array}{c}\text { CIS - } 1,2- \\
\text { DI - }\end{array}$ \\
\hline & $\begin{array}{c}\text { ETHANE } \\
\text { WATER } \\
\text { WHOLE }\end{array}$ & $\begin{array}{l}\text { CHLORO - } \\
\text { BROMO - } \\
\text { METHANE }\end{array}$ & $\begin{array}{c}\text { CHLORO- } \\
\text { ETHYL - } \\
\text { ENE }\end{array}$ & $\begin{array}{l}\text { PRO- } \\
\text { PENE, } \\
\text { WAT, WH }\end{array}$ & $\begin{array}{l}\text { CHLORO - } \\
\text { WATER } \\
\text { UNFITRD }\end{array}$ & $\begin{array}{l}\text { CHLORO- } \\
\text { WATER } \\
\text { UNFLTRD }\end{array}$ & $\begin{array}{l}\text { CHLORO- } \\
\text { WATER } \\
\text { UNFLTRD }\end{array}$ & $\begin{array}{l}\text { DI - } \\
\text { FLUORO- } \\
\text { METHANE }\end{array}$ & $\begin{array}{l}1,1-D I- \\
\text { CHLORO- } \\
\text { ETHANE }\end{array}$ & $\begin{array}{l}1,2 \text {-DI - } \\
\text { CHLORO - }\end{array}$ & $\begin{array}{l}\text { HYDRA - } \\
\text { ZINE }\end{array}$ & CHLORO- \\
\hline 210 & $\begin{array}{l}\text { TOTAL } \\
\text { (UG/L) }\end{array}$ & $\begin{array}{l}\text { TOTAL } \\
\text { (UG/L) }\end{array}$ & $\begin{array}{l}\text { TOTAL } \\
\text { (UG/L) }\end{array}$ & $\begin{array}{l}\text { TOTAL } \\
\text { (UG/L) }\end{array}$ & $\begin{array}{c}\text { REC } \\
(U G / L)\end{array}$ & $\begin{array}{c}\text { REC } \\
\text { (UG/L) }\end{array}$ & $\begin{array}{c}\text { REC } \\
\text { (UG/L) }\end{array}$ & $\begin{array}{l}\text { TOTAL } \\
\text { (UG/L) }\end{array}$ & $\begin{array}{l}\text { TOTAL } \\
\text { (UG/L) }\end{array}$ & $\begin{array}{l}\text { TOTAL } \\
\text { (UG/L) }\end{array}$ & $\begin{array}{l}\text { TOT.REC } \\
\text { (UG/L) }\end{array}$ & $\begin{array}{l}\text { TOTAL } \\
\text { (UG/L) }\end{array}$ \\
\hline
\end{tabular}

\begin{tabular}{|c|c|c|c|c|c|c|c|c|c|c|c|c|}
\hline OCT 1992 & & & & & & & & & & & & \\
\hline 07.07 & $<0.2$ & $<0.2$ & $<0.2$ & $<0.2$ & $<5.0$ & $<5.0$ & $<5.0$ & $<0.2$ & $<0.2$ & $<0.2$ & $<5.0$ & $<0.2$ \\
\hline $\mathrm{OCT}$ & & & & & & & & & & & & \\
\hline $15 \cdot 15$ & $<0.2$ & $<0.2$ & $<0.2$ & $<0.2$ & $<5.0$ & $<5.0$ & $<5.0$ & $<0.2$ & $<0.2$ & $<0.2$ & $<5.0$ & $<0.2$ \\
\hline NOV & & & & & & & & & & & & \\
\hline $10 \cdot 10$ & $<0.2$ & $<0.2$ & $<0.2$ & $<0.2$ & $<5.0$ & $<5.0$ & $<5.0$ & $<0.2$ & $<0.2$ & $<0.2$ & $<5.0$ & $<0.2$ \\
\hline DEC & & & & & & & & & & & & \\
\hline 09.09 & $<0.2$ & $<0.2$ & $<0.2$ & $<0.2$ & $<5.0$ & $<5.0$ & $<5.0$ & $<0.2$ & $<0.2$ & $<0.2$ & $<5.0$ & $<0.2$ \\
\hline JAN 1993 & & & & & & & & & & & & \\
\hline $09-09$ & $<0.2$ & $<0.2$ & $<0.2$ & $<0.2$ & $<5.0$ & $<5.0$ & $<5.0$ & $<0.2$ & $<0.2$ & $<0,2$ & $<5,0$ & $<0.2$ \\
\hline
\end{tabular}

\begin{tabular}{|c|c|c|c|c|c|c|c|c|c|c|c|}
\hline & & & & $2,2 \cdot D I$ & & & & & ISO- & P-ISO- & \\
\hline & $1,2-$ & & $1,3-D I$. & CHLORO - & CIS & TRANS - & & HEXA - & PROPYL - & PROPYL - & \\
\hline & TRANSDI & $1,2 \cdot D I-$ & CHLORO- & PRO - & $1,3-\mathrm{DI}$ & $1,3-\mathrm{DI}$ - & & CHLORO- & BENZENE & TOLUENE & \\
\hline & CHLORO - & CHLORO- & PROPANE & PANE & CHLORO- & CHLORO- & ETHYL - & BUT - & WATER & WATER & METHYL - \\
\hline & ETHENE & PROPANE & WAT. WH & WAT, WH & PROPENE & PROPENE & BENZENE & ADIENE & WHOLE & WHOLE & BROMIDE \\
\hline & $\begin{array}{l}\text { TOTAL } \\
\text { (UG/L) }\end{array}$ & $\begin{array}{l}\text { TOTAL } \\
\text { (UG/L) }\end{array}$ & $\begin{array}{l}\text { TOTAL } \\
\text { (UG/L) }\end{array}$ & $\begin{array}{l}\text { TOTAL } \\
\text { (UG/L) }\end{array}$ & $\begin{array}{c}\text { TOTAL } \\
\text { (UG/L) }\end{array}$ & $\begin{array}{l}\text { TOTAL } \\
\text { (UG/L) }\end{array}$ & $\begin{array}{l}\text { TOTAL } \\
\text { (UG/L) }\end{array}$ & $\begin{array}{l}\text { TOTAL } \\
\text { (UG/L) }\end{array}$ & $\begin{array}{c}\text { REC } \\
(U G / L)\end{array}$ & $\begin{array}{c}\text { REC } \\
(\mathrm{UG} / \mathrm{L})\end{array}$ & $\begin{array}{l}\text { TOTAL } \\
\text { (UG/L) }\end{array}$ \\
\hline
\end{tabular}

OCT 1992

$\begin{aligned} & 0 \text { OCT } 1992 \\ & 07-07\end{aligned}$

OCT

$15-15$

Nov

$10-10$

DEC

09.09

JAN 1993

09.09

\begin{tabular}{|c|c|c|c|}
\hline$<0.2$ & $<0.2$ & $<0.2$ & $<0.2$ \\
\hline$<0.2$ & $<0.2$ & $<0.2$ & $<0.2$ \\
\hline$<0.2$ & $<0.2$ & $<0.2$ & $<0.2$ \\
\hline$<0.2$ & $<0.2$ & $<0.2$ & $<0.2$ \\
\hline$<0.2$ & $<0.2$ & $<0.2$ & $<0.2$ \\
\hline \multicolumn{4}{|c|}{ METHYL } \\
\hline THYL - & ETHER & & BENZENE \\
\hline ENE & TERT - & & N-PROPY \\
\hline HLO - & BUTYL & NAPHTH - & WATER \\
\hline IDE & WAT UNF & ALENE & UNFLTRD \\
\hline $\begin{array}{l}\text { TAL } \\
\text { G/L }\end{array}$ & $\begin{array}{c}\text { REC } \\
\text { (UG/L) }\end{array}$ & $\begin{array}{c}\text { TOTAL } \\
\text { (UG/L) }\end{array}$ & $\begin{array}{c}\text { REC } \\
(U G / L)\end{array}$ \\
\hline
\end{tabular}

$<0.2$
$<0.2$
$<0.2$
$<0.2$
$<0.2$
OTAL
PRENE

\begin{tabular}{|c|c|}
\hline$<0.2$ & $<0.2$ \\
\hline$<0.2$ & $<0.2$ \\
\hline$<0.2$ & $<0.2$ \\
\hline$<0.2$ & $<0.2$ \\
\hline$<0.2$ & $<0.2$ \\
\hline $\begin{array}{l}\text { AANE. } \\
12- \\
\text { TRA. }\end{array}$ & $\begin{array}{c}\text { ETHANE, } \\
1,1,2,2 \\
\text { TETRA. }\end{array}$ \\
\hline $\begin{array}{l}\text { LORO - } \\
T \text { UNF }\end{array}$ & $\begin{array}{l}\text { CHLORO- } \\
\text { WAT UNE }\end{array}$ \\
\hline $\begin{array}{l}\text { REC } \\
G / L)\end{array}$ & $\begin{array}{c}\text { REC } \\
(U G / L)\end{array}$ \\
\hline
\end{tabular}

$<5.0$
$<5.0$
$<5.0$
$<5.0$
$<5.0$

ETRA.
HLORO.
ENE
TOTAL
UG /

$<0.20$

$<0.20$

$<0.2$

$<0.20$

$<0.20$

$<0.2$

$<0.20$

$<0.20$

$<0.2$

$<0.20$

$<0.20$

$<0.2$

$<0.20$

$<0.20$

$<0.2$

OCT 1992

$07-07$
OCT
$15-15$
NOV
$10-10$
DEC
09-09
JAN 1993

$\begin{array}{lllllllllll}<0.2 & <1.0 & <5.0 & <0.20 & <0.2 & <0.2 & <0.2 & <0.2 & <0.2 & <0.20 & <5.0 \\ <0.2 & <1.0 & <5.0 & <0.20 & <0.2 & <0.2 & <0.2 & <0.2 & <0.2 & <0.20 & <5.0 \\ <0.2 & <1.0 & <5.0 & <0.20 & <0.2 & <0.2 & <0.2 & <0.2 & <0.2 & <0.20 & <5.0 \\ <0.2 & <1.0 & <5.0 & <0.20 & <0.2 & <0.2 & <0.2 & <0.2 & <0.2 & <0.20 & <5.0 \\ <0.2 & <1.0 & <5.0 & <0.20 & <0.2 & <0.2 & <0.2 & <0.2 & <0.2 & <0.20 & <5.0\end{array}$


Table 6. Water-quality data for storm-sewer outfall stations, Irving, Texas, 1992-93-Continued

08055570 - HEREFORD ROAD OUTFALI AT WALNUT HILL ROAD, IRVING, TX (WY 1993)-Continued

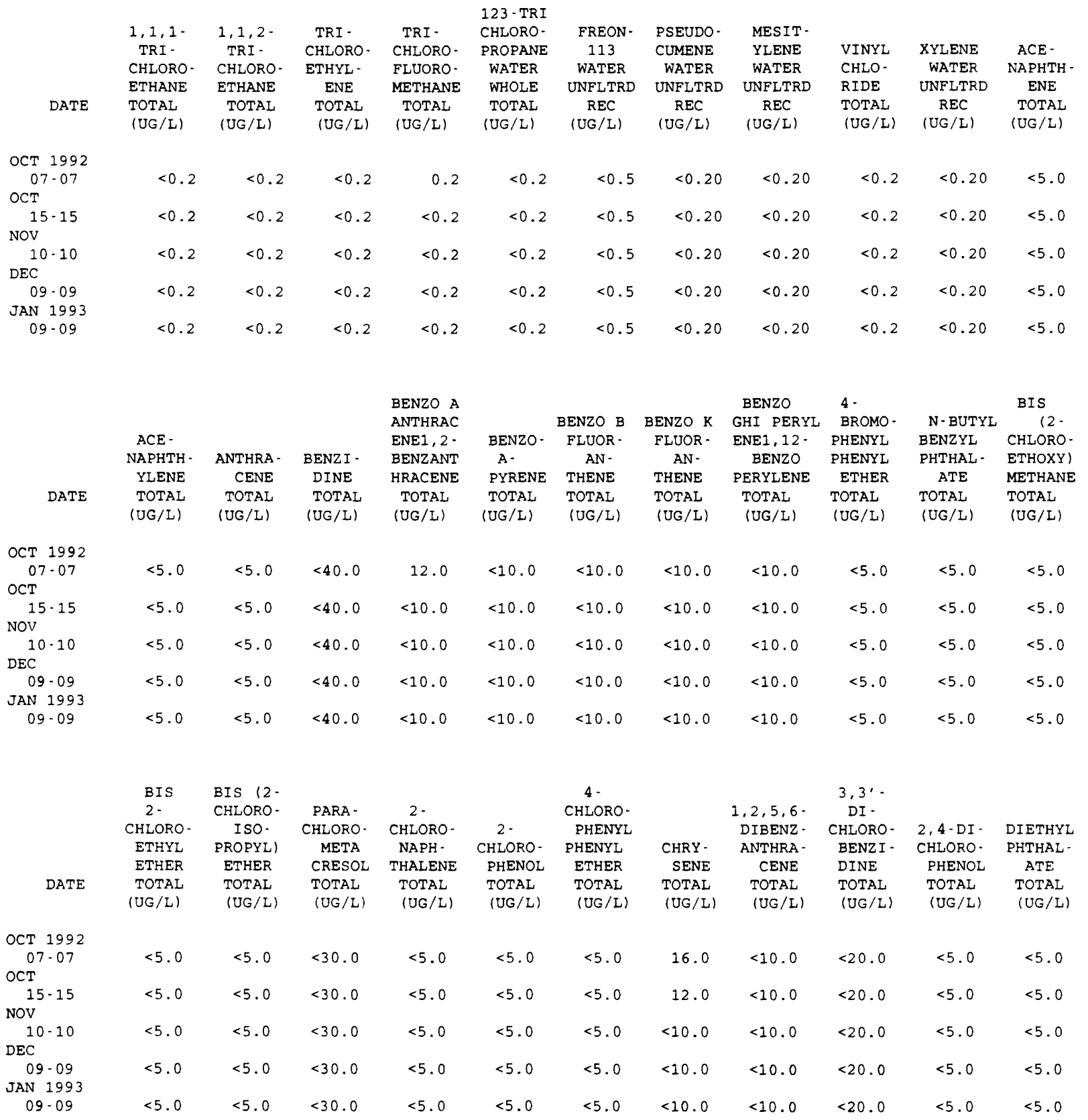


Table 6. Water-quality data for storm-sewer outfall stations, Irving, Texas, 1992-93-Continued

08055570 - HEREFORD ROAD OUTFALL AT WALNUT HILL ROAD, IRVING, TX (WY 1993)-Continued

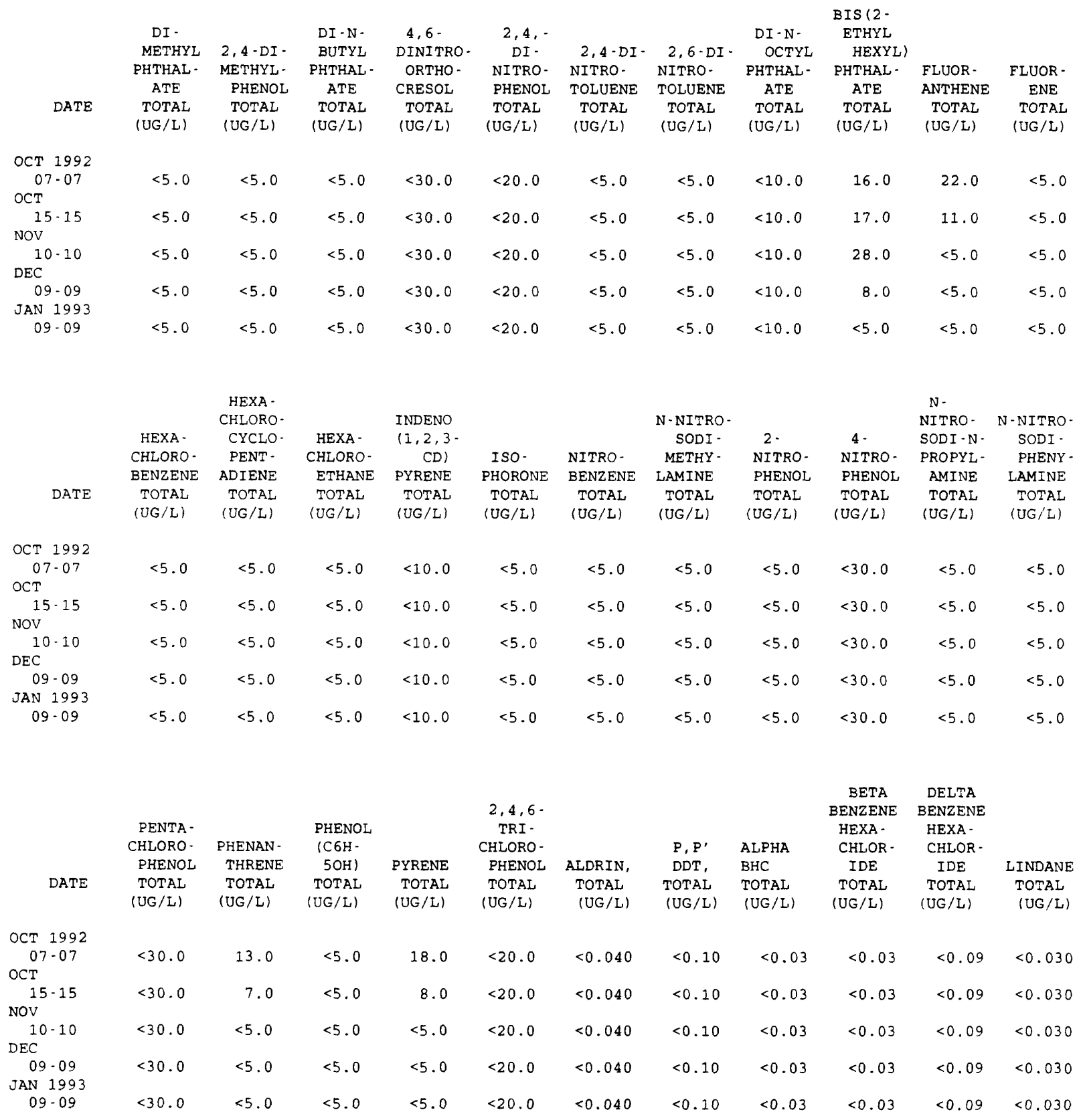


Table 6. Water-quality data for storm-sewer outfall stations, Irving, Texas, 1992-93-Continued

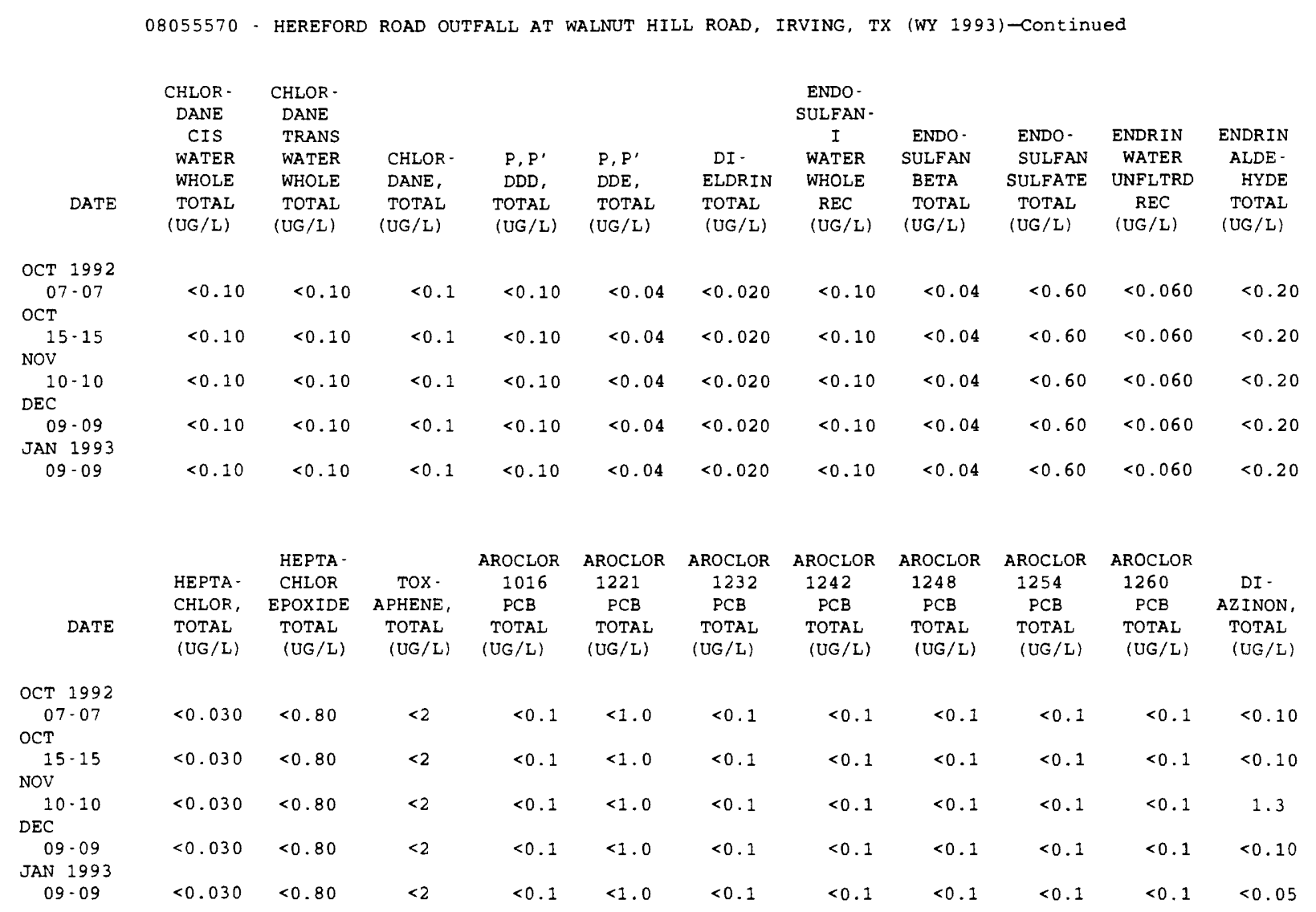


Table 6. Water-quality data for storm-sewer outfall stations, Irving, Texas, 1992-93-Continued

08056100 - TRIBUTARY TO ELM FORK TRINITY RIVER OUTFALL AT CASCADE STREET, IRVING, TX (WY 1992)

\begin{tabular}{|c|c|c|c|c|c|c|c|c|c|c|c|c|}
\hline DATE & TIME & $\begin{array}{l}\text { PRECIP- } \\
\text { ITATION } \\
\text { TOTAL } \\
\text { INCHES/ } \\
\text { STORM }\end{array}$ & $\begin{array}{l}\text { ELAPSED } \\
\text { TIME } \\
\text { OF } \\
\text { STORM } \\
\text { (HOURS) }\end{array}$ & $\begin{array}{l}\text { STORM } \\
\text { WATER } \\
\text { FLOW } \\
\text { (MGD) }\end{array}$ & $\begin{array}{l}\text { SPE- } \\
\text { CIFIC } \\
\text { CON- } \\
\text { DUCT- } \\
\text { ANCE } \\
\text { (US/CM) }\end{array}$ & $\begin{array}{c}\text { SPE- } \\
\text { CIFIC } \\
\text { CON- } \\
\text { DUCT- } \\
\text { ANCE } \\
\text { LAB } \\
\text { (US/CM) }\end{array}$ & $\begin{array}{c}\text { PH } \\
\text { WATER } \\
\text { WHOLE } \\
\text { FIELD } \\
\text { (STAND- } \\
\text { ARD } \\
\text { UNITS) }\end{array}$ & $\begin{array}{c}\text { PH } \\
\text { WATER } \\
\text { WHOLE } \\
\text { LAB } \\
\text { (STAND- } \\
\text { ARD } \\
\text { UNITS) }\end{array}$ & $\begin{array}{c}\text { TEMPER- } \\
\text { ATURE } \\
\text { WATER } \\
\text { (DEG C) }\end{array}$ & $\begin{array}{c}\text { OXYGEN } \\
\text { DEMAND, } \\
\text { CHEM- } \\
\text { ICAL } \\
\text { (HIGH } \\
\text { LEVEL) } \\
\text { (MG/L) }\end{array}$ & $\begin{array}{l}\text { OXYGEN } \\
\text { DEMAND, } \\
\text { BIO- } \\
\text { CHEM- } \\
\text { ICAL, } \\
5 \text { DAY } \\
\text { (MG/L) }\end{array}$ & $\begin{array}{l}\text { COLI- } \\
\text { FORM, } \\
\text { FECAL, } \\
0.7 \\
\text { UM-MF } \\
\text { (COLS./ } \\
100 \mathrm{ML} \text { ) }\end{array}$ \\
\hline $\begin{array}{c}\text { SEP } 1992 \\
21-21\end{array}$ & 0128 & 0.78 & 3.8 & 0.51 & 50 & 153 & 8.1 & 7.8 & 24.0 & 32 & 4.2 & $\mathrm{~K} 10000$ \\
\hline DATE & $\begin{array}{l}\text { STREP. } \\
\text { TOCOCCI } \\
\text { FECAL, } \\
\text { KF AGAR } \\
\text { (COLS. } \\
\text { PER } \\
100 \text { ML) }\end{array}$ & $\begin{array}{l}\text { HARD- } \\
\text { NESS } \\
\text { TOTAL } \\
\text { (MG/L } \\
\text { AS } \\
\text { CACO3) }\end{array}$ & $\begin{array}{l}\text { HARD- } \\
\text { NESS } \\
\text { NONCARB } \\
\text { DISSOLV } \\
\text { FLD. AS } \\
\text { CACO3 } \\
(\mathrm{MG} / \mathrm{L})\end{array}$ & $\begin{array}{l}\text { ALKA- } \\
\text { LINITY } \\
\text { WAT DIS } \\
\text { FIX END } \\
\text { FIELD } \\
\text { CACO3 } \\
\text { (MG } / L)\end{array}$ & $\begin{array}{l}\text { SOLIDS, } \\
\text { SUM OF } \\
\text { CONSTI - } \\
\text { TUENTS, } \\
\text { DIS- } \\
\text { SOLVED } \\
\text { (MG/L) }\end{array}$ & $\begin{array}{l}\text { RESIDUE } \\
\text { TOTAL } \\
\text { AT } 105 \\
\text { DEG. C, } \\
\text { SUS - } \\
\text { PENDED } \\
\text { (MG/L) }\end{array}$ & $\begin{array}{l}\text { SOLIDS, } \\
\text { RESIDUE } \\
\text { AT } 180 \\
\text { DEG. C } \\
\text { DIS - } \\
\text { SOLVED } \\
\text { (MG/L) }\end{array}$ & $\begin{array}{l}\text { CALCIUM } \\
\text { DIS- } \\
\text { SOLVED } \\
\text { (MG/L } \\
\text { AS CA) }\end{array}$ & $\begin{array}{l}\text { MAGNE- } \\
\text { SIUM, } \\
\text { DIS- } \\
\text { SOLVED } \\
\text { (MG/L } \\
\text { AS MG) }\end{array}$ & $\begin{array}{l}\text { SODIUM, } \\
\text { DIS - } \\
\text { SOLVED } \\
\text { (MG/L } \\
\text { AS NA) }\end{array}$ & $\begin{array}{r}\text { SODIUM } \\
\text { PERCENT }\end{array}$ & $\begin{array}{l}\text { SODIUM } \\
\text { AD- } \\
\text { SORP- } \\
\text { TION } \\
\text { RATIO }\end{array}$ \\
\hline $\begin{array}{c}\text { SEP } 1992 \\
21 \cdot 21\end{array}$ & 2500 & 36 & 6 & 30 & 63 & 72 & 42 & 13 & 0.92 & 3.3 & 16 & 0.2 \\
\hline DATE & $\begin{array}{l}\text { POTAS- } \\
\text { SIUM, } \\
\text { DIS - } \\
\text { SOLVED } \\
\text { (MG/L } \\
\text { AS K) }\end{array}$ & $\begin{array}{l}\text { SULFATE } \\
\text { DIS - } \\
\text { SOLVED } \\
\text { (MG/L } \\
\text { AS SO4) }\end{array}$ & $\begin{array}{l}\text { CHLO- } \\
\text { RIDE, } \\
\text { DIS- } \\
\text { SOLVED } \\
\text { (MG/L } \\
\text { AS CL) }\end{array}$ & $\begin{array}{l}\text { NITRO- } \\
\text { GEN, } \\
\text { NITRITE } \\
\text { TOTAL } \\
\text { (MG/L } \\
\text { AS N) }\end{array}$ & $\begin{array}{c}\text { NITRO- } \\
\text { GEN, } \\
\text { NO2+NO3 } \\
\text { TOTAL } \\
\text { (MG/L } \\
\text { AS N) }\end{array}$ & $\begin{array}{l}\text { NITRO- } \\
\text { GEN, } \\
\text { AMMONIA } \\
\text { TOTAL } \\
\text { (MG/L } \\
\text { AS N) }\end{array}$ & $\begin{array}{l}\text { NITRO- } \\
\text { GEN, AM- } \\
\text { MONIA + } \\
\text { ORGANIC } \\
\text { TOTAL } \\
\text { (MG/L } \\
\text { AS N) }\end{array}$ & $\begin{array}{l}\text { PHOS - } \\
\text { PHORUS } \\
\text { TOTAL } \\
\text { (MG/L } \\
\text { AS P) }\end{array}$ & $\begin{array}{c}\text { PHOS - } \\
\text { PHORUS } \\
\text { DIS - } \\
\text { SOLVED } \\
\text { (MG/L } \\
\text { AS P) }\end{array}$ & $\begin{array}{c}\text { ANTIMONY } \\
\text { TOTAL } \\
\text { EPA } \\
\text { (UG/L } \\
\text { AS SB) }\end{array}$ & $\begin{array}{c}\text { ARSENIC } \\
\text { TOTAL } \\
\text { (UG/L } \\
\text { AS AS) }\end{array}$ & $\begin{array}{l}\text { BERYL- } \\
\text { LIUM, } \\
\text { TOTAL } \\
\text { RECOV. } \\
\text { ERABLE } \\
\text { (UG/L } \\
\text { AS BE) }\end{array}$ \\
\hline $\begin{array}{c}\text { SEP } 1992 \\
21 \cdot 21\end{array}$ & 1.0 & 11 & 3.0 & 0.060 & 0.410 & 0.100 & 0.50 & 0.260 & 0.170 & $<10.0$ & 1 & $<10$ \\
\hline DATE & $\begin{array}{l}\text { CADMIUM } \\
\text { TOTAL } \\
\text { RECOV- } \\
\text { ERABLE } \\
\text { (UG/L } \\
\text { AS CD) }\end{array}$ & $\begin{array}{l}\text { CHRO- } \\
\text { MIUM, } \\
\text { TOTAL } \\
\text { RECOV- } \\
\text { ERABLE } \\
\text { (UG/L } \\
\text { AS CR) }\end{array}$ & $\begin{array}{l}\text { COPPER, } \\
\text { TOTAL } \\
\text { RECOV- } \\
\text { ERABLE } \\
\text { (UG/L } \\
\text { AS CU) }\end{array}$ & $\begin{array}{c}\text { CYANIDE } \\
\text { TOTAL } \\
\text { EPA } \\
\text { (MG/L } \\
\text { AS CN) }\end{array}$ & $\begin{array}{l}\text { CYANIDE } \\
\text { TOTAL } \\
\text { (MG/L } \\
\text { AS CN) }\end{array}$ & $\begin{array}{l}\text { LEAD, } \\
\text { TOTAL } \\
\text { RECOV- } \\
\text { ERABLE } \\
\text { (UG/L } \\
\text { AS PB) }\end{array}$ & $\begin{array}{l}\text { MERCURY } \\
\text { TOTAL } \\
\text { RECOV- } \\
\text { ERABLE } \\
\text { (UG/L } \\
\text { AS HG) }\end{array}$ & $\begin{array}{l}\text { NICKEL, } \\
\text { TOTAL } \\
\text { RECOV- } \\
\text { ERABLE } \\
\text { (UG/L } \\
\text { AS NI) }\end{array}$ & $\begin{array}{l}\text { SELE- } \\
\text { NIUM, } \\
\text { TOTAL } \\
\text { (UG/L } \\
\text { AS SE) }\end{array}$ & $\begin{array}{l}\text { SILVER, } \\
\text { TOTAL } \\
\text { RECOV- } \\
\text { ERABLE } \\
\text { (UG/L } \\
\text { AS AG) }\end{array}$ & $\begin{array}{l}\text { SILVER, } \\
\text { TOTAL } \\
\text { RECOV- } \\
\text { ERABLE } \\
\text { EPA } \\
\text { (UG/L } \\
\text { AS AG) }\end{array}$ & $\begin{array}{l}\text { THAL - } \\
\text { LIUM, } \\
\text { TOTAL } \\
\text { (UG/L }\end{array}$ \\
\hline $\begin{array}{c}\text { SEP } 1992 \\
21-21\end{array}$ & $<1$ & 5 & 7 & $<0.010$ & $<0.010$ & 15 & $<0.10$ & 7 & $<2$ & $<1$ & $<0.500$ & $<10$ \\
\hline DATE & $\begin{array}{l}\text { ZINC, } \\
\text { TOTAL } \\
\text { RECOV- } \\
\text { ERABLE } \\
\text { (UG/L } \\
\text { AS ZN) }\end{array}$ & $\begin{array}{l}\text { CARBON, } \\
\text { ORGANIC } \\
\text { TOTAL } \\
\text { (MG L L } \\
\text { AS C) }\end{array}$ & $\begin{array}{l}\text { OIL AND } \\
\text { GREASE, } \\
\text { TOTAL } \\
\text { RECOV. } \\
\text { GRAVI - } \\
\text { METRIC } \\
\text { (MG/L) }\end{array}$ & $\begin{array}{l}\text { PHENOLS } \\
\text { TOTAL } \\
\text { (UG/L) }\end{array}$ & $\begin{array}{l}\text { ACRO- } \\
\text { LEIN } \\
\text { TOTAL } \\
\text { (UG/L) }\end{array}$ & $\begin{array}{l}\text { ACRYLO- } \\
\text { NITRILE } \\
\text { TOTAL } \\
\text { (UG/L) }\end{array}$ & $\begin{array}{l}\text { BENZENE } \\
\text { TOTAL } \\
\text { (UG/L) }\end{array}$ & $\begin{array}{l}\text { BROMO- } \\
\text { BENZENE } \\
\text { WATER, } \\
\text { WHOLE, } \\
\text { TOTAL } \\
\text { (UG/L) }\end{array}$ & $\begin{array}{c}\text { BROMO- } \\
\text { FORM } \\
\text { TOTAL } \\
\text { (UG/L) }\end{array}$ & $\begin{array}{c}\text { BENZENE } \\
\text { N-BUTYL } \\
\text { WATER } \\
\text { UNFLTRD } \\
\text { REC } \\
(U G / L)\end{array}$ & $\begin{array}{l}\text { BENZENE } \\
\text { SEC } \\
\text { BUTYL- } \\
\text { WATER } \\
\text { UNFLTRD } \\
\text { REC } \\
\text { (UG/L) }\end{array}$ & $\begin{array}{c}\text { BENZENE } \\
\text { TERT- } \\
\text { BUTYL- } \\
\text { WATER } \\
\text { UNFLTRD } \\
\text { REC } \\
\text { (UG/L) }\end{array}$ \\
\hline $\begin{array}{c}\text { SEP } 1992 \\
21.21\end{array}$ & 140 & 9.6 & 3 & 4 & $<20$ & $<20$ & $<0.2$ & $<0.2$ & $<0.2$ & $<0.20$ & $<0.20$ & $<0.20$ \\
\hline DATE & $\begin{array}{l}\text { CARBON- } \\
\text { TETRA- } \\
\text { CHLO- } \\
\text { RIDE } \\
\text { TOTAL } \\
\text { (UG/L) }\end{array}$ & $\begin{array}{l}\text { CHLORO- } \\
\text { BENZENE } \\
\text { TOTAL } \\
\text { (UG/L) }\end{array}$ & $\begin{array}{c}\text { CHLORO - } \\
\text { DI - } \\
\text { BROMO - } \\
\text { METHANE } \\
\text { TOTAL } \\
\text { (UG/L) }\end{array}$ & $\begin{array}{l}\text { CHLORO- } \\
\text { ETHANE } \\
\text { TOTAL } \\
\text { (UG/L) }\end{array}$ & $\begin{array}{c}2- \\
\text { CHLORO- } \\
\text { ETHYL- } \\
\text { VINYL- } \\
\text { ETHER } \\
\text { TOTAL } \\
\text { (UG/L) }\end{array}$ & $\begin{array}{l}\text { CHLORO- } \\
\text { FORM } \\
\text { TOTAL } \\
\text { (UG/L) }\end{array}$ & $\begin{array}{l}\text { METHYL- } \\
\text { CHLO- } \\
\text { RIDE } \\
\text { TOTAL } \\
\text { (UG/L) }\end{array}$ & $\begin{array}{c}\text { O- } \\
\text { CHLORO- } \\
\text { TOLUENE } \\
\text { WATER } \\
\text { WHOLE } \\
\text { TOTAL } \\
\text { (UG/L) }\end{array}$ & $\begin{array}{l}\text { TOLUENE } \\
\text { P-CHLOR } \\
\text { WATER } \\
\text { UNFLTRD } \\
\text { REC } \\
(\text { UG } / L)\end{array}$ & $\begin{array}{l}\text { DIBROMO } \\
\text { CHLORO- } \\
\text { PROPANE } \\
\text { WATER } \\
\text { WHOLE } \\
\text { TOT.REC } \\
\text { (UG } / L \text { ) }\end{array}$ & $\begin{array}{c}\text { DI- } \\
\text { BROMO- } \\
\text { METHANE } \\
\text { WATER } \\
\text { WHOLE } \\
\text { RECOVER } \\
\text { (UG/L) }\end{array}$ & $\begin{array}{l}1,2- \\
\text { DIBROMO } \\
\text { ETHANE } \\
\text { WATER } \\
\text { WHOLE } \\
\text { TOTAL } \\
\text { (UG/L) }\end{array}$ \\
\hline $\begin{array}{c}\text { SEP } 1992 \\
21-21\end{array}$ & $<0.2$ & $<0.20$ & $<0.2$ & $<0.2$ & $<1.0$ & 0.2 & $<0.2$ & $<0.2$ & $<0.20$ & $<1.0$ & $<0.2$ & $<0.2$ \\
\hline
\end{tabular}


Table 6. Water-quality data for storm-sewer outfall stations, Irving, Texas, 1992-93-Continued

08056100 - TRIBUTARY TO ELM FORK TRINITY RIVER OUTFALL AT CASCADE STREET, IRVING, TX (WY 1992)-COntINUEd

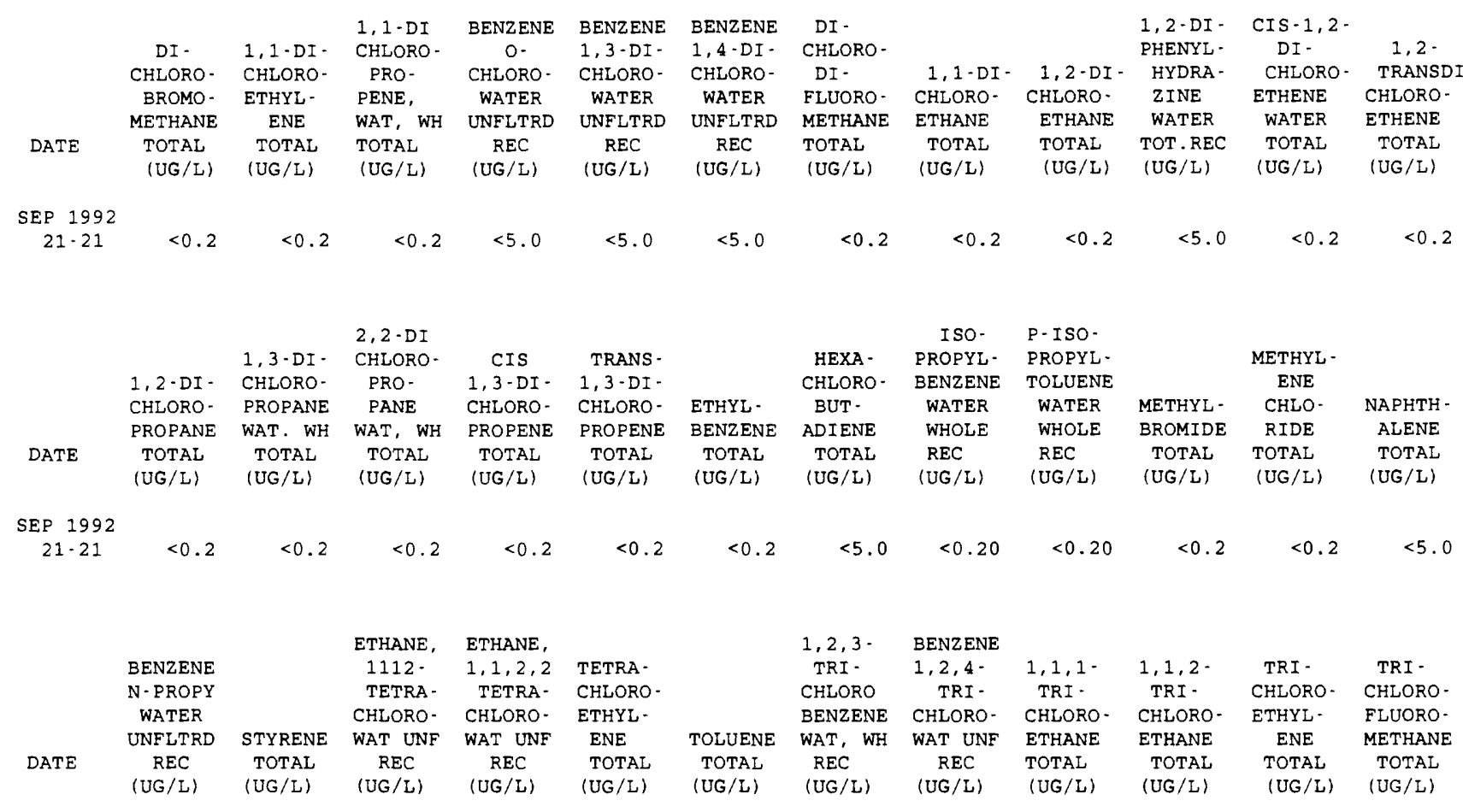

\begin{tabular}{|c|c|c|c|c|c|c|c|c|c|c|c|c|}
\hline $\begin{array}{c}\text { SEP } 1992 \\
21-21\end{array}$ & $<0.20$ & $<0.2$ & $<0.2$ & $<0.2$ & $<0.2$ & $<0.2$ & $<0.20$ & $<5.0$ & $<0.2$ & $<0.2$ & $<0.2$ & $<0.2$ \\
\hline DATE & $\begin{array}{l}123 \text {-TRI } \\
\text { CHLORO- } \\
\text { PROPANE } \\
\text { WATER } \\
\text { WHOLE } \\
\text { TOTAL } \\
\text { (UG/L) }\end{array}$ & $\begin{array}{l}\text { PSEUDO- } \\
\text { CUMENE } \\
\text { WATER } \\
\text { UNFLTRD } \\
\text { REC } \\
\text { (UG/L) }\end{array}$ & $\begin{array}{l}\text { MESIT - } \\
\text { YLENE } \\
\text { WATER } \\
\text { UNFLTRD } \\
\text { REC } \\
\text { (UG/L) }\end{array}$ & $\begin{array}{l}\text { VINYL } \\
\text { CHLO- } \\
\text { RIDE } \\
\text { TOTAL } \\
\text { (UG/L) }\end{array}$ & $\begin{array}{c}\text { XYLENE } \\
\text { WATER } \\
\text { UNFLTRD } \\
\text { REC } \\
(U G / L)\end{array}$ & $\begin{array}{c}\text { ACE- } \\
\text { NAPHTH - } \\
\text { ENE } \\
\text { TOTAL } \\
(\text { UG } / L)\end{array}$ & $\begin{array}{l}\text { ACE - } \\
\text { NAPHTH - } \\
\text { YLENE } \\
\text { TOTAL } \\
\text { (UG/L) }\end{array}$ & $\begin{array}{c}\text { ANTHRA- } \\
\text { CENE } \\
\text { TOTAL } \\
\text { (UG/L) }\end{array}$ & $\begin{array}{l}\text { BENZI - } \\
\text { DINE } \\
\text { TOTAL } \\
\text { (UG/L) }\end{array}$ & $\begin{array}{l}\text { BENZO A } \\
\text { ANTHRAC } \\
\text { ENE1,2- } \\
\text { BENZANT } \\
\text { HRACENE } \\
\text { TOTAL } \\
\text { (UG/L) }\end{array}$ & $\begin{array}{l}\text { BENZO- } \\
\text { A- } \\
\text { PYRENE } \\
\text { TOTAL } \\
\text { (UG/L) }\end{array}$ & $\begin{array}{l}\text { BENZO B } \\
\text { FLUOR- } \\
\text { AN- } \\
\text { THENE } \\
\text { TOTAL } \\
(\text { UG/L) }\end{array}$ \\
\hline $\begin{array}{c}\text { SEP } 1992 \\
21-21\end{array}$ & $<0.2$ & $<0.20$ & $<0.20$ & $<0.2$ & $<0.20$ & $<5.0$ & $<5.0$ & $<5.0$ & $<40.0$ & $<10.0$ & $<10.0$ & $<10.0$ \\
\hline DATE & $\begin{array}{l}\text { BENZO K } \\
\text { FLUOR- } \\
\text { AN- } \\
\text { THENE } \\
\text { TOTAL } \\
\text { (UG/L) }\end{array}$ & $\begin{array}{l}\text { BENZO } \\
\text { GHI PERYL } \\
\text { ENE1,12- } \\
\text { BENZO } \\
\text { PERYLENE } \\
\text { TOTAL } \\
(U G / L)\end{array}$ & $\begin{array}{c}4 \cdot- \\
\text { BROMO- } \\
\text { PHENYL } \\
\text { PHENYL } \\
\text { ETHER } \\
\text { TOTAL } \\
\text { (UG/L) }\end{array}$ & $\begin{array}{l}\text { N-BUTYL } \\
\text { BENZYL } \\
\text { PHTHAL- } \\
\text { ATE } \\
\text { TOTAL } \\
\text { (UG/L) }\end{array}$ & $\begin{array}{l}\text { BIS } \\
\quad \text { (2- } \\
\text { CHLORO- } \\
\text { ETHOXY) } \\
\text { METHANE } \\
\text { TOTAL } \\
\text { (UG/L) }\end{array}$ & $\begin{array}{c}\text { BIS } \\
2- \\
\text { CHLORO- } \\
\text { ETHYL } \\
\text { ETHER } \\
\text { TOTAL } \\
\text { (UG/L) }\end{array}$ & $\begin{array}{l}\text { BIS }(2- \\
\text { CHLORO- } \\
\text { ISO- } \\
\text { PROPYL) } \\
\text { ETHER } \\
\text { TOTAL } \\
\text { (UG/L) }\end{array}$ & $\begin{array}{l}\text { PARA- } \\
\text { CHLORO- } \\
\text { META } \\
\text { CRESOL } \\
\text { TOTAL } \\
\text { (UG/L) }\end{array}$ & $\begin{array}{c}2- \\
\text { CHLORO- } \\
\text { NAPH- } \\
\text { THALENE } \\
\text { TOTAL } \\
\text { (UG/L) }\end{array}$ & $\begin{array}{c}2- \\
\text { CHLORO- } \\
\text { PHENOL } \\
\text { TOTAL } \\
\text { (UG/L) }\end{array}$ & $\begin{array}{l}4- \\
\text { CHLORO- } \\
\text { PHENYL } \\
\text { PHENYL } \\
\text { ETHER } \\
\text { TOTAL } \\
\text { (UG/L) }\end{array}$ & $\begin{array}{l}\text { CHRY - } \\
\text { SENE } \\
\text { TOTAL } \\
\text { (UG/L) }\end{array}$ \\
\hline $\begin{array}{c}\text { SEP } 1992 \\
21-21\end{array}$ & $<10.0$ & $<10.0$ & $<5.0$ & $<5.0$ & $<5.0$ & $<5.0$ & $<5.0$ & $<30.0$ & $<5.0$ & $<5.0$ & $<5.0$ & $<10.0$ \\
\hline DATE & $\begin{array}{l}1,2,5,6- \\
\text { DIBENZ - } \\
\text { ANTHRA- } \\
\text { CENE } \\
\text { TOTAL } \\
\text { (UG/L) }\end{array}$ & $\begin{array}{c}3,3 \% \\
\text { DI - } \\
\text { CHLORO- } \\
\text { BENZI - } \\
\text { DINE } \\
\text { TOTAL } \\
\text { (UG/L) }\end{array}$ & $\begin{array}{l}2,4 \cdot D I- \\
\text { CHLORO- } \\
\text { PHENOL } \\
\text { TOTAL } \\
(U G / L)\end{array}$ & $\begin{array}{l}\text { DIETHYL } \\
\text { PHTHAL- } \\
\text { ATE } \\
\text { TOTAL } \\
\text { (UG/L) }\end{array}$ & $\begin{array}{c}\text { DI- } \\
\text { METHYL } \\
\text { PHTHAL- } \\
\text { ATE } \\
\text { TOTAL } \\
\text { (UG/L) }\end{array}$ & $\begin{array}{l}2,4-D I- \\
\text { METHYL- } \\
\text { PHENOL } \\
\text { TOTAL } \\
(U G / L)\end{array}$ & $\begin{array}{l}\text { DI - N - } \\
\text { BUTYL } \\
\text { PHTHAL- } \\
\text { ATE } \\
\text { TOTAL } \\
\text { (UG/L) }\end{array}$ & $\begin{array}{l}4,6- \\
\text { DINITRO- } \\
\text { ORTHO- } \\
\text { CRESOL } \\
\text { TOTAL } \\
\text { (UG/L) }\end{array}$ & $\begin{array}{c}2,4,- \\
\text { DI - } \\
\text { NITRO- } \\
\text { PHENOL } \\
\text { TOTAL } \\
\text { (UG/L) }\end{array}$ & $\begin{array}{l}2,4-D I- \\
\text { NITRO- } \\
\text { TOLUENE } \\
\text { TOTAL } \\
\text { (UG/L) }\end{array}$ & $\begin{array}{l}2,6-D I- \\
\text { NITRO- } \\
\text { TOLUENE } \\
\text { TOTAL } \\
\text { (UG/L) }\end{array}$ & $\begin{array}{c}\text { DI - N - } \\
\text { OCTYL } \\
\text { PHTHAL- } \\
\text { ATE } \\
\text { TOTAL } \\
(\text { UG } / L)\end{array}$ \\
\hline $\begin{array}{c}\text { SEP } 1992 \\
21-21\end{array}$ & $<10.0$ & $<20.0$ & $<5.0$ & $<5.0$ & $<5.0$ & $<5.0$ & $<5.0$ & $<30.0$ & $<20.0$ & $<5.0$ & $<5.0$ & $<10.0$ \\
\hline
\end{tabular}


Table 6. Water-quality data for storm-sewer outfall stations, Irving, Texas, 1992-93-Continued

08056100 - TRIBUTARY TO ELM FORK TRINITY RIVER OUTFALL AT CASCADE STREET, IRVING, TX (WY 1992)-CONEINUEd

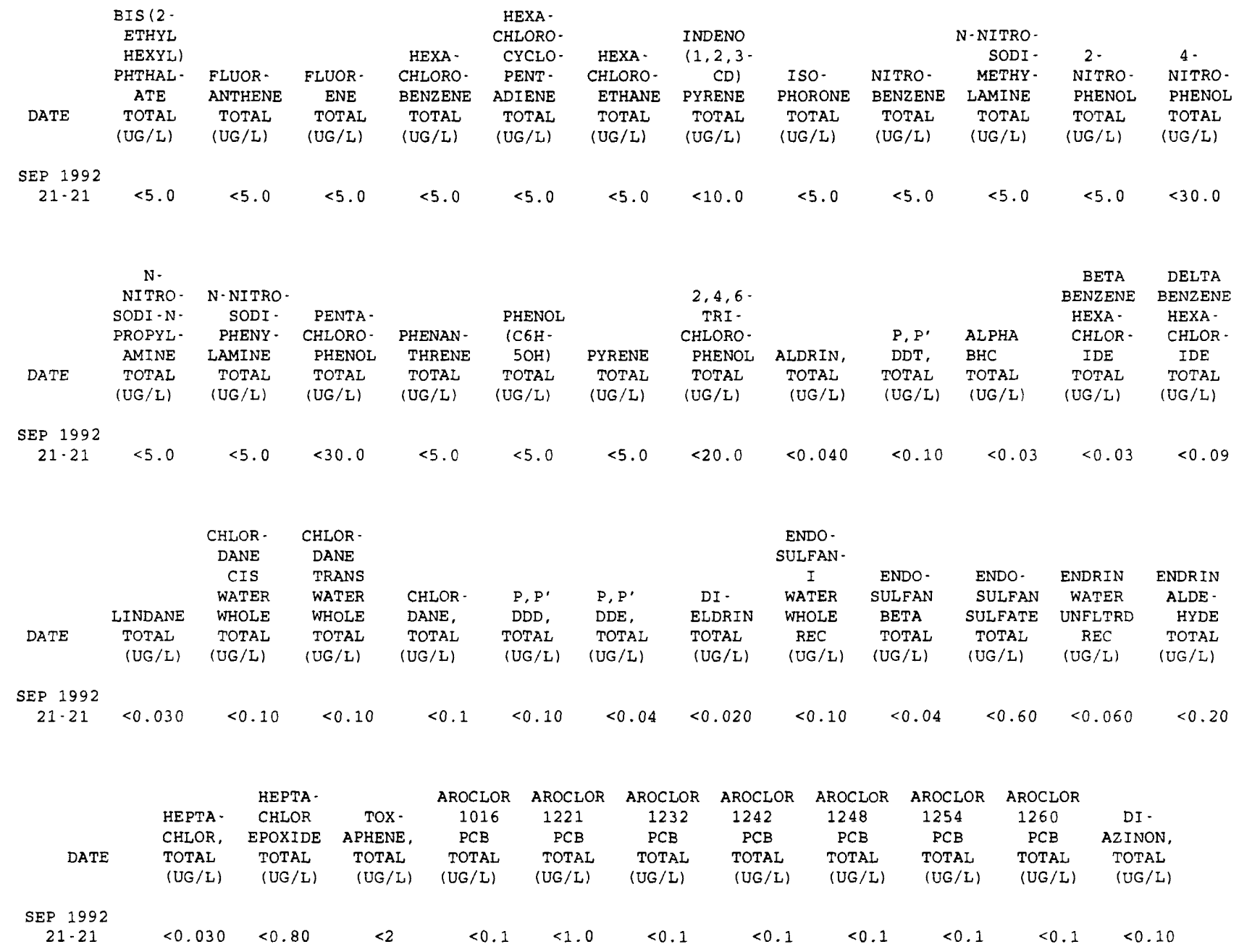


Table 6. Water-quality data for storm-sewer outfall stations, Irving, Texas, 1992-93-Continued

08056100 - TRIBUTARY TO ELM FORK TRINITY RIVER OUTFALL AT CASCADE STREET, IRVING, TX (WY 1993)

\begin{tabular}{|c|c|c|c|c|c|c|c|c|c|c|c|c|}
\hline DATE & TIME & $\begin{array}{l}\text { PRECIP- } \\
\text { ITATION } \\
\text { TOTAL } \\
\text { INCHES/ } \\
\text { STORM }\end{array}$ & $\begin{array}{l}\text { ELAPSED } \\
\text { TIME } \\
\text { OF } \\
\text { STORM } \\
\text { (HOURS) }\end{array}$ & $\begin{array}{l}\text { STORM } \\
\text { WATER } \\
\text { FLOW } \\
\text { (MGD) }\end{array}$ & $\begin{array}{l}\text { SPE - } \\
\text { CIFIC } \\
\text { CON- } \\
\text { DUCT - } \\
\text { ANCE } \\
\text { (US/CM) }\end{array}$ & $\begin{array}{c}\text { SPE - } \\
\text { CIFIC } \\
\text { CON - } \\
\text { DUCT - } \\
\text { ANCE } \\
\text { LAB } \\
\text { (US/CM) }\end{array}$ & $\begin{array}{c}\text { PH } \\
\text { WATER } \\
\text { WHOLE } \\
\text { FIELD } \\
\text { (STAND- } \\
\text { ARD } \\
\text { UNITS) }\end{array}$ & $\begin{array}{c}\text { PH } \\
\text { WATER } \\
\text { WHOLE } \\
\text { LAB } \\
\text { (STAND- } \\
\text { ARD } \\
\text { UNITS) }\end{array}$ & $\begin{array}{c}\text { TEMPER - } \\
\text { ATURE } \\
\text { WATER } \\
\text { (DEG C) }\end{array}$ & $\begin{array}{l}\text { OXYGEN } \\
\text { DEMAND, } \\
\text { CHEM- } \\
\text { ICAL } \\
\text { (HIGH } \\
\text { LEVEL) } \\
\text { (MG/L) }\end{array}$ & $\begin{array}{l}\text { OXYGEN } \\
\text { DEMAND, } \\
\text { BIO- } \\
\text { CHEM- } \\
\text { ICAL, } \\
5 \text { DAY } \\
\text { (MG/L) }\end{array}$ & $\begin{array}{l}\text { COLI - } \\
\text { FORM, } \\
\text { FECAL, } \\
0.7 \\
\text { UM-MF } \\
\text { (COLS./ } \\
100 \mathrm{ML} \text { ) }\end{array}$ \\
\hline OCT 1992 & & & & & & & & & & & & \\
\hline $\begin{array}{l}07-07 \\
O C T\end{array}$ & 1730 & 0.61 & 2.0 & 0.37 & 110 & 129 & 7.7 & 7.5 & 21.5 & 67 & 8.2 & 4300 \\
\hline $\begin{array}{l}28-29 \\
\text { NOV }\end{array}$ & 2335 & 0.45 & 6.0 & 0.27 & 80 & 168 & 7.9 & 7.4 & 18.5 & 61 & 6.0 & 14000 \\
\hline${ }_{\text {DEC }}^{19-19}$ & 0115 & 0.75 & 10.2 & 0.57 & 141 & 120 & 7.5 & 7.7 & 18.0 & 33 & 3.8 & 1400 \\
\hline $\begin{array}{c}09-09 \\
\text { JAN } 1993\end{array}$ & 0040 & 0.32 & 4.3 & 0.22 & 87 & 115 & 8.1 & 7.7 & 8.5 & 35 & 4.3 & $<1000$ \\
\hline $\begin{array}{l}09.09 \\
\text { JAN }\end{array}$ & 0230 & 0.29 & 5.9 & 0.17 & 132 & 169 & 8.2 & 7.9 & 10.5 & 81 & 4.0 & $K 870$ \\
\hline $28 \cdot 28$ & 1504 & 0.33 & 8.9 & 0.16 & 168 & 160 & 8.3 & 7.6 & 13.0 & $<10$ & . & 2700 \\
\hline & $\begin{array}{r}\text { STREP - } \\
\text { TOCOCCI }\end{array}$ & HARD - & $\begin{array}{l}\text { HARD- } \\
\text { NESS }\end{array}$ & $\begin{array}{l}\text { ALKA- } \\
\text { LINITY }\end{array}$ & $\begin{array}{l}\text { SOLIDS, } \\
\text { SUM OF }\end{array}$ & $\begin{array}{l}\text { RESIDUE } \\
\text { TOTAL }\end{array}$ & $\begin{array}{l}\text { SOLIDS, } \\
\text { RESIDUE }\end{array}$ & & MAGNE - & & & SODIUM \\
\hline & $\begin{array}{l}\text { FECAL, } \\
\text { KF AGAR } \\
\text { (COLS. }\end{array}$ & $\begin{array}{l}\text { NESS } \\
\text { TOTAL } \\
\text { (MG/L }\end{array}$ & $\begin{array}{l}\text { NONCARB } \\
\text { DISSOLV } \\
\text { FLD. AS }\end{array}$ & $\begin{array}{l}\text { WAT DIS } \\
\text { FIX END } \\
\text { FIELD }\end{array}$ & $\begin{array}{c}\text { CONSTI - } \\
\text { TUENTS, } \\
\text { DIS - }\end{array}$ & $\begin{array}{l}\text { AT } 105 \\
\text { DEG. C, } \\
\text { SUS. }\end{array}$ & $\begin{array}{l}\text { AT } 180 \\
\text { DEG. C } \\
\text { DIS - }\end{array}$ & $\begin{array}{l}\text { CALCIUM } \\
\text { DIS- } \\
\text { SOLVED }\end{array}$ & $\begin{array}{c}\text { SIUM, } \\
\text { DIS - } \\
\text { SOLVED }\end{array}$ & $\begin{array}{l}\text { SODIUM, } \\
\text { DIS - } \\
\text { SOLVED }\end{array}$ & & $\begin{array}{l}A D- \\
\text { SORP- } \\
\text { TION }\end{array}$ \\
\hline DATE & $\begin{array}{c}\text { PER } \\
100 \mathrm{ML})\end{array}$ & $\begin{array}{c}\text { AS } \\
\text { (ACO3) }\end{array}$ & $\begin{array}{c}\mathrm{CACO} 3 \\
(\mathrm{MG} / \mathrm{L})\end{array}$ & $\begin{array}{l}\mathrm{CACO} 3 \\
(\mathrm{MG} / \mathrm{L})\end{array}$ & $\begin{array}{l}\text { SOLVED } \\
\text { (MG/L) }\end{array}$ & $\begin{array}{l}\text { PENDED } \\
(M G / L)\end{array}$ & $\begin{array}{l}\text { SOLVED } \\
(M G / L)\end{array}$ & $\begin{array}{c}\text { (MG/L } \\
A S \quad C A)\end{array}$ & $\begin{array}{l}(\mathrm{MG} / \mathrm{L} \\
\mathrm{AS} \mathrm{MG})\end{array}$ & $\begin{array}{l}\text { (MG/L } \\
\text { AS NA) }\end{array}$ & $\begin{array}{l}\text { SODIUM } \\
\text { PERCENT }\end{array}$ & RATIO \\
\hline OCT 1992 & & & & & & & & & & & & \\
\hline $\begin{array}{l}07-07 \\
\text { OCT }\end{array}$ & 2500 & 39 & 14 & 25 & 61 & 97 & 82 & 14 & 0.91 & 3.1 & 14 & 0.2 \\
\hline $\begin{array}{l}28-29 \\
\text { NOV }\end{array}$ & 7800 & 59 & 21 & 38 & 95 & 64 & 104 & 21 & 1.6 & 6.2 & 18 & 0.4 \\
\hline $19 \cdot 19$ & 2400 & 35 & 3 & 32 & 58 & 15 & 41 & 13 & 0.70 & 3.7 & 18 & 0.3 \\
\hline $\begin{array}{c}09-09 \\
\text { JAN } 1993\end{array}$ & $\mathrm{~K} 5700$ & 33 & 7 & 26 & 60 & 56 & 56 & 12 & 0.74 & 3.8 & 20 & 0.3 \\
\hline $\begin{array}{l}09 \cdot 09 \\
\text { JAN }\end{array}$ & 5800 & 58 & 15 & 43 & 91 & 234 & 111 & 21 & 1.3 & 6.7 & 20 & 0.4 \\
\hline $28-28$ & 12000 & 56 & 8 & 48 & 86 & 64 & 87 & 19 & 2.0 & 6.2 & 19 & 0.4 \\
\hline
\end{tabular}

$\begin{array}{ccc} & \text { POTAS - } & \\ & \text { SIUM, } & \text { SULFATE } \\ & \text { DIS - } & \text { DIS - } \\ & \text { SOLVED } & \text { SOLVED } \\ \text { DATE } & (M G / L & (M G / L \\ & \text { AS K) } & \text { AS SO4) }\end{array}$

\begin{tabular}{|c|c|c|c|c|c|c|c|c|c|}
\hline & & & & NITRO - & & & & & BERYL - \\
\hline $\begin{array}{l}\text { CHLO- } \\
\text { RIDE, }\end{array}$ & $\begin{array}{c}\text { NITRO- } \\
\text { GEN, }\end{array}$ & $\begin{array}{l}\text { NITRO- } \\
\text { GEN, }\end{array}$ & $\begin{array}{l}\text { NITRO- } \\
\text { GEN, }\end{array}$ & $\begin{array}{l}\text { GEN, AM - } \\
\text { MONIA + }\end{array}$ & PHOS - & $\begin{array}{l}\text { PHOS - } \\
\text { PHORUS }\end{array}$ & ANT IMONY & & $\begin{array}{l}\text { LIUM, } \\
\text { TOTAL }\end{array}$ \\
\hline DIS - & NITRITE & $\mathrm{NO} 2+\mathrm{NO} 3$ & AMMONIA & ORGANIC & PHORUS & DIS- & TOTAL & ARSENIC & RECOV - \\
\hline $\begin{array}{l}\text { SOLVED } \\
\text { (MG/L }\end{array}$ & $\begin{array}{l}\text { TOTAL } \\
\text { (MG /L }\end{array}$ & $\begin{array}{l}\text { TOTAL } \\
\text { (MG/L }\end{array}$ & $\begin{array}{l}\text { TOTAL } \\
\text { (MG/L }\end{array}$ & $\begin{array}{l}\text { TOTAL } \\
\text { (MG/L }\end{array}$ & $\begin{array}{l}\text { TOTAL } \\
\text { (MG / L }\end{array}$ & $\begin{array}{l}\text { SOLVED } \\
\text { (MG/L }\end{array}$ & $\begin{array}{c}E P A \\
(U G / L\end{array}$ & $\begin{array}{l}\text { TOTAL } \\
\text { (UG/L }\end{array}$ & $\begin{array}{l}\text { ERABLE } \\
\text { (UG/L }\end{array}$ \\
\hline AS CL) & AS N) & AS N) & AS N) & AS N) & AS P) & AS P) & AS SB) & AS AS) & AS $B E$ ) \\
\hline
\end{tabular}

\begin{tabular}{|c|c|c|c|c|c|c|c|c|c|c|c|c|}
\hline \multicolumn{13}{|l|}{ OCT 1992} \\
\hline $07-07$ & 1.1 & 13 & 2.3 & 0.080 & 0.710 & 0.340 & 1.7 & 0.470 & 0.230 & $<10.0$ & 2 & $<10$ \\
\hline \multicolumn{13}{|l|}{ OCT } \\
\hline $28-29$ & 1.0 & 21 & 4.4 & 0.170 & 1.10 & 0.220 & 0.80 & 0.330 & 0.230 & $<10.0$ & 2 & $<10$ \\
\hline \multicolumn{13}{|l|}{ NOV } \\
\hline $19-19$ & 1.0 & 8.2 & 2.5 & 0.260 & 0.750 & 0.070 & 0.30 & 0.230 & 0.180 & $<10.0$ & $<1$ & $<10$ \\
\hline \multicolumn{13}{|l|}{ DEC } \\
\hline 09.09 & 0.80 & 11 & 2.6 & 0.090 & 0.540 & 0.140 & 0.50 & 0.330 & 0.200 & $<10.0$ & $<1$ & $<10$ \\
\hline \multicolumn{13}{|l|}{ JAN 1993} \\
\hline 09.09 & 1.5 & 11 & 4.2 & $\cdots$ & 1.20 & $\cdots$ & 0.60 & 0.450 & 0.190 & $<10.0$ & 2 & $<10$ \\
\hline \multicolumn{13}{|l|}{ JAN } \\
\hline $28-28$ & 1.1 & 17 & 4.0 & $\cdots$ & 0.550 & $\cdots$ & 0.40 & 0.420 & 0.270 & $<20.0$ & 2 & $<10$ \\
\hline
\end{tabular}


Table 6. Water-quality data for storm-sewer outfall stations, Irving, Texas, 1992-93-Continued

08056100 - TRIBUTARY TO ELM FORK TRINITY RIVER OUTFALL AT CASCADE STREET, IRVING, TX (WY 1993)-CONTINUEd

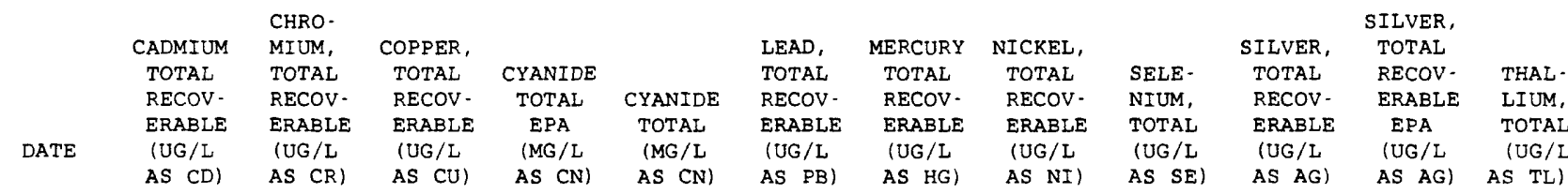

OCT 1992

$07-07$

OCT

$28-29$

NOV

$19-19$

$\mathrm{DEC}$

$09-09$

JAN 1993

09.09

JAN

$28-28$

$\begin{array}{lr}<1 & 13 \\ <1 & 11 \\ <1 & <1 \\ <1 & 10 \\ <1 & 23 \\ <1 & 6\end{array}$

6

$$
\begin{array}{lll}
13 & 0.040 & <0.010 \\
8 & <0.010 & 0.020 \\
4 & <0.010 & <0.010 \\
6 & 0.015 & <0.010 \\
8 & <0.010 & <0.010 \\
6 & <0.010 & <0.010
\end{array}
$$

OIL AND

ZINC, GREASE,

RECOV - ORGANIC RECOV.

ERABLE TOTAL GRAVI

(UG/L (MG/L METRIC

AS ZN) AS C) (MG/L)

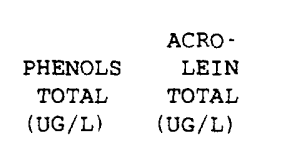

\begin{tabular}{|c|c|c|c|}
\hline \multicolumn{4}{|l|}{ OCT 1992} \\
\hline $07-07$ & 240 & 18 & 1 \\
\hline \multicolumn{4}{|l|}{ OCT } \\
\hline $28-29$ & 150 & 16 & 1 \\
\hline \multicolumn{4}{|l|}{ NOV } \\
\hline $19 \cdot 19$ & 70 & 6.9 & $<1$ \\
\hline \multicolumn{4}{|l|}{ DEC } \\
\hline $09-09$ & 90 & 9.6 & $<1$ \\
\hline \multicolumn{4}{|l|}{ JAN 1993} \\
\hline 09.09 & 170 & 14 & 1 \\
\hline \multicolumn{4}{|l|}{ JAN } \\
\hline $28-28$ & 80 & 8.9 & 2 \\
\hline
\end{tabular}

$\begin{array}{ll}29 & <0.10 \\ 18 & <0.10 \\ 7 & <0.10 \\ 13 & <0.10 \\ 29 & <0.10 \\ 13 & 0.20\end{array}$

13

AS AG)

AS AG) AS TL)

$\begin{array}{cccc} & \text { ERABLE } & \text { TOTAL } & \text { GRAVI } \\ \text { DATE } & \text { (UG } / L & \text { (MG } / L & \text { METRI } \\ & \text { AS ZN) } & \text { AS C) } & \text { (MG }\end{array}$

$\begin{array}{lll}5 & <20 & <20 \\ 5 & <20 & <20 \\ 3 & <20 & <20 \\ 4 & <20 & <20 \\ 3 & <20 & <20 \\ 8 & <20 & <20\end{array}$

$\begin{array}{cc} & \\ \text { ACRYLO- } & \\ \text { NITRILE } & \text { BENZENE } \\ \text { TOTAL } & \text { TOTAL } \\ \text { (UG } / L) & \text { (UG/L) }\end{array}$

BROMO-
BENZENE
WATER,
WHOLE,
TOTAL
(UG/L)

METHANE
BROMO
CHLORO-
WAT
UNFLTRD
REC
(UG/L)

$\begin{array}{lll}<0.2 & <0.2 & <0.20 \\ <0.2 & <0.2 & <0.20 \\ <0.2 & <0.2 & <0.20 \\ <0.2 & <0.2 & <0.20 \\ <0.2 & <0.2 & <0.20 \\ <0.2 & <0.2 & <0.20\end{array}$

$\begin{array}{rrr}<1 & <0.500 & <5 \\ <1 & <0.500 & <5 \\ <1 & <0.500 & <5 \\ <1 & <1.00 & <500 \\ <1 & <0.500 & <10 \\ <1 & <0.500 & <5\end{array}$

BENZENE

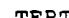

TERT -

BUTYL -

WATER
UNFLTRD

DATE

REC

(UG/L)

CARBON -

TETRA-

CHLO - CHLORO-

RIDE BENZENE

$\begin{array}{ll}\text { TOTAL } & \text { TOTAL } \\ \text { (UG/L) } & \text { (UG/L) }\end{array}$

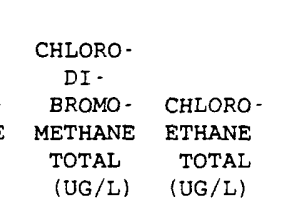

2 -

CHLORO-

ETHYL -

VINYL -

ETHER

TOTAL

(UG/L)

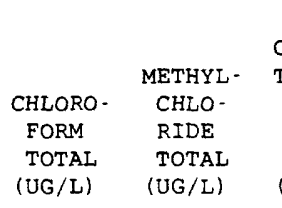

O.

CHLORO

\begin{tabular}{|c|c|c|c|c|c|c|c|c|c|c|c|c|}
\hline \multicolumn{13}{|l|}{ OCT 1992} \\
\hline 07.07 & $<0.20$ & $<0.2$ & $<0.20$ & $<0.2$ & $<0.2$ & $<1.0$ & 0.4 & $<0.2$ & $<0.2$ & $<0.20$ & $<1.0$ & $<0.2$ \\
\hline \multicolumn{13}{|l|}{ OCT } \\
\hline $28-29$ & $<0.20$ & $<0.2$ & $<0.20$ & $<0.2$ & $<0.2$ & $<1.0$ & 0.3 & $<0.2$ & $<0.2$ & $<0.20$ & $<1.0$ & $<0.2$ \\
\hline \multicolumn{13}{|l|}{ NoV } \\
\hline $19 \cdot 19$ & $<0.20$ & $<0.2$ & $<0.20$ & $<0.2$ & $<0.2$ & $<1.0$ & 0.7 & $<0.2$ & $<0.2$ & $<0.20$ & $<1.0$ & $<0.2$ \\
\hline \multicolumn{13}{|l|}{ DEC } \\
\hline $09-09$ & $<0.20$ & $<0.2$ & $<0.20$ & $<0.2$ & $<0.2$ & $<1.0$ & 0.4 & $<0.2$ & $<0.2$ & $<0.20$ & $<1.0$ & $<0.2$ \\
\hline \multicolumn{13}{|l|}{ JAN 1993} \\
\hline $09-09$ & $<0.20$ & $<0.2$ & $<0.20$ & $<0.2$ & $<0.2$ & $<1.0$ & 0.6 & $<0.2$ & $<0.2$ & $<0.20$ & $<1.0$ & $<0.2$ \\
\hline \multicolumn{13}{|l|}{ JAN } \\
\hline $28-28$ & $<0.20$ & $<0.2$ & $<0.20$ & $<0.2$ & $<0.2$ & $<1.0$ & 0.2 & $<0.2$ & $<0.2$ & $<0.20$ & $<1.0$ & $<0.2$ \\
\hline
\end{tabular}

TOLUEN

WATER

WHOLE

TOTAL

(UG/L) 
Table 6. Water-quality data for storm-sewer outfall stations, Irving, Texas, 1992-93-Continued

08056100 - TRIBUTARY TO ELM FORK TRINITY RIVER OUTFALL AT CASCADE STREET, IRVING, TX (WY 1993)-CONTINUEd

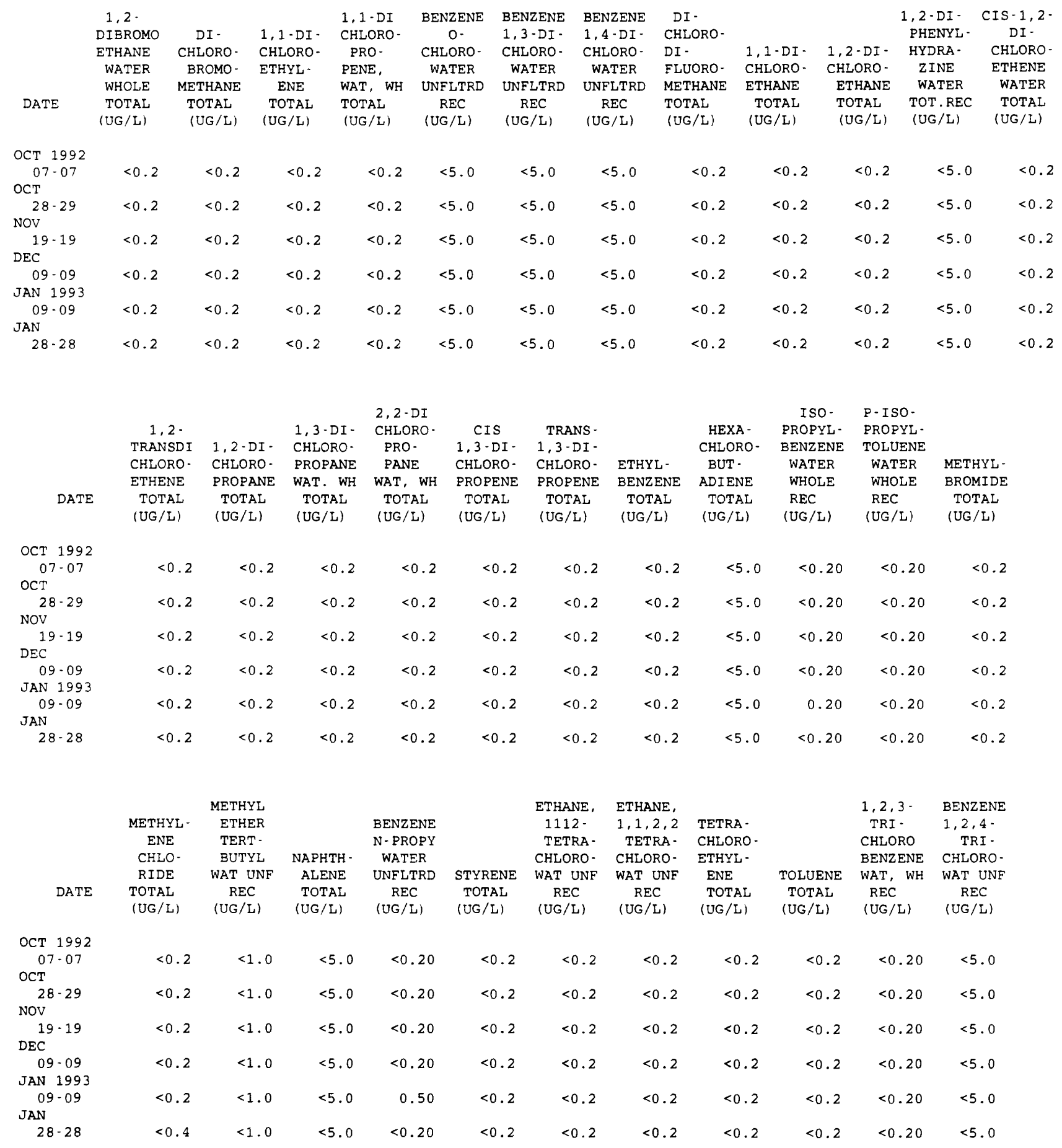


Table 6. Water-quality data for storm-sewer outfall stations, Irving, Texas, 1992-93-Continued

08056100 - TRIBUTARY TO ELM FORK TRINITY RIVER OUTFALI AT CASCADE STREET, IRVING, TX (WY 1993)-CONTINUEd

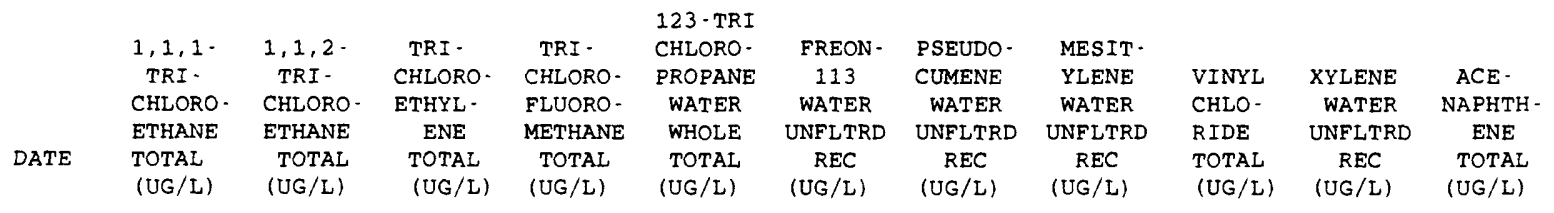

\begin{tabular}{|c|c|c|c|c|c|c|c|c|c|c|c|}
\hline \multicolumn{12}{|l|}{ OCT 1992} \\
\hline $07-07$ & $<0.3$ & $<0.2$ & $<0.2$ & $<0.2$ & $<0.2$ & $<0.5$ & $<0.20$ & $<0,20$ & $<0.2$ & $<0.20$ & $<5.0$ \\
\hline \multicolumn{12}{|l|}{ OCT } \\
\hline $28-29$ & $<0.2$ & $<0.2$ & $<0.2$ & $<0.2$ & $<0.2$ & $<0.5$ & $<0.20$ & $<0.20$ & $<0.2$ & $<0.20$ & $<5.0$ \\
\hline \multicolumn{12}{|l|}{ Nov } \\
\hline $19 \cdot 19$ & 0.7 & $<0.2$ & $<0.2$ & $<0.2$ & $<0.2$ & $<0.5$ & 0.20 & $<0.20$ & $<0.2$ & $<0.20$ & $<5.0$ \\
\hline \multicolumn{12}{|l|}{$D E C$} \\
\hline $\begin{array}{c}09-09 \\
\text { JAN } 1993\end{array}$ & $<0.2$ & $<0.2$ & $<0.2$ & $<0.2$ & $<0.2$ & $<0.5$ & $<0.20$ & $<0.20$ & $<0.2$ & $<0.20$ & $<5.0$ \\
\hline 09.09 & $<0.2$ & $<0.2$ & $<0.2$ & $<0.2$ & $<0.2$ & $<0.5$ & 0.60 & 1.7 & $<0.2$ & 0.30 & $<5.0$ \\
\hline \multicolumn{12}{|l|}{ JAN } \\
\hline $28 \cdot 28$ & $<0.2$ & $<0.2$ & $<0.2$ & $<0.2$ & $<0.2$ & $<0.5$ & 0.20 & $<0.20$ & $<0.2$ & $<0.20$ & $<5.0$ \\
\hline
\end{tabular}

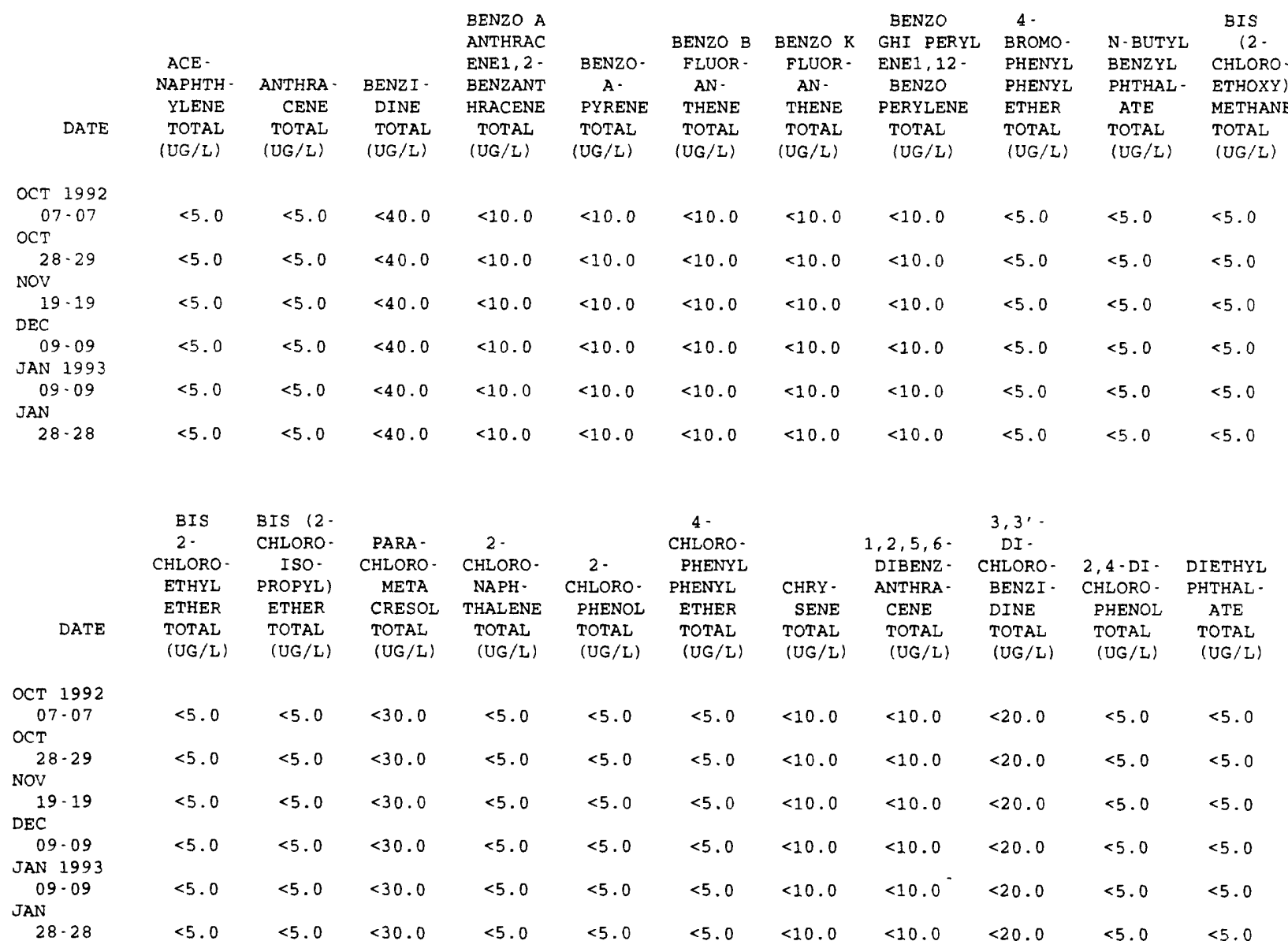


Table 6. Water-quality data for storm-sewer outfall stations, Irving, Texas, 1992-93-Continued

08056100 - TRIBUTARY TO ELM FORK TRINITY RIVER OUTFALL AT CASCADE STREET, IRVING, TX (WY 1993)-CONTINUEd

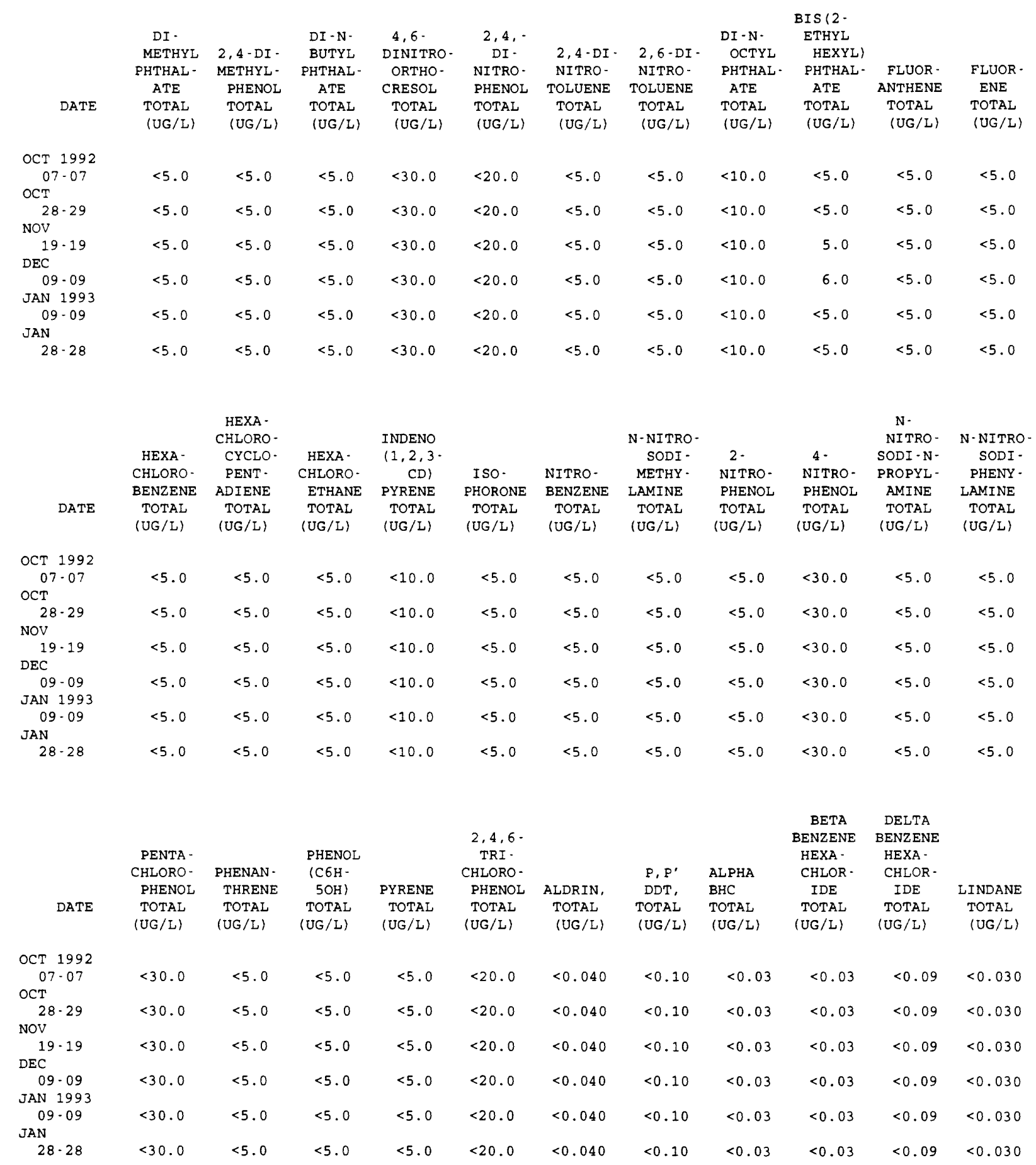


Table 6. Water-quality data for storm-sewer outfall stations, Irving, Texas, 1992-93-Continued

08056100 - TRIBUTARY TO ELM FORK TRINITY RIVER OUTFALL AT CASCADE STREET, IRVING, TX (WY 1993)-CONTINUEd

\begin{tabular}{|c|c|c|c|c|c|c|c|c|c|c|c|}
\hline & $\begin{array}{l}\text { CHLOR - } \\
\text { DANE }\end{array}$ & $\begin{array}{l}\text { CHLOR - } \\
\text { DANE }\end{array}$ & & & & & $\begin{array}{l}\text { ENDO- } \\
\text { SULFAN - }\end{array}$ & & & & \\
\hline & CIS & TRANS & & & & & $I$ & ENDO - & ENDO - & ENDRIN & ENDRIN \\
\hline & WATER & WATER & CHLOR - & $P, P^{\prime}$ & $P, P^{\prime}$ & DI - & WATER & SULFAN & SULFAN & WATER & ALDE - \\
\hline & WHOLE & WHOLE & DANE, & DDD & $\mathrm{DDE}$ & ELDRIN & WHOLE & BETA & SULFATE & UNFLTRD & HYDE \\
\hline DATE & $\begin{array}{l}\text { TOTAL } \\
\text { (UG/L) }\end{array}$ & $\begin{array}{l}\text { TOTAL } \\
\text { (UG/L) }\end{array}$ & $\begin{array}{l}\text { TOTAL } \\
\text { (UG/L) }\end{array}$ & $\begin{array}{l}\text { TOTAL } \\
\text { (UG/L) }\end{array}$ & $\begin{array}{l}\text { TOTAL } \\
\text { (UG/L) }\end{array}$ & $\begin{array}{l}\text { TOTAL } \\
\text { (UG/L) }\end{array}$ & $\begin{array}{l}\text { REC } \\
\text { (UG/L) }\end{array}$ & $\begin{array}{l}\text { TOTAL } \\
\text { (UG/L) }\end{array}$ & $\begin{array}{l}\text { TOTAL } \\
(U G / L)\end{array}$ & $\begin{array}{c}\text { REC } \\
\text { (UG/L) }\end{array}$ & $\begin{array}{l}\text { TOTAL } \\
(\mathrm{UG} / \mathrm{L})\end{array}$ \\
\hline Г 1992 & & & & & & & & & & & \\
\hline $\begin{array}{l}07-07 \\
\mathrm{~T}\end{array}$ & $<0.10$ & $<0.10$ & $<0.1$ & $<0.10$ & $<0.04$ & $<0.020$ & $<0.10$ & $<0.04$ & $<0.60$ & $<0.060$ & $<0.20$ \\
\hline $\begin{array}{l}28-29 \\
\mathrm{~V}\end{array}$ & $<0.10$ & $<0.10$ & $<0.1$ & $<0.10$ & $<0.04$ & $<0.020$ & $<0.10$ & $<0.04$ & $<0.60$ & $<0.060$ & $<0.20$ \\
\hline $\begin{array}{l}19 \cdot 19 \\
C\end{array}$ & $<0.10$ & $<0.10$ & $<0.1$ & $<0.10$ & $<0.04$ & $<0.020$ & $<0.10$ & $<0.04$ & $<0.60$ & $<0.060$ & $<0.20$ \\
\hline $\begin{array}{l}09-09 \\
\text { N } 1993\end{array}$ & $<0.10$ & $<0.10$ & $<0.1$ & $<0.10$ & $<0.04$ & $<0.020$ & $<0.10$ & $<0.04$ & $<0.60$ & $<0.060$ & $<0.20$ \\
\hline $\begin{array}{l}09 \cdot 09 \\
N\end{array}$ & $<0.10$ & $<0.10$ & $<0.1$ & $<0.10$ & $<0.04$ & $<0.020$ & $<0.10$ & $<0.04$ & $<0.60$ & $<0.060$ & $<0.20$ \\
\hline $28-28$ & $<0.10$ & $<0.10$ & $<0.1$ & $<0.10$ & $<0.04$ & $<0.020$ & $<0.10$ & $<0.04$ & $<0.60$ & $<0.060$ & $<0.20$ \\
\hline & & HEPTA - & & AROCLOR & AROCLOR & AROCLOR & AROCLOR & AROCLOR & AROCLOR & AROCLOR & \\
\hline & HEPTA - & CHLOR & TOX - & 1016 & 1221 & 1232 & 1242 & 1248 & 1254 & 1260 & DI - \\
\hline DATE & $\begin{array}{l}\text { CHLOR, } \\
\text { TOTAL } \\
\text { (UG/L) }\end{array}$ & $\begin{array}{c}\text { EPOXIDE } \\
\text { TOTAL } \\
\text { (UG/L) }\end{array}$ & $\begin{array}{c}\text { APHENE, } \\
\text { TOTAL } \\
\text { (UG/L) }\end{array}$ & $\begin{array}{c}\text { PCB } \\
\text { TOTAL } \\
\text { (UG/L) }\end{array}$ & $\begin{array}{c}\text { PCB } \\
\text { TOTAL } \\
\{U G / L\rangle\end{array}$ & $\begin{array}{c}\text { PCB } \\
\text { TOTAL } \\
\text { (UG/L) }\end{array}$ & $\begin{array}{c}\text { PCB } \\
\text { TOTAL } \\
(U G / L)\end{array}$ & $\begin{array}{c}\text { PCB } \\
\text { TOTAL } \\
(U G / L)\end{array}$ & $\begin{array}{c}\text { PCB } \\
\text { TOTAL } \\
\text { (UG/L) }\end{array}$ & $\begin{array}{c}\text { PCB } \\
\text { TOTAL } \\
\text { (UG/L) }\end{array}$ & $\begin{array}{c}\text { AZINON, } \\
\text { TOTAL } \\
\text { (UG/L) }\end{array}$ \\
\hline
\end{tabular}

\begin{tabular}{|c|c|c|c|c|c|c|c|c|c|c|c|}
\hline \multicolumn{12}{|l|}{ OCT 1992} \\
\hline 07.07 & $<0.030$ & $<0.80$ & $<2$ & $<0.1$ & $<1.0$ & $<0.1$ & $<0.1$ & $<0.1$ & $<0.1$ & 0.1 & $<0.10$ \\
\hline \multicolumn{12}{|l|}{ OCT } \\
\hline $28-29$ & $<0.030$ & $<0.80$ & $<2$ & $<0.1$ & $<1.0$ & $<0.1$ & $<0.1$ & $<0.1$ & $<0.1$ & $<0.1$ & $<0.10$ \\
\hline \multicolumn{12}{|l|}{ NOV } \\
\hline $19-19$ & $<0.030$ & $<0.80$ & $<2$ & $<0.1$ & $<1.0$ & $<0.1$ & $<0.1$ & $<0.1$ & $<0.1$ & $<0.1$ & $<0.10$ \\
\hline \multicolumn{12}{|l|}{ DEC } \\
\hline 09.09 & $<0.030$ & $<0.80$ & $<2$ & $<0.1$ & $<1.0$ & $<0.1$ & $<0.1$ & $<0.1$ & $<0.1$ & $<0.1$ & $<0.10$ \\
\hline \multicolumn{12}{|l|}{ JAN 1993} \\
\hline 09.09 & $<0.030$ & $<0.80$ & $<2$ & $<0.1$ & $<1.0$ & $<0.1$ & $<0.1$ & $<0.1$ & $<0.1$ & $<0.1$ & $<0.05$ \\
\hline \multicolumn{12}{|l|}{ JAN } \\
\hline $28-28$ & $<0.030$ & $<0.80$ & $<2$ & $<0.1$ & $<1.0$ & $<0.1$ & $<0.1$ & $<0.1$ & $<0.1$ & $<0.1$ & 0.10 \\
\hline
\end{tabular}


Table 7. Water-quality data for storm-sewer outfall stations, Mesquite, Texas, 1992-93

08061910 - SOUTH MESQUITE CREEK OUTFALL AT I-635, MESQUITE, TX (WY 1993)

\begin{tabular}{|c|c|c|c|c|c|c|c|c|c|c|c|c|}
\hline DATE & TIME & $\begin{array}{l}\text { PRECIP - } \\
\text { ITATION } \\
\text { TOTAL } \\
\text { INCHES/ } \\
\text { STORM }\end{array}$ & $\begin{array}{l}\text { ELAPSED } \\
\text { TIME } \\
\text { OF } \\
\text { STORM } \\
\text { (HOURS) }\end{array}$ & $\begin{array}{l}\text { STORM } \\
\text { WATER } \\
\text { FLOW } \\
\text { (MGD) }\end{array}$ & $\begin{array}{l}\text { SPE- } \\
\text { CIFIC } \\
\text { CON- } \\
\text { DUCT- } \\
\text { ANCE } \\
\text { (US/CM) }\end{array}$ & $\begin{array}{c}\text { SPE- } \\
\text { CIFIC } \\
\text { CON- } \\
\text { DUCT- } \\
\text { ANCE } \\
\text { LAB } \\
\text { (US/CM) }\end{array}$ & $\begin{array}{c}\text { PH } \\
\text { WATER } \\
\text { WHOLE } \\
\text { FIELD } \\
\text { (STAND- } \\
\text { ARD } \\
\text { UNITS) }\end{array}$ & $\begin{array}{c}\text { PH } \\
\text { WATER } \\
\text { WHOLE } \\
\text { LAB } \\
\text { (STAND- } \\
\text { ARD } \\
\text { UNITS) }\end{array}$ & $\begin{array}{c}\text { TEMPER - } \\
\text { ATURE } \\
\text { WATER } \\
\text { (DEG C) }\end{array}$ & $\begin{array}{l}\text { OXYGEN } \\
\text { DEMAND, } \\
\text { CHEM- } \\
\text { ICAL } \\
\text { (HIGH } \\
\text { LEVEL) } \\
\text { (MG/L) }\end{array}$ & $\begin{array}{l}\text { OXYGEN } \\
\text { DEMAND, } \\
\text { BIO- } \\
\text { CHEM- } \\
\text { ICAL, } \\
5 \text { DAY } \\
\text { (MG/L) }\end{array}$ & $\begin{array}{l}\text { COLI - } \\
\text { FORM, } \\
\text { FECAL, } \\
0.7 \\
\text { UM-MF } \\
\text { (COLS. } \\
100 \mathrm{ML} \text { ) }\end{array}$ \\
\hline \multicolumn{13}{|l|}{ FEB 1993} \\
\hline $24-24$ & 1637 & 0.31 & 6.1 & 0.22 & 202 & 168 & 7.8 & 7.5 & 13.0 & 57 & 7.0 & K490 \\
\hline \multicolumn{13}{|l|}{ MAR } \\
\hline $19-19$ & 1414 & 0.50 & 4.0 & 0.32 & 104 & 115 & 6.8 & 7.8 & 14.5 & 51 & 5.0 & 1100 \\
\hline \multicolumn{13}{|l|}{ MAR } \\
\hline $28-28$ & 1054 & 0.21 & 1.8 & 0.12 & 169 & $\cdots$ & 8.0 & $\cdots$ & 20.0 & 110 & 7.2 & 3000 \\
\hline \multicolumn{13}{|l|}{ APR } \\
\hline 03.04 & 1741 & 0.47 & 8.1 & 0.28 & 58 & 96 & 8.3 & 7.5 & 14.5 & 57 & 7.8 & 2500 \\
\hline \multicolumn{13}{|l|}{ APR } \\
\hline $29-29$ & 0010 & 0.79 & 3.7 & 0.65 & 384 & 74 & 7.6 & 6.9 & 21.0 & 54 & 7.8 & 90000 \\
\hline \multicolumn{13}{|l|}{ MAY } \\
\hline $23 \cdot 23$ & 1327 & 0.41 & 2.3 & 0.28 & 66 & 108 & 8.0 & 7.4 & 21.5 & 64 & 5.2 & 3300 \\
\hline \multicolumn{13}{|l|}{ JUN } \\
\hline 09.09 & 1930 & 0.92 & 1.3 & 0.65 & 240 & 86 & 8.0 & 6.7 & 24.5 & 49 & 7.0 & 8700 \\
\hline
\end{tabular}

\begin{tabular}{|c|c|c|c|c|c|c|c|c|c|c|c|c|}
\hline & $\begin{array}{r}\text { STREP - } \\
\text { TOCOCCI }\end{array}$ & HARD - & $\begin{array}{l}\text { HARD - } \\
\text { NESS }\end{array}$ & $\begin{array}{l}\text { ALKA- } \\
\text { LINITY }\end{array}$ & $\begin{array}{l}\text { SOLIDS, } \\
\text { SUM OF }\end{array}$ & $\begin{array}{l}\text { RESIDUE } \\
\text { TOTAL }\end{array}$ & $\begin{array}{l}\text { SOLIDS, } \\
\text { RESIDUE }\end{array}$ & & MAGNE - & & & SODIUM \\
\hline & FECAL, & NESS & NONCARB & WAT DIS & CONSTI - & AT 105 & AT 180 & CALCIUM & SIUM, & SODIUM, & & $\mathrm{AD}$ \\
\hline & $\begin{array}{l}\text { KF AGAR } \\
\text { (COLS. }\end{array}$ & $\begin{array}{l}\text { TOTAL } \\
\text { (MG/L }\end{array}$ & $\begin{array}{l}\text { DISSOLV } \\
\text { FLD. AS }\end{array}$ & $\begin{array}{l}\text { FIX END } \\
\text { FIELD }\end{array}$ & $\begin{array}{c}\text { TUENTS, } \\
\text { DIS - }\end{array}$ & $\begin{array}{l}\text { DEG . C, } \\
\text { SUS - }\end{array}$ & $\begin{array}{l}\text { DEG. C } \\
\text { DIS - }\end{array}$ & DIS - & $\begin{array}{l}\text { DIS - } \\
\text { SOLVED }\end{array}$ & $\begin{array}{l}\text { DIS - } \\
\text { SOLVED }\end{array}$ & & $\begin{array}{l}\text { SORP - } \\
\text { TION }\end{array}$ \\
\hline DATE & $\begin{array}{c}\text { PER } \\
100 \mathrm{ML})\end{array}$ & $\begin{array}{c}\text { AS } \\
\text { (ACO3) }\end{array}$ & $\begin{array}{r}\mathrm{CACO} 3 \\
(\mathrm{MG} / \mathrm{L})\end{array}$ & $\begin{array}{l}\mathrm{CACO} 3 \\
(\mathrm{MG} / \mathrm{L})\end{array}$ & $\begin{array}{c}\text { SOLVED } \\
(\mathrm{MG} / \mathrm{L})\end{array}$ & $\begin{array}{l}\text { PENDED } \\
(\mathrm{MG} / \mathrm{L})\end{array}$ & $\begin{array}{l}\text { SOLVED } \\
\text { (MG /L) }\end{array}$ & $\begin{array}{c}(\mathrm{MG} / \mathrm{L} \\
\mathrm{AS} \text { CA) }\end{array}$ & $\begin{array}{c}\text { (MG } / L \\
\text { AS MG) }\end{array}$ & $\begin{array}{c}\text { (MG/L } \\
\text { AS NA) }\end{array}$ & $\begin{array}{l}\text { SODIUM } \\
\text { PERCENT }\end{array}$ & RATIO \\
\hline
\end{tabular}

\begin{tabular}{|c|c|c|c|c|c|c|c|c|c|c|c|c|}
\hline \multicolumn{13}{|l|}{ FEB 1993} \\
\hline $24-24$ & 5900 & 54 & 19 & 35 & 88 & 25 & 92 & 20 & 0.96 & 7.6 & 23 & 0.5 \\
\hline \multicolumn{13}{|l|}{ MAR } \\
\hline $19-19$ & 1600 & 40 & 7 & 33 & 60 & 57 & 69 & 15 & 0.59 & 3.3 & 15 & 0.2 \\
\hline \multicolumn{13}{|l|}{ MAR } \\
\hline $28-28$ & 17000 & $\cdots$ & $\cdots$ & 30 & - & $\cdots$ & - & $\cdots$ & $\cdots$ & $\cdots$ & $\cdot$ & - \\
\hline \multicolumn{13}{|l|}{$A P R$} \\
\hline $03 \cdot 04$ & 8800 & 27 & 6 & 21 & 48 & 52 & 56 & 10 & 0.44 & 3.1 & 19 & 0.3 \\
\hline \multicolumn{13}{|l|}{ APR } \\
\hline $29-29$ & 28000 & 18 & 6 & 12 & 35 & 46 & 51 & 6.6 & 0.29 & 1.4 & 13 & 0.1 \\
\hline \multicolumn{13}{|l|}{ MAY } \\
\hline $23-23$ & 20000 & 26 & 10 & 16 & 46 & 26 & 52 & 9.8 & 0.39 & 2.6 & 17 & 0.2 \\
\hline \multicolumn{13}{|l|}{ JUN } \\
\hline 09.09 & 3700 & 22 & 4 & 18 & 40 & 21 & 50 & 8.0 & 0.37 & 2.0 & 16 & 0.2 \\
\hline
\end{tabular}

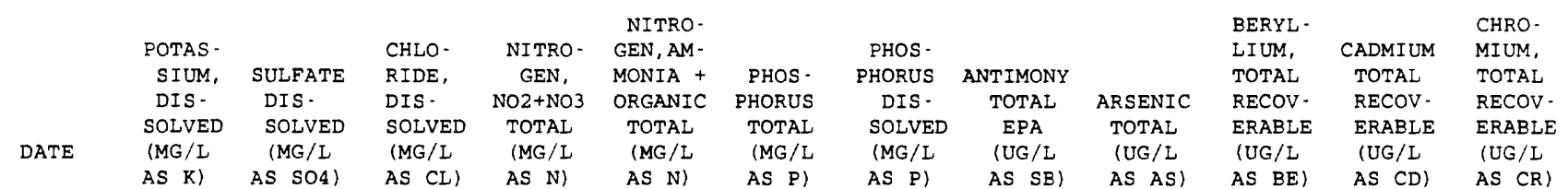

\begin{tabular}{|c|c|c|c|c|c|c|c|c|c|c|c|c|}
\hline $24-24$ & 1.5 & 18 & 11 & 1.50 & 1.0 & 0.090 & 0.050 & $<10.0$ & 1 & $<10$ & $<1$ & 7 \\
\hline \multicolumn{13}{|l|}{ MAR } \\
\hline $19 \cdot 19$ & 1.1 & 8.3 & 2.5 & 0.300 & 0.70 & 0.090 & 0.020 & $<20.0$ & 1 & $<10$ & $<1$ & 4 \\
\hline \multicolumn{13}{|l|}{ MAR } \\
\hline $28-28$ & $\cdots$ & $\cdots$ & $\cdots$ & 0.420 & 1.5 & 0.240 & 0.120 & $<10.0$ & 2 & $<10$ & $<1$ & 3 \\
\hline \multicolumn{13}{|l|}{ APR } \\
\hline $03-04$ & 1.0 & 7.2 & 2.4 & 0.350 & 1.0 & 0.160 & 0.080 & $<10.0$ & $<1$ & $<10$ & $<1$ & 3 \\
\hline \multicolumn{13}{|l|}{ APR } \\
\hline $29 \cdot 29$ & 1.7 & 3.9 & 2.1 & 0.280 & 0.90 & 0.170 & 0.090 & $<20.0$ & $<1$ & $<10$ & $<1$ & $<1$ \\
\hline \multicolumn{13}{|l|}{ MAY } \\
\hline $23-23$ & 1.3 & 5.9 & 2.2 & 0.410 & 0.80 & 0.150 & 0.090 & $<20.0$ & $<1$ & $<10$ & $<1$ & 4 \\
\hline \multicolumn{13}{|l|}{ JUN } \\
\hline $09 \cdot 09$ & 1.5 & 4.8 & 3.4 & 0.390 & 0.60 & 0.100 & 0.110 & $<10.0$ & 1 & $<10$ & $<1$ & 3 \\
\hline
\end{tabular}


Table 7. Water-quality data for storm-sewer outfall stations, Mesquite, Texas, 1992-93-Continued

08061910 - SOUTH MESQUITE CREEK OUTFALL AT I-635, MESQUITE, TX (WY 1993)-Continued

DATE

FEB 1993
$24-24$
MAR
$19-19$
MAR
$28-28$
APR
$03-04$
APR
$29-29$
MAY
$23-23$
JUN
$09-09$

COPPER,

TOTAL CYANTDE

RECOV- TOTAL CYANIDE RECOV- RECOV- RECOV-

ERABLE EPA TOTAL ERABLE ERABLE ERABLE

(UG/L (MG/L UMG/L

AS CU)

\section{(MG/L}

AS CN)

(UTG/L

AS PB)

(UG/L

(UG/L

AS NI)

$\begin{array}{lllll}6 & <0.010 & <0.010 & 17 & <0.10 \\ 4 & <0.010 & <0.010 & 29 & <0.10 \\ 7 & <0.010 & 0.010 & 38 & <0.10 \\ 6 & <0.010 & <0.010 & 30 & <0.10 \\ 8 & <0.010 & <0.010 & 35 & <0.10 \\ 5 & <0.010 & <0.010 & 23 & <0.10 \\ 8 & <0.010 & <0.010 & 29 & 0.10\end{array}$

$\begin{array}{ll}3 & <2 \\ 3 & <2 \\ 3 & <2 \\ 2 & <2 \\ 3 & <2 \\ 3 & <2 \\ 3 & <2\end{array}$

SELE-
NIUM,
TOTAL
(UG / L
AS SE)

$\begin{array}{lc} & \text { SILVER } \\ \text { SILVER, } & \text { TOTAL } \\ \text { TOTAL } & \text { RECOV- } \\ \text { RECOV- } & \text { ERABLE } \\ \text { ERABLE } & \text { EPA } \\ \text { (UG/L } & \text { (UG/L } \\ \text { AS AG) } & \text { AS AG) }\end{array}$

THAL -

LIUM,

TOTAL

(UG/L

AS TL)
ZINC

TOTAL CARBON,

RECOV - ORGANIC

ERABLE TOTAL

(UG/L (MG/L

AS $2 N$ ) AS C)
OIL AND

GREASE,

TOTAL

RECOV

GRAVI

DATE

(MG/L)

ACRO-
PHENOLS LEIN

TOTAL TOTAL

(UG/L) (UG/L)
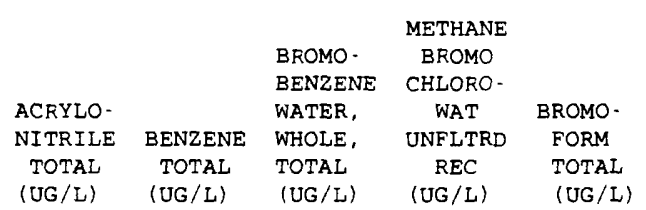$$
\text { N }
$$

BENZENE
N-BUTYL
WATER
UNFLTRD

BENZENE BENZENE

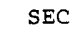
SEC
BUTYL BUTYL

WATER

REC

(UG/L)

REC
(UG/L)

(UG/L)
TERT -

TETRA

CHER -

INFLTRD RIDE

(UG/L) TOTAL

$\begin{array}{rrrrrr}6 & <20 & <20 & <0.2 & <0.2 & <0.20 \\ 2 & <20 & <20 & <0.2 & <0.2 & <0.20 \\ 4 & <20 & <20 & <0.2 & <0.2 & <0.20 \\ 1 & <20 & <20 & <0.2 & <0.2 & <0.20 \\ 12 & <20 & <20 & <0.2 & <0.2 & <0.20 \\ <1 & <500 & <500 & <5.0 & <5.0 & <5.00 \\ 4 & <1000 & <1000 & <10 & <10 & <10.0\end{array}$

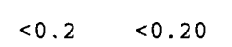

$<0.20$

$<0.20$

$<0.2$

$<0.2<0.20$

$<0.20$

$<0.20$

$<0.2$

$<0.2<0.20$

$<0.20$

$<0.20$

$<0.2$

$<0.2<0.20$

$<0.20$

$<0.20$

$<0.2$

$<0.2<0.20$

$<0.20$

$<0.20$

$<0.2$

$<5.0<5.0$

$<5.0$

$<5.0$

$<5.0$

$<10<10$

$<10$

$<10$

$<10$

$\begin{array}{clccc} & & & & 2- \\ & & \text { CHLORO- } & & \text { CHLORO- } \\ & & \text { DI- } & & \text { ETHYL- } \\ & \text { CHLORO- } & \text { BROMO- } & \text { CHLORO- } & \text { VINYL- } \\ \text { BATE } & \text { BENZENE } & \text { METHANE } & \text { ETHANE } & \text { ETHER } \\ & \text { TOTAL } & \text { TOTAL } & \text { TOTAL } & \text { TOTAL } \\ & (U G / L) & (U G / L) & (U G / L) & (U G / L)\end{array}$

$\begin{array}{cccc}\text { O- } & \\ & \text { CHLORO- } & \text { TOLUENE } \\ \text { CHLORO- } & \text { METHYL } & \text { TOLUENE } & \text { P-CHLOR } \\ \text { FORM } & \text { RIDE } & \text { WATER } & \text { WATER } \\ \text { TOTAL } & \text { THOTAL } & \text { TOTAL } & \text { RELTRD } \\ \text { (UG/L) } & \text { (UG/L) } & \text { (UG/L) } & \text { (UG/L) }\end{array}$

DIBROMO DI - 1,2 -

CHLORO - BROMO - DIBROMO DI.

PROPANE METHANE ETHANE CHLORO-

WATER WATER WATER BFOMO-

WHOLE WHOLE WHOLE METHANE

TOT.REC RECOVER TOTAL TOTAL

$\begin{array}{llll}(U G / L) & \text { (UG/L) } & \text { (UG/L) } & \text { (UG/L) }\end{array}$

\begin{tabular}{|c|c|c|c|c|c|c|c|c|c|c|c|c|}
\hline FEB 1993 & & & & & & & & & & & & \\
\hline $24-24$ & $<0.20$ & $<0.2$ & $<0.2$ & $<1.0$ & $<0.2$ & $<0.2$ & $<0.2$ & $<0.20$ & $<1.0$ & $<0.2$ & $<0.2$ & $<0.2$ \\
\hline MAR & & & & & & & & & & & & \\
\hline $19-19$ & $<0.20$ & $<0.2$ & $<0.2$ & $<1.0$ & $<0.2$ & $<0.2$ & $<0.2$ & $<0.20$ & $<1.0$ & $<0.2$ & $<0.2$ & $<0.2$ \\
\hline MAR & & & & & & & & & & & & \\
\hline $28-28$ & $<0.20$ & $<0.2$ & $<0.2$ & $<1.0$ & $<0.2$ & $<0.2$ & $<0.2$ & $<0.20$ & $<1.0$ & $<0.2$ & $<0.2$ & $<0.2$ \\
\hline APR & & & & & & & & & & & & \\
\hline 03.04 & $<0.20$ & $<0.2$ & $<0.2$ & $<1.0$ & $<0.2$ & $<0.2$ & $<0.2$ & $<0.20$ & $<1.0$ & $<0.2$ & $<0.2$ & $<0.2$ \\
\hline APR & & & & & & & & & & & & \\
\hline $29 \cdot 29$ & $<0.20$ & $<0.2$ & $<0.2$ & $<1.0$ & 0.2 & $<0.2$ & $<0.2$ & $<0.20$ & $<1.0$ & $<0.2$ & $<0.2$ & $<0.2$ \\
\hline MAY & & & & & & & & & & & & \\
\hline $23-23$ & $<5.0$ & $<5.0$ & $<5.0$ & $<25$ & $<5.0$ & $<5.0$ & $<5.0$ & $<5.0$ & $<25$ & $<5.0$ & $<5.0$ & $<5.0$ \\
\hline JUN & & & & & & & & & & & & \\
\hline 09.09 & $<10$ & $<10$ & $<10$ & $<50$ & $<10$ & $<10$ & $<10$ & $<10$ & $<50$ & $<10$ & $<10$ & $<10$ \\
\hline
\end{tabular}


Table 7. Water-quality data for storm-sewer outfall stations, Mesquite, Texas, 1992-93-Continued

08061910 - SOUTH MESQUITE CREEK OUTFALL AT I-635, MESQUITE, TX (WY 1993)-Continued

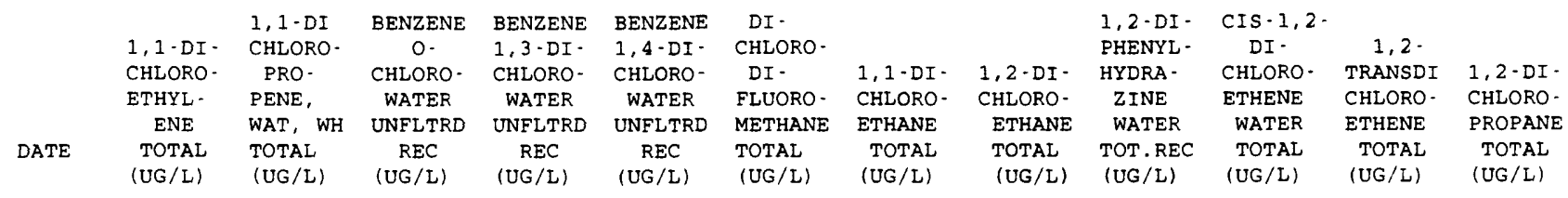

\begin{tabular}{|c|c|c|c|c|c|c|c|c|c|c|c|c|}
\hline \multicolumn{13}{|l|}{ FEB 1993} \\
\hline $\begin{array}{l}24 \cdot 24 \\
\text { MAR }\end{array}$ & $<0.2$ & $<0.2$ & $<5.0$ & $<5.0$ & $<5.0$ & $<0.2$ & $<0.2$ & $<0.2$ & $<5.0$ & $<0.2$ & $<0.2$ & $<0.2$ \\
\hline $\begin{array}{l}\text { MAR } \\
\quad 19-19\end{array}$ & $<0.2$ & $<0.2$ & $<0.20$ & $<0.20$ & $<0.20$ & $<0.2$ & $<0.2$ & $<0.2$ & $<5.0$ & $<0.2$ & $<0.2$ & $<0.2$ \\
\hline \multicolumn{13}{|l|}{ MAR } \\
\hline $28 \cdot 28$ & $<0.2$ & $<0.2$ & $<0.20$ & $<0.20$ & $<0.20$ & $<0.2$ & $<0.2$ & $<0.2$ & $<5.0$ & $<0.2$ & $<0.2$ & $<0.2$ \\
\hline \multicolumn{13}{|l|}{ APR } \\
\hline $03-04$ & $<0.2$ & $<0.2$ & $<5.0$ & $<5.0$ & $<5.0$ & $<0.2$ & $<0.2$ & $<0.2$ & $<5.0$ & $<0.2$ & $<0.2$ & $<0.2$ \\
\hline \multicolumn{13}{|l|}{ AFR } \\
\hline $29-29$ & $<0.2$ & $<0.2$ & $<0.20$ & $<0.20$ & $<0.20$ & $<0.2$ & $<0.2$ & $<0.2$ & $<5.0$ & $<0.2$ & $<0.2$ & $<0.2$ \\
\hline \multicolumn{13}{|l|}{ MAY } \\
\hline $23 \cdot 23$ & $<5.0$ & $<5.0$ & $<5.0$ & $<5.0$ & $<5.0$ & $<5.0$ & $<5.0$ & $<5.0$ & $<5.0$ & $<5.0$ & $<5.0$ & $<5.0$ \\
\hline JUN & & & & & & & & & & & & \\
\hline 09.09 & $<10$ & $<10$ & $<5.0$ & $<5.0$ & $<5,0$ & $<10$ & $<10$ & $<10$ & $<5.0$ & $<10$ & $<10$ & $<10$ \\
\hline
\end{tabular}

\begin{tabular}{|c|c|c|c|c|c|c|c|c|c|c|c|c|}
\hline & $\begin{array}{l}1,3 \cdot D I- \\
\text { CHLORO- }\end{array}$ & $\begin{array}{l}2,2-\mathrm{DI} \\
\text { CHLORO- } \\
\text { PRO- }\end{array}$ & $\begin{array}{c}\text { CIS } \\
1,3 \cdot D I .\end{array}$ & $\begin{array}{l}\text { TRANS - } \\
1,3 \text {-DI - }\end{array}$ & & $\begin{array}{c}\text { HEXA- } \\
\text { CHLORO- }\end{array}$ & $\begin{array}{l}\text { ISO- } \\
\text { PROPYL- } \\
\text { BENZENE }\end{array}$ & $\begin{array}{l}\text { P-ISO- } \\
\text { PROPYL - } \\
\text { TOLUENE }\end{array}$ & & $\begin{array}{l}\text { METHYL - } \\
\text { ENE }\end{array}$ & $\begin{array}{c}\text { METHYL } \\
\text { ETHER } \\
\text { TERT- }\end{array}$ & \\
\hline & PROPANE & PANE & CHLORO- & CHLORO- & ETHYL - & BUT - & WATER & WATER & METHYL - & CHLO- & BUTYL & NAPHTH - \\
\hline & WAT. WH & WAT, WH & PROPENE & PROPENE & BENZENE & ADIENE & WHOLE & WHOLE & BROMIDE & RIDE & WAT UNF & ALENE \\
\hline & $\begin{array}{l}\text { TOTAL } \\
\text { (UG/L) }\end{array}$ & $\begin{array}{l}\text { TOTAL } \\
\text { (UG/L) }\end{array}$ & $\begin{array}{l}\text { TOTAL } \\
\text { (UG/L) }\end{array}$ & $\begin{array}{l}\text { TOTAL } \\
\text { (UG/L) }\end{array}$ & $\begin{array}{l}\text { TOTAL } \\
(\mathrm{UG} / \mathrm{L})\end{array}$ & $\begin{array}{l}\text { TOTAL } \\
\text { (UG/L) }\end{array}$ & $\begin{array}{l}\text { REC } \\
(U G / L)\end{array}$ & $\begin{array}{l}\text { REC } \\
\text { (UG/L) }\end{array}$ & $\begin{array}{l}\text { TOTAL } \\
\text { (UG/L) }\end{array}$ & $\begin{array}{l}\text { TOTAL } \\
\text { (UG/L) }\end{array}$ & $\begin{array}{c}\text { REC } \\
(U G / L)\end{array}$ & $\begin{array}{l}\text { TOTAL } \\
\text { (UG/L) }\end{array}$ \\
\hline
\end{tabular}

\begin{tabular}{|c|c|c|c|c|c|c|c|c|c|c|c|c|}
\hline $\begin{array}{c}\text { FEB } 1993 \\
24 \cdot 24\end{array}$ & 15? & & & & & & & & & & & \\
\hline $\begin{array}{l}24 \cdot 24 \\
M A R\end{array}$ & $<0.2$ & $<0.2$ & $<0.2$ & $<0.2$ & $<0.2$ & $<5.0$ & $<0.20$ & $<0.20$ & $<0.2$ & $<0.2$ & $<1.0$ & $<5.0$ \\
\hline $19-19$ & $<0.2$ & $<0.2$ & $<0.2$ & $<0.2$ & $<0.2$ & $<0.2$ & $<0.20$ & $<0.20$ & $<0.2$ & $<0.2$ & $<1.0$ & $<0.2$ \\
\hline MAR & & & & & & & & & & & & \\
\hline$\underset{A P R}{28 \cdot 28}$ & $<0.2$ & $<0.2$ & $<0.2$ & $<0.2$ & $<0.2$ & $<0.2$ & $<0.20$ & $<0.20$ & $<0.2$ & $<0.2$ & $<1.0$ & $<0.2$ \\
\hline 03.04 & $<0.2$ & $<0.2$ & $<0.2$ & $<0.2$ & $<0.2$ & $<5.0$ & $<0.20$ & $<0.20$ & $<0.2$ & $<0.2$ & $<1.0$ & $<5.0$ \\
\hline $29-29$ & $<0.2$ & $<0.2$ & $<0.2$ & $<0.2$ & $<0.2$ & $<0.2$ & $<0.20$ & $<0.20$ & $<0.2$ & $<0.2$ & $<1.0$ & $<0.2$ \\
\hline $\begin{array}{l}\text { MAY } \\
23-23 \\
\text { JUN }\end{array}$ & $<5.0$ & $<5.0$ & $<5.0$ & $<5.0$ & $<5.0$ & $<5.0$ & $<5.0$ & $<5.0$ & $<5.0$ & $<5.0$ & $<25$ & $<5.0$ \\
\hline 09.09 & $<10$ & $<10$ & $<10$ & $<10$ & $<10$ & $<5.0$ & $<10$ & $<10$ & $<10$ & $<10$ & $<50$ & $<5.0$ \\
\hline
\end{tabular}

\begin{tabular}{|c|c|c|c|c|c|c|c|c|c|c|c|c|}
\hline & BENZENE & & $\begin{array}{c}\text { ETHANE, } \\
1112-\end{array}$ & $\begin{array}{l}\text { ETHANE, } \\
1,1,2,2\end{array}$ & TETRA - & & $\begin{array}{c}1,2,3- \\
\text { TRI. }\end{array}$ & $\begin{array}{l}\text { BENZENE } \\
1,2,4 \text { - }\end{array}$ & $1,1,1$. & $1,1,2-$ & & \\
\hline & $N$ - PROPY & & TETRA - & TETRA - & CHLORO - & & CHLORO & TRI - & TRI - & TRI - & CHLO & $\mathrm{CHLC}$ \\
\hline & WATER & & CHLORO - & CHLORO- & ETHYL - & & BENZENE & CHLORO- & CHLORO- & CHLORO- & ETHYL- & FLUORO- \\
\hline & $\begin{array}{c}\text { UNFLTRD } \\
\text { REC }\end{array}$ & STYRENE & WAT UNF & WAT UNF & ENE & TOLUENE & WAT, WH & WAT UNF & ETHANE & ETHANE & ENE & METHANE \\
\hline & $\begin{array}{c}\text { REC } \\
(U G / L)\end{array}$ & $\begin{array}{l}\text { TOTAL } \\
\text { (UG/L) }\end{array}$ & $\begin{array}{c}\text { REC } \\
(U G / L)\end{array}$ & $\begin{array}{c}\text { REC } \\
(U G / L)\end{array}$ & $\begin{array}{l}\text { TOTAL } \\
\text { (UG/L) }\end{array}$ & $\begin{array}{l}\text { TOTAL } \\
\text { (UG/L) }\end{array}$ & $\begin{array}{l}\text { REC } \\
(U G / L)\end{array}$ & $\begin{array}{c}\text { REC } \\
(\mathrm{UG} / \mathrm{L})\end{array}$ & $\begin{array}{l}\text { TOTAL } \\
\text { (UG/L) }\end{array}$ & $\begin{array}{l}\text { TOTAL } \\
(U G / L)\end{array}$ & $\begin{array}{l}\text { TOTAL } \\
\text { (UG/L) }\end{array}$ & $\begin{array}{l}\text { TOTAL } \\
\text { (UG/L) }\end{array}$ \\
\hline
\end{tabular}

\begin{tabular}{|c|c|c|c|c|c|c|c|c|c|c|c|c|}
\hline $\begin{array}{c}\text { FEB } 1993 \\
24-24\end{array}$ & $<0 \quad 20$ & $<02$ & $<02$ & $<0 ?$ & $50 ?$ & $10 ?$ & 20 & & & 80 & 8 & $<0,2$ \\
\hline MAR & & & & & & & & & -0.2 & & $<0.2$ & $<0.2$ \\
\hline $19-19$ & $<0.20$ & $<0.2$ & $<0.2$ & $<0.2$ & $<0.2$ & $<0.2$ & $<0.20$ & $<0.20$ & $<0.2$ & $<0.2$ & $<0.2$ & $<0.2$ \\
\hline MAR & & & & & & & & & & & & \\
\hline $28 \cdot 28$ & $<0.20$ & $<0.2$ & $<0.2$ & $<0.2$ & $<0.2$ & $<0.2$ & $<0.20$ & $<0.20$ & $<0.6$ & $<0.2$ & $<0.2$ & $<0.2$ \\
\hline $\begin{array}{l}03.04 \\
A P R\end{array}$ & $<0.20$ & $<0.2$ & $<0.2$ & $<0.2$ & $<0.2$ & $<0.2$ & $<0.20$ & $<5.0$ & $<0.2$ & $<0.2$ & $<0.2$ & $<0.2$ \\
\hline $\begin{array}{l}29-29 \\
\mathrm{MAY}\end{array}$ & $<0.20$ & $<0.2$ & $<0.2$ & $<0.2$ & $<0.2$ & $<0.2$ & $<0.20$ & $<0.20$ & $<0.2$ & $<0.2$ & $<0.2$ & $<0.2$ \\
\hline $\begin{array}{l}23 \cdot 23 \\
\text { JUN }\end{array}$ & $<5.0$ & $<5.0$ & $<5.0$ & $<5.0$ & $<5.0$ & $<5.0$ & $<5.0$ & $<5.0$ & $<5.0$ & $<5.0$ & $<5.0$ & $<5.0$ \\
\hline 09.09 & $<10$ & $<10$ & $<10$ & $<10$ & $<10$ & $<10$ & $<10$ & $<5.0$ & $<10$ & $<10$ & $<10$ & $<10$ \\
\hline
\end{tabular}


Table 7. Water-quality data for storm-sewer outfall stations, Mesquite, Texas, 1992-93-Continued

08061910 - SOUTH MESQUITE CREEK OUTFALL AT I-635, MESQUITE, TX (WY 1993)-Continued

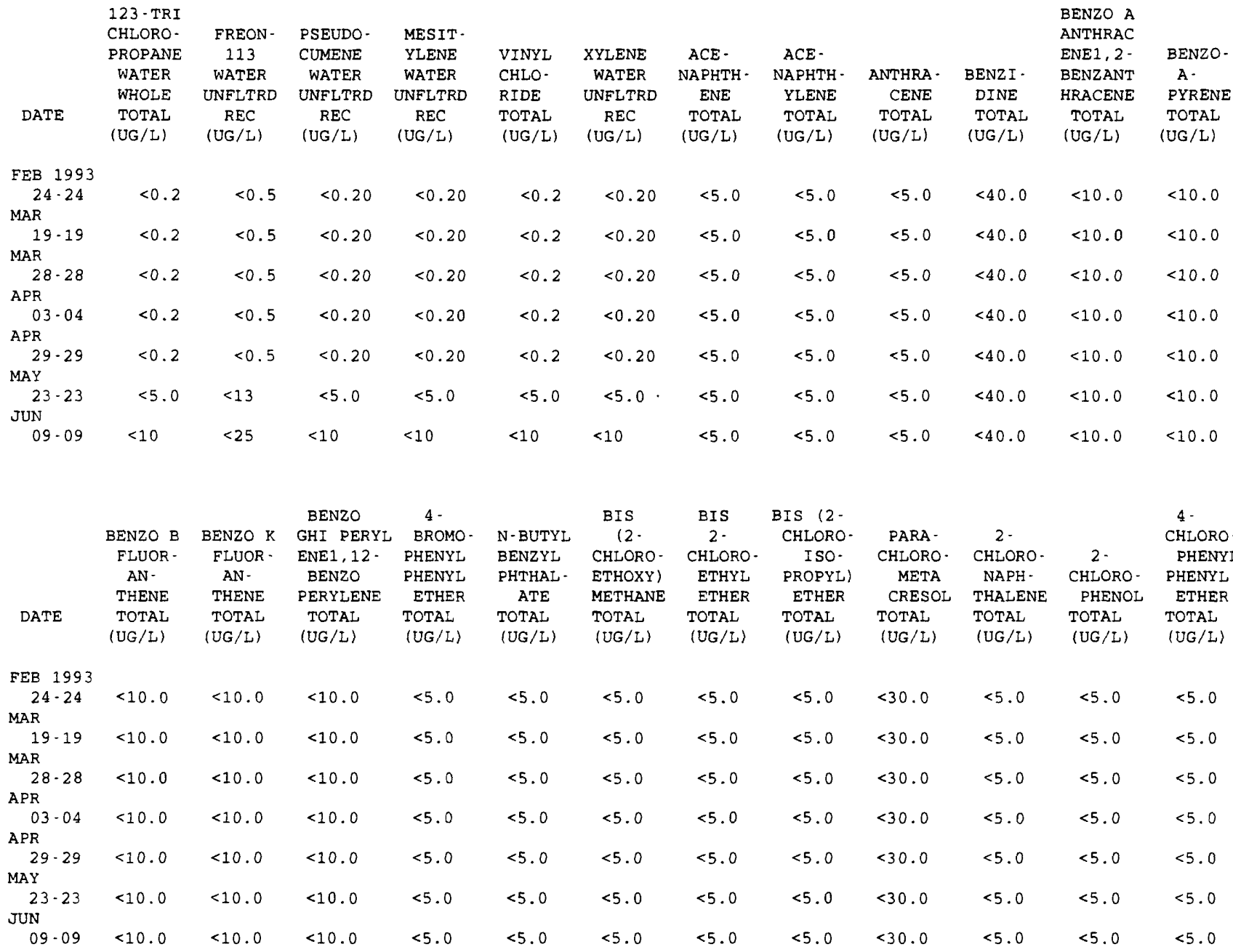

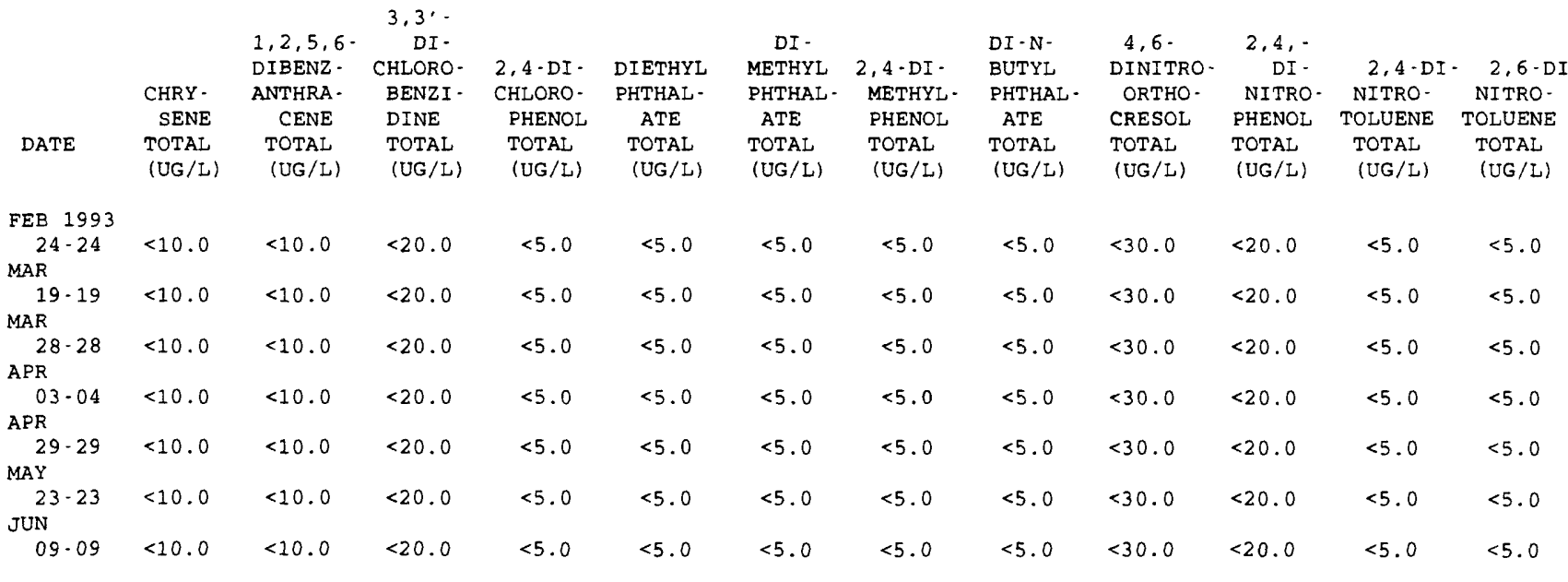




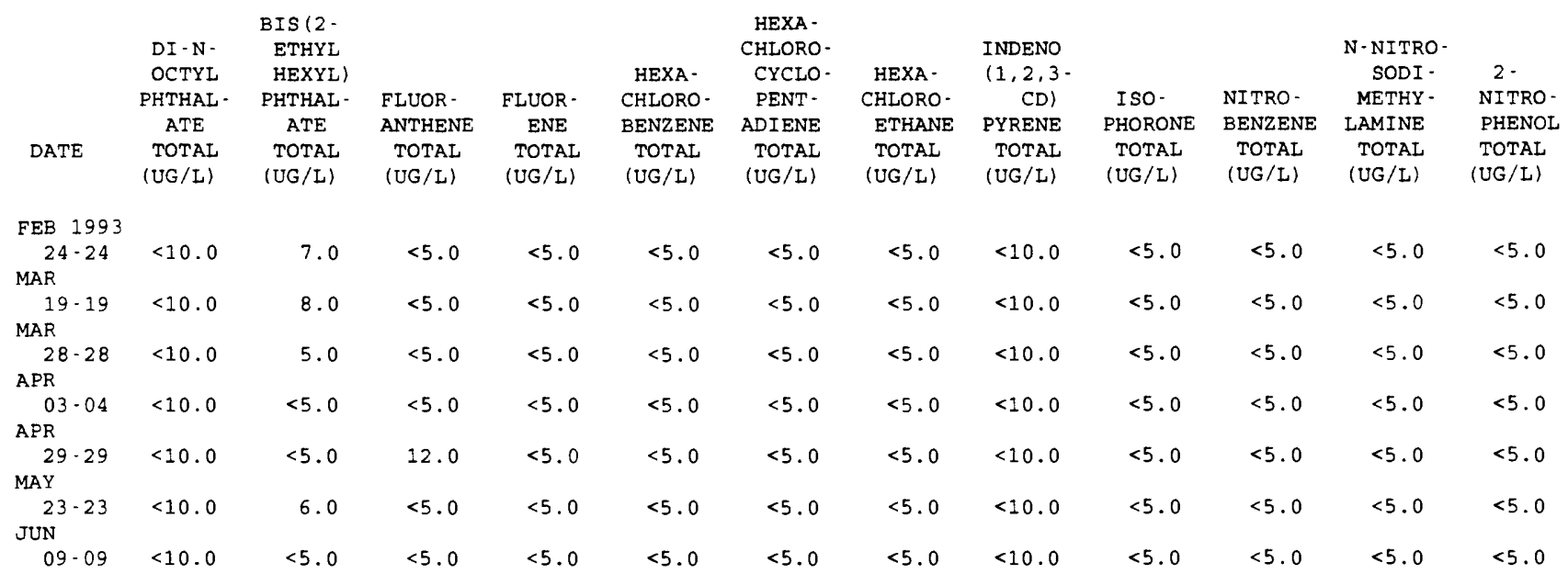

\begin{tabular}{|c|c|c|c|c|c|c|c|c|c|c|c|c|}
\hline DATE & $\begin{array}{c}4- \\
\text { NITRO- } \\
\text { PHENOL } \\
\text { TOTAL } \\
\text { (UG/L) }\end{array}$ & $\begin{array}{c}\text { N- } \\
\text { NITRO- } \\
\text { SODI-N- } \\
\text { PROPYL- } \\
\text { AMINE } \\
\text { TOTAL } \\
\text { (UG/L) }\end{array}$ & $\begin{array}{l}\text { N-NITRO- } \\
\text { SODI - } \\
\text { PHENY - } \\
\text { LAMINE } \\
\text { TOTAL } \\
(\text { UG/L) }\end{array}$ & $\begin{array}{l}\text { PENTA- } \\
\text { CHLORO- } \\
\text { PHENOL } \\
\text { TOTAL } \\
\text { (UG/L) }\end{array}$ & $\begin{array}{l}\text { PHENAN- } \\
\text { THRENE } \\
\text { TOTAL } \\
\text { (UG/L) }\end{array}$ & $\begin{array}{l}\text { PHENOL } \\
\text { (C6H- } \\
5 \mathrm{OH}) \\
\text { TOTAL } \\
\text { (UG/L) }\end{array}$ & $\begin{array}{c}\text { PYRENE } \\
\text { TOTAL } \\
\text { (UG/L) }\end{array}$ & $\begin{array}{c}2,4,6- \\
\text { TRI- } \\
\text { CHLORO- } \\
\text { PHENOL } \\
\text { TOTAL } \\
\text { (UG/L) }\end{array}$ & $\begin{array}{l}\text { ALDRIN, } \\
\text { TOTAL } \\
\text { (UG/L) }\end{array}$ & $\begin{array}{l}\text { P, } P^{\prime} \\
\text { DDT, } \\
\text { TOTAL } \\
\text { (UG/L) }\end{array}$ & $\begin{array}{l}\text { ALPHA } \\
\text { BHC } \\
\text { TOTAL } \\
\text { (UG/L) }\end{array}$ & $\begin{array}{c}\text { BETA } \\
\text { BENZENE } \\
\text { HEXA- } \\
\text { CHLOR- } \\
\text { IDE } \\
\text { TOTAL } \\
\text { (UG/L) }\end{array}$ \\
\hline FEB 1993 & & & & & & & & & & & & \\
\hline $\begin{array}{l}24-24 \\
\text { MAR }\end{array}$ & $<30.0$ & $<5.0$ & $<5.0$ & $<30.0$ & $<5.0$ & $<5.0$ & $<5.0$ & $<20.0$ & $<0.040$ & $<0.10$ & 0.03 & $<0.03$ \\
\hline $\operatorname{MAR}^{19-19}$ & $<30.0$ & $<5.0$ & $<5.0$ & $<30.0$ & $<5.0$ & $<5.0$ & $<5.0$ & $<20.0$ & $<0.040$ & $<0.10$ & $<0.03$ & $<0.03$ \\
\hline $\begin{array}{l}28-28 \\
\text { APR }\end{array}$ & $<30.0$ & $<5.0$ & $<5.0$ & $<30.0$ & $<5.0$ & $<5.0$ & $<5.0$ & $<20.0$ & $<0.040$ & $<0.10$ & $<0.03$ & $<0.03$ \\
\hline $\begin{array}{l}03-04 \\
\text { APR }\end{array}$ & $<30.0$ & $<5.0$ & $<5.0$ & $<30.0$ & $<5.0$ & $<5.0$ & $<5.0$ & $<20.0$ & $<0.040$ & $<0.10$ & $<0.03$ & $<0.03$ \\
\hline MAY & $<30.0$ & $<5.0$ & $<5.0$ & $<30.0$ & 10.0 & $<5.0$ & 10.0 & $<20.0$ & $<0.040$ & $<0.10$ & $<0.03$ & $<0.03$ \\
\hline $\begin{array}{l}23 \cdot 23 \\
\text { JUN }\end{array}$ & $<30.0$ & $<5.0$ & $<5.0$ & $<30.0$ & $<5.0$ & $<5.0$ & $<5.0$ & $<20.0$ & $<0.040$ & $<0.10$ & $<0.03$ & $<0.03$ \\
\hline $09-09$ & $<30.0$ & $<5.0$ & $<5.0$ & $<30.0$ & $<5.0$ & $<5.0$ & $<5.0$ & $<20.0$ & $<0.040$ & $<0.10$ & $<0.03$ & $<0.03$ \\
\hline & $\begin{array}{l}\text { DELTA } \\
\text { BENZENE }\end{array}$ & & $\begin{array}{l}\text { CHLOR - } \\
\text { DANE }\end{array}$ & $\begin{array}{l}\text { CHLOR - } \\
\text { DANE }\end{array}$ & & & & & $\begin{array}{l}\text { ENDO- } \\
\text { SULFAN- }\end{array}$ & & & \\
\hline & HEXA- & & CIS & TRANS & & & & & I & ENDO - & ENDO- & ENDRIN \\
\hline & $\begin{array}{l}\text { CHLOR- } \\
\text { IDE }\end{array}$ & LINDANE & $\begin{array}{l}\text { WATER } \\
\text { WHOLE }\end{array}$ & $\begin{array}{l}\text { WATER } \\
\text { WHOLE }\end{array}$ & $\begin{array}{l}\text { CHLOR - } \\
\text { DANE, }\end{array}$ & $\begin{array}{l}\mathrm{P}, \mathrm{P}^{\prime} \\
\mathrm{DDD}\end{array}$ & $\begin{array}{l}\mathrm{P}, \mathrm{P}^{\prime} \\
\mathrm{DDE}\end{array}$ & $\begin{array}{l}\text { DI- } \\
\text { ELDRIN }\end{array}$ & $\begin{array}{l}\text { WATER } \\
\text { WHOLE }\end{array}$ & $\begin{array}{l}\text { SULFAN } \\
\text { BETA }\end{array}$ & $\begin{array}{r}\text { SULFAN } \\
\text { SULFATE }\end{array}$ & $\begin{array}{l}\text { WATER } \\
\text { UNFLTRD }\end{array}$ \\
\hline DATE & $\begin{array}{l}\text { TOTAL } \\
\text { (UG/L) }\end{array}$ & $\begin{array}{l}\text { TOTAL } \\
\text { (UG/L) }\end{array}$ & $\begin{array}{l}\text { TOTAL } \\
\text { (UG/L) }\end{array}$ & $\begin{array}{l}\text { TOTAL } \\
(\mathrm{UG} / \mathrm{L})\end{array}$ & $\begin{array}{l}\text { TOTAL } \\
\text { (UG } / \mathrm{L})\end{array}$ & $\begin{array}{l}\text { TOTAL } \\
\text { (UG/L) }\end{array}$ & $\begin{array}{l}\text { TOTAL } \\
\text { (UG/L) }\end{array}$ & $\begin{array}{l}\text { TOTAL } \\
\text { (UG/L) }\end{array}$ & $\begin{array}{l}\mathrm{REC} \\
(\mathrm{UG} / \mathrm{L})\end{array}$ & $\begin{array}{l}\text { TOTAL } \\
\text { (UG/L) }\end{array}$ & $\begin{array}{l}\text { TOTAL } \\
\text { (UG/L) }\end{array}$ & $\begin{array}{c}\text { REC } \\
(U G / L)\end{array}$ \\
\hline FEB 19 & & & & & & & & & & & & \\
\hline $\begin{array}{l}24-24 \\
\operatorname{MAR}\end{array}$ & $<0.09$ & 0.030 & $<0.10$ & $<0.10$ & $<0.1$ & $<0.10$ & $<0.04$ & $<0.020$ & $<0.10$ & $<0.04$ & $<0.60$ & $<0.060$ \\
\hline${ }_{\text {MAR }}^{19-19}$ & $<0.09$ & $<0.030$ & $<0.10$ & $<0.10$ & $<0.1$ & $<0.10$ & $<0.04$ & $<0.020$ & $<0.10$ & $<0.04$ & $<0.60$ & $<0.060$ \\
\hline $\begin{array}{l}28-28 \\
\text { APR }\end{array}$ & $<0.09$ & $<0.030$ & $<0.10$ & $<0.10$ & $<0.1$ & $<0.10$ & $<0.04$ & $<0.020$ & $<0.10$ & $<0.04$ & $<0.60$ & $<0.060$ \\
\hline $\begin{array}{l}03 \cdot 04 \\
\text { APR }\end{array}$ & $<0.09$ & $<0.030$ & $<0.10$ & $<0.10$ & $<0.1$ & $<0.10$ & $<0.04$ & $<0.020$ & $<0.10$ & $<0.04$ & $<0.60$ & $<0.060$ \\
\hline${ }_{\mathrm{MAY}}^{29-29}$ & $<0.09$ & $<0.030$ & $<0.10$ & $<0.10$ & $<0.1$ & $<0.10$ & $<0.04$ & $<0.020$ & $<0.10$ & $<0.04$ & $<0.60$ & $<0.060$ \\
\hline $\begin{array}{l}23-23 \\
\text { IUN }\end{array}$ & $<0$. & $<0.030$ & $<0.10$ & $<0.10$ & $<0.1$ & $<0.10$ & $<0.04$ & $<0.020$ & $<0.10$ & $<0.04$ & $<0.60$ & $<0.060$ \\
\hline $09 \cdot 09$ & $<0.09$ & $<0.030$ & $<0.10$ & $<0.10$ & $<0.1$ & $<0.10$ & $<0.04$ & $<0.020$ & $<0.10$ & $<0.04$ & $<0.60$ & $<0.060$ \\
\hline
\end{tabular}


Table 7. Water-quality data for storm-sewer outfall stations, Mesquite, Texas, 1992-93-Continued 08061910 - SOUTH MESQUTTE CREEK OUTFALL AT I-635, MESQUITE, TX (WY 1993)-Continued

\begin{tabular}{|c|c|c|c|c|c|c|c|c|c|c|c|c|}
\hline & ENDRIN & & HEPTA - & & AROCLOR & AROCLOR & AROCLOR & AROCLOR & AROCLOR & AROCLOR & AROCLOR & \\
\hline & ALDE - & HEPTA - & CHLOR & TOX - & 1016 & 1221 & 1232 & 1242 & 1248 & 1254 & 1260 & DI - \\
\hline & HYDE & CHLOR, & EPOXIDE & APHENE, & PCB & PCB & PCB & $\mathrm{PCB}$ & $\mathrm{PCB}$ & PCB & PCB & AZINON, \\
\hline DATE & $\begin{array}{l}\text { TOTAL } \\
\text { (UG/L) }\end{array}$ & $\begin{array}{l}\text { TOTAL } \\
\text { (UG/L) }\end{array}$ & $\begin{array}{l}\text { TOTAL } \\
\text { (UG/L) }\end{array}$ & $\begin{array}{l}\text { TOTAL } \\
\text { (UG/L) }\end{array}$ & $\begin{array}{l}\text { TOTAL } \\
\text { (UG/L) }\end{array}$ & $\begin{array}{l}\text { TOTAL } \\
\text { (UG/L) }\end{array}$ & $\begin{array}{l}\text { TOTAL } \\
\text { (UG/L) }\end{array}$ & $\begin{array}{l}\text { TOTAL } \\
\text { (UG/L) }\end{array}$ & $\begin{array}{l}\text { TOTAL } \\
\text { (UG/L) }\end{array}$ & $\begin{array}{l}\text { TOTAL } \\
(U G / L)\end{array}$ & $\begin{array}{l}\text { TOTAL } \\
\text { (UG/L) }\end{array}$ & $\begin{array}{l}\text { TOTAL } \\
\text { (UG/L) }\end{array}$ \\
\hline \multicolumn{13}{|l|}{ FEB 1993} \\
\hline $24-24$ & $<0.20$ & $<0.030$ & $<0.80$ & $<2$ & $<0.1$ & $<1.0$ & $<0.1$ & $<0.1$ & $<0.1$ & $<0.1$ & $<0.1$ & $<0.05$ \\
\hline \multicolumn{13}{|l|}{ MAR } \\
\hline $19-19$ & $<0.20$ & $<0.030$ & $<0.80$ & $<2$ & $<0.1$ & $<1.0$ & $<0.1$ & $<0.1$ & $<0.1$ & $<0.1$ & $<0.1$ & $<0.05$ \\
\hline \multicolumn{13}{|l|}{ MAR } \\
\hline $28-28$ & $<0.20$ & $<0.030$ & $<0.80$ & $<2$ & $<0.1$ & $<1.0$ & $<0.1$ & $<0.1$ & $<0.1$ & $<0.1$ & $<0.1$ & $<0.05$ \\
\hline \multicolumn{13}{|l|}{ APR } \\
\hline $03-04$ & $<0.20$ & $<0.030$ & $<0.80$ & $<2$ & $<0.1$ & $<1.0$ & $<0.1$ & $<0.1$ & $<0.1$ & $<0.1$ & $<0.1$ & $\cdots$ \\
\hline \multicolumn{13}{|l|}{ APR } \\
\hline $29 \cdot 29$ & $<0.20$ & $<0.030$ & $<0.80$ & $<2$ & $<0.1$ & $<1.0$ & $<0.1$ & $<0.1$ & $<0.1$ & $<0.1$ & $<0.1$ & 0.20 \\
\hline \multicolumn{13}{|l|}{ MAY } \\
\hline $23-23$ & $<0.20$ & $<0.030$ & $<0.80$ & $<2$ & $<0.1$ & $<1.0$ & $<0.1$ & $<0.1$ & $<0.1$ & $<0.1$ & $<0.1$ & 0.20 \\
\hline \multicolumn{13}{|l|}{ JUN } \\
\hline 09.09 & $<0.20$ & $<0.030$ & $<0.80$ & $<2$ & $<0.1$ & $<1.0$ & $<0.1$ & $<0.1$ & $<0.1$ & $<0.1$ & $<0.1$ & 0.20 \\
\hline
\end{tabular}


Table 7. Water-quality data for storm-sewer outfall stations, Mesquite, Texas, 1992-93-Continued

08061915 - SOUTH MESQUITE CREEK OUTFALL AT SOUTH PARKWAY, MESQUITE, TX (WY 1993)

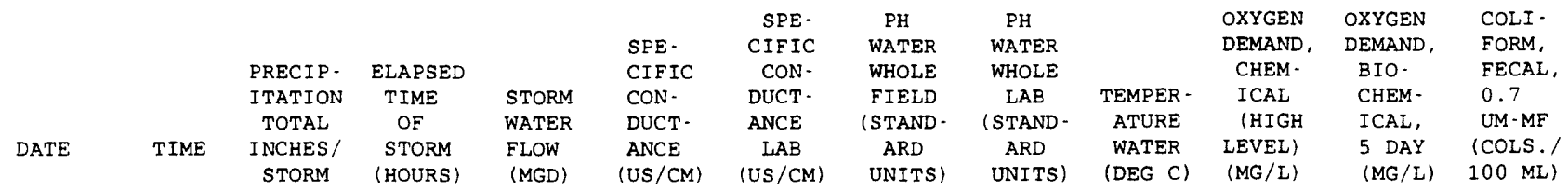

\begin{tabular}{|c|c|c|c|c|c|c|c|c|c|c|c|c|}
\hline \multicolumn{13}{|l|}{ MAR 1993} \\
\hline $11-12$ & 1630 & 0.31 & 9.0 & 0.11 & 237 & 257 & 8.3 & 7.6 & 13.0 & 75 & 7.5 & 3000 \\
\hline \multicolumn{13}{|l|}{ MAR } \\
\hline $28-28$ & 1041 & 0.20 & 2.9 & 0.09 & 266 & 281 & 7.7 & 7.4 & 18.0 & 90 & 7.3 & 110000 \\
\hline \multicolumn{13}{|l|}{ APR } \\
\hline $03-04$ & 1731 & 0.51 & 9.0 & 0.34 & 155 & 214 & 7.4 & 6.9 & 14.0 & 70 & 7.0 & $\mathrm{~K} 80000$ \\
\hline \multicolumn{13}{|l|}{ MAY } \\
\hline $18 \cdot 18$ & 1436 & 0.27 & 1.0 & 0.13 & 356 & 334 & 8.2 & 7.3 & 23.0 & 160 & 7.6 & K22000 \\
\hline \multicolumn{13}{|l|}{ MAY } \\
\hline $23 \cdot 23$ & 1302 & 0.44 & 3.0 & 0.25 & 105 & 253 & 7.9 & 7.2 & 21.5 & 120 & 3.8 & K31000 \\
\hline \multicolumn{13}{|l|}{ JUN } \\
\hline 09.09 & 1935 & 0.84 & 1.2 & 0.39 & 148 & 154 & 8.0 & 7.2 & 26.0 & 72 & 7.4 & 160000 \\
\hline \multicolumn{13}{|l|}{ JUN } \\
\hline $25 \cdot 25$ & 1915 & 0.58 & 3.8 & 0.37 & 55 & 187 & 7.8 & 7.0 & 25.5 & 53 & 7.8 & 270000 \\
\hline
\end{tabular}

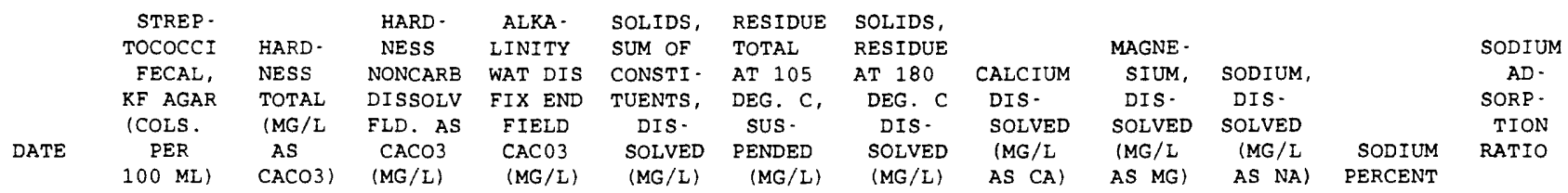

\begin{tabular}{|c|c|c|c|c|c|c|c|c|c|c|c|c|}
\hline MAR 199 & & & & & & & & & & & & \\
\hline${ }_{\operatorname{MAR}}^{11 \cdot 12}$ & 33000 & 78 & 12 & 66 & 136 & 97 & 142 & 27 & 2.6 & 13 & 25 & 0.6 \\
\hline $28-28$ & 96000 & 77 & 21 & 56 & 156 & 100 & 159 & 26 & 2.9 & 17 & 31 & 0.8 \\
\hline APR & & & & & & & & & & & & \\
\hline $\begin{array}{l}03 \cdot 04 \\
\text { MAY }\end{array}$ & K170000 & 54 & 16 & 38 & 101 & 139 & 135 & 19 & 1.7 & 6.4 & 19 & 0.4 \\
\hline $\operatorname{MAY}^{18-18}$ & K30000 & 43 & 13 & 30 & 135 & 608 & 92 & 15 & 1.4 & 8.0 & 26 & 0.5 \\
\hline $\begin{array}{l}23-23 \\
\text { JUN }\end{array}$ & K82000 & 37 & 4 & 33 & 88 & 155 & 75 & 13 & 1.1 & 5.4 & 22 & 0.4 \\
\hline $\begin{array}{l}09.09 \\
\text { JUN }\end{array}$ & 210000 & 31 & 5 & 26 & 82 & 99 & 65 & 11 & 0.81 & 4.2 & 20 & 0.3 \\
\hline $25-25$ & 490000 & 45 & 4 & 41 & 93 & 51 & 98 & 16 & 1.2 & 4.9 & 17 & 0.3 \\
\hline
\end{tabular}

\begin{tabular}{|c|c|c|c|c|c|c|c|c|c|c|c|c|}
\hline & $\begin{array}{l}\text { POTAS - } \\
\text { SIUM, } \\
\text { DIS - } \\
\text { SOLVED } \\
\text { (MG/L }\end{array}$ & $\begin{array}{l}\text { SULFATE } \\
\text { DIS - } \\
\text { SOLVED } \\
\text { (MG/L }\end{array}$ & $\begin{array}{l}\text { CHLO- } \\
\text { RIDE, } \\
\text { DIS- } \\
\text { SOLVED } \\
\text { MGG/L }\end{array}$ & $\begin{array}{c}\text { NITRO- } \\
\text { GEN, } \\
\text { NO2+NO3 } \\
\text { TOTAL } \\
\text { (MG/L }\end{array}$ & $\begin{array}{l}\text { NITRO- } \\
\text { GEN, AM- } \\
\text { MONIA + } \\
\text { ORGANIC } \\
\text { TOTAL } \\
\text { (MG/L }\end{array}$ & $\begin{array}{r}\text { PHOS - } \\
\text { PHORUS } \\
\text { TOTAL } \\
\text { (MG/L }\end{array}$ & $\begin{array}{c}\text { PHOS - } \\
\text { PHORUS } \\
\text { DIS- } \\
\text { SOLVED } \\
\text { (MG/L }\end{array}$ & $\begin{array}{c}\text { ANT IMONY } \\
\text { TOTAL } \\
\text { EPA } \\
\text { (UG/L }\end{array}$ & $\begin{array}{l}\text { ARSENIC } \\
\text { TOTAL } \\
\text { (UG /L }\end{array}$ & $\begin{array}{l}\text { BERYL- } \\
\text { LIUM, } \\
\text { TOTAL } \\
\text { RECOV- } \\
\text { ERABLE } \\
\text { (UG/L }\end{array}$ & $\begin{array}{l}\text { CADMIUM } \\
\text { TOTAL } \\
\text { RECOV- } \\
\text { ERABLE } \\
\text { (UG/L }\end{array}$ & $\begin{array}{l}\text { CHRO- } \\
\text { MIUM, } \\
\text { TOTAL } \\
\text { RECOV- } \\
\text { ERABLE } \\
\text { (UG/L }\end{array}$ \\
\hline & AS $\mathrm{K}$ ) & AS SO4) & AS CL) & AS N) & AS N) & AS $P$ ) & & $A S \quad S B$ ) & AS AS) & AS BE) & AS CD) & AS CR \\
\hline
\end{tabular}

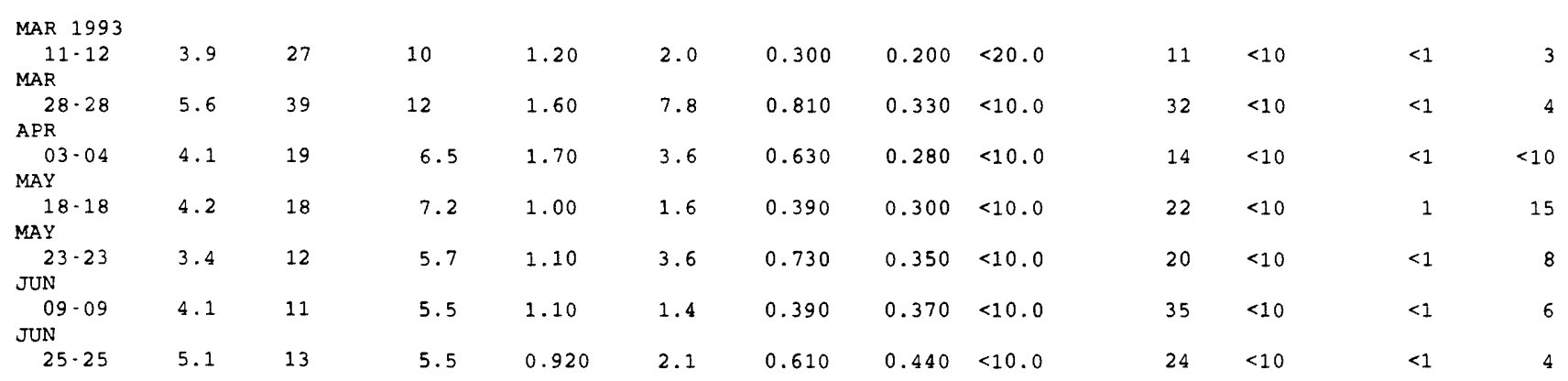


Table 7. Water-quality data for storm-sewer outfall stations, Mesquite, Texas, 1992-93-Continued

08061915 - SOUTH MESQUITE CREEK OUTFALL AT SOUTH PARKWAY, MESQUITE, TX (WY 1993)-CONTInUEd

\begin{tabular}{|c|c|c|c|c|c|c|c|c|c|c|c|c|}
\hline \multirow[t]{2}{*}{ DATE } & $\begin{array}{l}\text { COPPER, } \\
\text { TOTAL } \\
\text { RECOV- } \\
\text { ERABLE } \\
\text { (UG/L }\end{array}$ & $\begin{array}{c}\text { CYANIDE } \\
\text { TOTAL } \\
\text { EPA } \\
\text { (MG/L }\end{array}$ & $\begin{array}{l}\text { CYANIDE } \\
\text { TOTAL } \\
\text { (MG/L }\end{array}$ & $\begin{array}{l}\text { LEAD, } \\
\text { TOTAL } \\
\text { RECOV } \\
\text { ERABLE } \\
\text { (UG/L }\end{array}$ & $\begin{array}{l}\text { MERCURY } \\
\text { TOTAL } \\
\text { RECOV- } \\
\text { ERABLE } \\
\text { (UG/L }\end{array}$ & $\begin{array}{l}\text { NICKEL, } \\
\text { TOTAL } \\
\text { RECOV- } \\
\text { ERABLE } \\
\text { /UG/L }\end{array}$ & $\begin{array}{l}\text { SELE- } \\
\text { NIUM, } \\
\text { TOTAL } \\
\text { (UG /L }\end{array}$ & \multicolumn{2}{|c|}{$\begin{array}{cc} & \text { SILVER } \\
\text { SILVER, } & \text { TOTAL } \\
\text { TOTAL } & \text { RECOV- } \\
\text { RECOV- } & \text { ERABLE } \\
\text { ERABLE } & \text { EPA } \\
\text { (UG/L } & \text { (UG/L }\end{array}$} & $\begin{array}{l}\text { THAL - } \\
\text { LIUM, } \\
\text { TOTAL } \\
\text { (UG/L }\end{array}$ & $\begin{array}{l}\text { ZINC, } \\
\text { TOTAL } \\
\text { RECOV- } \\
\text { ERABLE } \\
\text { (UG/L }\end{array}$ & \multirow[t]{2}{*}{$\begin{array}{c}\text { CARBON, } \\
\text { ORGANIC } \\
\text { TOTAL } \\
\text { (MG/I } \\
\text { AS C) }\end{array}$} \\
\hline & AS CU) & AS CN) & AS CN) & AS PB) & AS HG) & AS NI) & AS SE) & AS AG) & AS AG) & AS TL) & AS $\quad \mathrm{ZN})$ & \\
\hline \multicolumn{13}{|l|}{ MAR 1993} \\
\hline $11 \cdot 12$ & 10 & $<0.010$ & $<0.010$ & 10 & $<0.10$ & 4 & $<2$ & $<1$ & $<0.500$ & $<10$ & 30 & 16 \\
\hline \multicolumn{13}{|l|}{ MAR } \\
\hline $28-28$ & 12 & $<0.010$ & $<0.010$ & 11 & $<0.10$ & 4 & $<2$ & $<1$ & $<0.500$ & $<5$ & 80 & 27 \\
\hline \multicolumn{13}{|l|}{ APR } \\
\hline $03-04$ & 8 & $<0.010$ & $<0.010$ & 12 & $<0.10$ & 4 & $<2$ & $<1$ & $<0.500$ & $<5$ & 70 & 23 \\
\hline \multicolumn{13}{|l|}{ MAY } \\
\hline $18-18$ & 25 & $<0.010$ & $<0.010$ & 43 & 0.40 & 16 & $<2$ & $<1$ & $<0.500$ & $<5$ & 230 & 43 \\
\hline \multicolumn{13}{|l|}{ MAY } \\
\hline $23-23$ & 12 & $<0.010$ & $<0.010$ & 19 & $<0.10$ & 8 & $<2$ & $<1$ & $<0.500$ & $<20$ & 110 & 24 \\
\hline \multicolumn{13}{|l|}{ JUN } \\
\hline 09.09 & 12 & $<0.010$ & $<0.010$ & 12 & 0.10 & 6 & $<2$ & $<1$ & $<0.500$ & $<10$ & 100 & 18 \\
\hline \multicolumn{13}{|l|}{ JUN } \\
\hline $25 \cdot 25$ & 12 & $<0.010$ & $<0.010$ & 7 & $<0.10$ & 5 & $<2$ & $<1$ & $<0.500$ & $<10$ & 60 & 18 \\
\hline & OIL AND & & & & & & METHANE & & & BENZENE & BENZENE & \\
\hline & $\begin{array}{c}\text { GREASE, } \\
\text { TOTAL }\end{array}$ & & & & & $\begin{array}{l}\text { BROMO- } \\
\text { BENZENE }\end{array}$ & $\begin{array}{l}\text { BROMO } \\
\text { CHLORO - }\end{array}$ & & $\begin{array}{l}\text { BENZENE } \\
\mathrm{N} \cdot \text { BUTYL }\end{array}$ & $\begin{array}{c}\text { SEC } \\
\text { BUTYL - }\end{array}$ & $\begin{array}{l}\text { TERT - } \\
\text { BUTYL - }\end{array}$ & $\begin{array}{l}\text { CARBON - } \\
\text { TETRA - }\end{array}$ \\
\hline & $\begin{array}{l}\text { RECOV. } \\
\text { GRAVI - }\end{array}$ & & ACRO - & ACRYLO - & & WATER, & WAT & BROMO - & WATER & WATER & WATER & CHLO - \\
\hline DATE & $\begin{array}{l}\text { GRAVI- } \\
\text { METRIC } \\
(\mathrm{MG} / \mathrm{L})\end{array}$ & $\begin{array}{l}\text { PHENOLS } \\
\text { TOTAL } \\
\text { (UG/L) }\end{array}$ & $\begin{array}{l}\text { LEIN } \\
\text { TOTAL } \\
\text { (UG/L) }\end{array}$ & $\begin{array}{l}\text { NITRILE } \\
\text { TOTAL } \\
\text { (UG/L) }\end{array}$ & $\begin{array}{l}\text { BENZENE } \\
\text { TOTAL } \\
\text { (UG/L) }\end{array}$ & $\begin{array}{l}\text { WHOLE, } \\
\text { TOTAL } \\
\text { (UG/L) }\end{array}$ & $\begin{array}{l}\text { UNFLTRD } \\
\text { REC } \\
\text { (UG/L) }\end{array}$ & $\begin{array}{l}\text { FORM } \\
\text { TOTAL } \\
\text { (UG/L) }\end{array}$ & $\begin{array}{l}\text { UNFLTRD } \\
\text { REC } \\
\text { (UG/L) }\end{array}$ & $\begin{array}{l}\text { UNFLTRD } \\
\text { REC } \\
\text { (UG/L) }\end{array}$ & $\begin{array}{l}\text { UNFLTRD } \\
\text { REC } \\
\text { (UG/L) }\end{array}$ & $\begin{array}{l}\text { RIDE } \\
\text { TOTAL } \\
\text { (UG/L) }\end{array}$ \\
\hline \multicolumn{13}{|l|}{ MAR 1993} \\
\hline $11-12$ & 1 & 3 & $<20$ & $<20$ & $<0.2$ & $<0.2$ & $<0.20$ & $<0.2$ & 0.20 & $<0.20$ & $<0.20$ & $<0.2$ \\
\hline \multicolumn{13}{|l|}{ MAR } \\
\hline $28-28$ & $<1$ & 5 & $<20$ & $<20$ & $<0.2$ & $<0.2$ & $<0.20$ & $<0.2$ & $<0.20$ & $<0.20$ & $<0.20$ & $<0.2$ \\
\hline \multicolumn{13}{|l|}{ APR } \\
\hline $03-04$ & $<1$ & 5 & $<20$ & $<20$ & $<0.2$ & $<0.2$ & $<0.20$ & $<0.2$ & $<0.20$ & $<0.20$ & $<0.20$ & $<0.2$ \\
\hline \multicolumn{13}{|l|}{ MAY } \\
\hline $18-18$ & 5 & 15 & $<200$ & $<200$ & $<2.0$ & $<2.0$ & $<2.00$ & $<2.0$ & $<2.0$ & $<2.0$ & $<2.0$ & $<2.0$ \\
\hline \multicolumn{13}{|l|}{ MAY } \\
\hline \multicolumn{5}{|l|}{ JUN } & $<5.0$ & $<5.0$ & $<5.00$ & $<5.0$ & $<5.0$ & $<5.0$ & $<5.0$ & $<5.0$ \\
\hline 09.09 & 4 & 5 & $<500$ & $<500$ & $<5.0$ & $<5.0$ & $<5.00$ & $<5.0$ & $<5.0$ & $<5.0$ & $<5.0$ & $<5.0$ \\
\hline \multicolumn{13}{|l|}{ JUN } \\
\hline $25 \cdot 25$ & 2 & 3 & $<500$ & $<500$ & $<5.0$ & $<5.0$ & $<5.00$ & $<5.0$ & $<5.0$ & $<5.0$ & $<5.0$ & $<5.0$ \\
\hline
\end{tabular}

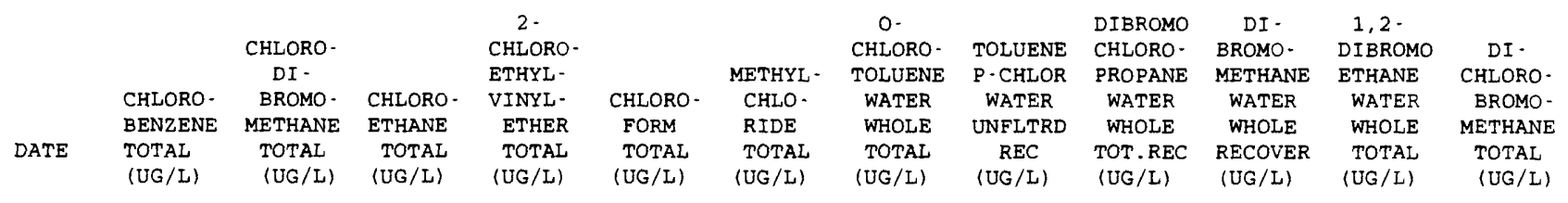

\begin{tabular}{|c|c|c|c|c|c|c|c|c|c|c|c|c|}
\hline & $<0.20$ & $<0.2$ & $<0.2$ & $<1.0$ & $<0.2$ & $<0.2$ & $<0.2$ & $<0.20$ & $<1,0$ & \multicolumn{3}{|c|}{ MAR 1993} \\
\hline \multicolumn{13}{|l|}{ MAR } \\
\hline $28-28$ & $<0.20$ & $<0.2$ & $<0.2$ & $<1.0$ & $<0.2$ & $<0.2$ & $<0.2$ & $<0.20$ & $<1.0$ & $<0.2$ & $<0.2$ & $<0.2$ \\
\hline \multicolumn{13}{|l|}{$A P R$} \\
\hline $03-04$ & $<0.20$ & $<0.2$ & $<0.2$ & $<1.0$ & $<0.2$ & $<0.2$ & $<0.2$ & $<0.20$ & $<1.0$ & $<0.2$ & $<0.2$ & $<0.2$ \\
\hline \multicolumn{13}{|l|}{ MAY } \\
\hline $18-18$ & $<2.0$ & $<2.0$ & $<2.0$ & $<10$ & $<2.0$ & $<2.0$ & $<2.0$ & $<2.0$ & $<10$ & $<2.0$ & $<2.0$ & $<2.0$ \\
\hline \multicolumn{13}{|l|}{ MAY } \\
\hline $23-23$ & $<5.0$ & $<5.0$ & $<5.0$ & $<25$ & $<5.0$ & $<5.0$ & $<5.0$ & $<5.0$ & $<25$ & $<5.0$ & $<5.0$ & $<5.0$ \\
\hline \multicolumn{13}{|l|}{ JUN } \\
\hline 09.09 & $<5.0$ & $<5.0$ & $<5.0$ & $<25$ & $<5.0$ & $<5.0$ & $<5.0$ & $<5.0$ & $<25$ & $<5.0$ & $<5.0$ & $<5.0$ \\
\hline \multicolumn{13}{|l|}{ JUN } \\
\hline $25 \cdot 25$ & $<5.0$ & $<5.0$ & $<5.0$ & $<25$ & $<5.0$ & $<5.0$ & $<5.0$ & $<5.0$ & $<25$ & $<5.0$ & $<5.0$ & $<5.0$ \\
\hline
\end{tabular}


Table 7. Water-quality data for storm-sewer outfall stations, Mesquite, Texas, 1992-93-Continued

08061915 - SOUTH MESQUITE CREEK OUTFALL AT SOUTH PARKWAY, MESQUITE, TX (WY 1993)-Continued

\begin{tabular}{|c|c|c|c|c|c|c|c|c|c|c|c|c|}
\hline & & $1,1-D I$ & BENZENE & BENZENE & BENZENE & DI - & & & $1,2-D I-$ & CIS-1,2- & & \\
\hline & $\begin{array}{l}1,1-D I- \\
\text { CHLORO- }\end{array}$ & $\begin{array}{l}\text { CHLORO- } \\
\text { PRO- }\end{array}$ & $\begin{array}{c}\text { O- } \\
\text { CHLORO. }\end{array}$ & $\begin{array}{l}1,3-D I- \\
\text { CHIORO - }\end{array}$ & 1,4-DI - & CHLORO - & & & $\begin{array}{l}\text { PHENYL - } \\
\text { HYDRA - }\end{array}$ & $\begin{array}{c}\text { DI - } \\
\text { CHLORO - }\end{array}$ & $\begin{array}{c}1,2- \\
\text { TRANSDI }\end{array}$ & \\
\hline & $\begin{array}{l}\text { ETHYL - } \\
\text { ENE }\end{array}$ & $\begin{array}{l}\text { PENE， } \\
\text { WAT， WH }\end{array}$ & $\begin{array}{l}\text { WATER } \\
\text { UNFLTRD }\end{array}$ & $\begin{array}{l}\text { WATER } \\
\text { UNFLTRD }\end{array}$ & $\begin{array}{l}\text { WATER } \\
\text { UNFLTRD }\end{array}$ & $\begin{array}{l}\text { FLUORO - } \\
\text { METHANE }\end{array}$ & EHLORO - & $\begin{array}{l}\text { CHLORO- } \\
\text { ETHANE }\end{array}$ & $\begin{array}{l}\text { ZINE } \\
\text { WATER }\end{array}$ & $\begin{array}{r}\text { ETHENE } \\
\text { WATER }\end{array}$ & $\begin{array}{l}\text { CHLORO - } \\
\text { ETHENE }\end{array}$ & $\begin{array}{l}\text { CHLORO - } \\
\text { PROPANE }\end{array}$ \\
\hline & $\begin{array}{l}\text { TOTAL } \\
\text { (UG/L) }\end{array}$ & $\begin{array}{l}\text { TOTAL } \\
\text { (UG/L) }\end{array}$ & $\begin{array}{c}\text { REC } \\
\text { (UG/L) }\end{array}$ & $\begin{array}{c}\text { REC } \\
(U G / L)\end{array}$ & $\begin{array}{c}\text { REC } \\
\text { (UG/L) }\end{array}$ & $\begin{array}{l}\text { TOTAL } \\
\text { (UG/L) }\end{array}$ & $\begin{array}{l}\text { TOTAL } \\
\text { (UG/L) }\end{array}$ & $\begin{array}{l}\text { TOTAL } \\
\text { (UG/L) }\end{array}$ & $\begin{array}{l}\text { TOT . REC } \\
\text { (UG/L) }\end{array}$ & $\begin{array}{l}\text { TOTAL } \\
\text { (UG/L) }\end{array}$ & $\begin{array}{l}\text { TOTAL } \\
\text { (UG/L) }\end{array}$ & $\begin{array}{l}\text { TOTAL } \\
(\mathrm{UG} / \mathrm{L})\end{array}$ \\
\hline
\end{tabular}

MAR 1993

\begin{tabular}{|c|c|c|c|c|c|c|c|c|c|c|c|c|}
\hline $11-12$ & $<0.2$ & $<0.2$ & $<5.0$ & $<5.0$ & $<5.0$ & $<0.2$ & $<0.2$ & $<0.2$ & $<5.0$ & $<0.2$ & $<0.2$ & $<0.2$ \\
\hline \multicolumn{13}{|l|}{ MAR } \\
\hline $28 \cdot 28$ & $<0.2$ & $<0.2$ & $<0.20$ & $<0.20$ & $<0.20$ & $<0.2$ & $<0.2$ & $<0.2$ & $<5.0$ & $<0.2$ & $<0.2$ & $<0.2$ \\
\hline \multicolumn{13}{|l|}{$A P R$} \\
\hline 03.04 & $<0.2$ & $<0.2$ & $<5.0$ & $<5.0$ & $<5.0$ & $<0.2$ & $<0.2$ & $<0.2$ & $<5.0$ & $<0.2$ & $<0.2$ & $<0.2$ \\
\hline \multicolumn{13}{|l|}{ MAY } \\
\hline $18-18$ & $<2.0$ & $<2.0$ & $<5.0$ & $<5.0$ & $<5.0$ & $<2.0$ & $<2.0$ & $<2.0$ & $<5.0$ & $<2.0$ & $<2.0$ & $<2.0$ \\
\hline \multicolumn{13}{|l|}{ MAY } \\
\hline $23-23$ & $<5.0$ & $<5.0$ & $<5.0$ & $<5.0$ & $<5.0$ & $<5.0$ & $<5.0$ & $<5.0$ & $<5.0$ & $<5.0$ & $<5.0$ & $<5.0$ \\
\hline \multicolumn{13}{|l|}{ JUN } \\
\hline 09.09 & $<5.0$ & $<5.0$ & $<5.0$ & $<5.0$ & $<5.0$ & $<5.0$ & $<5.0$ & $<5.0$ & $<5.0$ & $<5.0$ & $<5.0$ & $<5.0$ \\
\hline \multicolumn{13}{|l|}{ JUN } \\
\hline $25-25$ & $<5.0$ & $<5.0$ & $<5.0$ & $<5.0$ & $<5.0$ & $<5.0$ & $<5.0$ & $<5.0$ & $<5.0$ & $<5.0$ & $<5.0$ & $<5.0$ \\
\hline
\end{tabular}

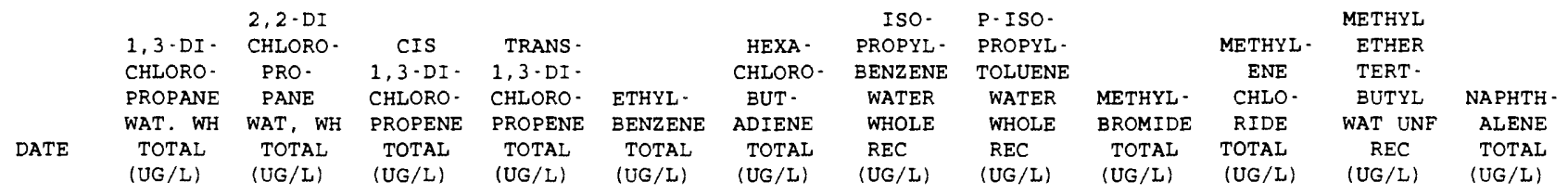

\begin{tabular}{|c|c|c|c|c|c|c|c|c|c|c|c|c|}
\hline $\begin{array}{c}\text { MAR } 1993 \\
11-12\end{array}$ & $<0.2$ & $<0.2$ & $<0.2$ & $<0.2$ & 0.2 & $<5.0$ & $<0.20$ & $<0.20$ & $<0.2$ & $<0.2$ & $<1.0$ & $<5.0$ \\
\hline \multicolumn{13}{|l|}{ MAR } \\
\hline $28 \cdot 28$ & $<0.2$ & $<0.2$ & $<0.2$ & $<0.2$ & $<0.2$ & $<0.2$ & $<0.20$ & $<0.20$ & $<0.2$ & $<0.2$ & $<1.0$ & 0.2 \\
\hline \multicolumn{13}{|l|}{ APR } \\
\hline $03 \cdot 04$ & $<0.2$ & $<0.2$ & $<0.2$ & $<0.2$ & $<0.2$ & $<5.0$ & $<0.20$ & $<0.20$ & $<0.2$ & $<0.2$ & $<1.0$ & $<5,0$ \\
\hline \multicolumn{13}{|l|}{ MAY } \\
\hline $18-18$ & $<2.0$ & $<2.0$ & $<2.0$ & $<2.0$ & $<2.0$ & $<5.0$ & $<2.0$ & $<2.0$ & $<2.0$ & $<2.0$ & $<10$ & $<5.0$ \\
\hline \multicolumn{13}{|l|}{ MAY } \\
\hline $23 \cdot 23$ & $<5.0$ & $<5.0$ & $<5.0$ & $<5.0$ & $<5.0$ & $<5.0$ & $<5.0$ & $<5.0$ & $<5.0$ & $<5.0$ & $<5.0$ & $<5.0$ \\
\hline \multicolumn{13}{|l|}{ JUN } \\
\hline 09.09 & $<5.0$ & $<5.0$ & $<5.0$ & $<5.0$ & $<5.0$ & $<5.0$ & $<5.0$ & $<5.0$ & $<5.0$ & $<5.0$ & $<25$ & $<5.0$ \\
\hline \multicolumn{13}{|l|}{ JUN } \\
\hline $25-25$ & $<5.0$ & $<5.0$ & $<5.0$ & $<5.0$ & $<5.0$ & $<5.0$ & $<5.0$ & $<5.0$ & $<5.0$ & $<5.0$ & $<25$ & $<5.0$ \\
\hline
\end{tabular}

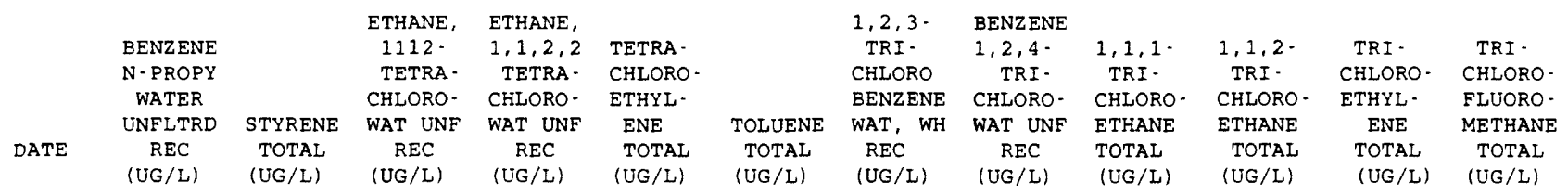

\begin{tabular}{|c|c|c|c|c|c|c|c|c|c|c|c|c|}
\hline \multicolumn{13}{|l|}{ MAR 1993} \\
\hline $11-12$ & $<0.20$ & $<0.2$ & $<0.2$ & $<0.2$ & $<0.2$ & $<0.2$ & $<0.20$ & $<5.0$ & $<0.2$ & $<0.2$ & $<0.2$ & $<0.2$ \\
\hline \multicolumn{13}{|l|}{ MAR } \\
\hline $28-28$ & $<0.20$ & $<0.2$ & $<0.2$ & $<0.2$ & $<0.2$ & $<0.2$ & $<0.20$ & $<0.20$ & $<0.2$ & $<0.2$ & $<0.2$ & $<0.2$ \\
\hline \multicolumn{13}{|l|}{$A P R$} \\
\hline $03 \cdot 04$ & $<0.20$ & $<0.2$ & $<0.2$ & $<0.2$ & $<0.2$ & $<0.2$ & $<0.20$ & $<5.0$ & $<0.2$ & $<0.2$ & $<0.2$ & $<0.2$ \\
\hline \multicolumn{13}{|l|}{ MAY } \\
\hline $18-18$ & $<2.0$ & $<2.0$ & $<2.0$ & $<2.0$ & $<2.0$ & $<2.0$ & $<2.0$ & $<5.0$ & $<2.0$ & $<2.0$ & $<2.0$ & $<2.0$ \\
\hline \multicolumn{13}{|l|}{ MAY } \\
\hline $23 \cdot 23$ & $<5.0$ & $<5.0$ & $<5.0$ & $<5.0$ & $<5.0$ & $<5.0$ & $<5.0$ & $<5.0$ & $<5.0$ & $<5.0$ & $<5.0$ & $<5.0$ \\
\hline \multicolumn{13}{|l|}{ JUN } \\
\hline 09.09 & $<5.0$ & $<5.0$ & $<5.0$ & $<5.0$ & $<5.0$ & $<5.0$ & $<5.0$ & $<5.0$ & $<5.0$ & $<5.0$ & $<5.0$ & $<5.0$ \\
\hline \multicolumn{13}{|l|}{ JUN } \\
\hline $25 \cdot 25$ & $<5.0$ & $<5.0$ & $<5.0$ & $<5.0$ & $<5.0$ & $<5.0$ & $<5.0$ & $<5.0$ & $<5.0$ & $<5.0$ & $<5.0$ & $<5.0$ \\
\hline
\end{tabular}


Table 7. Water-quality data for storm-sewer outfall stations, Mesquite, Texas, 1992-93-Continued

08061915 - SOUTH MESQUTTE CREEK OUTFALL AT SOUTH PARKWAY, MESQUITE, TX (WY 1993)-Continued

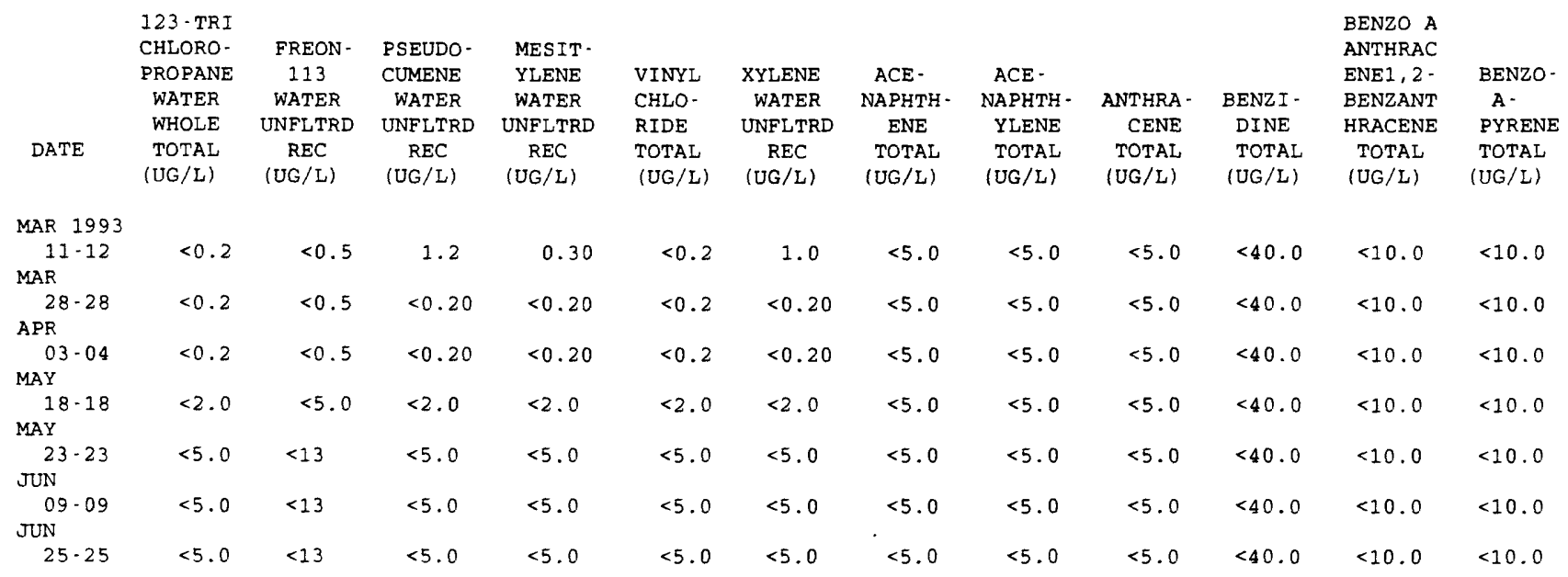

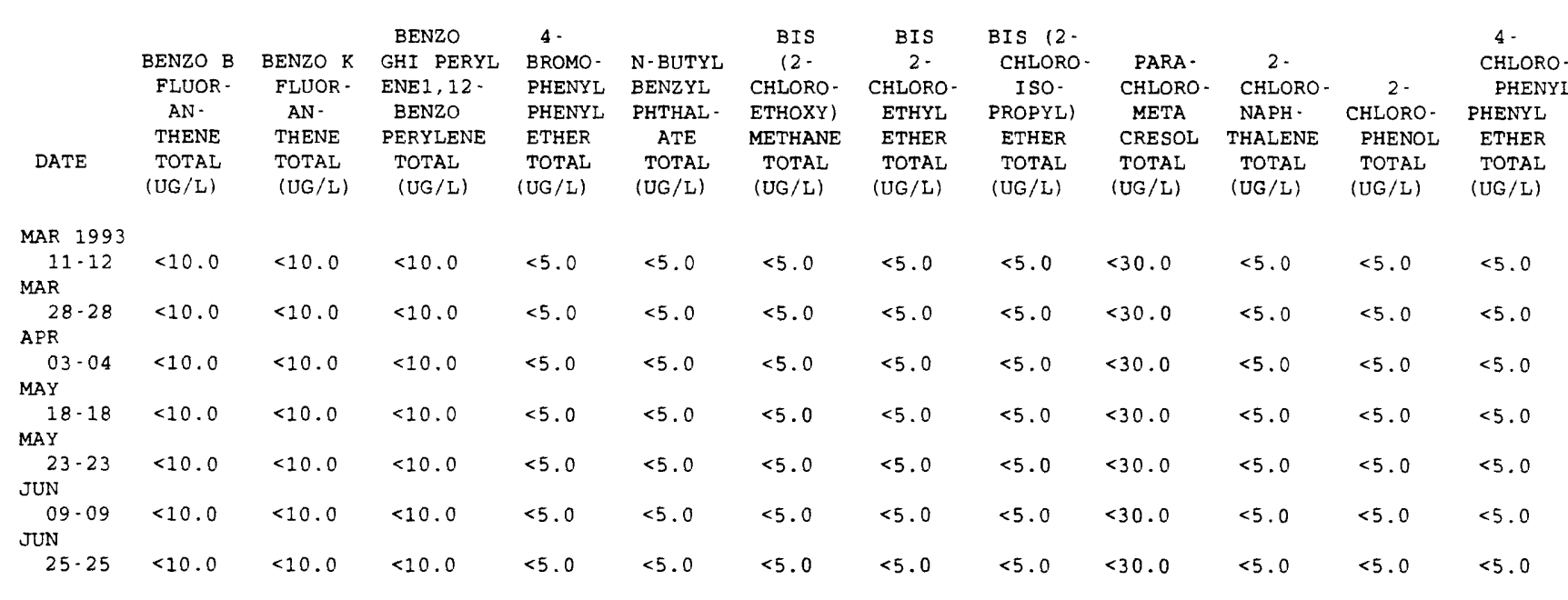

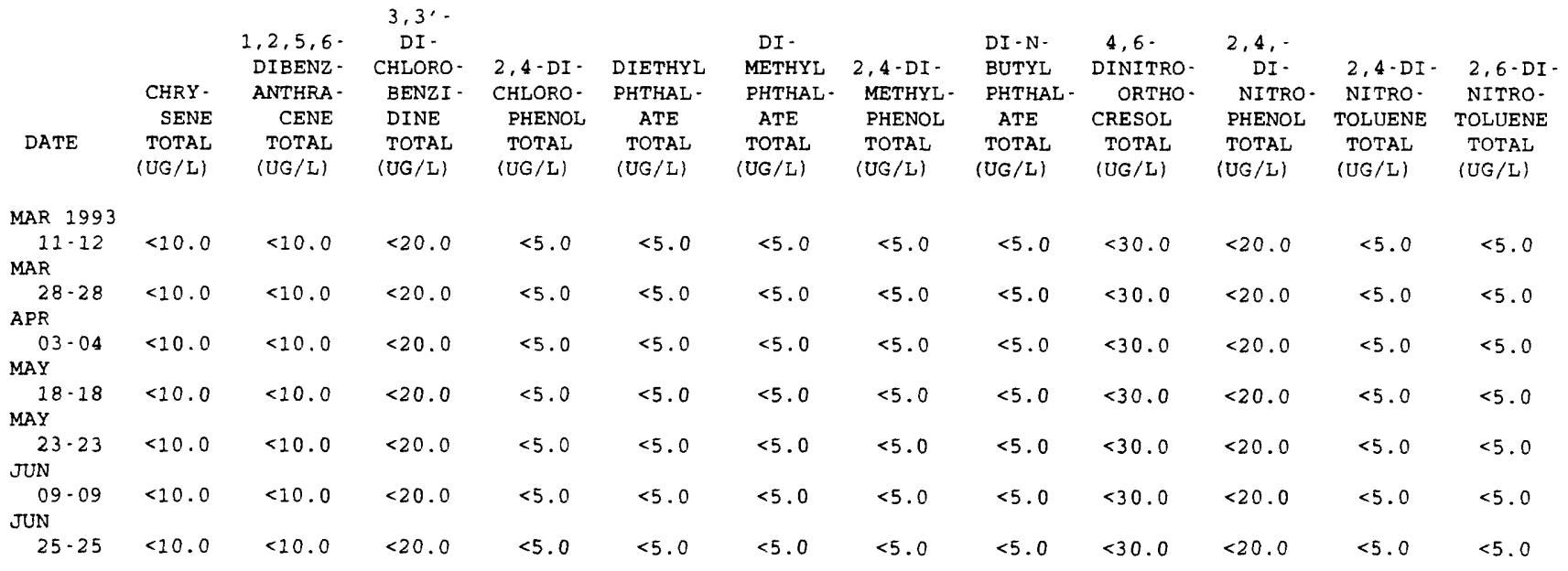


Table 7. Water-quality data for storm-sewer outfall stations, Mesquite, Texas, 1992-93-Continued

08061915 - SOUTH MESQUITE CREEK OUTFALI AT SOUTH PARKWAY, MESQUITE, TX (WY 1993)-ContinUed

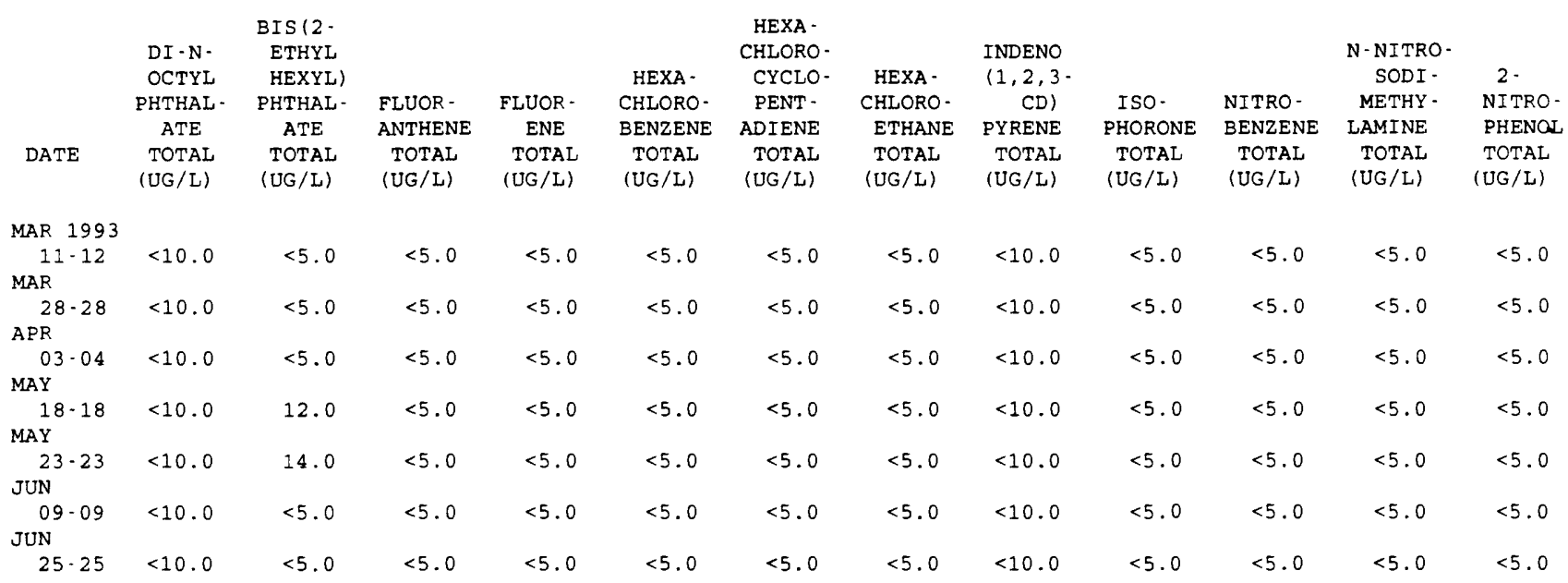

\begin{tabular}{|c|c|c|c|c|c|c|c|c|c|c|c|c|}
\hline DATE & $\begin{array}{c}4- \\
\text { NITRO- } \\
\text { PHENOL } \\
\text { TOTAL } \\
\text { (UG/L) }\end{array}$ & $\begin{array}{c}\text { N- } \\
\text { NITRO- } \\
\text { SODI-N- } \\
\text { PROPYL- } \\
\text { AMINE } \\
\text { TOTAL } \\
(U G / L)\end{array}$ & $\begin{array}{l}\text { N-NITRO- } \\
\text { SODI - } \\
\text { PHENY - } \\
\text { LAMINE } \\
\text { TOTAL } \\
\text { (UG/L) }\end{array}$ & $\begin{array}{l}\text { PENTA- } \\
\text { CHLORO- } \\
\text { PHENOL } \\
\text { TOTAL } \\
\text { (UG/L) }\end{array}$ & $\begin{array}{l}\text { PHENAN - } \\
\text { THRENE } \\
\text { TOTAL } \\
\text { (UG/L) }\end{array}$ & $\begin{array}{c}\text { PHENOL } \\
\text { (C6H- } \\
50 \mathrm{H}) \\
\text { TOTAL } \\
\text { (UG/L) }\end{array}$ & $\begin{array}{c}\text { PYRENE } \\
\text { TOTAL } \\
\text { (UG/L) }\end{array}$ & $\begin{array}{c}2,4,6- \\
\text { TRI- } \\
\text { CHLORO- } \\
\text { PHENOL } \\
\text { TOTAL } \\
\text { (UG/L) }\end{array}$ & $\begin{array}{l}\text { ALDRIN, } \\
\text { TOTAL } \\
\text { (UG/L) }\end{array}$ & $\begin{array}{l}\text { P, P' } \\
\text { DDT, } \\
\text { TOTAL } \\
\text { (UG/L) }\end{array}$ & $\begin{array}{l}\text { ALPHA } \\
\text { BHC } \\
\text { TOTAL } \\
\text { (UG/L) }\end{array}$ & $\begin{array}{c}\text { BETA } \\
\text { BENZENE } \\
\text { HEXA- } \\
\text { CHLOR- } \\
\text { IDE } \\
\text { TOTAL } \\
\text { (UG/L) }\end{array}$ \\
\hline MAR 1993 & & & & & & & & & & & & \\
\hline${ }_{\text {MAR }}^{11 \cdot 12}$ & $<30.0$ & $<5.0$ & $<5.0$ & $<30.0$ & $<5.0$ & $<5.0$ & $<5.0$ & $<20.0$ & $<0.040$ & $<0.10$ & 0.03 & $<0.03$ \\
\hline $\begin{array}{l}28 \cdot 28 \\
\text { APR }\end{array}$ & $<30.0$ & $<5.0$ & $<5.0$ & $<30.0$ & $<5.0$ & $<5.0$ & $<5.0$ & $<20.0$ & $<0.040$ & $<0.10$ & $<0.03$ & $<0.03$ \\
\hline $\begin{array}{l}03-04 \\
\text { MAY }\end{array}$ & $<30.0$ & $<5.0$ & $<5.0$ & $<30.0$ & $<5.0$ & $<5.0$ & $<5.0$ & $<20.0$ & $<0.040$ & $<0.10$ & $<0.03$ & $<0.03$ \\
\hline${ }_{\text {MAY }}^{18-18}$ & $<30.0$ & $<5.0$ & $<5.0$ & $<30.0$ & $<5.0$ & $<5.0$ & $<5.0$ & $<20.0$ & $<0.040$ & $<0.10$ & $<0.03$ & $<0.03$ \\
\hline $\begin{array}{l}23-23 \\
\text { JUN }\end{array}$ & $<30.0$ & $<5.0$ & $<5.0$ & $<30.0$ & $<5.0$ & $<5.0$ & $<5.0$ & $<20.0$ & $<0.040$ & $<0.10$ & $<0.03$ & $<0.03$ \\
\hline $\begin{array}{l}09.09 \\
\text { JUN }\end{array}$ & $<30.0$ & $<5.0$ & $<5.0$ & $<30.0$ & $<5.0$ & $<5.0$ & $<5.0$ & $<20.0$ & $<0.040$ & $<0.10$ & $<0.03$ & $<0.03$ \\
\hline $25 \cdot 25$ & $<30.0$ & $<5.0$ & $<5.0$ & $<30.0$ & $<5.0$ & $<5.0$ & $<5.0$ & $<20.0$ & $<0.040$ & $<0.10$ & $<0.03$ & $<0.03$ \\
\hline
\end{tabular}

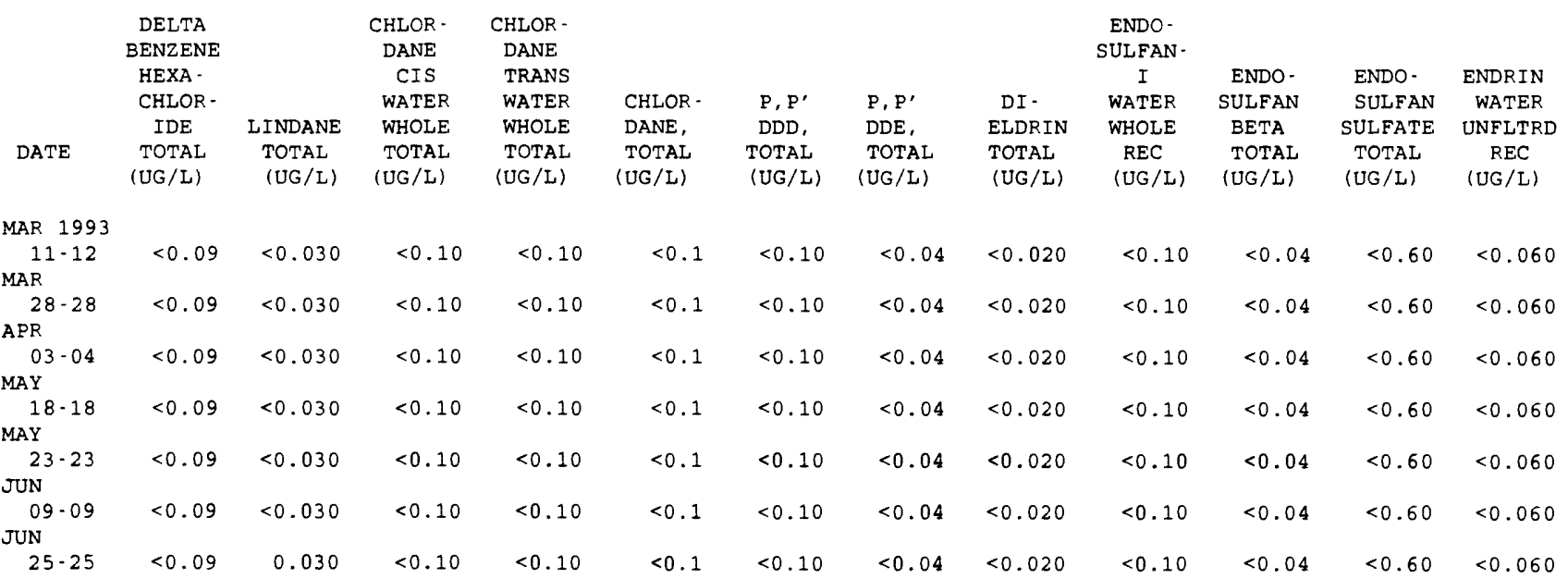


Table 7. Water-quality data for storm-sewer outfall stations, Mesquite, Texas, 1992-93-Continued 08061915 - SOUTH MESQUITE CREEK OUTFALI AT SOUTH PARKWAY, MESQUITE, TX (WY 1993)-ContinUed

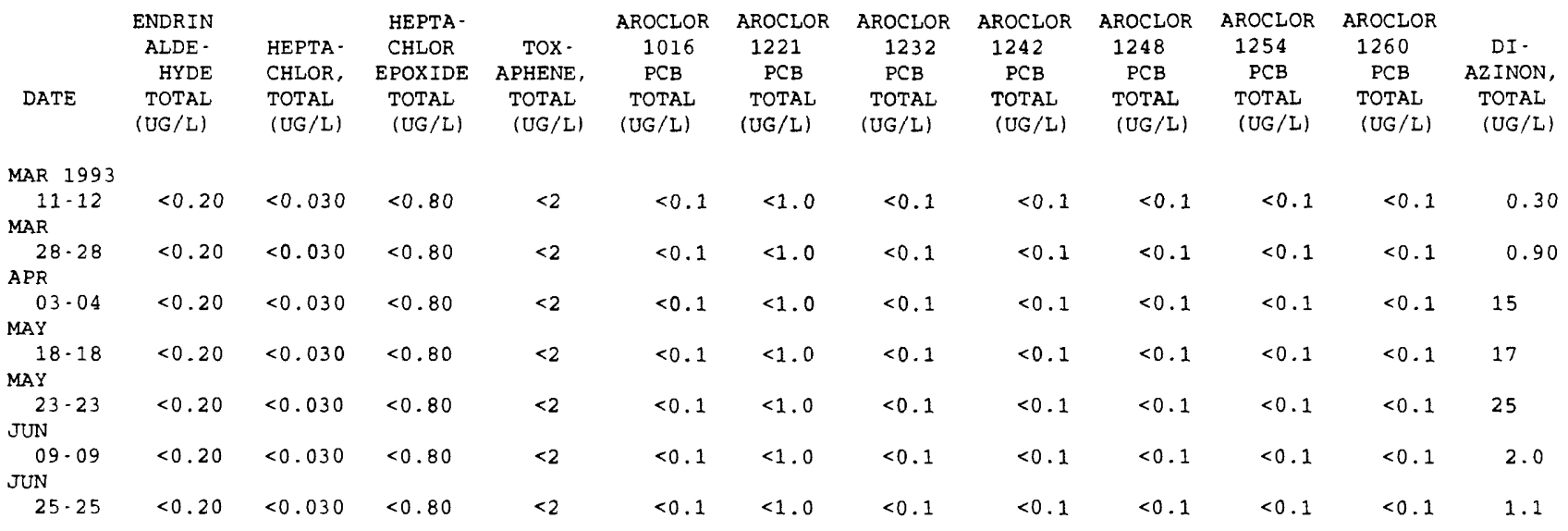


Table 7. Water-quality data for storm-sewer outfall stations, Mesquite, Texas, 1992-93-Continued

08061940 - SOUTH MESQUITE CREEK OUTFALL AT BRUTON ROAD, MESQUITE, TX (WY 1993)

\begin{tabular}{|c|c|c|c|c|c|c|c|c|c|c|c|c|}
\hline DATE & TIME & $\begin{array}{l}\text { PRECIP- } \\
\text { ITATION } \\
\text { TOTAL } \\
\text { INCHES / } \\
\text { STORM }\end{array}$ & $\begin{array}{l}\text { ELAPSED } \\
\text { TIME } \\
\text { OF } \\
\text { STORM } \\
\text { (HOURS) }\end{array}$ & $\begin{array}{l}\text { STORM } \\
\text { WATER } \\
\text { FLOW } \\
\text { (MGD) }\end{array}$ & $\begin{array}{l}\text { SPE- } \\
\text { CIFIC } \\
\text { CON- } \\
\text { DUCT- } \\
\text { ANCE } \\
\text { (US/CM) }\end{array}$ & $\begin{array}{c}\text { SPE- } \\
\text { CIFIC } \\
\text { CON- } \\
\text { DUCT- } \\
\text { ANCE } \\
\text { LAB } \\
\text { (US/CM) }\end{array}$ & $\begin{array}{c}\text { PH } \\
\text { WATER } \\
\text { WHOLE } \\
\text { FIELD } \\
\text { (STAND- } \\
\text { ARD } \\
\text { UNITS) }\end{array}$ & $\begin{array}{c}\text { PH } \\
\text { WATER } \\
\text { WHOLE } \\
\text { LAB } \\
\text { (STAND - } \\
\text { ARD } \\
\text { UNITS) }\end{array}$ & $\begin{array}{c}\text { TEMPER - } \\
\text { ATURE } \\
\text { WATER } \\
\text { (DEG C) }\end{array}$ & $\begin{array}{l}\text { OXYGEN } \\
\text { DEMAND, } \\
\text { CHEM- } \\
\text { ICAL } \\
\text { (HIGH } \\
\text { LEVEL) } \\
\text { (MG/L) }\end{array}$ & $\begin{array}{l}\text { OXYGEN } \\
\text { DEMAND, } \\
\text { BIO- } \\
\text { CHEM- } \\
\text { ICAL, } \\
5 \text { DAY } \\
\text { (MG/L) }\end{array}$ & $\begin{array}{l}\text { COLI - } \\
\text { FORM, } \\
\text { FECAL, } \\
0.7 \\
\text { UM-MF } \\
\text { (COLS. / } \\
100 \mathrm{ML})\end{array}$ \\
\hline \multicolumn{13}{|l|}{ FEB 1993} \\
\hline $10-10$ & 0447 & 0.21 & 3.7 & 0.05 & 130 & $\cdots$ & 7.6 & $\cdots$ & 13.5 & 53 & 4.5 & K1600 \\
\hline \multicolumn{13}{|l|}{ FEB } \\
\hline $24 \cdot 25$ & 1637 & 0.49 & 7.9 & 0.20 & 300 & 125 & 6.7 & 7.3 & 12.0 & 35 & 5.2 & K4200 \\
\hline \multicolumn{13}{|l|}{ MAR } \\
\hline 19.19 & 1400 & 0.40 & 4.7 & 0.14 & 74 & 106 & 7.0 & 7.6 & 14.5 & 45 & 5.6 & $\mathrm{~K} 20000$ \\
\hline \multicolumn{13}{|l|}{ APR } \\
\hline $03-03$ & 1718 & 0.35 & 2.2 & 0.10 & 100 & 94 & 6.4 & 7.1 & 17.0 & 69 & 8.0 & K53000 \\
\hline \multicolumn{13}{|l|}{ APR } \\
\hline $14 \cdot 14$ & 0700 & 1.1 & 3.1 & 0.56 & 495 & 95 & 7.6 & 6.4 & 19.5 & 93 & 7.0 & 56000 \\
\hline \multicolumn{13}{|l|}{ APR } \\
\hline $29-29$ & 0020 & 0.78 & 4.6 & 0.26 & 38 & 74 & 8.0 & 6.7 & 18.0 & 35 & 7.6 & 230000 \\
\hline \multicolumn{13}{|l|}{ MAY } \\
\hline $23-23$ & 1333 & 1.3 & 6.3 & 0.58 & 83 & 80 & 7.4 & 6.6 & 23.0 & 32 & 3.4 & $\mathrm{~K} 28000$ \\
\hline
\end{tabular}

\begin{tabular}{|c|c|c|c|c|c|c|c|c|c|c|c|c|}
\hline & $\begin{array}{l}\text { STREP - } \\
\text { TOCOCCI }\end{array}$ & HARD - & $\begin{array}{l}\text { HARD - } \\
\text { NESS }\end{array}$ & $\begin{array}{l}\text { ALKA - } \\
\text { LINITY }\end{array}$ & $\begin{array}{l}\text { SOLIDS, } \\
\text { SUM OF }\end{array}$ & $\begin{array}{l}\text { RESIDUE } \\
\text { TOTAL }\end{array}$ & $\begin{array}{l}\text { SOLIDS, } \\
\text { RESIDUE }\end{array}$ & & MAGNE - & & & SODIUM \\
\hline & FECAL, & NESS & NONCARB & WAT DIS & CONSTI - & AT 105 & AT 180 & CALCIUM & SIUM, & SODIUM, & & $\mathrm{AD}-$ \\
\hline & $\begin{array}{l}\text { KF AGAR } \\
\text { (COLS. }\end{array}$ & $\begin{array}{l}\text { TOTAL } \\
\text { (MG/L }\end{array}$ & $\begin{array}{l}\text { DISSOLV } \\
\text { FLD. AS }\end{array}$ & $\begin{array}{l}\text { FIX END } \\
\text { FIELD }\end{array}$ & $\begin{array}{c}\text { TUENTS, } \\
\text { DIS - }\end{array}$ & $\begin{array}{l}\text { DEG. C, } \\
\text { SUS - }\end{array}$ & $\begin{array}{l}\text { DEG. C } \\
\text { DIS - }\end{array}$ & $\begin{array}{l}\text { DIS - } \\
\text { SOLVED }\end{array}$ & $\begin{array}{l}\text { DIS - } \\
\text { SOLVED }\end{array}$ & $\begin{array}{l}\text { DIS - } \\
\text { SOLVED }\end{array}$ & SODIUM & SORP- \\
\hline ATE & $\begin{array}{c}\text { PER } \\
100 \mathrm{ML})\end{array}$ & CACO 31 & $(\mathrm{MG} / \mathrm{L})$ & (MG/L) & (MG/L) & (MG/L) & (MG/L) & AS CA) & AS MG) & AS NA) & PERCENT & \\
\hline
\end{tabular}

FEB 1993

\begin{tabular}{|c|c|c|c|c|c|c|c|c|c|c|c|c|}
\hline $10-10$ & $\mathrm{~K} 26000$ & $\cdots$ & $\cdots$ & 34 & $\cdots$ & $\cdots$ & $\cdots$ & $\cdots$ & $\cdots$ & $\cdots$ & $\cdots$ & $\cdots$ \\
\hline \multicolumn{13}{|l|}{ FEB } \\
\hline $24 \cdot 25$ & K71000 & 41 & 8 & 33 & 66 & 35 & 69 & 15 & 0.88 & 5.0 & 20 & 0.3 \\
\hline \multicolumn{13}{|l|}{ MAR } \\
\hline $19-19$ & $\mathrm{~K} 26000$ & 35 & 0 & 35 & 54 & 39 & 64 & 13 & 0.61 & 2.5 & 12 & 0.2 \\
\hline \multicolumn{13}{|l|}{ APR } \\
\hline 03.03 & $\mathrm{~K} 160000$ & 22 & 4 & 18 & 45 & 78 & 46 & 8.4 & 0.33 & 1.3 & 10 & 0.1 \\
\hline \multicolumn{13}{|l|}{ APR } \\
\hline $14-14$ & 290000 & 26 & 3 & 23 & 48 & 70 & 56 & 9.4 & 0.62 & 1.7 & 10 & 0.1 \\
\hline \multicolumn{13}{|l|}{ APR } \\
\hline $29 \cdot 29$ & 180000 & 17 & 4 & 13 & 30 & 59 & 55 & 6.3 & 0.37 & 1.0 & 11 & 0.1 \\
\hline \multicolumn{13}{|l|}{ MAY } \\
\hline $23-23$ & $\mathrm{~K} 74000$ & 22 & 1 & 21 & 39 & 39 & 49 & 8.1 & 0.50 & 1.6 & 12 & 0.1 \\
\hline DATE & $\begin{array}{l}\text { POTAS - } \\
\text { SIUM, } \\
\text { DIS - } \\
\text { SOLVED } \\
\text { (MG/L } \\
\text { AS K) }\end{array}$ & $\begin{array}{l}\text { SULFATE } \\
\text { DIS - } \\
\text { SOLVED } \\
\text { (MG/L } \\
\text { SS SO4) }\end{array}$ & $\begin{array}{l}\text { CHLO - } \\
\text { RIDE, } \\
\text { DIS - } \\
\text { SOLVED } \\
\text { (MG/L }\end{array}$ & $\begin{array}{l}\text { NITRO- } \\
\text { GEN, } \\
\text { NO2+NO3 } \\
\text { TOTAL } \\
\text { (MG/L }\end{array}$ & $\begin{array}{l}\text { NITRO- } \\
\text { GEN, AM- } \\
\text { MONIA + } \\
\text { ORGANIC } \\
\text { TOTAL } \\
\text { (MG/L }\end{array}$ & $\begin{array}{l}\text { PHOS - } \\
\text { PHORUS } \\
\text { TOTAL } \\
\text { (MG/L } \\
\text { AS P) }\end{array}$ & $\begin{array}{l}\text { PHOS - } \\
\text { PHORUS } \\
\text { DIS - } \\
\text { SOLVED } \\
\text { (MG/L }\end{array}$ & $\begin{array}{c}\text { ANTIMONY } \\
\text { TOTAL } \\
\text { EPA } \\
\text { (UG/L } \\
\text { AS SR }\end{array}$ & $\begin{array}{c}\text { ARSENIC } \\
\text { TOTAL } \\
\text { (UG/L }\end{array}$ & $\begin{array}{l}\text { BERYL- } \\
\text { LIUM, } \\
\text { TOTAL } \\
\text { RECOV- } \\
\text { ERABLE } \\
\text { (UG/L }\end{array}$ & $\begin{array}{l}\text { CADMIUM } \\
\text { TOTAL } \\
\text { RECOV- } \\
\text { ERABLE } \\
\text { (UG/L }\end{array}$ & $\begin{array}{l}\text { CHRO- } \\
\text { MIUM, } \\
\text { TOTAL } \\
\text { RECOV - } \\
\text { ERABLE } \\
\text { (UG/L }\end{array}$ \\
\hline & AS K $\mathrm{K}$ & AS SO4) & AS CL) & AS N) & AS N) & AS P) & AS P) & $A S \quad S B)$ & AS AS) & & AS CD) & AS CR) \\
\hline
\end{tabular}

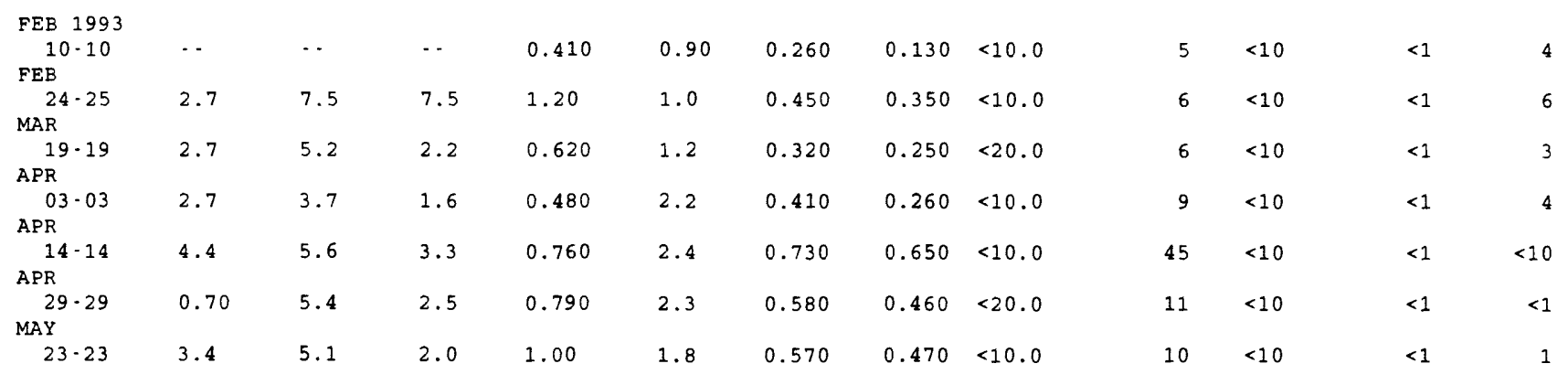


Table 7. Water-quality data for storm-sewer outfall stations, Mesquite, Texas, 1992-93-Continued

08061940 - SOUTH MESQUITE CREEK OUTFALL AT BRUTON ROAD, MESQUITE, TX (WY 1993)-Continued

\begin{tabular}{|c|c|c|c|c|c|c|c|c|c|c|c|c|}
\hline & \multirow[b]{2}{*}{$\begin{array}{l}\text { COPPER, } \\
\text { TOTAL }\end{array}$} & \multirow[b]{2}{*}{ CYANIDE } & & \multirow[b]{2}{*}{$\begin{array}{l}\text { LEAD, } \\
\text { TOTAL }\end{array}$} & \multirow[b]{2}{*}{$\begin{array}{l}\text { MERCURY } \\
\text { TOTAL }\end{array}$} & \multicolumn{7}{|c|}{ SILVER } \\
\hline & & & & & & $\begin{array}{l}\text { NICKEL, } \\
\text { TOTAL }\end{array}$ & SELE - & $\begin{array}{l}\text { SILVER, } \\
\text { TOTAL }\end{array}$ & $\begin{array}{l}\text { TOTAL } \\
\text { RECOV. }\end{array}$ & THAL - & $\begin{array}{l}\text { ZINC, } \\
\text { TOTAL }\end{array}$ & $A R B O N$ \\
\hline$T E$ & $\begin{array}{l}\text { RECOV- } \\
\text { ERABLE } \\
\text { (UG/L }\end{array}$ & $\begin{array}{c}\text { TOTAL } \\
\text { EPA } \\
\text { (MG/L }\end{array}$ & $\begin{array}{l}\text { CYANIDE } \\
\text { TOTAL } \\
\text { (MG/L }\end{array}$ & $\begin{array}{l}\text { RECOV- } \\
\text { ERABLE } \\
\text { (UG/L }\end{array}$ & $\begin{array}{l}\text { RECOV - } \\
\text { ERABLE } \\
\text { (UG/L }\end{array}$ & $\begin{array}{l}\text { RECOV- } \\
\text { ERABLE } \\
\text { (UG/L }\end{array}$ & $\begin{array}{l}\text { NIUM, } \\
\text { TOTAL } \\
\text { (UG/L }\end{array}$ & $\begin{array}{l}\text { RECOV- } \\
\text { ERABLE } \\
\text { (UG/L }\end{array}$ & $\begin{array}{l}\text { ERABLE } \\
\text { EPA } \\
\text { (UG/L }\end{array}$ & $\begin{array}{l}\text { LIUM, } \\
\text { TOTAL } \\
\text { (UG/L }\end{array}$ & $\begin{array}{l}\text { RECOV- } \\
\text { ERABLE } \\
\text { (UG/L }\end{array}$ & $\begin{array}{l}\text { ORGANI } \\
\text { TOTAL } \\
\text { (MG/L }\end{array}$ \\
\hline & AS $\mathrm{CU}$ ) & AS $\mathrm{CN}$ ) & AS CN) & AS PB) & AS HG) & AS NI) & AS SE) & AS AG) & AS AG) & AS TL) & AS $2 N$ ) & AS C) \\
\hline
\end{tabular}

FEB 1993
$10-10$
FEB
$24-25$
MAR
$19-19$
APR
$03-03$
APR
$14-14$
APR
$29-29$
MAY
$23-23$

$4<0.010<0.01000000$

$\begin{array}{ll}5 & <0.10 \\ 5 & <0.10 \\ 4 & <0.10 \\ 9 & <0.10 \\ 6 & <0.10 \\ 7 & <0.10 \\ 4 & <0.10\end{array}$

3
2
3
3
2
3
2

$\begin{array}{llllll}<2 & <1 & <0.500 & <10 & 30 & 12 \\ <2 & <1 & <0.500 & <5 & 30 & 12 \\ <2 & <1 & <0.500 & <5 & 40 & 11 \\ <2 & <1 & <0.500 & <5 & 60 & 22 \\ <2 & <1 & <0.500 & <5 & 30 & 16 \\ <2 & <1 & <0.500 & <5 & 40 & 12 \\ <2 & <1 & <0.500 & <5 & 30 & 11\end{array}$

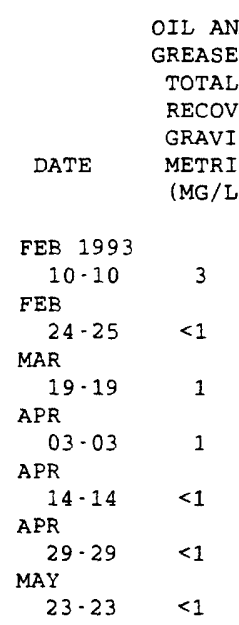

$\begin{array}{lrl}4 & <0.010 & <0.010 \\ 5 & 0.011 & <0.010 \\ 5 & <0.010 & <0.010 \\ 6 & <0.010 & <0.010 \\ 5 & <0.010 & <0.010 \\ 5 & <0.010 & <0.010 \\ 2 & <0.010 & <0.010\end{array}$

IL AND

RECOV.

PHENOIS ACRO- ACRYLO.

TETAL NITRILE

$(U G / L) \quad(U G / L) \quad(U G / L)$

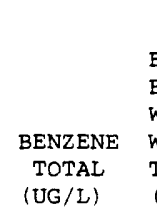

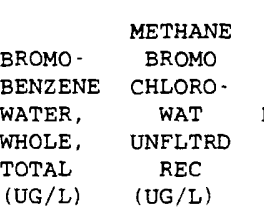

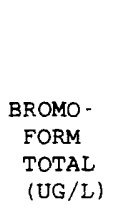

\begin{tabular}{|c|c|c|c|c|c|c|c|c|c|c|c|c|}
\hline & & & & 2 - & & & 0. & & DIBROMO & DI - & $1,2-$ & \\
\hline & & CHLORO- & & CHLORO- & & & CHLORO- & TOLUENE & CHLORO- & BROMO - & DIBROMO & DI - \\
\hline & & DI - & & ETHYL- & & METHYL - & TOLUENE & P. CHLOR & PROPANE & METHANE & ETHANE & CHLORO - \\
\hline & CHLORO- & BROMO - & CHLORO - & VINYL - & CHLORO - & CHLO- & WATER & WATER & WATER & WATER & WATER & BROMO - \\
\hline & BENZENE & METHANE & ETHANE & ETHER & FORM & RIDE & WHOLE & UNFLTRD & WHOLE & WHOLE & WHOLE & METHANE \\
\hline DATE & $\begin{array}{l}\text { TOTAL } \\
\text { (UG/L) }\end{array}$ & $\begin{array}{l}\text { TOTAL } \\
\text { (UG/L) }\end{array}$ & $\begin{array}{l}\text { TOTAL } \\
(\mathrm{UG} / \mathrm{L})\end{array}$ & $\begin{array}{l}\text { TOTAL } \\
(\mathrm{UG} / \mathrm{L})\end{array}$ & $\begin{array}{l}\text { TOTAL } \\
\text { (UG/L) }\end{array}$ & $\begin{array}{c}\text { TOTAL } \\
\text { (UG/L) }\end{array}$ & $\begin{array}{l}\text { TOTAL } \\
\text { (UG/L) }\end{array}$ & $\begin{array}{c}\text { REC } \\
(U G / L)\end{array}$ & $\begin{array}{l}\text { TOT.REC } \\
\text { (UG/L) }\end{array}$ & $\begin{array}{l}\text { RECOVER } \\
\text { (UG/L) }\end{array}$ & $\begin{array}{l}\text { TOTAL } \\
\text { (UG/L) }\end{array}$ & $\begin{array}{l}\text { TOTAL } \\
\text { (UG/L) }\end{array}$ \\
\hline
\end{tabular}

\begin{tabular}{|c|c|c|c|c|c|c|c|c|c|c|c|c|}
\hline \multicolumn{13}{|l|}{ FEB 1993} \\
\hline $10-10$ & $<0.20$ & $<0.2$ & $<0.2$ & $<1.0$ & $<0.2$ & 0.3 & $<0.2$ & $<0.20$ & $<1.0$ & $<0.2$ & $<0.2$ & $<0.2$ \\
\hline \multicolumn{13}{|l|}{ FEB } \\
\hline $24-25$ & $<0.20$ & $<0.2$ & $<0.2$ & $<1.0$ & $<0,2$ & $<0.2$ & $<0.2$ & $<0.20$ & $<1.0$ & $<0.2$ & $<0.2$ & $<0.2$ \\
\hline \multicolumn{13}{|l|}{ MAR } \\
\hline $19-19$ & $<0.20$ & $<0.2$ & $<0.2$ & $<1.0$ & $<0.2$ & $<0.2$ & $<0.2$ & $<0.20$ & $<1.0$ & $<0.2$ & $<0.2$ & $<0.2$ \\
\hline \multicolumn{13}{|l|}{ APR } \\
\hline 03.03 & $<0.20$ & $<0.2$ & $<0.2$ & $<1.0$ & $<0.2$ & $<0.2$ & $<0.2$ & $<0.20$ & $<1.0$ & $<0.2$ & $<0.2$ & $<0.2$ \\
\hline \multicolumn{13}{|l|}{ APR } \\
\hline $14-14$ & $<0.20$ & $<0.2$ & $<0.2$ & $<1.0$ & 0.2 & $<0.2$ & $<0.2$ & $<0.20$ & $<1.0$ & $<0.2$ & $<0.2$ & 0.2 \\
\hline \multicolumn{13}{|l|}{ APR } \\
\hline $29-29$ & $<0.20$ & $<0.2$ & $<0.2$ & $<1.0$ & $<0.2$ & $<0.2$ & $<0.2$ & $<0.20$ & $<1.0$ & $<0.2$ & $<0.2$ & $<0.2$ \\
\hline \multicolumn{13}{|l|}{ MAY } \\
\hline $23-23$ & $<5.0$ & $<5.0$ & $<5.0$ & $<25$ & $<5.0$ & $<5.0$ & $<5.0$ & $<5.0$ & $<25$ & $<5.0$ & $<5.0$ & $<5.0$ \\
\hline
\end{tabular}


Table 7. Water-quality data for storm-sewer outfall stations, Mesquite, Texas, 1992-93-Continued

08061940 - SOUTH MESQUITE CREEK OUTFALL AT BRUTON ROAD, MESQUITE, TX (WY 1993)-Continued

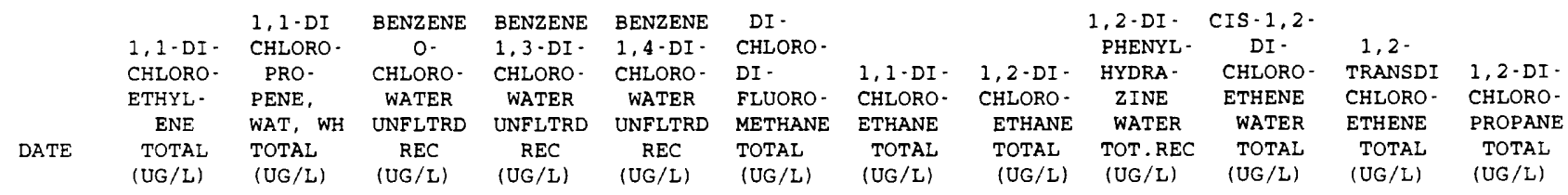

\begin{tabular}{|c|c|c|c|c|c|c|c|c|c|c|c|c|}
\hline \multicolumn{13}{|l|}{ FEB 1993} \\
\hline $10-10$ & $<0.2$ & $<0.2$ & $<5.0$ & $<5.0$ & $<5.0$ & $<0.2$ & $<0.2$ & $<0.2$ & $<5.0$ & $<0.2$ & $<0.2$ & $<0.2$ \\
\hline \multicolumn{13}{|l|}{ FEB } \\
\hline $24-25$ & $<0.2$ & $<0.2$ & $<5.0$ & $<5.0$ & $<5.0$ & $<0.2$ & $<0.2$ & $<0.2$ & $<5.0$ & $<0.2$ & $<0.2$ & $<0.2$ \\
\hline \multicolumn{13}{|l|}{ MAR } \\
\hline $19-19$ & $<0.2$ & $<0.2$ & $<0.20$ & $<0.20$ & $<0.20$ & $<0.2$ & $<0.2$ & $<0.2$ & $<5.0$ & $<0.2$ & $<0.2$ & $<0.2$ \\
\hline \multicolumn{13}{|l|}{ APR } \\
\hline 03.03 & $<0.2$ & $<0.2$ & $<5.0$ & $<5.0$ & $<5.0$ & $<0.2$ & $<0.2$ & $<0.2$ & $<5.0$ & $<0.2$ & $<0.2$ & $<0.2$ \\
\hline \multicolumn{13}{|l|}{ APR } \\
\hline $14-14$ & $<0.2$ & $<0.2$ & $<5.0$ & $<5.0$ & $<5.0$ & $<0.2$ & $<0.2$ & $<0.2$ & $<5.0$ & $<0.2$ & $<0.2$ & $<0.2$ \\
\hline \multicolumn{13}{|l|}{ APR } \\
\hline $29-29$ & $<0.2$ & $<0.2$ & $<0.20$ & $<0.20$ & $<0.20$ & $<0.2$ & $<0.2$ & $<0.2$ & $<5.0$ & $<0.2$ & $<0.2$ & $<0.2$ \\
\hline \multicolumn{13}{|l|}{ MAY } \\
\hline $23-23$ & $<5.0$ & $<5.0$ & $<5.0$ & $<5.0$ & $<5.0$ & $<5.0$ & $<5.0$ & $<5.0$ & $<5.0$ & $<5.0$ & $<5.0$ & $<5.0$ \\
\hline
\end{tabular}

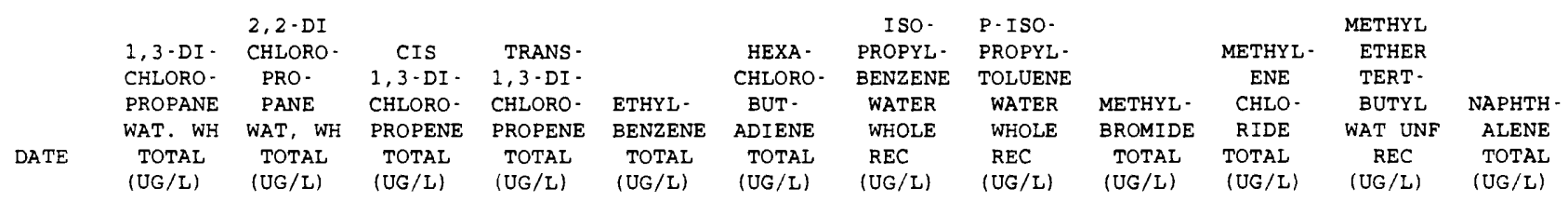

\begin{tabular}{|c|c|c|c|c|c|c|c|c|c|c|c|c|}
\hline \multicolumn{13}{|l|}{ FEB 1993} \\
\hline $10-10$ & $<0.2$ & $<0.2$ & $<0.2$ & $<0.2$ & $<0.2$ & $<5.0$ & $<0.20$ & $<0.20$ & $<0.2$ & $<0.2$ & $<1.0$ & $<5.0$ \\
\hline \multicolumn{13}{|l|}{ FEB } \\
\hline $24-25$ & $<0.2$ & $<0.2$ & $<0.2$ & $<0.2$ & $<0.2$ & $<5.0$ & $<0.20$ & $<0.20$ & $<0.2$ & $<0.3$ & $<1.0$ & $<5.0$ \\
\hline \multicolumn{13}{|l|}{ MAR } \\
\hline $19-19$ & $<0.2$ & $<0.2$ & $<0.2$ & $<0.2$ & $<0.2$ & $<0.2$ & $<0.20$ & $<0.20$ & $<0.2$ & $<0.2$ & $<1.0$ & $<0.2$ \\
\hline \multicolumn{13}{|l|}{ APR } \\
\hline 03.03 & $<0.2$ & $<0.2$ & $<0.2$ & $<0.2$ & $<0.2$ & $<5.0$ & $<0.20$ & $<0.20$ & $<0.2$ & $<0.2$ & $<1.0$ & $<5.0$ \\
\hline \multicolumn{13}{|l|}{ APR } \\
\hline $14 \cdot 14$ & $<0.2$ & $<0.2$ & $<0.2$ & $<0.2$ & $<0.2$ & $<5.0$ & $<0.20$ & $<0.20$ & $<0.2$ & $<0.2$ & $<1.0$ & $<5.0$ \\
\hline \multicolumn{13}{|l|}{ APR } \\
\hline $29 \cdot 29$ & $<0.2$ & $<0.2$ & $<0.2$ & $<0.2$ & $<0.2$ & $<0.2$ & $<0.20$ & $<0.20$ & $<0.2$ & $<0.2$ & $<1.0$ & $<0.2$ \\
\hline \multicolumn{13}{|l|}{ MAY } \\
\hline $23-23$ & $<5.0$ & $<5.0$ & $<5.0$ & $<5.0$ & $<5.0$ & $<5.0$ & $<5.0$ & $<5.0$ & $<5.0$ & $<5.0$ & $<25$ & $<5.0$ \\
\hline
\end{tabular}

\begin{tabular}{|c|c|c|c|c|c|c|c|c|c|c|c|c|}
\hline & $\begin{array}{c}\text { BENZENE } \\
\text { N-PROPY } \\
\text { WATER }\end{array}$ & & $\begin{array}{c}\text { ETHANE, } \\
1112- \\
\text { TETRA- } \\
\text { CHLORO- }\end{array}$ & $\begin{array}{r}\text { ETHANE, } \\
1,1,2,2 \\
\text { TETRA - } \\
\text { CHLORO - }\end{array}$ & $\begin{array}{l}\text { TETRA - } \\
\text { CHLORO- } \\
\text { ETHYL- }\end{array}$ & & $\begin{array}{c}1,2,3- \\
\text { TRI - } \\
\text { CHLORO } \\
\text { BENZENE }\end{array}$ & $\begin{array}{c}\text { BENZENE } \\
1,2,4- \\
\text { TRI - } \\
\text { CHLORO - }\end{array}$ & $\begin{array}{c}1,1,1- \\
\text { TRI - } \\
\text { CHLORO- }\end{array}$ & $\begin{array}{c}1,1,2- \\
\text { TRI- } \\
\text { CHLORO- }\end{array}$ & $\begin{array}{l}\text { TRI - } \\
\text { CHLORO - } \\
\text { ETHYL - }\end{array}$ & $\begin{array}{l}\text { TRI - } \\
\text { CHLORO- } \\
\text { FLUORO - }\end{array}$ \\
\hline DATE & $\begin{array}{l}\text { UNFLTRD } \\
\text { REC } \\
\text { (UG/L) }\end{array}$ & $\begin{array}{l}\text { STYRENE } \\
\text { TOTAL } \\
\text { (UG/L) }\end{array}$ & $\begin{array}{l}\text { WAT UNF } \\
\text { REC } \\
\text { (UG/L) }\end{array}$ & $\begin{array}{l}\text { WAT UNF } \\
\text { REC } \\
\text { (UG/L) }\end{array}$ & $\begin{array}{l}\text { ENE } \\
\text { TOTAL } \\
\text { (UG/L) }\end{array}$ & $\begin{array}{l}\text { TOLUENE } \\
\text { TOTAL } \\
\text { (UG/L) }\end{array}$ & $\begin{array}{l}\text { WAT, WH } \\
\text { REC } \\
(\mathrm{UG} / \mathrm{L})\end{array}$ & $\begin{array}{l}\text { WAT UNF } \\
\text { REC } \\
\text { (UG/L) }\end{array}$ & $\begin{array}{l}\text { ETHANE } \\
\text { TOTAL } \\
\text { (UG/L) }\end{array}$ & $\begin{array}{c}\text { ETHANE } \\
\text { TOTAL } \\
\text { (UG/L) }\end{array}$ & $\begin{array}{c}\text { ENE } \\
\text { TOTAL } \\
\text { (UG/L) }\end{array}$ & $\begin{array}{c}\text { METHANE } \\
\text { TOTAL } \\
\text { (UG/L) }\end{array}$ \\
\hline
\end{tabular}

\begin{tabular}{|c|c|c|c|c|c|c|c|c|c|c|c|c|}
\hline \multicolumn{13}{|l|}{ FEB 1993} \\
\hline $10-10$ & $<0.20$ & $<0.2$ & $<0.2$ & $<0.2$ & $<0.2$ & $<0.2$ & $<0.20$ & $<5.0$ & $<0.2$ & $<0.2$ & $<0.2$ & $<0.2$ \\
\hline \multicolumn{13}{|l|}{ FEB } \\
\hline $24-25$ & $<0.20$ & $<0.2$ & $<0.2$ & $<0.2$ & $<0.2$ & $<0.2$ & $<0.20$ & $<5.0$ & $<0.2$ & $<0.2$ & $<0.2$ & $<0.2$ \\
\hline \multicolumn{13}{|l|}{ MAR } \\
\hline $19-19$ & $<0.20$ & $<0.2$ & $<0.2$ & $<0.2$ & $<0.2$ & $<0.2$ & $<0.20$ & $<0.20$ & $<0.2$ & $<0.2$ & $<0.2$ & $<0.2$ \\
\hline \multicolumn{13}{|l|}{ APR } \\
\hline $03-03$ & $<0.20$ & $<0.2$ & $<0.2$ & $<0.2$ & $<0.2$ & $<0.2$ & $<0.20$ & $<5.0$ & $<0.2$ & $<0.2$ & $<0.2$ & $<0.2$ \\
\hline \multicolumn{13}{|l|}{ APR } \\
\hline $14-14$ & $<0.20$ & $<0.2$ & $<0.2$ & $<0.2$ & $<0.2$ & $<0.2$ & $<0.20$ & $<5.0$ & $<0.2$ & $<0.2$ & $<0.2$ & $<0.2$ \\
\hline \multicolumn{13}{|l|}{ APR } \\
\hline $29-29$ & $<0.20$ & $<0.2$ & $<0.2$ & $<0.2$ & $<0.2$ & $<0.2$ & $<0.20$ & $<0.20$ & $<0.2$ & $<0.2$ & $<0.2$ & $<0.2$ \\
\hline \multicolumn{13}{|l|}{ MAY } \\
\hline $23-23$ & $<5.0$ & $<5.0$ & $<5.0$ & $<5.0$ & $<5.0$ & $<5.0$ & $<5.0$ & $<5.0$ & $<5.0$ & $<5.0$ & $<5.0$ & $<5.0$ \\
\hline
\end{tabular}


Table 7. Water-quality data for storm-sewer outfall stations, Mesquite, Texas, 1992-93-Continued

08061940 - SOUTH MESQUITE CREEK OUTFALL AT BRUTON ROAD, MESQUITE, TX (WY 1993)-Continued

\begin{tabular}{|c|c|c|c|c|c|c|c|c|c|c|c|c|}
\hline DATE & $\begin{array}{c}123 \text { - TRI } \\
\text { CHLORO- } \\
\text { PROPANE } \\
\text { WATER } \\
\text { WHOLE } \\
\text { TOTAL } \\
\text { (UG/L) }\end{array}$ & $\begin{array}{l}\text { FREON- } \\
113 \\
\text { WATER } \\
\text { UNFLTRD } \\
\text { REC } \\
(U G / L)\end{array}$ & $\begin{array}{l}\text { PSEUDO- } \\
\text { CUMENE } \\
\text { WATER } \\
\text { UNFLTRD } \\
\text { REC } \\
\text { (UG/L) }\end{array}$ & $\begin{array}{l}\text { MESIT- } \\
\text { YLENE } \\
\text { WATER } \\
\text { UNFLTRD } \\
\text { REC } \\
\text { (UG/L) }\end{array}$ & $\begin{array}{l}\text { VINYL } \\
\text { CHLO- } \\
\text { RIDE } \\
\text { TOTAL } \\
\text { (UG/L) }\end{array}$ & $\begin{array}{c}\text { XYLENE } \\
\text { WATER } \\
\text { UNFLTRD } \\
\text { REC } \\
\text { (UG/L) }\end{array}$ & $\begin{array}{c}\text { ACE - } \\
\text { NAPHTH- } \\
\text { ENE } \\
\text { TOTAL } \\
(U G / L)\end{array}$ & $\begin{array}{l}\text { ACE - } \\
\text { NAPHTH - } \\
\text { YLENE } \\
\text { TOTAL } \\
\text { (UG/L) }\end{array}$ & $\begin{array}{l}\text { ANTHRA - } \\
\text { CENE } \\
\text { TOTAL } \\
\text { (UG/L) }\end{array}$ & $\begin{array}{l}\text { BENZI - } \\
\text { DINE } \\
\text { TOTAL } \\
\text { (UG/L) }\end{array}$ & $\begin{array}{l}\text { BENZO A } \\
\text { ANTHRAC } \\
\text { ENEI, } 2 \text { - } \\
\text { BENZANT } \\
\text { HRACENE } \\
\text { TOTAL } \\
\text { (UG/L) }\end{array}$ & $\begin{array}{l}\text { BENZO- } \\
\text { A- } \\
\text { PYRENE } \\
\text { TOTAL } \\
\text { (UG/L) }\end{array}$ \\
\hline \multicolumn{13}{|l|}{ FEB 1993} \\
\hline \multicolumn{12}{|l|}{ FEB } & $<10.0$ \\
\hline \multicolumn{12}{|l|}{ MAR } & $<10,0$ \\
\hline \multicolumn{9}{|l|}{ APR } & & & $<10.0$ & $<10.0$ \\
\hline $\begin{array}{l}03-03 \\
\text { APR }\end{array}$ & $<0.2$ & $<0.5$ & $<0.20$ & $<0.20$ & $<0.2$ & $<0.20$ & $<5.0$ & $<5.0$ & $<5.0$ & $<40.0$ & $<10.0$ & $<10.0$ \\
\hline $\begin{array}{l}14-14 \\
A P R\end{array}$ & $<0.2$ & $<0.5$ & $<0.20$ & $<0.20$ & $<0.2$ & $<0.20$ & $<5.0$ & $<5.0$ & $<5.0$ & $<40.0$ & $<10.0$ & $<10.0$ \\
\hline $\begin{array}{l}29 \cdot 29 \\
\text { MAY }\end{array}$ & $<0.2$ & $<0.5$ & $<0.20$ & $<0.20$ & $<0.2$ & $<0.20$ & $<5.0$ & $<5.0$ & $<5.0$ & $<40.0$ & $<10.0$ & $<10.0$ \\
\hline \multirow[t]{3}{*}{$23-23$} & $<5.0$ & $<13$ & $<5.0$ & $<5.0$ & $<5.0$ & $<5.0$ & $<5.0$ & $<5.0$ & $<5.0$ & $<40.0$ & $<10.0$ & $<10.0$ \\
\hline & & & BENZO & $4-$ & & BIS & BIS & BIS $12-$ & & & & $4-$ \\
\hline & $\begin{array}{l}\text { BENZO B } \\
\text { FLUOR - } \\
\text { AN- } \\
\text { THENE }\end{array}$ & $\begin{array}{l}\text { BENZO K } \\
\text { FLUOR- } \\
\text { AN- } \\
\text { THENE }\end{array}$ & $\begin{array}{l}\text { GHI PERYL } \\
\text { ENE1, 12- } \\
\text { BENZO } \\
\text { PERYLENE }\end{array}$ & $\begin{array}{l}\text { BROMO- } \\
\text { PHENYL } \\
\text { PHENYL } \\
\text { ETHER }\end{array}$ & $\begin{array}{l}\text { N-BUTYL } \\
\text { BENZYL } \\
\text { PHTHAL. } \\
\text { ATE }\end{array}$ & $\begin{array}{l}\text { (2- } \\
\text { CHLORO- } \\
\text { ETHOXY) } \\
\text { METHANE }\end{array}$ & $\begin{array}{c}2- \\
\text { CHLORO- } \\
\text { ETHYL } \\
\text { ETHER }\end{array}$ & $\begin{array}{l}\text { CHLORO- } \\
\text { ISO- } \\
\text { PROPYL) } \\
\text { ETHER }\end{array}$ & $\begin{array}{l}\text { PARA - } \\
\text { CHLORO- } \\
\text { META } \\
\text { CRESOL }\end{array}$ & $\begin{array}{c}2- \\
\text { CHLORO- } \\
\text { NAPH - } \\
\text { THALENE }\end{array}$ & $\begin{array}{l}2- \\
\text { CHLORO- } \\
\text { PHENOL }\end{array}$ & $\begin{array}{l}\text { CHLORO- } \\
\text { PHENYL } \\
\text { PHENYL } \\
\text { ETHER }\end{array}$ \\
\hline DATE & $\begin{array}{l}\text { TOTAL } \\
(U G / L)\end{array}$ & $\begin{array}{l}\text { TOTAL } \\
\text { (UG/L) }\end{array}$ & $\begin{array}{l}\text { TOTAL } \\
\text { (UG/L) }\end{array}$ & $\begin{array}{l}\text { TOTAL } \\
\text { (UG/L) }\end{array}$ & $\begin{array}{l}\text { TOTAL } \\
\text { (UG/L) }\end{array}$ & $\begin{array}{l}\text { TOTAL } \\
\text { (UG/L) }\end{array}$ & $\begin{array}{l}\text { TOTAL } \\
\text { (UG/L) }\end{array}$ & $\begin{array}{l}\text { TOTAL } \\
\text { (UG/L) }\end{array}$ & $\begin{array}{l}\text { TOTAL } \\
(U G / L)\end{array}$ & $\begin{array}{l}\text { TOTAL } \\
(\mathrm{UG} / \mathrm{L})\end{array}$ & $\begin{array}{l}\text { TOTAL } \\
\text { (UG/L) }\end{array}$ & $\begin{array}{l}\text { TOTAL } \\
\text { (UG/L) }\end{array}$ \\
\hline \multicolumn{13}{|l|}{ FEB 1993} \\
\hline $10-10$ & $<10.0$ & $<10.0$ & $<10.0$ & $<5.0$ & $<5.0$ & $<5.0$ & $<5.0$ & $<5.0$ & $<30.0$ & $<5.0$ & $<5.0$ & $<5.0$ \\
\hline \multicolumn{13}{|l|}{ FEB } \\
\hline $24-25$ & $<10.0$ & $<10.0$ & $<10.0$ & $<5.0$ & $<5.0$ & $<5.0$ & $<5.0$ & $<5.0$ & $<30.0$ & $<5.0$ & $<5.0$ & $<5.0$ \\
\hline \multicolumn{13}{|l|}{ MAR } \\
\hline $\begin{array}{l}19-19 \\
\text { APR }\end{array}$ & $<10.0$ & \multicolumn{7}{|c|}{ APR } & $<30.0$ & $<5.0$ & $<5.0$ & $<5.0$ \\
\hline $03-03$ & $<10.0$ & $<10.0$ & $<10.0$ & $<5.0$ & $<5.0$ & $<5.0$ & $<5.0$ & $<5.0$ & $<30.0$ & $<5.0$ & $<5.0$ & $<5.0$ \\
\hline \multicolumn{13}{|l|}{ APR } \\
\hline $14-14$ & $<10.0$ & $<10.0$ & $<10.0$ & $<5.0$ & $<5.0$ & $<5.0$ & $<5.0$ & $<5.0$ & $<30.0$ & $<5.0$ & $<5.0$ & $<5.0$ \\
\hline \multicolumn{13}{|l|}{$A P R$} \\
\hline $29-29$ & $<10.0$ & $<10.0$ & $<10.0$ & $<5.0$ & $<5.0$ & $<5.0$ & $<5.0$ & $<5.0$ & $<30.0$ & $<5.0$ & $<5.0$ & $<5.0$ \\
\hline \multicolumn{13}{|l|}{ MAY } \\
\hline $23-23$ & $<10.0$ & $<10.0$ & $<10.0$ & $<5.0$ & $<5.0$ & $<5.0$ & $<5.0$ & $<5.0$ & $<30.0$ & $<5.0$ & $<5.0$ & $<5.0$ \\
\hline
\end{tabular}

\begin{tabular}{|c|c|c|c|c|c|c|c|c|c|c|c|c|}
\hline DATE & $\begin{array}{l}\text { CHRY - } \\
\text { SENE } \\
\text { TOTAL } \\
\text { (UG/L) }\end{array}$ & $\begin{array}{r}1,2,5,6- \\
\text { DIBENZ- } \\
\text { ANTHRA- } \\
\text { CENE } \\
\text { TOTAL } \\
\text { (UG } / L \text { ) }\end{array}$ & $\begin{array}{c}3,3^{\prime}- \\
\text { DI - } \\
\text { CHLORO- } \\
\text { BENZI - } \\
\text { DINE } \\
\text { TOTAL } \\
\text { (UG/L) }\end{array}$ & $\begin{array}{l}2,4 \text { - DI - } \\
\text { CHLORO- } \\
\text { PHENOL } \\
\text { TOTAL } \\
\text { (UG/L) }\end{array}$ & $\begin{array}{c}\text { DIETHYL } \\
\text { PHTHAL- } \\
\text { ATE } \\
\text { TOTAL } \\
\text { (UG/L) }\end{array}$ & $\begin{array}{c}\text { DI - } \\
\text { METHYL } \\
\text { PHTHAL- } \\
\text { ATE } \\
\text { TOTAL } \\
\text { (UG/L) }\end{array}$ & $\begin{array}{l}2,4 \text { - DI - } \\
\text { METHYL - } \\
\text { PHENOL } \\
\text { TOTAL } \\
\text { (UG/L) }\end{array}$ & $\begin{array}{c}\text { DI - N- } \\
\text { BUTYL } \\
\text { PHTHAL- } \\
\text { ATE } \\
\text { TOTAL } \\
\text { (UG/L) }\end{array}$ & $\begin{array}{c}4,6- \\
\text { DINITRO- } \\
\text { ORTHO- } \\
\text { CRESOL } \\
\text { TOTAL } \\
\text { (UG/L) }\end{array}$ & $\begin{array}{c}2,4,- \\
\text { DI- } \\
\text { NITRO- } \\
\text { PHENOL } \\
\text { TOTAL } \\
\text { (UG/L) }\end{array}$ & $\begin{array}{l}2,4-\mathrm{DI}- \\
\text { NITRO- } \\
\text { TOLUENE } \\
\text { TOTAL } \\
(\mathrm{UG} / \mathrm{L})\end{array}$ & $\begin{array}{l}2,6-D I- \\
\text { NITRO- } \\
\text { TOLUENE } \\
\text { TOTAL } \\
\text { (UG/L) }\end{array}$ \\
\hline $\begin{array}{c}\text { FEB } 1993 \\
10-10\end{array}$ & $<10.0$ & $<10.0$ & $<20.0$ & $<5.0$ & $<5.0$ & $<5.0$ & $<5.0$ & $<5.0$ & $<30.0$ & $<20.0$ & $<5.0$ & $<5.0$ \\
\hline FEB & & & & & & & & & & & & \\
\hline $\begin{array}{l}24-25 \\
\text { MAR }\end{array}$ & $<10.0$ & $<10.0$ & $<20.0$ & $<5.0$ & $<5.0$ & $<5.0$ & $<5.0$ & $<5.0$ & $<30.0$ & $<20.0$ & $<5.0$ & $<5.0$ \\
\hline $\begin{array}{l}19 \cdot 19 \\
\text { APR }\end{array}$ & $<10.0$ & $<10.0$ & $<20.0$ & $<5.0$ & $<5.0$ & $<5.0$ & $<5.0$ & $<5.0$ & $<30.0$ & $<20.0$ & $<5.0$ & $<5.0$ \\
\hline $\begin{array}{l}03.03 \\
\text { APR }\end{array}$ & $<10.0$ & $<10.0$ & $<20.0$ & $<5.0$ & $<5.0$ & $<5.0$ & $<5.0$ & $<5.0$ & $<30.0$ & $<20.0$ & $<5.0$ & $<5.0$ \\
\hline $\begin{array}{l}14-14 \\
\text { APR }\end{array}$ & $<10.0$ & $<10.0$ & $<20.0$ & $<5.0$ & $<5.0$ & $<5.0$ & $<5.0$ & $<5.0$ & $<30.0$ & $<20.0$ & $<5.0$ & $<5.0$ \\
\hline $\begin{array}{l}29-29 \\
\text { MAY }\end{array}$ & $<10.0$ & $<10.0$ & $<20.0$ & $<5.0$ & $<5.0$ & $<5.0$ & $<5.0$ & $<5.0$ & $<30.0$ & $<20.0$ & $<5.0$ & $<5.0$ \\
\hline $23-23$ & $<10.0$ & $<10.0$ & $<20.0$ & $<5.0$ & $<5.0$ & $<5.0$ & $<5.0$ & $<5.0$ & $<30.0$ & $<20.0$ & $<5.0$ & $<5.0$ \\
\hline
\end{tabular}


Table 7. Water-quality data for storm-sewer outfall stations, Mesquite, Texas, 1992-93-Continued

08061940 - SOUTH MESQUITE CREEK OUTFALL AT BRUTON ROAD, MESQUiTE, TX (WY 1993)-Continued

\begin{tabular}{|c|c|c|c|c|c|c|c|c|c|c|c|c|}
\hline DATE & $\begin{array}{c}\text { DI - N- } \\
\text { OCTYL } \\
\text { PHTHAL - } \\
\text { ATE } \\
\text { TOTAL } \\
(\mathrm{UG} / \mathrm{L})\end{array}$ & $\begin{array}{l}\text { BIS }(2- \\
\text { ETHYL } \\
\text { HEXYL) } \\
\text { PHTHAL- } \\
\text { ATE } \\
\text { TOTAL } \\
\text { (UG/L) }\end{array}$ & $\begin{array}{l}\text { FLUOR - } \\
\text { ANTHENE } \\
\text { TOTAL } \\
\text { (UG/L) }\end{array}$ & $\begin{array}{c}\text { FLUOR - } \\
\text { ENE } \\
\text { TOTAL } \\
\text { (UG/L) }\end{array}$ & $\begin{array}{c}\text { HEXA- } \\
\text { CHLORO- } \\
\text { BENZENE } \\
\text { TOTAL } \\
\text { (UG/L) }\end{array}$ & $\begin{array}{l}\text { HEXA- } \\
\text { CHLORO- } \\
\text { CYCLO- } \\
\text { PENT- } \\
\text { ADIENE } \\
\text { TOTAL } \\
\text { (UG/L) }\end{array}$ & $\begin{array}{l}\text { HEXA- } \\
\text { CHLORO- } \\
\text { ETHANE } \\
\text { TOTAL } \\
\text { (UG/L) }\end{array}$ & $\begin{array}{c}\text { INDENO } \\
(1,2,3- \\
\text { CD }) \\
\text { PYRENE } \\
\text { TOTAL } \\
(U G / L)\end{array}$ & $\begin{array}{l}\text { ISO- } \\
\text { PHORONE } \\
\text { TOTAL } \\
\text { (UG/L) }\end{array}$ & $\begin{array}{l}\text { NITRO- } \\
\text { BENZENE } \\
\text { TOTAL } \\
(U G / L)\end{array}$ & $\begin{array}{l}\text { N- NITRO- } \\
\text { SODI - } \\
\text { METHY - } \\
\text { LAMINE } \\
\text { TOTAL } \\
\text { (UG/L) }\end{array}$ & $\begin{array}{c}2- \\
\text { NITRO- } \\
\text { PHENOL } \\
\text { TOTAL } \\
\text { (UG/L) }\end{array}$ \\
\hline FEB 1993 & & & & & & & & & & & & \\
\hline${ }_{\mathrm{FEB}}^{10 \cdot 10}$ & $<10.0$ & $<5.0$ & $<5.0$ & $<5.0$ & $<5.0$ & $<5.0$ & $<5.0$ & $<10.0$ & $<5.0$ & $<5.0$ & $<5.0$ & $<5.0$ \\
\hline $\begin{array}{l}24-25 \\
\text { MAR }\end{array}$ & $<10.0$ & $<5.0$ & $<5.0$ & $<5.0$ & $<5.0$ & $<5.0$ & $<5.0$ & $<10.0$ & $<5.0$ & $<5.0$ & $<5.0$ & $<5.0$ \\
\hline $\begin{array}{l}19-19 \\
\text { APR }\end{array}$ & $<10.0$ & $<5.0$ & $<5.0$ & $<5.0$ & $<5.0$ & $<5.0$ & $<5.0$ & $<10.0$ & $<5.0$ & $<5.0$ & $<5.0$ & $<5.0$ \\
\hline $\begin{array}{l}03.03 \\
\text { APR }\end{array}$ & $<10.0$ & $<5.0$ & $<5.0$ & $<5.0$ & $<5.0$ & $<5.0$ & $<5.0$ & $<10.0$ & $<5.0$ & $<5.0$ & $<5.0$ & $<5.0$ \\
\hline $\begin{array}{l}14-14 \\
\text { APR }\end{array}$ & $<10.0$ & $<5.0$ & $<5.0$ & $<5.0$ & $<5.0$ & $<5.0$ & $<5.0$ & $<10.0$ & $<5.0$ & $<5.0$ & $<5.0$ & $<5.0$ \\
\hline${ }_{\text {MAY }}^{29-29}$ & $<10.0$ & $<5.0$ & $<5.0$ & $<5.0$ & $<5.0$ & $<5.0$ & $<5.0$ & $<10.0$ & $<5.0$ & $<5.0$ & $<5.0$ & $<5.0$ \\
\hline $23-23$ & $<10.0$ & $<5.0$ & $<5.0$ & $<5.0$ & $<5.0$ & $<5.0$ & $<5.0$ & $<10.0$ & $<5.0$ & $<5.0$ & $<5.0$ & $<5.0$ \\
\hline DATE & $\begin{array}{l}\text { 4- } \\
\text { NITRO- } \\
\text { PHENOL } \\
\text { TOTAL } \\
\text { (UG/L) }\end{array}$ & $\begin{array}{c}\text { N- } \\
\text { NITRO- } \\
\text { SODI-N- } \\
\text { PROPYL- } \\
\text { AMINE } \\
\text { TOTAL } \\
\text { (UG/L) }\end{array}$ & $\begin{array}{c}N-N I T R O- \\
\text { SODI - } \\
\text { PHENY - } \\
\text { LAMINE } \\
\text { TOTAL } \\
(U G / L)\end{array}$ & $\begin{array}{l}\text { PENTA - } \\
\text { CHLORO- } \\
\text { PHENOL } \\
\text { TOTAL } \\
\text { (UG/L) }\end{array}$ & $\begin{array}{l}\text { PHENAN - } \\
\text { THRENE } \\
\text { TOTAL } \\
\text { (UG/L) }\end{array}$ & $\begin{array}{c}\text { PHENOL } \\
\text { (C6H- } \\
5 O H \text { ) } \\
\text { TOTAL } \\
\text { (UG/L) }\end{array}$ & $\begin{array}{c}\text { PYRENE } \\
\text { TOTAL } \\
\text { (UG/L) }\end{array}$ & $\begin{array}{c}2,4,6- \\
\text { TRI - } \\
\text { CHLORO- } \\
\text { PHENOL } \\
\text { TOTAL } \\
\text { (UG/L) }\end{array}$ & $\begin{array}{l}\text { ALDRIN, } \\
\text { TOTAL } \\
\text { (UG/L) }\end{array}$ & $\begin{array}{c}\text { P, } P^{\prime} \\
\text { DDT, } \\
\text { TOTAL } \\
(U G / L)\end{array}$ & $\begin{array}{l}\text { ALPHA } \\
\text { BHC } \\
\text { TOTAL } \\
\text { (UG/L) }\end{array}$ & $\begin{array}{c}\text { BETA } \\
\text { BENZENE } \\
\text { HEXA- } \\
\text { CHLOR- } \\
\text { IDE } \\
\text { TOTAL } \\
\text { (UG/L) }\end{array}$ \\
\hline FEB 1993 & & & & & & & & & & & & \\
\hline FEB & $<30.0$ & $<5.0$ & $<5.0$ & $<30.0$ & $<5.0$ & $<5.0$ & $<5.0$ & $<20.0$ & $<0.040$ & $<0.10$ & $<0.03$ & $<0.03$ \\
\hline${ }_{\text {MAR }}^{24-25}$ & $<30.0$ & $<5.0$ & $<5.0$ & $<30.0$ & $<5.0$ & $<5.0$ & $<5.0$ & $<20.0$ & $<0.040$ & $<0.10$ & $<0.03$ & $<0.03$ \\
\hline $\begin{array}{l}19-19 \\
\text { APR }\end{array}$ & $<30.0$ & $<5.0$ & $<5.0$ & $<30.0$ & $<5.0$ & $<5.0$ & $<5.0$ & $<20.0$ & $<0.040$ & $<0.10$ & $<0.03$ & $<0.03$ \\
\hline $\begin{array}{l}03.03 \\
A P R\end{array}$ & $<30.0$ & $<5.0$ & $<5.0$ & $<30.0$ & $<5.0$ & $<5.0$ & $<5.0$ & $<20.0$ & $<0.040$ & $<0.10$ & $<0.03$ & $<0.03$ \\
\hline${ }_{\mathrm{APR}}^{14-14}$ & $<30.0$ & $<5.0$ & $<5.0$ & $<30.0$ & $<5.0$ & $<5.0$ & $<5.0$ & $<20.0$ & $<0.040$ & $<0.10$ & $<0.03$ & $<0.03$ \\
\hline $\begin{array}{l}29-29 \\
\text { MAY }\end{array}$ & $<30.0$ & $<5.0$ & $<5.0$ & $<30.0$ & $<5.0$ & $<5.0$ & $<5.0$ & $<20.0$ & $<0.040$ & $<0.10$ & $<0.03$ & $<0.03$ \\
\hline $23 \cdot 23$ & $<30.0$ & $<5.0$ & $<5.0$ & $<30.0$ & $<5.0$ & $<5.0$ & $<5.0$ & $<20.0$ & $<0.040$ & $<0.10$ & $<0.03$ & $<0.03$ \\
\hline
\end{tabular}

\begin{tabular}{|c|c|c|c|c|c|c|c|c|c|c|c|c|}
\hline DATE & $\begin{array}{c}\text { DELTA } \\
\text { BENZENE } \\
\text { HEXA- } \\
\text { CHLOR- } \\
\text { IDE } \\
\text { TOTAL } \\
\text { (UG/L) }\end{array}$ & $\begin{array}{l}\text { LINDANE } \\
\text { TOTAL } \\
\text { (UG/L) }\end{array}$ & $\begin{array}{c}\text { CHLOR- } \\
\text { DANE } \\
\text { CIS } \\
\text { WATER } \\
\text { WHOLE } \\
\text { TOTAL } \\
\text { (UG/L) }\end{array}$ & $\begin{array}{l}\text { CHLOR- } \\
\text { DANE } \\
\text { TRANS } \\
\text { WATER } \\
\text { WHOLE } \\
\text { TOTAL } \\
\text { (UG/L) }\end{array}$ & $\begin{array}{l}\text { CHLOR - } \\
\text { DANE, } \\
\text { TOTAL } \\
\text { (UG/L) }\end{array}$ & $\begin{array}{l}\text { P, } P^{\prime} \\
\text { DDD, } \\
\text { TOTAL } \\
\text { (UG/L) }\end{array}$ & $\begin{array}{c}\text { P, } P^{\prime} \\
\text { DDE, } \\
\text { TOTAL } \\
(\mathrm{UG} / \mathrm{L})\end{array}$ & $\begin{array}{l}\text { DI- } \\
\text { ELDRIN } \\
\text { TOTAL } \\
\text { (UG/L) }\end{array}$ & $\begin{array}{c}\text { ENDO- } \\
\text { SULFAN- } \\
\text { I } \\
\text { WATER } \\
\text { WHOLE } \\
\text { REC } \\
(U G / L)\end{array}$ & $\begin{array}{l}\text { ENDO- } \\
\text { SULFAN } \\
\text { BETA } \\
\text { TOTAL } \\
\text { (UG/L) }\end{array}$ & $\begin{array}{l}\text { ENDO- } \\
\text { SULFAN } \\
\text { SULFATE } \\
\text { TOTAL } \\
\text { (UG/L) }\end{array}$ & $\begin{array}{c}\text { ENDRIN } \\
\text { WATER } \\
\text { UNFLTRD } \\
\text { REC } \\
(U G / L)\end{array}$ \\
\hline$E B \quad 199$ & & & & & & & & & & & & \\
\hline $\begin{array}{l}10-10 \\
\text { FEB }\end{array}$ & $<0.09$ & $<0.030$ & $<0.10$ & $<0.10$ & $<0.1$ & $<0.10$ & $<0.04$ & $<0.020$ & $<0.10$ & $<0.04$ & $<0.60$ & $<0.060$ \\
\hline $\begin{array}{l}24-25 \\
\text { MAR }\end{array}$ & $<0.09$ & $<0.030$ & $<0.10$ & $<0.10$ & $<0.1$ & $<0.10$ & $<0.04$ & $<0.020$ & $<0.10$ & $<0.04$ & $<0.60$ & $<0.060$ \\
\hline $\begin{array}{l}19-19 \\
A P R\end{array}$ & $<0.09$ & $<0.030$ & $<0.10$ & $<0.10$ & $<0.1$ & $<0.10$ & $<0.04$ & $<0.020$ & $<0.10$ & $<0.04$ & $<0.60$ & $<0.060$ \\
\hline $\begin{array}{l}03-03 \\
\text { APR }\end{array}$ & $<0.09$ & $<0.030$ & $<0.10$ & $<0.10$ & $<0.1$ & $<0.10$ & $<0.04$ & $<0.020$ & $<0.10$ & $<0.04$ & $<0.60$ & $<0.060$ \\
\hline $\begin{array}{l}14-14 \\
A P R\end{array}$ & $<0.09$ & $<0.030$ & $<0.10$ & $<0.10$ & $<0.1$ & $<0.10$ & $<0.04$ & $<0.020$ & $<0.10$ & $<0.04$ & $<0.60$ & $<0.060$ \\
\hline $\begin{array}{l}29-29 \\
\mathbb{A} Y\end{array}$ & $<0.09$ & $<0.030$ & $<0.10$ & $<0.10$ & $<0.1$ & $<0.10$ & $<0.04$ & $<0.020$ & $<0.10$ & $<0.04$ & $<0.60$ & $<0.060$ \\
\hline $23 \cdot 23$ & $<0.09$ & $<0.030$ & $<0.10$ & $<0.10$ & $<0.1$ & $<0.10$ & $<0.04$ & $<0.020$ & $<0.10$ & $<0.04$ & $<0.60$ & $<0.060$ \\
\hline
\end{tabular}


Table 7. Water-quality data for storm-sewer outfall stations, Mesquite, Texas, 1992-93-Continued 08061940 - SOUTH MESQUITE CREEK OUTFALL AT BRUTON ROAD, MESQUITE, TX (WY 1993)-Continued

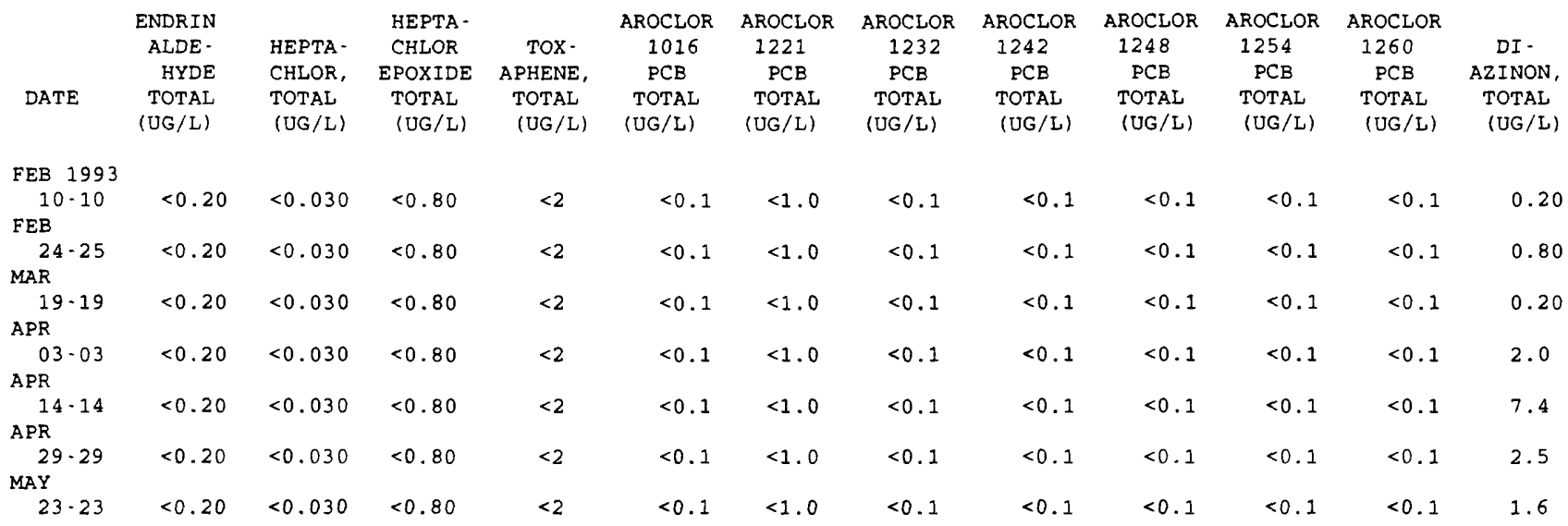


Table 8. Water-quality data for storm-sewer outfall stations, Plano, Texas, $1992-93$

08061510 - ROWLETT CREEK OUTFALL AT WILLOW CREEK PARK, PLANO, TX (WY 1993)

\begin{tabular}{|c|c|c|c|c|c|c|c|c|c|c|c|c|}
\hline DATE & TIME & $\begin{array}{l}\text { PRECIP. } \\
\text { ITATION } \\
\text { TOTAL } \\
\text { INCHES / } \\
\text { STORM }\end{array}$ & $\begin{array}{l}\text { ELAPSED } \\
\text { TIME } \\
\text { OF } \\
\text { STORM } \\
\text { (HOURS) }\end{array}$ & $\begin{array}{l}\text { STORM } \\
\text { WATER } \\
\text { FLOW } \\
\text { (MGD) }\end{array}$ & $\begin{array}{l}\text { SPE- } \\
\text { CIFIC } \\
\text { CON- } \\
\text { DUCT- } \\
\text { ANCE } \\
\text { (US/CM) }\end{array}$ & $\begin{array}{c}\text { SPE- } \\
\text { CIFIC } \\
\text { CON- } \\
\text { DUCT - } \\
\text { ANCE } \\
\text { LAB } \\
\text { (US/CM) }\end{array}$ & $\begin{array}{c}\text { PH } \\
\text { WATER } \\
\text { WHOLE } \\
\text { FIELD } \\
\text { (STAND- } \\
\text { ARD } \\
\text { UNITS) }\end{array}$ & $\begin{array}{c}\text { PH } \\
\text { WATER } \\
\text { WHOLE } \\
\text { LAB } \\
\text { (STAND- } \\
\text { ARD } \\
\text { UNITS) }\end{array}$ & $\begin{array}{c}\text { TEMPER - } \\
\text { ATURE } \\
\text { WATER } \\
\text { (DEG C) }\end{array}$ & $\begin{array}{l}\text { OXYGEN } \\
\text { DEMAND, } \\
\text { CHEM- } \\
\text { ICAL } \\
\text { (HIGH } \\
\text { LEVEL) } \\
\text { (MG } / L)\end{array}$ & $\begin{array}{l}\text { OXYGEN } \\
\text { DEMAND, } \\
\text { BIO- } \\
\text { CHEM- } \\
\text { ICAL, } \\
5 \text { DAY } \\
\text { (MG/L) }\end{array}$ & $\begin{array}{l}\text { COLI - } \\
\text { FORM, } \\
\text { FECAL, } \\
0.7 \\
\text { UM-MF } \\
\text { (COLS. } \\
100 \mathrm{ML} \text { ) }\end{array}$ \\
\hline $\begin{array}{c}\text { DEC } 1992 \\
09-09 \\
\text { JAN } 1993\end{array}$ & 0100 & 0.53 & 3.1 & 0.27 & 36 & 84 & 8.9 & 7.3 & 8.0 & 120 & 7.5 & 2600 \\
\hline $\begin{array}{l}09-09 \\
\text { JAN }\end{array}$ & 0310 & 0.49 & 5.0 & 0.27 & 66 & 86 & 8.3 & 8.1 & 9.5 & 79 & 4.8 & K5200 \\
\hline$\underset{F E B}{28-28}$ & 1450 & 0.30 & 7.7 & 0.16 & 76 & 127 & 7.4 & 7.8 & 11.5 & 71 & $\cdots$ & $\mathrm{K} 1000$ \\
\hline $\operatorname{MAR}^{10-10}$ & 0441 & 0.20 & 3.6 & 0.11 & 154 & 119 & 7.7 & 7.4 & 14.5 & 37 & 4.1 & K250 \\
\hline $\begin{array}{l}19-19 \\
\text { APR }\end{array}$ & 1330 & 0.41 & 5.5 & 0.24 & 80 & 115 & 8.0 & 7.8 & 13.5 & 150 & 7.3 & 2700 \\
\hline $\begin{array}{l}03-04 \\
\text { APR }\end{array}$ & 2335 & 0.47 & 2.7 & 0.31 & 63 & 95 & 7.4 & 7.5 & 13.0 & 70 & 5.0 & K8000 \\
\hline $14-14$ & 0630 & 0.83 & 4.5 & 0.54 & 133 & 110 & 8.0 & 7.4 & 13.5 & 90 & 7.6 & 41000 \\
\hline
\end{tabular}

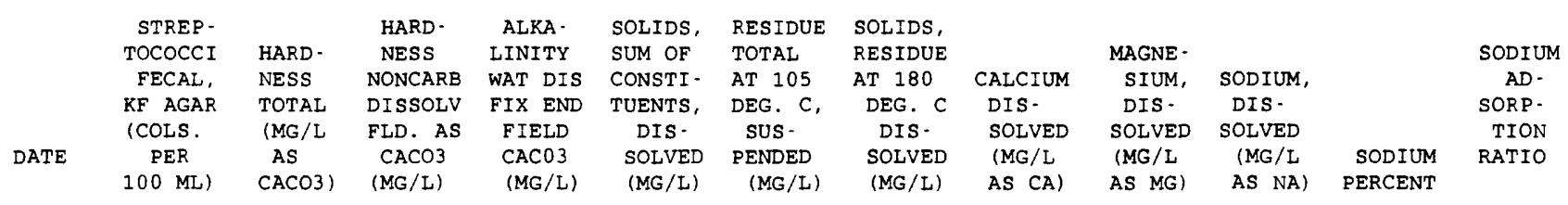

DEC 1992

$09-09$

18000

$\begin{array}{ccccc}18 & 0 & 21 & 39 & 44 \\ 23 & 0 & 28 & 60 & 130 \\ 31 & 0 & 38 & 66 & 44 \\ 32 & 0 & 39 & 60 & 38 \\ 36 & 0 & 37 & 63 & 108 \\ 20 & 0 & 20 & 47 & 124 \\ 29 & 1 & 28 & 54 & 53\end{array}$

$\begin{array}{cc}09-09 & 18000 \\ \text { JAN } 1993 & \\ 09-09 & 57000 \\ \text { JAN } & \\ 28-28 & 18000 \\ \text { FEB } & \\ 10-10 & 2600 \\ \text { MAR } & \\ 19-19 & 4800 \\ \text { APR } & \\ 03-04 & \mathrm{~K} 12000 \\ \text { APR } & \\ 14-14 & 100000\end{array}$

$\begin{array}{ll}36 & 7.0 \\ 40 & 8.9 \\ 55 & 12 \\ 44 & 12 \\ 55 & 14 \\ 41 & 7.5 \\ 65 & 11\end{array}$

$\begin{array}{ll}0.23 & 1.2 \\ 0.25 & 2.3 \\ 0.29 & 2.3 \\ 0.47 & 3.8 \\ 0.31 & 1.8 \\ 0.21 & 1.0 \\ 0.35 & 1.5\end{array}$

$\begin{array}{rl}10 & 0.1 \\ 15 & 0.2 \\ 12 & 0.2 \\ 18 & 0.3 \\ 9 & 0.1 \\ 9 & 0.1 \\ 9 & 0.1\end{array}$
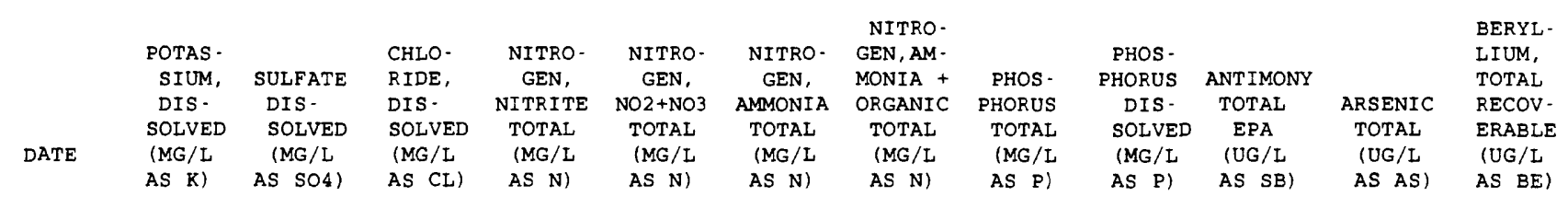

DEC 1992

\begin{tabular}{|c|c|c|c|c|c|c|c|c|c|c|c|c|}
\hline 09.09 & 3.1 & 2.6 & 0.80 & 0.050 & 0.320 & 0.150 & 0.60 & 0.210 & 0.100 & $<20.0$ & 1 & $<10$ \\
\hline \multicolumn{13}{|l|}{ JAN 1993} \\
\hline $09-09$ & 3.9 & 2.1 & 0.60 & $\cdots$ & 0.420 & $\cdots$ & 0.80 & 0.200 & 0.080 & $<10.0$ & 3 & $<10$ \\
\hline \multicolumn{13}{|l|}{ JAN } \\
\hline $28-28$ & 3.9 & 3.9 & 0.90 & $\cdots$ & 0.340 & $\cdots$ & 0.90 & 0.230 & 0.090 & $<20.0$ & 3 & $<10$ \\
\hline \multicolumn{13}{|l|}{ FEB } \\
\hline $10-10$ & 5.0 & 4.7 & 1.0 & $\cdots$ & 0.520 & $\cdots$ & 0.90 & 0.170 & 0.090 & $<10.0$ & 3 & $<10$ \\
\hline \multicolumn{13}{|l|}{ MAR } \\
\hline $19-19$ & 3.6 & 4.4 & 1.1 & $\cdots$ & 0.440 & $\cdots$ & 1.0 & 0.230 & 0.140 & $<20.0$ & 3 & $<10$ \\
\hline \multicolumn{13}{|l|}{ APR } \\
\hline 03.04 & 2.9 & 2.7 & 1.0 & $\cdots$ & 0.400 & $\cdots$ & 1.3 & 0.310 & 0.180 & $<10.0$ & 2 & $<10$ \\
\hline \multicolumn{13}{|l|}{ APR } \\
\hline $14-14$ & 3.6 & 3.6 & 1.6 & $\cdots$ & 0.460 & $\cdots$ & 1.1 & 0.270 & 0.210 & $<10.0$ & 3 & $<10$ \\
\hline
\end{tabular}


Table 8. Water-quality data for storm-sewer outfall stations, Plano, Texas, 1992-93-Continued

08061510 - ROWLETT CREEK OUTFALL AT WILLOW CREEK PARK, PLANO, TX (WY 1993)-Continued

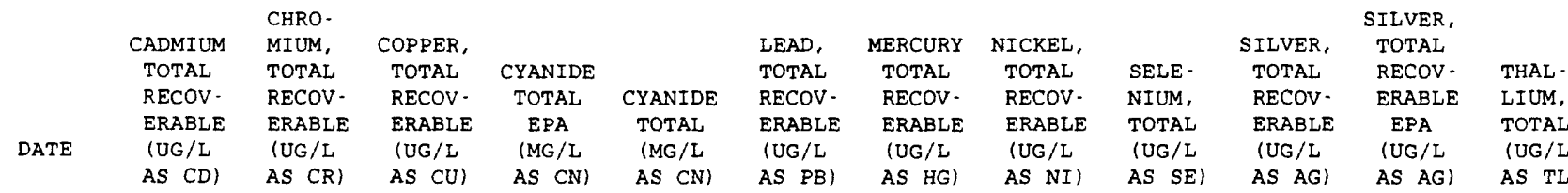

DEC 1992

$09-09$
JAN 1993

JAN 1993
$09-09$

JAN

$28-28$

FEB

$10-10$

MAR

$19-19$

APR

03.04

APR

$14 \cdot 14$

$<1$
$<1$
1
$<1$
$<1$
$<1$
$<1$

5
13
7
3
4
5
10

$\begin{array}{rrr}7 & 0.011 & <0.010 \\ 9 & <0.010 & <0.010 \\ 13 & <0.010 & <0.010 \\ 6 & <0.010 & <0.010 \\ 8 & 0.012 & <0.010 \\ 10 & <0.010 & <0.010 \\ 7 & <0.010 & <0.010\end{array}$

$\begin{aligned} 11 & <0.10 \\ 25 & <0.10 \\ 23 & <0.10 \\ 9 & <0.10 \\ 18 & <0.10 \\ 26 & <0.10 \\ 13 & <0.10\end{aligned}$

OIL AND

ZINC, GREASE

TOTAL CARBON, TOTAL

RECOV - ORGANIC RECOV.

ERABLE TOTAL GRAVI

DATE

METRIC (MG/L)
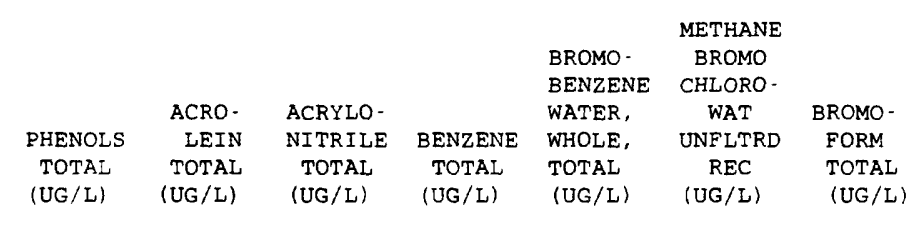

$$
\begin{array}{ll}
<1 & <0.500 \\
<1 & <0.500 \\
<1 & <0.500 \\
<1 & <0.500 \\
<1 & <0.500 \\
<1 & <0.500 \\
<1 & <0.500
\end{array}
$$

AS TL)

DEC 1992

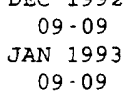

09.09

JAN

$28 \cdot 28$

FEB $10 \cdot 10$

MAR

$19 \cdot 19$

$A P R$

03.04

APR

$14-14$

$60 \quad 17$

120

18

21

50

14

110

110

60

21

15

7
2
5
4
$\cdots$
1
12

BENZENE

TERT -

BUTYL.

WATER

DATE

UNFLTRD

REC
(UG/L)

CARBON -

TETRA -

- CHLORO-

RIDE BENZENE

TOTAL

TOTAL

(UG /L)

$\begin{array}{lll}4 & <20 & <20 \\ 2 & <20 & <20 \\ 7 & <20 & <20 \\ 3 & <20 & <20 \\ 5 & <20 & <20 \\ 1 & <20 & <20 \\ 2 & <20 & <20\end{array}$

$<0.2$

$<0.2$

$<0.2<0.20$

$<0.2$

$<0.20$

$<0.2$

$<0.2$

$<0.20$

$<0.2$

$<0.2$

$<0.20$

$<0.2<0.2<0.20$

$<0.2$

$<0.2$

$<0.20$

$<0.2$

$<0.2$

$<0.20$

CHLORO-

ETHYL -

BROMO- CHLORO- VINYL

METHANE ETHANE ETHER

TOTAL TOTAL TOTAL

(UG/L)

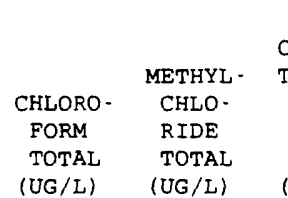

O- $\quad$ DIBROMO DI -

CHLORO- TOLUENE CHLORO- BROMO-

TOLUENE P-CHLOR PROPANE METHANE

WATER WATER WATER WATER

\begin{tabular}{|c|c|c|c|c|c|c|c|c|c|c|c|c|}
\hline \multicolumn{13}{|l|}{ DEC 1992} \\
\hline 09.09 & $<0.20$ & $<0.2$ & $<0.20$ & $<0.2$ & $<0.2$ & $<1.0$ & $<0.2$ & $<0.2$ & $<0.2$ & $<0.20$ & $<1.0$ & $<0.2$ \\
\hline \multicolumn{13}{|l|}{ JAN 1993} \\
\hline 09.09 & $<0.20$ & $<0.2$ & $<0.20$ & $<0.2$ & $<0.2$ & $<1.0$ & $<0.2$ & $<0.2$ & $<0.2$ & $<0.20$ & $<1.0$ & $<0.2$ \\
\hline \multicolumn{13}{|l|}{ JAN } \\
\hline $28 \cdot 28$ & $<0.20$ & $<0.2$ & $<0.20$ & $<0.2$ & $<0.2$ & $<1.0$ & $<0.2$ & $<0.2$ & $<0.2$ & $<0.20$ & $<1.0$ & $<0.2$ \\
\hline \multicolumn{13}{|l|}{ FEB } \\
\hline $10-10$ & $<0.20$ & $<0.2$ & $<0.20$ & $<0.2$ & $<0.2$ & $<1.0$ & $<0.2$ & $<0.2$ & $<0.2$ & $<0.20$ & $<1.0$ & $<0.2$ \\
\hline \multicolumn{13}{|l|}{ MAR } \\
\hline $19-19$ & $<0.20$ & $<0.2$ & $<0.20$ & $<0.2$ & $<0.2$ & $<1.0$ & $<0.2$ & $<0.2$ & $<0.2$ & $<0.20$ & $<1.0$ & $<0.2$ \\
\hline \multicolumn{13}{|l|}{ APR } \\
\hline $03-04$ & $<0.20$ & $<0.2$ & $<0.20$ & $<0.2$ & $<0.2$ & $<1.0$ & $<0.2$ & $<0.2$ & $<0.2$ & $<0.20$ & $<1.0$ & $<0.2$ \\
\hline \multicolumn{13}{|l|}{ APR } \\
\hline $14-14$ & $<0.20$ & $<0.2$ & $<0.20$ & $<0.2$ & $<0.2$ & $<1.0$ & $<0.2$ & $<0.2$ & $<0.2$ & $<0.20$ & $<1,0$ & $<0.2$ \\
\hline
\end{tabular}

WHOLE UNFLTRD WHOLE WHOLE

TOTAL REC TOT.REC RECOVER

(UG/L)

(UG/L)

(UG/L)

(UG/L) 
Table 8. Water-quality data for storm-sewer outfall stations, Plano, Texas, 1992-93-Continued

08061510 - ROWLETT CREEK OUTFALL AT WILLOW CREEK PARK, PLANO, TX (WY 1993)-Continued

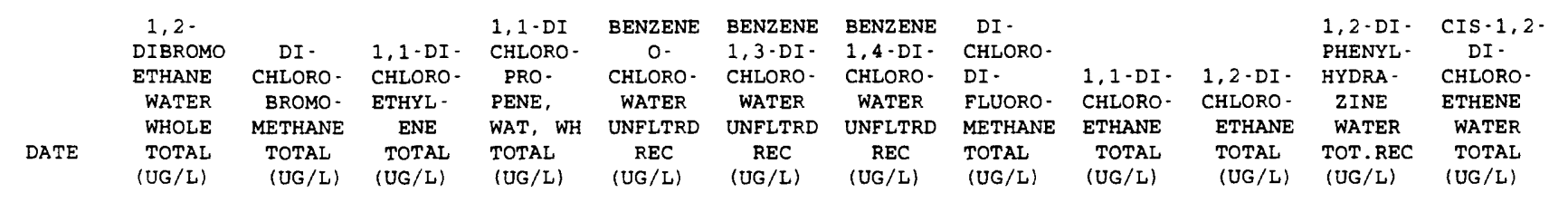

DEC 1992
$09-09$
JAN 1993
$09-09$
JAN
$28-28$
FEB
$10-10$
MAR
$19-19$
APR
$03-04$
APR
$14-14$

\begin{tabular}{|c|c|c|c|c|c|c|c|c|c|c|c|}
\hline$<0.2$ & $<0.2$ & $<0.2$ & $<0.2$ & $<5.0$ & $<5.0$ & $<5.0$ & $<0.2$ & $<0.2$ & $<0.2$ & $<5.0$ & $<0.2$ \\
\hline$<0.2$ & $<0.2$ & $<0.2$ & $<0.2$ & $<5.0$ & $<5.0$ & $<5.0$ & $<0.2$ & $<0.2$ & $<0.2$ & $<5.0$ & $<0.2$ \\
\hline$<0.2$ & $<0.2$ & $<0.2$ & $<0.2$ & $<5.0$ & $<5.0$ & $<5.0$ & $<0.2$ & $<0.2$ & $<0.2$ & $<5.0$ & $<0.2$ \\
\hline$<0.2$ & $<0.2$ & $<0.2$ & $<0.2$ & $<5.0$ & $<5.0$ & $<5.0$ & $<0.2$ & $<0.2$ & $<0.2$ & $<5.0$ & $<0.2$ \\
\hline$<0.2$ & $<0.2$ & $<0.2$ & $<0.2$ & $<0.20$ & $<0.20$ & $<0.20$ & $<0.2$ & $<0.2$ & $<0.2$ & $<5.0$ & $<0.2$ \\
\hline$<0.2$ & $<0.2$ & $<0.2$ & $<0.2$ & $<5.0$ & $<5.0$ & $<5.0$ & $<0.2$ & $<0.2$ & $<0.2$ & $<5.0$ & $<0.2$ \\
\hline$<0.2$ & $<0.2$ & $<0.2$ & $<0.2$ & $<5.0$ & $<5.0$ & $<5.0$ & $<0.2$ & $<0.2$ & $<0.2$ & $<5.0$ & $<0.2$ \\
\hline
\end{tabular}

DATE

\begin{tabular}{|c|c|c|c|c|c|c|c|c|c|c|}
\hline & & & $2,2-\mathrm{DI}$ & & & & & ISO- & P-ISO- & \\
\hline $1,2-$ & & $1,3-\mathrm{DI}$ - & CHLORO- & CIS & TRANS - & & HEXA - & PROPYL - & PROPYL - & \\
\hline TRANSDI & $1,2-D I-$ & CHLORO - & PRO - & $1,3-\mathrm{DI}-$ & $1,3-\mathrm{DI}=$ & & CHLORO - & BENZENE & TOLUENE & \\
\hline CHLORO - & CHLORO - & PROPANE & PANE & CHLORO- & CHLORO - & ЕTHYL- & BUT - & WATER & WATER & METHYL - \\
\hline $\begin{array}{r}\text { ETHENE } \\
\text { TOTAL } \\
\text { (UG/L) }\end{array}$ & $\begin{array}{l}\text { PROPANE } \\
\text { TOTAL } \\
\text { (UG/L) }\end{array}$ & $\begin{array}{l}\text { WAT. WH } \\
\text { TOTAL } \\
\text { (UG/L) }\end{array}$ & $\begin{array}{c}\text { WAT, WH } \\
\text { TOTAL } \\
\text { (UG/L) }\end{array}$ & $\begin{array}{l}\text { PROPENE } \\
\text { TOTAL } \\
\text { (UG/L) }\end{array}$ & $\begin{array}{l}\text { PROPENE } \\
\text { TOTAL } \\
\text { (UG/L) }\end{array}$ & $\begin{array}{l}\text { BENZENE } \\
\text { TOTAL } \\
\text { (UG/L) }\end{array}$ & $\begin{array}{c}\text { ADIENE } \\
\text { TOTAL } \\
\text { (UG/L) }\end{array}$ & $\begin{array}{l}\text { WHOLE } \\
\text { REC } \\
\text { (UG/L) }\end{array}$ & $\begin{array}{l}\text { WHOLE } \\
\text { REC } \\
\text { (UG/L) }\end{array}$ & $\begin{array}{c}\text { BROMIDE } \\
\text { TOTAL } \\
\text { (UG/L) }\end{array}$ \\
\hline
\end{tabular}

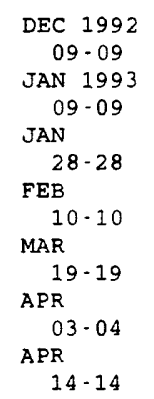

$\begin{array}{lllllllllll}<0.2 & <0.2 & <0.2 & <0.2 & <0.2 & <0.2 & <0.2 & <5.0 & <0.20 & <0.20 & <0.2 \\ <0.2 & <0.2 & <0.2 & <0.2 & <0.2 & <0.2 & <0.2 & <5.0 & <0.20 & <0.20 & <0.2 \\ <0.2 & <0.2 & <0.2 & <0.2 & <0.2 & <0.2 & <0.2 & <5.0 & <0.20 & <0.20 & <0.2 \\ <0.2 & <0.2 & <0.2 & <0.2 & <0.2 & <0.2 & <0.2 & <5.0 & <0.20 & <0.20 & <0.2 \\ <0.2 & <0.2 & <0.2 & <0.2 & <0.2 & <0.2 & <0.2 & <0.2 & <0.20 & <0.20 & <0.2 \\ <0.2 & <0.2 & <0.2 & <0.2 & <0.2 & <0.2 & <0.2 & <5.0 & <0.20 & <0.20 & <0.2 \\ <0.2 & <0.2 & <0.2 & <0.2 & <0.2 & <0.2 & <0.2 & <5.0 & <0.20 & <0.20 & <0.2\end{array}$

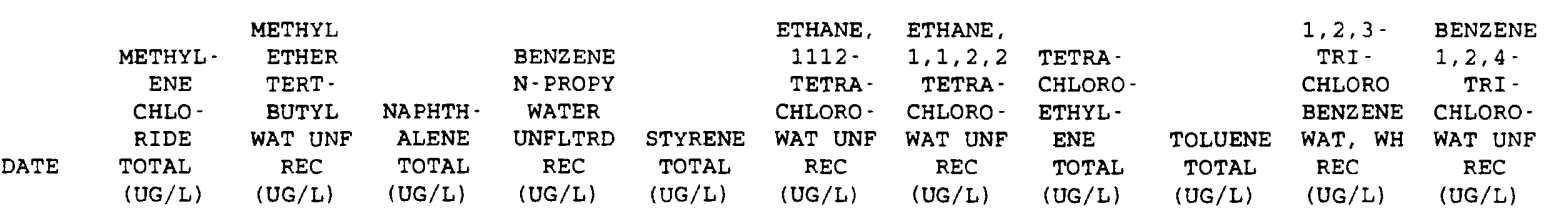

\begin{tabular}{|c|c|c|c|c|c|c|c|c|c|c|c|}
\hline \multicolumn{12}{|l|}{ DEC 1992} \\
\hline 09.09 & $<0.2$ & $<1.0$ & $<5.0$ & $<0.20$ & $<0.2$ & $<0.2$ & $<0.2$ & $<0.2$ & $<0.2$ & $<0.20$ & $<5.0$ \\
\hline \multicolumn{12}{|l|}{ JAN 1993} \\
\hline $09-09$ & $<0.2$ & $<1.0$ & $<5.0$ & $<0.20$ & $<0.2$ & $<0.2$ & $<0.2$ & $<0.2$ & $<0.2$ & $<0.20$ & $<5.0$ \\
\hline \multicolumn{12}{|l|}{ JAN } \\
\hline $28-28$ & $<0.4$ & $<1.0$ & $<5.0$ & $<0.20$ & $<0.2$ & $<0.2$ & $<0.2$ & $<0.2$ & 0.3 & $<0.20$ & $<5.0$ \\
\hline \multicolumn{12}{|l|}{ FEB } \\
\hline $10-10$ & $<0.4$ & $<1.0$ & $<5.0$ & $<0.20$ & $<0.2$ & $<0.2$ & $<0.2$ & $<0.2$ & $<0.2$ & $<0.20$ & $<5.0$ \\
\hline \multicolumn{12}{|l|}{ MAR } \\
\hline $19-19$ & $<0.2$ & $<1.0$ & $<0.2$ & $<0.20$ & $<0.2$ & $<0.2$ & $<0.2$ & $<0.2$ & $<0.2$ & $<0.20$ & $<0.20$ \\
\hline \multicolumn{12}{|l|}{$A P R$} \\
\hline $03-04$ & $<0.2$ & $<1.0$ & $<5.0$ & $<0.20$ & $<0.2$ & $<0.2$ & $<0.2$ & $<0.2$ & $<0.2$ & $<0.20$ & $<5.0$ \\
\hline \multicolumn{12}{|l|}{ APR } \\
\hline $14-14$ & $<0.2$ & $<1.0$ & $<5.0$ & $<0.20$ & $<0.2$ & $<0.2$ & $<0.2$ & $<0.2$ & $<0.2$ & $<0.20$ & $<5.0$ \\
\hline
\end{tabular}


Table 8. Water-quality data for storm-sewer outfall stations, Plano, Texas, 1992-93-Continued

08061510 - ROWLETT CREEK OUTFALL AT WILLOW CREEK PARK, PLANO, TX (WY 1993)-Continued

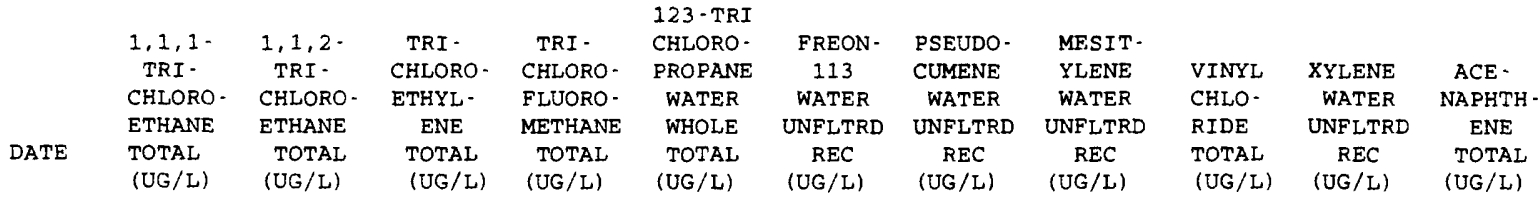

DEC 1992

DEC 1992
$09-09$
JAN 1993
$09-09$
JAN
$28-28$
FEB
$10-10$
MAR
$19-19$
APR
$03-04$
APR
$14-14$

$\begin{array}{lllllllllll}<0.2 & <0.2 & <0.2 & <0.2 & <0.2 & <0.5 & <0.20 & <0.20 & <0.2 & <0.20 & <5.0 \\ <0.2 & <0.2 & <0.2 & <0.2 & <0.2 & <0.5 & <0.20 & <0.20 & <0.2 & <0.20 & <5.0 \\ <0.2 & <0.2 & <0.2 & <0.2 & <0.2 & <0.5 & 0.20 & <0.20 & <0.2 & 0.30 & <5.0 \\ <0.2 & <0.2 & <0.2 & <0.2 & <0.2 & <0.5 & <0.20 & <0.20 & <0.2 & <0.20 & <5.0 \\ <0.2 & <0.2 & <0.2 & <0.2 & <0.2 & <0.5 & <0.20 & <0.20 & <0.2 & <0.20 & <5.0 \\ <0.2 & <0.2 & <0.2 & <0.2 & <0.2 & <0.5 & <0.20 & <0.20 & <0.2 & <0.20 & <5.0 \\ <0.2 & <0.2 & <0.2 & <0.2 & <0.2 & <0.5 & <0.20 & <0.20 & <0.2 & <0.20 & <5.0\end{array}$

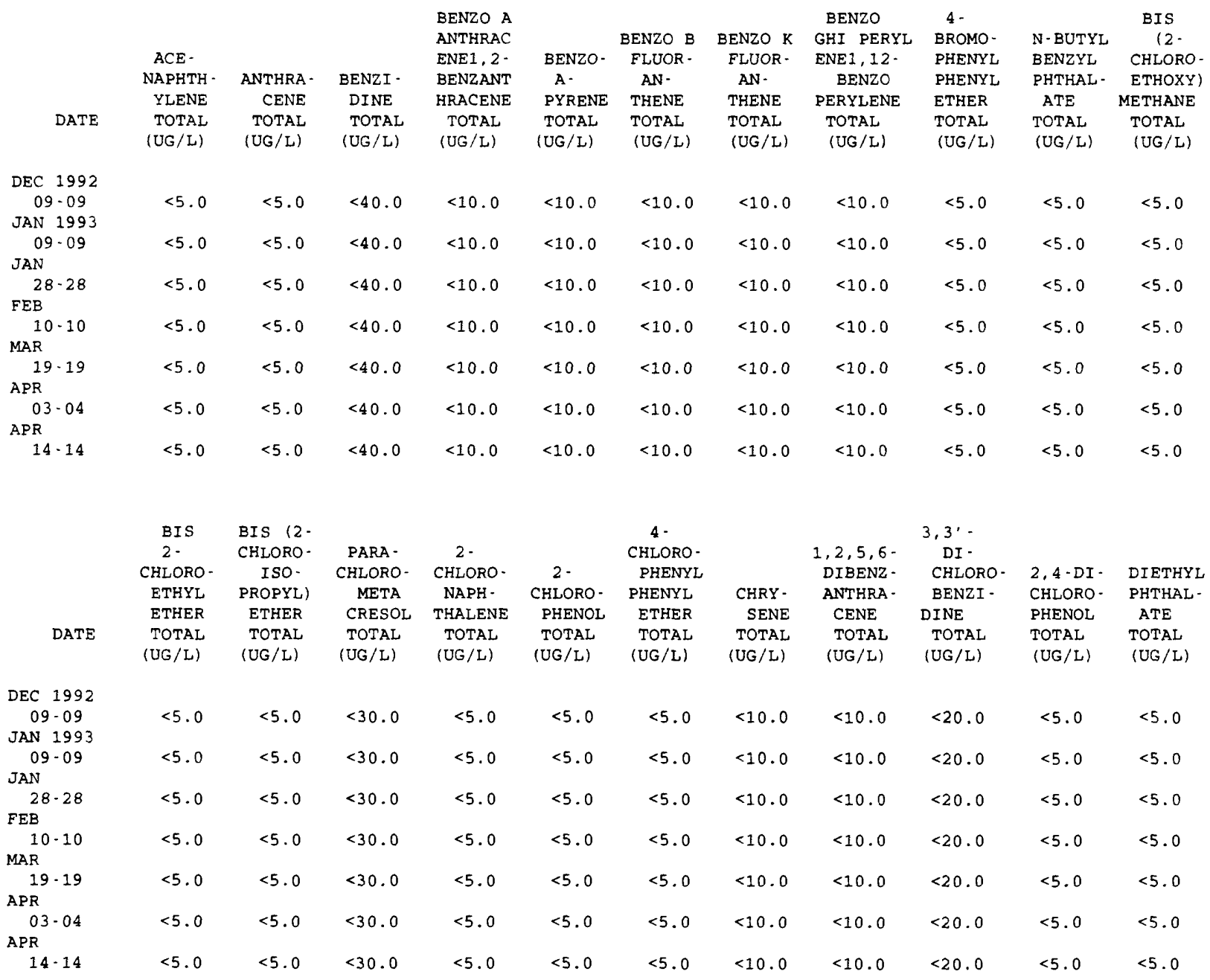


Table 8. Water-quality data for storm-sewer outfall stations, Plano, Texas, 1992-93-Continued

08061510 - ROWLETT CREEK OUTFALL AT WILLOW CREEK PARK, PLANO, TX (WY 1993)-Continued

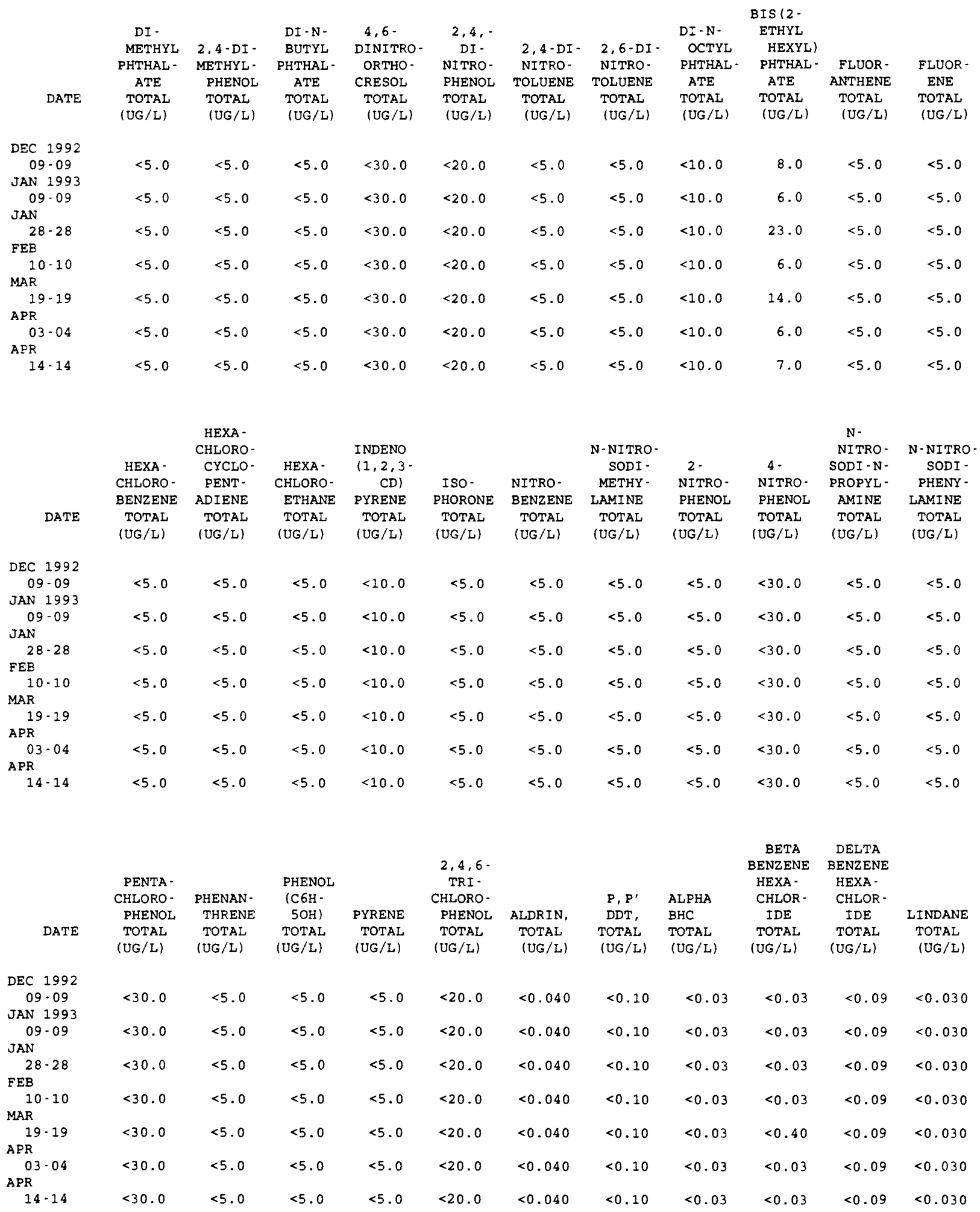


Table 8. Water-quality data for storm-sewer outfall stations, Plano, Texas, 1992-93-Continued

08061510 - ROWLETT CREEK OUTFALL AT WILLOW CREEK PARK, PLANO, TX (WY 1993)-Continued

\begin{tabular}{|c|c|c|c|c|c|c|c|c|c|c|c|}
\hline DATE & $\begin{array}{c}\text { CHLOR - } \\
\text { DANE } \\
\text { CIS } \\
\text { WATER } \\
\text { WHOLE } \\
\text { TOTAL } \\
\text { (UG/L) }\end{array}$ & $\begin{array}{c}\text { CHLOR - } \\
\text { DANE } \\
\text { TRANS } \\
\text { WATER } \\
\text { WHOLE } \\
\text { TOTAL } \\
\text { (UG/L) }\end{array}$ & $\begin{array}{l}\text { CHLOR- } \\
\text { DANE, } \\
\text { TOTAL } \\
\text { (UG/L) }\end{array}$ & $\begin{array}{c}P, P^{\prime} \\
\text { DDD, } \\
\text { TOTAL } \\
(U G / L)\end{array}$ & $\begin{array}{l}\text { P, } P^{\prime} \\
\text { DDE, } \\
\text { TOTAL } \\
\text { (UG/L) }\end{array}$ & $\begin{array}{l}\text { DI - } \\
\text { ELDRIN } \\
\text { TOTAL } \\
\text { (UG/L) }\end{array}$ & $\begin{array}{l}\text { ENDO- } \\
\text { SULFAN- } \\
\text { I } \\
\text { WATER } \\
\text { WHOLE } \\
\text { REC } \\
\text { (UG/L) }\end{array}$ & $\begin{array}{l}\text { ENDO- } \\
\text { SULFAN } \\
\text { BETA } \\
\text { TOTAL } \\
(U G / L)\end{array}$ & $\begin{array}{l}\text { ENDO- } \\
\text { SULFAN } \\
\text { SULFATE } \\
\text { TOTAL } \\
(U G / L)\end{array}$ & $\begin{array}{c}\text { ENDRIN } \\
\text { WATER } \\
\text { UNFLTRD } \\
\text { REC } \\
\text { (UG/L) }\end{array}$ & $\begin{array}{c}\text { ENDRIN } \\
\text { ALDE - } \\
\text { HYDE } \\
\text { TOTAL } \\
\text { (UG/L) }\end{array}$ \\
\hline DEC 1992 & & & & & & & & & & & \\
\hline $\begin{array}{c}09-09 \\
\text { JAN } 1993\end{array}$ & $<0.10$ & $<0.10$ & $<0.1$ & $<0.10$ & $<0.04$ & $<0.020$ & $<0.10$ & $<0.04$ & $<0.60$ & $<0.060$ & $<0.20$ \\
\hline $\begin{array}{l}09-09 \\
\text { JAN }\end{array}$ & $<0.10$ & $<0.10$ & 0.1 & $<0.10$ & $<0.04$ & $<0.020$ & $<0.10$ & $<0.04$ & $<0.60$ & $<0.060$ & $<0.20$ \\
\hline $\begin{array}{l}28-28 \\
\text { FEB }\end{array}$ & $<0.10$ & $<0.10$ & $<0.1$ & $<0.10$ & $<0.04$ & $<0.020$ & $<0.10$ & $<0.04$ & $<0.60$ & $<0.060$ & $<0.20$ \\
\hline $\begin{array}{l}10 \cdot 10 \\
\text { MAR }\end{array}$ & $<0.10$ & $<0.10$ & $<0.1$ & $<0.10$ & $<0.04$ & $<0.020$ & $<0.10$ & $<0.04$ & $<0.60$ & $<0.060$ & $<0.20$ \\
\hline${ }_{\text {APR }}^{19-19}$ & $<0.10$ & $<0.10$ & 0.1 & $<0.10$ & $<0.04$ & $<0.020$ & $<0.10$ & $<0.04$ & $<0.60$ & $<0.060$ & $<0.20$ \\
\hline $\begin{array}{l}03-04 \\
A P R\end{array}$ & $<0.10$ & $<0.10$ & 0.1 & $<0.10$ & $<0.04$ & $<0.020$ & $<0.10$ & $<0.04$ & $<0.60$ & $<0.060$ & $<0.20$ \\
\hline $14-14$ & $<0.10$ & $<0.10$ & 0.1 & $<0.10$ & $<0.04$ & $<0.020$ & $<0.10$ & $<0.04$ & $<0.60$ & $<0.060$ & $<0.20$ \\
\hline DATE & $\begin{array}{l}\text { HEPTA - } \\
\text { CHLOR, } \\
\text { TOTAL } \\
\text { (UG/L) }\end{array}$ & $\begin{array}{l}\text { HEPTA- } \\
\text { CHLOR } \\
\text { EPOXIDE } \\
\text { TOTAL } \\
\text { (UG/L) }\end{array}$ & $\begin{array}{c}\text { TOX - } \\
\text { APHENE, } \\
\text { TOTAL } \\
\text { (UG /L) }\end{array}$ & $\begin{array}{c}\text { AROCLOR } \\
1016 \\
\text { PCB } \\
\text { TOTAL } \\
\text { (UG/L) }\end{array}$ & $\begin{array}{c}\text { AROCLOR } \\
1221 \\
\text { PCB } \\
\text { TOTAL } \\
\text { (UG/L) }\end{array}$ & $\begin{array}{c}\text { AROCLOR } \\
1232 \\
\text { PCB } \\
\text { TOTAL } \\
\text { (UG/L) }\end{array}$ & $\begin{array}{c}\text { AROCLOR } \\
1242 \\
\text { PCB } \\
\text { TOTAL } \\
\text { (UG/L) }\end{array}$ & $\begin{array}{c}\text { AROCLOR } \\
1248 \\
\text { PCB } \\
\text { TOTAL } \\
\text { (UG/L) }\end{array}$ & $\begin{array}{c}\text { AROCLOR } \\
1254 \\
\text { PCB } \\
\text { TOTAL } \\
\text { (UG/L) }\end{array}$ & $\begin{array}{c}\text { AROCLOR } \\
1260 \\
\text { PCB } \\
\text { TOTAL } \\
\text { (UG/L) }\end{array}$ & $\begin{array}{c}\text { DI - } \\
\text { AZINON, } \\
\text { TOTAL } \\
\text { (UG/L) }\end{array}$ \\
\hline $\begin{array}{c}\text { DEC } 1992 \\
09-09 \\
\text { JAN } 1993\end{array}$ & $<0.030$ & $<0.80$ & $<2$ & $<0.1$ & $<1.0$ & $<0.1$ & $<0.1$ & $<0.1$ & $<0.1$ & $<0.1$ & 0.20 \\
\hline $\begin{array}{l}09-09 \\
\text { JAN }\end{array}$ & $<0.030$ & $<0.80$ & $<2$ & $<0.1$ & $<1.0$ & $<0.1$ & $<0.1$ & $<0.1$ & $<0.1$ & $<0.1$ & 0.10 \\
\hline $\begin{array}{l}28-28 \\
F E B\end{array}$ & $<0.030$ & $<0.80$ & $<2$ & $<0.1$ & $<1.0$ & $<0.1$ & $<0.1$ & $<0.1$ & $<0.1$ & $<0.1$ & 0.10 \\
\hline $\begin{array}{l}10-10 \\
M A R\end{array}$ & $<0.030$ & $<0.80$ & $<2$ & $<0.1$ & $<1.0$ & $<0.1$ & $<0.1$ & $<0.1$ & $<0.1$ & $<0.1$ & 0.10 \\
\hline $\begin{array}{l}19 \cdot 19 \\
A P R\end{array}$ & $<0.030$ & $<0.80$ & $<2$ & $<0.1$ & $<1.0$ & $<0.1$ & $<0.1$ & $<0.1$ & $<0.1$ & $<0.1$ & $<0.05$ \\
\hline $\begin{array}{l}03 \cdot 04 \\
A P R\end{array}$ & $<0.030$ & $<0.80$ & $<2$ & $<0.1$ & $<1.0$ & $<0.1$ & $<0.1$ & $<0.1$ & $<0.1$ & $<0.1$ & 0.60 \\
\hline $14-14$ & $<0.030$ & $<0.80$ & $<2$ & $<0.1$ & $<1.0$ & $<0.1$ & $<0,1$ & $<0.1$ & $<0.1$ & $<0.1$ & 3.0 \\
\hline
\end{tabular}


Table 8. Water-quality data for storm-sewer outfall stations, Plano, Texas, 1992-93-Continued

08061525 - SPRING CREEK OUTFALL AT PARK BOULEVARD, PLANO, TX (WY 1993)

\begin{tabular}{|c|c|c|c|c|c|c|c|c|c|c|c|c|}
\hline DATE & TIME & $\begin{array}{l}\text { PRECIP. } \\
\text { ITATION } \\
\text { TOTAL } \\
\text { INCHES/ } \\
\text { STORM }\end{array}$ & $\begin{array}{l}\text { ELAPSED } \\
\text { TIME } \\
\text { OF } \\
\text { STORM } \\
\text { (HOURS) }\end{array}$ & $\begin{array}{l}\text { STORM } \\
\text { WATER } \\
\text { FLOW } \\
\text { (MGD) }\end{array}$ & $\begin{array}{l}\text { SPE- } \\
\text { CIFIC } \\
\text { CON- } \\
\text { DUCT- } \\
\text { ANCE } \\
\text { (US/CM) }\end{array}$ & $\begin{array}{c}\text { SPE- } \\
\text { CIFIC } \\
\text { CON- } \\
\text { DUCT- } \\
\text { ANCE } \\
\text { LAB } \\
\text { (US/CM) }\end{array}$ & $\begin{array}{l}\text { PH } \\
\text { WATER } \\
\text { WHOLE } \\
\text { FIELD } \\
\text { (STAND- } \\
\text { ARD } \\
\text { UNITS) }\end{array}$ & $\begin{array}{c}\text { PH } \\
\text { WATER } \\
\text { WHOLE } \\
\text { LAB } \\
\text { (STAND- } \\
\text { ARD } \\
\text { UNITS) }\end{array}$ & $\begin{array}{c}\text { TEMPER - } \\
\text { ATURE } \\
\text { WATER } \\
\text { (DEG C) }\end{array}$ & $\begin{array}{l}\text { OXYGEN } \\
\text { DEMAND, } \\
\text { CHEM- } \\
\text { ICAL } \\
\text { (HIGH } \\
\text { LEVEL) } \\
\text { (MG/L) }\end{array}$ & $\begin{array}{l}\text { OXYGEN } \\
\text { DEMAND, } \\
\text { BIO- } \\
\text { CHEM- } \\
\text { ICAL, } \\
5 \text { DAY } \\
\text { (MG } / \mathrm{L} \text { ) }\end{array}$ & $\begin{array}{l}\text { COLI- } \\
\text { FORM, } \\
\text { FECAL, } \\
0.7 \\
\text { UM-MF } \\
\text { (COLS. } \\
100 \mathrm{ML} \text { ) }\end{array}$ \\
\hline $\begin{array}{c}\text { DEC } 1992 \\
09.09 \\
\text { JAN } 1993\end{array}$ & 0100 & 0.53 & 3.1 & 0.29 & 34 & 61 & 8.5 & 7.6 & 8.0 & 27 & 3.7 & $\mathrm{~K} 200$ \\
\hline $\begin{array}{l}28-28 \\
F E B\end{array}$ & 1610 & 0.28 & 6.3 & 0.15 & 73 & 122 & 6.3 & 8.0 & 11.0 & 59 & $\cdots$ & $\mathrm{K} 200$ \\
\hline MAR $10 \cdot 10$ & 0445 & 0.20 & 3.5 & 0.10 & 350 & $\cdots$ & 7.9 & $\cdots$ & 14.5 & 41 & 3.5 & K67 \\
\hline $\begin{array}{l}19-19 \\
\text { APR }\end{array}$ & 1333 & 0.35 & 5.0 & 0.17 & 94 & $\cdots$ & 7.8 & $\cdots$ & 13.5 & 47 & 4.7 & $\mathrm{~K} 280$ \\
\hline $\begin{array}{l}03 \cdot 04 \\
A P R\end{array}$ & 2336 & 0.46 & 2.4 & 0.27 & 48 & 71 & 7.3 & 7.5 & 14.0 & 40 & 2.8 & 970 \\
\hline $\begin{array}{l}14 \cdot 14 \\
\text { APR }\end{array}$ & 0610 & 0.68 & 4.3 & 0.40 & 250 & 84 & 7.6 & 7.4 & 13.5 & 39 & 4.9 & 7000 \\
\hline $28-29$ & 2347 & 1.3 & 4.8 & 0.70 & 201 & 73 & 7.6 & 7.5 & 21.0 & 26 & 4.6 & $\mathrm{~K} 47000$ \\
\hline & $\begin{array}{l}\text { STREP - } \\
\text { TOCOCCI }\end{array}$ & HARD - & $\begin{array}{l}\text { HARD- } \\
\text { NESS }\end{array}$ & $\begin{array}{l}\text { ALKA- } \\
\text { LINITY }\end{array}$ & $\begin{array}{l}\text { SOLIDS, } \\
\text { SUM OF }\end{array}$ & $\begin{array}{l}\text { RESIDUE } \\
\text { TOTAL }\end{array}$ & $\begin{array}{l}\text { SOLIDS, } \\
\text { RESIDUE }\end{array}$ & & MAGNE - & & & SODIUM \\
\hline & FECAL, & NESS & NONCARB & WAT DIS & CONSTI - & AT 105 & AT 180 & CALCIUM & SIUM, & SODIUM, & & $\mathrm{AD}$ - \\
\hline & $\begin{array}{l}\text { KF AGAR } \\
\text { (COLS. }\end{array}$ & $\begin{array}{l}\text { TOTAL } \\
\text { (MG/L }\end{array}$ & $\begin{array}{l}\text { DISSOLV } \\
\text { FLD. AS }\end{array}$ & $\begin{array}{l}\text { FIX END } \\
\text { FIELD }\end{array}$ & $\begin{array}{c}\text { TUENTS, } \\
\text { DIS - }\end{array}$ & $\begin{array}{l}\text { DEG. C, } \\
\text { SUS - }\end{array}$ & $\begin{array}{l}\text { DEG. C } \\
\text { DIS - }\end{array}$ & $\begin{array}{l}\text { DIS - } \\
\text { SOLVED }\end{array}$ & $\begin{array}{l}\text { DIS - } \\
\text { SOLVED }\end{array}$ & $\begin{array}{c}\text { DIS - } \\
\text { SOLVED }\end{array}$ & & $\begin{array}{l}\text { SORP- } \\
\text { TION }\end{array}$ \\
\hline DATE & $\begin{array}{c}\text { PER } \\
100 \mathrm{ML})\end{array}$ & $\begin{array}{c}\text { AS } \\
\text { CACO31 }\end{array}$ & $\begin{array}{r}\mathrm{CACO} 3 \\
(\mathrm{MG} / \mathrm{L})\end{array}$ & $\begin{array}{l}\mathrm{CACO} 3 \\
(\mathrm{MG} / \mathrm{L})\end{array}$ & $\begin{array}{l}\text { SOLVED } \\
(\mathrm{MG} / \mathrm{L})\end{array}$ & $\begin{array}{l}\text { PENDED } \\
(\mathrm{MG} / \mathrm{L})\end{array}$ & $\begin{array}{l}\text { SOLVED } \\
\text { (MG/L) }\end{array}$ & $\begin{array}{l}\text { (MG/L } \\
\text { AS CA) }\end{array}$ & $\begin{array}{c}\text { (MG /L } \\
\text { AS MG) }\end{array}$ & $\begin{array}{c}\text { (MG/L } \\
\text { AS NA) }\end{array}$ & $\begin{array}{l}\text { SODIUM } \\
\text { PERCENT }\end{array}$ & RATIO \\
\hline DEC 1992 & & & & & & & & & & & & \\
\hline $\begin{array}{l}09-09 \\
\text { JAN }\end{array}$ & 2200 & 17 & 0 & 26 & 31 & 22 & 23 & 6.7 & 0.12 & 0.90 & 9 & 0.1 \\
\hline $\begin{array}{l}28-28 \\
F E B\end{array}$ & K1700 & 47 & 8 & 39 & 86 & 69 & 249 & 18 & 0.39 & 20 & 46 & 1 \\
\hline $\begin{array}{l}10-10 \\
M A R\end{array}$ & 3400 & $\cdots$ & . & 39 & $\cdots$ & $\cdots$ & $\cdots$ & $\cdots$ & $\cdots$ & $\cdots$ & $\cdots$ & $\cdots$ \\
\hline $\begin{array}{l}19 \cdot 19 \\
A P R\end{array}$ & 7500 & $\cdots$ & .. & 31 & $\cdots$ & $\cdots$ & $\cdots$ & $\cdots$ & $\cdots$ & $\ldots$ & $\cdots$ & $\cdots$ \\
\hline $\begin{array}{l}03 \cdot 04 \\
A P R\end{array}$ & 5700 & 18 & 0 & 18 & 35 & 30 & 44 & 7.1 & 0.15 & 0.80 & 8 & 0.1 \\
\hline $\begin{array}{l}14-14 \\
\text { APR }\end{array}$ & 1400 & 25 & 0 & 25 & 43 & 42 & 50 & 9.5 & 0.20 & 1.3 & 9 & 0.1 \\
\hline
\end{tabular}

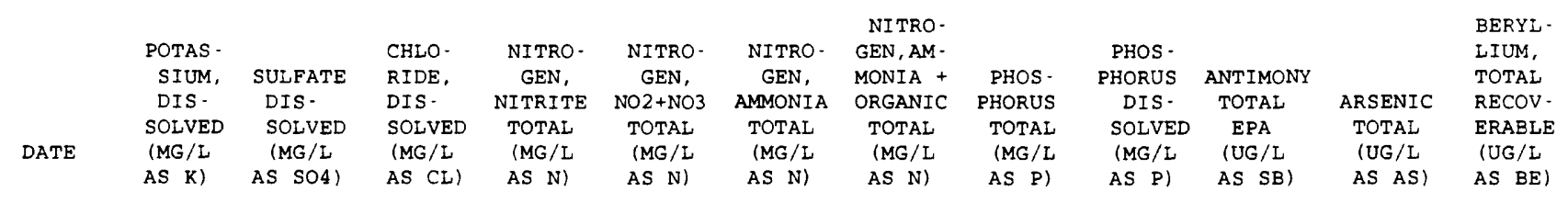

DEC 1992

\begin{tabular}{|c|c|c|c|c|c|c|c|c|c|c|c|c|}
\hline $\begin{array}{c}D E C \\
09.09\end{array}$ & 1.7 & 2.7 & 0.50 & 0.040 & 0.340 & 0.140 & 0.30 & 0.070 & 0.030 & $<10.0$ & 2 & $<10$ \\
\hline JAN 1993 & & & & & & & & & & & & \\
\hline $\begin{array}{l}28-28 \\
\text { FEB }\end{array}$ & 3.5 & 5.8 & 0.90 & $\cdots$ & 0.570 & $\cdots$ & 0.60 & 0.110 & 0.050 & $<20.0$ & 1 & $<10$ \\
\hline $\begin{array}{l}10-10 \\
\text { MAR }\end{array}$ & $\cdots$ & $\cdots$ & $\cdots$ & $\cdots$ & 0.510 & $\cdots$ & 0.40 & 0.080 & 0.030 & $<10.0$ & 2 & $<10$ \\
\hline $\begin{array}{l}19-19 \\
\text { APR }\end{array}$ & $\cdots$ & $<0.10$ & $\cdots$ & $\cdots$ & 0.400 & - & 0.70 & 0.100 & 0.030 & $<10.0$ & 3 & $<10$ \\
\hline $\begin{array}{l}03 \cdot 04 \\
A P R\end{array}$ & 1.8 & 2.4 & 0.60 & $\cdots$ & 0.410 & $\cdots$ & 0.80 & 0.100 & 0.040 & $<10.0$ & $<1$ & $<10$ \\
\hline $\begin{array}{l}14 \cdot 14 \\
A P R\end{array}$ & 2.0 & 2.9 & 1.0 & $\cdots$ & 0.360 & $\cdots$ & 0.70 & 0.110 & 0.060 & $<10.0$ & 2 & $<10$ \\
\hline $28-29$ & 1.9 & 3.3 & 0.90 & -. & 0.520 & $\cdots$ & 0.70 & 0.130 & 0.050 & $<20.0$ & $<1$ & $<10$ \\
\hline
\end{tabular}


Table 8. Water-quality data for storm-sewer outfall stations, Plano, Texas, 1992-93-Continued

08061525 - SPRING CREEK OUTFALL AT PARK BOULEVARD, PLANO, TX (WY 1993)-Continued

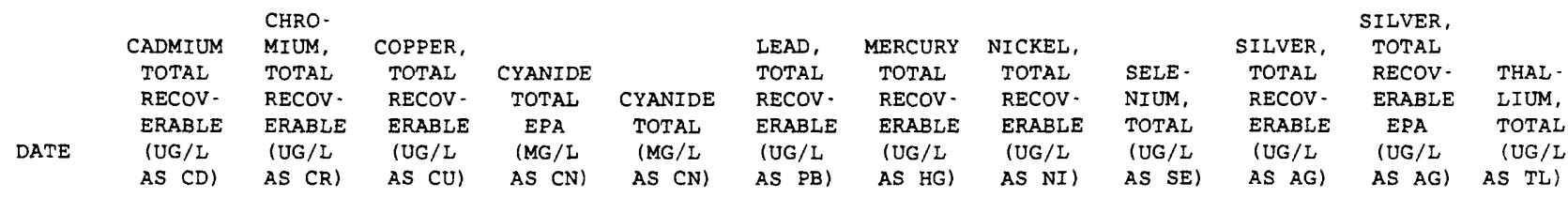

DEC 1992

$09-09$

JAN 1993

$28-28$

FEB

$10-10$

MAR

$19-19$

APR

$03 \cdot 04$

APR $14 \cdot 14$

APR

$28 \cdot 29$

$\begin{array}{rr}<1 & 6 \\ <1 & 5 \\ <1 & 4 \\ <1 & 4 \\ <1 & 2 \\ <1 & <10 \\ <1 & <1\end{array}$

6

$\begin{array}{ccc}8 & <0.010 & <0.010 \\ 12 & <0.010 & <0.010 \\ 6 & 0.015 & <0.010 \\ 8 & <0.010 & <0.010 \\ 6 & <0.010 & <0.010 \\ 9 & <0.010 & <0.010 \\ 9 & <0.010 & <0.010\end{array}$

$\begin{aligned} 11 & <0.10 \\ 15 & <0.10 \\ 7 & <0.10 \\ 13 & <0.10 \\ 6 & <0.10 \\ 10 & <0.10 \\ 20 & <0.10\end{aligned}$

OII AND

ZINC, GREASE,

TOTAL CARBON, TOTAL

RECOV - ORGANIC RECOV.

ERABLE TOTAL GRAVI

AS ZN) AS C)

(MG/L)

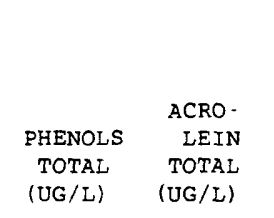

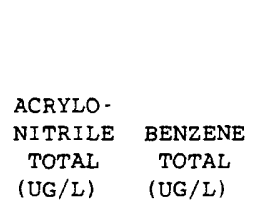

$\begin{array}{lc} & \text { METHANE } \\ \text { BROMO- } & \text { BROMO } \\ \text { BENZENE } & \text { CHLORO- } \\ \text { WATER, } & \text { WAT } \\ \text { WHOLE, } & \text { UNFLTRD } \\ \text { TOTAL } & \text { REC } \\ \text { (UG/L) } & \text { (UG/L) }\end{array}$

$\begin{array}{lll}<1 & <0.500 & <10 \\ <1 & <0.500 & <5 \\ <1 & <0.500 & <10 \\ <1 & <0.500 & <5 \\ <1 & <0.500 & <5 \\ <1 & <0.500 & <5 \\ <1 & <0.500 & <5\end{array}$

DEC 1992

09-09

JAN 1993

28- 28

FEB

MAR

$19 \cdot 19$

APR
$03-04$

APR

14-14

APR

$28 \cdot 29$

$\begin{array}{rlr}80 & 5.8 & <1 \\ 110 & 11 & 4\end{array}$

$\begin{array}{rll}2 & <20 & <20 \\ 8 & <20 & <20 \\ 17 & <20 & <20 \\ 8 & <20 & <20 \\ 5 & <20 & <20 \\ 10 & <20 & <20 \\ 3 & <20 & <20\end{array}$

$\begin{array}{lll}<0.2 & <0.2 & <0.20 \\ <0.2 & <0.2 & <0.20 \\ 0.2 & <0.2 & <0.20 \\ <0.2 & <0.2 & <0.20 \\ <0.2 & <0.2 & <0.20 \\ <0.2 & <0.2 & <0.20 \\ <0.2 & <0.2 & <0.20\end{array}$

CHLORO-

BENZENE

TERT -

BUTYL.

WATER

UNFLTRD

REC

(UG/L)

CARBON-
TETRA -
CHLO-
RIDE
TOTAL
(UG/L)

CHLORO-
BENZENE
TOTAL
(UG/L)

\begin{tabular}{|c|c|}
\hline $\begin{array}{c}\text { CHLORO- } \\
\text { DI - }\end{array}$ & \\
\hline BROMO - & CHLORO - \\
\hline METHANE & ETHANE \\
\hline $\begin{array}{l}\text { TOTAL } \\
\text { (UG/L) }\end{array}$ & $\begin{array}{l}\text { TOTAL } \\
\langle U G / L\rangle\end{array}$ \\
\hline
\end{tabular}

ETHYL -

VINYL.

ETHER

TOTAL

(UG/L)

$\begin{array}{cccccc}\text { O- } & & \text { DIBROMO } & \text { DI - } \\ & \text { CHLORO- } & \text { TOLUENE } & \text { CHLORO- } & \text { BROMO- } \\ \text { CHLORO- } & \text { CHLO- } & \text { TOLUENE } & \text { P-CHLOR } & \text { PROPANE } & \text { METHANE } \\ \text { FORM } & \text { RIDE } & \text { WHOLE } & \text { WATER } & \text { WATER } & \text { WATER } \\ \text { TOTAL } & \text { TOTAL } & \text { TOTAL } & \text { REC } & \text { WHOLE } & \text { WHOLE } \\ (U G / L) & (U G / L) & (U G / L) & (U G / L) & \text { (UG/L) } & \text { RECOVER } \\ \text { (UG/L) }\end{array}$

DEC 1992

09.09

$<0.20$

$<0.2<0.20$

$<0.2<0.2<1.0$

$<0.2$

$<0.2$

$<0.2<0.20$

$<1.0$

$<1.0$

$<0.2$

$28-28$

$<0.20$

$<0.2<0.20$

$<0.2$

$<0.2$

$<1.0$

$<0.2$

$<0.2$

$<0.2<0.20$

$<1$.

$<0.2$

$10-10$

$<0.20$

$<0.2<0.20$

$<0.2$

$<0.2$

$<1.0$

$<0.2$

$<0.2$

$<0$.

$<0.20$

$<1$.

$<0.2$

$19-19$

$<0.20$

$<0.2<0.20$

$<0.2$

$<0.2<1.0$

$<0.2$

$<0.2$

$<0.2$

$<0.20$

$<1.0$

$<0.2$

$03.04<0.20$

$<0.2<0.20$

$<0$.

$<0.2$

$<1.0$

$<0.2$

$<0.2$

$<0.2$

$<0.20$

$<1.0$

$<0.2$

$<0.2$

$<0.2$

$<0.20$

$<1.0$

$<0.2$

14-1

$28.29<0.20$

$<0.2<0.20$

$<0.2$

$<0.2<1.0$

$<0.2$

$<0.2$

$<0.2$

$<0.20$

$<1.0$

$<0.2$

$<0.2$ 
Table 8. Water-quality data for storm-sewer outfall stations, Plano, Texas, 1992-93-Continued

08061525 - SPRING CREEK OUTFALI AT PARK BOULEVARD, PLANO, TX (WY 1993)-Continued

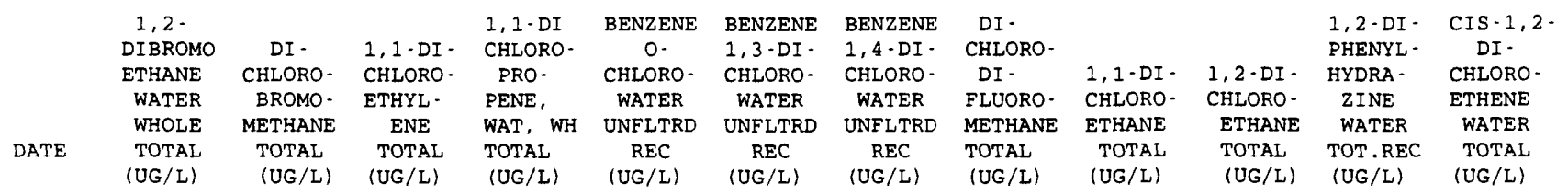

DEC 1992

09.09

JAN 1993

$28-28$

$<0.2$

$<0.20$

FEB

$10 \cdot 10$

$\begin{array}{lll}<0.2 & <0.2 & <0.2 \\ <0.2 & <0.2 & <0.2\end{array}$

$<0.2<5.0$

$<5.0<5.0$

$<0.2<0.2$

$<0.2$

$<5.0<0.2$

MAR
$19 \cdot 19$

APR

$03 \cdot 04$

$<0.2$

$<0.2<0.2$

$<0.2<5.0<5.0<5.0$

$<0.2<0.2$

$<0.2$

$<5.0<0.2$

$<0.2$

$<0.2<0.2$

$<0.2<5.0$

$<5.0<5.0$

$<0.2<0.2$

$<0.2$

$<5.0$

$<0.2$

$<0.2$

$<0.2$

$<0.2$

$<0.2<0.20$

$<0.20<0.20$

$<0.2<0$.

$<0.2$

$<5.0$

$<0.2$

APR $14-14$

$<0.2$

$<0.2<0.2$

$<0.2<5.0<5.0<5.0$

$<0.2<0.2<0.2$

$<5.0$

$<0.2$

APR

$<0.2$

$<0.2<5.0<5.0<5.0$

$<0.2$

$<0.2$

$<0.2$

$<5.0$

$<0.2$

$<0.2<0.20<0.20<0.20$

$<0$.

$<0$.

$<0.2$

$<5.0$

$<0.2$

\begin{tabular}{cccccc}
$1,2-$ & & \multicolumn{5}{c}{$2,2-D I$} \\
TRANSDI & $1,2-D I-$ & CHLORO- & PRO- & CIS & TRANS- \\
CHLORO- & CHLORO- & PROPANE & PANE & CHLORO- & CHLORO- \\
ETHENE & PROPANE & WAT. WH & WAT, WH & PROPENE & PROPENE \\
TOTAL & TOTAL & TOTAL & TOTAL & TOTAL & TOTAL \\
$(U G / L)$ & $(U G / L)$ & $(U G / L)$ & $(U G / L)$ & $(U G / L)$ & $(U G / L)$
\end{tabular}

\begin{tabular}{|c|c|c|c|c|}
\hline & & ISO- & P-ISO- & \\
\hline & HEXA - & PROPYL- & PROPYL - & \\
\hline ETHYL - & $\begin{array}{l}\text { CHLORO- } \\
\text { BUT - }\end{array}$ & $\begin{array}{c}\text { BENZENE } \\
\text { WATER }\end{array}$ & $\begin{array}{c}\text { TOLUENE } \\
\text { WATER }\end{array}$ & METHYL - \\
\hline BENZENE & ADIENE & WHOLE & WHOLE & BROMIDE \\
\hline $\begin{array}{l}\text { TOTAL } \\
\text { (UG/L) }\end{array}$ & $\begin{array}{l}\text { TOTAL } \\
\text { (UG/L) }\end{array}$ & $\begin{array}{l}\text { REC } \\
\text { (UG/L) }\end{array}$ & $\begin{array}{c}\text { REC } \\
(U G / L)\end{array}$ & $\begin{array}{l}\text { TOTAL } \\
\text { (UG/L) }\end{array}$ \\
\hline
\end{tabular}

DEC 1992

$09-09$

JAN 1993

$28-28$

FEB

$10-10$

$19-19$

APR

03-04

$14-14$

$A P R$

$28-29$

$\begin{array}{lllllllllll}<0.2 & <0.2 & <0.2 & <0.2 & <0.2 & <0.2 & <0.2 & <5.0 & <0.20 & <0.20 & <0.2 \\ <0.2 & <0.2 & <0.2 & <0.2 & <0.2 & <0.2 & <0.2 & <5.0 & <0.20 & <0.20 & <0.2 \\ <0.2 & <0.2 & <0.2 & <0.2 & <0.2 & <0.2 & 0.4 & <5.0 & <0.20 & <0.20 & <0.2 \\ <0.2 & <0.2 & <0.2 & <0.2 & <0.2 & <0.2 & <0.2 & <0.2 & <0.20 & <0.20 & <0.2 \\ <0.2 & <0.2 & <0.2 & <0.2 & <0.2 & <0.2 & <0.2 & <5.0 & <0.20 & <0.20 & <0.2 \\ <0.2 & <0.2 & <0.2 & <0.2 & <0.2 & <0.2 & <0.2 & <5.0 & <0.20 & <0.20 & <0.2 \\ <0.2 & <0.2 & <0.2 & <0.2 & <0.2 & <0.2 & <0.2 & <0.2 & <0.20 & <0.20 & <0.2\end{array}$

\begin{tabular}{|c|c|c|c|c|c|c|c|c|c|c|c|}
\hline 09.09 & $<0.2$ & $<1.0$ & $<5.0$ & $<0.20$ & $<0.2$ & $<0.2$ & $<0.2$ & $<0.2$ & $<0.2$ & $<0.20$ & $<5.0$ \\
\hline \multicolumn{12}{|l|}{ JAN 1993} \\
\hline $28-28$ & $<0.3$ & $<1.0$ & $<5.0$ & $<0.20$ & $<0.2$ & $<0.2$ & $<0.2$ & $<0.2$ & 0.2 & $<0.20$ & $<5.0$ \\
\hline \multicolumn{12}{|l|}{ FEB } \\
\hline $10 \cdot 10$ & $<0.2$ & $<1.0$ & $<5.0$ & $<0.20$ & 0.6 & $<0.2$ & $<0.2$ & $<0.2$ & $<0.2$ & $<0.20$ & $<5.0$ \\
\hline \multicolumn{12}{|l|}{ MAR } \\
\hline $19-19$ & $<0.3$ & $<1.0$ & $<0.2$ & $<0.20$ & $<0.2$ & $<0.2$ & $<0.2$ & $<0.2$ & $<0.2$ & $<0.20$ & $<0.20$ \\
\hline \multicolumn{12}{|l|}{ APR } \\
\hline 03.04 & $<0.2$ & $<1.0$ & $<5.0$ & $<0.20$ & $<0.2$ & $<0.2$ & $<0.2$ & $<0.2$ & $<0.2$ & $<0.20$ & $<5.0$ \\
\hline \multicolumn{12}{|l|}{ APR } \\
\hline $14-14$ & $<0.2$ & $<1.0$ & $<5.0$ & $<0.20$ & $<0.2$ & $<0.2$ & $<0.2$ & $<0.2$ & $<0.2$ & $<0.20$ & $<5.0$ \\
\hline \multicolumn{12}{|l|}{ APR } \\
\hline $28 \cdot 29$ & $<0.2$ & $<1.0$ & $<0.2$ & $<0.20$ & $<0.2$ & $<0.2$ & $<0.2$ & $<0.2$ & $<0.2$ & $<0.20$ & $<0.20$ \\
\hline
\end{tabular}


Table 8. Water-quality data for storm-sewer outfall stations, Plano, Texas, 1992-93-Continued

08061525 - SPRING CREEK OUTFALI AT PARK BOULEVARD, PLANO, TX (WY 1993)-Continued

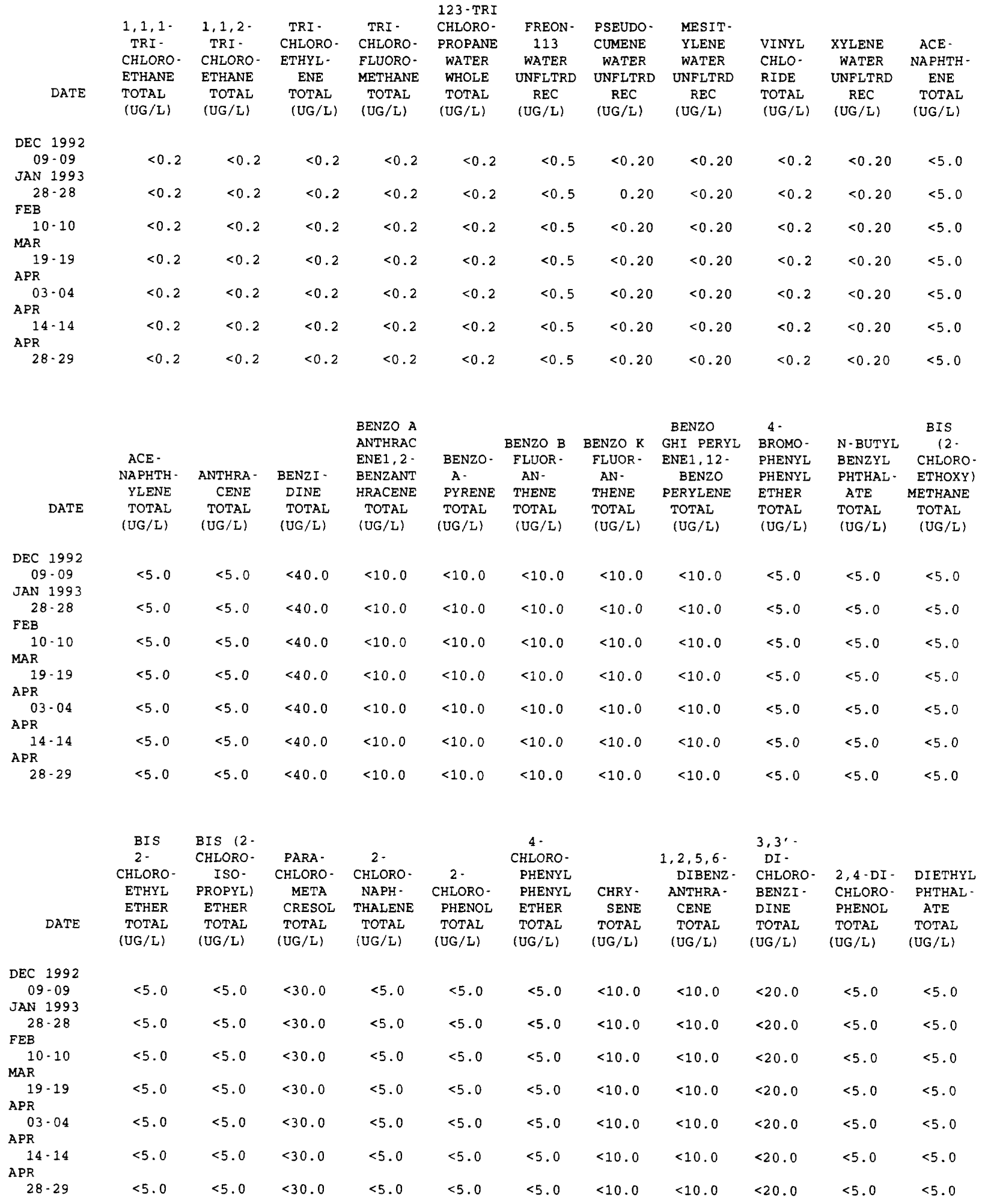


Table 8. Water-quality data for storm-sewer outfall stations, Plano, Texas, 1992-93-Continued

08061525 - SPRING CREEK OUTFALL AT PARK BOULEVARD, PLANO, TX (WY 1993)-Continued

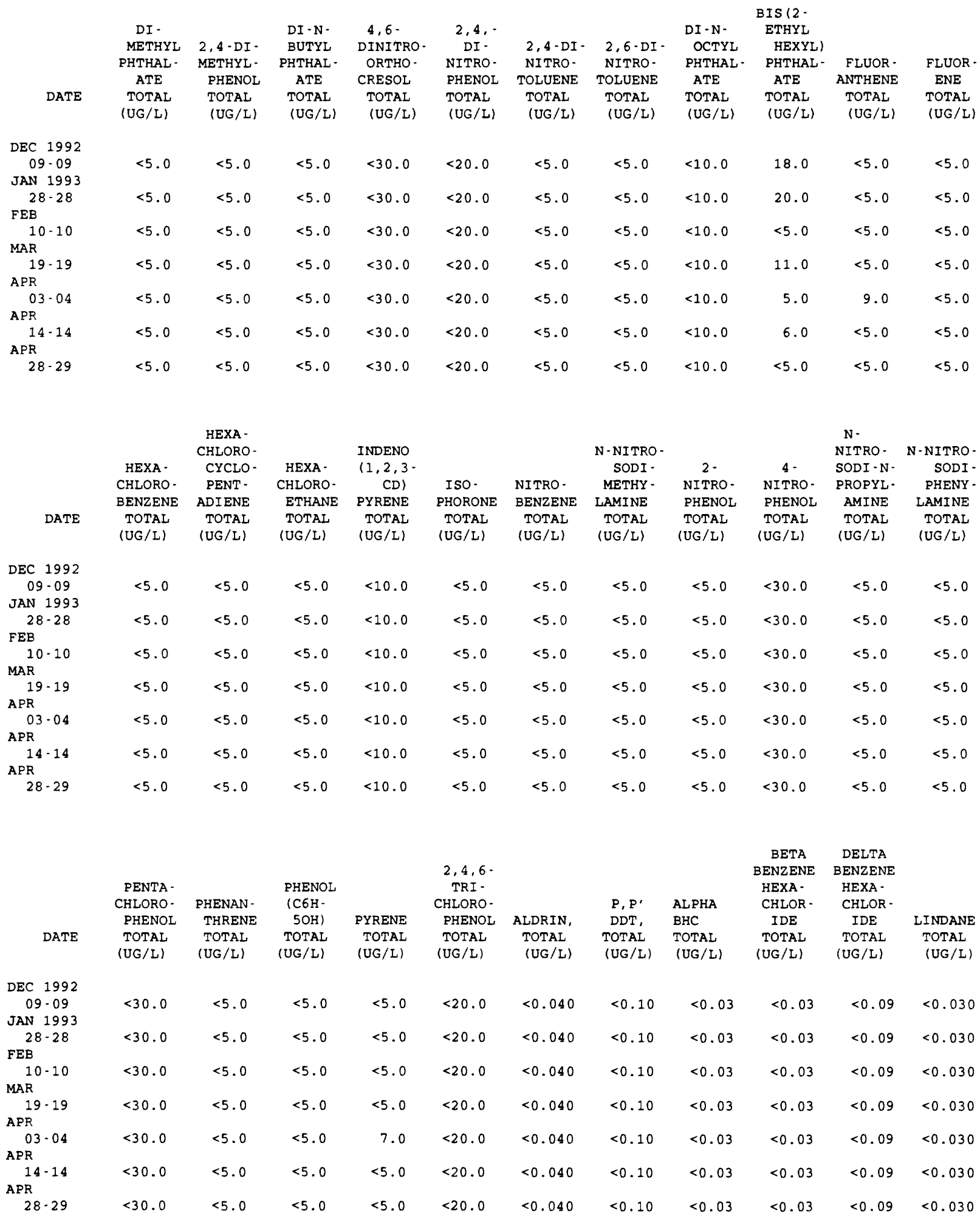


Table 8. Water-quality data for storm-sewer outfall stations, Plano, Texas, 1992-93-Continued

\begin{tabular}{|c|c|c|c|c|c|c|c|c|c|c|c|}
\hline & 0806152 & - SPRING & CREEK OI & JTFALI AT & PARK BOU & LEVARD， & ANO, TX & (WY 1993) & -Continued & & \\
\hline DATE & $\begin{array}{c}\text { CHLOR - } \\
\text { DANE } \\
\text { CIS } \\
\text { WATER } \\
\text { WHOLE } \\
\text { TOTAL } \\
\text { (UG/L) }\end{array}$ & $\begin{array}{l}\text { CHLOR - } \\
\text { DANE } \\
\text { TRANS } \\
\text { WATER } \\
\text { WHOLE } \\
\text { TOTAL } \\
\text { (UG/L) }\end{array}$ & $\begin{array}{l}\text { CHLOR- } \\
\text { DANE, } \\
\text { TOTAL } \\
\text { (UG/L) }\end{array}$ & $\begin{array}{l}\text { P, P } \\
\text { DDD, } \\
\text { TOTAL } \\
\text { (UG/L) }\end{array}$ & $\begin{array}{l}\text { P, } P^{\prime} \\
\text { DDE, } \\
\text { TOTAL } \\
(U G / L)\end{array}$ & $\begin{array}{l}\text { DI - } \\
\text { ELDRIN } \\
\text { TOTAL } \\
\text { (UG/L) }\end{array}$ & $\begin{array}{c}\text { ENDO- } \\
\text { SULFAN- } \\
\text { I } \\
\text { WATER } \\
\text { WHOLE } \\
\text { REC } \\
\text { (UG/L) }\end{array}$ & $\begin{array}{c}\text { ENDO- } \\
\text { SULFAN } \\
\text { BETA } \\
\text { TOTAL } \\
\text { (UG/L) }\end{array}$ & $\begin{array}{l}\text { ENDO- } \\
\text { SULFAN } \\
\text { SULFATE } \\
\text { TOTAL } \\
\text { (UG/L) }\end{array}$ & $\begin{array}{c}\text { ENDRIN } \\
\text { WATER } \\
\text { UNFLTRD } \\
\text { REC } \\
(U G / L)\end{array}$ & $\begin{array}{c}\text { ENDRIN } \\
\text { ALDE - } \\
\text { HYDE } \\
\text { TOTAL } \\
\text { (UG/L) }\end{array}$ \\
\hline DEC 1992 & & & & & & & & & & & \\
\hline $\begin{array}{c}09.09 \\
\text { JAN } 1993\end{array}$ & $<0.10$ & $<0.10$ & $<0.1$ & $<0.10$ & $<0.04$ & $<0.020$ & $<0.10$ & $<0.04$ & $<0.60$ & $<0.060$ & $<0.20$ \\
\hline $\begin{array}{l}28-28 \\
\text { FEB }\end{array}$ & $<0.10$ & $<0.10$ & $<0.1$ & $<0.10$ & $<0.04$ & $<0.020$ & $<0.10$ & $<0.04$ & $<0.60$ & $<0.060$ & $<0.20$ \\
\hline $\begin{array}{l}10-10 \\
\text { MAR }\end{array}$ & $<0.10$ & $<0.10$ & $<0.1$ & $<0.10$ & $<0.04$ & $<0.020$ & $<0.10$ & $<0.04$ & $<0.60$ & $<0.060$ & $<0.20$ \\
\hline $\begin{array}{l}19-19 \\
A P R\end{array}$ & $<0.10$ & $<0.10$ & $<0.1$ & $<0.10$ & $<0.04$ & $<0.020$ & $<0.10$ & $<0.04$ & $<0.60$ & $<0.060$ & $<0.20$ \\
\hline $\begin{array}{l}03-04 \\
A P R\end{array}$ & $<0.10$ & $<0.10$ & $<0.1$ & $<0.10$ & $<0.04$ & $<0.020$ & $<0.10$ & $<0.04$ & $<0.60$ & $<0.060$ & $<0.20$ \\
\hline $14-14$ & $<0.10$ & $<0.10$ & $<0.1$ & $<0.10$ & $<0.04$ & $<0.020$ & $<0.10$ & $<0.04$ & $<0.60$ & $<0.060$ & $<0.20$ \\
\hline $\begin{array}{l}\text { APR } \\
28-29\end{array}$ & $<0.10$ & $<0.10$ & $<0.1$ & $<0.10$ & $<0.04$ & $<0.020$ & $<0.10$ & $<0.04$ & $<0.60$ & $<0.060$ & $<0.20$ \\
\hline DATE & $\begin{array}{l}\text { HEPTA- } \\
\text { CHLOR, } \\
\text { TOTAL } \\
\text { (UG/L) }\end{array}$ & $\begin{array}{l}\text { HEPTA - } \\
\text { CHLOR } \\
\text { EPOXIDE } \\
\text { TOTAL } \\
\text { (UG/L) }\end{array}$ & $\begin{array}{c}\text { TOX - } \\
\text { APHENE, } \\
\text { TOTAL } \\
\text { (UG } / L \text { ) }\end{array}$ & $\begin{array}{c}\text { AROCLOR } \\
1016 \\
\text { PCB } \\
\text { TOTAL } \\
\text { (UG/L) }\end{array}$ & $\begin{array}{c}\text { AROCLOR } \\
1221 \\
\text { PCB } \\
\text { TOTAL } \\
(\mathrm{UG} / \mathrm{L})\end{array}$ & $\begin{array}{c}\text { AROCLOR } \\
1232 \\
\text { PCB } \\
\text { TOTAL } \\
\text { (UG/L) }\end{array}$ & $\begin{array}{c}\text { AROCLOR } \\
1242 \\
\text { PCB } \\
\text { TOTAL } \\
(U G / L)\end{array}$ & $\begin{array}{c}\text { AROCLOR } \\
1248 \\
\text { PCB } \\
\text { TOTAL } \\
\text { (UG/L) }\end{array}$ & $\begin{array}{c}\text { AROCLOR } \\
1254 \\
\text { PCB } \\
\text { TOTAL } \\
\text { (UG/L) }\end{array}$ & $\begin{array}{c}\text { AROCLOR } \\
1260 \\
\text { PCB } \\
\text { TOTAL } \\
\text { (UG/L) }\end{array}$ & $\begin{array}{c}\text { DI - } \\
\text { AZINON, } \\
\text { TOTAL } \\
\text { (UG/L) }\end{array}$ \\
\hline DEC 1992 & & & & & & & & & & & \\
\hline $\begin{array}{c}09.09 \\
\text { JAN } 1993\end{array}$ & $<0.030$ & $<0.80$ & $<2$ & $<0.1$ & $<1.0$ & $<0.1$ & $<0.1$ & $<0.1$ & $<0.1$ & $<0.1$ & $<0.10$ \\
\hline $\begin{array}{l}28-28 \\
F E B\end{array}$ & $<0.030$ & $<0.80$ & $<2$ & $<0.1$ & $<1.0$ & $<0.1$ & $<0.1$ & $<0.1$ & $<0.1$ & $<0.1$ & 0.10 \\
\hline $\begin{array}{l}10-10 \\
\text { MAR }\end{array}$ & $<0.030$ & $<0.80$ & $<2$ & $<0.1$ & $<1.0$ & $<0.1$ & $<0.1$ & $<0.1$ & $<0.1$ & $<0.1$ & $<0.05$ \\
\hline $19 \cdot 19$ & $<0.030$ & $<0.80$ & $<2$ & $<0.1$ & $<1.0$ & $<0.1$ & $<0.1$ & $<0.1$ & $<0.1$ & $<0.1$ & $<0.05$ \\
\hline APR & & & & & & & & & & & \\
\hline $\begin{array}{l}03-04 \\
\text { APR }\end{array}$ & $<0.030$ & $<0.80$ & $<2$ & $<0.1$ & $<1.0$ & $<0.1$ & $<0.1$ & $<0.1$ & $<0.1$ & $<0.1$ & $\cdots$ \\
\hline $\begin{array}{l}14-14 \\
A P R\end{array}$ & $<0.030$ & $<0.80$ & $<2$ & $<0.1$ & $<1.0$ & $<0.1$ & $<0.1$ & $<0.1$ & $<0.1$ & $<0.1$ & 0.20 \\
\hline $28-29$ & $<0.030$ & $<0.80$ & $<2$ & $<0.1$ & $<1,0$ & $<0.1$ & $<0.1$ & $<0.1$ & $<0.1$ & $<0.1$ & 0.20 \\
\hline
\end{tabular}


Table 8. Water-quality data for storm-sewer outfall stations, Plano, Texas, 1992-93-Continued

08061530 - SPRING CREEK OUTFALl AT AVENUE F, PLANO, TX (WY 1993)

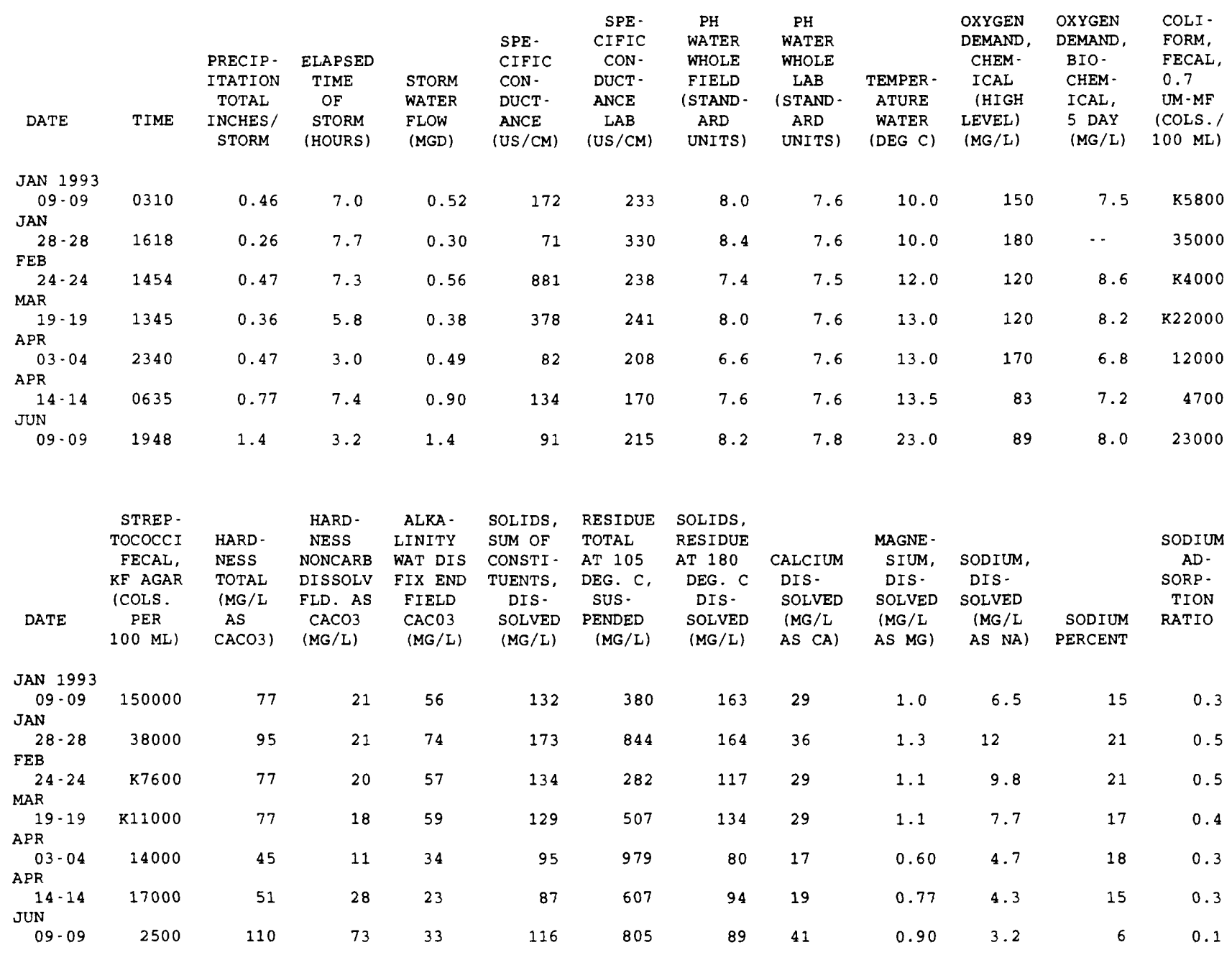

\begin{tabular}{|c|c|c|c|c|c|c|c|c|c|c|c|c|}
\hline DATE & $\begin{array}{l}\text { POTAS - } \\
\text { SIUM, } \\
\text { DIS- } \\
\text { SOLVED } \\
\text { (MG/L }\end{array}$ & $\begin{array}{l}\text { SULFATE } \\
\text { DIS- } \\
\text { SOLVED } \\
\text { (MG/L }\end{array}$ & $\begin{array}{l}\text { CHLO- } \\
\text { RIDE, } \\
\text { DIS- } \\
\text { SOLVED } \\
\text { (MG/L }\end{array}$ & $\begin{array}{c}\text { NITRO- } \\
\text { GEN, } \\
\text { NO2+NO3 } \\
\text { TOTAL } \\
\text { (MG/L }\end{array}$ & $\begin{array}{l}\text { NITRO- } \\
\text { GEN, AM- } \\
\text { MONIA + } \\
\text { ORGANIC } \\
\text { TOTAL } \\
\text { (MG/L }\end{array}$ & $\begin{array}{r}\text { PHOS - } \\
\text { PHORUS } \\
\text { TOTAL } \\
\text { (MG /L }\end{array}$ & $\begin{array}{c}\text { PHOS - } \\
\text { PHORUS } \\
\text { DIS - } \\
\text { SOLVED } \\
\text { (MG/L }\end{array}$ & $\begin{array}{l}\text { ANT IMONY } \\
\text { TOTAL } \\
\text { EPA } \\
\text { (UG/L }\end{array}$ & $\begin{array}{l}\text { ARSENIC } \\
\text { TOTAL } \\
\text { (UG/L }\end{array}$ & $\begin{array}{l}\text { BERYL- } \\
\text { LIUM, } \\
\text { TOTAL } \\
\text { RECOV- } \\
\text { ERABLE } \\
\text { (UG/L }\end{array}$ & $\begin{array}{l}\text { CADMIUM } \\
\text { TOTAL } \\
\text { RECOV- } \\
\text { ERABLE } \\
\text { (UG/L }\end{array}$ & $\begin{array}{l}\text { CHRO- } \\
\text { MIUM, } \\
\text { TOTAL } \\
\text { RECOV- } \\
\text { ERABLE } \\
\text { (UG/L }\end{array}$ \\
\hline & AS $\mathrm{K})$ & AS SO4) & AS CL) & AS N) & AS N) & AS P) & AS P) & AS $\quad S B$ ) & AS A.S) & $A S B E)$ & AS $C D)$ & AS $(R)$ \\
\hline
\end{tabular}

\begin{tabular}{|c|c|c|c|c|c|c|c|c|c|c|c|c|}
\hline \multicolumn{13}{|l|}{ JAN 1993} \\
\hline 09.09 & 3.1 & 27 & 5.4 & 0.860 & 1.3 & 0.700 & 0.150 & 53.0 & 3 & $<10$ & 3 & 26 \\
\hline \multicolumn{13}{|l|}{ JAN } \\
\hline $28-28$ & 3.5 & 32 & 12 & 0.730 & 1.3 & 0.710 & 0.120 & 20.0 & 6 & $<10$ & 3 & 28 \\
\hline \multicolumn{13}{|l|}{ FEB } \\
\hline $24-24$ & 2.6 & 22 & 11 & 1.10 & 1.5 & 0.920 & 0.450 & $<10.0$ & 4 & $<10$ & 2 & 20 \\
\hline \multicolumn{13}{|l|}{ MAR } \\
\hline $19-19$ & 3.4 & 22 & 7.9 & 0.740 & 0.70 & 0.380 & 0.300 & 44.0 & 6 & $<10$ & 1 & 12 \\
\hline \multicolumn{13}{|l|}{ APR } \\
\hline $03-04$ & 2.1 & 11 & 5.0 & 0.670 & 1.2 & 0.790 & 0.090 & 20.0 & 5 & $<10$ & 2 & 20 \\
\hline \multicolumn{13}{|l|}{ APR } \\
\hline $14-14$ & 2.2 & 13 & 4.2 & 0.660 & 0.80 & 0.380 & 0.230 & 22.0 & 3 & $<10$ & 1 & 10 \\
\hline \multicolumn{13}{|l|}{ JUN } \\
\hline $09-09$ & 3.5 & 9.6 & 5.2 & 0.530 & 0.80 & 0.320 & 0.300 & $<10.0$ & 7 & $<10$ & 2 & 12 \\
\hline
\end{tabular}


Table 8. Water-quality data for storm-sewer outfall stations, Plano, Texas, 1992-93-Continued

08061530 - SPRING CREEK OUTFALL AT AVENUE F, PLANO, TX (WY 1993)-Continued

\begin{tabular}{|c|c|c|c|c|c|c|c|c|c|c|c|c|}
\hline DATE & $\begin{array}{c}\text { COPPER, } \\
\text { TOTAL } \\
\text { RECOV- } \\
\text { ERABLE } \\
\text { (UG/L } \\
\text { AS CU) }\end{array}$ & $\begin{array}{c}\text { CYANIDE } \\
\text { TOTAL } \\
\text { EPA } \\
\text { (MG/L } \\
\text { AS CN) }\end{array}$ & $\begin{array}{c}\text { CYANIDE } \\
\text { TOTAL } \\
\text { (MG/L } \\
\text { AS CN) }\end{array}$ & $\begin{array}{l}\text { LEAD, } \\
\text { TOTAL } \\
\text { RECOV- } \\
\text { ERABLE } \\
\text { (UG/L } \\
\text { AS PB) }\end{array}$ & $\begin{array}{l}\text { MERCURY } \\
\text { TOTAL } \\
\text { RECOV- } \\
\text { ERABLE } \\
\text { (UG /L } \\
\text { AS HG) }\end{array}$ & $\begin{array}{l}\text { NICKEL, } \\
\text { TOTAL } \\
\text { RECOV- } \\
\text { ERABLE } \\
\text { (UG/L } \\
\text { AS NI) }\end{array}$ & $\begin{array}{l}\text { SELE- } \\
\text { NIUM, } \\
\text { TOTAL } \\
\text { (UG/L } \\
\text { AS SE) }\end{array}$ & $\begin{array}{l}\text { SILVER, } \\
\text { TOTAL } \\
\text { RECOV- } \\
\text { ERABLE } \\
\text { (UG/L } \\
\text { AS AG) }\end{array}$ & $\begin{array}{l}\text { SILVER, } \\
\text { TOTAL } \\
\text { RECOV- } \\
\text { ERABLE } \\
\text { EPA } \\
\text { (UG/L } \\
\text { AS AG) }\end{array}$ & $\begin{array}{l}\text { THAL- } \\
\text { LIUM, } \\
\text { TOTAL } \\
\text { (UG/L } \\
\text { AS TL) }\end{array}$ & $\begin{array}{l}\text { ZINC, } \\
\text { TOTAL } \\
\text { RECOV- } \\
\text { ERABLE } \\
\text { (UG/L } \\
\text { AS ZN) }\end{array}$ & $\begin{array}{c}\text { CARBON, } \\
\text { ORGANIC } \\
\text { TOTAL } \\
\text { (MG/L } \\
\text { AS C) }\end{array}$ \\
\hline JAN 1993 & & & & & & & & & & & & \\
\hline $\begin{array}{l}09-09 \\
\text { JAN }\end{array}$ & 700 & $<0.010$ & $<0.010$ & 160 & $<0.10$ & 14 & $<2$ & $<1$ & $<0.500$ & $<10$ & 340 & 30 \\
\hline $\begin{array}{l}28-28 \\
\text { FEB }\end{array}$ & 1100 & $<0.010$ & $<0.010$ & 170 & $<0.10$ & 23 & $<2$ & $<1$ & $<0.500$ & $<20$ & 390 & 49 \\
\hline $\begin{array}{l}24-24 \\
\text { MAR }\end{array}$ & 460 & $<0.010$ & $<0.010$ & 81 & $<0.10$ & 10 & $<2$ & $<1$ & $<0.500$ & $<5$ & 200 & 26 \\
\hline $\begin{array}{l}19-19 \\
\text { APR }\end{array}$ & 1200 & $<0.010$ & $<0.010$ & 100 & $<0.10$ & 12 & $<2$ & $<1$ & $<0.500$ & $<5$ & 230 & 16 \\
\hline $\begin{array}{l}03-04 \\
A P R\end{array}$ & 1300 & $<0.010$ & $<0.010$ & 180 & $<0.10$ & 19 & $<2$ & $<1$ & $<0.500$ & $<10$ & 340 & 36 \\
\hline $\mathrm{JUN}^{14-14}$ & 350 & $<0.010$ & $<0.010$ & 78 & $<0.10$ & 9 & $<2$ & $<1$ & $<0.500$ & $<10$ & 180 & 19 \\
\hline 09.09 & 11 & $<0.010$ & $<0.010$ & 160 & $<0.10$ & 16 & $<2$ & $<1$ & $<0.500$ & $<5$ & 390 & 30 \\
\hline & OIL AND & & & & & & METHANE & & & BENZENE & BENZENE & \\
\hline & $\begin{array}{c}\text { GREASE, } \\
\text { TOTAL } \\
\text { RECOV. } \\
\text { GRAVI - }\end{array}$ & PHENOLS & $\begin{array}{l}\text { ACRO- } \\
\text { LEIN }\end{array}$ & $\begin{array}{l}\text { ACRYLO- } \\
\text { NITRILE }\end{array}$ & BENZENE & $\begin{array}{l}\text { BROMO- } \\
\text { BENZENE } \\
\text { WATER, } \\
\text { WHOLE, }\end{array}$ & $\begin{array}{l}\text { BROMO } \\
\text { CHLORO- } \\
\text { WAT } \\
\text { UNFLTRD }\end{array}$ & $\begin{array}{l}\text { BROMO- } \\
\text { FORM }\end{array}$ & $\begin{array}{c}\text { BENZENE } \\
\text { N-BUTYL } \\
\text { WATER } \\
\text { UNFLTRD }\end{array}$ & $\begin{array}{c}\text { SEC } \\
\text { BUTYL- } \\
\text { WATER } \\
\text { UNFLTRD }\end{array}$ & $\begin{array}{l}\text { TERT - } \\
\text { BUTYL - } \\
\text { WATER } \\
\text { UNFLTRD }\end{array}$ & $\begin{array}{l}\text { CARBON - } \\
\text { TETRA- } \\
\text { CHLO- } \\
\text { RIDE }\end{array}$ \\
\hline DATE & $\begin{array}{c}\text { METRIC } \\
(\mathrm{MG} / \mathrm{L})\end{array}$ & $\begin{array}{l}\text { TOTAL } \\
(U G / L)\end{array}$ & $\begin{array}{l}\text { TOTAL } \\
\text { (UG/L) }\end{array}$ & $\begin{array}{l}\text { TOTAL } \\
\text { (UG/L) }\end{array}$ & $\begin{array}{l}\text { TOTAL } \\
\text { (UG/L) }\end{array}$ & $\begin{array}{l}\text { TOTAL } \\
\text { (UG/L) }\end{array}$ & $\begin{array}{c}\text { REC } \\
(\mathrm{UG} / \mathrm{L})\end{array}$ & $\begin{array}{l}\text { TOTAL } \\
\text { (UG/L) }\end{array}$ & $\begin{array}{c}\text { REC } \\
(U G / L)\end{array}$ & $\begin{array}{c}\text { REC } \\
(U G / L)\end{array}$ & $\begin{array}{c}\text { REC } \\
(U G / L)\end{array}$ & $\begin{array}{l}\text { TOTAL } \\
\text { (UG/L) }\end{array}$ \\
\hline JAN 1 & & & & & & & & & & & & \\
\hline $\begin{array}{l}09-09 \\
\text { JAN }\end{array}$ & 1 & 6 & $<20$ & $<20$ & $<0.2$ & $<0.2$ & $<0.20$ & $<0.2$ & $<0.20$ & $<0.20$ & $<0.20$ & $<0.2$ \\
\hline $\begin{array}{l}28-28 \\
\text { FEB }\end{array}$ & 4 & 19 & $<20$ & $<20$ & 0.6 & $<0.2$ & $<0.20$ & $<0.2$ & $<0.20$ & $<0.20$ & $<0.20$ & $<0.2$ \\
\hline $\begin{array}{l}24-24 \\
\text { MAR }\end{array}$ & 21 & 58 & $<20$ & $<20$ & $<0.2$ & $<0.2$ & $<0.20$ & $<0.2$ & $<0.20$ & $<0.20$ & $<0.20$ & $<0.2$ \\
\hline $\begin{array}{l}19-19 \\
\text { APR }\end{array}$ & 4 & 11 & $<20$ & $<20$ & 0,3 & $<0.2$ & $<0.20$ & $<0.2$ & $<0.20$ & $<0.20$ & $<0.20$ & $<0.2$ \\
\hline $\begin{array}{l}03.04 \\
\text { APR }\end{array}$ & 19 & 11 & $<20$ & $<20$ & $<0.2$ & $<0.2$ & $<0.20$ & $<0.2$ & $<0.20$ & $<0.20$ & $<0.20$ & $<0.2$ \\
\hline $\begin{array}{l}14 \cdot 14 \\
\text { JUN }\end{array}$ & 3 & 7 & $<20$ & $<20$ & $<0.2$ & $<0.2$ & $<0.20$ & $<0.2$ & $<0.20$ & $<0.20$ & $<0.20$ & $<0.2$ \\
\hline $09-09$ & 120 & 55 & $<1000$ & $<1000$ & $<10$ & $<10$ & $<10.0$ & $<10$ & $<10$ & $<10$ & $<10$ & $<10$ \\
\hline
\end{tabular}

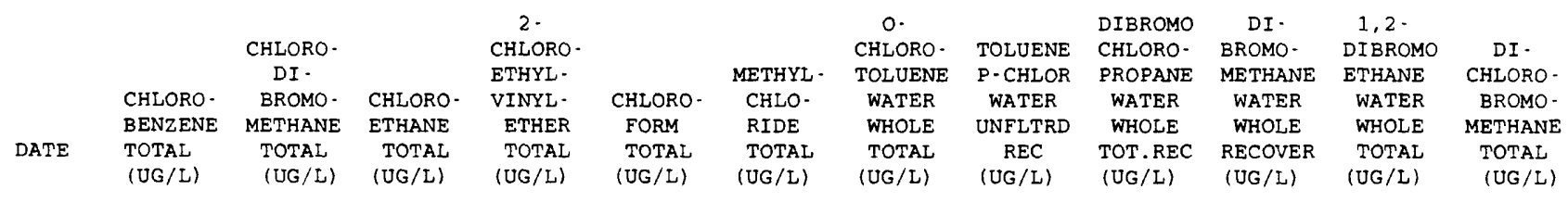

\begin{tabular}{|c|c|c|c|c|c|c|c|c|c|c|c|c|}
\hline JAN 1993 & & & & & & & & & & & & \\
\hline $\begin{array}{l}09-09 \\
\text { JAN }\end{array}$ & $<0.20$ & $<0.2$ & $<0.2$ & $<1.0$ & $<0.2$ & $<0.2$ & $<0.2$ & $<0.20$ & $<1.0$ & $<0.2$ & $<0.2$ & $<0.2$ \\
\hline $28-28$ & $<0.20$ & $<0,2$ & $<0.2$ & $<1.0$ & $<0.2$ & $<0.2$ & $<0.2$ & $<0.20$ & $<1.0$ & $<0.2$ & $<0.2$ & $<0.2$ \\
\hline FEB & & & & & & & & & & & & \\
\hline $24-24$ & $<0.20$ & $<0.2$ & $<0.2$ & $<1.0$ & 0.5 & $<0.2$ & $<0.2$ & $<0.20$ & $<1.0$ & $<0.2$ & $<0.2$ & 0.2 \\
\hline MAR & & & & & & & & & & & & \\
\hline $\begin{array}{c}19-19 \\
A P R\end{array}$ & $<0.20$ & $<0.2$ & $<0.2$ & $<1.0$ & 0.2 & $<0.2$ & $<0.2$ & $<0.20$ & $<1.0$ & $<0.2$ & $<0.2$ & $<0.2$ \\
\hline $03-04$ & $<0.20$ & $<0.2$ & $<0.2$ & $<1.0$ & $<0.2$ & $<0.2$ & $<0.2$ & $<0.20$ & $<1.0$ & $<0.2$ & $<0.2$ & $<0.2$ \\
\hline APR & & & & & & & & & & & & \\
\hline $\begin{array}{l}14-14 \\
\text { JUN }\end{array}$ & $<0.20$ & $<0.2$ & $<0.2$ & $<1.0$ & $<0.2$ & $<0.2$ & $<0.2$ & $<0.20$ & $<1.0$ & $<0.2$ & $<0.2$ & $<0.2$ \\
\hline 09.09 & $<10$ & $<10$ & $<10$ & $<50$ & $<10$ & $<10$ & $<10$ & $<10$ & $<50$ & $<10$ & $<10$ & $<10$ \\
\hline
\end{tabular}


08061530 - SPRING CREEK OUTFALl AT AVENUE F, PLANO, TX (WY 1993)-Continued

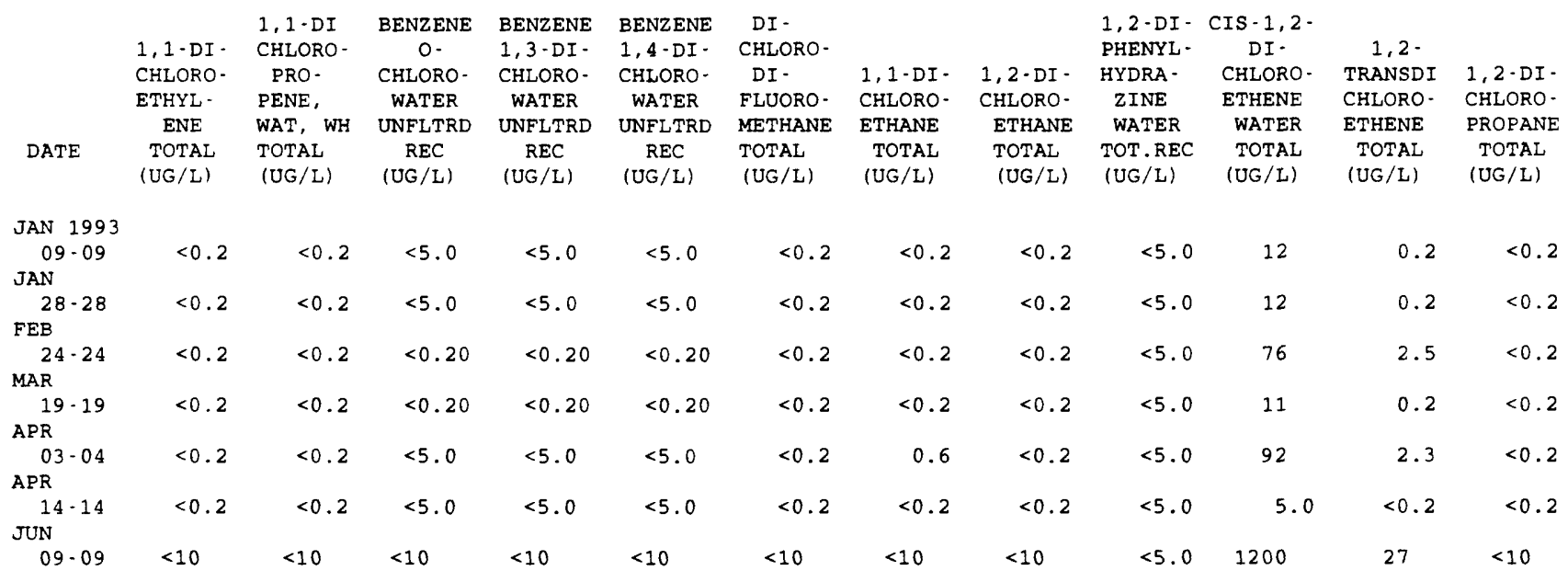

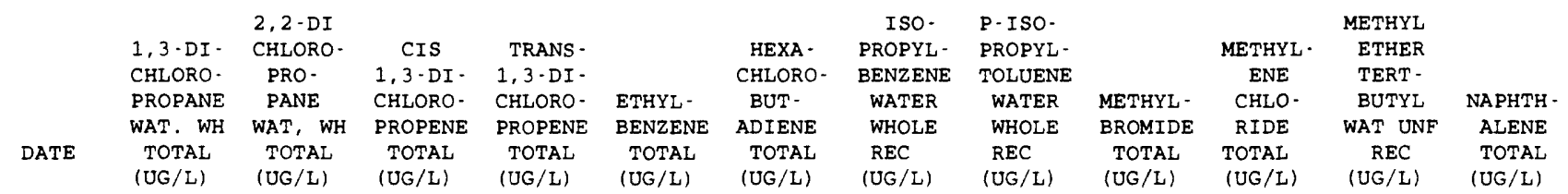

\begin{tabular}{|c|c|c|c|c|c|c|c|c|c|c|c|c|}
\hline $\begin{array}{c}\text { JAN } 1993 \\
09-09\end{array}$ & $<0.2$ & $<0.2$ & $<0.2$ & $<0.2$ & $<0.2$ & $<5.0$ & $<0.20$ & $<0.20$ & $<0.2$ & $<0.2$ & $<1.0$ & $<5.0$ \\
\hline \multicolumn{13}{|l|}{ JAN } \\
\hline $28-28$ & $<0.2$ & $<0.2$ & $<0.2$ & $<0.2$ & 1.2 & $<5.0$ & 0.30 & $<0.20$ & $<0.2$ & $<0.5$ & 5.4 & $<5.0$ \\
\hline \multicolumn{13}{|l|}{ FEB } \\
\hline $24 \cdot 24$ & $<0.2$ & $<0.2$ & $<0.2$ & $<0.2$ & $<0.2$ & $<0.2$ & 4.6 & $<0.20$ & $<0.2$ & $<0.3$ & $<1.0$ & $<0.2$ \\
\hline \multicolumn{13}{|l|}{ MAR } \\
\hline $19-19$ & $<0.2$ & $<0.2$ & $<0.2$ & $<0.2$ & 0.4 & $<0.2$ & 0.20 & $<0.20$ & $<0.2$ & $<0.2$ & $<1.0$ & 1.3 \\
\hline \multicolumn{13}{|l|}{ APR } \\
\hline $03-04$ & $<0.2$ & $<0.2$ & $<0.2$ & $<0.2$ & $<0.2$ & $<5.0$ & $<0.20$ & $<0.20$ & $<0.2$ & $<0.2$ & $<1.0$ & $<5.0$ \\
\hline \multicolumn{13}{|l|}{ APR } \\
\hline \multirow{2}{*}{\multicolumn{13}{|c|}{ JUN }} \\
\hline & & & & & & & & & & & & \\
\hline $09-09$ & $<10$ & $<10$ & $<10$ & $<10$ & $<10$ & $<10$ & $<10$ & $<10$ & $<10$ & $<10$ & $<50$ & $<10$ \\
\hline
\end{tabular}

\begin{tabular}{|c|c|c|c|c|c|c|c|c|c|c|c|c|}
\hline & BENZENE & & $\begin{array}{c}\text { ETHANE, } \\
1112 \text {. }\end{array}$ & $\begin{array}{l}\text { ETHANE, } \\
1,1,2,2\end{array}$ & TETRA & & $\begin{array}{c}1,2,3 . \\
\text { TRI. }\end{array}$ & $\begin{array}{l}\text { BENZENE } \\
1,2,4\end{array}$ & & & & $\mathrm{TR}$ \\
\hline & N-PROPY & & TETRA - & TETRA- & CHLORO- & & CHLORO & TRI - & TRI - & TRI - & CHLORO - & CHLORO- \\
\hline & $\begin{array}{l}\text { WATER } \\
\text { UNFLTRD }\end{array}$ & STYR & $\begin{array}{l}\text { CHLORO- } \\
\text { WAT UNF }\end{array}$ & $\begin{array}{l}\text { CHLORO- } \\
\text { WAT UNF }\end{array}$ & $\begin{array}{l}\text { ETHYL- } \\
\text { ENE }\end{array}$ & NE & $\begin{array}{l}\text { BENZENE } \\
\text { WAT, WH }\end{array}$ & $\begin{array}{l}\text { CHLORO- } \\
\text { WAT UNF }\end{array}$ & $\begin{array}{l}\text { CHLORO- } \\
\text { ETHANE }\end{array}$ & $\begin{array}{l}\text { CHLORO- } \\
\text { ETHANE }\end{array}$ & $\begin{array}{c}\text { ETHYL- } \\
\text { ENE }\end{array}$ & $\begin{array}{l}\text { FLUORO- } \\
\text { METHANE }\end{array}$ \\
\hline & $\begin{array}{c}\text { REC } \\
(\mathrm{UG} / \mathrm{L})\end{array}$ & $\begin{array}{l}\text { TOTAL } \\
\text { (UG/L) }\end{array}$ & $\begin{array}{c}\text { REC } \\
(U G / L)\end{array}$ & $\begin{array}{c}\text { REC } \\
(U G / L)\end{array}$ & $\begin{array}{c}\text { TOTAL } \\
\text { (UG/L) }\end{array}$ & $\begin{array}{l}\text { TOTAL } \\
\text { (UG/L) }\end{array}$ & $\begin{array}{l}\text { REC } \\
\text { (UG/L) }\end{array}$ & $\begin{array}{c}\text { REC } \\
(U G / L)\end{array}$ & $\begin{array}{l}\text { TOTAL } \\
\text { (UG/L) }\end{array}$ & $\begin{array}{l}\text { TOTAL } \\
\text { (UG/L) }\end{array}$ & $\begin{array}{l}\text { TOTAL } \\
\text { (UG/L) }\end{array}$ & $\begin{array}{l}\text { TOTAL } \\
\text { (UG/L) }\end{array}$ \\
\hline
\end{tabular}

\begin{tabular}{|c|c|c|c|c|c|c|c|c|c|c|c|c|}
\hline JAN 1993 & & & & & & & & & & & & \\
\hline JAN & $<0.20$ & $<0.2$ & $<0.2$ & $<0.2$ & $<0.2$ & $<0.2$ & $<0.20$ & $<5.0$ & $<0.2$ & $<0.2$ & 1.2 & $<0.2$ \\
\hline $28 \cdot 28$ & 0.40 & $<0.2$ & $<0.2$ & $<0.2$ & $<0.2$ & 1.8 & $<0.20$ & $<5.0$ & $<0.2$ & $<0.2$ & 1.0 & $<0.2$ \\
\hline FEB & & & & & & & & & & & & \\
\hline $\begin{array}{l}24-24 \\
\text { MAR }\end{array}$ & $<0.20$ & $<0.2$ & $<0.2$ & $<0.2$ & $<0.2$ & $<0.2$ & $<0.20$ & $<0.20$ & $<0.2$ & $<0.2$ & 2.4 & $<0.2$ \\
\hline $\begin{array}{l}\text { MAR } \\
\quad 19-19\end{array}$ & 0.20 & $<0.2$ & $<0.2$ & $<0.2$ & $<0.2$ & 1.3 & $<0.20$ & $<0.20$ & $<0.2$ & $<0.2$ & 1.0 & $<0.2$ \\
\hline APR & & & & & & & & & & & & \\
\hline $\begin{array}{c}03-04 \\
A P R\end{array}$ & $<0.20$ & $<0.2$ & $<0.2$ & $<0.2$ & $<0.2$ & $<0.2$ & $<0.20$ & $<5.0$ & $<0.2$ & $<0.2$ & 1.9 & $<0.2$ \\
\hline $\operatorname{JUN}^{14-14}$ & $<0.20$ & $<0.2$ & $<0.2$ & $<0.2$ & $<0.2$ & 0.2 & $<0.20$ & $<5.0$ & $<0.2$ & $<0.2$ & 0.3 & $<0.2$ \\
\hline $09-09$ & $<10$ & $<10$ & $<10$ & $<10$ & $<10$ & $<10$ & $<10$ & $<10$ & $<10$ & $<10$ & 28 & $<10$ \\
\hline
\end{tabular}


Table 8. Water-quality data for storm-sewer outfall stations, Plano, Texas, 1992-93-Continued

08061530 - SPRING CREEK OUTFALL AT AVENUE F, PLANO, TX (WY 1993)-Continued

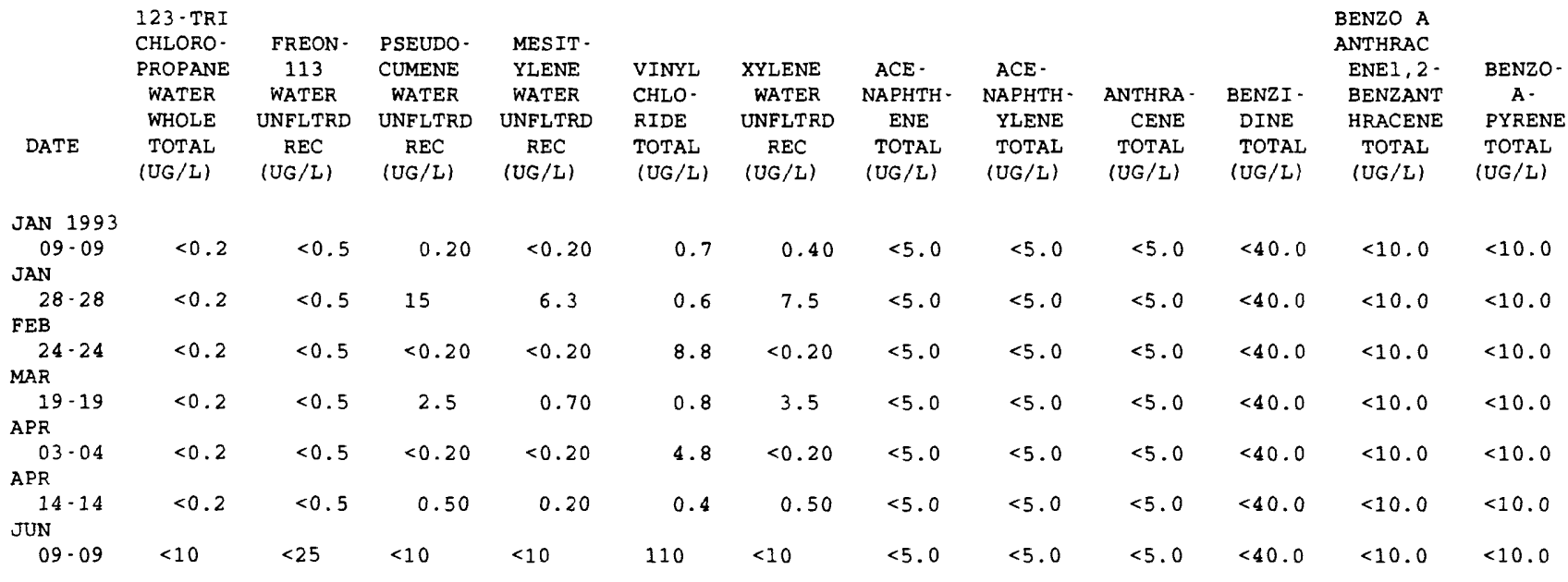

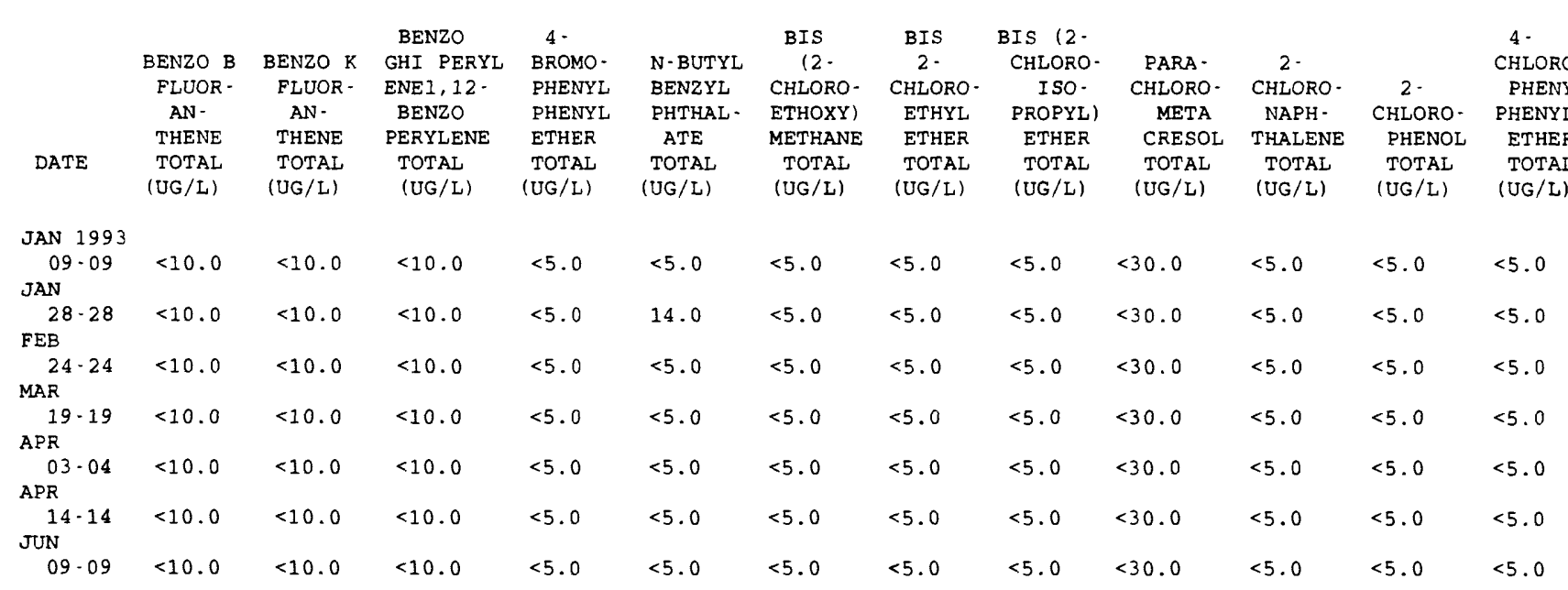

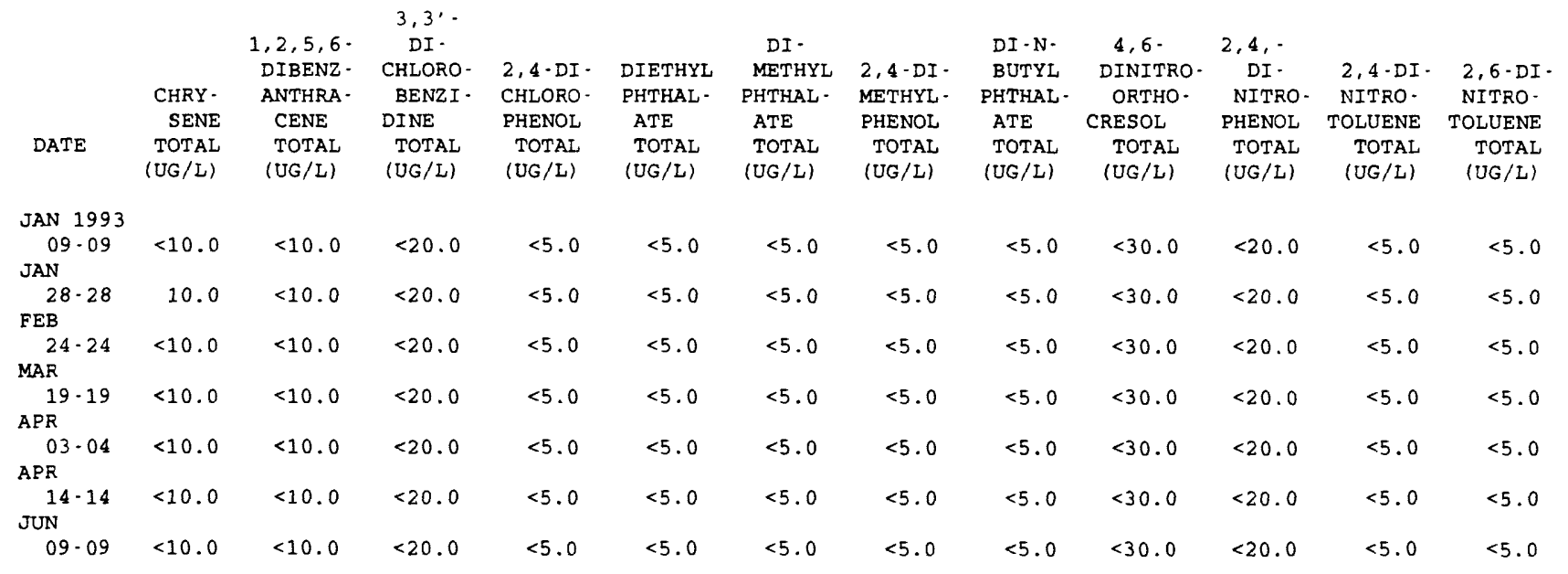




\begin{tabular}{|c|c|c|c|c|c|c|c|c|c|c|c|c|}
\hline DATE & $\begin{array}{c}\text { DI - N- } \\
\text { OCTYL } \\
\text { PHTHAL- } \\
\text { ATE } \\
\text { TOTAL } \\
\text { (UG/L) }\end{array}$ & $\begin{array}{l}\text { BIS }(2- \\
\text { ETHYL } \\
\text { HEXYL) } \\
\text { PHTHAL- } \\
\text { ATE } \\
\text { TOTAL } \\
\text { (UG/L) }\end{array}$ & $\begin{array}{c}\text { FLUOR - } \\
\text { ANTHENE } \\
\text { TOTAL } \\
\text { (UG/L) }\end{array}$ & $\begin{array}{l}\text { FLUOR- } \\
\text { ENE } \\
\text { TOTAL } \\
\text { (UG/L) }\end{array}$ & $\begin{array}{l}\text { HEXA- } \\
\text { CHLORO- } \\
\text { BENZENE } \\
\text { TOTAL } \\
\text { (UG/L) }\end{array}$ & $\begin{array}{l}\text { HEXA- } \\
\text { CHLORO- } \\
\text { CYCLO- } \\
\text { PENT- } \\
\text { ADIENE } \\
\text { TOTAL } \\
\text { (UG/L) }\end{array}$ & $\begin{array}{l}\text { HEXA- } \\
\text { CHLORO- } \\
\text { ETHANE } \\
\text { TOTAL } \\
\text { (UG/L) }\end{array}$ & $\begin{array}{c}\text { INDENO } \\
(1,2,3- \\
\text { CD) } \\
\text { PYRENE } \\
\text { TOTAL } \\
\text { (UG/L) }\end{array}$ & $\begin{array}{c}\text { ISO } \\
\text { PHORONE } \\
\text { TOTAL } \\
\text { (UG/L) }\end{array}$ & $\begin{array}{c}\text { NITRO- } \\
\text { BENZENE } \\
\text { TOTAL } \\
\text { (UG/L) }\end{array}$ & $\begin{array}{c}N \cdot \text { NITRO- } \\
\text { SODI- } \\
\text { METHY - } \\
\text { LAMINE } \\
\text { TOTAL } \\
(\text { UG } / L)\end{array}$ & $\begin{array}{l}2- \\
\text { NITRO } \\
\text { PHENOL } \\
\text { TOTAL } \\
\text { (UG/L) }\end{array}$ \\
\hline $\begin{array}{c}\text { JAN } 1993 \\
09-09\end{array}$ & $<10.0$ & 7.0 & $<5.0$ & $<5.0$ & $<5.0$ & $<5,0$ & $<5.0$ & $<10,0$ & $<5.0$ & $<5.0$ & $<5.0$ & $<5.0$ \\
\hline JAN & & & & & & & & & & & & \\
\hline $\begin{array}{l}28-28 \\
F E B\end{array}$ & $<10.0$ & 130 & $<5.0$ & $<5.0$ & $<5.0$ & $<5.0$ & $<5.0$ & $<10.0$ & $<5.0$ & $<5.0$ & $<5.0$ & $<5.0$ \\
\hline$\underset{\text { MAR }}{24 \cdot 24}$ & $<10.0$ & 10.0 & $<5.0$ & $<5.0$ & $<5.0$ & $<5.0$ & $<5.0$ & $<10.0$ & $<5.0$ & $<5.0$ & $<5.0$ & $<5.0$ \\
\hline $\begin{array}{l}19-19 \\
\text { APR }\end{array}$ & $<10.0$ & 11.0 & $<5.0$ & $<5.0$ & $<5.0$ & $<5.0$ & $<5.0$ & $<10.0$ & $<5.0$ & $<5.0$ & $<5.0$ & $<5.0$ \\
\hline $\begin{array}{l}03-04 \\
\text { APR }\end{array}$ & $<10.0$ & 7.0 & $<5.0$ & $<5.0$ & $<5.0$ & $<5.0$ & $<5.0$ & $<10.0$ & $<5.0$ & $<5.0$ & $<5.0$ & $<5.0$ \\
\hline $\begin{array}{l}14-14 \\
\text { JUN }\end{array}$ & $<10.0$ & 6.0 & $<5.0$ & $<5.0$ & $<5.0$ & $<5.0$ & $<5.0$ & $<10.0$ & $<5.0$ & $<5.0$ & $<5.0$ & $<5.0$ \\
\hline 09.09 & $<10.0$ & 5.0 & $<5.0$ & $<5.0$ & $<5.0$ & $<5.0$ & $<5.0$ & $<10.0$ & $<5.0$ & $<5.0$ & $<5.0$ & $<5.0$ \\
\hline DATE & $\begin{array}{l}4- \\
\text { NITRO- } \\
\text { PHENOL } \\
\text { TOTAL } \\
\text { (UG/L) }\end{array}$ & $\begin{array}{c}\text { N- } \\
\text { NITRO- } \\
\text { SODI -N- } \\
\text { PROPYL- } \\
\text { AMINE } \\
\text { TOTAL } \\
(\text { UG/L) }\end{array}$ & $\begin{array}{l}\text { N- NITRO- } \\
\text { SODI- } \\
\text { PHENY- } \\
\text { LAMINE } \\
\text { TOTAL } \\
(U G / L)\end{array}$ & $\begin{array}{l}\text { PENTA- } \\
\text { CHLORO- } \\
\text { PHENOL } \\
\text { TOTAL } \\
(\mathrm{UG} / \mathrm{L})\end{array}$ & $\begin{array}{l}\text { PHENAN- } \\
\text { THRENE } \\
\text { TOTAL } \\
\text { (UG/L) }\end{array}$ & $\begin{array}{c}\text { PHENOL } \\
\text { (C6H- } \\
50 \mathrm{H}) \\
\text { TOTAL } \\
\text { (UG/L) }\end{array}$ & $\begin{array}{c}\text { PYRENE } \\
\text { TOTAL } \\
\text { (UG/L) }\end{array}$ & $\begin{array}{c}2,4,6- \\
\text { TRI- } \\
\text { CHLORO- } \\
\text { PHENOL } \\
\text { TOTAL } \\
\text { (UG/L) }\end{array}$ & $\begin{array}{c}\text { ALDRIN, } \\
\text { TOTAL } \\
\text { (UG/L) }\end{array}$ & $\begin{array}{l}\text { P, P' } \\
\text { DDT, } \\
\text { TOTAL } \\
\text { (UG/L) }\end{array}$ & $\begin{array}{l}\text { ALPHA } \\
\text { BHC } \\
\text { TOTAL } \\
\text { (UG/L) }\end{array}$ & $\begin{array}{c}\text { BETA } \\
\text { BENZENE } \\
\text { HEXA- } \\
\text { CHLOR- } \\
\text { IDE } \\
\text { TOTAL } \\
\text { (UG/L) }\end{array}$ \\
\hline $\begin{array}{c}\text { JAN } 1993 \\
09 \cdot 09\end{array}$ & $<30.0$ & $<5.0$ & $<5.0$ & $<30.0$ & $<5.0$ & $<5.0$ & $<5.0$ & $<20.0$ & $<0.040$ & $<0.10$ & $<0.03$ & $<0.03$ \\
\hline $\begin{array}{l}\text { JAN } \\
\quad 28-28\end{array}$ & $<30.0$ & $<5.0$ & $<5.0$ & $<30.0$ & $<5.0$ & $<5.0$ & $<5.0$ & $<20.0$ & $<0.040$ & $<0.10$ & $<0.03$ & $<0.03$ \\
\hline FEB & & & & & & & & & & & & \\
\hline $\begin{array}{l}24-24 \\
\text { MAR }\end{array}$ & $<30.0$ & $<5.0$ & $<5.0$ & $<30.0$ & $<5.0$ & $<5.0$ & $<5.0$ & $<20.0$ & $<0.040$ & $<0.10$ & $<0.05$ & $<0.03$ \\
\hline $\begin{array}{l}19-19 \\
\text { APR }\end{array}$ & $<30.0$ & $<5.0$ & $<5.0$ & $<30.0$ & $<5.0$ & $<5.0$ & $<5.0$ & $<20.0$ & $<0.040$ & $<0.10$ & $<0.03$ & $<0.03$ \\
\hline $\begin{array}{l}03-04 \\
\text { APR }\end{array}$ & $<30.0$ & $<5.0$ & $<5.0$ & $<30.0$ & $<5.0$ & $<5.0$ & $<5.0$ & $<20.0$ & $<0.040$ & $<0.10$ & $<0.03$ & $<0.03$ \\
\hline $\begin{array}{l}14-14 \\
\text { JUN }\end{array}$ & $<30.0$ & $<5.0$ & $<5.0$ & $<30.0$ & $<5.0$ & $<5.0$ & $<5.0$ & $<20.0$ & $<0.040$ & $<0.10$ & $<0.03$ & $<0.03$ \\
\hline 09.09 & $<30.0$ & $<5.0$ & $<5.0$ & $<30.0$ & $<5.0$ & $<5.0$ & $<5.0$ & $<20.0$ & $<0.040$ & $<0.10$ & $<0.03$ & $<0.03$ \\
\hline
\end{tabular}

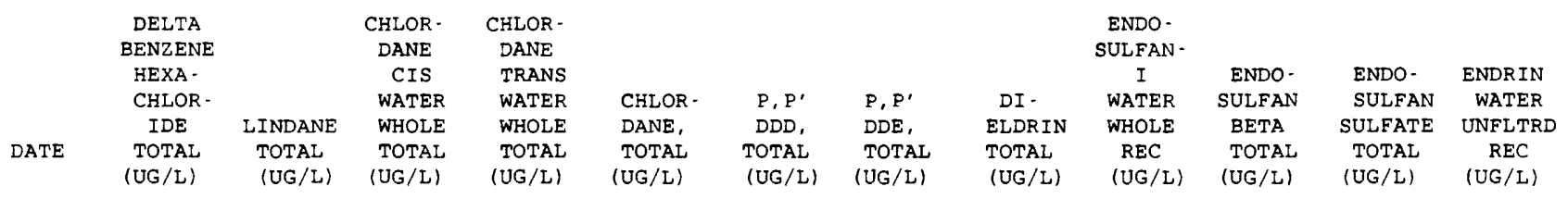

\begin{tabular}{|c|c|c|c|c|c|c|c|c|c|c|c|c|}
\hline $\begin{array}{c}\text { JAN } 1993 \\
09-09\end{array}$ & $<0.09$ & $<0.030$ & $<0.10$ & $<0.10$ & 0.1 & $<0.10$ & $<0.04$ & $<0.020$ & $<0.10$ & $<0.04$ & $<0.60$ & $<0.060$ \\
\hline JAN & & & & & & & & & & & & \\
\hline $28-28$ & $<0.09$ & $<0.030$ & $<0.10$ & $<0.10$ & 0.1 & $<0.10$ & $<0.04$ & $<0.020$ & $<0.10$ & $<0.04$ & $<0.60$ & $<0.060$ \\
\hline FEB & & & & & & & & & & & & \\
\hline $24-24$ & $<0.09$ & $<0.030$ & $<0.10$ & $<0.10$ & $<0.1$ & $<0.10$ & $<0.04$ & $<0.020$ & $<0.10$ & $<0.04$ & $<0.60$ & $<0.060$ \\
\hline MAR & & & & & & & & & & & & \\
\hline $19-19$ & $<0.09$ & $<0.030$ & $<0.10$ & $<0.10$ & 0.1 & $<0.10$ & $<0.04$ & $<0.020$ & $<0.10$ & $<0.04$ & $<0.60$ & $<0.060$ \\
\hline $\begin{array}{l}\text { APR } \\
03-04\end{array}$ & $<0.09$ & $<0.030$ & $<0.10$ & $<0.10$ & 0.1 & $<0.10$ & $<0.04$ & $<0.020$ & $<0.10$ & $<0.04$ & $<0,60$ & $<0.060$ \\
\hline APR & & & & & & & & & & & & \\
\hline $\begin{array}{l}14-14 \\
\text { JUN }\end{array}$ & $<0.09$ & $<0.030$ & $<0.10$ & $<0.10$ & 0.1 & $<0.10$ & $<0.04$ & $<0.020$ & $<0.10$ & $<0.04$ & $<0.60$ & $<0.060$ \\
\hline $09-09$ & $<0.09$ & $<0.030$ & $<0.10$ & $<0.10$ & $<0.1$ & $<0.10$ & $<0.04$ & $<0.020$ & $<0.10$ & $<0.04$ & $<0.60$ & $<0.060$ \\
\hline
\end{tabular}


Table 8. Water-quality data for storm-sewer outfall stations, Plano, Texas, 1992-93-Continued

08061530 - SPRING CREER OUTFALL AT AVENUE F, PLANO, TX (WY 1993)-Continued

\begin{tabular}{|c|c|c|c|c|c|c|c|c|c|c|c|c|}
\hline & ENDR IN & & HEPTA - & & AROCLOR & AROCLOR & AROCLOR & AROCLOR & AROCLOR & AROCLOR & AROCLOR & \\
\hline & ALDE - & HEPTA - & CHLOR & TOX - & 1016 & 1221 & 1232 & 1242 & 1248 & 1254 & 1260 & DI - \\
\hline & HYDE & CHLOR, & EPOXIDE & APHENE, & $P C B$ & PCB & PCB & PCB & PCB & PCB & PCB & AZINON \\
\hline DATE & $\begin{array}{l}\text { TOTAL } \\
\text { (UG/L) }\end{array}$ & $\begin{array}{l}\text { TOTAL } \\
\text { (UG/L) }\end{array}$ & $\begin{array}{l}\text { TOTAL } \\
\text { (UG/L) }\end{array}$ & $\begin{array}{l}\text { TOTAL } \\
\text { (UG/L) }\end{array}$ & $\begin{array}{l}\text { TOTAL } \\
\text { (UG/L) }\end{array}$ & $\begin{array}{l}\text { TOTAL } \\
\text { (UG/L) }\end{array}$ & $\begin{array}{l}\text { TOTAL } \\
\text { (UG/L) }\end{array}$ & $\begin{array}{l}\text { TOTAL } \\
\text { (UG/L) }\end{array}$ & $\begin{array}{l}\text { TOTAL } \\
\text { (UG/L) }\end{array}$ & $\begin{array}{l}\text { TOTAL } \\
\text { (UG/L) }\end{array}$ & $\begin{array}{l}\text { TOTAL } \\
\text { (UG/L) }\end{array}$ & $\begin{array}{l}\text { TOTAL } \\
\text { (UG/L) }\end{array}$ \\
\hline
\end{tabular}

\begin{tabular}{|c|c|c|c|c|c|c|c|c|c|c|c|c|}
\hline \multicolumn{13}{|l|}{ JAN 1993} \\
\hline 09.09 & $<0.20$ & $<0.030$ & $<0.80$ & $<2$ & $<0.1$ & $<1.0$ & $<0.1$ & $<0.1$ & $<0.1$ & $<0.1$ & $<0.1$ & 0.10 \\
\hline \multicolumn{13}{|l|}{ JAN } \\
\hline $28-28$ & $<0.20$ & $<0.030$ & $<0.80$ & $<2$ & $<0.1$ & $<1.0$ & $<0.1$ & $<0.1$ & $<0.1$ & $<0.1$ & $<0.1$ & 0.10 \\
\hline \multicolumn{13}{|l|}{ FEB } \\
\hline $24-24$ & $<0.20$ & $<0.030$ & $<0.80$ & $<2$ & $<0.1$ & $<1.0$ & $<0.1$ & $<0.1$ & $<0.1$ & $<0.1$ & $<0.1$ & $<0.05$ \\
\hline \multicolumn{13}{|l|}{ MAR } \\
\hline $19-19$ & $<0.20$ & $<0.030$ & $<0.80$ & $<2$ & $<0.1$ & $<1.0$ & $<0.1$ & $<0.1$ & $<0.1$ & $<0.1$ & $<0.1$ & $<0.05$ \\
\hline \multicolumn{13}{|l|}{ APR } \\
\hline 03.04 & $<0.20$ & $<0.030$ & $<0.80$ & $<2$ & $<0.1$ & $<1.0$ & $<0.1$ & $<0.1$ & $<0.1$ & $<0.1$ & $<0.1$ & 1.8 \\
\hline \multicolumn{13}{|l|}{ APR } \\
\hline $14 \cdot 14$ & $<0.20$ & $<0.030$ & $<0.80$ & $<2$ & $<0.1$ & $<1.0$ & $<0.1$ & $<0.1$ & $<0.1$ & $<0.1$ & $<0.1$ & 0.50 \\
\hline \multicolumn{13}{|l|}{ JUN } \\
\hline 09.09 & $<0.20$ & $<0.030$ & $<0.80$ & $<2$ & $<0.1$ & $<1.0$ & $<0.1$ & 0.2 & $<0.1$ & $<0.1$ & $<0.1$ & 0.20 \\
\hline
\end{tabular}


Table 9. Water-quality data for storm-sewer outfall stations, Texas Department of Transportation, Dallas and Fort Worth Districts, 1993-94

08048920 - DEER CREEK OUTFALL AT I-35W, FORT WORTH, TX (WY 1995)

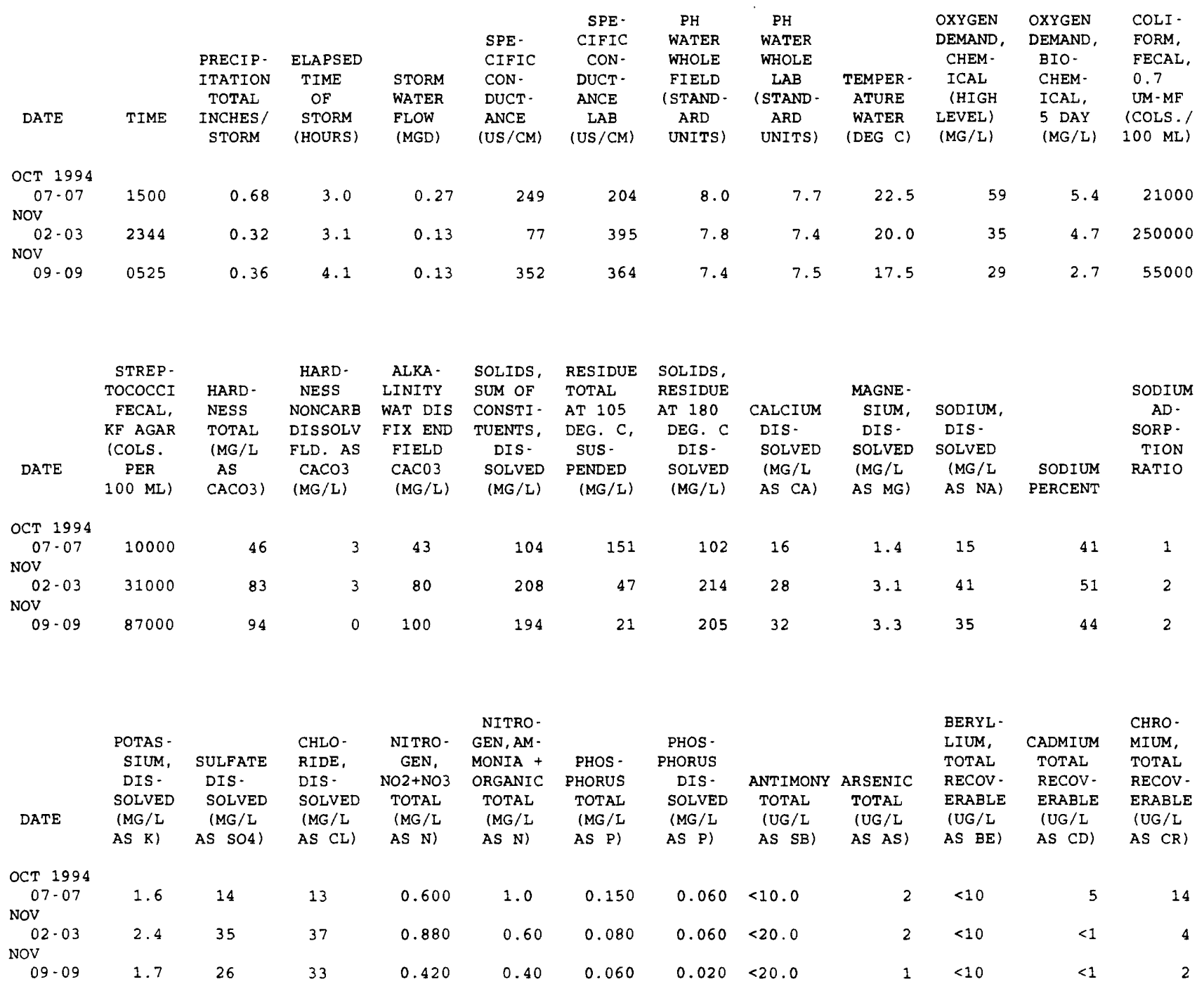

\begin{tabular}{|c|c|c|c|c|c|c|c|c|c|c|c|c|}
\hline DATE & $\begin{array}{l}\text { COPPER, } \\
\text { TOTAL } \\
\text { RECOV- } \\
\text { ERABLE } \\
\text { (UG/L } \\
\text { AS CU) }\end{array}$ & $\begin{array}{c}\text { CYANIDE, } \\
\text { TOTAL } \\
\text { EPA } \\
\text { (MG/L } \\
\text { AS CN) }\end{array}$ & $\begin{array}{c}\text { CYANIDE } \\
\text { TOTAL } \\
\text { (MG/L } \\
\text { AS CN) }\end{array}$ & $\begin{array}{l}\text { LEAD, } \\
\text { TOTAL } \\
\text { RECOV- } \\
\text { ERABLE } \\
\text { (UG/L } \\
\text { AS PB) }\end{array}$ & $\begin{array}{l}\text { MERCURY } \\
\text { TOTAL } \\
\text { RECOV- } \\
\text { ERABLE } \\
\text { (UG/L } \\
\text { AS HG) }\end{array}$ & $\begin{array}{l}\text { NICKEL, } \\
\text { TOTAL } \\
\text { RECOV- } \\
\text { ERABLE } \\
\text { (UG/L } \\
\text { AS NI) }\end{array}$ & $\begin{array}{l}\text { SELE- } \\
\text { NIUM, } \\
\text { TOTAL } \\
\text { (UG/L } \\
\text { AS SE) }\end{array}$ & $\begin{array}{l}\text { SILVER, } \\
\text { TOTAL } \\
\text { RECOV- } \\
\text { ERABLE } \\
\text { (UG/L } \\
\text { AS AG) }\end{array}$ & $\begin{array}{l}\text { SILVER, } \\
\text { TOTAL } \\
\text { RECOV- } \\
\text { ERABLE } \\
\text { EPA } \\
\text { (UG/L } \\
\text { AS AG) }\end{array}$ & $\begin{array}{l}\text { THAL- } \\
\text { LIUM, } \\
\text { TOTAL } \\
\text { (UG/L } \\
\text { AS TL) }\end{array}$ & $\begin{array}{l}\text { ZINC, } \\
\text { TOTAL } \\
\text { RECOV- } \\
\text { ERABLE } \\
\text { (UG/L } \\
\text { AS ZN) }\end{array}$ & $\begin{array}{c}\text { CARBON, } \\
\text { ORGANIC } \\
\text { TOTAL } \\
\text { (MG/L } \\
\text { AS C) }\end{array}$ \\
\hline OCT 1994 & & & & & & & & & & & & \\
\hline $\begin{array}{l}07-07 \\
\text { Nov }\end{array}$ & 17 & $<0.010$ & $<0.010$ & 14 & $<0.10$ & 44 & $<1$ & $<1$ & $<0.500$ & $<10$ & 60 & 14 \\
\hline $\begin{array}{l}02-03 \\
\text { NOV }\end{array}$ & 4 & $<0.010$ & $<0.010$ & 5 & $<0.10$ & 2 & $<1$ & $<1$ & $<0.500$ & $<10$ & 40 & 12 \\
\hline 09.09 & 3 & $<0.010$ & $<0.010$ & 3 & $<0.10$ & 1 & $<1$ & $<1$ & $<0.500$ & $<5$ & 30 & 7.9 \\
\hline
\end{tabular}


Table 9. Water-quality data for storm-sewer outfall stations, Texas Department of Transportation, Dallas and Fort Worth Districts, 1993-94-Continued

08048920 - DEER CREEK OUTFALI AT I-35W, FORT WORTH, TX (WY 1995)-Continued

\begin{tabular}{|c|c|c|c|c|c|c|c|c|c|c|c|c|}
\hline & OIL AND & & & & METHANE & & & BENZENE & BENZENE & & & \\
\hline & GREASE, & & & BROMO - & BROMO & & BENZENE & SEC & TERT - & CARBON - & & CHLORO \\
\hline & TOTAL & & & BENZENE & CHLORO- & & N-BUTYI & BUTYL - & BUTYL - & TETRA - & & DI - \\
\hline & RECOV. & & & WATER, & WAT & BROMO - & WATER & WATER & WATER & CHLO - & CHLORO - & BROMO \\
\hline & GRAVI - & PHENOLS & BENZENE & WHOLE, & UNFLTRD & FORM & UNFLTRD & UNFLTRD & UNFLTRD & RIDE & BENZENE & METHANE \\
\hline DATE & $\begin{array}{c}\text { METRIC } \\
\{\text { MG /L }\}\end{array}$ & $\begin{array}{l}\text { TOTAL } \\
\text { (UG/L) }\end{array}$ & $\begin{array}{l}\text { TOTAL } \\
\langle U G / L\rangle\end{array}$ & $\begin{array}{l}\text { TOTAL } \\
\text { (UG/L) }\end{array}$ & $\begin{array}{c}\text { REC } \\
(U G / L)\end{array}$ & $\begin{array}{l}\text { TOTAL } \\
\text { (UG/L) }\end{array}$ & $\begin{array}{c}\text { REC } \\
\text { (UG/L) }\end{array}$ & $\begin{array}{c}\text { REC } \\
\text { (UG/L) }\end{array}$ & $\begin{array}{c}\text { REC } \\
(U G / L)\end{array}$ & $\begin{array}{l}\text { TOTAL } \\
\text { (UG/L) }\end{array}$ & $\begin{array}{l}\text { TOTAL } \\
\text { (UG/L) }\end{array}$ & $\begin{array}{l}\text { TOTAL } \\
\text { (UG/L) }\end{array}$ \\
\hline
\end{tabular}

\begin{tabular}{|c|c|c|c|c|c|c|c|c|c|c|c|c|}
\hline \multicolumn{13}{|c|}{ OCT 1994} \\
\hline $07-07$ & $<1$ & 2 & $<4.0$ & $<4.0$ & $<4.00$ & $<4.0$ & $<4.0$ & $<4.0$ & $<4.0$ & $<4.0$ & $<4.0$ & $<4.0$ \\
\hline \multicolumn{13}{|l|}{ NOV } \\
\hline $02-03$ & $<1$ & 3 & $<0.2$ & $<0.2$ & $<0.20$ & $<0.2$ & $<0.20$ & $<0.20$ & $<0.20$ & $<0.2$ & $<0.20$ & $<0.2$ \\
\hline \multicolumn{13}{|l|}{ NOV } \\
\hline \multirow[t]{4}{*}{09.09} & $<1$ & $<1$ & $<1.0$ & $<1.0$ & $<1.00$ & $<1.0$ & $<1.0$ & $<1.0$ & $<1.0$ & $<1.0$ & $<1.0$ & $<1.0$ \\
\hline & & $2 \cdot$ & & & 0. & & DIBROMO & DI - & 1,2- & & & $1,1-D I$ \\
\hline & & $\begin{array}{l}\text { CHLORO- } \\
\text { ETHYL - }\end{array}$ & & METHYL - & $\begin{array}{l}\text { CHLORO - } \\
\text { TOLUENE }\end{array}$ & $\begin{array}{l}\text { TOLUENE } \\
\text { P-CHLOR }\end{array}$ & $\begin{array}{l}\text { CHLORO- } \\
\text { PROPANE }\end{array}$ & $\begin{array}{l}\text { BROMO- } \\
\text { METHANE }\end{array}$ & $\begin{array}{l}\text { DIBROMO } \\
\text { ETHANE }\end{array}$ & $\begin{array}{c}\text { DI- } \\
\text { CHLORO- }\end{array}$ & $\begin{array}{l}1,1-D I- \\
\text { CHLORO- }\end{array}$ & $\begin{array}{l}\text { CHLORO- } \\
\text { PRO - }\end{array}$ \\
\hline & CHLORO - & VINYL - & CHLORO - & CHLO - & WATER & WATER & WATER & WATER & WATER & BROMO - & ETHYL - & PENE, \\
\hline DATE & TOTAL & TOTAL & TOTAL & TOTAL & $\begin{array}{l}\text { WHOLE } \\
\text { TOTAL }\end{array}$ & REC & $\begin{array}{l}\text { WHOLE } \\
\text { TOT.REC }\end{array}$ & $\begin{array}{l}\text { WHOLE } \\
\text { RECOVER }\end{array}$ & $\begin{array}{l}\text { WHOLE } \\
\text { TOTAL }\end{array}$ & $\begin{array}{l}\text { METRANE } \\
\text { TOTAL }\end{array}$ & $\begin{array}{c}\text { ENE } \\
\text { TOTAL }\end{array}$ & $\begin{array}{l}\text { WAT, WH } \\
\text { TOTAL }\end{array}$ \\
\hline & $(U G / L)$ & $(U G / L)$ & $(U G / L)$ & $(U G / L)$ & $(U G / L)$ & (UG/L) & $(U G / L)$ & $(U G / L)$ & $\langle U G / L)$ & (UG/L) & $\langle U G / L\rangle$ & $\langle U G / L\rangle$ \\
\hline
\end{tabular}

\begin{tabular}{|c|c|c|c|c|c|c|c|c|c|c|c|c|}
\hline \multicolumn{13}{|l|}{ OCT 1994} \\
\hline 07.07 & $<4.0$ & $<20$ & $<4.0$ & $<4.0$ & $<4.0$ & $<4.0$ & $<20$ & $<4.0$ & $<4.0$ & $<4.0$ & $<4.0$ & $<4.0$ \\
\hline \multicolumn{13}{|l|}{ NOV } \\
\hline 02.03 & $<0.2$ & $<1.0$ & $<0.2$ & $<0.2$ & $<0.2$ & $<0.20$ & $<1.0$ & $<0.2$ & $<0.2$ & $<0.2$ & $<0.2$ & $<0.2$ \\
\hline \multicolumn{13}{|l|}{ NOV } \\
\hline \multirow[t]{6}{*}{09.09} & $<1.0$ & $<5.0$ & $<1.0$ & $<1.0$ & $<1.0$ & $<1.0$ & $<5.0$ & $<1.0$ & $<1.0$ & $<1.0$ & $<1.0$ & $<1.0$ \\
\hline & BENZENE & BENZENE & BENZENE & $\mathrm{DI}$. & & & $1,2 \cdot \mathrm{DI} \cdot$ & CIS-1,2- & & & & $2,2 \cdot D I$ \\
\hline & 0 & $1,3 \cdot D I-$ & $1,4-\mathrm{DI}$ - & CHLORO - & & & PHENYL - & DI - & 1,2 & & $1,3-\mathrm{DI}$ & CHLORO- \\
\hline & CHLORO- & CHLORO - & CHLORO - & DI - & $1,1-D I-$ & 1,2 -DI - & HYDRA - & CHLORO - & TRANSDI & $1,2 \cdot \mathrm{DI}$ - & CHLORO - & PRO- \\
\hline & WATER & WATER & WATER & FLUORO- & CHLORO - & CHLORO - & ZINE & ETHENE & CHLORO - & CHLORO - & PROPANE & PANE \\
\hline & UNFLTRD & UNFLTRD & UNFLTRD & METHANE & ETHANE & ETHANE & WATER & WATER & ETHENE & PROPANE & WAT. WH & WAT, WH \\
\hline DATE & $\begin{array}{c}\text { REC } \\
(U G / L)\end{array}$ & $\begin{array}{c}\text { REC } \\
\text { (UG/L) }\end{array}$ & $\begin{array}{c}\text { REC } \\
\text { (UG/L) }\end{array}$ & $\begin{array}{l}\text { TOTAL } \\
\text { (UG/L) }\end{array}$ & $\begin{array}{l}\text { TOTAL } \\
(\text { UG/L) }\end{array}$ & $\begin{array}{l}\text { TOTAL } \\
\text { (UG/L) }\end{array}$ & $\begin{array}{l}\text { TOT.REC } \\
\text { (UG/L) }\end{array}$ & $\begin{array}{l}\text { TOTAL } \\
\text { (UG/L) }\end{array}$ & $\begin{array}{l}\text { TOTAL } \\
\text { (UG/L) }\end{array}$ & $\begin{array}{l}\text { TOTAL } \\
\text { (UG/L) }\end{array}$ & $\begin{array}{l}\text { TOTAL } \\
(\mathrm{UG} / \mathrm{L})\end{array}$ & $\begin{array}{l}\text { TOTAL } \\
(\mathrm{UG} / \mathrm{L})\end{array}$ \\
\hline
\end{tabular}

\begin{tabular}{|c|c|c|c|c|c|c|c|c|c|c|c|c|}
\hline \multicolumn{13}{|l|}{ OCT 1994} \\
\hline 07.07 & $<5.0$ & $<5.0$ & $<5.0$ & $<4.0$ & $<4.0$ & $<4.0$ & $<5.0$ & $<4.0$ & $<4.0$ & $<4.0$ & $<4.0$ & $<4.0$ \\
\hline \multicolumn{13}{|l|}{ NOV } \\
\hline $02-03$ & $<5.0$ & $<5.0$ & $<5.0$ & $<0.2$ & $<0.2$ & $<0.2$ & $<5.0$ & $<0.2$ & $<0.2$ & $<0.2$ & $<0.2$ & $<0.2$ \\
\hline \multicolumn{13}{|l|}{ NoV } \\
\hline \multirow[t]{6}{*}{09.09} & $<5.0$ & $<5.0$ & $<5.0$ & $<1.0$ & $<1.0$ & $<1.0$ & $<5.0$ & $<1.0$ & $<1.0$ & $<1.0$ & $<1.0$ & $<1.0$ \\
\hline & & & & & ISO- & P-ISO- & \multicolumn{6}{|c|}{ METHYL } \\
\hline & CIS & TRANS - & & HEXA - & PROPYL - & PROPYL - & & METHYL - & ETHER & \multicolumn{3}{|c|}{ BENZENE } \\
\hline & $1,3 \cdot \mathrm{DI}=$ & $1,3-\mathrm{DI}-$ & & CHLORO - & BENZENE & TOLUENE & & ENE & TERT - & & $\mathrm{N} \cdot \mathrm{PROPY}$ & \\
\hline & CHLORO- & CHLORO - & ETHYL - & BUT - & WATER & WATER & METHYL - & CHLO - & BUTYL & NAPHTH - & WATER & \\
\hline & PROPENE & PROPENE & BENZENE & ADIENE & WHOLE & WHOLE & BROMIDE & RIDE & WAT UNF & ALENE & UNFLTRD & STYRENE \\
\hline DATE & $\begin{array}{l}\text { TOTAL } \\
\text { (UG/L) }\end{array}$ & $\begin{array}{l}\text { TOTAL } \\
\text { (UG/L) }\end{array}$ & $\begin{array}{l}\text { TOTAL } \\
\text { (UG/L) }\end{array}$ & $\begin{array}{l}\text { TOTAL } \\
\text { (UG/L) }\end{array}$ & $\begin{array}{c}\text { REC } \\
(U G / L)\end{array}$ & $\begin{array}{c}\mathrm{REC} \\
(\mathrm{UG} / \mathrm{L})\end{array}$ & $\begin{array}{l}\text { TOTAL } \\
\text { (UG/L) }\end{array}$ & $\begin{array}{l}\text { TOTAL } \\
\text { (UG/L) }\end{array}$ & $\begin{array}{c}\text { REC } \\
\text { (UG/L) }\end{array}$ & $\begin{array}{l}\text { TOTAL } \\
(\mathrm{UG} / \mathrm{L})\end{array}$ & $\begin{array}{c}\text { REC } \\
\langle U G / L\rangle\end{array}$ & $\begin{array}{l}\text { TOTAL } \\
\text { (UG/L) }\end{array}$ \\
\hline
\end{tabular}

\begin{tabular}{|c|c|c|c|c|c|c|c|c|c|c|c|c|}
\hline 07.07 & $<4.0$ & $<4.0$ & $<4.0$ & $<5.0$ & $<4.0$ & $<4.0$ & $<4.0$ & $<4.0$ & $<4.0$ & $<5.0$ & $<4.0$ & $<4.0$ \\
\hline \multicolumn{13}{|l|}{ NOV } \\
\hline $02-03$ & $<0.2$ & $<0.2$ & $<0.2$ & $<5.0$ & $<0.20$ & $<0.20$ & $<0.2$ & $<0.2$ & $<0.2$ & $<5.0$ & $<0.20$ & $<0.2$ \\
\hline \multicolumn{13}{|l|}{ NOV } \\
\hline 09.09 & $<1.0$ & $<1.0$ & $<1.0$ & $<5.0$ & $<1.0$ & $<1.0$ & $<1.0$ & $<1.0$ & $<1.0$ & $<5.0$ & $<1.0$ & $<1.0$ \\
\hline
\end{tabular}


Table 9. Water-quality data for storm-sewer outfall stations, Texas Department of Transportation, Dallas and Fort Worth Districts, 1993-94-Continued

08048920 - DEER CREEK OUTFALL AT I-35W, FORT WORTH, TX (WY 1995)-Continued

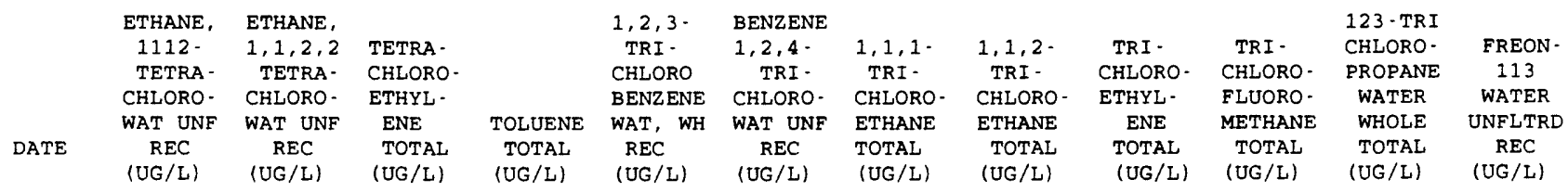

\begin{tabular}{|c|c|c|c|c|c|c|c|c|c|c|c|c|}
\hline \multicolumn{13}{|l|}{ OCT 1994} \\
\hline 07.07 & $<4.0$ & $<4.0$ & $<4.0$ & $<4.0$ & $<4.0$ & $<5.0$ & $<4.0$ & $<4.0$ & $<4.0$ & $<4.0$ & $<4.0$ & $<4.0$ \\
\hline \multicolumn{13}{|l|}{ NoV } \\
\hline 02.03 & $<0.2$ & $<0.2$ & $<0.2$ & $<0.2$ & $<0.20$ & $<5.0$ & $<0.2$ & $<0.2$ & $<0.2$ & $<0.2$ & $<0.2$ & $<0.2$ \\
\hline \multicolumn{13}{|l|}{ NoV } \\
\hline $09-09$ & $<1.0$ & $<1.0$ & $<1.0$ & $<1.0$ & $<1.0$ & $<5.0$ & $<1.0$ & $<1.0$ & $<1.0$ & $<1.0$ & $<1.0$ & $<1.0$ \\
\hline
\end{tabular}

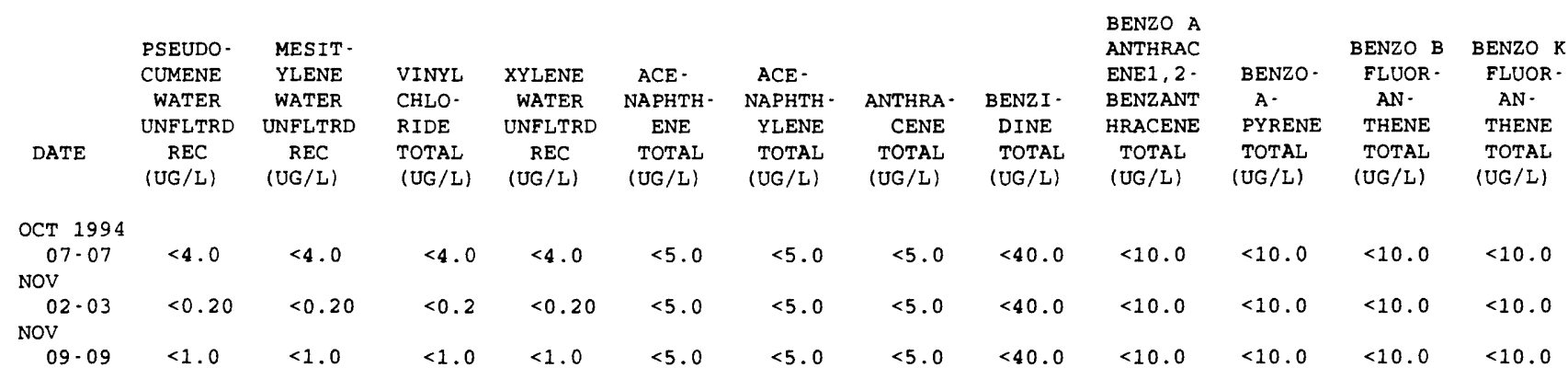

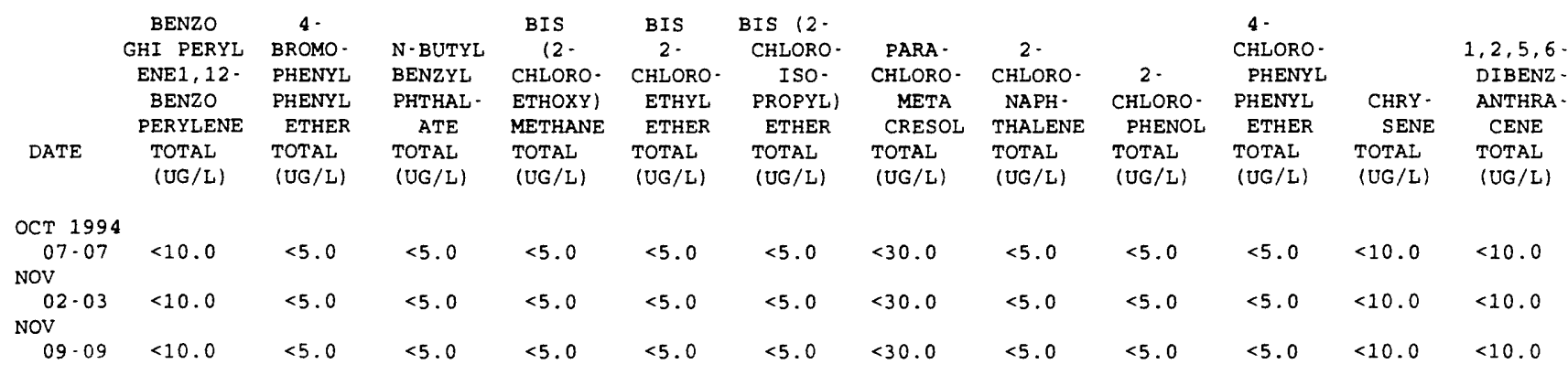

\begin{tabular}{|c|c|c|c|c|c|c|c|c|c|c|c|c|}
\hline DATE & $\begin{array}{c}3,3 \% \\
\text { DI - } \\
\text { CHLORO- } \\
\text { BENZI- } \\
\text { DINE } \\
\text { TOTAL } \\
\text { (UG/L) }\end{array}$ & $\begin{array}{l}2,4 \text {-DI - } \\
\text { CHLORO- } \\
\text { PHENOL } \\
\text { TOTAL } \\
\text { (UG/L) }\end{array}$ & $\begin{array}{l}\text { DIETHYL } \\
\text { PHTHAL. } \\
\text { ATE } \\
\text { TOTAL } \\
\text { (UG/L) }\end{array}$ & $\begin{array}{c}\text { DI - } \\
\text { METHYL } \\
\text { PHTHAL- } \\
\text { ATE } \\
\text { TOTAL } \\
\text { (UG/L) }\end{array}$ & $\begin{array}{l}2,4-D I- \\
\text { METHYL - } \\
\text { PHENOL } \\
\text { TOTAL } \\
(U G / L)\end{array}$ & $\begin{array}{c}\text { DI -N - } \\
\text { BUTYL } \\
\text { PHTHAL- } \\
\text { ATE } \\
\text { TOTAL } \\
\text { (UG/L) }\end{array}$ & $\begin{array}{c}4,6- \\
\text { DINITRO- } \\
\text { ORTHO- } \\
\text { CRESOL } \\
\text { TOTAL } \\
\text { (UG/L) }\end{array}$ & $\begin{array}{c}2,4,- \\
\text { DI- } \\
\text { NITRO- } \\
\text { PHENOL } \\
\text { TOTAL } \\
(U G / L)\end{array}$ & $\begin{array}{l}2,4 \cdot \mathrm{DI}- \\
\text { NITRO- } \\
\text { TOLUENE } \\
\text { TOTAL } \\
\text { (UG } / \mathrm{L} \text { ) }\end{array}$ & $\begin{array}{l}2,6-\mathrm{DI}- \\
\text { NITRO- } \\
\text { TOLUENE } \\
\text { TOTAL } \\
\text { (UG/L) }\end{array}$ & $\begin{array}{l}\text { DI - N - } \\
\text { OCTYL } \\
\text { PHTHAL- } \\
\text { ATE } \\
\text { TOTAL } \\
\text { (UG/L) }\end{array}$ & $\begin{array}{l}\text { BIS }(2 \cdot \\
\text { ETHYL } \\
\text { HEXYL) } \\
\text { PHTHAL - } \\
\text { ATE } \\
\text { TOTAL } \\
\text { (UG/L) }\end{array}$ \\
\hline CT 1994 & & & & & & & & & & & & \\
\hline $\begin{array}{l}07-07 \\
\text { Nov }\end{array}$ & $<20.0$ & $<5.0$ & $<5.0$ & $<5.0$ & $<5.0$ & $<5.0$ & $<30.0$ & $<20.0$ & $<5.0$ & $<5.0$ & $<10.0$ & $<5.0$ \\
\hline $\begin{array}{l}02 \cdot 03 \\
\text { NOV }\end{array}$ & $<20.0$ & $<5.0$ & $<5.0$ & $<5.0$ & $<5.0$ & $<5.0$ & $<30.0$ & $<20.0$ & $<5.0$ & $<5.0$ & $<10.0$ & $<5.0$ \\
\hline 09.09 & $<20.0$ & $<5.0$ & $<5.0$ & $<5.0$ & $<5.0$ & $<5.0$ & $<30.0$ & $<20.0$ & $<5.0$ & $<5.0$ & $<10.0$ & $<5.0$ \\
\hline
\end{tabular}


Table 9. Water-quality data for storm-sewer outfall stations, Texas Department of Transportation, Dallas and Fort Worth Districts, 1993-94-Continued

08048920 - DEER CREEK OUTFALL AT I-35W, FORT WORTH, TX (WY 1995)-CONtinued

\begin{tabular}{|c|c|c|c|c|c|c|c|c|c|c|c|c|}
\hline DATE & $\begin{array}{l}\text { FLUOR - } \\
\text { ANTHENE } \\
\text { TOTAL } \\
(U G / L)\end{array}$ & $\begin{array}{c}\text { FLUOR - } \\
\text { ENE } \\
\text { TOTAL } \\
\text { (UG/L) }\end{array}$ & $\begin{array}{l}\text { HEXA- } \\
\text { CHLORO- } \\
\text { BENZENE } \\
\text { TOTAL } \\
\text { (UG/L) }\end{array}$ & $\begin{array}{l}\text { HEXA- } \\
\text { CHLORO- } \\
\text { CYCLO- } \\
\text { PENT- } \\
\text { ADIENE } \\
\text { TOTAL } \\
\text { (UG/L) }\end{array}$ & $\begin{array}{l}\text { HEXA- } \\
\text { CHLORO- } \\
\text { ETHANE } \\
\text { TOTAL } \\
\text { (UG/L) }\end{array}$ & $\begin{array}{c}\text { INDENO } \\
(1,2,3- \\
\text { CD) } \\
\text { PYRENE } \\
\text { TOTAL } \\
\text { (UG/L) }\end{array}$ & $\begin{array}{l}\text { ISO- } \\
\text { PHORONE } \\
\text { TOTAL } \\
\text { (UG/L) }\end{array}$ & $\begin{array}{l}\text { NITRO- } \\
\text { BENZENE } \\
\text { TOTAL } \\
\text { (UG/L) }\end{array}$ & $\begin{array}{r}\text { N-NITRO- } \\
\text { SODI - } \\
\text { METHY - } \\
\text { LAMINE } \\
\text { TOTAL } \\
(\text { UG/L) }\end{array}$ & $\begin{array}{l}2- \\
\text { NITRO- } \\
\text { PHENOL } \\
\text { TOTAL } \\
(U G / L)\end{array}$ & $\begin{array}{c}4- \\
\text { NITRO- } \\
\text { PHENOL } \\
\text { TOTAL } \\
(\text { UG/L) }\end{array}$ & $\begin{array}{c}\text { N- } \\
\text { NITRO- } \\
\text { SODI-N- } \\
\text { PROPYL- } \\
\text { AMINE } \\
\text { TOTAL } \\
(U G / L)\end{array}$ \\
\hline \multicolumn{13}{|l|}{ OCT 1994} \\
\hline $\begin{array}{l}07-07 \\
\text { NOV }\end{array}$ & $<5.0$ & $<5.0$ & $<5.0$ & $<5.0$ & $<5.0$ & $<10.0$ & $<5.0$ & $<5.0$ & $<5.0$ & $<5.0$ & $<30.0$ & $<5.0$ \\
\hline $\begin{array}{l}02 \cdot 03 \\
\text { NoV }\end{array}$ & $<5.0$ & $<5.0$ & $<5.0$ & $<5.0$ & $<5.0$ & $<10.0$ & $<5.0$ & $<5.0$ & $<5.0$ & $<5.0$ & $<30.0$ & $<5.0$ \\
\hline 09.09 & $<5.0$ & $<5.0$ & $<5.0$ & $<5.0$ & $<5.0$ & $<10.0$ & $<5.0$ & $<5.0$ & $<5.0$ & $<5.0$ & $<30.0$ & $<5.0$ \\
\hline DATE & $\begin{array}{l}\text { N-NITRO- } \\
\text { SODI - } \\
\text { PHENY - } \\
\text { LAMINE } \\
\text { TOTAL } \\
\text { (UG/L) }\end{array}$ & $\begin{array}{l}\text { PENTA- } \\
\text { CHLORO- } \\
\text { PHENOL } \\
\text { TOTAL } \\
\text { (UG/L) }\end{array}$ & $\begin{array}{l}\text { PHENAN - } \\
\text { THRENE } \\
\text { TOTAL } \\
\text { (UG/L) }\end{array}$ & $\begin{array}{c}\text { PHENOL } \\
\text { (C6H- } \\
5 \mathrm{OH}) \\
\text { TOTAL } \\
\text { (UG/L) }\end{array}$ & $\begin{array}{c}\text { PYRENE } \\
\text { TOTAL } \\
\text { (UG/L) }\end{array}$ & $\begin{array}{c}2,4,6- \\
\text { TRI - } \\
\text { CHLORO- } \\
\text { PHENOL } \\
\text { TOTAL } \\
\text { (UG/L) }\end{array}$ & $\begin{array}{c}\text { ALDRIN, } \\
\text { TOTAL } \\
\text { (UG/L) }\end{array}$ & $\begin{array}{c}\text { P, } P^{\prime} \\
\text { DDT, } \\
\text { TOTAL } \\
\text { (UG / L) }\end{array}$ & $\begin{array}{l}\text { ALPHA } \\
\text { BHC } \\
\text { TOTAL } \\
\text { (UG/L) }\end{array}$ & $\begin{array}{c}\text { BETA } \\
\text { BENZENE } \\
\text { HEXA- } \\
\text { CHLOR- } \\
\text { IDE } \\
\text { TOTAL } \\
\text { (UG/L) }\end{array}$ & $\begin{array}{l}\text { DELTA } \\
\text { BENZENE } \\
\text { HEXA- } \\
\text { CHLOR- } \\
\text { IDE } \\
\text { TOTAL } \\
\text { (UG/L) }\end{array}$ & $\begin{array}{l}\text { IINDANE } \\
\text { TOTAL } \\
\text { (UG/L) }\end{array}$ \\
\hline \multicolumn{13}{|l|}{ OCT 1994} \\
\hline \multicolumn{13}{|l|}{ NOV } \\
\hline \multicolumn{13}{|l|}{ NOV } \\
\hline $09-09$ & $<5.0$ & $<30.0$ & $<5.0$ & $<5.0$ & $<5.0$ & $<20.0$ & $<0.040$ & $<0.10$ & $<0.03$ & $<0.03$ & $<0.09$ & $<0.030$ \\
\hline
\end{tabular}

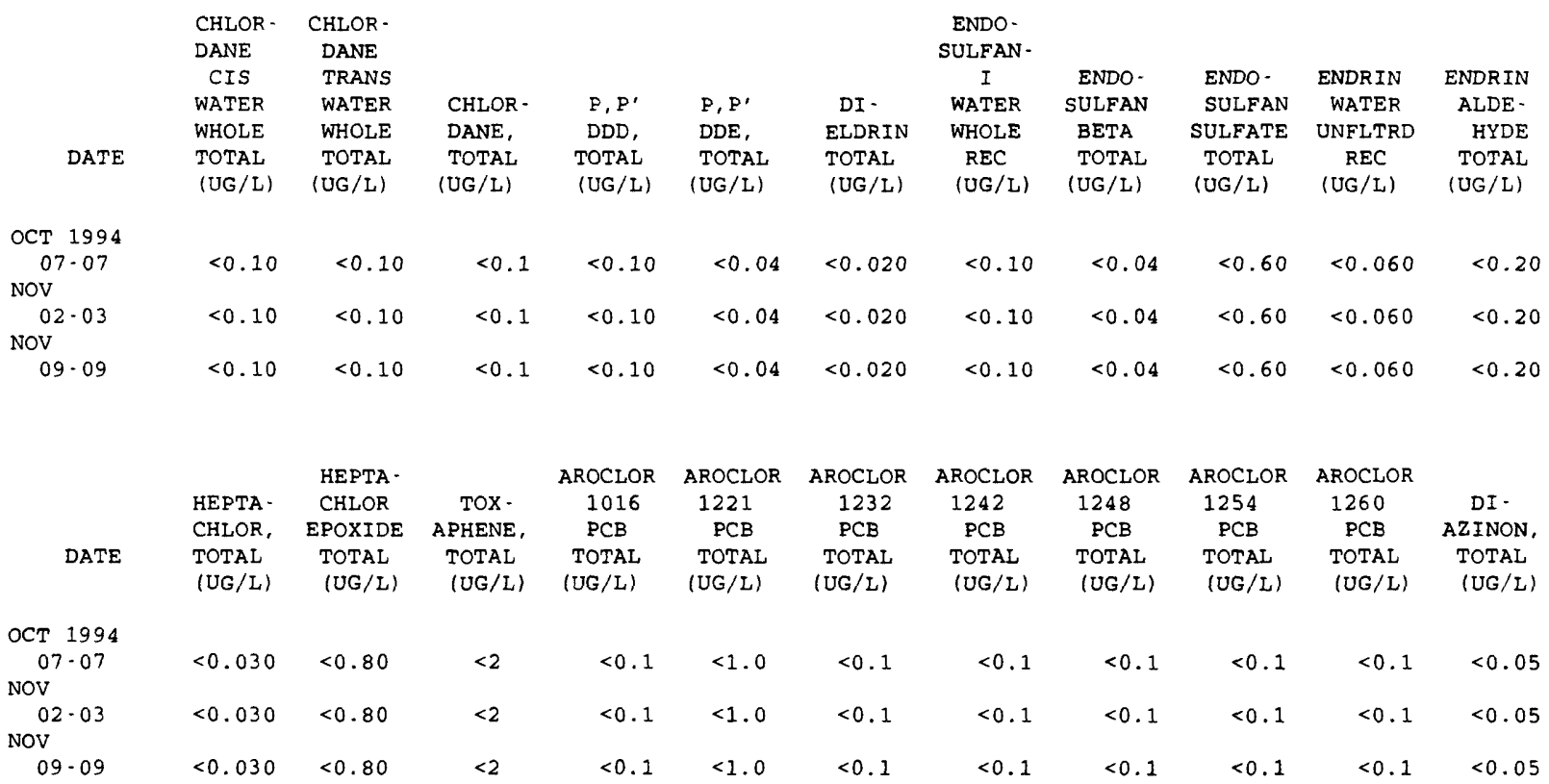


Table 9. Water-quality data for storm-sewer outfall stations, Texas Department of Transportation, Dallas and Fort Worth Districts, 1993-94-Continued

08049860 - MOUNTAIN CREEK OUTFALL AT I-20, DUNCANVILLE, TX (WY 1994)

\begin{tabular}{|c|c|c|c|c|c|c|c|c|c|c|c|c|}
\hline DATE & TIME & $\begin{array}{l}\text { PRECIP- } \\
\text { ITATION } \\
\text { TOTAL } \\
\text { INCHES/ } \\
\text { STORM }\end{array}$ & $\begin{array}{l}\text { ELAPSED } \\
\text { TIME } \\
\text { OF } \\
\text { STORM } \\
\text { (HOURS) }\end{array}$ & $\begin{array}{l}\text { STORM } \\
\text { WATER } \\
\text { FLOW } \\
\text { (MGD) }\end{array}$ & $\begin{array}{l}\text { SPE- } \\
\text { CIFIC } \\
\text { CON- } \\
\text { DUCT- } \\
\text { ANCE } \\
\text { (US/CM) }\end{array}$ & $\begin{array}{c}\text { SPE - } \\
\text { CIFIC } \\
\text { CON- } \\
\text { DUCT- } \\
\text { ANCE } \\
\text { LAB } \\
\text { (US/CM) }\end{array}$ & $\begin{array}{c}\text { PH } \\
\text { WATER } \\
\text { WHOLE } \\
\text { FIELD } \\
\text { (STAND- } \\
\text { ARD } \\
\text { UNITS) }\end{array}$ & $\begin{array}{c}\text { PH } \\
\text { WATER } \\
\text { WHOLE } \\
\text { LAB } \\
\text { (STAND- } \\
\text { ARD } \\
\text { UNITS) }\end{array}$ & $\begin{array}{c}\text { TEMPER - } \\
\text { ATURE } \\
\text { WATER } \\
\text { (DEG C) }\end{array}$ & $\begin{array}{l}\text { OXYGEN } \\
\text { DEMAND, } \\
\text { CHEM - } \\
\text { ICAL } \\
\text { (HIGH } \\
\text { LEVEL) } \\
\text { (MG/L) }\end{array}$ & $\begin{array}{l}\text { OXYGEN } \\
\text { DEMAND, } \\
\text { BIO- } \\
\text { CHEM- } \\
\text { ICAL, } \\
5 \text { DAY } \\
\text { (MG/L) }\end{array}$ & $\begin{array}{l}\text { COLI - } \\
\text { FORM, } \\
\text { FECAL, } \\
0.7 \\
\text { UM-MF } \\
\text { (COLS. } 1 \\
100 \mathrm{ML} \text { ) }\end{array}$ \\
\hline \multicolumn{13}{|l|}{ DEC 1993} \\
\hline \multicolumn{13}{|l|}{ JAN 1994} \\
\hline 11-11 & 0550 & 0.23 & 3.0 & 0.06 & 2240 & 1100 & 8.2 & 7.4 & 12.0 & 140 & $>9.2$ & K100 \\
\hline \multicolumn{13}{|l|}{ FEB } \\
\hline $28-28$ & 1800 & 0.29 & 2.8 & 0.14 & 886 & 664 & 7.8 & 7.6 & 11.5 & 87 & 8.4 & 2200 \\
\hline \multicolumn{13}{|l|}{ MAR } \\
\hline $08-08$ & 1145 & 0.20 & 3.8 & 0.08 & 905 & 906 & 7.5 & 7.5 & 16.0 & 56 & 4.3 & 1700 \\
\hline \multicolumn{13}{|l|}{ APR } \\
\hline $11-11$ & 1320 & 0.66 & 1.8 & 0.16 & 252 & 490 & 8.6 & 7.4 & 19.0 & 110 & 6.0 & 8000 \\
\hline \multicolumn{13}{|l|}{ MAY } \\
\hline 09.09 & 1230 & 0.56 & 4.5 & 0.28 & 513 & 546 & 7.5 & 7.6 & 20.0 & 49 & 6.5 & 11000 \\
\hline \multicolumn{13}{|l|}{ AUG } \\
\hline $20-20$ & 1820 & 0.85 & 1.7 & 0.15 & 351 & 443 & 7.6 & 7.4 & 29.0 & 120 & 9.2 & -- \\
\hline
\end{tabular}

\begin{tabular}{|c|c|c|c|c|c|c|c|c|c|c|c|c|}
\hline & $\begin{array}{r}\text { STREP - } \\
\text { TOCOCCI }\end{array}$ & HARD - & $\begin{array}{l}\text { HARD - } \\
\text { NESS }\end{array}$ & $\begin{array}{l}\text { ALKA- } \\
\text { LINITY }\end{array}$ & $\begin{array}{l}\text { SOLIDS, } \\
\text { SUM OF }\end{array}$ & $\begin{array}{l}\text { RESIDUE } \\
\text { TOTAL }\end{array}$ & $\begin{array}{l}\text { SOLIDS, } \\
\text { RESIDUE }\end{array}$ & & MAGNE - & & & SODIUM \\
\hline & FECAL, & NESS & NONCARB & WAT DIS & CONSTI - & AT 105 & AT 180 & CALCIUM & SIUM, & SODIUM, & & $A D-$ \\
\hline & $\begin{array}{l}\text { KF AGAR } \\
\text { (COLS. }\end{array}$ & $\begin{array}{l}\text { TOTAL } \\
\text { (MG/L }\end{array}$ & $\begin{array}{l}\text { DISSOLV } \\
\text { FLD. AS }\end{array}$ & $\begin{array}{l}\text { FIX END } \\
\text { FIELD }\end{array}$ & $\begin{array}{c}\text { TUENTS, } \\
\text { DIS - }\end{array}$ & $\begin{array}{l}\text { DEG . C, } \\
\text { SUS - }\end{array}$ & $\begin{array}{l}\text { DEG. C } \\
\text { DIS - }\end{array}$ & $\begin{array}{l}\text { DIS - } \\
\text { SOLVED }\end{array}$ & $\begin{array}{l}\text { DIS - } \\
\text { SOLVED }\end{array}$ & $\begin{array}{l}\text { DIS - } \\
\text { SOLVED }\end{array}$ & & $\begin{array}{l}\text { SORP - } \\
\text { TION }\end{array}$ \\
\hline & $\begin{array}{c}\text { PER } \\
100 \mathrm{ML})\end{array}$ & $\begin{array}{c}\text { AS } \\
\text { (ACO3) }\end{array}$ & $\begin{array}{r}\mathrm{CACO} 3 \\
(\mathrm{MG} / \mathrm{L})\end{array}$ & $\begin{array}{l}\mathrm{CACO} 3 \\
(\mathrm{MG} / \mathrm{L})\end{array}$ & $\begin{array}{l}\text { SOLVED } \\
\text { (MG/L) }\end{array}$ & $\begin{array}{l}\text { PENDED } \\
\text { (MG/L) }\end{array}$ & $\begin{array}{l}\text { SOLVED } \\
\text { (MG/L) }\end{array}$ & $\begin{array}{c}(\mathrm{MG} / \mathrm{L} \\
\mathrm{AS} \text { CA) }\end{array}$ & $\begin{array}{l}\text { (MG/L } \\
\text { AS MG) }\end{array}$ & $\begin{array}{l}\text { (MG/L } \\
\text { AS NA) }\end{array}$ & $\begin{array}{l}\text { SODIUM } \\
\text { PERCENT }\end{array}$ & RATIO \\
\hline
\end{tabular}

\begin{tabular}{|c|c|c|c|c|c|c|c|c|c|c|c|c|}
\hline DEC 1993 & & & & & & & & & & & & \\
\hline 02.03 & 28000 & 290 & 200 & 86 & 659 & 136 & 699 & 80 & 21 & 92 & 41 & 2 \\
\hline JAN 1994 & & & & & & & & & & & & \\
\hline $11-11$ & 1200 & 260 & 200 & 61 & 715 & 102 & 750 & 72 & 20 & 110 & 47 & 3 \\
\hline FEB & & & & & & & & & & & & \\
\hline $28-28$ & 5100 & 200 & 150 & 44 & 444 & 386 & 456 & 55 & 14 & 57 & 38 & 2 \\
\hline MAR & & & & & & & & & & & & \\
\hline 08.08 & 5800 & 270 & 190 & 84 & 599 & 73 & 647 & 77 & 20 & 82 & 39 & 2 \\
\hline APR & & & & & & & & & & & & \\
\hline $11-11$ & 26000 & 130 & 100 & 26 & 342 & 211 & 309 & 38 & 7.8 & 37 & 38 & 1 \\
\hline MAY & & & & & & & & & & & & \\
\hline $09-09$ & 68000 & 170 & 110 & 56 & 349 & 58 & 372 & 48 & 11 & 42 & 35 & 1 \\
\hline AUG & & & & & & & & & & & & \\
\hline $20-20$ & 18000 & 85 & 55 & 30 & 263 & 164 & 240 & 24 & 6.1 & 39 & 49 & 2 \\
\hline DATE & $\begin{array}{l}\text { POTAS - } \\
\text { SIUM, } \\
\text { DIS - } \\
\text { SOLVED } \\
\text { (MG/L } \\
\text { AS K) }\end{array}$ & $\begin{array}{l}\text { SULFATE } \\
\text { DIS - } \\
\text { SOLVED } \\
\text { (MG/L } \\
\text { AS SO4) }\end{array}$ & $\begin{array}{l}\text { CHLO- } \\
\text { RIDE, } \\
\text { DIS- } \\
\text { SOLVED } \\
\text { (MG/L } \\
\text { AS CL) }\end{array}$ & $\begin{array}{l}\text { NITRO- } \\
\text { GEN, } \\
\text { NO2+NO3 } \\
\text { TOTAL } \\
\text { (MG/L } \\
\text { AS N) }\end{array}$ & $\begin{array}{l}\text { NITRO- } \\
\text { GEN, AM- } \\
\text { MONIA + } \\
\text { ORGANIC } \\
\text { TOTAL } \\
\text { (MG/L } \\
\text { AS N) }\end{array}$ & $\begin{array}{l}\text { PHOS - } \\
\text { PHORUS } \\
\text { TOTAL } \\
\text { (MG/L } \\
\text { AS P) }\end{array}$ & $\begin{array}{c}\text { PHOS - } \\
\text { PHORUS } \\
\text { DIS - } \\
\text { SOLVED } \\
\text { (MG/L } \\
\text { AS P) }\end{array}$ & $\begin{array}{l}\text { ANTIMONY } \\
\text { TOTAL } \\
\text { (UG/L } \\
\text { AS SB) }\end{array}$ & $\begin{array}{c}\text { ARSENIC } \\
\text { TOTAL } \\
\text { (UG/L } \\
\text { AS AS) }\end{array}$ & $\begin{array}{l}\text { BERYL - } \\
\text { LIUM, } \\
\text { TOTAL } \\
\text { RECOV - } \\
\text { ERABLE } \\
\text { (UG/L } \\
\text { AS BE) }\end{array}$ & $\begin{array}{l}\text { CADMIUM } \\
\text { TOTAL } \\
\text { RECOV- } \\
\text { ERABLE } \\
\text { (UG/L } \\
\text { AS CD) }\end{array}$ & $\begin{array}{l}\text { CHRO- } \\
\text { MIUM, } \\
\text { TOTAL } \\
\text { RECOV- } \\
\text { ERABLE } \\
\text { (UG/L } \\
\text { AS CR) }\end{array}$ \\
\hline
\end{tabular}

\begin{tabular}{|c|c|c|c|c|c|c|c|c|c|c|c|c|}
\hline \multicolumn{13}{|l|}{ DEC 1993} \\
\hline 02.03 & 5.7 & 370 & 23 & 0.490 & 1.8 & 0.200 & 0.030 & $<10.0$ & 2 & $<10$ & $<1$ & 3 \\
\hline \multicolumn{13}{|l|}{ JAN 1994} \\
\hline $11-11$ & 4.4 & 420 & 11 & 1.30 & 2.9 & 0.250 & 0.050 & $<10.0$ & 2 & $<10$ & $<1$ & 5 \\
\hline \multicolumn{13}{|l|}{ FEB } \\
\hline $28-28$ & 2.8 & 260 & 6.6 & 0.320 & 2.1 & 0.430 & 0.020 & $<10.0$ & 5 & $<10$ & $<1$ & 12 \\
\hline \multicolumn{13}{|l|}{ MAR } \\
\hline $08-08$ & 3.1 & 350 & 10 & 0.400 & 1.2 & 0.090 & 0.020 & $<10.0$ & $<1$ & $<10$ & $<1$ & 3 \\
\hline \multicolumn{13}{|l|}{ APR } \\
\hline $11-11$ & 2.6 & 170 & 36 & 0.310 & 3.2 & 0.530 & 0.050 & $<10.0$ & 4 & $<10$ & $<1$ & 18 \\
\hline \multicolumn{13}{|l|}{ MAY } \\
\hline 09.09 & 3.4 & 200 & 3.6 & 0.160 & 1.8 & 0.110 & 0.010 & $<10.0$ & $<1$ & $<10$ & $<1$ & 3 \\
\hline \multicolumn{13}{|l|}{ AUG } \\
\hline $20-20$ & 3.4 & 110 & 5.2 & 0.830 & 3.6 & 0.560 & 0.210 & $<10.0$ & 3 & $<10$ & $<1$ & 9 \\
\hline
\end{tabular}


Table 9. Water-quality data for storm-sewer outfall stations, Texas Department of Transportation, Dallas and Fort Worth Districts, 1993-94-Continued

08049860 - MOUNTAIN CREEK OUTFALL AT I-20, DUNCANVILLE, TX (WY 1994)-Continued

\begin{tabular}{|c|c|c|c|c|c|c|c|c|c|c|c|c|}
\hline DATE & $\begin{array}{l}\text { COPPER, } \\
\text { TOTAL } \\
\text { RECOV- } \\
\text { ERABLE } \\
\text { (UG/L } \\
\text { AS CU) }\end{array}$ & $\begin{array}{l}\text { CYANIDE, } \\
\text { TOTAL } \\
\text { EPA } \\
\text { (MG/L } \\
\text { AS CN) }\end{array}$ & $\begin{array}{c}\text { CYANIDE } \\
\text { TOTAL } \\
\text { (MG/L } \\
\text { AS CN) }\end{array}$ & $\begin{array}{l}\text { LEAD, } \\
\text { TOTAL } \\
\text { RECOV- } \\
\text { ERABLE } \\
\text { (UG/L } \\
\text { AS PB) }\end{array}$ & $\begin{array}{l}\text { MERCURY } \\
\text { TOTAL } \\
\text { RECOV- } \\
\text { ERABLE } \\
\text { (UG/L } \\
\text { AS HG) }\end{array}$ & $\begin{array}{l}\text { NICKEL, } \\
\text { TOTAL } \\
\text { RECOV- } \\
\text { ERABLE } \\
\text { (UG/L } \\
\text { AS NI) }\end{array}$ & $\begin{array}{l}\text { SELE- } \\
\text { NIUM, } \\
\text { TOTAL } \\
\text { (UG /L } \\
\text { AS SE) }\end{array}$ & $\begin{array}{c}\text { SILVER, } \\
\text { TOTAL } \\
\text { RECOV- } \\
\text { ERABLE } \\
\text { (UG/L } \\
\text { AS AG) }\end{array}$ & $\begin{array}{c}\text { SILVER, } \\
\text { TOTAL } \\
\text { RECOV- } \\
\text { ERABLE } \\
\text { EPA } \\
\text { (UG/L } \\
\text { AS AG) }\end{array}$ & $\begin{array}{l}\text { THAL- } \\
\text { LIUM, } \\
\text { TOTAL } \\
\text { (UG/L } \\
\text { AS TL) }\end{array}$ & $\begin{array}{l}\text { ZINC, } \\
\text { TOTAL } \\
\text { RECOV- } \\
\text { ERABLE } \\
\text { (UG/L } \\
\text { AS ZN) }\end{array}$ & $\begin{array}{r}\text { CARBO } \\
\text { ORGAN } \\
\text { TOTA } \\
\text { (MG/ } \\
\text { AS C }\end{array}$ \\
\hline EC 1993 & & & & & & & & & & & & \\
\hline $\begin{array}{r}02-03 \\
\text { JAN } 1994\end{array}$ & 9 & $<0.010$ & $<0.010$ & 32 & $<0.10$ & 11 & $<1$ & $<1$ & $<0.500$ & $<10$ & 110 & 25 \\
\hline${ }_{F E B}^{11-11}$ & 13 & $<0.010$ & $<0.010$ & 35 & $<0.10$ & 21 & 1 & $<1$ & $<0.500$ & $<5$ & 190 & 38 \\
\hline $\begin{array}{l}28-28 \\
\text { MAR }\end{array}$ & 19 & $<0.010$ & $<0.010$ & 90 & $<0.10$ & 26 & $<1$ & $<1$ & $<0.500$ & $<20$ & 200 & 29 \\
\hline $\begin{array}{l}08-08 \\
\text { APR }\end{array}$ & 7 & $<0.010$ & $<0.010$ & 12 & $<0.10$ & 8 & $<1$ & $<1$ & $<0.500$ & $<5$ & 80 & 18 \\
\hline${ }_{\text {MAY }}^{11-11}$ & 20 & $<0.010$ & $<0.010$ & 120 & $<0.10$ & 47 & $<1$ & $<1$ & $<0.500$ & $<10$ & 260 & 31 \\
\hline $\begin{array}{l}09-09 \\
\text { AUG }\end{array}$ & 6 & $<0.010$ & $<0.010$ & 10 & $<0.10$ & 5 & $<1$ & $<1$ & $<0.500$ & $<5$ & 40 & 16 \\
\hline $20-20$ & 11 & $<0.010$ & $<0.010$ & 140 & $<0.10$ & 32 & $<1$ & $<1$ & $<0.500$ & $<5$ & 210 & \\
\hline
\end{tabular}

\begin{tabular}{|c|c|c|c|c|c|c|c|c|c|c|c|c|}
\hline & OIL AND & & & & & & METHANE & & & BENZENE & BENZENE & \\
\hline & GREASE, & & & & & BROMO - & BROMO & & BENZENE & SEC & TERT. & \\
\hline & TOTAL & & & & & BENZENE & CHLORO- & & $\mathrm{N}$-BUTYL & BUTYL - & BUTYL - & TETRA - \\
\hline & RECOV. & & ACRO - & ACRYLO- & & WATER, & WAT & BROMO - & WATER & WATER & WATER & CHLO- \\
\hline & GRAVI - & PHENOLS & LEIN & NITRILE & BENZENE & WHOLE, & UNFLTRD & FORM & UNFLTRD & UNFLTRD & UNFLTRD & RIDE \\
\hline & $\begin{array}{l}\text { METRIC } \\
\text { (MG/L) }\end{array}$ & $\begin{array}{l}\text { TOTAL } \\
\text { (UG/L) }\end{array}$ & $\begin{array}{l}\text { TOTAL } \\
\text { (UG/L) }\end{array}$ & $\begin{array}{l}\text { TOTAL } \\
\text { (UG/L) }\end{array}$ & $\begin{array}{l}\text { TOTAL } \\
\text { (UG/L) }\end{array}$ & $\begin{array}{l}\text { TOTAL } \\
\text { (UG } / L \text { ) }\end{array}$ & $\begin{array}{c}\text { REC } \\
(U G / L)\end{array}$ & $\begin{array}{l}\text { TOTAL } \\
\text { (UG/L) }\end{array}$ & $\begin{array}{c}\text { REC } \\
(U G / L)\end{array}$ & $\begin{array}{c}\text { REC } \\
(U G / L)\end{array}$ & $\begin{array}{c}\text { REC } \\
(U G / L)\end{array}$ & $\begin{array}{l}\text { TOTAL } \\
\text { (UG/L) }\end{array}$ \\
\hline
\end{tabular}

DEC 1993

\begin{tabular}{|c|c|c|c|c|c|c|c|c|c|c|c|c|}
\hline $\begin{array}{c}02-03 \\
\text { JAN }\end{array}$ & 6 & 10 & $<20$ & $<20$ & $<0.2$ & $<0.2$ & $<0.20$ & $<0.2$ & $<0.20$ & $<0.20$ & $<0.20$ & $<0.2$ \\
\hline $11 \cdot 11$ & $<1$ & 3 & $<20$ & $<20$ & $<0.2$ & $<0.2$ & $<0.20$ & $<0.2$ & $<0.20$ & $<0.20$ & $<0.20$ & $<0.2$ \\
\hline FEB & & & & & & & & & & & & \\
\hline $28-28$ & $<1$ & 1 & $<20$ & $<20$ & $<0.2$ & $<0.2$ & $<0.20$ & $<0.2$ & $<0.20$ & $<0.20$ & $<0.20$ & $<0.2$ \\
\hline MAR 08.08 & & & & & & & & & & & & \\
\hline APR & 2 & 2 & -20 & -20 & $<0.2$ & $<0.2$ & $<0.20$ & $<0.2$ & $<0.20$ & $<0.20$ & $<0.20$ & $<0.2$ \\
\hline $\begin{array}{l}11-11 \\
\text { MAY }\end{array}$ & $<1$ & 10 & $<20$ & $<20$ & $<0.2$ & $<0.2$ & $<0.20$ & $<0.2$ & $<0.20$ & $<0.20$ & $<0.20$ & $<0.2$ \\
\hline $\begin{array}{l}09 \cdot 09 \\
\text { AUG }\end{array}$ & $<1$ & 5 & $<20$ & $<20$ & $<0.2$ & $<0.2$ & $<0.20$ & $<0.2$ & $<0.20$ & $<0.20$ & $<0.20$ & $<0.2$ \\
\hline $20-20$ & $<1$ & 14 & $<20$ & $<20$ & $<0.2$ & $<0.2$ & $<0.20$ & $<0.2$ & $<0.20$ & $<0.20$ & $<0.20$ & $<0.2$ \\
\hline
\end{tabular}

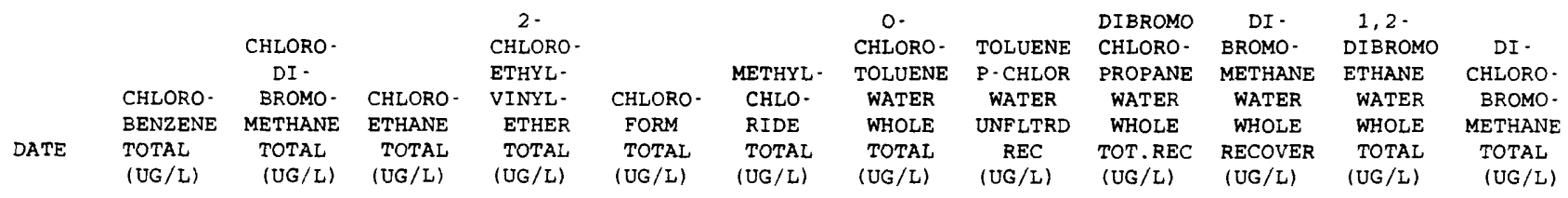

\begin{tabular}{|c|c|c|c|c|c|c|c|c|c|c|c|c|}
\hline DEC 1993 & & & & & & & & & & & & \\
\hline $02-03$ & $<0.20$ & $<0.2$ & $<0.2$ & $<1.0$ & $<0.2$ & 0.2 & $<0.2$ & $<0.20$ & $<1.0$ & $<0.2$ & $<0.2$ & $<0.2$ \\
\hline JAN 1994 & & & & & & & & & & & & \\
\hline $11-11$ & $<0.20$ & $<0.2$ & $<0.2$ & $<1.0$ & $<0.2$ & $<0.2$ & $<0.2$ & $<0.20$ & $<1.0$ & $<0.2$ & $<0.2$ & $<0.2$ \\
\hline FEB & & & & & & & & & & & & \\
\hline $28 \cdot 28$ & $<0.20$ & $<0.2$ & $<0.2$ & $<1.0$ & $<0.2$ & 0.3 & $<0.2$ & $<0.20$ & $<1.0$ & $<0.2$ & $<0.2$ & $<0.2$ \\
\hline MAR & & & & & & & & & & & & \\
\hline $08-08$ & $<0.20$ & $<0.2$ & $<0.2$ & $<1.0$ & $<0.2$ & 4.0 & $<0.2$ & $<0.20$ & $<1.0$ & $<0.2$ & $<0.2$ & $<0.2$ \\
\hline APR & & & & & & & & & & & & \\
\hline $11-11$ & $<0.20$ & $<0.2$ & $<0.2$ & $<1.0$ & $<0.2$ & 3.8 & $<0.2$ & $<0.20$ & $<1.0$ & $<0.2$ & $<0.2$ & $<0.2$ \\
\hline MAY & & & & & & & & & & & & \\
\hline 09.09 & $<0.20$ & $<0.2$ & $<0.2$ & $<1.0$ & $<0.2$ & 0.3 & $<0.2$ & $<0.20$ & $<1.0$ & $<0.2$ & $<0.2$ & $<0.2$ \\
\hline AUG & & & & & & & & & & & & \\
\hline $20-20$ & $<0.20$ & $<0.2$ & $<0.2$ & $<1.0$ & $<0.2$ & $<0.2$ & $<0.2$ & $<0.20$ & $<1.0$ & $<0.2$ & $<0.2$ & $<0.2$ \\
\hline
\end{tabular}


Table 9. Water-quality data for storm-sewer outfall stations, Texas Department of Transportation, Dallas and Fort Worth Districts, 1993-94-Continued

08049860 - MOUNTAIN CREEK OUTFALL AT I-20, DUNCANVILLE, TX (WY 1994)-Continued

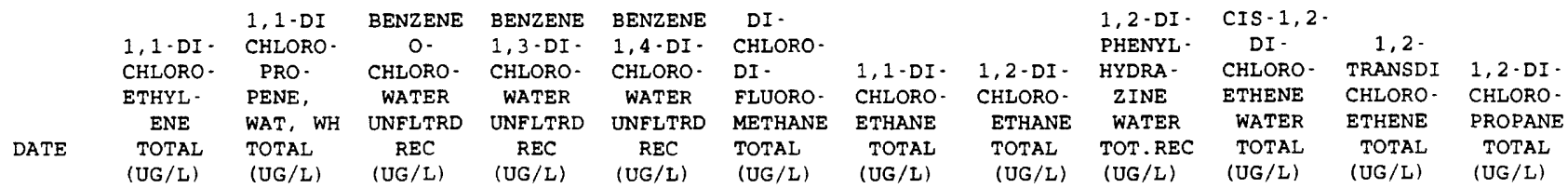

\begin{tabular}{|c|c|c|c|c|c|c|c|c|c|c|c|c|}
\hline $\begin{array}{c}\text { DEC } 1993 \\
02-03\end{array}$ & $<0.2$ & $<0.2$ & $<5.0$ & $<5.0$ & $<5.0$ & $<0.2$ & $<0.2$ & $<0.2$ & $<5.0$ & $<0.2$ & $<0.2$ & $<0.2$ \\
\hline JAN 1994 & & & & & & & & & & & & \\
\hline $11-11$ & $<0.2$ & $<0.2$ & $<5.0$ & $<5.0$ & $<5.0$ & $<0.2$ & $<0.2$ & $<0.2$ & $<5.0$ & $<0.2$ & $<0.2$ & $<0.2$ \\
\hline $\begin{array}{l}\text { FEB } \\
28-28\end{array}$ & $<0.2$ & $<0.2$ & $<5.0$ & $<5.0$ & $<5.0$ & $<0.2$ & $<0.2$ & $<0.2$ & $<5.0$ & $<0.2$ & $<0.2$ & $<0.2$ \\
\hline MAR & & & & & & & & & & & & \\
\hline $08 \cdot 08$ & $<0.2$ & $<0.2$ & $<5.0$ & $<5.0$ & $<5.0$ & $<0.2$ & $<0.2$ & $<0.2$ & $<5.0$ & $<0.2$ & $<0.2$ & $<0.2$ \\
\hline $\begin{array}{l}\text { APR } \\
11-11\end{array}$ & $<0.2$ & $<0.2$ & $<5.0$ & $<5.0$ & $<5.0$ & $<0.2$ & $<0.2$ & $<0.2$ & $<5.0$ & $<0.2$ & $<0.2$ & $<0.2$ \\
\hline MAY & & & & & & & & & & & & \\
\hline $\begin{array}{r}09-09 \\
\text { AUG }\end{array}$ & $<0.2$ & $<0.2$ & $<5.0$ & $<5.0$ & $<5.0$ & $<0.2$ & $<0.2$ & $<0.2$ & $<5.0$ & $<0.2$ & $<0.2$ & $<0.2$ \\
\hline $\begin{array}{l}\text { AUG } \\
20-20\end{array}$ & $<0.2$ & $<0.2$ & $<5.0$ & $<5.0$ & $<5.0$ & $<0.2$ & $<0.2$ & $<0.2$ & $<5.0$ & $<0.2$ & $<0.2$ & $<0.2$ \\
\hline
\end{tabular}

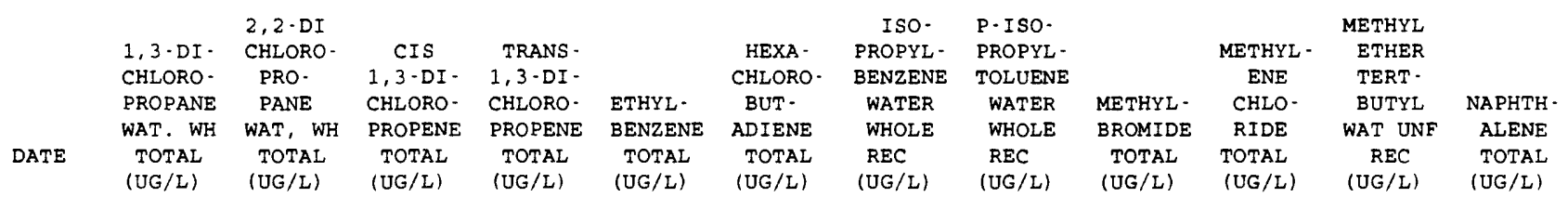

DEC 1993

\begin{tabular}{|c|c|c|c|c|c|c|c|c|c|c|c|c|}
\hline $02-03$ & $<0.2$ & $<0.2$ & $<0.2$ & $<0.2$ & $<0.2$ & $<5.0$ & $<0.20$ & $<0.20$ & $<0.2$ & $<0.2$ & $<1.0$ & $<5.0$ \\
\hline JAN 1994 & & & & & & & & & & & & \\
\hline $11 \cdot 11$ & $<0.2$ & $<0.2$ & $<0.2$ & $<0.2$ & $<0.2$ & $<5.0$ & $<0.20$ & $<0.20$ & $<0.2$ & $<0.2$ & $<1.0$ & $<5.0$ \\
\hline FEB & & & & & & & & & & & & \\
\hline$\underset{\text { MAR }}{28-28}$ & $<0.2$ & $<0.2$ & $<0.2$ & $<0.2$ & $<0.2$ & $<5.0$ & $<0.20$ & $<0.20$ & $<0.2$ & $<0.2$ & $<1.0$ & $<5.0$ \\
\hline $\begin{array}{l}\text { MAR } \\
08-08\end{array}$ & $<0.2$ & $<0,2$ & $<0.2$ & $<0.2$ & $<0,2$ & $<50$ & $<0,20$ & $<0,20$ & $<0 ?$ & $<0,2$ & $<10$ & $<5,0$ \\
\hline APR & & & & & & & & & & 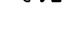 & . & \\
\hline $11-11$ & $<0.2$ & $<0.2$ & $<0.2$ & $<0.2$ & $<0.2$ & $<5.0$ & $<0.20$ & $<0.20$ & $<0.2$ & $<0.2$ & $<0.2$ & $<5.0$ \\
\hline & & & & & & & & & & & & \\
\hline $09-09$ & $<0.2$ & $<0.2$ & $<0.2$ & $<0.2$ & $<0.2$ & $<5.0$ & $<0.20$ & $<0.20$ & $<0.2$ & $<0.2$ & $<0.2$ & $<5.0$ \\
\hline JG & & & & & & & & & & & & \\
\hline $20-20$ & $<0.2$ & $<0.2$ & $<0.2$ & $<0.2$ & $<0.2$ & $<5.0$ & $<0.20$ & $<0.20$ & $<0.2$ & $<0.2$ & $<0.2$ & $<5.0$ \\
\hline
\end{tabular}

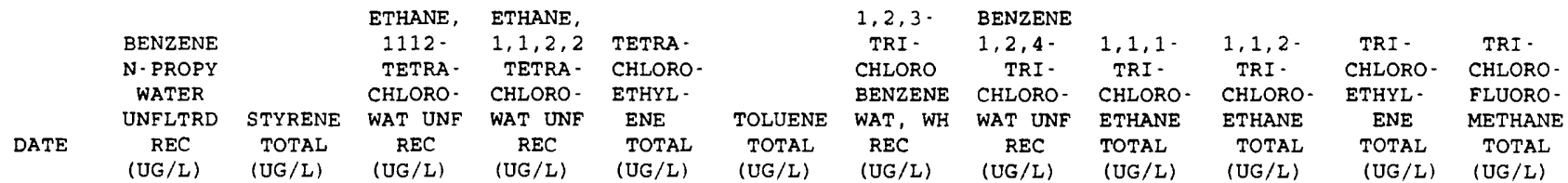

\begin{tabular}{|c|c|c|c|c|c|c|c|c|c|c|c|c|}
\hline \multicolumn{13}{|l|}{ DEC 1993} \\
\hline $\begin{array}{c}02-03 \\
\text { JAN } 1994\end{array}$ & $<0.20$ & $<0.2$ & $<0.2$ & $<0.2$ & $<0.2$ & $<0.2$ & $<0.20$ & $<5.0$ & $<0.2$ & $<0.2$ & $<0.2$ & $<0.2$ \\
\hline $11 \cdot 11$ & $<0.20$ & $<0.2$ & $<0.2$ & $<0.2$ & $<0.2$ & $<0.2$ & $<0.20$ & $<5.0$ & $<0.2$ & $<0.2$ & $<0.2$ & $<0.2$ \\
\hline FEB & & & & & & & & & & & & \\
\hline $28-28$ & $<0.20$ & $<0.2$ & $<0.2$ & $<0.2$ & $<0.2$ & $<0.2$ & $<0.20$ & $<5.0$ & $<0.2$ & $<0.2$ & $<0.2$ & $<0.2$ \\
\hline $\begin{array}{l}\text { MAR } \\
08-08\end{array}$ & $<0.20$ & $<0,2$ & $<02$ & $<0,2$ & $<02$ & $\leq 0$, & 10 & $5=0$ & & & 50 & $<0=$ \\
\hline APR & -0.00 & -0.2 & -0.2 & -0.2 & -0.4 & -0.2 & -0.20 & -3.0 & $<0.2$ & $<0.2$ & $<0.2$ & $<0.2$ \\
\hline $\begin{array}{c}11-11 \\
\text { MAY }\end{array}$ & $<0.20$ & $<0.2$ & $<0.2$ & $<0.2$ & $<0.2$ & $<0.2$ & $<0.20$ & $<5.0$ & $<0.2$ & $<0.2$ & $<0.2$ & $<0.2$ \\
\hline 09.09 & $<0.20$ & $<0.2$ & $<0.2$ & $<0.2$ & $<0.2$ & $<0.2$ & $<0.20$ & $<5.0$ & $<0.2$ & $<0.2$ & $<0.2$ & $<0.2$ \\
\hline AUG & & & & & & & & & & & & \\
\hline $20-20$ & $<0.20$ & $<0.2$ & $<0.2$ & $<0.2$ & $<0.2$ & $<0.2$ & $<0.20$ & $<5.0$ & $<0.2$ & $<0.2$ & $<0.2$ & $<0.2$ \\
\hline
\end{tabular}


Table 9. Water-quality data for storm-sewer outfall stations, Texas Department of Transportation, Dallas and Fort Worth Districts, 1993-94-Continued

08049860 - MOUNTAIN CREEK OUTFALL AT I-20, DUNCANVILLE, TX (WY 1994)-Continued

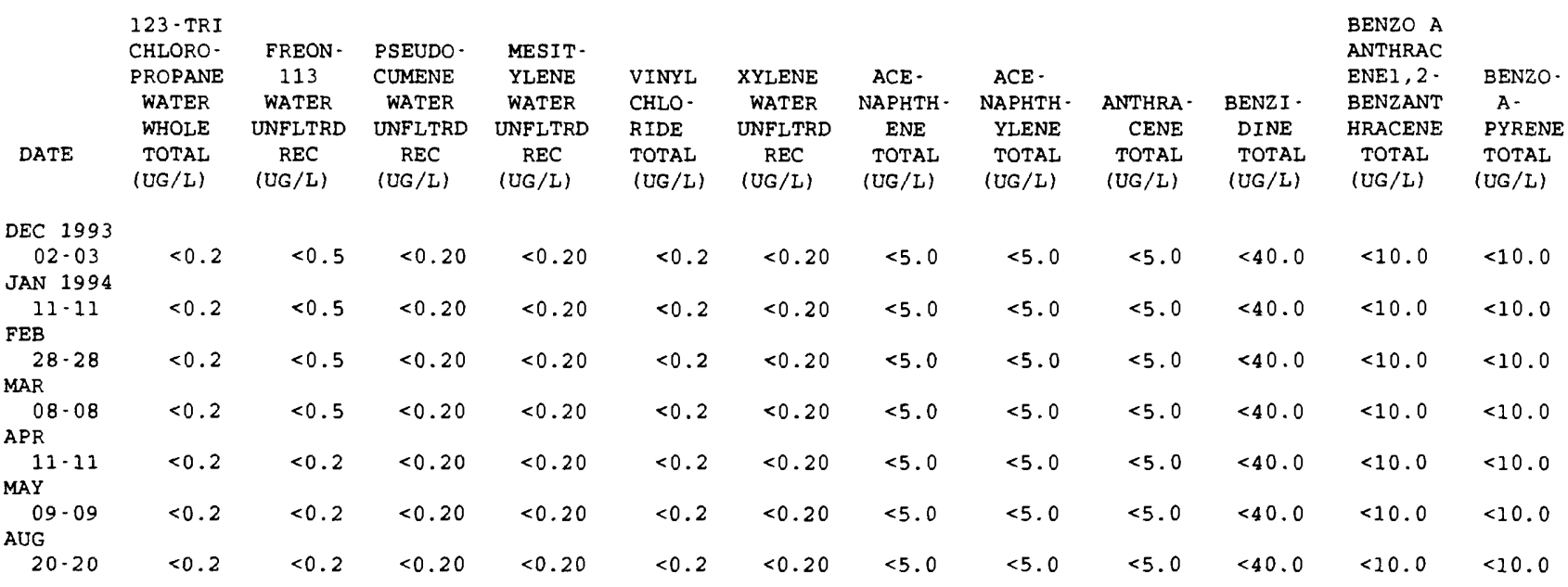

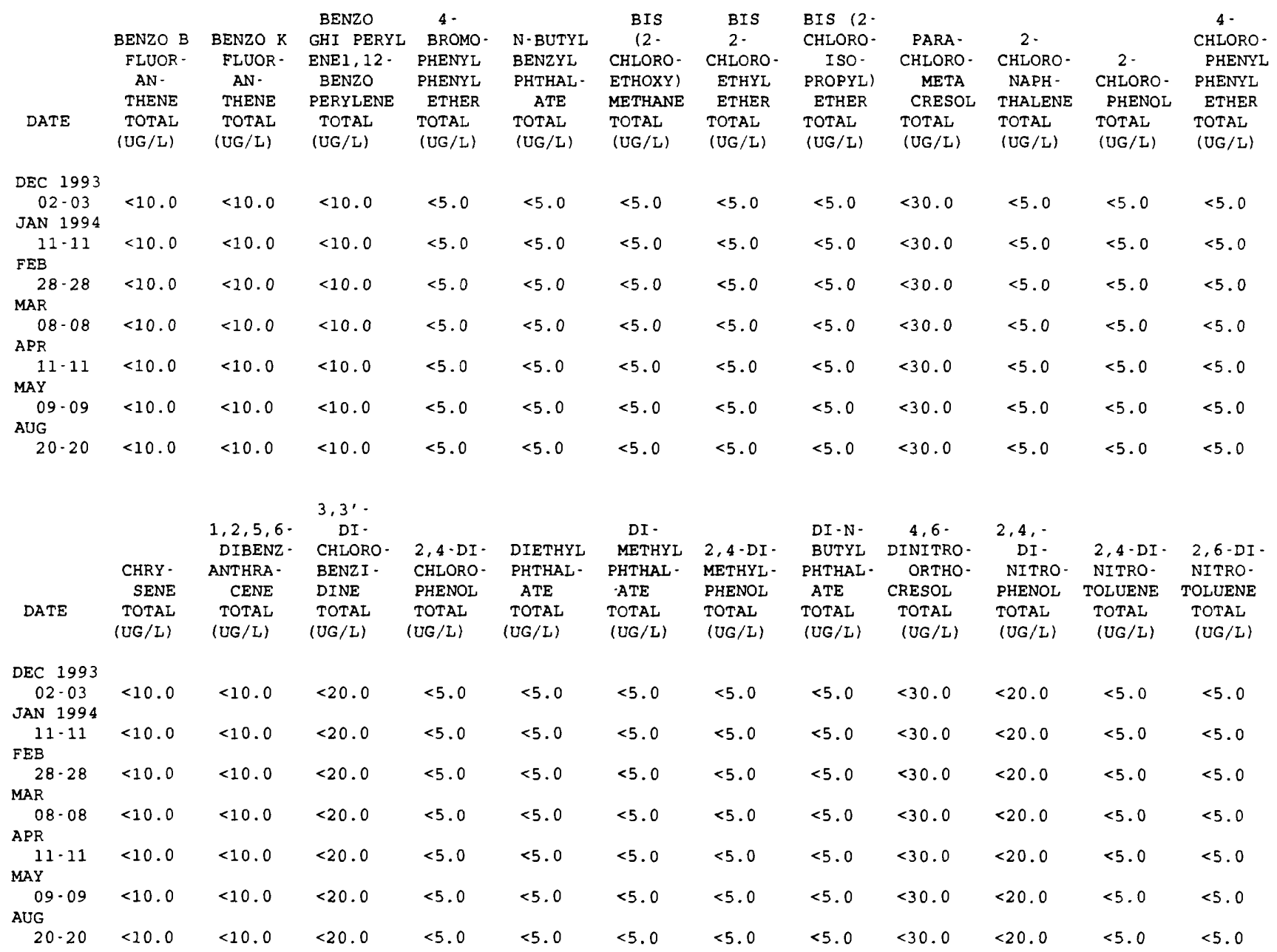


Table 9. Water-quality data for storm-sewer outfall stations, Texas Department of Transportation, Dallas and Fort Worth Districts, 1993-94-Continued

08049860 - MOUNTAIN CREEK OUTFALL AT I-20, DUNCANVILLE, TX (WY 1994)-Continued

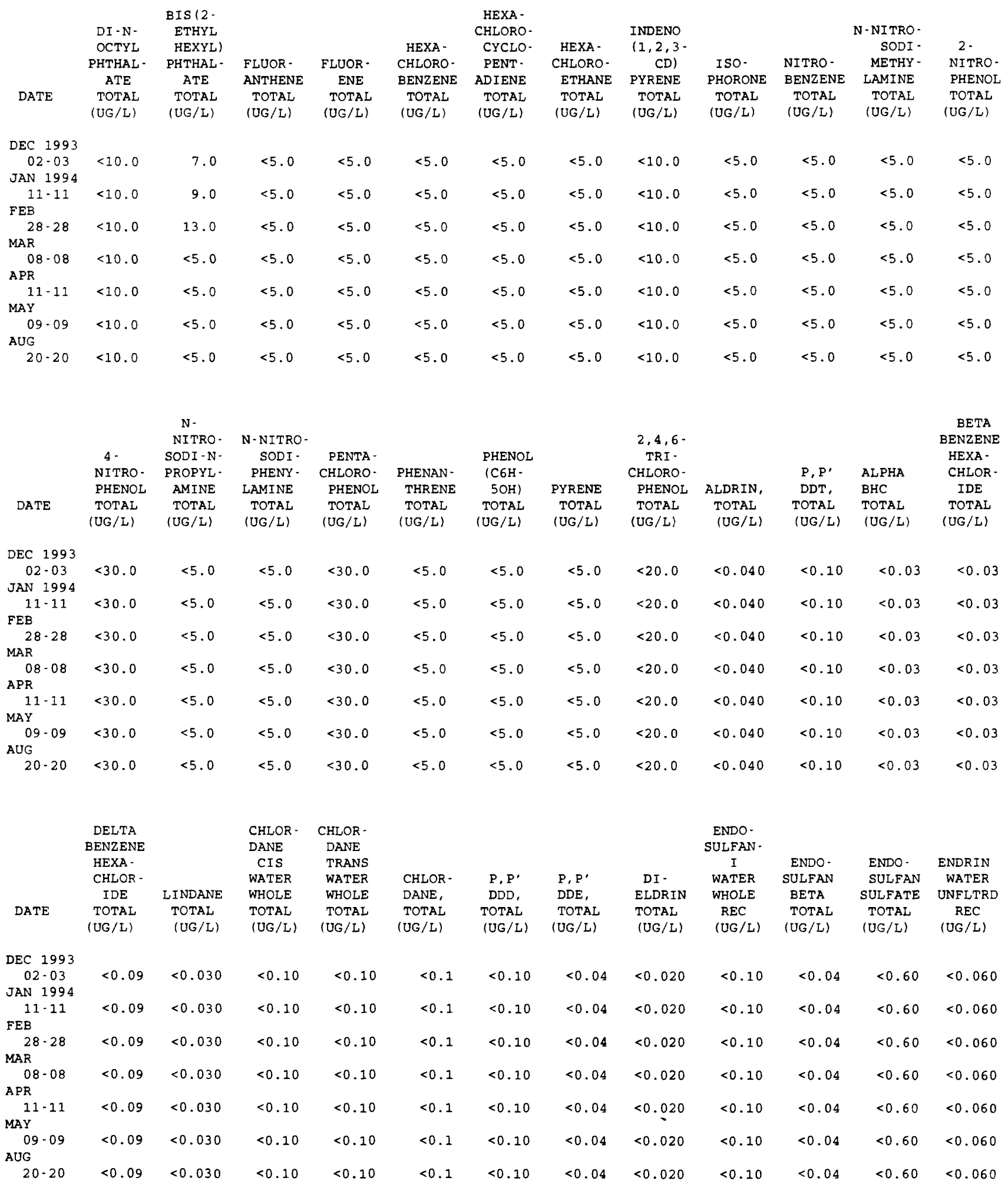


Table 9. Water-quality data for storm-sewer outfall stations, Texas Department of Transportation, Dallas and Fort Worth Districts, 1993-94-Continued

08049860 - MOUNTAIN CREEK OUTFALL AT I-20, DUNCANVILLE, TX (WY 1994)-Continued

\begin{tabular}{|c|c|c|c|c|c|c|c|c|c|c|c|c|}
\hline & ENDRIN & & HEPTA - & & AROCLOR & AROCLOR & AROCLOR & AROCLOR & AROCLOR & AROCLOR & AROCLOR & \\
\hline & ALDE - & HEPTA - & CHLOR & TOX - & 1016 & 1221 & 1232 & 1242 & 1248 & 1254 & 1260 & DI - \\
\hline & HYDE & CHLOR, & EPOXIDE & APHENE, & PCB & PCB & $\mathrm{PCB}$ & PCB & PCB & $\mathrm{PCB}$ & $\mathrm{PCB}$ & AZINON, \\
\hline DATE & $\begin{array}{l}\text { TOTAL } \\
\text { (UG/L) }\end{array}$ & $\begin{array}{l}\text { TOTAL } \\
\text { (UG/L) }\end{array}$ & $\begin{array}{l}\text { TOTAL } \\
\text { (UG/L) }\end{array}$ & $\begin{array}{l}\text { TOTAL } \\
(\text { UG/L) }\end{array}$ & $\begin{array}{l}\text { TOTAL } \\
\text { (UG/L) }\end{array}$ & $\begin{array}{l}\text { TOTAL } \\
\text { (UG/L) }\end{array}$ & $\begin{array}{c}\text { TOTAL } \\
\text { (UG/L) }\end{array}$ & $\begin{array}{l}\text { TOTAL } \\
\text { (UG/L) }\end{array}$ & $\begin{array}{l}\text { TOTAL } \\
\text { (UG/L) }\end{array}$ & $\begin{array}{l}\text { TOTAL } \\
\text { (UG/L) }\end{array}$ & $\begin{array}{l}\text { TOTAL } \\
\text { (UG/L) }\end{array}$ & $\begin{array}{l}\text { TOTAL } \\
\text { (UG/L) }\end{array}$ \\
\hline DEC 1993 & & & & & & & & & & & & \\
\hline 02.03 & $<0.20$ & $<0.030$ & $<0.80$ & $<2$ & $<0.1$ & $<1.0$ & $<0.1$ & $<0.1$ & $<0.1$ & $<0.1$ & $<0.1$ & $<0.05$ \\
\hline JAN 1994 & & & & & & & & & & & & \\
\hline $11 \cdot 11$ & $<0.20$ & $<0.030$ & $<0.80$ & $<2$ & $<0.1$ & $<1.0$ & $<0.1$ & $<0.1$ & $<0.1$ & $<0.1$ & $<0.1$ & $<0.05$ \\
\hline FEB & & & & & & & & & & & & \\
\hline $28 \cdot 28$ & $<0.20$ & $<0.030$ & $<0.80$ & $<2$ & $<0.1$ & $<1.0$ & $<0.1$ & $<0.1$ & $<0.1$ & $<0.1$ & $<0.1$ & $<0.05$ \\
\hline MAR & & & & & & & & & & & & \\
\hline $08-08$ & $<0.20$ & $<0.030$ & $<0.80$ & $<2$ & $<0.1$ & $<1.0$ & $<0.1$ & $<0.1$ & $<0.1$ & $<0.1$ & $<0.1$ & $<0.05$ \\
\hline APR & & & & & & & & & & & & \\
\hline $11-11$ & $<0.20$ & $<0.030$ & $<0.80$ & $<2$ & $<0.1$ & $<1.0$ & $<0.1$ & $<0.1$ & $<0.1$ & $<0.1$ & $<0.1$ & $<0.05$ \\
\hline MAY & & & & & & & & & & & & \\
\hline $09-09$ & $<0.20$ & $<0.030$ & $<0.80$ & $<2$ & $<0.1$ & $<1.0$ & $<0.1$ & $<0.1$ & $<0.1$ & $<0.1$ & $<0.1$ & $<0.05$ \\
\hline AUG & & & & & & & & & & & & \\
\hline $20 \cdot 20$ & $<0.20$ & $<0.030$ & $<0.80$ & $<2$ & $<0.1$ & $<1.0$ & $<0.1$ & $<0.1$ & $<0.1$ & $<0.1$ & $<0.1$ & $<0.05$ \\
\hline
\end{tabular}


Table 9. Water-quality data for storm-sewer outfall stations, Texas Department of Transportation, Dallas and Fort Worth Districts, 1993-94-Continued

08049950 - FISH CREEK OUTFALL AT I-20 ARLINGTON, TX (WY 1995)

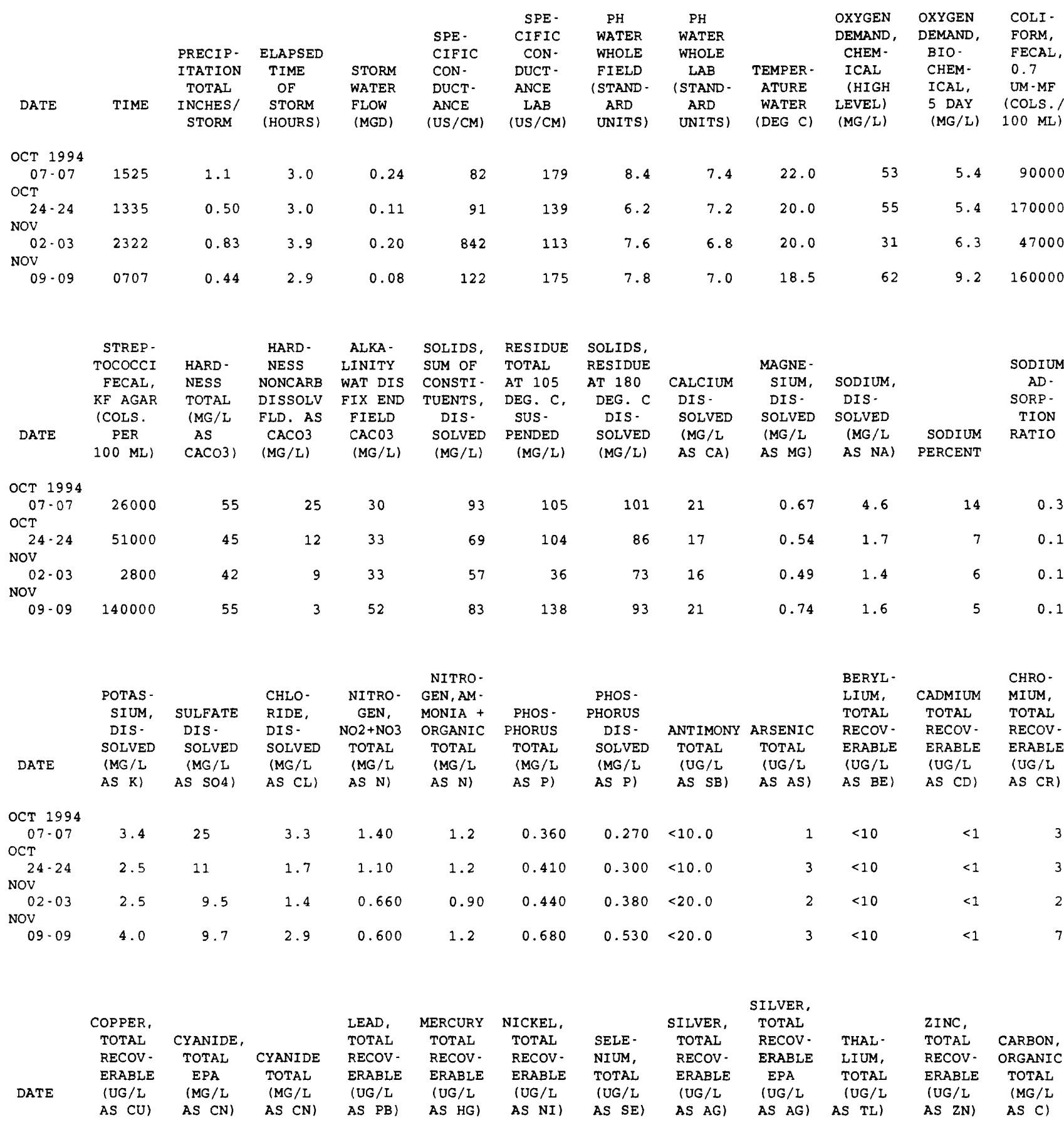

OCT 1994

OCT

$24-24$

NoV

$02 \cdot 03$

NOV

$09-09$

\begin{abstract}
$8<0.010<0.010$
$7<0.010<0.010$
\end{abstract}

$11<0.10$

5

$<1$

\begin{abstract}
$<1<0.500$
$<1<0.500$

$<1<0.500$
\end{abstract}

$<5$

$<5$

$50 \quad 18$

$21<0.10$

$8<0.10$

$<10$

$50 \quad 20$

$27<0.10$

$<1 \quad<0.500$

$<5$

$20 \quad 13$ 
Table 9. Water-quality data for storm-sewer outfall stations, Texas Department of Transportation, Dallas and Fort Worth Districts, 1993-94-Continued

08049950 - FISH CREEK OUTFALL AT I-20 ARLINGTON, TX (WY 1995)-Continued

\begin{tabular}{|c|c|c|c|c|c|c|c|c|c|c|c|c|}
\hline DATE & $\begin{array}{l}\text { OIL AND } \\
\text { GREASE, } \\
\text { TOTAL } \\
\text { RECOV. } \\
\text { GRAVI - } \\
\text { METRIC } \\
\text { (MG/L) }\end{array}$ & $\begin{array}{c}\text { PHENOLS } \\
\text { TOTAL } \\
\text { (UG/L) }\end{array}$ & $\begin{array}{c}\text { BENZENE } \\
\text { TOTAL } \\
\text { (UG/L) }\end{array}$ & $\begin{array}{l}\text { BROMO- } \\
\text { BENZENE } \\
\text { WATER, } \\
\text { WHOLE, } \\
\text { TOTAL } \\
\text { (UG/L) }\end{array}$ & $\begin{array}{l}\text { METHANE } \\
\text { BROMO } \\
\text { CHLORO- } \\
\text { WAT } \\
\text { UNFLTRD } \\
\text { REC } \\
\text { (UG/L) }\end{array}$ & $\begin{array}{l}\text { BROMO- } \\
\text { FORM } \\
\text { TOTAL } \\
\text { (UG/L) }\end{array}$ & $\begin{array}{c}\text { BENZENE } \\
\text { N-BUTYL } \\
\text { WATER } \\
\text { UNFLTRD } \\
\text { REC } \\
\text { (UG/L) }\end{array}$ & $\begin{array}{l}\text { BENZENE } \\
\text { SEC } \\
\text { BUTYL - } \\
\text { WATER } \\
\text { UNFLTRD } \\
\text { REC } \\
\text { (UG/L) }\end{array}$ & $\begin{array}{l}\text { BENZENE } \\
\text { TERT- } \\
\text { BUTYL- } \\
\text { WATER } \\
\text { UNFLTRD } \\
\text { REC } \\
\text { (UG/L) }\end{array}$ & $\begin{array}{l}\text { CARBON- } \\
\text { TETRA- } \\
\text { CHLO- } \\
\text { RIDE } \\
\text { TOTAL } \\
\text { (UG/L) }\end{array}$ & $\begin{array}{l}\text { CHLORO- } \\
\text { BENZENE } \\
\text { TOTAL } \\
\text { (UG/L) }\end{array}$ & $\begin{array}{c}\text { CHLORO- } \\
\text { DI - } \\
\text { BROMO- } \\
\text { METHANE } \\
\text { TOTAL } \\
\text { (UG/L) }\end{array}$ \\
\hline \multicolumn{13}{|l|}{ OCT 1994} \\
\hline $07-07$ & $<1$ & 4 & $<4.0$ & $<4.0$ & $<4.00$ & $<4.0$ & $<4.0$ & $<4.0$ & $<4.0$ & $<4.0$ & $<4.0$ & $<4.0$ \\
\hline \multicolumn{13}{|l|}{ OCT } \\
\hline $24-24$ & 2 & 3 & $<0.2$ & $<0.2$ & $<0.20$ & $<0.2$ & $<0.20$ & $<0.20$ & $<0.20$ & $<0.2$ & $<0.20$ & $<0.2$ \\
\hline \multicolumn{13}{|l|}{ Nov } \\
\hline 02.03 & $<1$ & $<1$ & $<0.2$ & $<0.2$ & $<0.20$ & $<0.2$ & $<0.20$ & $<0.20$ & $<0.20$ & $<0.2$ & $<0.20$ & $<0.2$ \\
\hline \multicolumn{13}{|l|}{ NOV } \\
\hline $09-09$ & $<1$ & 3 & $<0.2$ & $<0.2$ & $<0.20$ & $<0.2$ & $<0.20$ & $<0.20$ & $<0.20$ & $<0.2$ & $<0.20$ & $<0.2$ \\
\hline & & 2 & & & 0 & & DIBROMO & DI - & $1,2-$ & & & $1,1-D I$ \\
\hline & & $\begin{array}{l}\text { CHLORO - } \\
\text { ETHYL. }\end{array}$ & & METHYL - & $\begin{array}{l}\text { CHLORO - } \\
\text { TOLUENE }\end{array}$ & $\begin{array}{l}\text { TOLUENE } \\
\text { P-CHLOR }\end{array}$ & $\begin{array}{l}\text { CHLORO- } \\
\text { PROPANE }\end{array}$ & $\begin{array}{l}\text { BROMO - } \\
\text { METHANE }\end{array}$ & $\begin{array}{l}\text { DIBROMO } \\
\text { ETHANE }\end{array}$ & $\begin{array}{c}\text { DI- } \\
\text { CHLORO- }\end{array}$ & $\begin{array}{l}1,1 \text {-DI - } \\
\text { CHLORO- }\end{array}$ & $\begin{array}{c}\text { CHLORO- } \\
\text { PRO- }\end{array}$ \\
\hline & $\begin{array}{l}\text { CHLORO- } \\
\text { ETHANE }\end{array}$ & $\begin{array}{c}\text { VINYL- } \\
\text { ETHER }\end{array}$ & $\begin{array}{l}\text { CHLORO- } \\
\text { FORM }\end{array}$ & $\begin{array}{l}\text { CHLO- } \\
\text { RIDE }\end{array}$ & $\begin{array}{l}\text { WATER } \\
\text { WHOLE }\end{array}$ & $\begin{array}{l}\text { WATER } \\
\text { UNFLTRD }\end{array}$ & $\begin{array}{l}\text { WATER } \\
\text { WHOLE }\end{array}$ & $\begin{array}{l}\text { WATER } \\
\text { WHOLE }\end{array}$ & $\begin{array}{l}\text { WATER } \\
\text { WHOLE }\end{array}$ & $\begin{array}{l}\text { BROMO- } \\
\text { METHANE }\end{array}$ & $\begin{array}{c}\text { ETHYL- } \\
\text { ENE }\end{array}$ & $\begin{array}{l}\text { PENE, } \\
\text { WAT, WH }\end{array}$ \\
\hline DATE & $\begin{array}{l}\text { TOTAL } \\
\text { (UG/L) }\end{array}$ & $\begin{array}{l}\text { TOTAL } \\
\text { (UG/L) }\end{array}$ & $\begin{array}{l}\text { TOTAL } \\
\text { (UG/L) }\end{array}$ & $\begin{array}{l}\text { TOTAL } \\
\text { (UG/L) }\end{array}$ & $\begin{array}{l}\text { TOTAL } \\
\text { (UG/L) }\end{array}$ & $\begin{array}{c}\text { REC } \\
\text { (UG/L) }\end{array}$ & $\begin{array}{l}\text { TOT . REC } \\
\text { (UG/L) }\end{array}$ & $\begin{array}{l}\text { RECOVER } \\
\text { (UG/L) }\end{array}$ & $\begin{array}{l}\text { TOTAL } \\
\text { (UG/L) }\end{array}$ & $\begin{array}{l}\text { TOTAL } \\
\text { (UG/L) }\end{array}$ & $\begin{array}{l}\text { TOTAL } \\
\text { (UG/L) }\end{array}$ & $\begin{array}{l}\text { TOTAL } \\
(U G / L)\end{array}$ \\
\hline \multicolumn{13}{|l|}{ OCT 1994} \\
\hline $07-07$ & $<4.0$ & $<20$ & $<4.0$ & $<4.0$ & $<4.0$ & $<4.0$ & $<20$ & $<4.0$ & $<4.0$ & $<4.0$ & $<4.0$ & $<4.0$ \\
\hline \multicolumn{13}{|l|}{ OCT } \\
\hline $24-24$ & $<0.2$ & $<1.0$ & $<0.2$ & $<0.2$ & $<0.2$ & $<0.20$ & $<1.0$ & $<0.2$ & $<0.2$ & $<0.2$ & $<0.2$ & $<0.2$ \\
\hline \multicolumn{13}{|l|}{ Nov } \\
\hline $02-03$ & $<0.2$ & $<1.0$ & $<0.2$ & $<0.2$ & $<0.2$ & $<0.20$ & $<1.0$ & $<0.2$ & $<0.2$ & $<0.2$ & $<0.2$ & $<0.2$ \\
\hline
\end{tabular}

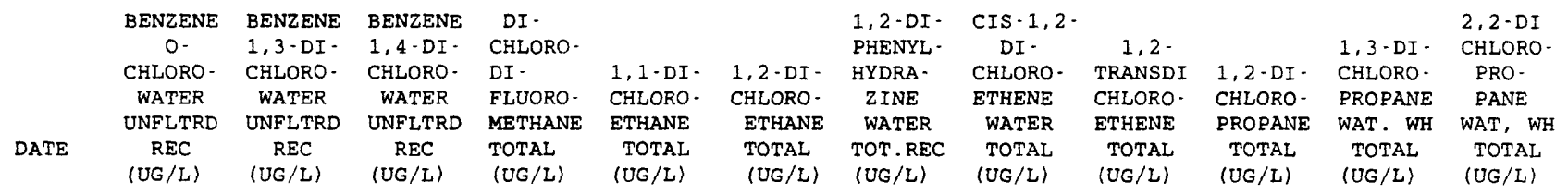

\begin{tabular}{|c|c|c|c|c|c|c|c|c|c|c|c|c|}
\hline \multicolumn{13}{|l|}{ OCT 1994} \\
\hline 07.07 & $<5.0$ & $<5.0$ & $<5.0$ & $<4.0$ & $<4.0$ & $<4.0$ & $<5.0$ & $<4.0$ & $<4.0$ & $<4.0$ & $<4.0$ & $<4.0$ \\
\hline \multicolumn{13}{|l|}{ OCT } \\
\hline $24-24$ & $<5.0$ & $<5.0$ & $<5.0$ & $<0.2$ & $<0.2$ & $<0.2$ & $<5.0$ & $<0.2$ & $<0.2$ & $<0.2$ & $<0.2$ & $<0.2$ \\
\hline \multicolumn{13}{|l|}{ NoV } \\
\hline $02 \cdot 03$ & $<5.0$ & $<5.0$ & $<5.0$ & $<0.2$ & $<0.2$ & $<0.2$ & $<5.0$ & $<0.2$ & $<0.2$ & $<0.2$ & $<0.2$ & $<0.2$ \\
\hline \multicolumn{13}{|l|}{ NOV } \\
\hline 09.09 & $<5.0$ & $<5.0$ & $<5.0$ & $<0.2$ & $<0.2$ & $<0.2$ & $<5.0$ & $<0.2$ & $<0.2$ & $<0.2$ & $<0.2$ & $<0.2$ \\
\hline
\end{tabular}

\begin{tabular}{|c|c|c|c|c|c|c|c|c|c|c|c|c|}
\hline & & & & & ISO - & P-ISO- & & & METHYL & & & \\
\hline & CIS & TRANS - & & HEXA - & PROPYL - & PROPYL - & & METHYL - & ETHER & & BENZENE & \\
\hline & $1,3-\mathrm{DI}$ - & $1,3-\mathrm{DI}$ - & & CHLORO- & BENZENE & TOLUENE & & ENE & TERT - & & N-PROPY & \\
\hline & CHLORO - & CHLORO- & ETHYL - & BUT - & WATER & WATER & METHYL - & CHLO - & BUTYL & NAPHTH - & WATER & \\
\hline & PROPENE & PROPENE & BENZENE & ADIENE & WHOLE & WHOLE & BROMIDE & RIDE & WAT UNF & ALENE & UNFLTRD & STYRENE \\
\hline DATE & $\begin{array}{l}\text { TOTAL } \\
\text { (UG/L) }\end{array}$ & $\begin{array}{l}\text { TOTAL } \\
\text { (UG/L) }\end{array}$ & $\begin{array}{l}\text { TOTAL } \\
(U G / L)\end{array}$ & $\begin{array}{l}\text { TOTAL } \\
\text { (UG/L) }\end{array}$ & $\begin{array}{c}\text { REC } \\
(U G / L)\end{array}$ & $\begin{array}{c}\text { REC } \\
(U G / L)\end{array}$ & $\begin{array}{l}\text { TOTAL } \\
\text { (UG/L) }\end{array}$ & $\begin{array}{l}\text { TOTAL } \\
\text { (UG/L) }\end{array}$ & $\begin{array}{c}\text { REC } \\
(U G / L)\end{array}$ & $\begin{array}{l}\text { TOTAL } \\
\text { (UG/L) }\end{array}$ & $\begin{array}{c}\text { REC } \\
\text { (UG/L) }\end{array}$ & $\begin{array}{l}\text { TOTAL } \\
\text { (UG/L) }\end{array}$ \\
\hline
\end{tabular}

\begin{tabular}{|c|c|c|c|c|c|c|c|c|c|c|c|c|}
\hline \multicolumn{13}{|l|}{ ОСт 1994} \\
\hline 07.07 & $<4.0$ & $<4.0$ & $<4,0$ & $<5,0$ & $<4.0$ & $<4.0$ & $<4.0$ & $<4.0$ & $<4.0$ & $<5.0$ & $<4.0$ & $<4.0$ \\
\hline \multicolumn{13}{|l|}{ OCT } \\
\hline $24-24$ & $<0.2$ & $<0.2$ & $<0.2$ & $<5.0$ & $<0.20$ & $<0.20$ & $<0.2$ & $<0.2$ & $<0.2$ & $<5.0$ & $<0.20$ & $<0.2$ \\
\hline \multicolumn{13}{|l|}{ Nov } \\
\hline $02-03$ & $<0.2$ & $<0.2$ & $<0.2$ & $<5.0$ & $<0.20$ & $<0.20$ & $<0.2$ & $<0.2$ & $<0.2$ & $<5.0$ & $<0.20$ & $<0.2$ \\
\hline \multicolumn{13}{|l|}{ NOV } \\
\hline 09.09 & $<0.2$ & $<0.2$ & $<0.2$ & $<5.0$ & $<0.20$ & $<0.20$ & $<0.2$ & $<0.2$ & $<0.2$ & $<5.0$ & $<0.20$ & $<0.2$ \\
\hline
\end{tabular}


Table 9. Water-quality data for storm-sewer outfall stations, Texas Department of Transportation, Dallas and Fort Worth Districts, 1993-94-Continued

08049950 - FISH CREEK OUTFALL AT I-20 ARLINGTON, TX (WY 1995)-Continued

\begin{tabular}{|c|c|c|c|c|c|c|c|c|c|c|c|c|}
\hline DATE & $\begin{array}{c}\text { ETHANE, } \\
1112- \\
\text { TETRA - } \\
\text { CHLORO- } \\
\text { WAT UNF } \\
\text { REC } \\
\text { (UG/L) }\end{array}$ & $\begin{array}{l}\text { ETHANE, } \\
1,1,2,2 \\
\text { TETRA- } \\
\text { CHLORO- } \\
\text { WAT UNF } \\
\text { REC } \\
\text { (UG/L) }\end{array}$ & $\begin{array}{l}\text { TETRA - } \\
\text { CHLORO- } \\
\text { ETHYL - } \\
\text { ENE } \\
\text { TOTAL } \\
\text { (UG/L) }\end{array}$ & $\begin{array}{l}\text { TOLUENE } \\
\text { TOTAL } \\
\text { (UG/L) }\end{array}$ & $\begin{array}{l}1,2,3- \\
\text { TRI - } \\
\text { CHLORO } \\
\text { BENZENE } \\
\text { WAT, WH } \\
\text { REC } \\
\text { (UG/L) }\end{array}$ & $\begin{array}{c}\text { BENZENE } \\
1,2,4 \text { - } \\
\text { TRI - } \\
\text { CHLORO- } \\
\text { WAT UNF } \\
\text { REC } \\
\text { (UG/L) }\end{array}$ & $\begin{array}{l}1,1,1 \text { - } \\
\text { TRI - } \\
\text { CHLORO- } \\
\text { ETHANE } \\
\text { TOTAL } \\
\text { (UG/L) }\end{array}$ & $\begin{array}{l}1,1,2- \\
\text { TRI - } \\
\text { CHLORO- } \\
\text { ETHANE } \\
\text { TOTAL } \\
\text { (UG/L) }\end{array}$ & $\begin{array}{l}\text { TRI- } \\
\text { CHLORO- } \\
\text { ETHYL- } \\
\text { ENE } \\
\text { TOTAL } \\
\text { (UG/L) }\end{array}$ & $\begin{array}{l}\text { TRI - } \\
\text { CHLORO- } \\
\text { FLUORO- } \\
\text { METHANE } \\
\text { TOTAL } \\
\text { (UG/L) }\end{array}$ & $\begin{array}{l}123 \text { - TRI } \\
\text { CHLORO- } \\
\text { PROPANE } \\
\text { WATER } \\
\text { WHOLE } \\
\text { TOTAL } \\
\text { (UG/L) }\end{array}$ & $\begin{array}{c}\text { FREON- } \\
113 \\
\text { WATER } \\
\text { UNFLTRD } \\
\text { REC } \\
\text { (UG/L) }\end{array}$ \\
\hline $\begin{array}{r}\text { OCT } 1994 \\
07-07\end{array}$ & $<4.0$ & $<4.0$ & $<4.0$ & $<4.0$ & $<4.0$ & $<5.0$ & $<4.0$ & $<4.0$ & $<4.0$ & $<4.0$ & $<4.0$ & $<4.0$ \\
\hline $\begin{array}{l}\text { OCT } \\
24-24\end{array}$ & $<0.2$ & $<0.2$ & $<0.2$ & $<0.2$ & $<0.20$ & $<5.0$ & $<0.2$ & $<0.2$ & $<0.2$ & $<0.2$ & $<0.2$ & $<0.2$ \\
\hline $\begin{array}{l}\text { NOV } \\
02-03\end{array}$ & $<0.2$ & $<0.2$ & $<0.2$ & $<0.2$ & $<0.20$ & $<5.0$ & $<0.2$ & $<0.2$ & $<0.2$ & $<0.2$ & $<0.2$ & $<0.2$ \\
\hline $09-09$ & $<0.2$ & $<0.2$ & $<0.2$ & $<0.2$ & $<0.20$ & $<5.0$ & $<0.2$ & $<0.2$ & $<0.2$ & $<0.2$ & $<0.2$ & $<0.2$ \\
\hline
\end{tabular}

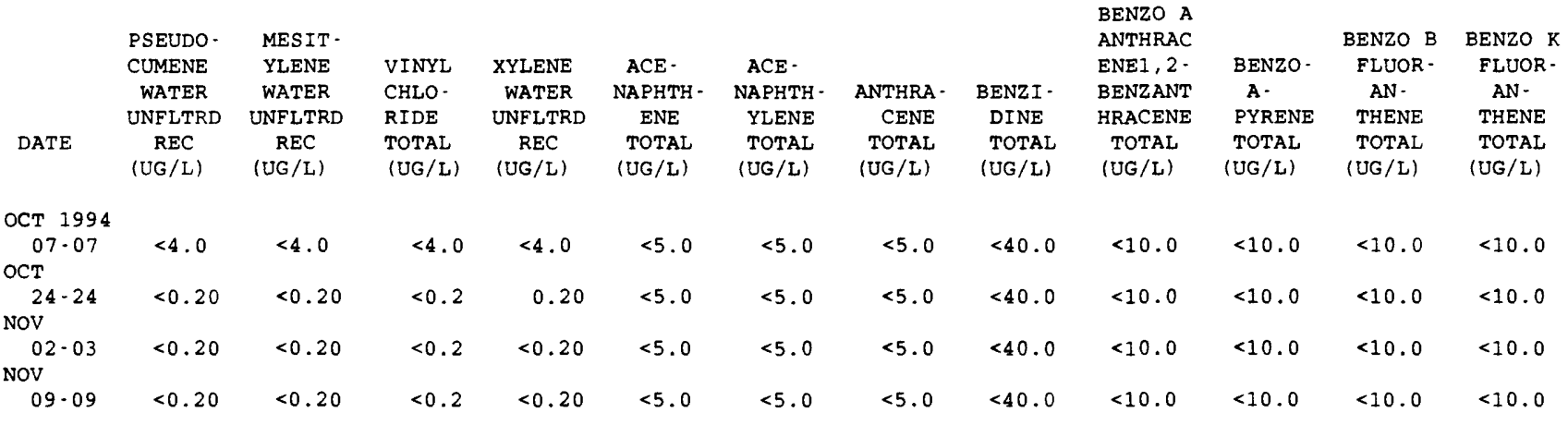

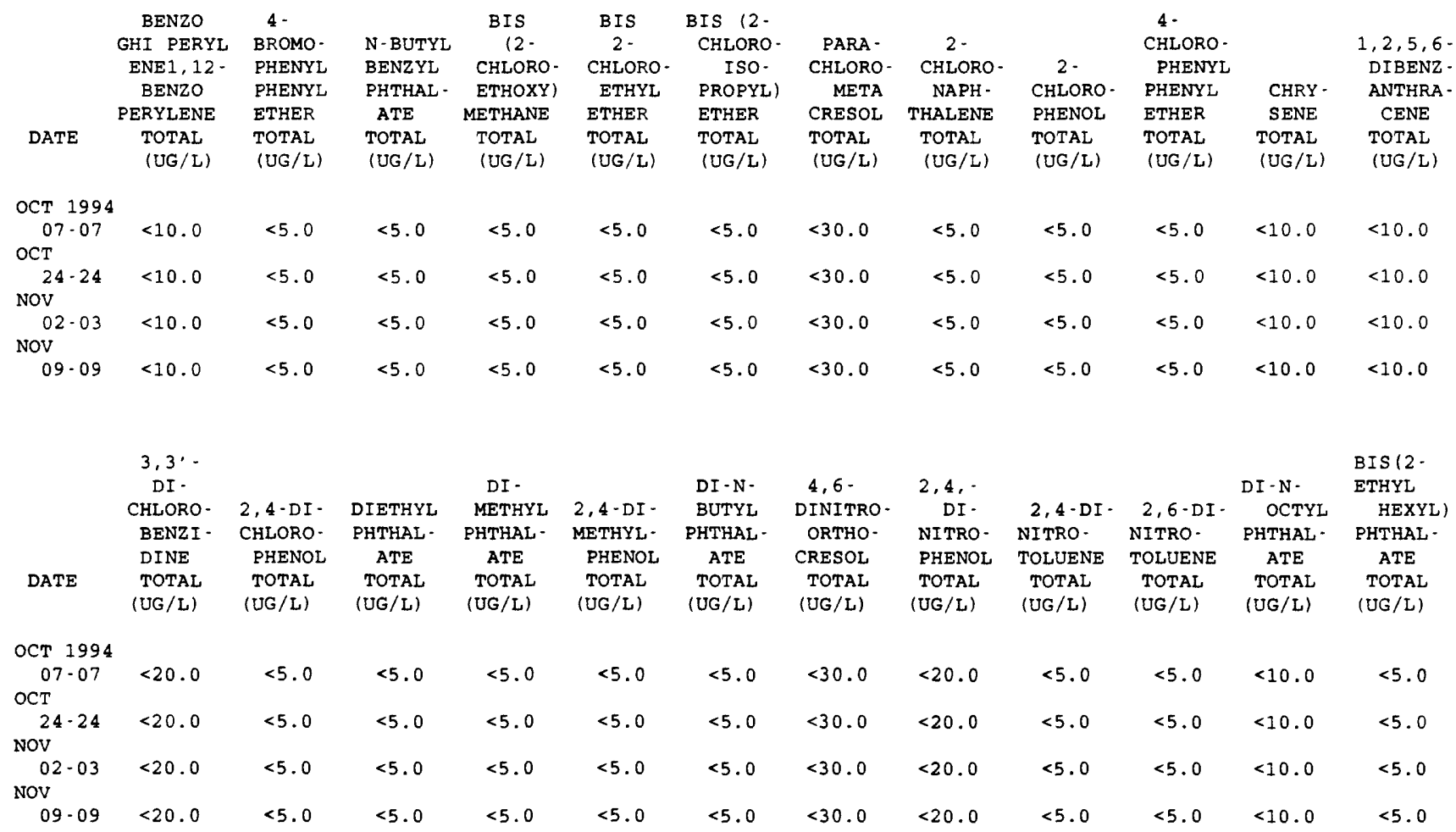


Table 9. Water-quality data for storm-sewer outfall stations, Texas Department of Transportation, Dallas and Fort Worth Districts, 1993-94-Continued.

08049950 - FISH CREEK OUTFALL AT I-20 ARLINGTON, TX (WY 1995)-Continued

\begin{tabular}{|c|c|c|c|c|c|c|c|c|c|c|c|c|}
\hline DATE & $\begin{array}{l}\text { FLUOR - } \\
\text { ANTHENE } \\
\text { TOTAL } \\
\text { (UG/L) }\end{array}$ & $\begin{array}{c}\text { FLUOR- } \\
\text { ENE } \\
\text { TOTAL } \\
\text { (UG/L) }\end{array}$ & $\begin{array}{l}\text { HEXA- } \\
\text { CHLORO- } \\
\text { BENZENE } \\
\text { TOTAL } \\
\text { (UG/L) }\end{array}$ & $\begin{array}{c}\text { HEXA- } \\
\text { CHLORO- } \\
\text { CYCLO- } \\
\text { PENT- } \\
\text { ADIENE } \\
\text { TOTAL } \\
\text { (UG/L) }\end{array}$ & $\begin{array}{l}\text { HEXA- } \\
\text { CHLORO- } \\
\text { ETHANE } \\
\text { TOTAL } \\
\text { (UG/L) }\end{array}$ & $\begin{array}{c}\text { INDENO } \\
\langle 1,2,3- \\
\text { CD }\rangle \\
\text { PYRENE } \\
\text { TOTAL } \\
\text { (UG/L) }\end{array}$ & $\begin{array}{l}\text { ISO- } \\
\text { PHORONE } \\
\text { TOTAL } \\
\text { (UG/L) }\end{array}$ & $\begin{array}{l}\text { NITRO- } \\
\text { BENZENE } \\
\text { TOTAL } \\
\text { (UG/L) }\end{array}$ & $\begin{array}{c}\text { N-NITRO- } \\
\text { SODI - } \\
\text { METHY - } \\
\text { LAMINE } \\
\text { TOTAL } \\
\text { (UG/L) }\end{array}$ & $\begin{array}{l}2- \\
\text { NITRO- } \\
\text { PHENOL } \\
\text { TOTAL } \\
\text { (UG } / L \text { ) }\end{array}$ & $\begin{array}{l}4- \\
\text { NITRO- } \\
\text { PHENOL } \\
\text { TOTAL } \\
\text { (UG/L) }\end{array}$ & $\begin{array}{c}\text { N- } \\
\text { NITRO- } \\
\text { SODI -N- } \\
\text { PROPYL- } \\
\text { AMINE } \\
\text { TOTAL } \\
\text { (UG/L) }\end{array}$ \\
\hline OCT 1994 & & & & & & & & & & & & \\
\hline $\begin{array}{l}07.07 \\
\text { OCT }\end{array}$ & $<5.0$ & $<5.0$ & $<5.0$ & $<5.0$ & $<5.0$ & $<10.0$ & $<5.0$ & $<5.0$ & $<5.0$ & $<5.0$ & $<30.0$ & $<5.0$ \\
\hline $\begin{array}{l}24-24 \\
\text { NoV }\end{array}$ & $<5.0$ & $<5.0$ & $<5.0$ & $<5.0$ & $<5.0$ & $<10.0$ & $<5.0$ & $<5.0$ & $<5.0$ & $<5.0$ & $<30.0$ & $<5.0$ \\
\hline $\begin{array}{l}02-03 \\
\text { Nov }\end{array}$ & $<5.0$ & $<5.0$ & $<5.0$ & $<5.0$ & $<5.0$ & $<10.0$ & $<5.0$ & $<5.0$ & $<5.0$ & $<5.0$ & $<30.0$ & $<5.0$ \\
\hline 09.09 & $<5.0$ & $<5.0$ & $<5.0$ & $<5.0$ & $<5.0$ & $<10.0$ & $<5.0$ & $<5.0$ & $<5.0$ & $<5.0$ & $<30.0$ & $<5.0$ \\
\hline DATE & $\begin{array}{l}\text { N-NITRO- } \\
\text { SODI - } \\
\text { PHENY - } \\
\text { LAMINE } \\
\text { TOTAL } \\
(U G / L)\end{array}$ & $\begin{array}{l}\text { PENTA- } \\
\text { CHLORO- } \\
\text { PHENOL } \\
\text { TOTAL } \\
\text { (UG/L) }\end{array}$ & $\begin{array}{l}\text { PHENAN- } \\
\text { THRENE } \\
\text { TOTAL } \\
(U G / L)\end{array}$ & $\begin{array}{c}\text { PHENOL } \\
\text { (C6H- } \\
5 O H) \\
\text { TOTAL } \\
\text { (UG/L) }\end{array}$ & $\begin{array}{c}\text { PYRENE } \\
\text { TOTAL } \\
\text { (UG/L) }\end{array}$ & $\begin{array}{c}2,4,6- \\
\text { TRI- } \\
\text { CHLORO- } \\
\text { PHENOL } \\
\text { TOTAL } \\
\text { (UG/L) }\end{array}$ & $\begin{array}{c}\text { ALDRIN, } \\
\text { TOTAL } \\
\text { (UG/L) }\end{array}$ & $\begin{array}{l}\text { P, P' } \\
\text { DDT, } \\
\text { TOTAL } \\
\text { (UG/L) }\end{array}$ & $\begin{array}{l}\text { ALPHA } \\
\text { BHC } \\
\text { TOTAL } \\
\text { (UG/L) }\end{array}$ & $\begin{array}{c}\text { BETA } \\
\text { BENZENE } \\
\text { HEXA- } \\
\text { CHLOR- } \\
\text { IDE } \\
\text { TOTAL } \\
\text { (UG/L) }\end{array}$ & $\begin{array}{l}\text { DELTA } \\
\text { BENZENE } \\
\text { HEXA- } \\
\text { CHLOR- } \\
\text { IDE } \\
\text { TOTAL } \\
\text { (UG/L) }\end{array}$ & $\begin{array}{l}\text { LINDANE } \\
\text { TOTAL } \\
\text { (UG } / L \text { ) }\end{array}$ \\
\hline OCT 1994 & & & & & & & & & & & & \\
\hline $\begin{array}{l}07.07 \\
\text { OCT }\end{array}$ & $<5.0$ & $<30.0$ & $<5.0$ & $<5.0$ & $<5.0$ & $<20.0$ & $<0.040$ & $<0.10$ & $<0.03$ & $<0.03$ & $<0.09$ & $<0.030$ \\
\hline $\begin{array}{l}24-24 \\
\text { NOV }\end{array}$ & $<5.0$ & $<30.0$ & $<5.0$ & $<5.0$ & $<5.0$ & $<20.0$ & $<0.040$ & $<0.10$ & $<0.03$ & $<0.03$ & $<0.09$ & $<0.030$ \\
\hline $\begin{array}{l}02-03 \\
\mathrm{NoV}\end{array}$ & $<5.0$ & $<30.0$ & $<5.0$ & $<5.0$ & $<5.0$ & $<20.0$ & $<0.040$ & $<0.10$ & $<0.03$ & $<0.03$ & $<0.09$ & $<0.030$ \\
\hline $09-09$ & $<5.0$ & $<30.0$ & $<5.0$ & $<5.0$ & $<5.0$ & $<20.0$ & $<0.040$ & $<0.10$ & $<0.03$ & $<0.03$ & $<0.09$ & $<0.030$ \\
\hline
\end{tabular}

\begin{tabular}{|c|c|c|c|c|c|c|c|c|c|c|}
\hline $\begin{array}{l}\text { CHLOR - } \\
\text { DANE }\end{array}$ & $\begin{array}{l}\text { CHLOR - } \\
\text { DANE }\end{array}$ & & & & & $\begin{array}{l}\text { ENDO- } \\
\text { SULFAN- }\end{array}$ & & & & \\
\hline CIS & TRANS & & & & & I & ENDO- & ENDO- & ENDRIN & ENDRIN \\
\hline WATER & WATER & CHLOR - & $P, P^{\prime}$ & $P, P^{\prime}$ & & WATER & SULFAN & SULFAN & WATER & ALDE- \\
\hline WHOLE & WHOLE & DANE, & DDD, & DDE, & ELDRIN & WHOLE & BETA & SULFATE & UNFLTRD & HYDE \\
\hline $\begin{array}{l}\text { TOTAL } \\
\text { (UG/L) }\end{array}$ & $\begin{array}{l}\text { TOTAL } \\
\text { (UG/L) }\end{array}$ & $\begin{array}{l}\text { TOTAL } \\
\text { (UG/L) }\end{array}$ & $\begin{array}{l}\text { TOTAL } \\
\text { (UG/L) }\end{array}$ & $\begin{array}{l}\text { TOTAL } \\
\text { (UG/L) }\end{array}$ & $\begin{array}{l}\text { TOTAL } \\
\text { (UG/L) }\end{array}$ & $\begin{array}{l}\text { REC } \\
(U G / L)\end{array}$ & $\begin{array}{l}\text { TOTAL } \\
\text { (UG/L) }\end{array}$ & $\begin{array}{l}\text { TOTAL } \\
(U G / L)\end{array}$ & $\begin{array}{c}\mathrm{REC} \\
(\mathrm{UG} / \mathrm{L}\rangle\end{array}$ & $\begin{array}{l}\text { TOTAL } \\
\text { (UG/L) }\end{array}$ \\
\hline
\end{tabular}

\begin{tabular}{|c|c|c|c|c|c|c|c|c|c|c|c|}
\hline $\begin{array}{l}\text { OCT } 1994 \\
07-07 \\
\text { OCT }\end{array}$ & $<0.10$ & $<0.10$ & $<0.1$ & $<0.10$ & $<0.04$ & $<0.020$ & $<0.10$ & $<0.04$ & $<0.60$ & $<0.060$ & $<0.20$ \\
\hline $\begin{array}{l}24-24 \\
\text { NoV }\end{array}$ & $<0.10$ & $<0.10$ & $<0.1$ & $<0.10$ & $<0.04$ & $<0.020$ & $<0.10$ & $<0.04$ & $<0.60$ & $<0.060$ & $<0.20$ \\
\hline $\begin{array}{l}02 \cdot 03 \\
\text { Nov }\end{array}$ & $<0.10$ & $<0.10$ & $<0.1$ & $<0.10$ & $<0.04$ & $<0.020$ & $<0.10$ & $<0.04$ & $<0.60$ & $<0.060$ & $<0.20$ \\
\hline $09-09$ & $<0.10$ & $<0.10$ & $<0.1$ & $<0.10$ & $<0.04$ & $<0.020$ & $<0.10$ & $<0.04$ & $<0.60$ & $<0.060$ & $<0.20$ \\
\hline DATE & $\begin{array}{l}\text { HEPTA - } \\
\text { CHLOR, } \\
\text { TOTAL } \\
\text { (UG/L) }\end{array}$ & $\begin{array}{l}\text { HEPTA - } \\
\text { CHLOR } \\
\text { EPOXIDE } \\
\text { TOTAL } \\
\text { (UG/L) }\end{array}$ & $\begin{array}{c}\text { TOX - } \\
\text { APHENE, } \\
\text { TOTAL } \\
\text { (UG /L) }\end{array}$ & $\begin{array}{c}\text { AROCLOR } \\
1016 \\
\text { PCB } \\
\text { TOTAL } \\
\text { (UG/L) }\end{array}$ & $\begin{array}{c}\text { AROCLOR } \\
1221 \\
\text { PCB } \\
\text { TOTAL } \\
\text { (UG/L) }\end{array}$ & $\begin{array}{l}\text { AROCLOR } \\
1232 \\
\text { PCB } \\
\text { TOTAL } \\
\text { (UG/L) }\end{array}$ & $\begin{array}{c}\text { AROCLOR } \\
1242 \\
\text { PCB } \\
\text { TOTAL } \\
\text { (UG/L) }\end{array}$ & $\begin{array}{c}\text { AROCLOR } \\
1248 \\
\text { PCB } \\
\text { TOTAL } \\
(U G / L)\end{array}$ & $\begin{array}{c}\text { AROCLOR } \\
1254 \\
\text { PCB } \\
\text { TOTAL } \\
(U G / L)\end{array}$ & $\begin{array}{c}\text { AROCLOR } \\
1260 \\
\text { PCB } \\
\text { TOTAL } \\
\text { (UG/L) }\end{array}$ & $\begin{array}{c}\text { DI - } \\
\text { AZINON, } \\
\text { TOTAL } \\
\text { (UG/L) }\end{array}$ \\
\hline
\end{tabular}

\begin{tabular}{|c|c|c|c|c|c|c|c|c|c|c|c|}
\hline $\begin{array}{c}\text { OCT } 1994 \\
07-07\end{array}$ & $<0.030$ & $<0.80$ & $<2$ & $<0.1$ & $<1.0$ & $<0.1$ & $<0.1$ & $<0.1$ & $<0.1$ & $<0,1$ & $<0.05$ \\
\hline OCT & & & & & & & & & & & \\
\hline $\begin{array}{l}24-24 \\
\text { Nov }\end{array}$ & $<0.030$ & $<0.80$ & $<2$ & $<0.1$ & $<1.0$ & $<0.1$ & $<0.1$ & $<0.1$ & $<0.1$ & $<0.1$ & $<0.05$ \\
\hline $\begin{array}{l}02 \cdot 03 \\
\text { Nov }\end{array}$ & $<0.030$ & $<0.80$ & $<2$ & $<0.1$ & $<1.0$ & $<0.1$ & $<0.1$ & $<0.1$ & $<0.1$ & $<0.1$ & $<0.05$ \\
\hline 09.09 & $<0.030$ & $<0.80$ & $<2$ & $<0.1$ & $<1.0$ & $<0.1$ & $<0.1$ & $<0.1$ & $<0.1$ & $<0.1$ & $<0.05$ \\
\hline
\end{tabular}


Table 9. Water-quality data for storm-sewer outfall stations, Texas Department of Transportation, Dallas and Fort Worth Districts, 1993-94-Continued

08055690 - BACHMAN BRANCH OUTFALL AT I-635, DALLAS, TX (WY 1994)

\begin{tabular}{|c|c|c|c|c|c|c|c|c|c|c|c|c|}
\hline DATE & TIME & $\begin{array}{l}\text { PRECIP- } \\
\text { ITATION } \\
\text { TOTAL } \\
\text { INCHES/ } \\
\text { STORM }\end{array}$ & $\begin{array}{l}\text { ELAPSED } \\
\text { TIME } \\
\text { OF } \\
\text { STORM } \\
\text { (HOURS) }\end{array}$ & $\begin{array}{l}\text { STORM } \\
\text { WATER } \\
\text { FLOW } \\
\text { (MGD) }\end{array}$ & $\begin{array}{l}\text { SPE- } \\
\text { CIFIC } \\
\text { CON- } \\
\text { DUCT- } \\
\text { ANCE } \\
\text { (US/CM) }\end{array}$ & $\begin{array}{c}\text { SPE- } \\
\text { CIFIC } \\
\text { CON- } \\
\text { DUCT- } \\
\text { ANCE } \\
\text { LAB } \\
\text { (US/CM) }\end{array}$ & $\begin{array}{l}\text { PH } \\
\text { WATER } \\
\text { WHOLE } \\
\text { FIELD } \\
\text { (STAND- } \\
\text { ARD } \\
\text { UNITS) }\end{array}$ & $\begin{array}{c}\text { PH } \\
\text { WATER } \\
\text { WHOLE } \\
\text { LAB } \\
\text { (STAND- } \\
\text { ARD } \\
\text { UNITS) }\end{array}$ & $\begin{array}{c}\text { TEMPER - } \\
\text { ATURE } \\
\text { WATER } \\
\text { (DEG C) }\end{array}$ & $\begin{array}{c}\text { OXYGEN } \\
\text { DEMAND, } \\
\text { CHEM- } \\
\text { ICAL } \\
\text { (HIGH } \\
\text { LEVEL) } \\
\text { (MG/L) }\end{array}$ & $\begin{array}{l}\text { OXYGEN } \\
\text { DEMAND, } \\
\text { BIO- } \\
\text { CHEM- } \\
\text { ICAL, } \\
5 \text { DAY } \\
\text { (MG/L) }\end{array}$ & $\begin{array}{l}\text { COLI- } \\
\text { FORM, } \\
\text { FECAL, } \\
0.7 \\
\text { UM-MF } \\
\text { (COLS. / } \\
100 \mathrm{ML} \text { ) }\end{array}$ \\
\hline DEC 1993 & & & & & & & & & & & & \\
\hline $\begin{array}{c}02-02 \\
\text { JAN } 1994\end{array}$ & 1652 & 0.20 & 2.6 & 0.06 & 307 & 306 & 8.0 & 7.5 & 18.0 & 99 & 10 & 120000 \\
\hline${ }_{\mathrm{FEB}}^{11-11}$ & 0142 & 0.22 & 8.3 & 0.04 & 286 & 308 & 7.8 & 6.9 & 12.0 & 93 & 8.7 & K53000 \\
\hline $\begin{array}{l}28-28 \\
\text { MAR }\end{array}$ & 1828 & 0.39 & 3.5 & 0.08 & 658 & 159 & 7.9 & 7.6 & 11.5 & 50 & 7.2 & 34000 \\
\hline $\begin{array}{l}\quad 08-08 \\
\text { APR }\end{array}$ & 1145 & 0.32 & 3.8 & 0.08 & 140 & 191 & 8.3 & 7.3 & 11.5 & 65 & 7.3 & K860000 \\
\hline $\operatorname{MAY}^{11-11}$ & 1327 & 0.30 & 1.3 & 0.06 & 231 & 198 & 7.5 & 6.6 & 20.0 & 84 & 10 & K620000 \\
\hline $\begin{array}{l}09-09 \\
\text { AUG }\end{array}$ & 1335 & 0.23 & 3.4 & 0.04 & 261 & $\cdots$ & 7.5 & $\cdots$ & 19.5 & $<10$ & 5.2 & K3200000 \\
\hline $05-05$ & 0801 & 0.31 & 1.5 & 0.08 & 390 & 343 & 6.7 & 7.1 & 24.5 & 93 & 8.3 & 250000 \\
\hline
\end{tabular}

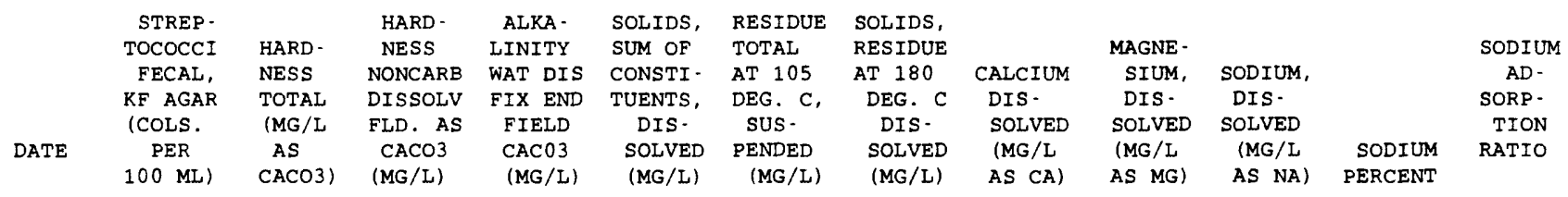

\begin{tabular}{|c|c|c|c|c|c|c|c|c|c|c|c|c|}
\hline DEC 1993 & & & & & & & & & & & & \\
\hline 02.02 & 100000 & 82 & 12 & 70 & 167 & 136 & 184 & 31 & 1.2 & 21 & 34 & 1 \\
\hline JAN 1994 & & & & & & & & & & & & \\
\hline${ }_{\text {FEB }}^{11-11}$ & 54000 & 100 & 62 & 41 & 167 & 42 & 185 & 39 & 1.3 & 9.5 & 16 & 0.4 \\
\hline $28 \cdot 28$ & 16000 & 47 & 9 & 38 & 87 & 44 & 94 & 18 & 0.61 & 6.7 & 22 & 0.4 \\
\hline MAR & & & & & & & & & & & & \\
\hline $08-08$ & 20000 & 50 & 6 & 44 & 93 & 104 & 92 & 19 & 0.63 & 5.5 & 18 & 0.3 \\
\hline APR & & & & & & & & & & & & \\
\hline $11-11$ & 310000 & 63 & 35 & 28 & 103 & 60 & 125 & 24 & 0.80 & 5.2 & 14 & 0.3 \\
\hline MAY & & & & & & & & & & & & \\
\hline 09.09 & 840000 & $\cdots$ & & 64 & - & 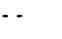 & - & - & $\cdots$ & $\cdots$ & & - - \\
\hline $\begin{array}{l}\text { AUG } \\
05-05\end{array}$ & $\mathrm{~K} 180000$ & 140 & . & - & 198 & 17 & 229 & 53 & 1.7 & 8.4 & 11 & 0.3 \\
\hline
\end{tabular}

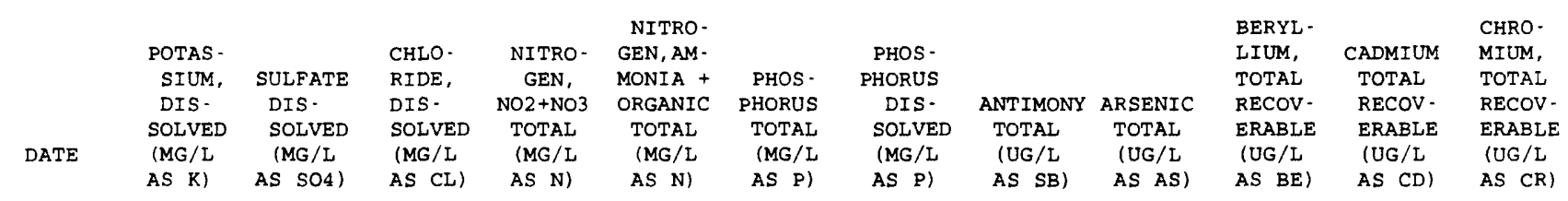

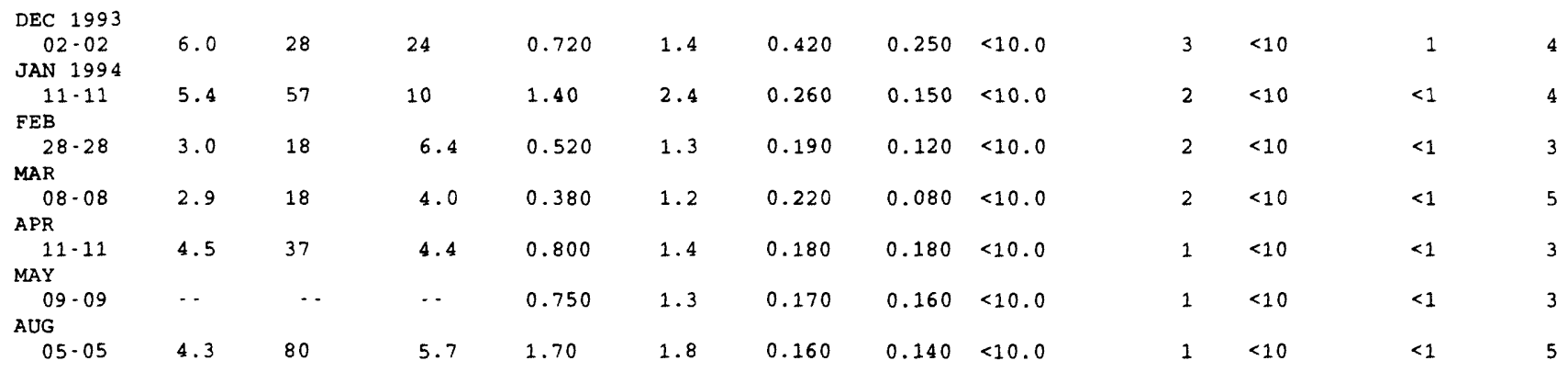


Table 9. Water-quality data for storm-sewer outfall stations, Texas Department of Transportation, Dallas and Fort Worth Districts, 1993-94-Continued

08055690 - BACHMAN BRANCH OUTFALL AT I.635, DALLAS, TX (WY 1994)-Continued

\begin{tabular}{|c|c|c|c|c|c|c|c|c|c|c|c|c|}
\hline & $\begin{array}{c}\text { COPPER, } \\
\text { TOTAL } \\
\text { RECOV } \\
\text { ERABLE } \\
\text { (UG/L }\end{array}$ & $\begin{array}{c}\text { CYANIDE, } \\
\text { TOTAL } \\
\text { EPA } \\
\text { (MG/L }\end{array}$ & $\begin{array}{l}\text { CYANIDE } \\
\text { TOTAL } \\
\text { (MG/L }\end{array}$ & $\begin{array}{l}\text { LEAD, } \\
\text { TOTAL } \\
\text { RECOV- } \\
\text { ERABLE } \\
\text { IUG/L }\end{array}$ & $\begin{array}{l}\text { MERCURY } \\
\text { TOTAL } \\
\text { RECOV - } \\
\text { ERABLE } \\
\text { (UG/L }\end{array}$ & $\begin{array}{c}\text { NICKEL, } \\
\text { TOTAL } \\
\text { RECOV- } \\
\text { ERABLE } \\
\text { (UG/L }\end{array}$ & $\begin{array}{l}\text { SELE- } \\
\text { NIUM, } \\
\text { TOTAL } \\
\text { (UG/L }\end{array}$ & $\begin{array}{l}\text { SILVER, } \\
\text { TOTAL } \\
\text { RECOV- } \\
\text { ERABLE } \\
\text { (UG/L }\end{array}$ & $\begin{array}{l}\text { SILVER, } \\
\text { TOTAL } \\
\text { RECOV- } \\
\text { ERABLE } \\
\text { ERA } \\
\text { (UG/L }\end{array}$ & $\begin{array}{l}\text { THAL- } \\
\text { LIUM, } \\
\text { TOTAL } \\
\text { (UG/L }\end{array}$ & $\begin{array}{l}\text { ZINC, } \\
\text { TOTAL } \\
\text { RECOV - } \\
\text { ERABLE } \\
\text { (UG/L }\end{array}$ & $\begin{array}{c}\text { CARBON, } \\
\text { ORGANIC } \\
\text { TOTAL } \\
\text { (MG/L }\end{array}$ \\
\hline & AS CU) & AS $\mathrm{CN}$ ) & AS CN) & AS PB) & AS HG) & AS NI) & AS SE) & AS AG) & AS AG) & AS TL) & AS $\quad \mathrm{ZN})$ & AS C) \\
\hline
\end{tabular}

DEC 1993

$02-02$

JAN 1994

$11 \cdot 11$

FEB

$28 \cdot 28$

$M A R$

$08-08$

APR

11- 11

MAY

$09-09$

AUG

05-05

$\begin{array}{lll}20 & <0.010 & <0.010 \\ 16 & <0.010 & <0.010 \\ 13 & <0.010 & <0.010 \\ 17 & <0.010 & <0.010 \\ 14 & 0.052 & <0.010 \\ 23 & <0.010 & 0.010 \\ 17 & <0.010 & <0.010\end{array}$

$\begin{aligned} 26 & <0.10 \\ 13 & <0.10 \\ 11 & <0.10 \\ 22 & <0.10 \\ 9 & <0.10 \\ 6 & <0.10 \\ 10 & <0.10\end{aligned}$

NI) AS SE

AS AG)

AS AG) AS TL)

AS $\mathrm{ZN})$ AS C)

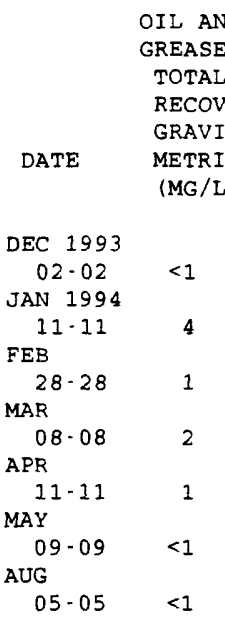

IL AND

REASE,

TOTAL

PHENOLS ACRO ACRYLO-

METRIC TOTAL TOTAL

(UG/L) (UG/L)

\begin{tabular}{|c|c|}
\hline ACRYLO- & \\
\hline NITRILE & BENZENE \\
\hline $\begin{array}{l}\text { TOTAL } \\
\text { (UG/L) }\end{array}$ & $\begin{array}{l}\text { TOTAL } \\
\text { (UG/L) }\end{array}$ \\
\hline
\end{tabular}

$\begin{array}{lc} & \text { METHANE } \\ \text { BROMO- } & \text { BROMO } \\ \text { BENZENE } & \text { CHLORO- } \\ \text { WATER, } & \text { WAT } \\ \text { WHOLE, } & \text { UNFLTRD } \\ \text { TOTAL } & \text { REC } \\ \text { (UG/L) } & \text { (UG/L) }\end{array}$

$\begin{array}{lr}<1 & <0.500 \\ <1 & 0.710 \\ <1 & <0.500 \\ <1 & <0.500 \\ <1 & <0.500 \\ <1 & <0.500 \\ <1 & <1.00\end{array}$

$\begin{array}{rrr}<10 & 240 & 25 \\ <10 & 100 & 24 \\ <5 & 80 & 13 \\ <5 & 120 & 16 \\ <10 & 80 & 23 \\ <10 & 50 & 20 \\ <5 & 120 & 24\end{array}$

$\begin{array}{rrrrrrrrrrr}4 & <20 & <20 & <0.2 & <0.2 & <0.20 & <0.2 & <0.20 & <0.20 & <0.20 & <0.2 \\ 12 & <20 & <20 & <0.2 & <0.2 & <0.20 & <0.2 & <0.20 & <0.20 & <0.20 & <0.2 \\ 12 & <20 & <20 & <0.2 & <0.2 & <0.20 & <0.2 & <0.20 & <0.20 & <0.20 & <0.2 \\ 2 & <20 & <20 & <0.2 & <0.2 & <0.20 & <0.2 & <0.20 & <0.20 & <0.20 & <0.2 \\ 8 & <20 & <20 & <0.2 & <0.2 & <0.20 & <0.2 & <0.20 & <0.20 & <0.20 & <0.2 \\ 16 & <20 & <20 & <0.2 & <0.2 & <0.20 & <0.2 & <0.20 & <0.20 & <0.20 & <0.2 \\ 9 & <20 & <20 & <0.2 & <0.2 & <0.20 & <0.2 & <0.20 & <0.20 & <0.20 & <0.2\end{array}$

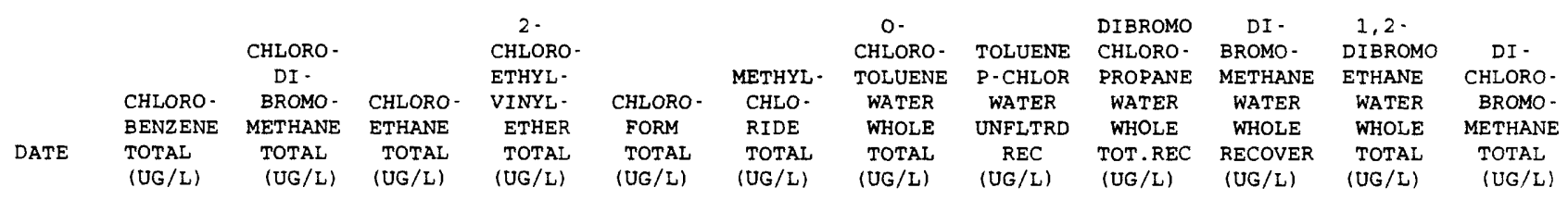

\begin{tabular}{|c|c|c|c|c|c|c|c|c|c|c|c|c|}
\hline $02 \cdot 02$ & $<0.20$ & $<0.2$ & $<0.2$ & $<1.0$ & $<0.2$ & $<0.2$ & $<0.2$ & $<0.20$ & $<1.0$ & $<0.2$ & $<0.2$ & $<0.2$ \\
\hline \multicolumn{13}{|l|}{ JAN 1994} \\
\hline $11 \cdot 11$ & $<0.20$ & $<0.2$ & $<0.2$ & $<1.0$ & $<0.2$ & $<0.2$ & $<0.2$ & $<0.20$ & $<1.0$ & $<0.2$ & $<0.2$ & $<0.2$ \\
\hline \multicolumn{13}{|l|}{ FEB } \\
\hline $28-28$ & $<0.20$ & $<0.2$ & $<0.2$ & $<1.0$ & $<0.2$ & $<0.2$ & $<0.2$ & $<0.20$ & $<1.0$ & $<0.2$ & $<0.2$ & $<0.2$ \\
\hline \multicolumn{13}{|l|}{ MAR } \\
\hline 08.08 & $<0.20$ & $<0.2$ & $<0.2$ & $<1.0$ & $<0.2$ & $<0.2$ & $<0.2$ & $<0.20$ & $<1.0$ & $<0.2$ & $<0.2$ & $<0.2$ \\
\hline \multicolumn{13}{|l|}{ APR } \\
\hline $11-11$ & $<0.20$ & $<0.2$ & $<0.2$ & $<1.0$ & $<0.2$ & $<0.2$ & $<0.2$ & $<0.20$ & $<1.0$ & $<0.2$ & $<0.2$ & $<0.2$ \\
\hline \multicolumn{13}{|l|}{ MAY } \\
\hline $09-09$ & $<0.20$ & $<0.2$ & $<0.2$ & $<1.0$ & $<0.2$ & $<0.2$ & $<0.2$ & $<0.20$ & $<1.0$ & $<0.2$ & $<0.2$ & $<0.2$ \\
\hline \multicolumn{13}{|l|}{ AUG } \\
\hline $05-05$ & $<0.20$ & $<0.2$ & $<0.2$ & $<1.0$ & $<0.2$ & 4.3 & $<0.2$ & $<0.20$ & $<1.0$ & $<0.2$ & $<0.2$ & $<0.2$ \\
\hline
\end{tabular}


Table 9. Water-quality data for storm-sewer outfall stations, Texas Department of Transportation, Dallas and Fort Worth Districts, 1993-94-Continued

08055690 - BACHMAN BRANCH OUTFALL AT I-635, DALLAS, TX (WY 1994)-Continued

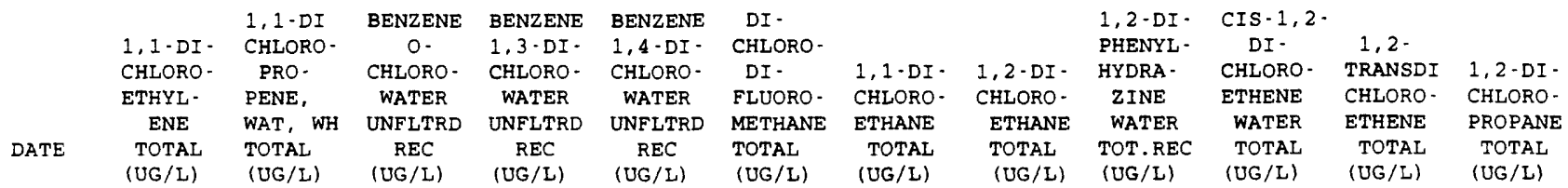

\begin{tabular}{|c|c|c|c|c|c|c|c|c|c|c|c|c|}
\hline DEC 1993 & & & & & & & & & & & & \\
\hline $\begin{array}{r}02-02 \\
\end{array}$ & $<0.2$ & $<0.2$ & $<0.20$ & $<0.20$ & $<0.20$ & $<0.2$ & $<0.2$ & $<0.2$ & $<5.0$ & $<0.2$ & $<0.2$ & $<0.2$ \\
\hline $11 \cdot 11$ & $<0.2$ & $<0.2$ & $<5.0$ & $<5.0$ & $<5.0$ & $<0.2$ & $<0.2$ & $<0.2$ & $<5.0$ & $<0.2$ & $<0.2$ & $<0.2$ \\
\hline FEB & & & & & & & & & & & & \\
\hline $28 \cdot 28$ & $<0.2$ & $<0.2$ & $<5.0$ & $<5.0$ & $<5.0$ & $<0.2$ & $<0.2$ & $<0.2$ & $<5.0$ & $<0.2$ & $<0.2$ & $<0.2$ \\
\hline $\begin{array}{l}\text { MAR } \\
\quad 08.08\end{array}$ & $<0.2$ & $<0.2$ & $<5.0$ & $<5.0$ & $<5.0$ & $<0.2$ & $<0.2$ & $<0.2$ & $<5.0$ & $<0.2$ & $<0.2$ & $<0.2$ \\
\hline $\begin{array}{l}\text { APR } \\
\quad 11-11\end{array}$ & $<0.2$ & $<0.2$ & $<5.0$ & $<5.0$ & $<5.0$ & $<0.2$ & $<0.2$ & $<0.2$ & $<5.0$ & $<0.2$ & $<0.2$ & $<0.2$ \\
\hline $\begin{array}{l}\text { MAY } \\
09-09\end{array}$ & $<0.2$ & $<0.2$ & $<5.0$ & $<5.0$ & $<5.0$ & $<0.2$ & $<0.2$ & $<0.2$ & $<5.0$ & $<0.2$ & $<0.2$ & $<0.2$ \\
\hline $\begin{array}{l}\text { AUG } \\
05-05\end{array}$ & $<0.2$ & $<0.2$ & $<5.0$ & $<5.0$ & $<5.0$ & $<0.2$ & $<0.2$ & $<0.2$ & $<5.0$ & $<0.2$ & $<0.2$ & $<0.2$ \\
\hline
\end{tabular}

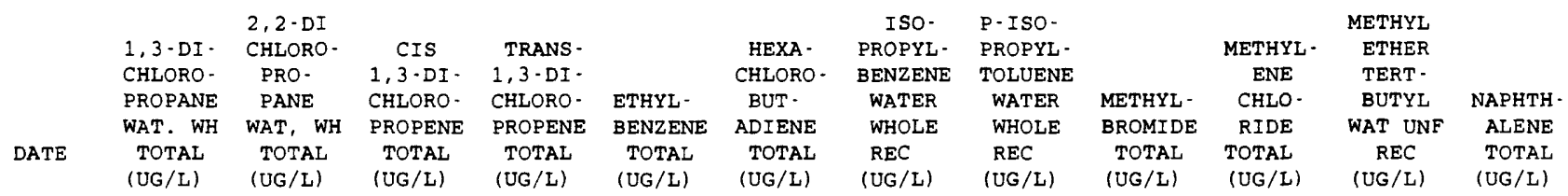

DEC 1993

\begin{tabular}{|c|c|c|c|c|c|c|c|c|c|c|c|c|}
\hline 02.02 & $<0.2$ & $<0.2$ & $<0.2$ & $<0.2$ & $<0.2$ & $<0.2$ & $<0.20$ & $<0.20$ & $<0.2$ & $<0.2$ & $<1.0$ & $<0.2$ \\
\hline JAN 1994 & & & & & & & & & & & & \\
\hline $11-11$ & $<0.2$ & $<0.2$ & $<0.2$ & $<0.2$ & $<0.2$ & $<5.0$ & $<0.20$ & $<0.20$ & $<0.2$ & $<0.2$ & $<1.0$ & $<5.0$ \\
\hline${ }_{\text {MAR }}^{28 \cdot 28}$ & $<0.2$ & $<0.2$ & $<0.2$ & $<0.2$ & $<0.2$ & $<5.0$ & $<0.20$ & $<0.20$ & $<0.2$ & $<0.2$ & $<1.0$ & $<5.0$ \\
\hline $\begin{array}{l}08.08 \\
\text { APR }\end{array}$ & $<0.2$ & $<0.2$ & $<0.2$ & $<0.2$ & $<0.2$ & $<5.0$ & $<0.20$ & $<0.20$ & $<0.2$ & $<0.2$ & $<1.0$ & $<5.0$ \\
\hline $\operatorname{MAY}^{11-11}$ & $<0.2$ & $<0.2$ & $<0.2$ & $<0.2$ & $<0.2$ & $<5.0$ & $<0.20$ & $<0.20$ & $<0.2$ & $<0.2$ & $<0.2$ & $<5.0$ \\
\hline $\begin{array}{l}09-09 \\
\text { AUG }\end{array}$ & $<0.2$ & $<0.2$ & $<0.2$ & $<0.2$ & $<0.2$ & $<5.0$ & $<0.20$ & $<0.20$ & $<0.2$ & $<0.2$ & $<0.2$ & $<5.0$ \\
\hline $05-05$ & $<0.2$ & $<0.2$ & $<0.2$ & $<0.2$ & $<0.2$ & $<5.0$ & $<0.20$ & $<0.20$ & $<0.2$ & $<0.2$ & $<0.2$ & $<5.0$ \\
\hline
\end{tabular}

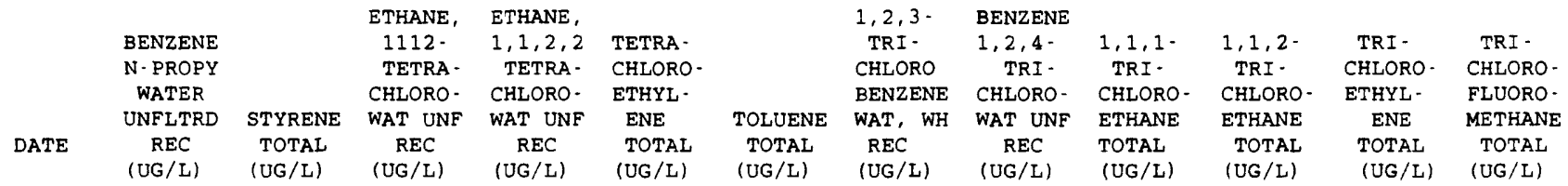

\begin{tabular}{|c|c|c|c|c|c|c|c|c|c|c|c|c|}
\hline DEC 1993 & & & & & & & & & & & & \\
\hline $\begin{array}{c}02-02 \\
\text { JAN } 1994\end{array}$ & $<0.20$ & $<0.2$ & $<0.2$ & $<0.2$ & $<0.2$ & $<0.2$ & $<0.20$ & $<0.20$ & $<0.2$ & $<0.2$ & $<0.2$ & $<0.2$ \\
\hline $11-11$ & $<0.20$ & $<0.2$ & $<0.2$ & $<0.2$ & $<0.2$ & $<0.2$ & $<0.20$ & $<5.0$ & $<0.2$ & $<0.2$ & $<0.2$ & $<0.2$ \\
\hline FEB & & & & & & & & & & & & \\
\hline $28-28$ & $<0.20$ & $<0.2$ & $<0.2$ & $<0.2$ & $<0.2$ & 0.2 & $<0.20$ & $<5.0$ & $<0.2$ & $<0.2$ & $<0.2$ & $<0.2$ \\
\hline MAR & & & & & & & & & & & & \\
\hline 08.08 & $<0.20$ & $<0.2$ & $<0.2$ & $<0.2$ & $<0.2$ & 0.2 & $<0.20$ & $<5.0$ & $<0.2$ & $<0.2$ & $<0.2$ & $<0.2$ \\
\hline APR & & & & & & & & & & & & \\
\hline $11-11$ & $<0.20$ & $<0.2$ & $<0.2$ & $<0.2$ & $<0.2$ & $<0.2$ & $<0.20$ & $<5.0$ & $<0.2$ & $<0.2$ & $<0.2$ & $<0.2$ \\
\hline MAY & & & & & & & & & & & & \\
\hline 09.09 & $<0.20$ & $<0.2$ & $<0.2$ & $<0.2$ & $<0.2$ & $<0.2$ & $<0.20$ & $<5.0$ & $<0.2$ & $<0.2$ & $<0.2$ & $<0.2$ \\
\hline AUG & & & & & & & & & & & & \\
\hline $05 \cdot 05$ & $<0.20$ & $<0.2$ & $<0.2$ & $<0.2$ & $<0.2$ & $<0.2$ & $<0.20$ & $<5.0$ & $<0.2$ & $<0.2$ & $<0.2$ & $<0.2$ \\
\hline
\end{tabular}


Table 9. Water-quality data for storm-sewer outfall stations, Texas Department of Transportation, Dallas and Fort Worth Districts, 1993-94-Continued

08055690 - BACHMAN BRANCH OUTFALL AT I-635, DALLAS, TX (WY 1994)-Continued

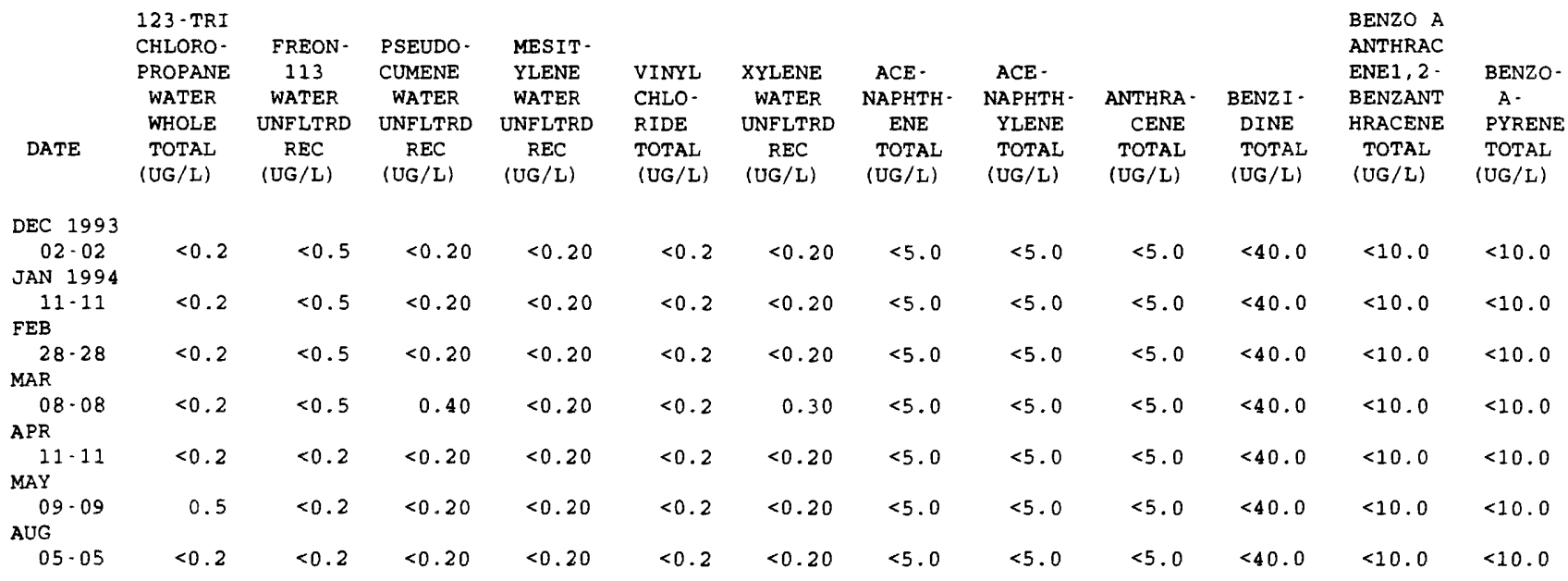

\begin{tabular}{|c|c|c|c|c|c|c|c|c|c|c|c|c|}
\hline DATE & $\begin{array}{l}\text { BENZO B } \\
\text { FLUOR- } \\
\text { AN- } \\
\text { THENE } \\
\text { TOTAL } \\
\text { (UG/L) }\end{array}$ & $\begin{array}{l}\text { BENZO K } \\
\text { FLUOR- } \\
\text { AN- } \\
\text { THENE } \\
\text { TOTAL } \\
\text { (UG/L) }\end{array}$ & $\begin{array}{l}\text { BENZO } \\
\text { GHI PERYL } \\
\text { ENE1, 12- } \\
\text { BENZO } \\
\text { PERYLENE } \\
\text { TOTAL } \\
\text { (UG/L) }\end{array}$ & $\begin{array}{c}4 \text { - } \\
\text { BROMO- } \\
\text { PHENYL } \\
\text { PHENYL } \\
\text { ETHER } \\
\text { TOTAL } \\
\text { (UG/L) }\end{array}$ & $\begin{array}{l}\text { N-BUTYL } \\
\text { BENZYL } \\
\text { PHTHAL- } \\
\text { ATE } \\
\text { TOTAL } \\
\text { (UG/L) }\end{array}$ & $\begin{array}{c}\text { BIS } \\
\text { (2- } \\
\text { CHLORO- } \\
\text { ETHOXY) } \\
\text { METHANE } \\
\text { TOTAL } \\
\text { (UG/L) }\end{array}$ & $\begin{array}{l}\text { BIS } \\
2 \cdot \\
\text { CHLORO- } \\
\text { ETHYL } \\
\text { ETHER } \\
\text { TOTAL } \\
\text { (UG/L) }\end{array}$ & $\begin{array}{l}\text { BIS } 12- \\
\text { CHLORO- } \\
\text { ISO- } \\
\text { PROPYL) } \\
\text { ETHER } \\
\text { TOTAL } \\
\text { (UG/L) }\end{array}$ & $\begin{array}{l}\text { PARA- } \\
\text { CHLORO- } \\
\text { META } \\
\text { CRESOL } \\
\text { TOTAL } \\
\text { (UG/L) }\end{array}$ & $\begin{array}{c}2- \\
\text { CHLORO- } \\
\text { NAPH- } \\
\text { THALENE } \\
\text { TOTAL } \\
\text { (UG/L) }\end{array}$ & $\begin{array}{l}2 \text { - } \\
\text { CHLORO- } \\
\text { PHENOL } \\
\text { TOTAL } \\
\text { (UG/L) }\end{array}$ & $\begin{array}{l}4 . \\
\text { CHLORO- } \\
\text { PHENYL } \\
\text { PHENYL } \\
\text { ETHER } \\
\text { TOTAL } \\
\text { (UG/L) }\end{array}$ \\
\hline DEC 1993 & & & & & & & & & & & & \\
\hline $\begin{array}{c}02-02 \\
\text { JAN } 1994\end{array}$ & $<10.0$ & $<10.0$ & $<10.0$ & $<5.0$ & $<5.0$ & $<5.0$ & $<5.0$ & $<5.0$ & $<30.0$ & $<5.0$ & $<5.0$ & $<5.0$ \\
\hline $\begin{array}{l}11-11 \\
\text { FEB }\end{array}$ & $<10.0$ & $<10.0$ & $<10.0$ & $<5.0$ & $<5.0$ & $<5.0$ & $<5.0$ & $<5.0$ & $<30.0$ & $<5.0$ & $<5.0$ & $<5.0$ \\
\hline $\begin{array}{l}28-28 \\
\text { MAR }\end{array}$ & $<10.0$ & $<10.0$ & $<10.0$ & $<5.0$ & $<5.0$ & $<5.0$ & $<5.0$ & $<5.0$ & $<30.0$ & $<5.0$ & $<5.0$ & $<5.0$ \\
\hline $\begin{array}{l}08.08 \\
\text { APR }\end{array}$ & $<10.0$ & $<10.0$ & $<10.0$ & $<5.0$ & $<5.0$ & $<5.0$ & $<5.0$ & $<5.0$ & $<30.0$ & $<5.0$ & $<5.0$ & $<5.0$ \\
\hline $\begin{array}{l}11-11 \\
\text { MAY }\end{array}$ & $<10.0$ & $<10.0$ & $<10.0$ & $<5.0$ & $<5.0$ & $<5.0$ & $<5.0$ & $<5.0$ & $<30.0$ & $<5.0$ & $<5.0$ & $<5.0$ \\
\hline $\begin{array}{l}09.09 \\
\text { AUG }\end{array}$ & $<10.0$ & $<10.0$ & $<10.0$ & $<5.0$ & $<5.0$ & $<5.0$ & $<5.0$ & $<5.0$ & $<30.0$ & $<5.0$ & $<5.0$ & $<5.0$ \\
\hline $05-05$ & $<10.0$ & $<10.0$ & $<10.0$ & $<5.0$ & $<5.0$ & $<5.0$ & $<5.0$ & $<5.0$ & $<30.0$ & $<5.0$ & $<5.0$ & $<5.0$ \\
\hline
\end{tabular}

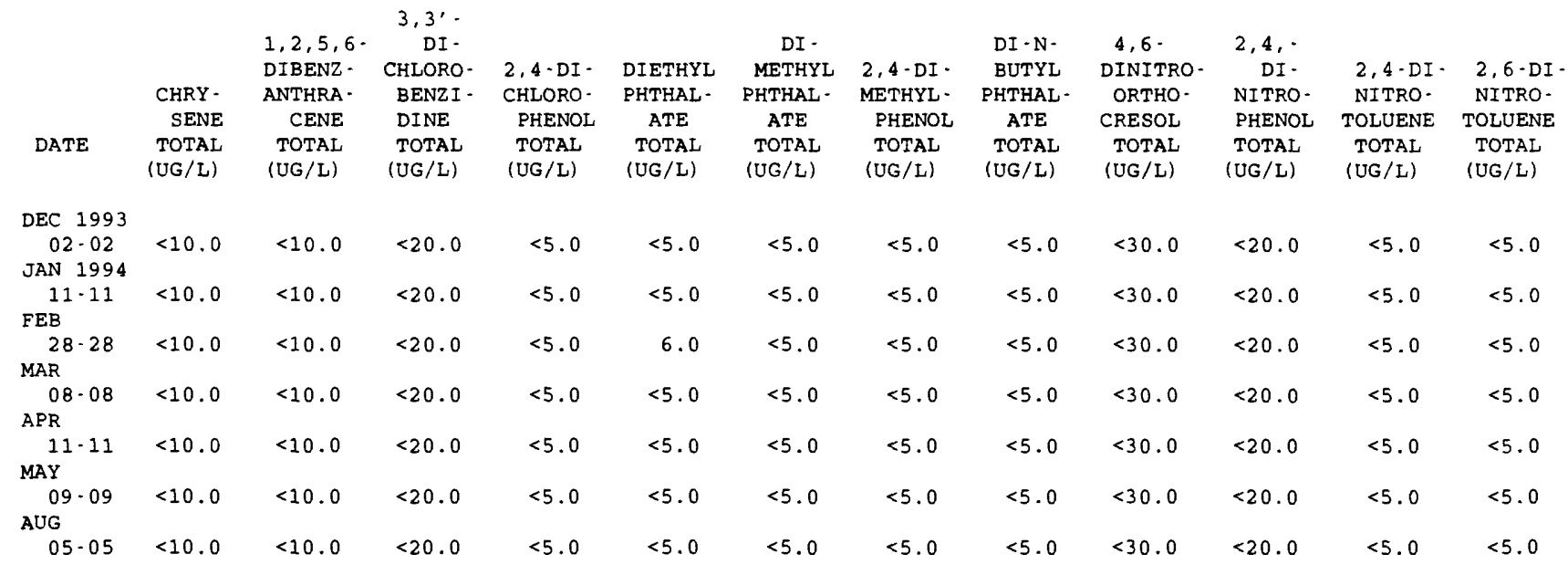


Table 9. Water-quality data for storm-sewer outfall stations, Texas Department of Transportation, Dallas and Fort Worth Districts, 1993-94-Continued

08055690 - BACHMAN BRANCH OUTFALL AT I-635, DALLAS, TX (WY 1994)-Continued

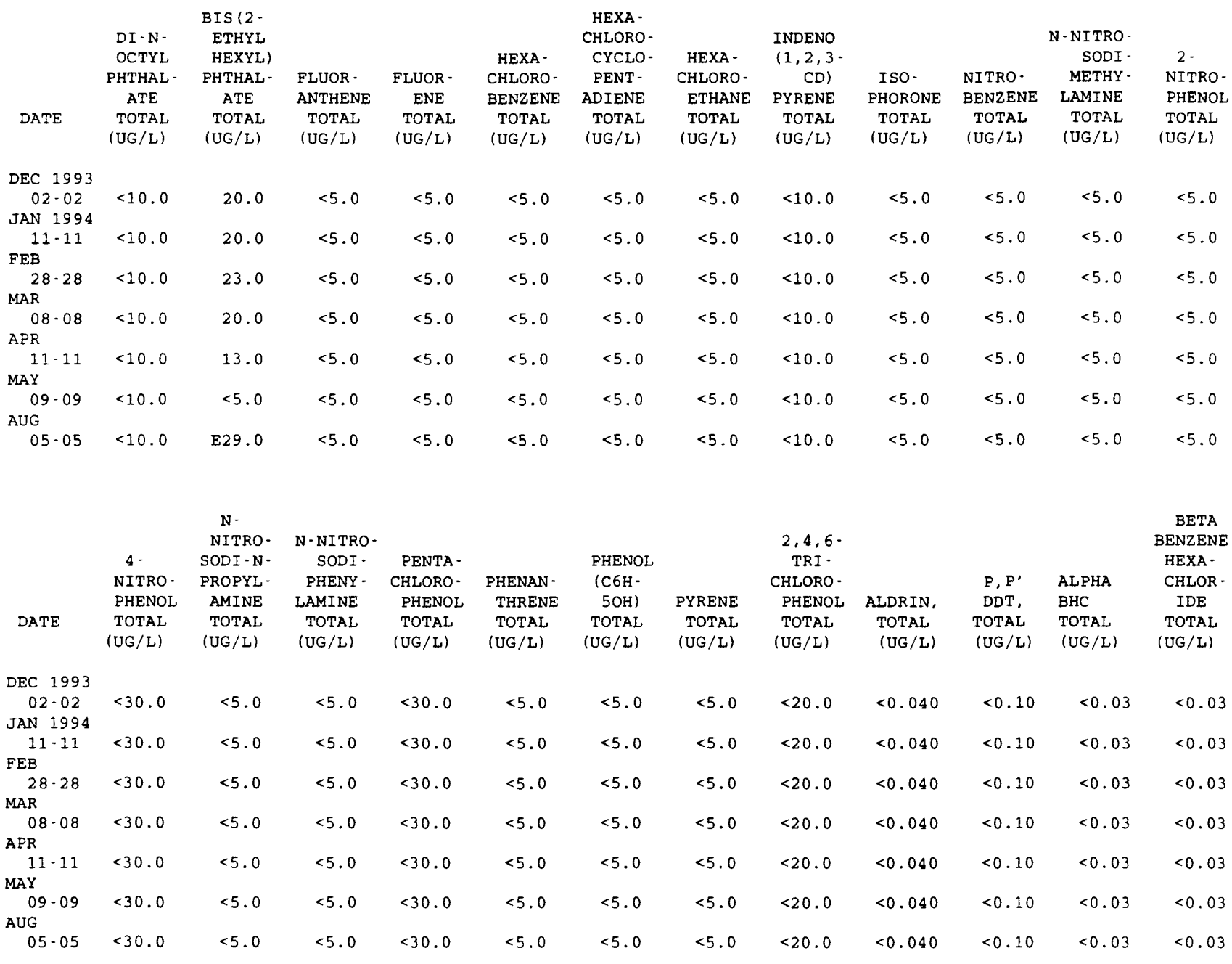

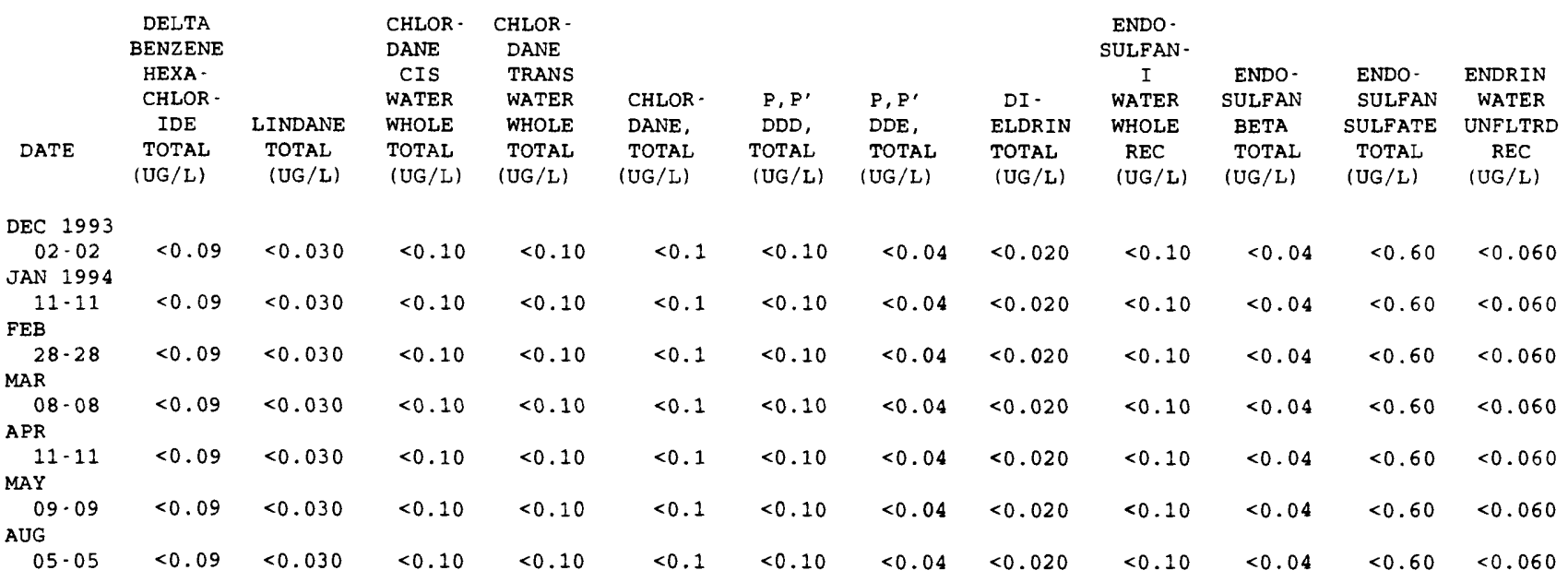


Table 9. Water-quality data for storm-sewer outfall stations, Texas Department of Transportation, Dallas and Fort Worth Districts, 1993-94-Continued

08055690 - BACHMAN BRANCH OUTFALL AT I-635, DALLAS, TX (WY 1994)-Continued

\begin{tabular}{|c|c|c|c|c|c|c|c|c|c|c|c|c|}
\hline & ENDRIN & & HEPTA - & & AROCLOR & AROCLOR & AROCLOR & AROCLOR & AROCLOR & AROCLOR & AROCLOR & \\
\hline & ALDE - & HEPTA - & CHLOR & TOX - & 1016 & 1221 & 1232 & 1242 & 1248 & 1254 & 1260 & DI - \\
\hline ATE & $\begin{array}{r}\text { HYDE } \\
\text { TOTAL }\end{array}$ & $\begin{array}{l}\text { CHLOR, } \\
\text { TOTAL }\end{array}$ & $\begin{array}{c}\text { EPOXIDE } \\
\text { TOTAL } \\
\text { (UG/L) }\end{array}$ & $\begin{array}{c}\text { APHENE, } \\
\text { TOTAL }\end{array}$ & $\begin{array}{c}\text { PCB } \\
\text { TOTAL }\end{array}$ & $\begin{array}{c}\text { PCB } \\
\text { TOTAL }\end{array}$ & $\begin{array}{c}\text { PCB } \\
\text { TOTAL }\end{array}$ & $\begin{array}{l}\text { PCB } \\
\text { TOTAL }\end{array}$ & $\begin{array}{c}\text { PCB } \\
\text { TOTAL }\end{array}$ & $\begin{array}{c}\text { PCB } \\
\text { TOTAL }\end{array}$ & $\begin{array}{c}\text { PCB } \\
\text { TOTAL }\end{array}$ & $\begin{array}{l}\text { AZINON, } \\
\text { TOTAL }\end{array}$ \\
\hline
\end{tabular}

\begin{tabular}{|c|c|c|c|c|c|c|c|c|c|c|c|c|}
\hline \multicolumn{13}{|l|}{ DEC 1993} \\
\hline 02.02 & $<0.20$ & $<0.030$ & $<0.80$ & $<2$ & $<0.1$ & $<1.0$ & $<0.1$ & $<0.1$ & $<0.1$ & $<0.1$ & $<0.1$ & $<0.05$ \\
\hline \multicolumn{13}{|l|}{ JAN 1994} \\
\hline $11-11$ & $<0.20$ & $<0.030$ & $<0.80$ & $<2$ & $<0.1$ & $<1.0$ & $<0.1$ & $<0.1$ & $<0.1$ & 0.2 & $<0.1$ & $<0.05$ \\
\hline \multicolumn{13}{|l|}{ FEB } \\
\hline $28-28$ & $<0.20$ & $<0.030$ & $<0.80$ & $<2$ & $<0.1$ & $<1.0$ & $<0.1$ & $<0.1$ & $<0.1$ & 0.1 & $<0.1$ & $<0.05$ \\
\hline \multicolumn{13}{|l|}{ MAR } \\
\hline 08.08 & $<0.20$ & $<0.030$ & $<0.80$ & $<2$ & $<0.1$ & $<1.0$ & $<0.1$ & $<0.1$ & $<0.1$ & $<0.1$ & $<0.1$ & $<0.05$ \\
\hline \multicolumn{13}{|l|}{ APR } \\
\hline $11-11$ & $<0.20$ & $<0.030$ & $<0.80$ & $<2$ & $<0.1$ & $<1.0$ & $<0.1$ & $<0.1$ & $<0.1$ & $<0.1$ & $<0.1$ & $<0.05$ \\
\hline \multicolumn{13}{|l|}{ MAY } \\
\hline $09-09$ & $<0.20$ & $<0.030$ & $<0.80$ & $<2$ & $<0.1$ & $<1.0$ & $<0.1$ & $<0.1$ & $<0.1$ & $<0.1$ & $<0.1$ & $<0.10$ \\
\hline \multicolumn{13}{|l|}{ AUG } \\
\hline $05-05$ & $<0.20$ & $<0.030$ & $<0.80$ & $<2$ & $<0.1$ & $<1.0$ & $<0.1$ & $<0.1$ & $<0.1$ & 0.2 & $<0.1$ & $<0.05$ \\
\hline
\end{tabular}


Tables 10-17. Quality-assurance/quality-control data for storm-sewer outfall stations

[TYpe of QA/QC sample: REP, replicate sample; LB, laboratory blank sample sent to U.S. Geological Survey National Water Quality Laboratory, Arvada, Colo.; EB, equipment blank sample; FMS, field matrix spike sample; LMS, laboratory matrix spike sample; REF, reference sample furnished by the U.S. Geological Survey Laboratory, Ocala, Fla.; TB, trip blank sample; FEB, field equipment blank sample; FLB, field laboratory blank sample. WY, water year; UG/L, micrograms per liter; <, less than; MG/L, milligrams per liter; NO2+NO3, nitrite plus nitrate; - - not determined; US/CM, microsiemens per centimeter at $25^{\circ} \mathrm{C}$; CACO3, calcium carbonate; DEG C, degrees Celsius; EPA, analyzed by U.S. Environmental Protection Agency method] 
Table 10. Quality-assurance/quality-control data for storm-sewer outfall stations, Arlington, Texas, 1992-93

08049220 - THE PARKS MALL OUTFALL AT I-20 WEST, ARLINGTON, TX (WY 1993)

\begin{tabular}{|c|c|c|c|c|c|c|c|c|c|c|c|c|c|}
\hline $\begin{array}{c}\text { TYPE } \\
\text { OF } \\
\text { QA/QC }\end{array}$ & DATE & TIME & $\begin{array}{c}\text { ARSENIC } \\
\text { TOTAL } \\
\text { (UG/L }\end{array}$ & $\begin{array}{l}\text { BERYL - } \\
\text { LIUM, } \\
\text { TOTAL } \\
\text { RECOV- } \\
\text { ERABLE } \\
\text { (UG/L }\end{array}$ & $\begin{array}{l}\text { CADMIUM } \\
\text { TOTAL } \\
\text { RECOV- } \\
\text { ERABLE } \\
\text { (UG/L }\end{array}$ & $\begin{array}{l}\text { CHRO- } \\
\text { MIUM, } \\
\text { TOTAL } \\
\text { RECOV- } \\
\text { ERABLE } \\
\text { (UG/L }\end{array}$ & $\begin{array}{l}\text { COPPER, } \\
\text { TOTAL } \\
\text { RECOV- } \\
\text { ERABLE } \\
\text { (UG/L }\end{array}$ & $\begin{array}{l}\text { LEAD, } \\
\text { TOTAL } \\
\text { RECOV- } \\
\text { ERABLE } \\
\text { (UG/L }\end{array}$ & $\begin{array}{l}\text { MERCURY } \\
\text { TOTAL } \\
\text { RECOV- } \\
\text { ERABLE } \\
\text { (UG/L }\end{array}$ & $\begin{array}{l}\text { NICKEL, } \\
\text { TOTAL } \\
\text { RECOV- } \\
\text { ERABLE } \\
\text { (UG/L }\end{array}$ & $\begin{array}{l}\text { SELE- } \\
\text { NIUM, } \\
\text { TOTAL } \\
\text { (UG/L }\end{array}$ & $\begin{array}{l}\text { SILVER, } \\
\text { TOTAL } \\
\text { RECOV - } \\
\text { ERABLE } \\
\text { (UG/L }\end{array}$ & $\begin{array}{l}\text { ZINC, } \\
\text { TOTAL } \\
\text { RECOV - } \\
\text { ERABLE } \\
(\mathrm{UG} / \mathrm{L}\end{array}$ \\
\hline SAMPLE & & & AS AS) & AS $\mathrm{BE}$ ) & AS CD) & AS CR) & $\mathrm{AS} \mathrm{CU})$ & AS PB) & AS HG) & AS NI) & AS SE) & AS AG) & AS $\mathrm{ZN}$ ) \\
\hline REP & $\begin{array}{l}\text { FEB } 1993 \\
24-24 \\
\text { MAR }\end{array}$ & 1420 & $<1$ & $<10$ & $<1$ & 4 & 6 & 19 & $<0.10$ & 3 & $<2$ & $<1$ & 30 \\
\hline REP & $11 \cdot 12$ & 1656 & 2 & $<10$ & $<1$ & 4 & 2 & 11 & $<0.10$ & 3 & $<2$ & $<1$ & 40 \\
\hline
\end{tabular}

08049320 - RIVER LEGACY PARK OUTFALL AT GREEN OAKS BOULEVARD, ARLINGTON, TX (WY 1993)

TYPE
OF
$Q A / Q C$

SAMPLE

REP

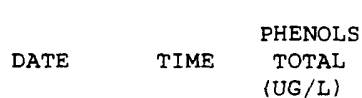

DEC 1992

$09-09$

0042
TOTAL
(UG/L)

6

08049360 - TRIBUTARY TO WEST FORK TRINITY RIVER OUTFALL AT BAIRD'S FARM ROAD, ARLINGTON, TX (WY 1993)

\begin{tabular}{|c|c|c|c|c|c|c|c|c|c|c|c|c|c|}
\hline $\begin{array}{c}\text { TYPE } \\
\text { OF } \\
\text { QA/QC } \\
\text { SAMPLE }\end{array}$ & DATE & TIME & $\begin{array}{l}\text { NITRO- } \\
\text { GEN, } \\
\text { NITRITE } \\
\text { TOTAL } \\
\text { (MG/L } \\
\text { AS N) }\end{array}$ & $\begin{array}{c}\text { NITRO- } \\
\text { GEN, } \\
\text { NO2+NO3 } \\
\text { TOTAL } \\
\text { (MG/L } \\
\text { AS N) }\end{array}$ & $\begin{array}{l}\text { NITRO- } \\
\text { GEN, } \\
\text { AMMONIA } \\
\text { TOTAL } \\
\text { (MG/L } \\
\text { AS N) }\end{array}$ & $\begin{array}{l}\text { NITRO- } \\
\text { GEN, AM- } \\
\text { MONIA + } \\
\text { ORGANIC } \\
\text { TOTAL } \\
\text { (MG/L } \\
\text { AS N) }\end{array}$ & $\begin{array}{l}\text { PHOS - } \\
\text { PHORUS } \\
\text { TOTAL } \\
\text { (MG/L } \\
\text { AS P) }\end{array}$ & $\begin{array}{c}\text { PHOS - } \\
\text { PHORUS } \\
\text { DIS - } \\
\text { SOLVED } \\
\text { (MG/L } \\
\text { AS P) }\end{array}$ & $\begin{array}{l}\text { OIL AND } \\
\text { GREASE, } \\
\text { TOTAL } \\
\text { RECOV. } \\
\text { GRAVI - } \\
\text { METRIC } \\
\text { (MG/L) }\end{array}$ & $\begin{array}{c}\text { BENZENE } \\
0- \\
\text { CHLORO- } \\
\text { WATER } \\
\text { UNFLTRD } \\
\text { REC } \\
\text { (UG/L) }\end{array}$ & $\begin{array}{c}\text { BENZENE } \\
1,3 \text { - DI - } \\
\text { CHLORO- } \\
\text { WATER } \\
\text { UNFLTRD } \\
\text { REC } \\
\text { (UG/L) }\end{array}$ & $\begin{array}{c}\text { BENZENE } \\
1,4-D I- \\
\text { CHLORO- } \\
\text { WATER } \\
\text { UNFLTRD } \\
\text { REC } \\
\text { (UG/L) }\end{array}$ & $\begin{array}{l}1,2-D I- \\
\text { PHENYL- } \\
\text { HYDRA- } \\
\text { ZINE } \\
\text { WATER } \\
\text { TOT.REC } \\
\text { (UG/L) }\end{array}$ \\
\hline & OCT 1992 & & & & & & & & & & & & \\
\hline REP & $\begin{array}{cc}29-29 \\
\text { FEB } \quad 1993\end{array}$ & 0007 & 0.040 & 0.510 & 0.110 & 0.80 & 0.290 & 0.220 & $<1$ & $\cdots$ & $\cdots$ & - & $\cdots$ \\
\hline REP & $\begin{array}{l}10-10 \\
\text { FEB }\end{array}$ & 0347 & $\cdots$ & $\cdots$ & $\cdots$ & $\cdots$ & $\cdots$ & $\cdots$ & $\cdots$ & $<5.0$ & $<5.0$ & $<5.0$ & $<5.0$ \\
\hline REP & $\begin{array}{l}10-10 \\
\text { MAR }\end{array}$ & 0348 & $\cdots$ & $\cdots$ & $\cdots$ & $\cdots$ & $\cdots$ & $\cdots$ & $\cdots$ & $\cdots$ & $\cdots$ & $\cdots$ & $\cdots$ \\
\hline LB & $28-28$ & 0740 & $\cdots$ & $\cdots$ & $\cdots$ & $\cdots$ & $\cdots$ & $\cdots$ & $\cdots$ & $<5.0$ & $<5.0$ & $<5.0$ & $<5.0$ \\
\hline
\end{tabular}

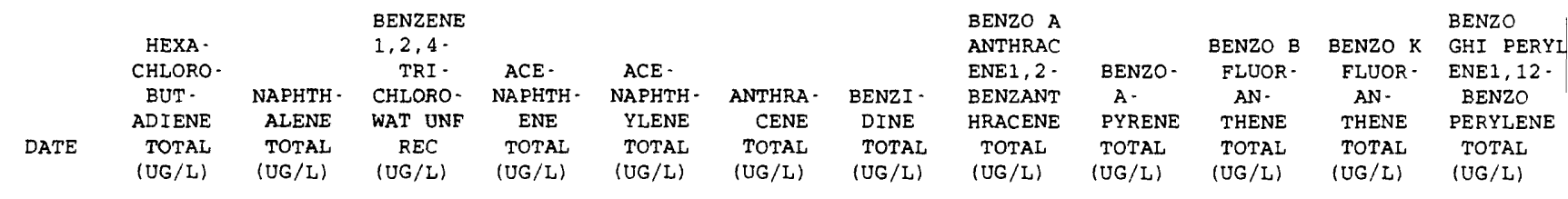

OCT 1992

$29 \cdot 29$
FEB 1993

FER 1993
$10 \cdot 10$

FEB

$10 \cdot 10$

MAR

$28 \cdot 28$

$<5.0$

$<5.0$

$<5.0$

$<5.0<5.0$

$<5.0<40.0$

$<10.0$

$<10$.

$<10$.

$<10.0<10.0$

$<5.0$

$<5.0$

$<5.0$

$<5.0$

$<5.0$

$<5.0$

$<40.0$

$<10.0$

$<10.0$

$<10.0$

$<10.0<10.0$ 
Table 10. Quality-assurance/quality-control data for storm-sewer outfall stations, Arlington, Texas, 1992-93-Continued 08049360 - TRIBUTARY TO WEST FORK TRINITY RIVER OUTFALL AT BAIRD'S FARM ROAD, ARLINGTON, TX (WY 1993)-CONEINUEd

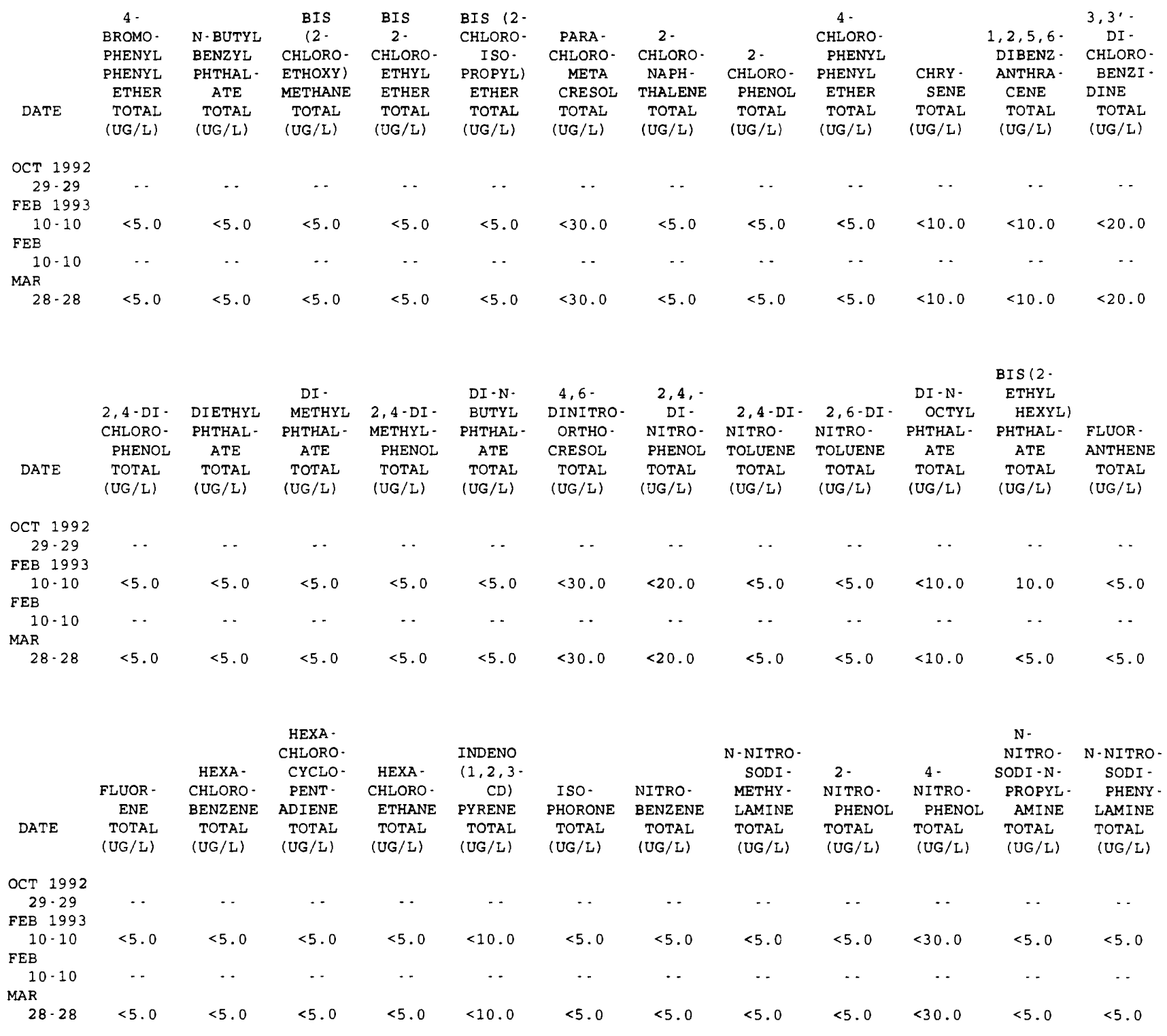

\begin{tabular}{|c|c|c|c|c|c|c|c|c|c|c|c|}
\hline DATE & $\begin{array}{l}\text { PENTA - } \\
\text { CHLORO - } \\
\text { PHENOL } \\
\text { TOTAL } \\
\text { (UG/L) }\end{array}$ & $\begin{array}{l}\text { PHENAN - } \\
\text { THRENE } \\
\text { TOTAL } \\
\text { (UG/L) }\end{array}$ & $\begin{array}{c}\text { PHENOL } \\
\text { (C6H - } \\
5 O H \text { ) } \\
\text { TOTAL } \\
\text { (UG/L) }\end{array}$ & $\begin{array}{c}\text { PYRENE } \\
\text { TOTAL } \\
\text { (UG/L) }\end{array}$ & $\begin{array}{c}2,4,6- \\
\text { TRI - } \\
\text { CHLORO- } \\
\text { PHENOL } \\
\text { TOTAL } \\
\text { (UG/L) }\end{array}$ & $\begin{array}{c}\text { ALDRIN, } \\
\text { TOTAL } \\
\text { (UG/L) }\end{array}$ & $\begin{array}{c}\text { P, } P^{\prime} \\
\text { DDT, } \\
\text { TOTAL } \\
\text { (UG/L) }\end{array}$ & $\begin{array}{l}\text { ALPHA } \\
\text { BHC } \\
\text { TOTAL } \\
\text { (UG/I) }\end{array}$ & $\begin{array}{c}\text { BETA } \\
\text { BENZENE } \\
\text { HEXA- } \\
\text { CHLOR - } \\
\text { IDE } \\
\text { TOTAL } \\
\text { (UG/L) }\end{array}$ & $\begin{array}{l}\text { DELTA } \\
\text { BENZENE } \\
\text { HEXA- } \\
\text { CHLOR - } \\
\text { IDE } \\
\text { TOTAL } \\
\text { (UG/L) }\end{array}$ & $\begin{array}{l}\text { LINDANE } \\
\text { TOTAL } \\
\text { (UG/L) }\end{array}$ \\
\hline OCT 1992 & & & & & & & & & & & \\
\hline $\begin{array}{c}29-29 \\
\text { FEB } 1993\end{array}$ & $\cdots$ & $\cdots$ & $\cdots$ & $\cdots$ & $\cdots$ & $\cdots$ & $\cdots$ & $\cdots$ & $\cdots$ & $\cdots$ & $\cdots$ \\
\hline $\begin{array}{l}10-10 \\
\text { FEB }\end{array}$ & $<30.0$ & $<5.0$ & $<5.0$ & $<5.0$ & $<20.0$ & $\cdots$ & $\cdots$ & $\cdots$ & $\cdots$ & $\cdots$ & $\cdots$ \\
\hline $\begin{array}{l}10-10 \\
\text { MAR }\end{array}$ & $\cdots$ & $\cdots$ & $\cdots$ & $\cdots$ & $\cdots$ & $<0.040$ & $<0.10$ & $<0.03$ & $<0.03$ & $<0.09$ & $<0.030$ \\
\hline $28-28$ & $<30.0$ & $<5.0$ & $<5.0$ & $<5.0$ & $<20.0$ & $\cdots$ & $\cdots$ & $\cdots$ & $\cdots$ & $\cdots$ & $\cdots$ \\
\hline
\end{tabular}


Table 10. Quality-assurance/quality-control data for storm-sewer outfall stations, Arlington, Texas, 1992-93-Continued 08049360 - TRIBUTARY TO WEST FORK TRINITY RIVER OUTFALL AT BAIRD'S FARM ROAD, ARLINGTON, TX (WY 1993)-CONEIIUEd

\begin{tabular}{|c|c|c|c|c|c|c|c|c|c|c|c|}
\hline DATE & $\begin{array}{c}\text { CHLOR - } \\
\text { DANE } \\
\text { CIS } \\
\text { WATER } \\
\text { WHOLE } \\
\text { TOTAL } \\
(\text { UG/L) }\end{array}$ & $\begin{array}{l}\text { CHLOR - } \\
\text { DANE } \\
\text { TRANS } \\
\text { WATER } \\
\text { WHOLE } \\
\text { TOTAL } \\
\text { (UG/L) }\end{array}$ & $\begin{array}{l}\text { CHLOR - } \\
\text { DANE, } \\
\text { TOTAL } \\
\text { (UG/L) }\end{array}$ & $\begin{array}{c}\text { P, } P^{\prime} \\
\text { DDD, } \\
\text { TOTAL } \\
\text { (UG/L) }\end{array}$ & $\begin{array}{l}P, P^{\prime} \\
\text { DDE, } \\
\text { TOTAL } \\
\text { (UG/L) }\end{array}$ & $\begin{array}{l}\text { DI - } \\
\text { ELDRIN } \\
\text { TOTAL } \\
\text { (UG/L) }\end{array}$ & $\begin{array}{c}\text { ENDO- } \\
\text { SULFAN- } \\
\text { I } \\
\text { WATER } \\
\text { WHOLE } \\
\text { REC } \\
\text { (UG/L) }\end{array}$ & $\begin{array}{l}\text { ENDO - } \\
\text { SULFAN } \\
\text { BETA } \\
\text { TOTAL } \\
\text { (UG/L) }\end{array}$ & $\begin{array}{l}\text { ENDO- } \\
\text { SULFAN } \\
\text { SULFATE } \\
\text { TOTAL } \\
\text { (UG/L) }\end{array}$ & $\begin{array}{c}\text { ENDRIN } \\
\text { WATER } \\
\text { UNFLTRD } \\
\text { REC } \\
\text { (UG/L) }\end{array}$ & $\begin{array}{c}\text { ENDRIN } \\
\text { ALDE- } \\
\text { HYDE } \\
\text { TOTAL } \\
(\text { UG/L) }\end{array}$ \\
\hline OCT 1992 & & & & & & & & & & & \\
\hline $\begin{array}{c}29-29 \\
\text { FEB } 1993\end{array}$ & $\cdots$ & $\cdots$ & $\cdots$ & $\cdots$ & $\cdots$ & $\cdots$ & $\cdots$ & $\cdots$ & $\cdots$ & $\cdots$ & $\cdots$ \\
\hline $\begin{array}{l}10-10 \\
F E B\end{array}$ & $\cdots$ & $\cdots$ & $\cdots$ & $\cdots$ & $\cdots$ & $\cdots$ & $\cdots$ & $\cdots$ & $\cdots$ & $\cdots$ & $\cdots$ \\
\hline $10-10$ & $<0.10$ & $<0.10$ & $<0.1$ & $<0.10$ & $<0.04$ & $<0.020$ & $<0.10$ & $<0.04$ & $<0.60$ & $<0.060$ & $<0.20$ \\
\hline $\begin{array}{l}\text { MAR } \\
28-28\end{array}$ & $\ldots$ & . & $\cdots$ & $\ldots$ & $\ldots$ & $\ldots$ & $\ldots$ & $\ldots$ & . & - . & \\
\hline DATE & $\begin{array}{l}\text { HEPTA- } \\
\text { CHLOR, } \\
\text { TOTAL } \\
\text { (UG/L) }\end{array}$ & $\begin{array}{l}\text { HEPTA - } \\
\text { CHLOR } \\
\text { EPOXIDE } \\
\text { TOTAL } \\
\text { (UG/L) }\end{array}$ & $\begin{array}{c}\text { TOX- } \\
\text { APHENE, } \\
\text { TOTAL } \\
\text { (UG/L) }\end{array}$ & $\begin{array}{c}\text { AROCLOR } \\
1016 \\
\text { PCB } \\
\text { TOTAL } \\
\text { (UG/L) }\end{array}$ & $\begin{array}{c}\text { AROCLOR } \\
1221 \\
\text { PCB } \\
\text { TOTAL } \\
\text { (UG/L) }\end{array}$ & $\begin{array}{c}\text { AROCLOR } \\
1232 \\
\text { PCB } \\
\text { TOTAL } \\
\text { (UG/L) }\end{array}$ & $\begin{array}{c}\text { AROCLOR } \\
1242 \\
\text { PCB } \\
\text { TOTAL } \\
\text { (UG/L) }\end{array}$ & $\begin{array}{c}\text { AROCLOR } \\
1248 \\
\text { PCB } \\
\text { TOTAL } \\
\text { (UG/L) }\end{array}$ & $\begin{array}{c}\text { AROCLOR } \\
1254 \\
\text { PCB } \\
\text { TOTAL } \\
\text { (UG/L) }\end{array}$ & $\begin{array}{c}\text { AROCLOR } \\
1260 \\
\text { PCB } \\
\text { TOTAL } \\
\text { (UG/L) }\end{array}$ & $\begin{array}{c}\text { DI - } \\
\text { AZINON, } \\
\text { TOTAL } \\
\text { (UG/L) }\end{array}$ \\
\hline OCT 1992 & & & & & & & & & & & \\
\hline $\begin{array}{c}29-29 \\
\text { FEB } 1993\end{array}$ & $\cdots$ & $\cdots$ & - & $\cdots$ & $\cdots$ & $\cdots$ & $\cdots$ & $\cdots$ & $\cdots$ & $\cdots$ & - \\
\hline $\begin{array}{l}10 \cdot 10 \\
\text { FEB }\end{array}$ & $\cdots$ & $\cdots$ & $\cdots$ & $\cdots$ & $\cdots$ & $\cdots$ & $\cdots$ & - & $\cdots$ & $\cdots$ & $\cdots$ \\
\hline $\begin{array}{l}10-10 \\
\text { MAR }\end{array}$ & $<0.030$ & $<0.80$ & $<2$ & $<0.1$ & $<1.0$ & $<0.1$ & $<0.1$ & $<0.1$ & $<0.1$ & $<0.1$ & 0.10 \\
\hline $28-28$ & $\cdots$ & $\cdots$ & $\cdots$ & $\cdots$ & $\ldots$ & $\ldots$ & $\cdots$ & $\cdots$ & $\cdots$ & $\cdots$ & $\cdots$ \\
\hline
\end{tabular}

08049470 - TRIBUTARY TO JOHNSON CREEK OUTFALI AT I-30 EAST, ARLINGTON, TX (WY 1993)

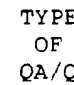

$O F$
$Q A / Q$

SAMPLE

REP

REP
DATE

MAR 1993
11.12
MAR

$11 \cdot 12$

\section{BENZENE}

$$
\begin{aligned}
& \text { O- } \\
& \text { CHLORO - } \\
& \text { WATER }
\end{aligned}
$$$$
\text { UNFLTRD }
$$

TIME

$$
\text { REC }
$$

BENZENE

1, 3-DI -

CHLORO-

WATER

UNFLTRD
REC

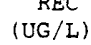

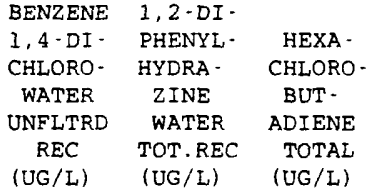
REC
(UG/I)

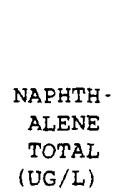

BENZENE

$1,2,4$ -

TRI -

ACE - ACE -

WAT UNF

REC

(UG/L)
RO - NAPHTH - NAPHTH - ANTHRA -

TOTAL TOTAL TOTAL

(UG/L) (UG/L) (UG/L)
1751

1752

$$
<5.0
$$$$
<5.0
$$$$
<5.0
$$$$
<5.0
$$$$
<5.0
$$$$
<5.0
$$$$
<5.0
$$$$
<5.0
$$$$
<5.0
$$$$
<5.0
$$

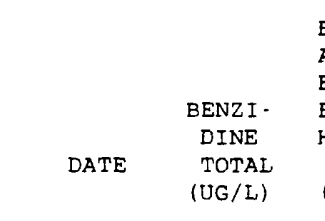

BENZO A ANTHRAC

ENE1, 2-

BENZANT

HRACENE

(UG/L)
-

MAR 1993

$11 \cdot 12$

MAR

$11-12$

BENZO 4 .

BENZO B BENZO $K$ GHI PERYL BROMO -

PHENYL

PHENYL

ETHER

TOTAL

(UG/L)
N-BUTYL

BENZYL

ATE

TOTAL

(UG/L)
BI

2 - $C H L O R O$

CHLORO - CHLORO - ISO-

ETHOXY) ETHYL PROPYL)

METHANE ETHER ETHER

TOTAL TOTAL TOTAL

(UG/L) (UG/L) (UG/L) 
Table 10. Quality-assurance/quality-control data for storm-sewer outfall stations, Arlington, Texas, 1992-93-Continued 08049470 - TRIBUTARY TO JOHNSON CREEK OUTFALL AT I-30 EAST, ARLINGTON, TX (WY 1993)-CONTInUEd

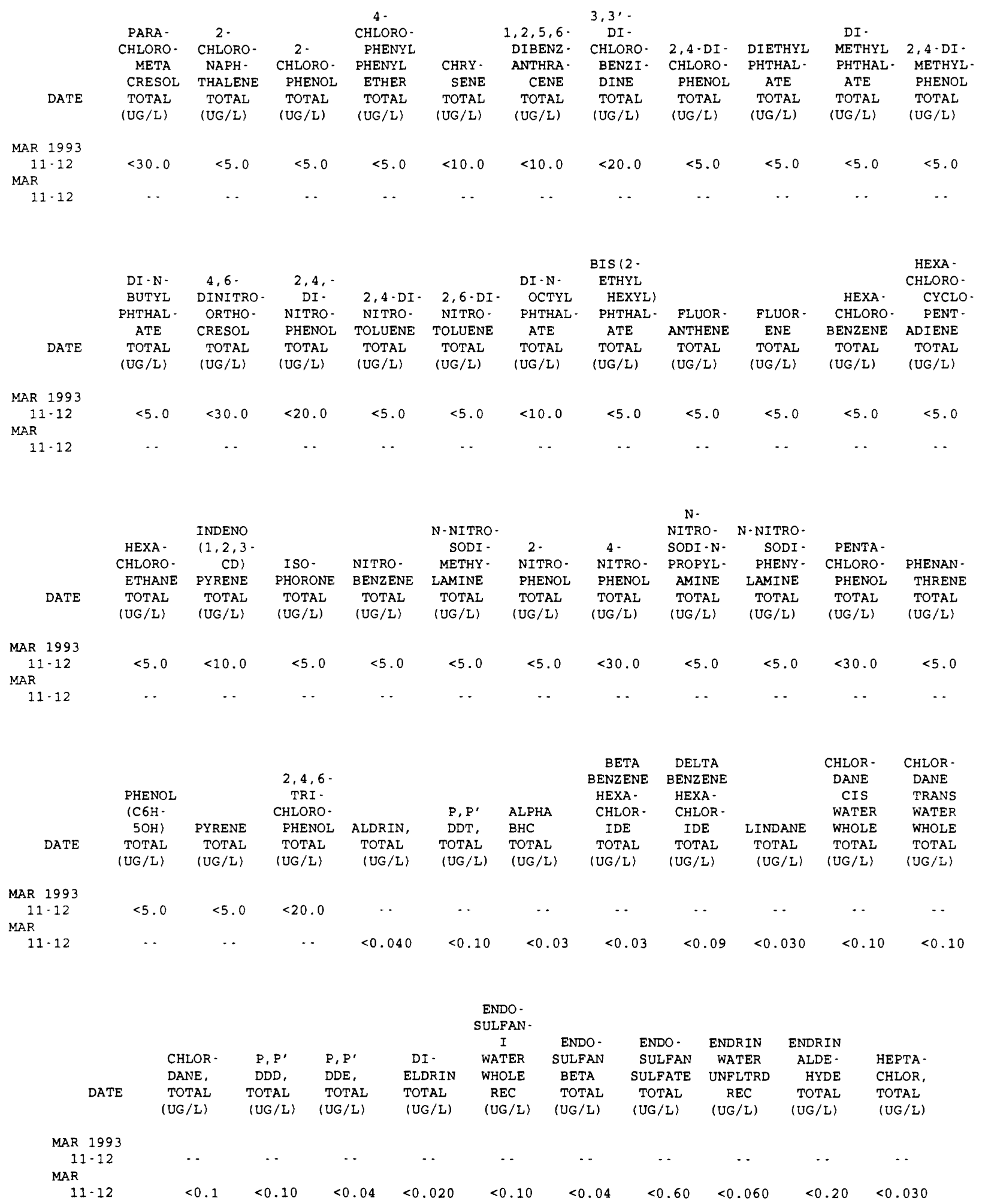


Table 10. Quality-assurance/quality-control data for storm-sewer outfall stations, Arlington, Texas, 1992-93-Continued 08049470 - TRIBUTARY TO JOHNSON CREEK OUTFALL AT I-30 EAST, ARLINGTON, TX (WY 1993)-CONTINUEd

\begin{tabular}{|c|c|c|c|c|c|c|c|c|c|c|}
\hline & HEPTA - & & AROCLOR & AROCLOR & AROCLOR & AROCLOR & AROCLOR & AROCLOR & AROCLOR & \\
\hline & CHLOR & TOX - & 1016 & 1221 & 1232 & 1242 & 1248 & 1254 & 1260 & DI - \\
\hline DATE & $\begin{array}{l}\text { EPOXIDE } \\
\text { TOTAL } \\
\text { (UG/L) }\end{array}$ & $\begin{array}{c}\text { APHENE, } \\
\text { TOTAL } \\
\text { (UG/L) }\end{array}$ & $\begin{array}{c}\text { PCB } \\
\text { TOTAL } \\
\text { (UG/L) }\end{array}$ & $\begin{array}{c}\text { PCB } \\
\text { TOTAL } \\
\text { (UG } / L)\end{array}$ & $\begin{array}{c}\text { PCB } \\
\text { TOTAL } \\
\text { (UG/L) }\end{array}$ & $\begin{array}{c}\text { PCB } \\
\text { TOTAL } \\
\text { (UG/L) }\end{array}$ & $\begin{array}{c}\text { PCB } \\
\text { TOTAL } \\
\text { (UG/L) }\end{array}$ & $\begin{array}{l}\text { PCB } \\
\text { TOTAL } \\
\text { (UG/L) }\end{array}$ & $\begin{array}{c}\text { PCB } \\
\text { TOTAL } \\
\text { (UG/L) }\end{array}$ & $\begin{array}{c}\text { AZINON, } \\
\text { TOTAL } \\
\text { (UG/L) }\end{array}$ \\
\hline
\end{tabular}

\begin{tabular}{|c|c|c|c|c|c|c|c|c|c|c|}
\hline MAR 1993 & & & & & & & & & & \\
\hline $11-12$ & $\cdots$ & $\cdots$ & $\cdots$ & $\cdots$ & $\cdots$ & $\cdots$ & $\cdots$ & - & $\cdots$ & - - \\
\hline \multicolumn{11}{|l|}{ MAR } \\
\hline $11-12$ & $<0.80$ & $<2$ & $<0.1$ & $<1.0$ & $<0.1$ & $<0.1$ & $<0.1$ & $<0.1$ & $<0.1$ & $<0.05$ \\
\hline
\end{tabular}


Table 11. Quality-assurance/quality-control data for storm-sewer outfall stations, Dallas, Texas, $1992-93$

08055590 - JOE'S CREEK OUTFALL AT DENTON DRIVE, DALLAS, TX (WY 1992)

TYPE

OF

$Q A / Q C$

SAMPLE

REP

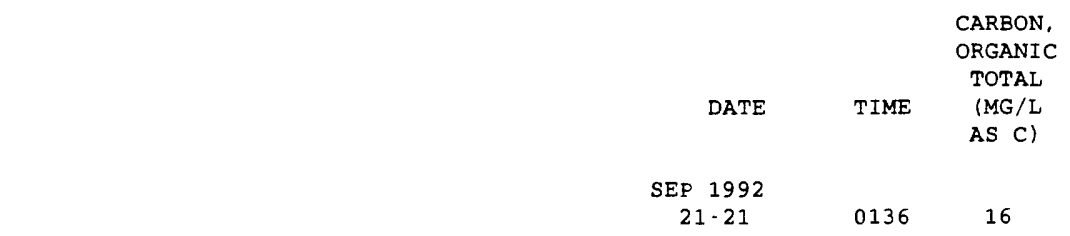

08056390 - BASTILLE STREET OUTFALL AT LA REUNION PARKWAY, DALLAS, TX (WY 1992)
TYPE

$O F$

$Q A / Q C$

SAMPLE

$E B$

EB
REP

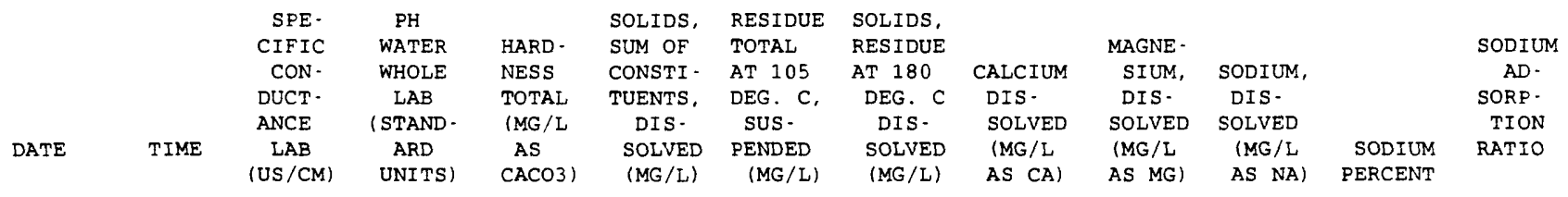

\begin{tabular}{|c|c|c|c|c|c|c|c|c|c|c|c|c|}
\hline $09 \ldots$ & 0930 & $\cdots$ & $\cdots$ & $\cdots$ & $\cdots$ & $\cdots$ & $\cdots$ & $\cdots$ & $\cdots$ & $\cdots$ & $\cdots$ & $\cdots$ \\
\hline \multicolumn{13}{|l|}{ JUN } \\
\hline \multirow[t]{4}{*}{$01-01$} & 2001 & 134 & 8.3 & 37 & 67 & 185 & 67 & 14 & 0.47 & 3.4 & 16 & 0.2 \\
\hline & & & & & BENZENE & BENZENE & BENZENE & $1,2-\mathrm{DI}-$ & & & BENZENE & \\
\hline & $\begin{array}{l}\text { POTAS - } \\
\text { SIUM, }\end{array}$ & SULFATE & $\begin{array}{l}\text { CHLO - } \\
\text { RIDE }\end{array}$ & CARBON & $\begin{array}{c}0- \\
\text { CHIO- }\end{array}$ & $\begin{array}{l}1,3-D I- \\
\text { CHLORO - }\end{array}$ & $\begin{array}{l}1,4-\mathrm{DI} \text { - } \\
\text { C }\end{array}$ & $\begin{array}{l}\text { PHENYL - } \\
\text { HYDRA - }\end{array}$ & HEXA - & & $\begin{array}{l}1,2,4- \\
\text { TRI - }\end{array}$ & $A C E$ \\
\hline & $\begin{array}{c}\text { DIS - } \\
\text { SOLVED }\end{array}$ & $\begin{array}{l}\text { DIS - } \\
\text { SOLVED }\end{array}$ & $\begin{array}{l}\text { DIS - } \\
\text { SOLVED }\end{array}$ & $\begin{array}{c}\text { ORGANIC } \\
\text { TOTAL }\end{array}$ & $\begin{array}{c}\text { WATER } \\
\text { UNFLTRD }\end{array}$ & $\begin{array}{l}\text { WATER } \\
\text { UNFLTRD }\end{array}$ & $\begin{array}{l}\text { WATER } \\
\text { UNFLTRD }\end{array}$ & $\begin{array}{l}\text { ZINE } \\
\text { WATER }\end{array}$ & $\begin{array}{c}\text { BUT - } \\
\text { ADIENE }\end{array}$ & $\begin{array}{c}\text { NAPHTH - } \\
\text { ALENE }\end{array}$ & $\begin{array}{l}\text { CHLORO- } \\
\text { WAT UNF }\end{array}$ & $\begin{array}{c}\text { NAPHTH - } \\
\text { ENE }\end{array}$ \\
\hline DATE & (MG/L & (MG/L & (MG / L & (MG/L & REC & REC & REC & TOT . REC & TOTAL & TOTAL & REC & TOTAL \\
\hline & AS K) & AS SO4) & AS CL) & AS C) & $(U G / L)$ & (UG/L) & (UG/L) & $\langle U G / L\rangle$ & (UG/L) & (UG/L) & (UG/L) & $(\mathrm{UG} / \mathrm{L})$ \\
\hline
\end{tabular}

\begin{tabular}{|c|c|c|c|c|c|c|c|c|c|c|c|c|}
\hline $09 \ldots$ & $\cdots$ & $\cdots$ & $\cdots$ & $\cdots$ & $<5.0$ & $<5.0$ & $<5.0$ & $<5.0$ & $<5.0$ & $<5.0$ & $<5.0$ & $<5.0$ \\
\hline JUN & & & & & & & & & & & & \\
\hline 01.01 & 1.9 & 15 & 2.1 & 9.9 & $\cdots$ & $\cdots$ & $\cdots$ & $\cdots$ & $\cdots$ & $\cdots$ & $\cdots$ & $\cdots$ \\
\hline
\end{tabular}

\begin{tabular}{|c|c|c|c|c|c|c|c|c|c|c|c|c|}
\hline & \multirow[b]{3}{*}{$\begin{array}{l}\text { ACE - } \\
\text { NAPHTH - } \\
\text { YLENE }\end{array}$} & \multirow[b]{3}{*}{$\begin{array}{c}\text { ANTHRA - } \\
\text { CENE }\end{array}$} & \multirow[b]{3}{*}{$\begin{array}{l}\text { BENZI - } \\
\text { DINE }\end{array}$} & \multicolumn{4}{|l|}{ BENZO A } & BENZO & \multicolumn{2}{|l|}{$4-$} & BIS & BIS \\
\hline & & & & ANTHRAC & & BENZO B & BENZO $\mathrm{K}$ & GHI PERYL & BROMO - & N-BUTYL & (2- & 2 \\
\hline & & & & $\begin{array}{l}\text { ENE1, 2- } \\
\text { BENZANT } \\
\text { HRACENE }\end{array}$ & $\begin{array}{l}\text { BENZO- } \\
\text { A- } \\
\text { PYRENE }\end{array}$ & $\begin{array}{l}\text { FLUOR - } \\
\text { AN - } \\
\text { THENE }\end{array}$ & $\begin{array}{l}\text { FLUOR - } \\
\text { AN- } \\
\text { THENE }\end{array}$ & $\begin{array}{l}\text { ENE1, } 12 \text { - } \\
\text { BENZO } \\
\text { PERYLENE }\end{array}$ & $\begin{array}{l}\text { PHENYL } \\
\text { PHENYL } \\
\text { ETHER }\end{array}$ & $\begin{array}{c}\text { BENZYL } \\
\text { PHTHAL- } \\
\text { ATE }\end{array}$ & $\begin{array}{l}\text { CHLORO- } \\
\text { ETHOXY) } \\
\text { METHANE }\end{array}$ & $\begin{array}{l}\text { CHLORO- } \\
\text { ETHYL } \\
\text { ETHER }\end{array}$ \\
\hline & $\begin{array}{l}\text { TOTAL } \\
\text { (UG/L) }\end{array}$ & $\begin{array}{l}\text { TOTAL } \\
\text { (UG/L) }\end{array}$ & $\begin{array}{l}\text { TOTAL } \\
\text { (UG/L) }\end{array}$ & $\begin{array}{l}\text { TOTAL } \\
\text { (UG/L) }\end{array}$ & $\begin{array}{l}\text { TOTAL } \\
\text { (UG/L) }\end{array}$ & $\begin{array}{l}\text { TOTAL } \\
\text { (UG/L) }\end{array}$ & $\begin{array}{l}\text { TOTAL } \\
\text { (UG/L) }\end{array}$ & $\begin{array}{l}\text { TOTAL } \\
\text { (UG/L) }\end{array}$ & $\begin{array}{l}\text { TOTAL } \\
\text { (UG/L) }\end{array}$ & $\begin{array}{l}\text { TOTAL } \\
\text { (UG/L) }\end{array}$ & $\begin{array}{l}\text { TOTAL } \\
(U G / L)\end{array}$ & $\begin{array}{l}\text { TOTAL } \\
\text { (UG/L) }\end{array}$ \\
\hline
\end{tabular}

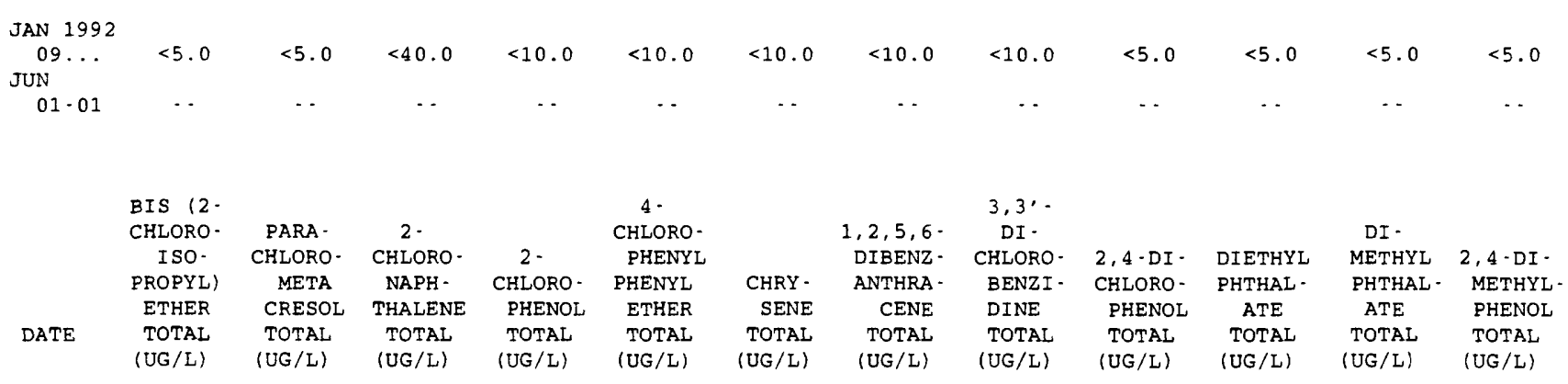

\begin{tabular}{|c|c|c|c|c|c|c|c|c|c|c|c|c|}
\hline $\begin{array}{c}\text { JAN } 1992 \\
09 \ldots\end{array}$ & $<5.0$ & $<30.0$ & $<5.0$ & $<5.0$ & $<5.0$ & $<10.0$ & $<10.0$ & $<20.0$ & $<5.0$ & $<5.0$ & $<5.0$ & $<5.0$ \\
\hline JUN & & & & & & & & & & & & \\
\hline $01-01$ & $\cdots$ & $\cdots$ & $\cdots$ & $\cdots$ & $\cdots$ & $\cdots$ & $\cdots$ & $\cdots$ & $\cdots$ & $\cdots$ & $\cdots$ & $\cdots$ \\
\hline
\end{tabular}


Table 11. Quality-assurance/quality-control data for storm-sewer outfall stations, Dallas, Texas, 1992-93-Continued

08056390 - BASTILLE STREET OUTFALL AT LA REUNION PARKWAY, DALLAS,TX (WY 1992)-COntinUed

\begin{tabular}{|c|c|c|c|c|c|c|c|c|c|c|c|c|}
\hline DATE & $\begin{array}{c}\text { DI - N- } \\
\text { BUTYL } \\
\text { PHTHAL- } \\
\text { ATE } \\
\text { TOTAL } \\
\text { (UG/L) }\end{array}$ & $\begin{array}{c}4,6- \\
\text { DINITRO- } \\
\text { ORTHO- } \\
\text { CRESOL } \\
\text { TOTAL } \\
\text { (UG/L) }\end{array}$ & $\begin{array}{c}2,4,- \\
\text { DI - } \\
\text { NITRO- } \\
\text { PHENOL } \\
\text { TOTAL } \\
\text { (UG/L) }\end{array}$ & $\begin{array}{l}2,4 \text {-DI- } \\
\text { NITRO- } \\
\text { TOLUENE } \\
\text { TOTAL } \\
\text { (UG/L) }\end{array}$ & $\begin{array}{l}2,6 \text {-DI - } \\
\text { NITRO- } \\
\text { TOLUENE } \\
\text { TOTAL } \\
\text { (UG/L) }\end{array}$ & $\begin{array}{c}\text { DI -N- } \\
\text { OCTYL } \\
\text { PHTHAL- } \\
\text { ATE } \\
\text { TOTAL } \\
(\text { UG/L) }\end{array}$ & $\begin{array}{c}\text { BIS }(2- \\
\text { ETHYL } \\
\text { HEXYL) } \\
\text { PHTHAL- } \\
\text { ATE } \\
\text { TOTAL } \\
\text { (UG/L) }\end{array}$ & $\begin{array}{l}\text { FLUOR - } \\
\text { ANTHENE } \\
\text { TOTAL } \\
(U G / L)\end{array}$ & $\begin{array}{l}\text { FLUOR- } \\
\text { ENE } \\
\text { TOTAL } \\
\text { (UG/L) }\end{array}$ & $\begin{array}{c}\text { HEXA- } \\
\text { CHLORO- } \\
\text { BENZENE } \\
\text { TOTAL } \\
\text { (UG/L) }\end{array}$ & $\begin{array}{c}\text { HEXA- } \\
\text { CHLORO- } \\
\text { CYCLO- } \\
\text { PENT - } \\
\text { ADIENE } \\
\text { TOTAL } \\
\text { (UG/L) }\end{array}$ & $\begin{array}{c}\text { HEXA- } \\
\text { CHLORO- } \\
\text { ETHANE } \\
\text { TOTAL } \\
\text { (UG/L) }\end{array}$ \\
\hline $\begin{array}{c}\text { JAN } 1992 \\
09 \ldots \\
\text { JUN } \\
01-01\end{array}$ & $<5.0$ & $<30.0$ & $<20.0$ & $<5.0$ & $<5.0$ & $<10.0$ & $<5.0$ & $<5.0$ & $<5.0$ & $<5.0$ & $<5.0$ & $<5.0$ \\
\hline DATE & $\begin{array}{r}\text { INDENO } \\
(1,2,3- \\
\text { CD) } \\
\text { PYRENE } \\
\text { TOTAL } \\
\text { (UG/L) }\end{array}$ & $\begin{array}{l}\text { ISO- } \\
\text { PHORONE } \\
\text { TOTAL } \\
\text { (UG/L) }\end{array}$ & $\begin{array}{l}\text { NITRO- } \\
\text { BENZENE } \\
\text { TOTAL } \\
\text { (UG/L) }\end{array}$ & $\begin{array}{l}\text { N-NITRO- } \\
\text { SODI - } \\
\text { METHY - } \\
\text { LAMINE } \\
\text { TOTAL } \\
\text { (UG/L) }\end{array}$ & $\begin{array}{l}2- \\
\text { NITRO- } \\
\text { PHENOL } \\
\text { TOTAL } \\
\text { (UG/L) }\end{array}$ & $\begin{array}{c}4- \\
\text { NITRO- } \\
\text { PHENOL } \\
\text { TOTAL } \\
\text { (UG/L) }\end{array}$ & $\begin{array}{l}\text { N-NITRO- } \\
\text { SODI - } \\
\text { PHENY - } \\
\text { LAMINE } \\
\text { TOTAL } \\
\text { (UG/L) }\end{array}$ & $\begin{array}{l}\text { PENTA- } \\
\text { CHLORO- } \\
\text { PHENOL } \\
\text { TOTAL } \\
\text { (UG/L) }\end{array}$ & $\begin{array}{l}\text { PHENAN - } \\
\text { THRENE } \\
\text { TOTAL } \\
\text { (UG/L) }\end{array}$ & $\begin{array}{c}\text { PHENOL } \\
\text { (C6H- } \\
50 \mathrm{H}) \\
\text { TOTAL } \\
\text { (UG/L) }\end{array}$ & $\begin{array}{l}\text { PYRENE } \\
\text { TOTAL } \\
\text { (UG/L) }\end{array}$ & $\begin{array}{c}2,4,6- \\
\text { TRI - } \\
\text { CHLORO- } \\
\text { PHENOL } \\
\text { TOTAL } \\
\text { (UG/L) }\end{array}$ \\
\hline $\begin{array}{c}\text { AN } 1992 \\
09 \ldots \\
\text { UN } \ldots 1-01\end{array}$ & $<10.0$ & $<5.0$ & $<5.0$ & $<5.0$ & $<5.0$ & $<30.0$ & $<5.0$ & $<30.0$ & $<5.0$ & $<5.0$ & $<5.0$ & $<20.0$ \\
\hline
\end{tabular}

08057135 - WHITE ROCK CREEK OUTFALL AT PRESTON ROAD, DALLAS, TX (WY 1993)

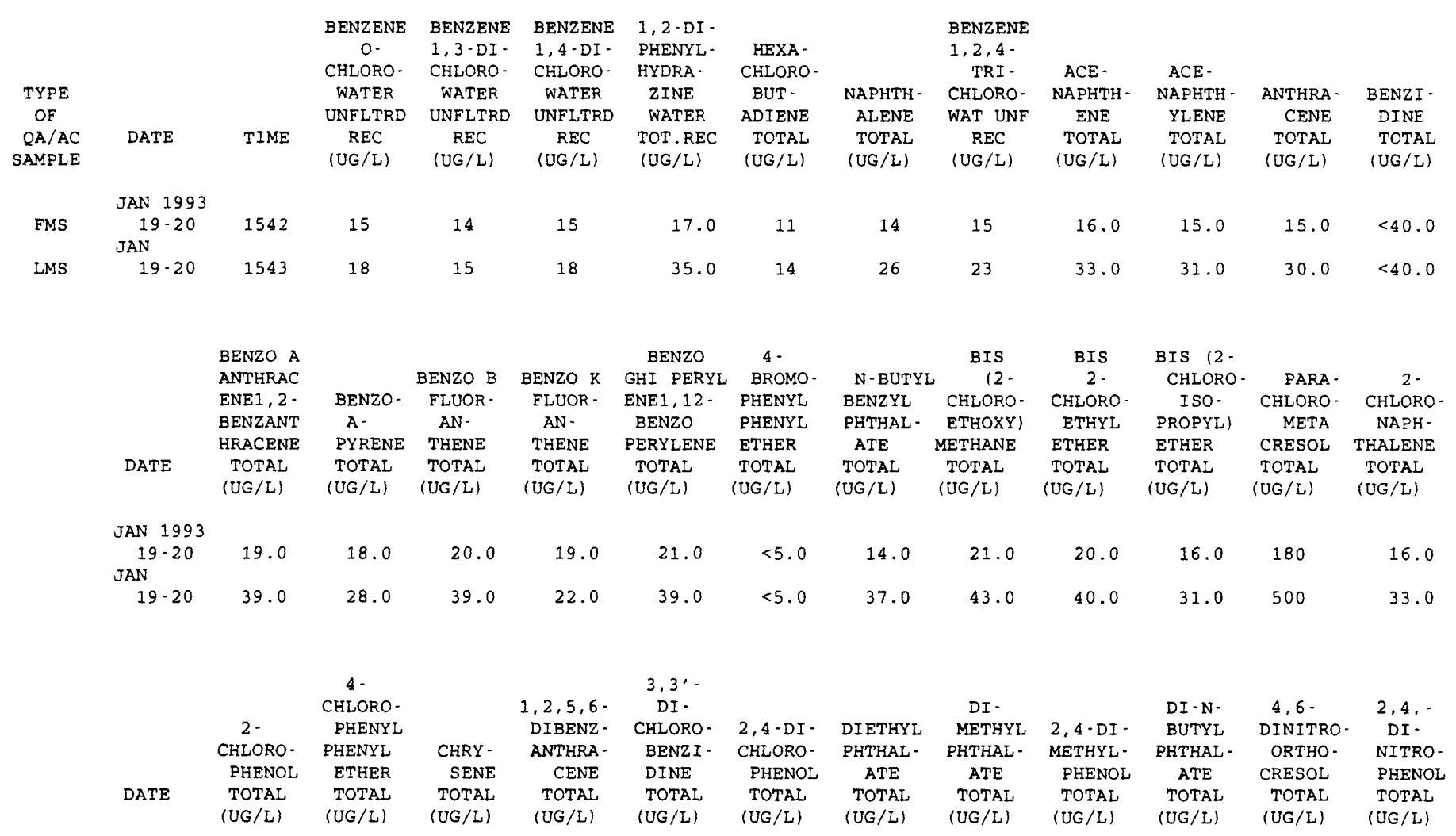

$\begin{array}{cccccccccccccc}\text { JAN } 1993 & & & & & & & & \\ 19-20 & 40.0 & 17.0 & 22.0 & 16.0 & <20.0 & 43.0 & 16.0 & 14.0 & 45.0 & 16.0 & 170 & 120 \\ \text { JAN } & 78.0 & 35.0 & 42.0 & 27.0 & <20.0 & 84.0 & 37.0 & 35.0 & 120 & 36.0 & 530 & 310\end{array}$


Table 11. Quality-assurance/quality-control data for storm-sewer outfall stations, Dallas, Texas, 1992-93-Continued

08057135 - WHITE ROCK CREEK OUTFALL AT PRESTON ROAD, DALLAS, TX (WY 1993)-Continued

\begin{tabular}{|c|c|c|c|c|c|c|c|c|c|c|c|}
\hline DATE & $\begin{array}{l}2,4 \cdot D I- \\
\text { NITRO- } \\
\text { TOLUENE } \\
\text { TOTAL } \\
(U G / L)\end{array}$ & $\begin{array}{l}2,6-D I- \\
\text { NITRO- } \\
\text { TOLUENE } \\
\text { TOTAL } \\
(\text { UG/L) }\end{array}$ & $\begin{array}{l}\text { DI - N- } \\
\text { OCTYL } \\
\text { PHTHAL- } \\
\text { ATE } \\
\text { TOTAL } \\
\text { (UG/L) }\end{array}$ & $\begin{array}{l}\text { BIS }(2- \\
\text { ETHYL } \\
\text { HEXYL) } \\
\text { PHTHAL- } \\
\text { ATE } \\
\text { TOTAL } \\
\text { (UG/L) }\end{array}$ & $\begin{array}{l}\text { FLUOR - } \\
\text { ANTHENE } \\
\text { TOTAL } \\
\text { (UG/L) }\end{array}$ & $\begin{array}{c}\text { FLUOR - } \\
\text { ENE } \\
\text { TOTAL } \\
\text { (UG/L) }\end{array}$ & $\begin{array}{l}\text { HEXA- } \\
\text { CHLORO- } \\
\text { BENZENE } \\
\text { TOTAL } \\
(\text { UG/L) }\end{array}$ & $\begin{array}{l}\text { HEXA- } \\
\text { CHLORO- } \\
\text { CYCLO- } \\
\text { PENT- } \\
\text { ADIENE } \\
\text { TOTAL } \\
\text { (UG/L) }\end{array}$ & $\begin{array}{l}\text { HEXA - } \\
\text { CHLORO- } \\
\text { ETHANE } \\
\text { TOTAL } \\
\text { (UG/L) }\end{array}$ & $\begin{array}{l}\text { INDENO } \\
(1,2,3- \\
\text { CD) } \\
\text { PYRENE } \\
\text { TOTAL } \\
(\text { UG/L) }\end{array}$ & $\begin{array}{l}\text { ISO- } \\
\text { PHORONE } \\
\text { TOTAL } \\
\text { (UG/L) }\end{array}$ \\
\hline JAN 1993 & & & & & & & & & & & \\
\hline $\begin{array}{l}19-20 \\
\text { JAN }\end{array}$ & 20.0 & 20.0 & 18.0 & 23.0 & 25.0 & 17.0 & 17.0 & $<5.0$ & 10.0 & 17.0 & $<5.0$ \\
\hline $19-20$ & 47.0 & 47.0 & 37.0 & 42.0 & 45.0 & 35.0 & 33.0 & 9.0 & 10.0 & 26.0 & 9.0 \\
\hline DATE & $\begin{array}{l}\text { NITRO- } \\
\text { BENZENE } \\
\text { TOTAL } \\
(\mathrm{UG} / \mathrm{L})\end{array}$ & $\begin{array}{l}\text { N-NITRO- } \\
\text { SODI - } \\
\text { METHY - } \\
\text { LAMINE } \\
\text { TOTAL } \\
\text { (UG/L) }\end{array}$ & $\begin{array}{c}2 \text { - } \\
\text { NITRO- } \\
\text { PHENOL } \\
\text { TOTAL } \\
\text { (UG/L) }\end{array}$ & $\begin{array}{l}4- \\
\text { NITRO - } \\
\text { PHENOL } \\
\text { TOTAL } \\
\text { (UG/L) }\end{array}$ & $\begin{array}{c}\text { N- } \\
\text { NITRO- } \\
\text { SODI - N- } \\
\text { PROPYL- } \\
\text { AMINE } \\
\text { TOTAL } \\
\text { (UG/L) }\end{array}$ & $\begin{array}{r}\text { N-NITRO- } \\
\text { SODI - } \\
\text { PHENY - } \\
\text { LAMINE } \\
\text { TOTAL } \\
\text { (UG/L) }\end{array}$ & $\begin{array}{l}\text { PENTA - } \\
\text { CHLORO - } \\
\text { PHENOL } \\
\text { TOTAL } \\
\text { (UG/L) }\end{array}$ & $\begin{array}{l}\text { PHENAN- } \\
\text { THRENE } \\
\text { TOTAL } \\
\text { (UG/L) }\end{array}$ & $\begin{array}{c}\text { PHENOL } \\
\text { (C6H- } \\
5 O H) \\
\text { TOTAL } \\
\text { (UG/L) }\end{array}$ & $\begin{array}{r}\text { PYRENE } \\
\text { TOTAL } \\
(\mathrm{UG} / \mathrm{L})\end{array}$ & $\begin{array}{c}2,4,6- \\
\text { TRI - } \\
\text { CHLORO- } \\
\text { PHENOL } \\
\text { TOTAL } \\
\text { (UG/L) }\end{array}$ \\
\hline JAN 1993 & & & & & & & & & & & \\
\hline $\begin{array}{l}19 \cdot 20 \\
\text { JAN }\end{array}$ & 20.0 & 19.0 & 44.0 & $<30.0$ & 16.0 & 16.0 & 160 & 19.0 & 25.0 & 23.0 & 130 \\
\hline $19-20$ & 40.0 & 36.0 & 88.0 & 310 & 22.0 & 30.0 & 470 & 36.0 & 65.0 & 41.0 & 350 \\
\hline
\end{tabular}

08057310 - ASH CREEK OUTFALL AT WHITTIER STREET, DALLAS, TX (WY 1992)

TYPE

OF

SAMPLE

REP

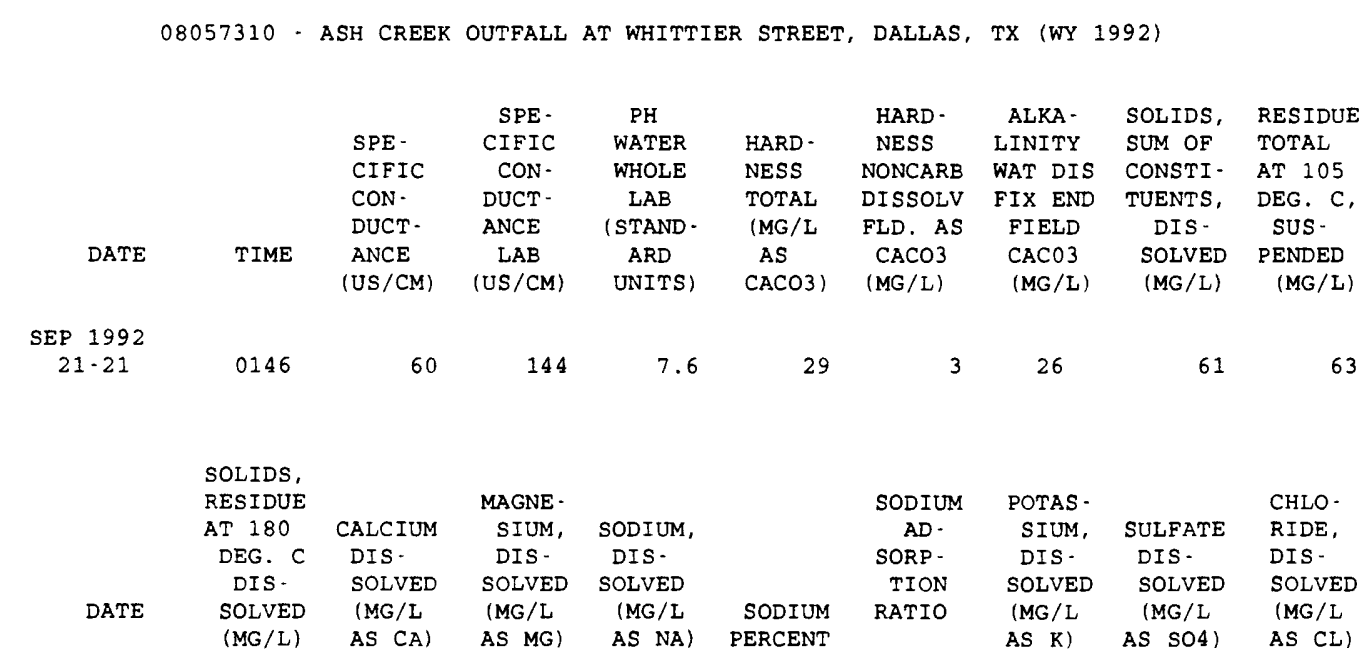

SEP 1992

$21-21$

$62 \quad 11$

$0.44 \quad 1.4$

8

0.1

4.7

4.8

2.5 
Table 11. Quality-assurance/quality-control data for storm-sewer outfall stations, Dallas, Texas, 1992-93-Continued 08057441 - NEWTON CREEK OUTFALL AT TIOGA STREET, DALLAS, TX (WY 1992)

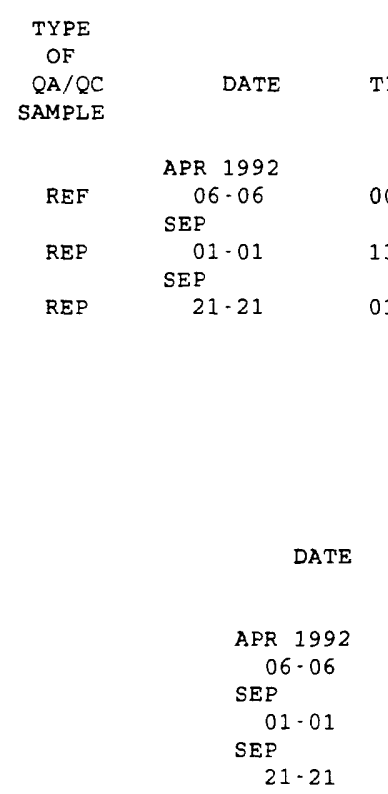

\begin{tabular}{|c|c|c|c|c|c|c|c|c|c|c|}
\hline IME & $\begin{array}{l}\text { NITRO- } \\
\text { GEN, } \\
\text { NITRITE } \\
\text { TOTAL } \\
\text { (MG/L }\end{array}$ & $\begin{array}{c}\text { NITRO- } \\
\text { GEN, } \\
\text { NO2+NO3 } \\
\text { TOTAL } \\
\text { (MG/L }\end{array}$ & $\begin{array}{l}\text { NITRO- } \\
\text { GEN, } \\
\text { AMMONIA } \\
\text { TOTAL } \\
\text { (MG/L }\end{array}$ & $\begin{array}{l}\text { NITRO- } \\
\text { GEN, AM- } \\
\text { MONIA + } \\
\text { ORGANIC } \\
\text { TOTAL } \\
\text { (MG/L }\end{array}$ & $\begin{array}{l}\text { PHOS - } \\
\text { PHORUS } \\
\text { TOTAL } \\
\text { (MG/L }\end{array}$ & $\begin{array}{c}\text { PHOS - } \\
\text { PHORUS } \\
\text { DIS - } \\
\text { SOLVED } \\
\text { (MG / L }\end{array}$ & $\begin{array}{c}\text { ARSENIC } \\
\text { TOTAL } \\
\text { (UG/L }\end{array}$ & $\begin{array}{l}\text { BERYL - } \\
\text { LIUM, } \\
\text { TOTAL } \\
\text { RECOV- } \\
\text { ERABLE } \\
\text { (UG/L }\end{array}$ & $\begin{array}{l}\text { CADMIUM } \\
\text { TOTAL } \\
\text { RECOV - } \\
\text { ERABLE } \\
\text { IUG/L }\end{array}$ & $\begin{array}{l}\text { CHRO- } \\
\text { MIUM, } \\
\text { TOTAL } \\
\text { RECOV- } \\
\text { ERABLE } \\
\text { (UG /L }\end{array}$ \\
\hline & AS N) & AS N) & AS N) & AS N) & AS P) & AS P) & AS AS) & AS BE) & AS CD) & AS CR) \\
\hline
\end{tabular}

$\begin{array}{ccccccc}0051 & 0.050 & 0.430 & 0.310 & 0.80 & 0.250 & 0.190 \\ 1323 & \ldots & \ldots & \ldots & \ldots & \ldots & \ldots \\ 0156 & \ldots & \ldots & \ldots & \ldots & \ldots & \ldots\end{array}$

$\begin{array}{rr}\cdots & \cdots \\ \cdots & \\ \quad & \cdots \\ & <10\end{array}$

$\begin{array}{ll}\cdots & \cdots \\ - & \cdots \\ <1 & \\ < & 7\end{array}$

\begin{tabular}{|c|c|c|c|c|c|c|c|c|c|}
\hline $\begin{array}{l}\text { OPPER, } \\
\text { TOTAL }\end{array}$ & $\begin{array}{l}\text { LEAD, } \\
\text { TOTAL }\end{array}$ & $\begin{array}{l}\text { MERCURY } \\
\text { TOTAL }\end{array}$ & $\begin{array}{l}\text { NICKEL, } \\
\text { TOTAL }\end{array}$ & SELE - & $\begin{array}{l}\text { SILVER, } \\
\text { TOTAL }\end{array}$ & $\begin{array}{l}\text { ZINC, } \\
\text { TOTAL }\end{array}$ & CARBON, & $\begin{array}{l}\text { OIL AND } \\
\text { GREASE, } \\
\text { TOTAL }\end{array}$ & \\
\hline RECOV - & RECOV - & RECOV - & RECOV - & NIUM, & RECOV - & RECOV - & ORGANIC & RECOV. & \\
\hline $\begin{array}{l}\text { ERABLE } \\
\text { /UG/L }\end{array}$ & $\begin{array}{l}\text { ERABLE } \\
\text { (UG/L }\end{array}$ & $\begin{array}{l}\text { ERABLE } \\
\text { /UG/L }\end{array}$ & $\begin{array}{l}\text { ERABLE } \\
(U G / L\end{array}$ & $\begin{array}{l}\text { TOTAL } \\
\text { (UG/L }\end{array}$ & $\begin{array}{l}\text { ERABLE } \\
\text { /UG/L }\end{array}$ & $\begin{array}{l}\text { ERABLE } \\
\text { (UG/L }\end{array}$ & $\begin{array}{l}\text { TOTAL } \\
\text { (MG/L }\end{array}$ & $\begin{array}{l}\text { GRAVI - } \\
\text { METRIC }\end{array}$ & $\begin{array}{c}\text { PHENOLS } \\
\text { TOTAL }\end{array}$ \\
\hline AS CU) & AS PB) & AS HG) & AS NI) & AS SE) & AS AG) & AS $\mathrm{ZN}$ ) & AS C) & $\langle M G / L\rangle$ & $(U G / L)$ \\
\hline
\end{tabular}
AS PB)

$\begin{array}{ll}\cdots & \cdots \\ \cdots & \cdots \\ 10 & 46\end{array}$
AS SE) 
Table 12. Quality-assurance/quality-control data for storm-sewer outfall stations, Fort worth, Texas, 1992-93

08047100 - CLEAR FORK TRINITY RIVER OUTFALL AT OAK HILL CIRCLE, FORT WORTH, TX (WY 1992)

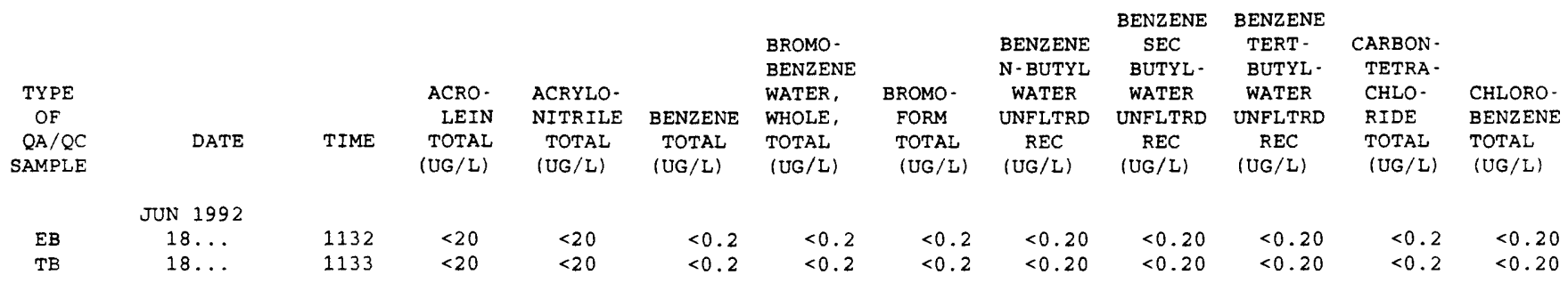

\begin{tabular}{|c|c|c|c|c|c|c|c|c|c|c|}
\hline DATE & $\begin{array}{l}\text { CHLORO- } \\
\text { DI- } \\
\text { BROMO- } \\
\text { METHANE } \\
\text { TOTAL } \\
\text { (UG } / \text { L) }\end{array}$ & $\begin{array}{l}\text { CHLORO- } \\
\text { ETHANE } \\
\text { TOTAL } \\
\text { (UG/L) }\end{array}$ & $\begin{array}{c}2- \\
\text { CHLORO- } \\
\text { ETHYL- } \\
\text { VINYL- } \\
\text { ETHER } \\
\text { TOTAL } \\
\text { (UG/L) }\end{array}$ & $\begin{array}{l}\text { CHLORO- } \\
\text { FORM } \\
\text { TOTAL } \\
\text { (UG/L) }\end{array}$ & $\begin{array}{l}\text { METHYL- } \\
\text { CHLO- } \\
\text { RIDE } \\
\text { TOTAL } \\
\text { (UG/L) }\end{array}$ & $\begin{array}{l}\text { O- } \\
\text { CHLORO- } \\
\text { TOLUENE } \\
\text { WATER } \\
\text { WHOLE } \\
\text { TOTAL } \\
\text { (UG/L) }\end{array}$ & $\begin{array}{c}\text { TOLUENE } \\
\text { P-CHLOR } \\
\text { WATER } \\
\text { UNFLTRD } \\
\text { REC } \\
\text { (UG/L) }\end{array}$ & $\begin{array}{l}\text { DIBROMO } \\
\text { CHLORO- } \\
\text { PROPANE } \\
\text { WATER } \\
\text { WHOLE } \\
\text { TOT. REC } \\
\text { (UG/L) }\end{array}$ & $\begin{array}{l}\text { DI - } \\
\text { BROMO- } \\
\text { METHANE } \\
\text { WATER } \\
\text { WHOLE } \\
\text { RECOVER } \\
\text { (UG/L) }\end{array}$ & $\begin{array}{l}1,2- \\
\text { DIBROMO } \\
\text { ETHANE } \\
\text { WATER } \\
\text { WHOLE } \\
\text { TOTAL } \\
\text { (UG/L) }\end{array}$ \\
\hline $\begin{array}{c}\text { JUN } 1992 \\
18 \ldots \\
18 \ldots\end{array}$ & $\begin{array}{l}<0.2 \\
<0.2\end{array}$ & $\begin{array}{l}<0.2 \\
<0.2\end{array}$ & $\begin{array}{l}<1.0 \\
<1.0\end{array}$ & $\begin{array}{r}0.8 \\
<0.2\end{array}$ & $\begin{array}{l}<0.2 \\
<0.2\end{array}$ & $\begin{array}{l}<0.2 \\
<0.2\end{array}$ & $\begin{array}{l}<0.20 \\
<0.20\end{array}$ & $\begin{array}{l}<1.0 \\
<1.0\end{array}$ & $\begin{array}{l}<0.2 \\
<0.2\end{array}$ & $\begin{array}{l}<0.2 \\
<0.2\end{array}$ \\
\hline DATE & $\begin{array}{c}\text { DI - } \\
\text { CHLORO- } \\
\text { BROMO- } \\
\text { METHANE } \\
\text { TOTAL } \\
\text { (UG/L) }\end{array}$ & $\begin{array}{c}1,1 \text {-DI- } \\
\text { CHLORO- } \\
\text { ETHYL- } \\
\text { ENE } \\
\text { TOTAL } \\
\text { (UG/L) }\end{array}$ & $\begin{array}{l}1,1-D I \\
\text { CHLORO- } \\
\text { PRO- } \\
\text { PENE, } \\
\text { WAT, WH } \\
\text { TOTAL } \\
\text { (UG } / L)\end{array}$ & $\begin{array}{c}\text { BENZENE } \\
0- \\
\text { CHLORO- } \\
\text { WATER } \\
\text { UNFLTRD } \\
\text { REC } \\
(U G / L)\end{array}$ & $\begin{array}{c}\text { BENZENE } \\
1,3-D I- \\
\text { CHLORO- } \\
\text { WATER } \\
\text { UNFLTRD } \\
\text { REC } \\
\text { (UG/L) }\end{array}$ & $\begin{array}{c}\text { BENZENE } \\
1,4-D I- \\
\text { CHLORO- } \\
\text { WATER } \\
\text { UNFLTRD } \\
\text { REC } \\
\text { (UG } / L \text { ) }\end{array}$ & $\begin{array}{l}\text { DI - } \\
\text { CHLORO- } \\
\text { DI- } \\
\text { FLUORO- } \\
\text { METHANE } \\
\text { TOTAL } \\
\text { (UG } / \text { L) }\end{array}$ & $\begin{array}{l}1,1 \text { - DI - } \\
\text { CHLORO- } \\
\text { ETHANE } \\
\text { TOTAL } \\
\text { (UG/L) }\end{array}$ & $\begin{array}{c}1,2 \cdot D I- \\
\text { CHLORO- } \\
\text { ETHANE } \\
\text { TOTAL } \\
\text { (UG/L) }\end{array}$ & $\begin{array}{c}\text { CIS- } 1,2- \\
\text { DI- } \\
\text { CHLORO- } \\
\text { ETHENE } \\
\text { WATER } \\
\text { TOTAL } \\
\text { (UG/L) }\end{array}$ \\
\hline $\begin{array}{c}\text { JUN } 1992 \\
18 \ldots \\
18 \ldots\end{array}$ & $\begin{array}{l}<0.2 \\
<0.2\end{array}$ & $\begin{array}{l}<0.2 \\
<0.2\end{array}$ & $\begin{array}{l}<0.2 \\
<0.2\end{array}$ & $\begin{array}{l}<0.20 \\
<0.20\end{array}$ & $\begin{array}{l}<0.20 \\
<0.20\end{array}$ & $\begin{array}{l}<0.20 \\
<0.20\end{array}$ & $\begin{array}{l}<0.2 \\
<0.2\end{array}$ & $\begin{array}{l}<0.2 \\
<0.2\end{array}$ & $\begin{array}{l}<0.2 \\
<0.2\end{array}$ & $\begin{array}{l}<0.2 \\
<0.2\end{array}$ \\
\hline DATE & $\begin{array}{c}1,2- \\
\text { TRANSDI } \\
\text { CHLORO- } \\
\text { ETHENE } \\
\text { TOTAL } \\
\text { (UG/L) }\end{array}$ & $\begin{array}{l}1,2-D I- \\
\text { CHLORO- } \\
\text { PROPANE } \\
\text { TOTAL } \\
\text { (UG/L) }\end{array}$ & $\begin{array}{c}1,3 \text {-DI - } \\
\text { CHLORO- } \\
\text { PROPANE } \\
\text { WAT. WH } \\
\text { TOTAL } \\
\text { (UG/L) }\end{array}$ & $\begin{array}{l}2,2-D I \\
\text { CHLORO- } \\
\text { PRO- } \\
\text { PANE } \\
\text { WAT, WH } \\
\text { TOTAL } \\
(U G / L)\end{array}$ & $\begin{array}{l}\text { CIS } \\
1,3-D I- \\
\text { CHLORO- } \\
\text { PROPENE } \\
\text { TOTAL } \\
\text { (UG/L) }\end{array}$ & $\begin{array}{l}\text { TRANS - } \\
1,3-\mathrm{DI} \text { - } \\
\text { CHLORO- } \\
\text { PROPENE } \\
\text { TOTAL } \\
\text { (UG/L) }\end{array}$ & $\begin{array}{l}\text { ETHYL - } \\
\text { BENZENE } \\
\text { TOTAL } \\
\text { (UG/L) }\end{array}$ & $\begin{array}{l}\text { HEXA- } \\
\text { CHLORO- } \\
\text { BUT - } \\
\text { ADIENE } \\
\text { TOTAL } \\
\text { (UG/L) }\end{array}$ & $\begin{array}{l}\text { ISO- } \\
\text { PROPYL- } \\
\text { BENZENE } \\
\text { WATER } \\
\text { WHOLE } \\
\text { REC } \\
\text { (UG/L) }\end{array}$ & $\begin{array}{l}\text { P-ISO- } \\
\text { PROPYL- } \\
\text { TOLUENE } \\
\text { WATER } \\
\text { WHOLE } \\
\text { REC } \\
\text { (UG/L) }\end{array}$ \\
\hline $\begin{array}{c}\text { JUN } 1992 \\
18 \ldots \\
18 \ldots\end{array}$ & $\begin{array}{l}<0.2 \\
<0.2\end{array}$ & $\begin{array}{l}<0.2 \\
<0.2\end{array}$ & $\begin{array}{l}<0.2 \\
<0.2\end{array}$ & $\begin{array}{l}<0.2 \\
<0.2\end{array}$ & $\begin{array}{l}<0.2 \\
<0.2\end{array}$ & $\begin{array}{l}<0.2 \\
<0.2\end{array}$ & $\begin{array}{l}<0.2 \\
<0.2\end{array}$ & $\begin{array}{l}<0.2 \\
<0.2\end{array}$ & $\begin{array}{l}<0.20 \\
<0.20\end{array}$ & $\begin{array}{l}<0.20 \\
<0.20\end{array}$ \\
\hline DATE & $\begin{array}{l}\text { METHYL- } \\
\text { BROMIDE } \\
\text { TOTAL } \\
\text { (UG/L) }\end{array}$ & $\begin{array}{l}\text { METHYL- } \\
\text { ENE } \\
\text { CHLO- } \\
\text { RIDE } \\
\text { TOTAL } \\
\text { (UG/L) }\end{array}$ & $\begin{array}{c}\text { NAPHTH - } \\
\text { ALENE } \\
\text { TOTAL } \\
\text { (UG/L) }\end{array}$ & $\begin{array}{c}\text { BENZENE } \\
\text { N- PROPY } \\
\text { WATER } \\
\text { UNFLTRD } \\
\text { REC } \\
(U G / L)\end{array}$ & $\begin{array}{l}\text { STYRENE } \\
\text { TOTAL } \\
\text { (UG/L) }\end{array}$ & $\begin{array}{c}\text { ETHANE, } \\
1112- \\
\text { TETRA- } \\
\text { CHLORO- } \\
\text { WAT UNF } \\
\text { REC } \\
\text { (UG/L) }\end{array}$ & $\begin{array}{c}\text { ETHANE, } \\
1,1,2,2 \\
\text { TETRA- } \\
\text { CHLORO- } \\
\text { WAT UNF } \\
\text { REC } \\
(U G / L)\end{array}$ & $\begin{array}{l}\text { TETRA- } \\
\text { CHLORO- } \\
\text { ETHYL- } \\
\text { ENE } \\
\text { TOTAL } \\
\text { (UG/L) }\end{array}$ & $\begin{array}{l}\text { TOLUENE } \\
\text { TOTAL } \\
\text { (UG/L) }\end{array}$ & $\begin{array}{l}1,2,3- \\
\text { TRI- } \\
\text { CHLORO } \\
\text { BENZENE } \\
\text { WAT, WH } \\
\text { REC } \\
\text { (UG/L) }\end{array}$ \\
\hline $\begin{array}{c}\text { JUN } 1992 \\
18 \ldots \\
18 \ldots\end{array}$ & $\begin{array}{l}<0.2 \\
<0.2\end{array}$ & $\begin{array}{l}<0.2 \\
<0.2\end{array}$ & $\begin{array}{l}<0.2 \\
<0.2\end{array}$ & $\begin{array}{l}<0.20 \\
<0.20\end{array}$ & $\begin{array}{l}<0.2 \\
<0.2\end{array}$ & $\begin{array}{l}<0.2 \\
<0.2\end{array}$ & $\begin{array}{l}<0.2 \\
<0.2\end{array}$ & $\begin{array}{l}<0.2 \\
<0.2\end{array}$ & $\begin{array}{l}<0.2 \\
<0.2\end{array}$ & $\begin{array}{l}<0.20 \\
<0.20\end{array}$ \\
\hline
\end{tabular}


Table 12. Quality-assurance/quality-control data for storm-sewer outfall stations, Fort worth, Texas, 1992-93-Continued 08047100 - CLEAR FORK TRINITY RIVER OUTFALL AT OAK HILL CIRCLE, FORT WORTH, TX (WY 1992)-CONTINUEd

\begin{tabular}{|c|c|c|c|c|c|c|c|c|c|c|}
\hline & BENZENE & & & & & $123-\mathrm{TRI}$ & & & & \\
\hline & $\begin{array}{c}1,2,4 \\
\text { TRI }\end{array}$ & $\begin{array}{c}1,1,1- \\
\text { TRI. }\end{array}$ & $\begin{array}{c}1,1,2- \\
\text { TRI - }\end{array}$ & $\begin{array}{c}\text { TRI - } \\
\text { CHLORO. }\end{array}$ & $\begin{array}{c}\text { TRI - } \\
\text { CHLORO- }\end{array}$ & $\begin{array}{l}\text { CHLORO- } \\
\text { PROPANE }\end{array}$ & $\begin{array}{l}\text { PSEUDO - } \\
\text { CUMENE }\end{array}$ & $\begin{array}{l}\text { MESIT - } \\
\text { YLENE }\end{array}$ & VINYL & XYLENE \\
\hline & CHLORO - & CHLORO- & CHLORO- & ETHYL - & FLUORO- & WATER & WATER & WATER & CHLO & WATER \\
\hline & WAT UNF & ETHANE & ETHANE & ENE & METHANE & WHOLE & UNFLTRD & UNFLTRD & RIDE & UNFLTRD \\
\hline DATE & $\begin{array}{c}\text { REC } \\
(U G / L)\end{array}$ & $\begin{array}{l}\text { TOTAL } \\
\text { (UG/L) }\end{array}$ & $\begin{array}{l}\text { TOTAL } \\
\text { (UG/L) }\end{array}$ & $\begin{array}{l}\text { TOTAL } \\
\text { (UG/L) }\end{array}$ & $\begin{array}{l}\text { TOTAL } \\
\text { (UG/L) }\end{array}$ & $\begin{array}{l}\text { TOTAL } \\
\text { (UG/L) }\end{array}$ & $\begin{array}{c}R E C \\
(U G / L)\end{array}$ & $\begin{array}{c}\text { REC } \\
(U G / L)\end{array}$ & $\begin{array}{l}\text { TOTAL } \\
\text { (UG/L) }\end{array}$ & $\begin{array}{c}\text { REC } \\
(U G / L)\end{array}$ \\
\hline $\mathrm{JN}$ & & & & & & & & & & \\
\hline $18 \ldots$ & $<0.20$ & $<0.2$ & $<0.2$ & $<0.2$ & 0.3 & $<0.2$ & $<0.20$ & $<0.20$ & $<0.2$ & $<0.20$ \\
\hline $18 \ldots$ & $<0.20$ & $<0.3$ & $<0.2$ & $<0.2$ & $<0.2$ & $<0.2$ & $<0.20$ & $<0.20$ & $<0.2$ & $<0.20$ \\
\hline
\end{tabular}

08048510 - WEST FORK TRINITY RIVER OUTFALL AT HIGHWAY 121, FORT WORTH, TX (WY 1992)

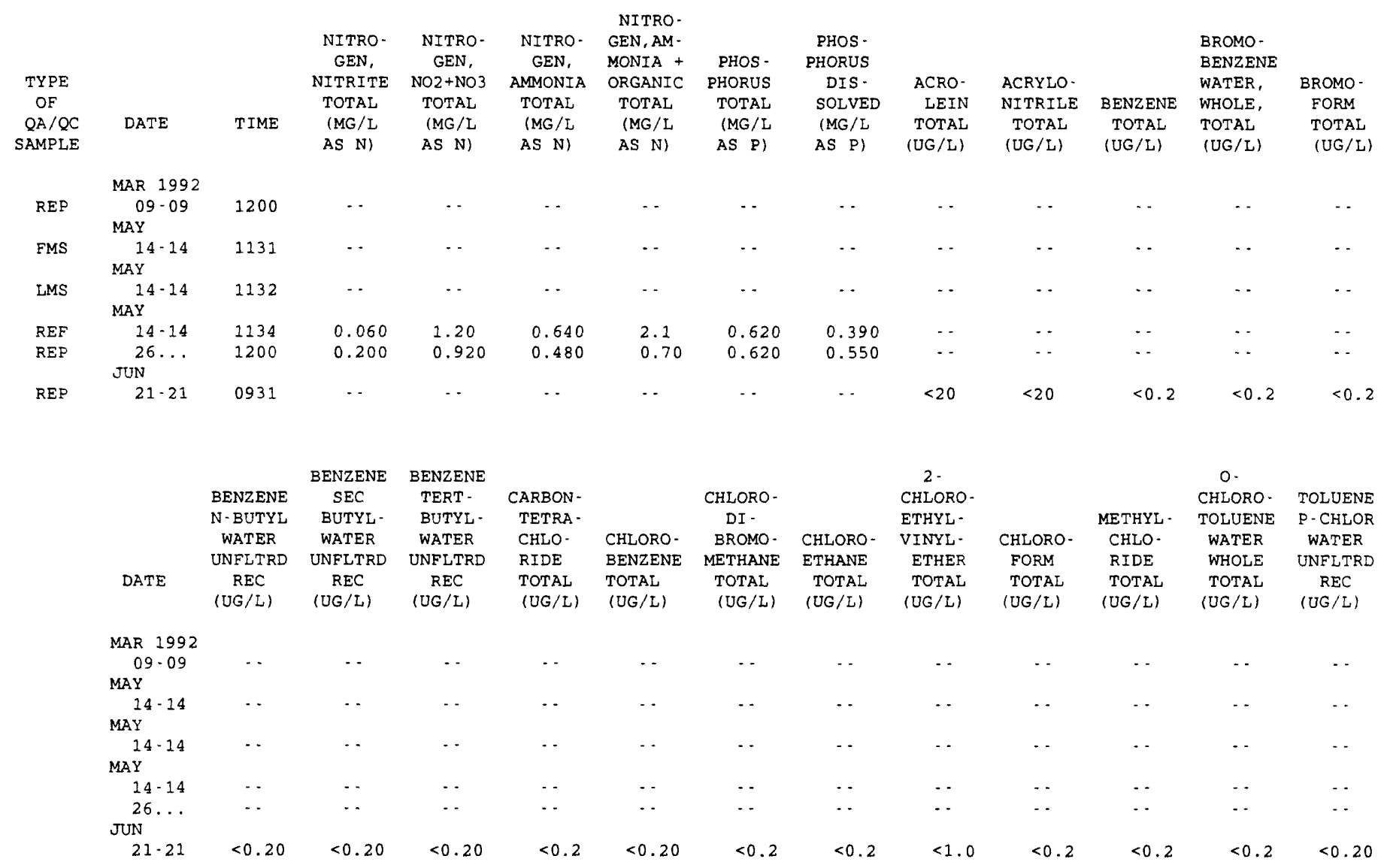


Table 12. Quality-assurance/quality-control data for storm-sewer outfall stations, Fort worth, Texas, 1992-93-Continued

08048510 - WEST FORK TRINITY RIVER OUTFALI AT HIGHWAY 121, FORT WORTH, TX (WY 1992-CONTINUEd)

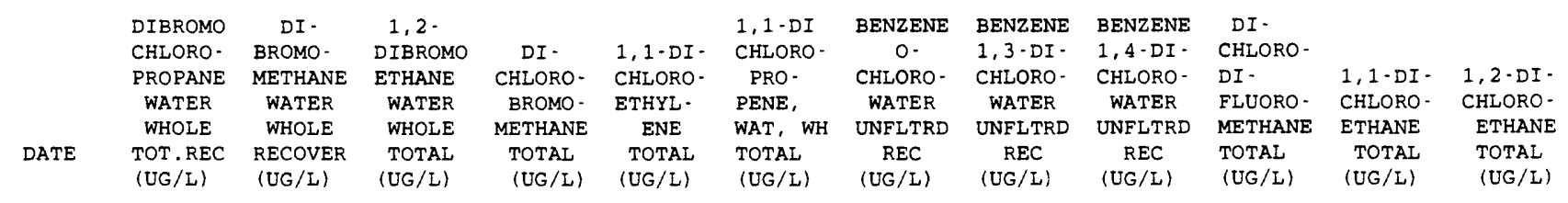

\begin{tabular}{|c|c|c|c|c|c|c|c|c|c|c|c|c|}
\hline \multicolumn{13}{|l|}{ MAR 1992} \\
\hline $\begin{array}{c}09-09 \\
\text { MAY }\end{array}$ & $\cdots$ & $\cdots$ & $\cdots$ & $\cdots$ & $\cdots$ & $\cdots$ & $<5.0$ & $<5.0$ & $<5.0$ & $\cdots$ & $\cdots$ & $\cdots$ \\
\hline $14-14$ & $\cdots$ & $\cdots$ & $\ldots$ & $\ldots$ & . & & & & & & & \\
\hline MAY & & & & & & & & 8.0 & 9.0 & & 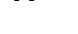 & $\cdots$ \\
\hline $14-14$ & $\cdots$ & $\cdots$ & - - & $\cdots$ & $\cdots$ & $\cdots$ & 9.0 & 8.0 & 9.0 & $\cdots$ & $\cdots$ & $\cdots$ \\
\hline MAY & & & & & & & & & & & & \\
\hline $14-14$ & -. & $\cdots$ & - & $\cdots$ & $\cdots$ & $\cdots$ & $\cdots$ & $\cdots$ & $\cdots$ & $\cdots$ & $\cdots$ & $\cdots$ \\
\hline $26 \ldots$ & $\cdots$ & $\cdots$ & $\cdots$ & $\cdots$ & $\cdots$ & $\cdots$ & $\cdots$ & $\cdots$ & $\cdots$ & $\cdots$ & $\cdots$ & $\cdots$ \\
\hline JUN & & & & & & & & & & & & \\
\hline $21 \cdot 21$ & $<1.0$ & $<0.2$ & $<0.2$ & $<0.2$ & $<0.2$ & $<0.2$ & $<0.20$ & $<0.20$ & $<0.20$ & $<0.2$ & $<0.2$ & $<0.2$ \\
\hline
\end{tabular}

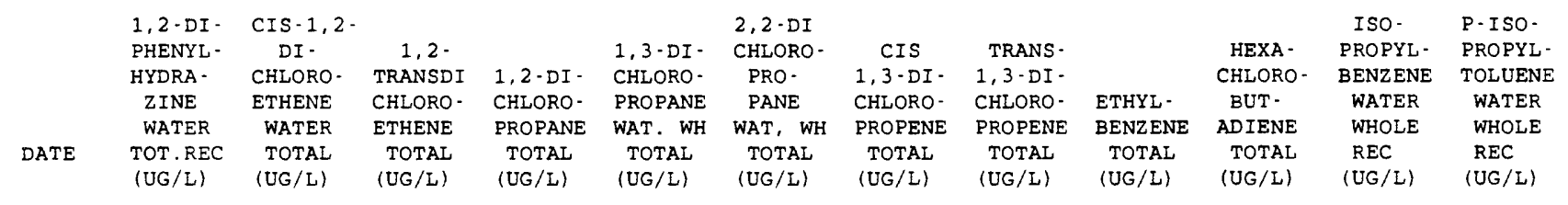

MAR 1992

$09-09$

MAY

$14 \cdot 14$

MAY

$14-14$

MAY

$14-14$

$26 \ldots$

JUN

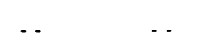

\begin{tabular}{|c|c|c|c|c|c|c|c|c|c|c|c|c|}
\hline \multicolumn{13}{|l|}{ MAR 1992} \\
\hline $09-09$ & $\cdots$ & - & $<5.0$ & $\cdots$ & $\cdots$ & $\cdots$ & $\cdots$ & $\cdots$ & $\cdots$ & $\cdots$ & $<5.0$ & $\cdots$ \\
\hline \multicolumn{13}{|l|}{ MAY } \\
\hline $14 \cdot 14$ & $\cdots$ & $\cdots$ & $<5.0$ & $\cdots$ & $\cdots$ & $\cdots$ & $\cdots$ & $\cdots$ & $\cdots$ & $\cdots$ & 12 & -- \\
\hline \multicolumn{13}{|l|}{ MAY } \\
\hline $14-14$ & $\cdots$ & $\cdots$ & $<5.0$ & $\cdots$ & $\cdots$ & -- & $\cdots$ & $\cdots$ & -- & $\cdots$ & 11 & $\cdots$ \\
\hline \multicolumn{13}{|l|}{ MAY } \\
\hline $14-14$ & $\cdots$ & -- & $\cdots$ & - & $\cdots$ & $\cdots$ & $\cdots$ & $\cdots$ & $\cdots$ & $\cdots$ & $\cdots$ & $\cdots$ \\
\hline $26 \ldots$ & $\cdots$ & $\cdots$ & -- & $\cdots$ & $\cdots$ & $\cdots$ & $\cdots$ & $\cdots$ & $\cdots$ & $\cdots$ & $\cdots$ & $\cdots$ \\
\hline \multicolumn{13}{|l|}{ JUN } \\
\hline $21-21$ & $<0.2$ & $<0.2$ & $<0.2$ & $<0.20$ & $<0.2$ & $<0.2$ & $<0.2$ & $<0.2$ & $<0.2$ & $<0.20$ & $<0.20$ & $<0.2$ \\
\hline
\end{tabular}


Table 12. Quality-assurance/quality-control data for storm-sewer outfall stations, Fort worth, Texas, 1992-93-Continued 08048510 - WEST FORK TRINITY RIVER OUTFALL AT HIGHWAY 121, FORT WORTH, TX (WY 1992-CONTINUEd)

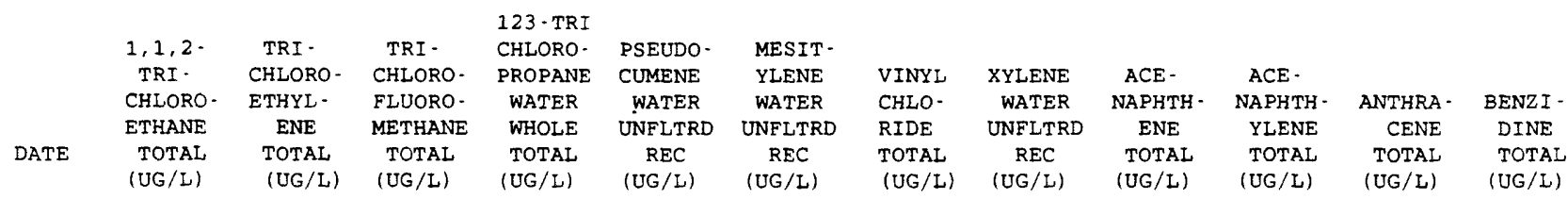

\begin{tabular}{|c|c|c|c|c|c|c|c|c|c|c|c|c|}
\hline $\begin{array}{r}\text { MAR } 1992 \\
09-09\end{array}$ & $\cdots$ & -- & $\cdots$ & $\cdots$ & $\cdots$ & $\cdots$ & $\cdots$ & $\cdots$ & $<5.0$ & $<5.0$ & $<5.0$ & $<40.0$ \\
\hline \multicolumn{13}{|l|}{ MAY } \\
\hline $14-14$ & $\cdots$ & $\cdots$ & $\cdots$ & $\cdots$ & $\cdots$ & $\cdots$ & $\cdots$ & $\cdots$ & 13.0 & 11.0 & 13.0 & $<40.0$ \\
\hline \multicolumn{13}{|l|}{ MAY } \\
\hline $14-14$ & $\cdots$ & $\cdots$ & $\cdots$ & $\cdots$ & $\cdots$ & $\cdots$ & $\cdots$ & $\cdots$ & 12.0 & 10.0 & 11.0 & $<40.0$ \\
\hline \multicolumn{13}{|l|}{ MAY } \\
\hline $14-14$ & $\cdots$ & $\cdots$ & $\cdots$ & $\cdots$ & $\cdots$ & $\cdots$ & $\cdots$ & -- & $-\cdot$ & - & $\cdots$ & $\cdots$ \\
\hline $26 \ldots$ & $\cdots$ & $\cdots$ & $\cdots$ & $\cdots$ & $\cdots$ & $\cdots$ & $\cdots$ & $\cdots$ & $\cdots$ & $\cdots$ & -- & $\cdots$ \\
\hline \multicolumn{13}{|l|}{ JUN } \\
\hline $21 \cdot 21$ & $<0.2$ & $<0.2$ & $<0.2$ & $<0.2$ & $<0.20$ & $<0.20$ & $<0.2$ & $<0.20$ & $\cdots$ & $\cdots$ & $\cdots$ & $\cdots$ \\
\hline
\end{tabular}

\begin{tabular}{|c|c|c|c|c|c|c|c|c|c|c|c|c|}
\hline & BENZO A & & & & BENZO & $4-$ & & BIS & BIS & BIS 12 - & & \\
\hline & ANTHRAC & & BENZO B & BENZO $\mathrm{K}$ & GHI PERYL & BROMO - & N-BUTYL & $12-$ & $2-$ & CHLORO - & PARA - & $2-$ \\
\hline & ENE1, 2- & BENZO - & FLUOR - & FLUOR - & ENE1, 12- & PHENYL & BENZYL & CHLORO - & CHLORO - & ISO & CHLORO - & CHLORO \\
\hline & BENZANT & A - & AN - & AN - & BENZO & PHENYL & PHTHAL - & ЕтноХY) & ETHYL & PROPYL) & META & NAPH - \\
\hline & HRACENE & PYRENE & THENE & THENE & PERYLENE & ETHER & ATE & METHANE & ETHER & ETHER & CRESOL & THALEN \\
\hline DATE & $\begin{array}{l}\text { TOTAL } \\
\text { (UG/L) }\end{array}$ & $\begin{array}{l}\text { TOTAL } \\
\text { (UG/L) }\end{array}$ & $\begin{array}{l}\text { TOTAL } \\
\text { (UG/L) }\end{array}$ & $\begin{array}{l}\text { TOTAL } \\
\text { (UG/L) }\end{array}$ & $\begin{array}{l}\text { TOTAL } \\
\text { (UG/L) }\end{array}$ & $\begin{array}{l}\text { TOTAL } \\
\text { (UG/L) }\end{array}$ & $\begin{array}{l}\text { TOTAL } \\
\text { (UG/L) }\end{array}$ & $\begin{array}{l}\text { TOTAL } \\
(U G / L)\end{array}$ & $\begin{array}{l}\text { TOTAL } \\
\text { (UG/L) }\end{array}$ & $\begin{array}{l}\text { TOTAL } \\
\text { (UG/L) }\end{array}$ & $\begin{array}{l}\text { TOTAL } \\
(\mathrm{UG} / \mathrm{L})\end{array}$ & $\begin{array}{l}\text { TOTAL } \\
\text { (UG/L) }\end{array}$ \\
\hline MAR 1992 & & & & & & & & & & & & \\
\hline $09-09$ & $<10.0$ & $<10.0$ & $<10.0$ & $<10.0$ & $<10,0$ & $<5.0$ & $<5.0$ & $<5.0$ & $<5.0$ & $<5.0$ & $<30.0$ & $<5.0$ \\
\hline MAY & & & & & & & & & & & & \\
\hline $14-14$ & 15.0 & 13.0 & 16.0 & 14.0 & 13.0 & $<5.0$ & 11.0 & 15.0 & 13.0 & 18.0 & 180 & $<5.0$ \\
\hline MAY & & & & & & & & & & & & \\
\hline $14-14$ & 13.0 & 11.0 & 14.0 & 13.0 & 11.0 & $<5.0$ & 9.0 & 13.0 & 11.0 & 15.0 & 120 & $<5.0$ \\
\hline MAY & & & & & & & & & & & & \\
\hline $14 \cdot 14$ & $-\cdot$ & - & $\cdots$ & $\cdots$ & $\cdots$ & $\cdots$ & $\cdots$ & $\cdots$ & $\cdots$ & - & $\cdots$ & $\cdots$ \\
\hline $26 \ldots$ & $\cdots$ & $\cdots$ & $\cdots$ & $\cdots$ & $-\cdots$ & $\cdots$ & $\cdots$ & - & $\cdots$ & $\cdots$ & $\cdots$ & $\cdots$ \\
\hline JUN & & & & & & & & & & & & \\
\hline $21-21$ & $\cdots$ & - & $\cdots$ & - & - - & $\cdots$ & $\cdots$ & $\therefore$ & $\cdots$ & $\cdots$ & $\cdots$ & $\cdots$ \\
\hline
\end{tabular}

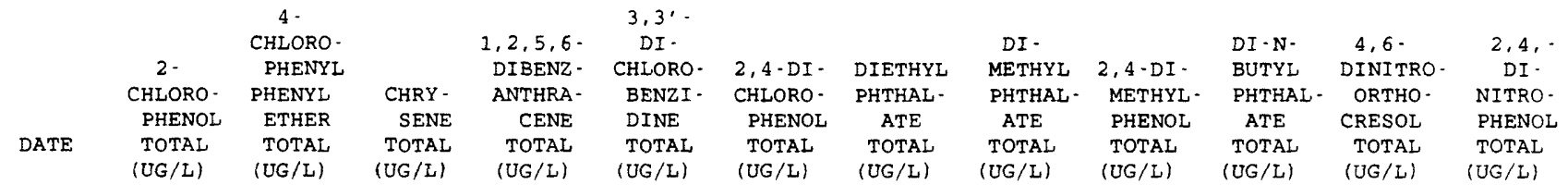

\begin{tabular}{|c|c|c|c|c|c|c|c|c|c|c|c|c|}
\hline \multicolumn{13}{|l|}{ MAR 1992} \\
\hline $09-09$ & $<5.0$ & $<5.0$ & $<10.0$ & $<10.0$ & $<20.0$ & $<5.0$ & $<5.0$ & $<5.0$ & $<5.0$ & $<5.0$ & $<30.0$ & $<20.0$ \\
\hline \multicolumn{13}{|l|}{ MAY } \\
\hline $14-14$ & 25.0 & 15.0 & 15.0 & 14.0 & $<20.0$ & 39.0 & 14.0 & 12.0 & 42.0 & 11.0 & 96.0 & 43.0 \\
\hline \multicolumn{13}{|l|}{ MAY } \\
\hline $14-14$ & 17.0 & 13.0 & 13.0 & 12.0 & $<20.0$ & 25.0 & 12.0 & 10.0 & 33.0 & 9.0 & 49.0 & 22.0 \\
\hline \multicolumn{13}{|l|}{ MAY } \\
\hline $14-14$ & $\cdots$ & $\cdots$ & $\cdots$ & $\cdots$ & -- & $\cdots$ & - & $\cdots$ & $\cdots$ & - & $\cdots$ & $\cdots$ \\
\hline $26 \ldots$ & $\cdots$ & $\cdots$ & $-\cdot$ & $\cdots$ & $\cdots$ & - & - & - & - & - & $\cdots$ & $\cdots$ \\
\hline \multicolumn{13}{|l|}{ JUN } \\
\hline $21-21$ & $\cdots$ & $\cdots$ & . & - & $\cdots$ & $\cdots$ & $\cdots$ & $\cdots$ & $\cdots$ & $\cdots$ & $\cdots$ & $\cdots$ \\
\hline
\end{tabular}


Table 12. Quality-assurance/quality-control data for storm-sewer outfall stations, Fort Worth, Texas, 1992-93-Continued

08048510 - WEST FORK TRINITY RIVER OUTFALL AT HIGHWAY 121, FORT WORTH, TX (WY 1992-CONTINUEd)

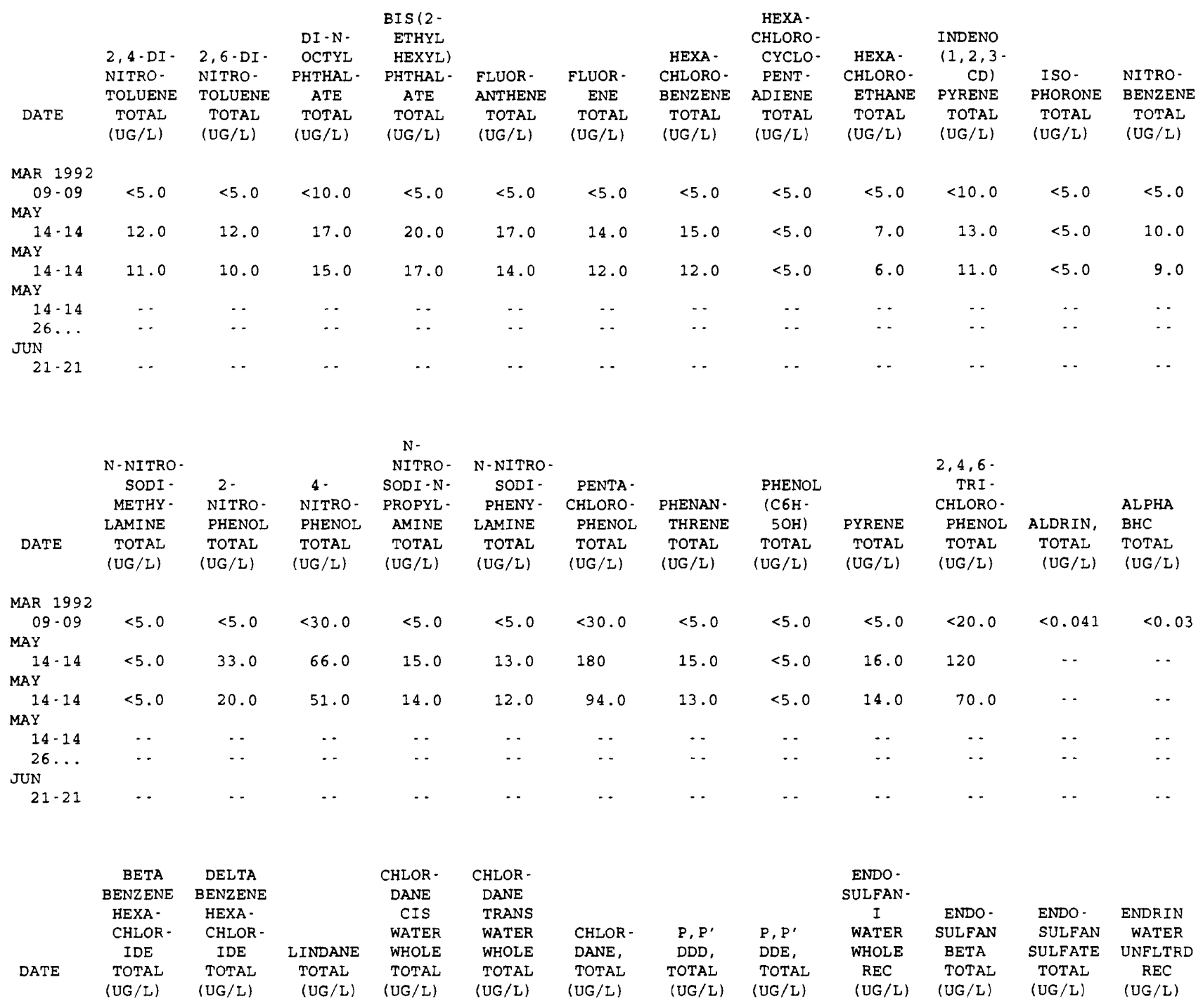

\begin{tabular}{|c|c|c|c|c|c|c|c|c|c|c|c|c|}
\hline MAR 1992 & & & & & & & & & & & & \\
\hline $09-09$ & $<0.03$ & $<0.09$ & $<0.030$ & $<0.10$ & $<0.10$ & $<0.1$ & $<0.10$ & $<0.04$ & $<0.10$ & $<0.04$ & $<0.60$ & $<0.060$ \\
\hline MAY & $\ldots$ & 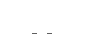 & 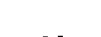 & . & . & 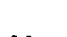 & & & & & & \\
\hline $\mathrm{MAY}^{14-14}$ & $\cdots$ & $\cdots$ & $\cdots$ & $\cdots$ & $\cdots$ & $\cdots$ & $\cdots$ & $\cdots$ & $\cdots$ & $\cdots$ & $\cdots$ & $\cdots$ \\
\hline $14-14$ & . & - & .. & -. & $\cdots$ & .. & .. & .. & .. & - & - & - \\
\hline MAY & & & & & & & & & & & & \\
\hline $14-14$ & -. & - & - & - & $\cdots$ & -. & - & $\cdots$ & -. & - & - & $\cdots$ \\
\hline $26 \ldots$ & $\cdots$ & $\cdots$ & $\cdots$ & .. & $\cdots$ & $\cdots$ & $\cdots$ & - & $\cdots$ & $\cdots$ & $\cdots$ & $\cdots$ \\
\hline $21-21$ & $\cdots$ & .. & . & 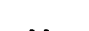 & $\ldots$ & . & . & . & 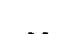 & 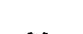 & . & 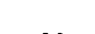 \\
\hline
\end{tabular}


Table 12. Quality-assurance/quality-control data for storm-sewer outfall stations, Fort worth, Texas, 1992-93-Continued 08048510 - WEST FORK TRINITY RIVER OUTFALL AT HIGHWAY 121, FORT WORTH, TX (WY 1992-CONTINUEd)

\begin{tabular}{|c|c|c|c|c|c|c|c|c|c|c|c|}
\hline & ENDRIN & & HEPTA - & & AROCLOR & AROCLOR & AROCLOR & AROCLOR & AROCLOR & AROCLOR & AROCLOR \\
\hline & ALDE - & HEPTA - & CHLOR & TOX - & 1016 & 1221 & 1232 & 1242 & 1248 & 1254 & 1260 \\
\hline & HYDE & CHLOR, & EPOXIDE & APHENE, & PCB & PCB & PCB & PCB & PCB & PCB & PCB \\
\hline DATE & $\begin{array}{l}\text { TOTAL } \\
\text { (UG/L) }\end{array}$ & $\begin{array}{l}\text { TOTAL } \\
\text { (UG/L) }\end{array}$ & $\begin{array}{l}\text { TOTAL } \\
\text { (UG/L) }\end{array}$ & $\begin{array}{l}\text { TOTAL } \\
\text { (UG/L) }\end{array}$ & $\begin{array}{l}\text { TOTAL } \\
\text { (UG/L) }\end{array}$ & $\begin{array}{l}\text { TOTAL } \\
\text { (UG/L) }\end{array}$ & $\begin{array}{l}\text { TOTAL } \\
\text { (UG/L) }\end{array}$ & $\begin{array}{l}\text { TOTAL } \\
\text { (UG/L) }\end{array}$ & $\begin{array}{l}\text { TOTAL } \\
(\mathrm{UG} / \mathrm{L})\end{array}$ & $\begin{array}{l}\text { TOTAL } \\
\text { (UG/L) }\end{array}$ & $\begin{array}{l}\text { TOTAL } \\
\text { (UG/L) }\end{array}$ \\
\hline \multicolumn{12}{|l|}{ MAR 1992} \\
\hline 09.09 & $<0.20$ & $<0.030$ & $<0.80$ & $<2$ & $<0.1$ & $<1.0$ & $<0.1$ & $<0.1$ & $<0.1$ & $<0.1$ & $<0.1$ \\
\hline \multicolumn{12}{|l|}{ MAY } \\
\hline $14-14$ & $\cdots$ & $\cdots$ & $\cdots$ & $\cdots$ & $\cdots$ & $\cdot$ & $\cdots$ & $\cdots$ & $\cdots$ & $\cdots$ & $\cdots$ \\
\hline \multicolumn{12}{|l|}{ MAY } \\
\hline $14 \cdot 14$ & $\cdots$ & $\cdots$ & $\cdots$ & $\cdots$ & $\cdots$ & $\cdots$ & $\cdots$ & $\cdots$ & $\cdots$ & $\cdots$ & $\cdots$ \\
\hline \multicolumn{12}{|l|}{ MAY } \\
\hline $14-14$ & $\cdots$ & $\cdots$ & $\cdots$ & $\cdots$ & $\cdots$ & $\cdots$ & $\cdots$ & $\cdots$ & $\cdots$ & $\cdots$ & $\cdots$ \\
\hline $26 \ldots$ & $\cdots$ & $\cdots$ & $\cdots$ & $\cdots$ & $\cdots$ & $\cdots$ & $\cdots$ & $\cdots$ & $\cdots$ & $\cdots$ & - \\
\hline \multicolumn{12}{|l|}{ JUN } \\
\hline $21-21$ & $\cdots$ & $\cdots$ & $\cdots$ & $\cdots$ & $\cdots$ & $\cdots$ & $\cdots$ & $\cdots$ & $\cdots$ & $\cdots$ & $\cdots$ \\
\hline
\end{tabular}

08048545 - DRY BRANCH OUTFALL AT 33RD STREET, FORT WORTH, TX (WY 1992)

\begin{tabular}{|c|c|c|c|c|c|c|c|c|c|c|c|}
\hline & & & SPE - & $\begin{array}{l}\text { SPE- } \\
\text { CIFIC }\end{array}$ & $\begin{array}{c}\text { PH } \\
\text { WATER }\end{array}$ & $\begin{array}{c}\text { PH } \\
\text { WATER }\end{array}$ & HARD - & $\begin{array}{l}\text { HARD- } \\
\text { NESS }\end{array}$ & $\begin{array}{l}\text { ALKA - } \\
\text { LINITY }\end{array}$ & $\begin{array}{l}\text { SOLIDS, } \\
\text { SUM OF }\end{array}$ & $\begin{array}{l}\text { RES IDUE } \\
\text { TOTAL }\end{array}$ \\
\hline & & & CIFIC & $\mathrm{CON}$ & WHOLE & WHOLE & NESS & NONCARB & WAT DIS & CONSTI - & AT 105 \\
\hline TYPE & & & CON- & DUCT - & FIELD & LAB & TOTAL & DISSOLV & FIX END & TUENTS, & DEG. C, \\
\hline OF & & & DUCT - & ANCE & (STAND - & (STAND - & (MG/L & FLD. AS & FIELD & DIS - & SUS - \\
\hline $\begin{array}{l}\text { QA/QC } \\
\text { SAMPLE }\end{array}$ & DATE & TIME & $\begin{array}{l}\text { ANCE } \\
\text { (US /CM) }\end{array}$ & $\begin{array}{c}\mathrm{LAB} \\
(\mathrm{US} / \mathrm{CM})\end{array}$ & $\begin{array}{c}\text { ARD } \\
\text { UNITS) }\end{array}$ & $\begin{array}{c}\text { ARD } \\
\text { UNITS) }\end{array}$ & $\begin{array}{c}\text { AS } \\
\text { (ACO3) }\end{array}$ & $\begin{array}{r}\mathrm{CACO} 3 \\
(\mathrm{MG} / \mathrm{L})\end{array}$ & $\begin{array}{l}\mathrm{CACO} 3 \\
(\mathrm{MG} / \mathrm{L})\end{array}$ & $\begin{array}{l}\text { SOLVED } \\
(M G / L)\end{array}$ & $\begin{array}{l}\text { PENDED } \\
(M G / L)\end{array}$ \\
\hline
\end{tabular}

$\begin{array}{cccccccccccc}\text { SEP } 1992 & & & & & & & & \\ \text { REP } & 10 \cdot 10 & 1401 & 64 & 110 & 8.3 & 8.2 & 27 & 0 & 27\end{array}$

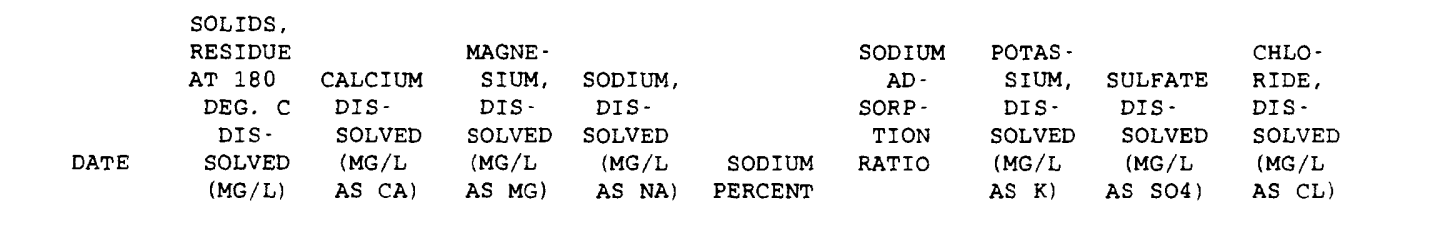

$\begin{array}{ccccccccc}\text { SEP } 1992 & & & & & & & \\ 10-10 & 37 & 10 & 0.47 & 3.2 & 19 & 0.3 & 1.6 & 4.1\end{array}$

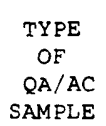

REP

REP

REP

08048545 - DRY BRANCH OUTFALL AT 33RD STREET, FORT WORTH, TX (WY 1993)

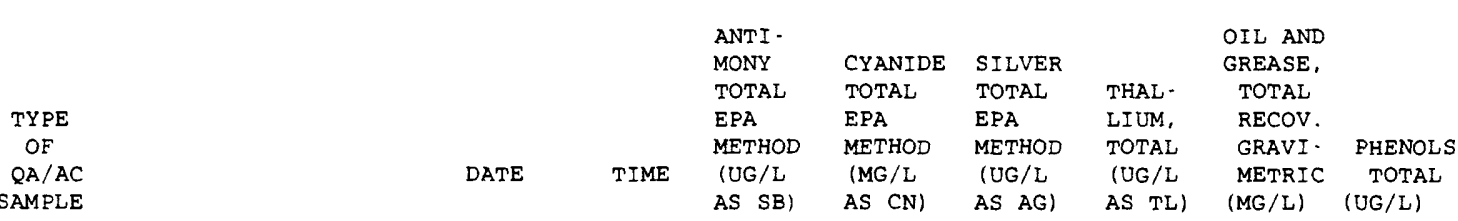

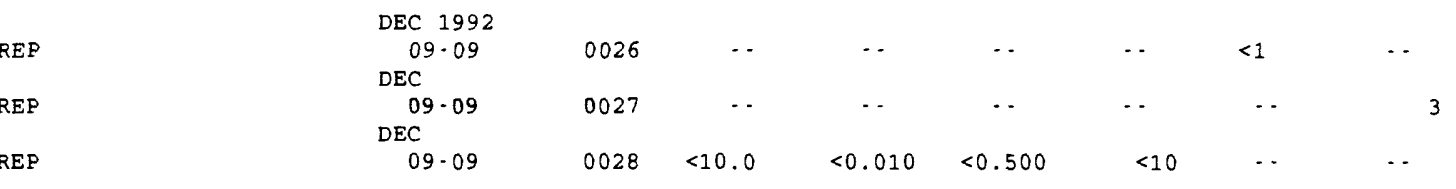


Table 12. Quality-assurance/quality-control data for storm-sewer outfall stations, Fort Worth, Texas, 1992-93-Continued

08048700 - EASTERN HILLS HIGH SCHOOL OUTFALL AT WEILER DRIVE, FORT WORTH, TX (WY 1992)

TYPE
OF

QA/QC

SAMPLE

REP

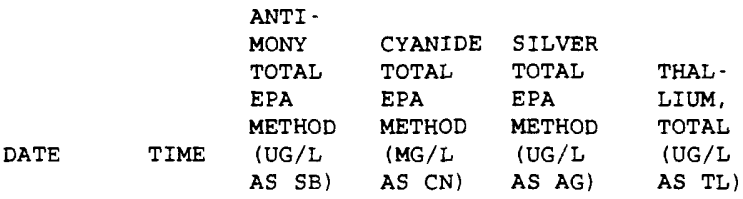

SEP 1992

21-21
$<0.010<0.500$

$0106<10.0$

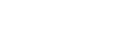

08048700 - EASTERN HILLS HIGH SCHOOL OUTFALL AT WEILER DRIVE, FORT WORTH, TX (WY 1993)

\begin{tabular}{|c|c|c|c|c|c|c|c|c|c|c|c|c|c|}
\hline $\begin{array}{c}\text { TYPE } \\
\text { OF } \\
\text { QA/QC } \\
\text { SAMPLE }\end{array}$ & DATE & TIME & $\begin{array}{c}\text { ARSENIC } \\
\text { TOTAL } \\
\text { (UG/L } \\
\text { AS AS) }\end{array}$ & $\begin{array}{l}\text { BERYL- } \\
\text { LIUM, } \\
\text { TOTAL } \\
\text { RECOV- } \\
\text { ERABLE } \\
\text { (UG/L } \\
\text { AS BE) }\end{array}$ & $\begin{array}{l}\text { CADMIUM } \\
\text { TOTAL } \\
\text { RECOV- } \\
\text { ERABLE } \\
\text { (UG/L } \\
\text { AS CD) }\end{array}$ & $\begin{array}{l}\text { CHRO- } \\
\text { MIUM, } \\
\text { TOTAL } \\
\text { RECOV- } \\
\text { ERABLE } \\
\text { (UG/L } \\
\text { AS CR) }\end{array}$ & $\begin{array}{l}\text { COPPER, } \\
\text { TOTAL } \\
\text { RECOV. } \\
\text { ERABLE } \\
\text { (UG/L } \\
\text { AS CU) }\end{array}$ & $\begin{array}{l}\text { LEAD, } \\
\text { TOTAL } \\
\text { RECOV- } \\
\text { ERABLE } \\
\text { (UG/L } \\
\text { AS PB) }\end{array}$ & $\begin{array}{l}\text { MERCURY } \\
\text { TOTAL } \\
\text { RECOV - } \\
\text { ERABLE } \\
\text { (UG/L } \\
\text { AS HG) }\end{array}$ & $\begin{array}{l}\text { NICKEL, } \\
\text { TOTAL } \\
\text { RECOV - } \\
\text { ERABLE } \\
\text { (UG/L } \\
\text { AS NI) }\end{array}$ & $\begin{array}{l}\text { SELE- } \\
\text { NIUM, } \\
\text { TOTAL } \\
\text { (UG/L } \\
\text { AS SE) }\end{array}$ & $\begin{array}{l}\text { SILVER, } \\
\text { TOTAL } \\
\text { RECOV- } \\
\text { ERABLE } \\
\text { (UG/L } \\
\text { AS AG) }\end{array}$ & $\begin{array}{l}\text { ZINC, } \\
\text { TOTAL } \\
\text { RECOV- } \\
\text { ERABLE } \\
\text { (UG/L } \\
\text { AS ZN) }\end{array}$ \\
\hline REP & $\begin{array}{c}\text { DEC } 1992 \\
09.09\end{array}$ & 0031 & $<1$ & $<10$ & $<1$ & $<1$ & 9 & 23 & $<0.10$ & 4 & $<2$ & $<1$ & 90 \\
\hline & DATE & TIME & $\begin{array}{c}\text { ARSENIC } \\
\text { TOTAL } \\
\text { (UG/L } \\
\text { AS AS) }\end{array}$ & $\begin{array}{l}\text { BERYL- } \\
\text { LIUM, } \\
\text { TOTAL } \\
\text { RECOV- } \\
\text { ERABLE } \\
\text { (UG/L } \\
\text { AS BE) }\end{array}$ & $\begin{array}{l}\text { CADMIUM } \\
\text { TOTAL } \\
\text { RECOV- } \\
\text { ERABLE } \\
\text { (UG/L } \\
\text { AS CD) }\end{array}$ & $\begin{array}{l}\text { CHRO- } \\
\text { MIUM, } \\
\text { TOTAL } \\
\text { RECOV- } \\
\text { ERABLE } \\
\text { (UG/L } \\
\text { AS CR) }\end{array}$ & $\begin{array}{l}\text { COPPER, } \\
\text { TOTAL } \\
\text { RECOV- } \\
\text { ERABLE } \\
\text { (UG/L } \\
\text { AS CU) }\end{array}$ & $\begin{array}{l}\text { LEAD, } \\
\text { TOTAL } \\
\text { RECOV. } \\
\text { ERABLE } \\
\text { (UG/L } \\
\text { AS PB) }\end{array}$ & $\begin{array}{l}\text { MERCURY } \\
\text { TOTAL } \\
\text { RECOV- } \\
\text { ERABLE } \\
\text { (UG/L } \\
\text { AS HG) }\end{array}$ & $\begin{array}{l}\text { NICKEL, } \\
\text { TOTAL } \\
\text { RECOV- } \\
\text { ERABLE } \\
\text { (UG/L } \\
\text { AS NI) }\end{array}$ & $\begin{array}{l}\text { SELE- } \\
\text { NIUM, } \\
\text { TOTAL } \\
\text { (UG/L } \\
\text { AS SE) }\end{array}$ & $\begin{array}{l}\text { SILVER, } \\
\text { TOTAL } \\
\text { RECOV. } \\
\text { ERABLE } \\
\text { (UG/L } \\
\text { AS AG) }\end{array}$ & $\begin{array}{l}\text { ZINC, } \\
\text { TOTAL } \\
\text { RECOV- } \\
\text { ERABLE } \\
\text { (UG/L } \\
\text { AS ZN) }\end{array}$ \\
\hline
\end{tabular}

DEC 1992

$09 \cdot 09$

0031

$<1<10$

$<1$

$<1$

9

$23<0.10 \quad 4 \quad<2$

$<1$

90 
Table 13. Quality-assurance/quality-control data for storm-sewer outfall stations, Garland, Texas, 1992-93

08061635 - TRIBUTARY TO DUCK CREEK OUTFALL AT HIGHTOWER ROAD, GARLAND, TX (WY 1992)

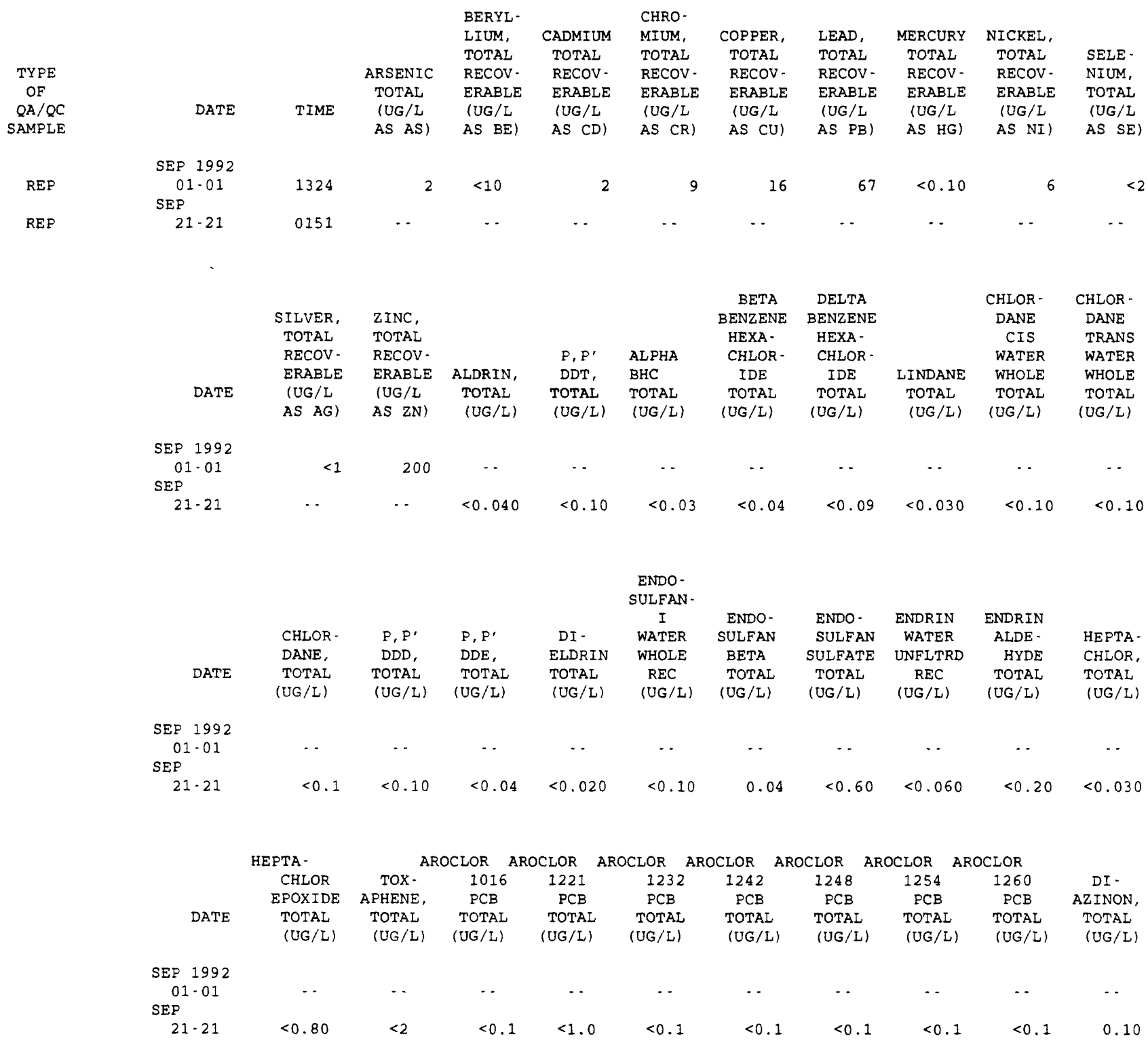

08061635 - TRIBUTARY TO DUCK CREEK OUTFALL AT HIGHTOWER ROAD, GARLAND, TX (WY 1993)

\begin{tabular}{|c|c|c|c|c|c|c|c|c|c|c|c|c|}
\hline & & & SPE - & $\begin{array}{r}\text { SPE- } \\
\text { CIFIC }\end{array}$ & $\begin{array}{c}\text { PH } \\
\text { WATER }\end{array}$ & $\begin{array}{c}\text { PH } \\
\text { WATER }\end{array}$ & HARD- & $\begin{array}{l}\text { HARD- } \\
\text { NESS }\end{array}$ & $\begin{array}{l}\text { ALKA - } \\
\text { LINITY }\end{array}$ & $\begin{array}{l}\text { SOLIDS, } \\
\text { SUM OF }\end{array}$ & $\begin{array}{l}\text { RESIDUE } \\
\text { TOTAL }\end{array}$ & $\begin{array}{l}\text { SOLIDS, } \\
\text { RES IDUE }\end{array}$ \\
\hline & & & CIFIC & $\mathrm{CON}-$ & WHOLE & WHOLE & NESS & NONCARB & WAT DIS & CONSTI- & AT 105 & AT 180 \\
\hline $\begin{array}{c}\text { TYPE } \\
\text { OF } \\
\text { QA/QC } \\
\text { SAMPLE }\end{array}$ & DATE & TIME & $\begin{array}{l}\text { CON- } \\
\text { DUCT - } \\
\text { ANCE } \\
\text { (US/CM) }\end{array}$ & $\begin{array}{c}\text { DUCT - } \\
\text { ANCE } \\
\text { LAB } \\
\text { (US/CM) }\end{array}$ & $\begin{array}{c}\text { FIELD } \\
\text { (STAND- } \\
\text { ARD } \\
\text { UNITS) }\end{array}$ & $\begin{array}{c}\text { LAB } \\
\text { (STAND- } \\
\text { ARD } \\
\text { UNITS) }\end{array}$ & $\begin{array}{l}\text { TOTAL } \\
\text { (MG/L } \\
\text { AS } \\
\text { CACO3) }\end{array}$ & $\begin{array}{c}\text { DISSOLV } \\
\text { FLD. AS } \\
\text { CACO3 } \\
\text { (MG /L) }\end{array}$ & $\begin{array}{c}\text { FIX END } \\
\text { FIELD } \\
\text { CAC03 } \\
\text { (MG/L) }\end{array}$ & $\begin{array}{c}\text { TUENTS, } \\
\text { DIS - } \\
\text { SOLVED } \\
\text { (MG / L) }\end{array}$ & $\begin{array}{l}\text { DEG. C, } \\
\text { SUS - } \\
\text { PENDED } \\
(M G / L)\end{array}$ & $\begin{array}{c}\text { DEG. C } \\
\text { DIS - } \\
\text { SOLVED } \\
\text { (MG/L) }\end{array}$ \\
\hline
\end{tabular}

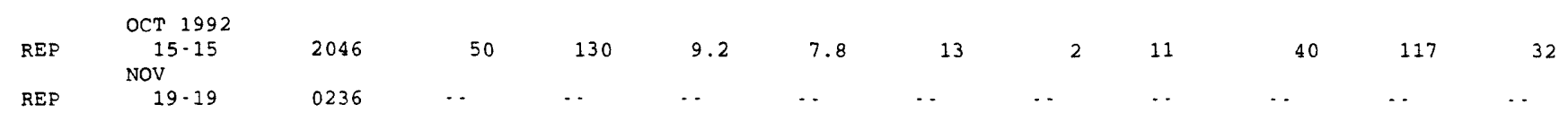


Table 13. Quality-assurance/quality-control data for storm-sewer outfall stations, Garland, Texas, 1992-93-Continued 08061635 - TRIBUTARY TO DUCK CREEK OUTFALL AT HIGHTOWER ROAD, GARLAND, TX (WY 1993)-CONTINUEd

\begin{tabular}{|c|c|c|c|c|c|c|c|c|c|c|}
\hline DATE & $\begin{array}{l}\text { CALCIUM } \\
\text { DIS - } \\
\text { SOLVED } \\
\text { (MG/L } \\
\text { AS CA) }\end{array}$ & $\begin{array}{l}\text { MAGNE. } \\
\text { SIUM, } \\
\text { DIS - } \\
\text { SOLVED } \\
\text { (MG/L } \\
\text { AS MG) }\end{array}$ & $\begin{array}{l}\text { SODIUM, } \\
\text { DIS - } \\
\text { SOLVED } \\
\text { (MG/L } \\
\text { AS NA) }\end{array}$ & $\begin{array}{l}\text { SODIUM } \\
\text { PERCENT }\end{array}$ & $\begin{array}{c}\text { SODIUM } \\
\text { AD - } \\
\text { SORP - } \\
\text { TION } \\
\text { RATIO }\end{array}$ & $\begin{array}{l}\text { POTAS - } \\
\text { SIUM, } \\
\text { DIS - } \\
\text { SOLVED } \\
\text { (MG/L } \\
\text { AS K) }\end{array}$ & $\begin{array}{l}\text { SULFATE } \\
\text { DIS - } \\
\text { SOLVED } \\
\text { (MG/L } \\
\text { AS SO4) }\end{array}$ & $\begin{array}{l}\text { CHLO- } \\
\text { RIDE, } \\
\text { DIS - } \\
\text { SOLVED } \\
\text { (MG/L } \\
\text { AS CL) }\end{array}$ & $\begin{array}{l}\text { OIL AND } \\
\text { GREASE, } \\
\text { TOTAL } \\
\text { RECOV. } \\
\text { GRAVI - } \\
\text { METRIC } \\
\text { (MG/L) }\end{array}$ & $\begin{array}{l}\text { PHENOLS } \\
\text { TOTAL } \\
\text { (UG/L) }\end{array}$ \\
\hline OCT 1992 & & & & & & & & & & \\
\hline $\begin{array}{l}15-15 \\
\text { Nov }\end{array}$ & 5.2 & 0.11 & 0.70 & 9 & 0.1 & 0.90 & 2.8 & 0.70 & $\cdots$ & $\cdots$ \\
\hline $19 \cdot 19$ & $\cdots$ & $\cdots$ & $\cdots$ & & $\cdots$ & $\cdots$ & $\cdots$ & $\cdots$ & 6 & 6 \\
\hline
\end{tabular}

08061660 - SLEEPY HOLLOW STREET OUTFALL AT NORTHWEST HIGHWAY, GARLAND, TX (WY 1993)

TYPE

OF

QA/QC

FMS

LMS

(n)

\section{- SLEE}


Table 13. Quality-assurance/quality-control data for storm-sewer outfall stations, Garland, Texas, 1992-93-Continued 08061690 - I-635 OUTFALI AT CENTERVILLE ROAD, GARLAND, TX (WY 1992)

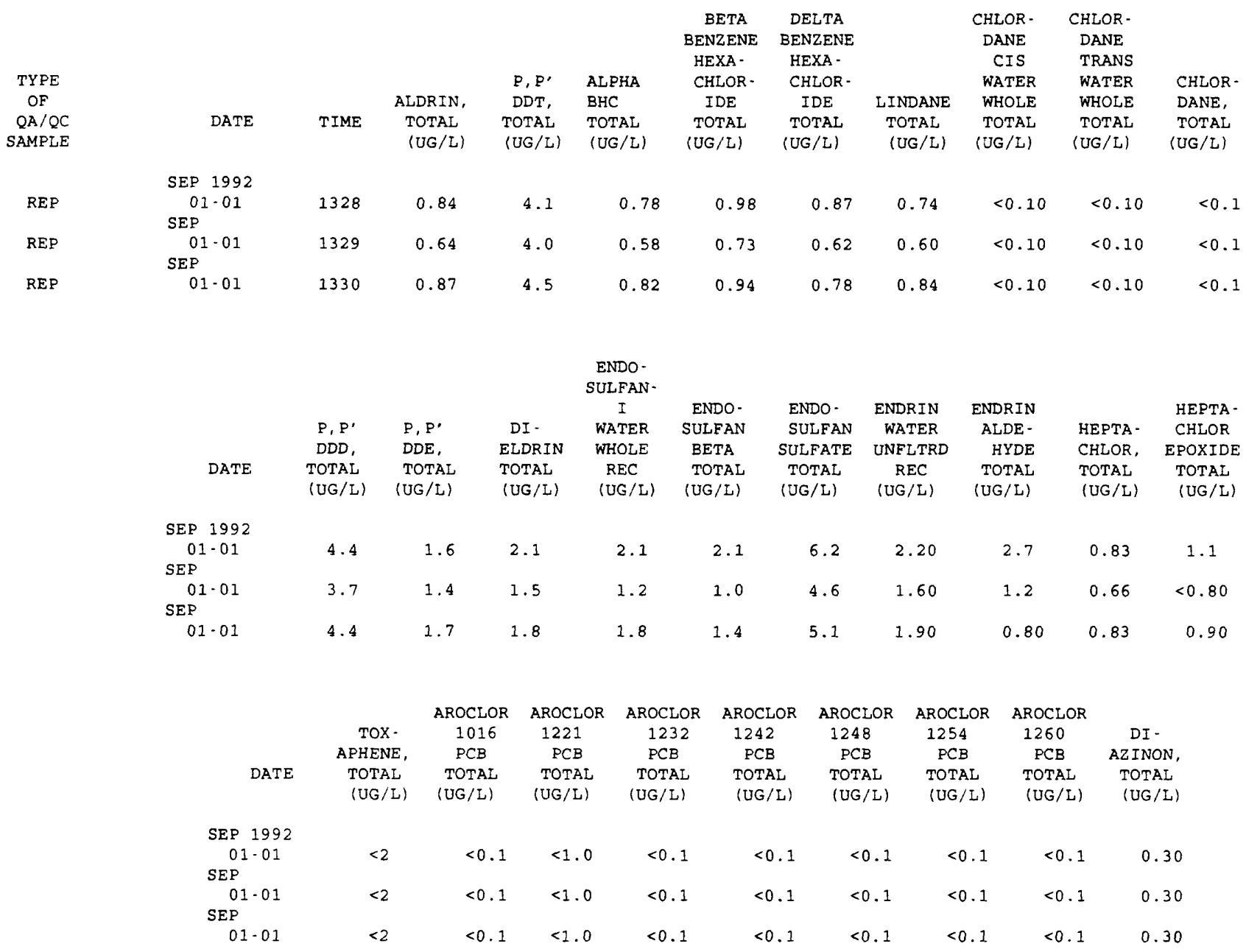

08061690 - I-635 OUTFALL AT CENTERVILLE ROAD, GARLAND, TX (WY 1993)

\begin{tabular}{|c|c|c|c|c|c|c|c|c|c|c|c|c|}
\hline $\begin{array}{c}\text { TYPE } \\
\text { OF } \\
Q A / Q C\end{array}$ & DATE & TIME & $\begin{array}{c}\text { GEN, } \\
\text { NITRITE } \\
\text { TOTAL } \\
\text { (MG/L }\end{array}$ & $\begin{array}{c}\text { GEN, } \\
\text { NO2+NO3 } \\
\text { TOTAL } \\
\text { (MG/L }\end{array}$ & $\begin{array}{c}\text { GEN, } \\
\text { AMMONIA } \\
\text { TOTAL } \\
\text { (MG } / \mathrm{L}\end{array}$ & $\begin{array}{c}\text { MONIA + } \\
\text { ORGANIC } \\
\text { TOTAL } \\
\text { (MG/L }\end{array}$ & $\begin{array}{l}\text { PHOS - } \\
\text { PHORUS } \\
\text { TOTAL } \\
\text { (MG/L }\end{array}$ & $\begin{array}{c}\text { PHORUS } \\
\text { DIS - } \\
\text { SOLVED } \\
\text { (MG/L }\end{array}$ & $\begin{array}{l}\text { TOTAL } \\
\text { EPA } \\
\text { METHOD } \\
\text { (UG/L }\end{array}$ & $\begin{array}{c}\text { ARSENIC } \\
\text { TOTAL } \\
\text { IUG/L }\end{array}$ & $\begin{array}{l}\text { TOTAL } \\
\text { RECOV - } \\
\text { ERABLE } \\
\text { (UG/L }\end{array}$ & $\begin{array}{l}\text { TOTAL } \\
\text { RECOV - } \\
\text { ERABLE } \\
\text { (UG / L }\end{array}$ \\
\hline SAMPLE & & & AS N) & AS N) & AS N) & AS N) & AS P) & $A S \quad P)$ & AS SB) & AS AS) & $A S B E$ ) & AS CD) \\
\hline
\end{tabular}

\begin{tabular}{|c|c|c|c|c|c|c|c|c|c|c|c|c|}
\hline & OCT 1992 & & & & & & & & & & & \\
\hline REP & $\mathrm{OCT}^{15 \cdot 15}$ & 2050 & $\cdots$ & $\cdots$ & $\cdots$ & $\cdots$ & $\cdots$ & $\cdots$ & $<10.0$ & $\cdots$ & $\cdots$ & $\cdots$ \\
\hline REP & $\begin{array}{l}15-15 \\
\text { NoV }\end{array}$ & 2051 & 0.020 & 0.220 & 0.160 & 1.1 & 0.230 & 0.090 & $\cdots$ & $\cdots$ & - & $\cdots$ \\
\hline REP & $19-19$ & 0231 & $\cdots$ & $\cdots$ & $\ldots$ & $\cdots$ & $\cdots$ & - & $\cdots$ & 2 & $<10$ & $<1$ \\
\hline
\end{tabular}


Table 13. Quality-assurance/quality-control data for storm-sewer outfall stations, Garland, Texas, 1992-93-Continued 08061690 - I-635 OUTFALL AT CENTERVILLE ROAD, GARLAND, TX (WY 1993)-Continued

\begin{tabular}{|c|c|c|c|c|c|c|c|c|c|c|}
\hline $\begin{array}{l}\text { CHRO- } \\
\text { MIUM, } \\
\text { TOTAL }\end{array}$ & $\begin{array}{l}\text { COPPER, } \\
\text { TOTAL }\end{array}$ & $\begin{array}{l}\text { ANTI - } \\
\text { MONY } \\
\text { TOTAL }\end{array}$ & $\begin{array}{l}\text { LEAD, } \\
\text { TOTAL }\end{array}$ & $\begin{array}{l}\text { MERCURY } \\
\text { TOTAL }\end{array}$ & $\begin{array}{l}\text { NICKEL, } \\
\text { TOTAL }\end{array}$ & SELE- & $\begin{array}{l}\text { SILVER, } \\
\text { TOTAL }\end{array}$ & $\begin{array}{l}\text { CYANIDE } \\
\text { TOTAL }\end{array}$ & THAL - & $\begin{array}{l}\text { ZINC, } \\
\text { TOTAL }\end{array}$ \\
\hline $\begin{array}{l}\text { RECOV - } \\
\text { ERABLE } \\
\text { (UG/L }\end{array}$ & $\begin{array}{l}\text { RECOV - } \\
\text { ERABLE } \\
\text { (UG / L }\end{array}$ & $\begin{array}{l}\text { EPA } \\
\text { METHOD } \\
\text { (UG/L }\end{array}$ & $\begin{array}{l}\text { RECOV - } \\
\text { ERABLE } \\
\text { (UG /L }\end{array}$ & $\begin{array}{l}\text { RECOV - } \\
\text { ERABLE } \\
\text { (UG /L }\end{array}$ & $\begin{array}{l}\text { RECOV - } \\
\text { ERABLE } \\
\text { (UG / L }\end{array}$ & $\begin{array}{l}\text { NIUM, } \\
\text { TOTAI } \\
\text { (UG/L }\end{array}$ & $\begin{array}{l}\text { RECOV - } \\
\text { ERABLE } \\
\text { (UG/L }\end{array}$ & $\begin{array}{l}\text { EPA } \\
\text { METHOD } \\
(\mathrm{MG} / \mathrm{L}\end{array}$ & $\begin{array}{l}\text { LIUM, } \\
\text { TOTAL } \\
\text { (UG/L }\end{array}$ & $\begin{array}{l}\text { RECOV - } \\
\text { ERABLE } \\
\text { (UG/L }\end{array}$ \\
\hline AS CR) & AS CU) & AS SB) & AS PB) & AS HG) & AS NI) & AS SE) & AS $A G)$ & AS $\mathrm{CN}$ ) & AS TL) & AS $\mathrm{ZN}$ ) \\
\hline
\end{tabular}
OCT 1992 $15-15$ OCT

$15 \cdot 15$ NOV

- $\quad$ -

$-$ 
Table 14. Quality-assurance/quality-control data for storm-sewer outfall stations, Irving, Texas, $1992-93$

08049590 - BEAR CREEK OUTFALL AT SHADY GROVE ROAD, IRVING, TX (WY 1993)

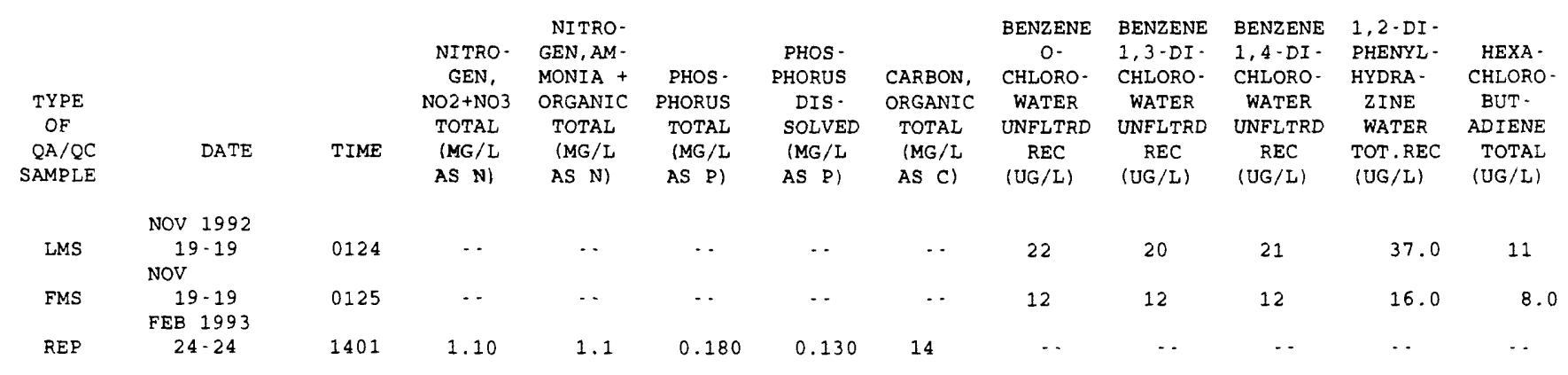

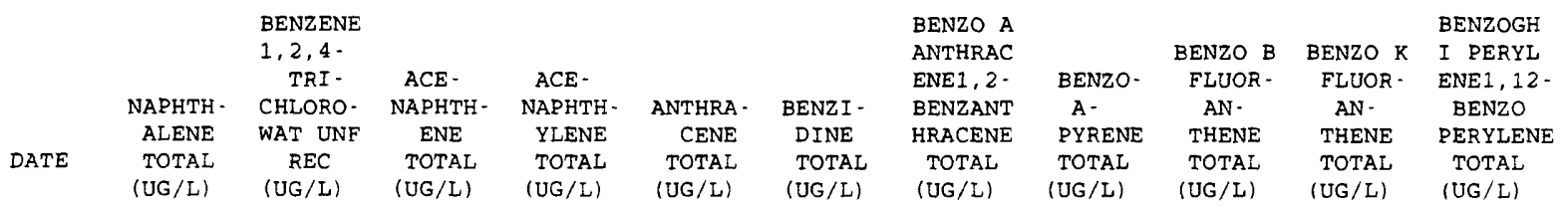

\begin{tabular}{|c|c|c|c|c|c|c|c|c|c|c|c|}
\hline \multicolumn{12}{|l|}{ Nov 1992} \\
\hline $19 \cdot 19$ & 25 & 21 & 29.0 & 28.0 & 28.0 & $<40.0$ & 61.0 & 27.0 & 36.0 & 25.0 & 23.0 \\
\hline \multicolumn{12}{|l|}{ NOV } \\
\hline $19 \cdot 19$ & $<5.0$ & 12 & 15.0 & 13.0 & 14.0 & $<40.0$ & 18.0 & 14.0 & 17.0 & 16.0 & 14.0 \\
\hline FEB 1993 & & & & & & & & & & & \\
\hline $24-24$ & - & - & $\cdots$ & $\cdots$ & . & . & $\cdots$ & . & - & $\cdots$ & $\cdots$ \\
\hline
\end{tabular}

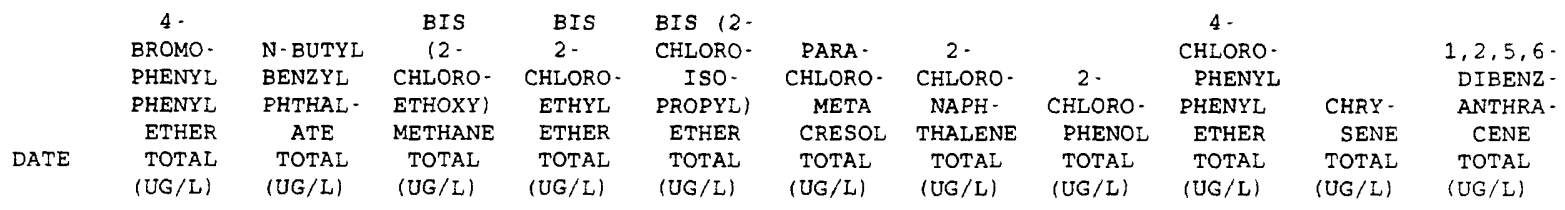

\begin{tabular}{|c|c|c|c|c|c|c|c|c|c|c|c|}
\hline \multicolumn{12}{|l|}{ NOV 1992} \\
\hline $19-19$ & $<5.0$ & 61.0 & 32.0 & 31.0 & 27.0 & 400 & 31.0 & 78.0 & 28.0 & 58.0 & 28.0 \\
\hline \multicolumn{12}{|l|}{ Nov } \\
\hline $19-19$ & $<5.0$ & 13.0 & 16.0 & 16.0 & 13.0 & 190 & 8.0 & 37.0 & 15.0 & 17.0 & 15.0 \\
\hline FEB 1993 & & & & & & & & & & & \\
\hline $24 \cdot 24$ & $\cdots$ & $\cdots$ & $\cdots$ & $\cdots$ & $\cdots$ & $\cdots$ & $\cdots$ & $\cdots$ & $\cdots$ & $\cdots$ & $\cdots$ \\
\hline
\end{tabular}

\begin{tabular}{|c|c|c|c|c|c|c|c|c|c|c|}
\hline DATE & $\begin{array}{c}3,3 \cdot- \\
\text { DI - } \\
\text { CHLORO- } \\
\text { BENZI - } \\
\text { DINE } \\
\text { TOTAL } \\
\text { (UG/L) }\end{array}$ & $\begin{array}{l}2,4-D I- \\
\text { CHLORO- } \\
\text { PHENOL } \\
\text { TOTAL } \\
\text { (UG/L) }\end{array}$ & $\begin{array}{l}\text { DIETHYL } \\
\text { PHTHAL - } \\
\text { ATE } \\
\text { TOTAL } \\
\text { (UG/L) }\end{array}$ & $\begin{array}{c}\text { DI - } \\
\text { METHYL } \\
\text { PHTHAL - } \\
\text { ATE } \\
\text { TOTAL } \\
\text { (UG/L) }\end{array}$ & $\begin{array}{l}2,4 \text {-DI - } \\
\text { METHYL - } \\
\text { PHENOL } \\
\text { TOTAL } \\
\text { (UG/L) }\end{array}$ & $\begin{array}{l}\text { DI - N- } \\
\text { BUTYL } \\
\text { PHTHAL- } \\
\text { ATE } \\
\text { TOTAL } \\
\text { (UG/L) }\end{array}$ & $\begin{array}{c}4,6- \\
\text { DINITRO- } \\
\text { ORTHO- } \\
\text { CRESOL } \\
\text { TOTAL } \\
\text { (UG/L) }\end{array}$ & $\begin{array}{c}2,4,- \\
\text { DI- } \\
\text { NITRO- } \\
\text { PHENOL } \\
\text { TOTAL } \\
\text { (UG/L) }\end{array}$ & $\begin{array}{l}2,4 \cdot \mathrm{DI}- \\
\text { NITRO- } \\
\text { TOLUENE } \\
\text { TOTAL } \\
\text { (UG/L) }\end{array}$ & $\begin{array}{l}2,6 \cdot \mathrm{DI}- \\
\text { NITRO- } \\
\text { TOLUENE } \\
\text { TOTAL } \\
\text { (UG/L) }\end{array}$ \\
\hline Nov 1992 & & & & & & & & & & \\
\hline $\begin{array}{l}19 \cdot 19 \\
\text { Nov }\end{array}$ & $<20.0$ & 77.0 & 22.0 & 15.0 & 72.0 & 37.0 & 310 & 200 & 45.0 & 44.0 \\
\hline $\begin{array}{c}19-19 \\
\text { FEB } 1993\end{array}$ & $<20.0$ & 36.0 & 8.0 & 8.0 & 37.0 & 15.0 & 150 & 79.0 & 19.0 & 25.0 \\
\hline $24-24$ & $\cdots$ & $\cdots$ & $\cdots$ & $\cdots$ & $\cdots$ & $\ldots$ & $\cdots$ & $\cdots$ & - & $\cdots$ \\
\hline
\end{tabular}


Table 14. Quality-assurance/quality-control data for storm-sewer outfall stations, Irving, Texas, 1992-93-Continued 08049590 - BEAR CREEK OUTFALI AT SHADY GROVE ROAD, IRVING, TX (WY 1993)-Continued

\begin{tabular}{|c|c|c|c|c|c|c|c|c|c|c|}
\hline DATE & $\begin{array}{l}\text { DI -N- } \\
\text { OCTYL } \\
\text { PHTHAL- } \\
\text { ATE } \\
\text { TOTAL } \\
(U G / L)\end{array}$ & $\begin{array}{l}\text { BIS (2- } \\
\text { ETHYL } \\
\text { HEXYL) } \\
\text { PHTHAL- } \\
\text { ATE } \\
\text { TOTAL } \\
\text { (UG/L) }\end{array}$ & $\begin{array}{l}\text { FLUOR - } \\
\text { ANTHENE } \\
\text { TOTAL } \\
\text { (UG/L) }\end{array}$ & $\begin{array}{c}\text { FLUOR - } \\
\text { ENE } \\
\text { TOTAL } \\
\text { (UG/L) }\end{array}$ & $\begin{array}{l}\text { HEXA- } \\
\text { CHLORO- } \\
\text { BENZENE } \\
\text { TOTAL } \\
\text { (UG/L) }\end{array}$ & $\begin{array}{l}\text { HEXA- } \\
\text { CHLORO- } \\
\text { CYCLO- } \\
\text { PENT - } \\
\text { ADIENE } \\
\text { TOTAL } \\
\text { (UG/L) }\end{array}$ & $\begin{array}{l}\text { HEXA- } \\
\text { CHLORO- } \\
\text { ETHANE } \\
\text { TOTAL } \\
\text { (UG/L) }\end{array}$ & $\begin{array}{c}\text { INDENO } \\
(1,2,3- \\
\text { CD) } \\
\text { PYRENE } \\
\text { TOTAL } \\
\text { (UG/L) }\end{array}$ & $\begin{array}{l}\text { ISO- } \\
\text { PHORONE } \\
\text { TOTAL } \\
\text { (UG/L) }\end{array}$ & $\begin{array}{l}\text { NITRO- } \\
\text { BENZENE } \\
\text { TOTAL } \\
(U G / L)\end{array}$ \\
\hline NOV 1992 & & & & & & & & & & \\
\hline $\begin{array}{l}19-19 \\
\text { NOV }\end{array}$ & 45.0 & 87.0 & 30.0 & 29.0 & 28.0 & 7.0 & 13.0 & 30.0 & 7.0 & 31.0 \\
\hline $\begin{array}{cc}19-19 \\
\text { FEB } 1993\end{array}$ & 24.0 & 28.0 & 16.0 & 15.0 & 15.0 & $<5.0$ & 9.0 & 14.0 & $<5.0$ & 16.0 \\
\hline $24-24$ & $\begin{array}{l}\text { N-NITRO- } \\
\text { SODI- } \\
\text { METHY - } \\
\text { LAMINE } \\
\text { TOTAL } \\
\text { (UG/L) }\end{array}$ & $\begin{array}{c}2 \text { - } \\
\text { NITRO- } \\
\text { PHENOL } \\
\text { TOTAL } \\
\text { (UG/L) }\end{array}$ & $\begin{array}{c}4- \\
\text { NITRO- } \\
\text { PHENOL } \\
\text { TOTAL } \\
\text { (UG/L) }\end{array}$ & $\begin{array}{l}\text { N- } \\
\text { NITRO- } \\
\text { SODI -N- } \\
\text { PROPYL- } \\
\text { AMINE } \\
\text { TOTAL } \\
\text { (UG/L) }\end{array}$ & $\begin{array}{l}\text { N-NITRO- } \\
\text { SODI - } \\
\text { PHENY - } \\
\text { LAMINE } \\
\text { TOTAL } \\
\text { (UG/L) }\end{array}$ & $\begin{array}{l}\text { PENTA - } \\
\text { CHLORO - } \\
\text { PHENOL } \\
\text { TOTAL } \\
\text { (UG/L) }\end{array}$ & $\begin{array}{l}\text { PHENAN - } \\
\text { THRENE } \\
\text { TOTAL } \\
\text { (UG/L) }\end{array}$ & $\begin{array}{l}\text { PHENOL } \\
\text { (C6H- } \\
5 O H) \\
\text { TOTAL } \\
\text { (UG/L) }\end{array}$ & $\begin{array}{r}\text { PYRENE } \\
\text { TOTAL } \\
(U G / L)\end{array}$ & $\begin{array}{c}2,4,6- \\
\text { TRI - } \\
\text { CHLORO- } \\
\text { PHENOL } \\
\text { TOTAL } \\
\text { (UG/L) }\end{array}$ \\
\hline NOV 1992 & & & & & & & & & & \\
\hline $\begin{array}{l}19-19 \\
\text { NOV }\end{array}$ & 16.0 & 98.0 & 260 & 23.0 & 33.0 & 380 & 30.0 & 45.0 & 30.0 & 300 \\
\hline $\begin{array}{c}19-19 \\
\text { FEB } 1993\end{array}$ & 9.0 & 45.0 & 95.0 & 13.0 & 12.0 & 150 & 15.0 & 15.0 & 16.0 & 150 \\
\hline $24-24$ & $\cdots$ & $\cdots$ & $\cdots$ & $\cdots$ & $\cdots$ & $\cdots$ & $\cdots$ & $\cdots$ & $\cdots$ & $\cdots$ \\
\hline
\end{tabular}

08055570 - HEREFORD ROAD OUTFALL AT WALNUT HILL ROAD, IRVING TX (WY 1993)

\begin{tabular}{|c|c|c|c|c|c|c|c|c|c|}
\hline DATE & TIME & $\begin{array}{c}\text { SPE- } \\
\text { CIFIC } \\
\text { CON- } \\
\text { DUCT- } \\
\text { ANCE } \\
\text { LAB } \\
\text { (US/CM) }\end{array}$ & $\begin{array}{c}\text { PH } \\
\text { WATER } \\
\text { WHOLE } \\
\text { LAB } \\
\text { (STAND. } \\
\text { ARD } \\
\text { UNITS) }\end{array}$ & $\begin{array}{l}\text { HARD - } \\
\text { NESS } \\
\text { TOTAL } \\
\text { (MG/L } \\
\text { AS } \\
\text { CACO3) }\end{array}$ & $\begin{array}{l}\text { SOLIDS, } \\
\text { SUM OF } \\
\text { CONSTI - } \\
\text { TUENTS, } \\
\text { DIS - } \\
\text { SOLVED } \\
\text { (MG/L) }\end{array}$ & $\begin{array}{l}\text { RESIDUE } \\
\text { TOTAL } \\
\text { AT } 105 \\
\text { DEG. C, } \\
\text { SUS. } \\
\text { PENDED } \\
\text { (MG/L) }\end{array}$ & $\begin{array}{l}\text { SOLIDS, } \\
\text { RESIDUE } \\
\text { AT } 180 \\
\text { DEG. C } \\
\text { DIS - } \\
\text { SOLVED } \\
\text { (MG/L) }\end{array}$ & $\begin{array}{l}\text { CALCIUM } \\
\text { DIS- } \\
\text { SOLVED } \\
\text { (MG/L } \\
\text { AS CA) }\end{array}$ & $\begin{array}{l}\text { MAGNE - } \\
\text { SIUM, } \\
\text { DIS- } \\
\text { SOLVED } \\
\text { (MG/L } \\
\text { AS MG) }\end{array}$ \\
\hline \multicolumn{10}{|l|}{ ОСт 1992} \\
\hline \multicolumn{10}{|l|}{ OCT } \\
\hline $07-07$ & 1722 & $\cdots$ & $\cdots$ & $\cdots$ & $\cdots$ & $\cdots$ & $\cdots$ & $\cdots$ & $\cdots$ \\
\hline \multicolumn{10}{|l|}{ Nov } \\
\hline \multicolumn{10}{|l|}{ NOV } \\
\hline $10-10$ & 1317 & 276 & 7.5 & 110 & 147 & 3 & 161 & 26 & 10 \\
\hline DATE & $\begin{array}{l}\text { SODIUM, } \\
\text { DIS - } \\
\text { SOLVED } \\
\text { (MG/L } \\
\text { AS NA) }\end{array}$ & $\begin{array}{l}\text { SODIUM } \\
\text { PERCENT }\end{array}$ & $\begin{array}{c}\text { SODIUM } \\
\text { AD- } \\
\text { SORP - } \\
\text { TION } \\
\text { RATIO }\end{array}$ & $\begin{array}{l}\text { POTAS - } \\
\text { SIUM, } \\
\text { DIS - } \\
\text { SOLVED } \\
\text { (MG/I } \\
\text { AS K) }\end{array}$ & $\begin{array}{l}\text { SULFATE } \\
\text { DIS - } \\
\text { SOLVED } \\
\text { (MG/L } \\
\text { AS SO4) }\end{array}$ & $\begin{array}{l}\text { CHLO- } \\
\text { RIDE, } \\
\text { DIS- } \\
\text { SOLVED } \\
\text { (MG/L } \\
\text { AS CL) }\end{array}$ & $\begin{array}{l}\text { NITRO- } \\
\text { GEN, } \\
\text { NITRITE } \\
\text { TOTAL } \\
\text { (MG/L } \\
\text { AS N) }\end{array}$ & $\begin{array}{l}\text { NITRO- } \\
\text { GEN, } \\
\text { NO2+NO3 } \\
\text { TOTAL } \\
\text { (MG/L } \\
\text { AS N) }\end{array}$ & $\begin{array}{l}\text { NITRO- } \\
\text { GEN, } \\
\text { AMMONIA } \\
\text { TOTAL } \\
\text { (MG/L } \\
\text { AS N) }\end{array}$ \\
\hline OCT 1992 & & & & & & & & & \\
\hline $07-07$ & $\cdots$ & $\cdots$ & $\cdots$ & $\cdots$ & $\cdots$ & $\cdots$ & 0.040 & 0.620 & 0.270 \\
\hline OCT & & & & & & & & & \\
\hline 07.07 & $\cdots$ & $\cdots$ & $\cdots$ & $\cdots$ & -- & -- & $\cdots$ & $\cdots$ & $-\cdot$ \\
\hline NOV & & & & & & & & & \\
\hline $10-10$ & $\cdots$ & $\cdots$ & $\cdots$ & $\cdots$ & $\cdots$ & $\cdots$ & 0.050 & 0.270 & 0.110 \\
\hline NOV & & & & & & & & & \\
\hline $10-10$ & 8.3 & 14 & 0.4 & 0.90 & 74 & 13 & $\cdots$ & $\cdots$ & $\cdots$ \\
\hline
\end{tabular}


Table 14. Quality-assurance/quality-control data for storm-sewer outfall stations, Irving, Texas, 1992-93-Continued 08055570 - HEREFORD ROAD OUTFALL AT WALNUT HILL ROAD, IRVING TX (WY 1993)-Continued

\begin{tabular}{|c|c|c|c|c|c|c|c|c|}
\hline \multirow{6}{*}{ DATE } & \multirow{5}{*}{$\begin{array}{l}\text { NITRO- } \\
\text { GEN, AM- } \\
\text { MONIA + } \\
\text { ORGANIC } \\
\text { TOTAL } \\
\text { (MG/L }\end{array}$} & \multirow{5}{*}{$\begin{array}{c}\text { PHOS - } \\
\text { PHORUS } \\
\text { TOTAL } \\
\text { (MG / L }\end{array}$} & \multirow{5}{*}{$\begin{array}{l}\text { PHOS - } \\
\text { PHORUS } \\
\text { DIS - } \\
\text { SOLVED } \\
\text { (MG / L }\end{array}$} & \multicolumn{2}{|l|}{ ANTI - } & \multirow[b]{2}{*}{ SILVER } & \multirow{5}{*}{$\begin{array}{l}\text { THAL - } \\
\text { LIUM, } \\
\text { TOTAL } \\
\text { (UG/L }\end{array}$} & \multirow{6}{*}{$\begin{array}{l}\text { CARBON, } \\
\text { ORGANIC } \\
\text { TOTAL } \\
\text { (MG/L } \\
\text { AS C) }\end{array}$} \\
\hline & & & & MONY & CYANIDE & & & \\
\hline & & & & TOTAL & TOTAL & TOTAL & & \\
\hline & & & & EPA & EPA & EPA & & \\
\hline & & & & $\begin{array}{l}\text { METHOD } \\
\text { (UG/L }\end{array}$ & $\begin{array}{l}\text { METHOD } \\
\text { (MG/L }\end{array}$ & $\begin{array}{l}\text { METHOD } \\
\text { (UG/L }\end{array}$ & & \\
\hline & AS N) & AS P) & AS P) & AS HB) & AS CN) & AS AG) & AS TL) & \\
\hline
\end{tabular}

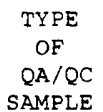

REP

REP

YPE

$\mathrm{QA} / \mathrm{QC}$

,

$2.0 \quad 0.370$

-

0.80

.
0.150

$\ldots \quad<10.0$

$<0.010<0.500$

0.170

..

..

.

$\cdots$

24

08056100 - TRIBUTARY TO ELM FORK TRINITY RIVER OUTFALL AT CASCADE STREET, IRVING, TX (WY 1993)

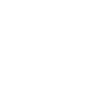

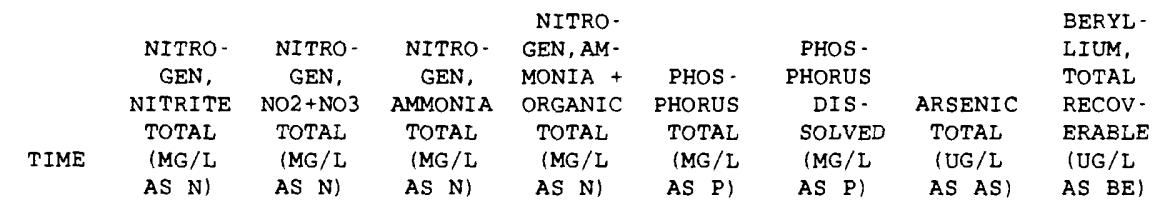

OCT 1992

NOV

$19 \cdot 19$

1731

0116

0.220

0.650

0.080

$<0.20$

0.220

CHRO -

MIUM

COPPER, TOTAL RECOV (UG/L AS CR)

CADMIUM
TOTAL
RECOV-
ERABLE
(UG/L
AS CD)

COPPER,
TOTAL
RECOV -
ERABLE
(UG / L

LEAD,
TOTAL
RECOV.
ERABLE
(UG/L
AS PB)

MERCURY
TOTAL
RECOV -
ERABLE
(UG/L
AS HG)

NICKEL,
TOTAL
RECOV-
ERABLE
(UG/L
AS NI)

SELENIUM,

TOTAL

(UG/L AS SE)

OCT 1992

$<1$

11

11

$23<0.10$

7

$<2$

$19-19$ 
Table 15. Quality-assurance/quality-control data for storm-sewer outfall stations, Mesquite, Texas, $1992-93$

08061915 - SOUTH MESQUITE CREEK OUTFALL AT SOUTH PARKWAY, MESQUITE, TX (WY 1993)

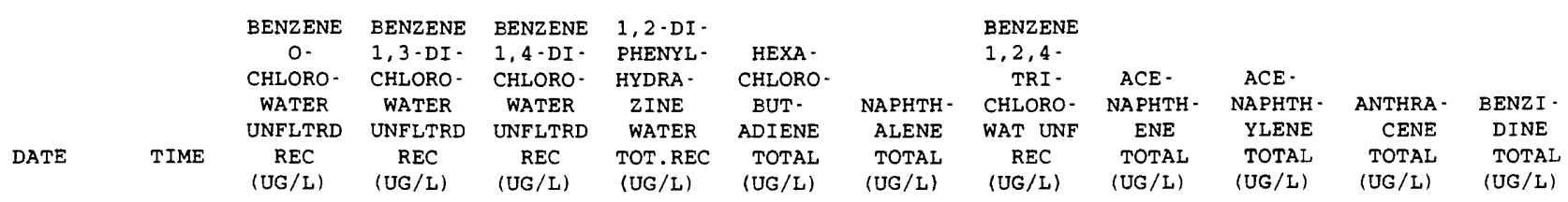

APR 1993

$$
08 \ldots
$$

1315

$<5.0$

$<5.0$

$<5.0$

$<5.0$

$<5.0$

$<5.0$

$<5.0$

$<5.0$

$<5.0$

$<5.0<40.0$

\begin{tabular}{|c|c|c|c|c|c|}
\hline \multicolumn{6}{|r|}{ BENZO } \\
\hline & ANTHRAC & & BENZO B & BENZO $\mathrm{K}$ & GHI PERYL \\
\hline & ENE1, 2- & BENZO - & FLUOR - & FLUOR - & ENE1,12- \\
\hline & BENZANT & & AN- & AN- & BENZO \\
\hline & HRACENE & PYRENE & THENE & THENE & PERYLENE \\
\hline$A 7$ & $\begin{array}{l}\text { TOTAL } \\
\text { (UG/L) }\end{array}$ & $\begin{array}{l}\text { TOTAL } \\
\text { (UG/L) }\end{array}$ & $\begin{array}{l}\text { TOTAL } \\
\text { (UG/L) }\end{array}$ & $\begin{array}{l}\text { TOTAL } \\
\text { (UG/L) }\end{array}$ & $\begin{array}{l}\text { TOTAL } \\
\text { (UG/L) }\end{array}$ \\
\hline
\end{tabular}

4 -

4- $\quad$ BIS BIS BIS 12 -

BROMO- N-BUTYL (2- 2 - CHLORO-

PHENYL BENZYL CHLORO- CHLORO- ISO-

PHENYL PHTHAL - ETHOXY) ETHYL PROPYL)

ETHER ATE METHANE ETHER ETHER

TOTAL

ATE

ETHER
TOTAL

$\begin{array}{ll}\text { (UG/L) } & \text { TOTAL } \\ & \end{array}$

(UG/L)

(UG/L)

PARA.

META NAPH.

CRESOL THALENE

TOTAL TOTAL

APR 1993

$08 \ldots<10.0$

$<10.0$

$<10.0<10.0<10.0$

$<5.0$

$<5.0$

$<5.0$

$<5.0$

$<5.0$

$<30.0$

$<5.0$

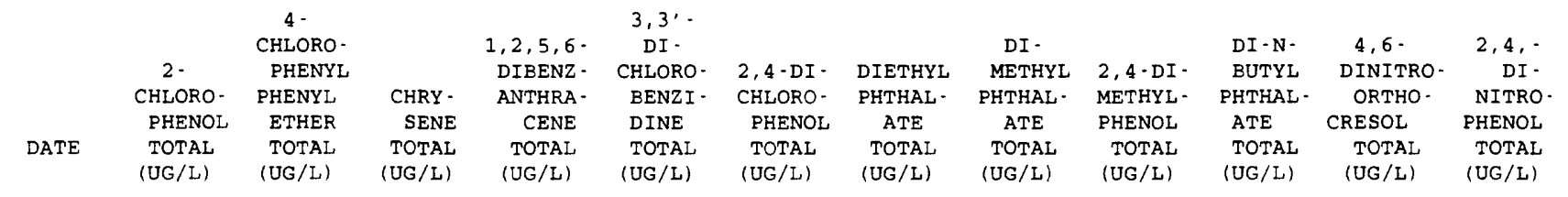

APR 1993

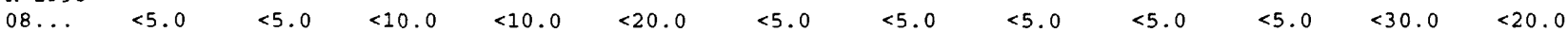

\begin{tabular}{|c|c|c|c|c|c|c|c|c|c|c|c|}
\hline DATE & $\begin{array}{l}2,4-\mathrm{DI} \text { - } \\
\text { NITRO- } \\
\text { TOLUENE } \\
\text { TOTAL } \\
\text { (UG/L) }\end{array}$ & $\begin{array}{l}2,6-\mathrm{DI} \text { - } \\
\text { NITRO- } \\
\text { TOLUENE } \\
\text { TOTAL } \\
(\mathrm{UG} / \mathrm{L})\end{array}$ & $\begin{array}{c}\text { DI - N- } \\
\text { OCTYL } \\
\text { PHTHAL- } \\
\text { ATE } \\
\text { TOTAL } \\
\text { (UG/L) }\end{array}$ & $\begin{array}{c}\text { BIS } 12- \\
\text { ETHYL } \\
\text { HEXYL) } \\
\text { PHTHAL- } \\
\text { ATE } \\
\text { TOTAL } \\
\text { (UG/L) }\end{array}$ & $\begin{array}{l}\text { FLUOR - } \\
\text { ANTHENE } \\
\text { TOTAL } \\
\text { (UG/L) }\end{array}$ & $\begin{array}{c}\text { FLUOR - } \\
\text { ENE } \\
\text { TOTAL } \\
\text { (UG/L) }\end{array}$ & $\begin{array}{l}\text { HEXA- } \\
\text { CHLORO- } \\
\text { BENZENE } \\
\text { TOTAL } \\
\text { (UG/L) }\end{array}$ & $\begin{array}{l}\text { HEXA- } \\
\text { CHLORO- } \\
\text { CYCLO- } \\
\text { PENT- } \\
\text { ADIENE } \\
\text { TOTAL } \\
\text { (UG/L) }\end{array}$ & $\begin{array}{l}\text { HEXA- } \\
\text { CHLORO- } \\
\text { ETHANE } \\
\text { TOTAL } \\
\text { (UG/L) }\end{array}$ & $\begin{array}{r}\text { INDENO } \\
(1,2,3- \\
\text { CD) } \\
\text { PYRENE } \\
\text { TOTAL } \\
\text { (UG/L) }\end{array}$ & $\begin{array}{l}\text { ISO- } \\
\text { PHORONE } \\
\text { TOTAL } \\
\text { (UG/L) }\end{array}$ \\
\hline $\begin{array}{c}\text { APR } 1993 \\
08 \ldots\end{array}$ & $<5.0$ & $<5.0$ & $<10.0$ & $<5.0$ & $<5.0$ & $<5.0$ & $<5.0$ & $<5.0$ & $<5.0$ & $<10.0$ & $<5.0$ \\
\hline DATE & $\begin{array}{l}\text { NITRO- } \\
\text { BENZENE } \\
\text { TOTAL } \\
\text { (UG/L) }\end{array}$ & $\begin{array}{l}\text { N-NITRO- } \\
\text { SODI - } \\
\text { METHY - } \\
\text { LAMINE } \\
\text { TOTAL } \\
\text { (UG/L) }\end{array}$ & $\begin{array}{l}2- \\
\text { NITRO- } \\
\text { PHENOL } \\
\text { TOTAL } \\
\text { (UG/L) }\end{array}$ & $\begin{array}{c}4- \\
\text { NITRO- } \\
\text { PHENOL } \\
\text { TOTAL } \\
\text { (UG/L) }\end{array}$ & $\begin{array}{c}\text { N- } \\
\text { NITRO- } \\
\text { SODI - N- } \\
\text { PROPYL- } \\
\text { AMINE } \\
\text { TOTAL } \\
\text { (UG/L) }\end{array}$ & $\begin{array}{l}\text { N-NITRO- } \\
\text { SODI - } \\
\text { PHENY - } \\
\text { LAMINE } \\
\text { TOTAL } \\
\text { (UG/L) }\end{array}$ & $\begin{array}{l}\text { PENTA - } \\
\text { CHLORO- } \\
\text { PHENOL } \\
\text { TOTAL } \\
\text { (UG/L) }\end{array}$ & $\begin{array}{c}\text { PHENAN - } \\
\text { THRENE } \\
\text { TOTAL } \\
\text { (UG/L) }\end{array}$ & $\begin{array}{l}\text { PHENOL } \\
\text { (C6H- } \\
5 O H) \\
\text { TOTAL } \\
\text { (UG/L) }\end{array}$ & $\begin{array}{c}\text { PYRENE } \\
\text { TOTAL } \\
\text { (UG/L) }\end{array}$ & $\begin{array}{c}2,4,6- \\
\text { TRI - } \\
\text { CHLORO- } \\
\text { PHENOL } \\
\text { TOTAL } \\
\text { (UG/L) }\end{array}$ \\
\hline $\begin{array}{c}\text { APR } 1993 \\
08 \ldots\end{array}$ & $<5.0$ & $<5.0$ & $<5.0$ & $<30.0$ & $<5.0$ & $<5.0$ & $<30.0$ & $<5.0$ & $<5.0$ & $<5.0$ & $<20.0$ \\
\hline
\end{tabular}


Table 15. Quality-assurance/quality-control data for storm-sewer outfall stations, Mesquite, Texas, 1992-93-Continued 08061940 - SOUTH MESQUITE CREEK OUTFALL AT BRUTON ROAD, MESQUITE, TX (WY 1993)

\section{TYPE \\ OF \\ $Q A / Q C$ \\ SAMPLE}

REP

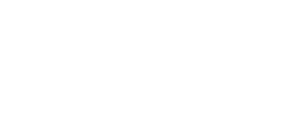

$\begin{array}{ccr} & \text { NITRO- } & \\ \text { NITRO- } & \text { GEN, AM- } & \\ \text { GEN, } & \text { MONIA + } & \text { PHOS- } \\ \text { NO2+NO3 } & \text { ORGANIC } & \text { PHORUS } \\ \text { TOTAL } & \text { TOTAL } & \text { TOTAL } \\ \text { (MG/L } & \text { (MG L } ~ & \text { (MG/L } \\ \text { AS N) } & \text { AS N) } & \text { AS P) }\end{array}$

$\begin{array}{lc}\text { PHOS - } & \\ \text { PHORUS } & \\ \text { DIS - } & \text { ARSENIC } \\ \text { SOLVED } & \text { TOTAL } \\ \text { (MG / L } & \text { (UG / L } \\ \text { AS P) } & \text { AS AS) }\end{array}$

BERYL -

LIUM,

TOTAL

TOTAL

RECOV -

ERABLE ERABLE

(UG/L (UG/L

DATE TIME

AS N)

AS N)

AS P)

AS P)

AS AS)

AS BE)

AS CD)

APR 1993

$14-14$

0701

0.770

2.2

0.700

0.630

48

$<10$

$<1$

CHRO -

MIUM,

TOTAL

RECOV -

ERABLE

DATE

(US/L

COPPER

TOTAL

RECOV -

ERABLE

AS CU)

LEA

TOTAL TOTAL

RECOV - RECOV - RECOV

ERABLE ERABLE ERABLE

(UG/L

(UG/L

(UG/L

AS NI)

AS HG)

SILVER, ZINC,

TOTAL TOTAL

RECOV - RECOV -

ERABLE ERABLE

(UG/L (UG/L

$<10$

6

$6<0.10$

APR 1993

$14-14$

$<2$

$<1$

50 
Table 16. Quality-assurance/quality-control data for storm-sewer outfall stations, Plano, Texas, 1992-93

08061510 - ROWLETT CREEK OUTFALL AT WILLOW CREEK PARK, PLANO, TX (WY 1993)

\begin{tabular}{|c|c|c|c|c|c|c|c|c|c|c|c|c|c|}
\hline & & & $\begin{array}{l}\text { SPE- } \\
\text { CIFIC }\end{array}$ & $\begin{array}{c}\text { SPE- } \\
\text { CIFIC } \\
\text { CON- }\end{array}$ & $\begin{array}{c}\text { PH } \\
\text { WATER } \\
\text { WHOLE }\end{array}$ & HARD - & $\begin{array}{l}\text { HARD - } \\
\text { NESS } \\
\text { NONCARB }\end{array}$ & $\begin{array}{l}\text { ALKA - } \\
\text { LINITY } \\
\text { WAT DIS }\end{array}$ & $\begin{array}{l}\text { SOLIDS, } \\
\text { SUM OF } \\
\text { CONSTI- }\end{array}$ & $\begin{array}{l}\text { RESIDUE } \\
\text { TOTAL } \\
\text { AT } 105\end{array}$ & $\begin{array}{l}\text { SOLIDS, } \\
\text { RESIDUE } \\
\text { AT } 180\end{array}$ & CALCIUM & $\begin{array}{l}\text { MAGNE } \\
\text { SIUM, }\end{array}$ \\
\hline TYPE & & & CON & DUCT - & LAB & TOTAL & DISSOLV & FIX END & TUENTS, & DEG. C, & DEG. C & DIS - & DIS - \\
\hline OF & & & DUCT - & ANCE & (STAND - & (MG /L & FLD. AS & FIELD & DIS- & & DIS- & SOLVED & SOLVED \\
\hline $\begin{array}{l}\text { QA/QC } \\
\text { AMPLE }\end{array}$ & DATE & IME & $\begin{array}{c}\text { ANCE } \\
\text { (US/CM) }\end{array}$ & $\begin{array}{c}\text { LAB } \\
\text { (US/CM) }\end{array}$ & $\begin{array}{c}\text { ARD } \\
\text { UNITS) }\end{array}$ & $\begin{array}{c}\text { AS } \\
\text { (ACO3) }\end{array}$ & $\begin{array}{c}\mathrm{CACO} 3 \\
(\mathrm{MG} / \mathrm{L})\end{array}$ & $\begin{array}{l}\mathrm{CACO3} \\
(\mathrm{MG} / \mathrm{L})\end{array}$ & $\begin{array}{l}\text { SOLVED } \\
(\mathrm{MG} / \mathrm{L})\end{array}$ & $\begin{array}{l}\text { PENDED } \\
\text { (MG/L) }\end{array}$ & $\begin{array}{l}\text { SOLVED } \\
\text { (MG/L) }\end{array}$ & $\begin{array}{l}\text { (MG/L } \\
A S C A)\end{array}$ & $\begin{array}{l}\text { (MG } / \mathrm{L} \\
\text { AS MG) }\end{array}$ \\
\hline
\end{tabular}

\begin{tabular}{|c|c|c|c|c|c|c|c|c|c|c|c|c|c|}
\hline & DEC 1992 & & & & & & & & & & & & \\
\hline \multirow{2}{*}{ REP } & 09.09 & 0101 & 54 & 92 & 7.2 & 18 & 0 & 21 & 40 & 43 & 18 & 7.0 & 0.22 \\
\hline & $\mathrm{FEB} 1993$ & & & & & & & & & & & & \\
\hline $\mathrm{TB}$ & $\begin{array}{l}10-10 \\
F E B\end{array}$ & 0442 & $\cdots$ & $\cdots$ & $\cdots$ & $\cdots$ & $\cdots$ & $\cdots$ & $\cdots$ & $\cdots$ & $\cdots$ & $\cdots$ & $\cdots$ \\
\hline FLB & $10-10$ & 0443 & $\cdots$ & $\cdots$ & $\cdots$ & $\cdots$ & - & $\cdots$ & $\cdots$ & $\cdots$ & $\cdots$ & $\cdots$ & $\cdots$ \\
\hline
\end{tabular}

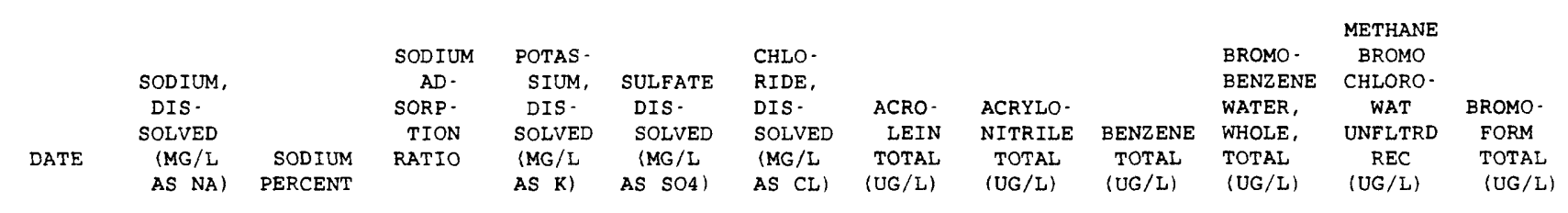

\begin{tabular}{|c|c|c|c|c|c|c|c|c|c|c|c|c|}
\hline \multicolumn{13}{|l|}{ DEC 1992} \\
\hline 09.09 & 1.3 & 11 & 0.1 & 3.1 & 2.6 & 0.70 & $\cdots$ & $\cdots$ & $\cdots$ & $\cdots$ & $\cdots$ & $\cdots$ \\
\hline \multicolumn{13}{|l|}{ FEB 1993} \\
\hline $10-10$ & $\cdots$ & $\cdots$ & $\cdots$ & $\cdots$ & $\cdots$ & $\cdots$ & $<20$ & $<20$ & $<0.2$ & $<0.2$ & $<0.20$ & $<0.2$ \\
\hline \multicolumn{13}{|l|}{ FEB } \\
\hline \multirow[t]{6}{*}{$10-10$} & $\cdots$ & $\cdots$ & $\cdots$ & $\cdots$ & $\cdots$ & $\cdots$ & $\cdots$ & $\cdots$ & $\cdots$ & $\cdots$ & $\cdots$ & $\cdots$ \\
\hline & & BENZENE & BENZENE & & & & & $2-$ & & & O- & \\
\hline & BENZENE & SEC & TERT - & CARBON - & & CHLORO- & & CHLORO - & & & CHLORO- & TOLUENE \\
\hline & N-BUTYL & BUTYL - & BUTYL - & TETRA - & & DI - & & ETHYL - & & METHYL - & TOLUENE & P.CHLOR \\
\hline & WATER & WATER & WATER & CHLO- & CHLORO- & BROMO- & CHLORO- & VINYL - & CHLORO - & CHLO - & WATER & WATER \\
\hline & UNFLTRD & UNFLTRD & UNFLTRD & RIDE & BENZENE & METHANE & ETHANE & ETHER & FORM & RIDE & WHOLE & UNFLTRD \\
\hline DATE & $\begin{array}{c}\text { REC } \\
\text { (UG/L) }\end{array}$ & $\begin{array}{c}\text { REC } \\
\text { (UG/L) }\end{array}$ & $\begin{array}{c}\text { REC } \\
\text { (UG/L) }\end{array}$ & $\begin{array}{l}\text { TOTAL } \\
\text { (UG/L) }\end{array}$ & $\begin{array}{l}\text { TOTAL } \\
\text { (UG/L) }\end{array}$ & $\begin{array}{l}\text { TOTAL } \\
\text { (UG/L) }\end{array}$ & $\begin{array}{l}\text { TOTAL } \\
\text { (UG/L) }\end{array}$ & $\begin{array}{l}\text { TOTAL } \\
\text { (UG/L) }\end{array}$ & $\begin{array}{l}\text { TOTAL } \\
\text { (UG/L) }\end{array}$ & $\begin{array}{l}\text { TOTAL } \\
\text { (UG/L) }\end{array}$ & $\begin{array}{l}\text { TOTAL } \\
\text { (UG/L) }\end{array}$ & $\begin{array}{c}\text { REC } \\
\text { (UG/I) }\end{array}$ \\
\hline
\end{tabular}

DEC 1992

$09 \cdot 09$
FEB 1993

$10-10$

FEB

$10-10$

$<0.20<0.20<0.20$

-.

-.

$<0.2<0.20$

$<0.2$

$\cdots$

-

$-$

-
- $\quad \cdots$
$<0.2<0.20$

\begin{tabular}{|c|c|c|c|c|c|c|c|c|c|c|c|c|}
\hline & DIBROMO & DI - & $1,2-$ & & & $1,1-\mathrm{DI}$ & BENZENE & BENZENE & BENZENE & DI - & & \\
\hline & CHLORO- & BROMO - & DIBROMO & DI - & $1,1-\mathrm{DI}$ & CHLORO - & 0 & $1,3-\mathrm{DI}-$ & $1,4-\mathrm{DI}$ & CHLORO- & & \\
\hline & $\begin{array}{l}\text { PROPANE } \\
\text { WATER }\end{array}$ & $\begin{array}{l}\text { METHANE } \\
\text { WATER }\end{array}$ & $\begin{array}{r}\text { ETHANE } \\
\text { WATER }\end{array}$ & $\begin{array}{l}\text { CHLORO - } \\
\text { BROMO - }\end{array}$ & $\begin{array}{l}\text { CHLORO- } \\
\text { ETHYL- }\end{array}$ & $\begin{array}{l}\text { PRO- } \\
\text { PENE }\end{array}$ & $\begin{array}{c}\text { CHLORO- } \\
\text { WATER }\end{array}$ & $\begin{array}{c}\text { CHLORO - } \\
\text { WATER }\end{array}$ & $\begin{array}{c}\text { CHLORO - } \\
\text { WATER }\end{array}$ & $\begin{array}{l}\text { DI - } \\
\text { FLUORO- }\end{array}$ & $\begin{array}{l}1,1-D I- \\
\text { CHLORO- }\end{array}$ & $\begin{array}{l}1,2 \text { - DI - } \\
\text { CHLORO- }\end{array}$ \\
\hline & WHOLE & WHOLE & WHOLE & METHANE & ENE & WAT, WH & UNFLTRD & UNFLTRD & UNFLTRD & METHANE & ETHANE & ETHANE \\
\hline DATE & $\begin{array}{l}\text { TOT.REC } \\
\text { (UG/L) }\end{array}$ & $\begin{array}{l}\text { RECOVER } \\
\text { (UG/L) }\end{array}$ & $\begin{array}{l}\text { TOTAL } \\
\text { (UG/L) }\end{array}$ & $\begin{array}{l}\text { TOTAL } \\
\text { (UG/L) }\end{array}$ & $\begin{array}{l}\text { TOTAL } \\
\text { (UG/L) }\end{array}$ & $\begin{array}{l}\text { TOTAL } \\
\text { (UG/L) }\end{array}$ & $\begin{array}{c}\text { REC } \\
\text { (UG/L) }\end{array}$ & $\begin{array}{c}\text { REC } \\
(U G / L)\end{array}$ & $\begin{array}{c}\text { REC } \\
(\mathrm{UG} / \mathrm{L})\end{array}$ & $\begin{array}{l}\text { TOTAL } \\
\text { (UG/L) }\end{array}$ & $\begin{array}{l}\text { TOTAL } \\
\text { (UG/L) }\end{array}$ & $\begin{array}{l}\text { TOTAL } \\
\text { (UG/L) }\end{array}$ \\
\hline
\end{tabular}

DEC 1992

$09-09$
FEB 1993

$10 \cdot 10$

FEB

$10 \cdot 10$

$<1.0$ 
Table 16. Quality-assurance/quality-control data for storm-sewer outfall stations, Plano, Texas, 1992-93-Contiued

08061510 - ROWLETT CREEK OUTFALL AT WILLOW CREEK PARK, PLANO, TX (WY 1993)-Continued

\begin{tabular}{|c|c|c|c|c|c|c|c|c|c|c|c|c|}
\hline & $\begin{array}{l}1,2-D I- \\
\text { PHENYL. }\end{array}$ & $\begin{array}{c}\text { CIS - } 1,2- \\
\text { DI. }\end{array}$ & 1,2 & & 1,3-DI- & $\begin{array}{l}2,2 \text { - DI } \\
\text { CHLORO- }\end{array}$ & CIS & TRANS - & & HEXA & $\begin{array}{l}\text { ISO- } \\
\text { PROPYL - }\end{array}$ & $\begin{array}{l}\text { P-ISO- } \\
\text { PROPYL. }\end{array}$ \\
\hline & HYDRA - & CHLORO- & TRANSDI & $1,2-D I-$ & CHLORO- & PRO- & 1,3-DI - & $1,3 \cdot D I$. & & CHLORO- & BENZENE & TOLUENE \\
\hline & ZINE & ETHENE & CHLORO- & CHLORO- & PROPANE & PANE & CHLORO- & CHLORO - & ETHYL - & BUT - & WATER & WATER \\
\hline & WATER & WATER & ETHENE & PROPANE & WAT. WH & WAT, WH & PROPENE & PROPENE & BENZENE & ADIENE & WHOLE & WHOLE \\
\hline DATE & $\begin{array}{l}\text { TOT.REC } \\
\text { (UG /L) }\end{array}$ & $\begin{array}{l}\text { TOTAL } \\
\text { (UG/L) }\end{array}$ & $\begin{array}{l}\text { TOTAL } \\
\text { (UG/L) }\end{array}$ & $\begin{array}{l}\text { TOTAL } \\
\text { (UG/L) }\end{array}$ & $\begin{array}{l}\text { TOTAL } \\
\text { (UG/L) }\end{array}$ & $\begin{array}{l}\text { TOTAL } \\
\text { (UG/L) }\end{array}$ & $\begin{array}{l}\text { TOTAL } \\
\text { (UG/L) }\end{array}$ & $\begin{array}{l}\text { TOTAL } \\
\text { (UG/L) }\end{array}$ & $\begin{array}{l}\text { TOTAL } \\
(\text { UG } / L)\end{array}$ & $\begin{array}{l}\text { TOTAL } \\
\text { (UG/L) }\end{array}$ & $\begin{array}{l}\text { REC } \\
\text { (UG/L) }\end{array}$ & $\begin{array}{l}\mathrm{REC} \\
\langle\mathrm{UG} / \mathrm{L}\rangle\end{array}$ \\
\hline
\end{tabular}

DEC 1992

09.09

FEB 1993

10-10

FEB

$10-10$

(UG/L)

G/L)

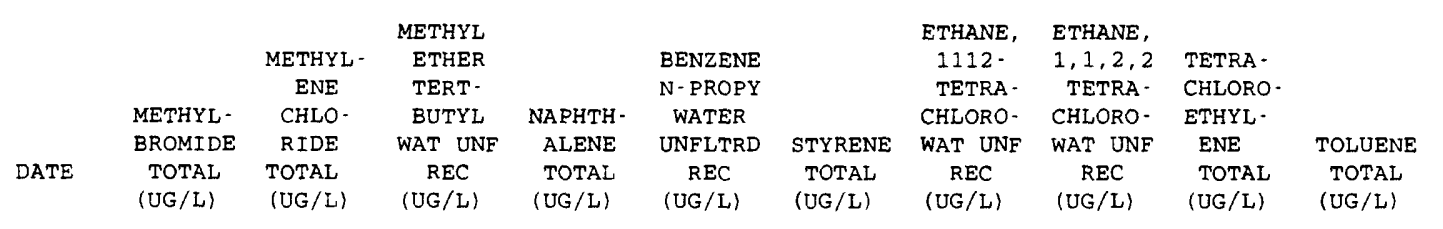

$\begin{array}{cc}\text { 1,2,3- } & \text { BENZENE } \\ \text { TRI- } & 1,2,4- \\ \text { CHLORO } & \text { TRI- } \\ \text { BENZENE } & \text { CHLORO- } \\ \text { WAT, WH } & \text { WAT UNF } \\ \text { REC } & \text { REC } \\ \text { (UG/L) } & \text { (UG/L) }\end{array}$

DEC 1992

$09-09$

FEB 1993

10-10

FEB

$10 \cdot 10$
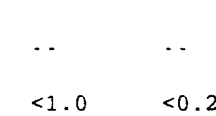

$<0.2$

$<5.0$

-

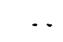

$<0.20$
-

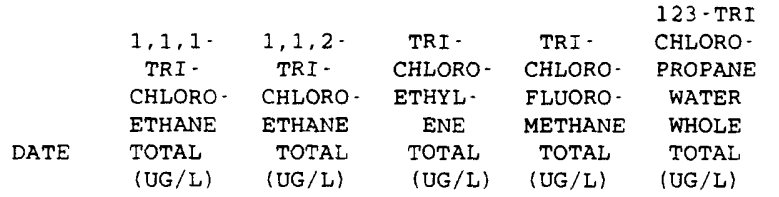

DEC 1992

09.09

FEB 1993

$10-10$

FEB

$10 \cdot 10$

\section{$<0.2$}

$<0.2$

$<0.2$

$<0.2$

$<0.2$

$<0.5$

$<0.20$

$<0.20$

..

$\begin{array}{ccc}\text { FREON- } & \text { PSEUDO- } & \text { MESIT- } \\ 113 & \text { CUMENE } & \text { YLENE } \\ \text { WATER } & \text { WATER } & \text { WATER } \\ \text { UNFLTRD } & \text { UNFLTRD } & \text { UNFLTRD } \\ \text { REC } & \text { REC } & \text { REC } \\ \text { (UG/L) } & \text { (UG/L) } & \text { (UG/L) }\end{array}$

$\begin{array}{lccc}\text { VINYL } & \text { XYLENE } & \text { ACE- } & \text { ACE - } \\ \text { CHLO- } & \text { WATER } & \text { NAPHTH - } & \text { NAPHTH - } \\ \text { RIDE } & \text { UNFLTRD } & \text { ENE } & \text { YLENE } \\ \text { TOTAL } & \text { REC } & \text { TOTAL } & \text { TOTAL } \\ \text { (UG/L) } & \text { (UG/L) } & \text { (UG/L) } & \text { (UG/L) }\end{array}$

$<0.2<0.20$

-
$<5.0$

$<5.0$

\begin{tabular}{|c|c|c|c|c|c|c|c|c|c|c|c|c|}
\hline & \multirow[b]{3}{*}{$\begin{array}{l}\text { ANTHRA - } \\
\text { CENE }\end{array}$} & \multicolumn{3}{|c|}{ BENZO A } & \multicolumn{3}{|r|}{ BENZO } & \multicolumn{2}{|l|}{$4-$} & \multirow{2}{*}{$\begin{array}{l}\text { BIS } \\
12-\end{array}$} & BIS & BIS \\
\hline & & & ANTHRAC & & BENZO B & BENZO $\mathrm{K}$ & GHI PERYL & BROMO - & $N-B U T Y L$ & & 2 & CHLORO- \\
\hline & & $\begin{array}{l}\text { BENZI - } \\
\text { DINE }\end{array}$ & $\begin{array}{l}\text { ENE 1, 2- } \\
\text { BENZANT } \\
\text { HRACENE }\end{array}$ & $\begin{array}{l}\text { BENZO- } \\
\text { A- } \\
\text { PYRENE }\end{array}$ & $\begin{array}{l}\text { FLUOR - } \\
\text { AN- } \\
\text { THENE }\end{array}$ & $\begin{array}{l}\text { FLUOR - } \\
\text { AN - } \\
\text { THENE }\end{array}$ & $\begin{array}{l}\text { ENE1, 12- } \\
\text { BENZO } \\
\text { PERYLENE }\end{array}$ & $\begin{array}{c}\text { PHENYL } \\
\text { PHENYL } \\
\text { ETHER }\end{array}$ & $\begin{array}{l}\text { BENZYL } \\
\text { PHTHAL - } \\
\text { ATE }\end{array}$ & $\begin{array}{l}\text { CHLORO- } \\
\text { ETHOXY) } \\
\text { METHANE }\end{array}$ & $\begin{array}{c}\text { CHLORO- } \\
\text { ETHYL } \\
\text { ETHER }\end{array}$ & $\begin{array}{c}\text { ISO- } \\
\text { PROPYL) } \\
\text { ETHER }\end{array}$ \\
\hline & $\begin{array}{l}\text { TOTAL } \\
\text { (UG/L) }\end{array}$ & $\begin{array}{l}\text { TOTAL } \\
\text { (UG/L) }\end{array}$ & $\begin{array}{l}\text { TOTAL } \\
\text { (UG/L) }\end{array}$ & $\begin{array}{l}\text { TOTAL } \\
\text { (UG/L) }\end{array}$ & $\begin{array}{l}\text { TOTAL } \\
\text { (UG/L) }\end{array}$ & $\begin{array}{l}\text { TOTAL } \\
\text { (UG /L) }\end{array}$ & $\begin{array}{l}\text { TOTAL } \\
\text { (UG/L) }\end{array}$ & $\begin{array}{l}\text { TOTAL } \\
\text { (UG/L) }\end{array}$ & $\begin{array}{l}\text { TOTAL, } \\
\text { (UG/L) }\end{array}$ & $\begin{array}{l}\text { TOTAL } \\
\text { (UG/L) }\end{array}$ & $\begin{array}{l}\text { TOTAL } \\
\text { (UG/L) }\end{array}$ & $\begin{array}{l}\text { TOTAL } \\
\text { (UG/L) }\end{array}$ \\
\hline
\end{tabular}

DEC 1992

09-09

FEB 1993

10-10

FEB

$10-10$

$<5.0<40.0$

$<10.0$

$<10.0<10.0$

$<10.0<10.0$

$<5.0$

$<5.0$

$<5.0$

$<5.0$

$<5.0$ 
Table 16. Quality-assurance/quality-control data for storm-sewer outfall stations, Plano, Texas, 1992-93-Continued

08061510 - ROWLETT CREEK OUTFALL AT WILLOW CREEK PARK, PLANO, TX (WY 1993)-Continued

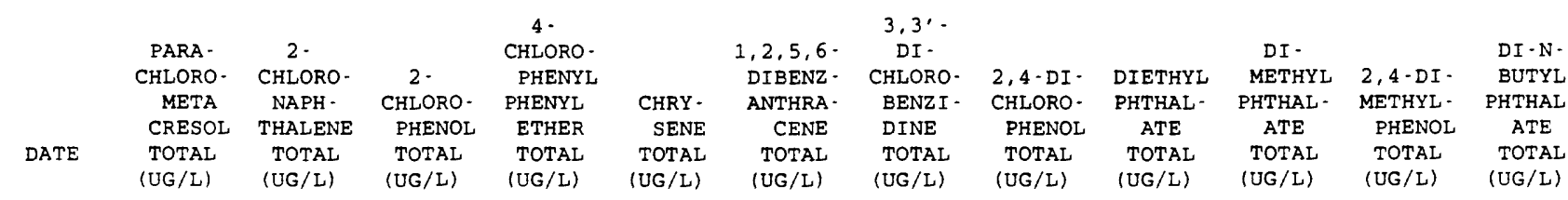

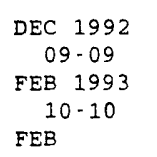

$<30.0$

$<5.0<5.0$

$<5.0$

$<10.0$

$<10.0$

$<20.0$

$<5.0$

$<5.0$

$<5.0$

$<5$.

$<5.0$

\begin{tabular}{|c|c|c|c|c|c|}
\hline & $\begin{array}{c}4,6- \\
\text { DINITRO- } \\
\text { ORTHO- } \\
\text { CRESOL }\end{array}$ & $\begin{array}{c}2,4,- \\
\text { DI- } \\
\text { NITRO- } \\
\text { PHENOL }\end{array}$ & $\begin{array}{l}2,4-\mathrm{DI} \text { - } \\
\text { NITRO- } \\
\text { TOLUENE }\end{array}$ & $\begin{array}{l}2,6-\mathrm{DI}- \\
\text { NITRO- } \\
\text { TOLUENE }\end{array}$ & $\begin{array}{c}\text { DI - N - } \\
\text { OCTYL } \\
\text { PHTHAL - } \\
\text { ATE }\end{array}$ \\
\hline ATE & $\begin{array}{l}\text { TOTAL } \\
\text { (UG/L) }\end{array}$ & $\begin{array}{l}\text { TOTAL } \\
\text { (UG/L) }\end{array}$ & $\begin{array}{l}\text { TOTAL } \\
\text { (UG/L) }\end{array}$ & $\begin{array}{l}\text { TOTAL } \\
\text { (UG/L) }\end{array}$ & $\begin{array}{l}\text { TOTAL } \\
\text { (UG/L) }\end{array}$ \\
\hline
\end{tabular}

BIS 12 -

ETHYL

HEXYL)

PHTHAL -

ATE

TOTAL

(UG/L)

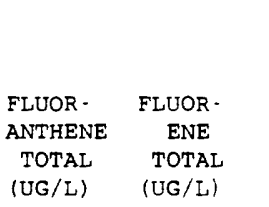

$\begin{array}{cc} & \text { HEXA- } \\ \text { CHLORO- } \\ \text { HEXA- } & \text { CYCLO- } \\ \text { CHLORO- } & \text { PENT- } \\ \text { BENZENE } & \text { ADIENE } \\ \text { TOTAL } & \text { TOTAL } \\ \text { (UG/L) } & (U G / L)\end{array}$

HEXA. CHLORO ETHANE PYRENE TOTAL TOTAL

DEC 1992

$09-09$

FEB 1993

$10-10$

FEB

$10-10<30.0<20.0<5.0<5.0$

$<10.0$

$<5.0$

$<5.0$

$<5.0$

$<5.0$

$<5.0$

$<5.0$

$<10.0$
N-

SODI - N - SODI- PENTA. PHENOL

N-NITRO SODI - 2- 4- SODI -

ISOMETHY NITROPHENOL NITRO. PHENOL TOTAL (UG/L)
TOTAL

(UG/L)
PHENY -

LAMINE

TOTAL

(UG/L)
CHLORO.

PHENOL

TOTAL

(UG/L)
NITRO- N-NITRO-

(UG/L)

(UG/L)

DEC 1992
09.09

$09 \cdot 09$
FEB 1993

$10-10$

FEB

$10 \cdot 10<5.0$

$<5.0$

$<5.0$

$<5.0<30.0$

$<5.0$

$<5.0<30.0$

$<5.0$

$<5.0$

$<5.0$

$<20.0$
TYP
$O F$
$Q A / Q C$ SAMPLE

REP

REP

FMS

LMS

08061525 - SPRING CREEK OUTFALL AT PARK BOULEVARD, PLANO, TX (WY 1993)

OIL AND

GREASE،

TOTAL

RECOV.

METRIC

(MG/L)

PHENOLS
TOTAL
(UG/L)

DEC 1992

09.09

DEC

$09-09$

FEB 1993

$10 \cdot 10$

FEB

$10 \cdot 10$
0103

0104

0446

0447
$<1$

$\cdots$

-

$\begin{array}{ccc} & \\ \text { ACRO- } & \text { ACRYLO- } & \\ \text { LEIN } & \text { NITRILE } & \text { BENZENE } \\ \text { TOTAL } & \text { TOTAL } & \text { TOTAL } \\ (U G / L) & \text { (UG/L) } & \text { (UG/L) }\end{array}$

$\begin{array}{lcc} & \text { METHANE } \\ \text { BROMO- } & \text { BROMO } & \\ \text { BENZENE } & \text { CHLORO- } \\ \text { WATER, } & \text { WAT } & \text { BROMO- } \\ \text { WHOLE, } & \text { UNFLTRD } & \text { FORM } \\ \text { TOTAL } & \text { REC } & \text { TOTAL } \\ \text { (UG/L) } & \text { (UG/L) } & \text { (UG/L) }\end{array}$

$\begin{array}{ccc} & \text { BENZENE } & \text { BENZENE } \\ \text { BENZENE } & \text { SEC } & \text { TERT- } \\ \text { N-BUTYL } & \text { BUTYL - } & \text { BUTYL- } \\ \text { WATER } & \text { WATER } & \text { WATER } \\ \text { UNFLTRD } & \text { UNFLTRD } & \text { UNFLTRD } \\ \text { REC } & \text { REC } & \text { REC } \\ \text { (UG/L) } & \text { (UG/L) } & \text { (UG/L) }\end{array}$

$<0.2$

$<0.20$

4.0

$<0.20$

$<0.20$

$<0.20$

$<20<20$
$<0$.

$<0.20$

10
$<0.20$

$<0.20$

$<0.20$ 
Table 16. Quality-assurance/quality-control data for storm-sewer outfall stations, Plano, Texas, 1992-93-Continued

08061525 - SPRING CREEK OUTFALL AT PARK BOULEVARD, PLANO, TX (WY 1993)-Continued

\begin{tabular}{|c|c|c|c|c|c|c|c|c|c|c|c|c|}
\hline & & & & & 2 . & & & 0. & & DIBROMO & DI. & $1,2$. \\
\hline & $\begin{array}{l}\text { CARBON - } \\
\text { TETRA - }\end{array}$ & & $\begin{array}{c}\text { CHLORO- } \\
\text { DI - }\end{array}$ & & $\begin{array}{l}\text { CHLORO- } \\
\text { ETHYL- }\end{array}$ & & METHYL- & $\begin{array}{l}\text { CHLORO- } \\
\text { TOLUENEE }\end{array}$ & $\begin{array}{l}\text { TOLUENE } \\
\text { P. CHLOR }\end{array}$ & CHLORO- & $\begin{array}{l}\text { BROMO- } \\
\text { METHANE }\end{array}$ & DIBROMO \\
\hline & CHLO - & CHLORO - & BROMO- & CHLORO- & VINYL - & CHLORO- & CHLO- & WATER & WATER & WATER & WATER & WATER \\
\hline & RIDE & BENZENE & METHANE & ETHANE & ETHER & FORM & RIDE & WHOLE & UNFLTRD & WHOLE & WHOLE & WHOLE \\
\hline DATE & $\begin{array}{l}\text { TOTAL } \\
\text { (UG/L) }\end{array}$ & $\begin{array}{l}\text { TOTAL } \\
\text { (UG/L) }\end{array}$ & $\begin{array}{l}\text { TOTAL } \\
\text { (UG/L) }\end{array}$ & $\begin{array}{l}\text { TOTAL } \\
\text { (UG/L) }\end{array}$ & $\begin{array}{l}\text { TOTAL } \\
\text { (UG/L) }\end{array}$ & $\begin{array}{l}\text { TOTAL } \\
\text { (UG/L) }\end{array}$ & $\begin{array}{l}\text { TOTAL } \\
\text { (UG/L) }\end{array}$ & $\begin{array}{l}\text { TOTAL } \\
\text { (UG/L) }\end{array}$ & $\begin{array}{c}\text { REC } \\
(U G / L)\end{array}$ & $\begin{array}{l}\text { TOT .REC } \\
\text { (UG/L) }\end{array}$ & $\begin{array}{l}\text { RECOVER } \\
\text { (UG/L) }\end{array}$ & $\begin{array}{l}\text { TOTAL } \\
\text { (UG/L) }\end{array}$ \\
\hline
\end{tabular}

DEC 1992

O9-09
DEC
$09-09$

FEB 1993

$10-1$

$10 \cdot 10$

$3.3 \quad 3.6$

3.9

3.0

$<1.0$

4.9

2.1

$<0.2$

$<0.20$

$<1.0$

$<0.2$

$<0.2$

7.7

8.7

9.7

$<1.0$

8.0

4.0

$<0.2<0.20$

$<1.0$

$<0.2$

$<0.2$

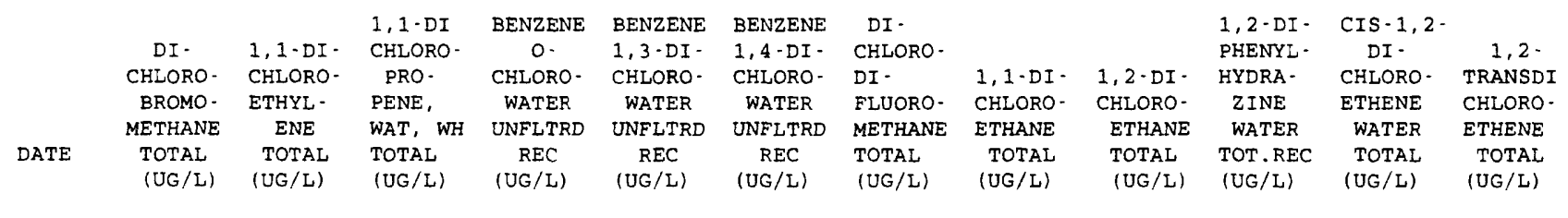

DEC 1992

09.09

DEC

09-09

FEB 1993

$10-10$

FEB

$10-10$

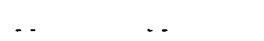

$(U G / L)$

UG/L)

(U)

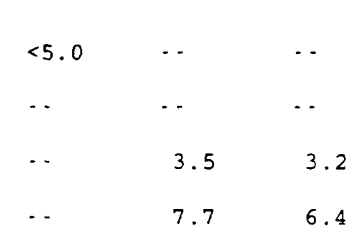

2, 2-DI

2,2 - DI
CHLORO-

$\begin{array}{lll}1,3-D I- & \text { CHLORO- } \\ 1,2-D I- & \text { CHLORO- } & \text { PRO- } \\ \text { CHLORO- } & \text { PROPANE } & \text { PANE } \\ \text { PROPANE } & \text { WAT. WH WAT, WH }\end{array}$

DATE

TOTAL TOTAL

(UG/L) (UG/L)
(UG/L)

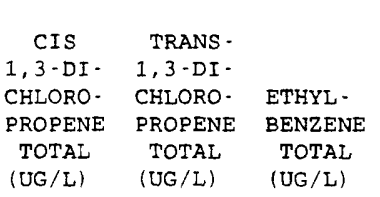

(UG/L)

$\begin{array}{ccc} & \text { ISO- } & \text { P-ISO- } \\ \text { HEXA- } & \text { PROPYL- } & \text { PROPYL- } \\ \text { CHLORO- } & \text { BENZENE } & \text { TOLUENE } \\ \text { BUT - } & \text { WATER } & \text { WATER } \\ \text { ADIENE } & \text { WHOLE } & \text { WHOLE } \\ \text { TOTAL } & \text { REC } & \text { REC } \\ \text { (UG/L) } & \text { (UG/L) } & \text { (UG/L) }\end{array}$

(UG/L)

(UG/L)
8.9

DEC 1992

O9. 09
DEC
$09-09$

FEB 1993

10-10

FEB
$10-10$

.

3

-

.

..

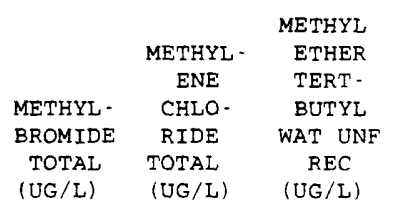


Table 16. Quality-assurance/quality-control data for storm-sewer outfall stations, Plano, Texas, 1992-93-Continued

08061525 - SPRING CREEK OUTFALL AT PARK BOULEVARD, PLANO, TX (WY 1993)-Continued

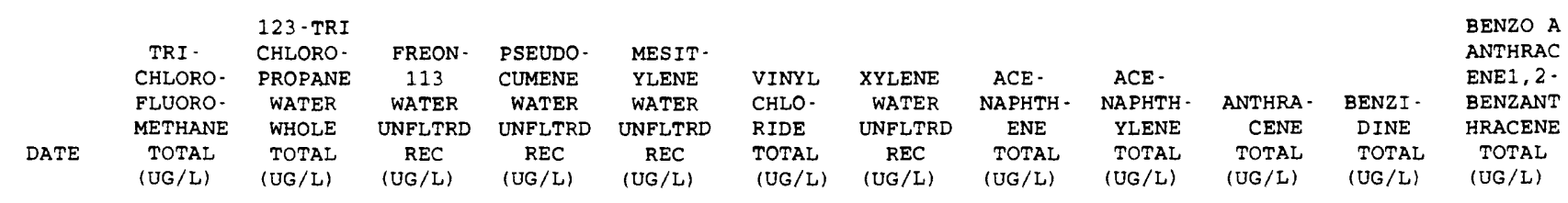

DEC 1992
$09-09$
DEC
$09-09$
FEB 1993
$10-10$
FEB
$10-10$

(1)

3.2

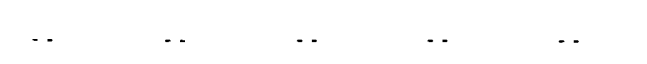

$3.2<0.2$
$<0.5$

$<0.5$
$<0.20$

$<0.20$
$<0.20$

$<0.20$
.

$\cdots$

$2.6<0.20$

$4.6<0.20$
$<5.0<5.0$

$<5$.

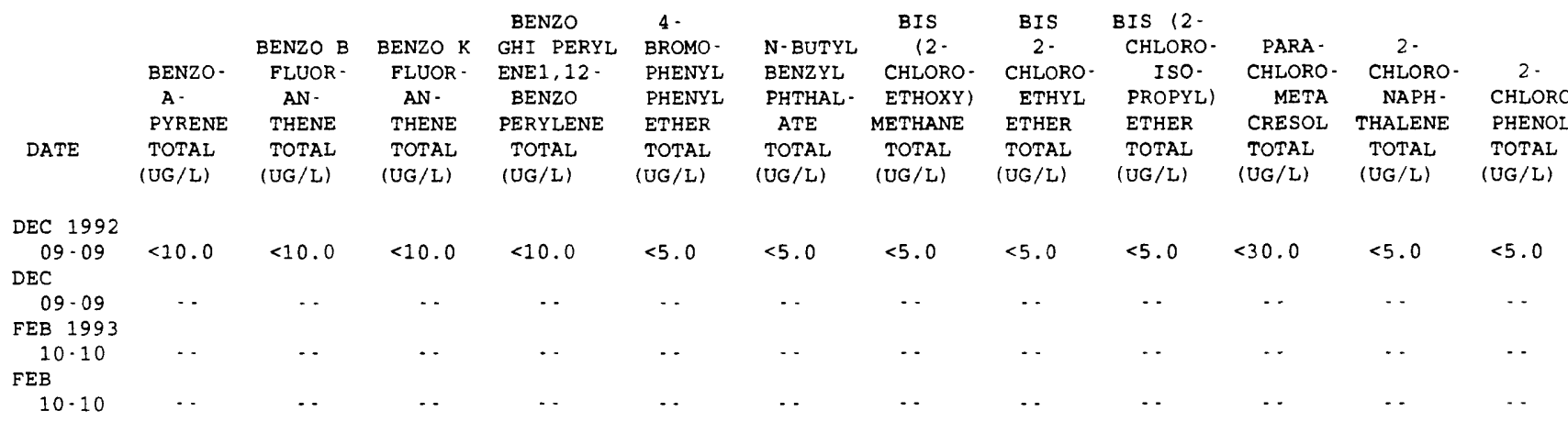

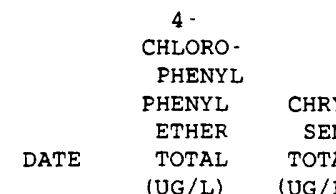

DEC 1992

09.09 DEC

$09-09$

FEB 1993

$10-10$

FEB

$10-10$
$<5$.

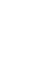

$\cdots$ $1,2,5,6-3,3^{\prime} \cdot$

DIBENZ - CHLORO -

CENE DINE

TOTAL TOTAL

(UG/I)
PHENOL

(UG/L)
DIETHYL METHYL

METHYL -

ATE ATE

TOTAL TOTAL

(UG / L)

$$
\begin{array}{cccc}
\text { DI-N- } & 4,6- & 2,4,- & \\
\text { BUTYL } & \text { DINITRO- } & \text { DI- } & 2,4-D I- \\
\text { PHTHAL- } & \text { ORTHO- } & \text { NITRO- } & \text { NITRO- } \\
\text { ATE } & \text { CRESOL } & \text { PHENOL } & \text { TOLUENE } \\
\text { TOTAL } & \text { TOTAL } & \text { TOTAL } & \text { TOTAL } \\
\text { (UG/L) } & \text { (UG/L) } & \text { (UG/L) } & \text { (UG/L) }
\end{array}
$$

\begin{tabular}{|c|c|c|c|c|c|c|c|c|c|c|c|c|}
\hline & & $D I-N-$ & $\begin{array}{l}\text { BIS (2- } \\
\text { ETHYL }\end{array}$ & & & & $\begin{array}{c}\text { HEXA - } \\
\text { CHLORO - }\end{array}$ & & INDENO & & & N-NITRO- \\
\hline & $2,6-\mathrm{DI}$ - & OCTYL & HEXYL) & & & HEXA - & CYCLO- & HEXA - & $(1,2,3-$ & & & SODI \\
\hline & $\begin{array}{l}\text { NITRO- } \\
\text { TOLUENE }\end{array}$ & $\begin{array}{c}\text { PHTHAL - } \\
\text { ATE }\end{array}$ & $\begin{array}{c}\text { PHTHAL - } \\
\text { ATE }\end{array}$ & $\begin{array}{l}\text { FLUOR- } \\
\text { ANTHENE }\end{array}$ & $\begin{array}{l}\text { FLUOR - } \\
\text { ENE }\end{array}$ & $\begin{array}{l}\text { CHLORO- } \\
\text { BENZENE }\end{array}$ & $\begin{array}{l}\text { PENT - } \\
\text { ADIENE }\end{array}$ & $\begin{array}{l}\text { CHLORO- } \\
\text { ETHANE }\end{array}$ & $\begin{array}{c}\text { CD) } \\
\text { PYRENE }\end{array}$ & $\begin{array}{l}\text { ISO - } \\
\text { PHORONE }\end{array}$ & $\begin{array}{l}\text { NITRO- } \\
\text { BENZENE }\end{array}$ & $\begin{array}{l}\text { METHY - } \\
\text { LAMINE }\end{array}$ \\
\hline DATE & $\begin{array}{l}\text { TOTAL } \\
(U G / L)\end{array}$ & $\begin{array}{l}\text { TOTAL } \\
\text { (UG/L) }\end{array}$ & $\begin{array}{l}\text { TOTAL } \\
(\mathrm{UG} / \mathrm{L})\end{array}$ & $\begin{array}{l}\text { TOTAL } \\
\text { (UG/L) }\end{array}$ & $\begin{array}{l}\text { TOTAL } \\
\text { (UG/L) }\end{array}$ & $\begin{array}{l}\text { TOTAL } \\
(U G / L)\end{array}$ & $\begin{array}{l}\text { TOTAL } \\
\text { (UG/L) }\end{array}$ & $\begin{array}{l}\text { TOTAL } \\
(\mathrm{UG} / \mathrm{L})\end{array}$ & $\begin{array}{l}\text { TOTAL } \\
\text { (UG/L) }\end{array}$ & $\begin{array}{c}\text { TOTAL } \\
\text { (UG/L) }\end{array}$ & $\begin{array}{l}\text { TOTAL } \\
\text { (UG/L) }\end{array}$ & $\begin{array}{l}\text { TOTAL } \\
\text { (UG/L) }\end{array}$ \\
\hline
\end{tabular}

\begin{tabular}{|c|c|c|c|c|c|c|c|c|c|c|c|c|}
\hline $09-09$ & $<5.0$ & $<10.0$ & $<5.0$ & $<5.0$ & $<5.0$ & $<5.0$ & $<5.0$ & $<5.0$ & $<10.0$ & $<5.0$ & $<5.0$ & $<5.0$ \\
\hline $\mathrm{DEC}$ & & & & & & & & & & & & \\
\hline $\begin{array}{c}09.09 \\
\text { FEB } 1993\end{array}$ & -- & $\cdots$ & -- & $\cdots$ & $\cdots$ & $\cdots$ & $\cdots$ & -- & $\cdots$ & $\cdots$ & $\cdots$ & $\cdots$ \\
\hline $10-10$ & $\cdots$ & $\cdots$ & $\cdots$ & $\cdots$ & $\cdots$ & $\cdots$ & $\cdots$ & $\cdots$ & $\cdots$ & - & $\cdots$ & $\cdots$ \\
\hline FEB & & & & & & & & & & & & \\
\hline $10-10$ & $\cdots$ & $\cdots$ & $\cdots$ & $\cdots$ & $\cdots$ & $\cdots$ & $\cdots$ & $\cdots$ & $\cdots$ & - & $\cdots$ & $\cdots$ \\
\hline
\end{tabular}

DEC 1992 
Table 16. Quality-assurance/quality-control data for storm-sewer outfall stations, Plano, Texas, 1992-93-Continued

08061525 - SPRING CREEK OUTFALL AT PARK BOULEVARD, PLANO, TX (WY 1993)-Continued

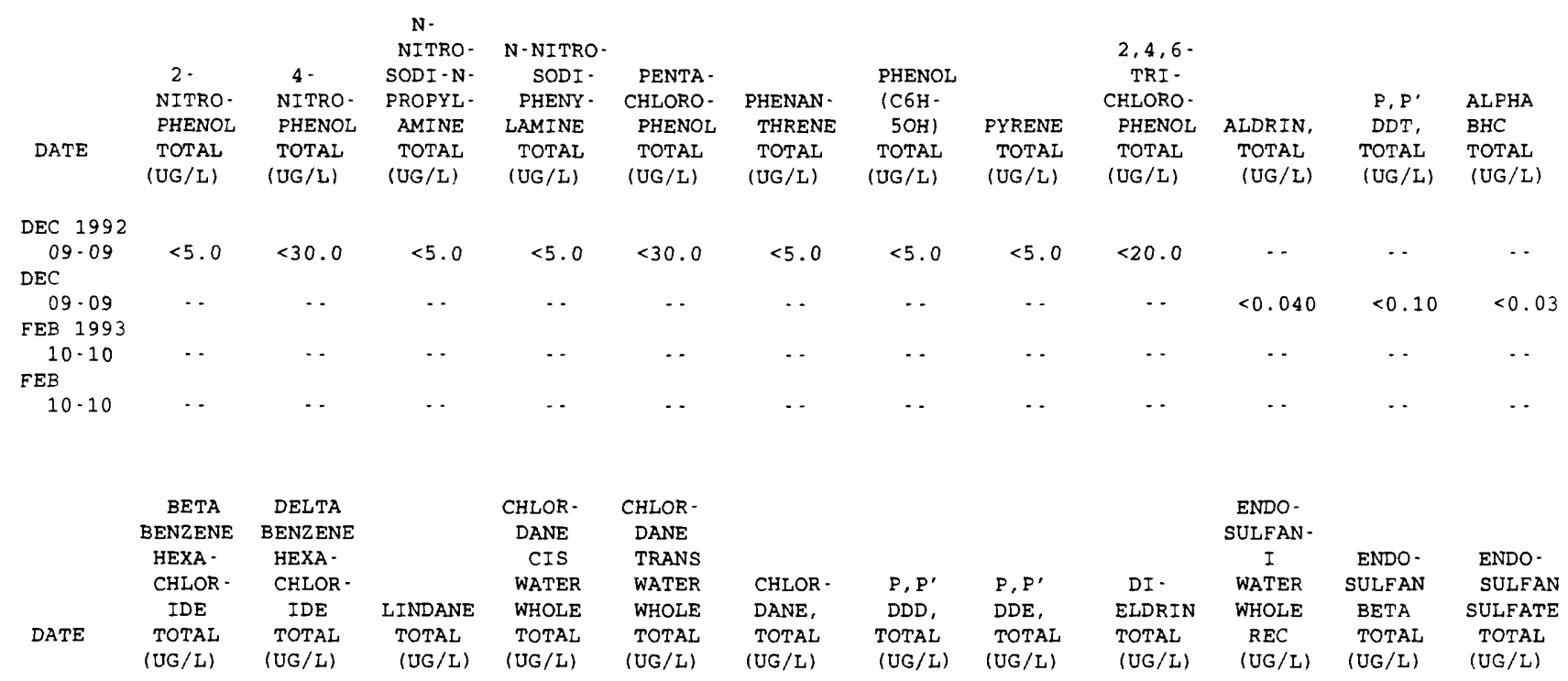

$\begin{array}{cccccc}\text { DEC } 1992 & & & & & \\ 09-09 & \ldots & \ldots & \ldots & \ldots & \ldots \\ \text { DEC } & & & & \\ 09-09 & <0.03 & <0.09 & <0.030 & <0.10 & <0.10 \\ \text { FEB } 1993 & \ldots & \ldots & \ldots & \ldots & \ldots \\ 10-10 & \ldots & \ldots & \ldots & \ldots & \ldots \\ \text { FEB } 10-10 & \ldots & \ldots & \ldots & \ldots & \end{array}$

\begin{tabular}{|c|c|c|c|c|c|c|c|c|c|c|c|c|}
\hline & ENDRIN & ENDRIN & & HEPTA - & & AROCLOR & AROCLOR & AROCLOR & AROCLOR & AROCLOR & AROCLOR & AROCLOR \\
\hline & WATER & ALDE - & HEPTA - & CHLOR & TOX. & 1016 & 1221 & 1232 & 1242 & 1248 & 1254 & 1260 \\
\hline TTE & $\begin{array}{l}\text { UNFLTRD } \\
\text { REC }\end{array}$ & $\begin{array}{r}\text { HYDE } \\
\text { TOTAL } \\
\text { (UG/I) }\end{array}$ & $\begin{array}{l}\text { CHLOR, } \\
\text { TOTAL }\end{array}$ & $\begin{array}{c}\text { EPOXIDE } \\
\text { TOTAL }\end{array}$ & $\begin{array}{c}\text { APHENE, } \\
\text { TOTAL } \\
\text { (UG/L, }\end{array}$ & $\begin{array}{c}\text { PCB } \\
\text { TOTAL }\end{array}$ & $\begin{array}{c}\text { PCB } \\
\text { TOTAL } \\
\text { (TG/T) }\end{array}$ & $\begin{array}{c}\text { PCB } \\
\text { TOTAL }\end{array}$ & $\begin{array}{c}\text { PCB } \\
\text { TOTAL }\end{array}$ & $\begin{array}{c}\text { PCB } \\
\text { TOTAL }\end{array}$ & $\begin{array}{c}\text { PCB } \\
\text { TOTAL }\end{array}$ & $\begin{array}{l}\text { PCB } \\
\text { TOTAL }\end{array}$ \\
\hline
\end{tabular}

DEC 1992

DEC

09.09

10.10

FEB

$10-10$

$<0.060<0.20<0.030<0.80<2$

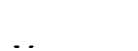

$<0.1<0.10$

$<0.04<0.020$

$<0.10$

$<0.04$

$<0.60$

-

.

$\cdots$

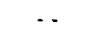

-
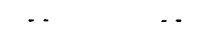
Table 16. Quality-assurance/quality-control data for storm-sewer outfall stations, Plano, Texas, 1992-93-Continued

08061530 - SPRING CREEK OUTFLOW AT AVENUE F, PLANO, TX (WY 1993)-Continued

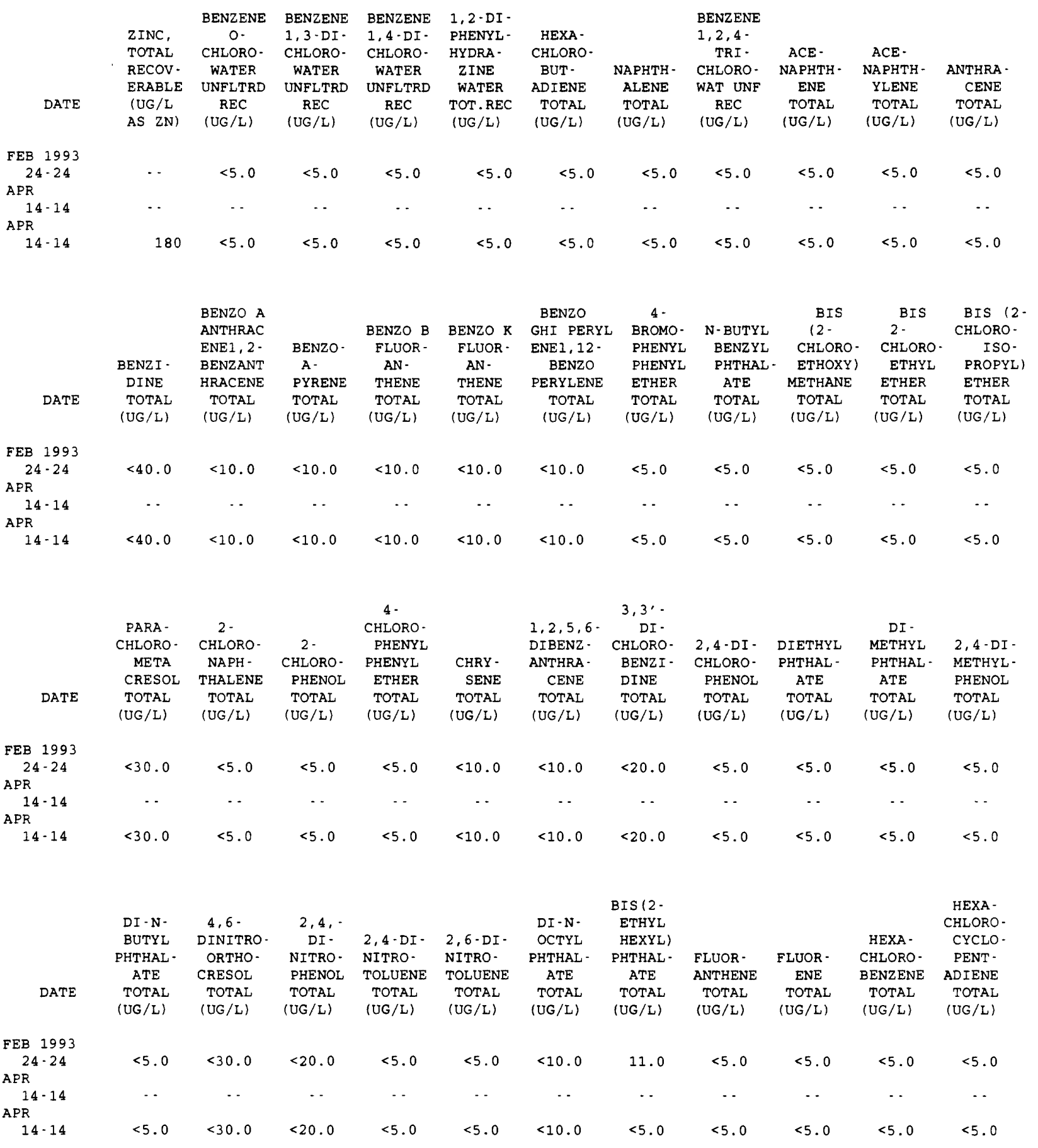


Table 16. Quality-assurance/quality-control data for storm-sewer outfall stations, Plano, Texas, 1992-93-Continued 08061530 - SPRING CREEK OUTFLOW AT AVENUE F, PLANO, TX (WY 1993)-Continued

\begin{tabular}{|c|c|c|c|c|c|c|c|c|c|c|c|}
\hline DATE & $\begin{array}{l}\text { HEXA- } \\
\text { CHLORO- } \\
\text { ETHANE } \\
\text { TOTAL } \\
\text { (UG/L) }\end{array}$ & $\begin{array}{r}\text { INDENO } \\
(1,2,3- \\
\text { CD }) \\
\text { PYRENE } \\
\text { TOTAL } \\
(\text { UG } / L)\end{array}$ & $\begin{array}{l}\text { ISO- } \\
\text { PHORONE } \\
\text { TOTAL } \\
\text { (UG/L) }\end{array}$ & $\begin{array}{l}\text { NITRO- } \\
\text { BENZENE } \\
\text { TOTAL } \\
(\mathrm{UG} / \mathrm{L})\end{array}$ & $\begin{array}{c}\text { N-NITRO- } \\
\text { SODI- } \\
\text { METHY- } \\
\text { LAMINE } \\
\text { TOTAL } \\
\text { (UG/L) }\end{array}$ & $\begin{array}{l}2- \\
\text { NITRO- } \\
\text { PHENOL } \\
\text { TOTAL } \\
\text { (UG/L) }\end{array}$ & $\begin{array}{c}4- \\
\text { NITRO- } \\
\text { PHENOL } \\
\text { TOTAL } \\
\text { (UG/L) }\end{array}$ & $\begin{array}{c}\text { N- } \\
\text { NITRO- } \\
\text { SODI-N- } \\
\text { PROPYL- } \\
\text { AMINE } \\
\text { TOTAL } \\
\text { (UG/L) }\end{array}$ & $\begin{array}{c}\text { N-NITRO- } \\
\text { SODI- } \\
\text { PHENY - } \\
\text { LAMINE } \\
\text { TOTAL } \\
(\text { UG } / L)\end{array}$ & $\begin{array}{l}\text { PENTA- } \\
\text { CHLORO- } \\
\text { PHENOL } \\
\text { TOTAL } \\
(U G / L)\end{array}$ & $\begin{array}{l}\text { PHENAN - } \\
\text { THRENE } \\
\text { TOTAL } \\
\text { (UG/L) }\end{array}$ \\
\hline FEB 1993 & & & & & & & & & & & \\
\hline $24-24$ & $<5.0$ & $<10.0$ & $<5.0$ & $<5.0$ & $<5.0$ & $<5.0$ & $<30.0$ & $<5.0$ & $<5.0$ & $<30.0$ & $<5.0$ \\
\hline APR & & & & & & & & & & & \\
\hline $14 \cdot 14$ & $\cdots$ & $\cdots$ & $\cdots$ & $\cdots$ & $\cdots$ & $\cdots$ & $\cdots$ & $\cdots$ & $\cdots$ & $\cdots$ & $\cdots$ \\
\hline APR & & & & & & & & & & & \\
\hline $14-14$ & $<5.0$ & $<10.0$ & $<5.0$ & $<5.0$ & $<5.0$ & $<5.0$ & $<30.0$ & $<5.0$ & $<5.0$ & $<30.0$ & $<5.0$ \\
\hline DATE & $\begin{array}{l}\text { PHENOL } \\
\text { (C6H- } \\
5 O H) \\
\text { TOTAL } \\
\text { (UG/L) }\end{array}$ & $\begin{array}{c}\text { PYRENE } \\
\text { TOTAL } \\
\text { (UG/L) }\end{array}$ & $\begin{array}{c}2,4,6- \\
\text { TRI - } \\
\text { CHLORO- } \\
\text { PHENOL } \\
\text { TOTAL } \\
\text { (UG/L) }\end{array}$ & $\begin{array}{l}\text { ALDRIN, } \\
\text { TOTAL } \\
\text { (UG/L) }\end{array}$ & $\begin{array}{c}\text { P, } \mathrm{P}^{\prime} \\
\text { DDT, } \\
\text { TOTAL } \\
\text { (UG/L) }\end{array}$ & $\begin{array}{l}\text { ALPHA } \\
\text { BHC } \\
\text { TOTAL } \\
\text { (UG/L) }\end{array}$ & $\begin{array}{c}\text { BETA } \\
\text { BENZENE } \\
\text { HEXA- } \\
\text { CHLOR- } \\
\text { IDE } \\
\text { TOTAL } \\
\text { (UG/L) }\end{array}$ & $\begin{array}{c}\text { DELTA } \\
\text { BENZENE } \\
\text { HEXA- } \\
\text { CHLOR- } \\
\text { IDE } \\
\text { TOTAL } \\
\text { (UG/L) }\end{array}$ & $\begin{array}{l}\text { LINDANE } \\
\text { TOTAL } \\
\text { (UG/L) }\end{array}$ & $\begin{array}{c}\text { CHLOR - } \\
\text { DANE } \\
\text { CIS } \\
\text { WATER } \\
\text { WHOLE } \\
\text { TOTAL } \\
\text { (UG/L) }\end{array}$ & $\begin{array}{c}\text { CHLOR - } \\
\text { DANE } \\
\text { TRANS } \\
\text { WATER } \\
\text { WHOLE } \\
\text { TOTAL } \\
\text { (UG/L) }\end{array}$ \\
\hline FEB 1993 & & & & & & & & & & & \\
\hline $\begin{array}{l}24-24 \\
\text { APR }\end{array}$ & $<5.0$ & $<5.0$ & $<20.0$ & $<0.040$ & $<0.10$ & $<0.03$ & $<0.03$ & $<0.09$ & $<0.030$ & $<0.10$ & $<0.10$ \\
\hline $14 \cdot 14$ & $\cdots$ & $\cdots$ & $\cdots$ & 0.73 & 4.0 & 0.86 & 1.1 & 0.88 & 0.85 & $<0.10$ & $<0.10$ \\
\hline $\begin{array}{l}\text { APR } \\
\qquad 14 \cdot 14\end{array}$ & $<5.0$ & $<5.0$ & $<20.0$ & 0.63 & 3.5 & 0.75 & 0.84 & 0.88 & 0.75 & $<0.10$ & $<0.10$ \\
\hline
\end{tabular}

\begin{tabular}{|c|c|c|c|c|c|c|c|c|c|c|}
\hline DATE & $\begin{array}{l}\text { CHLOR - } \\
\text { DANE, } \\
\text { TOTAL } \\
\text { (UG/L) }\end{array}$ & $\begin{array}{c}\text { P, P' } \\
\text { DDD } \\
\text { TOTAL } \\
(U G / L)\end{array}$ & $\begin{array}{l}\text { P, } P^{\prime} \\
\text { DDE, } \\
\text { TOTAL } \\
(U G / L)\end{array}$ & $\begin{array}{l}\text { DI - } \\
\text { ELDRIN } \\
\text { TOTAL } \\
(U G / L)\end{array}$ & $\begin{array}{c}\text { ENDO- } \\
\text { SULFAN- } \\
\text { I } \\
\text { WATER } \\
\text { WHOLE } \\
\text { REC } \\
(U G / L)\end{array}$ & $\begin{array}{l}\text { ENDO- } \\
\text { SULFAN } \\
\text { BETA } \\
\text { TOTAL } \\
\text { (UG/L) }\end{array}$ & $\begin{array}{l}\text { ENDO- } \\
\text { SULFAN } \\
\text { SULFATE } \\
\text { TOTAL } \\
\text { (UG/L) }\end{array}$ & $\begin{array}{c}\text { ENDRIN } \\
\text { WATER } \\
\text { UNFLTRD } \\
\text { REC } \\
(U G / L)\end{array}$ & $\begin{array}{c}\text { ENDRIN } \\
\text { ALDE- } \\
\text { HYDE } \\
\text { TOTAL } \\
\text { (UG/L) }\end{array}$ & $\begin{array}{l}\text { HEPTA- } \\
\text { CHLOR, } \\
\text { TOTAL } \\
\text { (UG/L) }\end{array}$ \\
\hline \multicolumn{11}{|l|}{ FEB 1993} \\
\hline $24-24$ & $<0.1$ & $<0.10$ & $<0.04$ & $<0.020$ & $<0.10$ & $<0.04$ & $<0.60$ & $<0.060$ & $<0.20$ & $<0.030$ \\
\hline \multicolumn{11}{|l|}{ APR } \\
\hline $14-14$ & 0.2 & 4.2 & 1.6 & 1.5 & 1.4 & 1.6 & 4.8 & 1.70 & 3.4 & 0.81 \\
\hline \multicolumn{11}{|l|}{ APR } \\
\hline \multirow[t]{4}{*}{$14-14$} & 0.2 & 3.6 & 1.3 & 1.3 & 1.3 & 1.5 & 4.7 & 1.50 & 1.3 & 0.65 \\
\hline & HEPTA - & & AROCLOR & AROCLOR & AROCLOR & AROCLOR & AROCLOR & AROCLOR & AROCLOR & \\
\hline & CHLOR & TOX - & 1016 & 1221 & 1232 & 1242 & 1248 & 1254 & 1260 & DI - \\
\hline & EPOXIDE & APHENE, & PCB & PCB & PCB & PCB & PCB & PCB & $\mathrm{PCB}$ & AZINON, \\
\hline DATE & $\begin{array}{l}\text { TOTAL } \\
(\text { UG } / L)\end{array}$ & $\begin{array}{l}\text { TOTAL } \\
\text { (UG/L) }\end{array}$ & $\begin{array}{l}\text { TOTAL } \\
\text { (UG/L) }\end{array}$ & $\begin{array}{l}\text { TOTAL } \\
\text { (UG/L) }\end{array}$ & $\begin{array}{l}\text { TOTAL } \\
\text { (UG/L) }\end{array}$ & $\begin{array}{l}\text { TOTAL } \\
\text { (UG/L) }\end{array}$ & $\begin{array}{l}\text { TOTAL } \\
\text { (UG/L) }\end{array}$ & $\begin{array}{l}\text { TOTAL } \\
\text { (UG/L) }\end{array}$ & $\begin{array}{l}\text { TOTAL } \\
\text { (UG/L) }\end{array}$ & $\begin{array}{l}\text { TOTAL } \\
\text { (UG/L) }\end{array}$ \\
\hline \multicolumn{11}{|l|}{ FEB 1993} \\
\hline $24-24$ & $<0.80$ & $<2$ & $<0.1$ & $<1.0$ & $<0.1$ & $<0.1$ & $<0.1$ & $<0.1$ & $<0.1$ & $<0.05$ \\
\hline \multicolumn{11}{|l|}{ APR } \\
\hline $14-14$ & 0.80 & $<2$ & $<0.1$ & $<1.0$ & $<0.1$ & $<0.1$ & $<0.1$ & $<0.1$ & $<0.1$ & $\cdots$ \\
\hline \multicolumn{11}{|l|}{$A P R$} \\
\hline $14 \cdot 14$ & $<0.80$ & $<2$ & $<0.1$ & $<1.0$ & $<0.1$ & $<0.1$ & $<0.1$ & $<0.1$ & $<0.1$ & $\cdots$ \\
\hline
\end{tabular}




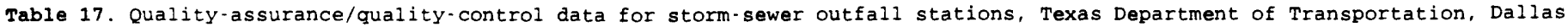
and Fort worth Districts, 1994

08048920 - DEER CREEK OUTFALL AT I-35W, FORT WORTH, TX (WY 1994)

$\begin{array}{ccc}\begin{array}{c}\text { TYPE } \\ \text { OF } \\ \text { QA/QC } \\ \text { SAMPLE }\end{array} & \text { DATE } & \text { TIME } \\ & & \\ & \text { MAY 1994 } & \\ \text { REP } & 09-09 & 1221 \\ & \text { MAY } \\ \text { LMS } & 09-09 & 1222 \\ & \text { AUG } \\ \text { FMS } & 31-31 & 1701 \\ & \text { AUG } & \\ \text { REP } & 31-31 & 1701 \\ & \text { AUG } \\ \text { TB } & 31-31 & 1703\end{array}$

\begin{tabular}{|c|c|c|c|c|c|c|c|c|c|c|}
\hline & $\begin{array}{l}\text { BERYL } \\
\text { LIUM, } \\
\text { TOTAL }\end{array}$ & $\begin{array}{l}\text { CADMIUM } \\
\text { WATER }\end{array}$ & $\begin{array}{l}\text { CHRO- } \\
\text { MIUM, } \\
\text { TOTAL }\end{array}$ & $\begin{array}{c}\text { COPPER, } \\
\text { TOTAL }\end{array}$ & $\begin{array}{l}\text { LEAD, } \\
\text { TOTAL }\end{array}$ & $\begin{array}{l}\text { MERCURY } \\
\text { TOTAL }\end{array}$ & $\begin{array}{l}\text { NICKEL, } \\
\text { TOTAL }\end{array}$ & SELE - & $\begin{array}{l}\text { SILVER, } \\
\text { TOTAL }\end{array}$ & $\begin{array}{l}\text { ZINC, } \\
\text { TOTAL }\end{array}$ \\
\hline $\begin{array}{l}\text { ARSENIC } \\
\text { TOTAL } \\
\text { (UG/L }\end{array}$ & $\begin{array}{l}\text { RECOV - } \\
\text { ERABLE } \\
\text { (UG / L }\end{array}$ & $\begin{array}{l}\text { UNFLTRD } \\
\text { TOTAL } \\
\text { (UG/L }\end{array}$ & $\begin{array}{l}\text { RECOV - } \\
\text { ERABLE } \\
\text { (UG / L }\end{array}$ & $\begin{array}{l}\text { RECOV - } \\
\text { ERABLE } \\
\text { (UG/L }\end{array}$ & $\begin{array}{l}\text { RECOV- } \\
\text { ERABLE } \\
(U G / L\end{array}$ & $\begin{array}{l}\text { RECOV- } \\
\text { ERABLE } \\
\text { (UG/L }\end{array}$ & $\begin{array}{l}\text { RECOV - } \\
\text { ERABLE } \\
\text { (UG /L }\end{array}$ & $\begin{array}{l}\text { NIUM, } \\
\text { TOTAL } \\
\text { (UG/L }\end{array}$ & $\begin{array}{l}\text { RECOV - } \\
\text { ERABLE } \\
\text { (UG/L }\end{array}$ & $\begin{array}{l}\text { RECOV - } \\
\text { ERABLE } \\
\text { (UG /L }\end{array}$ \\
\hline AS AS) & AS BE) & AS CD) & AS CR) & AS CU) & AS $\mathrm{PB})$ & AS HG) & AS NI) & AS SE) & AS AG) & AS $\mathrm{ZN}$ ) \\
\hline
\end{tabular}

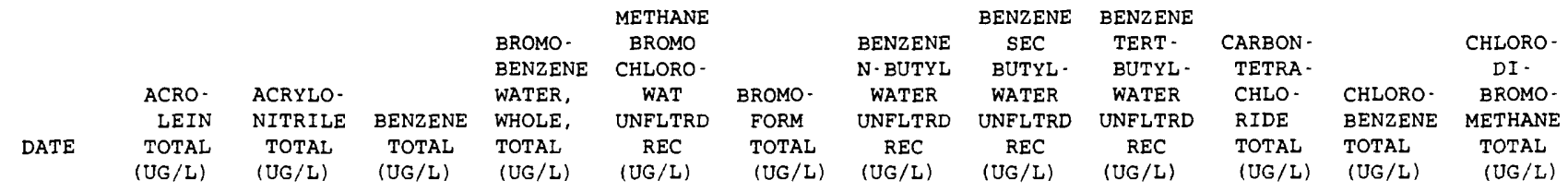

\begin{tabular}{|c|c|c|c|c|c|c|c|c|c|c|c|c|}
\hline $\begin{array}{c}\text { MAY } 1994 \\
09.09\end{array}$ & $\cdots$ & $\cdots$ & - & $\cdots$ & 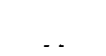 & $\cdots$ & - & & $\cdots$ & $\ldots$ & $\cdots$ & $\cdots$ \\
\hline \multicolumn{13}{|l|}{ MAY } \\
\hline $09-09$ & - & $\cdots$ & . & - & $\cdots$ & $\cdots$ & - & $\cdots$ & $\cdots$ & $\cdots$ & $\cdots$ & $\cdots$ \\
\hline \multicolumn{13}{|l|}{ AUG } \\
\hline $31-31$ & $<20.0$ & $<20.0$ & 6.30 & $<0.200$ & $<0.200$ & 8.60 & $<0.200$ & $<0.200$ & $<0.200$ & 6.00 & 6.60 & 8.40 \\
\hline \multicolumn{13}{|l|}{ AUG } \\
\hline $31-31$ & $\cdots$ & $\cdots$ & $\cdots$ & - & - & . & . & $\cdots$ & $\cdots$ & $\cdots$ & $\cdots$ & $\cdots$ \\
\hline \multicolumn{13}{|l|}{ AUG } \\
\hline $31 \cdot 31$ & $<20.0$ & $<20.0$ & $<0.200$ & $<0.200$ & $<0.200$ & $<0.200$ & $<0.200$ & $<0.200$ & $<0.200$ & $<0.200$ & $<0.200$ & $<0.200$ \\
\hline
\end{tabular}

\begin{tabular}{|c|c|c|c|c|c|c|c|c|c|c|c|c|}
\hline & & $\begin{array}{c}2- \\
\text { CHLORO- }\end{array}$ & & & $\begin{array}{c}\text { O- } \\
\text { CHLORO- }\end{array}$ & & $\begin{array}{l}\text { DIBROMO } \\
\text { CHLORO- }\end{array}$ & $\begin{array}{c}\text { DI- } \\
\text { BROMO- } \\
\text { METHANE }\end{array}$ & $\begin{array}{l}1,2- \\
\text { DIBROMO }\end{array}$ & DI - & & $\begin{array}{l}1,1-D I \\
\text { CHLORO- } \\
\text { PRO- }\end{array}$ \\
\hline & $\begin{array}{l}\text { CHLORO- } \\
\text { ETHANE }\end{array}$ & $\begin{array}{l}\text { VINYL- } \\
\text { ETHER }\end{array}$ & $\begin{array}{l}\text { CHLORO- } \\
\text { FORM }\end{array}$ & $\begin{array}{l}\text { CHLO- } \\
\text { RIDE }\end{array}$ & $\begin{array}{l}\text { WATER } \\
\text { WHOLE }\end{array}$ & $\begin{array}{l}\text { WATER } \\
\text { UNFLTRD }\end{array}$ & $\begin{array}{l}\text { WATER } \\
\text { WHOLE }\end{array}$ & $\begin{array}{l}\text { METHANE } \\
\text { WATER } \\
\text { WHOLE }\end{array}$ & $\begin{array}{c}\text { ETHANE } \\
\text { WATER } \\
\text { WHOLE }\end{array}$ & $\begin{array}{l}\text { CHLORO- } \\
\text { BROMO- } \\
\text { METHANE }\end{array}$ & $\begin{array}{l}\text { CHLORO- } \\
\text { ETHYL- } \\
\text { ENE }\end{array}$ & $\begin{array}{l}\text { PRO- } \\
\text { PENE, } \\
\text { WAT, WH }\end{array}$ \\
\hline & $\begin{array}{l}\text { TOTAL } \\
\text { (UG/L) }\end{array}$ & $\begin{array}{l}\text { TOTAL } \\
\text { (UG/L) }\end{array}$ & $\begin{array}{l}\text { TOTAL } \\
\text { (UG/L) }\end{array}$ & $\begin{array}{l}\text { TOTAL } \\
\text { (UG/L) }\end{array}$ & $\begin{array}{l}\text { TOTAL } \\
\text { (UG/L) }\end{array}$ & $\begin{array}{c}\text { REC } \\
(U G / L)\end{array}$ & $\begin{array}{l}\text { TOT.REC } \\
(\mathrm{UG} / \mathrm{L})\end{array}$ & $\begin{array}{l}\text { RECOVER } \\
\text { (UG/L) }\end{array}$ & $\begin{array}{l}\text { TOTAL } \\
\text { (UG/L) }\end{array}$ & $\begin{array}{l}\text { TOTAL } \\
\text { (UG/L) }\end{array}$ & $\begin{array}{l}\text { TOTAL } \\
\text { (UG/L) }\end{array}$ & $\begin{array}{l}\text { TOTAL } \\
\text { (UG/L) }\end{array}$ \\
\hline
\end{tabular}

\begin{tabular}{|c|c|c|c|c|c|c|c|c|c|c|c|c|}
\hline MAY 1994 & & & & & & & & & & & & \\
\hline $09-09$ & $\cdots$ & $\cdots$ & $\cdots$ & $\cdots$ & $\cdots$ & $\cdots$ & $\cdots$ & $\cdots$ & $\cdots$ & $\cdots$ & - & $\cdots$ \\
\hline $\begin{array}{l}\text { MAY } \\
09-09\end{array}$ & & & & & & & & & & & & \\
\hline $\begin{array}{l}09-09 \\
A U G\end{array}$ & $\cdots$ & $\cdots$ & $\cdots$ & $\cdots$ & $\cdots$ & $\cdots$ & $\cdots$ & $\cdots$ & $\cdots$ & $\cdots$ & $\cdots$ & $\cdots$ \\
\hline $\begin{array}{l}\text { AUG } \\
31 \cdot 31\end{array}$ & 6.70 & $<100$ & 9.70 & $<360$ & $<0-200$ & $\leq 0,200$ & $<100$ & $=030$ & 5030 & 780 & 40 & \\
\hline AUG & & & & & & & & & & & & \\
\hline $\begin{array}{l}31-31 \\
\text { AUG }\end{array}$ & $\cdots$ & $\cdots$ & $-\cdot$ & $\cdots$ & $\cdots$ & $\cdots$ & $\cdots$ & $\cdots$ & $\cdots$ & $-\cdot$ & $\cdots$ & $\cdots$ \\
\hline $31-31$ & $<0.200$ & $<1.00$ & $<0.200$ & $<0.200$ & $<0,200$ & $<0,200$ & $<100$ & 50200 & 10 & 80 & $<0200$ & $<0.200$ \\
\hline
\end{tabular}




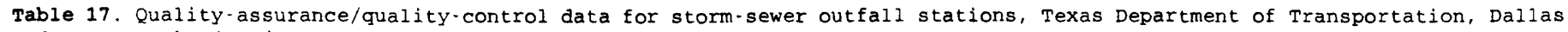
and Fort worth Districts, 1994-Continued

08048920 - DEER CREEK OUTFALL AT I-35W, FORT WORTH, TX (WY 1994)-Continued

\begin{tabular}{|c|c|c|c|c|c|c|c|c|c|c|c|c|}
\hline & $\begin{array}{c}\text { BENZENE } \\
0-D I \text { - }\end{array}$ & $\begin{array}{l}\text { BENZENE } \\
1,3-\mathrm{DI} \text { - }\end{array}$ & BENZENE & $\begin{array}{c}\text { DI - } \\
\text { CHLORO- }\end{array}$ & & & $\begin{array}{l}1,2 \cdot \mathrm{DI} \text { - } \\
\text { PHENYL - }\end{array}$ & $\begin{array}{c}\text { CIS - } 1,2- \\
\text { DI - }\end{array}$ & 1,2 & & $1,3$. & $\begin{array}{l}2,2-D I \\
\text { CHLORO. }\end{array}$ \\
\hline & CHLORO - & CHLORO- & CHLORO - & $\mathrm{DI}$ & $1,1-D I$ & $1,2 \cdot \mathrm{DI}=$ & HYDRA - & CHLORO- & TRANSDI & $1,2 \cdot \mathrm{DI}-$ & CHLORO- & PRO - \\
\hline & WATER & WATER & WATER & FLUORO- & CHLORO - & CHLORO- & ZINE & ETHENE & CHLORO - & CHLORO - & PROPANE & PANE \\
\hline & UNFLTRD & UNFLTRD & UNFLTRD & METHANE & ETHANE & ETHANE & WATER & WATER & ETHENE & PROPANE & WAT. WH & WAT, WH \\
\hline ATE & $\begin{array}{c}\text { REC } \\
(U G / L)\end{array}$ & $\begin{array}{c}\text { REC } \\
(\mathrm{UG} / \mathrm{L})\end{array}$ & $\begin{array}{c}\text { REC } \\
(U G / L)\end{array}$ & $\begin{array}{l}\text { TOTAL } \\
\text { (UG/L) }\end{array}$ & $\begin{array}{l}\text { TOTAL } \\
\text { (UG/L) }\end{array}$ & $\begin{array}{l}\text { TOTAL } \\
\text { (UG/L) }\end{array}$ & $\begin{array}{l}\text { TOT . REC } \\
\text { (UG/L) }\end{array}$ & $\begin{array}{l}\text { TOTAL } \\
(U G / L)\end{array}$ & $\begin{array}{l}\text { TOTAL } \\
\text { (UG/L) }\end{array}$ & $\begin{array}{l}\text { TOTAL } \\
\text { (UG/L) }\end{array}$ & $\begin{array}{l}\text { TOTAL } \\
\text { (UG/L) }\end{array}$ & $\begin{array}{l}\text { TOTAL } \\
\text { (UG/L) }\end{array}$ \\
\hline
\end{tabular}

MAY 1994

09.09

MAY

$09 \cdot 09$

AUG

AUG

$31 \cdot 31$

AUG

$31-31$

$<0.200$

-

$<0.200$

$<0.200$

$<0.200$

$<0.200$

$<0.200$

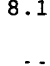

26.0

6.10

5.30

$<0.200$

-

$<0.200$

$<0.200$

$<0.200$

$<0.200$

$\begin{array}{ccc} & \text { CIS } & \text { TRANS - } \\ & 1,3-\mathrm{DI}- & 1,3-\mathrm{DI}- \\ & \text { CHLORO- } & \text { CHLORO- } \\ & \text { PROPENE } & \text { PROPENE } \\ \text { DATE } & \text { TOTAL } & \text { TOTAL } \\ & \text { (UG } / L) & (U G / L)\end{array}$

$\begin{array}{cccc} & & \text { ISO- } & \text { P-ISO- } \\ & \text { HEXA- } & \text { PROPYL- } & \text { PROPYL- } \\ & \text { CHLORO- } & \text { BENZENE } & \text { TOLUENE } \\ \text { ETHYL- } & \text { BUT- } & \text { WATER } & \text { WATER } \\ \text { BENZENE } & \text { ADIENE } & \text { WHOLE } & \text { WHOLE } \\ \text { TOTAL } & \text { TOTAL } & \text { REC } & \text { REC } \\ \text { (UG/L) } & \text { (UG/L) } & \text { (UG/L) } & \text { (UG/L) }\end{array}$

METHYL-
BROMIDE
TOTAL
(UG/L)

METHYL-
ENE
CHLO-
RIDE
TOTAL
(UG/L)

METHYI

$$
\text { TERT - }
$$

BUTYL

ETHER

WAT UNF
REC

(UG/L)

MAY 1994
$09-09$

$09-09$
MAY
$09-09$

AUG

$31-31$

AUG

$31 \cdot 31$

AUG

$31-31$

$<0.200<0.200$

$<0.200$

$<0.200$

$<0.200<0.200$

$<0.200$

$<0.200$

$<0.200$

$<0.200$

$<0.200$

$<0.200$

$<0.200$

NAPHTH
ALENE
TOTAL
(UG/L)

BENZENE

N-PROPY

WATER

UNFLTRD STYRENE

REC TOTAI

(UG/L) (UG/L)

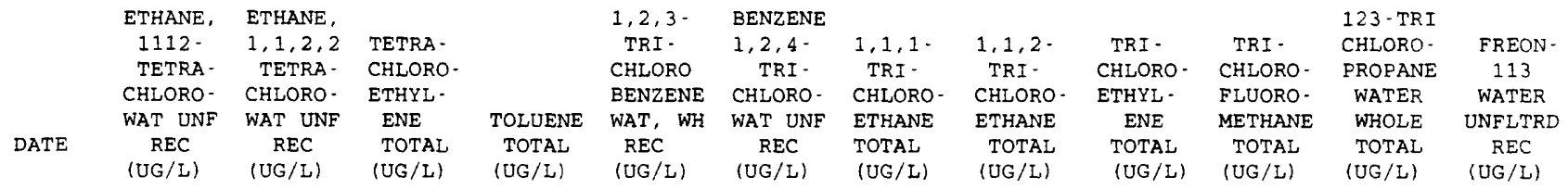

MAY 1994
09.09

MAY

$09-09$

AUG

$\begin{array}{llllll} & \cdots & \cdots & - & \ldots & \cdots\end{array}$

AUG

$31-31$

AUG

$31.31<0.200<0.200<0.200<0.200<0.200<0.200<0.200<0.200<0.200<0.200<0.200<0.200$ 


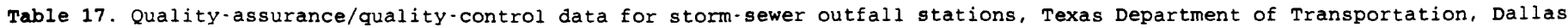
and Fort worth Districts, 1994-Continued

08048920 - DEER CREEK OUTFALL AT I-35W, FORT WORTH, TX (WY 1994)-Continued

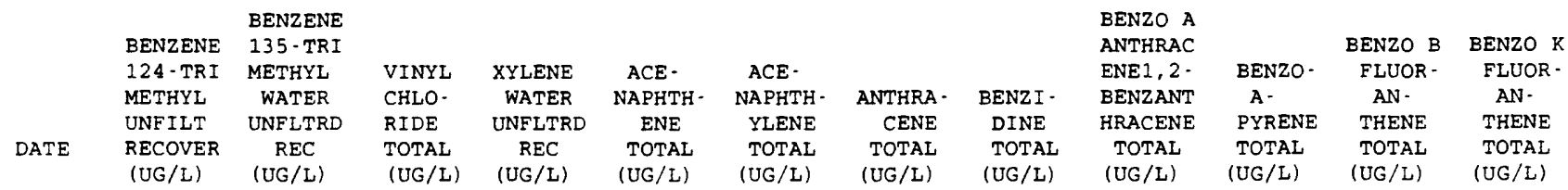

MAY 1994
$09-09$

MAY

09.09

AUG

$31 \cdot 31$

AUG

$31 \cdot 31$

AUG

$<0.200$

$<0.200$

$<0.200$

$<0.200$

13.0

14.0

12.0

$<40.0$

13.0

14.0

13.0

13.0

$31 \cdot 31$

$<0.200$

$<0.200$

$<0.200$

$<0.200$

BENZOGH 4

I PERYI BROMO

ENE1,12 PHENYL

- BENZOP PHENYL

TOTAL TOTAL

(UG/L)

$\begin{array}{ll} & \text { BIS } \\ \text { N-BUTYL } & \text { (2- } \\ \text { BENZYL } & \text { CHLORO- } \\ \text { PHTHAL- } & \text { ETHOXY) } \\ \text { ATE } & \text { METHANE } \\ \text { TOTAL } & \text { TOTAL } \\ (\text { UG } / L) & \text { (UG/L) }\end{array}$

BIS 2

CHLORO- CHLORO-

ETHYL ISO-

ETHER PROPYL)

UNFLTRD ETHER

RECOVER TOTAL

PARA -

CHLORO -

CHLORO-

CRESOL

TOTAL

(UG/L)

THALENE

TOTAL

(UG/L)

2 -
CHLORO-
PHENOL
TOTAL
(UG/L)

CHLORO -

PHENYL

PHENYL

ETHER

TOTAL

(UG/L)

CHRY

SENE

TOTAL

(UG/L)

$1,2,5,6$ DIBENZ -

ANTHRA -

CENE

TOTAL

(UG/L)

\section{MAY 1994 \\ $09-09$}

MAY

$09 \cdot 09$

AUG

$31-31$

AUG

$31 \cdot 31$

AUG

$31 \cdot 31$

15.0

25.0

14.0

16.0

28.0

22.

250

12.0

39.0

12.0

14.0

19.0

$3,3^{\prime}=$

DI -

CHLORO- 2,4 -DI.

BENZI - CHLORO-

DINE PHENO

DATE

TOTAL

TOTAL TOTAL
(UG/L) (UG/L)

DI -

METHYL 2,4-DI -

$D I-N-$

4,6

BUTYL DINITRO

PHTHAL - ORTHO-

CRESOL

PHENOL

TOTAL

(UG/L)

(UG/L)

$2,4, \cdot$ DI . NITRO PHENOL (UOTAL
MAY 1994

$09-09$

MAY

$09-09$

AUG

$31.31<20.0$

AUG

$31-31$

AUG

$31 \cdot 31$
41.

18.0

16.0

28.0

$17.0 \quad 370$

350

18.

17.0

$17.0 \quad 17.0$ 


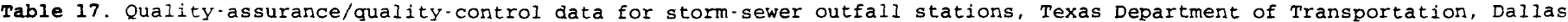
and Fort worth Districts, 1994-Continued

\section{0 - DEER CREEK OUTFALL AT I-35W, FORT WORTH, TX (WY 1994)-Continued}

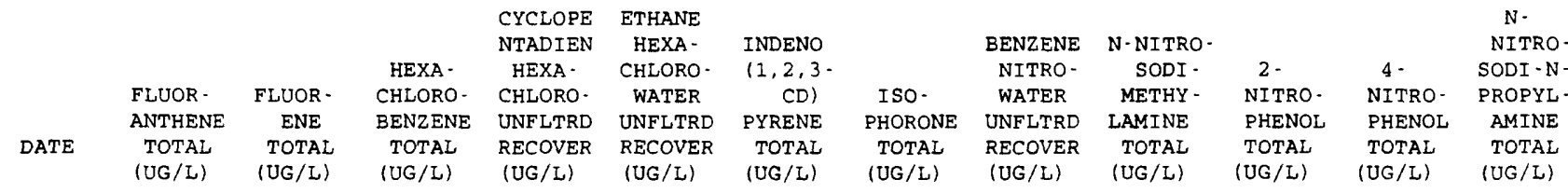

$\begin{array}{cccc}\text { MAY } 1994 & & & \\ 09.09 & \ldots & \ldots & \\ \text { MAY } & & & \\ 09.09 & \ldots & \ldots & \\ \text { AUG } & & & \\ 31.31 & 13.0 & 14.0 & 13 . \\ \text { AUG } & & & \\ 31-31 & \ldots & \ldots & \\ \text { AUG } & & & \\ 31.31 & \ldots & \ldots & \end{array}$

\begin{tabular}{|c|c|c|c|c|c|c|c|c|c|c|c|}
\hline DATE & $\begin{array}{l}\text { N-NITRO- } \\
\text { SODI - } \\
\text { PHENY - } \\
\text { LAMINE } \\
\text { TOTAL } \\
\text { (UG } / L \text { ) }\end{array}$ & $\begin{array}{l}\text { PENTA- } \\
\text { CHLORO- } \\
\text { PHENOL } \\
\text { TOTAL } \\
\text { (UG/L) }\end{array}$ & $\begin{array}{l}\text { PHENAN - } \\
\text { THRENE } \\
\text { TOTAL } \\
\text { (UG/L) }\end{array}$ & $\begin{array}{l}\text { PHENOL } \\
\text { (C6H- } \\
5 O H) \\
\text { TOTAL } \\
\text { (UG/L) }\end{array}$ & $\begin{array}{c}\text { PYRENE } \\
\text { TOTAL } \\
(\mathrm{UG} / \mathrm{L})\end{array}$ & $\begin{array}{c}2,4,6- \\
\text { TRI - } \\
\text { CHLORO- } \\
\text { PHENOL } \\
\text { TOTAL } \\
\text { (UG/L) }\end{array}$ & $\begin{array}{l}\text { ALDRIN, } \\
\text { TOTAL } \\
\text { (UG/L) }\end{array}$ & $\begin{array}{c}\text { P, } P^{\prime} \\
\text { DDT, } \\
\text { TOTAL } \\
\text { (UG/L) }\end{array}$ & $\begin{array}{l}\text { ALPHA } \\
\text { BHC } \\
\text { TOTAL } \\
\text { (UG/L) }\end{array}$ & $\begin{array}{l}\text { BENZENE } \\
\text { HEXA- } \\
\text { CHLOR- } \\
\text { IDE } \\
\text { TOTAL } \\
\text { (UG/L) }\end{array}$ & $\begin{array}{c}\text { BENZENE } \\
\text { HEXA - } \\
\text { CHLOR - } \\
\text { IDE } \\
\text { TOTAL } \\
\text { (UG/L) }\end{array}$ \\
\hline MAY 1994 & & & & & & & & & & & \\
\hline $\begin{array}{l}09.09 \\
\text { MAY }\end{array}$ & $\cdots$ & $\cdots$ & $\cdots$ & $\cdots$ & $\cdots$ & $\cdots$ & $<0.040$ & $<0.100$ & $<0.030$ & $<0.030$ & $<0.090$ \\
\hline $\begin{array}{l}09.09 \\
\text { AUG }\end{array}$ & $\cdots$ & $\cdots$ & $\cdots$ & $\cdots$ & $\cdots$ & $\cdots$ & 2.50 & 15.0 & 2.80 & 3.10 & 3.30 \\
\hline $\begin{array}{l}31 \cdot 31 \\
\text { AUG }\end{array}$ & 14.0 & 290 & 17.0 & 19.0 & 13.0 & 130 & - & $\cdots$ & - & - & $\cdots$ \\
\hline AUG $31-31$ & $\cdots$ & $\cdots$ & $\cdots$ & $\cdots$ & $\cdots$ & $\cdots$ & $<0.040$ & $<0.100$ & $<0.030$ & $<0.030$ & $<0.090$ \\
\hline $31-31$ & $\cdots$ & $\cdots$ & $\cdots$ & $\cdots$ & $\cdots$ & $\cdots$ & $\cdots$ & $\cdots$ & $\cdots$ & $\cdots$ & $\cdots$ \\
\hline & & $\begin{array}{l}\text { CHLOR - } \\
\text { DANE } \\
\text { CIS } \\
\text { WATER }\end{array}$ & $\begin{array}{l}\text { CHLOR - } \\
\text { DANE } \\
\text { TRANS } \\
\text { WATER }\end{array}$ & $\begin{array}{l}\text { CHLOR - } \\
\text { DANE, } \\
\text { TECH - }\end{array}$ & $P, P^{\prime}$ & $P, P^{\prime}$ & $D I-$ & $\begin{array}{c}\text { ENDO- } \\
\text { SULFAN- } \\
\text { I } \\
\text { WATER }\end{array}$ & $\begin{array}{l}\text { ENDO- } \\
\text { SULFAN }\end{array}$ & $\begin{array}{l}\text { ENDO- } \\
\text { SULFAN }\end{array}$ & $\begin{array}{r}\text { ENDRIN } \\
\text { WATER }\end{array}$ \\
\hline DATE & $\begin{array}{l}\text { LINDANE } \\
\text { TOTAL } \\
\text { (UG } / L \text { ) }\end{array}$ & $\begin{array}{l}\text { WHOLE } \\
\text { TOTAL } \\
\text { (UG/L) }\end{array}$ & $\begin{array}{l}\text { WHOLE } \\
\text { TOTAL } \\
\text { (UG/L) }\end{array}$ & $\begin{array}{l}\text { NICAL } \\
\text { TOTAL } \\
\text { (UG/L) }\end{array}$ & $\begin{array}{c}\text { DDD, } \\
\text { TOTAL } \\
\text { (UG/L) }\end{array}$ & $\begin{array}{l}\text { DDE, } \\
\text { TOTAL } \\
\text { (UG/L) }\end{array}$ & $\begin{array}{l}\text { ELDRIN } \\
\text { TOTAL } \\
\text { (UG/L) }\end{array}$ & $\begin{array}{c}\text { WHOLE } \\
\text { REC } \\
\text { (UG/L) }\end{array}$ & $\begin{array}{c}\text { II } \\
\text { TOTAL } \\
\text { (UG/L) }\end{array}$ & $\begin{array}{l}\text { SULFATE } \\
\text { TOTAL } \\
\text { (UG/L) }\end{array}$ & $\begin{array}{l}\text { UNFLTRD } \\
\text { REC } \\
(U G / L)\end{array}$ \\
\hline $\begin{array}{c}\text { MAY } 1994 \\
09-09\end{array}$ & $<0.030$ & $<0,100$ & & & & & & & & $<0 \quad 600$ & $<0 \quad 060$ \\
\hline MAY & $<0.030$ & $<0.100$ & $<0.100$ & $<0.100$ & $<0.100$ & $<0.040$ & $<0.0<0$ & $<0.100$ & $<0.040$ & $<0.600$ & $<0.060$ \\
\hline $\begin{array}{l}09-09 \\
\text { AUG }\end{array}$ & 2.80 & $<0.100$ & $<0.100$ & $<0.100$ & 18.0 & 6.40 & 5.90 & 4.30 & 5.10 & 18.0 & 5.20 \\
\hline AUG $^{31-31}$ & $\cdots$ & $\cdots$ & $\cdots$ & $\cdots$ & $\cdots$ & $\cdots$ & $\cdots$ & $\cdots$ & $\cdots$ & $\cdots$ & - \\
\hline $31 \cdot 31$ & $<0.030$ & $<0.100$ & $<0.100$ & $<0.100$ & $<0.100$ & $<0.040$ & $<0.020$ & $<0.100$ & $<0.040$ & $<0.600$ & $<0.060$ \\
\hline AUG & & & & & & & & & & & \\
\hline $31-31$ & $\cdots$ & $\cdots$ & $\cdots$ & $\cdots$ & $\cdots$ & $\cdots$ & $\cdots$ & $\cdots$ & $\cdots$ & $\cdots$ & $\cdots$ \\
\hline
\end{tabular}




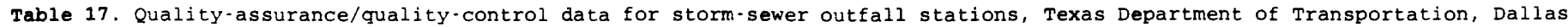
and Fort worth Districts, 1994-Continued

08048920 - DEER CREEK OUTFALL AT I-35W, FORT WORTH, TX (WY 1994)-Continued

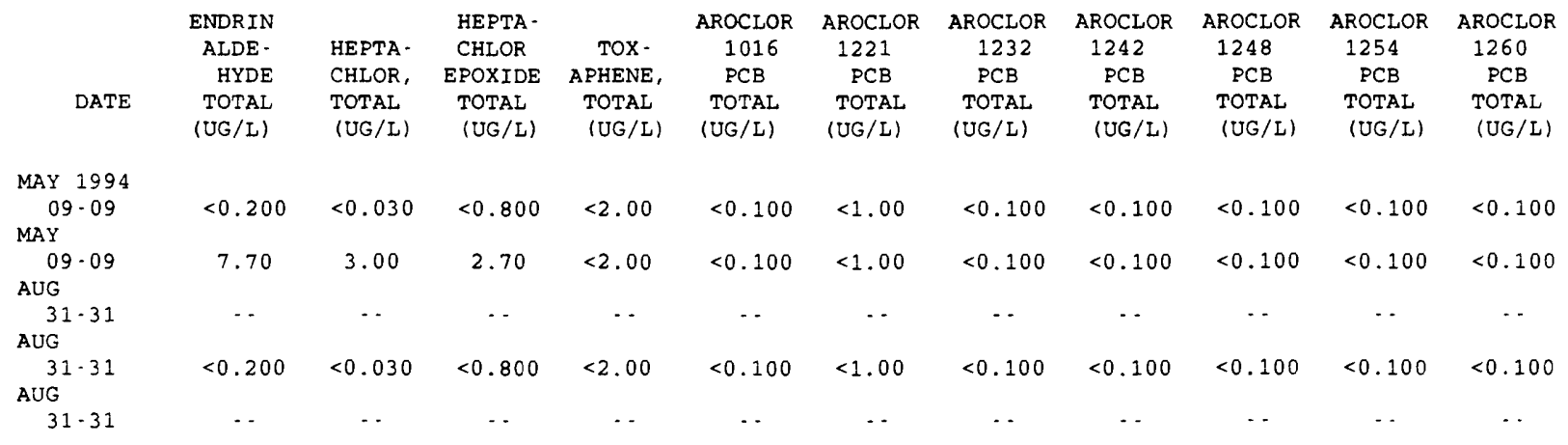

08049860 - MOUNTAIN CREEK OUTFALL AT I-20, DUNCANVILLE, TX (WY 1994)

TYPE

$O F$

$Q A / Q C$

SAMPLE

$\mathrm{EB}$

REP
DATE TIME

APR 1994

06... 1200

MAY

$09-09 \quad 1231$

$\begin{array}{ccc} & \text { NITRO- } & \\ \text { NITRO- } & \text { GEN,AM- } & \\ \text { GEN, } & \text { MONIA + } & \text { PHOS- } \\ \text { NO2+NO3 } & \text { ORGANIC } & \text { PHORUS } \\ \text { TOTAL } & \text { TOTAL } & \text { TOTAL } \\ \text { (MG/L } & \text { (MG/L } & \text { (MG/L } \\ \text { AS N) } & \text { AS N) } & \text { AS P) }\end{array}$

$\cdots$
0.180

$\cdots$

1.1

0.080

-

$<0.010$

$<5.00$

$<5.00$

$<5.00$

$<5.00$

BENZENE BENZENE BENZENE 1,2-DI.

O-DI - 1,3.DI- 1,4.DI- PHENYI

CHLORO- CHLORO-

WATER WATER WATER HYDRA

REC

REC

(UG/L)

(UG/L)

WATER

TOT. REC
(UG $/ \mathrm{L}$ )

HEXA-

CHLORO-

BUT -

ADIENE

TOTAL

(UG/L)

$<5.0$

.
.

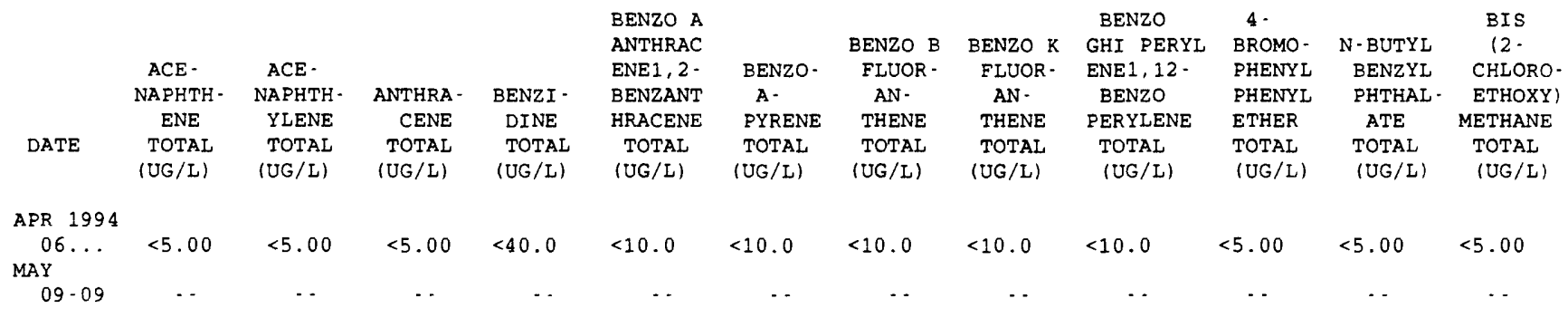

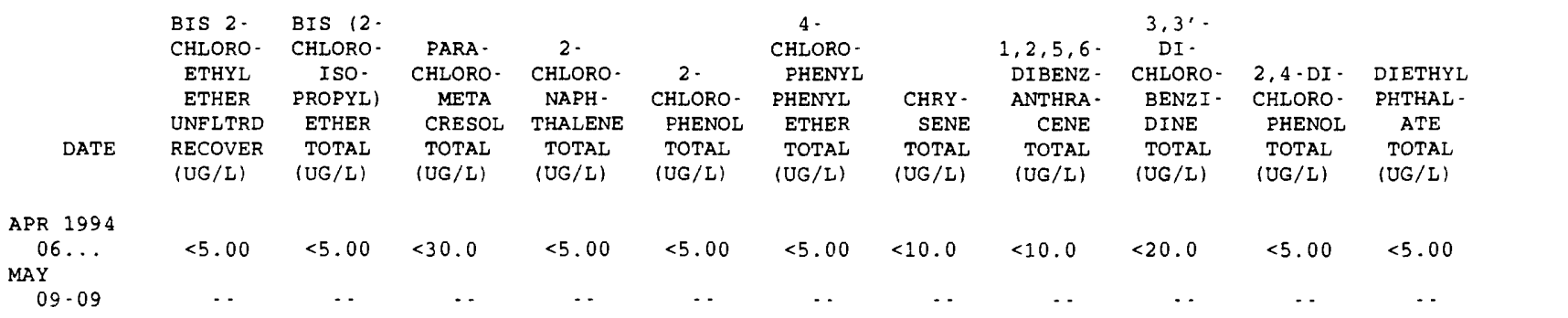




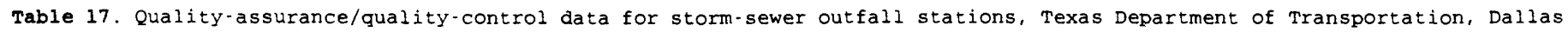
and Fort worth Districts, 1994-Continued

08049860 - MOUNTAIN CREEK OUTFALI AT I-20, DUNCANVILLE, TX (WY 1994)-Continued

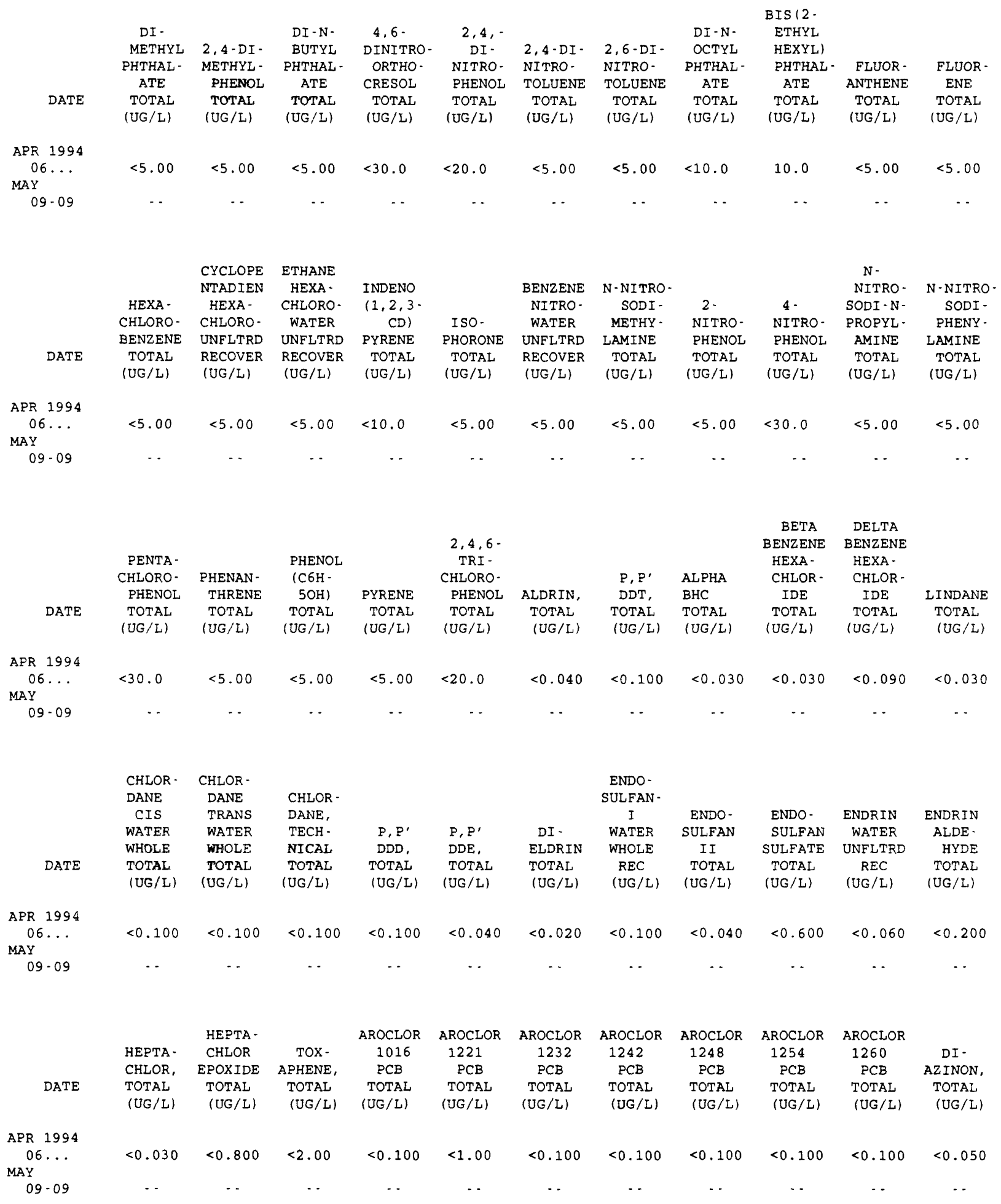




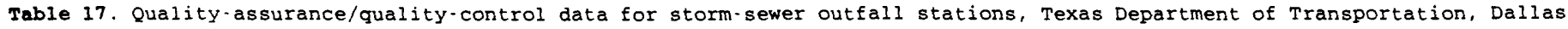
and Fort Worth Districts, 1994-Continued

08049950 - FISH CREEK OUTFALL AT I-20 ARLINGTON, TX (WY 1994)

TYPE

$O F$

$\mathrm{QA} / \mathrm{QC}$

SAMPLE

REP

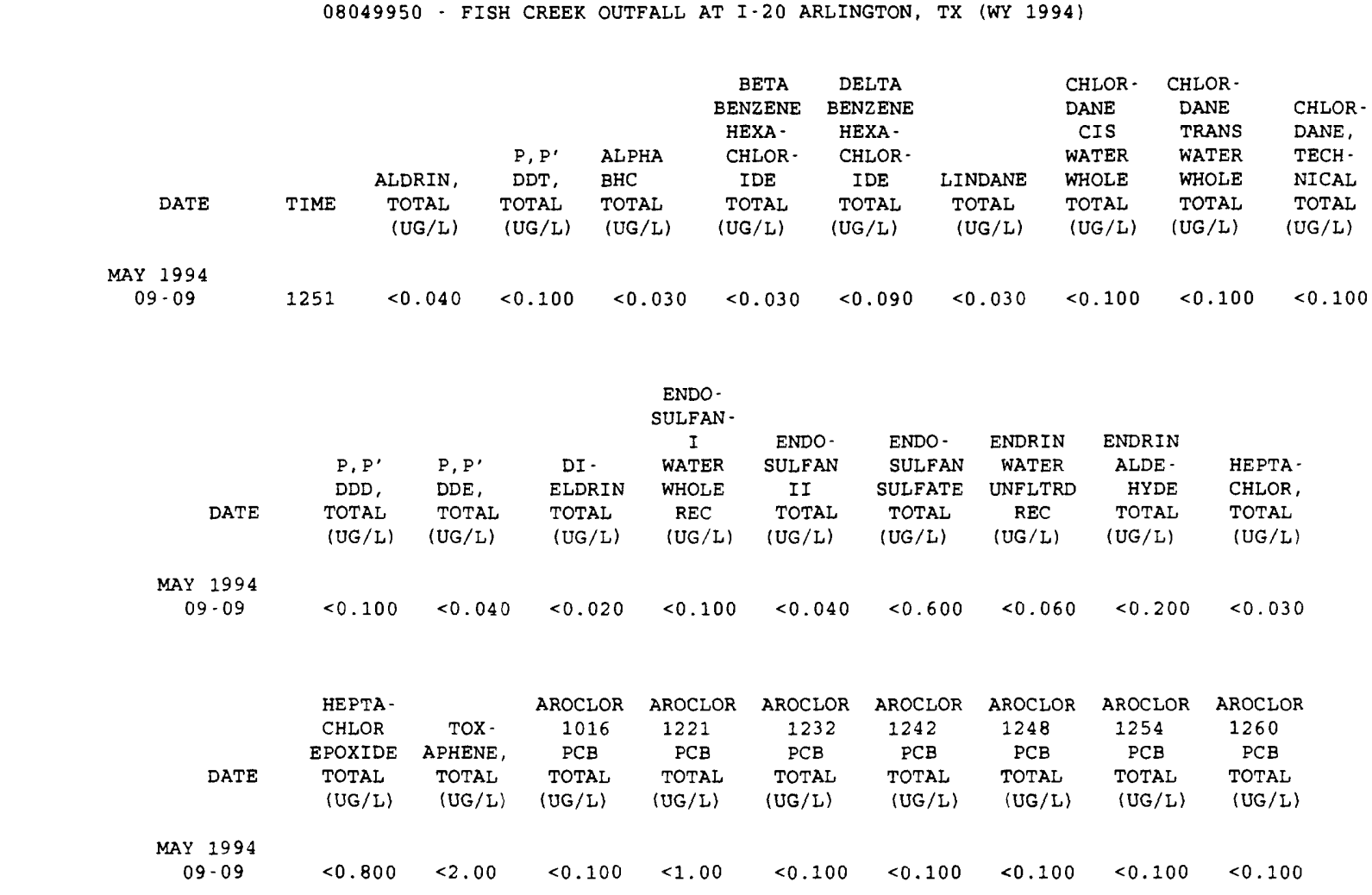

08055690 - BACHMAN BRANCH OUTFALL AT I-635, DALLAS, TX (WY 1994)

TYPE
OF

$Q A / Q C$

AMPLE

REP

APR 1994
$11-11$

BENZENE BENZENE BENZENE 1,2-DI-

O-DI - 1,3-DI- 1,4-DI- PHENYL - HEXA-

CHLORO- CHLORO- CHLORO- HYDRA- CHLORO-

UNFLTRD UNFLTRD UNFLTRD WATER ADIENE

REC REC

REC TOT.REC TOTAL

(UG/L)

(UG/L)

(UG/L)

(UG/L)

$\begin{array}{cc} & \text { BENZENE } \\ & 1,2,4- \\ & \text { TRI - } \\ \text { NAPHTH - } & \text { CHLORO- } \\ \text { ALENE } & \text { WAT UNF } \\ \text { TOTAL } & \text { REC } \\ \text { (UG } & \text { (UT) }\end{array}$

$\mathrm{ACE}$ -

NAPHTH - NAPHTH - ANTHRA - BENZI -

TOTAL TOTAL

(UG/L)

(UG/L)

(UG/L)

(UG/L)

CENE DINE

(UG/L) (UG/L)

1321

$<5.00<5.00$

$<5.00$

$<5.00<5.00$

$<5.00$

$<5.00$

$<5.00$

$<5.00$

$<5.00<40.0$

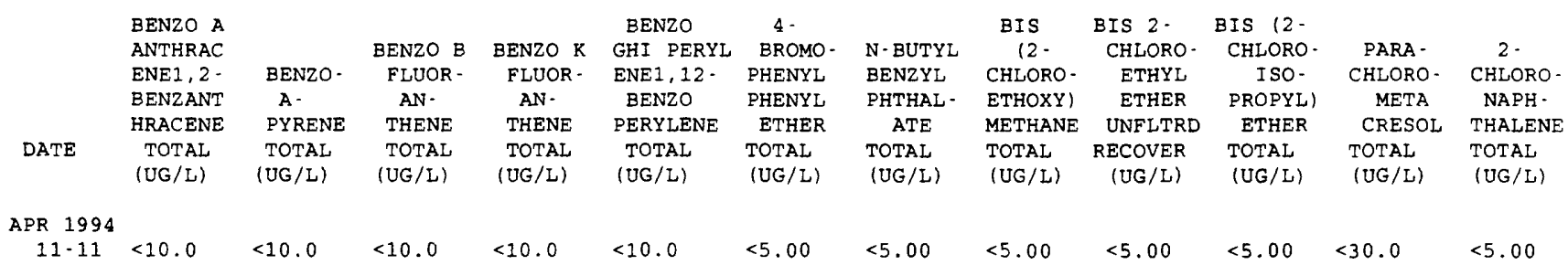




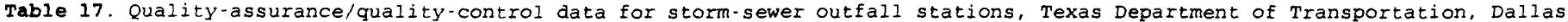
and Fort worth Districts, 1994-Continued

08055690 - BACHMAN BRANCH OUTFALL AT I-635, DALLAS, TX (WY 1994)-Continued

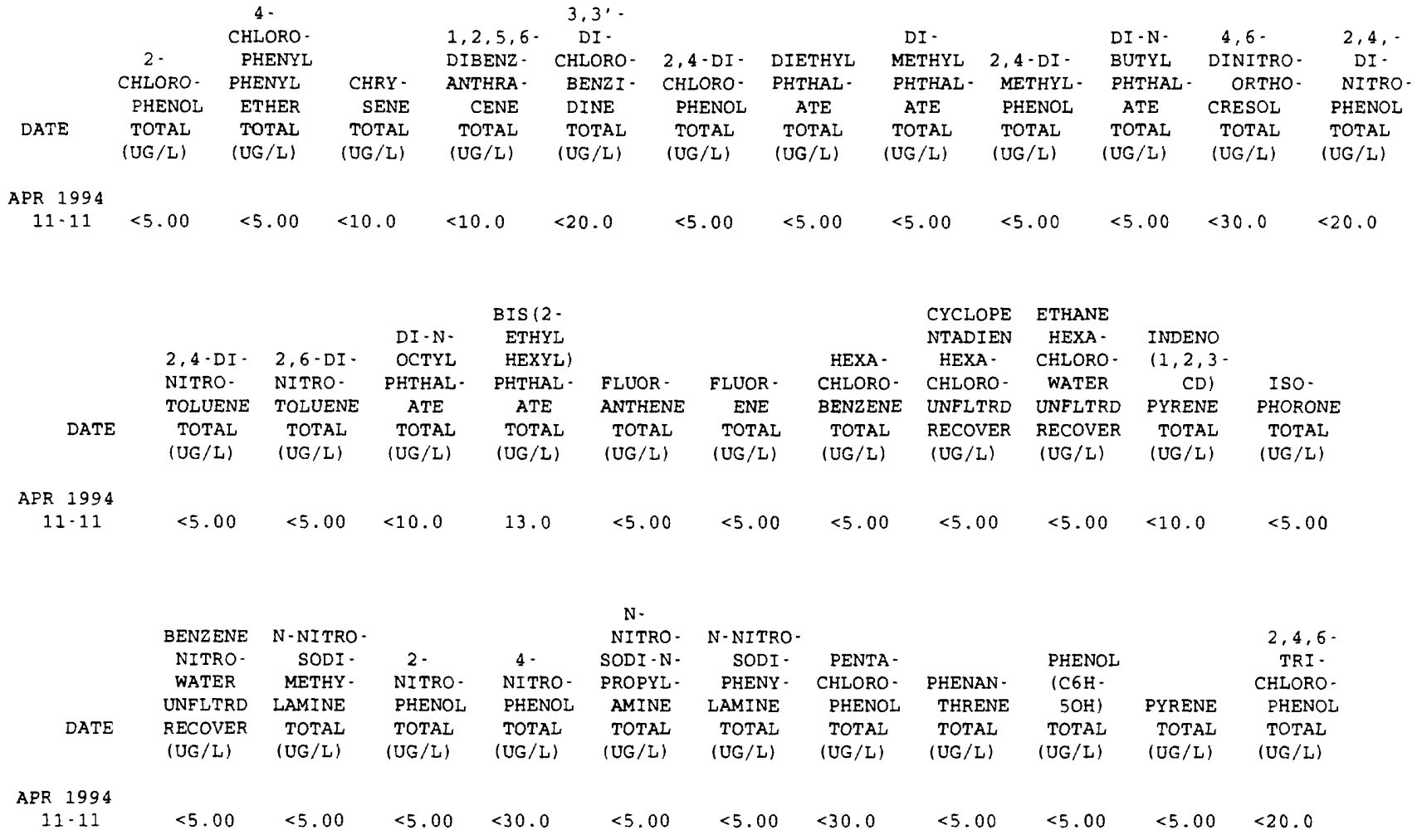

